\title{
Untersuchungen zur Struktur und Sukzession der saproxylen Käferfauna (Coleoptera) an Eichen- und Buchentotholz
}

\author{
Dissertation \\ zur Erlangung des Doktorgrades \\ der Fakultät für Forstwissenschaften und Waldökologie \\ der Georg-August-Universität Göttingen
}

vorgelegt von Norbert Menke

geboren in Emstek

Göttingen 2006 
Dieses Dokument wurde elektronisch veröffentlicht unter der Internetadresse

http://webdoc.sub.gwdg.de/diss/2006/menke/

D7

1. Berichterstatter: Prof. Dr. W. Sanders

2. Berichterstatter: Prof. Dr. R. Bürger-Arndt

Tag der mündlichen Prüfung: 20.01.2006 




\section{Abstract}

A study of structure and succession of saproxylic beetle fauna (Coleoptera) living in decaying oak and beech wood.

Decaying wood is a major source of biodiversity in forest ecosystems. It accounts for a large portion of the forest dwelling fauna and can be regarded as a functional important component of woodlands. In the past, forest cover has decreased dramatically in Europe. In contrast to virgin forests both the amount and structure of dead wood has been affected negatively by intensive forest management practices. As a consequence many dead wood associated species have become threatened. Despite the great importance of woody debris for the maintenance of a species rich fauna, little is known about their habitat requirements and the succession of species assemblages with ongoing degradation.

The main aims of this work were to get insight into 1 . the beetle species richness of an old forest reserve in the northwestern Germany using emergence and flight traps 2 . the community structure of dead wood associated beetle fauna on decaying snags and logs of oak and beech wood 3. their succession with advancing decay 4. the meaning of habitat qualities (e.g. tree species, bark cover, position, drill resistance) and 5. the phenology of flying and emerging of saproxylic beetles.

Due to habitat continuity and the high amount and structural diversity of woody debris, the study site harbours an uncommon species rich beetle fauna. Most of them were captured in low density wheras only few occured in high abundance. The association of saproxylic beetle species is variable to a high degree, even at very small scale. The occurence of species is largly influenced by habitat properties. Many species show clear preferences whereas others are using a wide range of substrate qualities. Beetle associations differ markedly between logs and snags and oak and beech wood provide habitat for different species communities. As wood decomposes, it is colonized by a succession of beetle assemblages. Differences in associations between tree species decreased with increasing decay stage.

Key words: Coarse woody debris, oak, beech, Coleoptera, saproxylic beetle diversity, succession, habitat preferences 



\section{Inhaltsverzeichnis}

1. Einleitung............................................................................................................................... 1

2. Das Untersuchungsgebiet .............................................................................................. 7

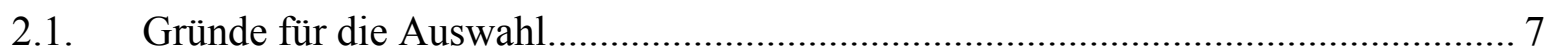

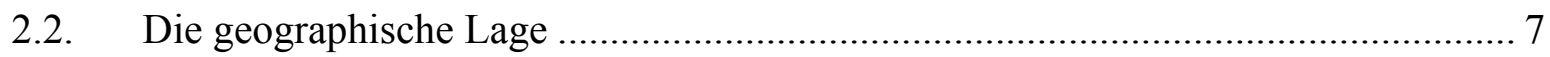

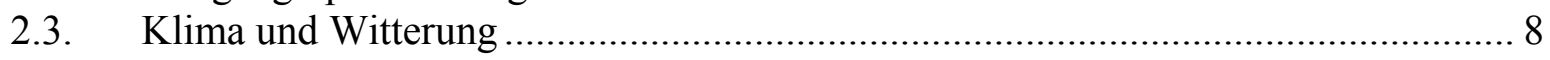

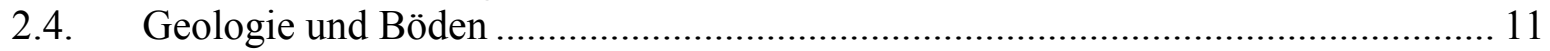

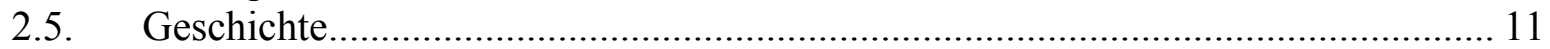

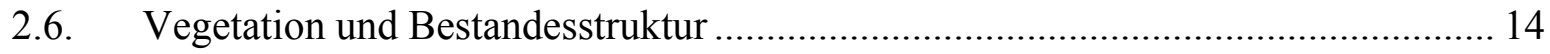

3. Untersuchungs- und Auswertungsmethoden ............................................................... 21

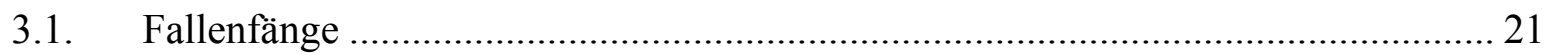

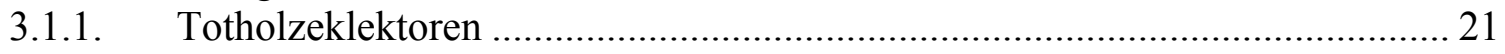

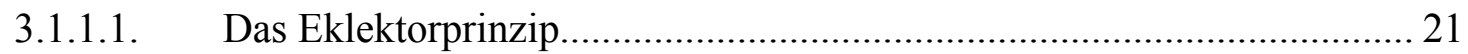

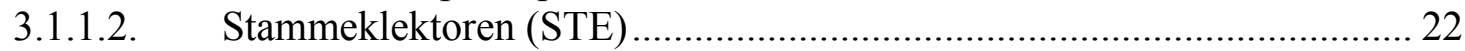

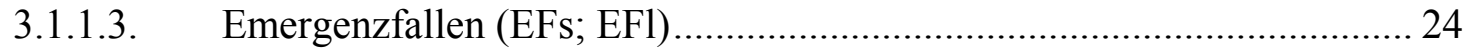

3.1.1.4. Modifizierte Bodeneklektoren (mBE) .................................................... 25

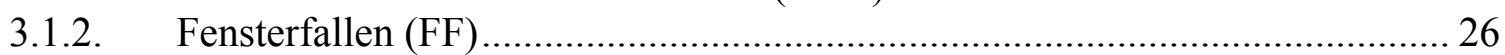

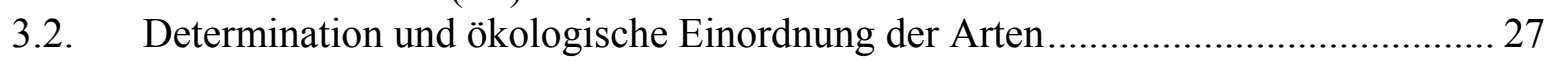

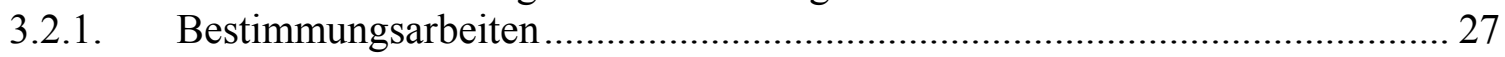

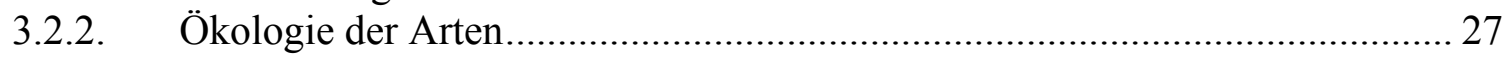

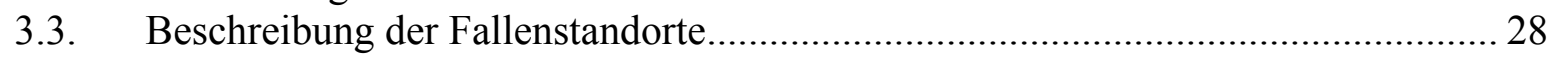

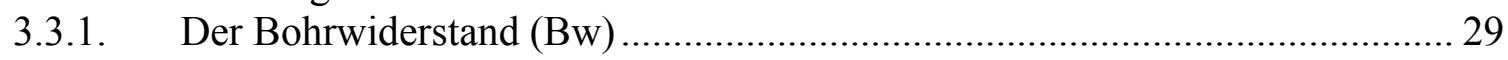

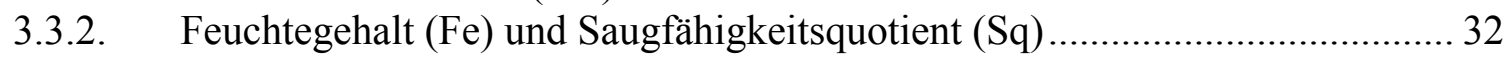

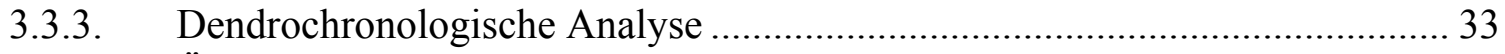

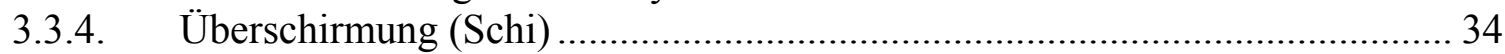

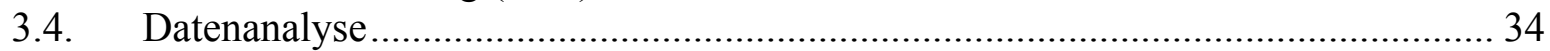

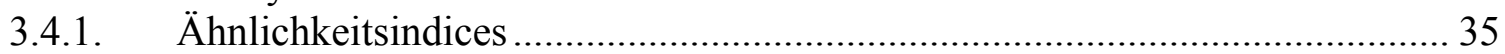

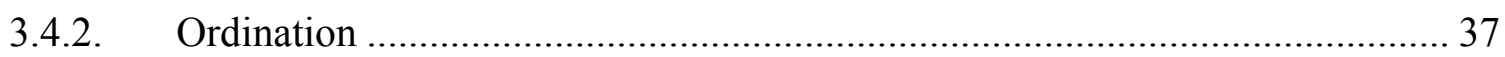

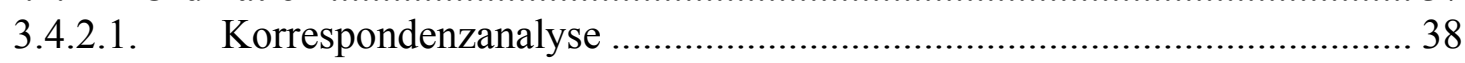

3.4.2.2. Kanonische Korrespondenzanalyse.................................................... 39

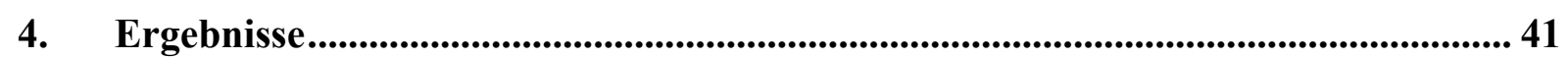

4.1. Überblick über Individuen-, Familien- und Artenzahlen ...................................... 41

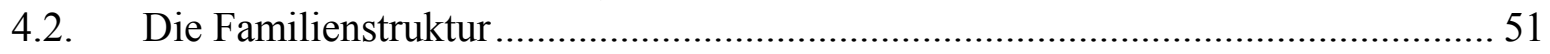

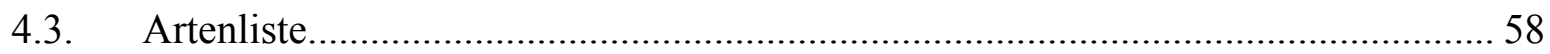

4.4. Die Struktur der Artengemeinschaften in den Eklektorproben ............................. 75

4.4.1. Frequenz- und Dominanzstruktur in Abhängigkeit von Eklektortyp und

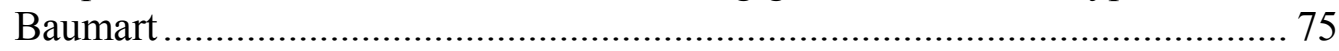

4.4.1.1. Frequenz- und Dominanzstruktur an liegendem Buchentotholz............. 87

4.4.1.2. Frequenz- und Dominanzstruktur an liegendem Eichentotholz.............. 92

4.4.1.3. Frequenz- und Dominanzstruktur an stehendem Buchentotholz ............ 96

4.4.1.4. Frequenz- und Dominanzstruktur an stehendem Eichentotholz ............. 101

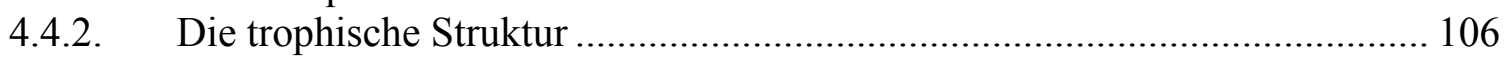

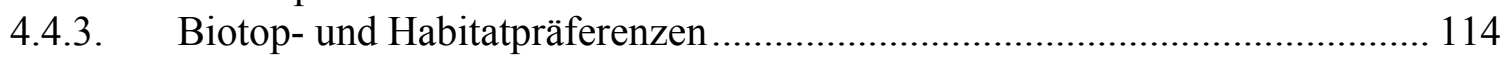

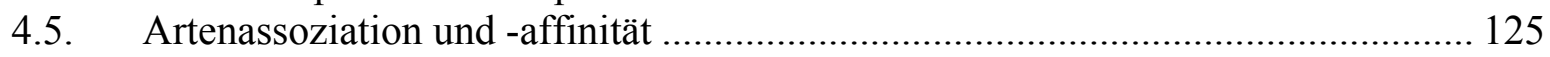

4.6. Die Sukzession der Artengemeinschaften .................................................. 131 
4.6.1. Verteilung, Vergesellschaftung und Sukzession der Arten an liegendem

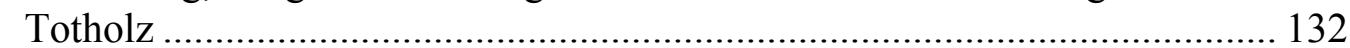

4.6.1.1. Sukzession der Artengemeinschaften an liegendem Buchentotholz...... 142

4.6.1.2. Sukzession der Artengemeinschaften an liegendem Eichentotholz....... 155

4.6.2. Verteilung, Vergesellschaftung und Sukzession der Arten an stehendem

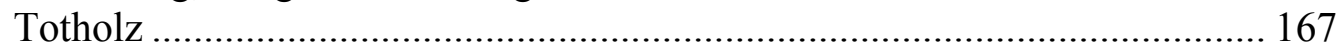

4.6.2.1. Sukzession der Artengemeinschaften an stehendem Buchentotholz ..... 174

4.6.2.2. Sukzession der Artengemeinschaften an stehendem Eichentotholz ...... 188

4.7. Die Struktur der Artengemeinschaften in den Fensterfallenproben ..................... 200

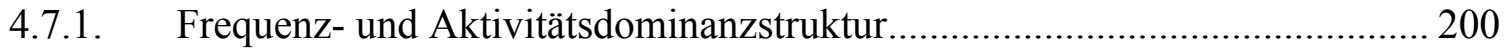

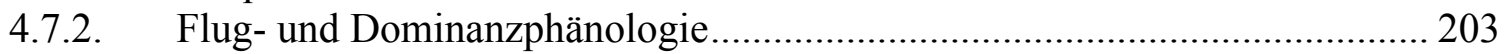

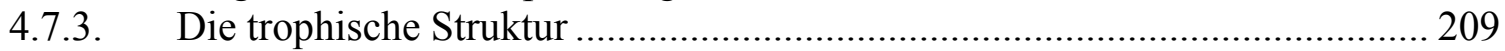

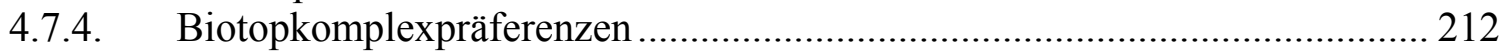

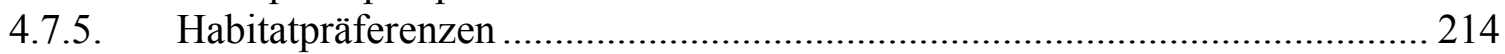

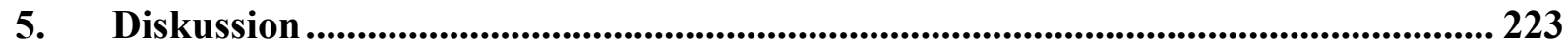

5.1. Die Käferfauna des NSG 'Neuenburger Urwald' ............................................. 223

5.2. Gründe für den Artenreichtum xylobionter Coleopteren...................................... 225

5.3. Vergleich der Fangergebnisse von Fensterfallen und Totholzeklektoren ............. 229

5.4. Die Bedeutung von Baumart, Lage und Zersetzungszustand ................................ 232

5.4.1. Einfluss der Baumart auf die Vergesellschaftung saproxyler Coleopteren ... 232

5.4.2. Unterschiede in der Besiedlung von stehendem und liegendem Totholz ...... 234

5.4.3. Einfluss des Zersetzungszustandes auf die Besiedlung durch Käfer ............. 237

6. Zusammenfassung ........................................................................................................ 243

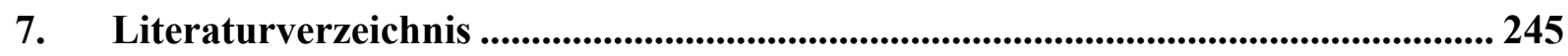

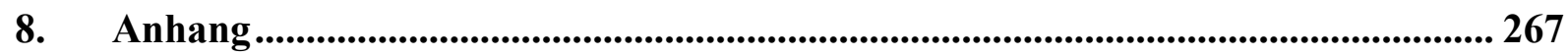

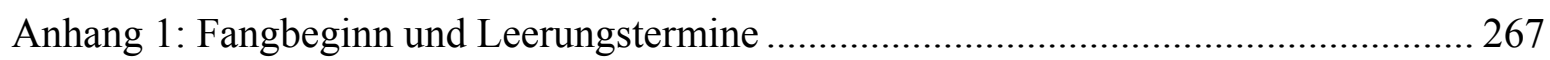

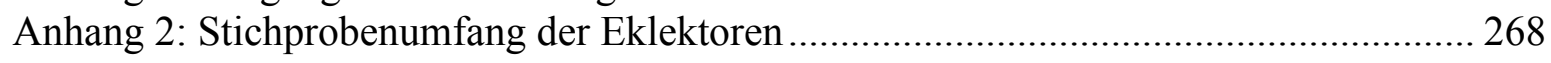

Anhang 3: Käferfamilien in verschiedenen Eklektortypen an Eiche und Buche ............... 270

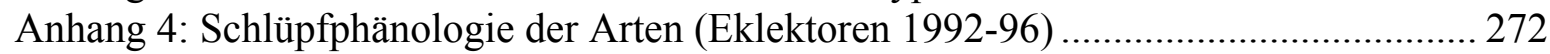

Anhang 5: Auftreten der Arten in STE- und EFs-Proben an Eiche und Buche ................. 281

Anhang 6: Artenaffinität: Sørensen-Quotient (SQ) für Artenpaarungen ............................ 290

Anhang 7a: Probenähnlichkeit: Sørensen-Quotient (SQ) für STE-Proben an Buche ........ 292

Anhang 7b: Probenähnlichkeit: Renkonen-Zahl (RE) für STE-Proben an Buche ............. 293

Anhang 8a: Probenähnlichkeit: Sørensen-Quotient (SQ) für STE-Proben an Eiche ......... 294

Anhang 8b: Probenähnlichkeit: Renkonen-Zahl (RE) für STE-Proben an Eiche.............. 295

Anhang 9a: Probenähnlichkeit: Sørensen-Quotient (SQ) für EFs-Proben an Buche......... 296

Anhang 9b: Probenähnlichkeit: Renkonen-Zahl (RE) für EFs-Proben an Buche.............. 298

Anhang 10a: Probenähnlichkeit: Sørensen-Quotient (SQ) für EFs-Proben an Eiche ........ 300

Anhang 10b: Probenähnlichkeit: Renkonen-Zahl (RE) für EFs-Proben an Eiche.............. 301

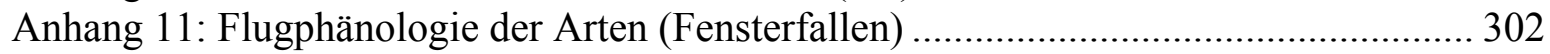

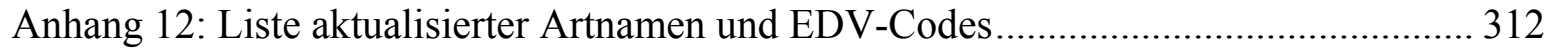

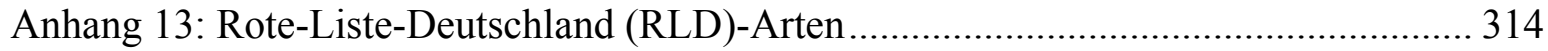

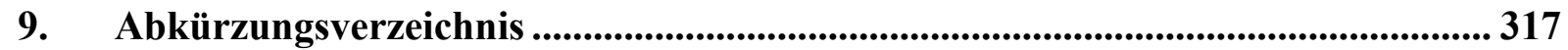




\section{Danksagung}

An erster Stelle gilt mein Dank meinem Doktorvater, Herrn Prof. Dr. W. Sanders. Er hat das Thema gestellt und den Fortgang der Untersuchung intensiv begleitet und gefördert. Besonders gerne denke ich an die vielen anregenden Diskussionen, die mir so manches Licht aufgehen ließen. Durch seine fachliche und menschliche Begleitung hat er ein Arbeitsklima geschaffen, das von nicht zu überschätzender Bedeutung für das Gelingen der Arbeit war. Vielen Dank.

Dem ehemaligen Direktor des Instituts für Forstzoologie, Herrn Prof. Dr. J. Lunderstädt, habe ich für die Bereitstellung von Arbeitsplatz und Werkstattkapazitäten sowie der Gewährung sachlicher und personeller Unterstützung zu danken.

Von den ehemaligen wissenschaftlichen Mitarbeitern des Instituts möchte ich insbesondere Dr. R. Hurling, Dr. J. Willig und Dr. J. Endres für die vielen anregenden Diskussionen über zoologische und ökologische Fragestellungen danken. Herr Dr. R. Hurling war darüber hinaus eine große und geduldige Hilfe bei Fragen zur Analyse der Daten. Er hat sich zudem die Mühe des Korrekturlesens des Manuskripts gemacht und viele Anregungen zu dessen Verbesserung gegeben. Dafür bin ich ihm sehr dankbar.

Für ihr Engagement bei der Entwicklung und dem Bau der Fallen möchte ich Frau Duntemann sowie den Herren Albrecht und Teichmann recht herzlich danken. Die graphische Darstellung der Fallen wurde freundlicherweise von Herrn Tambour (Universität Göttingen) übernommen.

Für die Bestimmung der Staphyliniden gilt mein großer Dank Herrn V. Assing (Hannover). Herrn Dr. K. Renner (Bielefeld) danke ich für die Bereitschaft, meine Determinationsergebnisse zu überprüfen und mich gegebenenfalls auf die richtige Fährte zu setzen.

Herrn Dr. Leuschner vom Institut für Palynologie und Quartärwissenschaften der Universität Göttingen und Frau Dipl. Biol. D. Schmidt waren bei der Bohrkernentnahme behilflich und haben die angesichts des Zustandes vieler Proben nicht leichte Aufgabe der dendrochronologischen Analyse übernommen. Hierfür ein herzliches Dankeschön.

Dank gebührt auch den Mitarbeitern der Fakultätsbibliothek. Insbesonderer der ehemaligen Leiterin, Frau Kollmeyer, sowie Herrn Spang bin ich dankbar für ihre Hilfe bei der Recherche und Bereitstellung der benötigten Literatur.

Für die Erteilung der Genehmigung zur Durchführung der Untersuchung in einem Naturschutzgebiet danke ich dem Naturschutzdezernat der ehemaligen Bezirksregierung Oldenburg. Den Mitarbeitern des Forstamtes Neuenburg, insbesondere dessen ehemaligem Leiter, Herrn Kriebitzsch, und dem zuständigen Revierbeamten, Herrn Smith, gilt mein Dank für die gewährte Unterstützung.

Viele weitere Kollegen, Freunde und Verwandte haben zum Gelingen der Arbeit beigetragen. Nicht alle konnten an dieser Stelle namentliche Erwähnung finden. Ihnen allen sei mein Dank ausgesprochen. 


\section{Einleitung}

Die in der Vergangenheit gestiegenen Ansprüche an den Wald, die zunehmende Nutzungsintensität und die Entwicklung der modernen Forstwirtschaft in Mitteleuropa haben unübersehbare Auswirkungen auf das heutige Erscheinungsbild der Wälder gehabt (HESMER \& SCHRÖDER 1963; CHRISTENSEN \& EMBORG 1996; KÜSTER 1995， 1998; BENGTSSON et al. 2000). Neben der Fläche und Verteilung des Waldes waren und sind auch die Struktur, Funktion und Dynamik von Waldökosystemen betroffen. Ungestörte Urwälder unterscheiden sich daher in vielfältiger Weise von genutzten Wirtschaftswäldern. Dies betrifft u. a. die Baumarten- und Altersklassenverteilung, die Strukturdynamik und das Bestandesklima. Im auffälligen Kontrast zum Wirtschaftswald steht auch das große und vielfältige Totholzangebot im Urwald.

Eine Vorstellung von den in unberührten Laubwaldökosystemen Mitteleuropas zu erwartenden Totholzvorräten vermitteln waldkundliche Aufnahmen in weitgehend unberührten Urwäldern, die v. a. in Süd- und Süd-Ost-Europa in Resten erhalten geblieben sind. BOBIEC (2002) nennt für den Urwald von Bialowieza (Polen) Totholzmengen von 87 bis $160 \mathrm{~m}^{3} / \mathrm{ha}$. BONCINA (2000) ermittelte in einem slovenischen Urwald einen Totholzvorrat von $138 \mathrm{~m}^{3} /$ ha. KORPEL (1997b) fand in slowakischen Eichen-Buchen-Urwäldern ein durchschnittliches Totholzvolumen von 70 bis $120 \mathrm{~m}^{3} / \mathrm{ha}$. Da Totholz oftmals nicht gleichmäßig auf der Fläche verteilt ist, sondern vielmehr geklumpt auftritt, werden auf Teilflächen deutlich höhere Mengen gefunden. Von Totholzvorräten in vergleichbarer Größenordnung berichten auch weitere Autoren wie z. B. FALINSKI 1978; LEIBUNDGUT 1982; KOOP \& HILGEN 1987; KORPEL 1992, 1995, 1997a; MOUNTFORD 2002; ÓDOR \& STANDOVÁR 2002; KRAIGHER et al. 2002; SANIGA \& SCHÜTZ 2002; WALANKIEWICZ 2002; CHRISTENSEN \& HAHN 2003; ÓDOR \& HEES 2004.

Entfällt im Urwald ein erheblicher Anteil des Holzbiomassevorrates auf Totholz, stellt sich die Situation im Wirtschaftswald ganz anders dar. Hier findet sich nur ein Bruchteil der natürlichen Totholzvorräte, wobei Bestände mit mehr als $10 \mathrm{~m}^{3} /$ ha schon als überdurchschnittlich totholzreich gelten müssen. Auch die Struktur des Totholzangebotes unterscheidet sich in erheblichem Maße, in dem z. B. ein Mangel an starkdimensionierten Stämmen fortgeschrittener Zersetzung zu verzeichnen ist. Statt dessen herrschen Holzernterückstände geringen Durchmessers und Baumstubben vor (ANDERSSON \& HYTTEBORN 1991; ALBRECHT 1991; RÖHRIG 1991; BURSCHEL 1992; ERDMANN \& WILKE 1997; KÜHNEL 1999; TABAKU \& MEYER 1999; BONCINA 2000; BOBIEC 2002).

Totholz ist insbesondere in natürlichen Waldökosystemen von großer Bedeutung. Es spielt eine wichtige Rolle im Nährstoff-, Kohlenstoff-, Wasser- und Energiehaushalt, beeinflusst die Bodeneigenschaften und -entwicklung, reduziert die Erosion, begünstigt die Bestandesverjüngung, trägt zu einer höheren Vielfalt des Mikroreliefs und der mikroklimatischen Bedingungen bei und ist ein Schlüsselfaktor für eine hohe Artenvielfalt. Die Bedeutung von Totholz für die nachhaltige Produktivität, Stabilität und Selbstregulationsfähigkeit von Waldökosystemen ist bislang unzureichend untersucht (SWIFT 1977a; FALINSKI 1978; HARMON et al. 1986; HAILA \& KOUKI 1994; SAMUELSON, GUSTAFSSON \& INGELÖG 1994; KAYAHARA, KLINKA \& LAVKULICH 1995; JURGENSEN et al. 1997; LOFROTH 1998; MACKENSEN \& BAUHUS 1999; KRANKINA, HARMON \& GRIAZKIN 1999; FERRIS 
\& HUMPHREY 1999; BENGTSSON et al. 2000; SIITONEN 2001; HARMON 2001; BODDY 2001; McCAY et al. 2002; ROUVINEN 2002; GROVE, MEGGS \& GODWIN 2002; JANISCH \& HARMON 2002; LAIHO \& PRESCOT 2004).

Totholz ist ein ausgesprochen vielgestaltiges Strukturelement, dass aufgrund der Vielzahl der Mikrohabitate Nischen für eine Vielzahl von Organismen bereitstellt und sich damit als ein Schlüsselfaktor für die Biodiversität in Waldökosystemen erweist (BADER, JANSSON \& JONSSON 1995; CHRISTENSEN \& EMBORG 1996; FREEDMAN et al. 1996; SVERDRUP-THYGESON \& MIDTGAARD 1998; DAJOZ 2000; SIITONEN 2001; HUMPHREY et al. 2002; GROVE 2002; KOMONEN 2003a). Es wird angenommen, dass ca. 20-25\% der waldbewohnenden Arten von einem angemessenen Totholzangebot abhängig sind (ELTON 1966; SIITONEN 2001; ALEXANDER 2003).Vertreter aus den unterschiedlichsten Artengruppen sind auf Totholz angewiesen bzw. werden durch diese Strukturkomponente begünstigt. Beispielhaft genannt seien

- $\quad$ Totholzpilze (z. B. JAHN 1990; MÖLLER 1994; FERRIS, PEACE \& NEWTON 2000; EDMANN \& JONSSON 2001; SIITONEN 2001; HEILMANN-CLAUSEN \& CHRISTENSEN 2003)

- $\quad$ epixyle Flechten und Moose (z. B. SÖDERSTRÖM 1988b; DANIELS 1991; ESSEEN et al. 1997; CRITES \& DALE 1998; RAMBO \& MUIR 1998; KRUYS et al. 1999; KRUYS \& JONSSON 1999; ODOR \& STANDOVAR 2001; HUMPHREY et al. 2002)

- $\quad$ Arthropoden (z. B. PALM 1951, 1959; SEASTEDT, REDDY \& CLINE 1989; BRECHTEL 1991; BUSCHINGER 1991; WESTRICH 1991; KLAUSNITZER 1996; CALDWELL 1996; HAMOND 1997; KÖHLER 2000; DOROW 2002; ALEXANDER 2003; KOMONEN 2003a, b; HÖVEMEYER \& SCHAUERMANN 2003; LINDHE 2004)

- Vögel (z. B. SCHERZINGER 1982; UTSCHIK 1991; SANDSTRÖM 1992; BLUME 1993; ANGELSTAM \& MIKUSINSKI 1994; PECHACEK 1995; BUNNEL et al. 2002; BURSELL 2002)

- $\quad$ Säugetiere (z. B. MASER \& TRAPPE 1984; JÜDES 1991; TAAKE 1991; ERICKSON \& WEST 2003)

Käfer haben sich in besonders vielfältiger Weise an die direkte oder indirekte Nutzung von Totholz angepaßt. Das abgestorbene Material wird u. a. als Nahrung genutzt und zur Fortpflanzung, zum Nahrungserwerb, als Schutzraum und zur Überwinterung aufgesucht. Dabei ist der Grad der Totholzbindung sehr unterschiedlich. Neben den Arten, die eine strenge Abhängigkeit von Totholz zeigen, stehen jene Arten, die von Totholz profitieren bzw. dieses fakultativ als zusätzlichen Lebensraum nutzen. $\mathrm{Zu}$ den Letzteren ist $\mathrm{z}$. B. eine Vielzahl von regelmäßig in Totholz anzutreffenden bodenbewohnenden und eurytopen Arten zu rechnen. 
Als saproxyl bzw. xylobiont ${ }^{1}$ werden im Folgenden jene Arten definiert, ,... die in ihrer Reproduktion obligatorisch an verletzte, absterbende oder tote verholzte Sproßteile von Bäumen und Sträuchern oder hieran lebenden Organismen gebunden sind" (KÖHLER 2000).

Von der ca. 8000 Arten umfassenden Käferfauna Mitteleuropas wird etwa jede vierte bis fünfte Art zu den Totholzbewohnern gezählt. In keiner anderen Insektenordnung findet sich eine vergleichbar hohe Zahl xylobionter Arten (RENNER 1991; GEISER 1994, 1998). KÖHLER (2000) beziffert die Zahl der obligaten Totholzbewohner in Deutschland mit 1371, das entspricht $21 \%$ der heimischen Käferfauna bzw. $56 \%$ der an Wald- und Gehölzbiotope gebundenen Käferarten.

Die reduzierte Menge und die verarmte Strukturvielfalt des Totholzangebotes im Wirtschaftswald, insbesondere das Fehlen der Zerfallsphase und der Mangel an starkdimensionierten stehenden Stämmen, hat die Lebensraumsituation für die Mehrzahl saproxyler Arten erheblich verschlechtert. In der Konsequenz ist diese ökologische Gruppe in hohem Maße gefährdet. Den Anteil gefährdeter Arten an der Gesamtheit der xylobionten Käferarten beziffert KÖHLER (2000) mit 59 \%. Charakteristisch ist auch, dass die xylobionten Coleopteren in den hohen Gefährdungsstufen besonders zahlreich vertreten sind. Als besonders kritisch ist die Gefährdungssituation bei den Besiedlern fortgeschrittener Zersetzungsgrade zu bewerten.

Die hohe Zahl xylobionter Käferarten geht mit einer starken ökologischen Differenzierung einher. Für die Mehrzahl der Arten sind die Ursachen der Habitatbindung allerdings nur sehr unzureichend bekannt (KLAUSNITZER 1995; KOMONEN 2001). Zu den zahlreichen Faktoren, denen eine große Bedeutung für das Vorkommen und die Vergesellschaftung der Arten in Waldökosystemen beigemessen wird, zählen u. a. geographische und klimatische Region, Totholzmenge, Faunentradition, Nutzungsgeschichte, Höhenlage, Baumart, Baumteil, Durchmesser, Lage, Zersetzungszustand und Substratqualität, Holzfeuchte, Pilzbesiedlung, Exposition und Mikroklima (DERKSEN 1941; PALM 1951, 1959; GEISER 1989, 1994; MÖLLER \& SCHNEIDER 1991; RAUH 1993; MÖLLER 1994; KÖHLER 1996; BENSE \& GEIS 1998; DAJOZ 2000; SCHUCK et al. 2004).

Unter mitteleuropäischen Bedingungen sind Mikroorganismen und Pilze die Hauptakteure des Holzabbaus (SWIFT 1977a; COOKE \& RAYNER 1984; RAYNER \& BODDY 1988; DIX 1995; BODDY 2001). Aber auch Tiere steuern in entscheidendem Maße Verlauf und Geschwindigkeit der Zersetzung. Für Käfer ermittelte DAJOZ (1966) einen Anteil von $80-90 \%$ an der tierischen Biomasse mit Konsumraten von $25 \%$ (Eichentotholz) bzw. $38 \%$ (Buchentotholz). Auch MAMAEV (1961, zit. nach DAJOZ 2000), und DERKSEN (1941) berichten von hohen Konsumptionsraten der Käfer an Eichen- bzw. Buchentotholz. Darüber hinaus schaffen Käfer Zutrittspforten, zerkleinern das Substrat und vergrößern damit die Oberfläche, fungieren als Vektoren z. B. von Pilzsporen und fördern auf vielfältige Weise die mikrobielle Aktivität (SCHIMITSCHEK 1953a, 1953b; BRAUNS 1954; AUSMUS 1977; SWIFT 1977b; ABBOTT \& CROSSLEY 1992; SWIFT, BODDY \& HEALEY 1984; BODDY \& SWIFT 1984; RAYNER \& BODDY 1988; EDMONDS \& EGLITES 1989;

\footnotetext{
${ }^{1}$ Während im deutschen Sprachraum der Begriff „xylobiont“ weit verbreitet ist, wird in englischsprachigen Publikationen der Terminus „saproxylic“ verwendet. Von Ausnahmen abgesehen, in denen dieser Terminus den Besiedlern später Zersetzungsstadien (Mulmkomplex) vorbehalten ist, bestehen i.d.R. keine grunsätzlichen Unterschiede in den Definitionen dieser beiden Begriffe (s. a. SPEIGHT 1989) und werden daher hier synonym verwendet.
} 
ZHONG \& SHOWALTER 1989; SETÄLÄ \& MARSHALL 1994; HAMMOND 1997; HÖVEMEYER 1998; HÅGVAR 1999; DAJOZ 2000; BODDY 2001; HÖVEMEYER \& SCHAUERMANN 2003).

Der Abbau toten Holzes erstreckt sich i. d. R. über einen sehr langen Zeitraum. Verlauf und Geschwindigkeit des Totholzabbaus sind sehr variabel, werden von zahlreichen Faktoren wie z. B. Baumart, Klima, Durchmesser und Zersetzergemeinschaft beeinflusst und gehen mit einer kontinuierlichen Veränderung der Substrateigenschaften (z. B. Dichte, Wasserhaltekapazität, Elementgehalte, Cellulose-Lignin-Verhältnis, C-N-Verhältnis, pH) einher (LAMBERT, LANG \& REINERS 1980; BODDY \& SWIFT 1984; HARMON et al. 1986; MACKENSEN \& BAUHUS 1999; HARMON, KRANKINA \& SEXTON 2000; TARASOV \& BIRDSEY 2001; STOKLAND 2001; SHOROHOVA \& SHOROHOV 2001; SCHÄFER 2002; KAHL 2003; MÜLLER-USING \& BARTSCH 2003; HÖVEMEYER \& SCHAUERMANN 2003; YATSKOV, HARMON \& KRANKINA 2003; LAIHO \& PRESCOT 2004).

Die mit der Dekomposition einhergehenden Veränderungen der Substrateigenschaften werden von einer Abbausukzession der das Totholz besiedelnden Organismen verursacht und/oder begleitet und enden mit der vollständigen Mineralisation des organischen Materials. Die überwiegende Zahl der Sukzessionsuntersuchungen befaßt sich mit der Entwicklung der Pilzgemeinschaften (z. B. RUNGE 1975; RENVALL 1995; HOILAND \& BENDIKSEN 1996; LINDBLAD 1998; DE MEULDER 1999; WINTERHOFF 2001; HEILMANNCLAUSEN 2001; SCHLECHTE 2002). Insekten waren hingegen nur vereinzelt Gegenstand von Sukzessionsuntersuchungen (z. B. BRAUNS 1954; DEYRUP 1981; SETÄLA \& MARSHALL 1994; HÖVEMEYER \& SCHAUERMANN 2003). Einige Untersuchungen, die sich mit der Käferfauna an Totholz von Laubbäumen befassen, sollen im Folgenden kurz erwähnt werden.

DERKSEN (1941) hat die Sukzession der Insekten an 2-10 Jahre alten Buchenstubben mit bekanntem Absterbezeitpunkt auf Kahlschlägen sowie im Bestandesinnern in der Nähe von Rostock untersucht. Neben manuellen Methoden kam dabei auch eine geringe Anzahl von Schlüpfkäfigen zum Einsatz. Ausschließlich mit manuellen Methoden hat SCHIMITSCHEK (1953, 1954) die Befallsfolgen der Tiere an Fichte-, Tannen- und Buchentotholz im Urwald Rotwald (Österreich) studiert. Als Ordnungskriterium orientiert er sich dabei am Zersetzungszustand der Kambialzone und des Holzkörpers. Mit der Sukzession der Käfer an Eichentotholz beschäftigen sich die Untersuchungen von MAMAEV (1961) (Ukraine) und DAJOZ (1966) (Süd-Frankreich). In der zuletzt genannten Untersuchung wurde auch die Artenvergesellschaftung der saproxylen Käfer im Holzkörper und unter der Rinde von Buchentotholz untersucht. Auf der Grundlage von Artenaffinitäten unterscheidet er jeweils drei Sukzessionsstadien. Die Entwicklung der Käfergemeinschaften an Kirschentotholz war Gegenstand der Untersuchungen von RENNER (1991).

Beruhen die Ergebnisse der genannten Untersuchungen ausschließlich oder doch zumindest überwiegend auf manuellen Erfassungsmethoden, wurden seit den 90er Jahren vermehrt verschiedene Typen von Eklektoren eingesetzt, so auch in den nachfolgend genannten Arbeiten.

Buchentotholz verschiedener Zersetzungsgrade und unterschiedlichen Durchmessers wurde von SCHMITT (1992) in einem Naturwaldreservat sowie in zwei Vergleichsflächen des Wirtschaftswaldes in Bayern beobachtet. Ebenfalls aus Bayern stammen die von HILT (1992) beschriebenen Ergebnisse. Gegenstand ihrer Aufnahmen waren die Insektengemein- 
schaften in Stubben, Schwach- und Starkholz von Eichen und Fichten verschiedener Zersetzungsgrade. Stehendes starkdimensioniertes Eichen- und Buchentotholz wurde in der Göhrde (Niedersachsen) von LACZNY (1993) untersucht. Zu den wenigen Untersuchungen, die sich mit stehendem Totholz beschäftigen, ist auch die von ZÖRNER (2003) zu rechnen, der stehendes starkes Eichentotholz mit überwiegend bekanntem Absterbejahr in einem niedersächsichen Naturwaldreservat untersucht hat. KLEINEVOSS (1995), HAASE, TOPP \& ZACH (1998) sowie KAPPES \& TOPP (2004) berichten über Fangergebnisse von überwiegend hochliegendem Buchen- und Eichentotholz eines Wirtschaftswaldes im Westerwald. Das befangene Totholz wurde hierbei nach Durchmesser gruppiert und verschiedenen Zersetzungklassen mittels Bohrwiderstandmessungen zugeordnet. Die Sukzession der Käfer an Buchentotholz auf einer Windwurffläche wird von FLECHTNER (2002) beschrieben.

Obwohl die xylobionten Käfer schon sehr früh das Interesse von Coleopterologen gefunden haben (z. B. SAALAS 1917, 1923; BLACKMANN \& STAGE 1924; KROGERUS 1927; PALM 1951), ist über ihre Sukzession im Abbauprozeß noch sehr wenig bekannt. So ist eine ,... exakte, statistisch abgesicherte Festlegung einzelner Käferarten auf bestimmte Sukzessionsstadien oder Altersstadien und Zersetzungsstadien von Totholz .. nach gegenwärtigem Kenntnisstand nicht zufriedenstellend möglich. Dies gilt insbesondere für die Folgegemeinschaften nach den primären Totholzbesiedlern" (KÖHLER 2000). Für die Mehrzahl der saproxylen Käferarten bestehen noch große Kenntnislücken hinsichtlich ihrer Ansprüche an den Lebensraum (DAJOZ 2000). Die hier vorgelegte Arbeit soll einen Beitrag zum besseren Verständnis der Vergesellschaftungen der Käfergemeinschaften an Totholz leisten.

Im Einzelnen werden folgende Ziele verfolgt:

1. Erfassung des Artenspektrums saproxyler Coleopteren in einem totholzreichen Waldschutzgebiet mit langer Biotoptradition

2. Quantitative Beschreibung und Vergleich der Struktur der Käferartengemeinschaften an liegendem und stehendem Eichen- und Buchentotholz

3. Beschreibung der Sukzession der Käfergemeinschaften mit fortschreitender Zersetzung

4. Analyse der Bedeutung verschiedener Habitatvariablen für das Auftreten der Arten und ihre Vergesellschaftung

5. Ermittlung schlüpf- und flugphänologischer Daten 


\section{Das Untersuchungsgebiet}

\subsection{Gründe für die Auswahl}

In Mitteleuropa sind weitgehend unbeeinflusste Urwälder nicht mehr zu finden. Zahlreiche faunistisch-ökologische Fragestellungen lassen sich aber in bewirtschafteten Waldökosystemen nicht oder nur unzureichend untersuchen. Dies gilt aufgrund des Habitatmangels auch für einen Großteil der Fragen zur Struktur und Sukzession der Tierlebensgemeinschaften an Totholz. Die vorliegenden Untersuchungsergebnisse zu den Einflussfaktoren auf die Struktur der Artengemeinschaften sowie die genannten Zielsetzungen dieser Arbeit hatten zur Konsequenz, dass nur solche Waldbestände für die Untersuchung geeignet erschienen, die mehrere, selten gleichzeitig anzutreffende Voraussetzungen erfüllen. Neben einem ausreichend hohen Angebot an starkdimensioniertem Totholz der verschiedenen Zersetzungsgrade sollte das Gebiet eine möglichst lange Biotoptradition haben. Alters- und Zerfallsphasen mußten in hinreichendem Umfang vorhanden sein, da nur hier die für Totholzbewohner günstige Vielfalt an Totholzstrukturen und Milieubedingungen zu erwarten war. Diese Ansprüche reduzierten die Auswahl auf eine kleine Zahl in Frage kommender Waldgebiete, die allesamt - nicht zuletzt gerade weil die genannten Voraussetzungen gegeben sind - unter Naturschutz stehen.

Die geforderten Bedingungen sind im Naturschutzgebiet (NSG) 'Neuenburger Urwald' erfüllt, sowohl was die Totholzausstattung als auch die Biotoptradition betrifft. KOOP (1982) zählt den 'Neuenburger Urwald' zu den ,... am weitgehendsten spontanen Wäldern der nordmitteleuropäischen Tiefebene" und SPEIGHT (1989) attestiert ihm eine potenzielle internationale Bedeutung aufgrund seiner saproxylischen Insektenfauna.

\subsection{Die geographische Lage}

Das 23,5 ha große NSG 'Neuenburger Urwald' liegt im nordwestniedersächsischen Tiefland, ca. $30 \mathrm{~km}$ nordnordwestlich von Oldenburg und $20 \mathrm{~km}$ südsüdwestlich von Wilhelmshaven (53²8' nördl. Breite, $7^{\circ} 59^{\prime}$ östl. Länge) und gehört somit zum Naturraum Ostfriesisch-Oldenburgische Geest, einem Altmoränengebiet mit vorwiegend ebener Oberfläche (MEYNEN \& SCHMITHÜSEN 1959-62) (Abb. 2-1). Das Untersuchungsgebiet ist Bestandteil des 670 ha großen staatlichen Forstreviers Neuenburgerholz (Forstamt Neuenburg, Abteilung 136), eines der wenigen größeren zusammenhängenden Waldgebiete in dieser ansonsten waldarmen Region. Die Reliefenergie des Gebietes ist sehr gering, die Höhenlage variiert zwischen fünf und zehn Metern. 


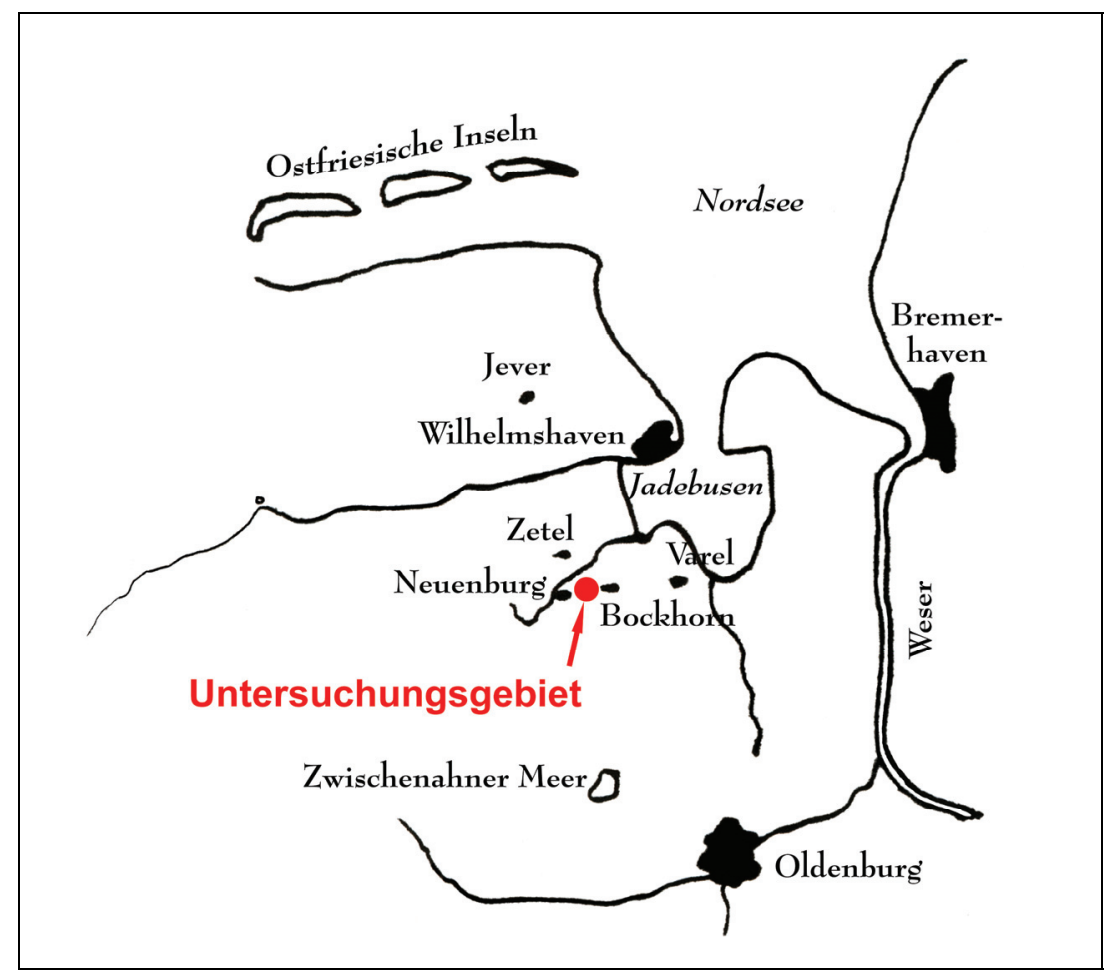

Abb. 2-1: Geographische Lage

\subsection{Klima und Witterung}

Das Klima des 'Neuenburger Urwaldes' ist bestimmt durch seine Nähe zum Meer. In nordwestliche Richtung beträgt die Entfernung zur Nordseeküste etwa $45 \mathrm{~km}$, bis zum östlich gelegenen Jadebusen sind es nur $10 \mathrm{~km}$. Reliefbedingte Einflüsse kommen hier kaum zum Tragen. Das atlantische Klima ist gekennzeichnet durch hohe Niederschlagsmengen, wovon etwa die Hälfte während der Vegetationszeit von Mai bis September fällt (Tab. 2-1). Die durchschnittliche relative Luftfeuchtigkeit ist ebenfalls hoch. Als deutlichstes Charakteristikum eines ozeanisch bestimmten Klimas sind die geringen Temperaturgegensätze zwischen Sommer und Winter zu nennen. Die mittlere Jahresschwankung beträgt in Jever 15,0 und in Oldenburg $15,7^{\circ} \mathrm{C}$. Zudem werden nur relativ wenige Frost- und Eistage gezählt.

Die Abbildungen 2-2 und 2-3 zeigen die Monatswerte der Temperatur (Wetterstationen Oldenburg und Jever) und des Niederschlages (Station Varel) in den Untersuchungsjahren sowie die Abweichungen der Monatsdurchschnittswerte vom langjährigen Mittel. Die Darstellungen und Ausführungen zum Witterungsverlauf im Untersuchungszeitraum beruhen auf den vom Deutschen Wetterdienst in den Jahren 1992 bis 1996 veröffentlichten monatlichen Witterungsberichten. 
Tabelle 2-1: Klimamerkmale des Wuchsbezirkes

Ostfriesisch-Oldenburgische Geest

(GÖRGES 1969 nach OTTO 1972)

\begin{tabular}{|l|l|}
\hline Mittl. Niederschlagssumme / Jahr & $780 \mathrm{~mm}$ \\
\hline Mittl. Niederschlagssumme Mai-Sept & $370 \mathrm{~mm}$ \\
\hline Mittl. rel. Luftfeuchtigkeit / Jahr & $84 \%$ \\
\hline Mittl. Jahrestemperatur & $8,5^{\circ} \mathrm{C}$ \\
\hline Mittl. Lufttemperatur Mai-Sept & $14,2^{\circ} \mathrm{C}$ \\
\hline Mittl. Zahl der Sommertage / Jahr & 12 \\
\hline Mittl. Zahl der Frosttage / Jahr & 75 \\
\hline
\end{tabular}

Das Jahr 1992 war überdurchschnittlich warm und etwas zu trocken. Mit einer Jahresdurchschnittstemperatur von über $10^{\circ} \mathrm{C}$ wurde das langjährige Mittel deutlich übertroffen. Über 30 Sommertage waren zu verzeichnen, am wärmsten Tag des Jahres wurde eine Temperatur von über $36{ }^{\circ} \mathrm{C}$ gemessen. Fast während der gesamten Fangzeit waren die Niederschlagsmengen unterdurchschnittlich.

1993 lag die Jahresdurchschnittstemperatur geringfügig über dem langjährigen Mittel, was insbesondere auf ein sehr warmes Frühjahr zurückzuführen war. Die Niederschläge fielen mit über $900 \mathrm{~mm}$ überdurchschnittlich aus. Besonders ab Juli war es sehr naß und kühl. Temperaturen von über $30^{\circ} \mathrm{C}$ wurden nicht erreicht, nur an wenigen Tagen wurde die $25{ }^{\circ} \mathrm{C}$ Marke überschritten.

Auch im Jahr 1995 war es überdurchschnittlich warm (um ca. 0,75 $\left.{ }^{\circ} \mathrm{C}\right)$ und naß $(105 \%)$. Die Sonnenscheindauer lag fast um ein Viertel über dem langjährigen Mittel. Höchsttemperaturen von $25{ }^{\circ} \mathrm{C}$ wurden in Jever an 28 und in Oldenburg an 47 Tagen registriert. Werte von $30{ }^{\circ} \mathrm{C}$ wurden an 2 bzw. 11 Tagen gemessen. Abgesehen vom September entsprachen die Niederschläge während der Untersuchungsmonate weitgehend den langjährigen Durchschnittswerten, während die Temperatur in den Monaten Juli und August über dem vieljährigen Mittel lag.

Der April und August des Jahres 1996 waren geringfügig zu warm. Ansonsten blieben die Temperaturen während der Untersuchung hinter dem langjährigen Mittel zurück. Für die Monate Februar bis Juni lagen keine Niederschlagswerte für die Station Varel vor. Den Meßergebnissen einiger Nachbarstationen zufolge waren die Monate Mai, August und September etwas zu naß, während in den übrigen Monaten zu wenig Niederschlag fiel. 


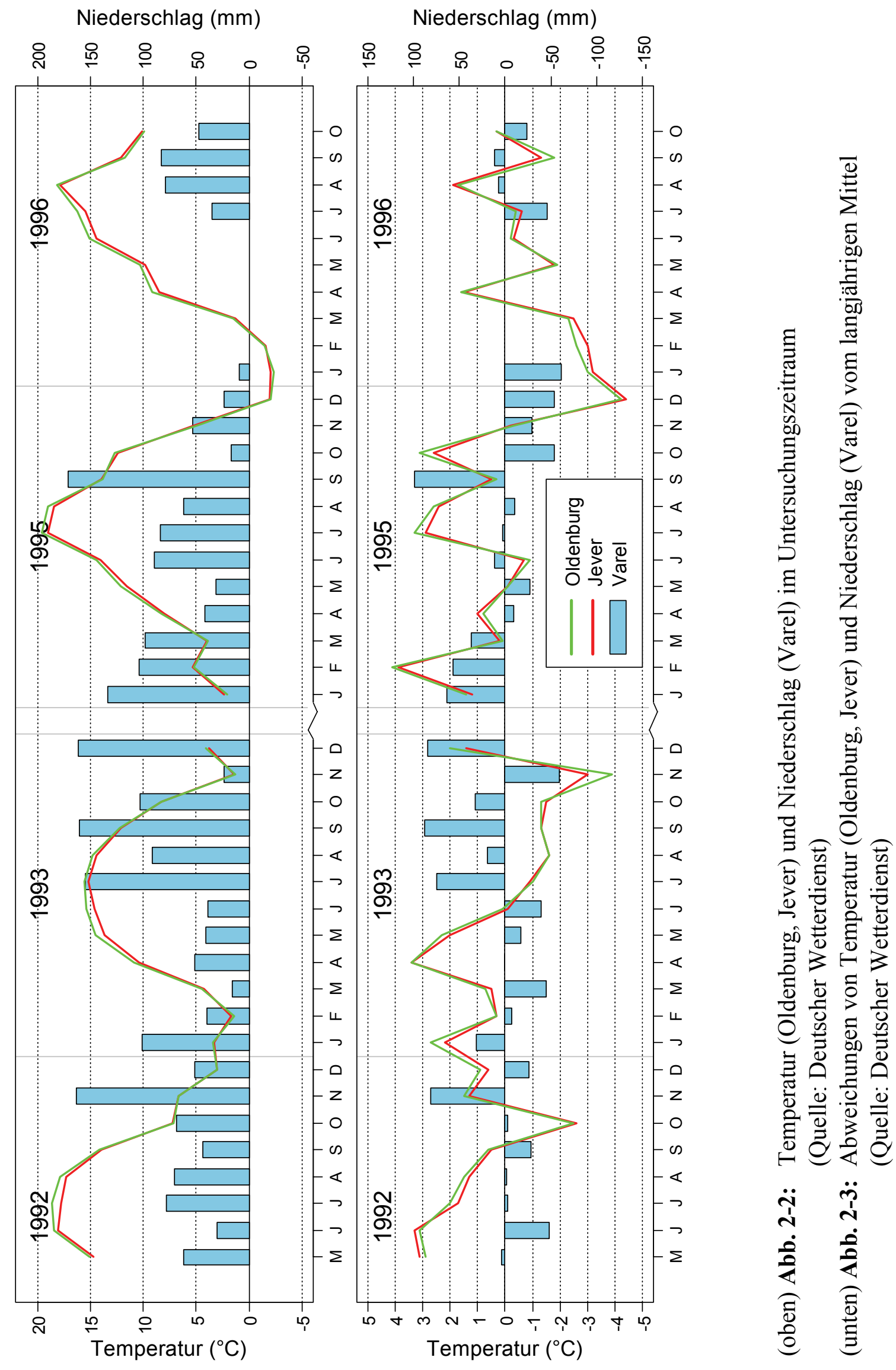




\subsection{Geologie und Böden}

Der 'Neuenburger Urwald' stockt auf einem Grundmoränenrücken. Unter einer Grundmoränendecke von geringer Mächtigkeit steht Lauenburger Ton an. Es handelt sich dabei um elstereiszeitliche Beckenabsätze, die im Neuenburgerholz eine Dicke von bis zu $60 \mathrm{~m}$ erreichen (SCHUCHT 1908, nach KOOP 1981). Der Lauenburger Ton wird von drenthestadialen Geschiebelehmen, Geschiebedecksanden oder Schmelzwassersanden überlagert (KRIEBITZSCH 1989). Auf dem größten Teil der Fläche überdeckt eine ca. $40-70 \mathrm{~cm}$ mächtige Schicht aus verlehmten Geschiebedecksanden den schwarzen Beckenton.

Der in etwa 40 bis $130 \mathrm{~cm}$ Tiefe anstehende Ton bildet einen Leithorizont und Grundwasserstauer. Daher herrschen im Gebiet des Neuenburgerholzes schwach wechselfeuchte Stauwasserstandorte vor. Die Standorttypenkarte weist für den größten Teil der Fläche eine ziemlich gute bis gute Nährstoffversorgung aus. Nur in einem kleineren Bereich des Naturschutzgebietes, wo die Decksande unverlehmt sind, ist die Nährstoffversorgung mäßig. Im Zentrum des Gebietes wird der Lauenburger Ton von einer bis zu $80 \mathrm{~cm}$ mächtigen Auflage aus Plaggenesch überlagert, die ihrerseits wiederum zum Teil von Sand bedeckt ist (KRIEBITZSCH 1989). Diese ehemals als Acker genutzte Fläche ist vermutlich vor über 400 Jahren wieder aus der landwirtschaftlichen Produktion herausgenommen worden (KOOP 1981). Der größte Teil des 'Neuenburger Urwaldes' weist ungestörte Böden auf, die keinen Hinweis auf frühere ackerbauliche Nutzung geben. Nach KOOP (1981) ist „,... de bodem .. voor het grootste deel ongestoord en heeft altijd onder bos gelegen".

Darüber hinaus finden sich verschiedene natürliche Senken im Untersuchungsgebiet, die als Viehtränken genutzt und zu diesem Zweck wahrscheinlich auch partiell vertieft worden sind.

\subsection{Geschichte}

Die große Bedeutung der Biotoptradition für die Struktur rezenter Biozönosen, auch und besonders der an Totholz gebundenen Arten, gibt Veranlassung, dem Werdegang des 'Neuenburger Urwaldes' etwas größere Aufmerksamkeit zu schenken.

Sowohl die Zusammensetzung der Vegetation als auch die Struktur dieses, irreführender Weise als Urwald bezeichneten, Waldes läßt die Spuren seiner Nutzungsgeschichte deutlich erkennen. Um das heutige Vegetationsbild zu verstehen, ,... ist es nötig, sich frühere Wirtschaftsweisen und ihre Auswirkungen stets vor Augen zu halten" (ELLENBERG 1986). Erst seit dem 17. Jahrhundert geben die Quellen näheren Aufschluß über die Nutzung und den Zustand des Neuenburgerholzes. Für den davorliegenden Zeitraum muss auf die allgemeinen Erkenntnisse der Forstgeschichte zurückgegriffen werden.

Bis in die jüngste Vergangenheit war der Wald im Wirtschaftssystem der ländlichen Bevölkerung von existentieller Bedeutung. Der Wald mußte den Bedarf an Brennholz decken. In den Geest-, Marsch- und Moorgebieten der Region mangelte es zudem an Steinen als Baumaterial, daher waren Holz und Lehm die wichtigsten Baustoffe. Darüber hinaus bestand ein großer Bedarf an Holz für den Bau von Schiffen, Brücken, Wegen und Deichen (NITZSCHKE 1932). Besonders die Eiche war wegen ihrer Dauerhaftigkeit als Bauholz sehr geschätzt. 
Kleinholz wurde durch Kopfholzbetrieb als eine Form der Niederwaldwirtschaft gewonnen. Hierzu wurde insbesondere die Hainbuche in 2-3 m Höhe auf den Stock gesetzt und die Stockausschläge in kurzen Umtriebszeiten geerntet. Diese Hiebsform bot ,,... geradezu ideale Voraussetzungen für eine gleichzeitige Kombination mit der Waldhude" (BURRICHTER 1984), da eine Verbißgefährdung durch das Vieh nicht bestand und gleichzeitig die Mastbäume geschont wurden.

Neben der Holzproduktion war der Wald eine unverzichtbare Grundlage für die Ernährung der Menschen. Die Viehwirtschaft war angewiesen auf die Waldweide und mit der Haltung von Schweinen gewann die Mastnutzung zunächst an Bedeutung. „In Breitenwirkung und Andauer ist keine Maßnahme des Menschen mit der extensiven und den Wald einbeziehenden Weidewirtschaft zu vergleichen" (ELLENBERG 1986). 1779 wurden 234 Pferde, 961 Stück Hornvieh, 660 Schweine und 1282 Gänse in das Neuenburgerholz getrieben (NITZSCHKE 1932). Hundert Jahre später hatte sich die Zahl bereits deutlich verringert. Erst zu Beginn des 20. Jahrhunderts ging die Zeit der Weidenutzung im Neuenburgerholz zu Ende.

Die Waldweide hatte eine Auflichtung der Bestände zur Folge, da aufkommender Jungwuchs von dem Vieh verbissen wurde. Höherer Licht- und Wärmegenuß bewirkten eine Ausbreitung von Gräsern und Kräutern, wodurch sich das Futterangebot deutlich verbesserte. Bisweilen wurde dieser gern gesehene Auflichtungsprozeß durch das Ringeln von Bäumen befördert, wie es auch aus dem Neuenburgerholz berichtet wird (NITZSCHKE 1932). Zugleich förderte der selektive Verbiß durch das Vieh jene Arten, die vom Vieh wegen ihrer Inhaltsstoffe oder aber aufgrund ihrer Ausstattung mit Dornen oder Stacheln gemieden wurden (z. B. Schlehe, Weißdorn, Stechpalme, Rubus-Arten). Im 'Neuenburger Urwald' war besonders der an mild-humides Klima gebundene Ilex begünstigt. In einer Forstbeschreibung aus dem Jahre 1705 ist denn auch von „vielem Hülsenunterbusch“ die Rede (nach NITZSCHKE 1932).

Auch die Mästung der Schweine fand, zumindest bis zur Einführung der Kartoffel, fast ausschließlich im Wald statt (HESMER \& SCHRÖDER 1963). Mit der Schweinezucht stieg die Bedeutung der mastproduzierenden Baumarten Eiche und Buche. Im nordwestdeutschen Tiefland wurde der Eiche der Vorzug gegeben (BURRICHTER 1984), die in den aufgelichteten Wäldern die Vorherrschaft errang und sich ,... in den geschützten alten 'Hudewäldern' in Form von mächtigen Stämmen erhalten" hat (WALTER 1979). Der Schweineeintrieb zur Mastnutzung wirkte sich in der Regel nicht negativ auf den Bestand aus. Vielmehr lockerte die Wühltätigkeit der Tiere den Boden und erleichterte die Naturverjüngung. Vor allem war die Notwendigkeit der Mastnutzung ein wichtiger Grund für den Erhalt und den Schutz der 'fruchtbaren Bäume' und trug somit zum Erhalt des Waldes bei.

In den Hudewäldern Nordwestdeutschlands war die Kopfschneitelung insbesondere der Hainbuche zur Gewinnung von Laubheu weit verbreitet (BURRICHTER 1984). Das im Sommer geerntete und getrocknete Laub wurde als Viehfutter für die Wintermonate verwendet. Die Kopfschneitelwirtschaft ähnelte sehr der Kopfholzwirtschaft zur Kleinholzgewinnung, lediglich die Umtriebszeiten waren auf ca. 3-4 Jahre verkürzt (POTT \& HÜPPE 1991).

Weit verbreitete Formen der Waldnutzung waren zudem die Streunutzung und das Plaggenstechen. Beide Nutzungsformen wurden vermutlich auch im Neuenburgerholz praktiziert (NITZSCHKE 1932). Sowohl die Laubstreu als auch die Plaggen wurden i. d. R. als Stallstreu in den Wintermonaten genutzt und anschließend als Dünger auf die Felder verbracht. Die Folgen für den Wald waren noch gravierender als die der Waldweide. Nährstoffexport, Aus- 
hagerung und Erosion des Bodens bedeuteten eine nachhaltige Schädigung der Leistungsfähigkeit des Standorts.

Die Summe der waldschädigenden Nutzungsformen, insbesondere ungeregelte Holznutzung, starker Weidebetrieb, das Plaggenstechen und die Nutzung der Streu, hatte eine Sukzession von Degradationsstadien zur Folge, die vielerorts mit der vollständigen Vernichtung des Waldes endete (HESMER \& SCHRÖDER 1963). Die Auswirkungen und die Geschwindigkeit dieses Prozesses waren einerseits abhängig von Art und Intensität der Eingriffe, andererseits von den Standortbedingungen und der Regenerationsfähigkeit und Verbißresistenz der Baumarten (HESMER \& SCHRÖDER 1963; POTT \& HÜPPE 1991; ELLENBERG 1986). Dass der 'Neuenburger Urwald' von der Vernichtug verschont geblieben ist, lag nach NITZSCHKE (1932) nicht zuletzt an der ,,... großen Regenerationskraft des Eichen-Hainbuchenwaldes auf gutem Boden". Laut Forstbeschreibung aus dem Jahre 1676 war das Neuenburgerholz zu diesem Zeitpunkt das größte und auch beste Waldgebiet der ehemaligen Grafschaft Oldenburg (nach WEHAGE 1930).

Lange Zeit gehörte das Neuenburgerholz zur gemeinen Mark, Boden und Aufwuchs waren gemeinschaftlicher Besitz der umliegenden Dorfschaften. Um eine Übernutzung der Bestände zu vermeiden, wurde das Recht der Markgenossenschaft auf Bauholzgewinnung schon früh reglementiert. „Uraltes Herkommen war die Anmeldung und Einholung der Erlaubnis, Bauholz zu hauen" (KREMSER 1990). Verstöße scheinen aber eher die Regel als die Ausnahme gewesen zu sein (WEHAGE 1930). Vermutlich schon seit dem frühen 15. Jahrhundert stand die Holzgerechtigkeit dem Landesherrn zu (NITZSCHKE 1932; KOOP 1981). Die Berechtigung zur Viehweide und zur Nutzung abgestorbener Bäume verblieb auch weiterhin bei den drei Ortschaften Bockhorn, Astede und Grabstede (TAUX 1986).

Um der ungeregelten Waldnutzung mit ihren negativen Auswirkungen auf den Zustand des Waldes entgegenzuwirken und wohl auch aufgrund jagdlicher Interessen erklärte der Oldenburger Graf Anton-Günther im Jahre 1654 größere Teile des Neuenburgerholzes ,„... als der Herrschaft gehörig” (nach KRIEBITZSCH 1989), ,... damit man bey begebenden Nothfällen $z u$ des gantzen Landes Ungelegenheit dessen keinen Mangel leiden und empfinden möge" (zit. nach NITSCHKE 1932). Gleichzeitig wurden die Nutzungsrechte der ländlichen Bevölkerung eingeschränkt. Eine Holzordnung aus dem Jahre 1656 gestattete nur die Nutzung von Weichhölzern und die gelegentliche Fällung von ,... sohren, unfruchtbaren Eichen- oder Büchenbaum zur Erhaltung ihrer Gebäude ... “ (zit. nach NITZSCHKE 1932). Um sich keiner Verstöße schuldig zu machen, wußte die Landbevölkerung sich zu helfen, indem sie kurzerhand einzelne Bäume ihrer Rinde entledigte um sie so in den „sohren” Zustand zu versetzen, was natürlich in späteren Holzordnungen entsprechende Strafandrohungen nach sich zog (NITZSCHKE 1932). Aus dem gleichen Jahr datiert auch die erste allgemeine Verfügung des Oldenburger Grafen über den Laubholzanbau, in der angeordnet wird, für jeden gefällten Eichen- bzw. Buchenbaum drei oder vier Heister zu pflanzen und zu schützen (HESMER \& SCHRÖDER 1963). Nicht eingeschränkt wurden dagegen die Berechtigungen zur Waldweide - mit der Ausnahme, dass Ziegen wegen ihrer besonders nachteiligen Wirkung auf den Wald nicht mehr eingetrieben werden durften - die Streunutzung und das Plaggenstechen.

Dass die unter Androhung von Leibesstrafen erlassenen und in kurzen Zeitabständen wiederholten Verordnungen offensichtlich nur wenig Erfolg hatten, davon legen die Holzordnungen und Forstbeschreibungen aus der Folgezeit reges Zeugnis ab (NITZSCHKE 1932). Insbesondere in den Jahren der 'Dänenzeit' (1667-1773) erfuhr das Neuenburgerholz eine starke Reduktion der bewaldeten Flächen. Da die Obrigkeit weit entfernt in Kopenhagen saß 
und die bestellten Holzknechte und -vögte eigene Interessen verfolgten, fand eine Kontrolle der Nutzungen kaum mehr statt. Trotz des Raubbaus wird in einer Forstbeschreibung aus dem Jahre 1705 der Forstort Schaar - zu dem das heutige Schutzgebiet gehört - als ,,... eine(r) der besten und größesten Örter des Neuenburger Buschs ..." bezeichnet, „... worinnen zwar eine große Menge der besten Bäume verhauen, wegen Versohrung verkaufet und vom Sturm umbgeworfen ..." sind, in dem aber noch viele alte Eichen in lückigem Bestand wachsen (nach NITZSCHKE 1932). Nicht nur Holzdiebstahl und Mißachtung der Anordnungen zur Verjüngung des Waldes scheinen also an der Tagesordnung gewesen zu sein, es gelang den Bauern auch, sich Waldbesitz anzueignen. Im 18. Jahrhundert waren ca. 80 \% der Fläche des Neuenburgerholzes waldfrei, nicht jedoch die Fläche des heutigen Schutzgebietes (KOOP 1981).

In der zweiten Hälfte des vorigen Jahrhunderts wurde die Fläche des 'Neuenburger Urwaldes' durch eine Verordnung des Oldenburger Großherzogs Nikolaus-Friedrich-Peter (1853-1900) aus der forstlichen Nutzung herausgenommen. Der 'Neuenburger Urwald' wurde als Ausschlußforst weitgehend sich selbst überlassen, totes Holz durfte allerdings zunächst weiter genutzt werden. 1938 wurde der 48,5 ha umfassende Forstort Große Schaar schließlich unter Naturschutz gestellt. Unberührt von der Schutzverordnung bleiben die Jagdausübung und ,die forstliche Nutzung kranker und abständiger Stämme sowie langschäftiger Eichen mit hohem Nutzholzwert" (Oldenburgisches Staatsministerium 1938), wovon die Forstverwaltung allerdings keinen Gebrauch macht. Lediglich die das Gebiet durchziehenden Wanderwege werden von umgestürzten Bäumen geräumt, ohne das Holz zu entnehmen. Nach dem zweiten Weltkrieg fiel mehr als die Hälfte des Gebietes der Säge zum Opfer, um das Holz für Brennholzzwecke nutzen zu können, so dass heute nur noch etwa 23 ha des alten Hutewaldes erhalten sind. $\mathrm{Zu}$ diesem Zeitpunkt wurden auch bereits abgestorbene Bäume der erhalten gebliebenen Fläche entfernt.

\subsection{Vegetation und Bestandesstruktur}

Das heutige Waldbild des 'Neuenburger Urwaldes' ist das Ergebnis natürlicher Standortbedingungen, anthropogener Überformungsprozesse und über einhundertjähriger spontaner Eigenentwicklung in Richtung auf die potenzielle natürliche Vegetation. Zwar zählen POTT \& HÜPPE (1991) den 'Neuenburger Urwald' zu den durch die Extensivwirtschaft ,... nur schwach überformten ehemaligen Bannwäldern ...", die Auswirkungen der historischen Waldnutzungsformen auf dessen floristische Zusammensetzung und Bestandesstruktur sind aber auch in der Gegenwart deutlich ablesbar.

Das Bestandesbild wird beherrscht von seinem sehr alten Baumbestand aus Stieleiche (Quercus robur) und Rotbuche (Fagus sylvatica), die in der Oberschicht dominieren. Das Alter der für das Gebiet so charakteristischen knorrigen Huteeichen, die allesamt aus Pflanzung hervorgegangen sind (KOOP 1982), wird auf 300 bis 500 Jahre geschätzt, einzelne Exemplare sollen sogar 800 Jahre alt sein. Für die Rotbuche wird ein Alter von bis zu 400 Jahren angegeben (KRIEBITZSCH 1989). Im Unterstand finden sich zahlreiche 200 bis 300 Jahre alte Hainbuchen (Carpinus betulus). Als weitere Baumarten sind überwiegend einzelstammweise Feld-, Spitz- und Bergahorn (Acer campestre, A. platanoides, A pseudoplatanus), Gold- und Warzen-Birke (Betula lutea, B. pendula) und Esche (Fraxinus exelsior) beigemischt. Ein von einem Windwurf betroffener Waldteil wurde um die Jahrhundertwende mit Roteiche (Quecus rubra) ausgepflanzt. 
Die Wuchsformen der Baumindividuen sind „.... Zeugen extensiver Betriebsformen des historischen Waldes mit seinen vielfältigen Funktionen" (BURRICHTER 1984). Dies gilt in besonderem Maße für die alten Huteeichen, die mit ihren mächtigen Stämmen und tief ansetzenden, ausladenden Kronen dem Habitus von Solitärbäumen entsprechen und damit Zeugnis ablegen von der ehemaligen offenen Struktur eines Hutewaldes. Vereinzelt finden sich noch sogenannte Armleuchterbuchen. Dabei handelt es sich um Mehrstammbäume, die aus Büschelpflanzungen hervorgegangen sind und deren Einzelstämme im unteren Bereich zu einem einzigen Stamm verwachsen sind. Von der ehemaligen Kopfholznutzung zeugen die kandalaberförmigen Kronenformen der Hainbuchen. In Abtriebshöhe, wo die zahlreichen Äste ansetzen, haben sich mächtige Überwallungswülste ausgebildet. Wegen der instabilen Baumarchitektur sind sie anfällig gegen Sturm und brechen, ebenso wie die Armleuchterbuchen, leicht auseinander, so dass nur noch wenige markante Einzelexemplare erhalten sind.

Im Unterstand ist eine artenreiche Kraut- und Strauchschicht ausgebildet. Beherrscht wird das Unterholz von der durch die Weideselektion begünstigten Stechpalme (Ilex aquifolium), die vielerorts ein kaum zu durchdringendes Dickicht bildet. An einigen Stellen haben sich hallenförmige Bestände von bis zu 15 m Höhe ausgebildet. Einzelne Exemplare erreichen einen Durchmesser von über $20 \mathrm{~cm}$ (KRIEBITZSCH 1989). Auf den zahlreichen Lichtungen, wo der schützende Überhalt für den früh- und spätfrostgefährdeten Ilex fehlt, wird die Strauchschicht von dichten Beständen aus Rubus-Arten dominiert ( $R$. fruticosus, $R$. idaeus). Des weiteren finden sich Hasel (Coryllus avellana), Weißdorn (Crataegus laevigata, $C$. monogyna), Faulbaum (Frangula alnus), Geißblatt (Lonicera periclymenum), Schlehe (Prunus spinosa) und Eberesche (Sorbus aucuparia). Entsprechend der kleinräumig sehr heterogenen Belichtungsverhältnisse ist auch die Ausprägung der artenreichen Kraut- und Strauchschicht sehr variabel und reicht von vegetationsfreien Partien bis hin zu stark vergrasten bzw. mit Rubus-Arten bewachsenen Lichtungen.

POTT \& HÜPPE (1991), an deren Ausführungen sich die folgende Darstellung orientiert, haben in ihrer vegetationskundlichen Untersuchung des 'Neuenburger Urwaldes' drei Waldtypen ausgeschieden, die fließend ineinander übergehen (Abb. 2-4). Die verschiedenen Stadien und Phasen der Waldentwicklung zeigen zudem deutliche Unterschiede in ihrer floristischen Zusammensetzung.

Auf meso- bis eutrophen, stau- und grundwasserbeeinflussten, vergleyten Lehmböden stockt der Waldziest-Eichen-Hainbuchenwald (Stellario-Carpinetum stachyetosum). Die Baumschicht dieser azonalen, nährstoffbedingten Subassoziation des Eichen-HainbuchenWaldes wird von der Eiche beherrscht, in deren Unterstand sich eine zweite Baumschicht aus Hainbuchen etabliert hat. Die Buche fehlt weitestgehend - auch in der Verjüngung - wegen ihrer Empfindlichkeit gegenüber Staunässe. Die Krautschicht ist artenreich. Vor allem anspruchsvolle Arten wie z. B. das Buschwindröschen (Anemone nemorosa), die Sternmiere (Stellaria holostea), der Waldziest (Stachys sylvatica), das Scharbockskraut (Ranunculus ficaria) und das Große Hexenkraut (Circaea lutetiana) prägen das Bild.

Auf den frischen, gut nährstoffversorgten Böden mit tieferliegendem Grundwasser findet die Buche sehr gute Wuchsbedingungen und verjüngt sich leicht. Sie ist hier der Eiche in der Wuchskraft deutlich überlegen und es hat sich ein Milio-Fagetum (Flattergras-Buchenwald) ausgebildet. Der immer noch geringe Buchenanteil im Oberstand ist das Ergebnis der früheren, eichenbegünstigenden Nutzungsformen, während in der Verjüngung bereits die Buche dominiert. In der Krautschicht finden sich u. a. das Flattergras (Milium effusum), das 
Buschwindröschen (Anemone nemorosa), die Wald-Segge (Carex sylvatica), der Waldmeister (Galium odoratum) und die Goldnessel (Lamiastrum galeobdolon).

Als dritter Waldtyp läßt sich auf mäßig trockenen bis frischen, anlehmigen und ärmeren (oligotrophen) Standorten das Fago-Quercetum (Buchen-Eichenwald) ausscheiden. Hier herrscht die Buche vor, während Eiche und Hainbuche in nur geringer Anzahl vertreten sind. In einigen kleineren Bereichen beteiligt sich die Sandbirke (Betula pendula) an der Oberschicht. In der artenarmen Krautschicht, deren Deckungsgrad gering ist, finden sich u. a. Hain-Veilchen (Viola riviniana), Rasen-Schmiele (Deschampsia caespitosa), Heidelbeere (Vaccinium myrtillus), Wiesen-Wachtelweizen (Melampyrum pratense) und Zweiblättrige Schattenblume (Maianthemum bifolium).

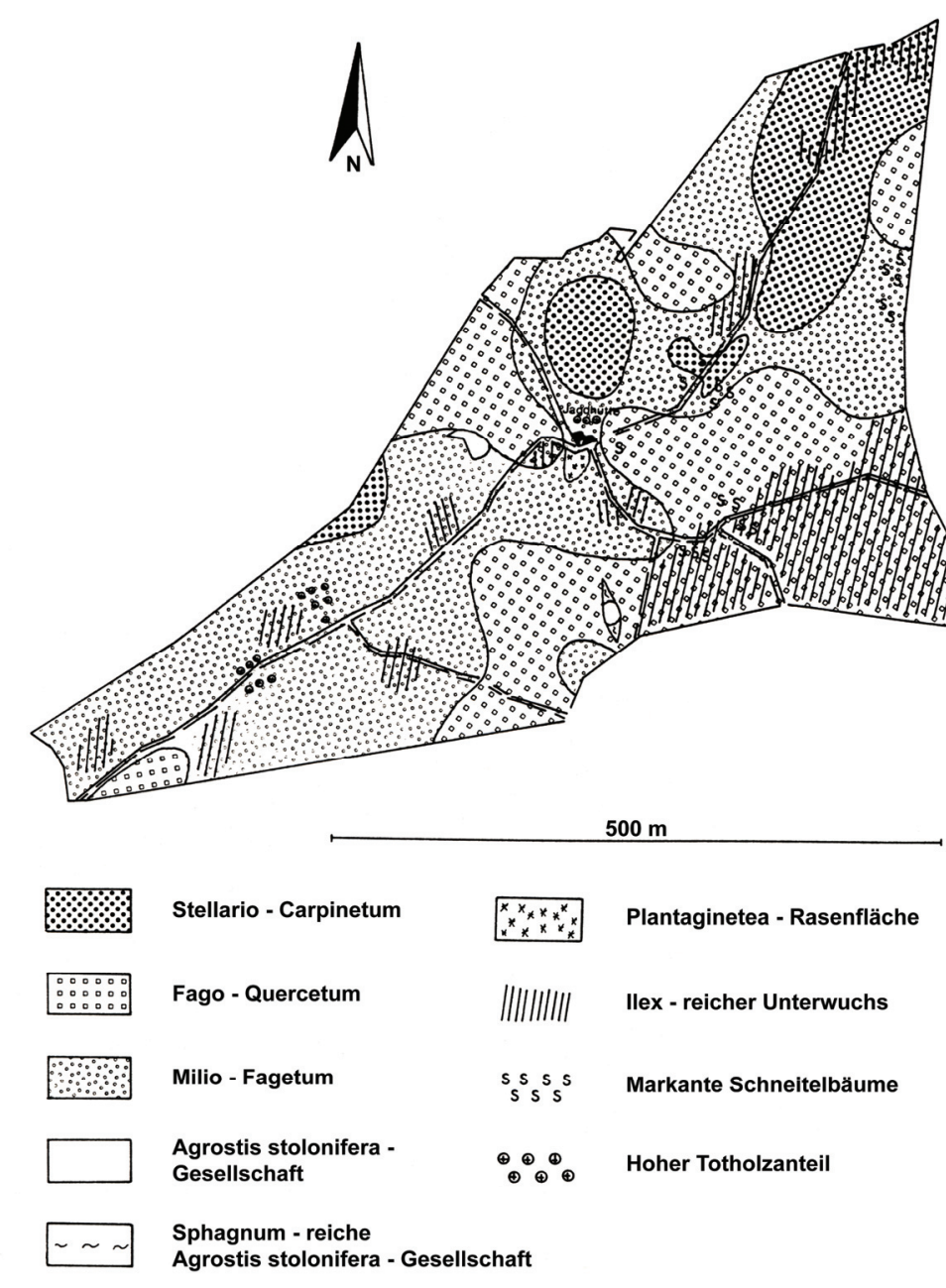

Abb. 2-4: Vegetationskarte (aus POTT \& HÜPPE 1991)

In feuchten, versumpften Bodensenken, die einstmals als Tränken für das Vieh genutzt wurden, hat sich eine feuchtigkeitsliebende Vegetation eingestellt, die sich u. a. aus Weißem Straußgras (Agrostis stolonifera), Winkel- und Schlank-Segge (Carex remota, C. gracilis), Flatterbinse (Juncus effusus), Flutender Schwaden (Glyceria fluitans), Sumpf-Labkraut (Galium palustre), Sumpf-Hornklee (Lotus uliginosus), Gemeines und Kleines Helmkraut (Scutellaria galericulata, Sc. minor), Faulbaum (Frangula alnus), Ohr- und Grau-Weide (Salix aurita, S. cinerea) sowie verschiedenen Sphagnum-Arten zusammensetzt. 
Mit abnehmender Bedeutung des Waldes für die Viehwirtschaft und aufgrund seiner Behandlung als Ausschlußforst, in dem forstwirtschaftliche Eingriffe weitgehend ausblieben, konnte sich der 'Neuenburger Urwald' (fast) ausschließlich seiner Eigendynamik entsprechend entwickeln, in dessen Folge sich sein Erscheinungsbild nachhaltig verändert hat. Der Bestand hat den ehemals offenen Charakter eines typischen Hutewaldes weitgehend eingebüßt und zeichnet sich heute durch seine hohe strukturelle Vielgestaltigkeit aus. „In seiner mosaikartigen Plenterstruktur zeigt der Wald ... verschiedenartige Zerfalls- und Verjüngungsphasen mit den spezifischen kleinstandörtlichen Differenzierungen" (POTT \& HÜPPE 1991). Die nachfolgenden Ausführungen zur Bestandesstruktur beruhen im Wesentlichen auf den Analysen von KOOP $(1981,1982)$.

Wie aus der Abbildung 2-5 ersichtlich wird, bilden die verschiedenen Entwicklungsphasen ein sehr kleinräumiges Sukzessionsmosaik. Die durchschnittiche Größe der verschiedenen Stadien und Phasen der Waldentwicklung und ihr Flächenanteil lassen eine Abhängigkeit vom Vegetationstyp erkennen. Im Stellario-Carpinetum ist die in Verjüngung befindliche Fläche und der mittlere Durchmesser der Verjüngungseinheiten am geringsten, während die entsprechenden Werte für das Fago-Quercetum deutlich höher ausfallen. Das Milio-Fagetum nimmt eine mittlere Position ein. Sie sind das Ergebnis des Zusammenbruchs einzelner Stämme (besonders im Stellario-Carpinetum) bzw. größerer Bestandesteile (häufiger im Fago-Quercetum). Dort, wo sich bereits Verjüngung im Unterstand etabliert hatte, wächst unmittelbar die nächste Baumgeneration heran, ansonsten entstehen vielfach Lichtungen, die oft über einen längeren Zeitraum von lichtliebenden Kräutern, Rubus-Arten oder Adlerfarn dominiert werden. Der Anteil dieser Kräuterstadien beträgt den Angaben von KOOP (1982) zufolge 10 bis $15 \%$. Fast ausschließlich im Fago-Quercetum lassen sich Pionierwaldstadien aus Birke und Eberesche beobachten. In späteren Stadien stellt sich eine stärkere Baumartenmischung und größere Ungleichaltrigkeit ein.

Auch in der Vertikalstruktur unterscheiden sich die Waldgesellschaften des 'Neuenburger Urwaldes', und zwar ist eine zunehmende Differenzierung vom Fago-Quercetum über das Milio-Fagetum zum Stellario-Carpinetum festzustellen. Die Bestände auf nährstoffärmeren Standorten tendieren zu größerer Einförmigkeit und Einschichtigkeit (KOOP 1982). 


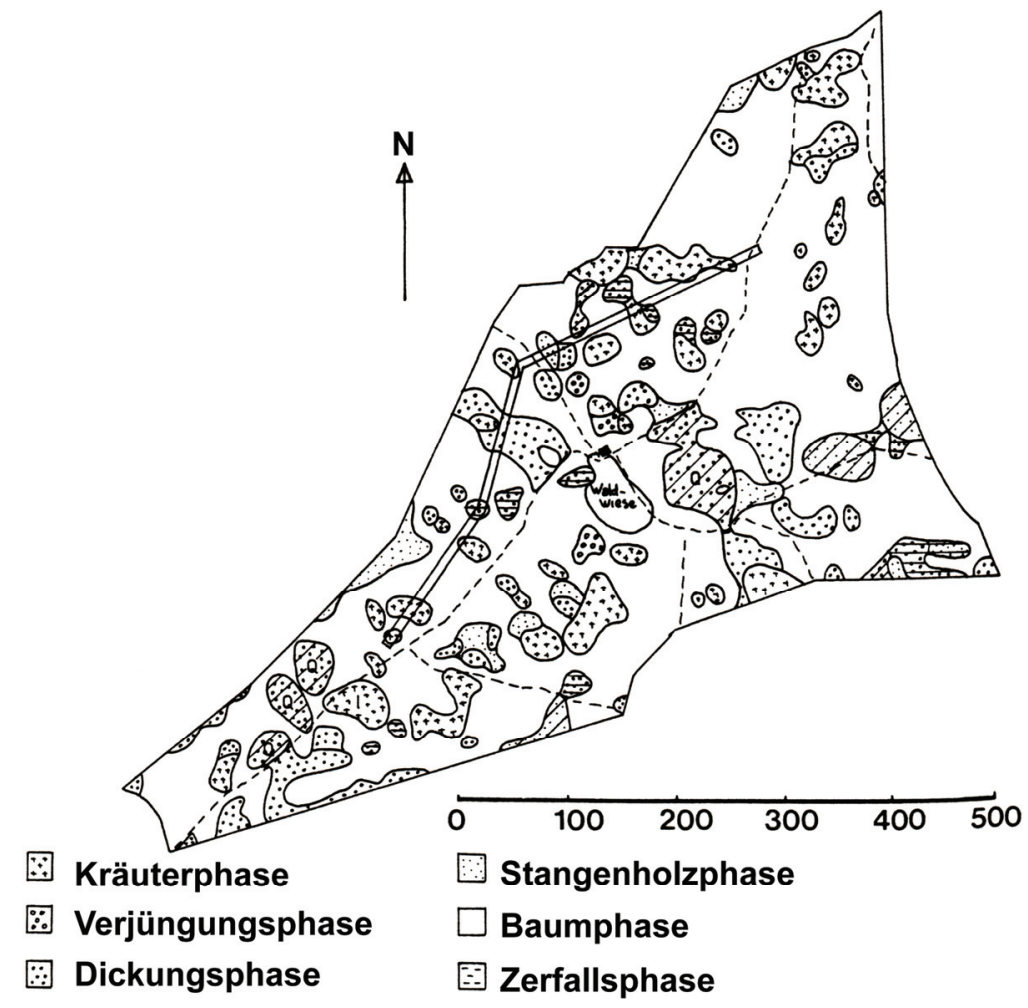

Abb. 2-5: Stadien und Phasen des Sukzessionsmosaiks (aus KOOP 1982)

Mit dem Ende der eichenbegünstigenden Nutzung des Waldes hat sich der Bestand geschlossen, wird aber immer wieder dort durchbrochen, wo ein umgestürzter Baum eine Lücke hinterlassen hat. Gleichzeitig hat sich die Baumartenzusammensetzung zu Gunsten der Buche verschoben. Sowohl WEHAGE (1930) als auch NITZSCHKE (1932) beobachteten bereits auf vielen Standorten des 'Neuenburger Urwaldes' die Konkurrenzüberlegenheit der Buche. Insbesondere in den jüngeren Altersklassen ist die Eiche kaum vertreten, weshalb NITZSCHKE (1932) eine Entwicklung zu einem reinen Buchenstadium prognostizierte. Nur auf den stark grundwasserbeeinflussten Standorten ist die Buche unterlegen (POTT \& HÜPPE 1991). Aufgrund des Umstandes, dass in alten Forstbereitungsprotokollen die Erle als häufig anzutreffende Baumart des Neuenburgerholzes genannt wurde, ist von verschiedenen Autoren (NITZSCHKE 1932; WEHAGE 1930; HESMER \& SCHRÖDER 1963) die Annahme geäußert worden, dass auch ohne die eichenfördernde Wirkungen der historischen Waldnutzungsformen von einer standortbedingten Vorherrschaft der Eiche in der ursprünglichen Waldgesellschaft ausgegangen werden kann (HESMER \& SCHRÖDER 1963). Als Erklärung für die sich abzeichnende Entwicklung zu einem von der Buche dominierten Wald auf dem überwiegenden Teil der Standorte läßt sich anführen, dass zum einen die im lichten Bestand aufgewachsenen Eichen nicht ihr volles Potenzial zum Höhenwachstum ausgeschöpft haben und deshalb leicht von nachwachsenden Buchen ausgedunkelt werden, zum anderen, dass vor allem durch irreversible Standortveränderungen in Folge der Anlage von Entwässerungsgräben und von Grundwasserabsenkung das Konkurrenzverhältnis zugunsten der Buche verschoben worden ist. „Im ganzen entsteht also der Eindruck, dass die Buche im ... Neuenburgerholz in früherer Zeit nie eine solche Dominanz erreicht hat, wie sie sich heute anbahn “ (HESMER \& SCHRÖDER 1963). Um dieser Entwicklung entgegenzutreten, wurden in jüngster Vergangenheit einige der alten Eichen freigestellt und einige größere Lücken mit Eichen ausgepflanzt. 

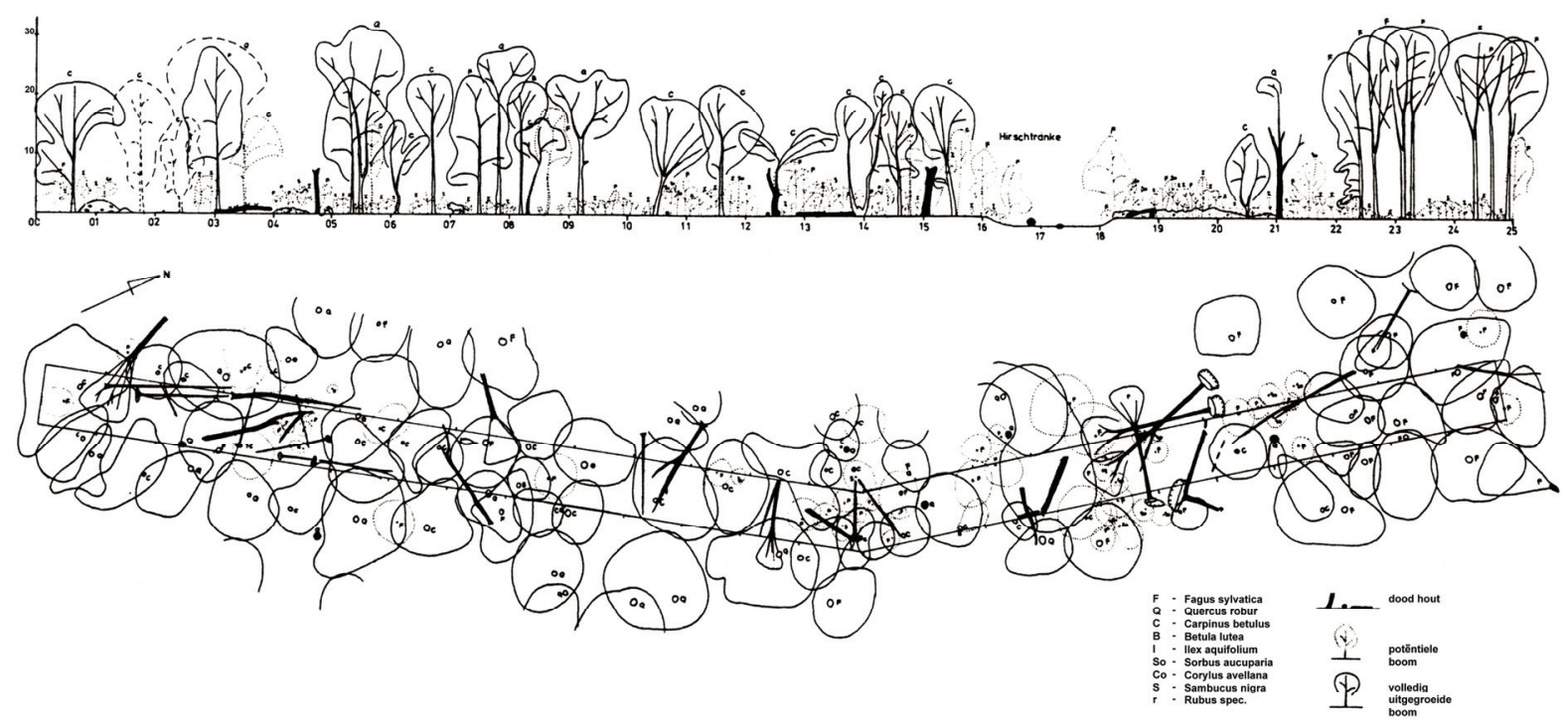

Abb. 2-6: Horizontal- und Vertikalstruktur (Grund- u. Aufriß) (aus KOOP 1982)

Ein wesentliches Strukturmerkmal des 'Neuenburger Urwaldes' ist das reichhaltige Angebot absterbender und abgestorbener Bäume. Nicht wenige der alten Baumveteranen sind in Folge von Überalterung und von Verletzungen partiell abgestorben und weisen in ihrem Innern deutliche Kernfäulen auf bzw. sind bereits mehr oder weniger stark ausgehöhlt und von Tieren, Moosen und Pilzen besiedelt. Überall im Bestand stehen und liegen starkdimensionierte Baumruinen verschiedener Zersetzungsgrade. Hinzu kommen größere Totholzvorräte im Kronenraum insbesondere der Eiche. Das Totholzangebot variiert nach Waldtyp und Entwicklungsphase. KOOP (1981) ermittelte im Milio-Fagetum eine Länge von 1000 m/ha, im Stellario-Carpinetum war es nur etwa die Hälfte. Auf einer 1,1 ha großen Kernfläche wurden von der Niedersächsischen Forstlichen Versuchsanstalt im Jahr 2000 die Vorräte des lebenden und abgestorbenen Bestandes ermittelt. Das Volumen des liegenden Totholzes belief sich hier auf 165, das des stehenden auf $47 \mathrm{~m}^{3} /$ ha. Die Holzbiomasse der lebenden Bäume betrug $488 \mathrm{~m}^{3} /$ ha (NFV 2005). Damit entfielen $30 \%$ der gesamten Holzbiomasse auf das Totholz. Da die letzte Totholzentnahme größeren Ausmaßes im 'Neuenburger Urwald' bereits mindestens 50 Jahre zurückliegt, ist der heutige Vorrat im Wesentlichen das Ergebnis natürlicher Absterbe- und Zersetzungsprozesse. In Verbindung mit der mosaikartigen Bestandesstruktur und deren Auswirkungen auf die Milieubedingungen stellt das große Totholzangebot im 'Neuenburger Urwald' ein vielfältiges Massenhabitat dar. 


\section{Untersuchungs- und Auswertungsmethoden}

\subsection{Fallenfänge}

Art und Anzahl der einzusetzenden Fallen sollten einerseits eine möglichst vollständige Erfassung des Artenspektrums xylobionter Käfer ermöglichen, andererseits aber auch Einblicke in die Vergesellschaftung dieser Arten sowohl in qualitativer als auch in quantitativer Hinsicht gewährleisten. Die Erfassung der Tiere erfolgte in den Jahren 1992, 1993, 1995 und 1996 mittels Verwendung verschiedener geschlossener Eklektortypen und Flugbarrierefallen. Während der Untersuchungsjahre waren die Fallen jeweils von April bis Oktober im Einsatz. Der Jahresfang einer Falle wird im folgenden als eine Probe behandelt. Die Leerungen und Kontrollen fanden in einem Drei-Wochen-Rhythmus statt, so dass eine Probe i. d. R. das Fangergebnis von acht Fangperioden beinhaltet (P1b-P8). Zu Beginn der Untersuchung kam es zu Verzögerungen bei der Fertigstellung einiger Fallen und in den Jahren 1992 und 1993 waren wenige Fallen schon früher (P1a) aktiviert worden (Tab. 3-1). Diesem Umstand wurde bei der Auswertung der Fänge Rechnung getragen, in vielen Darstellungen blieben die Fänge der Fangperiode P1a unberücksichtigt. Der erste Fangzeitraum der Untersuchungsjahre (P1a bzw. P1b) wich des öfteren geringfügig vom Drei-Wochen-Intervall ab, was auf den Witterungsverlauf im Frühjahr und auf die Dauer der Aufbauarbeiten zurückzuführen ist. Die Leerungstermine wurden so gelegt, dass die zeitliche Lage der einzelnen Fangperioden in den verschiedenen Untersuchungsjahren in etwa übereinstimmte, wodurch die Analyse phänologischer Daten deutlich erleichtert wurde. So umfaßte beispielsweise die dritte Fangperiode (P3) in allen Untersuchungsjahren einen Zeitraum von Anfang bis Ende Juni (vgl. auch Anhang 1).

Tabelle 3-1: Anzahl der in den einzelnen Fangperioden der Untersuchungsjahre fängischen Fallen

\begin{tabular}{|c|cccccccccc|}
\hline & \multicolumn{8}{|c|}{ Fa n g p e ri od e } \\
Jahr & P1a & P1b & P2 & P3 & P4 & P5 & P6 & P7 & P8 \\
\hline 1992 & 5 & 9 & 11 & 22 & 22 & 22 & 22 & 22 & 22 \\
1993 & 23 & 107 & 107 & 107 & 107 & 107 & 107 & 107 & 107 \\
1995 & & 121 & 121 & 121 & 121 & 121 & 121 & 121 & 121 \\
1996 & & 171 & 171 & 171 & 171 & 171 & 171 & 171 & 171 \\
\hline
\end{tabular}

\subsubsection{Totholzeklektoren}

\subsubsection{Das Eklektorprinzip}

Zur Erfassung der epi- und endotrunkalen Totholzfauna wurden wiederholt geschlossene Fallensysteme eingesetzt (z. B. DERKSEN 1941; REID 1963; NORD \& LEWIS 1970; GLEN 1976; BÜCHS 1988; OTTE 1989; OWEN 1989, 1992; WINTER 1991; SCHMITT 1992; 
RAUH 1993; NICOLAI 1995a). Entweder wird das zu untersuchende Totholz in den Eklektor verbracht oder die Fallen werden direkt am Objekt positioniert. Tiere, die das Substrat verlassen, fangen sich in angebrachten Behältern, die i. d. R. mit Fang- bzw. Konservierungsflüssigkeit (in der vorliegenden Untersuchung wurde $10 \%$-ige Essigsäure verwendet) gefüllt sind.

Durch die Möglichkeit, sowohl das Spektrum als auch die Häufigkeit der Arten zu erfassen und direkt mit dem durch sie besiedelten Totholzvolumen und dessen Eigenschaften in Beziehung zu bringen, sind Eklektoren hervorragend geeignet, Schlüpfabundanzen, die auftretenden Artenvergesellschaftungen und deren Veränderungen bei voranschreitender Sukzession zu analysieren. Durch den Bezug zum Entwicklungs- bzw. Aufenthaltsort der Tiere und durch den Betrieb über eine längere Fangperiode erlauben die Fänge Aussagen zur Schlüpfphänologie und Habitatbindung der Arten. Dem steht als Nachteil gegenüber, dass die Bewohner verschiedener Strukturteile wie Rinde, Splint- und Kernholz nicht separat erfaßt werden. Diese verfügen jedoch, trotz zahlreicher Übergänge, über mehr oder weniger eigenständige Artengemeinschaften und Sukzessionen (SCHIMITSCHEK 1953, 1954; DAJOZ 1966).

Da das vorrangige Interesse dieser Untersuchung der quantitativen Analyse der Käfergemeinschaften an Totholz galt, kam eine größere Anzahl unterschiedlicher Eklektortypen an liegendem und stehendem Totholz zum Einsatz. Wegen der beschränkten Anzahl der zur Verfügung stehenden Fallen und aus Gründen der Arbeitskapazität wurden die Eklektoren ausschließlich zur Untersuchung von Stieleiche (1992, 1993 und 1995) und Rotbuche (1995 und 1996) verwendet. Bei der Auswahl der Fallenstandorte wurde versucht, die Fallen an möglichst homogenen Stammabschnitten anzubringen.

\subsubsection{Stammeklektoren (STE)}

Für liegendes starkdimensioniertes Totholz sind stammumfassende, geschlossene Eklektoren entwickelt und in verschiedenen Untersuchungen erprobt worden (z. B. BEHRE 1989; OTTE 1989; RAUH 1993). Die in dieser Untersuchung eingesetzten STE orientierten sich an deren Fangprinzip, unterschieden sich jedoch durch die geänderte Konstruktion (Abb. 3-1).

Die $1 \mathrm{~m}$ langen STE wurden durch zwei $8 \mathrm{~cm}$ breite Aluminiumringe mit einem Durchmesser von $70 \mathrm{~cm}$ gehalten, die in einer Stofftasche geführt wurden. Zwischen den Ringen war Stoff gespannt. Die Ringe konnten auf einer Seite geöffnet werden und ließen sich so leicht um den Stamm legen. Ihr Abstand untereinander wurde durch Längs-, der Abstand zum Stamm durch Querabstandshalter reguliert. Die obere Längsverbindung trug eine Kopfdose (Fa. Ecotec, Bonn), auf der Unterseite waren zwei Weithalsflaschen angebracht. Sowohl der Stoffmantel als auch die Stirnseiten waren mit einem Klettverschluß befestigt. Die Stirnseiten wurden mit einem Spanndraht in eine gesägte stammumfassende Kerbe gezogen, die auch bei berindeten Stämmen bis auf den Holzkörper reichte, so dass ein dichter Abschluß zwischen Baumstamm und Falle weitgehend gewährleistet war und ein Ein- bzw. Auswandern über den subcorticalen Raum vermieden wurde. 


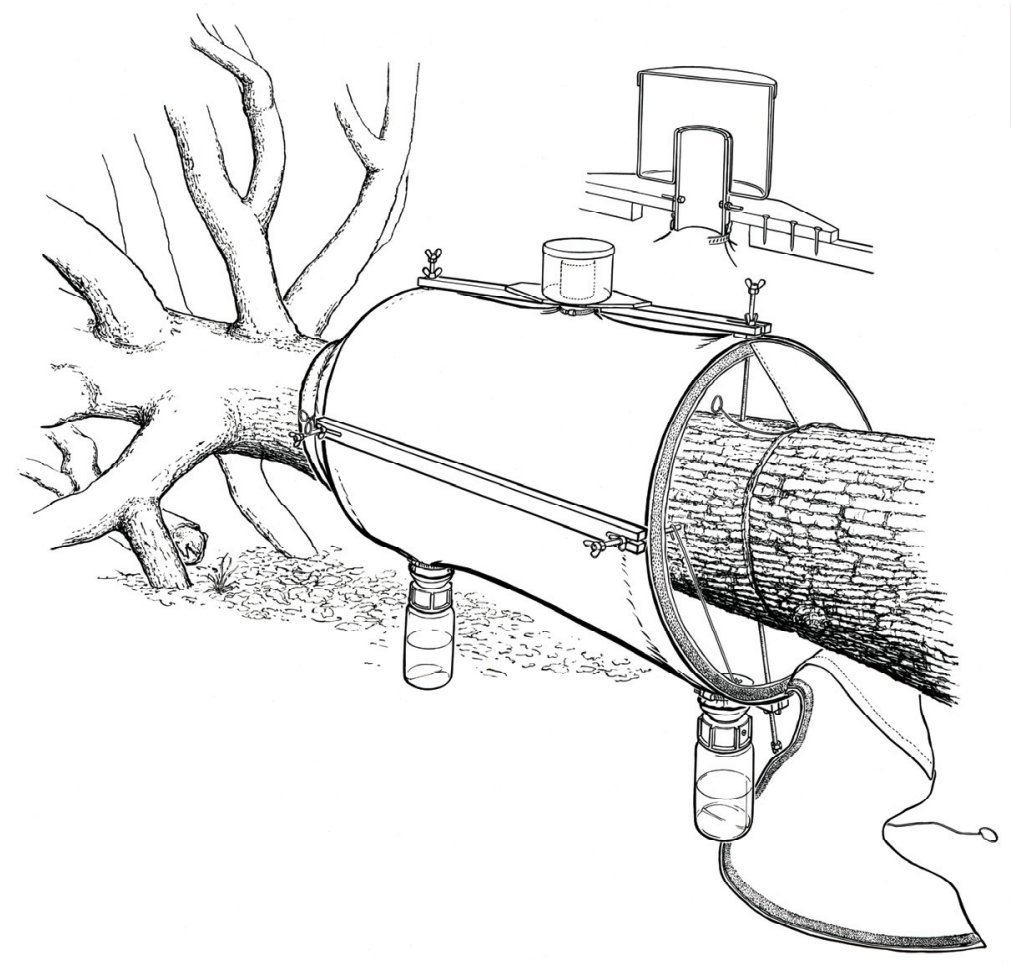

Abb. 3-1: Stammeklektor (STE)

Zahlreiche Stämme kamen wegen ihres Durchmessers für eine Beprobung nicht in Frage. Da aber trotzdem hinreichend viele geeignete Stämme zur Auswahl standen, wurde auf den Bau größerer Fallen verzichtet. Auch sehr stark zersetztes, mulmiges Totholz war für die Anbringung dieses Fallentyps vielfach nicht geeignet. Die Fallen wurden an auf- und hochliegenden Stämmen unterschiedlichen Zersetzungsgrades unter Berücksichtigung verschiedener Milieubedingungen aufgebaut. Dabei sollten die einzelnen Totholzabschnitte, soweit von außen ersichtlich, möglichst homogen sein.

Insgesamt wurden mit diesem Fallentyp 99 Proben genommen (Tab. 3-2). Die Oberfläche der befangenen Stammabschnitte belief sich auf 130,1 $\mathrm{m}^{2}$, von denen $85 \mathrm{~m}^{2}$ berindet waren. Das Volumen betrug 14,2 $\mathrm{m}^{3}$, der mittlere Durchmesser am Fallenstandort $42 \mathrm{~cm}$ o. R. Vereinzelt kam es zu Beeinträchtigungen der Probenahme durch Mäusefraß. Eine Falle fiel einer Brandstiftung zum Opfer. Nach starken Regenfällen sammelte sich gelegentlich Wasser in den Fangflaschen.

Tabelle 3-2: Anzahl der Proben pro Fallentyp und Untersuchungsjahr

\begin{tabular}{|c|c|c|c|c|c|}
\hline \multirow[b]{2}{*}{ Fallentyp } & \multicolumn{4}{|c|}{ Untersuchungsjahr } & \multirow[b]{2}{*}{ Summe } \\
\hline & 1992 & 1993 & 1995 & 1996 & \\
\hline STE & 11 & 27 & 35 & 26 & 99 \\
\hline EFs & & 50 & 36 & 55 & 141 \\
\hline EF1 & & & 11 & 50 & 61 \\
\hline mBE (Typ A) & 7 & 6 & & & 13 \\
\hline mBE (Typ B) & & & 19 & 20 & 39 \\
\hline FF (Typ A) & 4 & 4 & & & 8 \\
\hline FF (Typ B) & & 20 & 20 & 20 & 60 \\
\hline
\end{tabular}




\subsubsection{Emergenzfallen (EFs; EFI)}

Um die stehenden, starkdimensionierten, abgestorbenen Huteeichen und Buchen untersuchen zu können, mußte ein neuer Fallentyp entwickelt werden, da stammumfassende Konstruktionen hierfür nicht nur wegen ihres Durchmessers ungeeignet waren. Diese im Rahmen der Untersuchung neu entwickelte Emergenzfalle für stehendes Totholz (EFs) bestand aus zwei freien, $50 \mathrm{~cm}$ langen, beschichteten Sperrholzwänden, die mit einem Federstahlmesser direkt in der Stammoberfläche verankert wurden (Abb. 3-2). Der Abstand zwischen diesen Seitenteilen war variabel und konnte so den Erfordernissen des Stammes angepaßt werden. Zwischen den Wänden war Stoff gespannt, der durch U-Schienen gehalten wurde und auf dessen Unterseite sich eine Fangflasche befand. An der Ober- und Unterseite der Falle wurde der Stoff in einen angebrachten Sägeschnitt gezogen und somit das Falleninnere gegen die Außenwelt effektiv abgeschlossen.

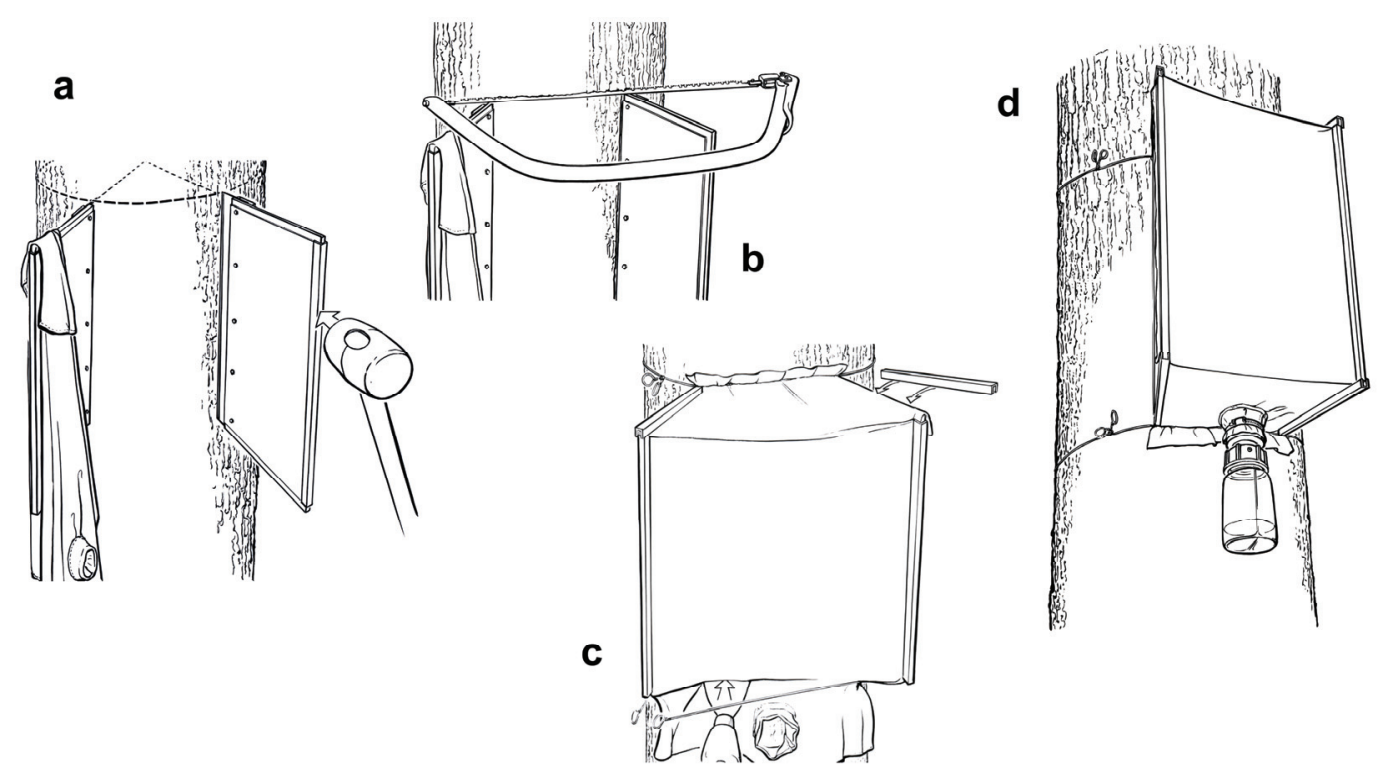

Abb. 3-2: Emergenzfallen (EFs), Aufbau: a-d

Wegen der erforderlichen Entwicklungsphase kam dieser Fallentyp im ersten Untersuchungsjahr noch nicht zum Einsatz. Die Fallen wurden an stehendem Eichen- und Buchentotholz im unteren Stammbereich in einer Höhe zwischen 0,5 und 2,5 m angebracht. Die Kriterien für die Verteilung der Fallen stimmten im wesentlichen mit den im vorangegangenen Abschnitt genannten überein. An den meisten Stämmen wurden mehrere Fallen installiert, um auch kleinräumige Unterschiede in der Besiedlung aufzeigen zu können. Es wurden 141 Proben an stehendem Totholz genommen (Tab. 3-2). Die befangene Oberfläche betrug insgesamt $22,8 \mathrm{~m}^{2}$, von denen $14,2 \mathrm{~m}^{2}$ noch berindet waren. Der mittlere Durchmesser der Stämme an den Fallenstandorten betrug $68,9 \mathrm{~cm}$ o. R. Im Mittel wurde pro Falle eine Fläche von $16,2 \mathrm{dm}^{2}(8,5-26,6)$ befangen. Störungen traten an diesem Fallentyp nur vereinzelt auf. 
In den Jahren 1995 und 1996 kam dieser Fallentyp probeweise auch an liegenden Buchenstämmen zum Einsatz (EFl). Im Gegensatz zur Konstruktion für stehende Stämme war die Fangflasche an der unteren Sperrholzwand angebracht. Die 61 Proben deckten eine Mantelfläche von $8,9 \mathrm{~m}^{2} \mathrm{ab}$, von der $4,8 \mathrm{~m}^{2}$ berindet waren.

\subsubsection{Modifizierte Bodeneklektoren (mBE)}

In ihrer Funktionsweise entsprachen diese Fallen weitgehend den üblichen Bodenphotoeklektoren (FUNKE 1971; MÜHLENBERG 1989), waren allerdings zum Boden hin durch eine engmaschige Gazematte abgeschlossen, um ein Eindringen u. a. von Bodenorganismen zu verhindern. Dadurch sollte gewährleistet werden, dass sich in der Kopfdose nur Tiere fingen, die zusammen mit dem Probenmaterial in das Falleninnere verbracht worden waren.

In den beiden ersten Untersuchungsjahren kamen pyramidenförmige Zeltfallen aus Stoff mit einer Grundfläche von $1 \mathrm{~m}^{2}$ zum Einsatz, deren reusenförmige Verengung von einer Kopfdose abgeschlossen wurde (Abb. 3-3). Diese Fallenkonstruktion verfügte über keinen Kunststoffring an der Basis, vielmehr war die Gazematte direkt mit den Seitenwänden vernäht. Mit diesem Typ wurden 13 Proben von Eichentotholz genommen (Tab. 3-2).

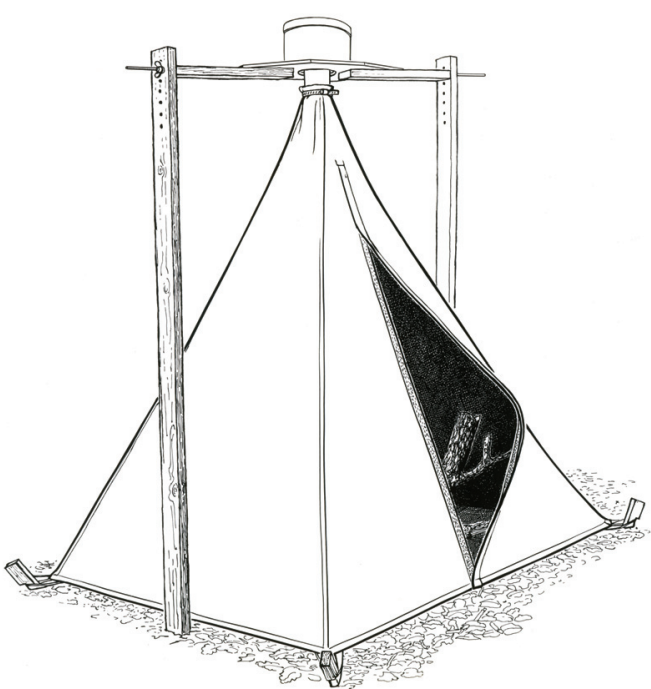

Abb. 3-3: Bodenphotoeklektor (mBE) Typ A

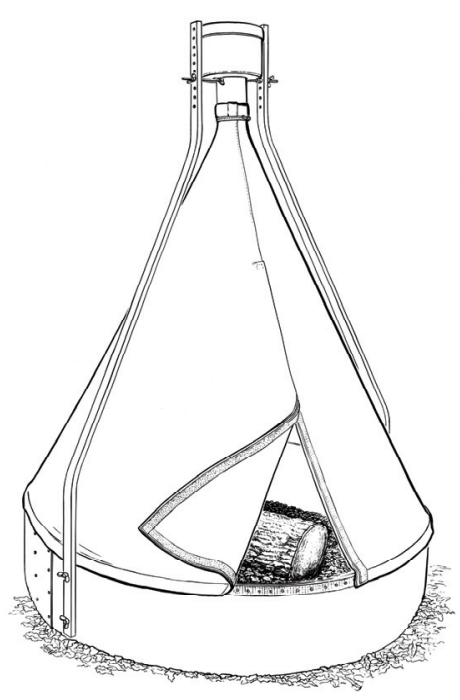

Abb. 3-4: (mBE) Typ B

Wegen der häufig festzustellenden Mäusefraßschäden wurde auf ihre Verwendung in den Jahren 1995 und 1996 verzichtet. Statt dessen wurde auf das Bauprinzip handelsüblicher Bodenphotoeklektoren zurückgegriffen (Abb. 3-4). An Stelle eines Kunststoffringes wurde ein auf der Innenseite besandeter Aluminiumring von $1 \mathrm{~m}$ Durchmesser verwendet. Es wurden insgesamt 39 Proben von Buchen genommen (Tab. 3-2).

Die Fallen wurden in ihrer Mehrzahl an beschatteten Standorten aufgestellt. Während die STE und EF fast ausschließlich an Totholz mit stärkerem Durchmesser positioniert wurden, dienten die mBE vor allem der Erfassung solcher Käferarten, die auch oder ausschließlich an dünnem Material zu finden sind. Darüber hinaus wurde auch starkdimensioniertes, in der 
Zersetzung weit fortgeschrittenes Totholz befangen, das für die Anbringung von STE ungeeignet war. Auch bei diesem Fallentyp wurde darauf geachtet, dass das in eine Falle eingebrachte Material möglichst homogen war. Die insgesamt befangene Mantelfläche betrug $131,7 \mathrm{~m}^{2}$ mit einer Rindenfläche von 53,4 $\mathrm{m}^{2}$ und einem Volumen von 4,4 $\mathrm{m}^{3}$.

Gegenüber Mäusefraß erwies sich der zuletzt beschriebene Fallentyp als deutlich weniger anfällig. Vereinzelt waren Löcher in den Stoffzelten, insbesondere aber in den Gazematten zu beobachten. Darüber hinaus waren einige Fallen durch Spaziergänger geöffnet und nur unvollständig wieder geschlossen worden.

\subsubsection{Fensterfallen (FF)}

Zur Erfassung flugaktiver Käferarten hat sich der Einsatz von Flugbarrierefallen bewährt (KÖHLER 1996; OTTE 1989). Es wird ein breites Spektrum u. a. auch an xylobionten Arten erfaßt. Ihr Einsatz in dieser Untersuchung diente in erster Linie der Vervollständigung des Artenspektrums. Es sollten auf diese Weise aber auch Daten zur Flugphänologie und zum Ausbreitungsverhalten gewonnen werden. Im Gegensatz zu geschlossenen Eklektoren ist der wirksame Einzugsbereich nicht bestimmbar. Die Effektivität dieses Fallentyps ist in hohem Maße von der Aktivität der Tiere abhängig (Aktivitätsdichte). In wie weit die Fangergebnisse Rückschlüsse auf die Dichte einzelner Arten bzw. deren Veränderungen zulassen, wurde bislang nicht untersucht.

Es kamen zwei verschiedene Fallentypen zum Einsatz. Der erste Typ, von dem 1992 und 1993 jeweils vier an der Zahl aufgestellt wurden, bestand aus einer durchgehenden Plexiglasscheibe mit einer Fläche von $0,5 \mathrm{~m}^{2}$, unter der ein Fangbehälter mit Weithalsflasche angebracht war, in die 50 \%iges Ethylenglycol gegeben wurde. Diese Fallen wurden in einem Rahmen aus Dachlatten aufgehängt (Abb. 3-5).

Da das Fangergebnis stark von der Ausrichtung der Prallfläche abhängig ist, wurden ab 1993 jährlich 20 Fensterfallen aufgestellt, deren beiden Prallflächen aus Plexiglas $(60 \times 25 \mathrm{~cm})$ rechtwinkelig zueinander ausgerichtet waren und so eine richtungsunabhängige Erfassung der fliegenden Käfer ermöglichte (Kreuzfallen). Oberhalb der Prallflächen war ein Plexiglasdach angebracht, um so zumindest einen Teil des Niederschlages abzuhalten. Unter der $0,3 \mathrm{~m}^{2}$ großen Prallfläche befand sich ein Trichter mit angeschraubter Fangflasche. Die Fallen wurden an Eisenständern in ca. 1,80 m Höhe aufgehängt (Abb. 3-6). Bevorzugt wurden die Fallen auf kleinen Bestandeslichtungen aufgestellt, da sie hier wegen des geringen Raumwiderstandes, der Wärme und des oft vorhandenen Blütenangebotes besonders effektiv sind. Aber auch die stärker beschatteten Bestandesteile wurden berücksichtigt und hier besonders die Nähe zum Totholz gesucht. Es wurden insgesamt 8 Proben à $0,5 \mathrm{~m}^{2}$ und 60 Proben à $0,3 \mathrm{~m}^{2}$ Prallfläche genommen (Tab. 3-2).

$\mathrm{Zu}$ Beeinträchtigungen kam es in einigen Fällen durch die Ansammlung von Regenwasser in den Fangflaschen, auch wenn in keinem Fall ein Überlaufen derselben zu verzeichnen war. Insbesondere zu Beginn und zu Ende der Vegetationszeit sammelten sich in den Trichtern größere Mengen an Laub und Blüten, wodurch die Fängigkeit der Fallen herabgesetzt und das Material in der Fangflasche stark verunreinigt wurde. 


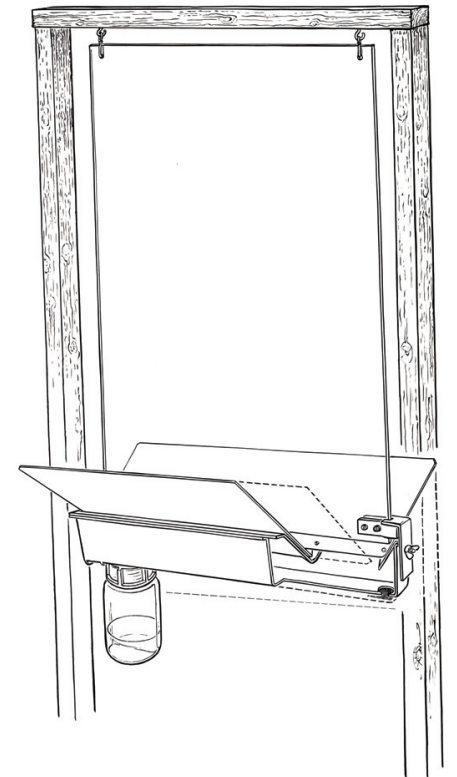

Abb. 3-5: Fensterfalle (FF) Typ A

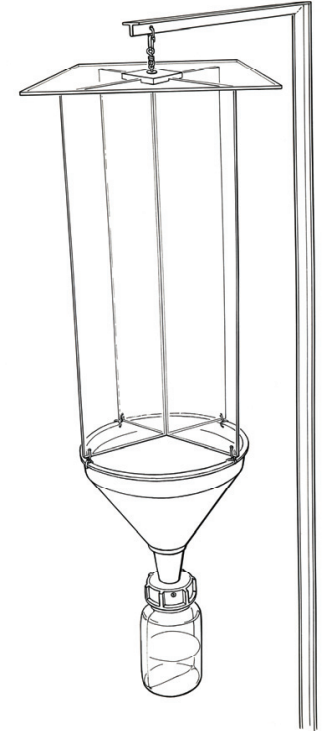

Abb. 3-6: Fensterfalle (FF) Typ B

\subsection{Determination und ökologische Einordnung der Arten}

\subsubsection{Bestimmungsarbeiten}

Die gefangenen Tiere wurden in Ethanol konserviert, später erfolgte die Bestimmung der Käfer. Hierzu wurde vor allem auf das Standardwerk zur Determination der mitteleuropäischen Käfer von FREUDE, HARDE \& LOHSE (1965-1983) und LOHSE \& LUCHT (19891994) zurückgegriffen. Die Nomenklatur richtet sich ebenfalls nach diesem Werk, die EDVCodierung nach LUCHT (1987) und LOHSE \& LUCHT (1989-1994). Der vierte Supplementband erschien zu einem Zeitpunkt, als der größere Teil der Auswertungen bereits abgeschlossen war (LUCHT \& KLAUSNITZER 1998). Da die Änderungen nicht nur die Nomenklatur, sondern auch die systematische Stellung verschiedener Arten betreffen, mußten diese in den Ergebnisteilen unberücksichtigt bleiben. Eine geringe Anzahl von Individuen, bei denen die Zuordnung zu einer Art nicht möglich war, weil z. B. für die Determination das Genital männlicher Exemplare erforderlich ist, blieb in der Auswertung unberücksichtigt. Von den nachgewiesenen Arten wurden Belegexemplare präpariert, von Fachleuten überprüft bzw. determiniert und in die Sammlung des Forstzoologischen Instituts in Göttingen aufgenommen.

\subsection{2. Ökologie der Arten}

Die Käferarten wurden nach ihrem Präferenzverhalten verschiedenen Biotopen und Habitaten zugeordnet. Darüber hinaus erfolgte eine Einteilung nach dem Konsumententyp, soweit hierzu Angaben in der Literatur gemacht werden, wobei das Augenmerk auf das Ernährungsverhalten der Larven gerichtet war. Die häufig widersprüchlichen Angaben zur Ökologie der Arten ist Ausdruck des geringen Kenntnisstandes und nicht zuletzt auf die verborgene 
Lebensweise der xylobionten Käferfauna zurückzuführen. Die hier getroffenen Einstufungen folgten in fast allen Fällen den Zuordnungen von KÖHLER (1991, 1996, 2000), die auf einer umfassenden Sichtung der einschlägigen Angaben in der Literatur beruhen. Damit sollte eine Vergleichbarkeit der Ergebnisse gewährleistet werden. Nur in wenigen begründeten Einzelfällen wurde eine abweichende Zuordnung getroffen. Für einige Arten war eine Eingruppierung nicht möglich. Die folgende Übersicht informiert über die unterschiedenen Klassen und die Bedeutung der Abkürzungen.

\section{Konsumententyp (Kon):}

$\begin{array}{llrl}\mathrm{p} & =\text { phytophag } & \mathrm{m} & =\text { mycetophag } \\ \mathrm{x} & =\text { xylophag }^{2} & \mathrm{z} & =\text { zoophag } \\ \mathrm{s} & = & \text { saprophag (einschl. necro- und coprophag) }\end{array}$

\section{Biotoppräferenz (Bio):}

$\begin{array}{ll}\mathrm{F}=\text { Feuchtbiotope } & \mathrm{O}=\text { Offenlandbiotope } \\ \mathrm{W}=\text { Waldbiotope } & \mathrm{E}=\text { eurytope Arten }\end{array}$

\section{Habitatpräferenz (Hab):}

\begin{tabular}{|c|c|c|c|}
\hline $\mathrm{V}=$ & Vegetation & $\mathrm{W}$ & $=$ Wasser \\
\hline $\mathrm{N}=$ & Nester $^{3}$ & $\mathrm{P}$ & $=$ Pilze $($ ohne TP) \\
\hline $\mathrm{B}=$ & Bodenstreu & $\mathrm{F}$ & $=$ Faulstoffe \\
\hline $\mathrm{T}=$ & festes Holz (lignicol) & $\mathrm{TM}$ & $=$ Holzmulm (xylodetricol) \\
\hline $\mathrm{TR}=$ & Totholzrinde (corticol) & $\mathrm{TP}$ & $=$ Totholzpilze (xylofungicol) \\
\hline $\mathrm{TS}=$ & Baumsaft (succicol) & $\mathrm{E}$ & $\begin{array}{l}=\text { 'euryöke' Arten ohne erkennbare } \\
\text { Habitatpräferenz }\end{array}$ \\
\hline
\end{tabular}

\subsection{Beschreibung der Fallenstandorte}

Im Mittelpunkt des Interesses dieser Untersuchung stand die Analyse der Artenvergesellschaftungen im Sukzessionsprozeß auf der Grundlage ihrer internen Datenstruktur. Die Interpretation der Ergebnisse bedarf aber der Hintergrundinformation sowohl über die Biologie und Ökologie der Arten als auch über Eigenschaften der Standorte, an denen die Artengemeinschaften erfaßt wurden. Aus der Vielzahl potenziell besiedlungsrelevanter Faktoren konnte nur eine begrenzte Auswahl näher untersucht werden. Die geschätzten oder gemessenen Werte wurden für die Auswertung in vielen Fällen in Klassen eingeteilt.

\footnotetext{
${ }^{2} \mathrm{Zu}$ diesem Konsumententyp werden von KÖHLER (2000) auch Arten gestellt, die verpilztes Holz fressen (xylomycetophag) sowie einige Arten, die sich xylophag und zusätzlich saprophag bzw. zoophag ernähren.

${ }^{3}$ Abweichend von der Klassifizierung von KÖHLER (2000) werden Arten, die bevorzugt in Totholznestern gefangen werden, hier nicht zu den Totholzkäfern (TN), sondern zu den Nestbewohnern (N) gestellt.
} 
An den Fallenstandorten wurden die folgenden Merkmale aufgenommen:

- die Baumart (Eiche, Buche)

- die Lage der Stämme (stehend, hochliegend, aufliegend) einschließlich dem Abstand zum Boden

- der Durchmesser

- das Rindenprozent (Klasse 1: 0-5 \%, Klasse 2: 6-50 \%, Klasse 3: 51-94 \%, Klasse $4: \geq 95 \%$ )

- die Beschattung (Klasse 1: stark schattig, Klasse 2: überwiegend schattig, Klasse 3: überwiegend sonnig)

- die Ausrichtung der Emergenzfallen an stehenden Stämmen nach der Himmelsrichtung (Nord, Ost, Süd, West)

Während sich bei den zuvor aufgezählten Merkmalen eine detaillierte Methodenbeschreibung erübrigt, sind für die nachfolgenden Einflussgrößen einige weitergehende Ausführungen erforderlich.

\subsubsection{Der Bohrwiderstand (Bw)}

Mit der voranschreitenden Zersetzung und der Zerstörung des Holzgefüges geht eine Abnahme der Festigkeitseigenschaften einher. Anstatt einer Einstufung des Zersetzungsgrades nach nur äußerlich wahrnehmbaren Kriterien, wurden Bohrwiderstandsmessungen mit dem von F. RINN entwickelten Resistographen 1410 durchgeführt (Abb. 3-7). Dieses Gerät läßt auch kleinräumige Änderungen in der Festigkeit des Holzkörpers innerhalb eines Stammes deutlich sichtbar werden (z. B. Zersetzungsstadien, Äste, Jahrringgrenzen) (RINN, SCHWEINEGRUBER \& SCHÄR 1994; ECKSTEIN \& SASS 1994; KLEINEVOSS, TOPP \& BOHAC 1996; KAHL 2003).

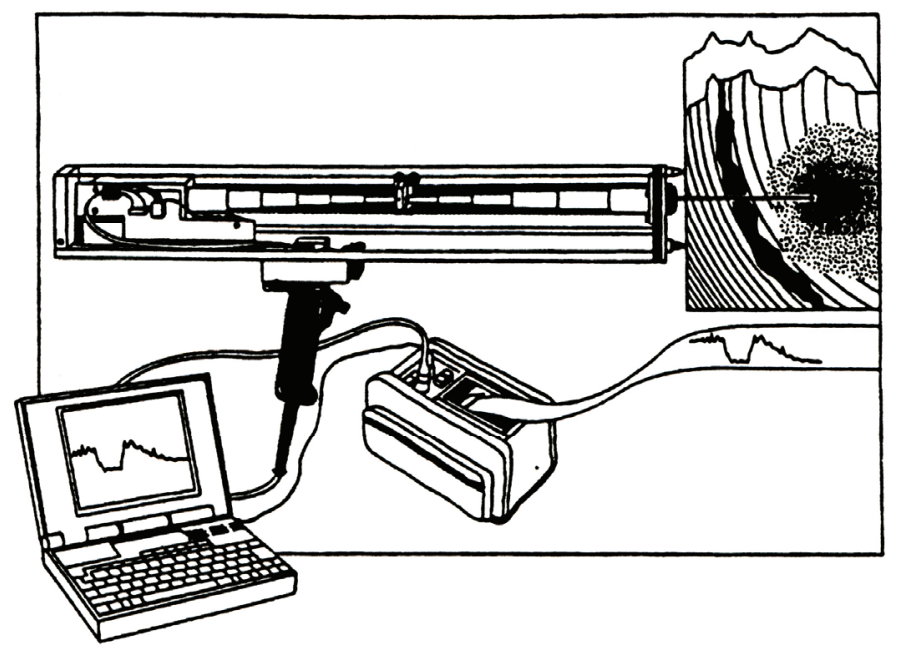

Abb. 3-7: Resistograph 1410 (aus RINN, SCHWEINEGRUBER \& SCHÄR 1996) 
Bei der Messung wird eine $41 \mathrm{~cm}$ lange und $3 \mathrm{~mm}$ breite Bohrnadel mit konstanter Geschwindigkeit und Drehzahl in das Holz vorgeschoben und die hierzu erforderliche Stromabnahme als dimensionsloser Wert gespeichert und auf einen Thermodrucker ausgegeben. Aufgrund der Form der Bohrnadel ist der gemessene Wert vor allem von der Dichte des Holzes an der Nadelspitze abhängig, wird darüber hinaus aber auch von der Reibung am Nadelschaft, der Vorschubgeschwindigkeit, der Holzfeuchte, der Art der Holzfäule und baumartenspezifischen Eigenschaften beeinflusst (RINN, SCHWEINEGRUBER \& SCHÄR 1994; ECKSTEIN \& SASS 1994; KELKENBERG 1995).

Insgesamt wurden 202 Messungen mit dem Resistographen an stehenden und liegenden Stammabschnitten durchgeführt. Das Material aus den modifizierten Bodeneklektoren blieb hiervon ausgenommen, da die erforderliche Homogenität des Materials nicht immer gegeben und somit eine Korrelation zwischen Artenvorkommen und Bohrwiderstand problematisch schien. In einigen Fällen, in denen zwei oder mehrere Fallen in unmittelbarer Nachbarschaft zueinander am gleichen Stamm angebracht waren, wurde nur eine Bohrung durchgeführt, soweit keine deutlichen Unterschiede in der Beschaffenheit des Stammes erkennbar waren. Hierauf ist es zurückzuführen, dass 290 Fallenproben einer Bohrwiderstandsklasse zugeordnet wurden. Die Abbildungen 3-8 und 3-9 zeigen beispielhaft einige Meßergebnisse von unterschiedlich stark zersetzten Eichen- und Buchenstämmen.

Sämtliche Messungen wurden mit einer Vorschubgeschwindigkeit von $70 \mathrm{~mm} / \mathrm{Min}$. durchgeführt. Um eine Beeinflussung der Meßergebnisse aufgrund von Abnutzungserscheinungen am Bohrnadelkopf auszuschließen, wurde die Nadel nach 20-25 Messungen gewechselt. Die Nadel wurde im Regelfall bis in die Nähe der Stammmitte, maximal jedoch $41 \mathrm{~cm}$ tief ins Stamminnere vorgeschoben. Vereinzelt kam es zum wiederholten automatischen Abbruch des Bohrvorganges, so dass in diesen Fällen mit den geringeren Bohrtiefen vorliebgenommen werden mußte. Da der mittlere Eindringwiderstand auch eine Funktion der Bohrtiefe ist, wurde, um Vergleiche zwischen den Probestämmen zu erlauben, für die Zuordnung der Proben zu einer Klasse der mittlere Eindringwiderstand der äußeren $15 \mathrm{~cm}$ herangezogen (Tab. 3-3).

Tabelle 3-3: Klassengrenzen des Bohrwiderstandes (Bw)

\begin{tabular}{|l|c|c|c|c|c|c|}
\hline & \multicolumn{6}{|c|}{ B o h rwiderstands k I as s e } \\
& $\mathbf{1}$ & $\mathbf{2}$ & $\mathbf{3}$ & $\mathbf{4}$ & $\mathbf{6}$ \\
\hline Bohrwiderstand & $<200$ & $200-299$ & $300-399$ & $400-499$ & $500-599$ & $\geq 600$ \\
\hline
\end{tabular}



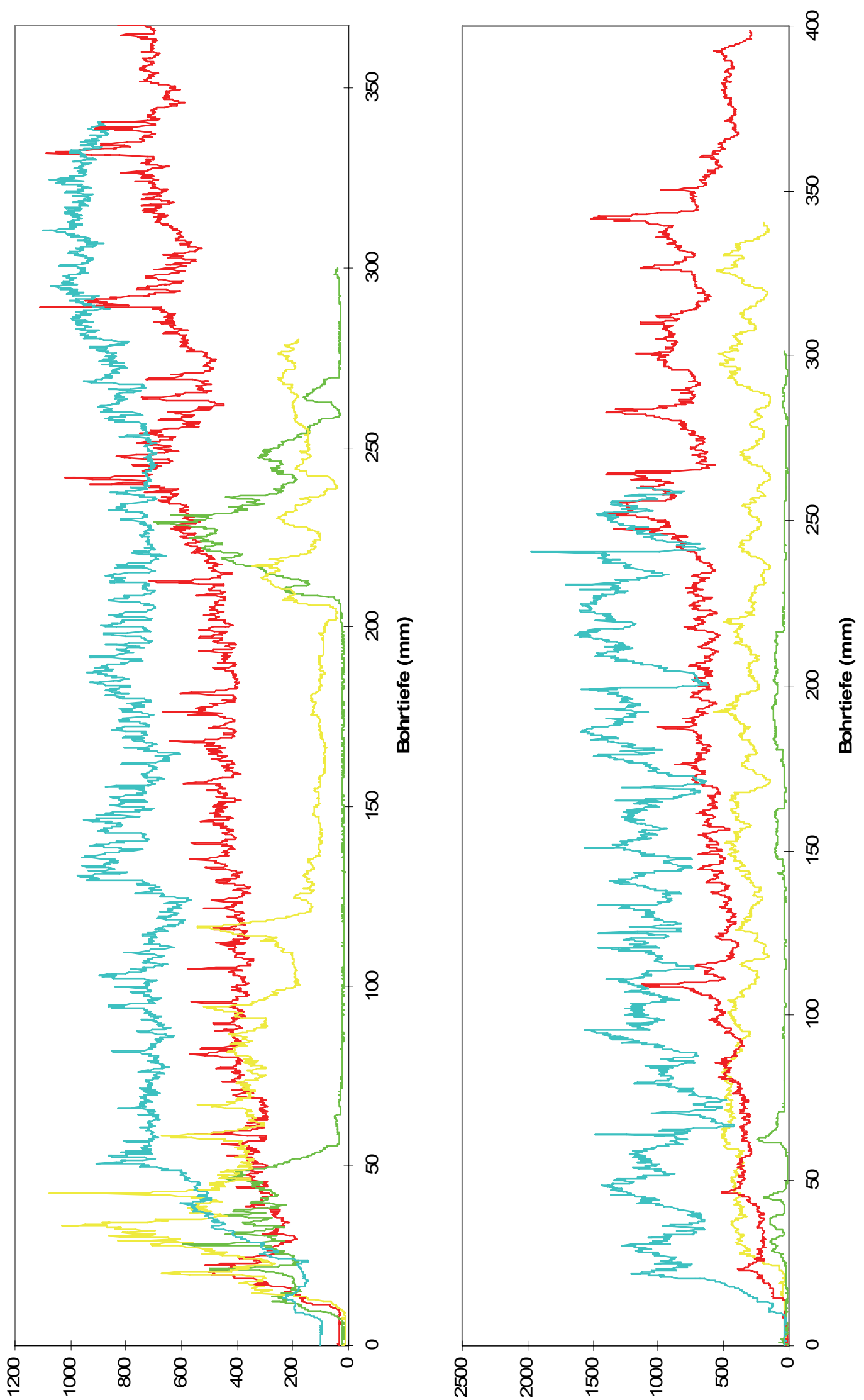

Abb. 3-8 u. 3-9: Beispiele für Bohrwiderstandsprofile an unterschiedlich stark zersetzten Eichen (links bzw. oben) und Buchen (rechts bzw. unten) 


\subsubsection{Feuchtegehalt (Fe) und Saugfähigkeitsquotient (Sq)}

In verschiedenen Untersuchungen zur Habitatbindung xylobionter Käfer wurde die Bedeutung der Feuchtigkeit am Standort und im Entwicklungssubstrat wiederholt hervorgehoben, so z. B. von DERKSEN (1941), BRAUNS (1951), PALM (1951, 1959), SCHIMITSCHEK (1953, 1954), SCHÖNBORN (1961) und DAJOZ (1966). Zu den Arten, die sich nur in feuchtem Holz entwickeln können, gehören z. B. die Ambrosiapilzzüchter aus den Gattungen Xyloterus und Xyleborus. Viele Larven mulmbewohnender Arten reagieren empfindlich auf Austrocknung. Andere Arten, wie z. B. einige Vertreter aus der Familie der Anobiidae, sind dagegen an eine Entwicklung im trockenen Holz angepaßt.

Um den Feuchtigkeitsgehalt der untersuchten Stämme zu bestimmen, wurden mit einem Hohlbohrer Bohrkerne mit einem Durchmesser von einem Zentimeter genommen. Pro Stamm konnte im Regelfall nur eine Bohrung durchgeführt werden. Die Stämme wurden horizontal und nach Möglichkeit im gesamten Querschnitt durchbohrt. In nicht wenigen Fällen war dies jedoch durch den Zustand des Holzes nicht möglich.

Geschützt vor Austrocknung wurden die Bohrkerne ins Labor gebracht und dort der Länge nach geteilt. Dies war notwendig, da eine Hälfte der Kerne für anderweitige Untersuchungen benötigt wurde (s. u.). Die Bohrkerne wurden, unterteilt in Abschnitte, gewogen, bei $105^{\circ} \mathrm{C}$ 72 Stunden lang getrocknet und anschließend die Gewichtsdifferenz festgestellt. Der Feuchtegehalt wurde als prozentuales Verhältnis von Gewichtsdifferenz zum Gewicht der getrockneten Proben berechnet. Die Zuordnung zu verschiedenen Feuchtegehaltsklassen beruhte, sofern möglich, auf den Werten für den halben Stammdurchmesser. Die festgelegten Klassengrenzen können der Tabelle 3-4 entnommen werden. Fallenstandorte vom gleichen Stamm wurden den gleichen Klassen zugeordnet, sofern sich dies nicht aufgrund erkennbarer Unterschiede verbot und der untersuchte Bohrkern keine sehr großen Feuchtegehaltsunterschiede im Stammquerschnitt erkennen ließ.

Tabelle 3-4: Klassengrenzen der Holzfeuchte (Fe)

\begin{tabular}{|l|c|c|c|c|}
\hline & \multicolumn{4}{|c|}{ Ho I z f e u c h t e k I a s s e } \\
& $\mathbf{1}$ & $\mathbf{2}$ & $\mathbf{3}$ & $\mathbf{4}$ \\
\hline Feuchtegehalt (\%) & $<35$ & $35-54,9$ & $55-74,9$ & $\geq 75$ \\
\hline
\end{tabular}

Der Feuchtegehalt des Holzes zeigt Schwankungen im Jahresverlauf und in Abhängigkeit von der aktuellen Wettersituation. Zu den zahlreichen beeinflussenden Faktoren zählen auch die Baumart, Lage und Holzdimension, das kleinstandörtliche Klima und der Zersetzungsgrad. Die mit fortschreitendem Abbau einhergehenden Änderungen der physikalischen Holzeigenschaften haben eine veränderte Wasserhalte- und Wasseraufnahmekapazität zur Folge (HARMON ET AL. 1986; MACKENSEN \& BAUHUS 1999; SCHÄFER 2002). SCHMITT (1992) machte sich diesen Umstand zunutze, um den Zersetzungszustand der von ihm untersuchten Hölzer zu charakterisieren. Der von ihm berechnete Saugfähigkeitsquotient gibt das Verhältnis der Wasseraufnahme während eines definierten Zeitraums zur Oberfläche einer 
getrockneten Holzprobe an. Hiervon abweichend wurde in dieser Untersuchung die Gewichtszunahme zum Gewicht der getrockneten Probe ins Verhältnis gesetzt.

Um einen günstigen Zeitraum für die Eintauchdauer der Bohrkerne festzulegen, wurde für 35 Probekerne die Wasseraufnahme in regelmäßigen Zeitabständen gemessen. Der Verlauf der Sättigungskurven machte deutlich, dass die relative Wasseraufnahme pro Zeiteinheit in den ersten Sekunden hoch ist, um danach deutlich zurückzugehen. Für die Berechnung des Saugfähigkeitsquotienten wurde daher die aufgenommene Wassermenge nach einer Eintauchdauer der Probestücke von $10 \mathrm{sec}$ herangezogen $\left(\mathrm{Sq}_{10}\right)$. Die Sättigungskurven ließen auch erkennen, dass, trotz ähnlichem Kurvenverlauf bei beiden Baumarten, die Eiche bezogen auf ihr Trockengewicht bei kurzer Eintauchdauer der Proben weniger Wasser aufgenommen hat als die Buche. Bei längeren Eintauchzeiten glichen sich die Kurven mehr und mehr an, bei gesättigten Proben lag der durchschnittliche Saugfähigkeitsquotient der Eiche sogar höher als der der Buche. Die Verteilungskurven der $\mathrm{Sq}_{10}$-Werte der beiden Baumarten hatten deutlich unterschiedliche Schwerpunkte. Infolgedessen wurden für jede Baumart eigene Klassengrenzen definiert (Tab. 3-5).

Tabelle 3-5: Klassengrenzen des Saugfähigkeitsquotienten (Sq)

\begin{tabular}{|l|c|c|c|c|}
\hline & \multicolumn{4}{|c|}{ S q-K I a s s e } \\
& $\mathbf{1}$ & $\mathbf{2}$ & $\mathbf{3}$ \\
\hline Sq (\%) Eiche & $<7,5$ & $7,5-9,9$ & $10-12,4$ & $\geq 12,5$ \\
Sq (\%) Buche & $<15$ & $15-24,9$ & $25-44,9$ & $\geq 45$ \\
\hline
\end{tabular}

\subsubsection{Dendrochronologische Analyse}

Der eigentliche Anlaß für die Entnahme der Bohrkerne war der Wunsch, nähere Informationen über den Zeitpunkt des Absterbens der Bäume und damit über die Dauer des Abbauprozesses zu erhalten. DERKSEN (1941) konnte in seiner Untersuchung zur Sukzession der Artengemeinschaften an Buchenstümpfen auf Angaben zum Fällzeitpunkt der Bäume zurückgreifen. Da die Sukzession der Artengemeinschaften nicht in erster Linie eine Funktion der Zeit ist, sondern der Abbauprozeß durch verschiedene Faktoren wie z. B. die standörtlichen Gegebenheiten, Pilzbefall etc. modifiziert wird, dienten die Ergebnisse der dendrochronologischen Analyse vor allem als Hintergrundinformation bei der Interpretation der vorgefundenen Artengemeinschaften.

Vor Ort läßt sich das Alter des Totholzes in vielen Fällen nur schwer oder gar nicht abschätzen. Während sich in den ersten Jahren noch vergleichsweise deutliche Veränderungen im Zustand des Totholzes zeigen, die - unter Berücksichtigung der Zersetzungsbedingungen Rückschlüsse auf den ungefähren Absterbezeitpunkt zulassen, gestaltet sich dieses Vorhaben in fortgeschrittenen Zersetzungsstadien mitunter als sehr schwierig, in vielen Fällen sogar als aussichtslos. So zeigten z. B. zahlreiche Eichenstümpfe des 'Neuenburger Urwaldes' über viele Jahre bis Jahrzehnte hinweg kaum äußerlich wahrnehmbare Merkmalsveränderungen. Der Zustand des äußeren Holzmantels verrät auch nicht, ob der Kernbereich noch von relativ fester Struktur ist, oder ob dieser schon vollständig zersetzt worden ist. 
Eine rückwirkende Datierung des Absterbezeitpunktes ist an eine Reihe von Vorraussetzungen geknüpft, die mit voranschreitender Zersetzung immer seltener erfüllt sind. Der Zustand der Bohrkerne muss die Messung der relevanten Parameter zumindest in Teilbereichen erlauben, ohne die die Suche nach Koinzidenzen mit Jahrringdiagrammen nicht möglich ist. Ist der äußere Holzkörper vollständig erhalten und lassen sich hinreichend viele Jahrringgrenzen und -breiten bestimmen, bereitet die dendrochronologische Analyse vergleichsweise geringe Schwierigkeiten. Ist nur der Splintbereich zersetzt, lassen Beobachtungen über die durchschnittliche Anzahl der Jahrringe im Splint der Baumart in der Region - besser des gleichen Waldbestandes - immer noch relativ genaue Aussagen zum Absterbezeitpunkt zu. In Fällen, in denen nur noch Teile des Holzkörpers im Stamminneren eine Mindestanzahl von Jahrringen erkennen lassen, kann zumindest festgestellt werden, wann der Baum noch nicht abgestorben war. In nicht wenigen Fällen war aber auch dies nicht möglich, da der Zustand des Holzkörpers aufgrund der fortgeschrittenen Zersetzung die Voraussetzungen einer dendrochronologischen Untersuchung nicht erfüllte.

\subsection{4. Überschirmung (Schi)}

Als Ergänzung zur Einschätzung der Beschattung der Fallenstandorte wurde der Anteil der überschirmten Fläche registriert. Hierzu wurden Fotografien des Kronenraumes mit einem schwachen Weitwinkel $(28 \mathrm{~mm})$ gemacht. Als Filmmaterial wurde ein Dokumentenfilm der Fa. AGFA (Ortho) mit einer Lichtempfindlichkeit von 25 ASA verwendet. Die Filmnegative wurden mit einem Computerprogramm zur Bildanalyse automatisch ausgewertet. Gemessen wurde der Anteil der Bildfläche mit einer Graustufe von 0 - 40 \%. Dieser Anteil war Grundlage der Einstufung der Fallenstandorte in Überschirmungsklassen (Tab. 3-6).

Tabelle 3-6: Überschirmungsklassen (Schi)

\begin{tabular}{|l|c|c|c|c|c|c|}
\hline & \multicolumn{6}{|c|}{ Ü b e r s c h i r m u n g s k l a s s e } \\
& $\mathbf{1}$ & $\mathbf{2}$ & $\mathbf{3}$ & $\mathbf{4}$ & $\mathbf{5}$ & $\mathbf{6}$ \\
\hline Überschirmung (\%) & $\geq 90$ & $80-89,9$ & $70-79,9$ & $60-69,9$ & $50-59,9$ & $<50$ \\
\hline
\end{tabular}

\subsection{Datenanalyse}

Die geeigneten Analysemethoden, um die Vergesellschaftung der Arten und deren sukzessive Veränderung mit voranschreitender Zersetzung des Totholzes zu beschreiben, lassen sich - mehr oder weniger eindeutig - Verfahren der direkten oder der indirekten Gradientenanalyse (WHITTAKER 1967) zuordnen. Die direkte Gradientenanalyse beschreibt die Verteilung von Arten entlang wichtiger Umweltgradienten (GAUCH 1982), setzt also eine möglichst exakte Erhebung relevanter Umweltfaktoren bzw. Faktorenkomplexe voraus, mit denen das Auftreten bzw. die Häufigkeit einzelner Arten unmittelbar in Beziehung gebracht werden kann. Direkte Verfahren leisten somit einen wesentlichen Beitrag zum Verständnis der beobachteten Verteilung der Arten. Um aus dem räumlichen Nebeneinander das zeitliche Nacheinander einer Sukzession zu rekonstruieren und zu erklären, sind Methoden der direkten Gradien- 
tenanalyse an die Erfassung des bzw. der Faktorengradienten gebunden. Die hinreichende Auswahl und Messung der besiedlungsbestimmenden Faktoren in der Sukzession der Totholzkäfergemeinschaften gestaltet sich jedoch als sehr problematisch. In Folge dessen haben manche Forscher den Versuch unternommen, den Faktorenkomplexgradienten an Totholz durch einfache Kenngrößen zu charakterisieren. So hat u. a. SCHIMITSCHEK $(1953,1954)$ den Abbaugradienten des Totholzes durch die Einteilung in Zersetzungsgrade umschrieben und DERKSEN (1941) nahm den Absterbezeitpunkt zum Ordnungsprinzip.

Ausgehend von der Annahme, dass das Vorkommen bzw. die Häufigkeit der Arten und die Veränderungen der Gesellschaftsstruktur im Sukzessionsprozeß vor allem eine Funktion der Umweltbedigungen und der Ressourcenqualität und damit auch der Sukzessionsdauer ist, sollte sich der Abbaugradient in der Komposition der Artengemeinschaften manifestieren, wodurch sich die Möglichkeit eröffnet, den oder die Gesellschaftsgradienten unabhängig von gemessenen Umweltvariablen, allein auf Grundlage der internen Datenstruktur, zu analysieren (TER BRAAK \& PRENTICE 1988). „Species composition may .. be a more informative indicator of environment than any given set of measured environmental variables" (TER BRAAK 1995). Die Vergesellschaftung der Arten wird somit zum Ordnungsprinzip.

Diese Verfahren der 'indirekten Gradientenanlyse' standen im Vordergrund dieser Untersuchung. Die auf diese Weise aufgedeckte Struktur der Artenvergesellschaftung kann in einem anschließenden separaten Arbeitsschritt mit an den Fallenstandorten erhobenen Umweltvariablen in Beziehung gesetzt werden. Einige Verfahren bieten aber auch die Möglichkeit, Informationen über einzelne Umweltvariablen unmittelbar in das Ordnungsverfahren $\mathrm{zu}$ integrieren. Auch wenn nur wenige Umweltvariablen erfaßt worden sind, kann so ihre Bedeutung für die Struktur und Variation der Artengemeinschaften eingeschätzt werden.

\subsection{1. Ähnlichkeitsindices}

Um Proben nach der Ähnlichkeit ihrer Artenzusammensetzung zu ordnen, sind zahlreiche numerische Indices entwickelt worden, die im Idealfall ihre ökologische Nähe zum Ausdruck bringen. Sie können auch zur Untersuchung von Sukzessionsprozessen herangezogen werden (HUTHA 1979). Die Beurteilung, wie gut welcher Index diese Aufgabe zu leisten vermag, ist schwierig, und so herrscht Unklarheit darüber, welcher Index für welche Fragestellung verwendet werden sollte (KREBS 1989). Die Eignung verschiedener Indices und ihre Abhängigkeit von unterschiedlichen Einflussgrößen wurde wiederholt diskutiert. HUTHA (1979) kommt zu dem Schluß, ,... that no measure yet devised is ideal'. Die Auswahl hat sich vielmehr an den konkreten Zielen der Untersuchung zu orientieren und verschiedene Einflussgrößen wie z. B. die Gradientenlänge, Stichprobengröße, Dominanzstruktur und Artenvielfalt zu berücksichtigen (TONGEREN 1995; WOLDA 1981; KREBS 1989).

Während einige der Ähnlichkeitsindices 'nur' die Präsenz bzw. Absenz der Arten berücksichtigen, fließen in andere Indices auch Informationen über deren Häufigkeit ein. Für die Analyse kurzer Gradienten sind quantitative Angaben in vielen Fällen von relativ großer Bedeutung, während andererseits bei langen Gradienten ein größeres Gewicht auf die An- und Abwesenheit der Arten zu legen ist (TONGEREN 1995). Aus der Gruppe der qualitativen Indices wurde der in der Tierökologie häufig verwendete SØRENSEN-Quotient (SQ \%) berechnet (SØRENSEN 1948). Er ist ein Maß für die Übereinstimmung des Arteninventars 
von Proben und kann Werte zwischen 0 (keine Übereinstimmung) und 100 (vollständige Artenidentität) einnehmen.

Während bei Vergleichen auf der Basis von Präsenz/Absenz-Daten 'seltenere' Arten den gleichen Einfluss auf den Indexwert haben wie die dominierenden, findet bei den Ähnlichkeitsparametern auf der Basis quantitativer Daten eine Gewichtung gemäß ihrer Häufigkeit Eingang. Zu dieser Gruppe von Indices gehört auch die nicht zuletzt wegen der einfachen Berechnung weit verbreitete Dominantenidentität nach RENKONEN (1938), die zu den besten quantitativen Ähnlichkeitskoeffizienten gerechnet wird (KREBS 1989) und deshalb auch in dieser Untersuchung Berücksichtigung fand (RE). Der Indexwert wird fast ausschließlich von den dominierenden Arten bestimmt und reagiert entsprechend sensitiv schon auf geringe Dichteschwankungen. In Anlehnung an die Ausführungen von HUTHA (1979), der eine Vielzahl von Ähnlichkeitsindices hinsichtlich ihrer Eignung zur Analyse einer natürlichen Sukzession verglichen hat, wurde auch die Dominantenidentität nach Logarithmierung der Daten berechnet $\left(\mathrm{RE}_{\mathrm{ln}}\right)$. Dadurch sollte der qualitativen Struktur der Artengemeinschaften ein größeres Gewicht verliehen werden, ohne die Häufigkeit der Arten gänzlich zu vernachlässigen. Die Berechnungen wurden als Grundlage für die Sortierung der Proben in einer Ähnlichkeitsmatrix herangezogen.

Ähnlichkeitsindices können auch für den paarweisen Vergleich der Verteilung der Artenpaaren (Affinität, Assoziation, Koordination) genutzt werden. DAJOZ (1966) hat in seiner Analyse der Sukzession der Totholzkäfergemeinschaften den Affinitätsindex von JACCARD verwendet. In der vorliegenden Untersuchung wurde, wie beim qualitativen Vergleich zweier Proben, der SØRENSEN-Quotient berechnet. Dieser Index berücksichtigt nur jene Proben, in denen wenigstens eine Art der zu untersuchenden Artenpaarung vorgefunden wurde.

Das Fehlen beider Arten kann aber ebenso von großer ökologischer Aussagekraft sein. Daher fließen bei verschiedene Indices auch diese Stichproben in die Analyse mit ein. Aus dieser Gruppe von Indices wurde der PHI-COEFFICIENT berechnet, der ebenfalls nur binäre Daten berücksichtigt. „PHI has a well-known relationship to $\chi^{2}$, and is most often used in ecology for measuring association between pairs of species" (PODANI 1994). Der PHICOEFFICIENT wurde in der komplementären Form als Unähnlichkeit berechnet und nimmt dann Werte zwischen 0 und 2 an. Hierbei steht der Wert 0 für perfekt positive Korrelationen, der Wert 2 für perfekt negative und der Wert 1 für fehlende Korrelationen. Die Interpretation der Ergebnisse muß berücksichtigen, dass im Rahmen einer Affinitätsanalyse die Einbeziehung von Proben, in denen keine der beiden zu untersuchenden Arten vorgefunden wurde, nur unter der Annahme gerechtfertigt ist, dass die Probenahmestellen potenzielle Besiedlungsorte der Arten sind, und somit die Wahrscheinlichkeit ihres Vorkommens in den Proben gleich hoch ist. Stammt aber ein größerer Anteil der Proben von Habitaten, die nicht zum natürlichen Siedlungsraum der Artenpaarung zu rechnen sind, hat dies zur Konsequenz, dass $\mathrm{zu}$ viele positive Assoziationen angezeigt werden (SOUTHWOOD 1978). Daher bedürfen die berechneten PHI-COEFFICIENTEN einer kritischen Prüfung, inwieweit die geforderten Bedingungen bei der Analyse jedes einzelnen Artenpaares erfüllt sind. Die Mißachtung dieser Voraussetzungen ist der Grund dafür, dass die Analyse von Artenaffinitäten mittels $\chi^{2}$-Test in vielen Fällen zu einer zu hohen Anzahl von signifikanten Affinitäten führt. Angesichts des gegenwärtig noch geringen Kenntnisstandes über die Ökologie zahlreicher Arten ist eine zutreffende Einschätzung, inwieweit die Voraussetzungen erfüllt sind, in vielen Fällen kaum möglich. Daher wird in der vorliegenden Untersuchung auf 
entsprechende Signifikanztests verzichtet. Vielmehr soll durch die Berechnung des PHICOEFFICIENTEN lediglich ein weiterer Gesichtspunkt in der Analyse der Artenvergesellschaftungen berücksichtigt werden.

Die Darstellung der Ergebnisse erfolgt in Form von Ähnlichkeitsmatrizen, die mit der Absicht geordnet wurden, 'natürliche Gruppierungen' herauszuarbeiten (JANETSCHEK 1982). Die Sortierung der Ähnlichkeitswerte erfolgte mittels der Seriation-Prozedur des Programmes SYNTAX 5.1 (PODANI 1994).

\subsubsection{Ordination}

Während der Einsatz verschiedener Ähnlichkeits- bzw. Unähnlichkeitsindices in der ökologischen Forschung auf eine lange Tradition zurückblicken kann, wurden einige bedeutsame multivariate Verfahren der indirekten Gradientenanalyse erst in jüngerer Zeit entwickelt (GAUCH 1982). Verschiedene Ordinationstechniken bieten die Möglichkeit, die Reaktion einer Vielzahl von Arten auf eine Vielzahl von Umweltvariablen simultan zu untersuchen. Anstatt die Reaktionen der einzelnen Arten im Gradienten zu betrachten, steht das Verhalten von Artengemeinschaften im Vordergrund.

Um die Arten nach ihrem Vorkommen und ihrer Häufigkeit bzw. die Habitate nach ihrer Artenstruktur zu ordnen, stehen verschiedene statistische Ordinationverfahren zur Verfügung. Ihnen gemeinsam ist das Ziel, die Gradientenstruktur herauszuarbeiten und eventuell auch den Einfluss einzelner ökologischer Faktoren aufzuzeigen.

Die Ordinationsverfahren unterscheiden sich u. a. in den ihnen zu Grunde liegenden Modellen und Algorithmen. In diese Untersuchung fanden Korrespondenzanalysen (CA, DCA) und kanonische Korrespondenzanalysen (CCA, DCCA) Eingang, die mit dem Programm CANOCO 3.1 berechnet und deren Ergebnisse mit CANODRAW 3.0 (SMILAUER 1992) graphisch dargestellt wurden ${ }^{4}$. Die Analysen wurden sowohl mit Präsenz-AbsenzDaten als auch mit den logarithmierten Schlüpfdichten der Arten vorgenommen. Arten mit keiner oder nur sehr geringer Bindung an Totholz (z. B. Rhynchaenus fagi) können i. d. R. nur wenig zum Verständnis der Gradientenstruktur der Sukzession an Totholz beitragen und wurden daher von der Analyse ausgenommen. Darüber hinaus war bei einigen Analysen eine Mindestfrequenz der Arten gefordert. Diese Selektion sollte zur Reduzierung der hohen Variabilität in den Artenkompositionen beitragen und war auch darin begründet, dass 'seltene' Arten - abhängig von der angewendeten Methode - nur geringen Einfluss auf das Ergebnis haben oder sich gar nachteilig auswirken können (GAUCH 1982; TER BRAAK \& VERDONSCHOT 1995).

\footnotetext{
${ }^{4}$ Allgemeine Einführungen in verschiedene Ordinationstechniken und Verweise zu weiterführender Literatur finden sich in GAUCH (1982), JONGMAN et al. (1995) und PALMER (2002). Zu den hier eingesetzten Verfahren siehe insbesondere HILL (1973), HILL \& GAUCH (1980), TER BRAAK (1986) und TER BRAAK \& VERDONSCHOT (1995).
} 


\subsubsection{Korrespondenzanalyse (Correspondence Analysis CA)}

HILL (1973) hat eine 'Analyse Factorielle des Correspondences' genannte Methode unter der Bezeichnung 'Reciprocal Averaging' in die ökologische Forschung übertragen. 1980 wurde von HILL \& GAUCH die Weiterentwicklung dieses Verfahrens als Detrended Correspondence Analysis (DCA) beschrieben, u. a. um den häufig auftretenden und als mathematisches Artefakt angesehenen 'arch effect' zu vermeiden (HILL \& GAUCH 1980). Da mit DECORANA ein effektives Computerprogramm zur Verfügung steht, fand diese Ordinationstechnik in der Ökologie vielfache Verwendung. „When an investigator desires a relatively objective community centered ordination, detrendet correspondence analysis has been found most effective and robust, especially with very heterogenous and difficult data sets" (GAUCH 1982).

Die Ordnung der Proben und Arten erfolgt in einem iterativen Anpassungsprozeß mit dem Ziel, die Hauptgradienten im Datenmaterial unabhängig von gemessenen Umweltvariablen aufzuzeigen, wobei ein unimodales Modell der Beziehung zwischen der Verteilung der Arten und den Faktorgradienten zu Grunde gelegt wird. Auf eine Berechnung von Ähnlichkeitsbzw. Distanzmatrizen wird dabei verzichtet. „Essentially, what is done is to take a set of species scores, then to define sample scores such that each sample score is the mean score of the species that occur in it, and finally to calculate new species scores so that the score of each species is the mean of the scores of the samples in which that species occurs. If this process, the two-way averaging iteration of reciprocal averaging, is repeated many times, then the scores will stabilize to a final solution which is independent of the initial scores and which has several desirable properties" (HILL 1979).

Im Ergebnis erfolgt eine Reduktion des n-dimensionalen Nischenraumes auf bis zu vier Ordinationsachsen, mathematisch handelt es sich dabei um Eigenvektoren, die als ,hypothetische“ (latente, theoretische, synthetische) Umweltgradienten betrachtet werden können und die die Variation der Artenverteilung bestmöglich erklären. Die berechneten Eigenwerte der Achsen sind ein Maß dafür, wie gut die „hypothetischen“ Variablen diese Erklärung leisten können, d. h. wie gut sich die Streuung der Daten auf wenige Dimensionen reduzieren lässt. Die Güte der Anpassung wird beeinflusst von der Anzahl der (Komplex-) Gradienten, die sich im Datenmaterial manifestieren.

Die Resultate werden als Ordinationsdiagramme dargestellt, die die Ähnlichkeit in der Artenstruktur der Proben bzw. die Ähnlichkeit in der Verteilung der Arten reflektieren sollen. Proben mit ähnlicher Artenstruktur bzw. Arten mit ähnlicher Verteilung erhalten auch ähnliche Positionen im Diagramm. Der Abstand zwischen Proben- und Artenpunkten verweist in der Tendenz auf die Häufigkeit bzw. die Wahrscheinlichkeit des Vorkommens der Art in der Probe (TER BRAAK \& PRENTICE 1988). Die zweidimensionale Darstellungsweise kann jedoch nur eine Annäherung an die reale Position der Objekte im vieldimensionalen Nischenraum sein. Insbesondere wenn die Streuung im Datenmaterial nur zu einem sehr geringen Teil auf die beiden dargestellten Achsen zurückgeführt werden kann, können sich auch nahe beieinanderliegende Objekte durch ein hohes Maß an Unähnlichkeit auszeichnen. Diese liegen dann entlang nicht dargestellter Achsen weit voneinander entfernt (TER BRAAK 1994). Deshalb muß die Interpretation nahe benachbarter Datenpunkte immer 
auf die Rohtabellen rekurrieren. Dagegen ist eine entfernte Position immer ein Ausdruck von Unähnlichkeit.

Artenpunkte in Zentrumsnähe des Diagramms bedürfen einer besonders kritischen Begutachtung. Neben der Möglichkeit, dass diese Arten hier ihr Optimum entlang der hypothetischen Variablen haben, kann diese Position auch von Arten eingenommen werden, die eine bimodale Verteilung zeigen oder aber keine deutliche Beziehung zu den Achsen erkennen lassen (TER BRAAK 1995).

Darüber hinaus ist das Ergebnis der Analyse empfindlich gegenüber artenarmen Proben mit einem relativ hohen Anteil 'seltener' Arten. In dem Bemühen, eine maximale Separation der Artenverteilungen entlang der ersten Ordinationsachse zu erreichen, werden diese Proben und Arten an die Enden der ersten Ordinationsachse gestellt und dadurch der Haupttrend in der Artenvergesellschaftung auf die zweite Ordinationsachse verwiesen (TER BRAAK 1995).

Zusammenfassend wird mittels der Korrespondenzanalyse die Struktur der Daten auf die Wirkung einiger weniger hypothetischer Variablen zurückgeführt. Die auf diese Weise erhaltene Ordination der Arten und Proben wird anschließend vor dem Hintergrund weiterer Informationen über die Fallenstandorte und über die Biologie und Ökologie der Arten interpretiert.

\subsubsection{Kanonische Korrespondenzanalyse (Canonical Correspondence Analysis CCA)}

TER BRAAK (1986) stellte mit der Kanonischen Korrespondenzanalyse (CCA) einen direkteren Weg vor, um die Vergesellschaftung der Arten in ihrer Abhängigkeit von gemessenen Umweltparametern aufzuzeigen. Die (D)CCA ist eine Erweiterung der (D)CA, weshalb die folgenden Ausführungen in erster Linie auf Unterschiede verweisen.

Ziel der kanonischen Ordination ist es, die Beziehung zwischen den an den Fallenstandorten erhobenen Variablen und der Verteilung der Arten und ihrer Vergesellschaftung aufzudecken (TER BRAAK 1995). Zu diesem Zweck erfährt der Algorithmus des 'Reciprocal Averaging' eine Modifikation, indem jeder Schritt des iterativen Anpassungsprozesses ergänzt wird durch eine multiple Regression zwischen den 'sample scores' und den Umweltvariablen. Auf diese Weise werden Ordinationsachsen in Abhängigkeit gemessener Umweltvariablen extrahiert, entlang derer eine maximale Seperation der Artenverteilungen gegeben ist (TER BRAAK 1987, 1988). Die Eigenwerte der Achsen sind Ausdruck dieser Separation und sie lassen Rückschlüsse zu auf den Anteil der Variation in der Artenverteilung, der durch die Kombination der gemessenen Faktoren erklärt werden kann.

Der Vergleich der Eigenwerte einer (D)CA und einer (D)CCA gibt Aufschluß darüber, wie gut der erhobene Faktorenkomplex zur Erklärung der Variation in der Artenverteilung beitragen kann. „If the results do not differ much, then we know, that no important environmental variables have been overlooked in the survey ... if the results of ordination and canonical ordination do differ much, then we may have overlooked major environmental variables, or important non-linear combinations of environmental variables included in the analysis" (TER BRAAK 1995).

Das resultierende Ordinationsdiagramm der (D)CCA vermittelt eine Vorstellung sowohl von der Variation der Artenvergesellschaftungen in Abhängigkeit der Variablen als auch von 
der Reaktion einzelner Arten auf verschiedene Umweltvariablen, die ebenfalls in der Grafik dargestellt werden. Nominale Variablen sind durch den Centroid derjenigen Proben, die der Klasse angehören, repräsentiert. Quantitative Variablen werden als Vektorpfeile dargestellt, deren Länge und Richtung durch ihre Korrelation mit den Ordinationsachsen bestimmt wird und somit - unter Berücksichtigung der Eigenwerte der Ordinationsachsen - ihren Beitrag zur Erklärung der Variation der Artenverteilung widerspiegeln (TER BRAAK 1995). Von Aussagekraft für die Interpretation des Ordinationsdiagramms ist auch die rechtwinkelige Projektion der Artenpunkte auf die (verlängerten) Vektorpfeile, da sie Hinweise auf die relative Verteilung der Arten in Abhängigkeit des jeweiligen Faktors geben (TER BRAAK 1986).

Nicht für alle Probenstandorte konnten die Umweltvariablen vollständig erfaßt werden. Beispielsweise waren einige der an stärker zersetztem Material genommen Bohrkerne nicht geeignet, um vergleichbare Saugfähigkeitsquotienten zu ermitteln. Proben mit unvollständigen Datensätzen wurden zunächst durch entsprechende Gewichtung von der kanonischen Korrespondenzanalyse ausgeschlossen und ihre Position im Ordinationsdiagramm anschließend auf der Grundlage ihrer Artenkomposition bestimmt. Da insbesondere für in der Zersetzung weit fortgeschrittene Probenstandorte eine auch nur annähernd genaue Datierung des Absterbezeitpunktes in vielen Fällen nicht möglich war, wurde dieser Faktor nicht in die (D)CCA aufgenommen.

Im Anschluß an die Analysen wurde, um die Bedeutung der einzelnen Habitatvariablen und ihrer Kombinationen für die Erklärung der Streuung im Datenmaterial zu prüfen, jeweils eine 'forward selection' durchgeführt. 


\section{Ergebnisse}

\section{1. Überblick über Individuen-, Familien- und Artenzahlen}

Für den 'Neuenburger Urwald' konnten während der Untersuchung insgesamt 711 Käferarten nachgewiesen werden. Die folgenden Ausführungen beschränken sich auf die Fänge der beschriebenen Eklektortypen und Flugbarrierefallen ${ }^{5}$. Insgesamt 64895 Käferindividuen aus 694 Arten wurden auf diese Weise erfaßt, von denen sich etwa jedes vierte Exemplar in den Fensterfallen fing (Tab. 4-1). Von den 75 festgestellten Käferfamilien wurden in den Fensterfallen und den Eklektoren jeweils 66 Familien gefunden. Trotz der deutlich höheren Individuenzahlen in den Eklektoren wurde die höchste Artenzahl in den Flugfallen festgestellt. Gemeinsam waren den beiden Fallensystemen 325 Arten, 237 Arten waren ausschließlich in Fensterfallen nachzuweisen, die Eklektoren verfügten über 132 exclusive Arten.

Tabelle 4-1: Übersicht: Individuen-, Familien- und Artenzahlen

\begin{tabular}{|l|ccc|ccc|ccc|}
\hline & \multicolumn{3}{|c|}{ F e n s t e r f a I I e n } & \multicolumn{3}{c|}{ E k I e k t o r e n } & \multicolumn{3}{c|}{ G e s a m t } \\
Jahr & Ind. & Fam. & Arten & Ind. & Fam. & Arten & Ind. & Fam. & Arten \\
\hline 1992 & 422 & 34 & 110 & 2653 & 35 & 145 & 3075 & 43 & 210 \\
1993 & 5564 & 60 & 411 & 12019 & 50 & 257 & 17583 & 63 & 495 \\
1995 & 5693 & 54 & 316 & 17019 & 52 & 303 & 22712 & 60 & 451 \\
1996 & 4679 & 57 & 343 & 16846 & 61 & 322 & 21525 & 66 & 463 \\
\hline Gesamt & 16358 & 66 & 562 & 48537 & 66 & 457 & 64895 & 75 & 694 \\
\hline
\end{tabular}

Der Einsatz von Fensterfallen (FF) hat sich hinsichtlich seiner Eignung zur Erfassung eines breiten Artenspektrums als ein sehr effektives Werkzeug erwiesen. Die in den 68 Proben gefangenen 16358 Exemplare verteilten sich auf 562 Käferarten. Damit wurde das Ergebnis aus den Eklektoren um mehr als 100 Arten übertroffen. Mit 411 Arten, das entspricht $73 \%$ der in FF und $59 \%$ der insgesamt im Gebiet registrierten Arten, war das Untersuchungsjahr 1993 am effektivsten.

Die höchste in einer Einzelprobe registrierte Artenzahl belief sich auf 112, das Minimum betrug 34 Arten. Im Mittel fingen sich 77 Arten pro Probe. Die Individuenzahl variierte zwischen 71 und 437 Tieren, der Mittelwert betrug 233,5 Individuen (Tab. 4-2).

\footnotetext{
${ }^{5}$ Die weiteren 17 Arten sind in der Gesamtartenliste (Kap. 4.3) aufgeführt.
} 
Tabelle 4-2: Individuen- und Artenzahlen in den Fensterfallenproben

\begin{tabular}{|c|r|c|r|}
\hline \multicolumn{2}{|c|}{ I N D I V I D U E N } & \multicolumn{2}{c|}{ A R T E N } \\
\hline $\begin{array}{c}\text { Individuen- } \\
\text { zahl }\end{array}$ & Proben & $\begin{array}{c}\text { Arten- } \\
\text { zahl }\end{array}$ & Proben \\
\hline$\geq 400$ & 4 & $\geq 100$ & 3 \\
$350-399$ & 4 & $90-99$ & 14 \\
$300-349$ & 11 & $80-89$ & 16 \\
$250-299$ & 12 & $70-79$ & 11 \\
$200-249$ & 15 & $60-69$ & 13 \\
$150-199$ & 11 & $50-59$ & 5 \\
$100-149$ & 6 & $40-49$ & 5 \\
$50-99$ & 5 & $30-39$ & 1 \\
\hline
\end{tabular}

Tabelle 4-3 gibt einen Überblick über die Fänge der FF in den Untersuchungsjahren und den einzelnen Fangperioden. Die Daten aus 1992 sind nur der Vollständigkeit halber aufgeführt, wegen der geringen Fallenzahl und ihres späteren Aufbaus aber von geringer Aussagekraft. Darüber hinaus waren die Fensterfallen nur im zweiten Untersuchungsjahr bereits ab Ende März (P1a) aktiviert. Eine Vergleichbarkeit ist am ehesten für die beiden letzten Untersuchungsjahre gegeben, in denen auch die Fallenstandorte beibehalten worden waren.

Tabelle 4-3: Fangergebnisse der Fensterfallen in den Fangperioden (1992-1996)

\begin{tabular}{|c|c|c|c|c|c|c|c|c|c|c|c|}
\hline \multirow{2}{*}{\multicolumn{2}{|c|}{ Jahr }} & \multicolumn{9}{|c|}{ Fangperiode } & \multirow[b]{2}{*}{ Ges. } \\
\hline & & P1a & P1b & P2 & P3 & P4 & P5 & P6 & P7 & P8 & \\
\hline \multirow[t]{3}{*}{1992} & Prallfl. $\left(\mathrm{m}^{2}\right)$ & & & & 2 & 2 & 2 & 2 & 2 & 2 & 2 \\
\hline & Ind. $/ \mathrm{m}^{2}$ & & & & 48 & 45 & 48 & 28 & 28 & 14 & 211 \\
\hline & Anz. Arten & & & & 43 & 41 & 43 & 23 & 24 & 13 & 110 \\
\hline \multirow[t]{3}{*}{1993} & Prallfl. $\left(\mathrm{m}^{2}\right)$ & 7,5 & 8 & 8 & 8 & 8 & 8 & 8 & 8 & 8 & 8 \\
\hline & Ind. $/ \mathrm{m}^{2}$ & 51 & 234 & 172 & 93 & 65 & 29 & 28 & 15 & 12 & 696 \\
\hline & Anz. Arten & 78 & 204 & 185 & 157 & 122 & 80 & 88 & 50 & 34 & 411 \\
\hline \multirow[t]{3}{*}{1995} & Prallfl. $\left(\mathrm{m}^{2}\right)$ & & 6 & 6 & 6 & 6 & 6 & 6 & 6 & 6 & 6 \\
\hline & Ind. $/ \mathrm{m}^{2}$ & & 220 & 374 & 123 & 123 & 53 & 35 & 11 & 12 & 949 \\
\hline & Anz. Arten & & 161 & 160 & 119 & 132 & 101 & 55 & 34 & 23 & 316 \\
\hline \multirow[t]{3}{*}{1996} & Prallfl. $\left(\mathrm{m}^{2}\right)$ & & 6 & 6 & 6 & 6 & 6 & 6 & 6 & 6 & 6 \\
\hline & Ind. $/ \mathrm{m}^{2}$ & & 97 & 184 & 158 & 86 & 150 & 69 & 22 & 15 & 780 \\
\hline & Anz. Arten & & 97 & 127 & 155 & 143 & 132 & 107 & 37 & 22 & 343 \\
\hline \multirow{5}{*}{$\begin{array}{l}1992- \\
1996\end{array}$} & Prallfl. $\left(\mathrm{m}^{2}\right)$ & 7,5 & 20 & 20 & 22 & 22 & 22 & 22 & 22 & 22 & 22 \\
\hline & Ind. $/ \mathrm{m}^{2}$ & 51 & 188 & 236 & 115 & 85 & 70 & 41 & 17 & 13 & 744 \\
\hline & Anz. Fam. & 24 & 46 & 55 & 56 & 52 & 47 & 38 & 23 & 14 & 66 \\
\hline & Anz. Arten & 78 & 275 & 282 & 262 & 245 & 211 & 167 & 90 & 57 & 562 \\
\hline & Artensumme & 78 & 289 & 389 & 452 & 500 & 535 & 547 & 555 & 562 & 562 \\
\hline
\end{tabular}


Die Fängigkeit war mit 949 Ind./m² Prallfläche 1995 am höchsten. Berücksichtigt man für 1993 aus Gründen der Vergleichbarkeit nur die Ergebnisse der 20 Kreuzfallen $\left(6 \mathrm{~m}^{2}\right.$ Prallfläche) und ignoriert die Periode P1a, so wurde mit 765 Ind. $/ \mathrm{m}^{2}$ ein Ergebnis ähnlich dem des Jahres 1996 erreicht. Mehr als 50 \% der Tiere wurden bis einschließlich Mai gefangenen. In den einzelnen Jahren kam es zu Verschiebungen, die zum einen auf den Witterungsverlauf zurückzuführen sind, die aber auch abhängig waren von der absoluten und relativen Häufigkeit einzelner dominierender Arten. 1993 wurde das Maximum aufgrund eines ungewöhnlich warmen und zugleich trockenen Frühjahres bereits Ende April / Anfang Mai (P1b) erreicht. 1996 war eine verzögerte Aktivität aufgrund eines zu kühlen Maimonats zu verzeichnen. So trat die dominierende Art der Fensterfallen, Meligethes denticulatus, im Vergleich zu den Vorjahren erst eine Fangperiode später in größerer Anzahl auf.

Auch hinsichtlich der Artenzahl war das Frühjahr am ergiebigsten. Bereits Mitte Mai waren über $50 \%$ der Arten nachgewiesen. In den folgenden Fangperioden flachte die Zunahme der Artensumme kontinuierlich ab. Nur etwa jede zehnte Art wurde ausschließlich ab Mitte Juli gefunden. Bezüglich der Artenzahlen wich die Entwicklung im letzten Untersuchungsjahr von der der Vorjahre ab, in dem die Monate Juni und Juli höhere Artenzahlen brachten als die Vormonate.

Mit den unterschiedlichen Eklektortypen wurden in den vier Untersuchungsjahren 353 Proben genommen. Das befangene Volumen betrug 23,8 $\mathrm{m}^{3}$, die Oberfläche belief sich auf 293,5 $\mathrm{m}^{2}$, von denen 53,6 \% berindet waren. Die Anzahl der eingesetzten Fallen und deren Verteilung z. B. auf die Baumarten und Zustandstypen variierte zwischen den Jahren erheblich. So wurde in den Jahren 1992 und 1993 ausschließlich Eichentotholz beprobt, 1995 entfielen nur noch $10 \%$ der befangenen Oberfläche auf diese Baumart und 1996 konzentrierten sich die Fänge ausschließlich auf Buchentotholz. Da in den beiden letzten Untersuchungsjahren eine deutlich höhere Fallenzahl zur Verfügung stand, war der Stichprobenumfang an Buche, sowohl hinsichtlich der Probenzahl als auch der befangenen Oberfläche, insgesamt mehr als doppelt so hoch wie an Eiche. Auch war der in den Einzeljahren auf die verschiedenen Eklektortypen entfallende Anteil an der Probenahme sehr unterschiedlich. Eine detaillierte Aufstellung des Stichprobenumfanges pro Eklektortyp und Baumart in den Untersuchungsjahren und Fangperioden findet sich in Anhang 2.

Die 48537 Individuen rekrutierten sich aus 66 Familien und 457 Käferarten. Die Schlüpfdichte betrug 165,4 Ind./m² Totholzoberfläche. Das Jahr 1993 war mit einer Dichte von 215,9 Ind. $/ \mathrm{m}^{2}$ am effektivsten. Die meisten Imagines wurden in der Fangperiode von Ende Juni bis Mitte Juli (P4) gefangen, die maximale Schlüpfdichte variierte in den einzelnen Untersuchungsjahren zwischen den Perioden P2 und P4 (Tab. 4-4). Erst ab August war ein merklicher Rückgang der Schlüpfdichte zu verzeichnen. Die Entwicklung der Artenzahlen im Jahresverlauf zeigte ein Bild ähnlich den Verhältnissen in den Fensterfallen. Mehr als die Hälfte der in Eklektoren gefangenen Käferarten war bereits bis Mitte Mai, $71 \%$ bis Anfang Juni nachgewiesen. Nur 51 Arten wurden ausschließlich nach Mitte Juli festgestellt. Während 1993 die maximale Artenzahl bereits in der Fangperiode P1b registriert wurde, war diese 1995 in P2 und 1996 erst in P3 zu verzeichnen. 
Tabelle 4-4: Fangergebnisse der Eklektoren in den Fangperioden (1992-96)

\begin{tabular}{|c|c|c|c|c|c|c|c|c|c|c|}
\hline & \multicolumn{9}{|c|}{ Fangperiode } & \multirow[b]{2}{*}{ Ges. } \\
\hline & P1a & P1b & $\mathbf{P 2}$ & P3 & P4 & P5 & P6 & P7 & P8 & \\
\hline Probefl. $\left(\mathrm{m}^{2}\right)$ & 4,9 & 274,9 & 276,7 & 293,5 & 293,5 & 293,5 & 293,5 & 293,5 & 293,5 & 293,5 \\
\hline Ind. $/ \mathrm{m}^{2}$ & 5,9 & 26,0 & 30,3 & 26,1 & 32,5 & 24,2 & 15,9 & 6,8 & 6,8 & 165,4 \\
\hline Anz. Fam. & 8 & 49 & 46 & 49 & 47 & 42 & 41 & 33 & 35 & 66 \\
\hline Anz. Arten & 15 & 263 & 247 & 240 & 221 & 179 & 157 & 131 & 150 & 457 \\
\hline Artensumme & 15 & 264 & 325 & 377 & 406 & 422 & 430 & 439 & 457 & 457 \\
\hline
\end{tabular}

15687 Tiere, verteilt auf 298 Arten, fingen sich in Fallen an bzw. mit Eichentotholz. Aus Buchentotholz schlüpften 32850 Individuen aus 399 Arten. Die Schlüpfdichte war an den beiden Baumarten mit 163,4 bzw. 166,3 Ind./m² sehr ähnlich (Tab. 4-5).

Tabelle 4-5: Fangergebnisse der Eklektoren getrennt nach Baumart, Fallentyp und Untersuchungsjahr

\begin{tabular}{|c|c|c|c|c|c|c|c|c|c|}
\hline & \multicolumn{4}{|c|}{ E I C H E } & \multicolumn{3}{|c|}{ B U C H E } & \multirow{2}{*}{ Ges. } \\
\hline & & 1992 & 1993 & 1995 & Ges. & 1995 & 1996 & Ges. & \\
\hline \multirow[t]{5}{*}{ STE } & Proben & 11 & 27 & 10 & 48 & 25 & 26 & 51 & 99 \\
\hline & Probefl. $\left(\mathrm{m}^{2}\right)$ & 11,9 & 32,3 & 11,6 & 55,8 & 35,4 & 38,9 & 74,3 & 130,1 \\
\hline & Individuen & 2594 & 4967 & 1015 & 8576 & 6626 & 7069 & 13695 & 22271 \\
\hline & Ind. / m² & 218,4 & 153,7 & 87,3 & 153,7 & 187,2 & 181,7 & 184,3 & 171,2 \\
\hline & Anz. Arten & 136 & 194 & 85 & 248 & 195 & 199 & 257 & 331 \\
\hline \multirow[t]{5}{*}{$\mathrm{mBE}$} & Proben & 7 & 6 & & 13 & 19 & 20 & 39 & 52 \\
\hline & Probefl. $\left(\mathrm{m}^{2}\right)$ & 16,8 & 16,5 & & 33,3 & 59,8 & 38,6 & 98,4 & 131,7 \\
\hline & Individuen & 59 & 2214 & & 2273 & 3477 & 1571 & 5048 & 7321 \\
\hline & Ind. / $\mathrm{m}^{2}$ & 3,5 & 134,4 & & 68,3 & 58,1 & 40,7 & 51,3 & 55,6 \\
\hline & Anz. Arten & 21 & 121 & & 124 & 197 & 184 & 262 & 294 \\
\hline \multirow[t]{5}{*}{ EFs } & Proben & & 50 & & 50 & 36 & 55 & 91 & 141 \\
\hline & Probefl. $\left(\mathrm{m}^{2}\right)$ & & 6,9 & & 6,9 & 6,5 & 9,4 & 15,9 & 22,8 \\
\hline & Individuen & & 4838 & & 4838 & 5728 & 7741 & 13469 & 18307 \\
\hline & Ind. / m² & & 700,7 & & 700,7 & 886,0 & 819,8 & 846,7 & 802,5 \\
\hline & Anz. Arten & & 112 & & 112 & 114 & 151 & 173 & 201 \\
\hline \multirow[t]{5}{*}{ EFl } & Proben & & & & & 11 & 50 & 61 & 61 \\
\hline & Probefl. $\left(\mathrm{m}^{2}\right)$ & & & & & 1,6 & 7,3 & 8,9 & 8,9 \\
\hline & Individuen & & & & & 173 & 465 & 638 & 638 \\
\hline & Ind. / $\mathrm{m}^{2}$ & & & & & 111,3 & 63,4 & 71,8 & 71,8 \\
\hline & Anz. Arten & & & & & 44 & 85 & 107 & 107 \\
\hline \multirow{5}{*}{$\begin{array}{l}\text { Ekl. } \\
\text { ges. }\end{array}$} & Proben & 18 & 83 & 10 & 111 & 91 & 151 & 242 & 353 \\
\hline & Probefl. $\left(\mathrm{m}^{2}\right)$ & 28,7 & 55,7 & 11,6 & 96,0 & 103,2 & 94,3 & 197,5 & 293,5 \\
\hline & Individuen & 2653 & 12019 & 1015 & 15687 & 16004 & 16846 & 32850 & 48537 \\
\hline & Ind. / $\mathrm{m}^{2}$ & 92,4 & 215,9 & 87,3 & 163,4 & 155,0 & 178,7 & 166,3 & 165,4 \\
\hline & Anz. Arten & 145 & 257 & 85 & 298 & 296 & 322 & 399 & 457 \\
\hline
\end{tabular}


Die meisten Individuen und Arten wurden mit Stammeklektoren an liegendem Totholz (S TE) erfaßt. In den 99 Proben fingen sich 22271 Individuen aus 331 Arten. Pro Quadratmeter Totholzoberfläche schlüpften 171,2 Individuen. Der Medianwert der Schlüpfdichten betrug 130,1, die höchste festgestellte Dichte 1192,1 Ind./ $\mathrm{m}^{2}$. Die maximale in einem STE vorgefundene Artenzahl belief sich auf 61, im Durchschnitt wurden 33 Arten gezählt.

Mit den 51 STE-Proben an liegendem Buchentotholz wurde ein Fläche von $74,3 \mathrm{~m}^{2}$ mit einem Rindenanteil von 79,5\% befangen. Das Volumen betrug 8,6 $\mathrm{m}^{3}$. Im Jahr 1995 belief sich die untersuchte Fläche auf $35,4 \mathrm{~m}^{2}$ und 1996 auf $38,9 \mathrm{~m}^{2}$.

257 Arten waren mit 13695 Exemplaren vertreten (Tab. 4-6). Die überwiegende Zahl der Individuen wurde in den ersten Fangperioden erfaßt. Die Artenzahlen war mit 195 bzw. 199 in beiden Jahren annähernd identisch und auch die registrierte Schlüpfdichte war sehr ähnlich (187,2 bzw. 181,7 Ind. $/ \mathrm{m}^{2}$ ). Die Schlüpfdichte in den Einzelproben variierte zwischen 37,9 und 450,3 Ind. $/ \mathrm{m}^{2}$. Der Median wurde mit 162,1, der Mittelwert mit 180,6 Ind.$/ \mathrm{m}^{2}$ festgestellt. In der großen Mehrzahl der Proben schlüpften zwischen 50 und 199 Ind./m² (Abb. 4-1).

Tabelle 4-6: Fangergebnisse der STE-Proben an Buche in den Fangperioden $(1995,1996)$

\begin{tabular}{|l|rrrrrrrr|r|}
\hline & \multicolumn{7}{|c|}{ F a n g p e r i o d e } & \\
& P1b & P2 & P3 & P4 & P5 & P6 & P7 & \multicolumn{1}{c|}{ P8 } & Ges. \\
\hline Probefl. $\left(\mathrm{m}^{2}\right)$ & 74,3 & 74,3 & 74,3 & 74,3 & 74,3 & 74,3 & 74,3 & 74,3 & 74,3 \\
Ind. $/ \mathrm{m}^{2}$ & 37,7 & 34,7 & 30,3 & 31,2 & 22,1 & 16,0 & 5,4 & 7,0 & 184,3 \\
Anz. Arten & 123 & 124 & 130 & 115 & 106 & 86 & 63 & 66 & 257 \\
Artensumme & 123 & 163 & 197 & 215 & 230 & 238 & 248 & 257 & 257 \\
\hline
\end{tabular}

Die in den Proben festgestellten Artenzahlen lagen zwischen 22 und 61 (Median $=37$; Mittelwert = 37,7). In 5 Proben waren über 49 Arten vertreten, in 2 Proben weniger als 25 (Abb. 4-2). Die meisten Arten wurden in den ersten Fangperioden festgestellt. Fast jede zweite Art war bereits bis Mitte Mai und 77 \% bis Ende Juni in den Fallen nachzuweisen. 


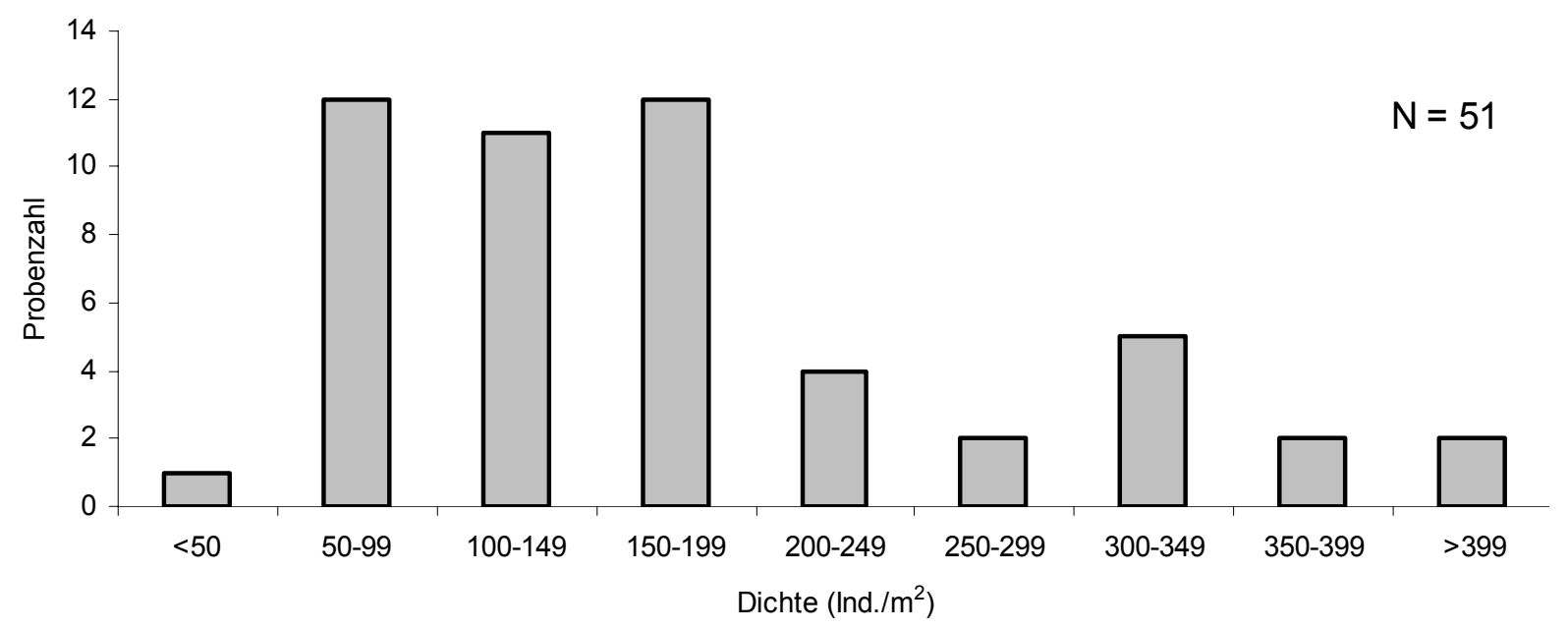

Abb. 4-1: Verteilung der Schlüpfdichten in STE-Proben an liegendem Buchentotholz

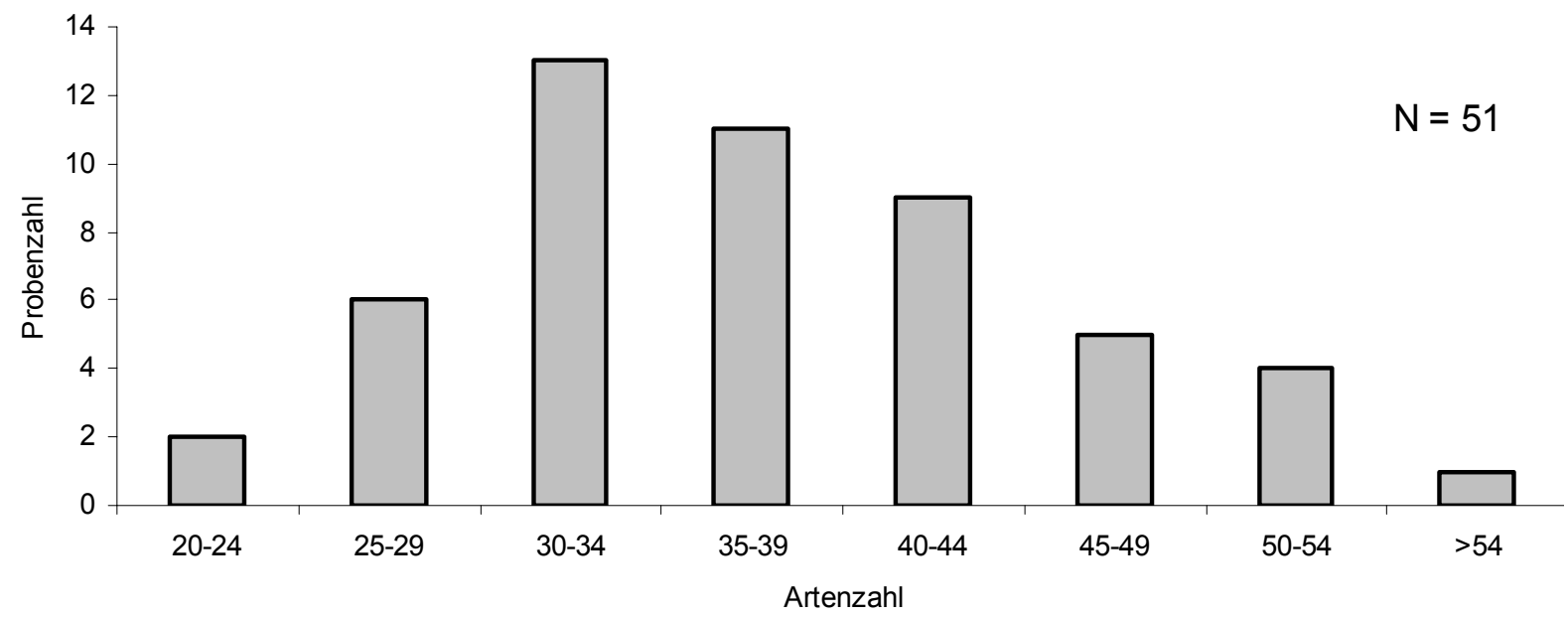

Abb. 4-2: Verteilung der Artenzahlenn in STE-Proben an liegendem Buchentotholz

Mit 48 STE-Proben an liegendem Eichentotholz wurde ein Fläche von $55,8 \mathrm{~m}^{2}$ mit einem Volumen von 5,6 $\mathrm{m}^{3}$ befangen. 46,6 \% der Fläche waren berindet. Im Jahr 1992 belief sich die Mantelfläche auf 11,9 $\mathrm{m}^{2}, 1993$ auf 32,3 $\mathrm{m}^{2}$ und 1995 auf 11,6 $\mathrm{m}^{2}$. Insgesamt wurden 8576 Individuen aus 248 Arten festgestellt, von denen 174 Arten auch in den STEProben an Buche gefunden worden waren. Die Schlüpfdichte belief sich auf $153,7 \mathrm{Ind} . / \mathrm{m}^{2}$ (Tab. 4-7). Im Vergleich zu den STE-Proben an Buchentotholz wurde in den Proben an Eiche sowohl eine im Durchschnitt niedrigere Individuen- als auch Artenzahl registriert.

Tabelle 4-7: Fangergebnisse der STE-Proben an Eiche in den Fangperioden $(1992,1993,1995)$

\begin{tabular}{|l|rcccccccc|r|}
\hline & \multicolumn{8}{|c|}{ F a n g p e r i o d e } & & \\
& P1a & P1b & P2 & P3 & P4 & P5 & P6 & P7 & \multicolumn{1}{c|}{ P8 } & Ges. \\
\hline Probefl. $\left(\mathrm{m}^{2}\right)$ & 4,9 & 54,0 & 55,8 & 55,8 & 55,8 & 55,8 & 55,8 & 55,8 & 55,8 & 55,8 \\
Ind. $/ \mathrm{m}^{2}$ & 5,9 & 24,4 & 24,2 & 20,2 & 35,4 & 15,8 & 14,1 & 8,1 & 11,6 & 153,7 \\
Anz. Arten & 15 & 138 & 122 & 104 & 97 & 73 & 68 & 61 & 63 & 248 \\
Artensumme & 15 & 139 & 182 & 206 & 217 & 227 & 235 & 239 & 248 & 248 \\
\hline
\end{tabular}


Die in den Einzelproben festgestellten Schlüpfdichten variierten zwischen 1192,1 und 15,6 Ind./m² (Abb. 4-3). Als Mittelwert ergaben sich 161,2, der Median betrug 97,4 Ind./ $\mathrm{m}^{2}$. In 25 Proben lag die Schlüpfdichte bei unter $100 \mathrm{Ind} . / \mathrm{m}^{2}$, in 11 Proben schlüpften 101-200, in 6 Proben 201-300, in 3 Proben 301-400 und in weiteren 3 Proben mehr als $400 \mathrm{Ind} . / \mathrm{m}^{2}$. Die überwiegende Zahl der Individuen wurde in den ersten Fangperioden gefangen, in denen die Schlüpfdichte - sieht man von dem geringen Stichprobenumfang der Periode P1a ab - bei jeweils über $20 \mathrm{Ind} . / \mathrm{m}^{2}$ lag.

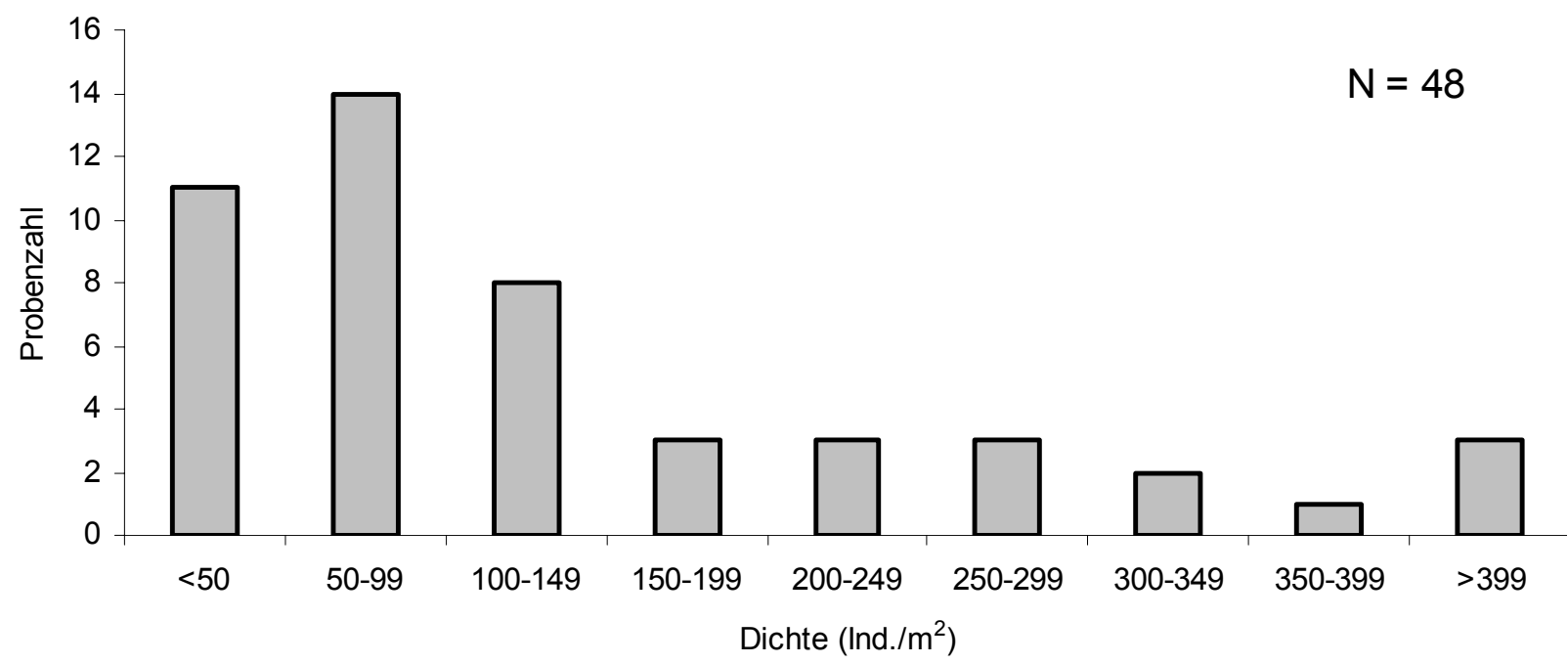

Abb. 4-3: Verteilung der Schlüpfdichten in STE-Proben an liegendem Eichentotholz

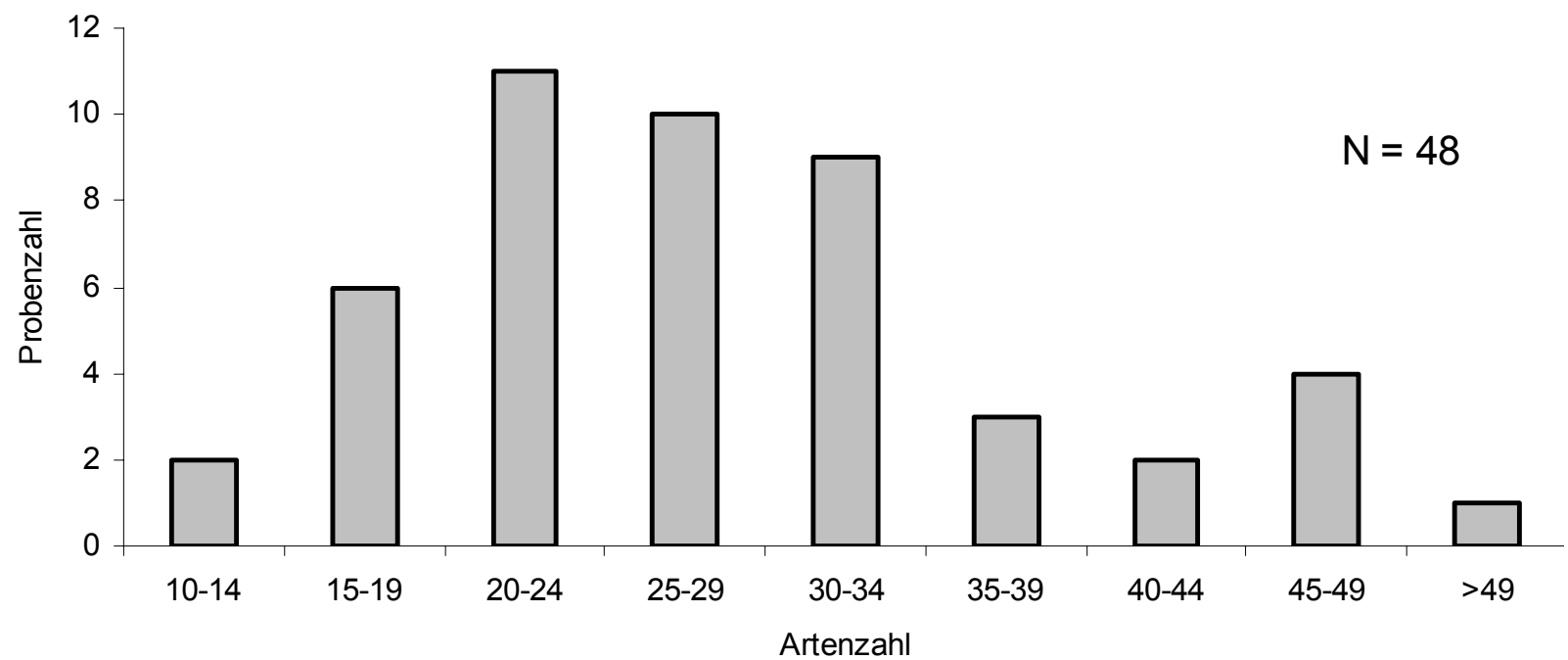

Abb. 4-4: Verteilung der Artenzahlen in STE-Proben an liegendem Eichentotholz

Die in den Proben vorgefundenen Artenzahlen lagen zwischen 10 und 55 (Median = 27; Mittelwert = 29). Am häufigsten wurden zwischen 20 und 34 Arten gefangen (Abb. 4-4). Die meisten Arten wurden, wie auch in den STE-Proben an Buche, in den ersten Fangperioden festgestellt. Mehr als die Hälfte der Arten (56 \%) war bereits bis Mitte Mai und $73 \%$ bis Ende Juni in den Fallen nachzuweisen. Das Maximum mit 138 Arten wurde in der Fangperiode $\mathrm{P} 1 \mathrm{~b}$ registriert. 
Mit den 141 Emergenzfallen an stehendem Totholz (EFs) wurde eine Mantelfläche von 22,8 $\mathrm{m}^{2}$ befangen, die zu 62,3 \% noch von Rinde bedeckt war. Die Schlüpfdichte belief sich auf 802,5 Ind. $/ \mathrm{m}^{2}$, der Medianwert betrug 423,5 Ind. $/ \mathrm{m}^{2} . \mathrm{Im}$ Durchschnitt traten in den Proben 15 Arten auf. Die 18307 Käferindividuen verteilten sich auf insgesamt 201 Arten.

An stehenden Buchenstämmen wurden 199536 und 199655 EFs-Proben genommen, die zusammen eine Fläche von 15,9 $\mathrm{m}^{2}$ abdeckten. Der Anteil der berindeten Fläche betrug im ersten Jahr $72 \%$ und im zweiten $69 \%$.

Insgesamt waren 173 Arten in den EFs-Proben nachzuweisen (Tab. 4-8). Die Schlüpfdichte lag 1995 mit 886,0 Ind. $/ \mathrm{m}^{2}$ etwas höher als im nachfolgenden Jahr, in dem 819,8 Ind. $/ \mathrm{m}^{2}$ registriert wurden. In beiden Untersuchungsjahren wurde die höchste Artendichte in der Fangperiode P3 festgestellt. Bis Ende Juni waren bereits 77 \% der Arten in den Proben nachgewiesen. Die höchste Schlüpfdichte wurde in der Fangperiode P4 gemessen.

Tabelle 4-8: Fangergebnisse der EFs-Proben an stehendem Buchentotholz (1995, 1996)

\begin{tabular}{|l|rrrrrrrr|r|}
\hline & \multicolumn{7}{|c|}{ F a n g p e r i o d e } & \\
& P1b & \multicolumn{1}{|c}{ P2 } & P3 & P4 & P5 & P6 & P7 & P8 & \multicolumn{1}{c|}{ Ges. } \\
\hline Probefl. $\left(\mathrm{m}^{2}\right)$ & 15,9 & 15,9 & 15,9 & 15,9 & 15,9 & 15,9 & 15,9 & 15,9 & 15,9 \\
Ind. $/ \mathrm{m}^{2}$ & 93,0 & 104,1 & 126,9 & 195,6 & 168,2 & 84,6 & 42,8 & 31,6 & 846,7 \\
Anz. Arten & 80 & 91 & 92 & 73 & 68 & 63 & 41 & 55 & 173 \\
Artensumme & 80 & 111 & 133 & 146 & 152 & 157 & 161 & 173 & 173 \\
\hline
\end{tabular}

Die höchste in einer Probe festgestellte Schlüpfdichte betrug 3830,0 Ind. $/ \mathrm{m}^{2}$, die niedrigste belief sich auf 45,3 Ind. $/ \mathrm{m}^{2}$. Der Mittelwert betrug 849,5 Ind. $/ \mathrm{m}^{2}$, der Median 576,5 Ind.$/ \mathrm{m}^{2}$. In etwa einem Drittel der Proben waren zwischen 200 und $600 \mathrm{Ind} . / \mathrm{m}^{2} \mathrm{zu}$ beobachten (Abb. 4-5), in 3 Proben wurden über $3500 \mathrm{Ind} . / \mathrm{m}^{2}$ registriert.

Die in den Proben vorgefundenen Artenzahlen variierten zwischen 3 und 42. Der Mittelwert und Median betrugen jeweils 17 Arten. In mehr als der Hälfte der Proben traten zwischen 10 und 19 Arten auf (Abb. 4-6).

An stehenden Eichenstämmen wurden 199350 EFs-Proben genommen, die zusammen eine Fläche von $6,9 \mathrm{~m}^{2}$ abdeckten. Der Anteil der berindeten Fläche betrug $44 \%$. Insgesamt wurden 4838 Tiere gefangen, die sich auf 112 Arten verteilten. Es wurde eine Schlüpfdichte von 700,7 Ind./ $\mathrm{m}^{2}$ registriert. Die Anzahl der gefangenen Individuen erreichte in der Fangperiode P2 mit einer Schlüpfdichte von $276 \mathrm{Ind} . / \mathrm{m}^{2}$ das Maximum. Anschließend gingen die Fangzahlen deutlich zurück (Tab. 4-9). Sowohl hinsichtlich der Schlüpfdichten als auch der in den Proben festgestellten Artenzahlen wurden in den EFs-Proben an Eiche niedrigere Werte als an stehendem Buchentotholz festgestellt. 


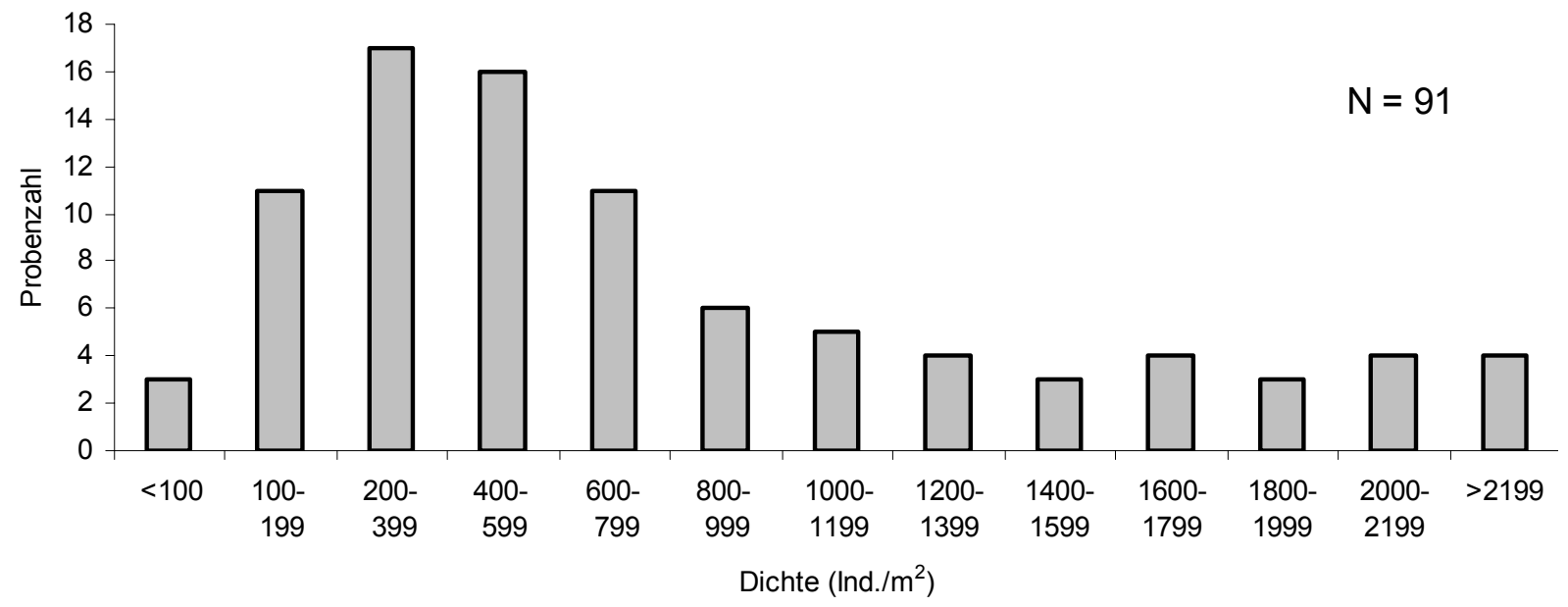

Abb. 4-5: Verteilung der Schlüpfdichten in EFs-Proben an stehendem Buchentotholz

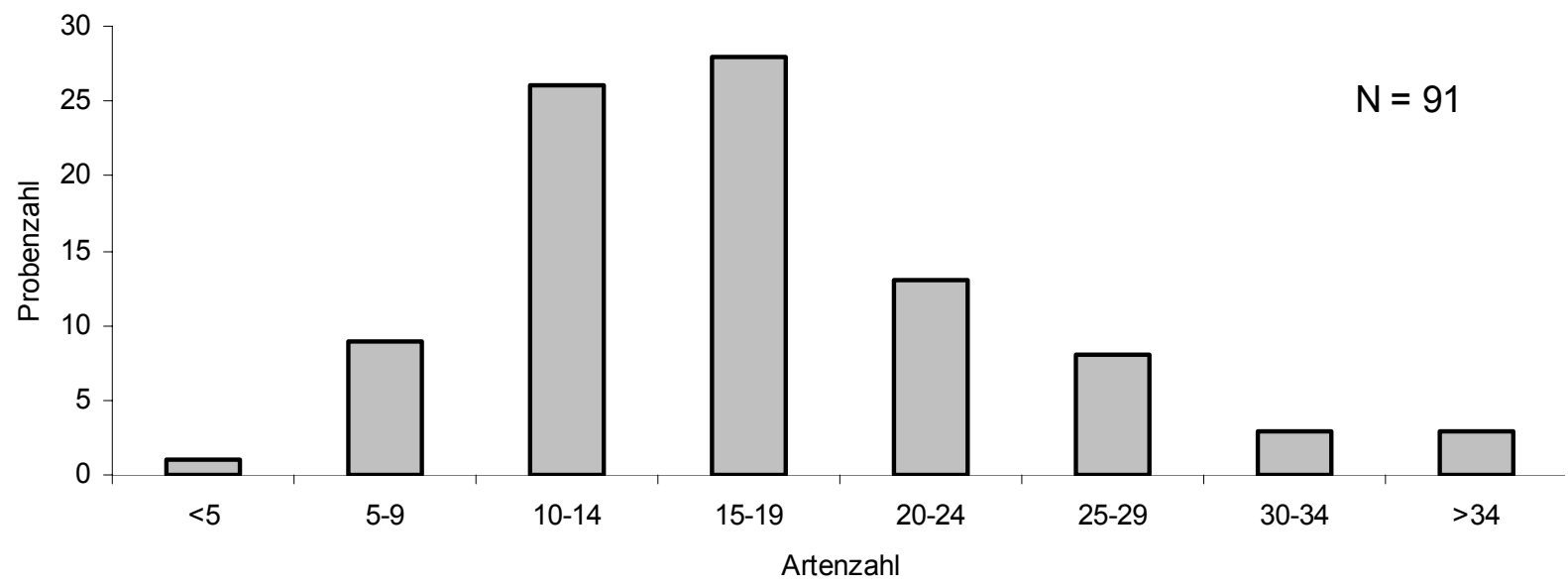

Abb. 4-6: Verteilung der Artenzahlen in EFs-Proben an stehendem Buchentotholz

Tabelle 4-9: Fangergebnisse der EFs-Proben an stehendem Eichentotholz (1993)

\begin{tabular}{|l|rrrrrrrr|r|}
\hline & \multicolumn{7}{|c|}{ F a n g p e r i o d e } & \\
& P1b & P2 & \multicolumn{1}{|c}{ P3 } & P4 & P5 & P6 & P7 & \multicolumn{1}{c|}{ P8 } & Ges. \\
\hline Probefl. $\left(\mathrm{m}^{2}\right)$ & 6,9 & 6,9 & 6,9 & 6,9 & 6,9 & 6,9 & 6,9 & 6,9 & 6,9 \\
Ind. $/ \mathrm{m}^{2}$ & 75,3 & 276,2 & 153,5 & 64,9 & 45,0 & 57,2 & 17,8 & 10,7 & 700,7 \\
Anz. Arten & 56 & 43 & 42 & 45 & 38 & 40 & 26 & 27 & 112 \\
Artensumme & 56 & 68 & 81 & 93 & 97 & 104 & 105 & 112 & 112 \\
\hline
\end{tabular}

Als maximale Dichte in einer Falle wurden 9093,3 Ind. $/ \mathrm{m}^{2}$ registriert, die niedrigste belief sich auf 23,1 Ind. $/ \mathrm{m}^{2}$. Der Mittelwert betrug 653,1 Ind. $/ \mathrm{m}^{2}$ und der Median lag bei 232,3 Ind. $/ \mathrm{m}^{2}$. In 8 Proben wurde eine Schlüpfdichte von unter 100, in 2 Proben von über 5000 Ind. $/ \mathrm{m}^{2}$ registriert. In der Mehrzahl waren es zwischen 100 und 300 Ind. $/ \mathrm{m}^{2}$ (Abb. 4-7). 


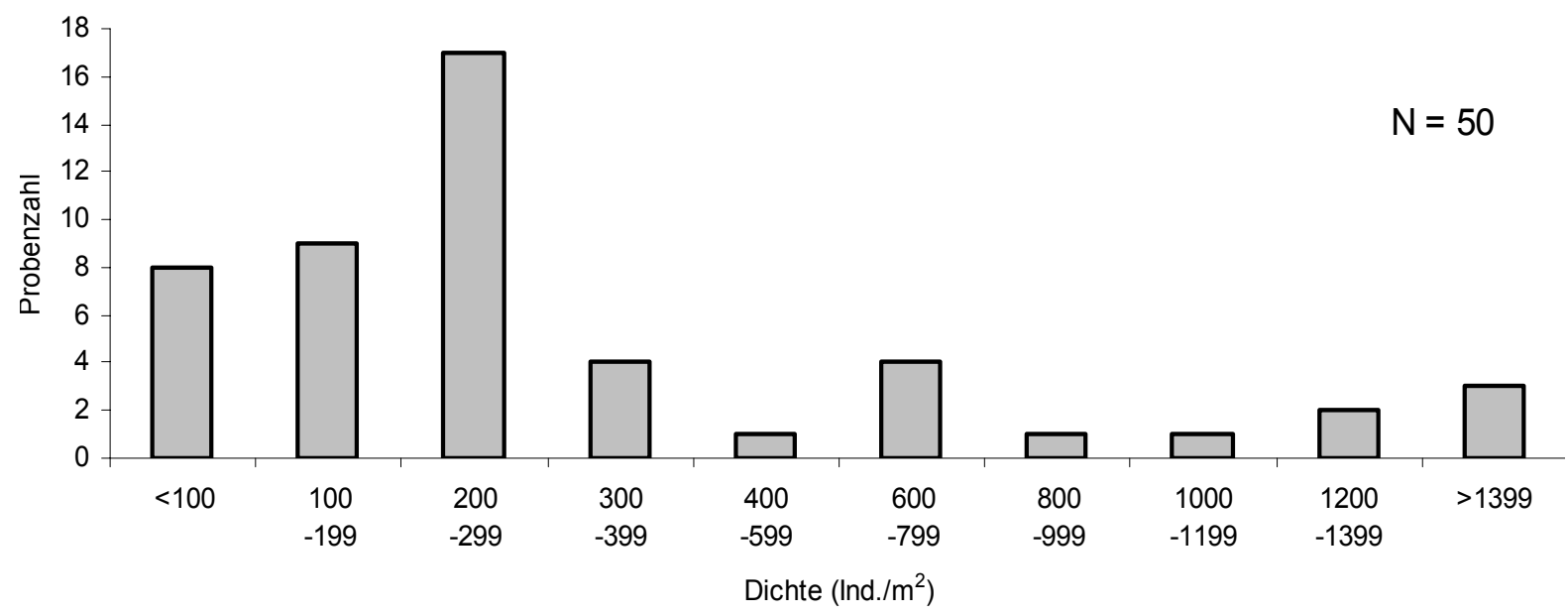

Abb. 4-7: Verteilung der Schlüpfdichten in EFs-Proben an stehendem Eichentotholz

Die in den Proben vorgefundenen Artenzahlen lagen zwischen 1 und 24. Der Mittelwert und Median betrugen jeweils 11 Arten. In der Hälfte der Proben wurden zwischen 10 und 19 Arten registriert, nur in 3 Proben lag die Artenzahl höher (Abb. 4-8). Die höchste Artendichte wurde in der Fangperiode P1b festgestellt, in der bereits $50 \%$ des Artenspektrums vorlagen. Fast drei Viertel der Arten waren, ähnlich wie in den EFs-Proben an Buche, bis zum Ende der Fangperiode P3 bereits in den Fallen aufgetreten.

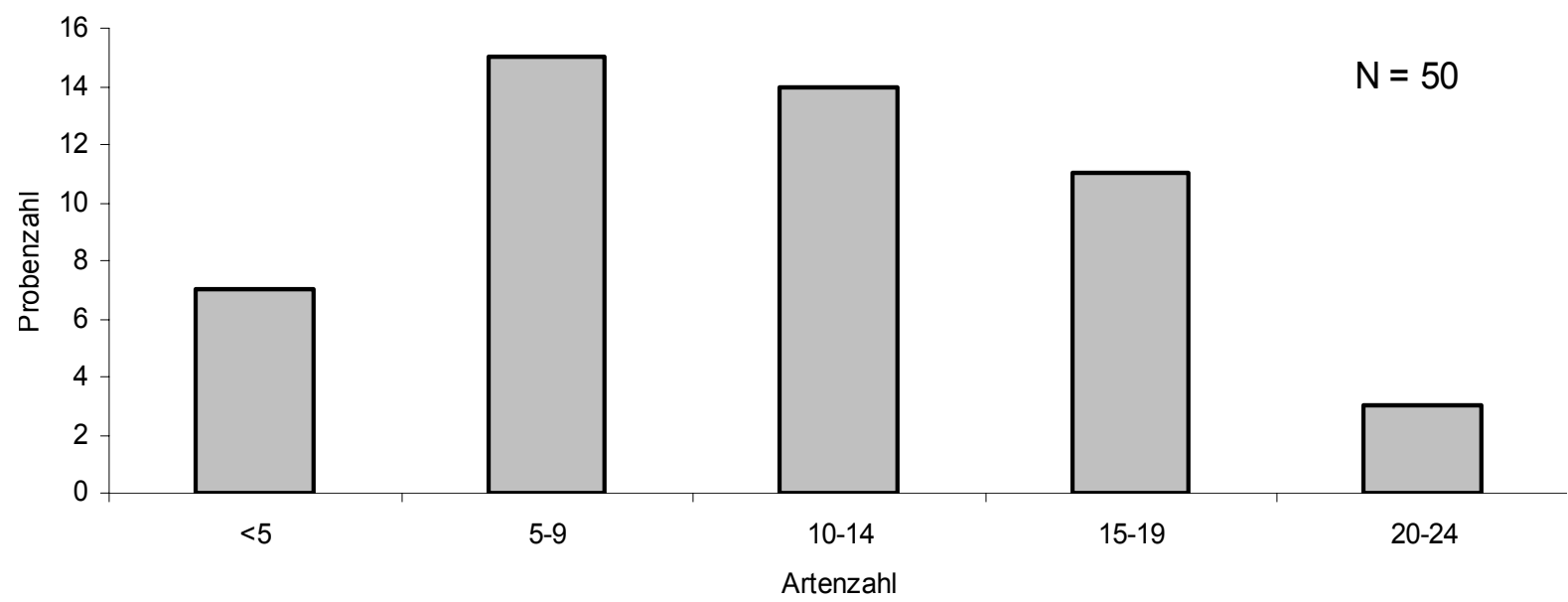

Abb. 4-8: Verteilung der Artenzahlen in EFs-Proben an stehendem Eichentotholz

Die modifizierten Bodeneklektoren (mBE) trugen mit 7321 Individuen aus 294 Arten zum Fangergebnis bei und wiesen mit 55,6 Ind. $/ \mathrm{m}^{2}$ die niedrigste Schlüpfdichte unter den eingesetzten Fallentypen auf. Die mBE wurden vor allem zur Beprobung dünneren Materials mit einem entsprechend hohen Oberflächen:Volumen-Verhältnis eingesetzt. Bezieht man die Individuenzahlen auf das befangene Totholzvolumen, wird mit 1645 Ind. $/ \mathrm{m}^{3}$ die Dichte für STE sogar überboten. Die höchste Dichte wurde in einer Probe mit Eichentotholz festgestellt (251,6 Ind. $/ \mathrm{m}^{2}$ ). Im Durchschnitt waren 27 Arten in den Proben vertreten. 
Die Emergenzfallen an liegendem Buchentotholz (EF1) erbrachten nur eine geringe Arten- und Individuenzahl. 638 Tiere, das entspricht einer Dichte von $71,8 \mathrm{Ind} . / \mathrm{m}^{2}$, verteilten sich auf 107 Arten.

\subsection{Die Familienstruktur}

Der bei weitem größte Teil der Familien war mit nur wenigen Individuen vertreten. 62 Familien stellten zusammen gerade einmal $19 \%$ der Tiere. 13 Familien erreichten jeweils Individuenanteile von über $2 \%$. Bezogen auf Fensterfallen und Eklektoren stellte sich das Bild als sehr ähnlich dar. In beiden Fällen waren 12 Familien mit jeweils mehr als $2 \%$ vertreten, wobei sich die Dominanzstruktur der Fensterfallen als die ausgeglichenere erwies. Acht Familien erlangten sowohl in den Fensterfallen als auch in den Eklektoren einen Anteil von über $2 \%$ (Tab. 4-10, Abb. 4-9 und Abb. 4-10).

Tabelle 4-10: Familienstruktur (systematisch geordnet)

\begin{tabular}{|l|rrr|rrr|rrr|}
\hline & \multicolumn{3}{|c|}{ Fensterfallen } & \multicolumn{3}{|c|}{ Eklektoren } & \multicolumn{3}{c|}{ Gesamt } \\
FAMILIE & Ind. & Ind.(\%) & Arten & Ind. & Ind.(\%) & Arten & Ind. & Ind.(\%) & Arten \\
\hline Carabidae & 201 & 1,23 & 28 & 525 & 1,08 & 19 & 726 & 1,12 & 39 \\
Dytiscidae & 98 & 0,60 & 6 & & & & 98 & 0,15 & 6 \\
Hydraenidae & 2 & 0,01 & 1 & 1 & 0,00 & 1 & 3 & 0,00 & 1 \\
Hydrophilidae & 178 & 1,09 & 17 & 14 & 0,03 & 5 & 192 & 0,30 & 18 \\
Histeridae & 32 & 0,20 & 7 & 918 & 1,89 & 6 & 950 & 1,46 & 9 \\
Silphidae & 82 & 0,50 & 5 & 10 & 0,02 & 2 & 92 & 0,14 & 6 \\
Leptinidae & & & & 9 & 0,02 & 1 & 9 & 0,01 & 1 \\
Cholevidae & 32 & 0,20 & 8 & 65 & 0,13 & 6 & 97 & 0,15 & 10 \\
Colonidae & 1 & 0,01 & 1 & & & & 1 & 0,00 & 1 \\
Leiodidae & 903 & 5,52 & 13 & 316 & 0,65 & 10 & 1219 & 1,88 & 15 \\
Scydmaenidae & 56 & 0,34 & 7 & 1104 & 2,27 & 12 & 1160 & 1,79 & 12 \\
Ptilidae & 609 & 3,72 & 14 & 2622 & 5,40 & 9 & 3231 & 4,98 & 15 \\
Scaphidiidae & 120 & 0,73 & 3 & 89 & 0,18 & 3 & 209 & 0,32 & 3 \\
Staphylinidae & 1864 & 11,40 & 164 & 5283 & 10,88 & 127 & 7147 & 11,01 & 201 \\
Pselaphidae & 449 & 2,74 & 13 & 592 & 1,22 & 12 & 1041 & 1,60 & 15 \\
Cantharidae & 99 & 0,61 & 10 & 173 & 0,36 & 10 & 272 & 0,42 & 14 \\
Malachiidae & 4 & 0,02 & 1 & 3 & 0,01 & 1 & 7 & 0,01 & 1 \\
Melyridae & 27 & 0,17 & 3 & 23 & 0,05 & 2 & 50 & 0,08 & 3 \\
Phloiophilidae & 1 & 0,01 & 1 & & & & 1 & 0,00 & 1 \\
Trogositidae & 2 & 0,01 & 1 & 3 & 0,01 & 1 & 5 & 0,01 & 1 \\
Lymexylonidae & 817 & 4,99 & 2 & 1325 & 2,73 & 2 & 2142 & 3,30 & 2 \\
Elateridae & 1927 & 11,78 & 19 & 460 & 0,95 & 17 & 2387 & 3,68 & 21 \\
Eucnemidae & 32 & 0,20 & 4 & 146 & 0,30 & 5 & 178 & 0,27 & 5 \\
Throscidae & 35 & 0,21 & 2 & 92 & 0,19 & 2 & 127 & 0,20 & 2 \\
Buprestidae & 1 & 0,01 & 1 & 9 & 0,02 & 2 & 10 & 0,02 & 2 \\
Clambidae & 10 & 0,06 & 2 & 2 & 0,00 & 1 & 12 & 0,02 & 2 \\
Scirtidae & 36 & 0,22 & 5 & 7 & 0,01 & 4 & 43 & 0,07 & 6 \\
Dryopidae & 1 & 0,01 & 1 & & & & 1 & 0,00 & 1 \\
Dermestidae & & & & 3 & 0,01 & 2 & 3 & 0,00 & 2 \\
\hline
\end{tabular}




\begin{tabular}{|c|c|c|c|c|c|c|c|c|c|}
\hline \multirow[b]{2}{*}{ FAMILIE } & \multicolumn{3}{|c|}{ Fensterfallen } & \multicolumn{3}{|c|}{ Eklektoren } & \multicolumn{3}{|c|}{ Gesamt } \\
\hline & Ind. & Ind.(\%) & Arten & Ind. & Ind.(\%) & Arten & Ind. & Ind.(\%) & Arten \\
\hline Byrrhidae & 3 & 0,02 & 1 & & & & 3 & 0,00 & 1 \\
\hline Byturidae & 261 & 1,60 & 2 & & & & 261 & 0,40 & 2 \\
\hline Cerylonidae & 492 & 3,01 & 3 & 1608 & 3,31 & 3 & 2100 & 3,24 & 3 \\
\hline Sphaerosomatidae & & & & 11 & 0,02 & 1 & 11 & 0,02 & 1 \\
\hline Nitidulidae & 1595 & 9,75 & 21 & 301 & 0,62 & 14 & 1896 & 2,92 & 25 \\
\hline Kateridae & 2 & 0,01 & 1 & 2 & 0,00 & 1 & 4 & 0,01 & 1 \\
\hline Rhizophagidae & 910 & 5,56 & 7 & 8701 & 17,93 & 6 & 9611 & 14,81 & 7 \\
\hline Cucujidae & 97 & 0,59 & 4 & 114 & 0,23 & 4 & 211 & 0,33 & 5 \\
\hline Silvanidae & 10 & 0,06 & 1 & 15 & 0,03 & 1 & 25 & 0,04 & 1 \\
\hline Erotylidae & 11 & 0,07 & 2 & & & & 11 & 0,02 & 2 \\
\hline Cryptophagidae & 587 & 3,59 & 30 & 1725 & 3,55 & 16 & 2312 & 3,56 & 34 \\
\hline Phalacridae & & & & 1 & 0,00 & 1 & 1 & 0,00 & 1 \\
\hline Laemophloeidae & 1 & 0,01 & 1 & 11 & 0,02 & 2 & 12 & 0,02 & 2 \\
\hline Lathridiidae & 1435 & 8,77 & 20 & 5668 & 11,68 & 16 & 7103 & 10,95 & 21 \\
\hline Merophysiidae & & & & 1 & 0,00 & 1 & 1 & 0,00 & 1 \\
\hline Mycetophagidae & 52 & 0,32 & 5 & 99 & 0,20 & 6 & 151 & 0,23 & 7 \\
\hline Colydiidae & 30 & 0,18 & 3 & 480 & 0,99 & 3 & 510 & 0,79 & 3 \\
\hline Corylophidae & 7 & 0,04 & 2 & 162 & 0,33 & 1 & 169 & 0,26 & 2 \\
\hline Endomychidae & & & & 4 & 0,01 & 1 & 4 & 0,01 & 1 \\
\hline Coccinellidae & 35 & 0,21 & 3 & 17 & 0,04 & 8 & 52 & 0,08 & 8 \\
\hline Sphindidae & 23 & 0,14 & 2 & 76 & 0,16 & 1 & 99 & 0,15 & 2 \\
\hline Cisidae & 227 & 1,39 & 11 & 1177 & 2,42 & 11 & 1404 & 2,16 & 13 \\
\hline Anobiidae & 132 & 0,81 & 7 & 3856 & 7,94 & 9 & 3988 & 6,15 & 9 \\
\hline Ptinidae & 1 & 0,01 & 1 & 10 & 0,02 & 2 & 11 & 0,02 & 2 \\
\hline Oedemeridae & & & & 2 & 0,00 & 1 & 2 & 0,00 & 1 \\
\hline Salpingidae & 229 & 1,40 & 4 & 307 & 0,63 & 4 & 536 & 0,83 & 4 \\
\hline Pyrochroidae & 117 & 0,72 & 2 & 105 & 0,22 & 2 & 222 & 0,34 & 2 \\
\hline Scraptiidae & 77 & 0,47 & 7 & 346 & 0,71 & 7 & 423 & 0,65 & 7 \\
\hline Aderidae & 10 & 0,06 & 1 & 563 & 1,16 & 1 & 573 & 0,88 & 1 \\
\hline Anthicidae & 1 & 0,01 & 1 & & & & 1 & 0,00 & 1 \\
\hline Mordellidae & 16 & 0,10 & 2 & 14 & 0,03 & 3 & 30 & 0,05 & 3 \\
\hline Melandryidae & 62 & 0,38 & 6 & 1796 & 3,70 & 6 & 1858 & 2,86 & 7 \\
\hline Tetratomidae & 10 & 0,06 & 1 & 4 & 0,01 & 2 & 14 & 0,02 & 2 \\
\hline Alleculidae & 12 & 0,07 & 2 & 12 & 0,02 & 3 & 24 & 0,04 & 3 \\
\hline Tenebrionidae & 27 & 0,17 & 2 & 835 & 1,72 & 4 & 862 & 1,33 & 4 \\
\hline Trogidae & & & & 1 & 0,00 & 1 & 1 & 0,00 & 1 \\
\hline Geotrupidae & 2 & 0,01 & 1 & & & & 2 & 0,00 & 1 \\
\hline Scarabaeidae & 290 & 1,77 & 13 & 18 & 0,04 & 1 & 308 & 0,47 & 14 \\
\hline Lucanidae & 36 & 0,22 & 1 & 125 & 0,26 & 1 & 161 & 0,25 & 1 \\
\hline Cerambycidae & 190 & 1,16 & 13 & 124 & 0,26 & 11 & 314 & 0,48 & 16 \\
\hline Chrysomelidae & 27 & 0,17 & 7 & 27 & 0,06 & 7 & 54 & 0,08 & 11 \\
\hline Anthribidae & 1 & 0,01 & 1 & 4 & 0,01 & 2 & 5 & 0,01 & 3 \\
\hline Scolytidae & 1477 & 9,03 & 15 & 6156 & 12,68 & 12 & 7633 & 11,76 & 18 \\
\hline Rhynchitidae & 12 & 0,07 & 3 & 2 & 0,00 & 1 & 14 & 0,02 & 3 \\
\hline Apionidae & & & & 3 & 0,01 & 2 & 3 & 0,00 & 2 \\
\hline Curculionidae & 232 & 1,42 & 14 & 260 & 0,54 & 16 & 492 & 0,76 & 20 \\
\hline
\end{tabular}


Am häufigsten fingen sich Vertreter der Rhizophagidae. Diese artenarme Käferfamilie ist in Mitteleuropa mit nur einer Gattung und 14 Arten vertreten, von denen 7 im Untersuchungsgebiet festgestellt wurden. Die flachen und gestreckten Käfer und ihre Larven sind häufig und in großen Dichten unter Rinde zu finden, wo sie sich räuberisch von anderen Insekten ernähren. Die oft genannte Affinität zu Borkenkäfern bedarf allerdings einer kritischen Prüfung (KÖHLER 2000). Zwar traten sie auch im 'Neuenburger Urwald' oftmals miteinander vergesellschaftet auf, doch ihre Verteilungen und Häufigkeiten am Totholz sprachen für eine eher lockere Beziehung.

Mehr als $90 \%$ der Individuen wurden in den Eklektoren gefangen, wo sie einen Anteil von $17,9 \%$ an den Gesamtfängen hatten. Ihre Anteile in den Untersuchungsjahren variierten zwischen 9,9 und 20,6 \%. Während in den mBE nur ein geringer Anteil der Fänge auf diese Familie entfiel (1,9\%), stellten sie 28,3 \% der STE- und 11,7 \% der EFs-Fänge. Unabhängig vom Fallentyp wurden die höheren Anteile jeweils an der Buche registriert. So gehörte jedes dritte in STE an Buche gefangene Individuum zu dieser Familie, während der Anteil an der Eiche 19,9 \% betrug. Der häufigste Vertreter der Rhizophagiden in den Eklektoren war mit $76 \%$ Rhizophagus dispar. Daneben erreichten Rh. bipustulatus (16\%) und Rh. nitidulus (7\%) noch nennenswerte Anteile.

In den Fensterfallen erreichten die Rhizophagiden einen Individuenanteil von 5,6 \%. Dieser war in den einzelnen Jahren mit 4,2 bis 6,9\% ziemlich konstant. Wie in den Eklektoren zeigten die Rhizophagiden die größte Aktivität im Frühling und Frühsommer. In den Flugbarrierefallen stellte Rh. bipustulatus $39 \%$ der Individuen dieser Familie, gefolgt von $R h$. dispar (28\%), Rh. nitidulus (14\%) und Rh. perforatus (13\%).

Die Scolytidae waren mit 11,8 \% Individuenanteil aus 18 Arten die zweithäufigste Käferfamilie im Untersuchungsgebiet. Ihr Anteil an den Eklektorfängen war mit 12,7 \% etwas höher als in den Fensterfallen (9,0 \%). Die Vertreter dieser Familie zeigen in den meisten Fällen eine Präferenz für bestimmte Baumarten und -teile. Nur wenige Arten besiedeln sowohl Nadel- als auch Laubholz, wie z. B. der im 'Neuenburger Urwald' nicht seltene Xyleborus saxeseni. Besonders in den ersten Phasen der Holzzersetzung spielen Scolytiden eine große Rolle, einige Arten besiedeln bereits noch lebende, i. d. R. aber stark geschwächte Bäume.

Die Mehrzahl der Arten kann zu den Rindenbrütern gerechnet werden, die sich von der Pflanzensubstanz ernähren. $45 \%$ der Scolytiden-Individuen, die in den Eklektoren gefangen wurden, gehörten zu dieser Gruppe, 41 \% wurden allein durch Dryocoetes villosus gestellt. In den Fensterfallen waren die Rindenbrüter mit $24 \%$ beteiligt, von denen Taphrorychus bicolor mit $13 \%$ den höchsten Individuenanteil hatte.

Die Holzbrüter ernähren sich nicht unmittelbar vom Holz der Bäume, statt dessen sind sie Züchter von Ambrosia-Pilzen, die ihnen die schwer verdauliche Holzsubstanz aufbereiten. Die vier im 'Neuenburger Urwald' gefundenen Vertreter stellten 56 \% (Eklektoren) bzw. $76 \%$ (Fensterfallen) der Individuen dieser Käferfamilie. War in den Fensterfallen Xyleborus dispar ihr häufigster Vertreter, herrschte in den Eklektoren Xyloterus signatus vor.

Der Anteil der Borkenkäfer an den Fängen der einzelnen Untersuchungsjahre zeigte starke Schwankungen. In den Fensterfallen variierte ihr Anteil am Jahresfang zwischen 4,8 und $15,3 \%$. In den Eklektoren ergaben sich die jährlichen Schwankungen vor allem durch den Einfluss der Baumart. Während an der Eiche 31,3 \% der Individuen aus dieser Familie stammten, belief sich ihr Anteil an der Buche auf nur 3,8 \%. In den EFs-Proben an Eiche 
waren die Borkenkäfer mit 57,6 \% besonders zahlreich vertreten, aber auch in den STE waren sie mit 24,8 \% die dominierende Familie. Dagegen belief sich ihr Anteil in den STE-Proben an Buche auf 6,7 \% und am stehenden Buchentotholz sogar auf nur 1,4 \%. Borkenkäfer sind beim Abbau von Buchentotholz allem Anschein nach von untergeordneter Bedeutung.

Die Staphylinidae gehörten mit jeweils $11 \%$ Anteil sowohl in den Eklektoren als auch in den Fensterfallen zu den dominierenden Familien. Die einzelnen Jahre zeigten kaum nennenswerte Unterschiede im Anteil dieser Familie (zwischen 9,9 und 11,4\%). Diese artenreichste Käferfamilie Mitteleuropas trägt auch im 'Neuenburger Urwald' mit insgesamt 201 festgestellten Arten, von denen 127 in den Eklektoren und 164 Arten in Fensterfallen gefunden wurden, am stärksten zur Artenvielfalt bei. Entsprechend vielfältig sind ihre Lebensweisen. Sie sind in fast allen Habitaten des Waldes zu finden, so an pflanzlichen und tierischen Faulstoffen, in Nestern u. a. auch von Ameisen, an Pilzen, im Boden und in der Laubstreu. Auch an Totholz werden sie regelmäßig zu den vorherrschenden Familien gezählt. Die Nahrungsverhältnisse erweisen sich ebenfalls als sehr vielfältig. Phyto-, myceto-, sapround zoophage Ernährungsweise sind vertreten. Viele Arten halten sich allerdings nicht an die Klassengrenzen, bei ihnen steht regelmäßig Mischkost auf dem Speiseplan. In ihrer Mehrheit scheinen sich die Arten, und zwar die Larven ebenso wie die Imagines, räuberisch zu ernähren.

Manche Arten lassen eine Bindung oder doch zumindest Präferenz für Totholz erkennen. Aus dieser Gruppe rekrutierten sich auch alle häufigeren Staphylinidenarten der Eklektoren. Fast jeder vierte Kurzdeckenflügler war ein Phloeonomus punctipennis, $18 \%$ entfielen auf Leptusa fumida, beides Besiedler von Totholzrinde. Der Individuenanteil der Staphylinidae war an beiden Baumarten mit 9,1 (Eiche) bzw. 11,7 \% (Buche) in etwa gleich hoch. Die geringsten Anteile wurden jeweils am stehenden Totholz registriert.

Nur wenige Arten waren gleichzeitig auch in den Fensterfallen häufig, wie z. B. der corticole Gabrius splendidulus. Boden- und pilzbewohnende Arten nehmen hier einen größeren Anteil ein. Der häufigste Kurzdeckenflügler der Fensterfallen war allerdings mit Leptusa pulchella ebenfalls ein Rindenbewohner. 


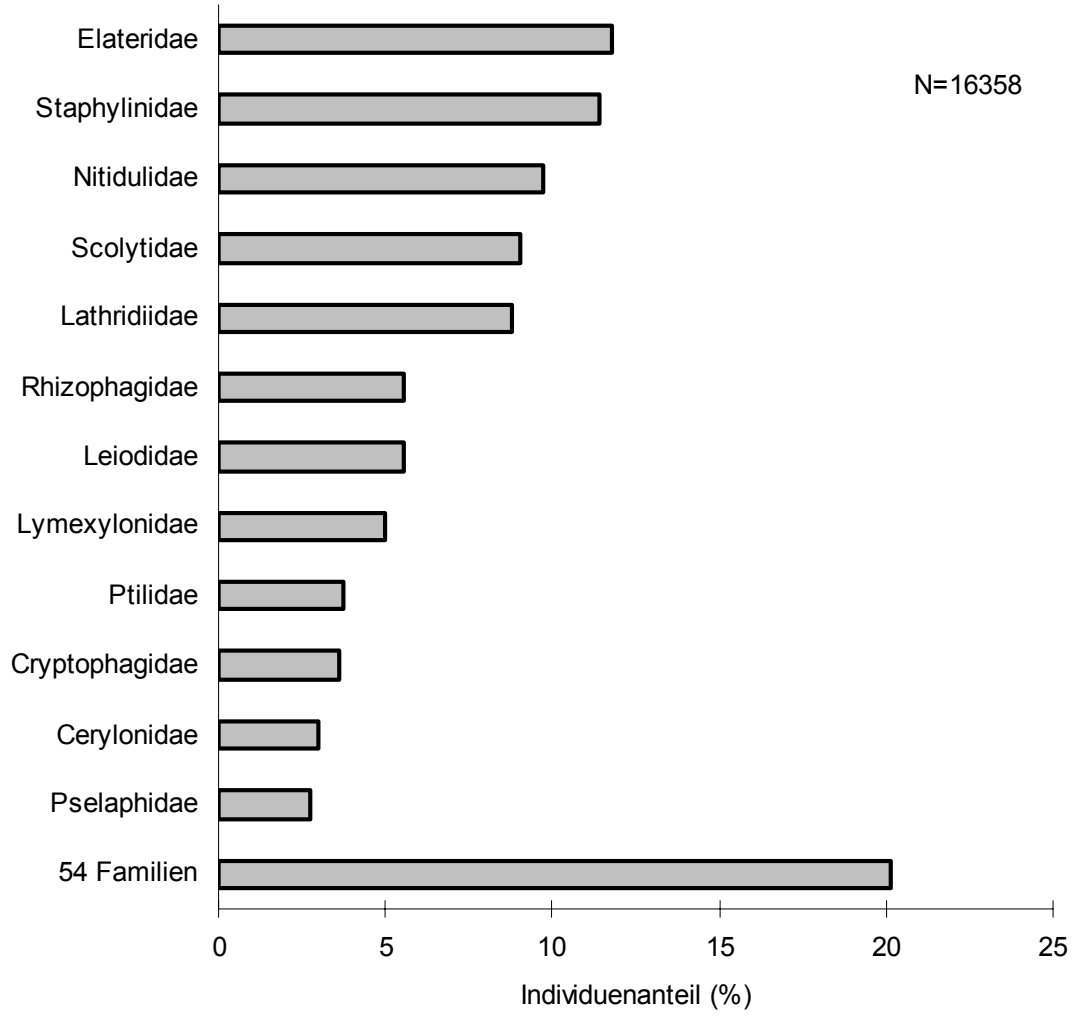

Abb. 4-9: Familiendominanz in den Fensterfallen (1992-96)

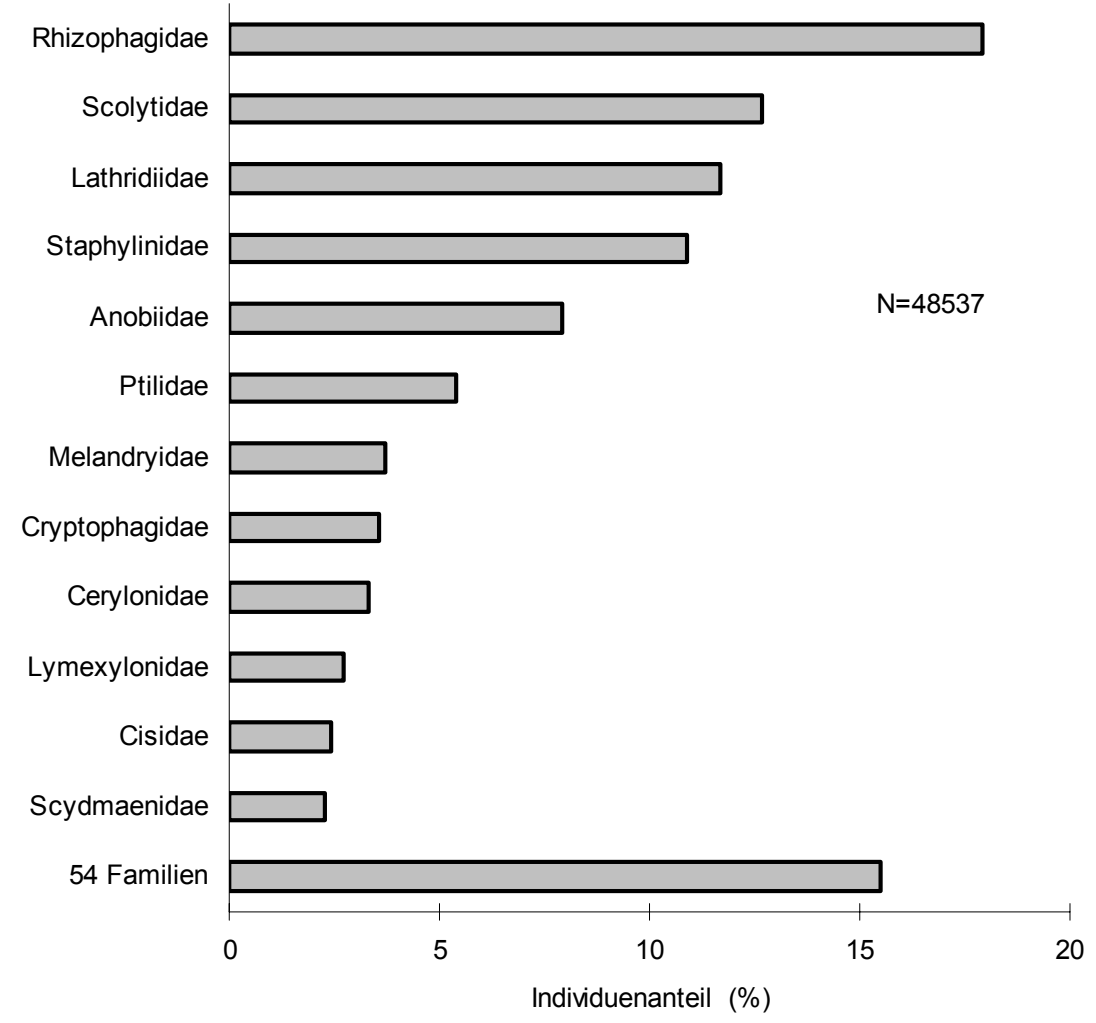

Abb. 4-10: Familiendominanz in den Eklektoren (1992-96) 
Lathridiidae sind in ihrer Mehrzahl sehr kleine Tiere von 1 - $2 \mathrm{~mm}$ und werden überall dort gefunden, wo sich Schimmelpilze entwickeln, also besonders häufig an feuchtem, sich zersetzendem Material, so auch an Totholz. Sie ernähren sich von Mycelien und Sporen von Holzpilzen und niederen Pilzen.

Von den 21 festgestellten Arten wurden 20 Arten mit einem Individuenanteil von zusammen $8,8 \%$ in Flugbarrierefallen gefangen. Vorherrschend waren hier Enicmus rugosus (21,6\%), E. testaceus (18,8 \%), E. fungicola (14,2 \%) und Aridius nodifer (17,5\%). Der Anteil dieser Familie an den Fensterfallenfängen variierte in den einzelnen Untersuchungsjahren zwischen 7,1 und 10,2\%. Nur A. nodifer hatte gleichzeitig auch einen hohen Anteil $(12,8 \%)$ an den Lathrididen aus den Eklektoren, in denen diese Familie 11,7 \% des Fanges stellte. Die eindeutig dominierende Art dieser Familie war jedoch Corticaria longicollis mit $72,1 \%$. Darüber hinaus trat nur noch Dinerella elongata mit einem nennenswerten Anteil $(8,8$ $\%)$ in Erscheinung. An den beiden untersuchten Baumarten stellten die Lathridiiden $11 \%$ (Eiche) bzw. $12 \%$ (Buche) des Gesamtfanges, wobei ihr Anteil in den EFs-Proben jeweils am höchsten ausfiel.

Zur Familie der Ptilidae gehören die kleinsten Käfer Mitteleuropas. Die meisten Arten erreichen gerade einmal eine Größe von 0,5 - 1,0 mm. Larven und Imagines ernähren sich von Pilzsporen, insbesondere von Schimmelpilzarten, aber auch anderer höherer Pilze. Sie sind überall dort zu finden, wo ihre Nahrung zur Verfügung steht. Dies sind besonders feuchte Örtlichkeiten wie z. B. faulende Pflanzenstoffe, Laubstreu und Totholzmulm.

Ptilidae waren mit 5,0\% an den Fängen aus dem 'Neuenburger Urwald' beteiligt. Ihr Anteil belief sich in den Fensterfallen auf 3,7\% und in den Eklektoren auf 5,4 \%. In den Fensterfallen variierte ihr Anteil in den Untersuchungsjahren zwischen 0,6 und 8,1\%. In den verschiedenen Eklektortypen waren die Individuenanteile jeweils an der Buche die höheren. Sowohl in den Flugbarrierefallen als auch in den Eklektoren stiegen die Fangzahlen ab Saisonbeginn kontinuierlich an und erreichten zwischen Mitte Juli und Anfang August ihr Maximum. In diesem Zeitraum waren sie mit 15,9 \% an den Fängen beteiligt. Arten mit einer Präferenz für Totholz beherrschten das Bild. Ihr Anteil in den Fensterfallen belief sich auf $79 \%$, in den Eklektoren sogar auf 98 \%. Von den 15 festgestellten Arten erreichten in beiden Fallensystemen Ptinella limbata, Pt. aptera und Pteryx suturalis höhere Anteile. Micridium halidaii war nur in den Eklektoren und Acrotrichis intermedia nur in den Flugbarrierefallen mit größeren Anteilen vertreten.

3,6 \% der gefangenen Individuen zählten zu den Cryptophagidae. Das galt sowohl für die Eklektor- als auch für die Fensterfallenfänge. Viele der meist kleinen Vertreter dieser artenreichen Familie sind an faulenden und schimmelnden Pflanzenresten zu finden, wo sie sich u. a. von Schimmelpilzen ernähren. Von den 34 Arten des 'Neuenburger Urwaldes' wurden 30 mit Fensterfallen erfaßt, nur 16 Arten mit Eklektoren. Der höchste Individuenanteil dieser Familie wurde mit 6,7 \% in den EFs-Proben an Buchentotholz gemessen. Der Individuenanteil jener Arten, für die eine Präferenz für Totholz und -pilze angegeben wird, war gering. In den Fensterfallen und den Eklektoren wurden jeweils nur ca. 50 Exemplare aus dieser Gruppe gefangen. Der größte Anteil entfiel auf Arten, die keine Präferenz für bestimmte Habitate erkennen lassen. Ihr Anteil an den Fängen dieser Familie erreichte in den Fensterfallen fast $50 \%$, in den Eklektoren sogar $98 \%$. In den Eklektoren war diese Gruppe fast ausschließlich durch Cryptophagus dentatus repräsentiert, der 93,7 \% der Individuen dieser Familie stellte und auch der häufigste Cryptophagidae der Fensterfallen war (37,3 \%). 
Darüber hinaus traten in den Flugbarrierefallen noch Atomaria nigrirostris, A. turgida und Antherophagus nigricornis häufiger auf.

Von den drei in Mitteleuropa beheimateten Arten aus der Familie der Lymexylonidae wurden zwei Arten mit einem Anteil von 5,0 \% in den Fensterfallen und von 2,7 \% in den Eklektoren gefangen. Der Anteil dieser Familie an den FF-Fängen zeigte in den einzelnen Untersuchungsjahren erhebliche Abweichungen. Die Larven dieser schmalen, langgestreckten Käfer bohren Gänge in das Holz noch lebender oder bereits abgestorbener Bäume. Hylecoetus dermestoides, deren Larven sich von eingetragenen Ambrosiapilzen ernähren, war mit 93,8 \% die bei weitem häufigste Art dieser Familie. Der xylophage Lymexylon navale erreichte nur in den Eklektoren einen Anteil von $10 \%$ an dieser Familie, in den Fensterfallen wurde lediglich ein Exemplar gefangen.

Als letzte Familie, die sowohl in den Eklektoren als auch in den Fensterfallen einen Individuenanteil von über $2 \%$ erreichte, sind hier die Cerylonidae mit einem Individuenanteil von 3,3 bzw. 3,0 \% zu nennen, der sich auf jeweils drei übereinstimmende Arten, allesamt zoophage Totholzpräferenten, verteilte. Der Anteil variierte in den Fensterfallen in den Untersuchungsjahren zwischen 2,4 und 3,4 \%. Mit einem Anteil von über $5 \%$ wurde der höchste Anteil in den STE-Proben an Buchentotholz registriert. Mit $79 \%$ in den Eklektoren bzw. $94 \%$ in den Fensterfallen bestimmte vor allem Cerylon ferrugineum das Bild dieser Familie.

Jeweils vier Familien erreichten ausschließlich in den Fensterfallen oder in den Eklektoren einen Anteil von über $2 \%$. Die dominierende Familie in den Fensterfallen waren die Elateridae, die mit 19 Arten 11,8 \% der gefangenen Tiere stellten. Ihr Anteil in den Untersuchungsjahren variierte zwischen 8,0 und 17,7 \%. Ein Drittel der Tiere wurde allein von Athous subfuscus gestellt. Daneben erreichten Athous haemorrhoidalis, Denticollis linearis, Melanotus castanipes und Agriotes aterrimus mit Anteilen von 10 bis $15 \%$ größere Bedeutung an den Fängen dieser Familie. Die Nitidulidae waren mit 9,8 \% am Fang beteiligt, die sich auf 21 Arten verteilten, von denen aber nur 3 in größeren Mengen gefangen wurden: Meligethes denticulatus (70 \%), M. aeneus (8\%) und Cychramus luteus (15\%). Die Leiodidae hatten in den Fensterfallen einen Anteil von 5,5 \%. 1993 entfielen 12,9 \%, 1995 nur 6,1 \% der Tiere auf diese Familie. Der häufigste Vertreter war Agathidium varians (31\%). Pselaphidae traten ebenfalls mit einem größeren Anteil in Erscheinung (2,7 \%). Der höchste Anteil wurde 1993 mit 3,5 \% registriert. Neben den beiden mulmbewohnenden Arten Euplectus bescidicus (34 \%) und E. punctatus (17\%) war der Rindenkäfer Bibloporus bicolor (28\%) häufiger in den Fensterfallen vertreten.

In den Eklektoren waren die Anobiidae mit einem Individuenanteil von 7,9 \% vertreten. Von den 3856 gefangenen Tieren wurden 3600 allein durch Ptilinus pectinicornis gestellt. Während sich der Individuenanteil an der Eiche auf unter $1 \%$ beschränkte, gehörten $11 \%$ der an Buchentotholz gefangenen Tiere zu dieser Familie. Besonders hoch war ihr Anteil in den EFs-Proben an Buche (20\%), wohingegen nur $2 \%$ der in STE-Proben gefunden Tiere zu den Anobiiden zählte. Melandryidae waren mit 3,7\% in den Eklektorfängen vertreten, die fast vollständig durch Orchesia undulata gestellt wurden. $85 \%$ der Melandryiden wurden in den mBE-Proben nachgewiesen, was auf eine Präferenz von $O$. undulata für schwächer dimensioniertes Material hindeutet. Cisidae waren an der Eiche mit einem Anteil von 0,9\% vertreten, während an der Buche ein Anteil von 3,1 \% registriert wurde. An beiden Baumarten wurden die höchsten Anteile am stehenden Totholz gefangen. Cis nitidus (42\%), C. castaneus (26\%) und Octotemnus glabriculus (16\%) waren die häufigsten Vertreter dieser Familie. Die 
Scydmaenidae waren an den Eklektorfängen mit 2,3\% beteiligt. $47 \%$ von ihnen entfielen auf Stenichnus collaris und $37 \%$ auf Neuraphes elongatulus.

Die Tabelle 4-10 zeigt auch den Anteil der übrigen Familien in den Fensterfallen und den Eklektoren. Eine weitergehende Differenzierung der Eklektorfänge nach Baumart und Fallentyp kann dem Anhang 3 entnommen werden.

\subsection{Artenliste}

Die folgende Tabelle 11 listet die im 'Neuenburger Urwald' vorgefundenen 711 Käferarten in systematischer Reihenfolge auf. Neben dem EDV-Code werden auch die vorgenommene Zuordnung zu den Konsumententypen und nach ihren Biotopkomplex- und Habitatpräferenzen aufgeführt ${ }^{6}$.

Tabelle 4-11: Liste der im 'Neuenburger Urwald' nachgewiesenen Käferarten einschließlich Angaben zur Nachweismethode $(\mathrm{FF}=$ Fensterfalle, Ekl = Eklektor) und $\mathrm{zu}$ ihrer ökologischen Klassifizierung.

\begin{tabular}{|c|c|c|c|c|c|c|}
\hline EDV-Code & A r t & FF & Ekl & Kon & Bio & Hab \\
\hline 01-.000-.000-. & Familie Carabidae & & & & & \\
\hline 01-.004-.001-. & Carabus coriaceus L., 1758 & & $\mathrm{x}$ & $\mathrm{Z}$ & W & B \\
\hline 01-.004-.010-. & Carabus problematicus Hbst.,1786 & & $\mathrm{x}$ & $\mathrm{z}$ & W & B \\
\hline 01-.004-.012-. & Carabus granulatus L., 1758 & & $\mathrm{x}$ & $\mathrm{z}$ & W & B \\
\hline 01-.004-.026-. & Carabus nemoralis Müll.,1764 & & & $\mathrm{z}$ & $\mathrm{E}$ & B \\
\hline 01-.005-.003-. & Cychrus caraboides (L., 1758) & & & $\mathrm{z}$ & W & B \\
\hline 01-.006-.002--. & Leistus rufomarginatus (Duft.,1812) & & $\mathrm{x}$ & $\mathrm{z}$ & W & B \\
\hline 01-.007-.006-. & Nebria brevicollis (F.,1792) & & $\mathrm{x}$ & $\mathrm{z}$ & W & B \\
\hline 01-.009-.007-. & Notiophilus rufipes Curt., 1829 & & & $\mathrm{z}$ & $\mathrm{O}$ & B \\
\hline 01-.009-.008-. & Notiophilus biguttatus (F.,1779) & $\mathrm{x}$ & $\mathrm{x}$ & $\mathrm{z}$ & $\mathrm{E}$ & $\mathrm{B}$ \\
\hline 01-.012-.002-. & Elaphrus cupreus Duft.,1812 & $\mathrm{x}$ & & $\mathrm{z}$ & $\mathrm{F}$ & B \\
\hline 01-.013-.001-. & Loricera pilicornis (F.,1775) & $\mathrm{x}$ & & $\mathrm{z}$ & $\mathrm{E}$ & $\mathrm{B}$ \\
\hline 01-.015-.001-. & Clivina fossor (L.,1758) & $\mathrm{x}$ & & $\mathrm{z}$ & $\mathrm{E}$ & $\mathrm{B}$ \\
\hline $01-.021-.007-$. & Trechus obtusus Er.,1837 & $\mathrm{x}$ & & $\mathrm{z}$ & $\mathrm{E}$ & B \\
\hline 01-.028-.001-. & Tachyta nana (Gyll.,1810) & & $\mathrm{x}$ & $\mathrm{z}$ & W & TR \\
\hline 01-.029-.042-. & Bembidion deletum Serv., 1821 & $\mathrm{x}$ & & $\mathrm{z}$ & $\mathrm{F}$ & $\mathrm{B}$ \\
\hline 01-.029-.090-. & Bembidion quadrimaculatum (L.,1761) & & & $\mathrm{z}$ & $\mathrm{E}$ & B \\
\hline 01-.029-.101-. & Bembidion mannerheimi Sahlb., 1827 & & & $\mathrm{z}$ & $\mathrm{F}$ & B \\
\hline 01-.029-.103-. & Bembidion lunulatum Fourcr., 1785 & $\mathrm{x}$ & $\mathrm{x}$ & $\mathrm{z}$ & $\mathrm{F}$ & $\mathrm{B}$ \\
\hline 01-.030-.004-. & Asaphidion flavipes (L.,1761) & $\mathrm{x}$ & & $\mathrm{z}$ & $\mathrm{O}$ & $\mathrm{B}$ \\
\hline 01-.032-.001-. & Patrobus australis Sahlb.,1875 & & & $\mathrm{z}$ & $\mathrm{F}$ & $\mathrm{B}$ \\
\hline 01-.037-.001-. & Anisodactylus binotatus (F.,1787) & $\mathrm{x}$ & & $\mathrm{z}$ & $\mathrm{O}$ & $\mathrm{B}$ \\
\hline 01-.041-.045-. & Harpalus latus (L.,1758) & & & $\mathrm{p}$ & $\mathrm{O}$ & B \\
\hline
\end{tabular}

${ }^{6}$ Die Liste berücksichtigt nicht die sich aus dem 4. Supplementband der Käfer Mitteleuropas (LUCHT \& KLAUSNITZER 1998) ergebenden Veränderungen. Die betroffenen Arten können dem Anhang 12 entnommen werden. 


\begin{tabular}{|c|c|c|c|c|c|c|}
\hline EDV-Code & A r t & FF & Ekl & Kon & Bio & Hab \\
\hline 01-.041-.047-. & Harpalus quadripunctatus Dej., 1829 & $\mathrm{x}$ & & $\mathrm{p}$ & W & B \\
\hline $01-.042-.001-$. & Stenolophus teutonus (Schrk.,1781) & $\mathrm{x}$ & & $\mathrm{z}$ & $\mathrm{O}$ & B \\
\hline 01-.045-.005-. & Bradycellus harpalinus (Serv.,1821) & $\mathrm{x}$ & & $\mathrm{p}$ & $\mathrm{O}$ & B \\
\hline 01-.046-.002-. & Acupalpus flavicollis (Sturm, 1825) & $\mathrm{x}$ & & $\mathrm{z}$ & $\mathrm{F}$ & B \\
\hline 01-.046-.010-. & Acupalpus exiguus (Dej.,1829) & $\mathrm{x}$ & & $\mathrm{z}$ & $\mathrm{F}$ & $\mathrm{B}$ \\
\hline 01-.049-.001-. & Stomis pumicatus (Panz.,1796) & & $\mathrm{x}$ & $\mathrm{z}$ & $\mathrm{F}$ & $\mathrm{B}$ \\
\hline 01-.051-.011-. & Pterostichus strenuus (Panz., 1797) & $\mathrm{x}$ & & $\mathrm{z}$ & $\mathrm{F}$ & $\mathrm{B}$ \\
\hline 01-.051-.0191. & Pterostichus rhaeticus Heer, 1837 & & & $\mathrm{z}$ & $\mathrm{W}$ & B \\
\hline 01-.051-.022-. & Pterostichus minor (Gyll.,1827) & $\mathrm{x}$ & $\mathrm{x}$ & $\mathrm{z}$ & $\mathrm{F}$ & B \\
\hline 01-.051-.024-. & Pterostichus oblongopunctatus (F.,1787) & & $\mathrm{x}$ & $\mathrm{z}$ & $\mathrm{W}$ & B \\
\hline 01-.051-.026-. & Pterostichus niger (Schall.,1783) & & $\mathrm{x}$ & $\mathrm{z}$ & $\mathrm{W}$ & B \\
\hline 01-.051-.027-. & Pterostichus melanarius (Ill.,1798) & & & $\mathrm{z}$ & $\mathrm{O}$ & $\mathrm{B}$ \\
\hline 01-.053-.002-. & Abax parallelepipedus (Pill.Mitt.,1783) & & $\mathrm{x}$ & $\mathrm{z}$ & $\mathrm{W}$ & $\mathrm{B}$ \\
\hline 01-.056-.008-. & Calathus rotundicollis Dej., 1828 & & & $\mathrm{z}$ & $\mathrm{W}$ & B \\
\hline 01-.062-.009-. & Agonum muelleri (Hbst.,1784) & $\mathrm{x}$ & $\mathrm{x}$ & $\mathrm{z}$ & $\mathrm{E}$ & B \\
\hline $01-.062-.028-$. & Agonum fuliginosum (Panz., 1809) & $\mathrm{x}$ & & $\mathrm{z}$ & $\mathrm{F}$ & $\mathrm{B}$ \\
\hline $01-.063-.002-$. & Platynus assimilis (Payk.,1790) & & & $\mathrm{z}$ & $\mathrm{W}$ & $\mathrm{B}$ \\
\hline 01-.065-.001-. & Amara plebeja (Gyll.,1810) & $\mathrm{x}$ & & $\mathrm{p}$ & $\mathrm{O}$ & $\mathrm{B}$ \\
\hline 01-.065-.008-. & Amara similata (Gyll.,1810) & $\mathrm{x}$ & $\mathrm{x}$ & $\mathrm{p}$ & $\mathrm{E}$ & B \\
\hline 01-.065-.014-. & Amara communis (Panz.,1797) & $\mathrm{x}$ & $\mathrm{x}$ & $\mathrm{p}$ & $\mathrm{E}$ & B \\
\hline 01-.065-.018-. & Amara lunicollis Schdte., 1837 & $\mathrm{x}$ & & $\mathrm{p}$ & $\mathrm{O}$ & $\mathrm{B}$ \\
\hline $01-.065-.021-$. & Amara aenea (Geer, 1774) & $\mathrm{x}$ & $\mathrm{x}$ & $\mathrm{p}$ & $\mathrm{E}$ & $\mathrm{B}$ \\
\hline 01-.065-.026-. & Amara familiaris (Duft.,1812) & $\mathrm{x}$ & & $\mathrm{p}$ & $\mathrm{E}$ & B \\
\hline 01-.065-.029-. & Amara tibialis (Payk.,1798) & $\mathrm{x}$ & & $\mathrm{p}$ & $\mathrm{O}$ & B \\
\hline 01-.070-.002-. & Badister bullatus (Schrk.,1798) & $\mathrm{x}$ & & $\mathrm{z}$ & $\mathrm{E}$ & B \\
\hline 01-.070-.003-. & Badister lacertosus Sturm, 1815 & $\mathrm{x}$ & & $\mathrm{z}$ & $\mathrm{F}$ & $\mathrm{B}$ \\
\hline 01-.079-.012-. & Dromius quadrimaculatus (L.,1758) & $\mathrm{x}$ & $\mathrm{x}$ & $\mathrm{z}$ & $\mathrm{W}$ & $\mathrm{V}$ \\
\hline 01-.079-.013-. & Dromius spilotus (Ill.,1798) & & $\mathrm{x}$ & $\mathrm{z}$ & $\mathrm{W}$ & $\mathrm{V}$ \\
\hline 04-.000-.000-. & Familie Dytiscidae & & & & & \\
\hline 04-.008-.004-. & Hydroporus umbrosus (Gyll.,1808) & $\mathrm{x}$ & & $\mathrm{z}$ & $\mathrm{W}$ & $\mathrm{W}$ \\
\hline 04-.008-.009-. & Hydroporus palustris (L., 1761) & $\mathrm{x}$ & & $\mathrm{z}$ & $\mathrm{F}$ & $\mathrm{W}$ \\
\hline 04-.008-.019-. & Hydroporus planus (F., 1781) & $\mathrm{x}$ & & $\mathrm{z}$ & $\mathrm{F}$ & $\mathrm{W}$ \\
\hline 04-.008-.027-. & Hydroporus memnonius Nicol.,1822 & $\mathrm{x}$ & & $\mathrm{z}$ & $\mathrm{F}$ & $\mathrm{W}$ \\
\hline 04-.023-.003-. & Agabus chalconotus (Panz.,1796) & $\mathrm{x}$ & & $\mathrm{z}$ & $\mathrm{F}$ & $\mathrm{W}$ \\
\hline 04-.023-.009-. & Agabus bipustulatus (L., 1767) & $\mathrm{x}$ & & $\mathrm{z}$ & $\mathrm{F}$ & $\mathrm{W}$ \\
\hline 07-.000-.000-. & Familie Hydraenidae & & & & & \\
\hline 07-.001-.000-. & Hydraena spec. & $\mathrm{x}$ & $\mathrm{x}$ & $\mathrm{p}$ & $\mathrm{F}$ & $\mathrm{W}$ \\
\hline 09-.000-.000-. & Familie Hydrophilidae & & & & & \\
\hline 09-.0011.008-. & Helophorus grandis Ill., 1798 & $\mathrm{x}$ & & $\mathrm{p}$ & $\mathrm{F}$ & $\mathrm{W}$ \\
\hline 09-.0011.0091. & Helophorus aequalis Thoms., 1868 & $\mathrm{x}$ & & $\mathrm{p}$ & $\mathrm{F}$ & $\mathrm{W}$ \\
\hline 09-.0011.0152. & Helophorus brevipalpis Bedel, 1881 & $\mathrm{x}$ & & $\mathrm{p}$ & $\mathrm{F}$ & $\mathrm{W}$ \\
\hline 09-.0011.022-. & Helophorus flavipes F., 1792 & $\mathrm{x}$ & & $\mathrm{p}$ & $\mathrm{F}$ & $\mathrm{W}$ \\
\hline 09-.0011.0221. & Helophorus obscurus Muls.,1844 & $\mathrm{x}$ & & $\mathrm{p}$ & $\mathrm{F}$ & $\mathrm{W}$ \\
\hline 09-.0011.028-. & Helophorus minutus F.,1775 & $\mathrm{x}$ & & $\mathrm{p}$ & $\mathrm{F}$ & $\mathrm{W}$ \\
\hline 09-.002-.001-. & Sphaeridium bipustulatum F.,1781 & $\mathrm{x}$ & & $\mathrm{s}$ & $\mathrm{E}$ & $\mathrm{F}$ \\
\hline 09-.002-.003-. & Sphaeridium scarabaeoides (L.,1758) & $\mathrm{x}$ & & $\mathrm{s}$ & $\mathrm{E}$ & $\mathrm{F}$ \\
\hline
\end{tabular}




\begin{tabular}{|c|c|c|c|c|c|c|}
\hline EDV-Code & Art & FF & Ekl & Kon & Bio & Hab \\
\hline 09-.002-.004-. & Sphaeridium lunatum F.,1792 & $\mathrm{x}$ & $\mathrm{x}$ & $\mathrm{s}$ & $\mathrm{E}$ & $\mathrm{F}$ \\
\hline 09-.003-.005-. & Cercyon impressus (Sturm, 1807) & $\mathrm{x}$ & & $\mathrm{s}$ & $\mathrm{E}$ & $\mathrm{F}$ \\
\hline 09-.003-.008-. & Cercyon melanocephalus (L.,1758) & $\mathrm{x}$ & $\mathrm{x}$ & $\mathrm{s}$ & $\mathrm{E}$ & $\mathrm{F}$ \\
\hline 09-.003-.011-. & Cercyon lateralis (Marsh., 1802) & $\mathrm{x}$ & & $\mathrm{s}$ & $\mathrm{E}$ & $\mathrm{F}$ \\
\hline 09-.003-.013-. & Cercyon unipunctatus (L.,1758) & & $\mathrm{x}$ & $\mathrm{s}$ & $\mathrm{E}$ & $\mathrm{F}$ \\
\hline 09-.003-.017-. & Cercyon pygmaeus (Ill.,1801) & $\mathrm{x}$ & $\mathrm{x}$ & $\mathrm{s}$ & $\mathrm{E}$ & $\mathrm{F}$ \\
\hline 09-.003-.021-. & Cercyon convexiusculus Steph.,1829 & $\mathrm{x}$ & & $\mathrm{s}$ & $\mathrm{E}$ & $\mathrm{F}$ \\
\hline 09-.004-.001-. & Megasternum obscurum (Marsh., 1802) & $\mathrm{x}$ & $\mathrm{x}$ & $\mathrm{s}$ & $\mathrm{E}$ & $\mathrm{F}$ \\
\hline 09-.008-.001-. & Hydrobius fuscipes (L., 1758) & $\mathrm{x}$ & & $\mathrm{s}$ & $\mathrm{F}$ & $\mathrm{W}$ \\
\hline 09-.010-.002-. & Anacaena limbata (F.,1792) & $\mathrm{x}$ & & $\mathrm{s}$ & $\mathrm{F}$ & $\mathrm{W}$ \\
\hline $10-.000-.000-$. & Familie Histeridae & & & & & \\
\hline $10-.002-.004-$. & Plegaderus dissectus Er., 1839 & $\mathrm{x}$ & $\mathrm{x}$ & $\mathrm{z}$ & $\mathrm{W}$ & $\mathrm{TM}$ \\
\hline $10-.005-.001-$. & Abraeus granulum Er., 1839 & $\mathrm{x}$ & $\mathrm{x}$ & $\mathrm{z}$ & $\mathrm{W}$ & $\mathrm{TM}$ \\
\hline $10-.009-.001-$. & Gnathoncus rotundatus (Kug., 1792) & & $\mathrm{x}$ & $\mathrm{z}$ & $\mathrm{E}$ & $\mathrm{N}$ \\
\hline $10-.009-.002-$. & Gnathoncus nannetensis (Mars.,1862) & & $\mathrm{x}$ & $\mathrm{z}$ & $\mathrm{E}$ & $\mathrm{N}$ \\
\hline 10-.009-.004-. & Gnathoncus buyssoni Auzat, 1917 & $\mathrm{x}$ & $\mathrm{x}$ & $\mathrm{z}$ & $\mathrm{E}$ & $\mathrm{N}$ \\
\hline $10-.020-.001-$. & Paromalus flavicornis (Hbst., 1792) & $\mathrm{x}$ & $\mathrm{x}$ & $\mathrm{z}$ & $\mathrm{W}$ & $\mathrm{TR}$ \\
\hline $10-.029-.005-$. & Margarinotus ventralis (Mars.,1854) & $\mathrm{x}$ & & $\mathrm{z}$ & $\mathrm{E}$ & $\mathrm{F}$ \\
\hline $10-.029-.008-$. & Margarinotus striola (Sahlb.,1819) & $\mathrm{x}$ & & $\mathrm{z}$ & $\mathrm{W}$ & $\mathrm{F}$ \\
\hline $10-.029-.013-$. & Margarinotus marginatus (Er., 1834) & $\mathrm{x}$ & & $\mathrm{z}$ & $\mathrm{E}$ & $\mathrm{N}$ \\
\hline $10-.032-.003-$. & Hister unicolor L., 1758 & & & $\mathrm{z}$ & $\mathrm{E}$ & $\mathrm{F}$ \\
\hline 11-.000-.000-. & Familie Sphaeritidae & & & & & \\
\hline $11-.001-.001-$. & Sphaerites glabratus (F., 1792) & & & - & $\mathrm{W}$ & TS \\
\hline $12-.000-.000-$. & Familie Silphidae & & & & & \\
\hline $12-.001-.002-$. & Necrophorus humator (Gled.,1767) & $\mathrm{x}$ & & $\mathrm{s}$ & $\mathrm{E}$ & $\mathrm{F}$ \\
\hline $12-.001-.006-$. & Necrophorus vespilloides Hbst.,1783 & $\mathrm{x}$ & & $\mathrm{s}$ & $\mathrm{W}$ & $\mathrm{F}$ \\
\hline $12-.003-.002-$. & Thanatophilus sinuatus (F.,1775) & $\mathrm{x}$ & & $\mathrm{s}$ & $\mathrm{E}$ & $\mathrm{F}$ \\
\hline $12-.004-.001-$. & Oeceoptoma thoracica (L.,1758) & $\mathrm{x}$ & $\mathrm{x}$ & $\mathrm{s}$ & $\mathrm{E}$ & $\mathrm{F}$ \\
\hline $12-.006-.001-$. & Xylodrepa quadrimaculata (Scop.,1772) & $\mathrm{x}$ & & $\mathrm{z}$ & $\mathrm{W}$ & $\mathrm{V}$ \\
\hline $12-.009-.001-$. & Phosphuga atrata (L.,1758) & & $\mathrm{x}$ & $\mathrm{z}$ & $\mathrm{W}$ & E \\
\hline $13-.000-.000-$. & Familie Leptinidae & & & & & \\
\hline $13-.001-.001-$. & Leptinus testaceus Müll.,1817 & & $\mathrm{x}$ & $\mathrm{s}$ & $\mathrm{E}$ & $\mathrm{N}$ \\
\hline $14-.000-.000-$. & Familie Cholevidae & & & & & \\
\hline $14-.002-.001-$. & Nemadus colonoides (Kr.,1851) & & $\mathrm{x}$ & $\mathrm{s}$ & $\mathrm{W}$ & $\mathrm{N}$ \\
\hline $14-.005-.001-$. & Nargus velox (Spence,1815) & $\mathrm{x}$ & $\mathrm{x}$ & $\mathrm{s}$ & $\mathrm{W}$ & B \\
\hline $14-.005-.003-$. & Nargus wilkini (Spence, 1815) & $\mathrm{x}$ & $\mathrm{x}$ & $\mathrm{s}$ & $\mathrm{W}$ & B \\
\hline 14-.010-.001-. & Sciodrepoides watsoni (Spence,1815) & $\mathrm{x}$ & & $\mathrm{s}$ & $\mathrm{E}$ & $\mathrm{F}$ \\
\hline $14-.010-.002-$. & Sciodrepoides fumatus (Spence,1815) & $\mathrm{x}$ & & $\mathrm{s}$ & $\mathrm{E}$ & $\mathrm{F}$ \\
\hline $14-.011-.007-$. & Catops tristis (Panz.,1793) & $\mathrm{x}$ & & $\mathrm{s}$ & $\mathrm{E}$ & $\mathrm{N}$ \\
\hline $14-.011-.012-$. & Catops nigrita Er., 1837 & $\mathrm{x}$ & $\mathrm{x}$ & $\mathrm{s}$ & $\mathrm{F}$ & $\mathrm{N}$ \\
\hline 14-.011-.016-. & Catops fuscus (Panz.,1794) & $\mathrm{x}$ & & $\mathrm{s}$ & $\mathrm{E}$ & $\mathrm{N}$ \\
\hline $14-.011-.017-$. & Catops fuliginosus Er.,1837 & $\mathrm{x}$ & $\mathrm{x}$ & $\mathrm{s}$ & $\mathrm{E}$ & $\mathrm{N}$ \\
\hline $14-.011-.020-$. & Catops picipes (F., 1792) & & $\mathrm{x}$ & $\mathrm{s}$ & $\mathrm{W}$ & $\mathrm{N}$ \\
\hline $15-.000-.000-$. & Familie Colonidae & & & & & \\
\hline $\begin{array}{l}\text { 15-.001-.000-. } \\
\text { 16-.000-.000-. }\end{array}$ & $\begin{array}{l}\text { Colon spec. } \\
\text { Familie Leiodidae }\end{array}$ & $\mathrm{x}$ & & $\mathrm{m}$ & $\mathrm{E}$ & $\mathrm{P}$ \\
\hline
\end{tabular}




\begin{tabular}{|c|c|c|c|c|c|c|}
\hline EDV-Code & A r t & FF & Ekl & Kon & Bio & Hab \\
\hline 16-.003-.0131. & Leiodes oblonga (Er.,1845) & $\mathrm{x}$ & & $\mathrm{m}$ & $\mathrm{W}$ & $\mathrm{P}$ \\
\hline 16-.003-.020-. & Leiodes polita (Marsh.,1802) & $\mathrm{x}$ & & $\mathrm{m}$ & $\mathrm{E}$ & $\mathrm{P}$ \\
\hline 16-.004-.001-. & Colenis immunda (Sturm,1807) & $\mathrm{x}$ & & $\mathrm{m}$ & $\mathrm{E}$ & $\mathrm{P}$ \\
\hline 16-.007-.001-. & Anisotoma humeralis (F.,1792) & $\mathrm{x}$ & $\mathrm{x}$ & $\mathrm{m}$ & $\mathrm{W}$ & $\mathrm{TP}$ \\
\hline 16-.007-.003-. & Anisotoma castanea (Hbst.,1792) & $\mathrm{x}$ & & $\mathrm{m}$ & $\mathrm{W}$ & $\mathrm{TP}$ \\
\hline 16-.007-.005-. & Anisotoma orbicularis (Hbst.,1792) & $\mathrm{x}$ & $\mathrm{x}$ & $\mathrm{m}$ & $\mathrm{W}$ & $\mathrm{TP}$ \\
\hline 16-.009-.001-. & Amphicyllis globus (F.,1792) & & $\mathrm{x}$ & $\mathrm{m}$ & $\mathrm{W}$ & $\mathrm{P}$ \\
\hline 16-.011-.003-. & Agathidium varians $($ Beck, 1817) & $\mathrm{x}$ & $\mathrm{x}$ & $\mathrm{m}$ & $\mathrm{W}$ & $\mathrm{TP}$ \\
\hline 16-.011-.007-. & Agathidium rotundatum (Gyll.,1827) & $\mathrm{x}$ & $\mathrm{x}$ & $\mathrm{m}$ & $\mathrm{W}$ & $\mathrm{P}$ \\
\hline 16-.011-.008-. & Agathidium confusum Bris., 1863 & $\mathrm{x}$ & $\mathrm{x}$ & $\mathrm{m}$ & $\mathrm{W}$ & $\mathrm{B}$ \\
\hline 16-.011-.010-. & Agathidium nigrinum Sturm, 1807 & & $\mathrm{x}$ & $\mathrm{m}$ & $\mathrm{W}$ & $\mathrm{P}$ \\
\hline 16-.011-.013-. & Agathidium nigripenne (F., 1792) & $\mathrm{x}$ & $\mathrm{x}$ & $\mathrm{m}$ & $\mathrm{W}$ & $\mathrm{TR}$ \\
\hline 16-.011-.014-. & Agathidium atrum (Payk.,1798) & $\mathrm{x}$ & & $\mathrm{m}$ & $\mathrm{W}$ & $\mathrm{P}$ \\
\hline 16-.011-.015-. & Agathidium seminulum (L., 1758) & $\mathrm{x}$ & $\mathrm{x}$ & $\mathrm{m}$ & $\mathrm{W}$ & $\mathrm{P}$ \\
\hline 16-.011-.018-. & Agathidium badium Er.,1845 & $\mathrm{x}$ & $\mathrm{x}$ & $\mathrm{m}$ & $\mathrm{W}$ & $\mathrm{P}$ \\
\hline 18-.000-.000-. & Familie Scydmaenidae & & & & & \\
\hline 18-.001-.001-. & Euthiconus conicicollis (Fairm.Lab.,1855) & $\mathrm{x}$ & $\mathrm{x}$ & $\mathrm{z}$ & $\mathrm{W}$ & $\mathrm{TM}$ \\
\hline 18-.002-.004-. & Euthia linearis Muls., 1861 & & $\mathrm{x}$ & $\mathrm{z}$ & $\mathrm{E}$ & $\mathrm{E}$ \\
\hline 18-.004-.003-. & Cephennium thoracicum Müll.Kunze, 1822 & & $\mathrm{x}$ & $\mathrm{z}$ & $\mathrm{W}$ & $\mathrm{B}$ \\
\hline 18-.005-.001-. & Neuraphes elongatulus (Müll.Kunze,1822) & $\mathrm{x}$ & $\mathrm{x}$ & $\mathrm{z}$ & $\mathrm{W}$ & $\mathrm{B}$ \\
\hline $18-.005-.005-$. & Neuraphes carinatus (Muls., 1861) & & $\mathrm{x}$ & $\mathrm{z}$ & $\mathrm{W}$ & $\mathrm{TM}$ \\
\hline 18-.006-.003-. & Scydmoraphes helvolus (Schaum,1844) & & $\mathrm{x}$ & $\mathrm{z}$ & $\mathrm{E}$ & $\mathrm{E}$ \\
\hline $18-.006-.004-$. & Scydmoraphes minutus (Chaud.,1845) & & $\mathrm{x}$ & $\mathrm{z}$ & $\mathrm{W}$ & $\mathrm{TM}$ \\
\hline 18-.007-.003-. & Stenichnus scutellaris (Müll.Kunze, 1822) & $\mathrm{x}$ & $\mathrm{x}$ & $\mathrm{z}$ & $\mathrm{E}$ & B \\
\hline 18-.007-.005-. & Stenichnus godarti (Latr.,1806) & $\mathrm{x}$ & $\mathrm{x}$ & $\mathrm{z}$ & $\mathrm{W}$ & $\mathrm{TM}$ \\
\hline 18-.007-.008-. & Stenichnus collaris (Müll.Kunze,1822) & $\mathrm{x}$ & $\mathrm{x}$ & $\mathrm{z}$ & $\mathrm{W}$ & B \\
\hline 18-.008-.001-. & Microscydmus nanus (Schaum,1844) & $\mathrm{x}$ & $\mathrm{x}$ & $\mathrm{z}$ & $\mathrm{W}$ & B \\
\hline 18-.008-.002-. & Microscydmus minimus (Chaud.,1845) & $\mathrm{x}$ & $\mathrm{x}$ & $\mathrm{z}$ & $\mathrm{W}$ & $\mathrm{TM}$ \\
\hline 21-.000-.000-. & Familie Ptilidae & & & & & \\
\hline 21-.002-.001-. & Ptenidium gressneri Er., 1845 & $\mathrm{x}$ & $\mathrm{x}$ & $\mathrm{m}$ & $\mathrm{W}$ & $\mathrm{TM}$ \\
\hline 21-.002-.002-. & Ptenidium laevigatum Er., 1845 & $\mathrm{x}$ & & $\mathrm{m}$ & $\mathrm{W}$ & $\mathrm{F}$ \\
\hline 21-.002-.004-. & Ptenidium intermedium Wank., 1869 & $\mathrm{x}$ & $\mathrm{x}$ & $\mathrm{m}$ & $\mathrm{F}$ & $\mathrm{F}$ \\
\hline 21-.002-.014-. & Ptenidium nitidum (Heer, 1841) & $\mathrm{x}$ & & $\mathrm{m}$ & $\mathrm{E}$ & $\mathrm{F}$ \\
\hline 21-.005-.003-. & Micridium halidaii (Matth.,1868) & $\mathrm{x}$ & $\mathrm{x}$ & $\mathrm{m}$ & $\mathrm{W}$ & $\mathrm{TM}$ \\
\hline 21-.007-.002-. & Euryptilium saxonicum (Gillm.,1845) & $\mathrm{x}$ & & $\mathrm{m}$ & $\mathrm{W}$ & $\mathrm{F}$ \\
\hline 21-.012-.002-. & Ptinella limbata $($ Heer, 1841$)$ & $\mathrm{x}$ & $\mathrm{x}$ & $\mathrm{m}$ & $\mathrm{W}$ & $\mathrm{TM}$ \\
\hline 21-.012-.004-. & Ptinella aptera (Guér.,1839) & $\mathrm{x}$ & $\mathrm{x}$ & $\mathrm{m}$ & $\mathrm{W}$ & $\mathrm{TM}$ \\
\hline 21-.013-.001-. & Pteryx suturalis (Heer, 1841) & $\mathrm{x}$ & $\mathrm{x}$ & $\mathrm{m}$ & $\mathrm{W}$ & $\mathrm{TM}$ \\
\hline 21-.019-.001-. & Acrotrichis grandicollis (Mannh.,1844) & $\mathrm{x}$ & & $\mathrm{m}$ & $\mathrm{E}$ & $\mathrm{F}$ \\
\hline 21-.019-.005-. & Acrotrichis sericans $($ Heer, 1841$)$ & $\mathrm{x}$ & & $\mathrm{m}$ & $\mathrm{E}$ & $\mathrm{F}$ \\
\hline 21-.019-.008-. & Acrotrichis pumila (Er.,1845) & $\mathrm{x}$ & & $\mathrm{m}$ & $\mathrm{E}$ & $\mathrm{F}$ \\
\hline 21-.019-.012-. & Acrotrichis insularis (Maek1.,1852) & $\mathrm{x}$ & $\mathrm{x}$ & $\mathrm{m}$ & $\mathrm{E}$ & $\mathrm{F}$ \\
\hline 21-.019-.015-. & Acrotrichis intermedia (Gillm.,1845) & $\mathrm{x}$ & $\mathrm{x}$ & $\mathrm{m}$ & $\mathrm{W}$ & B \\
\hline 21-.019-.021-. & Acrotrichis fascicularis (Hbst.,1792) & & $\mathrm{x}$ & $\mathrm{m}$ & $\mathrm{W}$ & $\mathrm{F}$ \\
\hline 22-.000-.000-. & Familie Scaphidiidae & & & & & \\
\hline 22-.002-.001-. & Scaphidium quadrimaculatum Ol.,1790 & $\mathrm{x}$ & $\mathrm{x}$ & $\mathrm{m}$ & $\mathrm{W}$ & $\mathrm{TP}$ \\
\hline
\end{tabular}




\begin{tabular}{|c|c|c|c|c|c|c|}
\hline EDV-Code & A r t & FF & Ekl & Kon & Bio & Hab \\
\hline 22-.003-.001-. & Scaphisoma agaricinum (L., 1758) & $\mathrm{x}$ & $\mathrm{x}$ & $\mathrm{m}$ & $\mathrm{W}$ & $\mathrm{TP}$ \\
\hline 22-.003-.007-. & Scaphisoma balcanicum Taman.,1954 & $\mathrm{x}$ & $\mathrm{x}$ & $\mathrm{m}$ & $\mathrm{W}$ & $\mathrm{TP}$ \\
\hline 23-.000-.000-. & Familie Staphylinidae & & & & & \\
\hline $23-.005-.001-$. & Phloeocharis subtilissima Mannh., 1830 & $\mathrm{x}$ & $\mathrm{x}$ & $\mathrm{z}$ & $\mathrm{W}$ & $\mathrm{TM}$ \\
\hline 23-.008-.001-. & Megarthrus depressus (Payk.,1789) & $\mathrm{x}$ & & $\mathrm{s}$ & $\mathrm{E}$ & $\mathrm{F}$ \\
\hline 23-.008-.004-. & Megarthrus sinuatocollis (Boisd.Lacord.,1835 & $\mathrm{x}$ & $\mathrm{x}$ & $\mathrm{s}$ & $\mathrm{E}$ & $\mathrm{F}$ \\
\hline 23-.009-.004-. & Proteinus brachypterus (F.,1792) & $\mathrm{x}$ & $\mathrm{x}$ & s & $\mathrm{E}$ & $\mathrm{F}$ \\
\hline 23-.009-.005-. & Proteinus atomarius Er., 1840 & & $\mathrm{x}$ & $\mathrm{s}$ & $\mathrm{W}$ & $\mathrm{F}$ \\
\hline 23-.009-.006-. & Proteinus macropterus (Grav.,1806) & $\mathrm{x}$ & & $\mathrm{s}$ & $\mathrm{E}$ & $\mathrm{F}$ \\
\hline 23-.014-.001-. & Phyllodrepa melanocephala (F., 1787) & & $\mathrm{x}$ & - & $\mathrm{W}$ & $\mathrm{N}$ \\
\hline 23-.014-.004-. & Phyllodrepa nigra (Grav.,1806) & & $\mathrm{x}$ & - & $\mathrm{W}$ & $\mathrm{N}$ \\
\hline 23-.014-.012-. & Phyllodrepa ioptera (Steph.,1834) & $\mathrm{x}$ & $\mathrm{x}$ & - & $\mathrm{W}$ & $\mathrm{TM}$ \\
\hline 23-.014-.015-. & Phyllodrepa gracilicornis (Fairm.Lab.,1856) & $\mathrm{x}$ & $\mathrm{x}$ & - & W & $\mathrm{TM}$ \\
\hline 23-.015-.005-. & Omalium rivulare (Payk.,1789) & $\mathrm{x}$ & $\mathrm{x}$ & $\mathrm{s}$ & $\mathrm{E}$ & $\mathrm{F}$ \\
\hline 23-.016-.001-. & Phloeonomus monilicornis (Gyll.,1810) & & $\mathrm{x}$ & $\mathrm{z}$ & $\mathrm{W}$ & TR \\
\hline 23-.016-.003-. & Phloeonomus planus (Payk., 1792) & $\mathrm{x}$ & $\mathrm{x}$ & $\mathrm{z}$ & $\mathrm{W}$ & TR \\
\hline 23-.016-.006-. & Phloeonomus punctipennis Thoms., 1867 & $\mathrm{x}$ & $\mathrm{x}$ & $\mathrm{z}$ & $\mathrm{W}$ & TR \\
\hline 23-.017-.003-. & Xylodromus concinnus (Marsh.,1802) & $\mathrm{x}$ & & - & $\mathrm{E}$ & $\mathrm{N}$ \\
\hline 23-.017-.004-. & Xylodromus testaceus (Er.,1840) & $\mathrm{x}$ & $\mathrm{x}$ & - & $\mathrm{W}$ & $\mathrm{N}$ \\
\hline 23-.023-.001-. & Phyllodrepoidea crenata (Grav.,1802) & $\mathrm{x}$ & $\mathrm{x}$ & - & $\mathrm{W}$ & TR \\
\hline 23-.025-.002-. & Lathrimaeum atrocephalum (Gyll.,1827) & & $\mathrm{x}$ & - & $\mathrm{W}$ & B \\
\hline 23-.025-.003-. & Lathrimaeum unicolor (Marsh.,1802) & $\mathrm{x}$ & $\mathrm{x}$ & - & $\mathrm{W}$ & B \\
\hline 23-.026-.001-. & Olophrum piceum (Gyll.,1810) & & $\mathrm{x}$ & - & $\mathrm{F}$ & B \\
\hline 23-.032-.001-. & Lesteva punctata Er.,1839 & $\mathrm{x}$ & & - & $\mathrm{F}$ & B \\
\hline 23-.032-.003-. & Lesteva longelytrata (Goeze, 1777) & $\mathrm{x}$ & $\mathrm{x}$ & - & $\mathrm{F}$ & B \\
\hline 23-.040-.001-. & Syntomium aeneum (Müll.,1821) & $\mathrm{x}$ & $\mathrm{x}$ & - & $\mathrm{W}$ & B \\
\hline 23-.042-.001-. & Coprophilus striatulus (F.,1792) & $\mathrm{x}$ & $\mathrm{x}$ & s & $\mathrm{E}$ & $\mathrm{F}$ \\
\hline $23-.046-.017-$. & Carpelimus corticinus (Grav.,1806) & $\mathrm{x}$ & $\mathrm{x}$ & $\mathrm{p}$ & $\mathrm{F}$ & B \\
\hline 23-.046-.030-. & Carpelimus gracilis (Mannh.,1830) & & $\mathrm{x}$ & $\mathrm{p}$ & $\mathrm{F}$ & B \\
\hline 23-.046-.032-. & Carpelimus elongatulus Er.,1839 & $\mathrm{x}$ & $\mathrm{x}$ & $\mathrm{p}$ & $\mathrm{F}$ & B \\
\hline 23-.048-.008-. & Oxytelus laqueatus (Marsh.,1802) & $\mathrm{x}$ & & $\mathrm{s}$ & $\mathrm{E}$ & $\mathrm{F}$ \\
\hline 23-.0481.003-. & Anotylus rugosus (F.,1775) & $\mathrm{x}$ & & s & $\mathrm{E}$ & $\mathrm{F}$ \\
\hline 23-.0481.008-. & Anotylus mutator (Lohse, 1963) & $\mathrm{x}$ & & $\mathrm{s}$ & $\mathrm{W}$ & $\mathrm{F}$ \\
\hline 23-.0481.022-. & Anotylus tetracarinatus (Block,1799) & $\mathrm{x}$ & $\mathrm{x}$ & $\mathrm{s}$ & $\mathrm{E}$ & $\mathrm{F}$ \\
\hline 23-.049-.001-. & Platystethus arenarius (Fourcr.,1785) & $\mathrm{x}$ & & $\mathrm{s}$ & $\mathrm{E}$ & $\mathrm{F}$ \\
\hline 23-.055-.006-. & Stenus fossulatus Er., 1840 & $\mathrm{x}$ & $\mathrm{x}$ & $\mathrm{z}$ & $\mathrm{F}$ & B \\
\hline 23-.055-.041-. & Stenus canaliculatus Gyll.,1827 & $\mathrm{x}$ & & $\mathrm{z}$ & $\mathrm{F}$ & B \\
\hline $23-.055-.050-$. & Stenus pusillus Steph., 1833 & $\mathrm{x}$ & & $\mathrm{z}$ & $\mathrm{F}$ & B \\
\hline $23-.055-.070-$. & Stenus fulvicornis Steph., 1833 & $\mathrm{x}$ & & $\mathrm{z}$ & $\mathrm{F}$ & B \\
\hline $23-.055-.094-$. & Stenus impressus Germ., 1824 & $\mathrm{x}$ & & $\mathrm{z}$ & $\mathrm{E}$ & B \\
\hline $23-.061-.003-$. & Rugilus rufipes Germ.,1836 & $\mathrm{x}$ & $\mathrm{x}$ & $\mathrm{z}$ & $\mathrm{E}$ & $\mathrm{F}$ \\
\hline $23-.068-.021-$. & Lathrobium fulvipenne (Grav.,1806) & $\mathrm{x}$ & $\mathrm{x}$ & $\mathrm{z}$ & $\mathrm{E}$ & B \\
\hline $23-.068-.023-$. & Lathrobium brunnipes (F.,1792) & & $\mathrm{x}$ & $\mathrm{z}$ & $\mathrm{F}$ & $\mathrm{B}$ \\
\hline 23-.078-.001-. & Nudobius lentus (Grav.,1806) & $\mathrm{x}$ & $\mathrm{x}$ & $\mathrm{z}$ & $\mathrm{W}$ & $\mathrm{TR}$ \\
\hline 23-.080-.010-. & Xantholinus linearis $(\mathrm{Ol} ., 1795)$ & $\mathrm{x}$ & $\mathrm{x}$ & $\mathrm{z}$ & $\mathrm{E}$ & B \\
\hline 23-.080-.015-. & Xantholinus longiventris Heer, 1839 & $\mathrm{x}$ & $\mathrm{x}$ & $\mathrm{z}$ & $\mathrm{E}$ & B \\
\hline
\end{tabular}




\begin{tabular}{|c|c|c|c|c|c|c|}
\hline EDV-Code & A r t & FF & Ekl & Kon & Bio & Hab \\
\hline $23-.081-.001-$. & Atrecus affinis (Payk.,1789) & $\mathrm{x}$ & $\mathrm{x}$ & $\mathrm{z}$ & $\mathrm{W}$ & $\mathrm{TM}$ \\
\hline 23-.082-.001-. & Othius punctulatus (Goeze, 1777) & & $\mathrm{x}$ & $\mathrm{z}$ & W & $\mathrm{B}$ \\
\hline 23-.082-.005-. & Othius myrmecophilus Kiesw., 1843 & & $\mathrm{x}$ & $\mathrm{z}$ & W & B \\
\hline 23-.088-.006-. & Philonthus subuliformis (Grav., 1802) & $\mathrm{x}$ & & $\mathrm{z}$ & $\mathrm{W}$ & $\mathrm{N}$ \\
\hline 23-.088-.020-. & Philonthus laminatus (Creutz.,1799) & $\mathrm{x}$ & & $\mathrm{z}$ & $\mathrm{O}$ & $\mathrm{F}$ \\
\hline 23-.088-.023-. & Philonthus cognatus Steph., 1832 & $\mathrm{x}$ & $\mathrm{x}$ & $\mathrm{z}$ & $\mathrm{E}$ & B \\
\hline $23-.088-.026-$. & Philonthus succicola Thoms., 1860 & & $\mathrm{x}$ & $\mathrm{z}$ & $\mathrm{E}$ & $\mathrm{F}$ \\
\hline $23-.088-.027-$. & Philonthus addendus Shp.,1867 & $\mathrm{x}$ & & $\mathrm{z}$ & $\mathrm{E}$ & $\mathrm{F}$ \\
\hline 23-.088-.029-. & Philonthus decorus (Grav.,1802) & $\mathrm{x}$ & $\mathrm{x}$ & $\mathrm{z}$ & W & $\mathrm{B}$ \\
\hline 23-.088-.033-. & Philonthus rotundicollis (Menetr.,1832) & $\mathrm{x}$ & & $\mathrm{z}$ & $\mathrm{E}$ & $\mathrm{F}$ \\
\hline 23-.088-.039-. & Philonthus carbonarius (Grav.,1810) & $\mathrm{x}$ & $\mathrm{x}$ & $\mathrm{z}$ & $\mathrm{E}$ & B \\
\hline 23-.088-.041-. & Philonthus cruentatus (Gm., 1789) & $\mathrm{x}$ & & $\mathrm{z}$ & $\mathrm{E}$ & $\mathrm{F}$ \\
\hline 23-.088-.044-. & Philonthus varians (Payk.,1789) & $\mathrm{x}$ & $\mathrm{x}$ & z & $\mathrm{E}$ & $\mathrm{F}$ \\
\hline 23-.088-.046-. & Philonthus splendens (F.,1792) & $\mathrm{x}$ & & $\mathrm{z}$ & $\mathrm{E}$ & $\mathrm{F}$ \\
\hline $23-.088-.047-$. & Philonthus fimetarius (Grav.,1802) & $\mathrm{x}$ & $\mathrm{x}$ & z & $\mathrm{E}$ & $\mathrm{F}$ \\
\hline 23-.088-.053-. & Philonthus quisquiliarius (Gyll.,1810) & $\mathrm{x}$ & & $\mathrm{z}$ & $\mathrm{F}$ & B \\
\hline $23-.088-.058-$. & Philonthus sanguinolentus (Grav.,1802) & $\mathrm{x}$ & & $\mathrm{z}$ & $\mathrm{E}$ & $\mathrm{F}$ \\
\hline 23-.088-.073-. & Philonthus marginatus (Ström,1768) & $\mathrm{x}$ & $\mathrm{x}$ & $\mathrm{z}$ & $\mathrm{E}$ & $\mathrm{F}$ \\
\hline 23-.090-.009-. & Gabrius splendidulus (Grav.,1802) & $\mathrm{x}$ & $\mathrm{x}$ & $\mathrm{z}$ & $\mathrm{W}$ & TR \\
\hline $23-.090-.024-$. & Gabrius subnigritulus (Rtt.,1909) & $\mathrm{x}$ & & $\mathrm{z}$ & $\mathrm{E}$ & B \\
\hline $23-.092-.002-$. & Ontholestes murinus (L.,1758) & $\mathrm{x}$ & & $\mathrm{z}$ & $\mathrm{O}$ & $\mathrm{F}$ \\
\hline 23-.099-.001-. & Ocypus olens (Müll.,1764) & & $\mathrm{x}$ & z & $\mathrm{E}$ & B \\
\hline 23-.099-.020-. & Ocypus compressus (Marsh.,1802) & & $\mathrm{x}$ & $\mathrm{z}$ & $\mathrm{W}$ & B \\
\hline $23-.104-.003-$. & Quedius infuscatus Er.,1840 & $\mathrm{x}$ & & $\mathrm{z}$ & $\mathrm{W}$ & $\mathrm{TM}$ \\
\hline 23-.104-.005-. & Quedius lateralis (Grav.,1802) & & $\mathrm{x}$ & $\mathrm{z}$ & W & $\mathrm{P}$ \\
\hline 23-.104-.013-. & Quedius cruentus (Ol.,1795) & $\mathrm{x}$ & $\mathrm{x}$ & $\mathrm{z}$ & $\mathrm{E}$ & $\mathrm{F}$ \\
\hline 23-.104-.014-. & Quedius brevicornis Thoms., 1860 & & $\mathrm{x}$ & $\mathrm{z}$ & $\mathrm{W}$ & $\mathrm{TM}$ \\
\hline 23-.104-.016-. & Quedius mesomelinus (Marsh.,1802) & $\mathrm{x}$ & $\mathrm{x}$ & $\mathrm{z}$ & $\mathrm{E}$ & $\mathrm{E}$ \\
\hline 23-.104-.018-. & Quedius maurus (Sahlb.,1830) & $\mathrm{x}$ & & $\mathrm{z}$ & $\mathrm{W}$ & $\mathrm{TM}$ \\
\hline 23-.104-.020-. & Quedius scitus (Grav.,1806) & $\mathrm{x}$ & $\mathrm{x}$ & $\mathrm{z}$ & W & $\mathrm{TM}$ \\
\hline $23-.104-.025-$. & Quedius fuliginosus (Grav.,1802) & $\mathrm{x}$ & & $\mathrm{z}$ & $\mathrm{F}$ & B \\
\hline $23-.104-.027-$. & Quedius tristis (Grav.,1802) & $\mathrm{x}$ & & $\mathrm{z}$ & $\mathrm{O}$ & B \\
\hline 23-.104-.048-. & Quedius fumatus (Steph.,1833) & $\mathrm{x}$ & & $\mathrm{z}$ & $\mathrm{W}$ & B \\
\hline $23-.104-.055-$. & Quedius lucidulus Er.,1839 & $\mathrm{x}$ & & $\mathrm{z}$ & $\mathrm{W}$ & $\mathrm{F}$ \\
\hline $23-.107-.001-$. & Habrocerus capillaricornis (Grav.,1806 & $\mathrm{x}$ & $\mathrm{x}$ & $\mathrm{z}$ & $\mathrm{W}$ & B \\
\hline $23-.108-.001-$. & Trichophya pilicornis (Gyll.,1810) & $\mathrm{x}$ & & $\mathrm{z}$ & $\mathrm{E}$ & $\mathrm{F}$ \\
\hline 23-.109-.008-. & Mycetoporus lepidus (Grav., 1802) & $\mathrm{x}$ & & $\mathrm{z}$ & $\mathrm{E}$ & B \\
\hline 23-.109-.017-. & Mycetoporus clavicornis (Steph.,1832) & & $\mathrm{x}$ & $\mathrm{z}$ & $\mathrm{O}$ & B \\
\hline $23-.109-.021-$. & Mycetoporus niger Fairm.Lab., 1856 & $\mathrm{x}$ & $\mathrm{x}$ & $\mathrm{z}$ & W & B \\
\hline $23-.111-.003-$. & Lordithon thoracicus (F.,1777) & $\mathrm{x}$ & & $\mathrm{z}$ & $\mathrm{W}$ & $\mathrm{P}$ \\
\hline 23-.111-.006-. & Lordithon trinotatus Er., 1839 & $\mathrm{x}$ & $\mathrm{x}$ & $\mathrm{z}$ & $\mathrm{W}$ & $\mathrm{P}$ \\
\hline $23-.111-.007-$. & Lordithon lunulatus (L.,1761) & $\mathrm{x}$ & $\mathrm{x}$ & $\mathrm{z}$ & W & $\mathrm{P}$ \\
\hline $23-.112-.001-$. & Bolitobius cingulata (Mannh.,1830) & $\mathrm{x}$ & $\mathrm{x}$ & $\mathrm{z}$ & E & B \\
\hline $23-.112-.003-$. & Bolitobius inclinans (Grav.,1806) & & $\mathrm{x}$ & $\mathrm{z}$ & W & $\mathrm{B}$ \\
\hline 23-.113-.001-. & Sepedophilus littoreus $(1 ., 1758)$ & $\mathrm{x}$ & $\mathrm{x}$ & $\mathrm{z}$ & $\mathrm{E}$ & $\mathrm{F}$ \\
\hline $23-.113-.002-$. & Sepedophilus testaceus (F., 1792) & $\mathrm{x}$ & $\mathrm{x}$ & $\mathrm{z}$ & W & $\mathrm{TM}$ \\
\hline
\end{tabular}


EDV-Code

23-.113-.005-.

23-.114-.001-.

23-.114-.002-.

23-.114-.007-.

23-.114-.008-.

23-.114-.0081.

23-.114-.012-.

23-.114-.013-.

23-.114-.015-.

23-.117-.004-.

23-.117-.006-.

23-.117-.010-.

23-.117-.013-.

23-.117-.014-.

23-.117-.015-.

23-.117-.020-.

23-.126-.004-.

23-.1261.002-.

23-.1262.001-.

23-.1262.005-.

23-.1262.013-.

23-.130-.004-.

23-.130-.009-.

23-.130-.011-.

23-.130-.016-.

23-.130-.021-.

23-.1301.001-.

23-.131-.001-.

23-.132-.002-.

23-.132-.003-.

23-.132-.006-.

23-.133-.001-.

23-.134-.001-.

23-.141-.001-.

23-.141-.004-.

23-.141-.006-.

23-.142-.001-.

23-.147-.001-.

23-.148-.001-.

23-.148-.002-.

23-.148-.003-.

23-.154-.006-.

23-.166-.014-.

23-.166-.015-.

23-.168-.001-.

23-.168-.002-.
A $\mathbf{r} \mathbf{t}$

Sepedophilus bipunctatus (Grav.,1802)

Tachyporus nitidulus (F.,1781)

Tachyporus obtusus (L.,1767)

Tachyporus hypnorum (F., 1775)

Tachyporus chrysomelinus (L., 1758)

Tachyporus dispar (Payk., 1789)

Tachyporus ruficollis Grav.,1802

Tachyporus transversalis Grav.,1806

Tachyporus pusillus Grav.,1806

Tachinus humeralis Grav., 1802

Tachinus subterraneus (L., 1758)

Tachinus pallipes Grav., 1806

Tachinus signatus Grav.,1802

Tachinus laticollis Grav.,1802

Tachinus marginellus (F.,1781)

Tachinus elongatus Gyll.,1810

Oligota parva Kr.,1862

Holobus apicatus Er., 1837

Cypha longicornis (Payk., 1800)

Cypha laeviuscula (Mannh.,1831)

Cypha hanseni (Palm,1949)

Gyrophaena affinis Mannh., 1830

Gyrophaena gentilis Er.,1839

Gyrophaena minima Er., 1837

Gyrophaena fasciata (Marsh.,1802)

Gyrophaena joyioides Wüsth.,1937

Agaricochara latissima (Steph.,1832)

Cyphaea curtula (Er., 1837)

Placusa depressa Maekl.,1845

Placusa tachyporoides (Waltl.,1838)

Placusa pumilio (Grav.,1802)

Homalota plana (Gyll.,1810)

Anomognathus cuspidatus (Er.,1839)

Leptusa pulchella (Mannh., 1830)

Leptusa fumida (Er.,1839)

Leptusa ruficollis (Er.,1839)

Euryusa castanoptera Kr., 1856

Bolitochara obliqua Er.,1837

Autalia impressa (O1.,1795)

Autalia longicornis Scheerp.,1947

Autalia rivularis (Grav.,1802)

Ischnopoda atra (Grav., 1806)

Aloconota gregaria (Er.,1839)

Aloconota languida (Er., 1837)

Amischa analis (Grav.,1802)

Amischa cavifrons (Shp.,1869)
FF Ekl Kon Bio Hab

$\begin{array}{llll}\mathrm{X} & \mathrm{z} & \mathrm{W} & \mathrm{TM}\end{array}$

$\mathrm{X} \quad-\mathrm{E} \quad \mathrm{B}$

$\mathrm{X} \quad-\mathrm{E} \quad \mathrm{B}$

$\mathrm{x} \quad \mathrm{X}-\mathrm{E}$ B

$\mathrm{X} x \quad-\mathrm{E}$ B

$\mathrm{x} \quad \mathrm{x}-\mathrm{E}$ B

$\mathrm{x} \quad-\mathrm{W} \quad \mathrm{B}$

X - E B

$\mathrm{X} \quad-\mathrm{E} \quad \mathrm{B}$

X $\quad-$ W F

$\mathrm{X} \quad-\mathrm{E} \quad \mathrm{F}$

X $\quad-$ E F

X $\quad-\quad$ E F

$x \quad-\quad$ E F

$\mathrm{X} \quad-\mathrm{E} \quad \mathrm{F}$

$\begin{array}{llll}\mathrm{X} & \mathrm{Z} & \mathrm{E} & \mathrm{F}\end{array}$

$\begin{array}{llll}\mathrm{X} & \mathrm{Z} & \mathrm{E} & \mathrm{F}\end{array}$

$\begin{array}{llll}x & \mathrm{Z} & \mathrm{W} & \mathrm{TP}\end{array}$

$\mathrm{x} \quad-\mathrm{E} \quad \mathrm{F}$

$\mathrm{x} \quad-\mathrm{E}$ B

$\mathrm{x} \quad-$ W TM

$\begin{array}{lllll}\mathrm{x} & \mathrm{E} & \mathrm{P}\end{array}$

$\begin{array}{lllll}\mathrm{X} & \mathrm{X} & \mathrm{m} & \mathrm{W} & \mathrm{P}\end{array}$

$\begin{array}{lllll}\mathrm{x} & \mathrm{x} & \mathrm{W} & \mathrm{P}\end{array}$

$\mathrm{x} \quad \mathrm{m} \quad \mathrm{W} \quad \mathrm{P}$

$\begin{array}{llllll}\mathrm{x} & \mathrm{X} & \mathrm{m} & \mathrm{W} & \mathrm{P}\end{array}$

$\begin{array}{lllll}\mathrm{X} & \mathrm{X} & \mathrm{m} & \mathrm{W} & \mathrm{TP}\end{array}$

$\begin{array}{llll}\mathrm{x} & - & \mathrm{W} & \mathrm{TR}\end{array}$

$\begin{array}{llll}\mathrm{X} & \mathrm{Z} & \mathrm{W} & \mathrm{TR}\end{array}$

$\begin{array}{lllll}\mathrm{X} & \mathrm{X} & \mathrm{Z} & \mathrm{W} & \mathrm{TR}\end{array}$

$\begin{array}{lllll}\mathrm{X} & \mathrm{X} & \mathrm{Z} & \mathrm{W} & \mathrm{TR}\end{array}$

$\mathrm{X} \quad \mathrm{X} \quad \mathrm{W}$ W TR

$\begin{array}{lllll}\mathrm{X} & \mathrm{X} & \mathrm{Z} & \mathrm{W} & \mathrm{TR}\end{array}$

$\mathrm{X} \quad \mathrm{X} \quad \mathrm{W}$ W TR

X $\quad \mathrm{x} \quad$ - W TR

$\mathrm{x} \quad \mathrm{x} \quad-\quad \mathrm{W} \quad \mathrm{V}$

$\begin{array}{lllll}\mathrm{X} & \mathrm{X} & \mathrm{Z} & \mathrm{W} & \mathrm{TR}\end{array}$

$\begin{array}{lllll}\mathrm{x} & \mathrm{x} & \mathrm{W} & \mathrm{TP}\end{array}$

$\begin{array}{llll}\mathrm{x} & \mathrm{m} & \mathrm{TP}\end{array}$

$m \quad$ W TP

$\begin{array}{llll}\mathrm{m} & \mathrm{E} & \mathrm{F}\end{array}$

$\mathrm{X} \quad-\quad \mathrm{F} \quad \mathrm{B}$

$\begin{array}{llll}\mathrm{X} & \mathrm{Z} & \mathrm{E} & \mathrm{B}\end{array}$

$\begin{array}{lllll}\mathrm{X} & \mathrm{Z} & \mathrm{F} & \mathrm{B}\end{array}$

$\begin{array}{lllll}\mathrm{X} & \mathrm{X} & \mathrm{Z} & \mathrm{E} & \mathrm{B}\end{array}$

$\begin{array}{llll}\mathrm{X} & \mathrm{Z} & \mathrm{B}\end{array}$ 


\section{EDV-Code}

23-.168-.004-.

23-.168-.007-.

23-.173-.001-.

23-.180-.003-.

23-.182-.002-

23-.182-.003-.

23-.184-.001-

23-.186-.005-.

23-.187-.007-.

23-.188-.004-.

23-.188-.008-

23-.188-.015-

23-.188-.020-

23-.188-.024-

23-.188-.025-

23-.188-.038-

23-.188-.045-.

23-.188-.046-

23-.188-.063-.

23-.188-.064-.

23-.188-.092-.

23-.188-.109-

23-.188-.110-

23-.188-.111-

23-.188-.126-

23-.188-.134-

23-.188-.136-

23-.188-.1361.

23-.188-.1362.

23-.188-.155-.

23-.188-.158-.

23-.188-.159-

23-.188-.165-

23-.188-.179-.

23-.188-.183-

23-.188-.196-

23-.188-.198-.

23-.188-.199-.

23-.188-.202-

23-.188-.208-.

23-.188-.210-.

23-.188-.223-.

23-.1881.005-.

23-.1881.006-.

23-.1881.011-.

23-.194-.001-.

\section{A r t}

Amischa soror (Kr.,1856)

Amischa decipiens (Shp.,1869)

Neohilara subterranea (Muls.Rey,1853)

Geostiba circellaris (Grav.,1806)

Dinaraea aequata (Er.,1837)

Dinaraea linearis (Grav.,1802)

Dadobia immersa (Er.,1837)

Plataraea brunnea (F.,1798)

Liogluta oblongiuscula (Shp.,1869)

Atheta elongatula (Grav.,1802)

Atheta terminalis (Grav.,1806)

Atheta melanocera (Thoms.,1856)

Atheta palustris (Kiesw.,1844)

Atheta parca (Muls.Rey,1873)

Atheta deformis (Kr.,1856)

Atheta monticola (Thoms.,1852)

Atheta nigricornis (Thoms.,1852)

Atheta harwoodi Will.,1930

Atheta palleola (Er.,1837)

Atheta benickiella Brundin,1948

Atheta boreella Brundin,1948

Atheta sodalis (Er.,1837)

Atheta gagatina (Baudi,1848)

Atheta pallidicornis (Thoms.,1856)

Atheta picipes (Thoms.,1856)

Atheta orphana (Er.,1837)

Atheta fungi (Grav.,1806)

Atheta negligens (Muls.Rey,1873)

Atheta amplicollis (Muls.Rey,1873)

Atheta dadopora (Thoms.,1867)

Atheta sordidula (Er.,1837)

Atheta celata (Er.,1837)

Atheta castanoptera (Mannh.,1830)

Atheta laticollis (Steph.,1832)

Atheta ravilla (Er., 1839)

Atheta pilicornis (Thoms.,1852)

Atheta britanniae Bernh.Scheerp.,1926

Atheta crassicornis (F.,1792)

Atheta macrocera (Thoms.,1856)

Atheta nigripes (Thoms.,1856)

Atheta atramentaria (Gyll.,1810)

Atheta longicornis (Grav.,1802)

Acrotona obfuscata (Grav.,1802)

Acrotona consanguinea (Epph.,1875)

Acrotona aterrima (Grav., 1802)

Thamiaraea cinnamomea (Grav.,1802)
FF Ekl Kon Bio Hab

$\begin{array}{lllll}x & x & z & E & B\end{array}$

$\begin{array}{lllll}X & X & Z & F & B\end{array}$

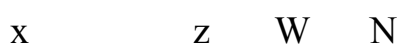

$\begin{array}{llll}x & z & W & B\end{array}$

$\begin{array}{lllll}\mathrm{x} & \mathrm{x} & \mathrm{Z} & \mathrm{W} & \mathrm{TR}\end{array}$

$\begin{array}{lllll}x & x & z & W & \text { TR }\end{array}$

$\begin{array}{lllll}\mathrm{X} & \mathrm{x} & \mathrm{Z} & \mathrm{W} & \mathrm{TR}\end{array}$

$\begin{array}{llll}\mathrm{X} & \mathrm{Z} & \mathrm{E} & \mathrm{N}\end{array}$

$\begin{array}{llll}x & z & W & B\end{array}$

$\begin{array}{lllll}x & x & z & F & B\end{array}$

$\begin{array}{llll}\mathrm{X} & \mathrm{Z} & \mathrm{F} & \mathrm{B}\end{array}$

$\begin{array}{llll}Z & F & B\end{array}$

$\begin{array}{llll}x & \mathrm{Z} & \mathrm{F} & \mathrm{B}\end{array}$

$\begin{array}{lllll}X & X & Z & F & B\end{array}$

$\begin{array}{llll}X & Z & F & B\end{array}$

$\begin{array}{llll}x & \mathrm{Z} & \mathrm{W} & \mathrm{F}\end{array}$

$\begin{array}{lllll}x & x & z & E & F\end{array}$

$\begin{array}{lllll}x & x & Z & E & N\end{array}$

$\begin{array}{lllll}x & z & W & P\end{array}$

$\begin{array}{llll}x & \text { Z } & \text { W } & \text { P }\end{array}$

$\begin{array}{lllll}x & \text { z } & \text { W } & \text { F }\end{array}$

$\begin{array}{lllll}x & x & Z & \text { W } & \text { F }\end{array}$

$\begin{array}{llll}\mathrm{x} & \mathrm{z} & \mathrm{W} & \mathrm{P}\end{array}$

$\begin{array}{llll}\mathrm{X} & \mathrm{Z} & \mathrm{W} & \mathrm{P}\end{array}$

$\begin{array}{llll}\mathrm{x} & \mathrm{z} & \mathrm{W} & \mathrm{TP}\end{array}$

$\begin{array}{llllllllllllll}x & z & W & F\end{array}$

$\begin{array}{lllll}\mathrm{X} & \mathrm{X} & \mathrm{Z} & \mathrm{E} & \mathrm{B}\end{array}$

$\begin{array}{llll}x & Z & W & B\end{array}$

$\begin{array}{lllll}\mathrm{X} & \mathrm{X} & \mathrm{Z} & \mathrm{F} & \mathrm{B}\end{array}$

$\begin{array}{lllll}\mathrm{X} & \mathrm{X} & \mathrm{Z} & \mathrm{W} & \mathrm{F}\end{array}$

$\begin{array}{lllll}x & x & z & O & F\end{array}$

$\begin{array}{llll}x & z & E & F\end{array}$

$\begin{array}{llll}x & z & W & P\end{array}$

$\begin{array}{llll}x & Z & E & F\end{array}$

$\begin{array}{lllll}x & x & Z & E & F\end{array}$

$\begin{array}{lllll}x & x & z & W & \text { TP }\end{array}$

$\begin{array}{lllll}\mathrm{X} & \mathrm{X} & \mathrm{Z} & \mathrm{W} & \mathrm{P}\end{array}$

$\begin{array}{lllll}\mathrm{X} & \mathrm{X} & \mathrm{Z} & \mathrm{W} & \mathrm{P}\end{array}$

$\begin{array}{lllll}x & x & z & O & F\end{array}$

$\begin{array}{lllll}x & x & z & E & F\end{array}$

$\begin{array}{lllll}x & x & z & E & F\end{array}$

$\begin{array}{llll}x & z & E & F\end{array}$

$\begin{array}{llll}\mathrm{X} & \mathrm{Z} & \mathrm{F} & \mathrm{F}\end{array}$

$\begin{array}{llll}\mathrm{X} & \mathrm{Z} & \mathrm{W} & \mathrm{B}\end{array}$

$\mathrm{X}$

$\begin{array}{cccc} & \mathrm{Z} & \mathrm{E} & \mathrm{F} \\ \mathrm{x} & \mathrm{z} & \mathrm{W} & \mathrm{TS}\end{array}$ 


\begin{tabular}{|c|c|c|c|c|c|c|}
\hline EDV-Code & A r t & FF & Ekl & Kon & Bio & Hab \\
\hline 23-.201-.004-. & Phloeopora testacea (Mannh.,1830) & $\mathrm{x}$ & $\mathrm{x}$ & $\mathrm{z}$ & $\mathrm{W}$ & $\mathrm{TR}$ \\
\hline 23-.201-.006-. & Phloeopora corticalis (Grav.,1802) & $\mathrm{x}$ & $\mathrm{x}$ & $\mathrm{z}$ & $\mathrm{W}$ & $\mathrm{TR}$ \\
\hline 23-.203-.003-. & Ilyobates nigricollis (Payk.,1800) & & $\mathrm{x}$ & $\mathrm{z}$ & $\mathrm{W}$ & $\mathrm{N}$ \\
\hline 23-.204-.005-. & Calodera aethiops (Grav.,1802) & $\mathrm{x}$ & $\mathrm{x}$ & $\mathrm{z}$ & $\mathrm{F}$ & B \\
\hline $23-.213-.006-$. & Meotica marchica Benick,1954 & $\mathrm{x}$ & & $\mathrm{z}$ & - & B \\
\hline 23-.219-.001-. & Mniusa incrassata (Muls.Rey,1852) & & $\mathrm{x}$ & $\mathrm{z}$ & $\mathrm{W}$ & B \\
\hline 23-.223-.003-. & Oxypoda procerula Mannh.,1830 & $\mathrm{x}$ & & $\mathrm{z}$ & $\mathrm{F}$ & $\mathrm{B}$ \\
\hline 23-.223-.007-. & Oxypoda vittata Mark.,1842 & & $\mathrm{x}$ & $\mathrm{z}$ & $\mathrm{W}$ & $\mathrm{N}$ \\
\hline 23-.223-.018-. & Oxypoda umbrata (Gyll.,1810) & $\mathrm{x}$ & & $\mathrm{z}$ & $\mathrm{E}$ & $\mathrm{F}$ \\
\hline $23-.223-.0331$ & Oxypoda arborea Zerche,1994 & $\mathrm{x}$ & $\mathrm{x}$ & $\mathrm{z}$ & $\mathrm{W}$ & $\mathrm{TP}$ \\
\hline 23-.223-.034-. & Oxypoda alternans (Grav.,1802) & $\mathrm{x}$ & $\mathrm{x}$ & $\mathrm{z}$ & $\mathrm{W}$ & $\mathrm{P}$ \\
\hline 23-.223-.046-. & Oxypoda brachyptera (Steph.,1832) & $\mathrm{x}$ & $\mathrm{x}$ & $\mathrm{z}$ & $\mathrm{O}$ & $\mathrm{B}$ \\
\hline 23-.223-.049-. & Oxypoda annularis Mannh.,1830 & & $\mathrm{x}$ & $\mathrm{z}$ & $\mathrm{W}$ & B \\
\hline 23-.223-.058-. & Oxypoda recondita Kr.,1856 & $\mathrm{x}$ & $\mathrm{x}$ & $\mathrm{z}$ & $\mathrm{W}$ & $\mathrm{TM}$ \\
\hline 23-.228-.001-. & Ischnoglossa prolixa (Grav.,1802) & $\mathrm{x}$ & $\mathrm{x}$ & $\mathrm{z}$ & $\mathrm{W}$ & $\mathrm{TR}$ \\
\hline 23-.229-.001-. & Dexiogya corticina (Er.,1837) & & $\mathrm{x}$ & $\mathrm{z}$ & $\mathrm{W}$ & $\mathrm{TR}$ \\
\hline 23-.234-.002-. & Haploglossa villosula (Steph.,1832) & $\mathrm{x}$ & $\mathrm{x}$ & $\mathrm{z}$ & $\mathrm{E}$ & $\mathrm{N}$ \\
\hline 23-.235-.001-. & Tinotus morion (Grav.,1802) & $\mathrm{x}$ & $\mathrm{x}$ & $\mathrm{z}$ & $\mathrm{E}$ & $\mathrm{F}$ \\
\hline 23-.237-.021-. & Aleochara lanuginosa Grav.,1802 & $\mathrm{x}$ & $\mathrm{x}$ & $\mathrm{z}$ & $\mathrm{E}$ & $\mathrm{F}$ \\
\hline 23-.237-.038-. & Aleochara ruficornis Grav.,1802 & & $\mathrm{x}$ & $\mathrm{z}$ & $\mathrm{E}$ & $\mathrm{F}$ \\
\hline 24-.000-.000-. & Familie Pselaphidae & & & & & \\
\hline 24-.002-.002-. & Bibloporus bicolor (Denny, 1825) & $\mathrm{x}$ & $\mathrm{x}$ & $\mathrm{z}$ & $\mathrm{W}$ & $\mathrm{TR}$ \\
\hline 24-.002-.003-. & Bibloporus minutus Raffr.,1914 & $\mathrm{x}$ & $\mathrm{x}$ & $\mathrm{z}$ & $\mathrm{W}$ & TR \\
\hline 24-.005-.006-. & Bibloplectus minutissimus (Aubé,1833) & & $\mathrm{x}$ & $\mathrm{z}$ & $\mathrm{F}$ & $\mathrm{B}$ \\
\hline 24-.006-.001-. & Euplectus nanus (Reichb.,1816) & $\mathrm{x}$ & $\mathrm{x}$ & $\mathrm{z}$ & $\mathrm{W}$ & $\mathrm{TM}$ \\
\hline 24-.006-.003-. & Euplectus piceus Motsch.,1835 & $\mathrm{x}$ & $\mathrm{x}$ & $\mathrm{z}$ & $\mathrm{W}$ & $\mathrm{TM}$ \\
\hline 24-.006-.007-. & Euplectus bescidicus Rtt., 1881 & $\mathrm{x}$ & $\mathrm{x}$ & $\mathrm{z}$ & $\mathrm{W}$ & $\mathrm{TM}$ \\
\hline 24-.006-.013-. & Euplectus punctatus Muls.,1861 & $\mathrm{x}$ & $\mathrm{x}$ & $\mathrm{z}$ & $\mathrm{W}$ & $\mathrm{TM}$ \\
\hline 24-.006-.015-. & Euplectus karsteni (Reichb.,1816) & $\mathrm{x}$ & $\mathrm{x}$ & $\mathrm{z}$ & $\mathrm{W}$ & $\mathrm{TM}$ \\
\hline 24-.008-.006-. & Plectophloeus nitidus (Fairm.,1857) & $\mathrm{x}$ & $\mathrm{x}$ & $\mathrm{z}$ & $\mathrm{W}$ & $\mathrm{TM}$ \\
\hline $24-.015-.002-$. & Batrisodes venustus (Reichb.,1816) & $\mathrm{x}$ & $\mathrm{x}$ & $\mathrm{z}$ & $\mathrm{W}$ & $\mathrm{N}$ \\
\hline 24-.017-.002-. & Bythinus burrelli Denny, 1825 & $\mathrm{x}$ & & $\mathrm{z}$ & $\mathrm{W}$ & $\mathrm{B}$ \\
\hline 24-.018-.008-. & Bryaxis puncticollis (Denny,1825) & $\mathrm{x}$ & & $\mathrm{z}$ & $\mathrm{E}$ & B \\
\hline 24-.019-.001-. & Tychus niger (Payk.,1800) & $\mathrm{x}$ & & $\mathrm{z}$ & $\mathrm{O}$ & $\mathrm{B}$ \\
\hline 24-.021-.001-. & Brachygluta fossulata (Reichb.,1816) & & $\mathrm{x}$ & $\mathrm{z}$ & $\mathrm{E}$ & B \\
\hline 24-.021-.007-. & Brachygluta haematica (Reichb.,1816) & $\mathrm{x}$ & $\mathrm{x}$ & $\mathrm{z}$ & $\mathrm{F}$ & B \\
\hline 27-.000-.000-. & Familie Cantharidae & & & & & \\
\hline $27-.001-.001-$. & Podabrus alpinus (Payk.,1798) & $\mathrm{x}$ & & $\mathrm{z}$ & $\mathrm{W}$ & V \\
\hline 27-.002-.008-. & Cantharis pellucida F.,1792 & $\mathrm{x}$ & $\mathrm{x}$ & $\mathrm{z}$ & $\mathrm{W}$ & V \\
\hline 27-.002-.018-. & Cantharis nigricans (Müll.,1776) & $\mathrm{x}$ & & $\mathrm{z}$ & $\mathrm{W}$ & V \\
\hline $27-.002-.025-$. & Cantharis decipiens Baudi, 1871 & $\mathrm{x}$ & $\mathrm{x}$ & $\mathrm{z}$ & $\mathrm{W}$ & V \\
\hline $27-.005-.002-$. & Rhagonycha fulva (Scop.,1763) & $\mathrm{x}$ & & $\mathrm{z}$ & $\mathrm{O}$ & $\mathrm{V}$ \\
\hline 27-.005-.008-. & Rhagonycha lignosa (Müll.,1764) & $\mathrm{x}$ & $\mathrm{x}$ & $\mathrm{z}$ & $\mathrm{E}$ & $\mathrm{V}$ \\
\hline 27-.005-.014-. & Rhagonycha gallica Pic, 1923 & $\mathrm{x}$ & $\mathrm{x}$ & $\mathrm{z}$ & $\mathrm{W}$ & $\mathrm{V}$ \\
\hline 27-.008-.001-. & Malthinus punctatus (Fourcr.,1785) & $\mathrm{x}$ & $\mathrm{x}$ & $\mathrm{z}$ & $\mathrm{W}$ & $\mathrm{TM}$ \\
\hline 27-.008-.002-. & Malthinus seriepunctatus Kiesw.,1851 & $\mathrm{x}$ & & $\mathrm{z}$ & $\mathrm{W}$ & $\mathrm{TM}$ \\
\hline
\end{tabular}




\section{EDV-Code}

27-.008-.010-.

27-.009-.012-

27-.009-.015-.

27-.009-.016-.

27-.009-.032-.

29-.000-.000-.

29-.006-.0032.

30-.000-.000-

30-.005-.005-.

30-.005-.008-.

30-.005-.009-.

301.000-.000-.

301.001-.001-.

321.000-.000-.

321.009-.001-.

33-.000-.000-.

33-.001-.001-.

33-.002-.001-.

34-.000-.000-.

34-.001-.004-.

34-.001-.013-.

34-.001-.014-.

34-.001-.015-.

34-.001-.019-.

34-.001-.021-

34-.004-.001-

34-.010-.001-.

34-.010-.003-.

34-.010-.009-.

34-.015-.004-

34-.016-.003-.

34-.022-.003-

34-.026-.003-.

34-.030-.001-

34-.031-.001-.

34-.033-.004-.

34-.038-.002-.

34-.041-.001-.

34-.041-.002-.

34-.041-.003-.

36-.000-.000-.

36-.001-.001-. 36-.003-.001-.

36-.008-.004-

36-.011-.001-.

36-.012-.001-.

\section{A $r$ t}

Malthinus frontalis (Marsh., 1802)

Malthodes minimus (L.,1758)

Malthodes guttifer Kiesw., 1852

Malthodes marginatus (Latr., 1806)

Malthodes brevicollis (Payk.,1798)

Familie Malachiidae

Malachius bipustulatus (L.,1758)

Familie Melyridae

Dasytes caeruleus (Geer,1774)

Dasytes plumbeus (Müll.,1776)

Dasytes aerosus Kiesw.,1867

\section{Familie Phloiophilidae}

Phloiophilus edwardsi Steph.,1830

Familie Trogositidae

Thymalus limbatus (F.,1787)

\section{Familie Lymexylonidae}

Hylecoetus dermestoides (L., 1761)

Lymexylon navale (L., 1758)

\section{Familie Elateridae}

Ampedus erythrogonus (Müll.,1821)

Ampedus brunnicornis Germ., 1844

Ampedus nigerrimus (Lacord.,1835)

Ampedus sanguineus (L., 1758)

Ampedus pomorum (Hbst.,1784)

Ampedus nigroflavus (Goeze, 1777)

Procraerus tibialis (Lacord.,1835)

Agriotes aterrimus (L.,1761)

Agriotes acuminatus (Steph., 1830)

Agriotes lineatus (L., 1767)

Adrastus pallens (F., 1792)

Melanotus castanipes (Payk.,1800)

Ctenicera pectinicornis (L., 1758)

Anostirus castaneus (L., 1758)

Calambus bipustulatus (L.,1767)

Hypoganus inunctus (Lacord.,1835)

Denticollis linearis (L., 1758)

Stenagostus rhombeus (O1.,1790)

Athous haemorrhoidalis (F.,1801)

Athous vittatus (F.,1792)

Athous subfuscus (Müll.,1767)

\section{Familie Eucnemidae}

Melasis buprestoides (L., 1761)

Eucnemis capucina Ahr.,1812

Dirhagus lepidus (Rosh., 1847)

Hylis olexai (Palm,1955)

Xylophilus corticalis (Payk., 1800)
FF Ekl Kon Bio Hab

$\begin{array}{ccccc} & \text { x } & \text { z } & \text { W } & \text { TM } \\ & \text { x } & \text { z } & \text { W } & \text { TM } \\ \text { x } & \text { x } & \text { z } & \text { W } & \text { TM } \\ & \text { x } & \text { z } & \text { W } & \text { TM } \\ & \text { x } & \text { z } & \text { W } & \text { TM }\end{array}$

$\begin{array}{lllll}\mathrm{X} & \mathrm{X} & \mathrm{Z} & \mathrm{W} & \mathrm{TM}\end{array}$

$\begin{array}{ccccc}\text { X } & \text { X } & \text { z } & \text { W } & \text { T } \\ \text { x } & & \text { z } & \text { W } & \text { T } \\ \text { x } & \text { x } & \text { z } & \text { W } & \text { T }\end{array}$

$\mathrm{X}$

$\begin{array}{lll}\mathrm{z} & \mathrm{W} & \mathrm{T}\end{array}$

$\begin{array}{lllll}\mathrm{x} & \mathrm{x} & \mathrm{m} & \mathrm{W} & \mathrm{TP}\end{array}$

$\begin{array}{lllll}\mathrm{x} & \mathrm{x} & \mathrm{m} & \mathrm{W} & \mathrm{T}\end{array}$

$\begin{array}{lllll}\mathrm{x} & \mathrm{x} & \mathrm{x} & \mathrm{W} & \mathrm{T}\end{array}$

$\begin{array}{lllll}\mathrm{X} & \mathrm{X} & \mathrm{Z} & \mathrm{W} & \mathrm{TM}\end{array}$

$\begin{array}{lllll}\mathrm{X} & \mathrm{X} & \mathrm{Z} & \mathrm{W} & \text { TM }\end{array}$

$\begin{array}{lllll}\mathrm{X} & \mathrm{X} & \mathrm{Z} & \mathrm{W} & \mathrm{TM}\end{array}$

$\begin{array}{lllll}\mathrm{X} & \mathrm{X} & \mathrm{Z} & \mathrm{W} & \mathrm{TM}\end{array}$

$\begin{array}{lllll}\mathrm{x} & \mathrm{X} & \mathrm{Z} & \mathrm{W} & \mathrm{T}\end{array}$

$\begin{array}{lllll}\mathrm{X} & \mathrm{X} & \mathrm{Z} & \mathrm{W} & \mathrm{T}\end{array}$

$\begin{array}{llll}\mathrm{X} & \mathrm{X} & \mathrm{W} & \mathrm{T}\end{array}$

$\begin{array}{lllll}x & x & p & \text { W } & \text { V }\end{array}$

$\begin{array}{llllll}x & x & p & O & V\end{array}$

$\begin{array}{lllll}\mathrm{x} & \mathrm{p} & \mathrm{O} & \mathrm{V}\end{array}$

$\begin{array}{lllll}\mathrm{X} & \mathrm{E} & \mathrm{V}\end{array}$

$\begin{array}{lllll}\mathrm{X} & \mathrm{X} & \mathrm{X} & \mathrm{W} & \mathrm{TM}\end{array}$

$\begin{array}{lllll}x & p & F & V\end{array}$

$\begin{array}{lllll}\mathrm{X} & \mathrm{X} & \mathrm{W} & \mathrm{T}\end{array}$

$\begin{array}{lllll}\mathrm{X} & \mathrm{X} & \mathrm{X} & \mathrm{W} & \mathrm{T}\end{array}$

$\begin{array}{lllll}\mathrm{x} & \mathrm{X} & \mathrm{x} & \mathrm{W} & \mathrm{T}\end{array}$

$\begin{array}{lllll}\mathrm{X} & \mathrm{X} & \mathrm{X} & \mathrm{W} & \mathrm{T}\end{array}$

$\begin{array}{llll}x & x & \text { W } & \text { TM }\end{array}$

$\begin{array}{lllll}x & x & p & E & V\end{array}$

$\begin{array}{llll}x & p & \text { W } & \text { V }\end{array}$

$\begin{array}{lllll}\mathrm{X} & \mathrm{X} & \mathrm{p} & \mathrm{W} & \mathrm{V}\end{array}$

$\begin{array}{lllll}\mathrm{x} & \mathrm{x} & \mathrm{x} & \mathrm{W} & \mathrm{T}\end{array}$

$\begin{array}{llll}x & \mathrm{X} & \mathrm{W} & \mathrm{T}\end{array}$

$\begin{array}{llllll}\mathrm{X} & \mathrm{X} & \mathrm{X} & \mathrm{W} & \mathrm{T}\end{array}$

$\begin{array}{lllll}\mathrm{x} & \mathrm{X} & \mathrm{x} & \mathrm{W} & \mathrm{T}\end{array}$

$\begin{array}{lllll}\mathrm{x} & \mathrm{x} & \mathrm{x} & \mathrm{W} & \mathrm{T}\end{array}$ 
EDV-Code

37-.000-.000-.

37-.001-.002-.

Familie Throscidae

37-.001-.003-.

38-.000-.000-.

38-.020-.003-.

38-.020-.007-.

381.000-.000-.

381.002-.002-.

381.002-.008-.

40-.000-.000-.

40-.002-.001-.

40-.003-.001-.

40-.003-.002-.

40-.003-.006-.

40-.003-.011-.

40-.004-.001-.

42-.000-.000-.

42-.002-.002-

45-.000-.000-.

45-.003-.001-.

45-.008-.010-.

47-.000-.000-.

47-.010-.001-.

49-.000-.000-.

49-.001-.001-.

49-.001-.002-.

492.000-.000-.

492.002-.001-.

492.002-.002-.

492.002-.003-.

493.000-.000-.

493.001-.007-.

50-.000-.000-.

50-.008-.003-.

50-.008-.014-.

50-.008-.024-.

50-.008-.026-.

50-.008-.028-.

50-.008-.030-.

50-.008-.034-

50-.008-.039-.

50-.008-.058-.

50-.009-.002-.

50-.009-.005-.

50-.009-.015-.

50-.009-.016-.

Familie Clambidae

Familie Scirtidae

Familie Dryopidae

\section{Familie Byrrhidae}

\section{Familie Byturidae}

Familie Nitidulidae

\section{A r t}

FF Ekl Kon Bio Hab

Trixagus dermestoides (L.,1767)

Trixagus carinifrons Bonv.,1859

\section{Familie Buprestidae}

Agrilus biguttatus (F., 1777)

Agrilus sulcicollis Lacord.,1835

Clambus punctulum (Beck,1817)

Clambus nigrellus Rtt., 1914

Microcara testacea (L.,1767)

Cyphon coarctatus Payk.,1799

Cyphon palustris Thoms., 1855

Cyphon ochraceus Steph.,1830

Cyphon padi (L.,1758)

Prionocyphon serricornis (Müll.,1821)

Dryops ernesti Goz., 1886

\section{Familie Dermestidae}

Trogoderma angustum (Sol.,1849)

Anthrenus museorum (L., 1761)

Cytilus sericeus (Forst.,1771)

Byturus tomentosus (Geer, 1774)

Byturus ochraceus (Scriba, 1790)

Familie Cerylonidae

Cerylon fagi Bris., 1867

Cerylon histeroides (F., 1792)

Cerylon ferrugineum Steph.,1830

Familie Sphaerosomatidae

Sphaerosoma pilosum (Panz.,1793)

Meligethes denticulatus (Heer,1841)

Meligethes aeneus (F.,1775)

Meligethes sulcatus Bris., 1863

Meligethes difficilis (Heer,1841)

Meligethes ochropus Sturm, 1845

Meligethes brunnicornis Sturm, 1845

Meligethes viduatus (Heer,1841)

Meligethes ovatus Sturm, 1845

Meligethes nigrescens Steph., 1830

Epuraea guttata (O1.,1811)

Epuraea neglecta (Heer,1841)

Epuraea marseuli Rtt., 1872

Epuraea pygmaea (Gyll.,1808)

$\begin{array}{ccccc}\mathrm{x} & \mathrm{x} & - & \mathrm{E} & \mathrm{E} \\ \mathrm{x} & \mathrm{x} & - & \mathrm{E} & \mathrm{E} \\ & & & & \\ \mathrm{x} & \mathrm{x} & \mathrm{x} & \mathrm{W} & \mathrm{TR} \\ & \mathrm{x} & \mathrm{x} & \mathrm{W} & \mathrm{TR}\end{array}$

$\begin{array}{ccccc}\mathrm{x} & \mathrm{x} & \mathrm{m} & \mathrm{W} & \mathrm{F} \\ \mathrm{x} & & \mathrm{m} & \mathrm{F} & \mathrm{F} \\ & & & & \\ \mathrm{x} & & \mathrm{s} & \mathrm{W} & \mathrm{V} \\ \mathrm{x} & \mathrm{x} & \mathrm{s} & \mathrm{F} & \mathrm{V} \\ \mathrm{x} & & \mathrm{s} & \mathrm{F} & \mathrm{V} \\ \mathrm{x} & \mathrm{x} & \mathrm{s} & \mathrm{W} & \mathrm{V} \\ \mathrm{x} & \mathrm{x} & \mathrm{s} & \mathrm{W} & \mathrm{V} \\ & \mathrm{x} & \mathrm{s} & \mathrm{W} & \mathrm{V}\end{array}$

$\mathrm{X}$

W

$\begin{array}{ccccc} & \mathrm{x} & \mathrm{s} & - & \mathrm{N} \\ & \mathrm{x} & \mathrm{s} & \mathrm{O} & \mathrm{V} \\ \mathrm{x} & & \mathrm{p} & \mathrm{E} & \mathrm{V} \\ & & & & \\ \mathrm{x} & & \mathrm{p} & \mathrm{E} & \mathrm{V} \\ \mathrm{x} & & \mathrm{p} & \mathrm{E} & \mathrm{V}\end{array}$

$\begin{array}{lllll}\mathrm{X} & \mathrm{X} & \mathrm{Z} & \mathrm{W} & \mathrm{TM}\end{array}$

$\begin{array}{lllll}\mathrm{X} & \mathrm{X} & \mathrm{Z} & \mathrm{W} & \mathrm{TM}\end{array}$

$\begin{array}{lllll}\text { X } & \mathrm{X} & \mathrm{Z} & \mathrm{W} & \text { TM }\end{array}$

$\begin{array}{ccccc} & \mathrm{x} & \mathrm{m} & \mathrm{W} & \mathrm{B} \\ \mathrm{x} & \mathrm{x} & \mathrm{p} & \mathrm{E} & \mathrm{V} \\ \mathrm{x} & \mathrm{x} & \mathrm{p} & \mathrm{E} & \mathrm{V} \\ \mathrm{x} & & \mathrm{p} & \mathrm{O} & \mathrm{V} \\ \mathrm{x} & & \mathrm{p} & \mathrm{E} & \mathrm{V} \\ \mathrm{x} & & \mathrm{p} & \mathrm{W} & \mathrm{V} \\ \mathrm{x} & & \mathrm{p} & \mathrm{W} & \mathrm{V} \\ \mathrm{x} & & \mathrm{p} & \mathrm{E} & \mathrm{V} \\ \mathrm{x} & & \mathrm{p} & \mathrm{E} & \mathrm{V} \\ \mathrm{x} & & \mathrm{p} & \mathrm{F} & \mathrm{V} \\ & & \mathrm{z} & \mathrm{W} & \mathrm{TS} \\ \mathrm{x} & \mathrm{x} & \mathrm{z} & \mathrm{W} & \mathrm{TR} \\ \mathrm{x} & \mathrm{x} & \mathrm{z} & \mathrm{W} & \mathrm{TR} \\ \mathrm{x} & \mathrm{x} & \mathrm{z} & \mathrm{W} & \mathrm{TR}\end{array}$




\begin{tabular}{|c|c|c|c|c|c|c|}
\hline EDV-Code & A r t & FF & Ekl & Kon & Bio & Hab \\
\hline 50-.009-.017-. & Epuraea longula Er.,1845 & $\mathrm{x}$ & $\mathrm{x}$ & $\mathrm{z}$ & $\mathrm{W}$ & V \\
\hline 50-.009-.020-. & Epuraea terminalis (Mannh.,1843) & $\mathrm{x}$ & & $\mathrm{z}$ & $\mathrm{W}$ & TR \\
\hline 50-.009-.027-. & Epuraea unicolor (Ol.,1790) & $\mathrm{x}$ & $\mathrm{x}$ & $\mathrm{z}$ & $\mathrm{E}$ & E \\
\hline 50-.009-.028-. & Epuraea variegata (Hbst.,1793) & $\mathrm{x}$ & $\mathrm{x}$ & $\mathrm{z}$ & $\mathrm{W}$ & TP \\
\hline 50-.009-.033-. & Epuraea depressa (Ill.,1798) & $\mathrm{x}$ & & $\mathrm{z}$ & E & $\mathrm{N}$ \\
\hline $50-.013-.002-$. & Soronia grisea (L.,1758) & $\mathrm{x}$ & & - & $\mathrm{W}$ & TS \\
\hline $50-.015-.001-$. & Pocadius ferrugineus (F.,1775) & $\mathrm{x}$ & & $\mathrm{m}$ & $\mathrm{W}$ & $\mathrm{P}$ \\
\hline 50-.019-.002-. & Cychramus luteus (F.,1787) & $\mathrm{x}$ & $\mathrm{x}$ & $\mathrm{m}$ & $\mathrm{W}$ & $\mathrm{P}$ \\
\hline 50-.020-.001-. & Cryptarcha strigata (F., 1787) & & $\mathrm{x}$ & $\mathrm{z}$ & $\mathrm{W}$ & TS \\
\hline 50-.020-.002-. & Cryptarcha undata (Ol.,1790) & & $\mathrm{x}$ & $\mathrm{z}$ & $\mathrm{W}$ & TS \\
\hline $50-.021-.001-$. & Glischrochilus quadriguttatus (F.,1776) & $\mathrm{x}$ & $\mathrm{x}$ & $\mathrm{z}$ & $\mathrm{W}$ & TR \\
\hline $50-.021-.002-$. & Glischrochilus hortensis (Fourcr., 1785) & & $\mathrm{x}$ & $\mathrm{z}$ & $\mathrm{E}$ & $\mathrm{F}$ \\
\hline $50-.022-.001-$. & Pityophagus ferrugineus (L.,1761) & & $\mathrm{x}$ & $\mathrm{z}$ & $\mathrm{W}$ & TR \\
\hline 501.000-.000-. & Familie Kateridae & & & & & \\
\hline 501.003-.001-. & Brachypterus urticae (F.,1792) & $\mathrm{x}$ & $\mathrm{x}$ & $\mathrm{p}$ & E & V \\
\hline $52-.000-.000-$. & Familie Rhizophagidae & & & & & \\
\hline $52-.001-.003-$. & Rhizophagus depressus (F.,1792) & $\mathrm{x}$ & & $\mathrm{z}$ & $\mathrm{W}$ & TR \\
\hline $52-.001-.004-$. & Rhizophagus ferrugineus (Payk.,1800) & $\mathrm{x}$ & $\mathrm{x}$ & $\mathrm{z}$ & $\mathrm{W}$ & TR \\
\hline $52-.001-.006-$. & Rhizophagus perforatus Er., 1845 & $\mathrm{x}$ & $\mathrm{x}$ & $\mathrm{z}$ & $\mathrm{W}$ & TR \\
\hline $52-.001-.008-$. & Rhizophagus dispar (Payk.,1800) & $\mathrm{x}$ & $\mathrm{x}$ & $\mathrm{z}$ & $\mathrm{W}$ & TR \\
\hline 52-.001-.009-. & Rhizophagus bipustulatus (F.,1792) & $\mathrm{x}$ & $\mathrm{x}$ & $\mathrm{z}$ & $\mathrm{W}$ & TR \\
\hline $52-.001-.010-$. & Rhizophagus nitidulus (F.,1798) & $\mathrm{x}$ & $\mathrm{x}$ & $\mathrm{z}$ & $\mathrm{W}$ & TR \\
\hline $52-.001-.013-$. & Rhizophagus cribratus Gyll.,1827 & $\mathrm{x}$ & $\mathrm{x}$ & $\mathrm{z}$ & $\mathrm{W}$ & TR \\
\hline 53-.000-.000-. & Familie Cucujidae & & & & & \\
\hline 53-.001-.005-. & Monotoma picipes Hbst.,1793 & $\mathrm{x}$ & & $\mathrm{m}$ & E & $\mathrm{F}$ \\
\hline 53-.001-.009-. & Monotoma longicollis (Gyll.,1827) & $\mathrm{x}$ & $\mathrm{x}$ & $\mathrm{m}$ & E & $\mathrm{F}$ \\
\hline $53-.012-.001-$. & Uleiota planata (L.,1761) & $\mathrm{x}$ & $\mathrm{x}$ & $\mathrm{z}$ & $\mathrm{W}$ & TR \\
\hline 53-.015-.001-. & Pediacus depressus (Hbst., 1797) & & $\mathrm{x}$ & $\mathrm{z}$ & $\mathrm{W}$ & TR \\
\hline $53-.015-.002-$. & Pediacus dermestoides (F.,1792) & $\mathrm{x}$ & $\mathrm{x}$ & $\mathrm{z}$ & $\mathrm{W}$ & TR \\
\hline 531.000-.000-. & Familie Silvanidae & & & & & \\
\hline 531.006-.001-. & Silvanus bidentatus (F.,1792) & $\mathrm{x}$ & $\mathrm{x}$ & $\mathrm{z}$ & $\mathrm{W}$ & TR \\
\hline 54-.000-.000-. & Familie Erotylidae & & & & & \\
\hline $54-.001-.001-$. & Tritoma bipustulata F., 1775 & $\mathrm{x}$ & & $\mathrm{m}$ & $\mathrm{W}$ & $\mathrm{TP}$ \\
\hline $54-.002-.003-$. & Triplax russica (L.,1758) & $\mathrm{x}$ & & $\mathrm{m}$ & $\mathrm{W}$ & TP \\
\hline $55-.000-.000-$. & Familie Cryptophagidae & & & & & \\
\hline 55-.008-.007-. & Cryptophagus acutangulus Gyll.,1827 & $\mathrm{x}$ & & $\mathrm{m}$ & E & $\mathrm{P}$ \\
\hline 55-.008-.016-. & Cryptophagus lapponicus Gyll.,1827 & $\mathrm{x}$ & & $\mathrm{m}$ & $\mathrm{W}$ & $\mathrm{N}$ \\
\hline 55-.008-.019-. & Cryptophagus pubescens Sturm, 1845 & $\mathrm{x}$ & $\mathrm{x}$ & $\mathrm{m}$ & $\mathrm{E}$ & $\mathrm{N}$ \\
\hline $55-.008-.021-$. & Cryptophagus saginatus Sturm, 1845 & & $\mathrm{x}$ & $\mathrm{m}$ & E & $\mathrm{F}$ \\
\hline 55-.008-.027-. & Cryptophagus dentatus (Hbst.,1793) & $\mathrm{x}$ & $\mathrm{x}$ & $\mathrm{m}$ & E & $\mathrm{E}$ \\
\hline 55-.008-.030-. & Cryptophagus distinguendus Sturm, 1845 & $\mathrm{x}$ & $\mathrm{x}$ & $\mathrm{m}$ & E & $\mathrm{E}$ \\
\hline 55-.008-.034-. & Cryptophagus scanicus (L.,1758) & $\mathrm{x}$ & $\mathrm{x}$ & $\mathrm{m}$ & E & $\mathrm{E}$ \\
\hline 55-.008-.039-. & Cryptophagus scutellatus Newm.,1834 & $\mathrm{x}$ & & $\mathrm{m}$ & E & $\mathrm{F}$ \\
\hline 55-.008-.040-. & Cryptophagus lycoperdi (Scop.,1763) & $\mathrm{x}$ & $\mathrm{x}$ & $\mathrm{m}$ & $\mathrm{W}$ & $\mathrm{P}$ \\
\hline $55-.008-.042-$. & Cryptophagus pilosus Gyll.,1827 & $\mathrm{x}$ & & $\mathrm{m}$ & E & $\mathrm{E}$ \\
\hline 55-.0081.005-. & Micrambe abietis (Payk.,1798) & & $\mathrm{x}$ & $\mathrm{m}$ & $\mathrm{W}$ & TP \\
\hline
\end{tabular}




\begin{tabular}{|c|c|c|c|c|c|c|}
\hline EDV-Code & A r t & FF & Ekl & Kon & Bio & Hab \\
\hline 55-.011-.001-. & Antherophagus nigricornis (F.,1787) & $\mathrm{x}$ & & $\mathrm{m}$ & $\mathrm{E}$ & $\mathrm{N}$ \\
\hline $55-.011-.003-$. & Antherophagus pallens (L.,1758) & $\mathrm{x}$ & & $\mathrm{m}$ & $\mathrm{E}$ & $\mathrm{N}$ \\
\hline $55-.012-.001-$. & Caenoscelis subdeplanata Bris., 1882 & $\mathrm{x}$ & & $\mathrm{m}$ & $\mathrm{W}$ & $\mathrm{TM}$ \\
\hline 55-.014-.001-. & Atomaria impressa Er., 1846 & $\mathrm{x}$ & & $\mathrm{m}$ & $\mathrm{F}$ & B \\
\hline 55-.014-.006-. & Atomaria ornata Heer, 1841 & $\mathrm{x}$ & & $\mathrm{m}$ & $\mathrm{W}$ & TP \\
\hline 55-.014-.011-. & Atomaria pusilla (Payk., 1798) & & $\mathrm{x}$ & $\mathrm{m}$ & $\mathrm{E}$ & $\mathrm{E}$ \\
\hline 55-.014-.014-. & Atomaria fuscata (Schönh.,1808) & $\mathrm{x}$ & $\mathrm{x}$ & $\mathrm{m}$ & $\mathrm{E}$ & $\mathrm{E}$ \\
\hline 55-.014-.016-. & Atomaria lewisi Rtt., 1877 & $\mathrm{x}$ & & $\mathrm{m}$ & $\mathrm{E}$ & $\mathrm{F}$ \\
\hline 55-.014-.021-. & Atomaria basalis Er., 1846 & $\mathrm{x}$ & & $\mathrm{m}$ & $\mathrm{F}$ & $\mathrm{B}$ \\
\hline 55-.014-.024-. & Atomaria rubella Heer, 1841 & $\mathrm{x}$ & & $\mathrm{m}$ & $\mathrm{E}$ & $\mathrm{F}$ \\
\hline $55-.014-.025-$. & Atomaria atricapilla Steph., 1830 & $\mathrm{x}$ & & $\mathrm{m}$ & $\mathrm{E}$ & $\mathrm{E}$ \\
\hline $55-.014-.028-$. & Atomaria analis Er.,1846 & $\mathrm{x}$ & & $\mathrm{m}$ & $\mathrm{E}$ & $\mathrm{E}$ \\
\hline 55-.014-.0281. & Atomaria nitidula (Marsh.,1802) & $\mathrm{x}$ & & $\mathrm{m}$ & E & $\mathrm{F}$ \\
\hline 55-.014-.033-. & Atomaria turgida Er.,1846 & $\mathrm{x}$ & $\mathrm{x}$ & $\mathrm{m}$ & $\mathrm{W}$ & $\mathrm{B}$ \\
\hline 55-.014-.036-. & Atomaria testacea Steph., 1830 & $\mathrm{x}$ & $\mathrm{x}$ & $\mathrm{m}$ & E & $\mathrm{E}$ \\
\hline 55-.014-.038-. & Atomaria umbrina (Gyll.,1827) & & $\mathrm{x}$ & $\mathrm{m}$ & $\mathrm{W}$ & TP \\
\hline 55-.014-.044-. & Atomaria puncticollis Thoms., 1868 & $\mathrm{x}$ & & $\mathrm{m}$ & $\mathrm{O}$ & $\mathrm{F}$ \\
\hline $55-.014-.045-$. & Atomaria nigrirostris Steph., 1830 & $\mathrm{x}$ & $\mathrm{x}$ & $\mathrm{m}$ & E & $\mathrm{B}$ \\
\hline 55-.014-.046-. & Atomaria linearis Steph.,1830 & $\mathrm{x}$ & $\mathrm{x}$ & $\mathrm{m}$ & E & $\mathrm{F}$ \\
\hline 55-.014-.051-. & Atomaria pulchra Er.,1846 & $\mathrm{x}$ & $\mathrm{x}$ & $\mathrm{m}$ & $\mathrm{W}$ & TP \\
\hline $55-.014-.052-$. & Atomaria atrata Rtt., 1875 & $\mathrm{x}$ & & $\mathrm{m}$ & $\mathrm{W}$ & TP \\
\hline $55-.014-.053-$. & Atomaria procerula Er.,1846 & $\mathrm{x}$ & & $\mathrm{m}$ & $\mathrm{W}$ & TP \\
\hline $55-.014-.054-$. & Atomaria bella Rtt., 1875 & $\mathrm{x}$ & $\mathrm{x}$ & $\mathrm{m}$ & $\mathrm{W}$ & TP \\
\hline 55-.014-.0541. & Atomaria lohsei Johns.Strand,1968 & & & $\mathrm{m}$ & $\mathrm{W}$ & TP \\
\hline $56-.000-.000-$. & Familie Phalacridae & & & & & \\
\hline 56-.002-.001-. & Olibrus aeneus (F.,1792) & & $\mathrm{x}$ & $\mathrm{p}$ & $\mathrm{O}$ & $\mathrm{V}$ \\
\hline 561.000-.000-. & Familie Laemophloeidae & & & & & \\
\hline 561.004-.001-. & Cryptolestes duplicatus (Waltl,1839) & $\mathrm{x}$ & $\mathrm{x}$ & $\mathrm{z}$ & $\mathrm{W}$ & TR \\
\hline 561.004-.005-. & Cryptolestes ferrugineus (Steph.,1831) & & $\mathrm{x}$ & $\mathrm{z}$ & E & $\mathrm{E}$ \\
\hline 58-.000-.000-. & Familie Lathridiidae & & & & & \\
\hline 58-.003-.0021. & Latridius minutus (L.,1767) & $\mathrm{x}$ & $\mathrm{x}$ & $\mathrm{m}$ & E & $\mathrm{F}$ \\
\hline 58-.004-.010-. & Enicmus fungicola Thoms., 1868 & $\mathrm{x}$ & $\mathrm{x}$ & $\mathrm{m}$ & $\mathrm{W}$ & TP \\
\hline 58-.004-.012-. & Enicmus rugosus (Hbst., 1793) & $\mathrm{x}$ & $\mathrm{x}$ & $\mathrm{m}$ & E & $\mathrm{P}$ \\
\hline 58-.004-.013-. & Enicmus testaceus (Steph.,1830) & $\mathrm{x}$ & $\mathrm{x}$ & $\mathrm{m}$ & $\mathrm{W}$ & TP \\
\hline 58-.004-.014-. & Enicmus transversus $(\mathrm{Ol} ., 1790)$ & $\mathrm{x}$ & $\mathrm{x}$ & $\mathrm{m}$ & $\mathrm{E}$ & $\mathrm{E}$ \\
\hline 58-.004-.015-. & Enicmus histrio Tomlin, 1910 & $\mathrm{x}$ & & $\mathrm{m}$ & $\mathrm{E}$ & $\mathrm{E}$ \\
\hline 58-.0041.001-. & Dienerella elongata (Curt.,1830) & $\mathrm{x}$ & $\mathrm{x}$ & $\mathrm{m}$ & $\mathrm{E}$ & $\mathrm{E}$ \\
\hline 58-.0041.0021. & Dienerella clathrata (Mannh.,1844) & & $\mathrm{x}$ & $\mathrm{m}$ & $\mathrm{W}$ & $\mathrm{E}$ \\
\hline 58-.005-.0011. & Cartodere constricta (Gyll.,1827) & $\mathrm{x}$ & & $\mathrm{m}$ & $\mathrm{E}$ & $\mathrm{F}$ \\
\hline 58-.0061.001-. & Stephostethus lardarius (Deg.,1775) & $\mathrm{x}$ & $\mathrm{x}$ & $\mathrm{m}$ & E & $\mathrm{E}$ \\
\hline 58-.0061.002-. & Stephostethus angusticollis (Gyll.,1827) & $\mathrm{x}$ & $\mathrm{x}$ & $\mathrm{m}$ & $\mathrm{W}$ & $\mathrm{E}$ \\
\hline 58-.0061.007-. & Stephostethus rugicollis $(\mathrm{Ol} ., 1790)$ & $\mathrm{x}$ & & $\mathrm{m}$ & $\mathrm{W}$ & TP \\
\hline 58-.0063.0011. & Aridius norvegicus (Belon, 1887) & $\mathrm{x}$ & & $\mathrm{m}$ & $\mathrm{W}$ & $\mathrm{TM}$ \\
\hline 58-.0063.003-. & Aridius nodifer (Westw.,1839) & $\mathrm{x}$ & $\mathrm{x}$ & $\mathrm{m}$ & $\mathrm{E}$ & $\mathrm{E}$ \\
\hline $58-.007-.002-$. & Corticaria punctulata Marsh,1802 & $\mathrm{x}$ & & $\mathrm{m}$ & $\mathrm{E}$ & $\mathrm{E}$ \\
\hline 58-.007-.016-. & Corticaria linearis (Payk.,1798) & $\mathrm{x}$ & $\mathrm{x}$ & $\mathrm{m}$ & $\mathrm{W}$ & TP \\
\hline
\end{tabular}




\begin{tabular}{|c|c|c|c|c|c|c|}
\hline EDV-Code & A r t & FF & Ekl & Kon & Bio & Hab \\
\hline 58-.007-.018-. & Corticaria longicollis (Zett.,1838) & $\mathrm{x}$ & $\mathrm{x}$ & $\mathrm{m}$ & $\mathrm{W}$ & $\mathrm{TM}$ \\
\hline 58-.007-.021-. & Corticaria elongata (Gyll.,1827) & $\mathrm{x}$ & $\mathrm{x}$ & $\mathrm{m}$ & $\mathrm{E}$ & $\mathrm{E}$ \\
\hline 58-.008-.002-. & Corticarina similata (Gyll.,1827) & $\mathrm{x}$ & $\mathrm{x}$ & $\mathrm{m}$ & $\mathrm{E}$ & $\mathrm{E}$ \\
\hline 58-.008-.005-. & Corticarina fuscula (Gyll.,1827) & $\mathrm{x}$ & $\mathrm{x}$ & $\mathrm{m}$ & $\mathrm{E}$ & $\mathrm{E}$ \\
\hline 58-.0081.001-. & Cortinicara gibbosa (Hbst.,1793) & $\mathrm{x}$ & $\mathrm{x}$ & $\mathrm{m}$ & $\mathrm{E}$ & $\mathrm{E}$ \\
\hline 581.000-.000-. & Familie Merophysiidae & & & & & \\
\hline 581.001-.001-. & Holoparamecus caularum Aube, 1843 & & $\mathrm{x}$ & $\mathrm{m}$ & $\mathrm{O}$ & $\mathrm{F}$ \\
\hline 59-.000-.000-. & Familie Mycetophagidae & & & & & \\
\hline 59-.002-.001-. & Triphyllus bicolor (F.,1792) & $\mathrm{x}$ & $\mathrm{x}$ & $\mathrm{m}$ & W & $\mathrm{TP}$ \\
\hline 59-.003-.001-. & Litargus connexus (Fourcr., 1785) & $\mathrm{x}$ & $\mathrm{x}$ & $\mathrm{m}$ & $\mathrm{W}$ & TR \\
\hline 59-.004-.001-. & Mycetophagus quadripustulatus (L.,1761) & & $\mathrm{x}$ & $\mathrm{m}$ & W & TP \\
\hline 59-.004-.003-. & Mycetophagus piceus (F.,1792) & $\mathrm{x}$ & $\mathrm{x}$ & $\mathrm{m}$ & $\mathrm{W}$ & TP \\
\hline 59-.004-.006-. & Mycetophagus atomarius (F., 1792) & $\mathrm{x}$ & $\mathrm{x}$ & $\mathrm{m}$ & $\mathrm{W}$ & $\mathrm{TP}$ \\
\hline 59-.004-.010-. & Mycetophagus populi F.,1798 & & $\mathrm{x}$ & $\mathrm{m}$ & $\mathrm{W}$ & $\mathrm{TP}$ \\
\hline 59-.005-.001-. & Typhaea stercorea (L.,1758) & $\mathrm{x}$ & & $\mathrm{m}$ & $\mathrm{E}$ & $\mathrm{F}$ \\
\hline $60-.000-.000-$. & Familie Colydiidae & & & & & \\
\hline 60-.014-.001-. & Cicones variegatus (Hellw.,1792) & $\mathrm{x}$ & $\mathrm{x}$ & $\mathrm{z}$ & W & $\mathrm{TP}$ \\
\hline 60-.016-.001-. & Bitoma crenata (F.,1775) & $\mathrm{x}$ & $\mathrm{x}$ & $\mathrm{z}$ & W & TR \\
\hline 60-.020-.001-. & Teredus cylindricus $(\mathrm{O} ., 1790)$ & $\mathrm{x}$ & $\mathrm{x}$ & $\mathrm{z}$ & $\mathrm{W}$ & $\mathrm{T}$ \\
\hline 601.000-.000-. & Familie Corylophidae & & & & & \\
\hline 601.008-.003-. & Orthoperus atomus (Gyll.,1808) & $\mathrm{x}$ & & $\mathrm{m}$ & W & TP \\
\hline 601.008-.004-. & Orthoperus mundus Matth.,1885 & $\mathrm{x}$ & $\mathrm{x}$ & $\mathrm{m}$ & $\mathrm{W}$ & TP \\
\hline 61-.000-.000-. & Familie Endomychidae & & & & & \\
\hline 61-.013-.001-. & Endomychus coccineus (L.,1758) & & $\mathrm{x}$ & $\mathrm{m}$ & W & $\mathrm{TP}$ \\
\hline 62-.000-.000-. & Familie Coccinellidae & & & & & \\
\hline 62-.0081.004-. & Nephus bipunctatus (Kug.,1794) & & $\mathrm{x}$ & $\mathrm{z}$ & $\mathrm{O}$ & $\mathrm{V}$ \\
\hline 62-.012-.002-. & Chilocorus renipustulatus (Scriba,1790) & & $\mathrm{x}$ & $\mathrm{z}$ & $\mathrm{W}$ & $\mathrm{V}$ \\
\hline 62-.017-.001-. & Aphidecta obliterata (L.,1758) & & $\mathrm{x}$ & $\mathrm{z}$ & W & V \\
\hline 62-.023-.002-. & Adalia decempunctata (L.,1758) & $\mathrm{x}$ & $\mathrm{x}$ & $\mathrm{z}$ & $\mathrm{E}$ & V \\
\hline 62-.023-.003-. & Adalia bipunctata (L.,1758) & & $\mathrm{x}$ & $\mathrm{z}$ & $\mathrm{E}$ & V \\
\hline 62-.025-.003-. & Coccinella septempunctata L., 1758 & $\mathrm{x}$ & $\mathrm{x}$ & $\mathrm{z}$ & E & $\mathrm{V}$ \\
\hline 62-.025-.005-. & Coccinella quinquepunctata L., 1758 & & $\mathrm{x}$ & $\mathrm{z}$ & $\mathrm{O}$ & V \\
\hline 62-.032-.001-. & Propylea quatuordecimpunctata (L.,1758) & $\mathrm{x}$ & $\mathrm{x}$ & $\mathrm{z}$ & $\mathrm{E}$ & V \\
\hline 63-.000-.000-. & Familie Sphindidae & & & & & \\
\hline 63-.001-.001-. & Sphindus dubius (Gyll.,1808) & $\mathrm{x}$ & & $\mathrm{m}$ & $\mathrm{W}$ & TP \\
\hline 63-.002-.001-. & Arpidiphorus orbiculatus (Gyll.,1808) & $\mathrm{x}$ & $\mathrm{x}$ & $\mathrm{m}$ & W & TP \\
\hline 65-.000-.000-. & Familie Cisidae & & & & & \\
\hline 65-.001-.001-. & Octotemnus glabriculus (Gyll.,1827) & $\mathrm{x}$ & $\mathrm{x}$ & $\mathrm{m}$ & $\mathrm{W}$ & TP \\
\hline 65-.005-.002-. & Sulcacis bidentulus (Rosh.,1847) & & $\mathrm{x}$ & $\mathrm{m}$ & $\mathrm{W}$ & TP \\
\hline 65-.006-.002-. & Cis nitidus (F., 1792) & $\mathrm{x}$ & $\mathrm{x}$ & $\mathrm{m}$ & W & TP \\
\hline 65-.006-.004-. & Cis glabratus Mell.,1848 & $\mathrm{x}$ & $\mathrm{x}$ & $\mathrm{m}$ & $\mathrm{W}$ & TP \\
\hline 65-.006-.007-. & Cis hispidus (Payk., 1798) & $\mathrm{x}$ & $\mathrm{x}$ & $\mathrm{m}$ & $\mathrm{W}$ & TP \\
\hline 65-.006-.011-. & Cis boleti (Scop.,1763) & $\mathrm{x}$ & $\mathrm{x}$ & $\mathrm{m}$ & $\mathrm{W}$ & $\mathrm{TP}$ \\
\hline 65-.006-.0111. & Cis rugolosus Mell., 1848 & $\mathrm{x}$ & & $\mathrm{m}$ & $\mathrm{W}$ & TP \\
\hline 65-.006-.015-. & Cis castaneus Mell.,1848 & $\mathrm{x}$ & $\mathrm{x}$ & $\mathrm{m}$ & $\mathrm{W}$ & $\mathrm{TP}$ \\
\hline 65-.006-.017-. & Cis bidentatus $(\mathrm{Ol} ., 1790)$ & $\mathrm{x}$ & $\mathrm{x}$ & $\mathrm{m}$ & W & TP \\
\hline
\end{tabular}




\begin{tabular}{|c|c|c|c|c|c|c|}
\hline EDV-Code & A r t & FF & Ekl & Kon & Bio & Hab \\
\hline 65-.006-.022-. & Cis alni Gyll., 1813 & $\mathrm{x}$ & & $\mathrm{m}$ & $\mathrm{W}$ & $\mathrm{TP}$ \\
\hline 65-.006-.024-. & Cis pygmaeus (Marsh.,1802) & & $\mathrm{x}$ & $\mathrm{m}$ & $\mathrm{W}$ & TP \\
\hline 65-.006-.028-. & Cis festivus (Panz.,1793) & $\mathrm{x}$ & $\mathrm{x}$ & $\mathrm{m}$ & $\mathrm{W}$ & TP \\
\hline 65-.007-.002-. & Ennearthron cornutum (Gyll.,1827) & $\mathrm{x}$ & $\mathrm{x}$ & $\mathrm{m}$ & $\mathrm{W}$ & $\mathrm{TP}$ \\
\hline 68-.000-.000-. & Familie Anobiidae & & & & & \\
\hline 68-.001-.002-. & Hedobia imperialis (L., 1767) & & $\mathrm{x}$ & $\mathrm{x}$ & $\mathrm{W}$ & $\mathrm{T}$ \\
\hline $68-.003-.003-$. & Dryophilus pusillus (Gyll.,1808) & & $\mathrm{x}$ & $\mathrm{x}$ & $\mathrm{W}$ & $\mathrm{T}$ \\
\hline 68-.005-.001-. & Xestobium plumbeum (Ill.,1801) & $\mathrm{x}$ & $\mathrm{x}$ & $\mathrm{x}$ & $\mathrm{W}$ & $\mathrm{T}$ \\
\hline 68-.005-.002-. & Xestobium rufovillosum (Geer,1774) & $\mathrm{x}$ & $\mathrm{x}$ & $\mathrm{x}$ & $\mathrm{W}$ & $\mathrm{T}$ \\
\hline 68-.012-.005-. & Anobium costatum Arrag.,1830 & $\mathrm{x}$ & $\mathrm{x}$ & $\mathrm{x}$ & $\mathrm{W}$ & $\mathrm{T}$ \\
\hline 68-.014-.001-. & Ptilinus pectinicornis (L.,1758) & $\mathrm{x}$ & $\mathrm{x}$ & $\mathrm{x}$ & $\mathrm{W}$ & $\mathrm{T}$ \\
\hline 68-.022-.001-. & Dorcatoma flavicornis (F.,1792) & $\mathrm{x}$ & $\mathrm{x}$ & $\mathrm{x}$ & $\mathrm{W}$ & $\mathrm{T}$ \\
\hline 68-.022-.003-. & Dorcatoma chrysomelina Sturm, 1837 & $\mathrm{x}$ & $\mathrm{x}$ & $\mathrm{x}$ & $\mathrm{W}$ & $\mathrm{T}$ \\
\hline 68-.022-.007-. & Dorcatoma robusta Strand,1938 & $\mathrm{x}$ & $\mathrm{x}$ & $\mathrm{m}$ & $\mathrm{W}$ & $\mathrm{TP}$ \\
\hline 69-.000-.000-. & Familie Ptinidae & & & & & \\
\hline 69-.008-.005-. & Ptinus fur (L., 1758) & & $\mathrm{x}$ & s & $\mathrm{E}$ & $\mathrm{F}$ \\
\hline 69-.008-.013-. & Ptinus subpilosus Sturm, 1837 & $\mathrm{x}$ & $\mathrm{x}$ & s & $\mathrm{W}$ & B \\
\hline 70-.000-.000-. & Familie Oedemeridae & & & & & \\
\hline 70-.007-.0021. & Ischnomera cyanea (F., 1792) & & $\mathrm{x}$ & $\mathrm{x}$ & $\mathrm{W}$ & $\mathrm{T}$ \\
\hline 711.000-.000-. & Familie Salpingidae & & & & & \\
\hline 711.001-.002-. & Lissodema quadripustulatum (Marsh.,1802) & $\mathrm{x}$ & $\mathrm{x}$ & $\mathrm{z}$ & $\mathrm{W}$ & TR \\
\hline 711.005-.001-. & Vincenzellus ruficollis (Panz., 1794) & $\mathrm{x}$ & $\mathrm{x}$ & $\mathrm{z}$ & $\mathrm{W}$ & TR \\
\hline 711.006-.002-. & Rhinosimus planirostris (F., 1787) & $\mathrm{x}$ & $\mathrm{x}$ & $\mathrm{z}$ & $\mathrm{W}$ & TR \\
\hline 711.006-.003-. & Rhinosimus ruficollis (L.,1761) & $\mathrm{x}$ & $\mathrm{x}$ & $\mathrm{z}$ & $\mathrm{W}$ & TR \\
\hline 72-.000-.000-. & Familie Pyrochroidae & & & & & \\
\hline 72-.001-.001-. & Pyrochroa coccinea (L., 1761) & $\mathrm{x}$ & $\mathrm{x}$ & $\mathrm{z}$ & $\mathrm{W}$ & TR \\
\hline 72-.002-.001-. & Schizotus pectinicornis (L.,1758) & $\mathrm{x}$ & $\mathrm{x}$ & $\mathrm{z}$ & $\mathrm{W}$ & TR \\
\hline 73-.000-.000-. & Familie Scraptiidae & & & & & \\
\hline 73-.004-.001-. & Anaspis humeralis (F.,1775) & $\mathrm{x}$ & $\mathrm{x}$ & $\mathrm{x}$ & $\mathrm{W}$ & $\mathrm{T}$ \\
\hline 73-.004-.009-. & Anaspis frontalis (L., 1758) & $\mathrm{x}$ & $\mathrm{x}$ & $\mathrm{x}$ & $\mathrm{W}$ & $\mathrm{T}$ \\
\hline 73-.004-.010-. & Anaspis maculata Fourcr.,1785 & $\mathrm{x}$ & $\mathrm{x}$ & $\mathrm{x}$ & $\mathrm{W}$ & $\mathrm{T}$ \\
\hline 73-.004-.011-. & Anaspis schilskyana Csiki,1915 & $\mathrm{x}$ & $\mathrm{x}$ & $\mathrm{x}$ & $\mathrm{W}$ & $\mathrm{T}$ \\
\hline 73-.004-.012-. & Anaspis thoracica (L.,1758) & $\mathrm{x}$ & $\mathrm{x}$ & $\mathrm{x}$ & $\mathrm{W}$ & $\mathrm{T}$ \\
\hline 73-.004-.019-. & Anaspis rufilabris (Gyll.,1827) & $\mathrm{x}$ & $\mathrm{x}$ & $\mathrm{x}$ & $\mathrm{W}$ & $\mathrm{T}$ \\
\hline 73-.004-.022-. & Anaspis flava (L.,1758) & $\mathrm{x}$ & $\mathrm{x}$ & $\mathrm{x}$ & $\mathrm{W}$ & $\mathrm{T}$ \\
\hline 74-.000-.000-. & Familie Aderidae & & & & & \\
\hline 74-.002-.007-. & Aderus oculatus (Panz.,1796) & $\mathrm{x}$ & $\mathrm{x}$ & $\mathrm{x}$ & $\mathrm{W}$ & $\mathrm{TM}$ \\
\hline 75-.000-.000-. & Familie Anthicidae & & & & & \\
\hline 75-.0043.002-. & Omonadus floralis (L.,1758) & $\mathrm{x}$ & & - & $\mathrm{E}$ & $\mathrm{F}$ \\
\hline 79-.000-.000-. & Familie Mordellidae & & & & & \\
\hline 79-.001-.001-. & Tomoxia biguttata (Gyll.,1827) & $\mathrm{x}$ & $\mathrm{x}$ & $\mathrm{x}$ & $\mathrm{W}$ & $\mathrm{T}$ \\
\hline 79-.011-.053-. & Mordellistena variegata (F., 1798) & & $\mathrm{x}$ & $\mathrm{x}$ & $\mathrm{W}$ & $\mathrm{T}$ \\
\hline 79-.012-.001-. & Mordellochroa abdominalis (F.,1775) & $\mathrm{x}$ & $\mathrm{x}$ & $\mathrm{x}$ & $\mathrm{W}$ & $\mathrm{T}$ \\
\hline 80-.000-.000-. & Familie Melandryidae & & & & & \\
\hline 80-.004-.001-. & Hallomenus binotatus (Quensel,1790) & $\mathrm{x}$ & $\mathrm{x}$ & $\mathrm{m}$ & $\mathrm{W}$ & $\mathrm{TP}$ \\
\hline 80-.005-.004-. & Orchesia minor Walk., 1837 & $\mathrm{x}$ & $\mathrm{x}$ & $\mathrm{m}$ & $\mathrm{W}$ & $\mathrm{T}$ \\
\hline
\end{tabular}




\begin{tabular}{|c|c|c|c|c|c|c|}
\hline EDV-Code & A r t & FF & Ekl & Kon & Bio & Hab \\
\hline 80-.005-.006-. & Orchesia undulata Kr.,1853 & & $\mathrm{x}$ & $\mathrm{m}$ & $\mathrm{W}$ & TR \\
\hline 80-.007-.001-. & Abdera affinis (Payk., 1799) & $\mathrm{x}$ & & $\mathrm{m}$ & $\mathrm{W}$ & $\mathrm{TP}$ \\
\hline 80-.013-.001-. & Hypulus quercinus (Quensel,1790) & $\mathrm{x}$ & $\mathrm{x}$ & $\mathrm{x}$ & $\mathrm{W}$ & $\mathrm{TM}$ \\
\hline 80-.016-.001-. & Melandrya caraboides (L.,1761) & $\mathrm{x}$ & $\mathrm{x}$ & $\mathrm{x}$ & $\mathrm{W}$ & $\mathrm{T}$ \\
\hline 80-.018-.001-. & Conopalpus testaceus $(\mathrm{Ol} ., 1790)$ & $\mathrm{x}$ & $\mathrm{x}$ & $\mathrm{x}$ & $\mathrm{W}$ & $\mathrm{T}$ \\
\hline 801.000-.000-. & Familie Tetratomidae & & & & & \\
\hline $801.001-.002-$. & Tetratoma desmaresti Latr.,1807 & & $\mathrm{x}$ & $\mathrm{m}$ & $\mathrm{W}$ & $\mathrm{TP}$ \\
\hline 801.001-.003-. & Tetratoma ancora F., 1790 & $\mathrm{x}$ & $\mathrm{x}$ & $\mathrm{m}$ & $\mathrm{W}$ & $\mathrm{TP}$ \\
\hline 82-.000-.000-. & Familie Alleculidae & & & & & \\
\hline 82-.001-.002-. & Allecula morio (F., 1787) & & $\mathrm{x}$ & $\mathrm{x}$ & $\mathrm{W}$ & $\mathrm{TM}$ \\
\hline 82-.005-.001-. & Pseudocistela ceramboides (L.,1761) & $\mathrm{x}$ & $\mathrm{x}$ & $\mathrm{x}$ & $\mathrm{W}$ & $\mathrm{TM}$ \\
\hline 82-.008-.011-. & Mycetochara linearis (Ill.,1794) & $\mathrm{x}$ & $\mathrm{x}$ & $\mathrm{x}$ & $\mathrm{W}$ & $\mathrm{T}$ \\
\hline 83-.000-.000-. & Familie Tenebrionidae & & & & & \\
\hline 83-.019-.001-. & Scaphidema metallicum (F., 1792) & & $\mathrm{x}$ & $\mathrm{m}$ & $\mathrm{W}$ & $\mathrm{TP}$ \\
\hline 83-.023-.001-. & Corticeus unicolor (Pill.Mitt.,1783) & $\mathrm{x}$ & $\mathrm{x}$ & $\mathrm{z}$ & $\mathrm{W}$ & TR \\
\hline 83-.023-.008-. & Corticeus fasciatus F., 1790 & $\mathrm{x}$ & $\mathrm{x}$ & $\mathrm{z}$ & $\mathrm{W}$ & $\mathrm{T}$ \\
\hline 83-.025-.002-. & Tribolium castaneum (Hbst.,1797) & & $\mathrm{x}$ & $\mathrm{s}$ & $\mathrm{E}$ & $\mathrm{F}$ \\
\hline 841.000-.000-. & Familie Trogidae & & & & & \\
\hline 841.001-.004-. & Trox scaber (L.,1767) & & $\mathrm{x}$ & $\mathrm{s}$ & $\mathrm{E}$ & $\mathrm{N}$ \\
\hline 842.000-.000-. & Familie Geotrupidae & & & & & \\
\hline 842.005-.001-. & Anoplotrupes stercorosus (Scriba,1791) & $\mathrm{x}$ & & $\mathrm{s}$ & $\mathrm{W}$ & $\mathrm{F}$ \\
\hline 85-.000-.000-. & Familie Scarabaeidae & & & & & \\
\hline 85-.014-.018-. & Onthophagus similis (Scriba,1790) & $\mathrm{x}$ & & $\mathrm{s}$ & $\mathrm{O}$ & $\mathrm{F}$ \\
\hline 85-.019-.004-. & Aphodius fossor (L.,1758) & $\mathrm{x}$ & & $\mathrm{s}$ & $\mathrm{O}$ & $\mathrm{F}$ \\
\hline 85-.019-.005-. & Aphodius haemorrhoidalis (L.,1758) & $\mathrm{x}$ & & $\mathrm{s}$ & $\mathrm{O}$ & $\mathrm{F}$ \\
\hline 85-.019-.012-. & Aphodius rufipes (L.,1758) & $\mathrm{x}$ & & $\mathrm{s}$ & $\mathrm{E}$ & $\mathrm{F}$ \\
\hline 85-.019-.022-. & Aphodius maculatus Sturm, 1800 & $\mathrm{x}$ & & $\mathrm{s}$ & $\mathrm{W}$ & $\mathrm{F}$ \\
\hline 85-.019-.024-. & Aphodius pusillus (Hbst.,1789) & $\mathrm{x}$ & & $\mathrm{s}$ & $\mathrm{O}$ & $\mathrm{F}$ \\
\hline 85-.019-.039-. & Aphodius contaminatus (Hbst., 1783) & $\mathrm{x}$ & & $\mathrm{s}$ & $\mathrm{E}$ & $\mathrm{F}$ \\
\hline 85-.019-.044-. & Aphodius prodromus $($ Brahm, 1790) & $\mathrm{x}$ & & $\mathrm{s}$ & $\mathrm{E}$ & $\mathrm{F}$ \\
\hline 85-.019-.060-. & Aphodius fimetarius (L.,1758) & $\mathrm{x}$ & & $\mathrm{s}$ & $\mathrm{E}$ & $\mathrm{F}$ \\
\hline 85-.019-.066-. & Aphodius ater (Geer,1774) & $\mathrm{x}$ & & $\mathrm{s}$ & $\mathrm{E}$ & $\mathrm{F}$ \\
\hline 85-.025-.001-. & Serica brunnea (L., 1758) & $\mathrm{x}$ & & $\mathrm{p}$ & $\mathrm{O}$ & V \\
\hline 85-.033-.002-. & Melolontha melolontha (L.,1758) & $\mathrm{x}$ & & $\mathrm{p}$ & $\mathrm{O}$ & $\mathrm{V}$ \\
\hline 85-.037-.001-. & Phyllopertha horticola (L.,1758) & $\mathrm{x}$ & & $\mathrm{p}$ & $\mathrm{E}$ & V \\
\hline 85-.050-.002-. & Gnorimus variabilis (L., 1758) & & $\mathrm{x}$ & $\mathrm{x}$ & $\mathrm{W}$ & $\mathrm{TM}$ \\
\hline 86-.000-.000-. & Familie Lucanidae & & & & & \\
\hline 86-.005-.001-. & Sinodendron cylindricum (L.,1758) & $\mathrm{x}$ & $\mathrm{x}$ & $\mathrm{x}$ & $\mathrm{W}$ & $\mathrm{T}$ \\
\hline 87-.000-.000-. & Familie Cerambycidae & & & & & \\
\hline 87-.011-.001-. & Rhagium bifasciatum F.,1775 & $\mathrm{x}$ & $\mathrm{x}$ & $\mathrm{x}$ & $\mathrm{W}$ & $\mathrm{T}$ \\
\hline 87-.011-.002-. & Rhagium sycophanta (Schrk.,1781) & & $\mathrm{x}$ & $\mathrm{x}$ & $\mathrm{W}$ & TR \\
\hline 87-.011-.003-. & Rhagium mordax (Geer,1775) & $\mathrm{x}$ & $\mathrm{x}$ & $\mathrm{x}$ & $\mathrm{W}$ & TR \\
\hline 87-.023-.002-. & Grammoptera ruficornis (F.,1781) & $\mathrm{x}$ & & $\mathrm{x}$ & $\mathrm{W}$ & $\mathrm{T}$ \\
\hline 87-.024-.001-. & Alosterna tabacicolor (Geer, 1775) & $\mathrm{x}$ & $\mathrm{x}$ & $\mathrm{x}$ & $\mathrm{W}$ & $\mathrm{T}$ \\
\hline 87-.028-.002-. & Judolia cerambyciformis (Schrk.,1781) & $\mathrm{x}$ & & $\mathrm{x}$ & $\mathrm{W}$ & $\mathrm{T}$ \\
\hline 87-.029-.006-. & Strangalia quadrifasciata (L.,1758) & $\mathrm{x}$ & & $\mathrm{x}$ & $\mathrm{W}$ & $\mathrm{T}$ \\
\hline
\end{tabular}




\begin{tabular}{|c|c|c|c|c|c|c|}
\hline EDV-Code & A r t & FF & Ekl & Kon & Bio & Hab \\
\hline 87-.029-.007-. & Strangalia maculata (Poda,1761) & $\mathrm{x}$ & $\mathrm{x}$ & $\mathrm{x}$ & W & $\mathrm{T}$ \\
\hline 87-.029-.009-. & Strangalia aethiops (Poda,1761) & $\mathrm{x}$ & & $\mathrm{x}$ & W & $\mathrm{T}$ \\
\hline 87-.029-.010-. & Strangalia melanura (L., 1758) & $\mathrm{x}$ & $\mathrm{x}$ & $\mathrm{x}$ & $\mathrm{W}$ & $\mathrm{T}$ \\
\hline 87-.054-.001-. & Pyrrhidium sanguineum (L.,1758) & $\mathrm{x}$ & & $\mathrm{x}$ & $\mathrm{W}$ & $\mathrm{T}$ \\
\hline 87-.058-.003-. & Clytus arietis (L.,1758) & $\mathrm{x}$ & $\mathrm{x}$ & $\mathrm{x}$ & $\mathrm{W}$ & $\mathrm{T}$ \\
\hline 87-.071-.002-. & Mesosa nebulosa (F.,1781) & & $\mathrm{x}$ & $\mathrm{x}$ & $\mathrm{W}$ & $\mathrm{T}$ \\
\hline $87-.075-.002-$. & Pogonocherus hispidus (L.,1758) & $\mathrm{x}$ & $\mathrm{x}$ & $\mathrm{x}$ & $\mathrm{W}$ & $\mathrm{T}$ \\
\hline 87-.078-.001-. & Leiopus nebulosus (L.,1758) & $\mathrm{x}$ & $\mathrm{x}$ & $\mathrm{x}$ & $\mathrm{W}$ & $\mathrm{T}$ \\
\hline 87-.082-.004-. & Saperda scalaris (L., 1758) & & $\mathrm{x}$ & $\mathrm{x}$ & $\mathrm{W}$ & $\mathrm{T}$ \\
\hline 88-.000-.000-. & Familie Chrysomelidae & & & & & \\
\hline 88-.0061.003-. & Oulema gallaeciana (Heyden,1870) & $\mathrm{x}$ & & $\mathrm{p}$ & $\mathrm{E}$ & $\mathrm{V}$ \\
\hline 88-.0061.005-. & Oulema melanopus (L.,1758) & $\mathrm{x}$ & $\mathrm{x}$ & $\mathrm{p}$ & $\mathrm{E}$ & $\mathrm{V}$ \\
\hline 88-.017-.071-. & Cryptocephalus pusillus F.,1777 & $\mathrm{x}$ & $\mathrm{x}$ & $\mathrm{p}$ & $\mathrm{W}$ & $\mathrm{V}$ \\
\hline 88-.023-.0061. & Chrysolina fastuosa (Scop.,1763) & & & $\mathrm{p}$ & $\mathrm{O}$ & $\mathrm{V}$ \\
\hline 88-.028-.001-. & Gastrophysa polygoni (L.,1758) & $\mathrm{x}$ & & $\mathrm{p}$ & $\mathrm{O}$ & $\mathrm{V}$ \\
\hline 88-.035-.011-. & Gonioctena quinquepunctata (F., 1787) & $\mathrm{x}$ & $\mathrm{x}$ & $\mathrm{p}$ & $\mathrm{W}$ & $\mathrm{V}$ \\
\hline 88-.036-.004-. & Phratora laticollis Suffr., 1851 & $\mathrm{x}$ & & $\mathrm{p}$ & $\mathrm{W}$ & $\mathrm{V}$ \\
\hline 88-.042-.001-. & Lochmaea capreae (L.,1758) & $\mathrm{x}$ & & $\mathrm{p}$ & $\mathrm{E}$ & V \\
\hline 88-.049-.005-. & Phyllotreta undulata Kutsch., 1860 & & $\mathrm{x}$ & $\mathrm{p}$ & E & $\mathrm{V}$ \\
\hline 88-.049-.010-. & Phyllotreta striolata (F.,1803) & & $\mathrm{x}$ & $\mathrm{p}$ & E & V \\
\hline 88-.066-.004-. & Chaetocnema laevicollis (Thoms.,1866) & & $\mathrm{x}$ & $\mathrm{p}$ & $\mathrm{E}$ & V \\
\hline 88-.066-.017-. & Chaetocnema hortensis (Fourcr.,1785) & & $\mathrm{x}$ & $\mathrm{p}$ & $\mathrm{E}$ & $\mathrm{V}$ \\
\hline 90-.000-.000-. & Familie Anthribidae & & & & & \\
\hline $90-.006-.001-$. & Enedreutes sepicola (F., 1792) & & $\mathrm{x}$ & $\mathrm{x}$ & $\mathrm{W}$ & $\mathrm{T}$ \\
\hline 90-.010-.001-. & Anthribus albinus (L., 1758) & $\mathrm{x}$ & & $\mathrm{x}$ & $\mathrm{W}$ & $\mathrm{T}$ \\
\hline $90-.015-.002-$. & Choragus sheppardi Kirby,1818 & & $\mathrm{x}$ & $\mathrm{x}$ & W & TP \\
\hline 91-.000-.000-. & Familie Scolytidae & & & & & \\
\hline $91-.001-.003-$. & Scolytus intricatus (Ratz.,1837) & $\mathrm{x}$ & $\mathrm{x}$ & $\mathrm{x}$ & W & TR \\
\hline $91-.004-.002-$. & Hylastes opacus Er.,1836 & & $\mathrm{x}$ & $\mathrm{x}$ & $\mathrm{W}$ & TR \\
\hline $91-.004-.003-$. & Hylastes cunicularius Er.,1836 & $\mathrm{x}$ & & $\mathrm{x}$ & $\mathrm{W}$ & TR \\
\hline $91-.005-.002-$. & Hylurgops palliatus (Gyll.,1813) & & $\mathrm{x}$ & $\mathrm{x}$ & W & TR \\
\hline $91-.008-.001-$. & Dendroctonus micans (Kug.,1794) & $\mathrm{x}$ & & $\mathrm{x}$ & $\mathrm{W}$ & $\mathrm{TR}$ \\
\hline $91-.020-.002-$. & Crypturgus hispidulus Thoms., 1870 & $\mathrm{x}$ & $\mathrm{x}$ & $\mathrm{x}$ & W & TR \\
\hline $91-.024-.001-$. & Dryocoetes autographus (Ratz.,1837) & $\mathrm{x}$ & & $\mathrm{x}$ & W & TR \\
\hline $91-.024-.002-$. & Dryocoetes villosus (F., 1792) & $\mathrm{x}$ & $\mathrm{x}$ & $\mathrm{x}$ & $\mathrm{W}$ & TR \\
\hline 91-.024-.003-. & Dryocoetes alni (Georg, 1856) & $\mathrm{x}$ & & $\mathrm{x}$ & $\mathrm{W}$ & TR \\
\hline 91-.026-.004-. & Cryphalus abietis (Ratz., 1837) & $\mathrm{x}$ & $\mathrm{x}$ & $\mathrm{x}$ & W & TR \\
\hline $91-.027-.001-$. & Ernoporicus fagi (F., 1778) & $\mathrm{x}$ & & $\mathrm{x}$ & $\mathrm{W}$ & TR \\
\hline $91-.031-.003-$. & Taphrorychus bicolor (Hbst., 1793) & $\mathrm{x}$ & $\mathrm{x}$ & $\mathrm{x}$ & W & TR \\
\hline $91-.032-.001-$. & Pityogenes chalcographus (L.,1761) & $\mathrm{x}$ & $\mathrm{x}$ & $\mathrm{x}$ & W & TR \\
\hline 91-.035-.004-. & Ips typographus (L.,1758) & & $\mathrm{x}$ & $\mathrm{x}$ & $\mathrm{W}$ & TR \\
\hline $91-.036-.001-$. & Xyleborus dispar (F.,1792) & $\mathrm{x}$ & & $\mathrm{m}$ & $\mathrm{W}$ & $\mathrm{T}$ \\
\hline 91-.036-.004-. & Xyleborus saxeseni (Ratz.,1837) & $\mathrm{x}$ & $\mathrm{x}$ & $\mathrm{m}$ & W & $\mathrm{T}$ \\
\hline $91-.038-.001-$. & Xyloterus domesticus (L.,1758) & $\mathrm{x}$ & $\mathrm{x}$ & $\mathrm{m}$ & W & $\mathrm{T}$ \\
\hline $91-.038-.002-$. & Xyloterus signatus (F.,1787) & $\mathrm{x}$ & $\mathrm{x}$ & $\mathrm{m}$ & $\mathrm{W}$ & $\mathrm{T}$ \\
\hline
\end{tabular}

923.000-.000-. Familie Rhynchitidae 


\begin{tabular}{|c|c|c|c|c|c|c|}
\hline EDV-Code & A r t & FF & Ekl & Kon & Bio & Hab \\
\hline $923.003-.001-$. & Lasiorhynchites sericeus (Hbst.,1797) & $\mathrm{x}$ & & $\mathrm{p}$ & W & V \\
\hline 923.004-.005-. & Caenorhinus aequatus (L.,1767) & $\mathrm{x}$ & & $\mathrm{p}$ & $\mathrm{O}$ & $\mathrm{V}$ \\
\hline 923.007-.004-. & Deporaus betulae (L.,1758) & $\mathrm{x}$ & $\mathrm{x}$ & $\mathrm{p}$ & $\mathrm{E}$ & $\mathrm{V}$ \\
\hline 925.000-.000-. & Familie Apionidae & & & & & \\
\hline $925.021-.002-$. & Protapion fulvipes (Fourcr., 1785) & & $\mathrm{x}$ & $\mathrm{p}$ & $\mathrm{O}$ & $\mathrm{V}$ \\
\hline 925.034-.001-. & Ischnopterapion loti (Kirby,1808) & & $\mathrm{x}$ & $\mathrm{p}$ & $\mathrm{O}$ & V \\
\hline 93-.000-.000-. & Familie Curculionidae & & & & & \\
\hline 93-.015-.104-. & Otiorhynchus singularis (L.,1767) & & $\mathrm{x}$ & $\mathrm{p}$ & $\mathrm{E}$ & V \\
\hline 93-.019-.002-. & Caenopsis waltoni (Boh.,1843) & & $\mathrm{x}$ & $\mathrm{p}$ & $\mathrm{O}$ & B \\
\hline $93-.021-.015-$. & Phyllobius calcaratus (F.,1792) & $\mathrm{x}$ & $\mathrm{x}$ & $\mathrm{p}$ & $\mathrm{W}$ & $\mathrm{V}$ \\
\hline 93-.021-.019-. & Phyllobius argentatus (L.,1758) & $\mathrm{x}$ & $\mathrm{x}$ & $\mathrm{p}$ & $\mathrm{W}$ & $\mathrm{V}$ \\
\hline $93-.027-.011-$. & Polydrusus cervinus (L.,1758) & $\mathrm{x}$ & & $\mathrm{p}$ & $\mathrm{W}$ & V \\
\hline 93-.027-.016-. & Polydrusus undatus (F.,1781) & $\mathrm{x}$ & $\mathrm{x}$ & $\mathrm{p}$ & $\mathrm{W}$ & $\mathrm{V}$ \\
\hline $93-.037-.011-$. & Barypeithes pellucidus (Boh.,1834) & & $\mathrm{x}$ & $\mathrm{p}$ & $\mathrm{E}$ & $\mathrm{V}$ \\
\hline $93-.040-.002-$. & Strophosoma melanogrammum (Forst., 1771) & $\mathrm{x}$ & $\mathrm{x}$ & $\mathrm{p}$ & $\mathrm{W}$ & V \\
\hline $93-.040-.003-$. & Strophosoma capitatum (Geer,1775) & $\mathrm{x}$ & $\mathrm{x}$ & $\mathrm{p}$ & $\mathrm{W}$ & V \\
\hline 93-.044-.016-. & Sitona lepidus Gyll.,1834 & $\mathrm{x}$ & $\mathrm{x}$ & $\mathrm{p}$ & $\mathrm{O}$ & V \\
\hline 93-.092-.004-. & Notaris acridulus (L.,1758) & $\mathrm{x}$ & & $\mathrm{p}$ & $\mathrm{O}$ & V \\
\hline 93-.104-.019-. & Tychius picirostris (F.,1787) & & $\mathrm{x}$ & $\mathrm{p}$ & $\mathrm{O}$ & $\mathrm{V}$ \\
\hline $93-.106-.015-$. & Anthonomus rubi (Hbst.,1795) & $\mathrm{x}$ & & $\mathrm{p}$ & $\mathrm{E}$ & $\mathrm{V}$ \\
\hline $93-.110-.011-$. & Curculio pyrrhoceras Marsh.,1802 & $\mathrm{x}$ & $\mathrm{x}$ & $\mathrm{p}$ & $\mathrm{W}$ & V \\
\hline 93-.157-.003-. & Coeliodes dryados (Gm.,1790) & & $\mathrm{x}$ & $\mathrm{p}$ & $\mathrm{W}$ & V \\
\hline 93-.157-.008-. & Coeliodes erythroleucos (Gmel., 1790) & $\mathrm{x}$ & $\mathrm{x}$ & $\mathrm{p}$ & $\mathrm{W}$ & V \\
\hline $93-.163-.0601$. & Ceutorhynchus floralis (Payk.,1792) & $\mathrm{x}$ & $\mathrm{x}$ & $\mathrm{p}$ & $\mathrm{E}$ & $\mathrm{V}$ \\
\hline $93-.169-.001-$. & Nedyus quadrimaculatus (L.,1758) & & & $\mathrm{p}$ & $\mathrm{E}$ & V \\
\hline $93-.180-.005-$. & Rhynchaenus quercus (L.,1758) & & $\mathrm{x}$ & $\mathrm{p}$ & $\mathrm{W}$ & V \\
\hline 93-.180-.013-. & Rhynchaenus fagi (L.,1758) & $\mathrm{x}$ & $\mathrm{x}$ & $\mathrm{p}$ & $\mathrm{W}$ & V \\
\hline 93-.180-.025-. & Rhynchaenus salicis (L.,1758) & $\mathrm{x}$ & & $\mathrm{p}$ & $\mathrm{W}$ & V \\
\hline
\end{tabular}

\subsection{Die Struktur der Artengemeinschaften in den Eklektorproben}

\subsubsection{Frequenz- und Dominanzstruktur in Abhängigkeit von Eklektortyp und Baumart}

Die überwiegende Zahl der Arten war in nur wenigen der insgesamt 353 Eklektorproben anzutreffen (Tab. 4-12). 125 Arten (27\%) traten in nur einer Falle auf und waren gemeinsam mit $0,3 \%$ an der Gesamtzahl gefangener Individuen beteiligt. 403 Arten (88 \%) wurden in weniger als $10 \%$ der Proben festgestellt, nur drei Arten mit einem gemeinsamen Individuenanteil von $25 \%$ wurden in mehr als der Hälfte der Proben gefunden.

Die beiden untersuchten Baumarten zeigten große Übereinstimmungen in der Frequenzstruktur der Arten. Größere Abweichungen traten jedoch bei den auf die Frequenzklassen entfallenden Individuenanteile auf. Jeweils $7 \%$ der Arten waren in mindestens $20 \%$ und über $80 \%$ der Arten in weniger als $10 \%$ der Proben anzutreffen. Läßt man die EFl-Proben wegen 
der geringen Fangzahlen unberücksichtigt, zeigte sich auch bei einer Gegenüberstellung der Eklektortypen hinsichtlich der auf die Frequenzklassen entfallenden Artenanteile eine große Ähnlichkeit.

Tabelle 4-12: Frequenzstruktur Eklektorproben

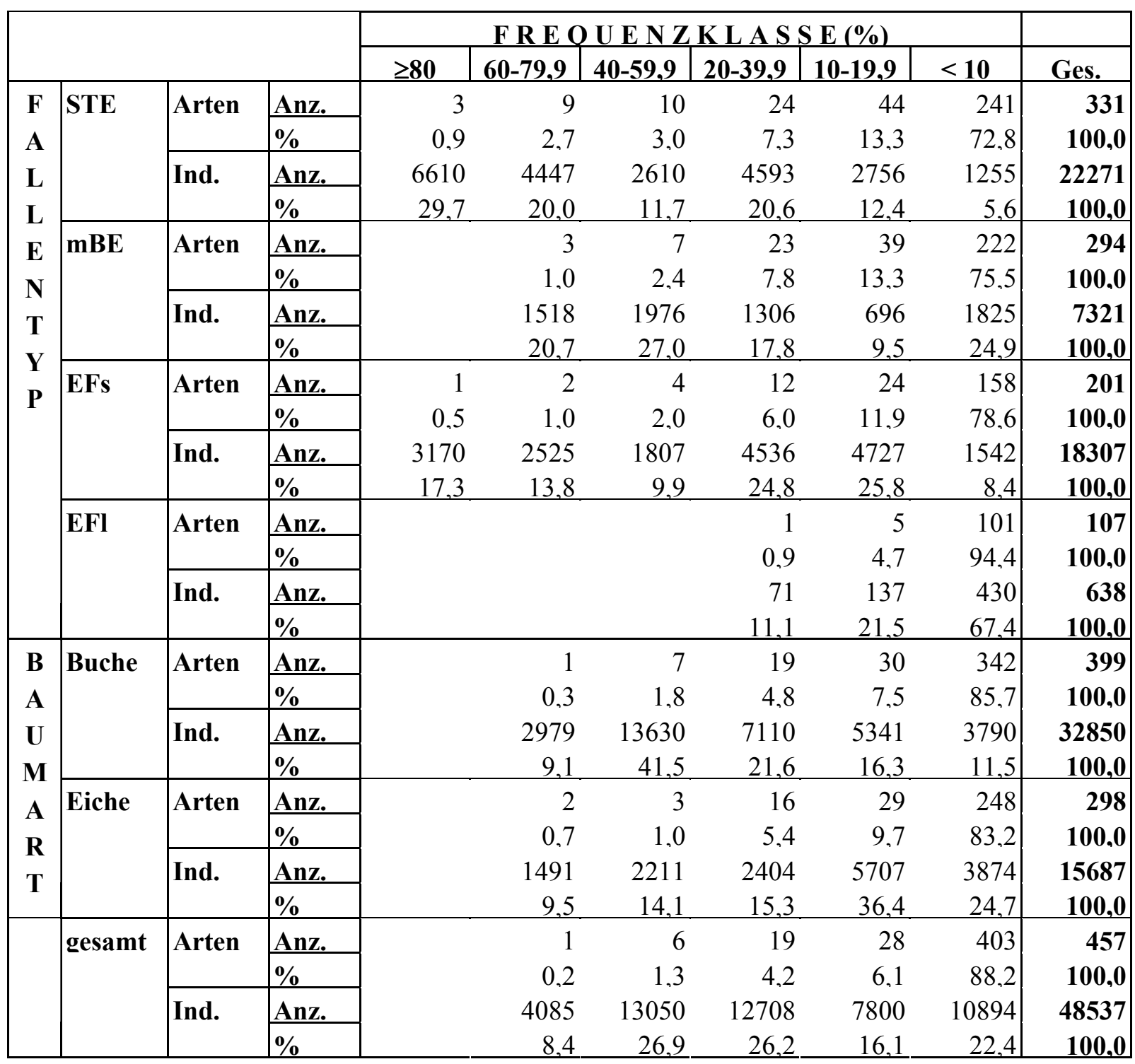

In der Tabelle 4-13 sind diejenigen Arten aufgelistet, die wenigstens in $30 \%$ der Proben eines Eklektortyps registriert wurden. Einige Arten wurden an der Buche mit deutlich größerer Regelmäßigkeit festgestellt als an der Eiche, so z. B. Ptilinus pectinicornis, Corticeus unicolor, Paromalus flavicornis und Plegaderus dissectus. Auf der anderen Seite traten Neuraphes elongatulus, Rhynchaenus fagi und Teredus cylindricus regelmäßiger an Eiche in Erscheinung. Unterschiede waren auch zwischen den Eklektortypen zu beobachten. Als Beispiele für Arten, die in einer Vielzahl der STE, aber in vergleichsweise wenigen EFsProben vertreten waren, stehen Phloeonomus punctipennis, Leptusa pulchella, Pteryx suturalis und Ptinella limbata. Cis castaneus und Micridium halidaii erreichten dagegen in den EFs-Proben deutlich höhere Frequenzprozentwerte als in den STE. 
Tabelle 4-13: Frequenzanteil (\%) von Arten mit hohem Frequenzanteil (>30\%) in wenigstens einem Eklektortyp

\begin{tabular}{|c|c|c|c|c|c|c|c|}
\hline \multirow[b]{2}{*}{$\mathbf{A R}_{\mathbf{T}}$} & \multicolumn{4}{|c|}{ Eklektortyp } & \multicolumn{2}{|c|}{ Baumart } & \multirow{2}{*}{$\begin{array}{c}\text { Eklektoren } \\
\text { gesamt }\end{array}$} \\
\hline & STE & mBE & EFs & EFI & $\mathbf{E i}$ & Bu & \\
\hline Corticaria longicollis & 86,9 & 26,9 & 87,9 & 16,4 & 79,3 & 60,3 & 66,3 \\
\hline Cryptophagus dentatus & 79,8 & 44,2 & 69,5 & 6,6 & 60,4 & 56,6 & 57,8 \\
\hline Rhizophagus dispar & 92,9 & 15,4 & 61,0 & 27,9 & 57,7 & 57,4 & 57,5 \\
\hline Rhizophagus bipustulatus & 68,7 & 36,5 & 56,0 & 11,5 & 52,3 & 47,5 & 49,0 \\
\hline Leptusa fumida & 68,7 & 67,3 & 37,6 & 9,8 & 38,7 & 49,2 & 45,9 \\
\hline Cerylon ferrugineum & 68,7 & 46,2 & 44,0 & 4,9 & 34,2 & 49,2 & 44,5 \\
\hline Phloeonomus punctipennis & 88,9 & 76,9 & 14,2 & 8,2 & 49,5 & 40,5 & 43,3 \\
\hline Ptilinus pectinicornis & 43,4 & 65,4 & 36,9 & 6,6 & 12,6 & 49,2 & 37,7 \\
\hline Stenichnus collaris & 69,7 & 1,9 & 27,7 & 13,1 & 35,1 & 32,2 & 33,1 \\
\hline Aridius nodifer & 64,6 & 36,5 & 12,8 & 4,9 & 37,8 & 25,6 & 29,5 \\
\hline Corticeus unicolor & 38,4 & 32,7 & 31,2 & 0,0 & 15,3 & 33,9 & 28,0 \\
\hline Paromalus flavicornis & 22,2 & 11,5 & 46,1 & 4,9 & 13,5 & 33,5 & 27,2 \\
\hline Pteryx suturalis & 68,7 & 15,4 & 9,9 & 6,6 & 26,1 & 26,9 & 26,6 \\
\hline Dienerella elongata & 31,3 & 1,9 & 43,3 & 1,6 & 29,7 & 25,2 & 26,6 \\
\hline Phloeocharis subtilissima & 65,7 & 17,3 & 9,9 & 1,6 & 28,8 & 23,6 & 25,2 \\
\hline Plegaderus dissectus & 26,3 & 34,6 & 30,5 & 1,6 & 2,7 & 35,1 & 24,9 \\
\hline Bibloporus bicolor & 48,5 & 25,0 & 17,0 & 3,3 & 30,6 & 21,9 & 24,6 \\
\hline Neuraphes elongatulus & 63,6 & 0,0 & 15,6 & 0,0 & 39,6 & 16,9 & 24,1 \\
\hline Gabrius splendidulus & 48,5 & 32,7 & 4,3 & 18,0 & 17,1 & 26,0 & 23,2 \\
\hline Sepedophilus testaceus & 57,6 & 3,8 & 11,3 & 8,2 & 16,2 & 25,6 & 22,7 \\
\hline Rhizophagus nitidulus & 45,5 & 17,3 & 16,3 & 3,3 & 11,7 & 27,3 & 22,4 \\
\hline Cerylon histeroides & 34,3 & 21,2 & 21,3 & 1,6 & 14,4 & 24,8 & 21,5 \\
\hline Orchesia undulata & 30,3 & 50,0 & 11,3 & 3,3 & 9,9 & 26,0 & 21,0 \\
\hline Ptinella limbata & 57,6 & 9,6 & 6,4 & 3,3 & 18,9 & 21,5 & 20,7 \\
\hline Rhynchaenus fagi & 17,2 & 38,5 & 25,5 & 0,0 & 36,9 & 13,2 & 20,7 \\
\hline Phyllodrepa ioptera & 28,3 & 42,3 & 14,2 & 1,6 & 27,9 & 16,5 & 20,1 \\
\hline Teredus cylindricus & 21,2 & 3,8 & 32,6 & 1,6 & 36,9 & 12,0 & 19,8 \\
\hline Pterostichus oblongopunctatus & 54,5 & 25,0 & 0,7 & 0,0 & 27,9 & 15,3 & 19,3 \\
\hline Leptusa pulchella & 51,5 & 19,2 & 4,3 & 0,0 & 26,1 & 15,7 & 19,0 \\
\hline Cerylon fagi & 51,5 & 7,7 & 5,7 & 3,3 & 20,7 & 17,4 & 18,4 \\
\hline Denticollis linearis & 34,3 & 48,1 & 1,4 & 4,9 & 19,8 & 17,4 & 18,1 \\
\hline Melanotus castanipes & 31,3 & 44,2 & 6,4 & 0,0 & 12,6 & 20,2 & 17,8 \\
\hline Cis castaneus & 5,1 & 9,6 & 36,9 & 0,0 & 14,4 & 19,0 & 17,6 \\
\hline Pterostichus niger & 50,5 & 5,8 & 1,4 & 6,6 & 24,3 & 13,2 & 16,7 \\
\hline Anaspis schilskyana & 13,1 & 38,5 & 17,7 & 0,0 & 15,3 & 16,9 & 16,4 \\
\hline Bolitochara obliqua & 33,3 & 34,6 & 0,7 & 1,6 & 9,9 & 17,4 & 15,0 \\
\hline Agathidium seminulum & 30,3 & 7,7 & 3,5 & 1,6 & 18,9 & 7,9 & 11,3 \\
\hline Corticarina similata & 6,1 & 46,2 & 3,5 & 0,0 & 0,0 & 14,5 & 9,9 \\
\hline Athous subfuscus & 12,1 & 34,6 & 1,4 & 0,0 & 9,0 & 9,1 & 9,1 \\
\hline Trixagus dermestoides & 9,1 & 34,6 & 1,4 & 0,0 & 5,4 & 9,5 & 8,2 \\
\hline Ampedus pomorum & 11,1 & 32,7 & 0,0 & 0,0 & 4,5 & 9,5 & 7,9 \\
\hline
\end{tabular}


Mit der größten Regelmäßigkeit wurde Corticaria longicollis in den Eklektorproben beobachtet. Dieser Lathridiidae war in $66 \%$ der Proben anwesend, das Frequenzprozent für die STE-Proben betrug 87, dasjenige für die EFs-Proben an stehendem Totholz 88 \%. Cryptophagus dentatus trat ebenso wie Rhizophagus dispar in zahlreichen Proben auf (204 bzw. 203). Letztere Art erreichte dabei aber einen viermal höheren Individuenanteil. Arten, die sich durch ein hohes Frequenzprozent auszeichneten, waren i. d. R. auch in größerer Individuenzahl vertreten. Andererseits waren einige der dominierenden Arten in vergleichsweise wenigen Proben zu finden. Hier sind vor allem Frischholzbesiedler wie Hylecoetus dermestoides, Xyloterus signatus und Xyl. domesticus sowie Dryocoetes villosus zu erwähnen. Obwohl in nur 22 Proben vertreten, gehörte letztere zu den wenigen dominanten Arten. Häufiger war es der Fall, dass Arten zwar in vielen Proben, aber nur in geringer Dichte beobachtet wurden. Phloeocharis subtilissima, Plegaderus dissectus und Bibloporus bicolor waren jeweils in ca. $25 \%$ der Proben anwesend, erreichten aber mit Individuenanteilen von 0,4 bis 0,5\% lediglich den Status von subrezedenten Begleitarten.

Auf den gesamten Stichprobenumfang bezogen, erreichten 12 Arten den Status von Hauptarten, während 445 Arten nur mit einem Individuenanteil von jeweils unter 2,0 \% am Gesamtergebnis beteiligt waren. Zusammen stellten die Begleitarten 41,2\% der gefangenen Individuen. Sowohl an der Eiche als auch an der Buche entfielen $64 \%$ der Individuen auf nur $3 \%$ der Arten, wohingegen 87 bzw. $89 \%$ der Arten mit zusammen 12 bzw. $13 \%$ der Individuen vertreten waren (Tab. 4-14). 
Tabelle 4-14: Dominanzklassenstruktur Eklektorproben

\begin{tabular}{|c|c|c|c|c|c|c|c|c|c|c|}
\hline & \multicolumn{6}{|c|}{ D O M I N A N Z KLA S S E (\%) } & \multirow[b]{3}{*}{ Ges. } \\
\hline & & & & \multicolumn{3}{|c|}{ Hauptarten } & \multicolumn{3}{|c|}{ Begleitarten } & \\
\hline & & & & $\geq 10$ & $5-9,99$ & $2-4,99$ & $1-1,99$ & 0,3-0,99 & $<\mathbf{0 , 3 0}$ & \\
\hline \multirow{16}{*}{$\begin{array}{l}\mathbf{F} \\
\mathbf{A} \\
\mathbf{L} \\
\mathbf{L} \\
\mathbf{E} \\
\mathbf{N} \\
\mathbf{T} \\
\mathbf{Y} \\
\mathbf{P}\end{array}$} & \multirow[t]{4}{*}{ STE } & \multirow[t]{2}{*}{ Arten } & Anz. & 1 & 2 & 9 & 7 & 24 & 288 & 331 \\
\hline & & & $\%$ & 0,3 & 0,6 & 2,7 & 2,1 & 7,3 & 87,0 & 100,0 \\
\hline & & \multirow[t]{2}{*}{ Ind. } & Anz. & 5022 & 2835 & 6372 & 2409 & 3090 & 2543 & 22271 \\
\hline & & & $\%$ & 22,5 & 12,7 & 28,6 & 10,8 & 13,9 & 11,4 & 100,0 \\
\hline & \multirow[t]{4}{*}{ mBE } & \multirow[t]{2}{*}{ Arten } & Anz. & 1 & 4 & 1 & 11 & 36 & 241 & 294 \\
\hline & & & $\%$ & 0,3 & 1,4 & 0,3 & 3,7 & 12,2 & 82,0 & 100,0 \\
\hline & & \multirow[t]{2}{*}{ Ind. } & Anz. & 1514 & 2048 & 274 & 989 & 1360 & 1136 & 7321 \\
\hline & & & $\%$ & 20,7 & 28,0 & 3,7 & 13,5 & 18,6 & 15,5 & 100,0 \\
\hline & \multirow[t]{4}{*}{ EFs } & \multirow[t]{2}{*}{ Arten } & Anz. & 3 & 2 & 6 & 7 & 14 & 169 & 201 \\
\hline & & & $\%$ & 1,5 & 1,0 & 3,0 & 3,5 & 7,0 & 84,1 & 100,0 \\
\hline & & \multirow[t]{2}{*}{ Ind. } & Anz. & 8358 & 2525 & 2842 & 1857 & 1253 & 1472 & 18307 \\
\hline & & & $\%$ & 45,7 & 13,8 & 15,5 & 10,1 & 6,8 & 8,0 & 100,0 \\
\hline & \multirow[t]{4}{*}{ EFI } & \multirow[t]{2}{*}{ Arten } & Anz. & 3 & 1 & 7 & 11 & 32 & 53 & 107 \\
\hline & & & $\%$ & 2,8 & 0,9 & 6,5 & 10,3 & 29,9 & 49,5 & 100,0 \\
\hline & & \multirow[t]{2}{*}{ Ind. } & Anz. & 207 & 35 & 155 & 94 & 94 & 53 & 638 \\
\hline & & & $\%$ & 32,4 & 5,5 & 24,3 & 14,7 & 14,7 & 8,3 & 100,0 \\
\hline \multirow{8}{*}{$\begin{array}{l}\mathbf{B} \\
\mathbf{A} \\
\mathbf{U} \\
\mathbf{M} \\
\mathbf{A} \\
\mathbf{R} \\
\mathbf{T}\end{array}$} & \multirow[t]{4}{*}{ Buche } & \multirow[t]{2}{*}{ Arten } & Anz. & 2 & 1 & 10 & 9 & 23 & 354 & 399 \\
\hline & & & $\%$ & 0,5 & 0,3 & 2,5 & 2,3 & 5,8 & 88,7 & 100,0 \\
\hline & & \multirow[t]{2}{*}{ Ind. } & Anz. & 8886 & 2979 & 9004 & 3864 & 3809 & 4308 & 32850 \\
\hline & & & $\%$ & 27,1 & 9,1 & 27,4 & 11,8 & 11,6 & 13,1 & 100,0 \\
\hline & \multirow[t]{4}{*}{ Eiche } & \multirow[t]{2}{*}{ Arten } & Anz. & 2 & 3 & 5 & 7 & 23 & 258 & 298 \\
\hline & & & $\%$ & 0,7 & 1,0 & 1,7 & 2,3 & 7,7 & 86,6 & 100,0 \\
\hline & & \multirow[t]{2}{*}{ Ind. } & Anz. & 4324 & 3291 & 2440 & 1814 & 1964 & 1854 & 15687 \\
\hline & & & $\%$ & 27,6 & 21,0 & 15,6 & 11,6 & 12,5 & 11,8 & 100,0 \\
\hline \multirow{4}{*}{\multicolumn{2}{|c|}{ Gesamt }} & \multirow[t]{2}{*}{ Arten } & Anz. & 1 & 3 & 8 & 12 & 23 & 410 & 457 \\
\hline & & & $\%$ & 0,2 & 0,7 & 1,8 & 2,6 & 5,0 & 89,7 & 100,0 \\
\hline & & \multirow[t]{2}{*}{ Ind. } & Anz. & 6612 & 10194 & 11738 & 7902 & 5424 & 6667 & 48537 \\
\hline & & & $\%$ & 13,6 & 21,0 & 24,2 & 16,3 & 11,2 & 13,7 & 100,0 \\
\hline
\end{tabular}


Die Abbildung 4-11 stellt den Individuenanteil jener 25 Arten (sortiert nach Gesamtanteil) dar, die zumindest an einer Baumart mit über $1 \%$ am Fangergebnis beteiligt waren.

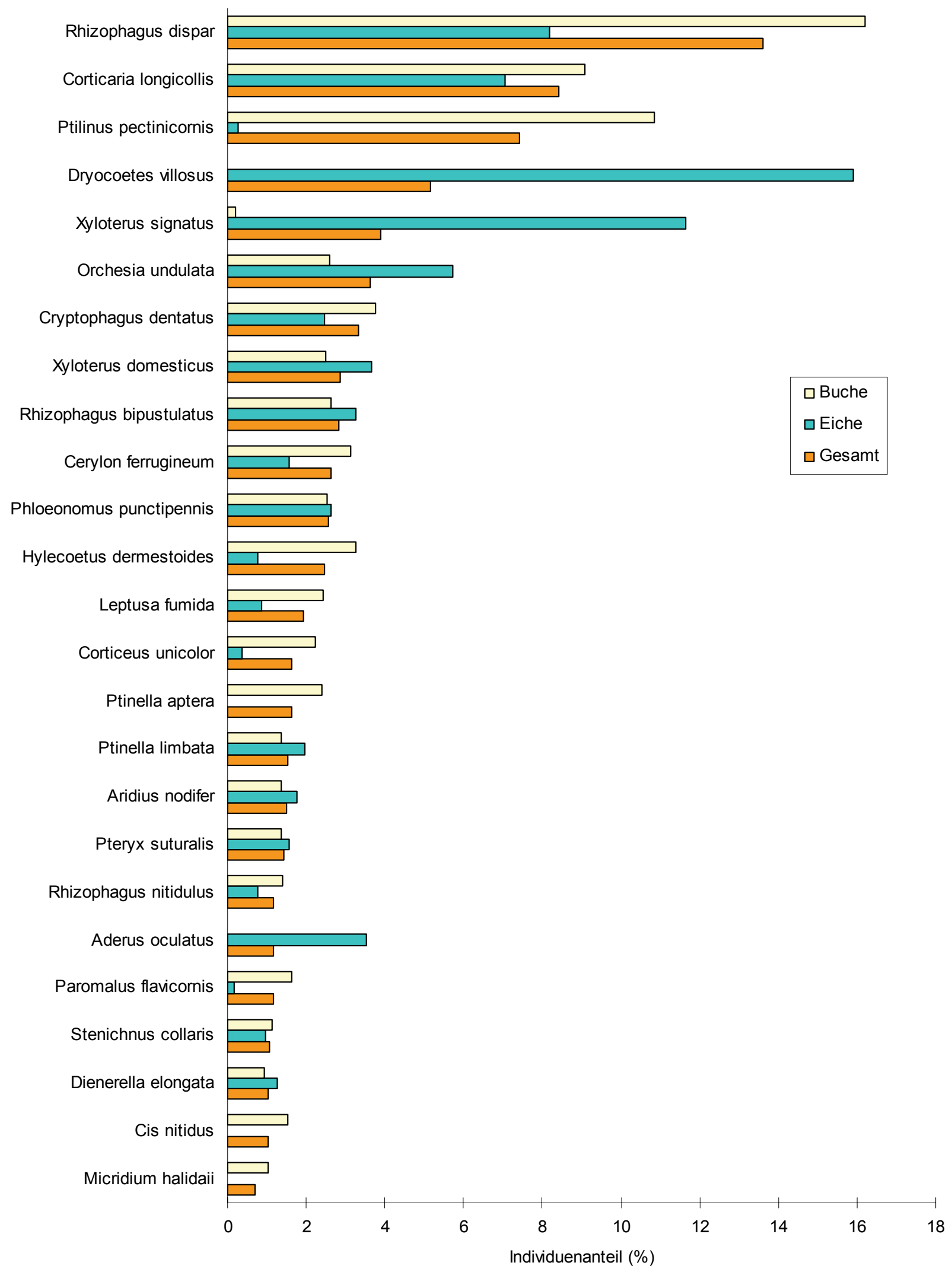

Abb. 4-11: Individuenanteil dominanter Arten an Eiche und Buche 
Auf die einzige eudominante Art, Rhizophagus dispar, entfielen 13,6 \% der Individuen. Über $80 \%$ der gefangenen Tiere stammten aus Eklektoren an Buchentotholz. In den Fängen an Eichentotholz war dieser Rindenbewohner mit einem Anteil von 8,2 \% die dritthäufigste Art, an Buchentotholz mit 16,2 \% die häufigste. Besonders hoch war ihr Anteil mit 22,6 \% in den STE-Proben, während sich dieser in den EFs-Proben auf 8,2 und in den mBE-Proben auf nur $0,2 \%$ belief. $76 \%$ der Exemplare wurden mit STE, $23 \%$ mit EFs gefangen. In den Fangperioden P1b bis P4 wurden gleichermaßen hohe Individuenzahlen registriert. Über $70 \%$ der Individuen wurden in diesem Zeitraum erfaßt (Abb. 4-12a).

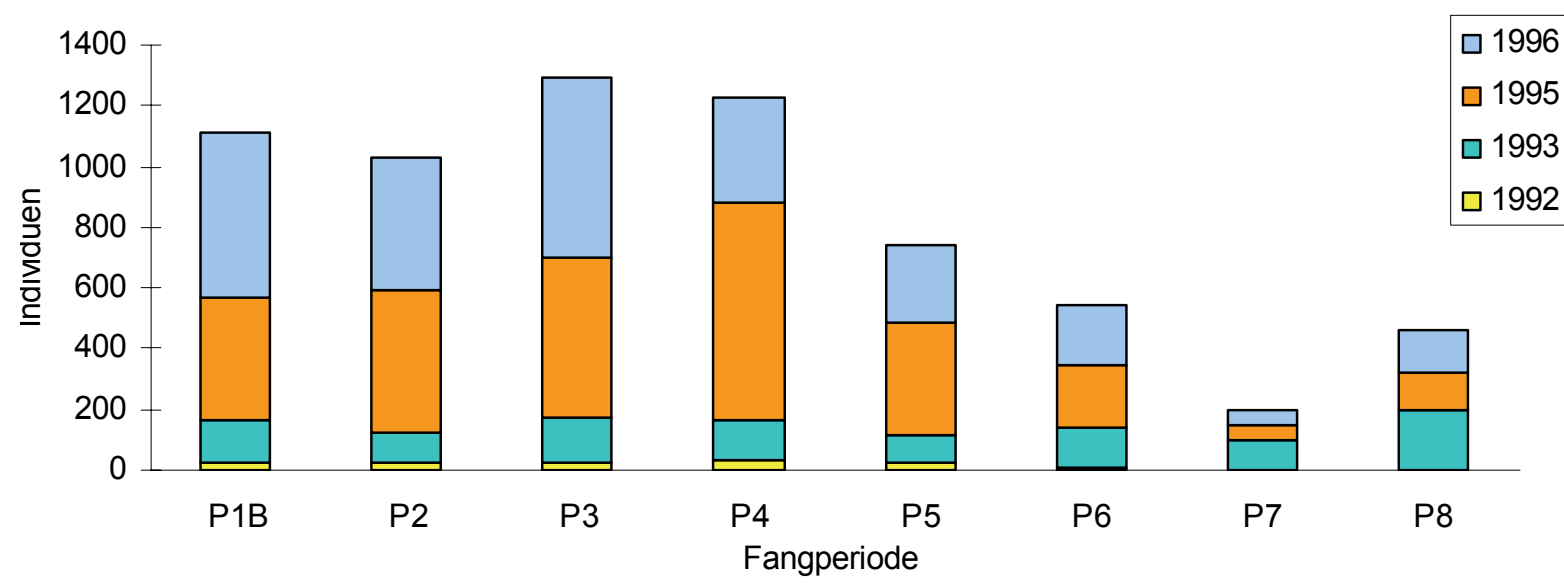

Abb. 4-12a: Schlüpfphänologie Rhizophagus dispar

Corticaria longicollis war in allen Untersuchungsjahren dominant. $27 \%$ der Individuen wurden an Eichentotholz gefangen. Der Individuenanteil an dieser Baumart betrug 7,1 \%, an Buche 9,1\%. $78 \%$ der Tiere stammten aus EFs-Proben, wo C. longicollis mit einem Anteil von $17,3 \%$ als häufigste Art in Erscheinung trat, während in den STE nur ein Anteil von $3,9 \%$ und in den $\mathrm{mBE}$ von $0,3 \%$ erreicht wurde. C. longicollis war in allen Fangperioden am Fang beteiligt, die maximale Individuenzahl wurde im August gefangen (Abb. 4-12b).

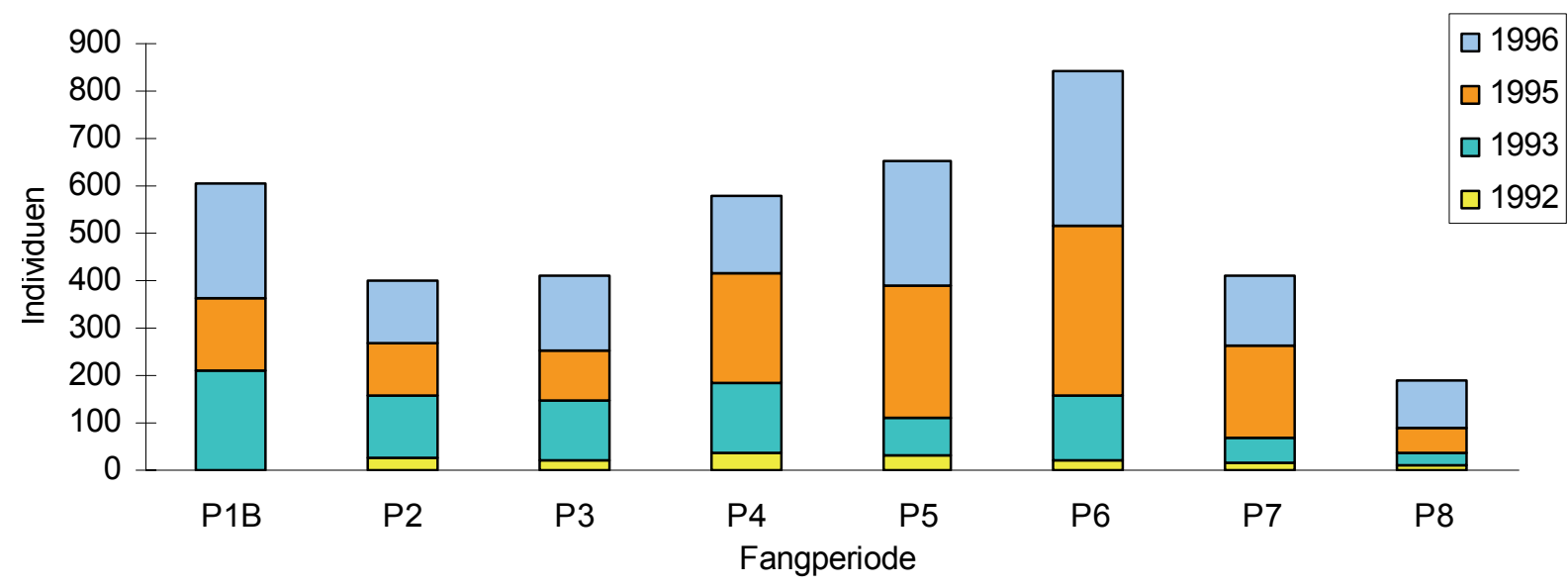

Abb. 4-12b: Schlüpfphänologie Corticaria longicollis 
$10,8 \%$ der an Buchentotholz gefangenen Käferindividuen wurden von Ptilinus pectinicornis gestellt. An der Eiche trat dieser Anobiidae nur sporadisch (0,26 \%) in Erscheinung. Am Gesamtergebnis war er mit einem Individuenanteil von 7,4 \% beteiligt. $75 \%$ der Individuen wurden an stehendem Totholz gefangen, an dem diese Art einen Individuenanteil von $17,3 \%$ stellte. $63 \%$ der insgesamt 3600 gefangenen Individuen wurden im Juli erfaßt, $33 \%$ entfielen auf die sich anschließende Fangperiode P5 (Abb. 4-12c).

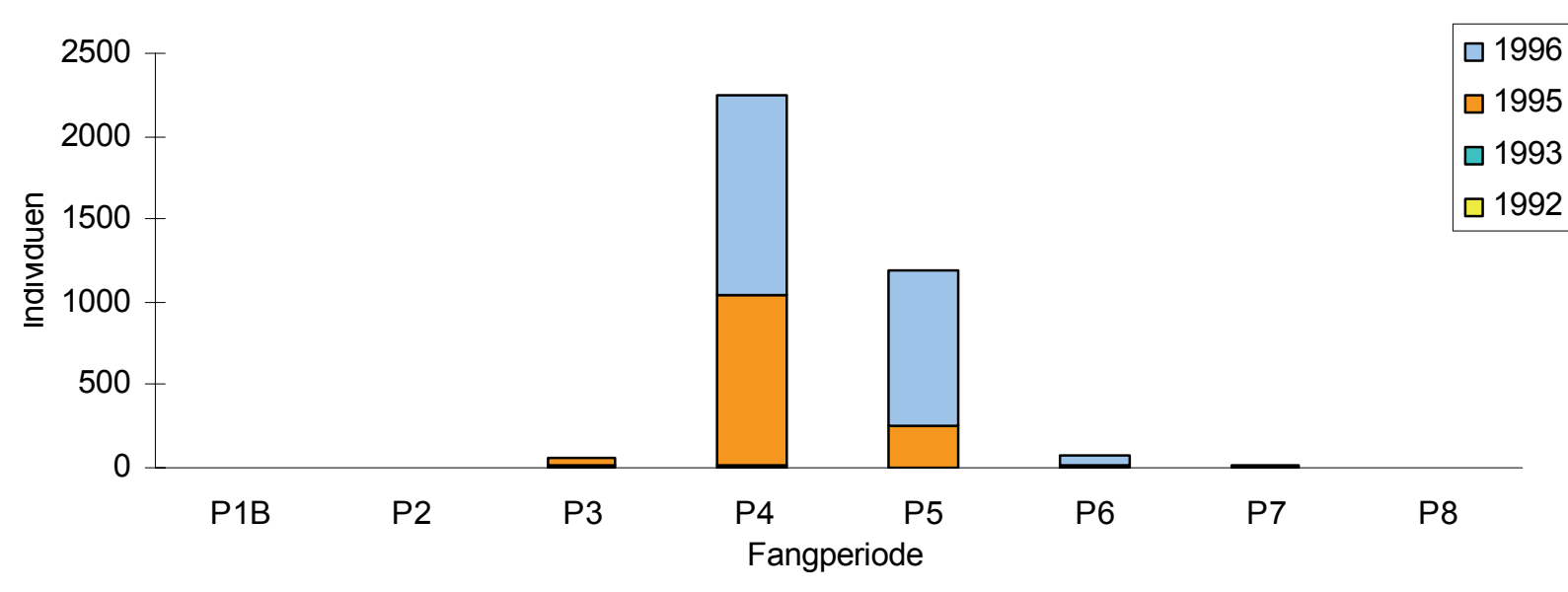

Abb. 4-12c: Schlüpfphänologie Ptilinus pectinicornis

Der Borkenkäfer Dryocoetes villosus, der bevorzugt in der Borke anbrüchiger oder frisch abgestorbener Eichen brütet, wurde vor allem an stehenden Eichen erfaßt. Da diese Totholzstruktur nur 1993 beprobt wurde, stammten fast alle Fänge aus diesem Jahr, in dem $D$. villosus als einzige Art mit einem Individuenanteil von 20,8 \% den Status einer eudominanten Hauptart erreichte. Auch wenn man den gesamten an Eiche erhobenen Fang zu Grunde legt, bleibt der Zottige Eichenborkenkäfer mit 15,9 \% Individuenanteil die häufigste Art. Am Gesamtfang sämtlicher Untersuchungsjahre war er immerhin noch mit über $5 \%$ beteiligt. Die höchste Schlüpfaktivität war in der Fangperiode P2 zu verzeichnen (Abb. 4-12d).

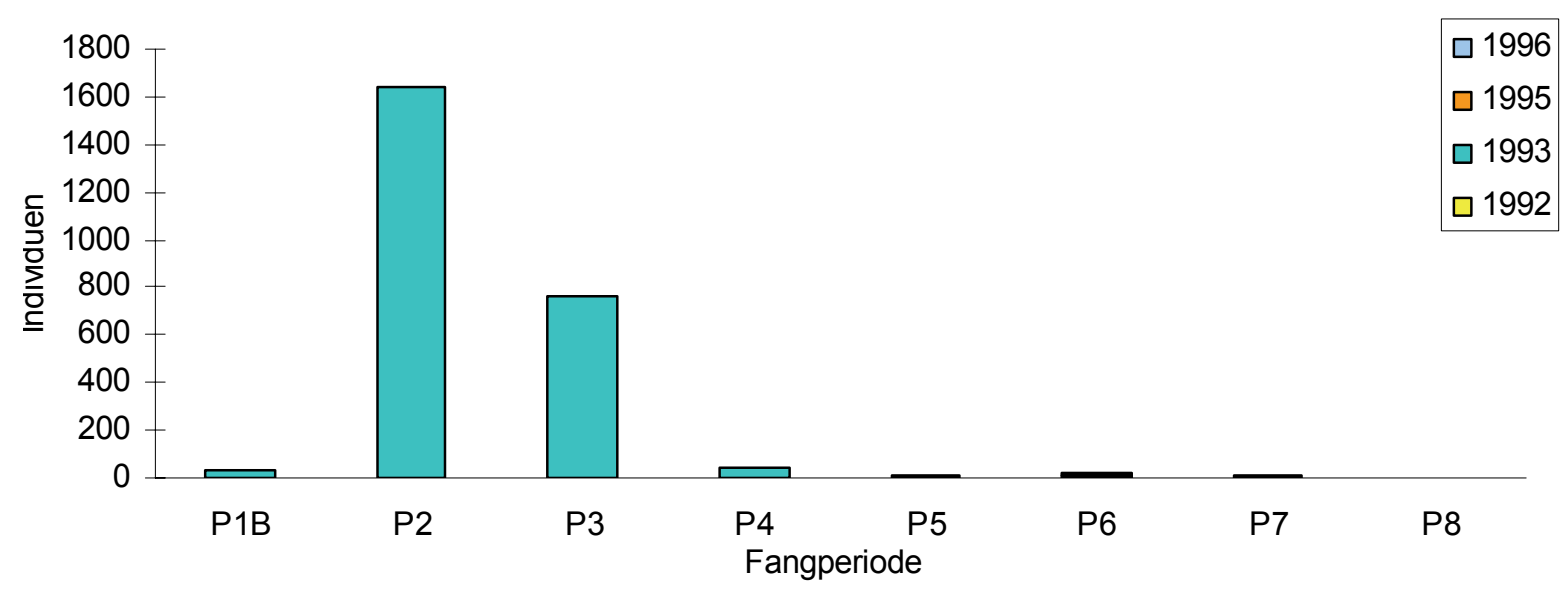

Abb. 4-12d: Schlüpfphänologie Dryocoetes villosus 
Auch Xyloterus signatus, der Eichennutzholzborkenkäfer, bevorzugt die Eiche als Brutsubstrat. $11,7 \%$ der an dieser Baumart gemachten Fänge entfielen auf diesen Holzbrüter, während am Buchentotholz nur ein Anteil von 0,2 \% erreicht wurde. Am Gesamtfang war er mit $3,9 \%$ beteiligt. $82 \%$ der Individuen wurden mit STE erfaßt. Die Anzahl gefangener Individuen wurde in hohem Maße vom Zeitpunkt der Fallenaufstellung beeinflusst. Fast $60 \%$ der Individuen stammten aus einer STE-Probe aus dem Jahr 1992. Diese Falle war erst Mitte Mai installiert worden, einem Zeitpunkt also, zu dem Xyl. signatus die Besiedlungsphase bereits abgeschlossen hatte, während in den Folgejahren die STE noch während der Schwärmphase angebracht worden waren und somit auch keine vollständige Besiedlung hatte stattfinden können. Die Käfer überwintern, anders als Xyl. domesticus, außerhalb ihres Brutsystems. Um solche Überwinterer handelte es sich auch bei den wenigen an Buchen gefangenen Exemplaren, die in ihrer Mehrzahl in der Fangperiode P1b das Winterquartier verließen (Abb. 4-12e).

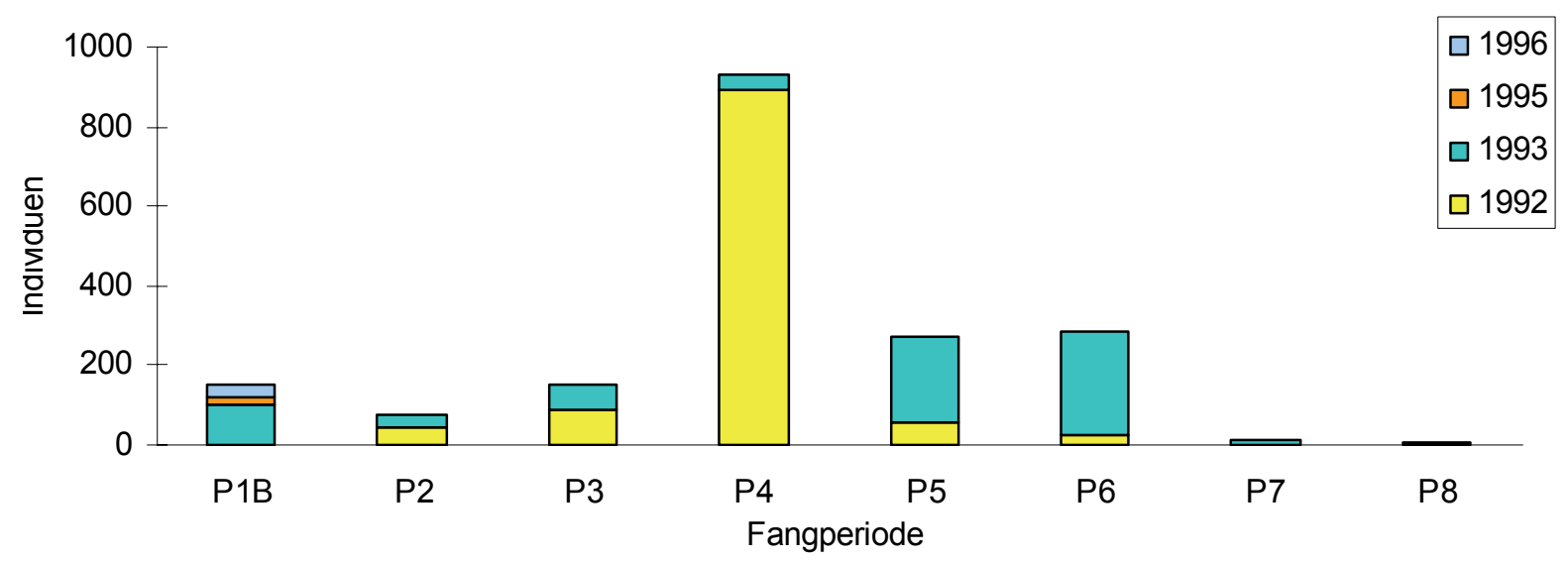

Abb. 4-12e: Schlüpfphänologie Xyloterus signatus

Xyloterus domesticus gehörte sowohl an der Eiche mit einem Individuenanteil von 3,7 \% als auch an der Buche mit einem Wert von 2,5\% zu den dominierenden Arten. $91 \%$ der Individuen wurden mit STE erfaßt, 59 \% fingen sich an Buchentotholz. Da Xyl. domesticus überwiegend im Brutsystem überwintert und dieses häufig schon im März verläßt, dürfte dieser Käfer den Entwicklungsort zum Zeitpunkt des Fallenaufbaus in der überwiegenden Zahl bereits verlassen gehabt haben und damit in den Eklektorfängen unterrepräsentiert gewesen sein (Abb. 4-12f).

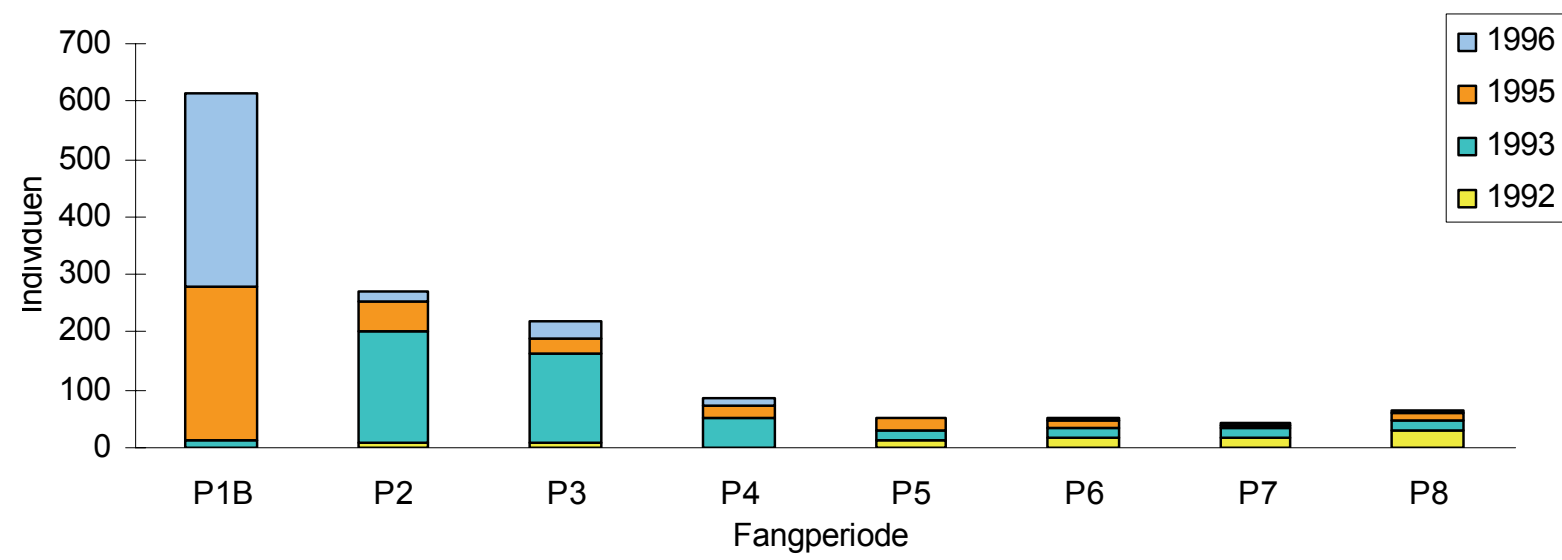

Abb. 4-12f: Schlüpfphänologie Xyloterus domesticus 
Mit einem Anteil von 3,6 \% war Orchesia undulata am Gesamtfang beteiligt. An den beiden Baumarten wurde eine annähernd gleiche Anzahl von Tieren gefangen. An der Eiche gehörte dieser Melandryidae mit 5,7 \% zu den dominanten und an der Buche mit 2,6 \% zu den subdominanten Arten. $86 \%$ der Tiere wurden in den mBE-Proben nachgewiesen, da das bevorzugte Habitat dieser Art - schwächerdimensioniertes Material mit lockerer, verpilzter Rinde - ausschließlich mit diesem Fallentyp untersucht wurde. Dieser Pilzfresser war mit über $20 \%$ an den mBE-Fängen beteiligt. $O$. undulata war zumindest in Einzelexemplaren während der gesamten Vegetationsperiode in den Eklektoren vertreten. In allen Untersuchungsjahren konzentrierten sich die Fänge auf die Monate Juli und August (Abb. 4-12g).

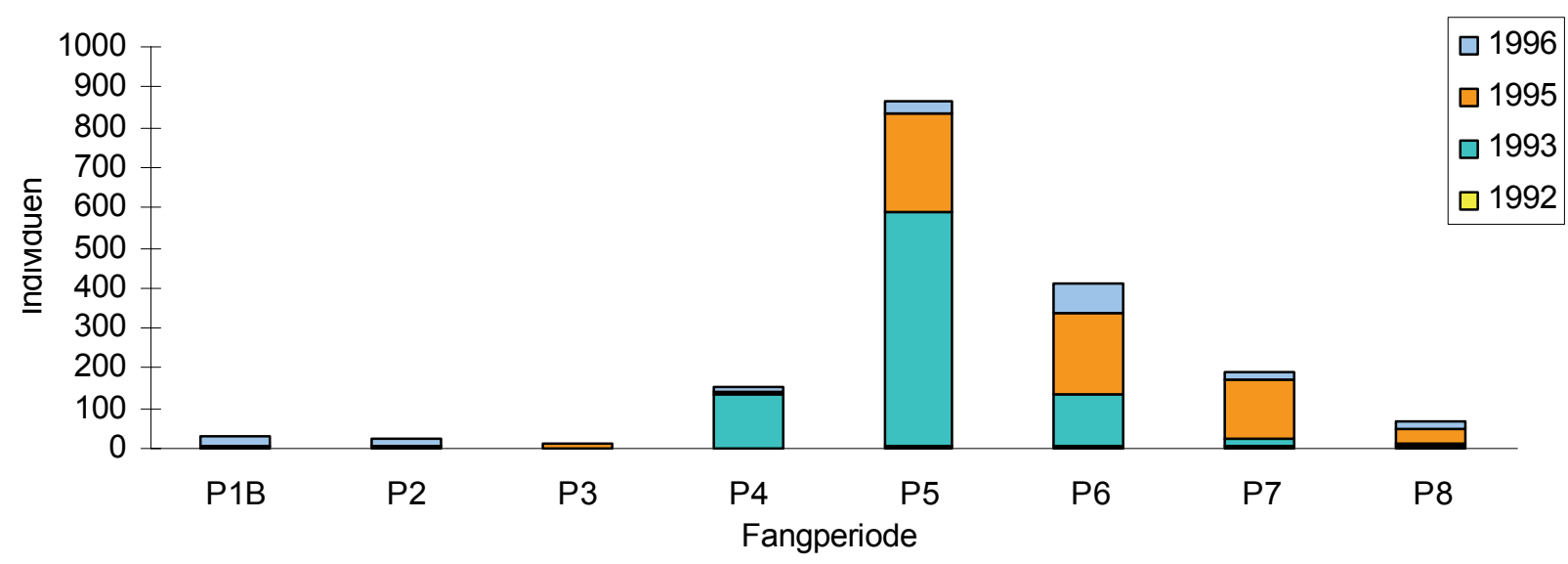

Abb. 4-12g: Schlüpfphänologie Orchesia undulata

Ebenfalls Pilzfresser, aber nicht streng an Totholz gebunden, ist die subdominante $(3,3 \%)$ Art Cryptophagus dentatus. In den einzelnen Jahren wurden Anteile von 2,4 bis 4,5\% verzeichnet. $76 \%$ der Exemplare wurden an Buche gefangen. Hier wurde ein Individuenanteil von 3,7\% erreicht, an der Eiche von 2,5\%. $63 \%$ der Tiere stammten aus EFs-Proben. Die Fänge verteilten sich sehr gleichmäßig auf die Fangperioden (Abb. 4-12h).

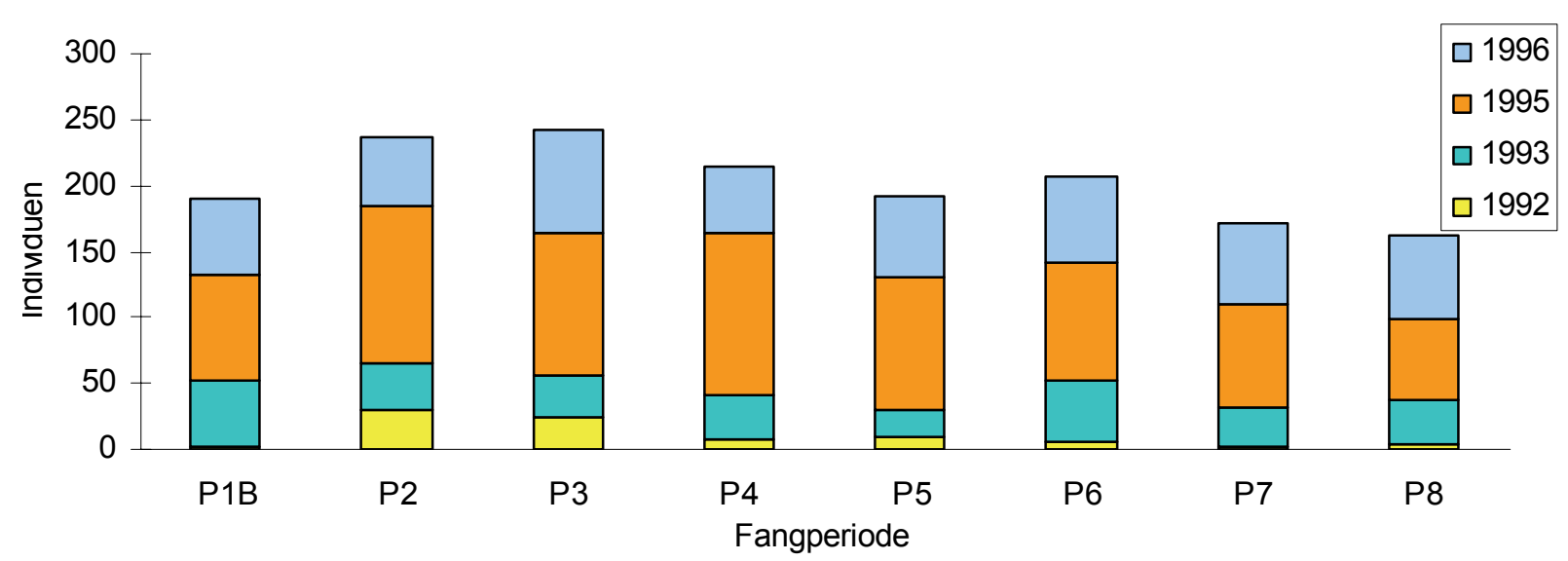

Abb. 4-12h: Schlüpfphänologie Cryptophagus dentatus 
Nach Rhizophagus dispar war Rh. bipustulatus der zweithäufigste in den Eklektoren gefangene Räuber. Der jährliche Individuenanteil lag zwischen 2,3 und 3,5 \%. Anders als bei $R h$. dispar war die relative Häufigkeit von Rh. bipustulatus an den beiden Baumarten mit 3,3\% an Eiche und 2,6\% an Buche sehr ähnlich. $57 \%$ der Tiere wurden mit STE, $38 \%$ mit EFs erfaßt. Die meisten Individuen fingen sich in den ersten Fangperioden, das Maximum wurde im April/Mai registriert. Danach gingen die Fänge kontinuierlich zurück (Abb. 4-12i).

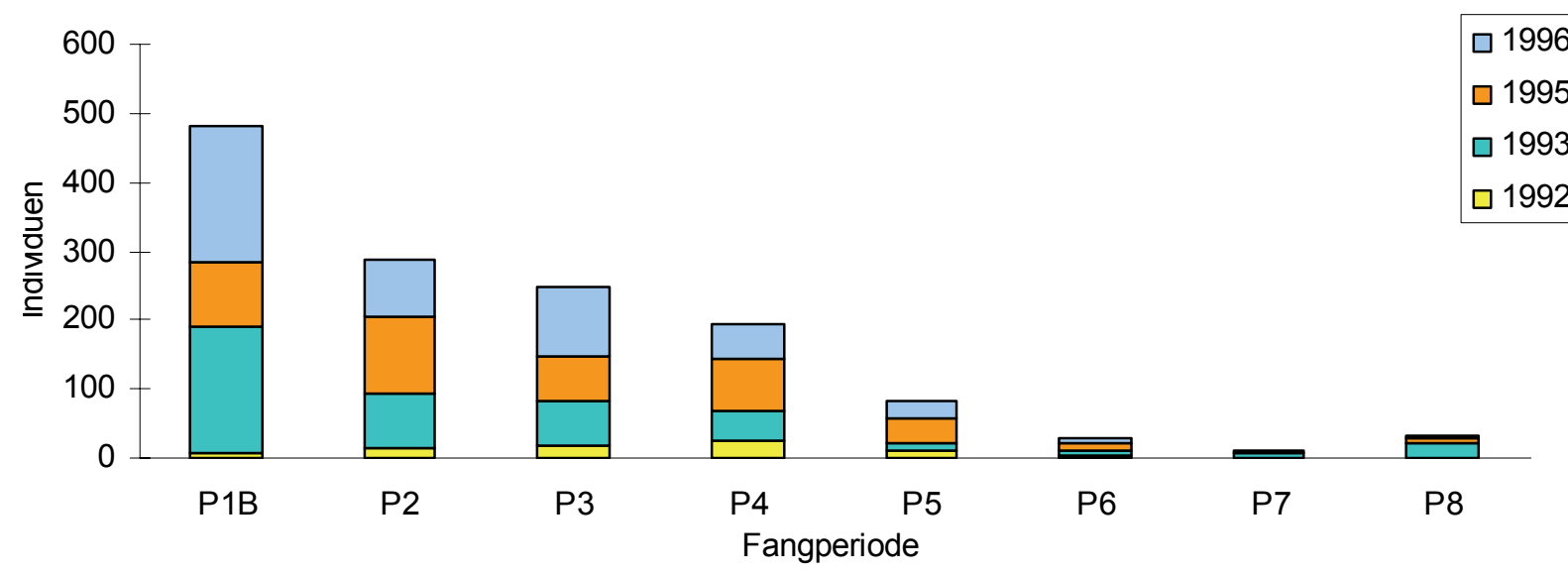

Abb. 4-12i: Schlüpfphänologie Rhizophagus bipustulatus

2,6 \% der gefangenen Individuen wurde von Cerylon ferrugineum gestellt. Dieser Käfer lebt räuberisch unter der Rinde und im morschen Holz. 1,6 \% der an Eiche und 3,1 \% der an Buche gefangenen Individuen gehörten zu dieser Art. Die höchste Anzahl fing sich in der Fangperiode P2. Zwei Drittel der insgesamt 1272 Individuen wurden von Mitte April bis einschließlich Juni registriert (Abb. 4-12k).

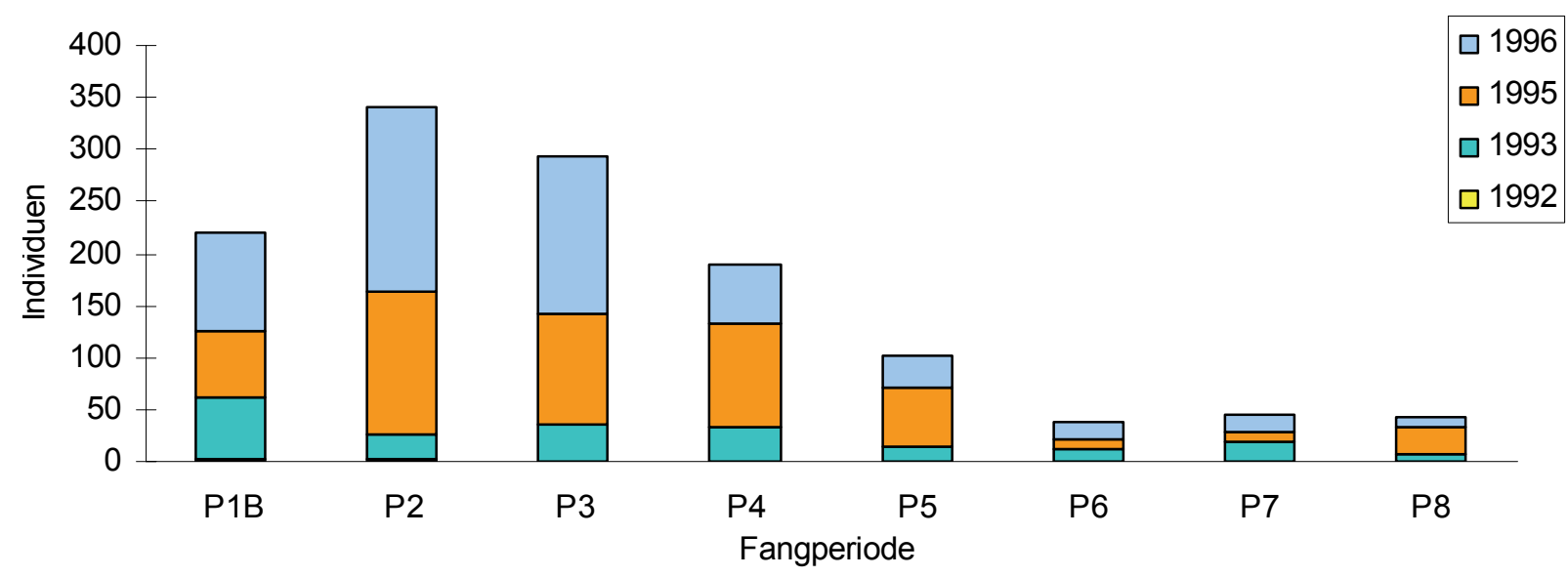

Abb. 4-12k: Schlüpfphänologie Cerylon ferrugineum

Der vor allem unter der Rinde von Laubbaumarten vorkommende Staphylinidae Phloeonomus punctipennis erreichte ebenfalls einen Anteil von 2,6\%. Dieser Wert wurde annähernd sowohl für die beiden Baumarten als auch in den einzelnen Untersuchungsjahren 
gemessen. An liegendem Totholz war diese Art in höherem Maße am Fangergebnis beteiligt als an stehendem. Im Jahresverlauf nahmen die Fangzahlen kontinuierlich ab. Annähernd ein Drittel der Individuen fingen sich in der Fangperiode P1b (Abb. 4-121).

Als letzte der Hauptarten bleibt noch Hylecoetus dermestoides zu erwähnen. Dieser pilzzüchtende Holzbrüter besiedelt frische Stämme verschiedener Baumarten mit einer Präferenz für die Buche. 3,3\% der an Buche gefangenen Tiere wurden von dieser Art gestellt, im Vergleich zu $0,8 \%$ an Eichentotholz. Die meisten Individuen verließen im Mai ihren Entwicklungsort (Abb. 4-12m).

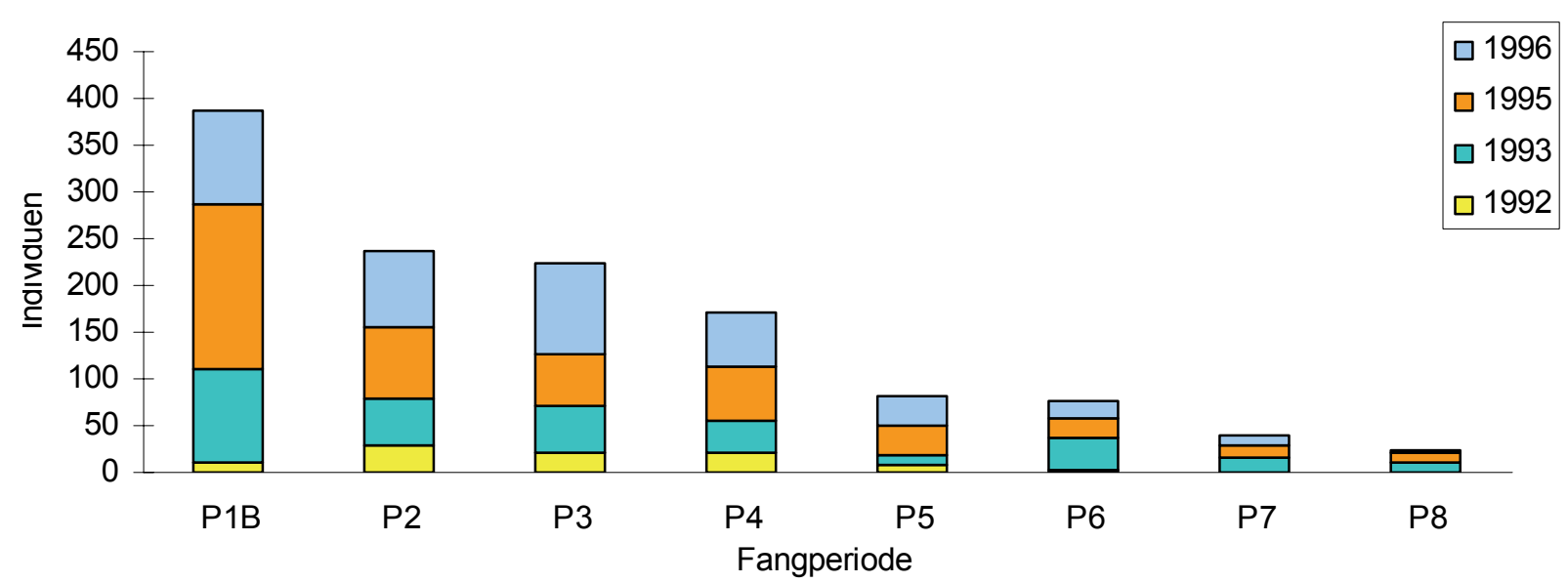

Abb. 4-12I: Schlüpfphänologie Phloeonomus punctipennis

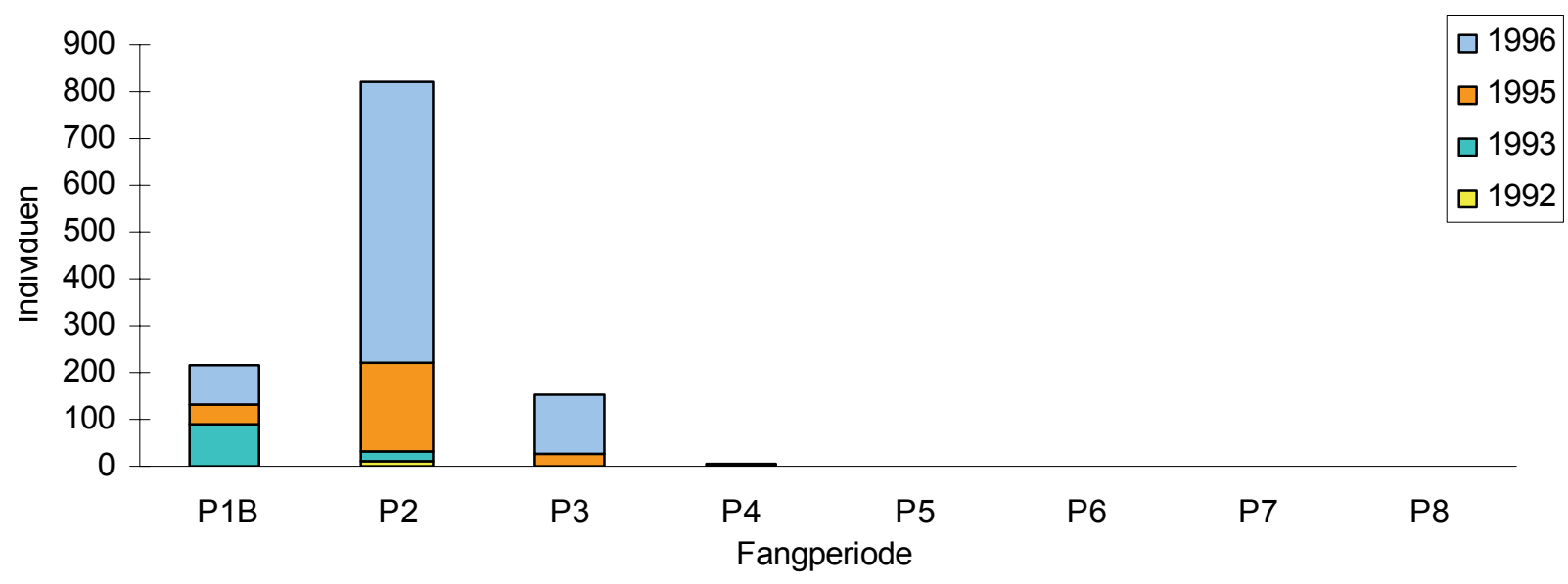

Abb. 4-12m: Schlüpfphänologie Hylecoetus dermestoides

Neben den genannten Arten, die am Gesamtfang mit mindestens $2 \%$ beteiligt waren, traten einige Arten nur an einer Baumart als Hauptarten in Erscheinung. An Eiche war dies Aderus oculatus (3,5\%), an Buche traten Leptusa fumida (2,4\%), Ptinella aptera (2,4 \%) und Corticeus unicolor (2,2\%) in Anzahl auf.

Weitere Informationen zur Frequenz, zum Individuenanteil und zur Schlüpfphänologie auch der übrigen Arten können dem Anhang 4 entnommen werden. 


\subsubsection{Frequenz- und Dominanzstruktur an liegendem Buchentotholz}

Die überwiegende Zahl der Arten wurde nur vereinzelt in den STE-Proben nachgewiesen, annähernd jede dritte Käferart war in nur einer Falle festzustellen. 169 Arten mit einem Individuenanteil von 4,6 \% waren in bis zu 5 Proben vertreten. Dagegen entfielen 43,7 \% der Individuen auf 7 Arten, die in mehr als 40 Proben in Erscheinung traten (Tab. 4-15). Fünf dieser Arten zählten auch zu den Hauptarten.

Tabelle 4-15: Frequenzstruktur (STE Buche)

\begin{tabular}{|c|c|c|c|c|c|c|c|c|}
\hline & \multicolumn{6}{|c|}{ F R E Q U E N Z KL A S S E (\%) } & \multirow[b]{2}{*}{$\Sigma$} \\
\hline & & $\geq 80$ & $60-79,9$ & $40-59,9$ & $20-39,9$ & $10-19,9$ & $<10$ & \\
\hline \multirow[t]{2}{*}{ Arten } & Anz. & 7 & 9 & 13 & 23 & 36 & 169 & 257 \\
\hline & $\%$ & 2,7 & 3,5 & 5,1 & 8,9 & 14,0 & 65,8 & 100,0 \\
\hline \multirow[t]{2}{*}{ Ind. } & Anz. & 5981 & 2497 & 1468 & 2548 & 573 & 628 & 13695 \\
\hline & $\%$ & 43,7 & 18,2 & 10,7 & 18,6 & 4,2 & 4,6 & 100,0 \\
\hline
\end{tabular}

Zwölf Arten waren mit mindestens 2,0 \% am Gesamtfang beteiligt. Gemeinsam hatten diese einen Individuenanteil von 63,6 \% (Tab. 4-16). 1995 erreichten 13 Arten den Status einer Hauptart, 1996 waren es 12 Arten. Die von diesen gestellten Individuenanteile betrugen 68,0 \% bzw. 64,2 \%. Dagegen waren die 245 Begleitarten mit nur 36,4 \% am Gesamtergebnis beteiligt. In den Einzeljahren waren 182 bzw. 187 Begleitarten in den Fallen vertreten. In den einzelnen Fangperioden variierte die Anzahl der Hauptarten zwischen 8 (P2) und 15 (P7), der von ihnen gestellte Individuenanteil zwischen 67,2 \% (P2) und 74,8 \% (P7) (Tab. 4-18).

Tabelle 4-16: Dominanzklassenstruktur (STE Buche)

\begin{tabular}{|c|c|c|c|c|c|c|c|c|c|}
\hline & \multicolumn{6}{|c|}{ DO M I N A NZKLA S S E (\%) } & \multirow[b]{3}{*}{$\Sigma$} \\
\hline & & & \multicolumn{3}{|c|}{ Hauptarten } & \multicolumn{3}{|c|}{ Begleitarten } & \\
\hline & & & $\geq 10$ & $5-9,99$ & $2-4,99$ & $1-1,99$ & $0,3-0,99$ & $<\mathbf{0 , 3 0}$ & \\
\hline \multirow[t]{4}{*}{1995} & \multirow[t]{2}{*}{ Arten } & Anz. & 1 & 1 & 11 & 9 & 21 & 152 & 195 \\
\hline & & $\%$ & 0,5 & 0,5 & 5,6 & 4,6 & 10,8 & 77,9 & 100,0 \\
\hline & \multirow[t]{2}{*}{ Ind. } & Anz. & 1910 & 398 & 2196 & 817 & 751 & 554 & 6626 \\
\hline & & $\%$ & 28,8 & 6,0 & 33,1 & 12,3 & 11,3 & 8,4 & 100,0 \\
\hline \multirow[t]{4}{*}{1996} & \multirow[t]{2}{*}{ Arten } & Anz. & 1 & 1 & 10 & 11 & 21 & 155 & 199 \\
\hline & & $\%$ & 0,5 & 0,5 & 5,0 & 5,5 & 10,6 & 77,9 & 100,0 \\
\hline & \multirow[t]{2}{*}{ Ind. } & Anz. & 1941 & 641 & 1958 & 1173 & 736 & 620 & 7069 \\
\hline & & $\%$ & 27,5 & 9,1 & 27,7 & 16,6 & 10,4 & 8,8 & 100,0 \\
\hline \multirow[t]{4}{*}{ gesamt } & \multirow[t]{2}{*}{ Arten } & Anz. & 1 & 2 & 9 & 8 & 23 & 214 & 257 \\
\hline & & $\%$ & 0,4 & 0,8 & 3,5 & 3,1 & 8,9 & 83,3 & 100,0 \\
\hline & \multirow[t]{2}{*}{ Ind. } & Anz. & 3851 & 1483 & 3375 & 1783 & 1926 & 1277 & 13695 \\
\hline & & $\%$ & 28,1 & 10,8 & 24,6 & 13,0 & 14,1 & 9,3 & 100,0 \\
\hline
\end{tabular}


Die einzige eudominante Art, Rhizophagus dispar, war mit 28,1\% am Fangergebnis beteiligt (Tab. 4-17). Der Individuenanteil dieses Räubers war in beiden Untersuchungsjahren mit 28,8 und 27,5 \% sehr hoch. In allen Fangperioden war Rh. dispar mit Anteilen zwischen 18,6 und 41,2 \% die häufigste Art (Tab. 4-18). In den 50 Proben, in denen er auftrat, wurde eine spezifische Schlüpfdichte ${ }^{7}$ von 52,5 Ind. $/ \mathrm{m}^{2}$ ermittelt. In der Mehrzahl der Proben wurden bis zu 40 Ind. $/ \mathrm{m}^{2}$ festgestellt, die maximale gemessene Dichte betrug 201,9 Ind. $/ \mathrm{m}^{2}$ (Abb. 4-13a).

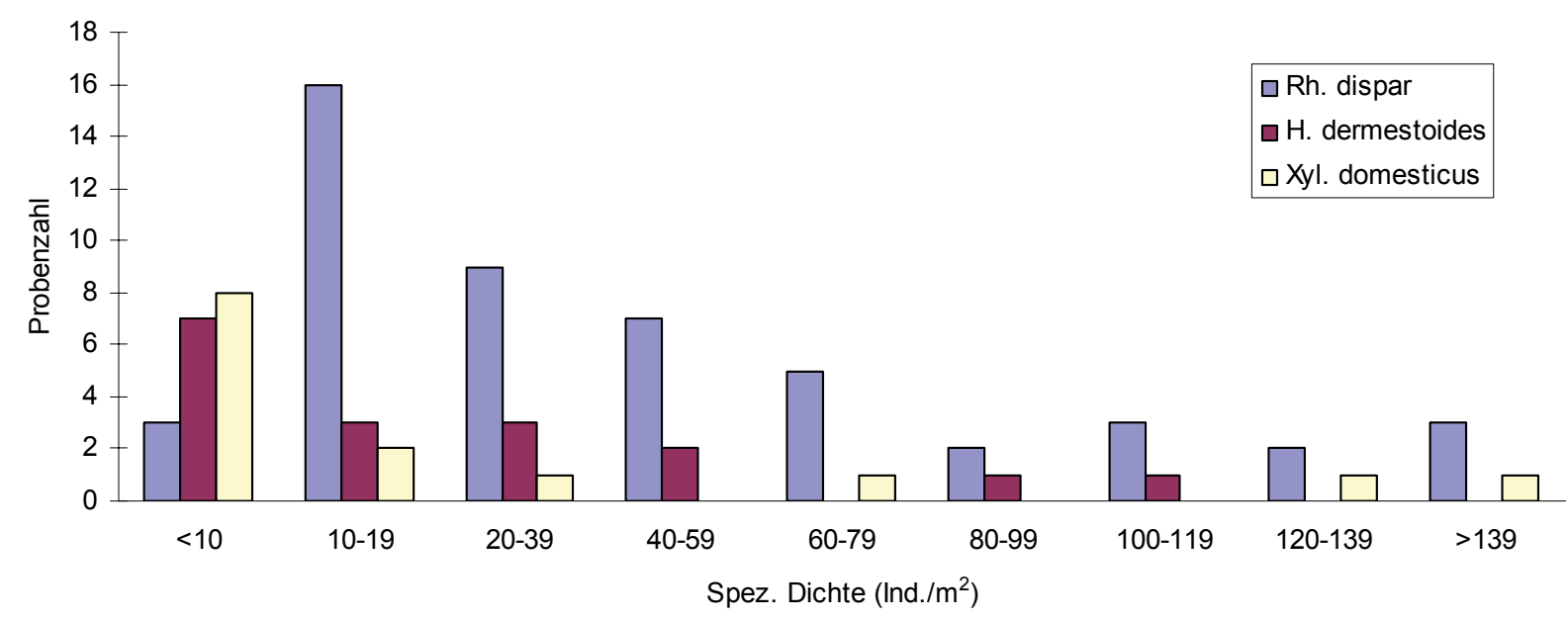

Abb. 4-13a: Spezifische Schlüpfdichten ausgewählter Hauptarten (STE Buche)

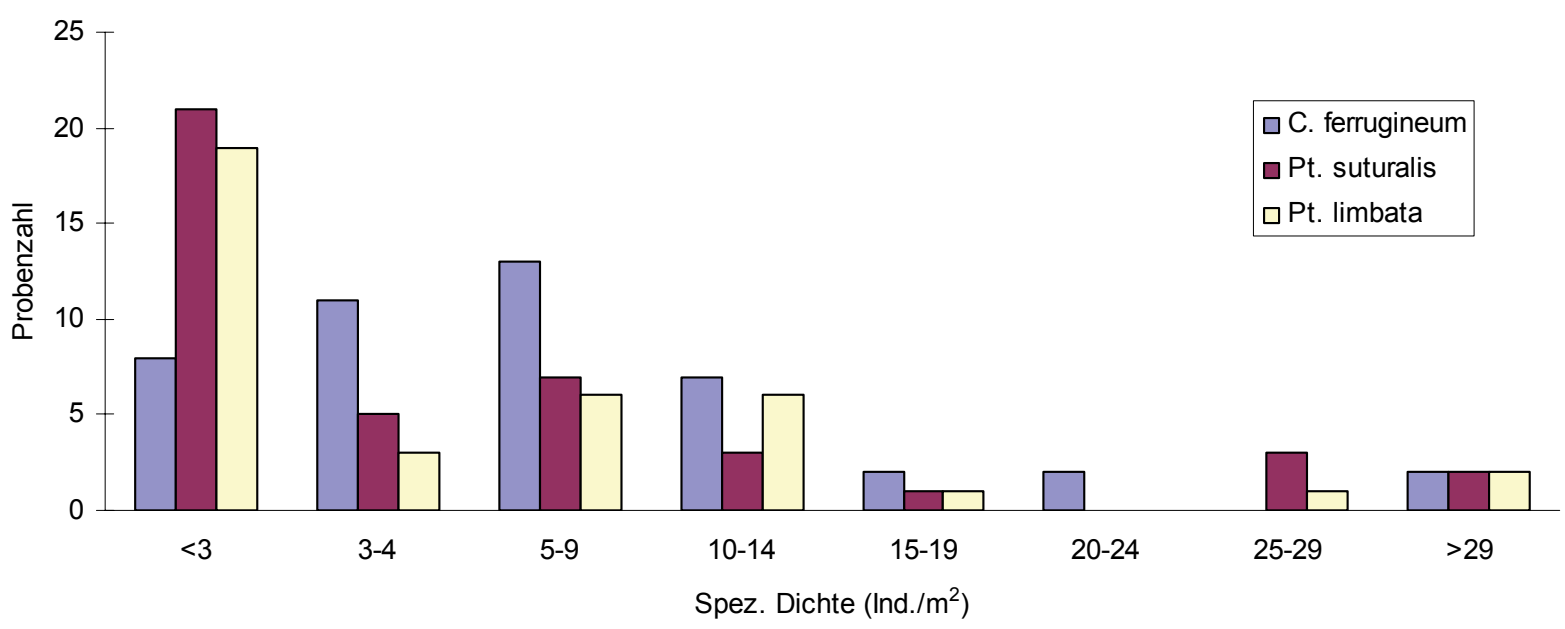

Abb. 4-13b: Spezifische Schlüpfdichten ausgewählter Hauptarten (STE Buche)

\footnotetext{
${ }^{7}$ Berechnungsgrundlage für die 'spezifische Dichte' war die befangene Mantelfläche derjenigen Proben, in denen die Art nachgewiesen werden konnte. Im Vergleich hierzu bezieht sich die 'allgemeine Dichte' jeweils auf die gesamte beprobte Mantelfläche.
} 
Tabelle 4-17: Frequenz, Dominanz und Schlüpfdichte häufiger Arten (STE Buche)

\begin{tabular}{|c|c|c|c|c|c|c|}
\hline \multirow[b]{2}{*}{ Art } & \multicolumn{2}{|c|}{ Frequenz } & \multicolumn{2}{|c|}{ Individuen } & \multicolumn{2}{|c|}{ Dichte (Ind./ $\mathbf{m}^{2}$ ) } \\
\hline & Anz. & $\%$ & Anz. & $\%$ & allg. & \\
\hline Rhizophagus dispar & 50 & 98,0 & 3851 & 28,12 & 51,8 & 52,5 \\
\hline Hylecoetus dermestoides & 17 & 33,3 & 765 & 5,59 & 10,3 & 27,1 \\
\hline Xyloterus domesticus & 14 & 27,5 & 718 & 5,24 & 9,7 & 31,8 \\
\hline Cerylon ferrugineum & 45 & 88,2 & 566 & 4,13 & 7,6 & 8,5 \\
\hline Pteryx suturalis & 42 & 82,4 & 421 & 3,07 & 5,7 & 6,9 \\
\hline Ptinella limbata & 38 & 74,5 & 411 & 3,00 & 5,5 & 7,6 \\
\hline Rhizophagus bipustulatus & 35 & 68,6 & 406 & 2,96 & 5,5 & 7,6 \\
\hline Phloeonomus punctipennis & 47 & 92,2 & 399 & 2,91 & 5,4 & 5,7 \\
\hline Rhizophagus nitidulus & 33 & 64,7 & 305 & 2,23 & 4,1 & 6,1 \\
\hline Corticaria longicollis & 45 & 88,2 & 295 & 2,15 & 4,0 & 4,5 \\
\hline Corticeus unicolor & 29 & 56,9 & 294 & 2,15 & 4,0 & 6,8 \\
\hline Stenichnus collaris & 37 & 72,5 & 278 & 2,03 & 3,7 & 5,2 \\
\hline Sepedophilus testaceus & 40 & 78,4 & 273 & 1,99 & 3,7 & 4,7 \\
\hline Aridius nodifer & 35 & 68,6 & 272 & 1,99 & 3,7 & 5,3 \\
\hline Ptilinus pectinicornis & 39 & 76,5 & 268 & 1,96 & 3,6 & 4,7 \\
\hline Cryptophagus dentatus & 43 & 84,3 & 242 & 1,77 & 3,3 & 3,8 \\
\hline Leptusa fumida & 41 & 80,4 & 207 & 1,51 & 2,8 & 3,4 \\
\hline Ptinella aptera & 23 & 45,1 & 182 & 1,33 & 2,4 & 5,6 \\
\hline Gabrius splendidulus & 34 & 66,7 & 170 & 1,24 & 2,3 & 3,3 \\
\hline Orchesia undulata & 27 & 52,9 & 169 & 1,23 & 2,3 & 4,2 \\
\hline Vincenzellus ruficollis & 13 & 25,5 & 136 & 0,99 & 1,8 & 6,8 \\
\hline Plegaderus dissectus & 24 & 47,1 & 127 & 0,93 & 1,7 & 3,5 \\
\hline Neuraphes elongatulus & 30 & 58,8 & 125 & 0,91 & 1,7 & 2,9 \\
\hline Xyleborus saxeseni & 4 & 7,8 & 124 & 0,91 & 1,7 & 20,2 \\
\hline Octotemnus glabriculus & 16 & 31,4 & 122 & 0,89 & 1,6 & 5,2 \\
\hline Euryusa castanoptera & 20 & 39,2 & 121 & 0,88 & 1,6 & 3,9 \\
\hline Phloeocharis subtilissima & 40 & 78,4 & 114 & 0,83 & 1,5 & 1,9 \\
\hline Pterostichus niger & 24 & 47,1 & 104 & 0,76 & 1,4 & 3,0 \\
\hline Bolitochara obliqua & 26 & 51,0 & 101 & 0,74 & 1,4 & 2,6 \\
\hline Bibloporus bicolor & 22 & 43,1 & 72 & 0,53 & 1,0 & 2,2 \\
\hline Epuraea variegata & 16 & 31,4 & 72 & 0,53 & 1,0 & 3,1 \\
\hline Cerylon fagi & 30 & 58,8 & 70 & 0,51 & 0,9 & 1,6 \\
\hline Cerylon histeroides & 22 & 43,1 & 69 & 0,50 & 0,9 & 2,2 \\
\hline Leptusa pulchella & 25 & 49,0 & 69 & 0,50 & 0,9 & 1,8 \\
\hline Agathidium nigripenne & 10 & 19,6 & 66 & 0,48 & 0,9 & 4,1 \\
\hline Orthoperus mundus & 14 & 27,5 & 65 & 0,47 & 0,9 & 3,0 \\
\hline Paromalus flavicornis & 18 & 35,3 & 59 & 0,43 & 0,8 & 2,2 \\
\hline Anomognathus cuspidatus & 15 & 29,4 & 55 & 0,40 & 0,7 & 2,4 \\
\hline Dienerella elongata & 16 & 31,4 & 54 & 0,39 & 0,7 & 2,3 \\
\hline Pterostichus oblongopunctatus & 27 & 52,9 & 54 & 0,39 & 0,7 & 1,4 \\
\hline Enicmus rugosus & 14 & 27,5 & 51 & 0,37 & 0,7 & 2,4 \\
\hline Cis nitidus & 12 & 23,5 & 50 & 0,37 & 0,7 & 2,9 \\
\hline Taphrorychus bicolor & 10 & 19,6 & 46 & 0,34 & 0,6 & 3,0 \\
\hline
\end{tabular}


Tabelle 4-18: Dominanzphänologie: Die Hauptarten der Fangperioden (STE Buche)

\begin{tabular}{|c|c|c|c|c|c|c|c|c|}
\hline \multirow[b]{2}{*}{ ART } & \multicolumn{8}{|c|}{ Fange eriode } \\
\hline & P1b & $\mathbf{P 2}$ & P3 & P4 & P5 & P6 & P7 & P8 \\
\hline Rhizophagus dispar & 25,6 & 25,5 & 32,2 & 32,9 & 25,9 & 23,2 & 18,6 & 41,2 \\
\hline Xyloterus domesticus & 19,2 & 2,1 & & & & & 2,2 & 2,5 \\
\hline Rhizophagus bipustulatus & 5,2 & 3,9 & 2,9 & 2,4 & & & & \\
\hline Cerylon ferrugineum & 4,1 & 6,1 & 4,5 & 4,7 & 2,4 & & 3,2 & 3,5 \\
\hline Xyleborus saxeseni & 3,8 & & & & & & & \\
\hline Phloeonomus punctipennis & 3,5 & 2,7 & 3,6 & 2,9 & & 2,7 & 4,2 & \\
\hline Corticaria longicollis & 3,5 & & & & & 2,6 & 2,5 & \\
\hline Hylecoetus dermestoides & 3,0 & 21,9 & 5,1 & & & & & \\
\hline Gabrius splendidulus & 2,2 & & & & & & & \\
\hline Cryptophagus dentatus & 2,2 & & & & & 2,5 & & \\
\hline Rhizophagus nitidulus & & 2,5 & 4,1 & & & 3,2 & & 3,7 \\
\hline Corticeus unicolor & & 2,4 & 5,2 & 2,0 & & & & \\
\hline Stenichnus collaris & & & 4,2 & 2,8 & 2,6 & & & \\
\hline Sepedophilus testaceus & & & 4,1 & 2,3 & & & 4,0 & 3,1 \\
\hline Leptusa fumida & & & 3,6 & & & & & \\
\hline Aridius nodifer & & & & 4,9 & 4,3 & & 2,5 & \\
\hline Ptilinus pectinicornis & & & & 7,9 & 4,2 & & & \\
\hline Ptinella limbata & & & & 3,7 & 11,2 & 9,1 & 4,7 & 2,1 \\
\hline Pteryx suturalis & & & & 3,9 & 8,2 & 11,8 & 5,9 & 3,5 \\
\hline Ptinella aptera & & & & & 5,7 & 3,1 & 3,7 & 3,7 \\
\hline Orchesia undulata & & & & & 2,3 & 5,1 & 5,7 & \\
\hline Plegaderus dissectus & & & & & 2,0 & & & \\
\hline Pterostichus niger & & & & & & 3,5 & & \\
\hline Bibloporus bicolor & & & & & & 2,1 & & \\
\hline Orthoperus mundus & & & & & & & 6,2 & \\
\hline Octotemnus glabriculus & & & & & & & 4,7 & 3,9 \\
\hline Enicmus rugosus & & & & & & & 4,0 & 3,1 \\
\hline Cis nitidus & & & & & & & 2,7 & \\
\hline Catops picipes & & & & & & & & 2,1 \\
\hline Anzahl Hauptarten & 10 & 8 & 10 & 11 & 10 & 11 & 15 & 11 \\
\hline Ind.anteil Hauptarten & 72,3 & 67,2 & 69,5 & 70,3 & 68,7 & 68,9 & 74,8 & 72,3 \\
\hline Individuen gesamt & 2803 & 2576 & 2251 & 2315 & 1643 & 1186 & 404 & 517 \\
\hline
\end{tabular}

Die beiden dominanten Arten in den STE-Proben an Buche waren Xyloterus domesticus und Hylecoetus dermestoides. Die erste Art erreichte 1995 einen Individuenanteil von 6,0 \% und war 1996 mit 4,5 \% subdominant. In der Fangperiode P1b war dieser Borkenkäfer mit einem Individuenanteil von 19,2 \% vertreten. Xyl. domesticus trat in 14 Proben auf und schlüpfte in einer spezifischen Dichte von 31,8 Ind. $/ \mathrm{m}^{2}$, als Maximum wurden $161,8 \mathrm{Ind} . / \mathrm{m}^{2}$ registriert (Abb. 4-13a). H. dermestoides erreichte 1995 mit einem Individuenanteil von 1,9 \% nur den Status einer rezedenten Begleitart. Dagegen lag der Anteil 1996 bei über $9 \%$. Die höchste Dominanz wurde in der Fangperiode P2 erreicht. Für die 17 Proben wurde eine Schlüpfdichte von 27,1 Ind./m² (Maximum 112,2 Ind./m²) ermittelt (Abb. 4-13a). 
Von den übrigen Hauptarten waren Cerylon ferrugineum, Pteryx suturalis, Rhizophagus bipustulatus, Phloeonomus punctipennis, Rhizophagus nitidulus und Corticaria longicollis in beiden Untersuchungsjahren subdominant, Ptinella limbata erreichte diesen Status nur 1995, Corticeus unicolor und Stenichnus collaris nur 1996. Die spezifischen Schlüpfdichten dieser Arten variierten zwischen 4,5 und 8,5 Ind. $/ \mathrm{m}^{2}$. In der Mehrzahl der Proben traten die genannten Arten mit jeweils weniger als $5 \mathrm{Ind} . / \mathrm{m}^{2}$ auf, nur Cerylon ferrugineum wurde des öfteren in etwas höheren Dichten beobachtet (Abb. 4-13b-d)

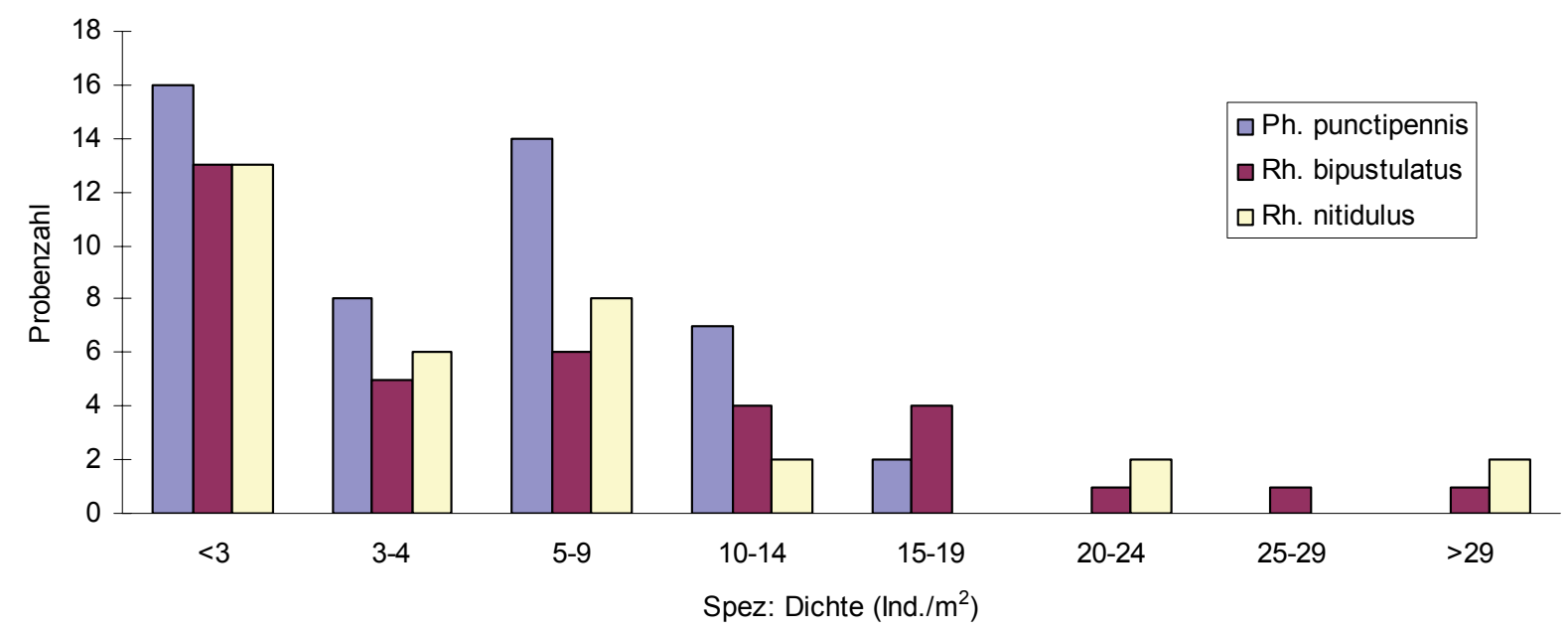

Abb. 4-13c: Spezifische Schlüpfdichten ausgewählter Hauptarten (STE Buche)

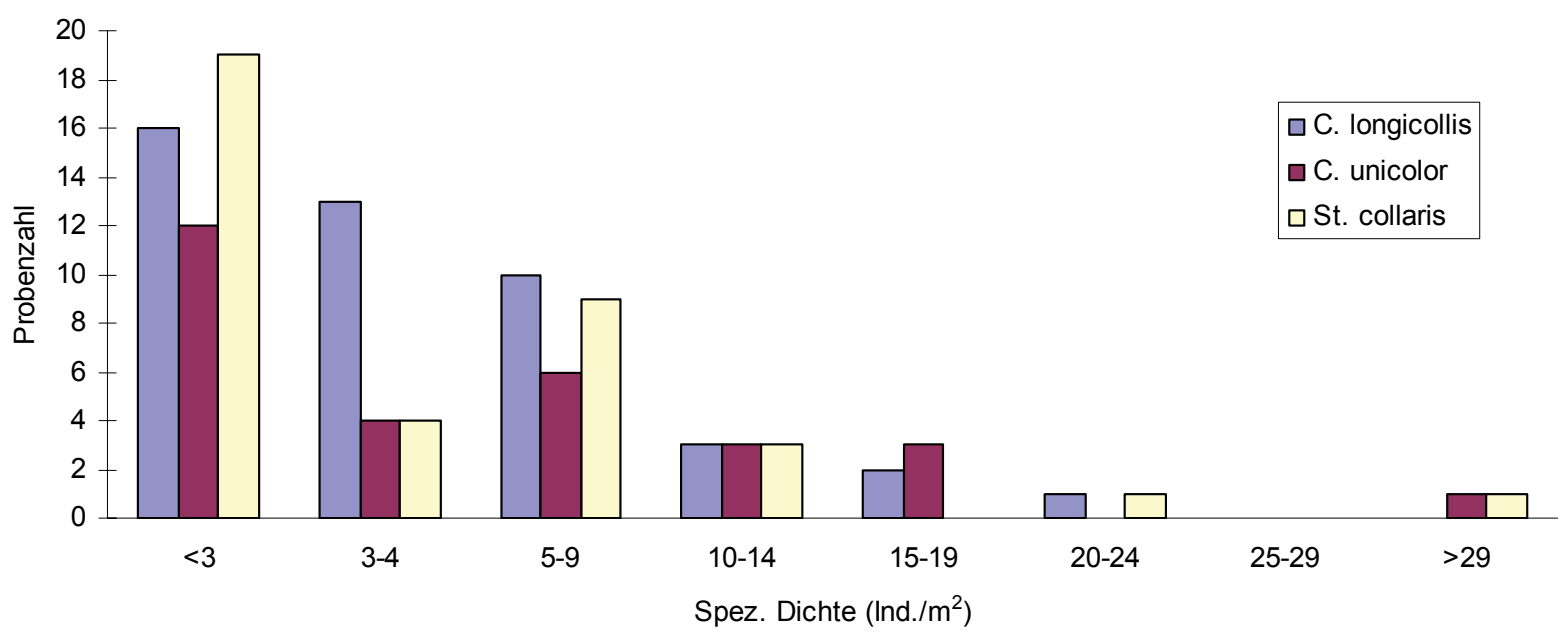

Abb. 4-13d: Spezifische Schlüpfdichten ausgewählter Hauptarten (STE Buche)

Weitere Informationen über das Spektrum xylobionter Arten an liegendem Buchentotholz und ihre Dominanz können dem Anhang 5 entnommen werden. 


\subsubsection{Frequenz- und Dominanzstruktur an liegendem Eichentotholz}

Mehr als ein Drittel der Käferarten aus STE-Proben an Eiche trat in nur einer Probe in Erscheinung. 170 Arten mit einem Individuenanteil von 12,4\% wurden in weniger als $10 \%$ der Proben festgestellt. Dagegen entfielen 24,1 \% der Individuen auf nur 3 Arten, die in mehr als 40 Proben in Erscheinung traten (Tab. 4-19).

Tabelle 4-19: Frequenzstruktur (STE Eiche)

\begin{tabular}{|c|c|c|c|c|c|c|c|c|}
\hline & \multicolumn{6}{|c|}{ F R E Q U E N Z K L A S S E (\%) } & \multirow[b]{2}{*}{$\sum$} \\
\hline & & $\geq \mathbf{8 0}$ & 60-79,9 & 40-59,9 & $20-39,9$ & $10-19,9$ & $<10$ & \\
\hline \multirow[t]{2}{*}{ Arten } & Anz. & 3 & 5 & 11 & 19 & 40 & 170 & 248 \\
\hline & $\%$ & 1,2 & 2,0 & 4,4 & 7,7 & 16,1 & 68,5 & 100,0 \\
\hline \multirow[t]{2}{*}{ Ind. } & Anz. & 2065 & 1291 & 914 & 2564 & 682 & 1060 & 8576 \\
\hline & $\%$ & 24,1 & 15,1 & 10,7 & 29,9 & 8,0 & 12,4 & 100,0 \\
\hline
\end{tabular}

Von den 19 Arten, die in mindestens $40 \%$ der STE-Proben an Eiche gefangen wurden, waren 17 Arten auch in den STE-Proben an Buche mit hoher Regelmäßigkeit zu beobachten. Nur für wenige Arten zeichneten sich deutlichere Unterschiede ab. So erreichten Agathidium seminulum und Mniusa incrassata eine deutlich höhere Frequenz an Eiche, während Plegaderus dissectus, Ptinella aptera, Orchesia undulata, Ptilinus pectinicornis, Corticeus unicolor und Rhizophagus nitidulus in größerer Regelmäßigkeit in den STE-Proben an Buche vertreten waren.

11 Arten waren mit mindestens 2,0\% am Gesamtfang beteiligt, gemeinsam stellten sie einen Individuenanteil von 68,2 \% (Tab. 4-20). Die Dominanzklassenstruktur war damit den in den STE-Proben an Buche beobachteten Verhältnissen sehr ähnlich. 1992 erreichten 9 Arten den Status einer Hauptart, 1993 waren es 11 und 199512 Arten. Die von diesen gestellten Individuenanteile betrugen 74, 67 bzw. 77 \%. Dagegen waren die 237 Begleitarten mit nur 31,8 \% am Gesamtergebnis beteiligt. In den einzelnen Fangperioden wurden zwischen 9 und 12 Hauptarten registriert, die einen Individuenanteil von 60,6 \% (P1b) bis 82,1 \% (P6) stellten (Tab. 4-22). Von den 11 Hauptarten in den STE-Proben an Eichentotholz zählten 7 Arten auch zu den Hauptarten an Buche.

Tabelle 4-20: Dominanzklassen (STE Eiche)

\begin{tabular}{|c|c|c|c|c|c|c|c|c|}
\hline & \multicolumn{6}{|c|}{ DOM I N A NZKLASSE (\%) } & \multirow[b]{3}{*}{$\Sigma$} \\
\hline & & \multicolumn{3}{|c|}{ Hauptarten } & \multicolumn{3}{|c|}{ Begleitarten } & \\
\hline & & $\geq 10$ & $5-9,99$ & $2-4,99$ & $1-1,99$ & 0,3-0,99 & $<\mathbf{0 , 3 0}$ & \\
\hline \multirow[t]{2}{*}{ Arten } & Anz. & 2 & 2 & 7 & 5 & 21 & 211 & 248 \\
\hline & $\%$ & 0,8 & 0,8 & 2,8 & 2,0 & 8,5 & 85,1 & 100,0 \\
\hline \multirow[t]{2}{*}{ Ind. } & Anz. & 2704 & 1140 & 2008 & 628 & 1085 & 1011 & 8576 \\
\hline & $\%$ & 31,5 & 13,3 & 23,4 & 7,3 & 12,7 & 11,8 & 100,0 \\
\hline
\end{tabular}


Tabelle 4-21: Frequenz, Dominanz und Schlüpfdichte häufiger Arten (STE Eiche)

\begin{tabular}{|c|c|c|c|c|c|c|}
\hline \multirow[b]{2}{*}{ Art } & \multicolumn{2}{|c|}{ Frequenz } & \multicolumn{2}{|c|}{ Individuen } & \multicolumn{2}{|c|}{ Dichte (Ind./m²) } \\
\hline & Anz. & $\%$ & Anz. & $\%$ & allg. & spez. \\
\hline Xyloterus signatus & 13 & 27,1 & 1533 & 17,88 & 27,5 & 93,8 \\
\hline Rhizophagus dispar & 42 & 87,5 & 1171 & 13,65 & 21,0 & 24,1 \\
\hline Corticaria longicollis & 41 & 85,4 & 582 & 6,79 & 10,4 & 12,4 \\
\hline Xyloterus domesticus & 4 & 8,3 & 558 & 6,51 & 10,0 & 117,1 \\
\hline Rhizophagus bipustulatus & 33 & 68,8 & 380 & 4,43 & 6,8 & 9,7 \\
\hline Phloeonomus punctipennis & 41 & 85,4 & 312 & 3,64 & 5,6 & 6,7 \\
\hline Ptinella limbata & 19 & 39,6 & 305 & 3,56 & 5,5 & 14,3 \\
\hline Cryptophagus dentatus & 36 & 75,0 & 266 & 3,10 & 4,8 & 6,3 \\
\hline Neuraphes elongatulus & 33 & 68,8 & 258 & 3,01 & 4,6 & 6,5 \\
\hline Aridius nodifer & 29 & 60,4 & 247 & 2,88 & 4,4 & 7,1 \\
\hline Pteryx suturalis & 26 & 54,2 & 240 & 2,80 & 4,3 & 7,9 \\
\hline Cerylon ferrugineum & 23 & 47,9 & 143 & 1,67 & 2,6 & 5,6 \\
\hline Stenichnus collaris & 32 & 66,7 & 140 & 1,63 & 2,5 & 3,8 \\
\hline Hylecoetus dermestoides & 7 & 14,6 & 120 & 1,40 & 2,2 & 17,2 \\
\hline Rhizophagus nitidulus & 12 & 25,0 & 116 & 1,35 & 2,1 & 9,5 \\
\hline Dienerella elongata & 15 & 31,3 & 109 & 1,27 & 2,0 & 5,6 \\
\hline Teredus cylindricus & 17 & 35,4 & 84 & 0,98 & 1,5 & 3,9 \\
\hline Pterostichus oblongopunctatus & 27 & 56,3 & 83 & 0,97 & 1,5 & 2,7 \\
\hline Leptusa fumida & 27 & 56,3 & 74 & 0,86 & 1,3 & 2,4 \\
\hline Leptusa pulchella & 26 & 54,2 & 70 & 0,82 & 1,3 & 2,4 \\
\hline Phloeocharis subtilissima & 25 & 52,1 & 68 & 0,79 & 1,2 & 2,3 \\
\hline Rhinosimus ruficollis & 5 & 10,4 & 67 & 0,78 & 1,2 & 11,4 \\
\hline Pterostichus niger & 26 & 54,2 & 62 & 0,72 & 1,1 & 2,0 \\
\hline Cerylon fagi & 21 & 43,8 & 56 & 0,65 & 1,0 & 2,2 \\
\hline Bolitochara obliqua & 7 & 14,6 & 55 & 0,64 & 1,0 & 8,1 \\
\hline Phyllodrepa ioptera & 15 & 31,3 & 51 & 0,59 & 0,9 & 2,6 \\
\hline Bibloporus bicolor & 26 & 54,2 & 49 & 0,57 & 0,9 & 1,6 \\
\hline Sepedophilus testaceus & 17 & 35,4 & 47 & 0,55 & 0,8 & 2,6 \\
\hline Xestobium rufovillosum & 2 & 4,2 & 46 & 0,54 & 0,8 & 17,3 \\
\hline Orthoperus mundus & 12 & 25,0 & 45 & 0,52 & 0,8 & 3,4 \\
\hline Mniusa incrassata & 20 & 41,7 & 37 & 0,43 & 0,7 & 1,6 \\
\hline Rhynchaenus fagi & 15 & 31,3 & 35 & 0,41 & 0,6 & 1,9 \\
\hline Euplectus piceus & 11 & 22,9 & 34 & 0,40 & 0,6 & 2,6 \\
\hline Agathidium seminulum & 21 & 43,8 & 32 & 0,37 & 0,6 & 1,3 \\
\hline Denticollis linearis & 19 & 39,6 & 32 & 0,37 & 0,6 & 1,4 \\
\hline Cerylon histeroides & 12 & 25,0 & 31 & 0,36 & 0,6 & 2,1 \\
\hline Atomaria bella & 17 & 35,4 & 27 & 0,31 & 0,5 & 1,2 \\
\hline
\end{tabular}

Die eudominante Art Xyloterus signatus war in 13 Proben vertreten und stellte 17,9\% der gefangenen Käferindividuen (Tab. 4-21). In fast allen Fangperioden war dieser Borkenkäfer zu den Hauptarten zu zählen. Sein höchster Individuenanteil war mit 46,5 \% in der Fangperiode P4 zu verzeichnen (Tab. 4-22). Die spezifische Schlüpfdichte belief sich auf 
93,8 Ind. $/ \mathrm{m}^{2}$ und war vor allem bedingt durch hohe Fangzahlen in nur zwei Proben, in denen bis zu 975 Ind. $/ \mathrm{m}^{2}$ erfaßt wurden. In der Mehrzahl der Proben war eine nur geringe Schlüpfdichte zu verzeichnen (Abb. 4-14a).

Tabelle 4-22: Dominanzphänologie: Die Hauptarten der Fangperioden (STE Eiche)

\begin{tabular}{|c|c|c|c|c|c|c|c|c|}
\hline \multirow[b]{2}{*}{ ART } & \multicolumn{8}{|c|}{ Fange eriode } \\
\hline & P1b & $\mathbf{P 2}$ & $\mathbf{P 3}$ & P4 & P5 & P6 & P7 & P8 \\
\hline Rhizophagus dispar & 12,0 & 8,7 & 13,9 & 9,3 & 13,6 & 16,7 & 21,4 & 30,3 \\
\hline Corticaria longicollis & 7,1 & 7,1 & 7,6 & 4,8 & 9,0 & 8,5 & 8,4 & 4,0 \\
\hline Xyloterus signatus & 6,5 & 5,6 & 13,1 & 46,5 & 13,9 & 21,2 & 2,4 & \\
\hline Rhizophagus bipustulatus & 9,8 & 5,8 & 5,4 & 3,0 & & & & 3,2 \\
\hline Hylecoetus dermestoides & 6,5 & 2,4 & & & & & & \\
\hline Phloeonomus punctipennis & 4,8 & 4,7 & 4,8 & 2,7 & 2,1 & 4,3 & 2,9 & \\
\hline Neuraphes elongatulus & 3,6 & 2,6 & 3,0 & 3,0 & & 2,8 & & 5,5 \\
\hline Bolitochara obliqua & 3,1 & & & & & & & \\
\hline Phloeocharis subtilissima & 2,1 & & & & & & & \\
\hline Rhynchaenus fagi & 2,0 & & & & & & & \\
\hline Rhizophagus nitidulus & 3,0 & 3,7 & & & & & & \\
\hline Xyloterus domesticus & & 14,8 & 14,4 & 2,6 & 3,1 & 3,8 & 6,6 & 7,5 \\
\hline Denticollis linearis & & 2,2 & & & & & & \\
\hline Pterostichus oblongopunctatus & & 2,1 & & & & & & \\
\hline Cryptophagus dentatus & & 3,9 & 4,4 & & 2,1 & 4,2 & 5,5 & 4,9 \\
\hline Stenichnus collaris & & & 3,7 & & & & & \\
\hline Ptinella limbata & & & & 5,3 & 16,5 & 3,5 & & \\
\hline Pteryx suturalis & & & & 4,0 & 7,9 & 6,2 & & \\
\hline Orthoperus mundus & & & & & & 2,0 & & \\
\hline Pterostichus niger & & & & & & 2,0 & & \\
\hline Aridius nodifer & & & & & 5,4 & 6,8 & 13,2 & 4,6 \\
\hline Cerylon ferrugineum & & & & & 3,2 & & 4,4 & \\
\hline Dienerella elongata & & & & & & & 3,5 & 2,6 \\
\hline Rhinosimus ruficollis & & & & & & & 3,3 & 5,8 \\
\hline Teredus cylindricus & & & & & & & & 2,8 \\
\hline Catops picipes & & & & & & & & 3,1 \\
\hline Sepedophilus testaceus & & & & & & & & 2,2 \\
\hline Anzahl Hauptarten & 11 & 12 & 9 & 9 & 10 & 12 & 10 & 12 \\
\hline Ind.anteil Hauptarten & 60,6 & 63,6 & 70,4 & 81,2 & 76,9 & 82,1 & 71,6 & 76,6 \\
\hline Individuen gesamt & 1321 & 1348 & 1126 & 1975 & 884 & 789 & 454 & 650 \\
\hline
\end{tabular}

Rhizophagus dispar zählte, ebenso wie an der Buche, mit einem Anteil von 13,7 \% zu den eudominanten Arten. Auch in den einzelnen Fangperioden gehörte er zu den häufigsten Arten. Der Anteil dieses Räubers variierte zwischen 6,3 \% im ersten Untersuchungsjahr und 18,5 \% im Jahr 1993. In den 42 Proben, in denen er auftrat, wurde eine Schlüpfdichte von 24,1 Ind. $/ \mathrm{m}^{2}$ ermittelt, die maximale gemessene Dichte betrug $175 \mathrm{Ind} . / \mathrm{m}^{2}$ (Abb. $4-14 \mathrm{a}$ ). 
Die beiden dominanten Arten waren Corticaria longicollis und Xyloterus domesticus. Die erste Art erreichte 1992 und 1993 einen Individuenanteil von 6,2 bzw. 7,7 \% und war 1995 mit 3,7\% subdominant. C. longicollis war in allen Fangperioden zu den Hauptarten zu rechnen. Die Schlüpfdichte belief sich auf 12,4 Ind. $/ \mathrm{m}^{2}$, das Maximum betrug $57,3 \mathrm{Ind} . / \mathrm{m}^{2}$. In annähernd der Hälfte der Proben mit Nachweisen von C. longicollis wurden zwischen 5 und $14 \mathrm{Ind} . / \mathrm{m}^{2}$ registriert (Abb. 4-14b). Xyl. domesticus trat in nur 4 Proben auf, erreichte aber dennoch einen Individuenanteil von $6,5 \%$. Für die vier Proben wurde eine Dichte von 117 Ind. $/ \mathrm{m}^{2}$ ermittelt (Abb. 4-14a). Die höchsten Individuenanteile wurden in den Fangperioden $\mathrm{P} 2$ und $\mathrm{P} 3$ gemessen.

$\mathrm{Zu}$ den Hauptarten waren ferner Rhizophagus bipustulatus $(4,4 \%)$, Phloeonomus punctipennis (3,6 \%), die beiden Ptiliden Ptinella limbata (3,6 \%) und Pteryx suturalis $(2,8 \%)$ sowie Cryptophagus dentatus (3,1\%), Neuraphes elongatulus (3,0\%) und Aridius nodifer (2,9 \%) zu rechnen. Sie schlüpften in Dichten zwischen 6,3 und 14,3 Ind./m² (Abb. 4-14b-c).

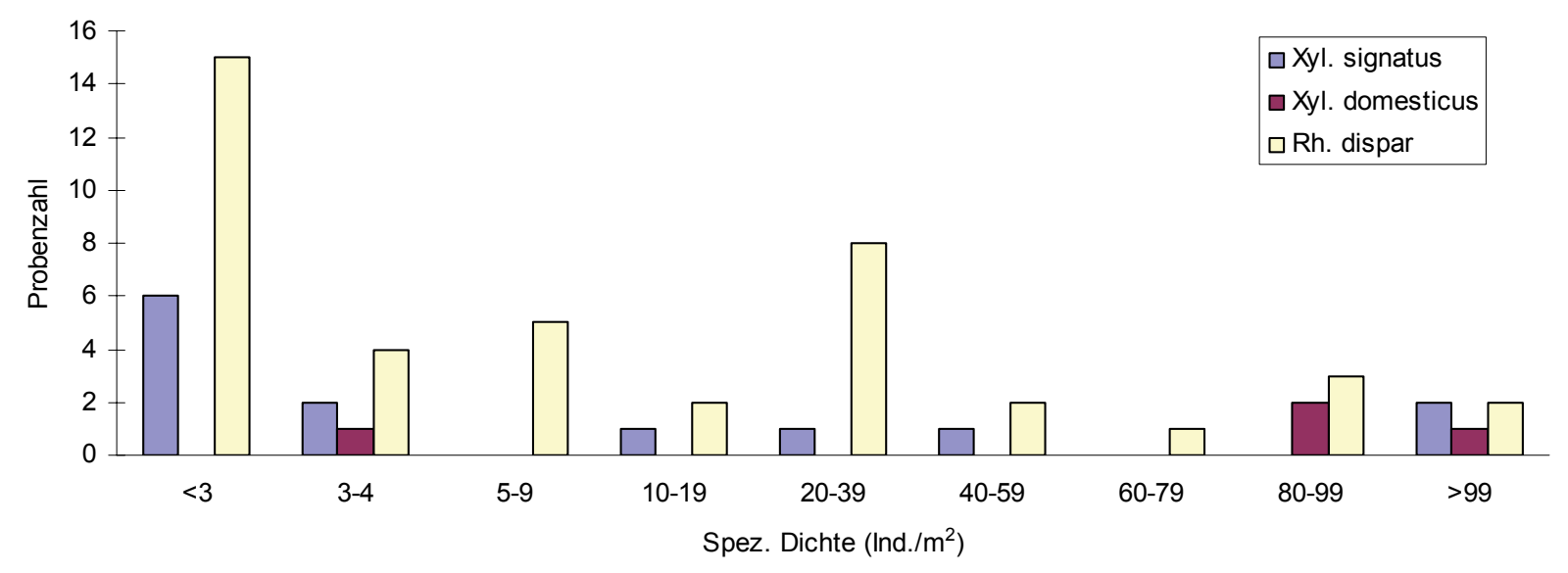

Abb. 4-14a: Spezifische Schlüpfdichten ausgewählter Hauptarten (STE Eiche)

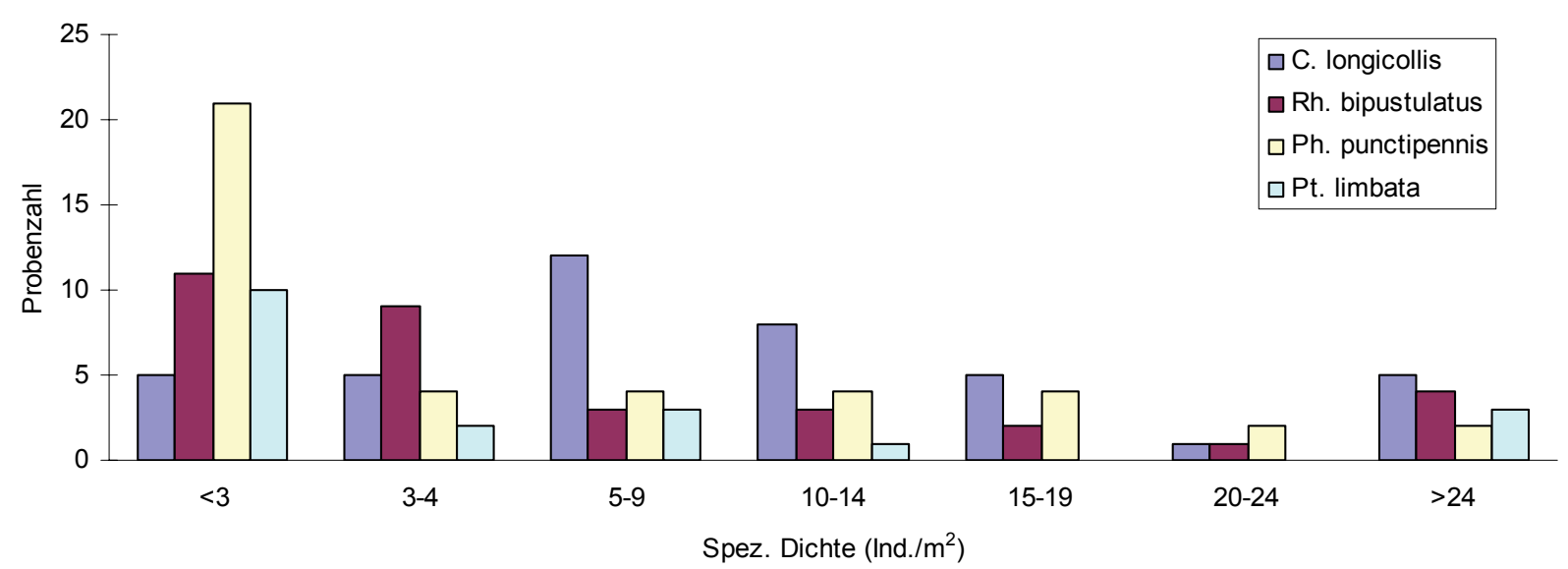

Abb. 4-14b: Spezifische Schlüpfdichten ausgewählter Hauptarten (STE Eiche) 


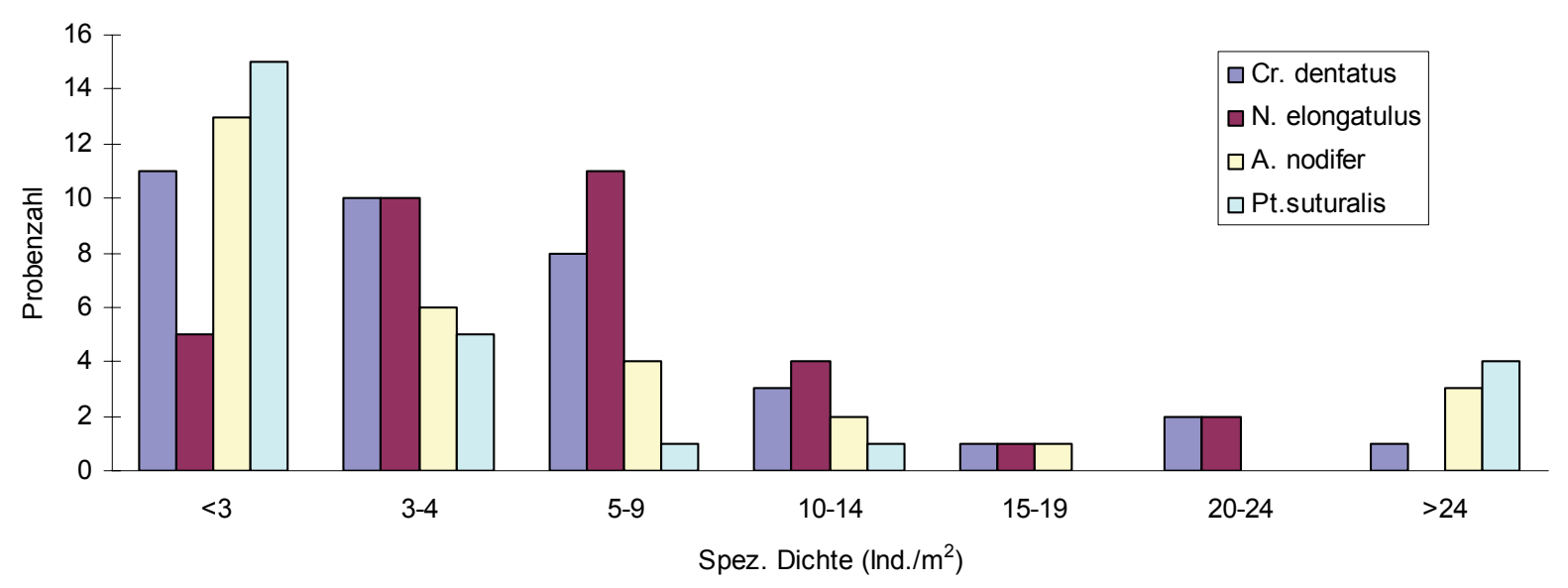

Abb. 4-14c: Spezifische Schlüpfdichten ausgewählter Hauptarten (STE Eiche)

Weitere Informationen zur Struktur der Artengemeinschaft an liegendem Eichentotholz können dem Anhang 5 entnommen werden.

\subsubsection{Frequenz- und Dominanzstruktur an stehendem Buchentotholz}

Nur wenige der 173 aufgefundenen Käferarten waren regelmäßiger Bestandteil der an stehendem Buchentotholz festgestellten Artenvergesellschaftungen (Tab. 4-23). 65 Arten wurden in nur einer Falle beobachtet, 126 Arten in bis zu 9 Proben. Diese trugen mit nur 4,2\% zum Fangergebnis bei. Hingegen hatten 4 Arten, die in über $60 \%$ der Proben auftraten, einen Individuenanteil von $39,6 \%$.

Tabelle 4-23: Frequenzstruktur (EFs Buche)

\begin{tabular}{|c|c|c|c|c|c|c|c|c|}
\hline & \multicolumn{6}{|c|}{ F R E Q UE N Z K L A S S E (\%) } & \multirow[b]{2}{*}{$\sum$} \\
\hline & & $\geq \mathbf{8 0}$ & $60-79,9$ & $40-59,9$ & $20-39,9$ & 10-19,9 & $<\mathbf{1 0}$ & \\
\hline \multirow[t]{2}{*}{ Arten } & Anz. & 1 & 3 & 9 & 12 & 22 & 126 & 173 \\
\hline & $\%$ & 0,6 & 1,7 & 5,2 & 6,9 & 12,7 & 72,8 & 100,0 \\
\hline \multirow[t]{2}{*}{ Ind. } & Anz. & 2649 & 2688 & 4911 & 1279 & 1372 & 570 & 13469 \\
\hline & $\%$ & 19,7 & 20,0 & 36,5 & 9,5 & 10,2 & 4,2 & 100,0 \\
\hline
\end{tabular}

Sechs von sieben Arten, die in mindestens $50 \%$ der EFs-Proben auftraten, waren auch in den STE-Proben an liegendem Buchentotholz mit einem Frequenzwert von über $50 \%$ vertreten. Die einzige Ausnahme war Paromalus flavicornis, der in $59 \%$ der EFs-Proben, aber in nur $35 \%$ der STE-Proben zu beobachten war. Der mycetophage Mulmbewohner Corticaria longicollis war in $86 \%$ der Proben anzutreffen. Ebenfalls mit hoher Regelmäßigkeit festzustellen waren Cryptophagus dentatus (77\%) und die beiden Rhizophagus-Arten dispar (71\%) und bipustulatus (62\%) (Tab. 4-25). 
Elf Hauptarten erreichten gemeinsam einen Anteil von 78,2 \% (Tab. 4-24). 1995 waren 11 und 199610 Arten dieser Gruppe zuzurechnen, die 82,4 bzw. 76,0 \% der Tiere stellten. Dagegen erreichten die 162 Begleitarten lediglich einen Anteil von 21,8 \%. Die Mehrzahl gehörte zur Klasse der sporadischen Arten, die $83 \%$ der Arten aber nur 7,5 \% der Individuen stellten. In den einzelnen Fangperioden wurden zwischen 6 und 11 Hauptarten mit einem gemeinsamen Individuenanteil von 74,1 (P1b) bis 83,8 \% (P7) registriert (Tab. 4-26). Fünf der 11 Hauptarten hatten auch in den STE-Proben an liegendem Buchentotholz einen Anteil von mindestens $2 \%$.

Tabelle 4-24: Dominanzklassen (EFs Buche)

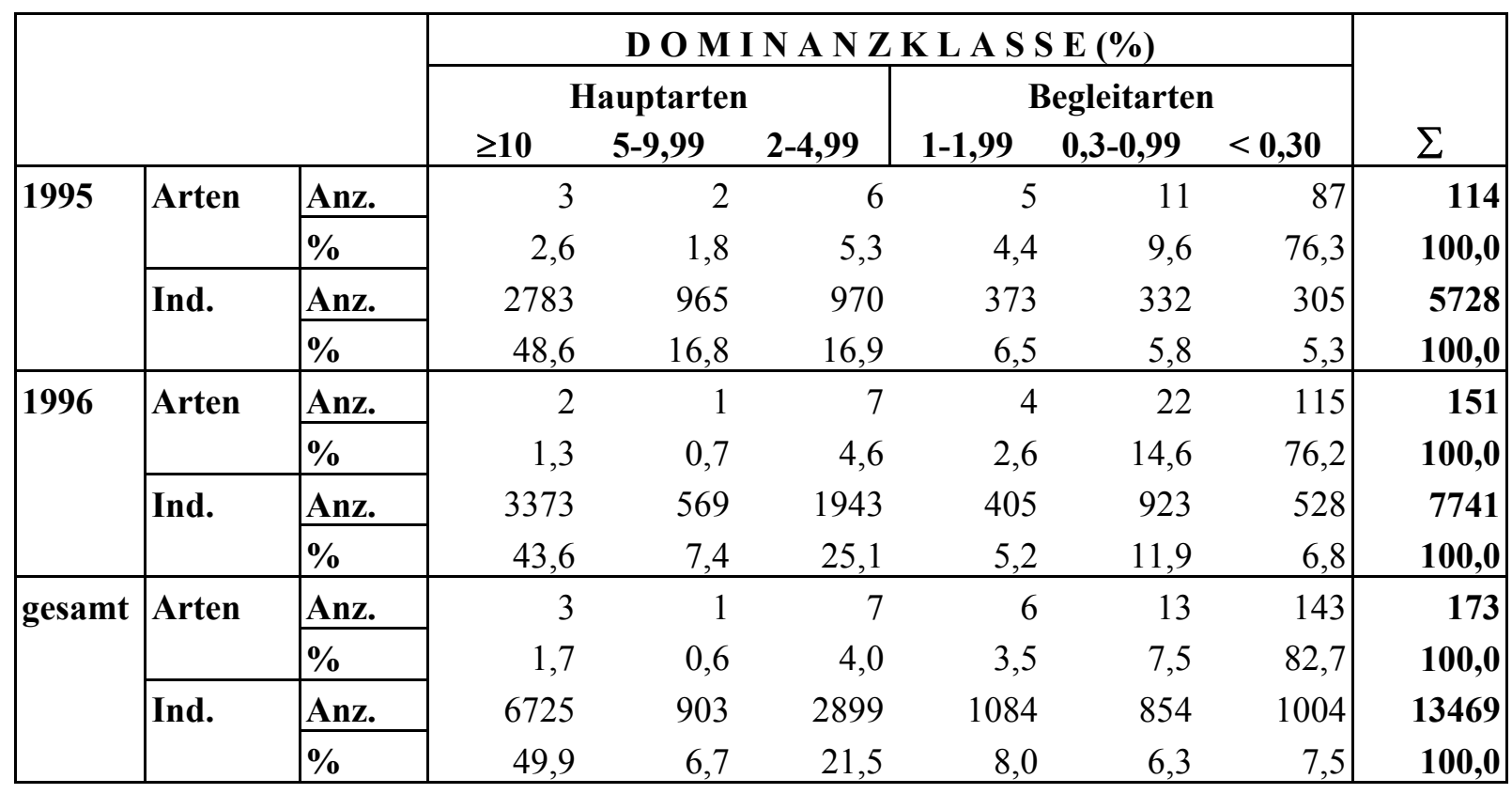


Tabelle 4-25: Frequenz, Dominanz und Schlüpfdichte häufiger Arten (EFs Buche)

\begin{tabular}{|c|c|c|c|c|c|c|}
\hline \multirow[b]{2}{*}{ Art } & \multicolumn{2}{|c|}{ Frequenz } & \multicolumn{2}{|c|}{ Individuen } & \multicolumn{2}{|c|}{ Dichte (Ind./m²) } \\
\hline & Anz. & $\%$ & Anz. & $\%$ & allg. & spez. \\
\hline Ptilinus pectinicornis & 49 & 53,8 & 2681 & 19,90 & 168,5 & 316,7 \\
\hline Corticaria longicollis & 78 & 85,7 & 2649 & 19,67 & 166,5 & 197,2 \\
\hline Rhizophagus dispar & 65 & 71,4 & 1395 & 10,36 & 87,7 & 120,4 \\
\hline Cryptophagus dentatus & 70 & 76,9 & 903 & 6,70 & 56,8 & 73,2 \\
\hline Ptinella aptera & 24 & 26,4 & 535 & 3,97 & 33,6 & 120,6 \\
\hline Paromalus flavicornis & 54 & 59,3 & 466 & 3,46 & 29,3 & 48,7 \\
\hline Cis nitidus & 17 & 18,7 & 445 & 3,30 & 28,0 & 143,9 \\
\hline Cerylon ferrugineum & 51 & 56,0 & 410 & 3,04 & 25,8 & 45,5 \\
\hline Rhizophagus bipustulatus & 56 & 61,5 & 390 & 2,90 & 24,5 & 38,4 \\
\hline Corticeus unicolor & 39 & 42,9 & 375 & 2,78 & 23,6 & 54,1 \\
\hline Micridium halidaii & 37 & 40,7 & 278 & 2,06 & 17,5 & 44,2 \\
\hline Dienerella elongata & 44 & 48,4 & 247 & 1,83 & 15,5 & 31,9 \\
\hline Hylecoetus dermestoides & 15 & 16,5 & 196 & 1,46 & 12,3 & 73,6 \\
\hline Cis castaneus & 38 & 41,8 & 186 & 1,38 & 11,7 & 27,3 \\
\hline Leptusa fumida & 41 & 45,1 & 174 & 1,29 & 10,9 & 23,9 \\
\hline Cicones variegatus & 13 & 14,3 & 146 & 1,08 & 9,2 & 64,9 \\
\hline Taphrorychus bicolor & 19 & 20,9 & 135 & 1,00 & 8,5 & 38,7 \\
\hline Rhizophagus nitidulus & 23 & 25,3 & 119 & 0,88 & 7,5 & 28,4 \\
\hline Plegaderus dissectus & 42 & 46,2 & 94 & 0,70 & 5,9 & 12,5 \\
\hline Stenichnus collaris & 33 & 36,3 & 79 & 0,59 & 5,0 & 14,3 \\
\hline Anaspis schilskyana & 19 & 20,9 & 75 & 0,56 & 4,7 & 23,4 \\
\hline Cerylon histeroides & 27 & 29,7 & 69 & 0,51 & 4,3 & 14,8 \\
\hline Phloeopora testacea & 23 & 25,3 & 66 & 0,49 & 4,1 & 15,5 \\
\hline Cis bidentatus & 18 & 19,8 & 58 & 0,43 & 3,6 & 17,8 \\
\hline Abraeus granulum & 26 & 28,6 & 55 & 0,41 & 3,5 & 12,4 \\
\hline Orchesia undulata & 14 & 15,4 & 51 & 0,38 & 3,2 & 20,4 \\
\hline Mycetophagus atomarius & 10 & 11,0 & 51 & 0,38 & 3,2 & 29,6 \\
\hline Phloeonomus punctipennis & 13 & 14,3 & 47 & 0,35 & 3,0 & 19,1 \\
\hline Sepedophilus testaceus & 16 & 17,6 & 46 & 0,34 & 2,9 & 17,3 \\
\hline Teredus cylindricus & 22 & 24,2 & 44 & 0,33 & 2,8 & 11,2 \\
\hline Orthoperus mundus & 17 & 18,7 & 38 & 0,28 & 2,4 & 12,7 \\
\hline Euplectus punctatus & 22 & 24,2 & 37 & 0,27 & 2,3 & 9,5 \\
\hline Pediacus dermestoides & 19 & 20,9 & 35 & 0,26 & 2,2 & 10,8 \\
\hline Sinodendron cylindricum & 10 & 11,0 & 35 & 0,26 & 2,2 & 22,1 \\
\hline
\end{tabular}

Obwohl in nur 49 Proben anwesend, war Ptilinus pectinicornis die häufigste Art in den EFs-Proben an stehendem Buchentotholz. 1995 war dieser Anobiidae mit 11,4 \% und 1996 mit 26,2 \% am Fangergebnis beteiligt. Pro Quadratmeter schlüpften 316,7 Tiere (spez. Dichte). In 24 Proben lag die Dichte bei unter 50 Ind. $/ \mathrm{m}^{2}$ (Abb. 4-15a). Die höchste in einer Probe gemessene Dichte belief sich auf 3405,7 Ind. $/ \mathrm{m}^{2}$. In der Fangperiode P4 wurden 52,6 \% und in der Fangperiode P5 35,2 \% der gefangenen Tiere von dieser Art gestellt (Tab. 4-26). 
Tabelle 4-26: Dominanzphänologie: Die Hauptarten der Fangperioden (EFs Buche)

\begin{tabular}{|l|rrrrrrrrr|}
\hline & \multicolumn{7}{|c}{ F a n g p e r i o d e } \\
ART & P1b & P2 & P3 & P4 & P5 & P6 & P7 & P8 \\
\hline Corticaria longicollis & 19,4 & 10,7 & 11,4 & 11,0 & 18,9 & 47,5 & 48,2 & 27,9 \\
Rhizophagus dispar & 14,1 & 13,8 & 18,6 & 8,0 & 6,2 & 8,2 & 2,2 & 8,4 \\
Rhizophagus bipustulatus & 9,1 & 4,6 & 3,8 & & & & & \\
Leptusa fumida & 6,8 & & & & & & & \\
Cryptophagus dentatus & 4,8 & 7,1 & 7,8 & 3,7 & 4,6 & 6,3 & 18,1 & 22,3 \\
Hylecoetus dermestoides & 2,8 & 8,2 & & & & & & \\
Cerylon ferrugineum & 2,8 & 8,5 & 6,0 & & & & & 3,4 \\
Corticeus unicolor & 3,7 & 5,6 & 5,5 & & & & & \\
Cicones variegatus & & 3,6 & & & & & & \\
Anaspis schilskyana & & 3,6 & & & & & & \\
Paromalus flavicornis & 3,9 & 6,1 & 5,0 & 2,6 & 2,3 & & 3,1 & 4,2 \\
Micridium halidaii & & & 9,7 & & & & & \\
Taphrorychus bicolor & 2,4 & & 2,0 & & & & & \\
Ptilinus pectinicornis & & & & 52,6 & 35,2 & 4,2 & & \\
Ptinella aptera & & & & 2,6 & 12,3 & 6,6 & & \\
Dienerella elongata & & & 2,5 & & & 3,0 & 3,7 & 4,8 \\
Cis castaneus & & & & & & 2,5 & & \\
Cis nitidus & 4,4 & 3,1 & 3,4 & & 2,6 & 2,7 & 8,7 & 8,0 \\
\hline Anzahl Hauptarten & $\mathbf{1 1}$ & $\mathbf{1 1}$ & $\mathbf{1 1}$ & $\mathbf{6}$ & $\mathbf{7}$ & $\mathbf{8}$ & $\mathbf{6}$ & $\mathbf{7}$ \\
Ind.anteil Hauptarten & $\mathbf{7 4 , 1}$ & $\mathbf{7 5 , 1}$ & $\mathbf{7 5 , 6}$ & $\mathbf{8 0 , 3}$ & $\mathbf{8 2 , 2}$ & $\mathbf{8 1 , 1}$ & $\mathbf{8 3 , 8}$ & $\mathbf{7 8 , 9}$ \\
Individuen gesamt & $\mathbf{1 4 8 0}$ & $\mathbf{1 6 5 6}$ & $\mathbf{2 0 1 8}$ & $\mathbf{3 1 1 1}$ & $\mathbf{2 6 7 5}$ & $\mathbf{1 3 4 6}$ & $\mathbf{6 8 1}$ & $\mathbf{5 0 2}$ \\
\hline
\end{tabular}

Neben Ptilinus pectinicornis war Corticaria longicollis die zweite Art, die in beiden Untersuchungsjahren als eudominant einzustufen war. 1995 war sie mit 22,7 \% Individuenanteil dominierend, 1996 belief sich der Anteil auf 17,4 \%. Zugleich war dieser Lathridiidae die einzige Art, die in allen Fangperioden einen Anteil von über $10 \%$ erreichte. C. longicollis schlüpfte in einer spezifischen Dichte von 197 Ind./ $\mathrm{m}^{2}$, das Maximum betrug 2151,4 Ind./ $\mathrm{m}^{2}$. In der Mehrzahl der Proben waren zwischen 10 und 99 Ind. $/ \mathrm{m}^{2}$ zu verzeichnen (Abb. 4-15a). Der Individuenanteil an stehendem Buchentotholz lag deutlich höher als in den STE-Proben an Buche, obwohl in letzteren ein ähnlich hoher Frequenzprozentwert beobachtet worden war.

Als dritte eudominante Art trat Rhizophagus dispar in Erscheinung, der auch an liegendem Buchentotholz einen sehr hohen Anteil am Fangergebnis hatte. 1995 wurde ein Individuenanteil von 14,4 \%, im Folgejahr von 7,4 \% beobachtet. In den Fangperioden P2 und P3 war dieser Räuber die vorherrschende Art in den Proben. Für die 65 Proben, in denen diese Art auftrat, wurde eine Dichte von 120,4 Ind. $/ \mathrm{m}^{2}$ ermittelt, das Maximum betrug $782,4 \mathrm{Ind} . / \mathrm{m}^{2}$ (Abb. 4-15a). 


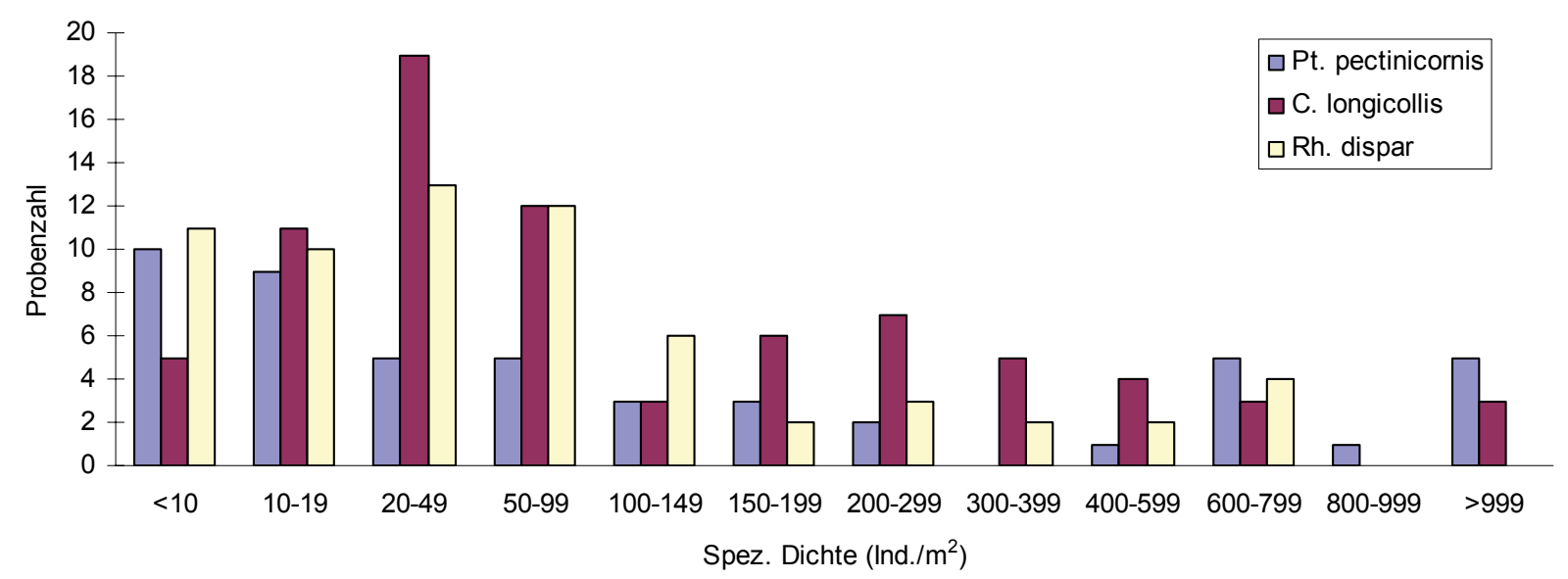

Abb. 4-15a: Spezifische Schlüpfdichten ausgewählter Hauptarten (EFs Buche)

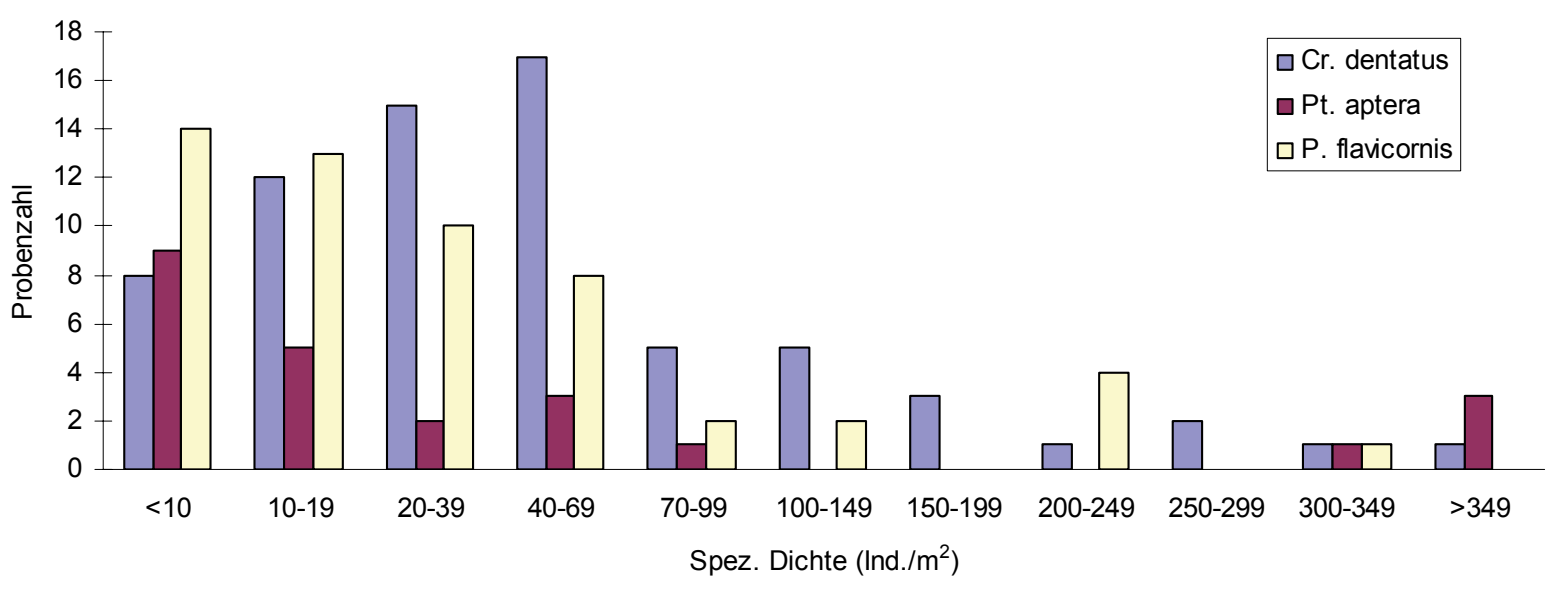

Abb. 4-15b: Spezifische Schlüpfdichten ausgewählter Hauptarten (EFs Buche)

Cryptophagus dentatus war mit einem Individuenanteil von 6,7 \% die einzige dominante Art. 1995 entfielen 9,2 und 1996 4,9\% der Tiere auf diesen Cryptophagiden. Cr. dentatus gehörte zu den wenigen Arten, die in allen Fangperioden zu den Hauptarten zu rechnen waren. In der Hälfte der Proben wurden Schlüpfdichten von unter $40 \mathrm{Ind} . / \mathrm{m}^{2}$ registriert, die höchste Dichte belief sich auf 875,7 Ind./m² (Abb. 4-15b).

1995 trat auch Ptinella aptera als dominante Art (7,6 \%) in Erscheinung. 1996 wurde aber nur ein Anteil von 1,3\% erreicht. Cerylon ferrugineum, Rhizophagus bipustulatus, Corticeus unicolor und Paromalus flavicornis waren in beiden Untersuchungsjahren subdominant, Micridium halidaii und Hylecoetus dermestoides nur 1995, Cis nitidus und Dinerella elongata nur 1996. Erstere drei Arten waren auch in den STE-Proben an Buche subdominant. 


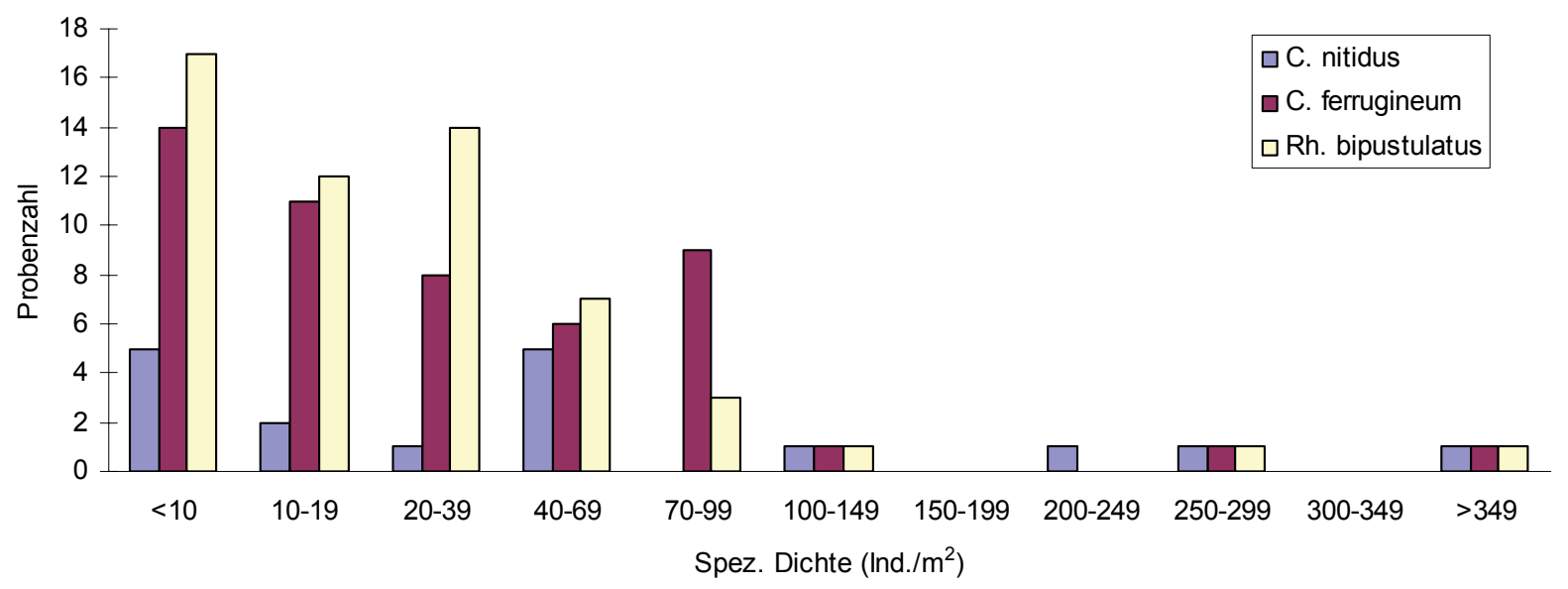

Abb. 4-15c: Spezifische Schlüpfdichten ausgewählter Hauptarten (EFs Buche)

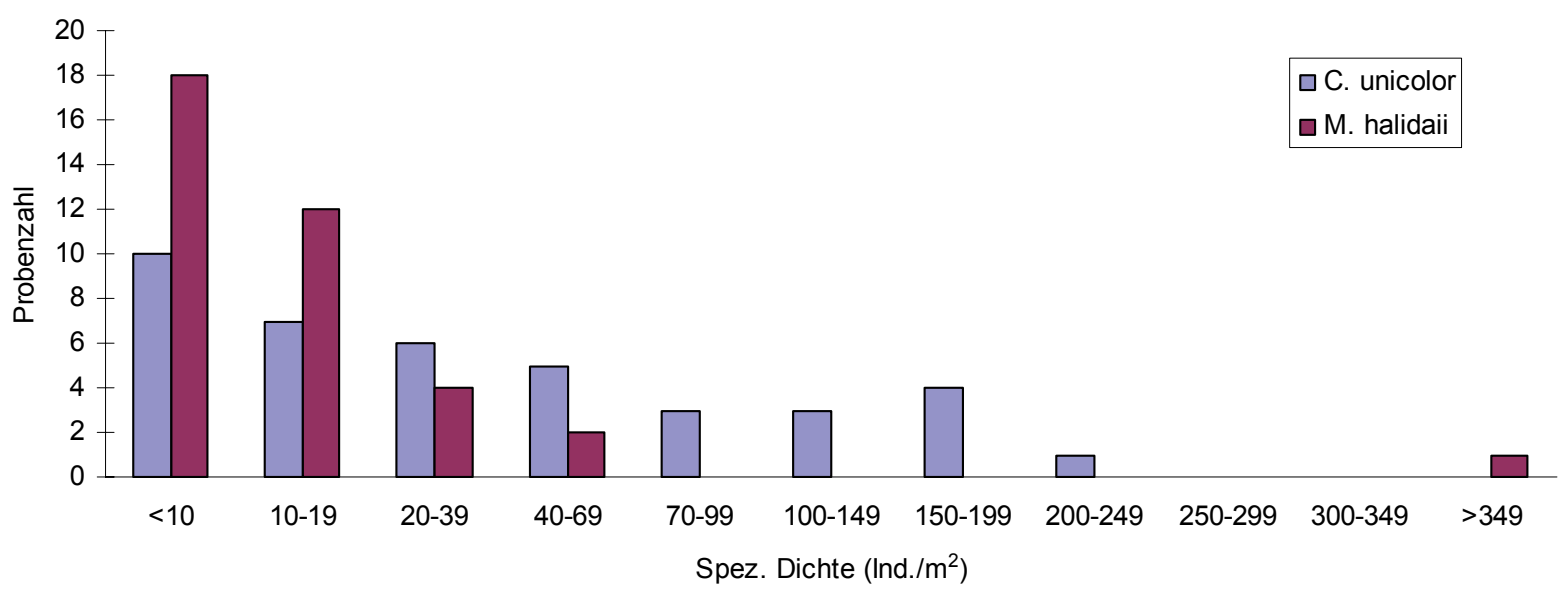

Abb. 4-15d: Spezifische Schlüpfdichten ausgewählter Hauptarten (EFs Buche)

Weitergehende Informationen zum Vorkommen der Arten an stehendem Buchentotholz können dem Anhang 5 entnommen werden.

\subsubsection{Frequenz- und Dominanzstruktur an stehendem Eichentotholz}

Auch in den EFs-Proben an Eichentotholz traten nur wenige Arten regelmäßig in Erscheinung (Tab. 4-27). 44 Arten wurden in nur einer Falle festgestellt, 74 Arten in bis zu 4 Proben. Diese waren mit 7,2 \% am Fangergebnis beteiligt. 58,7\% der Individuen wurden von 10 Arten gestellt, die in 10 bis 19 Proben zu finden waren, 23,2\% entfielen auf 6 Arten, die in mehr als 20 Fallen gefangen wurden. Corticaria longicollis, Cryptophagus dentatus, Rhizophagus dispar und Rh. bipustulatus waren auch in den EFs-Proben an Buche mit hoher Regelmäßigkeit anzutreffen. Dagegen wurden Rhynchaenus fagi und Teredus cylindricus verhältnismäßig häufiger an der Eiche festgestellt. Während für die letztere, sehr seltene Art, eine Präferenz für Eiche genannt wird, scheint Rh. fagi als phytophager Rüsselkäfer an Buche das berindete Eichentotholz regelmäßig zur Überwinterung aufzusuchen. 
Tabelle 4-27: Frequenzstruktur (EFs Eiche)

\begin{tabular}{|c|c|c|c|c|c|c|c|c|}
\hline & \multicolumn{6}{|c|}{ F R E Q U E N Z K L A S S E (\%) } & \multirow[b]{2}{*}{2} \\
\hline & & $\geq \mathbf{8 0}$ & $60-79,9$ & 40-59,9 & $20-39,9$ & $10-19,9$ & $<\mathbf{1 0}$ & \\
\hline \multirow[t]{2}{*}{ Arten } & Anz. & 1 & 0 & 5 & 10 & 22 & 74 & 112 \\
\hline & $\%$ & 0,9 & 0,0 & 4,5 & 8,9 & 19,6 & 66,1 & 100,0 \\
\hline \multirow[t]{2}{*}{ Ind. } & Anz. & 521 & 0 & 602 & 2840 & 528 & 347 & 4838 \\
\hline & $\%$ & 10,8 & 0,0 & 12,4 & 58,7 & 10,9 & 7,2 & 100,0 \\
\hline
\end{tabular}

Neun Hauparten hatten einen gemeinsamen Individuenanteil von 83,9 \% (Tab. 4-28). 76 \% der Arten waren als sporadisch zu klassifizieren, ihr Individuenanteil belief sich auf 5,2\%. In den einzelnen Fangperioden wurden zwischen 2 und 16 Hauptarten registriert, die zwischen 73,5 und 91,1 \% der Individuen stellten (Tab. 4-30).

Tabelle 4-28: Dominanzklassen (EFs Eiche)

\begin{tabular}{|c|c|c|c|c|c|c|c|c|}
\hline & \multicolumn{6}{|c|}{ D O M I N A N Z K L A S S E (\%) } & \multirow[b]{3}{*}{ 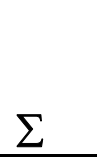 } \\
\hline & & \multicolumn{3}{|c|}{ Hauptarten } & \multicolumn{3}{|c|}{ Begleitarten } & \\
\hline & & $\geq \mathbf{1 0}$ & $5-9,99$ & $2-4,99$ & $1-1,99$ & 0,3-0,99 & $<\mathbf{0 , 3 0}$ & \\
\hline \multirow[t]{2}{*}{ Arten } & Anz. & 2 & 1 & 6 & 3 & 15 & 85 & 112 \\
\hline & $\%$ & 1,8 & 0,9 & 5,4 & 2,7 & 13,4 & 75,9 & 100,0 \\
\hline \multirow[t]{2}{*}{ Ind. } & Anz. & 2992 & 295 & 772 & 206 & 321 & 252 & 4838 \\
\hline & $\%$ & 61,8 & 6,1 & 16,0 & 4,3 & 6,6 & 5,2 & 100,0 \\
\hline
\end{tabular}

Die Dominanzstruktur wurde in erster Linie durch eine Art geprägt, die in wenigen Proben in sehr hoher Dichte in Erscheinung trat. Mit einem Individuenanteil von 51,1 \% war der Borkenkäfer Dryocoetes villosus die häufigste am stehenden Eichentotholz gefangene Art. In den 16 Proben mit D. villosus-Befall wurde eine Dichte von 1042,6 Ind. $/ \mathrm{m}^{2}$ registriert (Tab. 4-29). $96 \%$ der Individuen entstammten drei Proben, die alle an einem Stamm genommen worden waren. Hier beliefen sich die Schlüpfdichten auf 1812,9, 4662,5 und 8940,0 Ind./. $\mathrm{m}^{2}$ (Abb. 32a). Die Tiere schlüpften zu 66,2 \% in der Fangperiode P2 und zu 30,2 \% in P3. In diesen Fangperioden stellte D. villosus 85,8 bzw. $70,5 \%$ der gefangenen Individuen (Tab. 4-30). 
Tabelle 4-29: Frequenz, Dominanz und Schlüpfdichte häufiger Arten (EFs Eiche)

\begin{tabular}{|c|c|c|c|c|c|c|}
\hline \multirow[b]{2}{*}{ Art } & \multicolumn{2}{|c|}{ Frequenz } & \multicolumn{2}{|c|}{ Individuen } & \multicolumn{2}{|c|}{ Dichte (Ind./m²) } \\
\hline & Anz. & $\%$ & Anz. & $\%$ & allg. & spez. \\
\hline Dryocoetes villosus & 16 & 32,0 & 2471 & 51,07 & 357,9 & 1042,6 \\
\hline Corticaria longicollis & 46 & 92,0 & 521 & 10,77 & 75,5 & 82,4 \\
\hline Xyloterus signatus & 8 & 16,0 & 295 & 6,10 & 42,7 & 241,8 \\
\hline Teredus cylindricus & 24 & 48,0 & 187 & 3,87 & 27,1 & 56,8 \\
\hline Rhizophagus bipustulatus & 23 & 46,0 & 129 & 2,67 & 18,7 & 38,7 \\
\hline Lymexylon navale & 3 & 6,0 & 129 & 2,67 & 18,7 & 266,0 \\
\hline Cryptophagus dentatus & 28 & 56,0 & 115 & 2,38 & 16,7 & 29,5 \\
\hline Rhizophagus dispar & 21 & 42,0 & 112 & 2,32 & 16,2 & 36,8 \\
\hline Cis castaneus & 14 & 28,0 & 100 & 2,07 & 14,5 & 50,5 \\
\hline Dienerella elongata & 17 & 34,0 & 89 & 1,84 & 12,9 & 37,8 \\
\hline Rhynchaenus fagi & 23 & 46,0 & 59 & 1,22 & 8,5 & 17,7 \\
\hline Cerylon ferrugineum & 11 & 22,0 & 58 & 1,20 & 8,4 & 37,4 \\
\hline Enicmus testaceus & 3 & 6,0 & 39 & 0,81 & 5,6 & 85,7 \\
\hline Corticeus fasciatus & 9 & 18,0 & 29 & 0,60 & 4,2 & 22,2 \\
\hline Leptusa fumida & 12 & 24,0 & 27 & 0,56 & 3,9 & 15,2 \\
\hline Ptilinus pectinicornis & 3 & 6,0 & 24 & 0,50 & 3,5 & 59,3 \\
\hline Aderus oculatus & 5 & 10,0 & 23 & 0,48 & 3,3 & 32,6 \\
\hline Leptusa ruficollis & 12 & 24,0 & 22 & 0,45 & 3,2 & 13,1 \\
\hline Phyllodrepa ioptera & 10 & 20,0 & 22 & 0,45 & 3,2 & 16,1 \\
\hline Euplectus punctatus & 12 & 24,0 & 20 & 0,41 & 2,9 & 12,8 \\
\hline
\end{tabular}

Als zweite eudominante Art war der mycetophage Mulmbewohner Corticaria longicollis in $92 \%$ der Proben anzutreffen und erzielte mit einer spezifischen Schlüpfdichte von 82,4 Ind. $/ \mathrm{m}^{2}$ einen Individuenanteil von 10,8 \%. Die höchste festgestellte Schlüpfdichte belief sich auf 540,7 Ind. $/ \mathrm{m}^{2}$, in der Mehrzahl der Proben wurden zwischen 20 und $99 \mathrm{Ind} . / \mathrm{m}^{2}$ verzeichnet (Abb. 4-16a). Der Status einer Hauptart wurde während der gesamten Vegetationsperiode eingenommen.

Xyloterus signatus wurde in nur 8 Proben festgestellt, schlüpfte hier aber in einer Dichte von $241,8 \mathrm{Ind} . / \mathrm{m}^{2}$ und erreichte so einen Individuenanteil von $6,1 \%$ (Abb. 4-16a). Die höchsten Anteile wurden in den Fangperioden P5 (46,9 \%) und P6 (29,6 \%) erreicht.

Mit 187 gefangenen Individuen (3,9\%) aus 24 Proben war der vom Aussterben bedrohte Colydiidae Teredus cylindricus die vierthäufigste Art am stehenden Eichentotholz. Die spezifische Schlüpfdichte war mit 56,8 Ind. $/ \mathrm{m}^{2}$ deutlich höher als in den STE-Proben und den EFs-Proben an stehendem Buchentotholz. Es wurden bis zu 56 Exemplare $\left(329,4 \mathrm{Ind} . / \mathrm{m}^{2}\right)$ in einer Falle festgestellt (Abb. 4-16b). Die höchsten Schlüpfdichten wurden an unberindeten Stammabschnitten verzeichnet. $38 \%$ der Tiere fingen sich in der Fangperiode P3, jeweils $20 \%$ in P2 und P4. In der Fangperiode P3 war T. cylindricus die zweit- und in der Fangperiode $\mathrm{P} 4$ die dritthäufigste Art in den EFs-Proben. 
Tabelle 4-30: Dominanzphänologie: Die Hauptarten der Fangperioden (EFs Eiche)

\begin{tabular}{|c|c|c|c|c|c|c|c|c|}
\hline \multirow[b]{2}{*}{ ART } & \multicolumn{8}{|c|}{ F a g g e riode } \\
\hline & P1b & $\mathbf{P 2}$ & $\mathbf{P 3}$ & $\mathbf{P 4}$ & P5 & P6 & P7 & P8 \\
\hline Corticaria longicollis & 22,3 & 3,7 & 5,8 & 21,0 & 11,6 & 25,3 & 25,2 & 17,6 \\
\hline Rhizophagus bipustulatus & 11,5 & & 2,2 & 2,2 & 2,3 & & 2,4 & 2,7 \\
\hline Rhynchaenus fagi & 11,2 & & & & & & & \\
\hline Cryptophagus dentatus & 6,3 & & & 3,1 & 3,5 & 5,3 & 4,9 & 9,5 \\
\hline Cerylon ferrugineum & 4,8 & & & 2,7 & & & & \\
\hline Dienerella elongata & 3,7 & & & 2,2 & & 5,8 & 8,9 & 9,5 \\
\hline Xyloterus signatus & 3,5 & & & 2,0 & 46,9 & 29,6 & 2,4 & \\
\hline Teredus cylindricus & 3,1 & & 6,8 & 8,3 & 3,2 & & 3,3 & 4,1 \\
\hline Rhizophagus dispar & 2,1 & & 2,5 & 3,8 & 3,2 & 4,8 & 4,9 & 6,8 \\
\hline Dryocoetes villosus & 5,0 & 85,8 & 70,5 & 8,0 & 3,5 & 3,0 & 2,4 & \\
\hline Cis castaneus & & & 2,2 & 2,7 & 2,6 & 5,1 & 13,0 & 4,1 \\
\hline Lymexylon navale & & & & 26,3 & 3,2 & & & \\
\hline Enicmus testaceus & & & & 2,5 & 5,1 & & & \\
\hline Carabus problematicus & & & & & & 2,3 & 4,9 & 2,7 \\
\hline Aridius nodifer & & & & & & & 4,1 & \\
\hline Leptusa ruficollis & & & & & & & 4,1 & \\
\hline Aderus oculatus & & & & & & & 3,3 & \\
\hline Phyllodrepa ioptera & & & & & & & 2,4 & 2,7 \\
\hline Xyloterus domesticus & & & & & & & 2,4 & \\
\hline Cis bidentatus & & & & & & & 2,4 & \\
\hline Neuraphes elongatulus & & & & & & & & 6,8 \\
\hline Catops picipes & & & & & & & & 10,8 \\
\hline Rhizophagus perforatus & & & & & & & & 2,7 \\
\hline Anzahl Hauptarten & 10 & 2 & 6 & 12 & 10 & 8 & 16 & 12 \\
\hline Ind.anteil Hauptarten & 73,5 & 89,5 & 89,9 & 84,8 & 85,2 & 81,3 & 91,1 & 79,7 \\
\hline Individuen gesamt & 520 & 1907 & 1060 & 448 & 311 & 395 & 123 & 74 \\
\hline
\end{tabular}

Die beiden Rhizophagus-Arten dispar und bipustulatus waren in 21 bzw. 23 Proben anwesend. Während erstere Art in den STE-Proben an beiden Baumarten und in den EFsProben an stehendem Buchentotholz deutlich häufiger war, traten in den EFs-Proben an Eiche beide Arten in einer etwa übereinstimmenden Dichte in Erscheinung. Die höchsten Schlüpfdichten wurden für beide Arten an Stammabschnitten mit Borkenkäferbefall registriert (Abb. 4-16b-c).

$\mathrm{Zu}$ den Hauptarten zählten ferner Cryptophagus dentatus (2,4\%), Cis castaneus (2,1\%) und auch Lymexylon navale (2,7\%), obwohl letztere in nur drei Proben festgestellt werden konnte. 


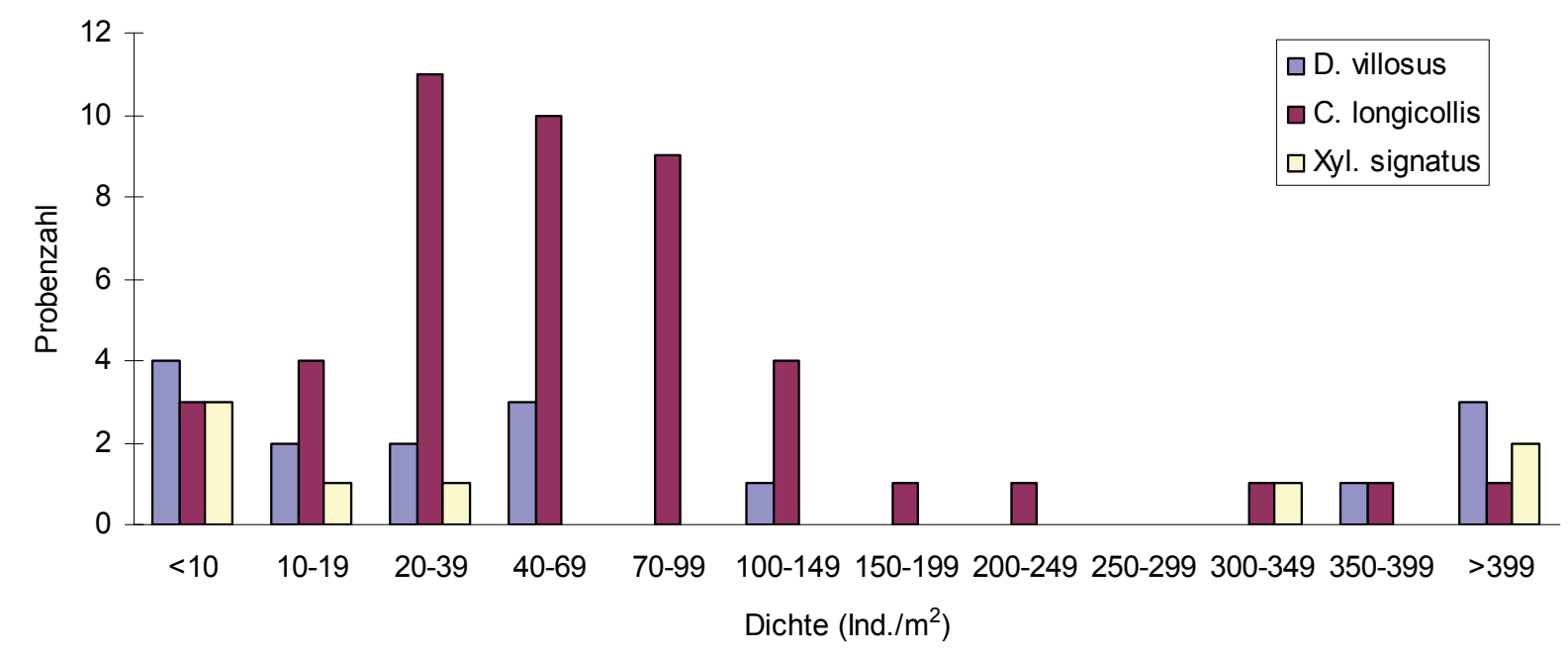

Abb. 4-16a: Spezifische Schlüpfdichten ausgewählter Hauptarten (EFs Eiche)

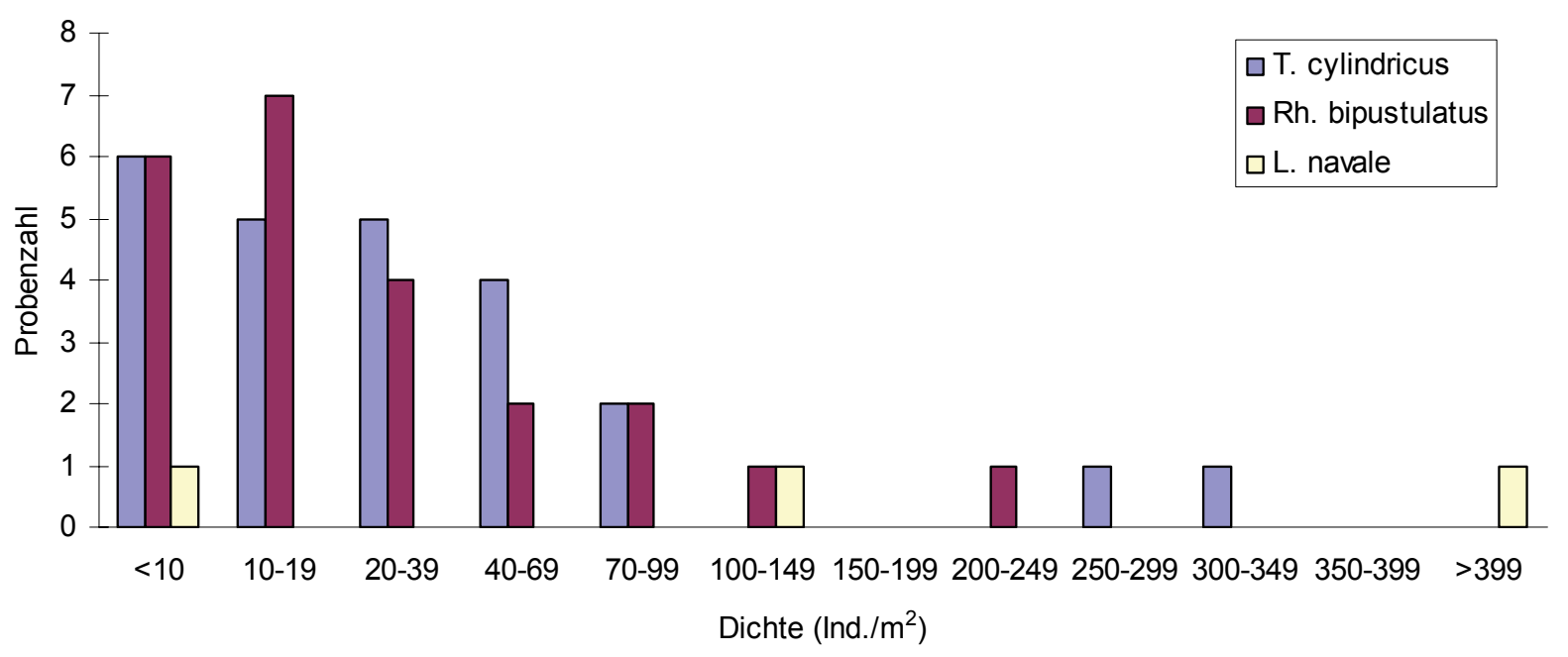

Abb. 4-16b: Spezifische Schlüpfdichten ausgewählter Hauptarten (EFs Eiche)

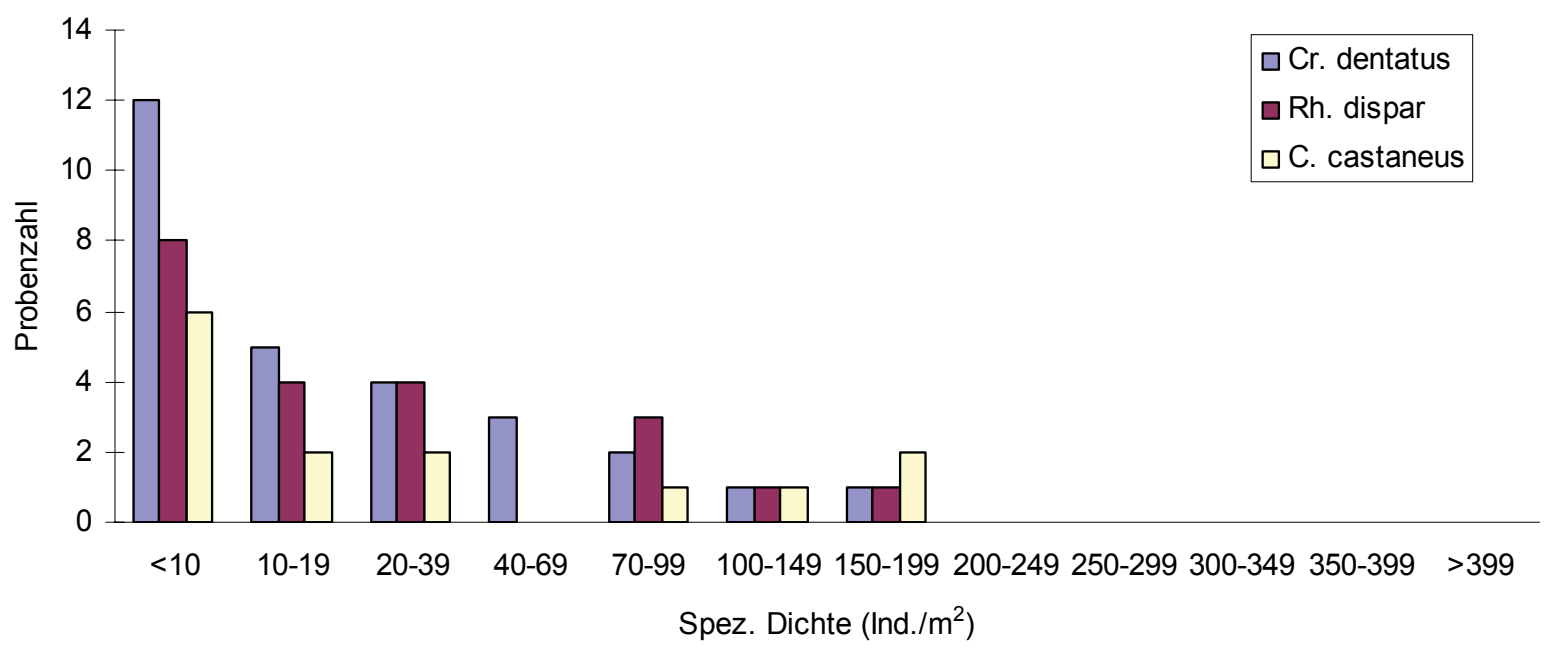

Abb. 4-16c: Spezifische Schlüpfdichten ausgewählter Hauptarten (EFs Eiche)

Weitere Angaben zur Dominanzstruktur und Dichte der Arten finden sich im Anhang 5. 


\subsubsection{Die trophische Struktur}

Mit 203 Arten (44 \%) und einem Individuenanteil von 39,6 \% waren die Zoophagen die dominierende Gruppe in den Eklektoren (Tab. 4-31, Abb. 4-17 u. 4-18). In den Fängen an Eichentotholz gehörte jede zweite Art zu dieser Gruppe, an Buchentotholz belief sich der Artenanteil auf 44,1\%. Auch in den einzelnen Fallentypen stellten die Räuber das Gros der Arten, gleiches gilt für die einzelnen Fangperioden, in denen der Artenanteil zwischen 46,6 und $49,7 \%$ variierte (Tab. 4-32).

Deutliche Unterschiede waren allerdings bei den Individuenanteilen dieses Konsumententyps zu verzeichnen. An der Buche schlüpften die Räuber in einer Dichte von $72,7 \mathrm{Ind} . / \mathrm{m}^{2}$ und waren mit einem Anteil von 43,7 \% die individuenstärkste Gruppe. An Eichentotholz waren die Zoophagen nur die zweitstärkste Fraktion. Die Schlüpfdichte betrug 50,7 Ind./ $\mathrm{m}^{2}$ und der Individuenanteil belief sich auf 31,0 \%. Der höchste Individuenanteil wurde in den STE-Proben registriert (54,6 \%), wohingegen die räuberischen Arten in den EFs-Proben mit einem Individuenanteil von 27,3 \% nur den dritten Rang belegten. Besonders in den ersten Fangperioden nahmen die Zoophagen in den Eklektoren eine dominierende Position ein. Etwa ab der zweiten Julihälfte war ein deutlicher Rückgang der Fangzahlen zu beobachten.

Tabelle 4-31: Arten- und Individuenzahlen der Konsumententypen

\begin{tabular}{|c|c|c|c|c|c|c|c|c|}
\hline \multirow{2}{*}{\multicolumn{2}{|c|}{ Kon.-typ }} & \multicolumn{2}{|c|}{ Baumart } & \multicolumn{4}{|c|}{ Fallentyp } & \multirow[b]{2}{*}{ gesamt } \\
\hline & & $\mathrm{Bu}$ & Ei & STE & mBE & EFs & EFI & \\
\hline \multirow{7}{*}{ 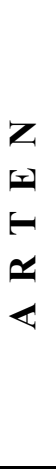 } & phytophag & 38 & 23 & 25 & 24 & 9 & 9 & 42 \\
\hline & mycetophag & 88 & 63 & 76 & 62 & 58 & 28 & 97 \\
\hline & saprophag & 21 & 15 & 17 & 13 & 10 & 4 & 29 \\
\hline & xylophag & 55 & 33 & 35 & 50 & 32 & 7 & 64 \\
\hline & zoophag & 176 & 149 & 163 & 132 & 84 & 52 & 203 \\
\hline & o. Angabe & 21 & 15 & 15 & 13 & 8 & 7 & 22 \\
\hline & $\Sigma$ & 399 & 298 & 331 & 294 & 201 & 107 & 457 \\
\hline \multirow{7}{*}{ 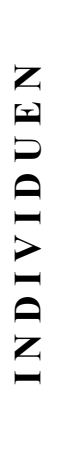 } & phytophag & 218 & 182 & 111 & 180 & 95 & 14 & 400 \\
\hline & mycetophag & 12180 & 6637 & 8693 & 2750 & 7067 & 307 & 18817 \\
\hline & saprophag & 80 & 58 & 68 & 25 & 36 & 9 & 138 \\
\hline & xylophag & 4827 & 3574 & 670 & 1909 & 5806 & 16 & 8401 \\
\hline & zoophag & 14351 & 4869 & 12165 & 1782 & 4997 & 276 & 19220 \\
\hline & o. Angabe & 1194 & 367 & 564 & 675 & 306 & 16 & 1561 \\
\hline & $\Sigma$ & 32850 & 15687 & 22271 & 7321 & 18307 & 638 & 48537 \\
\hline
\end{tabular}


$46 \%$ der 206 Arten mit räuberischer Ernährungsweise waren den Totholzbewohnern zuzurechnen. Diese stellten $89 \%$ der gefangenen Individuen mit zoophager Ernährungsweise. Unter diesen dominierten die Rindenkäfer, die mit 44 Arten vertreten waren und $68 \%$ der Individuen stellten. $17 \%$ der Arten und Individuen gelten als Totholzmulmbewohner. Darüber hinaus erreichten nur noch Arten der Bodenoberfläche nennenswerte Anteile an den Fängen dieses Konsumententyps. $26 \%$ der Arten und $9 \%$ der Individuen rekrutierten sich aus dieser Fraktion.

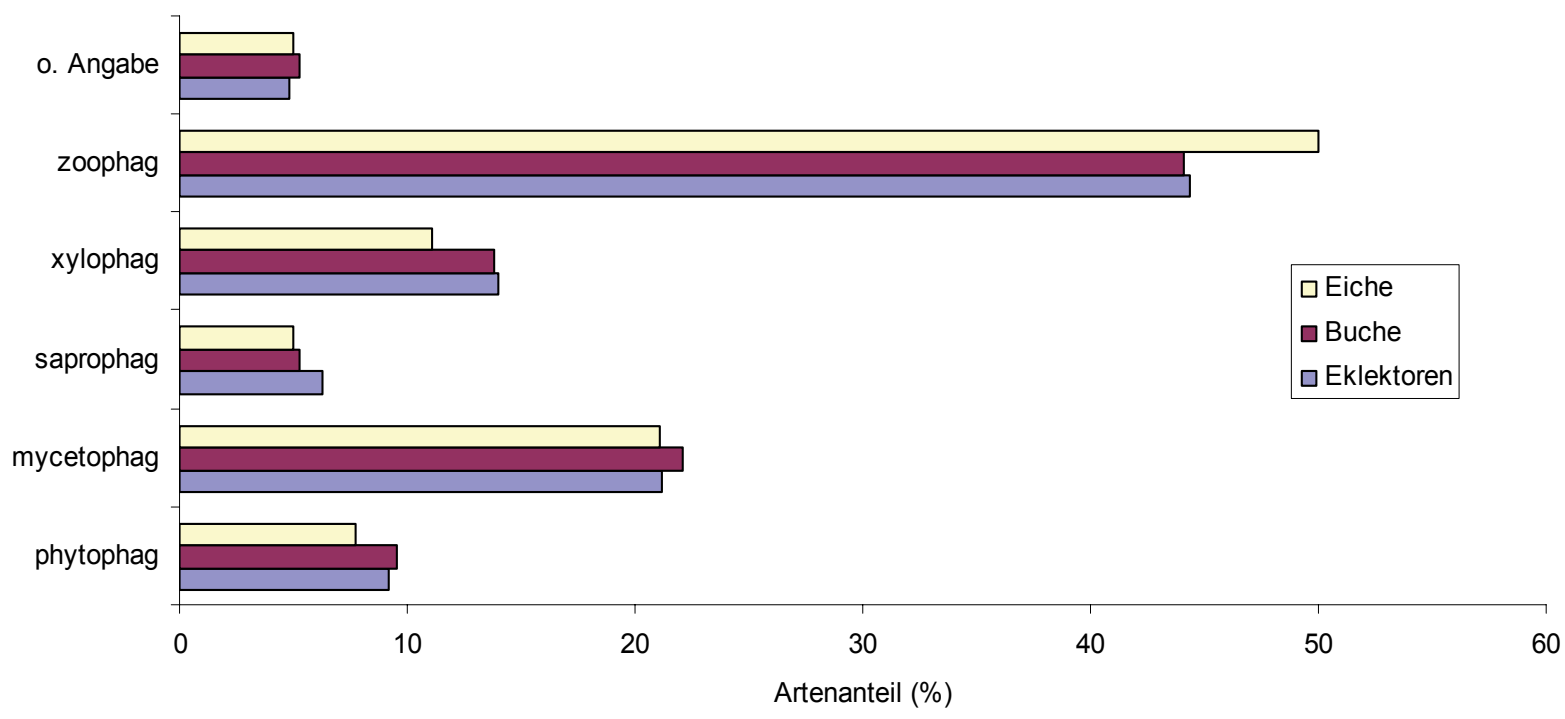

Abb. 4-17: Artenanteile der Konsumententypen

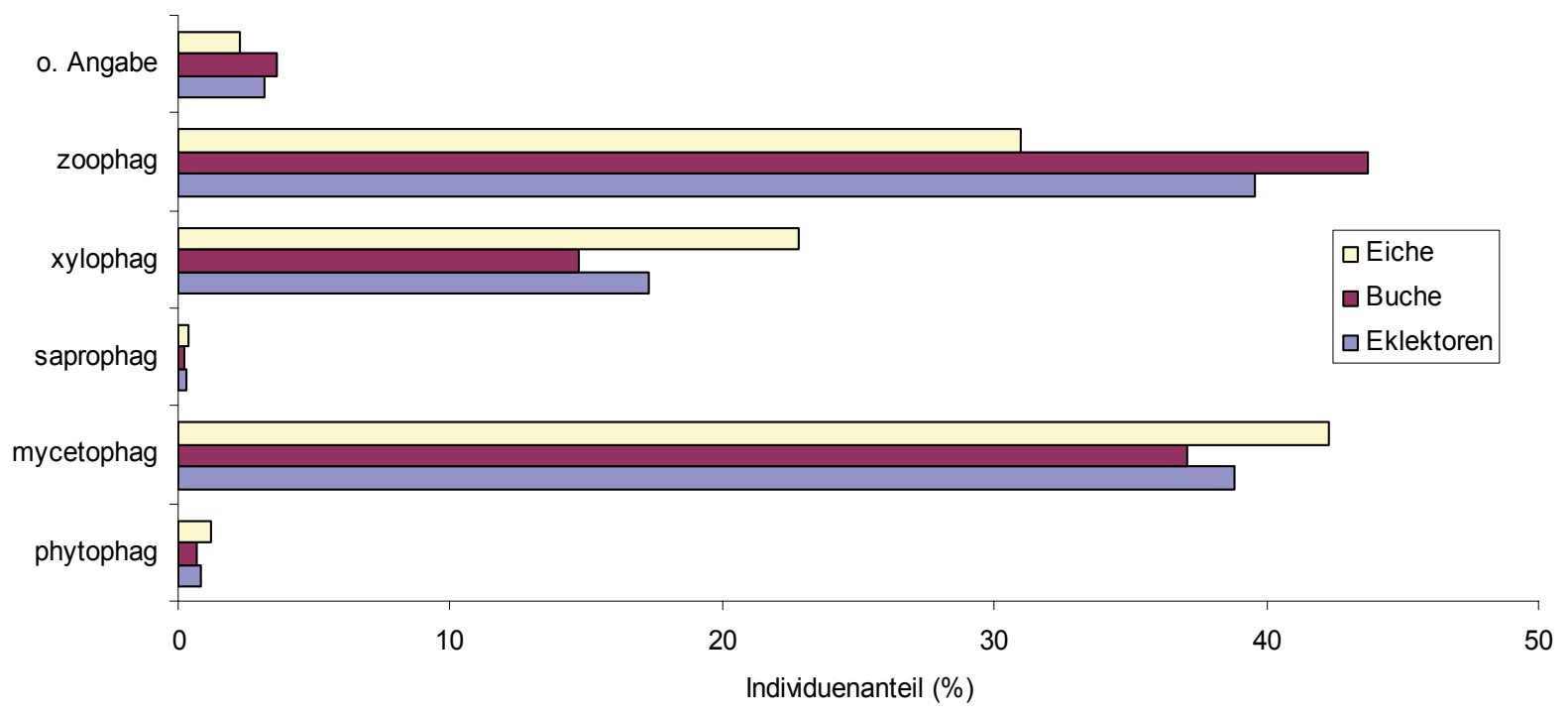

Abb. 4-18: Individuenanteile der Konsumententypen 
Mit einem Anteil von 34,4 \% war Rhizophagus dispar der klar vorherrschende Vertreter unter den zoophagen Arten (Abb. 4-19). Mit deutlichem Abstand folgten Rh. bipustulatus (7,1\%), Cerylon ferrugineum (6,6 \%) und Phloeonomus punctipennis (6,5\%). Neben den genannten Arten, die an beiden Baumarten zu den häufigeren Vertretern gehörten, erreichten Teredus cylindricus und Neuraphes elongatulus nur an Eichentotholz und Corticeus unicolor nur an Buchentotholz höhere Individuenanteile an den gefangenen Räubern.

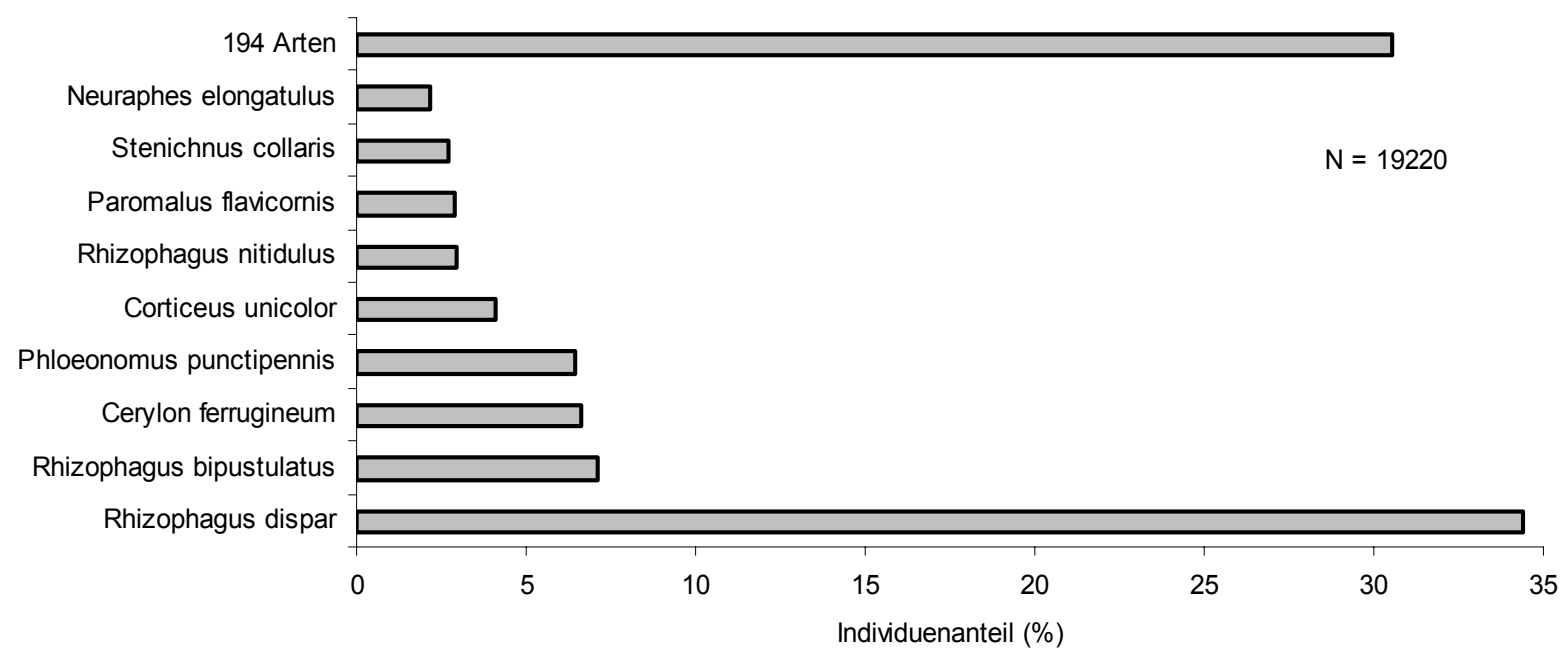

Abb. 4-19: Dominanzstruktur zoophager Arten

97 Arten (21\%) wurden den Mycetophagen zugeordnet. Mit einem Individuenanteil von 38,8 \% waren die Pilzfresser fast ebenso zahlreich in den Eklektoren vertreten wie die räuberischen Arten. In den eingesetzten Fallentypen waren die Mycetophagen jeweils die zweitstärkste Artengruppe mit Artenanteilen zwischen 21,1 \% (mBE) und 28,9 \% (EFs). Ebenso wurde in den einzelnen Fangperioden jeweils der zweite Rang eingenommen. Während die zoophagen Arten vor allem in der ersten Jahreshälfte dominierten, waren die Pilzfresser die vorherrschende Gruppe besonders in der zweiten Hälfte der Vegetationsperiode. Der Artenanteil stimmte an den beiden untersuchten Baumarten fast überein. An Buchentotholz schlüpften die Mycetophagen in einer Dichte von $61,7 \mathrm{Ind} . / \mathrm{m}^{2}$, ihr Individuenanteil belief sich auf $37,1 \%$. Mit einer Schlüpfdichte von $69,1 \mathrm{Ind} . / \mathrm{m}^{2}$ und einem Individuenanteil von 42,3 \% war dieser Konsumententyp an Eichentotholz individuenreicher vertreten als die Gruppe der zoophagen Arten. Abgesehen von den STE-Proben stellten die Arten mit mycetophager Ernährungsweise in den verschiedenen Eklektortypen jeweils die höchsten Individuenanteile.

$16 \%$ der gefangenen mycetophagen Tiere entfielen auf Arten, die keine Präferenz für bestimmte Habitate erkennen lassen. Xylobionte Arten stellten $82 \%$ der Individuen dieses Ernährungstyps. Hinsichtlich der Artenzahl dominierten die Totholzpilzbewohner, die allerdings nur mit einem Individuenanteil von 12,2 \% zum Gesamtfang dieses Konsumententyps beitrugen. Dagegen erreichten sechs xylodetricolen Arten mit mycetophager Ernährungsweise einen Individuenanteil von 35, 4 \% und die lignicolen Arten einen Anteil von 24,6 \%.

Mit einem Individuenanteil von 21,7 \% war Corticaria longicollis der häufigste Vertreter unter den mycetophagen Arten (Abb. 4-20). An Buchentotholz belief sich sein Anteil sogar 
auf fast $25 \%$ (Eiche 16,7\%). An Eichentotholz dominierte dagegen der pilzzüchtende Borkenkäfer Xyloterus signatus (27,5\%). Orchesia undulata, Xyloterus domesticus und Cryptophagus dentatus waren an beiden Baumarten mit höheren Anteilen (> $5 \%$ ) an den Fängen dieses Konsumententyps vertreten, während Hylecoetus dermestoides und Ptinella aptera nur an Buchentotholz einen Individuenanteil von über $5 \%$ an den gefangenen Pilzfressern erzielten.

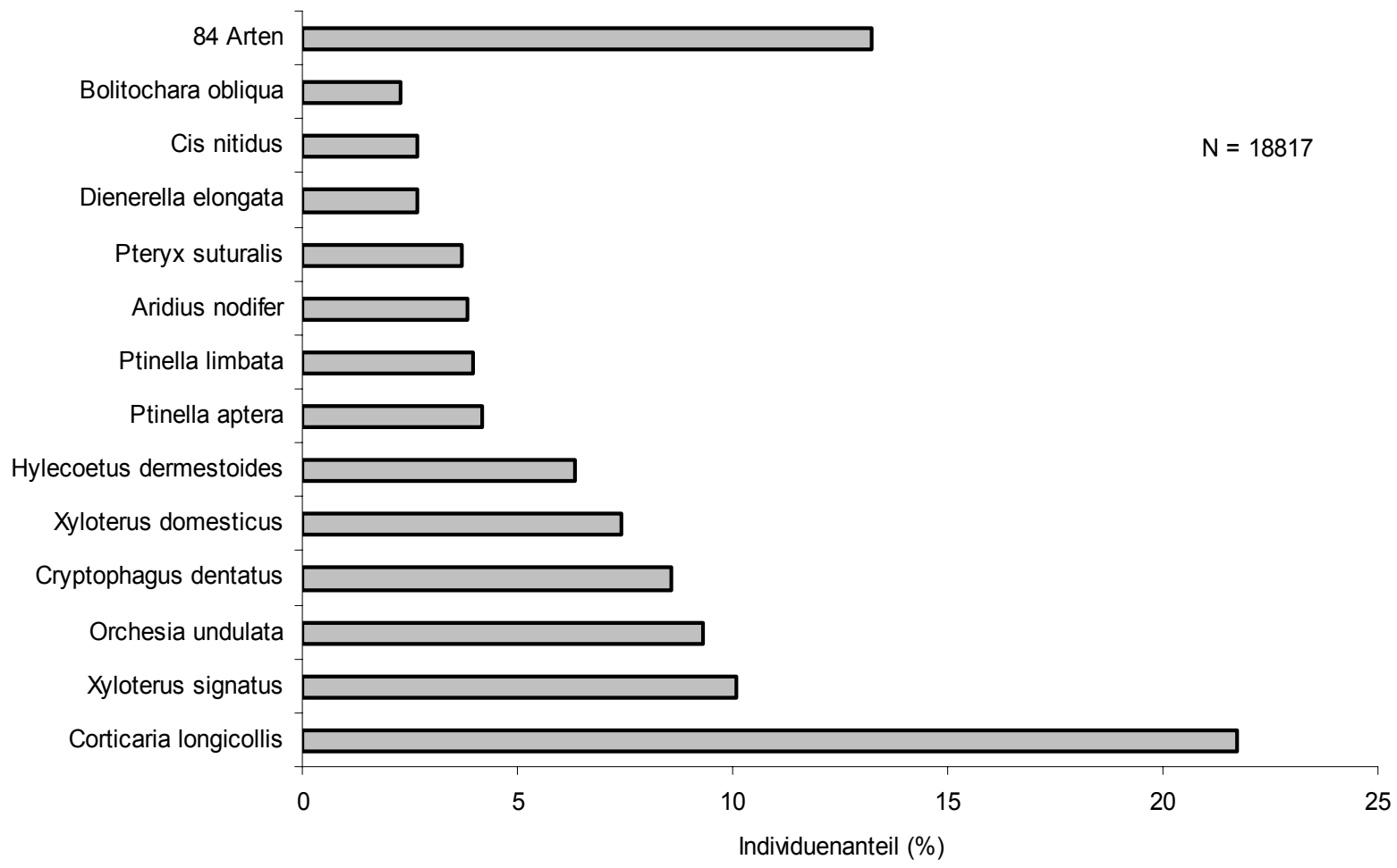

Abb. 4-20: Dominanzstruktur mycetophager Arten 
Tabelle 4-32: Arten- und Individuenanteile der Konsumententypen in den Fangperioden

\begin{tabular}{|c|c|c|c|c|c|c|c|c|c|}
\hline & & & & & $\operatorname{ang}_{\mathbf{I}}$ & i o d e & & & \\
\hline & Kon.-typ & P1b & $\mathbf{P 2}$ & $\mathbf{P 3}$ & $\mathbf{P 4}$ & P5 & P6 & P7 & P8 \\
\hline Ar- & phytophag & 26 & 16 & 16 & 11 & 5 & 4 & 7 & 9 \\
\hline ten- & mycetophag & 58 & 64 & 55 & 60 & 54 & 47 & 44 & 41 \\
\hline & saprophag & 13 & 7 & 7 & 5 & 2 & 3 & 4 & 10 \\
\hline & xylophag & 27 & 27 & 33 & 34 & 22 & 19 & 5 & 4 \\
\hline & zoophag & 124 & 122 & 119 & 103 & 88 & 78 & 64 & 73 \\
\hline & o. Angabe & 15 & 11 & 10 & 8 & 8 & 6 & 7 & 13 \\
\hline & $\Sigma$ & 263 & 247 & 240 & 221 & 179 & 157 & 131 & 150 \\
\hline Ar- & phytophag & 9,9 & 6,5 & 6,7 & 5,0 & 2,8 & 2,5 & 5,3 & 6,0 \\
\hline ten- & mycetophag & 22,1 & 25,9 & 22,9 & 27,1 & 30,2 & 29,9 & 33,6 & 27,3 \\
\hline an- & saprophag & 4,9 & 2,8 & 2,9 & 2,3 & 1,1 & 1,9 & 3,1 & 6,7 \\
\hline & xylophag & 10,3 & 10,9 & 13,8 & 15,4 & 12,3 & 12,1 & 3,8 & 2,7 \\
\hline & zoophag & 47,1 & 49,4 & 49,6 & 46,6 & 49,2 & 49,7 & 48,9 & 48,7 \\
\hline & o. Angabe & 5,7 & 4,5 & 4,2 & 3,6 & 4,5 & 3,8 & 5,3 & 8,7 \\
\hline & $\Sigma$ & 100,0 & 100,0 & 100,0 & 100,0 & 100,0 & 100,0 & 100,0 & 100,0 \\
\hline In- & phytophag & 168 & 68 & 44 & 32 & 18 & 5 & 19 & 44 \\
\hline di- & mycetophag & 2509 & 2369 & 2066 & 3117 & 3761 & 2845 & 1297 & 848 \\
\hline vi- & saprophag & 17 & 10 & 10 & 6 & 2 & 9 & 5 & 79 \\
\hline du- & xylophag & 315 & 2063 & 1357 & 3070 & 1388 & 172 & 27 & 9 \\
\hline zahl & zoophag & 3805 & 3615 & 3970 & 3124 & 1813 & 1314 & 585 & 973 \\
\hline & o. Angabe & 339 & 253 & 217 & 190 & 107 & 334 & 72 & 48 \\
\hline & $\Sigma$ & 7153 & 8378 & 7664 & 9539 & 7089 & 4679 & 2005 & 2001 \\
\hline In- & phytophag & 2,3 & 0,8 & 0,6 & 0,3 & 0,3 & 0,1 & 0,9 & 2,2 \\
\hline di- & mycetophag & 35,1 & 28,3 & 27,0 & 32,7 & 53,1 & 60,8 & 64,7 & 42,4 \\
\hline Vl- & saprophag & 0,2 & 0,1 & 0,1 & 0,1 & 0,0 & 0,2 & 0,2 & 3,9 \\
\hline en- & xylophag & 4,4 & 24,6 & 17,7 & 32,2 & 19,6 & 3,7 & 1,3 & 0,4 \\
\hline an- & zoophag & 53,2 & 43,1 & 51,8 & 32,7 & 25,6 & 28,1 & 29,2 & 48,6 \\
\hline teil & o. Angabe & 4,7 & 3,0 & 2,8 & 2,0 & 1,5 & 7,1 & 3,6 & 2,4 \\
\hline & $\Sigma$ & 100,0 & 100,0 & 100,0 & 100,0 & 100,0 & 100,0 & 100,0 & 100,0 \\
\hline
\end{tabular}

Die Xylophagen waren mit 64 Arten (14,0 \%) in den Eklektorproben vertreten und stellten $17,3 \%$ der gefangenen Individuen. An Eichentotholz gehörten 33 Arten (11,1\%) und 22,8 \% der Individuen zu diesem Konsumententyp. Die Schlüpfdichte belief sich auf $22,8 \mathrm{Ind} . / \mathrm{m}^{2}$. An Buchentotholz wurde ein Artenanteil von 13,8 (55 Arten) und ein Individuenanteil von $14,7 \%$ registriert, die Schlüpfdichte betrug 14,7 Ind. $/ \mathrm{m}^{2}$. Während in den STE-Proben nur 3,0 \% der gefangenen Individuen diesem Ernährungstyp zugerechnet werden konnten, belief sich ihr Anteil in den mBE-Proben auf 26,1 \% und in den EFs-Proben sogar auf 31,7 \%. Die höchsten Artenzahlen waren in den Fangperioden P3 und P4 zu beobachten. In der zuletzt 
genannten Fangperiode wurde auch der höchste Individuenanteil registriert. Mit 32,2 \% wurde ein fast ebenso hoher Wert wie für die Gruppen der Zoophagen und Mycetophagen verzeichnet.

67,2 \% der xylophagen Arten mit einem Individuenanteil von 58,6\% werden zu den lignicolen Arten gerechnet. Die Rindenbewohner stellten ein Drittel der Individuen dieses Konsumententyps. Von den insgesamt 64 Arten mit xylophager Ernährungsweise waren nur 5 Arten mit wenigstens $2 \%$ Anteil am Fangergebnis dieser Gruppe beteiligt (Abb. 4-21). 42,9\% der Individuen entfielen auf den Anobiiden Ptilinus pectinicornis. Dryocoetes villosus und Aderus oculatus waren die dominierenden Arten dieser Konsumentengruppe an Eichentotholz.

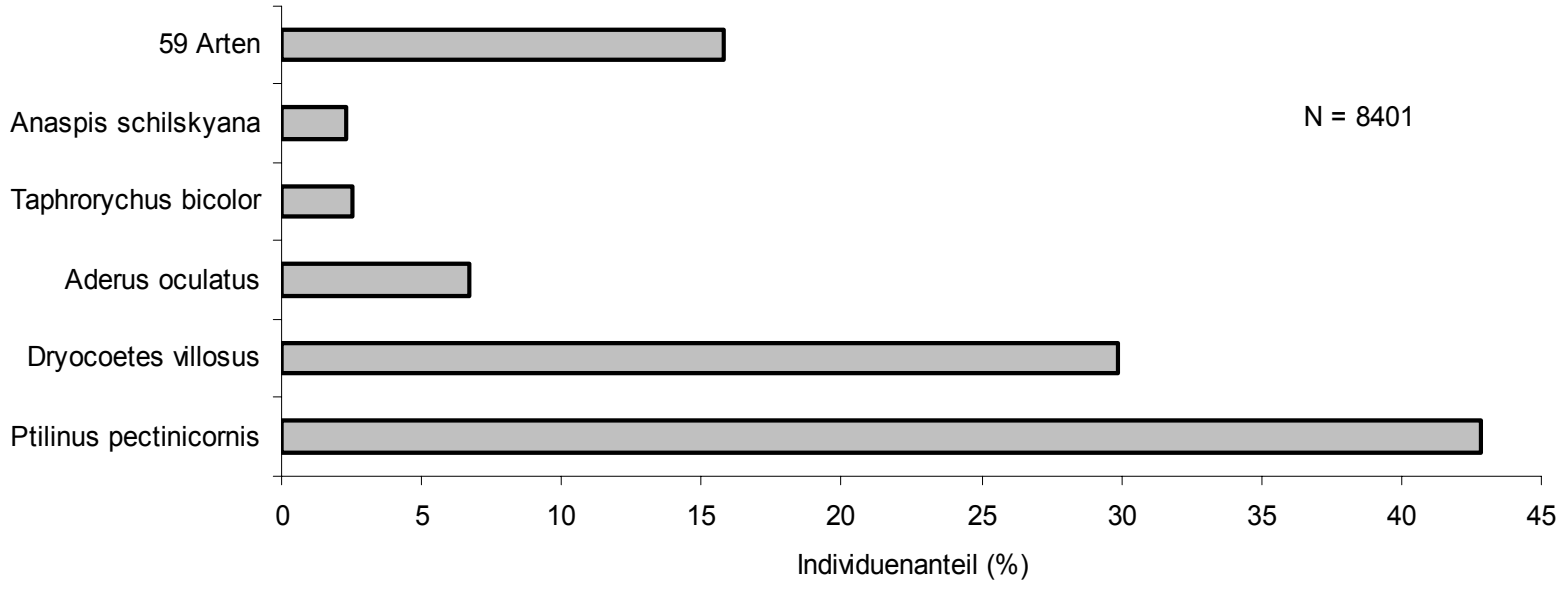

Abb. 4-21: Dominanzstruktur xylophager Arten

Mit 29 Arten erreichten die Saprophagen nur einen Individuenanteil von 0,3\% in den Eklektoren. Die Phytophagen stellten mit 42 Arten 0,8 \% der Individuen. Für 22 Arten mit einem Individuenanteil von 3,2 \% war eine Zuordnung zu einem Konsumententyp nicht möglich. Unter diesen waren insbesondere Leptusa fumida, L. pulchella und Phylodrepa ioptera in größerer Zahl vertreten.

In den STE-Proben an liegendem Buchentotholz wurden 129 zoophage Arten (50,2 \%) mit einem Individuenanteil von 61,6 \% gefangen (Abb. 4-22 u. 4-23). Die Schlüpfdichte belief sich auf 113,5 Ind./m². 1995 erreichten 106 Arten (54,4\%) einen Individuenanteil von 61,1 \%, 1996 waren 101 Arten (50,8 \%) mit einem Individuenanteil von 62,1 \% in den Fallen vertreten. 45,7 \% der Individuen mit räuberischer Ernährungsweise entfielen allein auf Rhizophagus dispar.

Mit 64 Arten (24,9\%) und einem Individuenanteil von 32,1 \% waren die mycetophagen Arten der zweithäufigste Ernährungstyp in den STE-Proben an liegendem Buchentotholz. Die Tiere schlüpften in einer Dichte von $59,1 \mathrm{Ind} . / \mathrm{m}^{2}$. In den beiden Untersuchungsjahren entfielen 24,6 bzw. 26,1 \% der Arten und 33,3 bzw. 30,9 \% der Individuen auf diesen Ernährungstyp. Die häufigsten Vertreter dieser Gruppe waren Hylecoetus dermestoides $(17,4 \%)$ und Xyloterus domesticus (16,4 \%). 


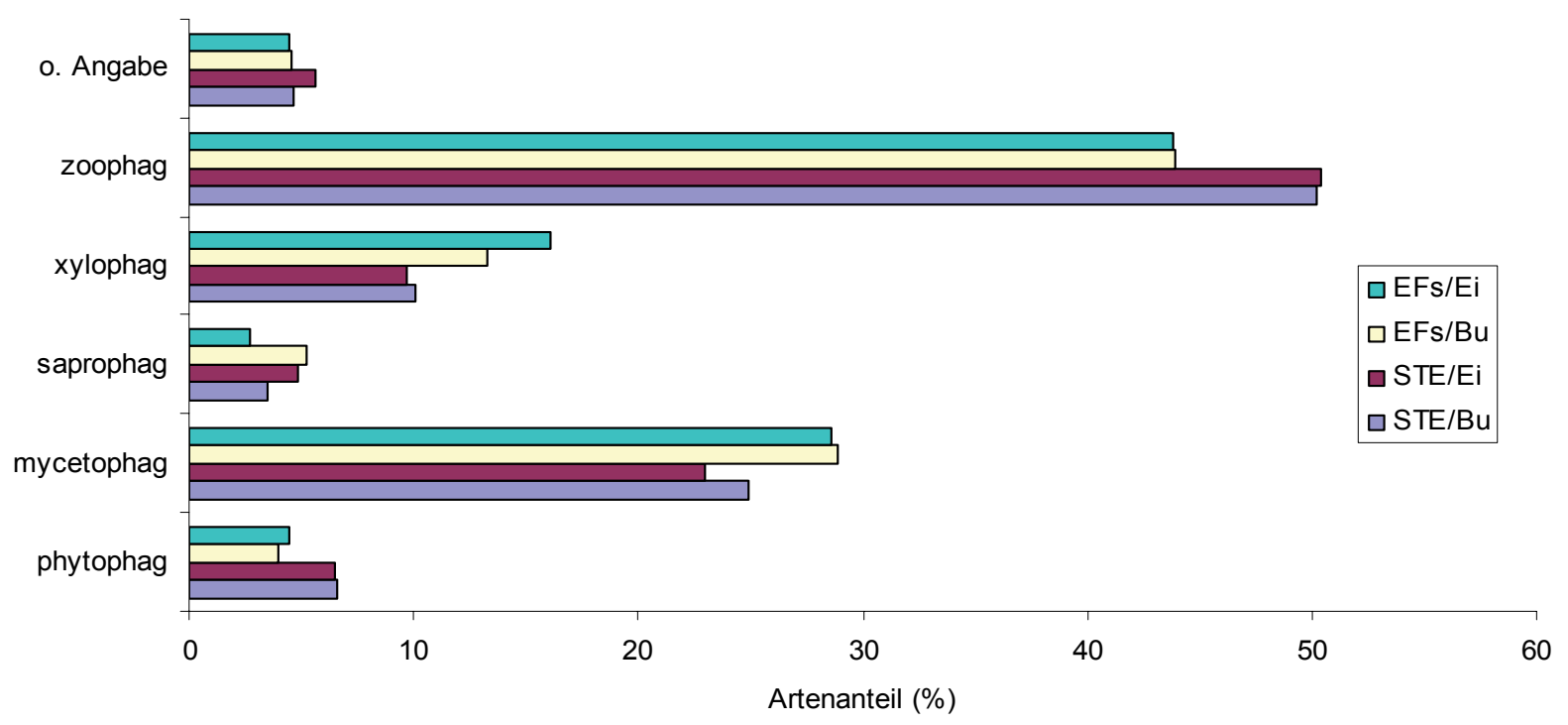

Abb. 4-22: Artenanteile der Konsumententypen an liegendem und stehendem Eichen- und Buchentotholz

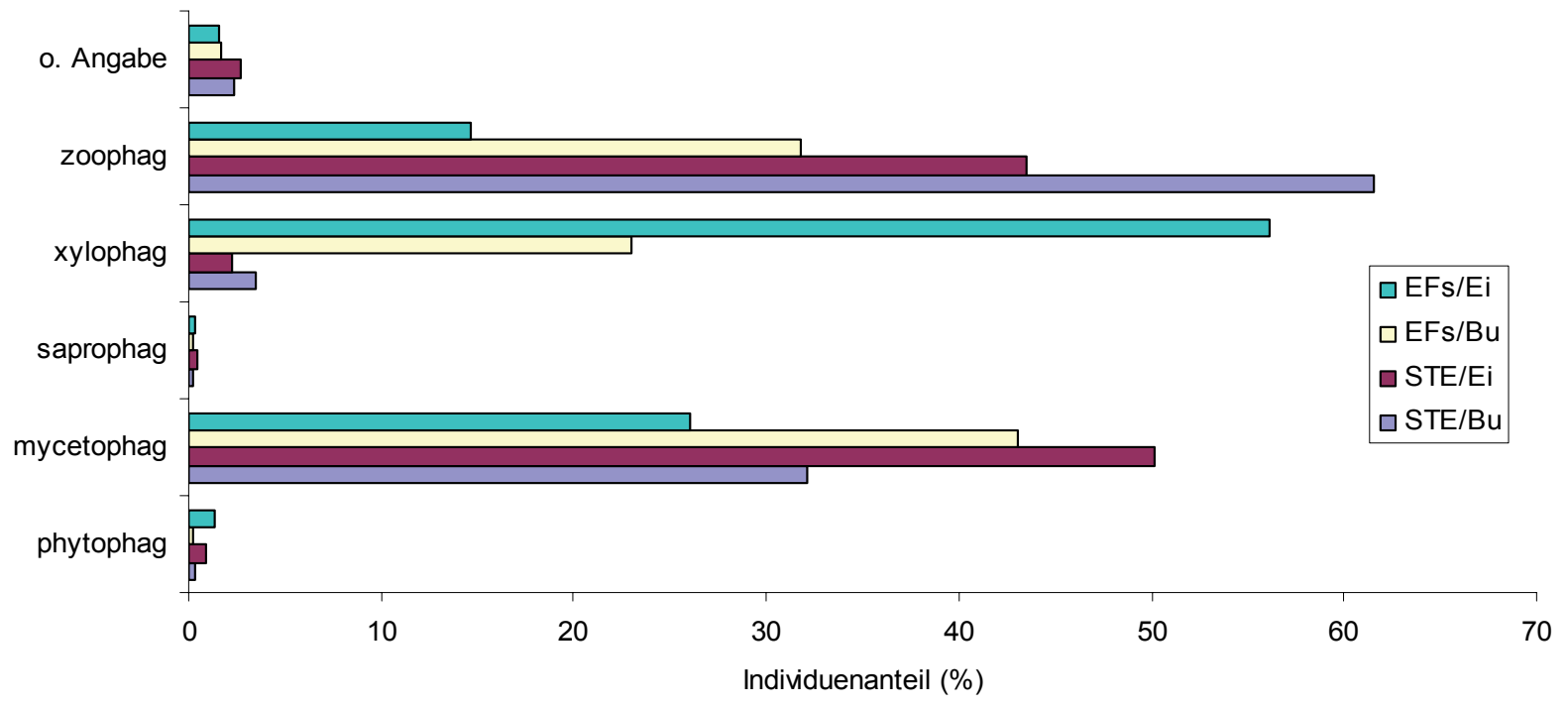

Abb. 4-23: Individuenanteile der Konsumententypen an liegendem und stehendem Eichenund Buchentotholz

Die 26 xylophagen Arten (10,1\%) hatten nur einen Individuenanteil von 3,5 \%, von denen wiederum 56,3\% von Ptilinus pectinicornis gestellt wurden. Zwischen den beiden Untersuchungsjahren gab es, wie schon bei den vorangegangenen Konsumentengruppen, nur geringfügige Abweichungen. Gleiches war auch für die Gruppe der Phytophagen und Saprophagen zu beobachten, die in nur geringen Individuenzahlen in Erscheinung traten.

Auch in den STE-Proben an liegendem Eichentotholz gehörten über die Hälfte der Arten zu den Zoophagen. Diese 125 Arten erreichten aber nur einen Individuenateil von 43,5 \%. Mit 66,8 Ind. $/ \mathrm{m}^{2}$ lag die Schlüpfdichte deutlich niedriger als an der Buche. 31,4 \% der Individuen entfielen auf Rhizophagus dispar, 10,2\% auf Rh. bipustulatus. 
Die individuenstärkste Gruppe waren die Mycetophagen, die mit 57 Arten $(23,0 \%)$ und einem Individuenanteil von 50,2 \% vertreten waren. Die Schlüpfdichte betrug $77,1 \mathrm{Ind} . / \mathrm{m}^{2}$. Am häufigsten war Xyloterus signatus festzustellen (35,6\%), gefolgt von Corticaria longicollis $(13,5 \%)$ und Xyloterus domesticus (13,0\%).

Die Xylophagen waren auch in den STE-Proben an Eiche nur in geringer Anzahl anzutreffen. Auf die 24 Arten (9,7 \%) entfielen 2,3 \% der Individuen. Mit 46 Individuen war Xestobium rufovillosum der häufigste Vertreter dieses Konsumententyps.

76 (43,9 \%) der in EFs-Proben an stehendem Buchentotholz gefangenen Arten waren den Zoophagen zuzurechnen. Sie stellten 31,8 \% der Individuen (1995: 33,4 \%; 1996: 30,7 \%). Die ermittelte Schlüpfdichte belief sich auf 269,4 Ind. $/ \mathrm{m}^{2}$. Ein Drittel der Individuen entfiel auf Rhizophagus dispar, daneben waren Paromalus flavicornis (10,9\%), Cerylon ferrugineum (9,6 \%), Rhizophagus bipustulatus (9,1 \%) und Corticeus unicolor (8,7 \%) in beiden Untersuchungsjahren in größerem Ausmaße am Fang dieses Konsumententyps beteiligt.

Individuenreicher als die Räuber traten die mycetophagen Arten in Erscheinung. Sie schlüpften in einer Dichte von 365,1 Ind. $/ \mathrm{m}^{2}$ und erreichten so einen Individuenanteil von $43,1 \%$, die sich auf 50 Arten $(28,9 \%)$ verteilten. In beiden Untersuchungsjahren waren Corticaria longicollis (45,6 \%) und Cryptophagus dentatus (15,5\%) die häufigsten Vertreter dieser Gruppe.

Die xylophagen Arten erreichten, anders als in den STE-Proben an Buche, am stehenden Totholz größere Bedeutung. Die 23 Arten (13,3\%) waren mit einem Individuenanteil von $23,0 \%$ in den Proben vertreten. Es wurde eine Schlüpfdichte von 194,5 Ind. $/ \mathrm{m}^{2}$ festgestellt. Der Individuenanteil belief sich 1995 auf 13,6 und 1996 auf 29,9 \%. 86,7 \% der gefangenen Tiere wurden von Ptilinus pectinicornis gestellt.

Die Artenanteile der einzelnen Konsumententypen in den EFs-Proben an stehendem Eichentotholz entsprachen weitgehend den Verhältnissen an der Buche. Deutliche Unterschiede waren jedoch bei den Individuenanteilen zu verzeichnen. Mit einer Schlüpfdichte von 103,0 Ind. $/ \mathrm{m}^{2}$ waren die 49 räuberischen Arten (43,8 \%) mit nur 14,7\% am Fangergebnis beteiligt. Der häufigste Vertreter dieses Ernährungstyps war Teredus cylindricus $(26,3 \%)$, gefolgt von Rhizophagus bipustulatus (18,1\%) und Rh. dispar (15,8 \%).

Die mycetophagen Arten stellten mit einem Artenanteil von 28,6 \% einen Individuenanteil von 26,0 \%. Die Schlüpfdichte betrug 182,3 Ind. $/ \mathrm{m}^{2}$. Corticaria longicollis $(41,4 \%)$ und Xyloterus signatus $(23,4 \%)$ waren vorherrschend.

Mit 392,8 Ind. $/ \mathrm{m}^{2}$ erreichten die xylophagen Arten die höchste Schlüpfdichte. Die 18 festgestellten Arten waren mit 56,1 \% an den gefangenen Individuen beteiligt. 91,1\% der Individuen wurden allein von Dryocoetes villosus gestellt, weitere 4,8\% entfielen auf Lymexylon navale. 


\subsubsection{Biotop- und Habitatpräferenzen}

In den Eklektoren fingen sich 16 Arten, die eine Präferenz für offene Biotope zeigen. Mit nur 33 Individuen war ihr Anteil am Gesamtfang (0,07 \%) von untergeordneter Bedeutung. Die Bewohner verschiedener Feuchtbiotope, wie sie sich auch innerhalb des Schutzgebietes finden, wurden mit 56 Individuen $(0,12 \%)$ und 23 Arten festgestellt. Die häufigste Art dieser Gruppe, der Kurzdeckenflügler Amischa decipiens, ist auch in feuchten Wäldern zu Hause und wird hier u. a. in Baummulm gefunden. Mit 104 Arten und einem Individuenanteil von 7,4 \% waren eurytope Arten in den Eklektoren vertreten. Die überwiegende Zahl der Arten wurde nur in Einzelexemplaren gefangen. Lediglich Cryptophagus dentatus, Aridius nodifer und Dinerella elongata traten in größerer Anzahl auf. Zusammen waren diese drei Arten mit $5,8 \%$ an den Fängen beteiligt. Mehr als $92 \%$ der gefangenen Tiere verteilten sich auf 313 Arten, die eine Präferenz für verschiedene Waldbiotope zeigen.

Die Bewohner der Vegetation waren mit 55 Arten (12,0\%) und einem Individuenanteil von 1,2\% in den Eklektoren vertreten (Tab. 4-33, Abb. 4-24 u. 4-25). An Buchentotholz wurden 49 Arten (12,3\%) und an der Eiche 31 Arten (10,4\%) registriert, die aber nur 1,1 bzw. 1,5 \% der Individuen stellten. Mit 40 Arten wurde der höchste Arten- (13,6 \%) und Individuenanteil $(3,4 \%)$ in den mBE-Proben festgestellt. In den einzelnen Untersuchungsjahren variierte der Artenanteil zwischen 8,3 und 12,4\%, der Individuenanteil zwischen 0,8 und 1,7 \%. Der überwiegende Teil der Fänge fiel in die ersten Fangperioden. An den Fängen der Fangperiode P1b waren die Bewohner der Vegetation mit 30 Arten vertreten, die 3,3\% der Individuen stellten (Tab. 4-34). Allein 40,0 \% der Individuen dieser Präferentengruppe wurden in dieser Fangperiode gefangen. Im April/Mai fingen sich viele Exemplare von Rhynchaenus fagi in den Eklektoren, der in nicht geringer Anzahl das Totholz zur Überwinterung nutzte. Er stellte etwa ein Viertel der Individuen dieser Präferentengruppe.

Bodenbewohner wurden mit 1928 Individuen aus 77 Arten (16,8 \%) gefangen. Mit einem Individuenanteil von 4,0 \% waren sie die dritthäufigste Gruppe in den Eklektoren. Die Schlüpfdichte betrug 6,6 Ind. $/ \mathrm{m}^{2}$. An Buchentotholz waren 16,8 \% der Arten und 3,2\% der Individuen zu den Bodenbewohnern zu rechnen, an Eichentotholz beliefen sich die Anteile auf 18,1 und 5,6 \%. Die höchsten Arten- und Individuenanteile waren an liegendem Totholz stärkerer Dimension zu verzeichnen. An den Fangergebnissen der einzelnen Fangperioden waren die Bodenbewohner mit 12,7 bis $20,1 \%$ der Arten und mit 2,9 bis 6,8\% der Individuen beteiligt. 50,0 \% der Individuen wurden in den Fangperioden P1b bis P3 gefangen. 68,8 \% der Arten und 92,6 \% der Individuen werden den Räubern zugerechnet. Sowohl an Eichen- als auch an Buchentotholz waren die Scydmaeniden Stenichnus collaris und Neuraphes elongatulus die häufigsten Vertreter dieser Habitatpräferentengruppe.

Sieht man von den durch ein Einzelexemplar vertretenen Wasserbewohnern ab, hatten die Arten mit einer Präferenz für Nester unterschiedlichen Ursprungs den geringsten Individuenanteil. 0,2 \% der Individuen verteilten sich auf 19 Arten. Ebenfalls gering war der Anteil der Faulstoffbesiedler, die lediglich in 147 Exemplaren (0,3\%) gefangen wurden, die aber auf 44 Arten verteilt waren, so dass fast jede 10. Art dieser Gruppe zuzurechnen war. Pilze (ohne Totholzpilze) werden von 18 Arten bevorzugt als Lebensstätte gewählt. Gemeinsam hatten sie einen Individuenanteil von 0,7 \%. Die häufigsten Repräsentanten dieser Gruppe waren Enicmus rugosus und Agathidium seminulum. 
Tabelle 4-33: Arten- und Individuenzahlen der Habitatpräferenztypen

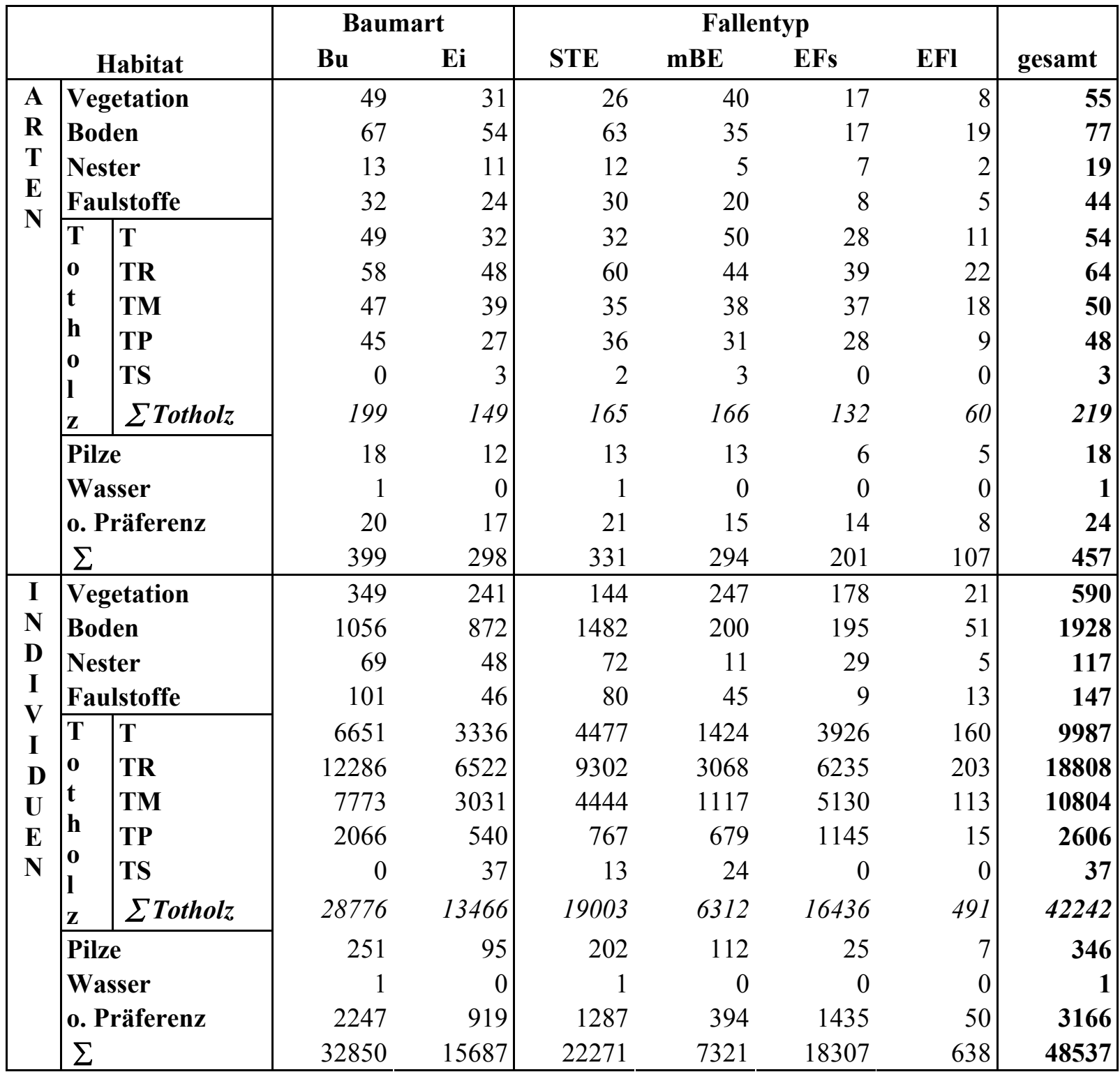

24 Arten (5,3\%) mit einem Individuenanteil von 6,5\% lassen keine Präferenzen für bestimmte Habitate erkennen. Sie finden geeignete Lebensbedingungen an sehr unterschiedlichen Orten. Die 3166 gefangenen Individuen schlüpften in einer Dichte von 10,8 Ind./ $\mathrm{m}^{2}$. 20 Arten (5,0 \%) wurden an Buchentotholz gefangen, die 6,8 \% der Individuen stellten. An Eichentotholz wurden 17 Arten (5,7 \%) mit einem Individuenanteil von 5,9\% festgestellt. Der Artenanteil in den eingesetzten Fallentypen lag zwischen 5,1 und 7,5 \%, der Individuenanteil zwischen 5,4 und 7,8 \%. In den einzelnen Untersuchungsjahren wurden zwischen 5,1 und $7,7 \%$ der Individuen von dieser Gruppe gestellt. Die Fangzahlen nahmen von Beginn der Vegetationsperiode an zunächst zu, erreichten in der Fangperiode P5 das Maximum und gingen danach kontinuierlich zurück. 66,7 \% der Arten waren den Mycetophagen zuzurechnen, die 95,2 \% der Individuen dieser Gruppe stellten. Die dominierenden Arten waren an beiden Baumarten Cryptophagus dentatus, Aridius nodifer und Dinerella elongata. 


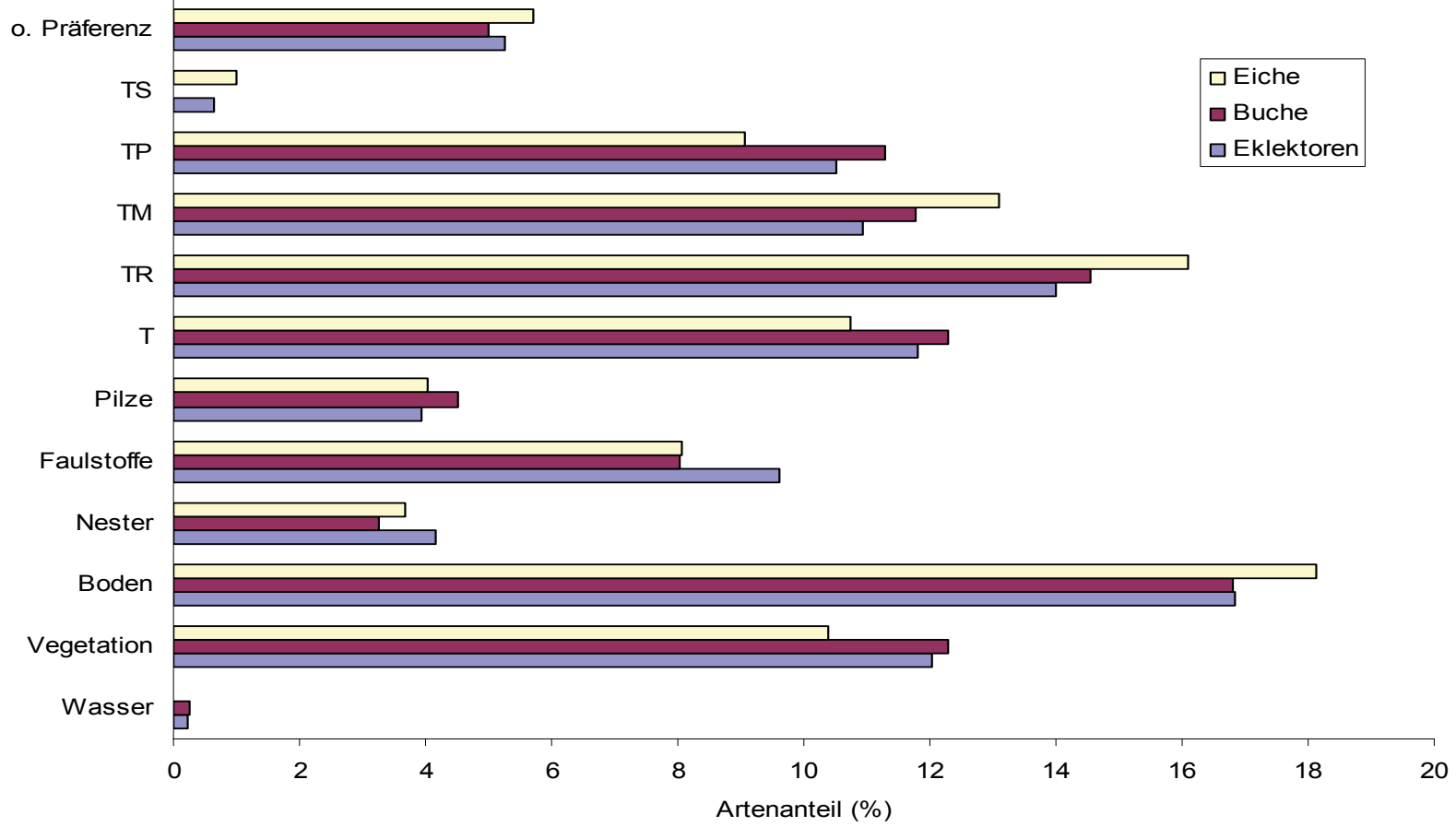

Abb. 4-24: Artenanteile der Habitatpräferenztypen

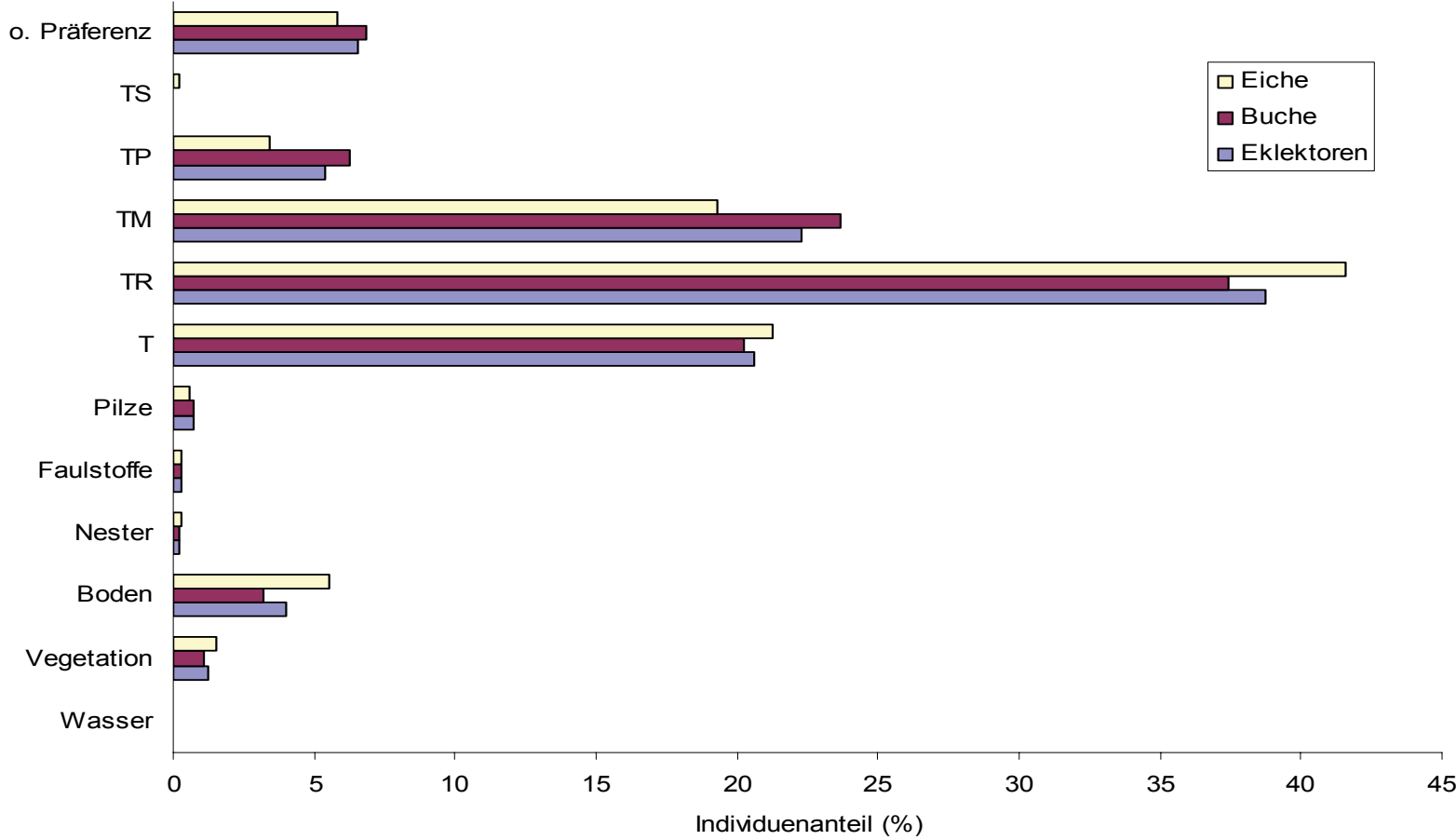

Abb. 4-25: Individuenanteile der Habitatpräferenztypen 
Tabelle 4-34: Arten- und Individuenanteile der Habitatpräferenztypen in den Fangperioden

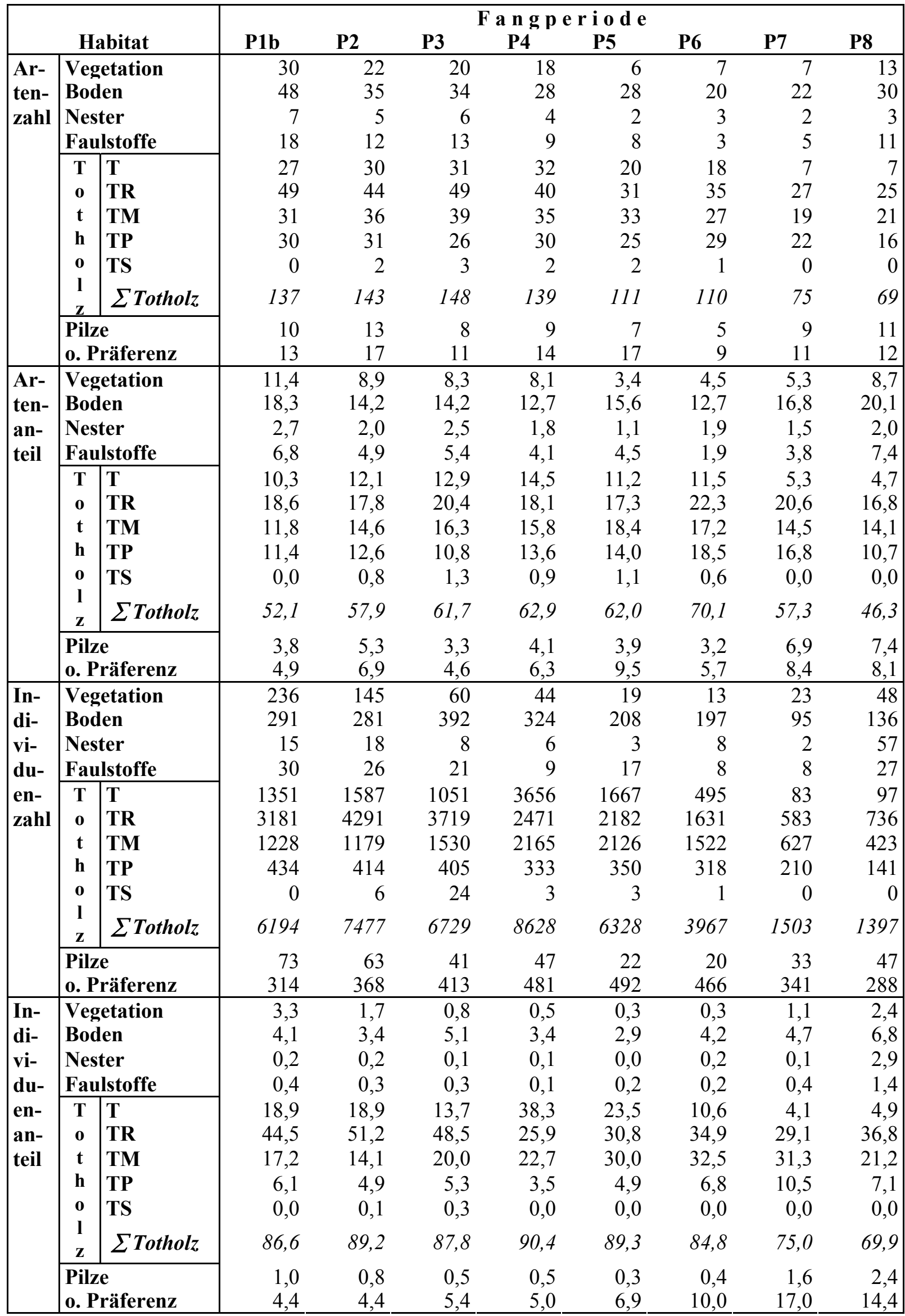


Die obligaten Totholzbewohner nahmen sowohl hinsichtlich ihrer Artenzahl als auch der Individuenmenge die eindeutig vorherrschende Stellung ein. Insgesamt wurden 219 xylobionte Arten, das entspricht einem Artenanteil von 47,9 \%, mit den Eklektoren erfaßt. Sie stellten 87,0 \% der Individuen. An Buchentotholz waren 49,9\% der Arten und 87,6 \% der Individuen den obligaten Totholzbewohnern zuzurechnen, an Eichentotholz beliefen sich die Anteile auf 50,0 und 85,8 \%. Der höchste Arten- und Individuenanteil wurde jeweils in den EFs-Proben registriert. Auch in den einzelnen Untersuchungsjahren waren nur geringe Unterschiede festzustellen. Der Artenanteil an den Jahresergebnissen variierte zwischen 52,2 und $54,5 \%$, der Individuenanteil zwischen 81,1 und 88,0 \%.

Die Anzahl gefangener Totholzkäfer war in der ersten Hälfte der Vegetationsperiode am höchsten. Der Artenanteil der xylobionten Käfer in den einzelnen Fangperioden bewegte sich zwischen 46,3 (P8) und 70,1 \% (P6), die Artenzahlen zwischen 69 (P8) und 148 (P3). Der Individuenanteil variierte zwischen 69,9 (P8) und 90,4\% (P4). Mit einem Artenanteil von 42,5\% und einem Individuenanteil von 40,3 \% waren die Zoophagen die dominierende Konsumentengruppe unter den Totholzbewohnern. Der Individuenanteil der Mycetophagen betrug 36,6 \%, der der Xylophagen 19,9 \% (Abb. 4-27 u. 4-28).

Für die 219 xylobionten Käferarten läßt sich eine weitergehende Differenzierung nach den bevorzugt besiedelten Strukturteilen des Totholzes vornehmen. Corticole Arten (TR) halten sich vor allem in oder unter der Rinde bzw. Borke auf. Lignicole Arten (T) finden sich vermehrt in noch relativ festen Holzteilen, während die xylodetricolen Arten (TM) mulmartiges Material präferieren. $\mathrm{Zu}$ den Totholzbewohnern werden auch die zahlreichen an Baumpilze gebundene Arten (TP) gerechnet, sowie Arten, die sich vor allem an austretendem Baumsaft finden.

Die höchste Arten- und Individuenzahl wurde von den Rindenbewohnern gestellt. 64 corticole Arten (14,0 \%) waren mit 38,7 \% der Individuen am Fangergebnis beteiligt. An Buchentotholz gehörten 14,5 \% der Arten und 37,4 \% der Individuen zu dieser Präferentengruppe, an Eichentotholz beliefen sich die Anteile auf 16,1 und 41,6 \%. Pro $\mathrm{m}^{2}$ Rindenfläche schlüpften an Eiche 150,4, an Buche 111,6 Individuen. Der Artenanteil in den einzelnen Fangperioden variierte zwischen 16,8 (P8) und 22,3\% (P6), der Individuenanteil zwischen 25,9 (P4) und 51,2 \% (P2). 59,5\% der Individuen wurden in den Fangperioden P1b bis P3 gefangen. $69 \%$ der Arten und Individuen dieser Gruppe werden den Räubern zugerechnet (Abb. 4-27 und 4-28). 13 Arten mit einem Individuenanteil von 14,7 \% gelten als xylophag, die mycetophagen Arten stellten 9,9\% der Individuen. Die dominierenden Arten waren Rhizophagus dispar, Dryocoetes villosus und Orchesia undulata (Abb. 4-26). 


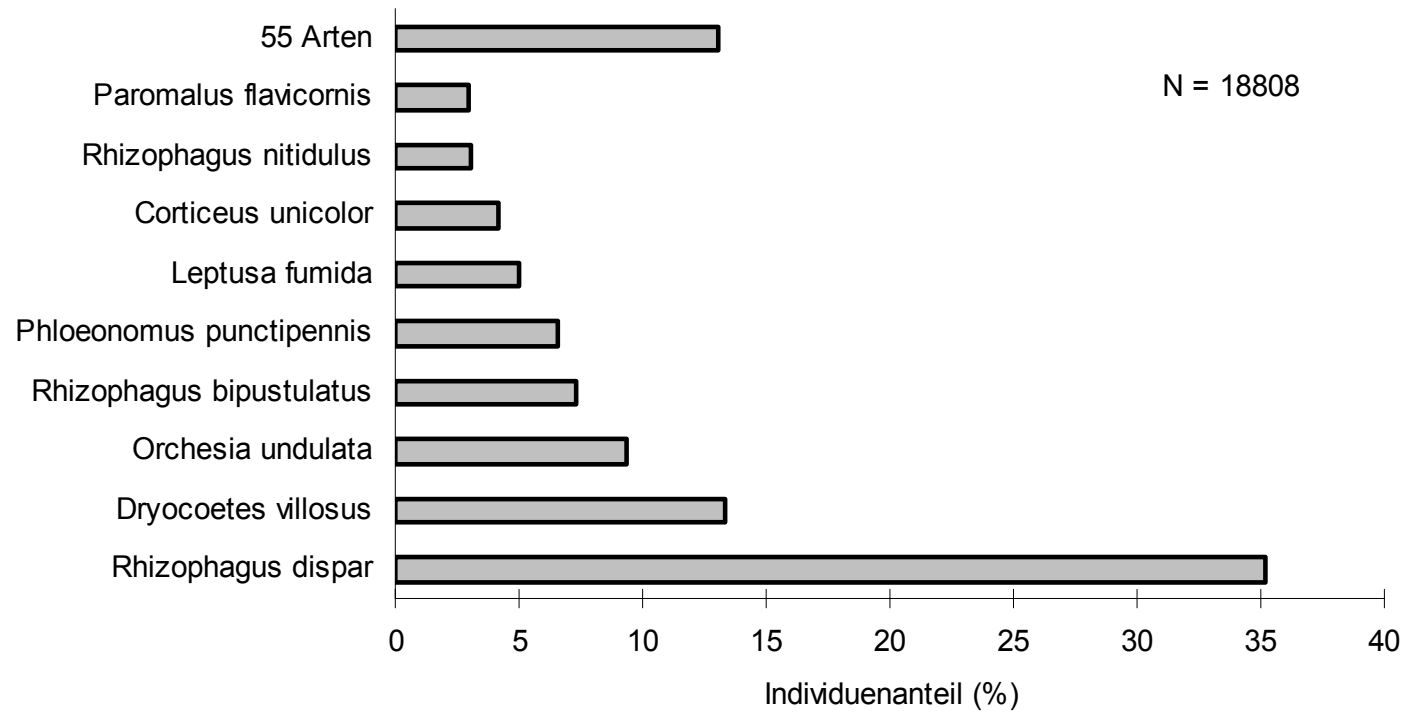

Abb. 4-26: Dominanzstruktur der corticolen Arten

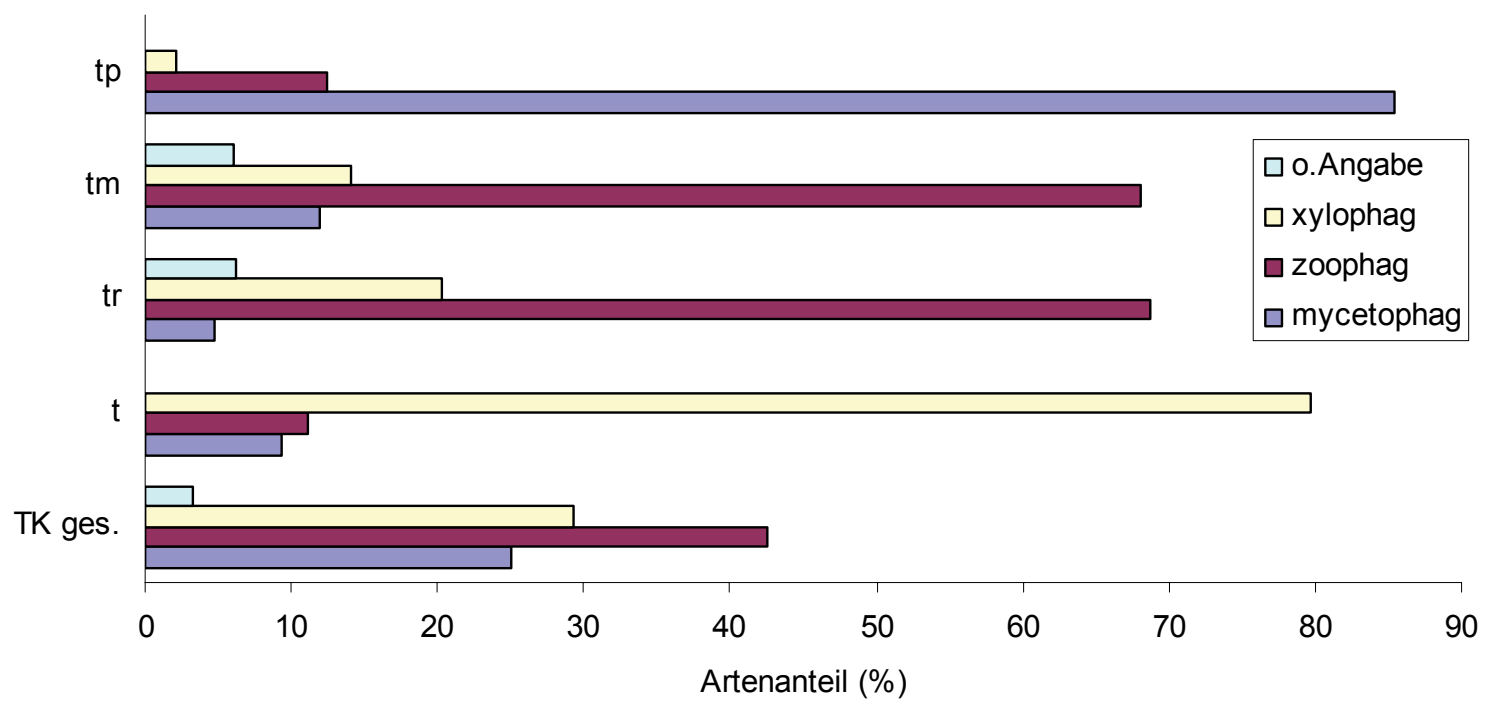

Abb. 4-27: Artenanteile der Konsumentengilden an den Habitatpräferenztypen

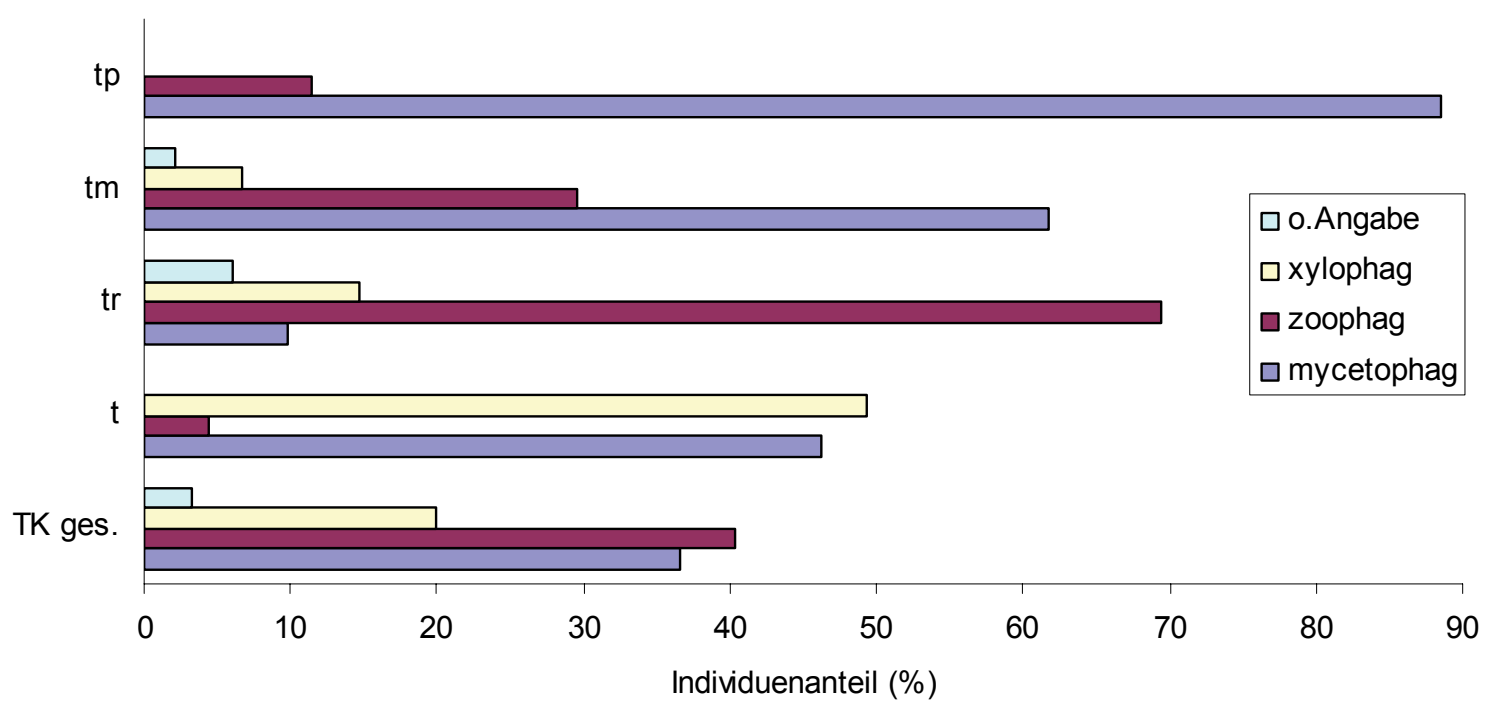

Abb. 4-28: Individuenanteile der Konsumentengilden an den Habitatpräferenztypen 
54 lignicole Arten (11,8 \%) hatten einen Individuenanteil von 20,6 \%. Arten- und Individuenanteil stimmten an beiden untersuchten Baumarten annähernd überein. Die höchsten Artenzahlen und -anteile wurden in den Fangperioden P2 bis P4 verzeichnet. In der Fangperiode P4 wurde auch der höchste Individuenanteil mit 38,3 \% erreicht. Mehr als ein Drittel der gefangenen Tiere dieser Gruppe wurden alleine in diesem Fangzeitraum erfaßt. 79,6 \% der Arten mit einem Individuenanteil von 49,3\% gelten als xylophag. Die Mycetophagen hatten einen Individuenanteil von 46,3 \%, waren aber mit nur 5 Arten vertreten. Die restlichen 4,4 \% entfielen auf 6 zoophage Arten. Die häufigsten lignicolen Arten waren Ptilinus pectinicornis, Xyloterus signatus, Xyl. domesticus und Hylecoetus dermestoides (Abb. 4-29).

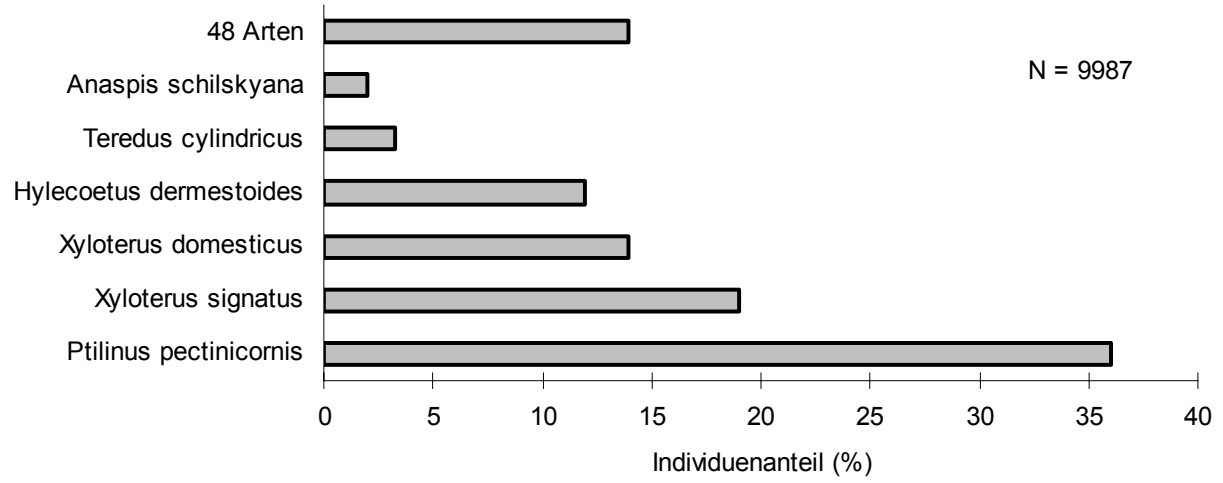

Abb. 4-29: Dominanzstruktur der lignicolen Arten

$22,3 \%$ der Individuen wurden von den 50 (10,9\%) festgestellten xylodetricolen Arten zum Fangergebnis der Eklektoren beigetragen. Der Individuenanteil an der Buche betrug 23,7 \%, der an Eichentotholz 19,3 \%. Der höchste Arten- und Individuenanteil wurde am stehenden Totholz registriert. In den Fangperioden P4 und P5 wurden jeweils $20 \%$ der Fänge dieser Gruppe gemacht. 34 Arten mit einem Individuenanteil von 29,6 \% gelten als zoophag. $61,7 \%$ der Individuen entfielen auf nur 6 mycetophage Arten. An beiden Baumarten häufig waren Corticaria longicollis, Cerylon ferrugineum, Ptinella limbata und Pteryx suturalis. Daneben waren Ptinella aptera an Buchentotholz und Aderus oculatus an Eichentotholz zahlreich vertreten (Abb. 4-30).

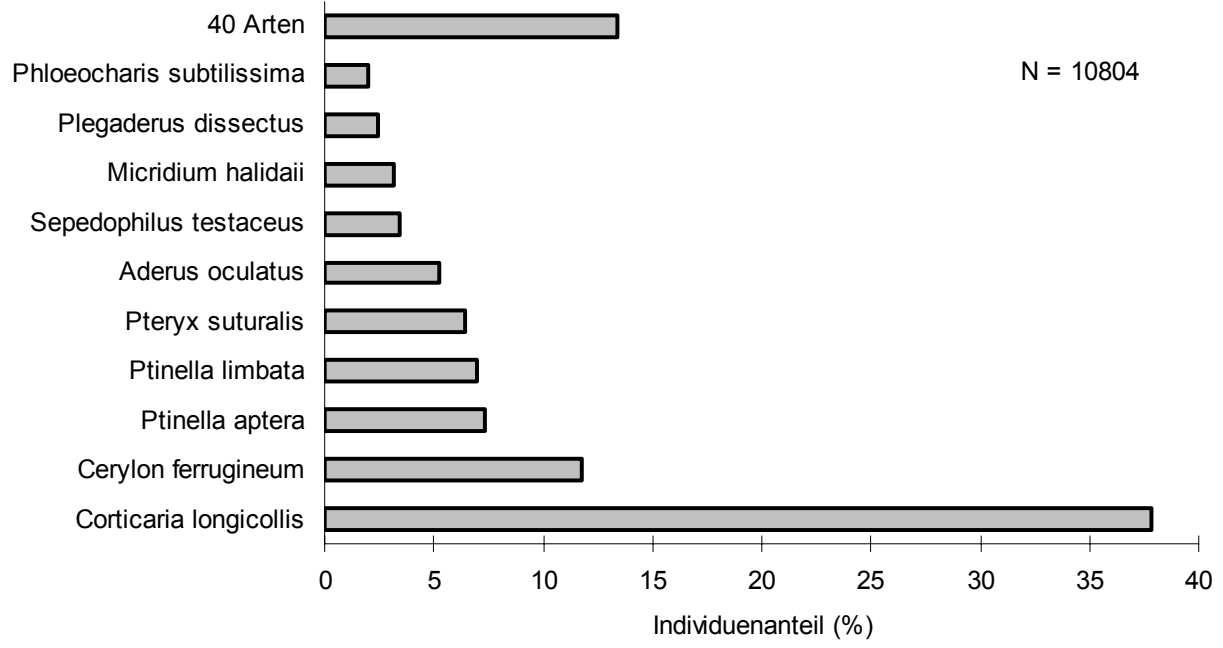

Abb. 4-30: Dominanzstruktur der xylodetricolen Arten 
48 Arten mit einem Individuenanteil von 5,4 \% zeigen eine Präferenz für Totholzpilze. An Eichentotholz waren 27 Arten mit einem Individuenanteil von 3,4 \%, an Buchentotholz 45 Arten mit einem Anteil von 6,3\% vertreten. In den einzelnen Fangperioden variierte der Artenanteil zwischen 10,7 (P8) und 18,5 \% (P6), der Individuenanteil zwischen 3,5 (P4) und $10,5 \%$ (P7). Im Verlauf der Vegetationsperiode war ein kontinuierlicher Rückgang der Fangzahlen festzustellen. 88,5 \% der Individuen wurden von 41 mycetophagen Arten gestellt, die restlichen 11,4 \% der Individuen entfielen auf Zoophage. Die dominierende Art war Cis nitidus, die allerdings fast ausschließlich an Buchentotholz gefangen wurde. Bolitochara obliqua und Cis castaneus waren an beiden Baumarten zahlreich vertreten (Abb. 4-31).

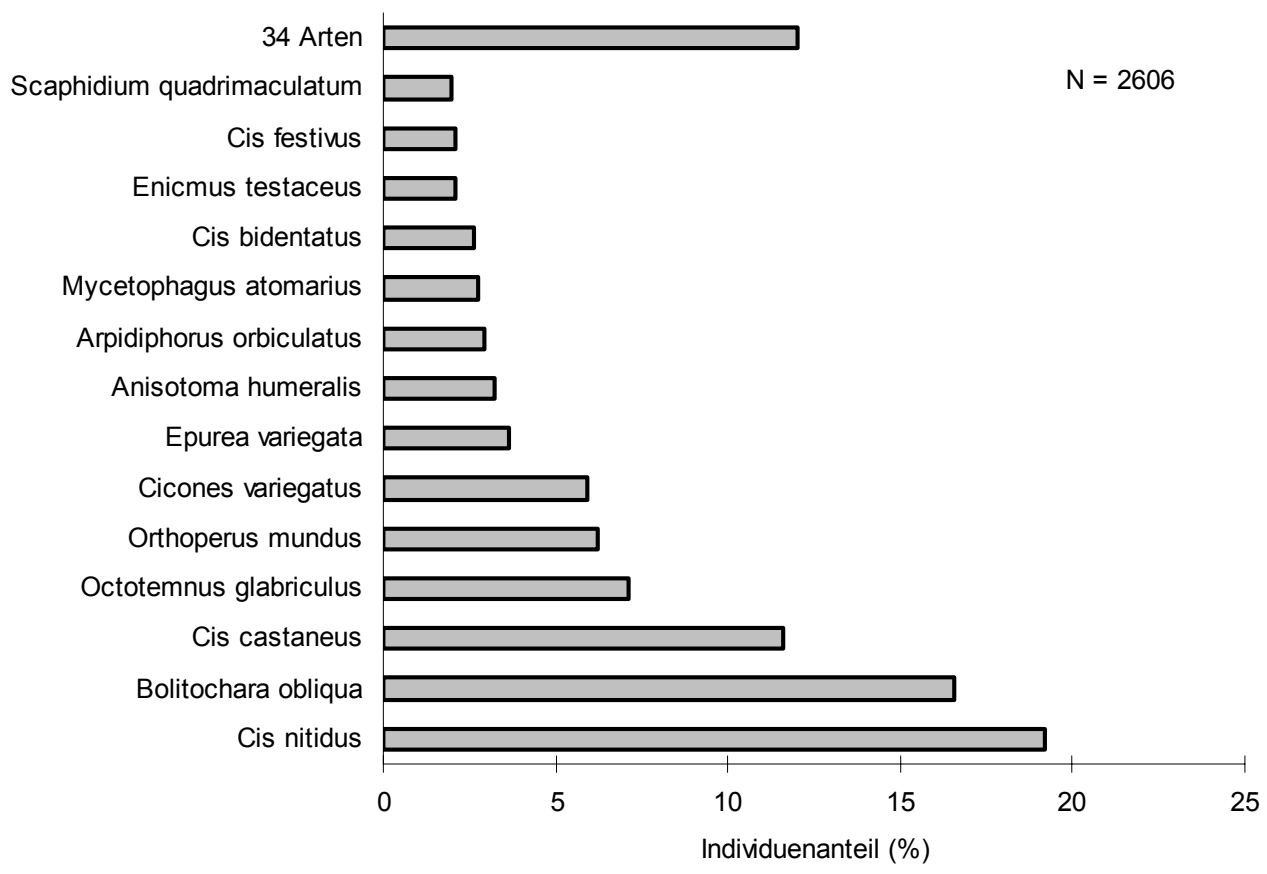

Abb. 4-31: Dominanzstruktur der xylofungicolen Arten

In den STE-Proben an liegendem Buchentotholz waren die Totholzkäfer mit 143 Arten (55,6 \%) vertreten, die 88,3 \% der Individuen stellten. Die Schlüpfdichte belief sich auf 162,7 Ind. $/ \mathrm{m}^{2}$. 1995 stellten 107 Arten 86,4 \% der Individuen, 1996 erreichten 126 Arten gemeinsam einen Individuenanteil von $90,0 \%$.

Vorherrschend waren die Rindenbewohner, die allein mit 21,4\% der Arten und 49,5\% der Individuen zum Fangergebnis beitrugen (Abb. 4-32 u. 4-33). Mit 23,6 \% war der Artenanteil in beiden Untersuchungsjahren übereinstimmend, die Individuenanteile beliefen sich auf 47,8 (1995) und 51,0 \% (1996). Auf die berindete Fläche bezogen schlüpften 114,8 Ind./ $\mathrm{m}^{2}$. $56,8 \%$ der Tiere wurden durch Rhizophagus dispar repräsentiert.

Mulmbewohner traten mit 31 Arten (12,1\%) auf, die 19,9\% der Individuen stellten. 1995 wurden 26 Arten und 199628 Arten festgestellt, die mit 23,4 bzw. 16,7 \% der Individuen zum Fangergebnis beitrugen. Die Schlüpfdichte belief sich auf 36,7 Ind. $/ \mathrm{m}^{2}$. Die häufigsten Vertreter dieser Gruppe waren Cerylon ferrugineum, Pteryx suturalis und Ptinella limbata. 
24 lignicole Arten (9,3\%) trugen mit 14,8 \% zum Fangergebnis bei. Pro $\mathrm{m}^{2}$ befangener Mantelfläche wurden 27,3 Individuen erfaßt. 1995 entfielen 11,1 \% der gefangenen Individuen auf diese Präferentengruppe, 1996 wurde ein Anteil von 18,3 \% registriert. Mit 765 gefangenen Exemplaren war Hylecoetus dermestoides die dominierende lignicole Art, 718 Individuen entfielen auf Xyloterus domesticus, 268 auf Ptilinus pectinicornis.

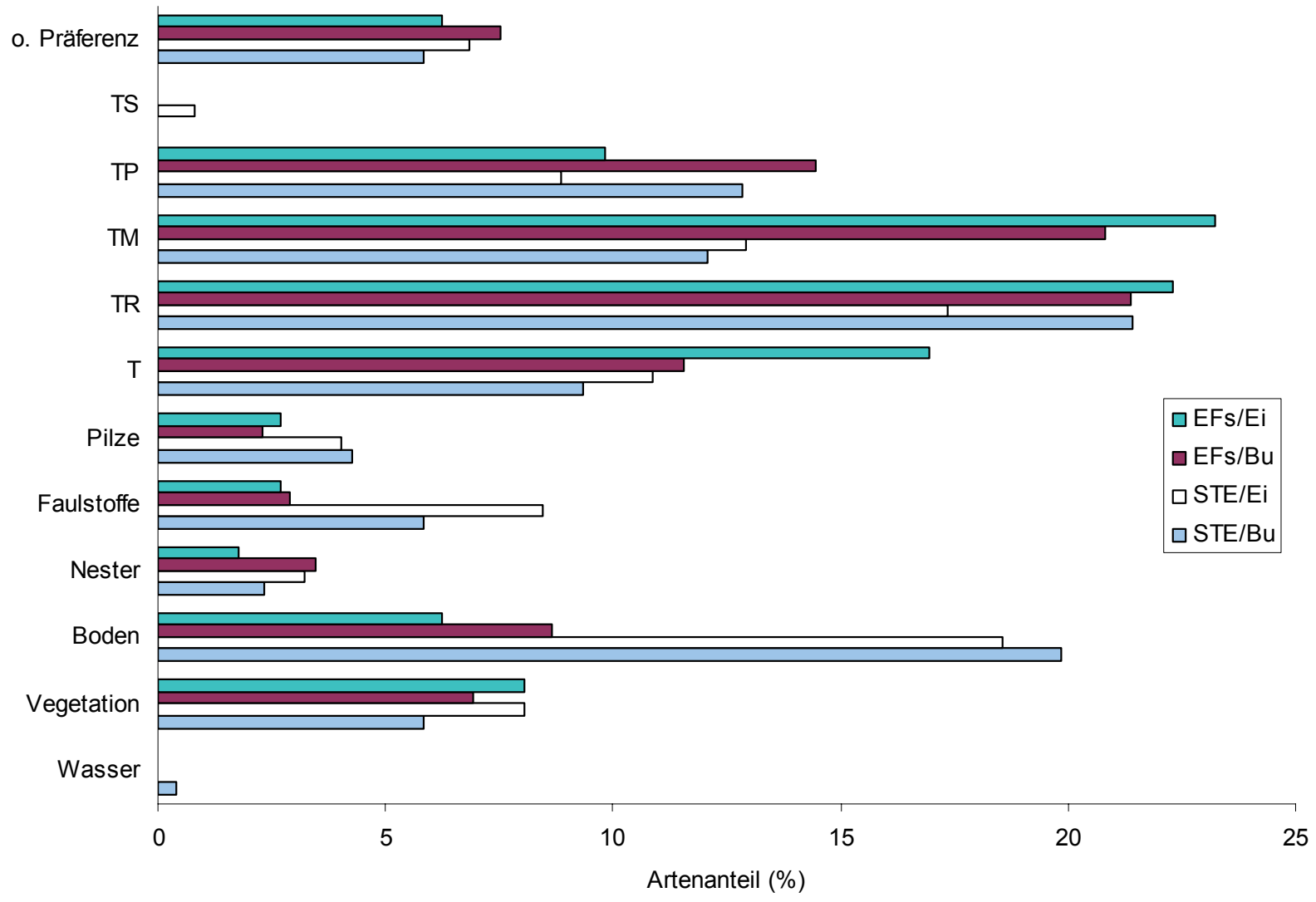

Abb. 4-32: Artenanteile der Habitatpräferenztypen an liegendem und stehendem Buchenund Eichentotholz

Bewohner von Totholzpilzen wurden mit 33 Arten (12,8\%) und einem Individuenanteil von $4,1 \%$ festgestellt. Die häufigsten Vertreter waren Octotemnus glabriculus und Bolitochara obliqua.

Die übrigen Präferentengruppen hatten gemeinsam einen Artenanteil von 44,4\%, ihr Individuenanteil belief sich aber auf nur 11,7\%. Die Bodenbewohner waren mit 51 Arten $(19,8 \%)$ und einem Individuenanteil von 5,4 \% vertreten. Stenichnus collaris war der häufigste Vertreter dieser Gruppe. 4,5 \% der Individuen wurden von Arten gestellt, die keine Präferenz für ein bestimmtes Habitat erkennen lassen. Unter diesen waren vor allem Aridius nodifer und Cryptophagus dentatus in größerer Anzahl beteiligt.

In den STE-Proben an liegendem Eichentotholz hatten 126 xylobionte Arten (50,8\%) einen Individuenanteil von 80,6 \%. Es schlüpften 123,9 Ind./m². 43 Arten (17,3\%) werden zu den Rindenbewohnern gezählt, die $29,5 \%$ der Individuen stellten. Pro $\mathrm{m}^{2}$ Rindenfläche wurden 97,3 Individuen erfaßt. Wie auch am liegenden Buchentotholz waren Rhizophagus dispar, Rh. bipustulatus und Phloeonomus punctipennis die häufigsten Vertreter. 
Die lignicolen Arten hatten einen Individuenanteil von 28,5\%, die sich auf 27 Arten (10,9\%) verteilten. Die Schlüpfdichte belief sich auf 43,9 Ind. $/ \mathrm{m}^{2}$. Dominierend waren die pilzzüchtenden Borkenkäferarten Xyloterus signatus und Xyl. domesticus.

Die Mulmbewohner wurden mit 32 Arten (12,9\%) und einem Individuenanteil von 20,0 \% festgestellt. 30,8 Individuen wurden pro $\mathrm{m}^{2}$ befangener Mantelfläche erfaßt. Ein Drittel der gefangenen Tiere wurde von Corticaria longicollis gestellt. Daneben waren Ptinella limbata, Pteryx suturalis und Cerylon ferrugineum häufige Vertreter dieser Gruppe.

Besiedler von Totholzpilzen waren in den STE-Proben an Eichentotholz in geringerer Artenzahl und Individuendichte vertreten als an der Buche. 22 Arten hatten einen Anteil von 2,4 \% am Fangergebnis. Es wurden nur 3,8 Ind./ $\mathrm{m}^{2}$ registriert. Am häufigsten waren Bolitochara obliqua und Orthoperus mundus zu beobachten.

Unter den Nicht-Totholzkäfern waren insbesondere die Bodenbewohner artenreich vertreten. Die 46 Arten (18,5\%) hatten gemeinsam einen Individuenanteil von 8,7 \%. Die am häufigsten vorgefundenen Arten entsprachen denen an Buchentotholz. Vorherrschend war hier allerdings Neuraphes elongatulus. Die 17 Arten (6,9\%) ohne erkennbare Präferenz für bestimmte Habitate hatten einen Individunanteil von 7,8 \%. Cryptophagus dentatus und Aridius nodifer waren auch an der Eiche vorherrschende Vertreter dieser Gruppe.

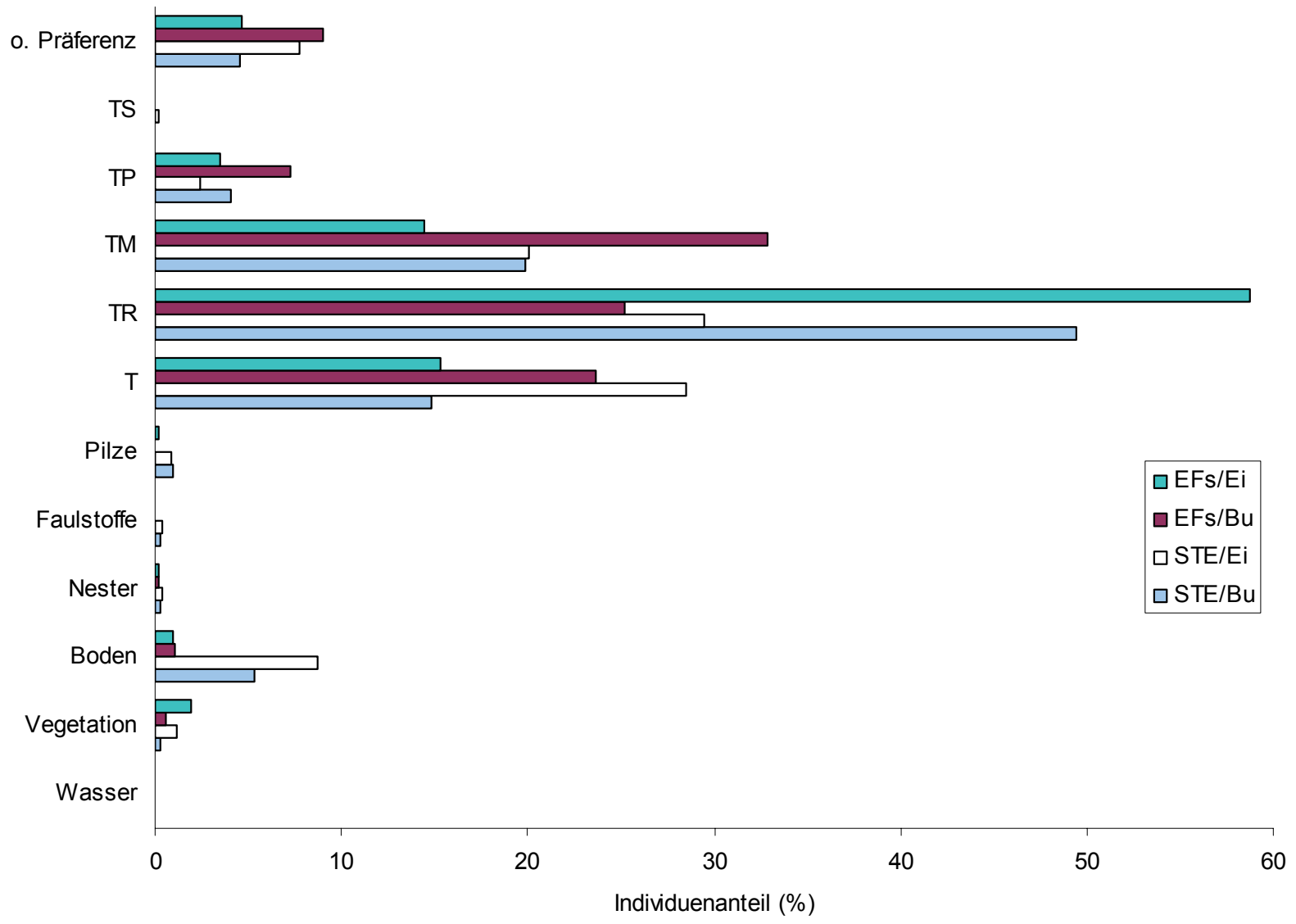

Abb. 4-33: Individuenanteile der Habitatpräferenztypen an liegendem und stehendem Buchen- und Eichentotholz 
In den EFs-Proben an stehendem Buchentotholz hatten die Totholzkäfer einen Artenanteil von 68,2 \% (118 Arten) und einen Individuenanteil von 89,0\%. Pro $\mathrm{m}^{2}$ Probefläche schlüften 753,5 Tiere.

Die Rindenkäfer waren mit 37 festgestellten Arten (21,4\%) die artenreichste Gruppe, auf die ein Viertel der gefangenen Tiere entfielen. 1995 wurde von 28 Arten ein Individuenanteil von $28,0 \%$ erreicht, 1996 stellten 31 Arten 23,1\% der Individuen. Pro $\mathrm{m}^{2}$ Rindenfläche wurde im ersten Jahr eine Schlüpfdichte von 344,7, im zweiten von 274,5 Individuen gemessen. 41,1\% der gefangenen Rindenkäfer entfielen auf Rhizophagus dispar, 13,7 \% auf Paromalus flavicornis, 11,5\% auf Rhizophagus bipustulatus und 11,1\% auf Corticeus unicolor.

Ein Drittel der gefangenen Individuen waren den Mulmbewohnern zuzurechnen. Die 36 Arten traten in einer Dichte von 278,5 Ind. $/ \mathrm{m}^{2}$. 1995 wurden 31 Arten mit einem Individuenanteil von $39,7 \%$ in einer Dichte von 351,4 Ind. $/ \mathrm{m}^{2}$ festgestellt, 1996 traten 33 Arten mit einem Anteil von 27,9 \% und einer Dichte von 228,6 Ind./ $\mathrm{m}^{2}$ auf. $60 \%$ der Individuen waren allein auf die Fänge von Corticaria longicollis zurückzuführen.

20 lignicole Arten stellten 23,7 \% der Individuen. 1995 entfielen 14,9 \% des Fangergebnisses auf diese Habitatpräferentengruppe, 1996 war sie mit einem Anteil von 30,1 \% vorherrschend in den EFs-Proben an Buche. Die Tiere traten in einer Dichte von 200,4 Ind./ $\mathrm{m}^{2}$ auf (1995: 132,1 Ind. $/ \mathrm{m}^{2}$, 1996: 247,1 Ind./m²). Ptilinus pectinicornis war die dominierende Art, die mit 84,1 \% der Individuen zum Fangergebnis dieser Guppe beitrug.

Die Präferenten für Totholzpilze traten mit 25 Arten in Erscheinung und hatten einen Individuenanteil von 7,2 \%. 61,4 Individuen wurden pro $\mathrm{m}^{2}$ Probefläche registriert (1995: 50,0 Ind. $/ \mathrm{m}^{2}, 1996:$ 69,2 Ind. $\left./ \mathrm{m}^{2}\right)$. Cis nitidus, C. castaneus und Cicones variegatus waren die häufigsten Vertreter dieser Gruppe an stehendem Buchentotholz.

Neben den obligaten Totholzbewohnern hatten nur einige wenige Arten, die sich keiner Präferentengruppe zuordnen ließen, höhere Anteile am Fangergebnis. Neben Cryptophagus dentatus war, anders als in den STE-Proben, Dinerella elongata in Anzahl zu verzeichnen, wohingegn Aridius nodifer nur in geringer Dichte auftrat.

In den EFs-Proben an stehendem Eichentotholz waren die Totholzkäfer mit 81 Arten und 4450 Individuen vertreten. Damit wurde hier der höchste Arten- (72,3\%) und Individuenanteil (92,0 \%) registriert. Die Schlüpfdichte belief sich auf 644,5 Ind. $/ \mathrm{m}^{2}$ und lag damit um über 100 Ind. $/ \mathrm{m}^{2}$ niedriger als an der Buche.

$58,7 \%$ der Individuen wurde von 25 rindenbewohnenden Arten (22,3\%) gestellt. Bedingt durch das massenhafte Auftreten von Dryocoetes villosus in einigen wenigen Proben wurde mit 939,7 Individuen pro $\mathrm{m}^{2}$ Rindenfläche eine deutlich höhere Schlüpfdichte als an stehendem Buchentotholz registriert.

26 Arten (23,2 \%) mit einem Individuenanteil von 14,5 \% waren zu den Mulmbewohnern zu zählen. Es wurden 101,4 Ind. $/ \mathrm{m}^{2}$ gefangen. $74,4 \%$ der Tiere wurden von Corticaria longicollis gestellt, $8,3 \%$ von Cerylon ferrugineum.

Die 19 lignicolen Arten (17\%) schlüpften in einer Dichte von 107,0 Ind. $/ \mathrm{m}^{2}$ und erreichten so einen Individuenanteil von 15,3\%. Auf Xyloterus signatus entfielen 39,9\% der Individuen, Teredus cylindricus trug mit 25,3 und Lymexylon navale mit 17,5 \% zum Fangergebnis dieser Gruppe bei. 
3,5 \% der Individuen entfielen auf die Präferenten für Totholzpilze, die mit 11 Arten vertreten waren. Cis castaneus und Enicmus testaceus herrschten vor.

Wie schon an stehendem Buchentotholz waren nur noch Arten ohne erkennbare Habitatpräferenz in größerer Anzahl vertreten. Die 7 festgestellten Arten hatten einen Individuenanteil von 4,7 \%. Wie auch in den EFs-Proben an Buchentotholz war Cryptophagus dentatus am häufigsten anzutreffen, gefolgt von Dienerella elongata und Aridius nodifer.

\subsection{Artenassoziation und -affinität}

Das gemeinschaftliche räumliche Auftreten der Arten kann u. a. durch unmittelbare Wechselwirkungen (z. B. Räuber-Beute-Beziehungen) oder aber indirekt durch die Nutzung identischer Ressourcen verursacht sein. Das räumliche Nebeneinander von Artenpaaren wurde unter zwei Aspekten untersucht. Zum einen sollte der Frage nachgegangen werden, inwieweit eine Art zur biotischen Umwelt einer anderen Art gehört. Als Ausdruck dieses hier als Assoziation bezeichneten gemeinsamen Vorkommens der Arten wurde das gemeinsame Auftreten zweier Arten als prozentuale Überlappung berechnet. Beispielsweise war Rhizophagus dispar in allen Proben zu finden, in denen Hylecoetus dermestoides auftrat, während andererseits $H$. dermestoides in nur $22 \%$ der Proben mit Rh. dispar - Besiedlung schlüpfte (Tab. 4-39).

Der zweite, hier als Affinität bezeichnete Aspekt untersucht die Ähnlichkeit in der Verteilung von Artenpaaren. Als Maße hierfür wurden der Sørensen-Quotient (SQ) und der PHIKoeffizient ermittelt. Berechnungsgrundlage war jeweils die An- bzw. Abwesenheit der Arten unter Nichtbeachtung ihrer Häufigkeit. Es wurden nur die Fänge der STE- und EFs-Proben berücksichtigt und hieraus ausschließlich Arten herangezogen, die in mindestens 10 Proben zugegen waren. Unberücksichtigt blieb die Frage der phänologischen Überlappung.

Bei 103 berücksichtigten Arten ergaben sich 5253 mögliche Artenpaarungen, für die in der großen Mehrheit nur niedrige SQ-Werte ermittelt wurden (Tab. 4-35). In 34,9 \% der Fälle wurde ein SQ-Wert von 10,0 \% nicht überschritten, bei weiteren 32,7 \% der Artenpaarungen lag dieser Wert zwischen 10,0 und 20,0 \%. Nur in 2,8 \% der möglichen Artenkombinationen wurde ein SQ-Wert von mindestens 50,0 \% erreicht. In die Tabelle 4-37 sind in erster Linie diese Artenkombinationen aufgenommen worden.

Tabelle 4-35: Verteilung der Artenpaarungen auf die Ähnlichkeitsklassen (Sørensen-Quotient)

\begin{tabular}{|c|c|c|c|c|c|c|c|c|c|}
\hline & \multicolumn{9}{|c|}{$S Q-K I$ asse } \\
\hline & $\leq 10,0$ & $\begin{array}{c}10,1- \\
20,0 \\
\end{array}$ & $\begin{array}{c}20,1- \\
30,0\end{array}$ & $\begin{array}{c}30,1- \\
40,0 \\
\end{array}$ & $\begin{array}{c}40,1- \\
50,0 \\
\end{array}$ & $\begin{array}{c}50,1 \text { - } \\
60,0 \\
\end{array}$ & $\begin{array}{c}60,1- \\
70,0 \\
\end{array}$ & $\begin{array}{c}70,1 \text { - } \\
80,0 \\
\end{array}$ & $\begin{array}{c}80,1 \text { - } \\
90,0 \\
\end{array}$ \\
\hline Anz. & 1835 & 1717 & 919 & 435 & 202 & 100 & 36 & 6 & 3 \\
\hline$\%$ & 34,9 & 32,7 & 17,5 & 8,3 & 3,8 & 1,9 & 0,7 & 0,1 & 0,1 \\
\hline
\end{tabular}


Die berechneten PHI-Koeffizienten deuteten in ihrer Mehrheit auf fehlende Korrelationen zwischen den Arten hin (Tab. 4-36). In 61,5 \% der Fälle lag dieser Wert zwischen 0,9 und 1,1, während für nur 12 Artenpaarungen der Koeffizient unter 0,5 lag und somit am ehesten auf positive Korrelationen hinwies (Tab. 4-38). Auch hohe PHI-Koeffizienten als Hinweis auf negative Korrelationen waren die Ausnahme.

Tabelle 4-36: Verteilung der Artenpaarungen auf die PHI-Koeffizient-Klassen

\begin{tabular}{|l|ccccccc|}
\hline & \multicolumn{7}{|c|}{ P H I - K o e f f i z i e n t - K I a s s e } \\
& & $\mathbf{0 , 3 0 1}-$ & $\mathbf{0 , 5 0 1}-$ & $\mathbf{0 , 7 0 1}-$ & $\mathbf{0 , 9 0 1}-$ & $\mathbf{1 , 1 0 1}-$ & \\
& $\leq \mathbf{0 , 3 0 0}$ & $\mathbf{0 , 5 0 0}$ & $\mathbf{0 , 7 0 0}$ & $\mathbf{0 , 9 0 0}$ & $\mathbf{1 , 1 0 0}$ & $\mathbf{1 , 3 0 0}$ & $>\mathbf{1 , 3 0 0}$ \\
\hline Anz. & 0 & 12 & 204 & 1475 & 3231 & 330 & 1 \\
$\mathbf{\%}$ & 0,0 & 0,2 & 3,9 & 28,1 & 61,5 & 6,3 & 0,0 \\
\hline
\end{tabular}

Der Sørensen-Quotient wird maßgeblich von der Frequenz der Arten in den Proben beeinflusst. Nur Arten mit ähnlichen Frequenzprozentwerten können auch hohe SQ-Werte erreichen. Gleichzeitig erreichen Arten, die in der Mehrzahl der Proben auftreten, am leichtesten hohe Ähnlichkeitswerte. Mit zunehmender Frequenz der Arten war folglich ein tendenzieller Anstieg der SQ-Werte zu verzeichnen. Andererseits sind hohe SQ-Werte bei geringem Frequenzprozent am ehesten im Sinne einer signifikanten Affinität zu interpretieren.

Die höchsten SQ-Werte wurden denn auch von Arten erreicht, die in hoher Frequenz in den Proben in Erscheinung traten. So ließ sich eine große Übereinstimmung im räumlichen Auftreten von Corticaria longicollis und Cryptophagus dentatus mit einem SQ von $82 \%$ feststellen, während der PHI-Koeffizient 0,911 betrug und somit weder eine positive noch eine negative Korrelation anzeigte. Für die möglichen Paarbildungen der Arten mit einer Frequenz von über 100 überschritt der Sørensen-Quotient in allen Fällen den Wert von 50 \%. Gleichzeitig waren diese Arten regelmäßiger Bestandteil der biotischen Umwelt der meisten übrigen Arten. Für die überwiegende Mehrzahl der Arten galt, dass Corticaria longicollis, Rhizophagus dispar und Cryprophagus dentatus in großer Regelmäßigkeit gemeinsam mit ihnen in den Proben auftraten. Ebenfalls traten die meisten Arten häufig gemeinsam mit Rhizophagus bipustulatus, Cerylon ferrugineum, Leptusa fumida und Phloeonomus punctipennis auf.

Im Folgenden soll beispielhaft auf die Vergesellschaftung einiger Arten eingegangen werden. Dabei wird auf die Vergesellschaftung mit den hochfrequenten Arten i. d. R. nicht mehr ausdrücklich hingewiesen. Ergebnisse für weitere Artenkombinationen (Sørensen-Quotient) finden sich im Anhang 6.

In 18 von 22 Proben (82\%) trat der Borkenkäfer Dryocoetes villosus gemeinsam mit Corticaria longicollis und dem Räuber Rhizophagus dispar auf. Ebenfalls wurden Cryptophagus dentatus und Rhizophagus bipustulatus häufig angetroffen. Keine Art zeigte aber ein ähnliches Verteilungsmuster wie D. villosus. Die größte Übereinstimmung zeigte der ebenfalls vornehmlich an Eiche zu findende Borkenkäfer Xyloterus signatus. Der SQ-Wert betrug aber nur $30 \%(\mathrm{PHI}=0,778)$.

Der ebenfalls an frischer Eiche brütende Xyloterus signatus trat in vielen Fällen gemeinsam mit Aridius nodifer, Phloeocharis subtilissima, Rhizophagus nitidulus und Xyl. domes- 
ticus in den Proben auf. Mit der letztgenannten Art wurde auch die größte Übereinstimmung in der Verteilung festgestellt (SQ $=61 \%$; PHI =0,44). Es wurde bereits darauf hingewiesen, dass dies nicht auf identische Entwicklungsorte hindeutet, sondern vielmehr durch das Überwinterungsverhalten von Xyl. signatus erklärt werden kann. Darüber hinaus zeigten noch Rhinosimus ruficollis (SQ $=46 \%$; PHI $=0,603$ ) und Glischrochilus quadriguttatus $(\mathrm{SQ}=40 \%$; PHI = 0,619) ein ähnliches Verteilungsmuster.

Tabelle 4-37: Sørensen-Quotienten für ausgewählte Artenkombinationen

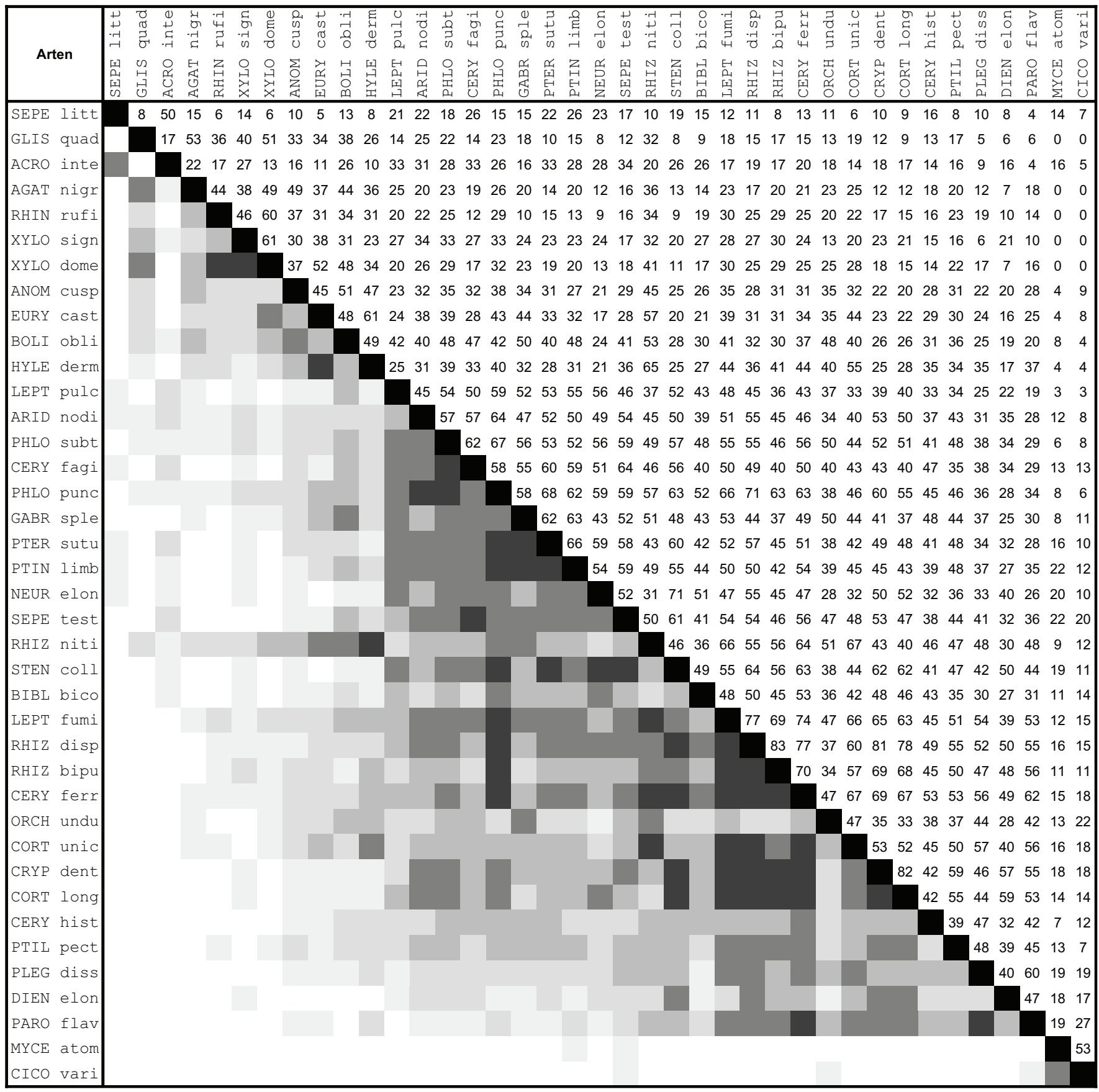

$\square<20 \square$ 20-29,9 $\square 30-39,9 \square$ प0-49,9 $\square 50-59,9 \quad \square \geq 60$ 
Die beiden zuletzt genannten Arten zeigten eine größere Übereinstimmumg mit dem Vorkommen von Xyloterus domesticus (SQ = $60 \%$; PHI = 0,447 bzw. $51 \%$; PHI =0,503). Auf $52 \%$ belief sich der SQ-Wert mit Euryusa castanoptera (PHI = 0,542). In $76 \%$ der Proben mit Xyl. domesticus - Besiedlung wurde auch Rhizophagus nitidulus festgestellt. Darüber hinaus waren Corticeus unicolor und Phloeocharis subtilissima regelmäßig in den Proben anzutreffen.

Tabelle 4-38: PHI-Koeffizienten für ausgewählte Artenpaarungen

\begin{tabular}{|c|c|c|c|c|c|c|c|c|}
\hline \multicolumn{3}{|c|}{ Artenparung } & \multirow{2}{*}{$\frac{\text { P H I }}{0,504}$} & \multicolumn{4}{|c|}{ Artenpa arung } & \multirow{2}{*}{$\begin{array}{c}\text { P H I } \\
0,478\end{array}$} \\
\hline Acro & inte & Sepe litt & & Cort & unic & : & Rhiz niti & \\
\hline Agat & badi & Cery fagi & 0,561 & Epur & $\operatorname{mars}$ & : & Glis quad & 0,568 \\
\hline Agat & nigr & : Anom cusp & 0,535 & Epur & mars & 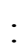 & xylo dome & 0,544 \\
\hline Agat & nigr & Boli obli & 0,582 & Eury & cast & : & Hyle derm & 0,454 \\
\hline Agat & nigr & : Glis quad & 0,496 & Eury & cast & : & Rhiz niti & 0,472 \\
\hline Agat & nigr & : Rhin rufi & 0,599 & Eury & cast & : & Xylo dome & 0,542 \\
\hline Agat & nigr & : Xylo dome & 0,544 & Gabr & sple & : & Octo glab & 0,565 \\
\hline Ampe & eryt & : Anas rufi & 0,572 & Gabr & sple & : & Phlo punc & 0,545 \\
\hline Ampe & eryt & : Atom bell & 0,533 & Gabr & sple & : & Phlo subt & 0,592 \\
\hline Anom & cusp & : Boli obli & 0,564 & Gabr & sple & : & Pter sutu & 0,505 \\
\hline Anom & cusp & : Taph bico & 0,588 & Gabr & sple & : & Ptin limb & 0,483 \\
\hline Arid & nodi & : Cery fagi & 0,595 & Glis & quad & : & Xylo dome & 0,503 \\
\hline Arid & nodi & : Phlo punc & 0,574 & Hyle & derm & : & Rhiz niti & 0,400 \\
\hline Boli & obli & : Dina aequ & 0,553 & Lept & fumi & : & Rhiz disp & 0,519 \\
\hline Boli & obli & : Gabr sple & 0,589 & Lept & fumi & : & Rhiz niti & 0,487 \\
\hline Boli & obli & : Hyle derm & 0,596 & Lept & pulc & : & Mniu incr & 0,579 \\
\hline Boli & obli & : Phlo subt & 0,598 & Lept & pulc & ( & Neur elon & 0,594 \\
\hline Boli & obli & : Rhiz niti & 0,539 & Lept & pulc & : & Phlo punc & 0,540 \\
\hline Boli & obli & : Xylo dome & 0,591 & Lept & pulc & $\frac{1}{2}$ & Ptin limb & 0,598 \\
\hline Cery & fagi & : Gabr sple & 0,589 & Neur & elon & : & Sten coll & 0,479 \\
\hline Cery & fagi & : Phlo punc & 0,583 & Paro & flav & : & Pleg diss & 0,579 \\
\hline Cery & fagi & : Phlo subt & 0,514 & Phlo & punc & : & Phlo subt & 0,511 \\
\hline Cery & fagi & : Pter sutu & 0,554 & $\mathrm{Phlo}$ & punc & : & Pter sutu & 0,504 \\
\hline Cery & fagi & : Ptin limb & 0,550 & $\mathrm{Phlo}$ & punc & : & Ptin limb & 0,544 \\
\hline Cery & fagi & : Sepe test & 0,494 & $\mathrm{Phlo}$ & punc & : & Rhiz disp & 0,581 \\
\hline Cery & ferr & : Cort unic & 0,531 & $\mathrm{Phlo}$ & subt & $\begin{array}{l}\text { e } \\
\text { e }\end{array}$ & Sepe test & 0,596 \\
\hline Cery & ferr & : Lept fumi & 0,541 & Pter & sutu & : & Ptin limb & 0,480 \\
\hline Cery & ferr & : Rhiz disp & 0,588 & Ptin & $1 \mathrm{imb}$ & ( & Sepe test & 0,576 \\
\hline Cery & ferr & : Rhiz niti & 0,514 & Rhin & rufi & : & Xylo dome & 0,447 \\
\hline Cico & vari & : Myce atom & 0,506 & Rhiz & bipu & : & Rhiz disp & 0,512 \\
\hline Cort & unic & : Hyle derm & 0,531 & Sepe & test & : & Sten coll & 0,597 \\
\hline Cort & unic & : Lept fumi & 0,549 & Xylo & dome & : & Xylo sign & 0,440 \\
\hline
\end{tabular}

Der Borkenkäfer Taphrorychus bicolor zeigte die größten Affinitäten zu Anomognathus cuspidatus $(\mathrm{SQ}=48 \%$; PHI = 0,558), Hylecoetus dermestoides $(\mathrm{SQ}=47$ \%; PHI = 0,609) 
und Phloeopora testacea $(\mathrm{SQ}=44 \%$; PHI =0,646). Rhizophagus nitidulus, Corticeus unicolor und Ptilinus pectinicornis waren häufig in den Proben mit T. bicolor vergesellschaftet.

Hylecoetus dermestoides wies eine hohe Affinität zu Rhizophagus nitidulus auf (SQ = $65 \%$; PHI = 0,4). In 35 von 39 Proben mit Hylecoetus-Befall war auch dieser Räuber zugegen. In $85 \%$ der Proben war zudem Corticeus unicolor anwesend. Mit der letzteren Art wurde ein SQ-Wert von $55 \%$ festgestellt $(\mathrm{PHI}=0,531)$. Euryusa castanoptera war eine weitere Art, deren räumliche Verteilung der von $H$. dermestoides relativ ähnlich war $(\mathrm{SQ}-\mathrm{Wert}=61 \%$; PHI $=0,454)$.

Ptilinus pectinicornis war in 95 Proben anzutreffen. Dementsprechend konnten nur hochfrequente Arten höhere SQ - Werte erreichen, während die PHI - Werte keine Korrelationen anzeigten. Es waren dies Cryptophagus dentatus ( $\mathrm{SQ}=59 \%$; PHI $=0,808)$, Corticaria longicollis $(\mathrm{SQ}=55 \%$; PHI = 0,977), Rhizophagus dispar (SQ $=55 \%$; PHI = 0,912) und Rh. bipustulatus $(\mathrm{SQ}=50 \%$; PHI = 0,951), Cerylon ferrugineum $(\mathrm{SQ}=53 \%$; $\mathrm{PHI}=0,854)$, Leptusa fumida $(\mathrm{SQ}=51 \%$; PHI = 0,879) und Corticeus unicolor $(\mathrm{SQ}=50 \%$; $\mathrm{PHI}=0,793)$. Corticaria longicollis und Cryptophagus dentatus wurden in den meisten Fällen (88 bzw. $84 \%)$ in den gleichen Proben angetroffen.

Nur geringe Übereinstimmung in der räumlichen Verteilung war zwischen Denticollis linearis und anderen Arten festzustellen. Die höchsten Ähnlichkeiten waren mit Neuraphes elongatulus $(\mathrm{SQ}=41 \%$; PHI $=0,701)$, Leptusa pulchella $(\mathrm{SQ}=39 \%$; $\mathrm{PHI}=0,741)$, Stenichnus collaris $(\mathrm{SQ}=39 \%$; PHI = 0,745), Sepedophilus testaceus $(\mathrm{SQ}=39 \%$; $\mathrm{PHI}=$ $0,745)$ und Phloeonomus punctipennis $(\mathrm{SQ}=39 \%$; PHI =0,723) zu verzeichnen. Abgesehen von den hochfrequenten Arten traten noch Pteryx suturalis und Phloeocharis subtilissima in großer Regelmäßigkeit in den gleichen Proben auf.

Der mulmbewohnende Schnellkäfer Melanotus castanipes wurde in zahlreichen Fällen gemeinsam mit Stenichnus collaris (75\%) und Sepedophilus testaceus (70 \%) gefangen. Die letztere Art zeigt auch die größte Ähnlichkeit im räumlichen Auftreten (SQ = 50\%; PHI = 0,615). Pteryx suturalis und Aridius nodifer waren jeweils in $68 \%$ der Proben mit MelanotusBefall zugegen.

Die Verteilung von Orchesia undulata wies die größte Übereinstimmung mit der von Rhizophagus nitidulus ( $\mathrm{SQ}=51 \%$; PHI =0,625), Gabrius splendidulus $(\mathrm{SQ}=50 \%$; $\mathrm{PHI}=$ 0,629) und Phloeocharis subtillissima (SQ $50 \%$; PHI = 0,643) auf. Häufig wurden auch Sepedophilus testaceus, Corticeus unicolor und Paromalus flavicornis in den gleichen Proben festgestellt.

Als letzte Art soll hier Teredus cylindricus Erwähnung finden. Obwohl diese Art als vom Aussterben bedroht gilt, wurde sie in jeder 4. Probe festgestellt. Kaum eine andere Art verfügte über so wenige regelmäßige Begleiter wie dieser Colydiidae. Nur die vier Arten mit den höchsten Frequenzprozenten waren regelmäßig an den gleichen Standorten zu finden. Ihrerseits war diese als Räuber eingeschätzte Art aber auch nur in wenigen Fällen regelmäßiger Bestandteil der biotischen Umwelt anderer Arten. Hierbei handelte es sich ausschließlich um Arten, die in einer viel geringeren Anzahl von Proben gefangen worden waren, wie z. B. Corticeus fasciatus, Atomaria bella und Ampedus erythrogonus. Die höchste positive Korrelation wurde für die Artenkombination mit Corticeus fasciatus registriert (PHI =0,737). 
Tabelle 4-39: Prozentuale Überlappung im Auftreten ausgewählter Arten

(Lesebeispiel: Bibloporus bicolor war in $45 \%$ der Proben mit Anomognathus cuspidatus-Vorkommen vertreten, andererseits trat $A$. cuspidatus in $18 \%$ der Proben mit B. bicolor-Besiedlung auf.)

\begin{tabular}{|c|c|c|c|c|c|c|c|c|c|c|c|c|c|c|c|c|c|c|c|c|c|c|c|c|c|c|c|c|c|c|}
\hline$+\frac{1}{a}$ & 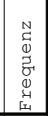 & & 4 & $\mid \begin{array}{l}0 \\
0 \\
0\end{array}$ & 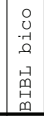 & 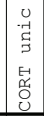 & 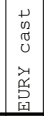 & 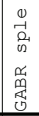 & 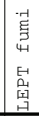 & 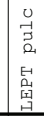 & 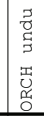 & 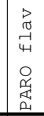 & 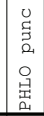 & 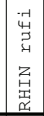 & 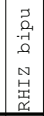 & 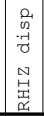 & 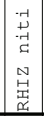 & 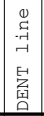 & 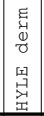 & 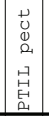 & 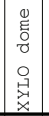 & 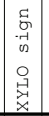 & 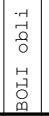 & \begin{tabular}{|c|} 
\\
0 \\
0 \\
0 \\
0 \\
0 \\
0 \\
0 \\
0 \\
\end{tabular} & 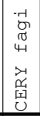 & 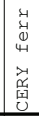 & 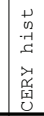 & 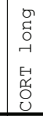 & 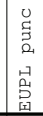 & 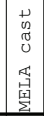 \\
\hline TR & 29 & $\mathrm{OOM}$ & cusp & & 115 & 62 & 48 & 48 & 90 & 34 & 45 & 55 & 90 & 34 & 93 & 100 & 76 & $\overline{10}$ & 55 & \begin{tabular}{l|l|}
66 &
\end{tabular} & 34 & 31 & \begin{tabular}{|l|}
55 \\
\end{tabular} & \begin{tabular}{|l|}
14 \\
\end{tabular} & 48 & 86 & 45 & 83 & 38 & 24 \\
\hline & 72 & $B L$ & bico & & & 4 & 15 & 38 & 64 & 39 & 29 & 35 & 65 & 13 & 68 & 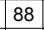 & 5 & & & 0 & 11 & 19 & 22 & 17 & 36 & 74 & 40 & 90 & 35 & 15 \\
\hline & 82 & $\mathrm{RT}$ & unic & & & & 30 & 37 & 2 & 28 & 37 & 57 & 54 & 15 & 9 & 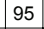 & 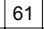 & 7 & 0 & & 18 & 13 & 28 & 29 & 37 & 87 & 40 & 93 & 33 & 23 \\
\hline & 22 & YO & vill & & 3 & 23 & 9 & 5 & 50 & 14 & 0 & 23 & 45 & 27 & 7 & 82 & 20 & & & 8 & 27 & 36 & 14 & 32 & 18 & 32 & 9 & 82 & 18 & 18 \\
\hline $\mathrm{TR}$ & 33 & RY & cast & & & & & 58 & 91 & 33 & 42 & 45 & 91 & 27 & 5 & 00 & 88 & 21 & $67 \mid$ & 58 & 45 & 36 & 48 & 15 & 39 & 85 & 42 & 82 & 21 & 21 \\
\hline TR & 54 & $\mathrm{ABR}$ & sple & & & 56 & 35 & & 5 & 54 & 46 & 39 & 87 & 7 & 69 & 94 & 57 & 28 & 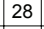 & 61 & 17 & 19 & 41 & 15 & 57 & 83 & 52 & 91 & 39 & 30 \\
\hline 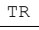 & 21 & PT & fumi & & 8 & 55 & 5 & 38 & & 6 & 32 & 45 & 62 & 18 & 6 & 95 & 51 & 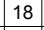 & $29 \mid$ & 5 & 18 & 17 & 26 & 20 & 37 & 77 & 35 & 86 & 32 & 19 \\
\hline 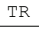 & 57 & PT & pulc & & 9 & 40 & 19 & 1 & 75 & & 33 & 25 & 86 & 14 & 5 & 93 & 40 & -1 & 4 & 46 & 14 & 21 & 33 & 5 & 51 & 70 & 35 & 95 & 35 & 28 \\
\hline TR & 46 & $\mathrm{RCH}$ & undu & & 6 & 65 & 30 & 4 & 85 & 41 & & 1 & 63 & 15 & 2 & \begin{tabular}{|l|}
89 \\
\end{tabular} & 63 & 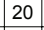 & $\theta$ & 57 & 20 & 11 & 41 & 1 & 46 & 89 & 46 & 91 & 43 & 26 \\
\hline TR & 87 & ARO & flav & & & 54 & 17 & 4 & 63 & 16 & 32 & & 38 & 9 & 6 & 84 & 43 & 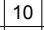 & 26 & 7 & 10 & 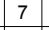 & 14 & 38 & 24 & 77 & 37 & 91 & 40 & 14 \\
\hline R & 45 & EDI & derm & & & 51 & 27 & 1 & 62 & 20 & 27 & 40 & 58 & 16 & 76 & 87 & 49 & 1 & 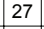 & 67 & 22 & 13 & 24 & 2 & 33 & 76 & 38 & 91 & 20 & 27 \\
\hline TR & 108 & $\mathrm{HLO}$ & punc & & 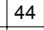 & 41 & 28 & 4 & 69 & 45 & 27 & 31 & & 18 & 74 & 94 & 46 & $P$ & $27 \mid$ & 44 & 19 & 21 & 28 & 12 & 44 & 69 & 36 & 81 & 26 & 25 \\
\hline & 35 & HLO & test & & 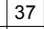 & 77 & 37 & 6 & 86 & 26 & 43 & 66 & 54 & 26 & 94 & 97 & 66 & & 9 & 49 & 17 & 9 & 23 & 2 & 17 & 89 & 19 & 77 & 40 & 6 \\
\hline $\mathrm{TT}$ & 25 & $\mathrm{HIN}$ & rufi & & 6 & 48 & 36 & 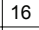 & 8 & 32 & 28 & 32 & 76 & & 100 & 100 & 6 & 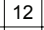 & 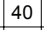 & 56 & 60 & 52 & 4 & ( & 20 & 76 & 28 & 88 & 12 & 16 \\
\hline , & 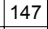 & $\mathrm{HIZ}$ & bipu & & 3 & 44 & 19 & 5 & 63 & 25 & 22 & 45 & 54 & 17 & & 91 & 4 & 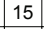 & 6 & 41 & 17 & 18 & 8 & 24 & 28 & 66 & 32 & 82 & 29 & 18 \\
\hline 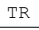 & 2 & HIZ & disp & & & 4 & 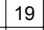 & D. & 65 & 30 & 23 & 41 & 57 & 14 & 75 & & & & & 42 & 14 & 16 & 19 & 24 & 3 & 66 & 33 & 35 & 28 & 20 \\
\hline TR & 68 & $\mathrm{HIZ}$ & niti & 2 & & 74 & 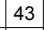 & 46 & 1 & 34 & 43 & 54 & 74 & 24 & 88 & 100 & & 4 & 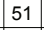 & 56 & 28 & 24 & 40 & 22 & 43 & 93 & 44 & 81 & 35 & 26 \\
\hline TR & 29 & $\mathrm{APH}$ & bico & & 2 & 66 & 38 & 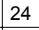 & 9 & 24 & 38 & 2 & 2 & 34 & 00 & 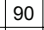 & & & & 9 & 34 & 24 & 31 & 10 & 4 & 3 & 41 & 6 & 1 & - \\
\hline TR & 2 & INC & rufi & & & 80 & 45 & 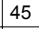 & 5 & 45 & 45 & 55 & 5 & 35 & 85 & 0 & $\varepsilon$ & 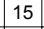 & $f^{2}$ & 85 & 30 & 25 & 50 & coll & 40 & 35 & 30 & 80 & 50 & 30 \\
\hline $\mathrm{T}$ & 38 & NAS & $\operatorname{sch} i$ & & & & & 3 & 42 & 13 & 16 & 7 & 2 & 1 & 42 & 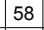 & & 1 & & & & 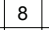 & 13 & 32 & 6 & 5 & 26 & 0 & ) & 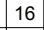 \\
\hline $\mathrm{T}$ & 34 & NAS & thor & 1 & & 5 & 18 & 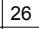 & 56 & 15 & 35 & 56 & 41 & 2 & 9 & 1 & 6 & & & 44 & 18 & 15 & 15 & 5 & 4 & 5 & 6 & 97 & 32 & 2 \\
\hline $\mathrm{T}$ & 36 & ENT & ne & & & & 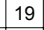 & & 61 & 50 & 25 & 25 & 78 & 8 & 1 & 92 & & & & 9 & 7 & 19 & 7 & $\varepsilon$ & 5 & 4 & 6 & 6 & 2 & 22 \\
\hline$T$ & 3 & LLE & derm & 1 & 3 & & 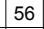 & 3 & 90 & 31 & 44 & 9 & 4 & 6 & ST & 0 & 9 & & & & 28 & 21 & \begin{tabular}{|l|} 
\\
\end{tabular} & 23 & 1 & 5 & 6 & 0 & 3 & 1 \\
\hline $\mathrm{T}$ & 9 & IIL & pect & 2 & 1 & & 20 & 35 & 8 & 27 & 27 & 3 & 9 & 5 & 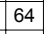 & 79 & 40 & P & & & 1 & 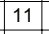 & 12 & 6 & 28 & 3 & 33 & 38 & 4 & 23 \\
\hline$T$ & 67 & RE & cyli & & 0 & 2 & & 12 & 9 & 22 & 0 & 4 & 37 & 4 & 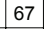 & 69 & 3 & & & 31 & & \begin{tabular}{|l|l|}
7 \\
\end{tabular} & & & 9 & 6 & 4 & 87 & 1 & 12 \\
\hline T & 18 & ST & rufo & 0 & 8 & & 0 & 2 & 9 & 17 & 22 & 7 & 17 & 6 & $y$ & 14 & 6 & 1 & & 6 & & 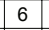 & 7 & 2 & 7 & 8 & 22 & 00 & 11 & 8 \\
\hline $\mathrm{T}$ & 2 & LIO & dome & & & & 60 & $p$ & 88 & 32 & 36 & 6 & 4 & 60 & 0 & 100 & 7 & & & & & 6 & 5 & 1 & 3 & 5 & 4 & 72 & 5 & 2 \\
\hline$T$ & 31 & LO & sign & & & & & & 68 & 39 & 16 & 9 & 4 & 42 & 87 & 90 & 52 & 3 & & 2 & 55 & & & 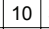 & 9 & 1 & 23 & 81 & 3 & 29 \\
\hline TP & 34 & OLI & obli & & & & & & 4 & 56 & 56 & 5 & 88 & 29 & 9 & 0 & 7 & & & & 1 & 2 & & I & 65 & 88 & 44 & 94 & 9 & 29 \\
\hline $\mathrm{T}$ & & IS & ide & & & & & & 48 & 13 & 26 & 1 & 2 & 4 & 8 & & & & & & & & & & 7 & 3 & 35 & 100 & 3 & \\
\hline $\mathrm{T}$ & 5 & IS & cast & & & & 9 & & 2 & 5 & 4 & 8 & 2 & 0 & & & & 5 & & & & & & & & 5 & 30 & 91 & {[} & \\
\hline$T$ & 3 & IS & niti & 7 & & & 0 & & 3 & 23 & 20 & 7 & 53 & 3 & 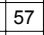 & 7 & & & & & & 3 & 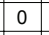 & 5 & & 7 & 43 & 97 & 7 & 17 \\
\hline $\mathrm{T}$ & & RTH & mund & 2 & & & & & 61 & 30 & 23 & 0 & 4 & 11 & ( & & & & & & $t$ & 20 & 2 & & 3 & 5 & 2 & 86 & j) & 25 \\
\hline TM & & BRA & gran & & & & & & \begin{tabular}{|l|}
57 \\
\end{tabular} & 24 & 3 & 9 & 38 & 7 & 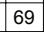 & & & & & & & \begin{tabular}{|l|}
2 \\
\end{tabular} & & 3 & & + & 36 & 8 & 3 & 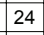 \\
\hline TM & & QRY & $g i$ & & & & & & & 49 & o & 6 & 81 & 8 & & & & & & & 2 & 0 & 7 & & & & 9 & 2 & 32 & 37 \\
\hline TM & & RT & long & & & & & & 5 & 26 & 0 & 8 & 41 & 8 & & & & & & & & 1 & & & 5 & 4 & 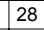 & & 2 & 17 \\
\hline $\mathrm{Tl}$ & & L & besc & & & & 02 & & 61 & 54 & 29 & 9 & 71 & 14 & 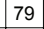 & & & & & & 25 & 8 & 36 & & 3 & 9 & 46 & 82 & 3 & 21 \\
\hline TI & & JPL & 1 & & & & & & 1 & 28 & 25 & 2 & 4 & 6 & & & & & & & & & & & 3 & 1 & y & 4 & & 22 \\
\hline TM & 5 & JPL & punc & 1 & & & 12 & 6 & 66 & 34 & 34 & 9 & 47 & 5 & & 3 & 1. & & & & & 2 & 7 & & 2 & 69 & 36 & 88 & & 17 \\
\hline TM & 2 & ALT & con & & & & 5 & 5 & & 0 & 9 & 7 & 18 & 5 & 0 & & & & & & & 1 & & & & 1 & 14 & 100 & 9 & \\
\hline TM & & $\mathrm{ELA}$ & ast & & & & 18 & & 3 & 40 & 30 & 0 & 68 & 10 & tr & & & & & & 3 & 20 & 12 & & 55 & 3 & 38 & 90 & 25 & \\
\hline TM & & $\mathrm{CR}$ & hali & & & & & & & 7 & 2 & 9 & 13 & 9 & & & & & & & & & & & & 7 & $?$ & 9 & 24 & \\
\hline $\mathrm{Tl}$ & 2 & $\mathrm{CR}$ & $n i$ & & & & & & & 5 & ) & 5 & 12 & 0 & & & & & & & & & & & D & 5 & 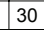 & 85 & 20 & 10 \\
\hline $\mathrm{TI}$ & & $\mathrm{LIO}$ & $\mathrm{abt}$ & & & & & & D & 47 & 9 & ) & 80 & 16 & 66 & & & & & & & 2 & & & + & 73 & 7 & ${ }^{7}$ & 33 & 2 \\
\hline TM & 4 & HYL & pt & & & & & & B & 29 & 25 & 8 & 58 & 5 & & & & & & & 1 & 7 & 1 & & 3 & 0 & 7 & 31 & 1 & 23 \\
\hline $\mathrm{Tl}$ & 2 & C & 1 & & & & & & 6 & 38 & 21 & 42 & 67 & 4 & 67 & & & & & & & & & & 3 & 3 & $?$ & 96 & 25 & 13 \\
\hline $\mathrm{Tl}$ & & $G$ & ss & & & & & & & & 36 & 8 & 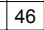 & 13 & & & & & & & & & & & 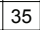 & 81 & 5 & 0 & 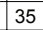 & 20 \\
\hline $\mathrm{TT}$ & & $\mathrm{R}$ & 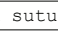 & & & & & & & & 29 & 29 & the & , & & & & & & & & 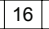 & & & & 66 & 7 & 5 & 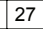 & t \\
\hline $\mathrm{T}$ & & $\mathrm{N}$ & 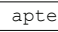 & & & & & & & & 29 & 53 & 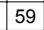 & 2 & & & & & & & & & & & & 80 & 7 & 6 & 35 & 22 \\
\hline & & $\mathrm{N}$ & 7 & & & & & & & & & 1 & 82 & 9 & & & & & & & & & & & & 80 & 38 & 9 & t & t \\
\hline $\mathrm{TT}$ & & EPE & 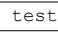 & & & & & & & & & & & & & & & & & & & & & & & 3 & 5 & 2 & 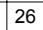 & 38 \\
\hline - & 8 & D & 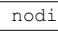 & & & & & & & 38 & 27 & & 77 & & & & & & & & & & & & 3 & 5 & 3 & 9 & 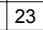 & 33 \\
\hline- & & RYP & & & & & & & & & & & & 5 & & & & & & & & & & & & 60 & & & & t \\
\hline $\mathrm{E}$ & & & & & & & & & & & 21 & & & & & & & & & & & & & & & 9 & 7 & 7 & & 21 \\
\hline 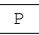 & 2 & 11 & 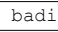 & & & & & & & 44 & 48 & 36 & De & 8 & 6 & & & & & & 4 & + & & 2 & 5 & 0 & 4 & 84 & 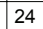 & 48 \\
\hline$E^{F}$ & & $\mathrm{~T}$ & 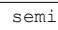 & & & & & & & 9 & 5 & 2 & 71 & 3 & & & & & & & & 1 & 2 & 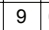 & & 57 & 34 & 91 & 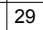 & 31 \\
\hline & & & Iugo & & & & & & & 2 & 48 & + & 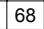 & 4 & & & & & & & 2 & 8 & 3 & 2 & & 4 & 8 & 88 & 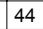 & 32 \\
\hline & & & -3 & & & & & & & & 2 & 0 & 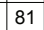 & 9 & & & & & & & & & & 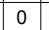 & & 1 & 9 & 0 & & 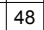 \\
\hline & 2 & & $1 d \pm$ & & & & & & & & 27 & 3 & 91 & 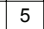 & 5 & & & & & & \begin{tabular}{|l|}
14 \\
\end{tabular} & \begin{tabular}{|l|}
14 \\
\end{tabular} & 6 & 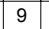 & & 1 & 36 & 91 & 23 & 27 \\
\hline & 3 & $\mathrm{R}$ & 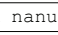 & & & & & & & + & 27 & 1 & 3 & - & +2 & & & & & 61 & 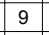 & 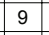 & 5 & 24 & 5 & 3 & 2 & 8 & 3 & $t$ \\
\hline & 2 & IIU & -1 & & & & t & & & & 5 & 1 & t & 0 & 9 & 9 & & & 1 & 19 & & 1 & 5 & 2 & & 9 & 6 & 0 & - & 20 \\
\hline & o & URR & Lon & & & & 12 & & & & 1 & 26 & 67 & 6 & 6 & 85 & 8 & & 5 & 38 & 7 & 16 & 16 & 4 & & 9 & 8 & 1 & 8 & 27 \\
\hline${ }_{1}$ & & EN & 11 & & & & & & & 40 & 27 & 40 & 63 & 6 & & & 3 & 26 & 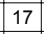 & ]$^{4}$ & & 3 & 19 & 1 & & 9 & 32 & 91 & 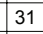 & 28 \\
\hline & $\sqrt{4}$ & PT & 111 & & & & & & & 0 & 19 & 61 & 33 & II & 15 & 75 & & & 2 & \begin{tabular}{|l|}
33 \\
\end{tabular} & & 11 & 11 & | & & & 0 & 20 & & 8 \\
\hline
\end{tabular}




\subsection{Die Sukzession der Artengemeinschaften}

Um die Reaktion von Arten und -gemeinschaften auf die sich im Abbauprozeß verändernden Habitateigenschaften zu untersuchen, wurden die Ergebnisse der Probenerhebung verschiedenen Verfahren der Gradientenanalyse unterzogen. Neben einigen Ordinationstechniken sollten Ähnlichkeitsindices Einblicke in die Vergesellschaftungen der Käferarten erlauben, die Gradientenstruktur des Probenkollektivs sichtbar machen und gleichzeitig Hinweise auf die sie verursachenden Faktoren(komplexe) geben. Den eingesetzten Verfahren lag die Annahme zu Grunde, dass sich der Zersetzungsgradient in der qualitativen und quantitativen Struktur der Artengemeinschaften manifestiert, wodurch die Möglichkeit eröffnet wird, die interne Datenstruktur der Proben zum Ausgangspunkt für die Analyse der Sukzession zu machen.

Neben der Bedeutung der erhobenen Umweltvariablen für die Vergesellschaftung der Arten wird auch die Verteilung xylobionter Käferarten auf einzelne Faktorenklassen dargestellt, soweit die entsprechenden Faktoren einen nennenswerten Beitrag zur Erklärung der Streuung im Datenmaterial beitrugen. Es sei aber angemerkt, dass es nicht das Ziel der Untersuchung war, Präferenzen und Ursachen für die Habitatbindung der Arten zu ermitteln. Dies hätte ein völlig anderes Methodendesign erforderlich gemacht. Vielmehr sollten lediglich Hinweise auf die möglichen Ursachen für die vorgefundenen Gesellschaftsstrukturen gegeben werden. Unabhängig von diesen Einschränkungen können die gemachten Beobachtungen den bisherigen Kenntnisstand zu den Habitatpräferenzen xylobionter Arten in mancherlei Hinsicht bestätigen, ergänzen oder aber auch in Frage stellen.

Wenn in den nachfolgenden Abschnitten dennoch der Begriff 'Präferenz' Verwendung findet, soll damit lediglich auf einen, unter Beachtung des auf die jeweilige Faktorenklasse entfallenden Stichprobenanteils, relativen Nachweisschwerpunkt in der Verteilung der Art innerhalb des Probenkollektivs hingewiesen werden. In den entsprechenden Tabellen wurden derartige relative Schwerpunkte durch Fettdruck kenntlich gemacht. Dabei wurde die Frequenz der Arten und der auf die Klassen entfallende Proben- und Flächenanteil berücksichtigt. Die subjektiv festgelegten Präferenzkriterien sind in Tabelle 4-40 aufgeführt. Je geringer die Anzahl der Proben war, in der eine Art auftrat, desto strengere Anforderungen wurden gestellt. Als erstes Präferenzkriterium sollte der auf eine Klasse entfallende Frequenz- bzw. Individuenanteil einer Art ein Mehrfaches des auf diese Klasse entfallenden Proben- bzw. Flächenanteils (Erwartungswert) betragen. Da dieses Kriterium z. T. zu unerfüllbar hohen Anforderungen führte, wie z. B. im Falle der Baumarten, wurde als alternatives Kriterium, wiederum in Abhängigkeit von der Frequenz der Arten, gefordert, dass der beobachtete Frequenz- bzw. Individuenanteil den Erwartungswert zumindest um einen festgelegten Wert übertraf.

Welches der beiden Kriterien leichter zu erfüllen war, hing sowohl von der Anzahl der Klassen als auch von der Gleichverteilung der Proben auf diese Klassen ab. So sind die Hervorhebungen in den Spalten für die Baumartenpräferenz fast ausschließlich auf das zweite Kriterium zurückzuführen, während bei den Bohrwiderstandsklassen in der überwiegenden Zahl der Fälle das erste Kriterium leichter erfüllbar war. Davon beeinflusst ist auch die Anzahl der markierten Arten. Da die Klassen z. T. in sehr unterschiedlichen Anteilen am Stichprobenumfang beteiligt waren, sind zudem die nach obigen Kriterien ausgeschiedenen Präferenzbereiche in vielen Fällen nicht identisch mit den maximalen auf eine Klasse 
entfallenden Individuen- bzw. Frequenzanteilen. In der Konsequenz wurden einige Arten fast ausschließlich in Proben einer einzelnen Variablenklasse festgestellt, ohne das sie einem der beiden Präferenzkriterien genügten, andererseits wurden diese z. T. erfüllt, obwohl ein nur geringer Teil der Fänge auf diese Klasse entfiel.

Aufgrund methodischer Erwägungen wurden die STE- und EFs-Proben getrennt behandelt. Zudem wurde die Verteilung der Arten auf die Faktorenklassen nur für diejeniegen Arten analysiert, die in den STE- bzw. EFs-Proben jeweils mit mindestens 10 Individuen und in mindestens 5 Proben vertreten waren.

Tabelle 4-40: Präferenzkriterien

\begin{tabular}{|c|c|c|}
\hline Frequenz & $\begin{array}{c}\text { Präferenzkriterium 1 } \\
\mathrm{x} \text {-facher Erwartungswert }\end{array}$ & $\begin{array}{c}\text { Präferenzkriterium 2 } \\
\text { Erwartungswert } \\
\text { plus x Prozent }\end{array}$ \\
\hline $5-10$ & $\mathrm{x}=4$ & $\mathrm{x}=40 \%$ \\
\hline $11-20$ & $\mathrm{x}=3$ & $\mathrm{x}=35 \%$ \\
\hline$>20$ & $\mathrm{x}=2$ & $\mathrm{x}=30 \%$ \\
\hline
\end{tabular}

Die hier geschilderte Vorgehensweise soll an einem kurzen Beispiel erläutert werden. Die Art A wurde in 15 Proben festgestellt. Von den Nachweisen entfielen 10 (67\%) auf die Eiche und $5(34 \%)$ auf die Buche. Der auf diese beiden Baumarten entfallende Probenanteil soll hier $25 \%$ für Eiche und $75 \%$ für Buche betragen. Die Erwartungswerte betragen demnach ebenfalls 25 und $75 \%$. Um das 'Präferenz'kriterium für Eiche zu erfüllen, sollten die Nachweise der Art A entweder mindestens das dreifache des Erwartungswertes betragen ('Präferenz'kriterium 1: 3 × $25 \%=75 \%$ ) oder den Erwartungswert um mindestens $35 \%$ übertreffen ('Präferenz'kriterium 2: $25 \%+35 \%=60 \%$ ). Da $67 \%$ der Nachweise von Art A auf die Eiche entfallen, wird in diesem Beispiel nur das zweite Kriterium erfüllt.

\subsubsection{Verteilung, Vergesellschaftung und Sukzession der Arten an liegendem Totholz}

Insgesamt wurden 22271 Käferindividuen aus 331 Arten, darunter 165 obligate Totholzkäferarten, die $85 \%$ der Individuen stellten, mit Stammeklektoren erfaßt. Arten mit fehlender oder nur sehr geringer Beziehung zu Totholz (z. B. Rhynchaenus fagi) fanden in der weiteren Auswertung keine Berücksichtigung. Die Daten wurden zunächst mehreren DCA's unterzogen, wobei alternativ die logarithmierten Schlüpfdichten oder lediglich die An- bzw. Abwesenheit der Arten Eingang in die Berechnungen fanden. Darüber hinaus wurden die Analysen mit einem reduzierten Artenspektrum wiederholt, wobei nur Arten, die in mindestens 10 STE-Proben angetroffen worden waren, berücksichtigt wurden.

Um zu prüfen, ob die richtigen Habitatvariablen erhoben worden sind, diese also einen nennenswerten Beitrag zur Erklärung der Streuung im Datenmaterial zu leisten vermögen, wurde mit den gleichen Datensätzen zudem jeweils eine DCCA durchgeführt. 
Die zusammenfassenden Ergebnisse dieser Analysen sind in der Tabelle 4-41 wiedergegeben. Die Abbildungen 4-34 und 4-35 zeigen die Ordinationsdiagramme einer DCA, resultierend aus den logarithmierten Schlüpfdichten derjenigen Arten, die in mindestens 10 STEProben angetroffen worden waren. Das Ordinationsergebnis beruht somit auf der Verteilung von insgesamt 83 Arten. Aber auch wenn keine Bedingungen an die Mindestfrequenz der Arten gestellt wurden und/oder nur das Vorkommen der Arten, nicht aber ihre Dichte in die Berechnung eingingen, ließen die daraus resultierenden Ordinationsdiagramme vergleichbare Verteilungsmuster sichtbar werden, auch wenn der durch die Ordinationsachsen erklärbare Anteil an der Streuung des Datenmaterials z. T. deutlich niedriger ausfiel. Der Vergleich der Ergebnisse der DCA's mit den Resultaten aus den DCCA's, insbesondere der Eigenwerte der Ordinationsachsen und der Arten-Umwelt-Korrelationen, erlaubte den Schluß, dass die erhobenen Umweltvariablen geeignet waren, einen nennenswerten Beitrag zur Erklärung der Streuung im Datenmaterial zu leisten (Tab. 4-41).

Das Ordinationsdiagramm der Probenverteilung (Abb. 4-34) läßt deutlich eine ausgeprägte Separierung der Proben entlang der zweiten Ordinationsachse $(\lambda 2=0.151)$ erkennen. Dieses Muster war vor allem auf den Einfluss der Baumart auf die Zusammensetzung der Käfergemeinschaften zurückzuführen. Als Ergebnis der durchgeführten 'forward selection' wurde, und zwar unabhängig von der Berechnungsgrundlage, der Variablen Baumart als Einzelfaktor die wichtigste Rolle zur Erklärung der Streuung im Datenmaterial zugewiesen. Die untersuchte Baumart spielt für die qualitative und quantitative Struktur der Artenvergesellschaftungen offensichtlich eine nicht unbedeutende Rolle.

Tabelle 4-41: Zusammenfassung der Ergebnisse verschiedener Analysemethoden mit den Fangdaten aus STE-Proben an liegendem Buchen- und Eichentotholz

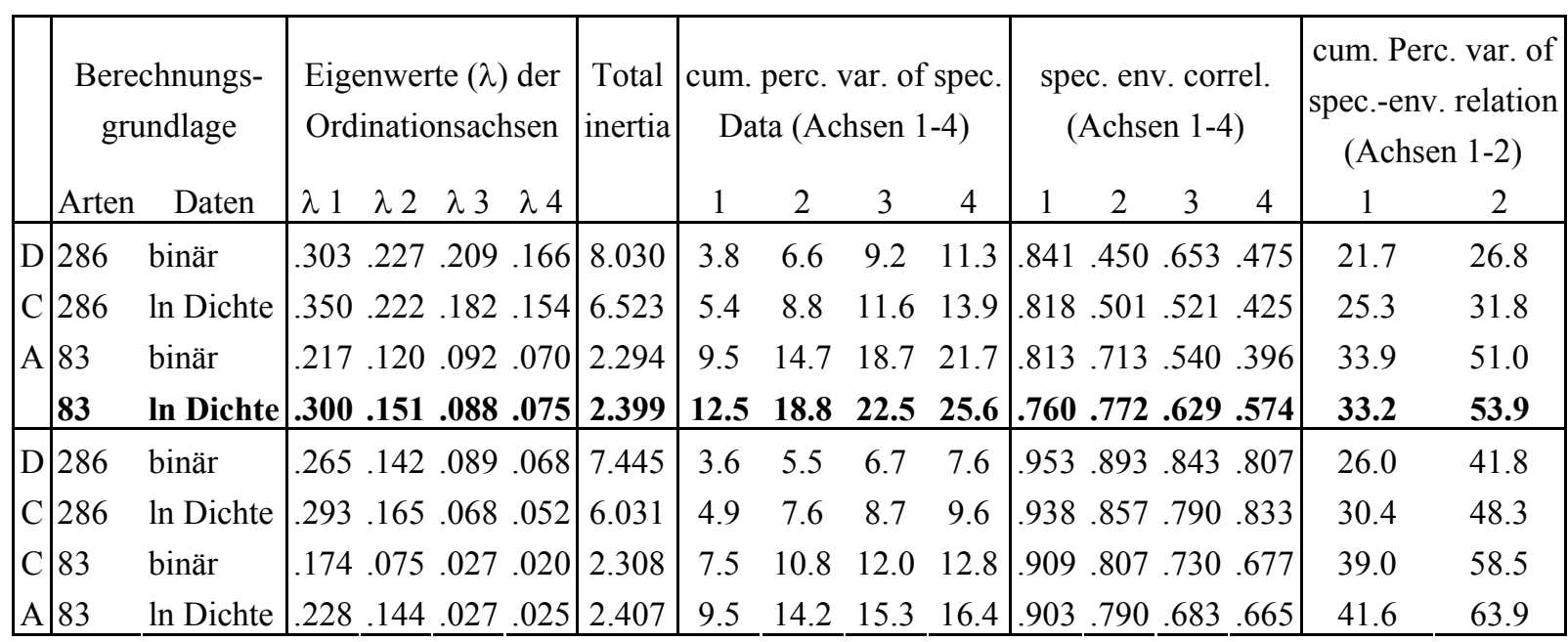

Die Diagramme machen aber auch deutlich, dass der ersten Ordinationsachse für die Erklärung der Streuung im Datenmaterial die größere Bedeutung zukam $(\lambda 1=0.300)$. Hinter dieser hypothetischen Variablen verbarg sich vor allem ein Gradient aus Bohrwiderstandsund Rindenklasse, zwei miteinander positiv korrelierte Faktoren. Während sich im linken Teil des Diagramms vor allem Proben mit hohem Bohrwiderstand und Rindenprozent finden, sind diese Werte entlang der ersten Ordinationsachse tendenziell rückläufig. 
Die Ordinationsachsen drei und vier und die mit ihnen korrelierten Habitatvariablen trugen nur noch in vergleichsweise geringem Maße zur Klärung der Streuung im Datenmaterial bei ( $\lambda 3=0.088, \lambda 4=0.075)$. Unter Berücksichtigung der Korrelationen zwischen den Habitatvariablen ergab sich folgende Rangfolge der Faktoren für die Erklärung der verbleibenden Reststreuungen: Baumart, Berindung und Bohrwiderstand, Lage, Beschirmung, Saugfähigkeit, Beschattung und Holzfeuchte. Die deutlichsten Korrelationen wurden, wie nicht anders zu erwarten, für die Faktoren Bohrwiderstand und Berindung, Feuchte und Saugfähigkeit sowie Beschirmung und Beschattung verzeichnet.

Über das Vorkommen der Arten und die Verteilung der Individuen auf die beiden untersuchten Baumarten sowie in Abhängigkeit von den Faktoren Bohrwiderstand und Berindung geben die Tabellen 4-42 und 4-43 Auskunft. Zur Beurteilung dieser Werte ist zudem der auf die Klassen entfallende Anteil der Stichproben bzw. der befangenen Mantel- und Rindenfläche angegeben. Einen ersten Hinweis auf die Verteilung der Arten in Abhängigkeit vom Faktor Baumart gibt auch ihre Position im Ordinationsdiagramm (Abb. 4-35). 


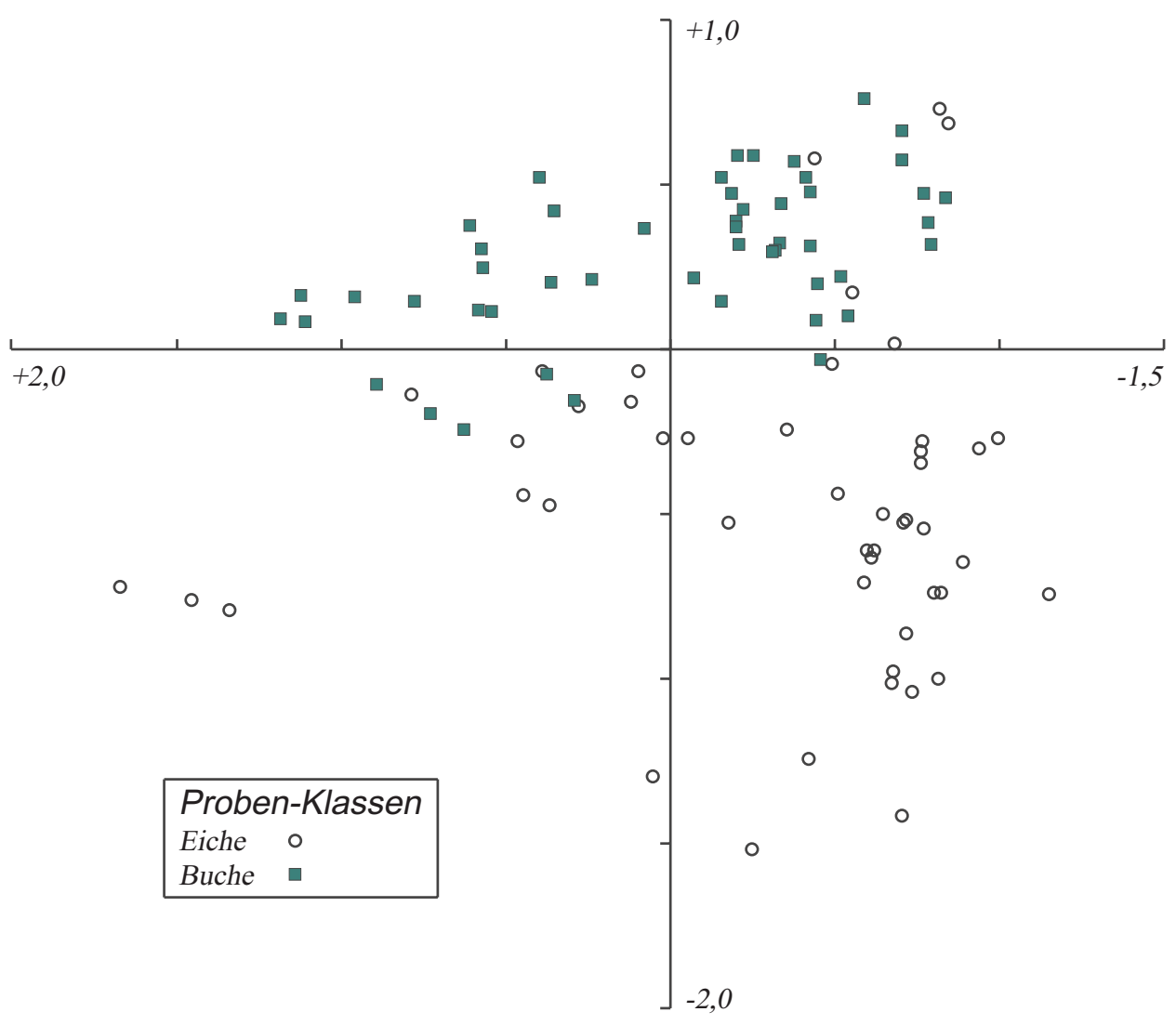

Abb. 4-34: Ordinationsdiagramm (DCA) der STE-Proben von Eichen- und Buchentotholz

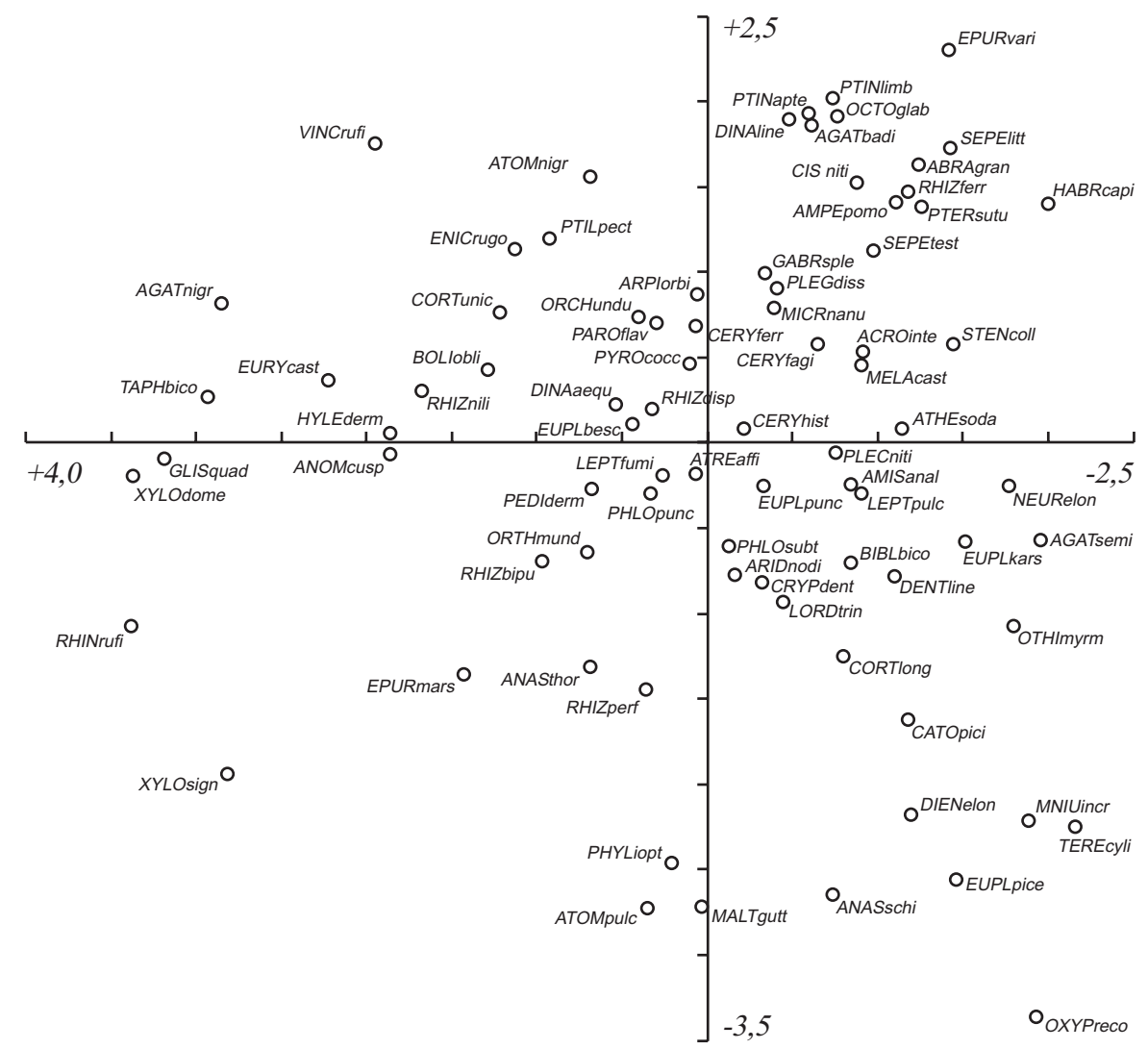

Abb. 4-35: Ordinationsdiagramm (DCA) der Arten aus STE-Proben von Eichen- und Buchentotholz 
Tabelle 4-42: Prozentuale Verteilung der Nachweise (Frequenz) in Abhängigkeit von Baumart, Rinden- und Bohrwiderstandsklassen ('Präferenzen' hervorgehoben)

\begin{tabular}{|c|c|c|c|c|c|c|c|c|c|c|c|c|c|}
\hline \multirow[b]{2}{*}{ Art } & \multirow[b]{2}{*}{ Frequenz } & \multicolumn{2}{|c|}{ Baumart } & \multicolumn{4}{|c|}{ Rindenklasse } & \multicolumn{6}{|c|}{ Bohrwiderstandsklasse } \\
\hline & & Ei & Bu & Ri 4 & Ri 3 & Ri 2 & Ri 1 & Bw 6 & Bw 5 & Bw 4 & Bw 3 & Bw 2 & Bw 1 \\
\hline Abraeus granulum & 15 & 13,3 & 86,7 & 46,7 & 20,0 & 20,0 & 13,3 & 6,7 & 26,7 & 0,0 & 33,3 & 13,3 & 20,0 \\
\hline Agathidium nigripenne & 12 & 16,7 & 83,3 & 91,7 & 8,3 & 0,0 & 0,0 & 50,0 & 41,7 & 8,3 & 0,0 & 0,0 & 0,0 \\
\hline Ampedus erythrogonus & 9 & 100,0 & 0,0 & 0,0 & 0,0 & 0,0 & 100,0 & 0,0 & 11,1 & 11,1 & 44,4 & 22,2 & 11,1 \\
\hline Ampedus pomorum & 11 & 27,3 & 72,7 & 27,3 & 18,2 & 27,3 & 27,3 & 9,1 & 27,3 & 0,0 & 18,2 & 27,3 & 18,2 \\
\hline Anaspis schilskyana & 13 & 53,8 & 46,2 & 30,8 & 7,7 & 7,7 & 53,8 & 15,4 & 23,1 & 23,1 & 7,7 & 15,4 & 15,4 \\
\hline Anaspis thoracica & 11 & 27,3 & 72,7 & 36,4 & 36,4 & 0,0 & 27,3 & 27,3 & 36,4 & 9,1 & 18,2 & 9,1 & 0,0 \\
\hline Anisotoma humeralis & 6 & 83,3 & 16,7 & 50,0 & 16,7 & 0,0 & 33,3 & 0,0 & 50,0 & 0,0 & 16,7 & 16,7 & 16,7 \\
\hline Anisotoma orbicularis & 7 & 28,6 & 71,4 & 42,9 & 28,6 & 14,3 & 14,3 & 0,0 & 57,1 & 0,0 & 0,0 & 42,9 & 0,0 \\
\hline Anomognathus cuspidatus & 22 & 31,8 & 68,2 & 77,3 & 9,1 & 4,5 & 9,1 & 31,8 & 31,8 & 18,2 & 9,1 & 4,5 & 4,5 \\
\hline Arpidiphorus orbiculatus & 11 & 9,1 & 90,9 & 54,5 & 9,1 & 9,1 & 27,3 & 27,3 & 36,4 & 9,1 & 9,1 & 9,1 & 9,1 \\
\hline Atomaria bella & 18 & 94,4 & 5,6 & 27,8 & 0,0 & 0,0 & 72,2 & 0,0 & 22,2 & 27,8 & 33,3 & 16,7 & 0,0 \\
\hline Atomaria pulchra & 11 & 36,4 & 63,6 & 45,5 & 9,1 & 9,1 & 36,4 & 27,3 & 18,2 & 18,2 & 18,2 & 9,1 & 9,1 \\
\hline Atrecus affinis & 10 & 50,0 & 50,0 & 70,0 & 10,0 & 10,0 & 10,0 & 0,0 & 50,0 & 10,0 & 10,0 & 10,0 & 20,0 \\
\hline Bibloporus bicolor & 48 & 54,2 & 45,8 & 41,7 & 22,9 & 8,3 & 27,1 & 6,3 & 41,7 & 12,5 & 22,9 & 14,6 & 2,1 \\
\hline Bolitochara obliqua & 33 & 21,2 & 78,8 & 75,8 & 21,2 & 0,0 & 3,0 & 27,3 & 42,4 & 12,1 & 9,1 & 6,1 & 3,0 \\
\hline Cerylon fagi & 51 & 41,2 & 58,8 & 58,8 & 25,5 & 3,9 & 11,8 & 7,8 & 39,2 & 11,8 & 17,6 & 13,7 & 9,8 \\
\hline Cerylon ferrugineum & 68 & 33,8 & 66,2 & 60,3 & 20,6 & 5,9 & 13,2 & 14,7 & 38,2 & 11,8 & 17,6 & 13,2 & 4,4 \\
\hline Cerylon histeroides & 34 & 35,3 & 64,7 & 47,1 & 29,4 & 8,8 & 14,7 & 8,8 & 35,3 & 11,8 & 20,6 & 17,6 & 5,9 \\
\hline Cis boleti & 7 & 0,0 & 100,0 & 14,3 & 42,9 & 14,3 & 28,6 & 0,0 & 42,9 & 14,3 & 42,9 & 0,0 & 0,0 \\
\hline Cis nitidus & 13 & 7,7 & 92,3 & 53,8 & 23,1 & 15,4 & 7,7 & 0,0 & 30,8 & 0,0 & 7,7 & 38,5 & 23,1 \\
\hline Corticaria linearis & 7 & 71,4 & 28,6 & 71,4 & 14,3 & 0,0 & 14,3 & 14,3 & 57,1 & 14,3 & 14,3 & 0,0 & 0,0 \\
\hline Corticaria longicollis & 86 & 47,7 & 52,3 & 45,3 & 16,3 & 9,3 & 29,1 & 9,3 & 32,6 & 17,4 & 20,9 & 11,6 & 8,1 \\
\hline Corticeus fasciatus & 6 & 66,7 & 33,3 & 16,7 & 16,7 & 16,7 & 50,0 & 0,0 & 16,7 & 16,7 & 16,7 & 33,3 & 16,7 \\
\hline Corticeus unicolor & 38 & 23,7 & 76,3 & 68,4 & 23,7 & 2,6 & 5,3 & 15,8 & 50,0 & 10,5 & 7,9 & 10,5 & 5,3 \\
\hline Denticollis linearis & 34 & 55,9 & 44,1 & 35,3 & 20,6 & 11,8 & 32,4 & 2,9 & 41,2 & 11,8 & 17,6 & 11,8 & 14,7 \\
\hline Dinaraea aequata & 17 & 29,4 & 70,6 & 70,6 & 23,5 & 5,9 & 0,0 & 23,5 & 29,4 & 17,6 & 5,9 & 17,6 & 5,9 \\
\hline Dinaraea linearis & 17 & 23,5 & 76,5 & 76,5 & 17,6 & 0,0 & 5,9 & 17,6 & 47,1 & 5,9 & 0,0 & 17,6 & 11,8 \\
\hline Epuraea marseuli & 14 & 50,0 & 50,0 & 71,4 & 0,0 & 0,0 & 28,6 & 21,4 & 35,7 & 0,0 & 28,6 & 7,1 & 7,1 \\
\hline Epuraea variegata & 16 & 0,0 & 100,0 & 18,8 & 37,5 & 31,3 & 12,5 & 6,3 & 25,0 & 6,3 & 12,5 & 12,5 & $\mathbf{3 7 , 5}$ \\
\hline Euplectus bescidicus & 19 & 31,6 & 68,4 & 73,7 & 10,5 & 5,3 & 10,5 & 21,1 & 47,4 & 21,1 & 0,0 & 5,3 & 5,3 \\
\hline Euplectus karsteni & 12 & 50,0 & 50,0 & 16,7 & 25,0 & 8,3 & 50,0 & 0,0 & 41,7 & 16,7 & 25,0 & 16,7 & 0,0 \\
\hline Euplectus piceus & 17 & 64,7 & 35,3 & 41,2 & 23,5 & 5,9 & 29,4 & 11,8 & 17,6 & 5,9 & 29,4 & 23,5 & 11,8 \\
\hline Euplectus punctatus & 25 & 40,0 & 60,0 & 60,0 & 16,0 & 4,0 & 20,0 & 16,0 & 32,0 & 16,0 & 12,0 & 20,0 & 4,0 \\
\hline Euryusa castanoptera & 26 & 23,1 & 76,9 & 76,9 & 15,4 & 0,0 & 7,7 & 30,8 & 46,2 & 15,4 & 7,7 & 0,0 & 0,0 \\
\hline Gabrius splendidulus & 48 & 29,2 & 70,8 & 58,3 & 20,8 & 6,3 & 14,6 & 16,7 & 33,3 & 8,3 & 18,8 & 16,7 & 6,3 \\
\hline Glischrochilus 4-guttatus & 13 & 38,5 & 61,5 & 84,6 & 7,7 & 0,0 & 7,7 & 46,2 & 46,2 & 0,0 & 0,0 & 7,7 & 0,0 \\
\hline Hylecoetus dermestoides & 24 & 29,2 & 70,8 & 83,3 & 16,7 & 0,0 & 0,0 & 33,3 & 45,8 & 16,7 & 4,2 & 0,0 & 0,0 \\
\hline Hypulus quercinus & 5 & 100,0 & 0,0 & 0,0 & 0,0 & 0,0 & 100,0 & 0,0 & 0,0 & 40,0 & 40,0 & 20,0 & 0,0 \\
\hline Leptusa fumida & 68 & 39,7 & 60,3 & 55,9 & 19,1 & 4,4 & 20,6 & 13,2 & 39,7 & 10,3 & 17,6 & 13,2 & 5,9 \\
\hline Leptusa pulchella & 51 & 51,0 & 49,0 & 51,0 & 13,7 & 3,9 & 31,4 & 7,8 & 41,2 & 11,8 & 17,6 & 13,7 & 7,8 \\
\hline Malthodes guttifer & 14 & 92,9 & 7,1 & 64,3 & 7,1 & 7,1 & 21,4 & 0,0 & 57,1 & 21,4 & 14,3 & 7,1 & 0,0 \\
\hline Melanotus castanipes & 31 & 29,0 & 71,0 & 45,2 & 12,9 & 16,1 & 25,8 & 16,1 & 32,3 & 9,7 & 9,7 & 16,1 & 16,1 \\
\hline Mycetophagus atomarius & 7 & 14,3 & 85,7 & 14,3 & 28,6 & 57,1 & 0,0 & 0,0 & 14,3 & 0,0 & 28,6 & 0,0 & 57,1 \\
\hline
\end{tabular}




\begin{tabular}{|c|c|c|c|c|c|c|c|c|c|c|c|c|c|}
\hline \multirow{2}{*}{\multicolumn{2}{|c|}{ Art }} & \multicolumn{2}{|c|}{ Baumart } & \multicolumn{4}{|c|}{ Rindenklasse } & \multicolumn{6}{|c|}{ Bohrwiderstandsklasse } \\
\hline & & Ei & Bu & Ri 4 & Ri 3 & Ri 2 & Ri 1 & Bw 6 & Bw 5 & Bw 4 & Bw 3 & Bw 2 & Bw 1 \\
\hline Octotemnus glabriculus & 16 & 0,0 & 100,0 & 25,0 & 43,8 & 18,8 & 12,5 & 6,3 & 25,0 & 0,0 & 31,3 & 31,3 & 6,3 \\
\hline Orchesia undulata & 30 & 10,0 & $\mathbf{9 0 , 0}$ & 60,0 & 20,0 & 6,7 & 13,3 & 23,3 & 33,3 & 10,0 & 13,3 & 13,3 & ,7 \\
\hline Orthoperus mundus & 26 & 46,2 & 53,8 & 65,4 & 19,2 & 7,7 & 7,7 & 15,4 & 34,6 & 19,2 & 15,4 & 7,7 & 7,7 \\
\hline Oxypoda recondita & 10 & 80,0 & 20,0 & 20,0 & 0,0 & 0,0 & 80,0 & 0,0 & 30,0 & 30,0 & 10,0 & 20,0 & 10,0 \\
\hline Paromalus flavicornis & 22 & 18,2 & 81,8 & 59,1 & 27,3 & 9,1 & 4,5 & 18,2 & 36,4 & 9,1 & 9,1 & 13,6 & 13,6 \\
\hline Pediacus dermestoides & 25 & 20,0 & 80,0 & 48,0 & 20,0 & 16,0 & 16,0 & 20,0 & 36,0 & 16,0 & 24,0 & 0,0 & 4,0 \\
\hline Phloeocharis subtilissima & 65 & 38,5 & 61,5 & 52,3 & 15,4 & 7,7 & 24,6 & 15,4 & 30,8 & 20,0 & 15,4 & 12,3 & 6,2 \\
\hline Phloeonomus punctipennis & 88 & 46,6 & 53,4 & 54,5 & 11,4 & 10,2 & 23,9 & 11,4 & 34,1 & 12,5 & 20,5 & 13,6 & 8,0 \\
\hline Phloeopora corticalis & 5 & 60,0 & 40,0 & 80,0 & 20,0 & 0,0 & 0,0 & 20,0 & 60,0 & 20,0 & 0,0 & 0,0 & 0,0 \\
\hline testacea & 10 & 20,0 & 80,0 & 80,0 & 0 & 10,0 & 10,0 & ,0 & 20,0 & 30,0 & 10,0 & 0,0 & 10,0 \\
\hline Phyllodrepa ioptera & 28 & 53,6 & 46,4 & 46,4 & 14,3 & 3,6 & 35,7 & 21,4 & 21,4 & 14,3 & 25,0 & 10,7 & 7,1 \\
\hline Phyllodrepoidea crenata & 1 & 42,9 & 57,1 & 85,7 & 0,0 & 14,3 & 0,0 & 28,6 & 57,1 & 0,0 & 14,3 & 0,0 & 0,0 \\
\hline Plectophloeus nitidus & 13 & 53,8 & 46,2 & 53,8 & 15,4 & 0,0 & 30,8 & 0,0 & 38,5 & 23,1 & 38,5 & 0,0 & 0,0 \\
\hline Plegaderus dissectus & 26 & 7,7 & 92,3 & 50,0 & 26,9 & 11,5 & 11,5 & 15,4 & 30,8 & 3,8 & 23,1 & 11,5 & 15,4 \\
\hline Pteryx suturalis & 68 & 38,2 & 61,8 & 50,0 & 20,6 & 8,8 & 20,6 & 10,3 & 30,9 & 13,2 & 17,6 & 16,2 & 11,8 \\
\hline Ptilinus pectinicornis & 43 & 9,3 & $\mathbf{9 0 , 7}$ & 62,8 & 16,3 & 11,6 & 9,3 & 18,6 & 34,9 & 9,3 & 14,0 & 14,0 & 9,3 \\
\hline Ptinella aptera & 26 & 11,5 & $\mathbf{8 8 , 5}$ & 38,5 & $\mathbf{3 0 , 8}$ & 23,1 & 7,7 & ,5 & 30,8 & 11,5 & 19,2 & 15,4 & 11,5 \\
\hline Ptinella limbata & 57 & 33,3 & 66,7 & 61,4 & 19,3 & 10,5 & 8,8 & 10,5 & 36,8 & 12,3 & 14,0 & 17,5 & 8,8 \\
\hline Pyrochroa coccinea & 13 & 23,1 & 76,9 & 61,5 & 23,1 & 7,7 & 7,7 & 7,7 & 38,5 & 7,7 & 15,4 & 23,1 & 7,7 \\
\hline Rhagium mordax & 7 & 0,0 & 100,0 & 42,9 & 57,1 & 0,0 & 0,0 & 14,3 & 28,6 & 14,3 & 28,6 & 14,3 & 0,0 \\
\hline Rhinosimus ruficollis & 14 & 35,7 & 64,3 & 78,6 & 14,3 & 0,0 & 7,1 & 28,6 & 64,3 & 0,0 & 0,0 & 7,1 & 0,0 \\
\hline Rhizophagus bipustulatus & 68 & 48,5 & 51,5 & 58,8 & 11,8 & 8,8 & 20,6 & 14,7 & 38,2 & 13,2 & 17,6 & 8,8 & 7,4 \\
\hline Rhizophagus dispar & 92 & 45,7 & 54,3 & 52,2 & 16,3 & 8,7 & 22,8 & 10,9 & 34,8 & 14,1 & 19,6 & 12,0 & 8,7 \\
\hline Rhizophagus ferrugineus & 18 & 33,3 & 66,7 & 38,9 & 11,1 & 22,2 & 27,8 & 11,1 & 16,7 & 16,7 & 22,2 & 16,7 & 16,7 \\
\hline Rhizophagus nitidulus & 45 & 26,7 & 73,3 & 71,1 & 20,0 & 4,4 & 4,4 & 22,2 & 53,3 & 8,9 & 6,7 & 6,7 & 2,2 \\
\hline Rhizophagus perforatus & 16 & 56,3 & 43,8 & 62,5 & 6,3 & 6,3 & 25,0 & 18,8 & 37,5 & 6,3 & 12,5 & 6,3 & 18,8 \\
\hline Scaphisoma balcanicum & 6 & 83,3 & 16,7 & 16,7 & 16,7 & 0,0 & 66,7 & 0,0 & 16,7 & 0,0 & 83,3 & 0,0 & 0,0 \\
\hline Schizotus pectinicornis & 5 & 20,0 & 80,0 & 40,0 & 40,0 & 20,0 & 0,0 & 0,0 & 40,0 & 0,0 & 60,0 & 0,0 & 0,0 \\
\hline Sepedophilus testaceus & 57 & 29,8 & 70,2 & 50,9 & 19,3 & 14,0 & 15,8 & 12,3 & 29,8 & 8,8 & 21,1 & 14,0 & 14,0 \\
\hline Sinodendron cylindricum & 9 & 22,2 & 77,8 & 22,2 & 11,1 & 22,2 & 44,4 & 11,1 & 11,1 & 22,2 & 22,2 & 11,1 & 22,2 \\
\hline Taphrorychus bicolor & 10 & 0,0 & 100,0 & 100,0 & 0,0 & 0,0 & 0,0 & 60,0 & 40,0 & 0,0 & 0,0 & 0,0 & 0,0 \\
\hline Teredus cylindricus & 21 & 81,0 & 19,0 & 28,6 & 4,8 & 4,8 & 61,9 & 0,0 & 14,3 & 23,8 & 38,1 & 14,3 & 9,5 \\
\hline Vincenzellus ruficollis & 13 & 0,0 & 100,0 & 92,3 & 7,7 & 0,0 & 0,0 & 23,1 & 46,2 & 15,4 & 7,7 & 0,0 & 7,7 \\
\hline Xyleborus saxeseni & 5 & 20,0 & 80,0 & 100,0 & 0,0 & 0,0 & 0,0 & 60,0 & 20,0 & 20,0 & 0,0 & 0,0 & 0,0 \\
\hline Xyloterus domesticus & 18 & 22,2 & 77,8 & 94,4 & 5,6 & 0,0 & 0,0 & 44,4 & 55,6 & 0,0 & 0,0 & 0,0 & 0,0 \\
\hline Xyloterus signatus & 22 & 59,1 & 40,9 & 81,8 & 4,5 & 4,5 & 9,1 & 27,3 & 50,0 & 9,1 & 4,5 & 9,1 & 0,0 \\
\hline Proben (99) in \% & & 48,5 & 51,5 & 48,5 & 15,2 & 9,1 & 27,3 & 10,1 & $\mathbf{3 4 , 3}$ & 15,2 & 20,2 & 12,1 & 8,1 \\
\hline
\end{tabular}

Von den insgesamt 99 mit STE an liegendem starkdimensionierten Totholz genommenen Proben entfielen 48,5 \% auf die Eiche, 51,5\% auf die Buche. An der befangenen Oberfläche hatte die Eiche einen Anteil von 42,9, die Buche von 57,1\%. 126 der insgesamt 165 in STEProben festgestellten obligaten Totholzarten wurden an Eichen- und 143 an Buchentotholz festgestellt. 
Von den 22 xylobionten Käferarten, die ausschließlich in STE-Proben an Eichentotholz aufraten, fand sich keine in mehr als 10 Proben, 20 wurden in weniger als 5 Fallen und in nur wenigen Einzelexemplaren festgestellt. Folglich sind nur 2 Arten in die Tabellen 4-42 und 4-43 aufgenommen worden. Ampedus erythrogonus wurde in 9 Proben mit insgesamt 13 Individuen gefangen. Die Stämme waren allesamt rindenlos, zu mindestens $80 \%$ überschirmt und gehörten in der Mehrzahl zu den BW-Klassen 3 und 2 und den SQ-Klassen 3 und 4. Hypulus quercinus wurde in 5 Proben mit 24 Individuen ausschließlich an Eiche registriert, in 2 Proben gemeinsam mit der vorhergehenden Art. Die Fangorte wiesen hinsichtlich Berindung, Überschirmung und SQ-Klasse im wesentlichen die gleichen Eigenschaften auf wie die von A. erythrogonus, allerdings wurde Hypulus quercinus in der Mehrheit an etwas festerem Holz (BW-Klasse 4) beobachtet.

Alle übrigen xylobionten Arten traten zumindest in Einzelexemplaren auch in STE-Proben an Buchentotholz in Erscheinung. Nur wenige Arten erfüllten die Präferenzkriterien für Eichentotholz. Neben dem Borkenkäfer Xyloterus signatus sind hier Atomaria bella, Scaphisoma balcanicum, Malthodes guttifer, Teredus cylindricus, Corticeus fasciatus, Anisotoma humeralis, Euplectus piceus und Phyllodrepa ioptera zu nennen.

39 saproxyle Arten waren in STE-Proben ausschließlich an liegendem Buchentotholz zu finden. Von ihnen waren 32 Arten in weniger als 5 Proben zugegen. Nur 4 Arten, Taphrorychus bicolor, Vincenzellus ruficollis, Octotemnus glabriculus und Epuraea variegata traten in mindestens 10 Proben in Erscheinung. 3 weitere Arten waren jeweils in 7 Proben nachzuweisen. Diese waren Cis boleti, Rhagium mordax und Scaphisoma agaricinum. Letzterer ist wegen der geringen Individuenzahl nicht in die Tabellen 4-42 und 4-43 aufgenommen worden. Im Vergleich zur Eiche beherbergten die STE-Proben an Buche eine deutlich größere Anzahl von Arten, die zumindest ein Präferenzkriterium für diese Baumart erfüllten. Eine besonders ausgeprägte Bevorzugung der Buche war für Ptilinus pectinicornis, Plegaderus dissectus, Ptinella aptera, Cis nitidus, Agathidium nigripenne, Corticeus unicolor und Orchesia undulata zu beobachten.

Die Faktoren Bohrwiderstand und Berindung sind miteinander positiv korreliert. Die Verteilung der Arten in Abhängigkeit dieser Faktoren wird deshalb im Folgenden gemeinsam behandelt.

Fast die Hälfte der aufgenommenen Proben bzw. der untersuchten Mantelfläche entfiel auf die Rindenklasse 4. Somit kommt hier vor allem das zweite Präferenzkriterium zum tragen. Sieben Arten erfüllten sowohl hinsichtlich der Frequenz- als auch der Individuenverteilung dieses Kriterium. Für acht weitere Arten gab nur die Individuenverteilung und für eine Art nur die Frequenzverteilung einen Hinweis auf eine Präferenz für die Ri-Klasse 4. Alle 16 Arten erfüllten zugleich ein Präferenzkriterium für die BW-Klassen 6 oder 5 oder hatten hier zumindest einen Verteilungsschwerpunkt. Hierher gehörten die Rindenkäfer Agathidium nigripenne, Glischrochilus quadriguttatus, Taphrorychus bicolor, Vincenzellus ruficollis, Anomognathus cuspidatus, Euryusa castanoptera, Phyllodrepoidea crenata, Rhinosimus ruficollis, Rhizophagus bipustulatus und Rhizophagus nitidulus. Darüber hinaus traten die Frischholzbesiedler Xyleborus saxeseni, Xyloterus domesticus und signatus und Hylecoetus dermestoides als 'Präferenten' für Proben der Ri-Klasse 4 und für Stammabschnitte mit hohem Bohrwiderstand in Erscheinung. Aber auch Ptilinus pectinicornis war in den STE-Proben am häufigsten in Stammabschnitten mit hohem Rindenprozent und hohem Bohrwiderstand zu beobachten. 
Fast sämtliche als Rindenbesiedler eingestufte Arten, die keines der beiden Präferenzkriterien für die Ri-Klasse 4 erfüllten, wurden dennoch in ihrer Mehrheit an Stammabschnitten der Ri-Klasse 4 und 3 gefangen. Nur Rhizophagus ferrugineus wurde in Stammabschnitten der Rindenklasse 1 und 2 ebenso häufig wie in denen der Klasse 3 und 4 gefangen und erfüllte sogar das Präferenzkriterium für die Ri-Klasse 2.

Einige Arten mit einer 'Präferenz' für Stammabschnitte mit hohem Bohrwiderstand erfüllten zwar nicht die Präferenzkriterien für die Ri-Klasse 4, hatten dort aber sehr wohl einen Verteilungsschwerpunkt. Zu nennen sind hier Corticeus unicolor, Pediacus dermestoides, Rhizophagus dispar und der als Mulmkäfer eingestufte Ptinella limbata.

Abschließend sollen noch einige Arten Erwähnung finden, die eine 'Präferenz' für rindenlose Stammteile und/oder Abschnitte mit niedrigem Bohrwiderstand zeigten. Hierbei handelt es sich überwiegend um Besiedler von Totholzpilzen oder um Mulmbewohner. Die Präferenzkriterien für die Ri-Klasse 1 wurden von Ampedus erythrogonus, Atomaria bella, Hypulus quercinus und Oxypoda recondita erfüllt. Für Scaphisoma balcanicum gilt dies nur hinsichtlich seiner Individuen- und für Teredus cylindricus nur hinsichtlich der Frequenzverteilung. 13 von 21 Proben mit Teredus-Befall entfielen auf Stammabschnitte der RiKlasse 1, dennoch wurden 65 von 89 Individuen an Abschnitten der Ri-Klasse 4 gefangen. Unabhängig von der Berindung wurden Stämme der BW-Klasse 3 bevorzugt. Beispiele für 'Präferenten' für Stämme mit niedrigem Bohrwiderstand sind ferner Cis nitidus, Epuraea varigata, Mycetophagus atomarius und Plegaderus dissectus.

Die klare Trennung der STE-Proben von Eichen- und Buchentotholz aufgrund ihrer Artenvergesellschaftungen war der Anlaß, den Sukzessionsprozeß an den beiden Baumarten im Folgenden getrennt zu untersuchen. 
Tabelle 4-43: Prozentuale Verteilung der Individuen in Abhängigkeit von Baumart, Rindenund Bohrwiderstandsklassen ('Präferenzen' hervorgehoben)

\begin{tabular}{|c|c|c|c|c|c|c|c|c|c|c|c|c|}
\hline $\begin{array}{l}\text { Art } \\
\text { Individuen }\end{array}$ & & Baun & $\begin{array}{l}\text { mart } \\
\mathrm{Bu}\end{array}$ & $\begin{array}{r}\text { Rinden } \\
\text { Ri } 4 \text { Ri } 3\end{array}$ & nklas & Ri 1 & Bw 6 & Bohry & viders & tands & klasse & Bw 1 \\
\hline Abraeus granulum & 29 & 10,3 & 89,7 & $48,324,1$ & 10,3 & 17,2 & 3,4 & 24,1 & 0,0 & 31,0 & 24,1 & 17,2 \\
\hline Agathidium nigripenne & 68 & 2,9 & 97,1 & $\mathbf{9 8 , 5} \quad 1,5$ & 0,0 & 0,0 & 63,2 & 35,3 & 1,5 & 0,0 & 0,0 & 0,0 \\
\hline Ampedus erythrogonus & 13 & 100,0 & 0,0 & $0,0 \quad 0,0$ & 0,0 & 100,0 & 0,0 & 7,7 & 7,7 & 53,8 & 23,1 & 7,7 \\
\hline Ampedus pomorum & 14 & 21,4 & 78,6 & $28,614,3$ & 21,4 & 35,7 & 14,3 & 28,6 & 0,0 & 21,4 & 21,4 & 14,3 \\
\hline Anaspis schilskyana & 20 & 55,0 & 45,0 & $35,0 \quad 5,0$ & 5,0 & 55,0 & 25,0 & 15,0 & 25,0 & 5,0 & 20,0 & 10,0 \\
\hline Anaspis thoracica & 16 & 37,5 & 62,5 & $37,531,3$ & 0,0 & 31,3 & 31,3 & 31,3 & 18,8 & 12,5 & 6,3 & 0,0 \\
\hline Anisotoma humeralis & 15 & 93,3 & 6,7 & $33,3 \quad 6,7$ & 0,0 & 60,0 & 0,0 & 33,3 & 0,0 & 6,7 & 26,7 & 33,3 \\
\hline Anisotoma orbicularis & 14 & 14,3 & 85,7 & $21,414,3$ & 42,9 & 21,4 & 0,0 & 64,3 & 0,0 & 0,0 & 35,7 & 0,0 \\
\hline Anomognathus cuspidatus & 63 & 12,7 & 87,3 & $\mathbf{9 2 , 1} 3,2$ & 1,6 & 3,2 & 39,7 & 41,3 & 12,7 & 3,2 & 1,6 & 1,6 \\
\hline Arpidiphorus orbiculatus & 19 & 10,5 & 89,5 & $52,610,5$ & 5,3 & 31,6 & 21,1 & 36,8 & 5,3 & 10,5 & 10,5 & 15,8 \\
\hline Atomaria bella & 28 & 96,4 & 3,6 & $17,9 \quad 0,0$ & 0,0 & 82,1 & 0,0 & 14,3 & 28,6 & 39,3 & 17,9 & 0,0 \\
\hline Atomaria pulchra & 13 & 46,2 & 53,8 & $38,5 \quad 7,7$ & 7,7 & 46,2 & 23,1 & 15,4 & 23,1 & 15,4 & 15,4 & 7,7 \\
\hline Atrecus affinis & 10 & 50,0 & 50,0 & $70,010,0$ & 10,0 & 10,0 & 0,0 & 50,0 & 10,0 & 10,0 & 10,0 & 20,0 \\
\hline Bibloporus bicolor & 121 & 40,5 & 59,5 & $29,8 \mathbf{3 0 , 6}$ & 9,9 & 29,8 & 3,3 & 31,4 & 9,1 & 35,5 & 19,8 & 0,8 \\
\hline Bolitochara obliqua & 156 & 35,3 & 64,7 & $73,126,3$ & 0,0 & 0,6 & 25,6 & 55,8 & 10,9 & 2,6 & 4,5 & 0,6 \\
\hline Cerylon fagi & 126 & 44,4 & 55,6 & $66,723,8$ & 2,4 & 7,1 & 7,1 & 37,3 & 16,7 & 16,7 & 14,3 & 7,9 \\
\hline Cerylon ferrugineum & 709 & 20,2 & 79,8 & $65,626,4$ & 4,4 & 3,7 & 13,8 & 32,9 & 23,6 & 14,1 & 13,1 & 2,5 \\
\hline Cerylon histeroides & 100 & 31,0 & 69,0 & $45,026,0$ & 19,0 & 10,0 & 5,0 & 22,0 & 20,0 & 32,0 & 15,0 & 6,0 \\
\hline Cis boleti & 21 & 0,0 & 100,0 & $4,828,6$ & 28,6 & 38,1 & 0,0 & 33,3 & 4,8 & 61,9 & 0,0 & 0,0 \\
\hline Cis nitidus & 51 & 2,0 & 98,0 & $70,6 \quad 9,8$ & 13,7 & 5,9 & 0,0 & 13,7 & 0,0 & 5,9 & 62,7 & 17,6 \\
\hline Corticaria linearis & 14 & 71,4 & 28,6 & $71,421,4$ & 0,0 & 7,1 & 21,4 & 64,3 & 7,1 & 7,1 & 0,0 & 0,0 \\
\hline Corticaria longicollis & 877 & 66,4 & 33,6 & $39,017,0$ & 5,5 & 38,5 & 6,0 & 30,4 & 24,3 & 20,3 & 13,6 & 5,4 \\
\hline Corticeus fasciatus & 13 & 84,6 & 15,4 & $53,8 \quad 7,7$ & 7,7 & 30,8 & 0,0 & 7,7 & 15,4 & 53,8 & 15,4 & 7,7 \\
\hline Corticeus unicolor & 314 & 6,4 & 93,6 & $77,420,1$ & 0,3 & 2,2 & 55,1 & 21,7 & 10,2 & 3,2 & 9,2 & 0,6 \\
\hline Denticollis linearis & 54 & 59,3 & 40,7 & $44,416,7$ & 9,3 & 29,6 & 1,9 & 44,4 & 11,1 & 14,8 & 13,0 & 14,8 \\
\hline Dinaraea aequata & 34 & 52,9 & 47,1 & $76,520,6$ & 2,9 & 0,0 & 11,8 & 35,3 & 26,5 & 2,9 & 20,6 & 2,9 \\
\hline Dinaraea linearis & 37 & 24,3 & 75,7 & $83,810,8$ & 0,0 & 5,4 & 18,9 & 37,8 & 2,7 & 0,0 & 32,4 & 8,1 \\
\hline Epuraea marseuli & 30 & 66,7 & 33,3 & $80,0 \quad 0,0$ & 0,0 & 20,0 & 20,0 & 46,7 & 0,0 & 20,0 & 10,0 & 3,3 \\
\hline Epuraea variegata & 72 & 0,0 & 100,0 & $38,927,8$ & 27,8 & 5,6 & 1,4 & 13,9 & 1,4 & 23,6 & 8,3 & 51,4 \\
\hline Euplectus bescidicus & 24 & 29,2 & 70,8 & $70,812,5$ & 4,2 & 12,5 & 20,8 & 45,8 & 25,0 & 0,0 & 4,2 & 4,2 \\
\hline Euplectus karsteni & 22 & 45,5 & 54,5 & $9,122,7$ & 9,1 & 59,1 & 0,0 & 27,3 & 13,6 & 50,0 & 9,1 & 0,0 \\
\hline Euplectus piceus & 41 & 82,9 & 17,1 & $36,614,6$ & 2,4 & 46,3 & 4,9 & 12,2 & 2,4 & 34,1 & 26,8 & 19,5 \\
\hline Euplectus punctatus & 53 & 26,4 & 73,6 & $52,8 \mathbf{3 4 , 0}$ & 3,8 & 9,4 & 20,8 & 24,5 & 15,1 & 26,4 & 11,3 & 1,9 \\
\hline Euryusa castanoptera & 145 & 16,6 & 83,4 & $\mathbf{9 2 , 4} 6,2$ & 0,0 & 1,4 & 47,6 & 36,6 & 13,1 & 2,8 & 0,0 & 0,0 \\
\hline Gabrius splendidulus & 192 & 11,5 & 88,5 & $53,1 \mathbf{3 1 , 8}$ & 3,6 & 11,5 & 13,5 & 28,1 & 6,8 & 24,0 & 23,4 & 4,2 \\
\hline Glischrochilus quadriguttatus & 56 & 32,1 & 67,9 & $\mathbf{9 4 , 6} \quad 3,6$ & 0,0 & 1,8 & 50,0 & 48,2 & 0,0 & 0,0 & 1,8 & 0,0 \\
\hline Hylecoetus dermestoides & 885 & 13,6 & 86,4 & $78,421,6$ & 0,0 & 0,0 & 66,9 & 20,6 & 7,6 & 5,0 & 0,0 & 0,0 \\
\hline Hypulus quercinus & 24 & 100,0 & 0,0 & $0,0 \quad 0,0$ & 0,0 & 100,0 & 0,0 & 0,0 & 75,0 & 20,8 & 4,2 & 0,0 \\
\hline Leptusa fumida & 281 & 26,3 & 73,7 & $56,6 \mathbf{3 2 , 0}$ & 2,8 & 8,5 & 19,6 & 38,1 & 8,9 & 13,9 & 17,4 & 2,1 \\
\hline Leptusa pulchella & 139 & 50,4 & 49,6 & $56,823,0$ & 2,9 & 17,3 & 4,3 & 46,8 & 12,2 & 10,8 & 20,9 & 5,0 \\
\hline
\end{tabular}




\begin{tabular}{|c|c|c|c|c|c|c|c|c|c|c|c|c|c|}
\hline \multicolumn{2}{|l|}{$\begin{array}{l}\text { Art } \\
\text { Individuen } \\
\end{array}$} & Baun & $\begin{array}{l}\text { mart } \\
\qquad \mathrm{Bu}\end{array}$ & & Rindenl & klass & $\mathbf{R i} 1$ & Bw 6 & Bohry & viders & standsk & klasse & Bw 1 \\
\hline Malthodes guttifer & 26 & 96,2 & & 57,7 & 715,4 & 3,8 & 23,1 & 0,0 & 65,4 & 15,4 & 15,4 & 3,8 & 0,0 \\
\hline Melanotus castanipes & 44 & 27,3 & 72,7 & 40,9 & 920,51 & 15,9 & 22,7 & 15,9 & 29,5 & 11,4 & 11,4 & 18,2 & 13,6 \\
\hline Mycetophagus atomarius & 17 & 5,9 & 94,1 & 17,6 & 623,55 & 58,8 & 0,0 & 0,0 & 5,9 & 0,0 & 35,3 & 0,0 & $\mathbf{5 8 , 8}$ \\
\hline Octotemnus glabriculus & 122 & 0,0 & 100,0 & 3,3 & $3 \mathbf{5 8 , 2} 1$ & 17,2 & 21,3 & 1,6 & 59,0 & 0,0 & 33,6 & 4,9 & 0,8 \\
\hline Orchesia undulata & 183 & 7,7 & 92,3 & 41,5 & 528,4 & 9,3 & 20,8 & 20,2 & 53,0 & 9,8 & 6,6 & 7,7 & 2,7 \\
\hline Orthoperus mundus & 110 & 40,9 & 59,1 & 83,6 & 612,7 & 1,8 & 1,8 & 35,5 & 36,4 & 12,7 & 11,8 & 1,8 & 1,8 \\
\hline Oxypoda recondita & 10 & 80,0 & 20,0 & 20,0 & $0 \quad 0,0$ & 0,0 & $\mathbf{8 0 , 0}$ & 0,0 & 30,0 & 30,0 & 10,0 & 20,0 & 10,0 \\
\hline Paromalus flavicornis & 68 & 13,2 & 86,8 & 48,5 & 542,6 & 7,4 & 1,5 & 22,1 & 26,5 & 4,4 & 23,5 & 14,7 & 8,8 \\
\hline Pediacus derm & 42 & 11,9 & 88,1 & 69,0 & 011,9 & 9,5 & 9,5 & 33,3 & 40,5 & 9,5 & 14,3 & 0,0 & 2,4 \\
\hline Phloeocharis subtilissima & 182 & 37,4 & 62,6 & 57,7 & 715,4 & 4,9 & 22,0 & 20,3 & 33,0 & 14,3 & 9,3 & 15,4 & 7,7 \\
\hline Phloeonomus pun & 711 & 43,9 & 56,1 & 68,1 & 116,0 & 8,4 & 7,5 & 18,7 & 40,1 & 16,3 & 7,7 & 11,3 & 5,9 \\
\hline Phloeopora corticalis & 12 & 58,3 & 41,7 & 75,0 & 025,0 & 0,0 & 0,0 & 8,3 & 66,7 & 25,0 & 0,0 & 0,0 & 0,0 \\
\hline Phloeopora testacea & 14 & 21,4 & 78,6 & 85,7 & $7 \quad 0,0$ & 7,1 & 7,1 & 35,7 & 21,4 & 28,6 & 7,1 & 0,0 & 7,1 \\
\hline Phyllodrepa ioptera & 67 & 76,1 & 23,9 & 37,3 & 328,4 & 1,5 & 32,8 & 10,4 & 37,3 & 26,9 & 16,4 & 6,0 & 3,0 \\
\hline Phyllpdrepoidea cr & 11 & 54,5 & 45,5 & 90,0 & $\mathbf{0} \quad 0,0$ & 9,1 & 0,0 & 27,3 & 63,6 & 0,0 & 9,1 & 0,0 & 0,0 \\
\hline Plecto & 22 & 50,0 & 50,0 & 59,1 & 113,6 & 0,0 & 27,3 & 0,0 & 22,7 & 40,9 & 36,4 & 0,0 & 0, \\
\hline Plegaderus dissectus & 129 & 1,6 & 98,4 & 62,8 & 820,21 & 11,6 & 5,4 & 3,9 & 9,3 & 0,8 & 15,5 & 63,6 & 7,0 \\
\hline Pteryx suturalis & 661 & 36,3 & 63,7 & 51,3 & 319,71 & 13,8 & 15,3 & 11,0 & 28,3 & 16,5 & 8,2 & 15,4 & 20,6 \\
\hline Ptilinus pectinicor & 272 & 1,5 & 98,5 & 80,1 & 112,5 & 4,8 & 2,6 & 41,2 & 36,4 & 6,6 & 7,0 & 6,3 & 2,6 \\
\hline Ptinella aptera & 185 & 1,6 & 98,4 & 43,8 & 35,71 & 17,8 & 2,7 & 9,2 & 54,6 & 7,0 & 8,6 & 18,4 & 2,2 \\
\hline Ptinella limbata & 716 & 42,6 & 57,4 & 64,5 & 521,51 & 12,2 & 1,8 & 8,8 & 67,5 & 6,4 & 2,5 & 11,7 & 3,1 \\
\hline Pyrochroa coccinea & 29 & 51,7 & 48,3 & 69,0 & 20,7 & 3,4 & 6,9 & 3,4 & 55,2 & 10,3 & 17,2 & 10,3 & 3,4 \\
\hline Rhagium mordax & 12 & 0,0 & 100,0 & 25,0 & 75,0 & 0,0 & 0,0 & 8,3 & 50,0 & 8,3 & 16,7 & 16,7 & 0,0 \\
\hline Rhinosimus ruficollis & 105 & 63,8 & 36,2 & 94,3 & 34,8 & 0,0 & 1,0 & 22,9 & 76,2 & 0,0 & 0,0 & 1,0 & 0,0 \\
\hline Rhizophagus bipustu & 786 & 48,3 & 51,7 & 85,4 & 49,0 & ,7 & 3,9 & 32,7 & 44,4 & 12,6 & 6,2 & 2,2 & 1,9 \\
\hline Rhizop & 5022 & 23,3 & 76,7 & 77,7 & 716,9 & 2,1 & 3,2 & 30,9 & 41,9 & 10,7 & 5,1 & 8,8 & 2,7 \\
\hline Rhizophagus ferrugineus & 35 & 20,0 & 80,0 & 22,9 & 25,73 & 34,3 & 17,1 & 25,7 & 28,6 & 8,6 & 17,1 & 11,4 & 8,6 \\
\hline Rhizophagus nitidulus & 421 & 27,6 & 72,4 & 80,8 & 18,3 & 0,5 & 0,5 & 28,3 & 44,7 & 11,2 & 12,8 & 2,9 & 0,2 \\
\hline Rhizophagus perforatus & 41 & 61,0 & 39,0 & 73,2 & 2,4 & 4,9 & 19,5 & 24,4 & 34,1 & 2,4 & 17,1 & 12,2 & 9,8 \\
\hline Scaphisoma balcanicum & 21 & 95,2 & 4,8 & 9,5 & $5 \quad 4,8$ & 0,0 & $\mathbf{8 5 , 7}$ & 0,0 & 9,5 & 0,0 & 90,5 & 0,0 & 0,0 \\
\hline Schizotus pectinicornis & 19 & 5,3 & 94,7 & 26,3 & 68,4 & 5,3 & 0,0 & 0,0 & 26,3 & 0,0 & 73,7 & 0,0 & 0,0 \\
\hline Sepedophilus testaceus & 320 & 14,7 & 85,3 & 41,3 & 25,91 & 14,1 & 18,8 & 3,8 & 22,8 & 7,5 & 17,2 & 25,3 & 23,4 \\
\hline Sinodendron cylindricum & 26 & 15,4 & 84,6 & 15,4 & 38,5 & 7,7 & 38,5 & 3,8 & 19,2 & 15,4 & 42,3 & 3,8 & 15,4 \\
\hline Taphrorychus bicolor & 46 & 0,0 & 100,0 & 100,0 & 0,0 & 0,0 & 0,0 & 67,4 & 32,6 & 0,0 & 0,0 & 0,0 & 0,0 \\
\hline Teredus cylindricus & 89 & $\mathbf{9 4 , 4}$ & 5,6 & 73,0 & 2,2 & 1,1 & 23,6 & 0,0 & 3,4 & 7,9 & 82,0 & 4,5 & 2,2 \\
\hline Vincenzellus ruficollis & 136 & 0,0 & 100,0 & 99,3 & 30,7 & 0,0 & 0,0 & 16,9 & 61,8 & 19,9 & 0,7 & 0,0 & 0,7 \\
\hline Xyleborus saxeseni & 129 & 3,9 & 96,1 & 100,0 & 0,0 & 0,0 & 0,0 & 17,1 & 79,1 & 3,9 & 0,0 & 0,0 & 0,0 \\
\hline Xyloterus domesticus & 1276 & 43,7 & 56,3 & 99,6 & $5 \quad 0,4$ & 0,0 & 0,0 & 30,6 & 69,4 & 0,0 & 0,0 & 0,0 & 0,0 \\
\hline Xyloterus signatus & 1559 & $\mathbf{9 8 , 3}$ & 1,7 & 98,9 & 0,7 & 0,1 & 0,3 & 1,3 & $\mathbf{9 8 , 0}$ & 0,3 & 0,1 & 0,3 & 0,0 \\
\hline $\begin{array}{l}\text { Mantelfläche }\left(130 \mathrm{~m}^{2}\right) \text { in \% } \\
\text { Rindenfläche }\left(85 \mathrm{~m}^{2}\right) \text { in \% }\end{array}$ & & $\begin{array}{l}42,9 \\
30,6\end{array}$ & $\begin{array}{l}57,1 \\
69,4\end{array}$ & $\begin{array}{l}49,6 \\
75,9\end{array}$ & $\begin{array}{l}6 \\
15,2 \\
19,0\end{array}$ & $\begin{array}{l}9,2 \\
5,0\end{array}$ & $\begin{array}{r}26,0 \\
0,0\end{array}$ & $\begin{array}{l}13,2 \\
20,0\end{array}$ & $\begin{array}{l}32,0 \\
40,1\end{array}$ & $\begin{array}{r}14,8 \\
9,8\end{array}$ & $\begin{array}{l}18,7 \\
11,2\end{array}$ & $\begin{array}{l}12,8 \\
13,1\end{array}$ & $\begin{array}{l}8,7 \\
5,8\end{array}$ \\
\hline
\end{tabular}




\subsubsection{Sukzession der Artengemeinschaften an liegendem Buchentotholz}

Zunächst wurde die Ähnlichkeit der an liegendem Buchentotholz genommenen STE-Proben in ihrer Artenvergesellschaftung untersucht. Berechnet wurden die Dominantenidentitäten nach Renkonen einerseits auf Grundlage der logarithmierten Schlüpfdichten ( $\left.\mathrm{RE}_{\mathrm{ln}}\right)$ (Tab. 4-44), andererseits ohne Logarithmierung (RE) (Anhang 7b) sowie die Sørensenquotienten (SQ) (Anhang 7a).

Tabelle 4-44 läßt entlang der Diagonalen die geforderte Konzentration hoher Ähnlichkeitswerte erkennen. Im linken oberen Teil der Tabelle ist eine Häufung von Proben festzustellen, die sich untereinander durch hohe Ähnlichkeitswerte auszeichnen. Sie waren von Stämmen genommen worden, die erst am Beginn der Zersetzung standen und i. d. R. noch keinen Bodenkontakt hatten, da die Kronenäste als Abstandshalter fungierten. Das Holz wies eine noch große Festgkeit auf und die Stämme waren in der weit überwiegenden Zahl der Fälle vollständig berindet. Eine in der Tendenz fortschreitende Zersetzung des beprobten Materials gibt sich entlang der Diagonalen zu erkennen. Damit einhergehend wurden nur noch vereinzelt hohe Ähnlichkeitswerte ( 60 \%) erreicht. Sieht man von der ersten Zersetzungsstufe $\mathrm{ab}$, waren anhand der Dominantenidentitäten keine eindeutigen Fallengruppierungen zu erkennen, die sich durch große Ähnlichkeit untereinander bei gleichzeitig geringer Übereinstimmung in der Struktur der Artengemeinschaften mit den Mitgliedern benachbarter Gruppen hervorgehoben hätten. Am ehesten wären hier die Fallengruppen 17b/96 - 17a/96, 11/95 01b/96, 18a/96 - 15/96, 05a/96 - 20/95 und 03/95 - 19b/95 abzugrenzen. Der Wechsel in der Zusammensetzung der Käferzönosen entlang des Zersetzungsgradienten stellte sich insgesamt eher als ein Kontinuum dar. Gleichzeitig ließ die Anordnung der Proben vermuten, dass sich verschiedene, voneinander mehr oder weniger unabhängige Gradienten in dem erhobenen Datenmaterial manifestieren, bzw. dass die beobachteten Artenvergesellschaftungen die Folge von Faktorenkombinationen sind, die nur z. T. eng mit der Zersetzungsdauer korreliert sind.

Eine den vorangegangenen Ausführungen sehr ähnliche Anordnung der Proben ergab sich auch als Ergebnis einer DCA (Abb. 4-36). Da bei diesem Verfahren insgesamt vier Achsen (hypothetische Variablen) berechnet werden, ließen sich in vielen Fällen Fallengruppen deutlicher voneinander abgrenzen. Von den 229 Arten, deren logarithmierte Schlüpfdichten Grundlage für die Berechnungen waren, sind aus Gründen der Darstellbarkeit vor allem Arten mit geringer Frequenz nicht in das Ordinationsdiagramm (Abb. 4-37) aufgenommen worden. Die wichtigsten Ergebnisse verschiedener Korrespondenzanalysen werden in der Tabelle 4-45 vergleichend gegenübergestellt.

Die Eigenwerte der in den Abbildungen 4-36 und 4-37 dargestellten Ordinationsachsen betrugen 0,353 (Achse 1) und 0,190 (Achse 2), die der nicht dargestellten dritten und vierten Achse beliefen sich auf 0,142 und 0,121. Damit konnten die vier hypothetischen Variablen gemeinsam 19,4 \% der Gesamtvarianz des Datenmaterials erklären. Blieben die zahlreichen nur in Einzelproben auftretenden Arten unberücksichtigt, erhöhte sich der erklärbare Anteil auf $29,1 \%$. 
Tabelle 4-44: Ähnlichkeitsmatrix $\left(\mathrm{RE}_{\mathrm{ln}}\right)$ der STE-Proben an liegendem Buchentotholz

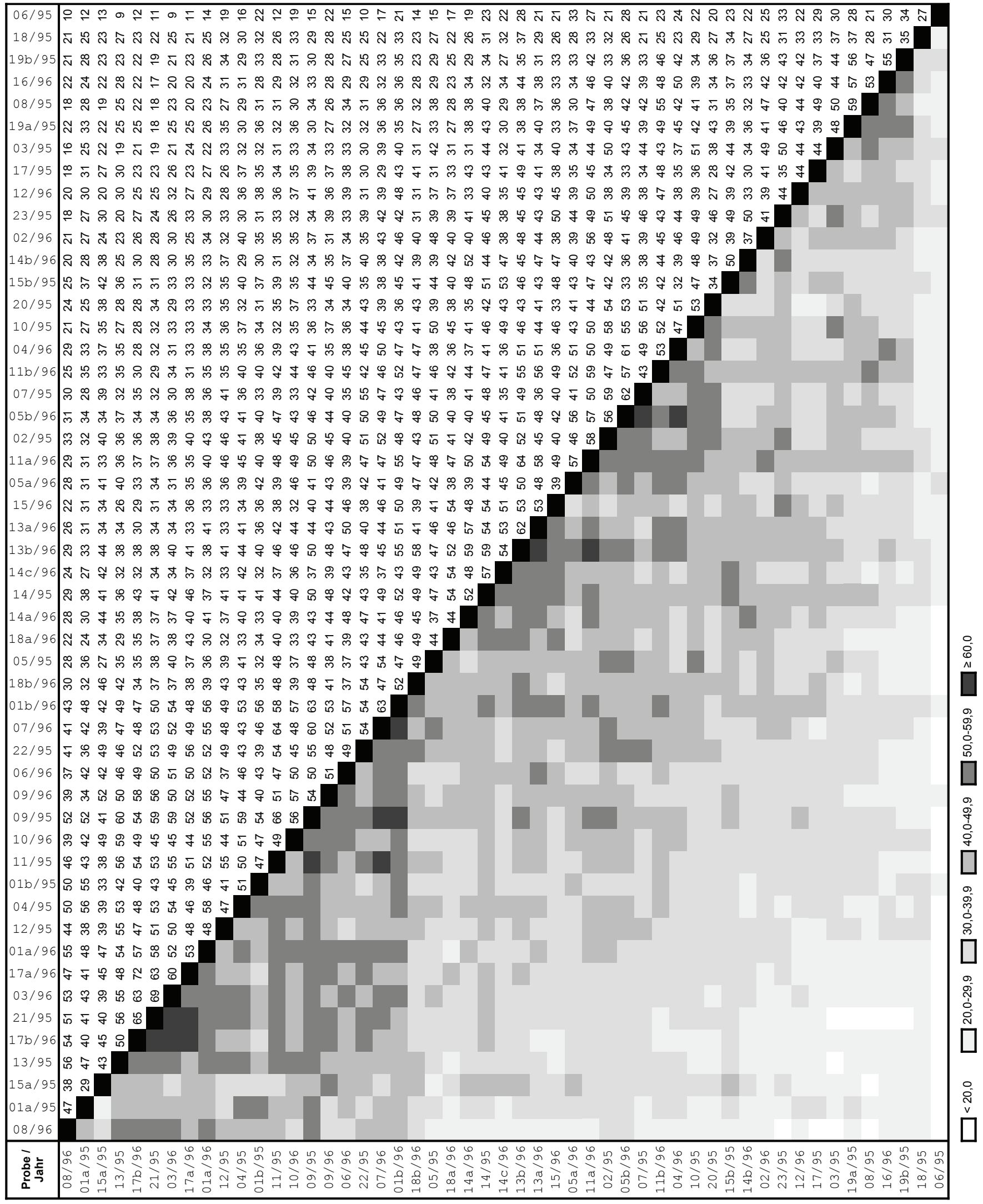




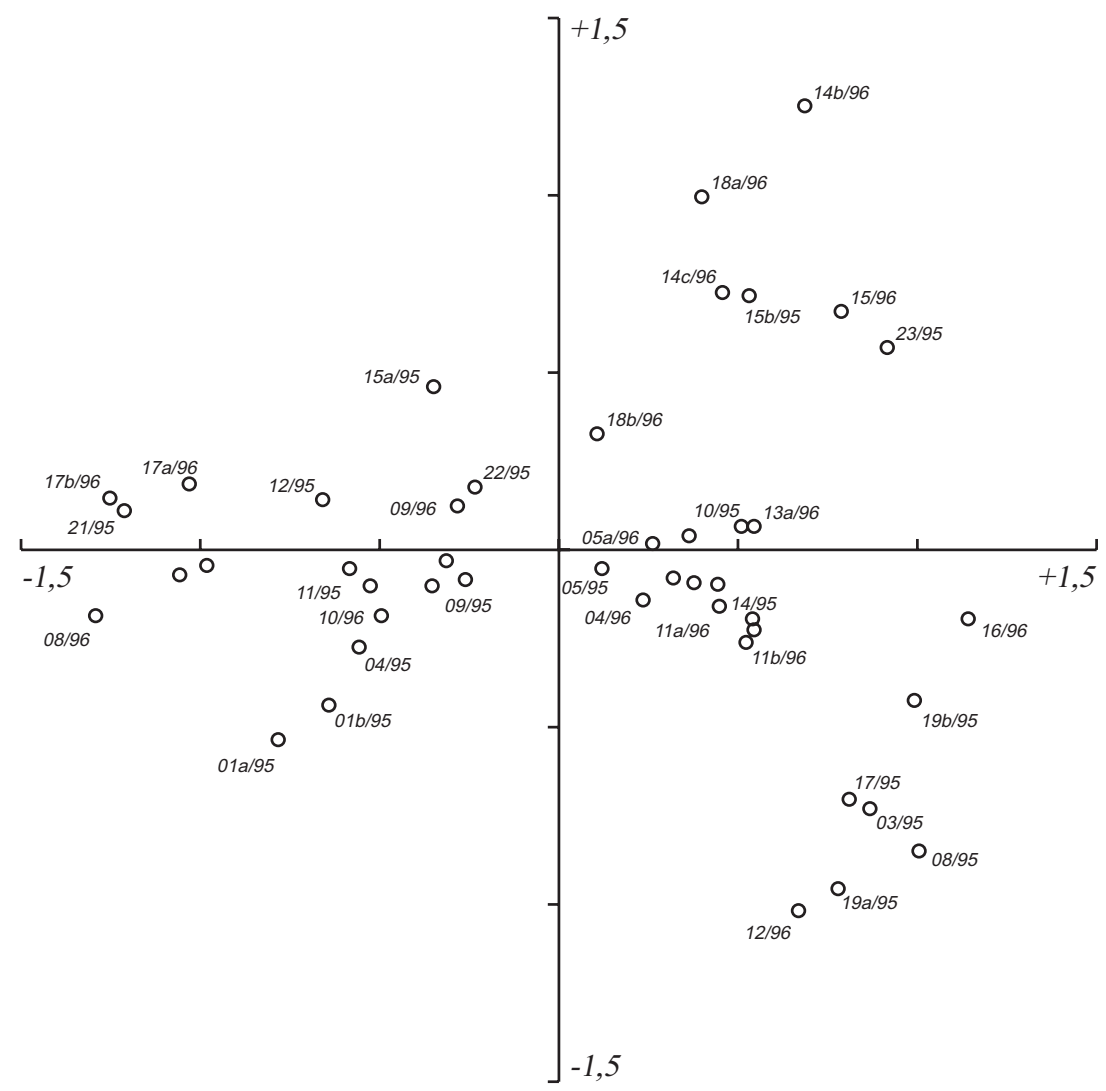

Abb. 4-36: Ordinationsdiagramm (DCA): STE-Proben an liegendem Buchentotholz

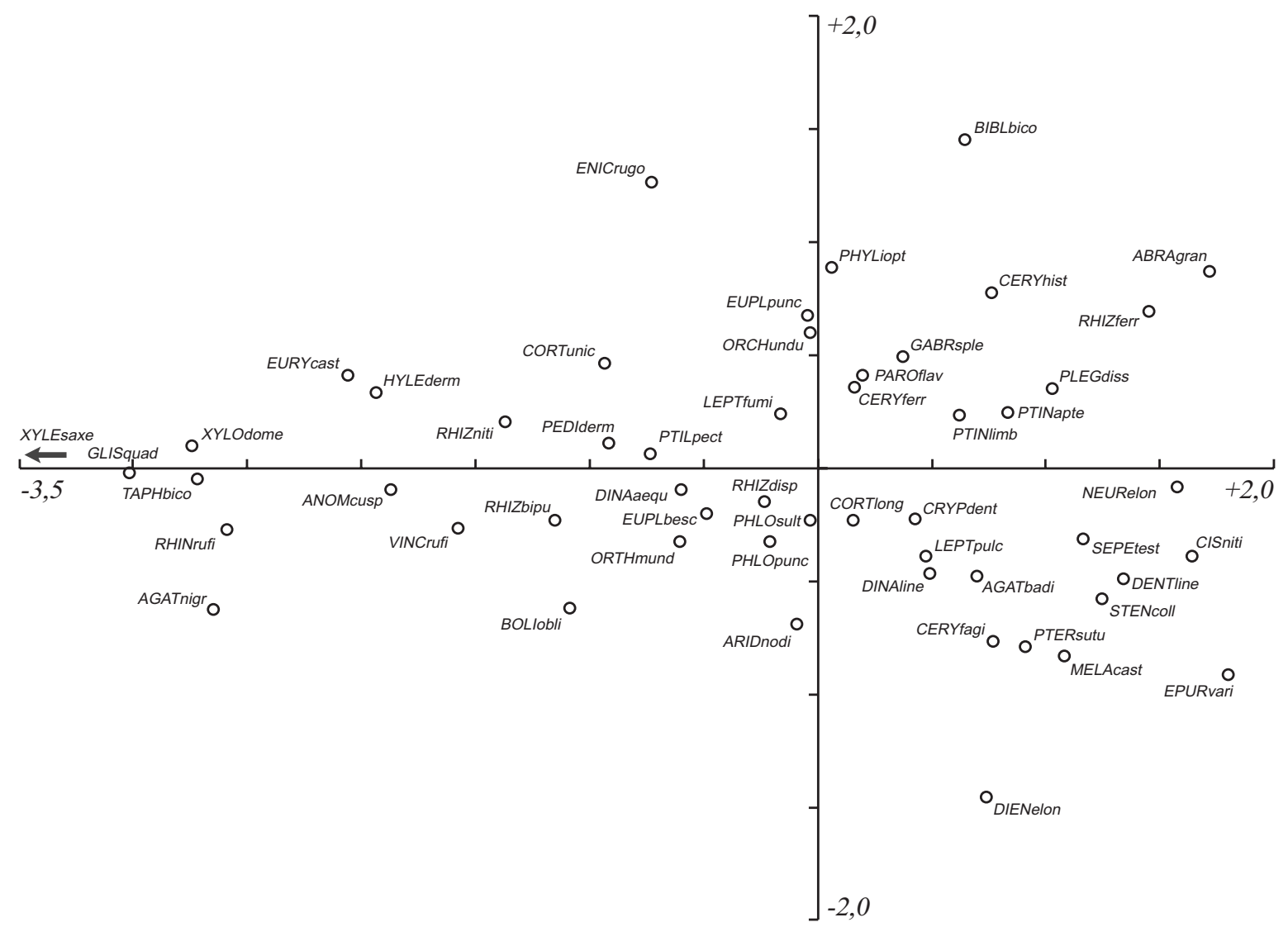

Abb. 4-37: Ordinationsdiagramm (DCA): Arten aus STE-Proben an liegendem Buchentotholz 
Tabelle 4-45: Ergebnisse verschiedener Korrespondenzanalysen mit den Fangdaten aus STE-Proben an Buchentotholz

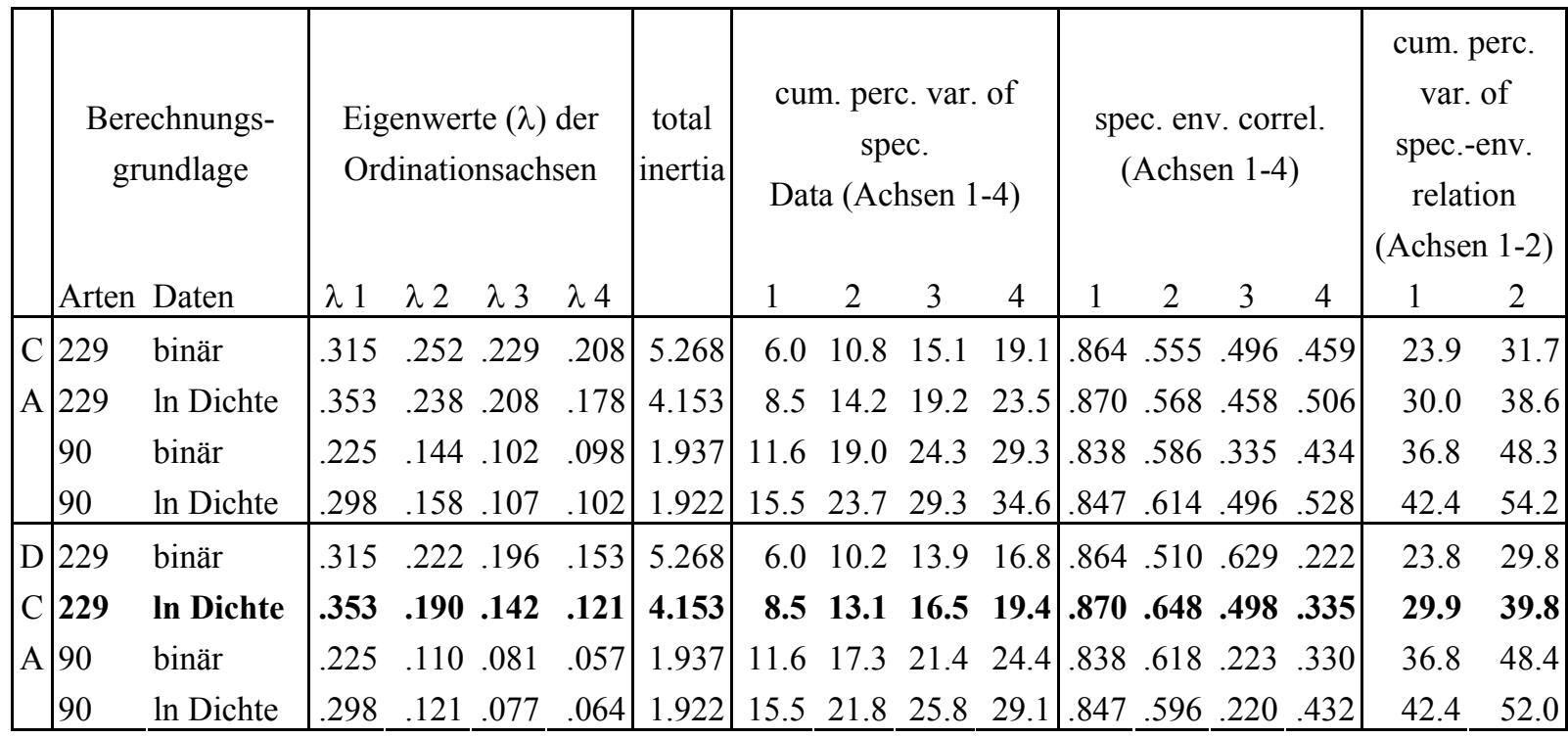

Die vorgefundenen Artenvergesellschaftungen lassen sich zum Teil durch die Dauer des Zersetzungsprozesses erklären. Im linken Teil des Ordinationsdiagrammes ist eine Konzentration von Proben zu beobachten, die zum Zeitpunkt der Beprobung erst seit wenigen Jahren abgestorben waren. Entsprechend wurden hier jene Arten positioniert, die eine Präferenz für vergleichsweise frisches Totholz erkennen lassen, wie z. B. die Borkenkäferarten Xyleborus saxeseni, Xyloterus domesticus und Taphrorychus bicolor. Im rechten Teil der Grafik findet sich hingegen der größte Teil jener Proben, die bereits seit mindestens 6 Jahren abgestorben waren. Arten mit einer Präferenz für Mulm und Totholzpilze finden sich hier in größerer Anzahl.

Deutlich zeigt sich aber auch, dass bei fortgeschrittener Zersetzungsdauer die Streuung in der Artenvergesellschaftung zunahm, was sich bereits in den sinkenden Werten der Dominantenidentitäten angedeutet hatte. Abgesehen von den ersten Jahren ist der Absterbezeitpunkt offenbar nur noch in geringerem Maße geeignet, die Struktur der Artengemeinschaften zu erklären, was möglicherweise darauf zurückgeführt werden kann, dass die Zersetzungsgeschwindigkeit der einzelnen Totholzobjekte stark variiert und die Korrelation zwischen Absterbezeitpunkt und Zersetzungszustand zunehmend geringer wird. Die Vergesellschaftung der Arten wurde allem Anschein nach von anderen Faktoren als der Zersetzungsdauer beeinflusst.

Um die Beziehung zwischen den an den Fallenstandorten erhobenen Habitatvariablen und den festgestellten Artenkompositionen aufzudecken und deren Eignung zur Erklärung der vorgefundenen Vergesellschaftungen zu prüfen, wurden die Daten verschiedenen Verfahren der kanonischen Korrespondenzanalyse unterzogen. Da für eine große Anzahl von Proben keine Informationen zum Absterbezeitpunkt vorlagen und hiervon besonders stärker zersetzte Stämme betroffen waren, blieb dieser Faktor in der weiteren Auswertung unberücksichtigt.

Die wichtigsten Ergebnisse verschiedener kanonischer Korrespondenzanalysen auf unterschiedlicher Datenbasis sind in der Tabelle 4-46 vergleichend gegenübergestellt. Die 
Abbildungen 4-38 und 4-39 zeigen die Ordinationsdiagramme einer CCA, bei der die Proben und Arten in Abhängigkeit von den an den Fallenstandorten erhobenen Variablen dargestellt werden. Grundlage für die Berechnungen waren die logarithmierten Schlüpfdichten jener 90 Arten, die in mindestens 5 Proben angetroffen worden waren. Nur ein Teil von ihnen fand in der graphischen Darstellung Platz. Für die als passiv gekennzeichneten Proben lagen keine vollständigen Angaben zu den Habitatvariablen vor. Ihre Positionen wurden in einem zweiten Schritt einzig und allein aufgrund ihrer Artenkombinationen bestimmt.

Tabelle 4-46: Ergebnisse verschiedener kanonischer Korrespondenzanalysen mit den Fangdaten aus STE-Proben an liegendem Buchentotholz

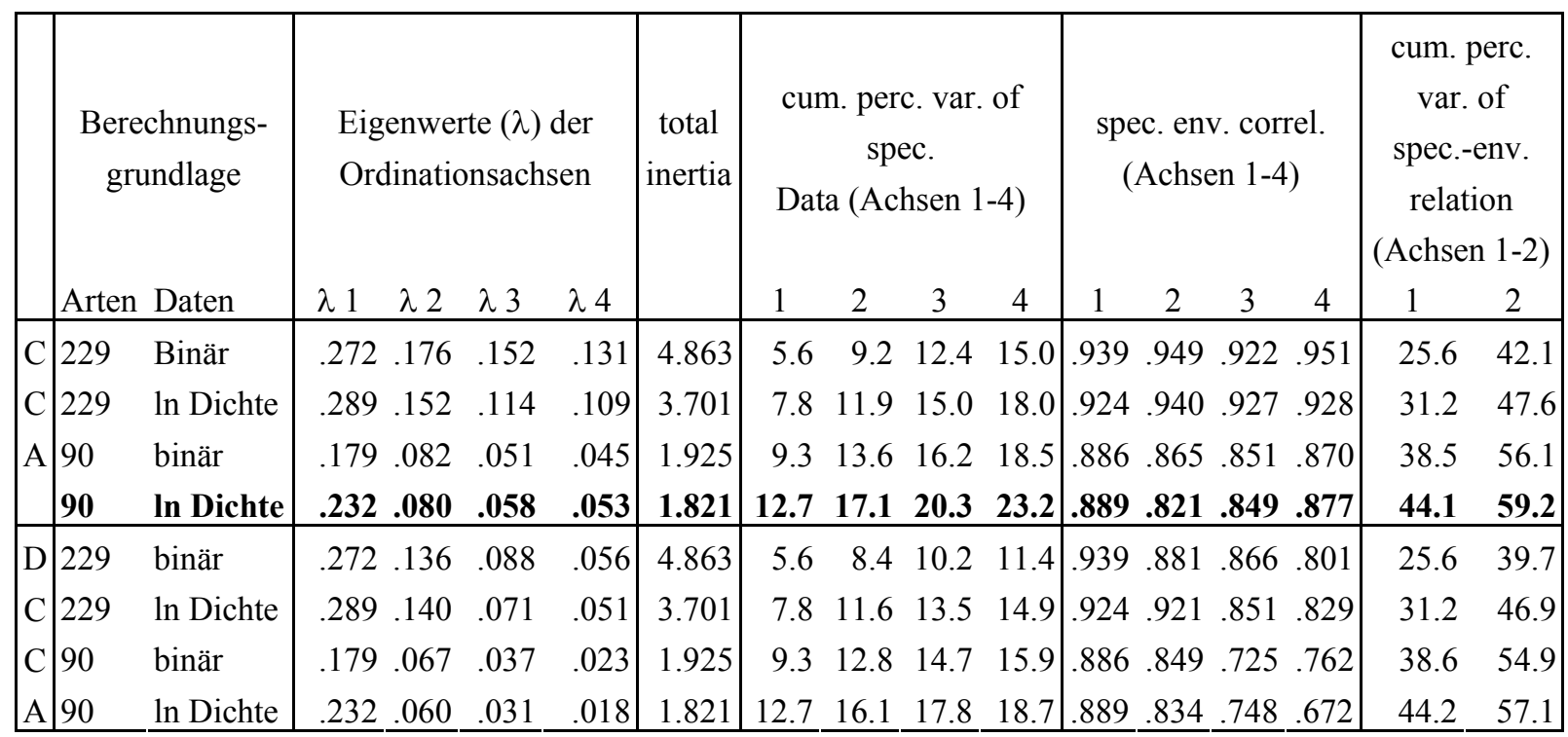




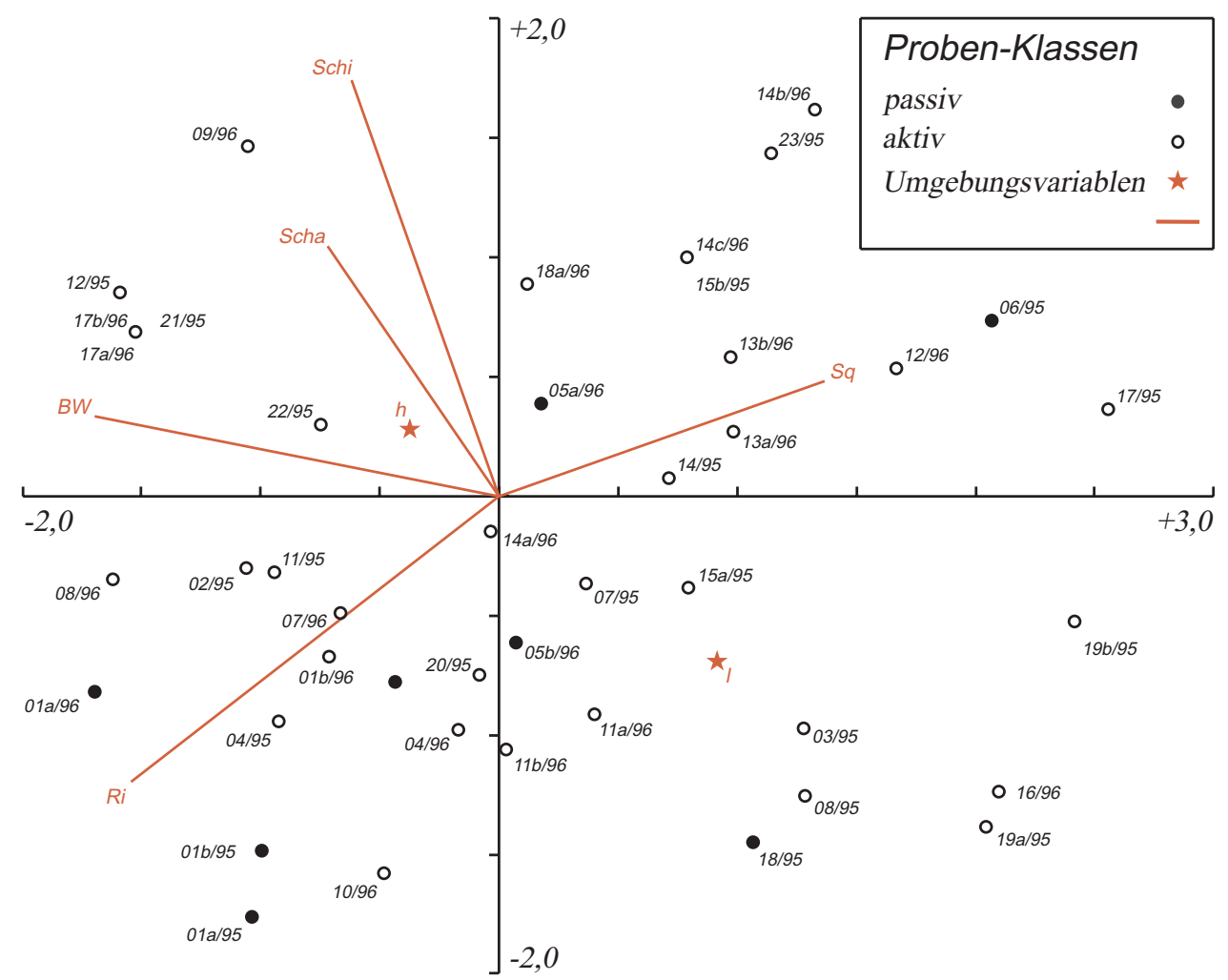

Abb. 4-38: Ordinationsdiagramm (CCA): STE-Proben an liegendem Buchentotholz und Habitatvariablen

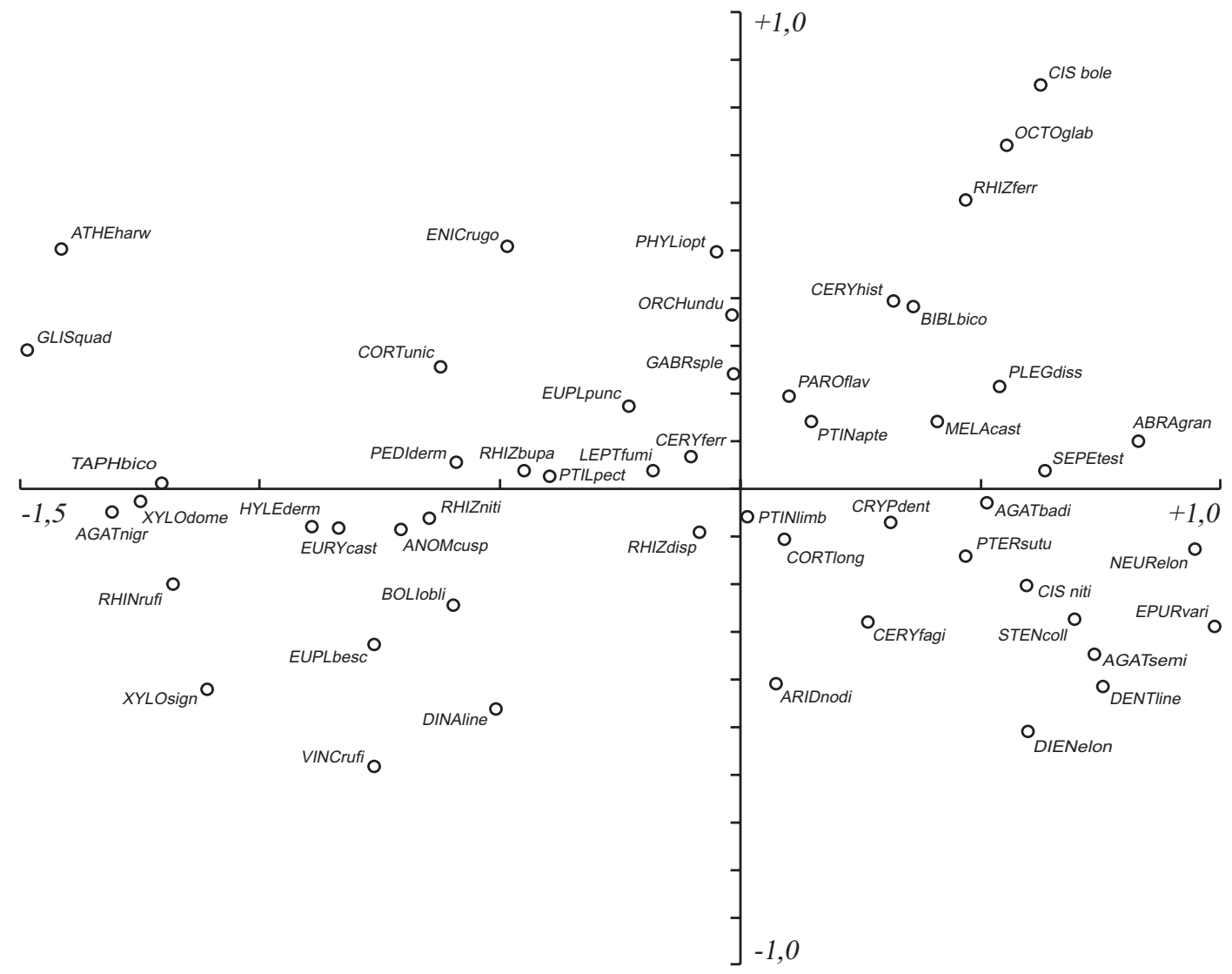

Abb. 4-39: Ordinationsdiagramm (CCA): Arten aus STE-Proben an liegendem Buchentotholz 
Der Eigenwert der 1. Achse betrug 0,232. Im Vergleich hierzu war die Bedeutung der übrigen Achsen mit Eigenwerten von 0,080, 0,058 und 0,053 deutlich geringer. Ein nennenswerter Gradient war somit fast ausschließlich entlang der ersten Ordinationsachse ausgeprägt. Eine Gegenüberstellung der Ergebnisse der Korrespondenzanalysen und der kanonischen Korrespondenzanalysen ließ den Schluß zu, dass die gemessenen Variablen durchaus geeignet waren, einen nicht unwesentlichen Anteil an der in den Proben vorgefundenen Variation in den Artenkompositionen zu erklären.

Das höchste Erklärungspotenzial von den in der CCA berücksichtigten Faktoren wies der Bohrwiderstand auf, gefolgt von Berindung und Saugfähigkeitsquotient. Diese drei Einflussgrößen waren am engsten mit der 1. Ordinationsachse korreliert, während die 2. Achse die engste Korrelation zum Faktor Beschirmung aufwies (Tab. 4-47). Der geringste Beitrag zur Erklärung der Artenstreuung wurde durch den Faktor Holzfeuchtigkeit beigesteuert.

Um die Bedeutung der Faktorenkombinationen für die Erklärung der Artenstreuung zu untersuchen, müssen Korrelationen zwischen diesen berücksichtigt werden (Tab. 4-48). Zu diesem Zweck wurden die Variablen einer 'forward selection' unterzogen. Der Bohrwiderstand lieferte mit 30,2 \% den größten Beitrag zum insgesamt durch die aufgenommenen Faktoren erklärbaren Anteil. Bohrwiderstand und Berindung waren positiv miteinander korreliert. Gemeinsam erreichten sie einen Anteil von 49,1 \%. Im Gegensatz zur isolierten Betrachtung der Einzelfaktoren erwies sich in der kumulativen Betrachtung der Faktor Lage als bedeutsamer als der Saugfähigkeitsquotient, da letzterer vergleichsweise eng mit den Faktoren Bohrwiderstand und Berindung korreliert war. Gemeinsam erreichten die vier genannten Faktoren einen Wert von 73,6 \%. Die restlichen 26,4\% wurden durch die Variablen Überschirmung, Beschattung und Holzfeuchte beigetragen.

Im linken oberen Bereich des Ordinationsdiagramms sind vor allem jene Proben positioniert, die an vollständig berindeten Stammabschnitten mit sehr hohem Bohrwiderstand genommen wurden. Im linken unteren Sektor sind ebenfalls fast ausschließlich Proben der Rindenklasse $4 \mathrm{zu}$ finden, allerdings waren viele von ihnen der Bohrwiderstandsklasse 5 zuzuordnen. Proben mit geringem Bohrwiderstand, hoher Saugfähigkeit und in vielen Fällen geringem Rindenprozent sind schwerpunktmäßig am rechten Rand des Diagramms zu finden. Gleichzeitig ist im rechten Teil des Ordinationsdiagramm eine stärkere Streuung insbesondere auch entlang der 2. Achse zu beobachten. Noch ausgeprägter war diese Verteilung bereits als Ergebnis der Korrespondenzanalysen in Erscheinung getreten. Offensichtlich nahm mit voranschreitender Zersetzung das Erklärungspotenzial der Faktoren Bohrwiderstand und Berindung tendenziell ab. Zum einen sanken die Korrelation zwischen diesen beiden Faktoren, zum anderen erlangten andere Variablen allem Anschein nach ein größeres Gewicht. Im unteren rechten Sektor des Ordinationsdiagramms ist eine Anhäufung von Proben zu beobachten, die bei sehr niedrigem Bohrwiderstand den Rindenklassen 2 und 3 zuzuordnen waren und die zudem Kontakt zur Bodenoberfläche hatten. Im rechten oberen Teil des Ordinationsdiagramms finden sich dagegen vermehrt Proben, die keinen Bodenkontakt hatten, die bei geringem Rindenprozent einen größeren Bohrwiderstand aufwiesen und in vielen Fällen weniger stark überschirmt waren. 
Tabelle 4-47: Kanonische Koeffizienten und Korrelationskoeffizienten

\begin{tabular}{|l|cc|cc|}
\hline & \multicolumn{2}{|c|}{ Kanonischer Koeffizient } & \multicolumn{2}{c|}{ Korrelationskoeffizient } \\
Variable & $-0,50$ & $-0,58$ & $-0,73$ & $-0,52$ \\
Ri & $-0,47$ & $+0,29$ & $-0,80$ & $+0,14$ \\
Bw & $+0,25$ & $+0,30$ & $+0,65$ & $+0,21$ \\
Sq & $-0,06$ & $+0,16$ & $+0,05$ & $+0,04$ \\
Fe & $-0,02$ & $+0,63$ & $-0,29$ & $+0,75$ \\
Schi & $-0,16$ & $-0,06$ & $-0,34$ & $+0,45$ \\
Scha & $-0,36$ & $+0,44$ & $-0,51$ & $+0,43$ \\
Lage: hochliegend & & & $+0,51$ & $-0,43$ \\
\hline
\end{tabular}

Tabelle 4-48: Korrelationen zwischen den Variablen

\begin{tabular}{|c|c|c|c|c|c|c|}
\hline Variable & Bw & Sq & $\mathrm{Fe}$ & Schi & Scha & Lage \\
\hline $\mathbf{R i}$ & \multirow[t]{6}{*}{$+0,41$} & $-0,33$ & $+0,07$ & $-0,14$ & $-0,11$ & $\pm 0,20$ \\
\hline Bw & & \multirow{5}{*}{$-0,57$} & $-0,12$ & $+0,37$ & $+0,27$ & $\pm 0,16$ \\
\hline Sq & & & \multirow[t]{4}{*}{$+0,12$} & $-0,19$ & $-0,27$ & $\pm 0,05$ \\
\hline $\mathrm{Fe}$ & & & & \multirow[t]{3}{*}{$-0,10$} & $-0,25$ & $\pm 0,08$ \\
\hline Schi & & & & & \multirow[t]{2}{*}{$+0,63$} & $\pm 0,18$ \\
\hline Scha & & & & & & $\pm 0,25$ \\
\hline
\end{tabular}

Die folgenden Ausführungen beschränken sich weitestgehend auf die beiden wichtigsten der an den Fallenstandorten erhobenen Variablen. Die Tabelle 4-49 informiert über den auf die Bw- und Ri-Klassen entfallenden Stichprobenumfang und über die dort angetroffenen Arten- und Individuenzahlen.

Tabelle 4-49: Probenumfang, Arten- und Individuenzahl einzelner Bw- und Ri-Klassen

\begin{tabular}{|c|c|c|c|c|c|c|c|c|c|c|c|}
\hline & \multicolumn{6}{|c|}{ Bohrwiderstandsklasse } & \multicolumn{4}{|c|}{ Rindenklasse } & \multirow[b]{2}{*}{ ges. } \\
\hline & Bw 6 & Bw 5 & Bw 4 & Bw 3 & Bw 2 & Bw 1 & Ri 4 & Ri 3 & Ri 2 & Ri 1 & \\
\hline Proben & 10 & 16 & 4 & 7 & 7 & 7 & 27 & 12 & 8 & 4 & 51 \\
\hline Mantelfläche $\left(\mathrm{dm}^{2}\right)$ & 1714 & 2282 & 573 & 910 & 947 & 1003 & 4115 & 1708 & 1103 & 503 & 7430 \\
\hline Volumen $\left(\mathbf{d m}^{3}\right)$ & 2289 & 2566 & 641 & 923 & 1050 & 1116 & 4923 & 1929 & 1193 & 540 & 8585 \\
\hline Rindenfläche $\left(\mathrm{dm}^{2}\right)$ & 1698 & 1924 & 424 & 550 & 810 & 497 & 4115 & 1406 & 382 & 0 & 5903 \\
\hline Ri \% & 99,0 & 84,3 & 74,0 & 60,5 & 85,5 & 49,5 & 100,0 & 82,3 & 34,6 & 0,0 & 79,5 \\
\hline Individuen & 4673 & 4491 & 840 & 1207 & 1431 & 1053 & 9094 & 2802 & 1068 & 731 & 13695 \\
\hline Ind. $/ \mathrm{m}^{2}$ & 273 & 197 & 147 & 133 & 151 & 105 & 221 & 164 & 97 & 145 & 184 \\
\hline Arten & 137 & 163 & 80 & 110 & 103 & 112 & 186 & 144 & 119 & 103 & 257 \\
\hline
\end{tabular}


Unabhängig von der $\mathrm{Bw}$ - und Ri-Klasse waren die räuberischen Arten dominierend (Tab. 4-50). Sie stellten jeweils über $50 \%$ der Arten und Individuen. Die höchsten Schlüpfdichten wurden in der Bw-Klasse 6 und der Ri-Klasse 4 registriert. Bei sinkendem Berindungsprozent war ein Rückgang des Individuenanteils zu verzeichnen. Die zweithäufigste Konsumentengruppe waren die Mycetophagen. Ihr Artenanteil lag in fast allen Klassen relativ konstant zwischen 26,1 und 28,2\%. Lediglich in der Bw-Klasse 1 war er mit 22,3\% geringfügig niedriger. Der Individuenanteil wurde wenig von der Ri-Klasse beeinflusst und variierte nur zwischen 31,1 und 38,1\%. Deutlicher waren die Unterschiede in den einzelnen Bw-Klassen. Hier lagen die Individuenanteile zwischen 18,0 (Klasse 2) und 36,9\% (Klasse 5). Auf 2,2 bis 4,8\% belief sich der Individuenanteil der xylophagen Arten in den einzelnen Bw-Klassen, während der Artenanteil zwischen 6,3 und 10,0 \% variierte. Der relativ höchste Arten- und Individuenanteil und die höchste Schlüpfdichte wurde für Proben der Ri-Klasse 1 registriert. Die übrigen Konsumtengruppen waren in nur geringem Umfang in den STE-Proben an Buchentotholz vertreten.

Tabelle 4-50: Anteile der Konsumententypen in den Bw- und Ri-Klassen

\begin{tabular}{|c|c|c|c|c|c|c|c|c|c|c|c|c|}
\hline \multirow{2}{*}{\multicolumn{2}{|c|}{ Kon.-typ }} & \multicolumn{6}{|c|}{ Bohrwiderstandsklasse } & \multicolumn{4}{|c|}{ Rindenklasse } & \multirow[b]{2}{*}{ ges. } \\
\hline & & Bw 6 & Bw 5 & Bw 4 & Bw 3 & Bw 2 & Bw 1 & $\mathbf{R i} 4$ & Ri 3 & Ri 2 & Ri 1 & \\
\hline $\mathbf{A}$ & _. & 7 & 7 & 2 & 5 & 4 & 6 & 8 & 7 & 6 & 3 & 12 \\
\hline $\mathbf{R}$ & mycetophag & 37 & 44 & 22 & 31 & 27 & 25 & 51 & 39 & 31 & 27 & 64 \\
\hline $\mathbf{T}$ & phytophag & 2 & 7 & 4 & 4 & 4 & 6 & 8 & 6 & 5 & 5 & 17 \\
\hline $\mathbf{E}$ & saprophag & 4 & 2 & 0 & 2 & 1 & 4 & 7 & 1 & 2 & 3 & 9 \\
\hline \multirow[t]{2}{*}{$\mathbf{N}$} & xylophag & 11 & 16 & 5 & 11 & 10 & 8 & 13 & 13 & 12 & 12 & 26 \\
\hline & zoophag & 76 & 87 & 47 & 57 & 57 & 63 & 99 & 78 & 63 & 53 & 129 \\
\hline D & - & 2,0 & 2,3 & 0,7 & 3,4 & 5,1 & 1,3 & 2,1 & 4,0 & 1,4 & 1,9 & 2,4 \\
\hline $\mathbf{O}$ & mycetophag & 33,7 & 36,9 & 31,4 & 24,8 & 18,0 & 32,1 & 31,1 & 32,6 & 38,1 & 32,6 & 32,1 \\
\hline $\mathbf{M}$ & phytophag & 0,1 & 0,3 & 0,8 & 0,3 & 0,3 & 0,7 & 0,1 & 0,3 & 0,9 & 0,8 & 0,3 \\
\hline $\mathbf{I}$ & saprophag & 0,1 & 0,1 & 0,0 & 0,2 & 0,1 & 1,4 & 0,1 & 0,1 & 0,5 & 1,5 & 0,2 \\
\hline \multirow[t]{2}{*}{$\mathbf{N}$. } & xylophag & 3,6 & 3,7 & 2,9 & 4,8 & 2,2 & 2,8 & 3,4 & 2,9 & 3,1 & 7,7 & 3,5 \\
\hline & zoophag & 60,6 & 56,7 & 64,2 & 66,4 & 74,4 & 61,7 & 63,2 & 60,2 & 56,0 & 55,5 & 61,6 \\
\hline $\mathbf{I}$ & 1 & 5,4 & 4,6 & 1,0 & 4,5 & 7,7 & 1,4 & 4,6 & 6,5 & 1,4 & 2,8 & 4,4 \\
\hline $\mathbf{N}$ & mycet & 91,8 & 72,6 & 46,1 & 32,8 & 27,2 & 33,7 & 68,8 & 53,5 & 36,9 & 47,3 & 59,1 \\
\hline D. & phytophag & 0,2 & 0,5 & 1,2 & 0,4 & 0,4 & 0,7 & 0,3 & 0,5 & 0,9 & 1,2 & 0,5 \\
\hline / & saprophag & 0,2 & 0,2 & 0,0 & 0,3 & 0,1 & 1,5 & 0,2 & 0,1 & 0,5 & 2,2 & 0,4 \\
\hline $\mathbf{q}$ & xylophag & 9,8 & 7,3 & 4,2 & 6,4 & 3,3 & 2,9 & 7,4 & 4,7 & 3,0 & 11,1 & 6,4 \\
\hline $\mathbf{m}$ & zoophag & 165,3 & 111,6 & 94,0 & 88,1 & 112,4 & 64,8 & 139,6 & 98,7 & 54,2 & 80,7 & 113,5 \\
\hline
\end{tabular}

Der größte Teil der nachgewiesenen Arten gehörte zur Gruppe der Rindenbewohner (Tab. 4-51). Sie waren in den Bw-Klassen 6 bis 3 und den Ri-Klassen 4 bis 2 die artenreichste Fraktion. Selbst in den Proben der Ri-Klasse 1 waren 15,5 \% der Arten und 30,8 \% der Individuen den corticolen Arten zuzurechnen. Der höchste Individuenanteil wurde mit 58,2\% in den Proben der Bw-Klasse 6 bzw. mit 55,3\% in denen der Ri-Klasse 4 registriert. In den Proben dieser Klassen schlüpften 158,7 bzw. 122,1 Ind $/ \mathrm{m}^{2}$. Der relativ hohe Individuenanteil in der Bw-Klasse 2 war darin begründet, dass die untersuchten Stammabschnitte dieser Klasse vor allem den Ri-Klassen 3 und 4 angehörten. 
Die lignicolen Arten waren mit 9,3\% am Artenspektrum beteiligt und stellten gemeinsam einen Individuenanteil von 14,8 \%. Mit abnehmendem Bohrwiderstand war eine sinkende Schlüpfdichte dieser Präferentengruppe zu beobachten. Der höchste Individuenanteil wurde mit 24,7 \% in Proben der Bw-Klasse 6 bzw. mit 19,1\% in Stammabschnitten der Ri-Klasse 4 gemessen.

Mit fortschreitender Zersetzung erreichten die an Totholzpilze gebundenen Arten höhere Individuen- und Artenanteile. Die höchsten Schlüpfdichten wurden in Stammabschnitten der Bw-Klasse 3 bzw. der Ri-Klasse 1 verzeichnet. In der Bw-Klasse 6 belief sich ihr Individuenanteil auf nur 2,3\%, in der Ri-Klasse 4 auf 2,8 \%.

Tabelle 4-51: Anteile der Habitatpräferenztypen in den Bw- und Ri-Klassen

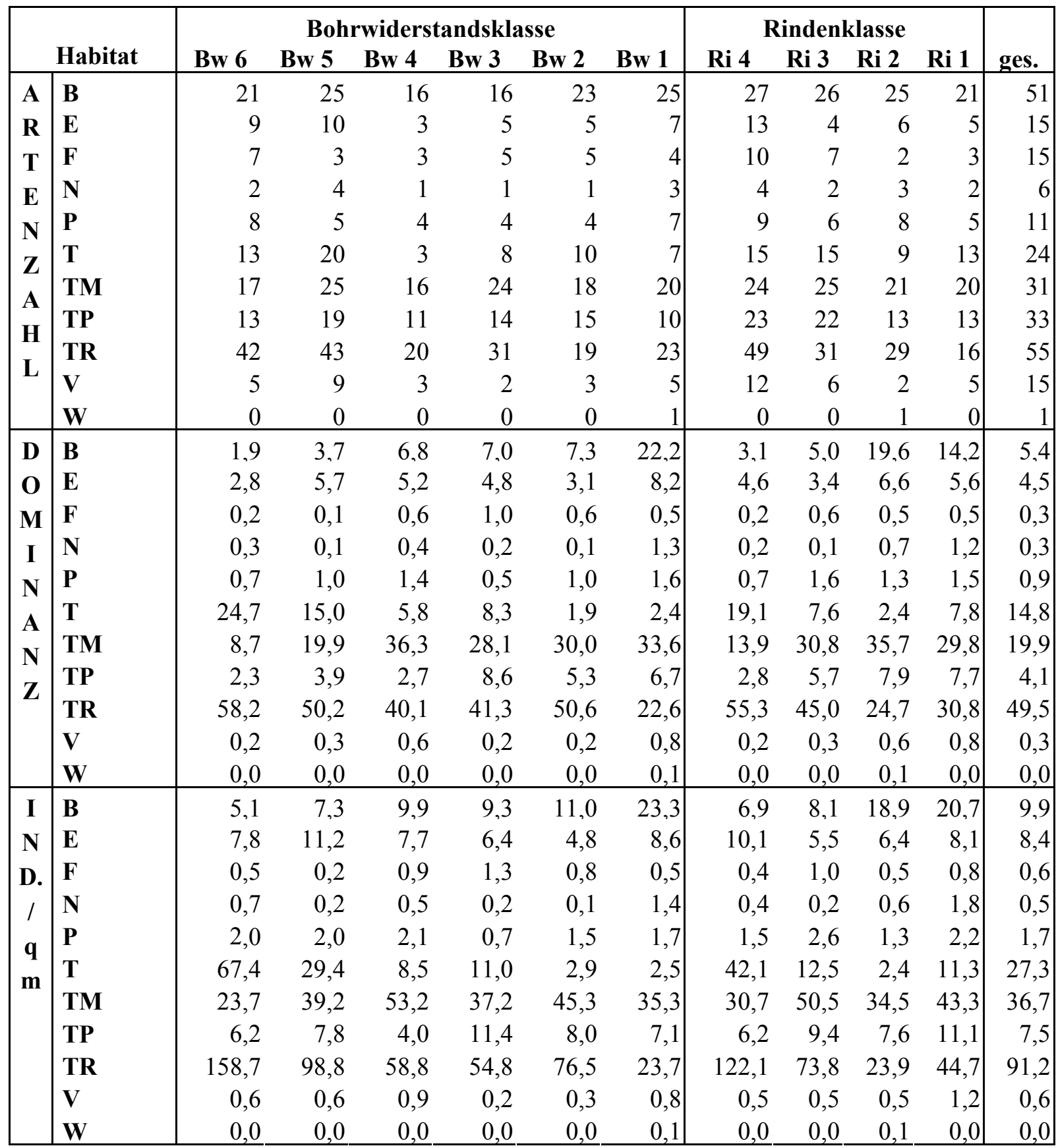


Mit einen Individuenanteil von 19,9 \% waren die Mulmbewohner nach den Rindenkäfern die zweithäufigste Präferentengruppe. Die höchste Schlüfdichte wurde mit $53,2 \mathrm{Ind} / \mathrm{m}^{2}$ in Proben der Bw-Klasse 4 festgestellt. Dieser Fraktion waren $20 \%$ der Arten und 36,3\% der Individuen zuzurechnen. In den Stammabschnitten der Bw-Klasse 1 und der Ri-Klasse 2 waren die Mulmbewohner sogar die individuenstärkste Fraktion.

Wie die Mulmbewohner erlangten auch die bodenbewohnenden Arten mit fortschreitender Zersetzung eine größere Bedeutung. Sie erreichten in fast allen Klassen einen hohen Artenanteil, waren aber erst in den Bw-Klassen 2 und 1 und der Ri-Klasse 1 vorherrschend. Der Individuenanteil stieg von 1,9\% in Proben der Bw-Klasse 6 auf 22,2 \% in der Klasse 1. Mit 19,6 bzw. 14,2 \% waren sie am Fangergebnis der Ri-Klassen 2 und 1 beteiligt. Mit abnehmendem Bohrwiderstand und sinkendem Rindenprozent stieg die registrierte Schlüpfdichte an. Den größten Anteil hatte diese Gruppe an schattigen Stammabschnitten mit Bodenkontakt.

Obwohl sich frisches und stark zersetztes liegendes Buchentotholz deutlich in der beobachteten qualitativen und quantitativen Struktur der Artenvergesellschaftungen unterschieden, ließen sich eng abgegrenzte Artengemeinschaften im Sukzessionsprozeß kaum ausmachen. Die Übergänge erwiesen sich vielmehr als fließend. Mit fortschreitender Zersetzung traten neue Arten hinzu, andere verschwanden, viele waren an Stammabschnitten sehr unterschiedlichen Zustandes zu finden und ließen lediglich Veränderungen in der erfaßten Schlüpfdichte erkennen. Die Positionen der Arten entlang der Achsen im Ordinationsdiagramm verweisen somit in vielen Fällen nur auf unterschiedliche Schwerpunkte in der Häufigkeitsverteilung der Arten (Abb. 4-38).

Die Koordinaten der Arten im Ordinationsdiagramm der kanonischen Korrespondenzanalyse (Abb. 4-39) ergaben sich aus ihrer Verteilung auf die Proben. Die Nähe der Arten zueinander erlaubt daher in begrenztem Maße Rückschlüsse auf die Ähnlichkeit ihres Vorkommens. Es gilt allerdings zu berücksichtigen, dass die zweidimensionale Darstellung eine Dimensionenreduktion bedeutet und folglich Arten mit ähnlicher Position durchaus erhebliche Unterschiede in ihrer Verteilung z. B. entlang der dritten Ordinationsachse aufweisen können. Zudem sind die sehr verschiedenen Eigenwerte der beiden Ordinationsachsen zu beachten. Des weiteren besteht die Möglichkeit, dass die Bedeutung der Variablen für die Verteilung einzelner Arten erheblich größer oder kleiner sein kann als für die Erklärung der Artenvergesellschaftungen. Die Koordinaten der Arten im Ordinationsdiagramm sind somit lediglich als bestmögliche Abbildung ihrer Verteilung im zweidimensionalen Raum zu interpretieren.

An frischen Stämmen fanden sich verschiedene Arten, die an den Zustand des Holzes vergleichsweise enge Ansprüche stellen. Atheta harwoodi und Glischrochilus quadriguttatus waren fast ausschließlich in Proben der Bw-Klasse 6 und Ri-Klasse 4 zu finden. Agathidium nigripenne, Taphrorychus bicolor, Rhinosimus ruficollis, Xyloterus signatus und Xyloterus domesticus hatten den Schwerpunkt ihrers Vorkommens an liegendem Buchentotholz in Proben der Bw-Klassen 5 und 6 und der Ri-Klasse 4. Xyloterus domesticus erreichte in diesen Klassen Individuenanteile von 8,4 und 7,3\% (Tab. 4-52). 
Tabelle 4-52: Dominante Arten in einzelnen Variablenklassen (Ind. \%)

\begin{tabular}{|c|c|c|c|c|c|c|c|c|c|c|}
\hline \multirow[b]{2}{*}{ ART } & \multicolumn{6}{|c|}{ Bohrwiderstandsklasse } & \multicolumn{4}{|c|}{ Rindenklasse } \\
\hline & Bw 6 & Bw 5 & Bw 4 & Bw 3 & Bw 2 & Bw 1 & Ri 4 & Ri 3 & Ri 2 & Ri 1 \\
\hline Rhizophagus dispar & 33,2 & 29,8 & 23,8 & 17,0 & 29,5 & 12,6 & 32,4 & 24,5 & 10,0 & 14,6 \\
\hline Hylecoetus dermestoides & 12,7 & 2,2 & 3,7 & 3,6 & 0,0 & 0,0 & 6,9 & 5,1 & 0,0 & 0,0 \\
\hline Xyloterus domesticus & 8,4 & 7,3 & 0,0 & 0,0 & 0,0 & 0,0 & 7,8 & 0,2 & 0,0 & 0,0 \\
\hline Rhizophagus bipustulatus & 5,5 & 2,2 & 1,2 & 1,4 & 0,6 & 1,4 & 3,9 & 1,0 & 1,2 & 1,1 \\
\hline Corticeus unicolor & 3,7 & 1,2 & 3,1 & 0,8 & 2,0 & 0,1 & 2,5 & 2,2 & 0,1 & 0,8 \\
\hline Rhizophagus nitidulus & 2,5 & 2,4 & 1,4 & 4,4 & 0,8 & 0,1 & 2,6 & 2,4 & 0,2 & 0,1 \\
\hline Ptilinus pectinicornis & 2,4 & 2,2 & 2,0 & 1,6 & 1,0 & 0,7 & 2,4 & 1,2 & 1,2 & 0,8 \\
\hline Ptinella limbata & 1,3 & 5,4 & 4,3 & 1,2 & 2,2 & 2,1 & 1,8 & 5,5 & 8,1 & 1,1 \\
\hline Aridius nodifer & 1,3 & 2,9 & 1,3 & 1,3 & 1,0 & 3,5 & 2,4 & 0,9 & 1,9 & 1,8 \\
\hline Ptinella aptera & 0,4 & 2,2 & 1,5 & 1,3 & 2,3 & 0,4 & 0,9 & 2,4 & 3,1 & 0,5 \\
\hline Cryptophagus dentatus & 0,8 & 2,0 & 1,8 & 3,1 & 1,5 & 3,7 & 1,5 & 1,4 & 3,9 & 3,3 \\
\hline Orchesia undulata & 0,8 & 1,9 & 1,9 & 1,0 & 0,9 & 0,5 & 0,8 & 1,5 & 1,6 & 5,1 \\
\hline Cerylon ferrugineum & 2,1 & 3,5 & 12,6 & 7,9 & 6,4 & 1,7 & 3,8 & 6,2 & 2,9 & 2,5 \\
\hline Vincenzellus ruficollis & 0,5 & 1,9 & 3,2 & 0,1 & 0,0 & 0,1 & 1,5 & 0,0 & 0,0 & 0,0 \\
\hline Corticaria longicollis & 1,1 & 2,3 & 3,1 & 3,3 & 2,1 & 4,0 & 1,7 & 3,7 & 2,8 & 1,5 \\
\hline Sepedophilus testaceus & 0,3 & 1,3 & 2,5 & 3,1 & 4,8 & 7,0 & 1,1 & 2,8 & 4,2 & 7,1 \\
\hline Dienerella elongata & 0,1 & 0,4 & 2,1 & 0,2 & 0,3 & 0,5 & 0,2 & 1,0 & 0,3 & 0,1 \\
\hline Pteryx suturalis & 1,6 & 2,2 & 7,6 & 1,3 & 2,3 & 12,8 & 1,4 & 4,5 & 8,5 & 10,0 \\
\hline Gabrius splendidulus & 0,6 & 1,0 & 1,0 & 3,5 & 3,0 & 0,7 & 0,9 & 2,1 & 0,6 & 2,5 \\
\hline Octotemnus glabriculus & 0,0 & 1,6 & 0,0 & 3,4 & 0,4 & 0,1 & 0,0 & 2,5 & 2,0 & 3,6 \\
\hline Bibloporus bicolor & 0,1 & 0,4 & 0,0 & 2,7 & 1,2 & 0,1 & 0,1 & 1,2 & 1,0 & 2,2 \\
\hline Cerylon histeroides & 0,1 & 0,4 & 0,2 & 2,3 & 0,8 & 0,6 & 0,2 & 0,9 & 1,8 & 1,0 \\
\hline Leptusa fumida & 1,2 & 1,5 & 0,6 & 2,6 & 3,1 & 0,4 & 1,3 & 2,6 & 0,7 & 1,0 \\
\hline Plegaderus dissectus & 0,1 & 0,2 & 0,1 & 1,6 & 5,7 & 0,9 & 0,9 & 0,9 & 1,4 & 1,0 \\
\hline Phloeonomus punctipennis & 2,8 & 2,6 & 1,0 & 2,3 & 5,0 & 4,0 & 2,7 & 3,0 & 5,3 & 1,6 \\
\hline Cis nitidus & 0,0 & 0,1 & 0,0 & 0,2 & 2,2 & 0,9 & 0,4 & 0,2 & 0,7 & 0,4 \\
\hline Stenichnus collaris & 0,2 & 1,5 & 1,7 & 3,0 & 2,2 & 11,3 & 1,6 & 1,9 & 5,5 & 3,3 \\
\hline Neuraphes elongatulus & 0,2 & 0,8 & 0,7 & 1,7 & 0,7 & 4,5 & 0,3 & 1,0 & 6,1 & 1,1 \\
\hline Epuraea variegata & 0,0 & 0,2 & 0,1 & 1,4 & 0,4 & 3,5 & 0,3 & 0,7 & 1,9 & 0,5 \\
\hline
\end{tabular}

Hylecoetus dermestoides, Euryusa castanoptera, Corticeus unicolor, Pediacus dermesteoides, Rhizophagus nitidulus, Rhizophagus bipustulatus und Anomognathus cuspidatus sind Beispiele für Arten, die in ihrer überwiegenden Mehrheit zwar ebenfalls in Proben der BwKlassen 6 und 5 bzw. der Ri-Klasse 4 gefangen wurden, die aber schon vermehrt auch in noch relativ festem, aber in der Zersetzung bereits etwas weiter fortgeschrittenem Material auftraten. Rh. bipustulatus war in allen Bw- und Ri-Klassen vertreten, gehörte aber nur in Proben der Bw-Klasse 6 und 5 bzw. der Ri-Klasse 4 zu den Hauptarten. 77,4 \% der gefangenen Tiere von Hylecoetus dermestoides entfielen auf die Bw-Klasse 6, der Individuenanteil belief sich hier auf $12,7 \%$. Auch in den Bw-Klassen 5 bis 3 wurde der Status einer Hauptart erreicht. Von Corticeus unicolor wurden 58,8 \% und von Rhizophagus nitidulus 39,0\% der Tiere in 
Proben der Bw-Klasse 6 registriert. Auch in geringeren Bw-Klassen erreichten sie noch höhere Individuenanteile. Die drei zuletzt genannten Arten zählten sowohl in den Proben der Ri-Klasse 4 als auch denen der Klasse 3 zu den Hauptarten. Ptilinus pectinicornis gehörte zu den häufigeren Arten in den Bw-Klassen 6 bis 4. Mit abnehmendem Bohrwiderstand und Rindenprozent gingen die Fangzahlen und die registrierten Schlüpfdichten zurück.

Vincenzellus ruficollis wurde mit den höchsten Schlüpfdichten in Stammabschnitten der Bw-Klassen 5 und 4 festgestellt, 99 \% der Individuen entfielen auf Proben der Ri-Klasse 4. Der höchste Dominanzwert wurde für die Bw-Klasse 4 registriert. Die höchste Dichte und den höchsten Individuenanteil hatte Cerylon ferrugineum in Proben der Bw-Klasse 4 und war in den Klassen 4 bis 2 jeweils die zweithäufigste Art. Euplectus punctatus erreichte die höchsten Individuenanteile in den Bw-Klassen 4 und 3, Gabrius splendidulus zählte zu den dominierenden Arten in den Bw-Klassen 3 und 2.

$\mathrm{Zu}$ den Arten, die, zumindest soweit es die STE-Proben an liegendem Buchentotholz betrifft, besonders in stärker zersetzten Stammabschnitten ein relativ größeres Gewicht erlangten, zählten u. a. die Mulmbewohner Cerylon histeroides, Plegaderus dissectus, Sepedophilus testaceus und Abraeus granulum. Plegaderus dissectus wurde vor allem an weichen, aber noch berindeten Stammabschnitten gefangen und erreichte so in den Proben der BwKlasse 2 einen Individuenanteil von 5,7 \%. Mit sinkendem Bohrwiderstand und abnehmender Berindung stieg der Individuenanteil und die festgestellte Dichte von Sepedophilus testaceus fast kontinuierlich an. Einige totholzpilz- und bodenbewohnende Arten erlangten in den Stadien fortgeschrittener Zersetzung ebenfalls größere Bedeutung. Beispiele sind Cis nitidus, Epuraea variegata und Neuraphes elongatulus. Stenichnus collaris wurde in den größten Schlüpfdichten an Stämmen niedriger Ri- und Bw-Klassen registriert, wo sie auch die höchsten Dominanzwerte erreichte.

Eine ganze Reihe von Arten zeigte im Bohrwiderstandsgradienten eine eher bimodale Verteilung. Mit fortschreitender Zersetzung nahm ihre absolute und/oder relative Häufigkeit zunächst zu, fiel dann wieder ab und stieg bei niedrigen Bohrwiderständen erneut an. Ein Beispiel hierfür ist Pteryx suturalis, der die höchsten Dichten in den Bw-Klassen 4 und 1 erreichte. Weitere Beispiele sind Ptinella aptera, Aridius nodifer, Bibloporus bicolor und Octotemnus glabriculus.

Abschließend sollen noch einige Arten Erwähnung finden, deren Verteilung keine deutlichen Schwerpunkte erkennen ließen und die in Stammabschnitten von sehr unterschiedlichem Zustand auftraten bzw. die in zahlreichen Klassen dominierten. Sie finden sich im Ordinationsdiagramm vor allem in der Nähe des Zentrums abgebildet. Nur eine Art, Rhizophagus dispar, war in allen Bw- und Ri-Klassen als Hauptart vertreten. Auch an unberindeten Stammabschnitten erreichte dieser räuberische Rindenkäfer den höchsten Individuenanteil von allen Arten. Die weitaus überwiegende Anzahl der Tiere wurde allerdings an Stämmen der Bw-Klassen 6 und 5 bzw. der Ri-Klassen 4 und 3 gefangen. Recht ausgeglichen war die Verteilung von Corticaria longicollis. Die festgestellten Dichten dieses Mulmbewohners waren in allen Bw-Klassen annähernd gleich hoch. Als weitere Arten können Leptusa fumida und Cryptophagus dentatus zu dieser Gruppe gestellt werden. 


\subsubsection{Sukzession der Artengemeinschaften an liegendem Eichentotholz}

Für die 48 STE-Proben, die an liegendem Eichentotholz genommen worden waren, wurden nur vereinzelt hohe Ähnlichkeitswerte ermittelt (Tab. 4-53, Anhang 8a, b). Wie am liegenden Buchentotholz waren höhere Werte in erster Linie zwischen relativ frischen Stammabschnitten zu beobachten. Die höchsten Übereinstimmungen erreichten Proben, die am gleichen Stamm genommen worden waren. Dies waren die Proben 20a und 20b/93 sowie die Proben 9/92, 10b und 10c/93. In vielen Fällen wiesen aber auch Proben vom gleichen Stamm deutliche Differenzen in der Struktur der Artenvergesellschaftungen auf. Entlang der Diagonalen der Ähnlichkeitsmatrix zeichnet sich eine, wenn auch nur gering ausgeprägte, Tendenz fortschreitender Zersetzung ab, wobei die Position der Proben an den Enden der Matrix ihrer geringen Ähnlichkeit mit allen übrigen Proben zuzuschreiben ist. Gruppen von Proben, die sich untereinander durch hohe Ähnlichkeit bei gleichzeitig geringer Übereinstimmung mit den übrigen Proben auszeichneten, lassen sich kaum ausmachen. Wie schon im Falle der STEProben an Buchentotholz zeichneten sich derartige Gruppierungen vor allem bei vergleichsweise frischem Material ab. Eine solche Gruppenbildung deutet sich am ehesten für die Proben 20b/93, 10/92 und 20a/93 sowie für die Proben 10c/93, 14/93, 18b/93, 05b/93, 05a/93, $17 / 93,10 \mathrm{~b} / 93,18 \mathrm{a} / 93$ und $09 / 92$ an.

Diese Proben wurden auch als Ergebnis der DCA's in räumliche Nähe zueinander gestellt. Die wiedergegebenen Ordinationsdiagramme (Abb. 4-40 und 4-41) wurden auf der Grundlage logarithmierter Werte von insgesamt 216 Arten ermittelt. Aus Gründen der Darstellbarkeit wurde vor allem auf die Wiedergabe der Position von Arten mit geringer Frequenz verzichtet. Die wichtigsten Ergebnisse verschiedener Korrespondenzanalyseverfahren (CA, DCA) auf unterschiedlicher Berechnungsgrundlage sind in der Tabelle 4-54 zusammengefaßt. 
Tabelle 4-53: Ähnlichkeitsmatrix $\left(\mathrm{RE}_{\mathrm{ln}}\right)$ der STE-Proben an liegendem Eichentotholz

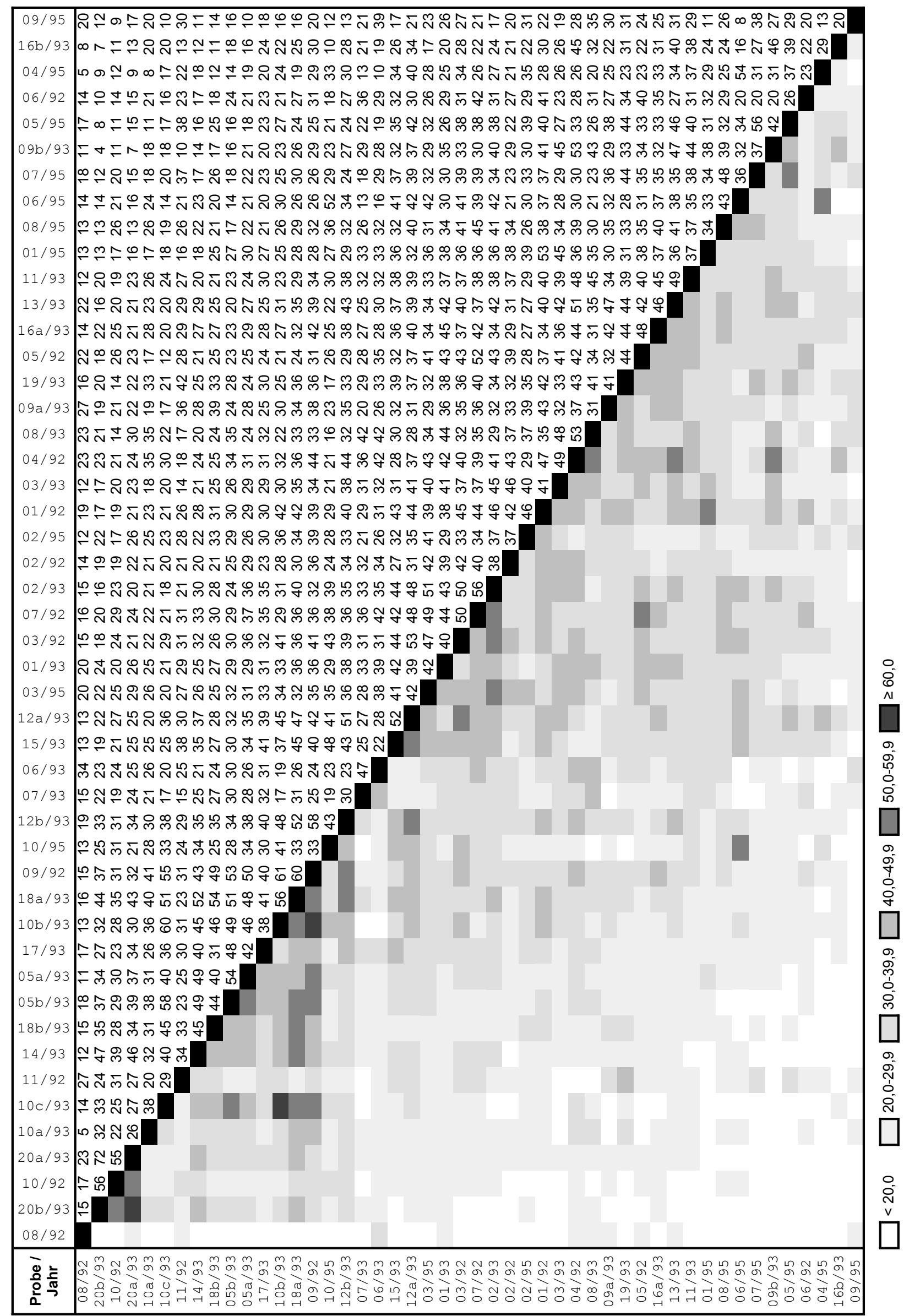


Tabelle 4-54: Ergebnisse verschiedener Korrespondenzanalysen mit den Fangdaten aus STEProben an Eichentotholz

\begin{tabular}{|c|c|c|c|c|c|c|c|c|c|c|c|c|c|c|}
\hline \multirow{2}{*}{\multicolumn{3}{|c|}{$\begin{array}{l}\begin{array}{c}\text { Berechnungs- } \\
\text { grundlage }\end{array} \\
\text { Arten Daten }\end{array}$}} & \multirow{2}{*}{$\begin{array}{l}\text { Eigenwerte }(\lambda) \text { der } \\
\text { Ordinationsachsen } \\
\begin{array}{llll}\lambda 1 & \lambda 2 & \lambda 3 & \lambda 4\end{array}\end{array}$} & \multirow[t]{2}{*}{$\begin{array}{c}\text { total } \\
\text { Inertia }\end{array}$} & \multicolumn{4}{|c|}{$\begin{array}{c}\text { cum. perc. var. of spec. } \\
\text { data (Achsen 1-4) }\end{array}$} & \multicolumn{4}{|c|}{$\begin{array}{l}\text { spec. env. correl. } \\
\text { (Achsen 1-4) }\end{array}$} & \multicolumn{2}{|c|}{$\begin{array}{c}\text { cum. perc. var. of } \\
\text { spec.-env. relation } \\
\text { (Achsen 1-2) }\end{array}$} \\
\hline & & & & & 1 & 2 & 3 & 4 & 1 & 2 & 3 & 4 & 1 & 2 \\
\hline & 216 & inär & $\begin{array}{llll}.357 .344 .308 & .383\end{array}$ & 7.009 & 5.1 & 10.0 & 14.4 & 18.4 & .710 & 39 & .658 & 502 & 13 & 18. \\
\hline A & 216 & In Dichte & .408 .325 .295$. & 5.783 & 7.1 & 12.7 & 17.8 & 22.3 & .656 & .479 & .677 & .575 & 14.8 & 21.1 \\
\hline & 70 & binär & 133. 158. 196 . 233 & 2.414 & 9.7 & 17.8 & 24.3 & 29.9 & .688 & .458 & .586 & .433 & 21.8 & 29.9 \\
\hline & 70 & ln Dichte & .320 .221 .189 .138 & 2.454 & 13.1 & 22.1 & 29.8 & 35.4 & .699 & .575 & & .606 & & 39.1 \\
\hline & 216 & när & 238. 357.299 .258$. & 7.009 & 5.1 & 9.4 & 13.1 & 16.4 & .710 & .6 & .550 & .267 & 13 & 23.5 \\
\hline & 216 & In Dichte & 193 408.313 .221$. & 5.783 & 7.1 & 12.5 & 16.3 & 19.6 & .656 & .473 & .5 & .698 & 14.8 & 20.8 \\
\hline & 70 & binär & . 079. 169. 169. & 2.414 & 9.7 & 16.7 & 21.2 & 24.5 & .688 & .410 & .658 & .566 & 21.8 & 27.8 \\
\hline & 70 & In Dichte & .320 .201 .105 .084 & 2.454 & 13.1 & 21.2 & 25.5 & 28.9 & .699 & .665 & .579 & .505 & 26 & 42.4 \\
\hline
\end{tabular}

Die Eigenwerte der beiden ersten Ordinationsachsen betrugen 0,408 und 0,313, die der nicht abgebildeten 3. und 4. Achse beliefen sich auf 0,221 und 0,193. Der gemeinsame Erklärungsanteil dieser vier hypothetischen Variablen betrug 19,6\%. Wurden in der Analyse nur jene 70 Arten berücksichtigt, die in mindestens fünf Proben vertreten waren, erhöhte sich der Erklärungsanteil auf 28,9\%. Trotz der Schwierigkeiten und den daher nicht auszuschließenden Ungenauigkeiten der dendrochronologischen Analyse läßt die Anordnung der Proben im Ordinationsdiagramm einen Einfluss der Dauer des Zersetzungsprozesses auf die Artenvergesellschaftungen erkennen. Am linken Rand sind Proben positioniert, die zum Zeitpunkt der Aufnahme bis zu fünf Jahre abgestorben waren. Dem entsprechend sind hier Arten abgebildet, die noch relativ frisches Material präferieren, wie z. B. Glischrochilus quadriguttatus, Rhinosimus ruficollis und die beiden Xyloterus-Arten. Zum Zentrum hin schließen sich Proben von Stammabschnitten mit einer Zersetzungsdauer von bis zu zehn Jahren an. Hier sind insbesondere corticole Arten in größerer Anzahl positioniert. Im rechten Teil des Ordinationsdiagramms sind in erster Linie Proben von Stämmen fortgeschrittener Zersetzungsstadien dargestellt, ebenso jene Probestämme, bei denen eine dendrochronologische Analyse nicht möglich war. In diesem Bereich wird eine größere Anzahl boden- und mulmbewohnender Arten, wie z. B. Stenichnus collaris, Neuraphes elongatulus, Melanotus castanipes und Hypulus quercinus, dargestellt. 


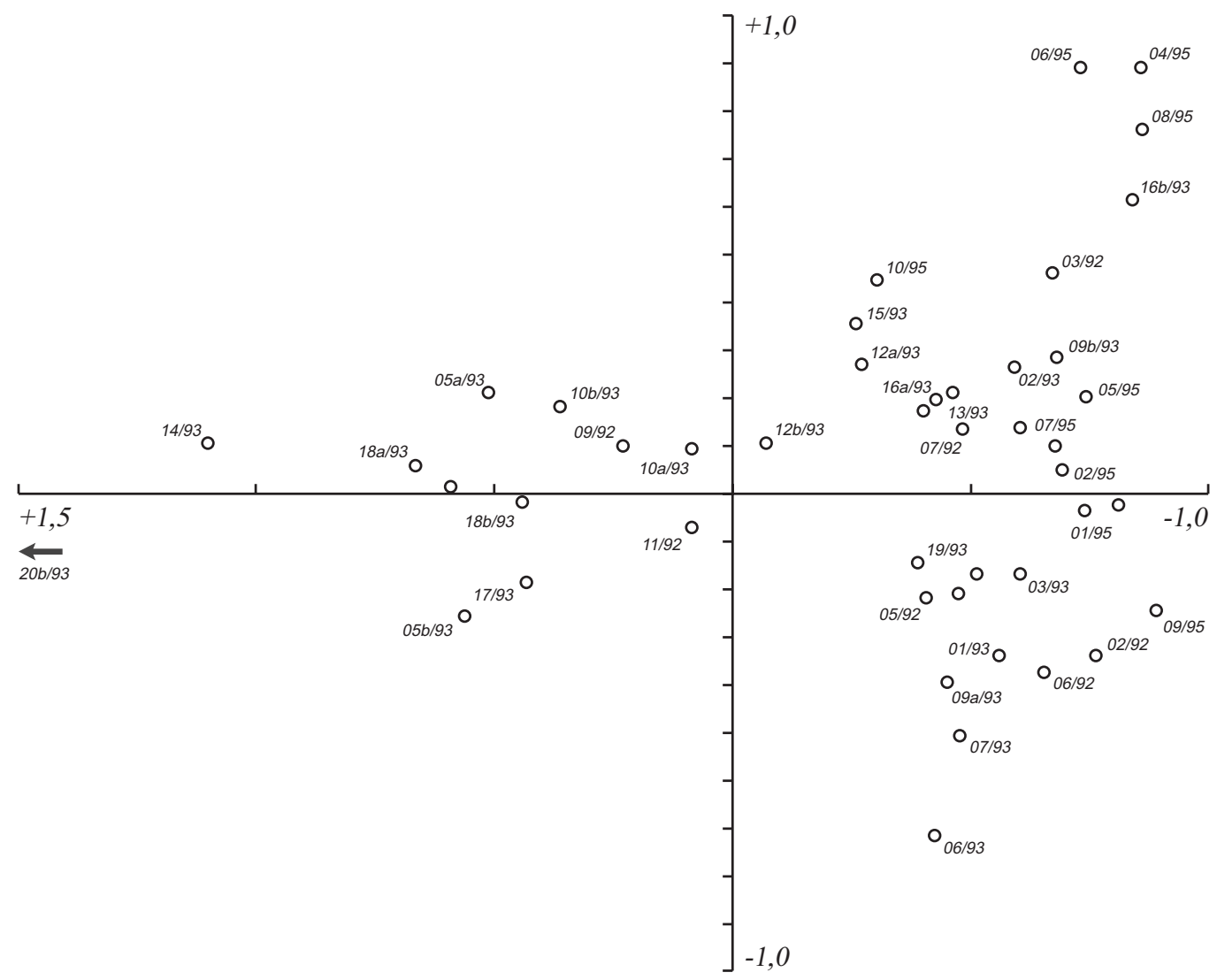

Abb. 4-40: Ordinationsdiagramm (DCA): STE-Proben an liegendem Eichentotholz

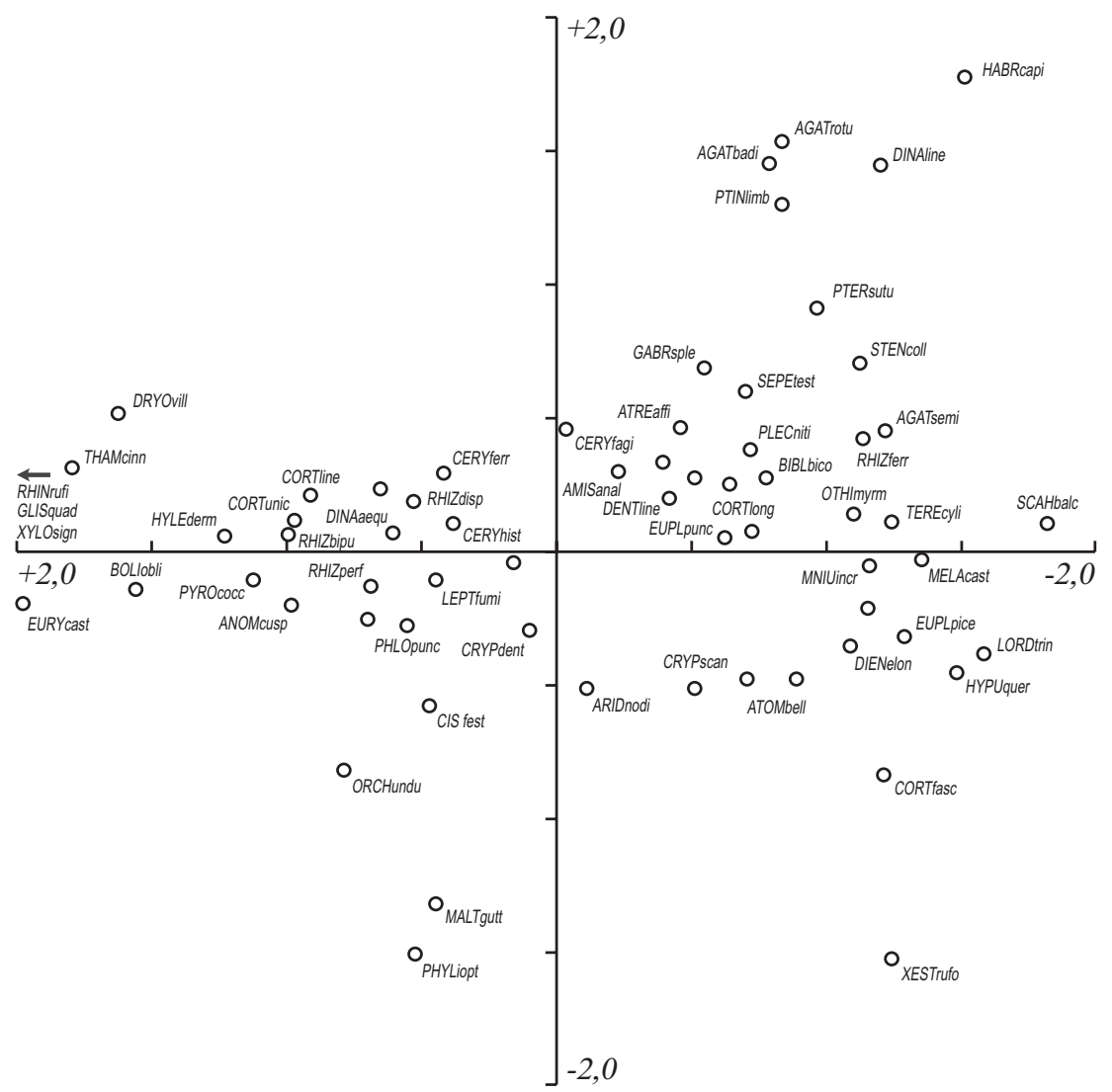

Abb. 4-41: Ordinationsdiagramm (DCA): Arten aus STE-Proben an liegendem Eichentotholz 
Wie schon bei den STE-Proben an liegendem Buchentotholz, so zeigte sich auch an der Eiche, dass mit fortschreitender Zersetzung eine stärkere Streuung entlang der zweiten Ordinationsachse zu registrieren war. Sieht man von den ersten Jahren der Zersetzung ab, in denen eine vergleichsweise enge Beziehung zwischen den vorgefundenen Artenvergesellschaftungen und der Dauer des Abbauprozesses zu beobachten war, ist dieser Faktor in den Phasen fortgeschrittener Zersetzung in nur geringem Maße zur Erklärung der Streuung in den Artenvergesellschaftungen geeignet. Auch hier scheinen andere Faktoren ein größeres Gewicht zu erlangen, die nur locker mit der Zersetzungsdauer korreliert sind.

Um die vorgefundene Struktur der Artengemeinschaften mit weiteren an den Fallenstandorten aufgenommenen Variablen in Beziehung zu setzen und deren Beitrag zur Erklärung der Streuung zu untersuchen, kamen wiederum Verfahren der kanonischen Korrespondenzanalyse zur Anwendung. Auch diesmal wurde aus den gleichen Gründen wie bei der Analyse der STE-Proben an liegendem Buchentotholz der Faktor Absterbezeitpunkt nicht berücksichtigt. Die wichtigsten Ergebnisse werden in der Tabelle 4-55 vergleichend gegenübergestellt. Die Ordinationsdiagramme (Abb. 4-42 und 4-43) zeigen das Ergebnis einer CCA, wobei nur die Schlüpfdichten jener 70 Arten Berücksichtigung fanden, die in mindestens fünf Proben angetroffen worden waren. Für die als passiv gekennzeichneten Proben lagen wiederum nur unvollständige Datensätze der Habitatvariablen vor, ihre Positionierung erfolgte ausschließlich auf der Grundlage ihrer Artenvergesellschaftungen.

Die Eigenwerte der beiden ersten Ordinationsachsen betrugen 0,231 und 0,106. Der Vergleich der Ergebnisse der Korrespondenzanalysen mit denen der kanonischen Korrespondenzanalysen läßt erkennen, dass die an den Fallenstandorten aufgenommenen Variablen geeignet sind, einen nenneswerten Beitrag zur Erklärung der vorgefundenen Streuung in den Artenkompositionen zu leisten.

Tabelle 4-55: Ergebnisse verschiedener kanonischer Korrespondenzanalysen mit den Fangdaten aus den STE-Proben an Eichentotholz

\begin{tabular}{|c|c|c|c|c|c|c|c|c|c|c|c|c|c|c|}
\hline \multirow{2}{*}{\multicolumn{3}{|c|}{$\begin{array}{l}\begin{array}{c}\text { Berechnungs- } \\
\text { grundlage }\end{array} \\
\text { Arten Daten } \\
\end{array}$}} & \multirow{2}{*}{$\begin{array}{l}\text { Eigenwerte }(\lambda) \text { der } \\
\text { Ordinationsachsen } \\
\begin{array}{llll}\lambda 1 & \lambda 2 & \lambda 3 & \lambda 4\end{array}\end{array}$} & \multirow[t]{2}{*}{$\begin{array}{l}\text { total } \\
\text { Inertia }\end{array}$} & \multicolumn{4}{|c|}{$\begin{array}{c}\text { cum. perc. var. of spec. } \\
\text { data (Achsen 1-4) }\end{array}$} & \multicolumn{4}{|c|}{$\begin{array}{l}\text { spec. env. correl. } \\
\text { (Achsen 1-4) }\end{array}$} & \multicolumn{2}{|c|}{$\begin{array}{l}\text { cum. perc. var. of } \\
\text { spec.-env. relation } \\
\text { (Achsen 1-2) }\end{array}$} \\
\hline & & & & & 1 & 2 & 3 & 4 & 1 & 2 & 3 & 4 & 1 & 2 \\
\hline 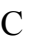 & 216 & binär & 166. 177. 208. 289. & 6.457 & 4.5 & 7.7 & 10.5 & 13.0 & .970 & .964 & .915 & .909 & 23.0 & 39.6 \\
\hline $\mathrm{C}$ & 216 & Dichte & 139. 161. 179. 318. & 5.342 & 5.9 & 0 & 3 & 14.9 & . & 025 & 905 & .903 & 28.8 & 44. \\
\hline A & 70 & binär & 064. 077. 096. 158. & 2.376 & 6.6 & 10.7 & 13.9 & 16.6 & .881 & .821 & .822 & .850 & 30.3 & 48.7 \\
\hline & 70 & In Dichte & 231. 106 231.082 .068 & 2.415 & 9.6 & 13.9 & 17.3 & 20.1 & .899 & .010 & 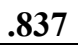 & .865 & 50.2 & 55.8 \\
\hline 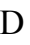 & 216 & 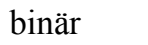 & .043. 289.082. & 6.457 & 4.5 & 6.7 & 8.0 & 17 & .970 & & .809 & .729 & & 35. \\
\hline $\mathrm{C}$ & 216 & ln Dichte & .035. 072. 155. & 5.342 & 5.9 & 8.8 & 10.2 & 10.8 & .953 & .895 & .868 & .667 & 28.2 & 42.5 \\
\hline C & 70 & binär & .024. 158.068. & 2.376 & 6.6 & 9.5 & 10.9 & 11.9 & .881 & .847 & .700 & .701 & 30.3 & 44.7 \\
\hline & 70 & In Dichte & .231 .069 .045 .026 & 2.415 & 9.6 & 12.4 & 14.3 & 15 & .899 & .830 & .789 & .582 & 38.3 & 50. \\
\hline
\end{tabular}


4.6. Die Sukzession der Artengemeinschaften

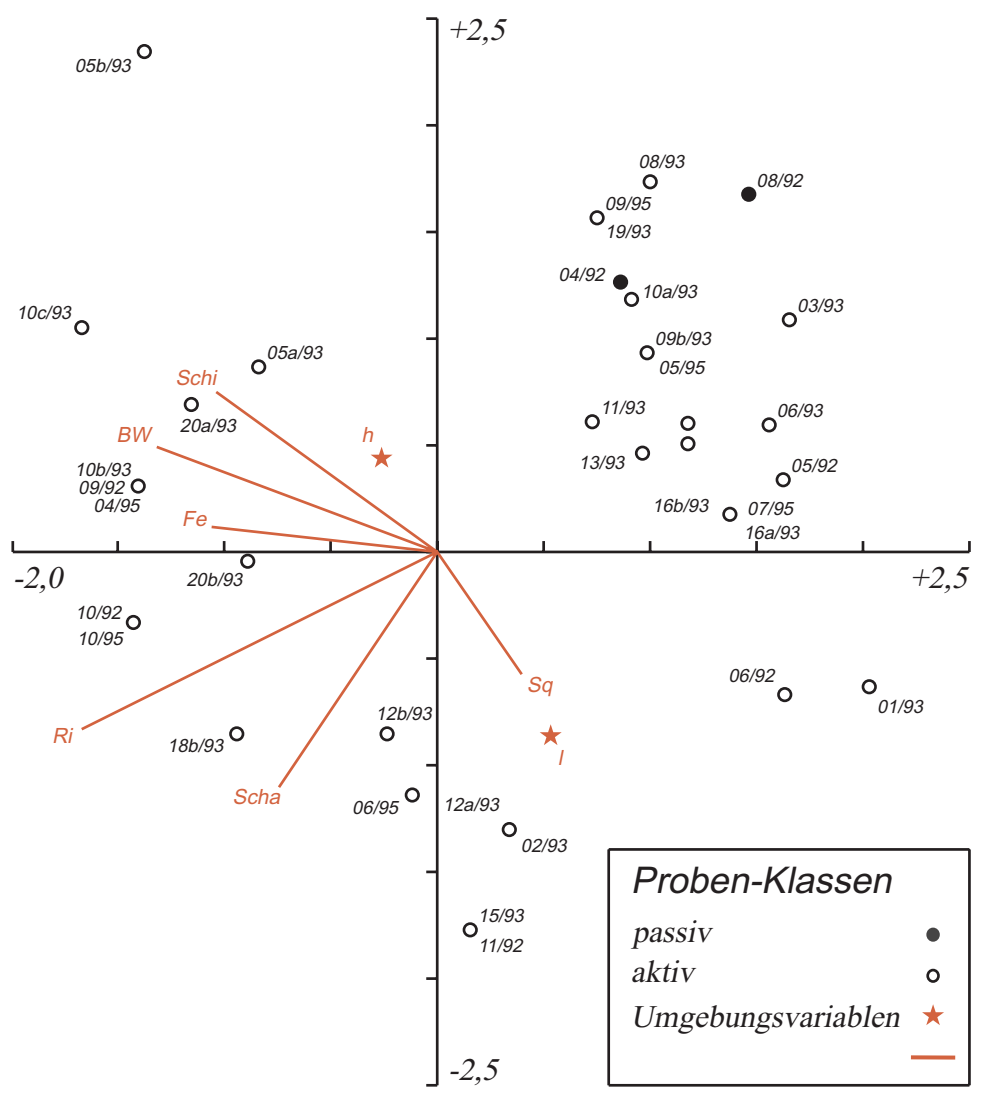

Abb. 4-42: Ordinationsdiagramm (CCA): STE-Proben an liegendem Eichentotholz und Habitatvariablen

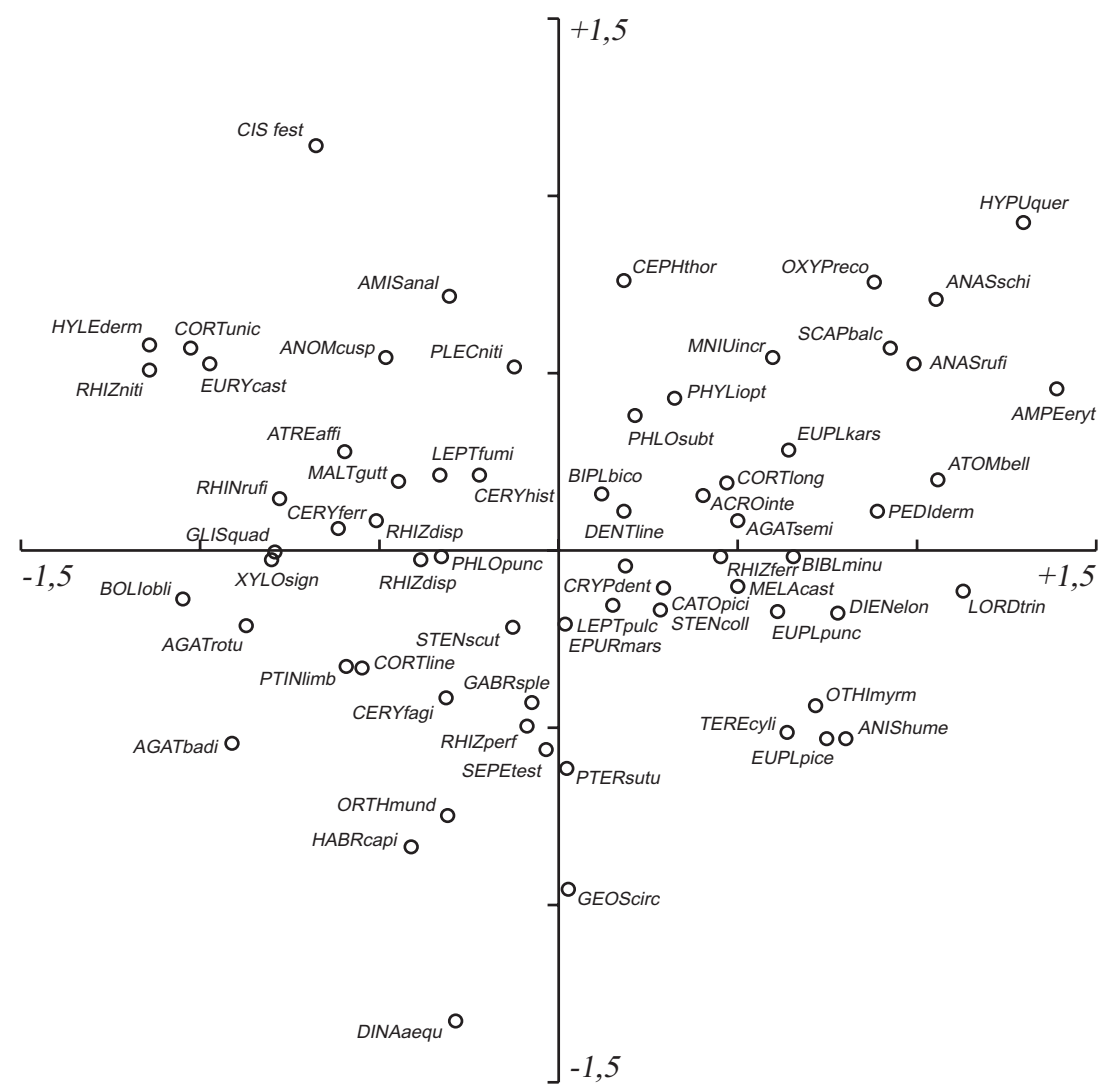

Abb. 4-43: Ordinationsdiagramm (CCA): Arten aus STE-Proben an liegendem Eichentotholz 
Von den berücksichtigten Variablen wurde dem Faktor Berindung das größte Erklärungspotenzial zugewiesen, gefolgt vom Bohrwiderstand, dem Grad der Überschirmung, den Faktoren Feuchte, Beschattung und Lage mit jeweils übereinstimmendem Erklärungspotenzial und dem ermittelten Saugfähigkeitsquotienten an letzter Position. Die vier zuerst genannten Einflussgrößen wiesen die engste Korrelation zur ersten Ordinationsachse auf, während die übrigen Faktoren in größerem Maße mit der zweiten Achse korreliert waren (Tab. 4-56).

Tabelle 4-56: Kanonische Koeffizienten und Korrelationskoeffizienten

\begin{tabular}{|c|c|c|c|c|}
\hline \multirow[b]{2}{*}{ Variable } & \multicolumn{2}{|c|}{ Kanonischer Koeffizient } & \multicolumn{2}{|c|}{ Korrelationskoeffizient } \\
\hline & Achse 1 & Achse 2 & Achse 1 & Achse 2 \\
\hline $\mathbf{R i}$ & $-0,67$ & $-0,66$ & $-0,86$ & $-0,40$ \\
\hline Bw & $-0,07$ & $+0,22$ & $-0,68$ & $+0,23$ \\
\hline Sq & $+0,50$ & $-0,34$ & $+0,20$ & $-0,27$ \\
\hline $\mathbf{F e}$ & $-0,34$ & $+0,06$ & $-0,55$ & $+0,06$ \\
\hline Schi & $-0,29$ & $+0,80$ & $-0,54$ & $+0,36$ \\
\hline Scha & $-0,12$ & $-0,46$ & $-0,38$ & $-0,53$ \\
\hline Lage: hochliegend & $-0,09$ & $+0,20$ & $-0,33$ & $+0,58$ \\
\hline aufliegend & & & $+0,33$ & $-0,58$ \\
\hline
\end{tabular}

Um die Bedeutung von Faktorkombinationen für die Erklärung der Streuung in den Artenvergesellschaftungen zu klären, waren auch hier die Korrelationen zwischen den Variablen zu berücksichtigen (Tab. 4-57). Als Ergebnis einer 'forward selection' wurde dem Faktor Berindung mit 31,7 \% der größte Beitrag zum insgesamt durch die untersuchten Variablen erklärbaren Anteil zugewiesen. Da der Bohrwiderstand die engste Korrelation zu diesem Faktor aufwies, erlangte die Lage der Probestämme das größere Erklärungspotenzial für die verbleibende Reststreuung, gleichrangig gefolgt von Bohrwiderstand und Überschirmung. Der gemeinsame Anteil dieser vier Variablen am insgesamt durch die berücksichtigten Einflussgrößen erklärbaren Anteil belief sich auf 71,7\%. Die restlichen 28,3\% waren auf die verbleibenden Variablen Beschattung, Saugfähigkeitsquotient und Feuchte zurückzuführen.

Tabelle 4-57: Korrelationen zwischen den Variablen

\begin{tabular}{|c|c|c|c|c|c|c|}
\hline Variable & Bw & $\mathrm{Sq}$ & $\mathrm{Fe}$ & Schi & Scha & Lage \\
\hline $\mathbf{R i}$ & \multirow[t]{6}{*}{$+0,46$} & $+0,10$ & $+0,45$ & $+0,43$ & $+0,45$ & $\pm 0,02$ \\
\hline Bw & & \multirow[t]{5}{*}{$-0,32$} & $+0,44$ & $+0,17$ & $+0,04$ & $\pm 0,38$ \\
\hline $\mathbf{S q}$ & & & \multirow[t]{4}{*}{$+0,34$} & $+0,35$ & $+0,17$ & $\pm 0,17$ \\
\hline $\mathrm{Fe}$ & & & & \multirow[t]{3}{*}{$+0,37$} & $+0,03$ & $\pm 0,17$ \\
\hline Schi & & & & & \multirow[t]{2}{*}{$+0,31$} & $\pm 0,35$ \\
\hline Scha & & & & & & $\pm 0,00$ \\
\hline
\end{tabular}


In dem Bemühen, die Proben an möglichst homogenen Stammabschnitten zu nehmen, entfielen nur 4 Proben auf die Rindenklassen 2 und 3. 21 Proben mit einem Mantelflächenanteil von $42 \%$ wurden an vollständig berindeten Stammabschnitten genommen, 23 Proben mit einem Anteil von $52 \%$ entfielen auf die Rindenklasse 1 (Tab. 4-58). Im CCA-Ordinationsdiagramm ist eine deutliche Separierung der Proben in zwei Gruppen zu erkennen (Abb. 4-42). In der rechten Gruppe sind alle Stammabschnitte der Rindenklassen eins und zwei, in der linken die Abschnitte der Klassen drei und vier vereint. Gleichzeitig gehörte der größte Teil der berindeten Stammabschnitte höheren Bohrwiderstandsklassen an. An berindeten Stammabschnitten wurde eine höhere Arten- und Individuendichte registriert als an unberindeten. Ein deutlicher Rückgang der Schlüpfdichten ging auch einher mit sinkenden Bohrwiderstandswerten an den Fallenstandorten. Zudem wurden die höheren Schlüpfdichten an Stammabschnitten ohne Bodenkontakt registriert.

Tabelle 4-58: Probenumfang, Arten- und Individuenzahl einzelner Variablenklassen

\begin{tabular}{|c|c|c|c|c|c|c|c|c|c|c|c|c|}
\hline & \multicolumn{5}{|c|}{ Bohrwiderstandsklasse } & \multicolumn{4}{|c|}{ Rindenklasse } & \multicolumn{2}{|c|}{ Lage } & \multirow[b]{2}{*}{ ges. } \\
\hline & Bw 5 & Bw 4 & Bw 3 & Bw 2 & Bw 1 & Ri 4 & Ri 3 & Ri 2 & Ri 1 & $\mathbf{h}$ & 1 & \\
\hline Proben & 18 & 11 & 13 & 5 & 1 & 21 & 3 & 1 & 23 & 30 & 18 & 48 \\
\hline Mantelfläche $\left(\mathrm{dm}^{2}\right)$ & 1875 & 1351 & 1516 & 712 & 127 & 2341 & 267 & 91 & 2881 & 3354 & 2226 & 5581 \\
\hline Volumen $\left(\mathbf{d m}^{3}\right)$ & 1737 & 1420 & 1482 & 807 & 122 & 2353 & 184 & 66 & 2965 & 3338 & 2230 & 5568 \\
\hline Rindenfläche $\left(\mathrm{dm}^{2}\right)$ & 1485 & 408 & 405 & 301 & 0 & 2341 & 209 & 46 & 2 & 1648 & 950 & 2598 \\
\hline Ri \% & 79,2 & 30,2 & 26,7 & 42,2 & 0,0 & 100,0 & 78,4 & 50,1 & 0,1 & 49,1 & 42,7 & 46,6 \\
\hline Individuen & 5138 & 1685 & 1073 & 634 & 46 & 6150 & 606 & 89 & 1731 & 6658 & 1918 & 8576 \\
\hline Ind./m ${ }^{2}$ & 274 & 125 & 71 & 89 & 36 & 263 & 227 & 98 & 60 & 198 & 86 & 154 \\
\hline Arten & 159 & 132 & 127 & 90 & 27 & 181 & 71 & 27 & 154 & 200 & 159 & 248 \\
\hline
\end{tabular}

Die überwiegende Zahl der vorgefundenen Käferarten war den Räubern zuzuordnen (Tab. 4-59). Unabhängig von der Bw- und Ri-Klasse bzw. der Lage der Stämme entfielen zwischen 50 und $60 \%$ der Arten auf diese Konsumentengruppe. Mit fortschreitender Zersetzung war ein Sinken der Schlüpfdichten zu verzeichnen. In den Proben der Bw-Klasse 5 wurde mit 101,9 Ind. $/ \mathrm{m}^{2}$ die höchste Dichte festgestellt, dennoch hatte der von den Räubern gestellte Individuenanteil in dieser Klasse mit 37,2 \% einen vergleichsweise niedrigen Wert. Die individuenstärkste Konsumentengruppe waren allerdings die Arten mit mycetophager Ernährungsweise. Ihr Artenanteil in den aufgeführten Klassen variierte zwischen 20 und $26 \%$. Die höchsten Schlüpfdichten wurden an berindeten und noch sehr festen Stammabschnitten festgestellt. Der höchste Individuenanteil wurde in Proben der Bw-Klasse 5 mit $58,9 \%$ registriert und war vor allem durch den Borkenkäfer Xyloterus signatus verursacht. Die xylophagen Arten stellten zwischen 6 und $11 \%$ der Arten. Auf diese entfielen zwischen 1 und $10 \%$ der Individuen der aufgeführten Klassen. Die höchste Schlüpfdichte wurde in Proben der Bw-Klasse 2 registriert. 
Tabelle 4-59: Anteile der Konsumententypen in einzelnen Variablenklassen

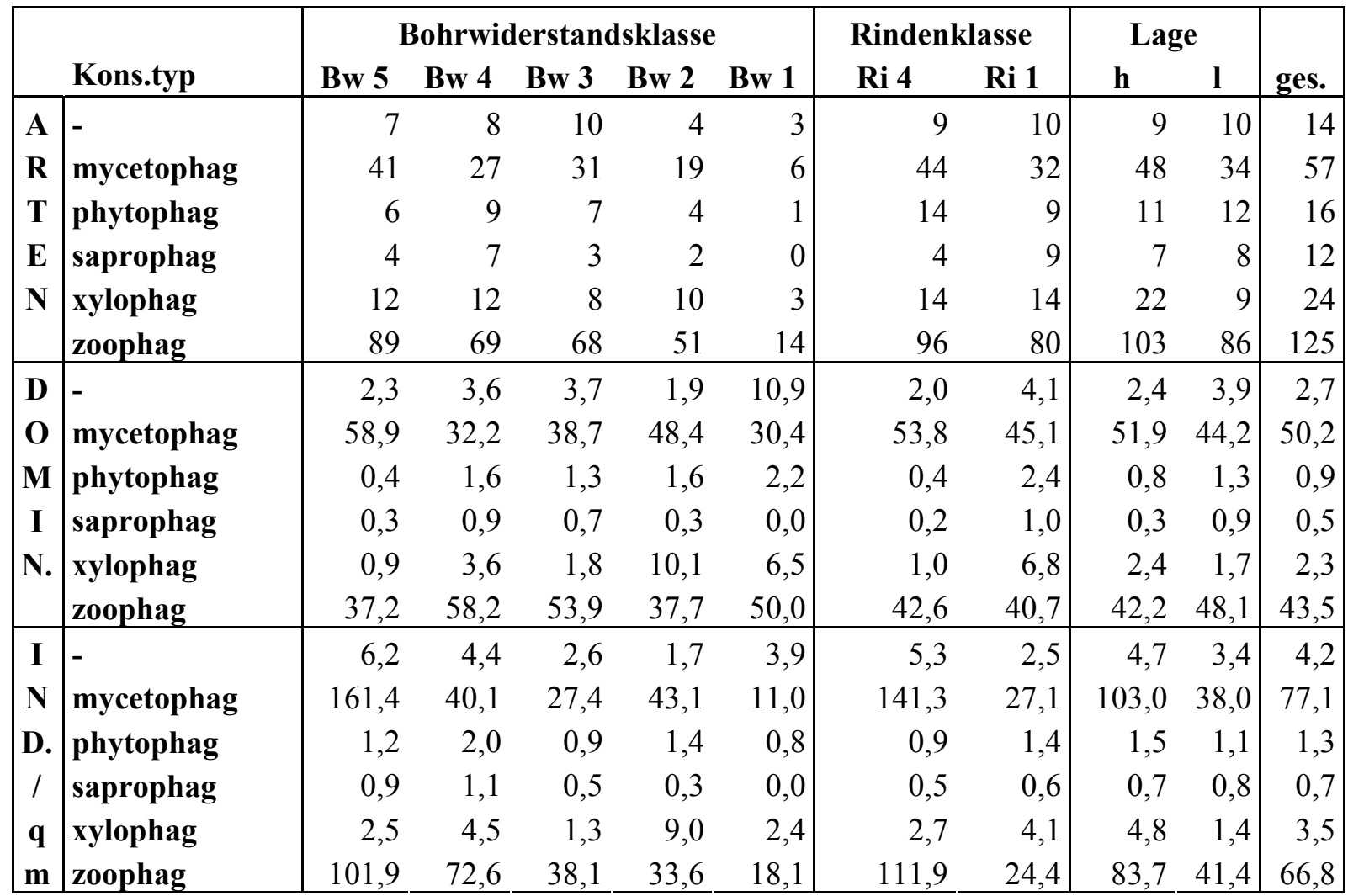

Der Artenanteil der Totholzbewohner variierte zwischen den einzelnen Bw-Klassen zwischen 49 \% (Bw-Klasse 3) und 67 \% (Bw-Klasse 1) (Tab. 4-60). Der höchste Individuenanteil wurde in Stammabschnitten der Bw-Klasse 5 mit $90 \%$ registriert, der niedrigste mit $55 \%$ in Proben der Klasse 3. An berindeten Stämmen war der Artenanteil der Totholzkäfer geringfügig, der Individuenanteil hingegen deutlich höher als an unberindeten. Auch waren an aufliegenden Stammabschnitten die Arten- und Individuenanteile niedriger als an solchen ohne Bodenkontakt.

Am individuenreichsten trat die Gruppe der Rindenkäfer in Erscheinung. Die 43 Arten waren mit 29,5 \% an den Fängen beteiligt. In den Proben der Bw-Klassen 5 und 4 sowie der Ri-Klasse 4 waren sie die artenreichste Gruppe. Hier wurden auch hohe Individuenanteile festgestellt. Die Stellung als häufigste Präferentengruppe wurde allerdings nur für die Proben der Bw-Klasse 4 festgestellt. Die Schlüpfdichte sank mit abnehmendem Bohrwiderstand kontinuierlich ab. In Proben der Ri-Klasse 1 entfielen immerhin noch $14 \%$ der Arten und $12,5 \%$ der Individuen auf diesen Präferenztyp.

Die 27 lignicolen Arten waren mit einem Individuenanteil von 28,5\% die insgesamt zweithäufigste Gruppe in den STE-Proben von Eichentotholz. Die Individuen wurden in ihrer großen Mehrheit an festen, berindeten und hochliegenden Stammabschnitten gefangen, in denen die höchsten Dominanzwerte erreicht wurden. Die Schlüpfdichte erreichte in den Proben der Bw-Klasse 5 ein Maximum von 117,7 Ind. $/ \mathrm{m}^{2}$. 
Die Mulmbewohner hatten einen Individuenanteil von 20,0 \%, der von 32 Arten gestellt wurde. Die höchsten Schlüpfdichten wurden in den Bw-Klassen 5 und 2 bzw. der Ri-Klasse 4 festgestellt. Sie erwiesen sich als dominierende Präferentengruppe in den Proben der RiKlasse 1 sowie an aufliegenden Stammabschnitten. Ihr Individuenanteil stieg mit sinkendem Bohrwiderstand tentenziell an.

Tabelle 4-60: Anteile der Habitatpräferenztypen in einzelnen Variablenklassen

\begin{tabular}{|c|c|c|c|c|c|c|c|c|c|c|c|}
\hline & \multirow[b]{2}{*}{ Habitat } & \multicolumn{5}{|c|}{ Bohrwiderstandsklasse } & \multicolumn{2}{|c|}{ Rindenklasse } & \multicolumn{2}{|c|}{ Lage } & \multirow[b]{2}{*}{ ges. } \\
\hline & & Bw 5 & Bw 4 & Bw 3 & Bw 2 & Bw 1 & Ri 4 & Ri 1 & $\mathbf{h}$ & $\mathbf{L}$ & \\
\hline $\mathbf{A}$ & B & 21 & 22 & 34 & 13 & 3 & 29 & 32 & 30 & 36 & 46 \\
\hline $\mathbf{R}$ & $\mathbf{E}$ & 11 & 8 & 8 & 6 & 4 & 13 & 11 & 12 & 11 & 17 \\
\hline $\mathbf{T}$ & $\mathbf{F}$ & 10 & 10 & 5 & 5 & 0 & 12 & 11 & 15 & 10 & 21 \\
\hline $\mathbf{E}$ & $\mathbf{N}$ & 4 & 3 & 4 & 0 & 0 & 3 & 5 & 5 & 5 & 8 \\
\hline $\mathbf{N}$ & $\mathbf{P}$ & 7 & 4 & 6 & 3 & 0 & 8 & 4 & 7 & 7 & 10 \\
\hline $\mathbf{Z}$ & $\mathbf{T}$ & 15 & 15 & 8 & 12 & 4 & 18 & 16 & 26 & 12 & 27 \\
\hline $\mathbf{A}$ & TM & 24 & 23 & 26 & 22 & 6 & 26 & 27 & 29 & 26 & 32 \\
\hline $\mathbf{H}$ & $\mathbf{T P}$ & 16 & 8 & 10 & 6 & 1 & 16 & 12 & 19 & 12 & 22 \\
\hline \multirow[t]{3}{*}{$\mathbf{L}$} & TR & 39 & 26 & 17 & 18 & 7 & 39 & 22 & 42 & 23 & 43 \\
\hline & TS & 2 & 2 & 1 & 0 & 0 & 2 & 1 & 1 & 2 & 2 \\
\hline & $\mathbf{V}$ & 10 & 11 & 8 & 5 & 2 & 15 & 13 & 14 & 15 & 20 \\
\hline D & B & 3,8 & 10,7 & 24,5 & 15,9 & 13,0 & 5,3 & 20,2 & 5,7 & 19,1 & 8,7 \\
\hline $\mathbf{O}$ & $\mathbf{E}$ & 4,4 & 12,2 & 14,4 & 11,5 & 8,7 & 5,3 & 18,1 & 5,0 & 17,3 & 7,8 \\
\hline $\mathbf{M}$ & F & 0,3 & 0,8 & 0,5 & 0,8 & 0,0 & 0,3 & 0,9 & $\beta, 3$ & 0,8 & 0,4 \\
\hline I & $\mathbf{N}$ & 0,4 & 0,5 & 0,9 & 0,0 & 0,0 & 0,1 & 0,8 & 0,3 & 0,7 & 0,4 \\
\hline $\mathbf{N}$ & $\mathbf{P}$ & 0,4 & 1,0 & 2,6 & 0,8 & 0,0 & 0,5 & 2,0 & 0,6 & 1,8 & 0,8 \\
\hline $\mathbf{A}$ & $\mathbf{T}$ & 42,9 & 4,7 & 8,1 & 11,2 & 8,7 & 36,7 & 6,8 & 35,0 & 6,0 & 28,5 \\
\hline $\mathbf{N}$ & $\mathbf{T M}$ & 13,9 & 24,9 & 26,7 & 44,2 & 34,8 & 16,9 & 31,4 & 17,3 & 29,4 & 20,0 \\
\hline \multirow[t]{4}{*}{$\mathbf{Z}$} & TP & 2,1 & 2,0 & 4,5 & 2,4 & 10,9 & 1,6 & 4,0 & 2,3 & 2,8 & 2,4 \\
\hline & TR & 31,0 & 40,9 & 15,6 & 11,0 & 19,6 & 32,5 & 12,5 & 32,2 & 20,2 & 29,5 \\
\hline & TS & 0,1 & 0,3 & 0,3 & 0,0 & 0,0 & 0,2 & 0,1 & 0,1 & 0,4 & 0,2 \\
\hline & $\mathbf{V}$ & 0,7 & 1,8 & 1,9 & 2,2 & 4,3 & 0,6 & 3,2 & 1,1 & 1,7 & 1,2 \\
\hline I & B & 10,5 & 13,4 & 17,3 & 14,2 & 4,7 & 13,8 & 12,1 & 11,4 & 16,4 & 13,4 \\
\hline $\mathbf{N}$ & $\mathbf{E}$ & 12,1 & 15,3 & 10,2 & 10,2 & 3,1 & 14,0 & 10,9 & 10,0 & 14,9 & 11,9 \\
\hline D. & F & 0,7 & 1,0 & 0,3 & 0,7 & 0,0 & 0,9 & 0,5 & 0,6 & 0,7 & 0,7 \\
\hline I & $\mathbf{N}$ & 1,0 & 0,6 & 0,7 & 0,0 & 0,0 & 0,3 & 0,5 & 0,7 & 0,6 & 0,6 \\
\hline $\mathbf{q}$ & $\mathbf{P}$ & 1,2 & 1,3 & 1,8 & 0,7 & 0,0 & 1,4 & 1,2 & 1,1 & 1,5 & 1,3 \\
\hline \multirow[t]{6}{*}{$\mathbf{m}$} & $\mathbf{T}$ & 117,7 & 5,9 & 5,7 & 10,0 & 3,1 & 96,3 & 4,1 & 69,6 & 5,2 & 43,9 \\
\hline & $\mathbf{T M}$ & 38,1 & 31,1 & 18,9 & 39,3 & 12,6 & 44,5 & 18,9 & 34,4 & 25,3 & 30,8 \\
\hline & TP & 5,8 & 2,5 & 3,2 & 2,1 & 3,9 & 4,2 & 2,4 & 4,7 & 2,4 & 3,8 \\
\hline & TR & 84,9 & 51,1 & 11,0 & 9,8 & 7,1 & 85,3 & 7,5 & 63,8 & 17,4 & 45,3 \\
\hline & TS & 0,3 & 0,4 & 0,2 & 0,0 & 0,0 & 0,5 & 0,0 & 0,2 & 0,3 & 0,2 \\
\hline & $\mathbf{V}$ & 1,9 & 2,2 & 1,3 & 2,0 & 1,6 & 1,5 & 1,9 & 2,1 & 1,4 & 1,8 \\
\hline
\end{tabular}


Auch die bodenbewohnenden Käferarten waren bei fortgeschrittener Zersetzung mit höheren Individuenanteilen am Fangergebnis beteiligt. In den Proben der Bw-Klasse 3, der RiKlasse 1 und an aufliegenden Stammabschnitten waren die Bodenbewohner die artenreichste Gruppe. Hier erreichten sie auch ihre höchsten Individuenanteile und Schlüpfdichten, sieht man von der Ri-Klasse $1 \mathrm{ab}$, wo sie geringfügig unter der in Proben der Ri-Klasse 4 festgestellten lag.

Die in Abbildung 4-43 dargestellten Positionen der Arten im CCA-Ordinationsdiagramm gibt in Verbindung mit den Vektorpfeilen aus der Abbildung 4-42 erste Hinweise auf ihre Verteilung in Abhängigkeit von den Variablenklassen. Die Tabelle 4-61 informiert über die Hauptarten einzelner Faktorklassen.

In der linken Hälfte des Ordinationsdiagramms sind fast ausschließlich Arten positioniert, die vor allem an berindeten Stammabschnitten nachgewiesen wurden. Innerhalb dieses Kollektivs wird die Position der Arten vor allem von ihrer Verteilung in Abhängigkeit von der Lage der Stämme und der Bw-Klasse bestimmt. Die dominierende Art an berindeten Stammabschnitten war Xyloterus signatus. Über 99 \% der Individuen wurden an hochliegendem Material der Bw-Klasse 5 gefangen. Auch die Individuen von Bolitochara obliqua, Glischrochilus quadriguttatus und Rhinosimus ruficollis entfielen zu über $90 \%$ auf diese Variablenklassen. Hylecoetus dermestoides, Rhizophagus nitidulus, Corticeus unicolor und Euryusa castanoptera wurden ebenfalls fast ausschließlich an berindeten und hochliegenden Stammabschnitten gefangen, ein größerer Anteil der Fänge (30 - $42 \%$ ) entfiel dabei auf die BwKlasse 4, in der erstere beiden Arten zu den Hauptarten zu rechnen waren.

Näher am Zentrum des Diagramms sind viele Arten angesiedelt, die zwar vor allem an berindeten Abschnitten auftraten, die aber zu größeren Anteilen auch an unberindeten und/oder Proben geringeren Bohrwiderstandes zu finden waren. $\mathrm{Zu}$ nennen sind hier $\mathrm{u}$. a. Cerylon histeroides, Leptusa fumida, Phloeonomus punctipennis und Leptusa pulchella. In fast allen Variablenklassen zu den dominierenden Arten zu zählen waren Rhizophagus dispar und Rh. bipustulatus.

Arten, die in erster Linie an berindeten und aufliegenden Stammpartien nachgewiesen wurden, finden sich vor allem im unteren Teil der Grafik. In den meisten Fällen war damit auch ein gehäuftes Auftreten an Abschnitten geringeren Bohrwiderstandes verbunden. Beispiele sind Stenichnus scutellaris, Sepedophilus testaceus und Pteryx suturalis. Letztere gehörte zu den dominierenden Arten an aufliegenden und berindeten Eichenstämmen sowie in den Bw-Klassen 4 bis 1.

Von den übrigen Arten mit einem bevorzugten Auftreten an berindeten Stammabschnitten soll noch auf die Position des Mulmkäfers Ptinella limbata und von Teredus cylindricus hingewiesen werden. Erstere wurde zu fast $80 \%$ an Stammabschnitten der Bw-Klasse 5 festgestellt. Sie zählte zugleich aber auch zu den Hauptarten der Bw-Klasse 2 und war sowohl an Stammabschnitten mit als auch ohne Bodenkontakt eine der dominierenden Arten. Teredus cylindricus wurde in 17 Proben mit zusammen 84 Individuen registriert. Ca. $75 \%$ der Individuen fingen sich an berindeten Stammabschnitten mit Bodenkontakt. Die Mehrheit der Individuen ( $83 \%)$ wurde an Stammteilen der Bw-Klasse 3 gesammelt. Hier erreichte Teredus cylindricus einen Individuenanteil von $6,5 \%$. 
Tabelle 4-61: Dominante Arten in einzelnen Variablenklassen (Ind. \%)

\begin{tabular}{|c|c|c|c|c|c|c|c|c|}
\hline \multirow[b]{2}{*}{ ART } & \multicolumn{4}{|c|}{ Bohrwiderstandsklasse } & \multicolumn{2}{|c|}{ Rindenklasse } & \multicolumn{2}{|c|}{ Lage } \\
\hline & Bw 5 & Bw 4 & Bw 3 & Bw 2+1 & Ri 4 & Ri 1 & $\mathbf{h}$ & 1 \\
\hline Xyloterus signatus & 29,6 & 0,3 & 0,1 & 0,7 & 24,7 & 0,3 & 23,0 & 0,2 \\
\hline Rhizophagus dispar & 14,9 & 20,1 & 4,6 & 2,8 & 15,5 & 3,2 & 15,4 & 7,7 \\
\hline Rhizophagus bipustulatus & 4,9 & 5,3 & 3,0 & 1,2 & 5,1 & 1,3 & 5,1 & 2,0 \\
\hline Ptinella limbata & 4,7 & 0,6 & 0,4 & 7,6 & 4,8 & 0,3 & 3,7 & 3,1 \\
\hline Phloeonomus punctipennis & 3,3 & 6,4 & 2,5 & 1,3 & 3,9 & 2,4 & 3,4 & 4,4 \\
\hline Cerylon ferrugineum & 1,5 & 3,6 & 0,5 & 0,3 & 2,0 & 0,5 & 2,0 & 0,4 \\
\hline Hylecoetus dermestoides & 1,6 & 2,1 & 0,0 & 0,0 & 1,2 & 0,0 & 1,6 & 0,6 \\
\hline Rhizophagus nitidulus & 1,6 & 2,1 & 0,1 & 0,0 & 1,7 & 0,1 & 1,7 & 0,2 \\
\hline Corticaria longicollis & 3,2 & 11,1 & 12,9 & 13,8 & 3,1 & 18,9 & 5,4 & 11,7 \\
\hline Aridius nodifer & 1,8 & 7,2 & 2,5 & 1,0 & 1,7 & 7,5 & 1,3 & 8,4 \\
\hline Teredus cylindricus & 0,0 & 0,4 & 6,5 & 0,7 & 1,0 & 1,2 & 0,3 & 3,3 \\
\hline Cryptophagus dentatus & 2,3 & 2,8 & 6,4 & 4,6 & 2,5 & 6,0 & 2,5 & 5,2 \\
\hline Pteryx suturalis & 1,7 & 2,7 & 3,5 & 10,3 & 3,4 & 1,6 & 1,5 & 7,1 \\
\hline Neuraphes elongatulus & 1,1 & 3,7 & 8,5 & 7,1 & 1,5 & 7,8 & 2,0 & 6,6 \\
\hline Stenichnus collaris & 1,0 & 1,2 & 4,9 & 2,2 & 1,3 & 2,4 & 1,1 & 3,5 \\
\hline Dienerella elongata & 0,1 & 1,6 & 4,2 & 4,7 & 0,8 & 3,5 & 1,0 & 2,3 \\
\hline
\end{tabular}

Einige wenige Arten wurden fast ausschließlich an rindenlosen Stammsegmenten festgestellt. Im Ordinationsdiagramm wurden diese am rechten oberen Rand positioniert. Damit verbunden war ein gehäufter Nachweis in Proben geringeren Bohrwiderstandes. Mniusa incrassata, Hypulus quercinus und Oxypoda recondita sind Beispiele für Arten, die in ihrer Mehrheit an hochliegenden Stammabschnitten erfaßt wurden, Anaspis rufilabris, Pediacus dermestoides und Lordithon trinotatus waren vor allem an aufliegenden Partien zu finden. Keine dieser hier genannten Arten trat in sehr hoher Indivduenzahl auf.

Näher zum Zentrum des Ordinationsdiagramms sind vor allem jene Arten dargestellt, deren Verteilungen wenig von der Berindung der Stämme beeinflusst schienen. Corticaria longicollis, Neuraphes elongatulus und Cryptophagus dentatus waren sowohl in aufliegenden als auch in hochliegenden Stammabschnitten dominant. Gleichzeitig zählten sie auch in vielen Bw-Klassen zu den Hauptarten. Phyllodrepa ioptera und Phloeocharis subtilissima wurden vor allem an Stammabschnitten ohne Bodenkontakt festgestellt.

Im rechten unteren Viertel der Abbildung sind in erster Linie Arten zu finden, die in der Mehrzahl der Fälle an aufliegenden Eichenstämmen und/oder Stammabschnitten mit geringem Bohrwiderstand gefangen wurden. Sowohl Stenichnus collaris als auch Dienerella elongata zählten hier zu den Hauptarten. Auch Melanotus castanipes, Othius myrmecophilus, Euplectus piceus und Anisotoma humeralis wurden hier gehäuft beobachtet. 


\subsubsection{Verteilung, Vergesellschaftung und Sukzession der Arten an stehendem Totholz}

Fünfzig der insgesamt 141 an stehendem Totholz genommenen EFs-Proben entfielen auf die Eiche. Diese deckten eine Oberfläche von $6,9 \mathrm{~m}^{2} \mathrm{ab}$, von denen $3 \mathrm{~m}^{2}$ berindet waren. Die 91 an Buchentotholz genommenen Proben befingen eine Mantelfläche von 15,9 $\mathrm{m}^{2}, 11,2 \mathrm{~m}^{2}$ waren berindet. Die 18307 gefangenen Käferindividuen verteilten sich auf 201 Arten, darunter 132 obligate Totholzkäferarten mit einem Individuenanteil von zusammen $90 \%$.

Die Fangergebnisse wurden mehreren DCA's und DCCA's unterzogen, wobei auch diesmal alternativ die An- bzw. Abwesenheit oder die logarithmierte Schlüpfdichte Berechnungsgrundlage war, darüber hinaus war in einigen Analysen ein Nachweis der Arten in mindestens 10 Proben gefordert. Eine zusammenfassende Übersicht der Ergebnisse ist in der Tabelle 4-62 wiedergegeben. Beispielhaft zeigen die Abbildungen 4-44 und 4-45 das Ergebnis einer DCA, beruhend auf den logarithmierten Schlüpfdichten jener 53 Arten, die in wenigstens 10 Proben angetroffen worden waren. Aber auch wenn alle Arten und/oder nur ihre Präsenz Berücksichtigung fanden, zeigten die entsprechenden Ordinationsdiagramme ein sehr ähnliches Verteilungsmuster.

Tabelle 4-62: Zusammenfassung der Ergebnisse verschiedener Analysemethoden mit den Fangdaten aus EFs-Proben an stehendem Eichen- und Buchentotholz

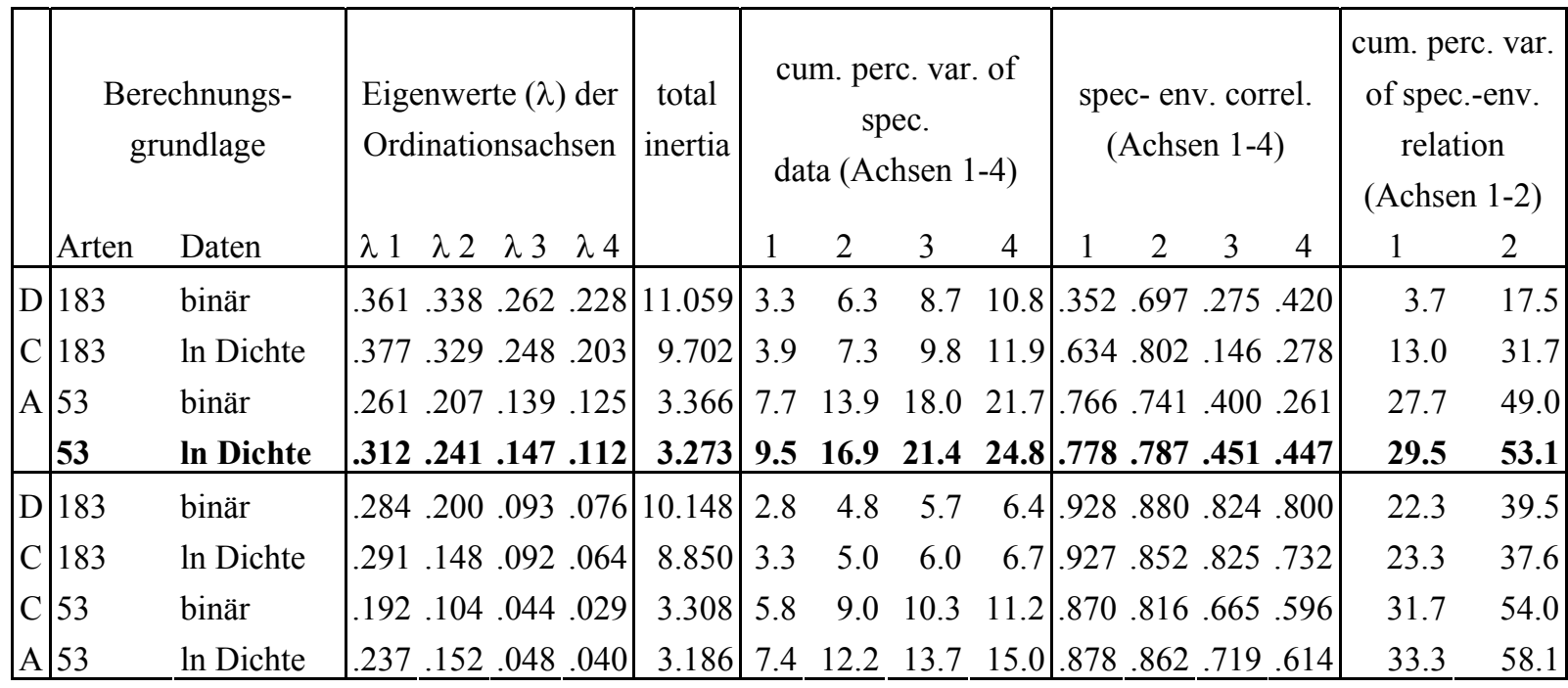

In der Abbildung 4-44 wurde kenntlich gemacht, von welcher der beiden untersuchten Baumarten die Proben stammten. Es wird, wie dies auch bei den STE-Proben an liegendem Totholz der Fall war, eine ausgeprägte Separierung der Proben entlang der zweiten Ordinationsachse $(\lambda 2=0.241)$ deutlich. Auch bei den EFs-Proben trug der Faktor Baumart am stärksten zur Erklärung der vorgefundenen Artenvergesellschaftungen bei, unabhängig von der Berechnungsgrundlage.

Von den 132 Totholzkäferarten aus EFs-Proben wurden 81 an Eiche und 118 an Buche festgestellt. Die Verteilung der Nachweise und Individuenzahlen von 61 Arten, die in mindestens 5 Proben mit wenigstens 10 Exemplaren festgestellt worden waren, ist in den Tabellen 4-63 und 4-64 aufgeführt. 


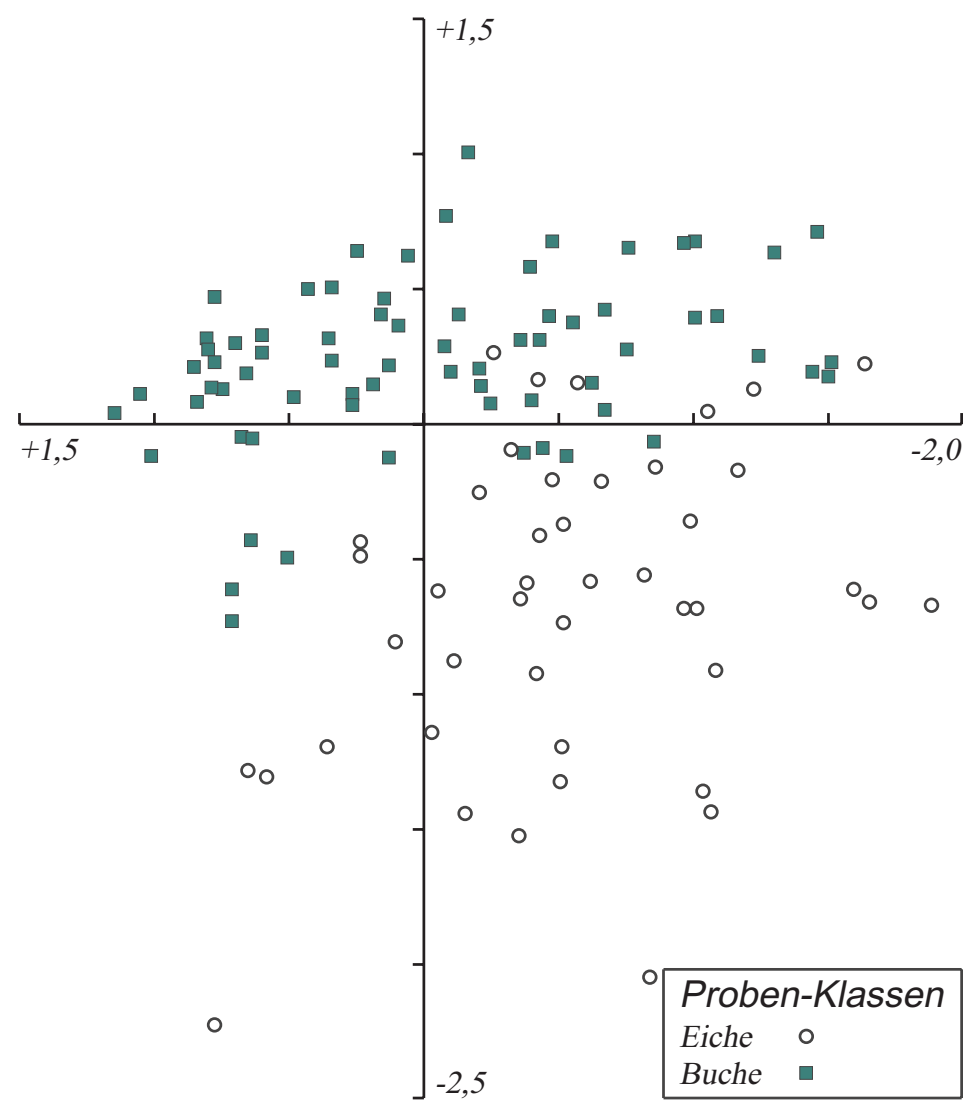

Abb. 4-44: Ordinationsdiagramm (DCA) der EFs-Proben von Eichen- und Buchentotholz

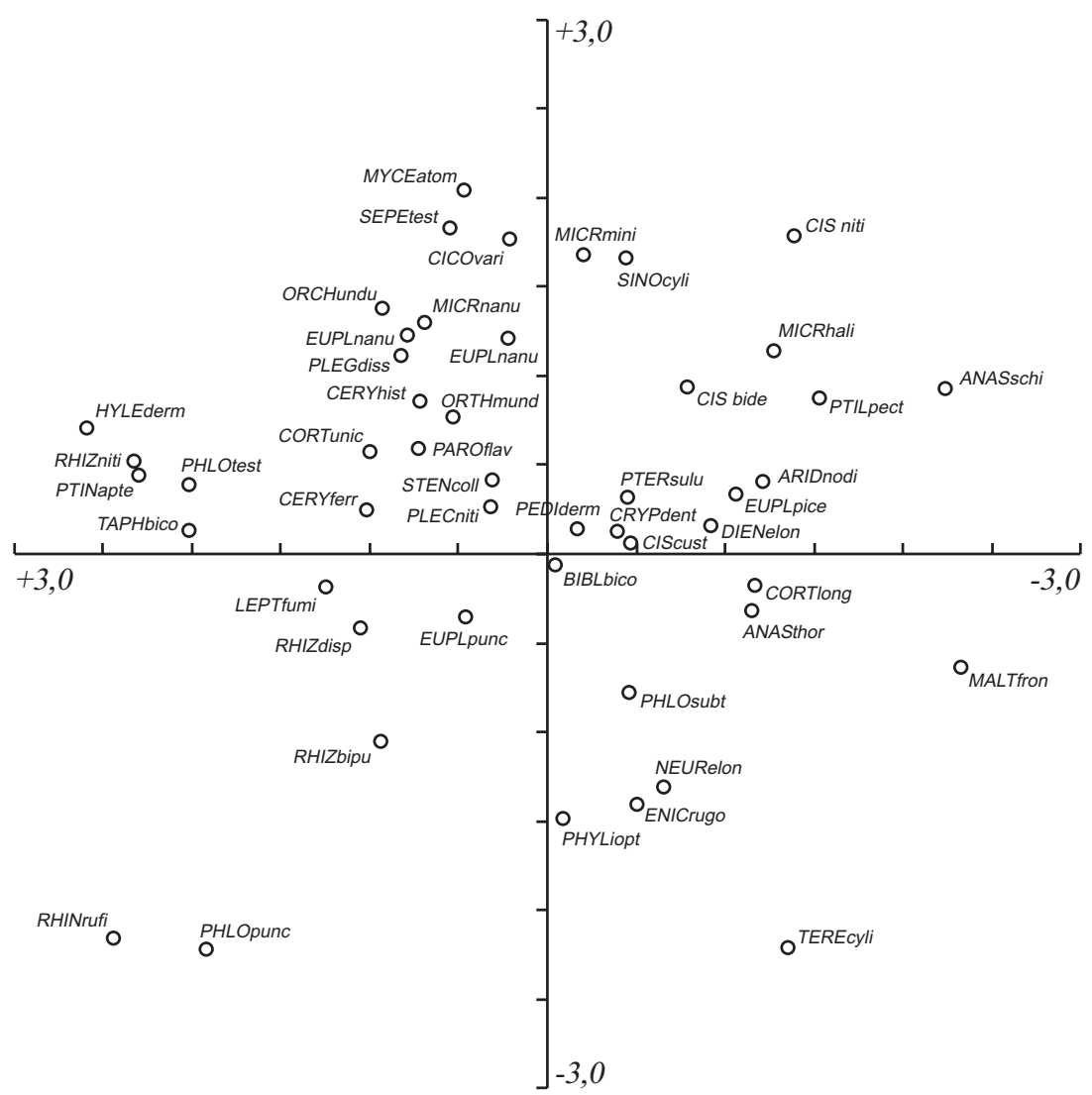

Abb. 4-45: Ordinationsdiagramm (DCA) der Arten aus EFs-Proben von Eichen- und Buchentotholz 
Die 14 ausschließlich an Eiche angetroffenen Arten traten in maximal 5 EFs-Proben auf. Anaspis rufilabris und Dorcatoma chrysomelina waren in geringer Individuenzahl in 5, Agrilus biguttatus in 4 und Lymexylon navale in 3 Proben anwesend. Obwohl nur $35 \%$ der Proben und 30,3\% der befangenen Oberfläche auf die Eiche entfielen, wurden einige Arten zu über $80 \%$ der gesammelten Individuen und der Nachweise an dieser Baumart festgestellt. Hierzu zählten u. a. Dryocoetes villosus, Corticeus fasciatus und Xyloterus signatus. Teredus cylindricus war in 46 EFs-Proben mit 231 Individuen vertreten. 24 Nachweise entfielen auf die Baumart Eiche, 22 auf die Buche. An ersterer war eine deutlich höhere Schlüpfdichte zu verzeichnen, so dass $81 \%$ der Individuen an Eiche gefangen wurden.

Eine größere Anzahl von Arten wurde in erster Linie an Buchentotholz gefangen. Im Ordinationsdiagramm (Abb. 4-45) sind diese in erster Linie in der Nähe des oberen Randes positioniert. Da jedoch ein Großteil der Stichproben auf diese Baumart entfiel, ließen sich diese Verteilungen in Anbetracht der gewählten Präferenzkriterien nur in Ausnahmefällen als Hinweis auf eine Bevorzugung dieser Baumart deuten.

51 xylobionte Käferarten waren ausschließlich in EFs-Proben von Buchentotholz vertreten, von denen wiederum 27 Arten in nur einer Probe nachgewiesen wurden. 9 Arten wurden in mindestens 10 Proben vorgefunden. Diese waren Rhizophagus nitidulus, Taphrorychus bicolor, Cis nitidus, Sepedophilus testaceus, Hylecoetus dermestoides, Cicones variegatus, Euplectus nanus, Mycetophagus atomarius und Sinodendron cylindricum. Vier Arten, Hedobia imperialis, Sepedophilus bipunctatus, Stenichnus godarti und Vincenzellus ruficollis, wurden in 5 bis 8 Proben ausschließlich an Buche registriert.

Eine größere Anzahl von Arten war auch mit wenigen Exemplaren in wenigen Proben an Eichentotholz vertreten. U. a. waren dies Ptinella aptera, Ptilinus pectinicornis, Plegaderus dissectus, Abraeus granulum, Micridium halidaii, Pediacus dermestoides, Paromalus flavicornis, Orchesia undulata und Corticeus unicolor.

Soweit die genannten Arten auch in den STE-Proben in größerer Zahl angetroffen worden waren, war auch dort ein gehäuftes Auftreten an Buchentotholz zu verzeichnen gewesen. Für die 45 xylobionten Käferarten, die sowohl in den EFs- als auch in den STE-Proben in mindestens 5 Proben mit mindestens 10 Individuen vertreten waren, ließen sich, unter Berücksichtigung der jeweils auf die Baumarten entfallenden Stichprobenanteile, kaum auffällige Differenzen feststellen. Beispiele für deutlichere Abweichungen in der Verteilung der Nachweise und/oder der Individuenzahlen sind Euplectus piceus, Orthoperus mundus, Anaspis schilskyana und Corticaria longicollis. 
Tabelle 4-63: Prozentuale Verteilung der Nachweise (Frequenz) in Abhängigkeit von Baumart, Rinden- und Bohrwiderstandsklasse ('Präferenzen' hervorgehoben)

\begin{tabular}{|c|c|c|c|c|c|c|c|c|c|c|c|c|c|}
\hline \multirow[b]{2}{*}{ Art } & \multirow[b]{2}{*}{ Frequenz } & \multicolumn{2}{|c|}{ Baumart } & \multicolumn{4}{|c|}{ Rindenklasse } & \multicolumn{6}{|c|}{ Bohrwiderstandsklasse } \\
\hline & & Ei & Bu & Ri 4 & & & Ri 1 & Bw 6 & Bw 5 & Bw 4 & Bw 3 & Bw 2 & Bw 1 \\
\hline Abraeus granulum & 27 & 3,7 & 96,3 & 50,0 & 11,5 & 7,7 & 30,8 & 3,7 & 7,4 & 18,5 & 11,1 & 29,6 & 29,6 \\
\hline Aderus oculatus & 9 & 55,6 & 44,4 & 11,1 & 0,0 & 11,1 & 77,8 & 0,0 & & 0,0 & 0,0 & 33,3 & 55,6 \\
\hline Anaspis schilskyana & 25 & 24,0 & 76,0 & 37,5 & 8,3 & 16,7 & 37,5 & 8,0 & 8,0 & 24,0 & 4,0 & 12,0 & 44,0 \\
\hline Anaspis thoracica & 23 & 39,1 & 60,9 & 59,1 & 4,5 & 9,1 & 27,3 & 4,3 & 4,3 & 30,4 & & 21,7 & 21,7 \\
\hline Anomognathus cuspidatus & 7 & 14,3 & 85,7 & 100,0 & 0,0 & 0,0 & 0,0 & 0,0 & 71,4 & 0,0 & 14,3 & 14,3 & 0,0 \\
\hline Bibloporus bicolor & 24 & 29,2 & 70,8 & 56,5 & 4,3 & 13,0 & 26,1 & 4,2 & 12,5 & 25,0 & 20,8 & 12,5 & 25,0 \\
\hline Cerylon ferrugineum & 62 & 17,7 & 82,3 & 68,9 & 8,2 & 11,5 & & 4,8 & 14,5 & 29,0 & 19,4 & 21,0 & 11,3 \\
\hline Cerylon histeroides & 30 & 10,0 & 90,0 & 63,3 & 6,7 & 10,0 & 20,0 & 0,0 & 20,0 & 26,7 & 13,3 & 20,0 & 20,0 \\
\hline Cicones variegatus & 13 & 0,0 & 100,0 & 46,2 & 7,7 & 23,1 & & 7,7 & 0,0 & 0,0 & & 69,2 & 7,7 \\
\hline Cis bidentatus & 20 & 10,0 & 90,0 & 55,0 & 15,0 & 15,0 & 15,0 & 0,0 & 0,0 & 40,0 & 10,0 & 30,0 & 20,0 \\
\hline Cis castaneus & 52 & 26,9 & 73,1 & 61,5 & 7,7 & 13,5 & & 1,9 & 9,6 & 32,7 & & 19,2 & 19,2 \\
\hline Cis nitidus & 17 & 0,0 & 100,0 & 47,1 & 29,4 & 17,6 & 5,9 & 0,0 & 0,0 & 29,4 & 0,0 & 41,2 & 29,4 \\
\hline Corticaria longicollis & 124 & 37,1 & 62,9 & 44,7 & 7,3 & 10,6 & 37,4 & 4,8 & 5,6 & 24,2 & 21,0 & 21,0 & 23,4 \\
\hline Corticeus fasciatus & 11 & 81,8 & 18,2 & 0,0 & 0,0 & 18,2 & & 9,1 & 0,0 & & 36,4 & 27,3 & 9,1 \\
\hline Corticeus unicolor & 44 & 11,4 & 88,6 & 65,1 & 7,0 & 11,6 & 16,3 & 9,1 & 15,9 & 29,5 & 20,5 & 22,7 & 2,3 \\
\hline & 18 & 88,9 & & 88,9 & 0,0 & 0,0 & & 5,6 & 16,7 & 55,6 & & 6 & 0,0 \\
\hline Enicmus fungicola & 7 & 71,4 & 28,6 & 57,1 & 0,0 & 0,0 & 42,9 & 0,0 & 0,0 & 57,1 & 14,3 & 14,3 & 14,3 \\
\hline Enicmus testaceus & 8 & 37,5 & 62,5 & 62,5 & 0,0 & 0,0 & 37,5 & 0,0 & 12,5 & 37,5 & 0,0 & 25,0 & 25,0 \\
\hline Euplectus bescidicu & 9 & 11,1 & 88,9 & 55,6 & 11,1 & 22,2 & & 0,0 & 22,2 & 44,4 & 11,1 & 11,1 & 11,1 \\
\hline Euplectus nanus & 12 & 0,0 & 100,0 & 81,8 & 9,1 & 9,1 & 0,0 & 8,3 & 8,3 & 25,0 & 8,3 & 33,3 & 16,7 \\
\hline & 19 & 26,3 & & 38,9 & 11,1 & 11,1 & 38,9 & 0,0 & 0,0 & 10,5 & 21,1 & 21,1 & 47,4 \\
\hline & 34 & 35,3 & 64,7 & 66,7 & 9,1 & 6,1 & 18,2 & 5,9 & 17,6 & 38,2 & 17,6 & 14,7 & 5,9 \\
\hline & 7 & 14,3 & 85,7 & 100,0 & 0,0 & 0,0 & 0,0 & 28,6 & 28,6 & 28,6 & 0,0 & 14,3 & 0,0 \\
\hline & 5 & 0,0 & 100,0 & 60,0 & 20,0 & 20,0 & 0,0 & & 0,0 & 20,0 & 20,0 & 20,0 & 40,0 \\
\hline Hylecoetus dermestoides & 15 & 0,0 & 100,0 & 93,3 & 6,7 & 0,0 & 0,0 & 13,3 & 20,0 & 40,0 & 20,0 & 6,7 & 0,0 \\
\hline & 53 & 22,6 & & 73,6 & 3,8 & 7,5 & 15,1 & & 18,9 & 34,0 & 15,1 & 20,8 & 5,7 \\
\hline & 20 & 30,0 & 70,0 & 47,4 & 5,3 & 15,8 & & 0,0 & 0,0 & 15,0 & 20,0 & 20,0 & 45,0 \\
\hline & 9 & 33,3 & 66,7 & 66,7 & 0,0 & 0,0 & 33,3 & 22,2 & 22,2 & 11,1 & 11,1 & 22,2 & 11,1 \\
\hline & 42 & 11,9 & & 52,4 & 9,5 & 11,9 & & & 2,4 & 28,6 & 9,5 & 28,6 & 28,6 \\
\hline Microscydmus minimus & 18 & 5,6 & 94,4 & 52,9 & 5,9 & 11,8 & 29,4 & 11,1 & 11,1 & 5,6 & 11,1 & 27,8 & 33,3 \\
\hline & 10 & 0,0 & 100,0 & 40,0 & 10,0 & 30,0 & & 20,0 & 0,0 & 10,0 & 10,0 & 60,0 & 0,0 \\
\hline Orchesia undulata & 16 & 12,5 & 87,5 & 62,5 & 12,5 & 0,0 & 25,0 & 6,3 & 6,3 & 31,3 & 25,0 & 25,0 & 6,3 \\
\hline Orthoperus mundus & 18 & 5,6 & 94,4 & 72,2 & 5,6 & 16,7 & & 16,7 & 11,1 & 33,3 & 0,0 & 27,8 & 11,1 \\
\hline Paromalus flavicornis & 65 & 16,9 & 83,1 & 67,2 & 9,4 & 9,4 & 14,1 & 4,6 & 15,4 & 27,7 & 15,4 & 24,6 & 12,3 \\
\hline Pediacus dermestoides & 20 & 5,0 & 95,0 & 57,9 & 5,3 & 0,0 & 36,8 & 10,0 & 25,0 & 5,0 & 5,0 & 15,0 & 40,0 \\
\hline & 14 & 35,7 & & 50,0 & 7,1 & 7,1 & 35,7 & 14,3 & & 0,0 & 21,4 & 21,4 & 21,4 \\
\hline Phloeonomus punctipennis & 20 & 35,0 & 65,0 & 90,0 & 5,0 & 0,0 & 5,0 & 5,0 & 35,0 & 30,0 & 10,0 & 20,0 & 0,0 \\
\hline Phloeopora corticalis & 6 & 83,3 & 16,7 & 83,3 & 0,0 & 0,0 & 16,7 & 0,0 & 33,3 & 33,3 & 16,7 & 16,7 & 0,0 \\
\hline Phloeopora testacea & 25 & 8,0 & 92,0 & 95,8 & 0,0 & 4,2 & 0,0 & 8,0 & 32,0 & 28,0 & 12,0 & 12,0 & 8,0 \\
\hline Phyllodrepa ioptera & 20 & 50,0 & 50,0 & 70,0 & 0,0 & 5,0 & 25,0 & 0,0 & 30,0 & 15,0 & 25,0 & 20,0 & 10,0 \\
\hline Plectophloeus nitidus & 11 & 27,3 & 72,7 & 72,7 & 9,1 & 9,1 & 9,1 & 18,2 & 18,2 & 27,3 & 9,1 & 9,1 & 18,2 \\
\hline
\end{tabular}




\begin{tabular}{|c|c|c|c|c|c|c|c|c|c|c|c|c|c|}
\hline \multirow[b]{2}{*}{ Art } & \multirow[b]{2}{*}{ Frequenz } & \multicolumn{2}{|c|}{ Baumart } & \multicolumn{4}{|c|}{ Rindenklasse } & \multicolumn{6}{|c|}{ Bohrwiderstandsklasse } \\
\hline & & Ei & Bu & Ri 4 & Ri 3 & Ri 2 & Ri 1 & Bw 6 & Bw 5 & Bw 4 & Bw 3 & Bw 2 & Bw 1 \\
\hline Plegaderus dissectus & 43 & 2,3 & $\mathbf{9 7 , 7}$ & 74,4 & 9,3 & 7,0 & 9,3 & 4,7 & 14,0 & 23,3 & 16,3 & 27,9 & 14,0 \\
\hline Pteryx suturalis & 14 & 21,4 & 78,6 & 42,9 & 7,1 & 14,3 & 35,7 & 7,1 & 7,1 & 28,6 & 7,1 & 42,9 & 7,1 \\
\hline Ptilinus pectinicornis & 52 & 5,8 & 94,2 & 41,2 & 5,9 & 21,6 & 31,4 & 1,9 & 13,5 & 15,4 & 7,7 & 21,2 & 40,4 \\
\hline Ptinella aptera & 25 & 4,0 & 96,0 & 88,0 & 4,0 & 4,0 & 4,0 & 8,0 & 28,0 & 20,0 & 16,0 & 24,0 & 4,0 \\
\hline Ptinella limbata & & 22,2 & 77,8 & 88,9 & 0,0 & 11,1 & 0,0 & 22,2 & 0,0 & 33,3 & 22,2 & 11,1 & 11,1 \\
\hline Rhinosimus ruficollis & 11 & 27,3 & 72,7 & 90,9 & 9,1 & 0,0 & 0,0 & 0,0 & 36,4 & 36,4 & 18,2 & 9,1 & 0,0 \\
\hline Rhizophagus bipustulatus & 79 & 29,1 & 70,9 & 73,1 & 5,1 & 5,1 & 16,7 & 3,8 & 21,5 & 29,1 & 16,5 & 16,5 & 12,7 \\
\hline Rhizophagus dispar & 86 & 24,4 & 75,6 & 72,1 & 5,8 & 7,0 & 15,1 & 3,5 & 17,4 & 30,2 & 15,1 & 22,1 & 11,6 \\
\hline Rhizophagus nitidulus & 23 & 0,0 & 100,0 & 100,0 & 0,0 & 0,0 & 0,0 & 13,0 & 34,8 & 30,4 & 8,7 & 13,0 & 0,0 \\
\hline Scydmoraphes minutus & 9 & 22,2 & 77,8 & 77,8 & 0,0 & 0,0 & 22,2 & 0,0 & 0,0 & 11,1 & 11,1 & 55,6 & 22,2 \\
\hline Sepedophilus bipunctatus & 5 & 0,0 & 100,0 & 40,0 & 0,0 & 0,0 & 60,0 & 0,0 & 0,0 & 20,0 & 0,0 & 60,0 & 20,0 \\
\hline Sepedophilus testaceus & 16 & 0,0 & 100,0 & 62,5 & 0,0 & 6,3 & 31,3 & 12,5 & 18,8 & 12,5 & 12,5 & 31,3 & 12,5 \\
\hline Sinodendron cylindricum & 10 & 0,0 & 100,0 & 22,2 & 0,0 & 22,2 & 55,6 & 10,0 & 0,0 & 20,0 & 0,0 & 20,0 & 50,0 \\
\hline Stenichnus godarti & 8 & 0,0 & 100,0 & 50,0 & 12,5 & 12,5 & 25,0 & 0,0 & 12,5 & 12,5 & 12,5 & 37,5 & 25,0 \\
\hline Taphrorychus bicolor & 19 & 0,0 & 100,0 & 94,7 & 0,0 & 5,3 & 0,0 & 10,5 & 36,8 & 31,6 & 10,5 & 10,5 & 0,0 \\
\hline Teredus cylindricus & 46 & 52,2 & 47,8 & 37,8 & 2,2 & 2,2 & 57,8 & 2,2 & 15,2 & 13,0 & 21,7 & 23,9 & 23,9 \\
\hline Vincenzellus ruficollis & 7 & 0,0 & 100,0 & 71,4 & 14,3 & 14,3 & 0,0 & 0,0 & 28,6 & 14,3 & 42,9 & 14,3 & 0,0 \\
\hline Xestobium rufovillosum & 14 & 35,7 & 64,3 & 7,1 & 0,0 & 28,6 & 64,3 & 7,1 & 0,0 & 7,1 & 14,3 & 21,4 & 50,0 \\
\hline Xyloterus domesticus & 7 & 57,1 & 42,9 & 100,0 & 0,0 & 0,0 & 0,0 & 0,0 & 28,6 & 57,1 & 14,3 & 0,0 & 0,0 \\
\hline Xyloterus signatus & & 88,9 & 11,1 & 88,9 & 0,0 & 0,0 & 11,1 & 0,0 & 22,2 & 44,4 & 33,3 & 0,0 & 0,0 \\
\hline Probenverteilung & 141 & 35,5 & 64,5 & $\mathbf{5 0 , 7}$ & 6,4 & 9,3 & 33,6 & 4,3 & 12,8 & 22,7 & 18,4 & 20,6 & 21,3 \\
\hline
\end{tabular}

Der Faktor Baumart war am stärksten mit der 2. Ordinationsachse korreliert. Ein größerer Erklärungsanteil für die vorgefundenen Artenvergesellschaftungen wurde von der ersten hypothetischen Variablen geliefert $(\lambda 1=0.312)$. Dahinter war vor allem der gemeinsame Einfluss mehrerer miteinander positiv korrelierter Einflussgrößen verborgen, insbesondere der Faktoren Berindung, Feuchte und Bohrwiderstand.

Im abgebildeten Ordinationsdiagramm (Abb. 4-44) sind Proben von berindeten Stammabschnitten mit hohem Bohrwiderstand und Feuchtegehalt vor allem im linken Bereich positioniert. Dementsprechend sind in diesem Bereich jene Arten zu finden, die in erster Linie an frischerem Material gefangen wurden, während Arten, die in ihrer Mehrheit an unberindeten Stammabschnitten mit niedrigem Bohrwiderstand und Feuchtegehalt festgestellt wurden, vermehrt im rechten Teil positioniert sind (Abb. 4-45).

$\mathrm{Zu}$ den Arten, die vor allem an berindeten Stammteilen mit hohem Bohrwiderstand gefangen wurden, zählten u. a. Phloeonomus punctipennis, Rhizophagus dispar, Rh. bipustulatus, Rh. nitidulus, Taphrorychus bicolor, Anomognathus cuspidatus und Ptinella aptera. An berindeten Stammabschnitten mittleren Bohrwiderstandes zeigten u. a. Dryocoetes villosus, Xyloterus signatus, Cerylon ferrugineum, Leptusa fumida, Orchesia undulata und Paromalus flavicornis ein gehäuftes Auftreten. Die genannten Arten wurden fast ausnahmslos in Proben der Feuchteklasse 3 festgestellt.

In Proben von rindenlosen Stammteilen mit geringem Bohrwiderstand und niedrigem Feuchtegehalt hatten u. a. Corticeus fasciatus, Xestobium rufovillosum und Ptilinus 
pectinicornis ihren Verteilungsschwerpunkt, während Anaspis schilskyana und Micridium halidaii eher in feuchteren Bereichen zu finden waren. Teredus cylindricus trat in erster Linie an rindenlosen Eichenstämmen der Bohrwiderstandsklasse 3 und den Feuchteklassen 2 bis 4 in Erscheinung. Aderus oculatus, Sepedophilus testaceus und Sinodendron cylindricum wurden ebenfalls gehäuft an unberindeten Stammsegmenten geringen Bohrwiderstandes festgestellt. Angaben zu ihrer Verteilung in Abhängigkeit vom Feuchtegehalt waren jedoch nicht möglich, da sie zum größten Teil von Probenahmepunkten stammten, für die keine entsprechenden Messungen durchgeführt werden konnten.

Die Verteilung der Arten auf die Bw- und Ri-Klassen wich in einigen Fällen mehr oder weniger deutlich von den in den STE-Proben beobachteten Gegebenheiten ab. So wurde z. B. Ptilinus pectinicornis an liegendem Totholz fast ausschließlich an berindeten Stämmen mit hohem Bohrwiderstand gefangen, an stehendem Totholz dagegen an Stämmen mit sehr geringem Bohrwidestand. Auch Xyloterus signatus, und X. domesticus fingen sich an liegendem Totholz häufiger an Stämmen mit höherer Festigkeit.

Tabelle 4-64: Prozentuale Verteilung der Individuen in Abhängigkeit von Baumart, Rindenund Bohrwiderstandsklasse ('Präferenzen' hervorgehoben)

\begin{tabular}{|c|c|c|c|c|c|c|c|c|c|c|c|c|c|}
\hline \multirow[b]{2}{*}{ Art } & \multirow[b]{2}{*}{ Individuen } & \multicolumn{2}{|c|}{ Baumart } & \multicolumn{4}{|c|}{ Rindenklasse } & \multicolumn{6}{|c|}{ Bohrwiderstandsklasse } \\
\hline & & Ei & Bu & $\mathbf{R}$ & $\mathbf{R}$ & & Ri 1 & Bw 6 & & & & Bw 2 & Bw 1 \\
\hline Abraeus granulum & 56 & 1,8 & 98,2 & 46,3 & 7,4 & 5,6 & 40,7 & 1,8 & 16,1 & 14,3 & 8,9 & 28,6 & 30,4 \\
\hline Aderus oculatus & 33 & 69,7 & 30,3 & 6,1 & 0,0 & 12,1 & 81,8 & 0,0 & 6,1 & 0,0 & 0,0 & 24,2 & 69,7 \\
\hline Anaspis schilskyana & 83 & 9,6 & 90,4 & 23,5 & 9,9 & 27,2 & 39,5 & 3,6 & 3,6 & 31,3 & 1,2 & 9,6 & 50,6 \\
\hline Anaspis thoracica & 37 & 40,5 & 59,5 & 55,6 & 2,8 & 13,9 & 27,8 & 2,7 & 2,7 & 45,9 & 10,8 & 13,5 & 24,3 \\
\hline Anomognathus cuspidatus & 14 & 7,1 & 92,9 & 100,0 & 0,0 & 0,0 & 0,0 & 0,0 & 64,3 & 0,0 & 7,1 & 28,6 & 0,0 \\
\hline Bibloporus bicolor & 35 & 22,9 & 77,1 & 64,7 & 2,9 & 11,8 & 20,6 & 2,9 & 8,6 & 25,7 & 31,4 & 14,3 & 17,1 \\
\hline Cerylon ferrugineum & 468 & 12,4 & 87,6 & 84,3 & 5,6 & 3,2 & 6,9 & 6,0 & 19,2 & 33,5 & 25,0 & 11,3 & 4,9 \\
\hline Cerylon histeroides & 73 & 5,5 & 94,5 & 43,8 & 13,7 & 4,1 & 38,4 & 0,0 & 12,3 & 16,4 & 8,2 & 34,2 & 28,8 \\
\hline Cicones variegatus & 146 & 0,0 & 100,0 & 63,7 & 7,5 & 23,3 & 5,5 & 0,7 & 0,0 & 0,0 & 2,7 & 95,2 & 1,4 \\
\hline Cis bidentatus & 66 & 12,1 & 87,9 & 69,7 & 12,1 & 12,1 & 6,1 & 0,0 & 0,0 & 31,8 & 9,1 & 47,0 & 12,1 \\
\hline Cis castaneus & 286 & 35,0 & 65,0 & 69,6 & 5,6 & 13,6 & 611,2 & 0,3 & 8,4 & 42,7 & 7,0 & 30,1 & 11,5 \\
\hline Cis nitidus & 445 & 0,0 & 100,0 & 73,3 & 11,9 & 14,6 & 0,2 & 0,0 & 0,0 & 15,1 & 0,0 & 71,2 & 13,7 \\
\hline Corticaria longicollis & 3170 & 16,4 & 83,6 & 38,9 & 5,3 & 34,5 & 21,3 & 0,7 & 1,0 & 23,6 & 13,9 & 38,0 & 22,7 \\
\hline Corticeus fasciatus & 31 & 93,5 & 6,5 & 0,0 & 0,0 & 6,5 & 93,5 & 12,9 & 0,0 & 9,7 & 48,4 & 25,8 & 3,2 \\
\hline Corticeus unicolor & 390 & 3,8 & 96,2 & 61,7 & 5,7 & 14,9 & 17,7 & 3,8 & 9,7 & 44,6 & 14,4 & 27,2 & 0,3 \\
\hline Dryocoetes villosus & 2483 & 99,5 & 0,5 & 99,8 & 0,0 & 0,0 & 0,2 & 0,1 & 0,6 & 68,7 & 30,5 & 0,2 & 0,0 \\
\hline Enicmus fungicola & 11 & 63,6 & 36,4 & 54,5 & 0,0 & 0,0 & 45,5 & 0,0 & 0,0 & 36,4 & 27,3 & 27,3 & 9,1 \\
\hline Enicmus testaceus & 47 & 83,0 & 17,0 & 19,1 & 0,0 & 0,0 & 80,9 & 0,0 & 6,4 & $\mathbf{8 3 , 0}$ & 0,0 & 4,3 & 6,4 \\
\hline Euplectus bescidicus & 10 & 10,0 & 90,0 & 60,0 & 10,0 & 20,0 & 10,0 & 0,0 & 30,0 & 40,0 & 10,0 & 10,0 & 10,0 \\
\hline Euplectus nanus & 17 & 0,0 & 100,0 & 87,5 & 6,3 & 6,3 & 0,0 & 5,9 & 5,9 & 29,4 & 11,8 & 35,3 & 11,8 \\
\hline Euplectus piceus & 25 & 20,0 & 80,0 & 34,8 & 8,7 & 13,0 & 43,5 & 0,0 & 0,0 & 8,0 & 16,0 & 32,0 & 44,0 \\
\hline Euplectus punctatus & 57 & 35,1 & 64,9 & 71,4 & 5,4 & 5,4 & 17,9 & 7,0 & 22,8 & 38,6 & 15,8 & 12,3 & 3,5 \\
\hline Euryusa castanoptera & 13 & 7,7 & 92,3 & 100,0 & 0,0 & 0,0 & 0,0 & 30,8 & 15,4 & 15,4 & 0,0 & 38,5 & 0,0 \\
\hline Hedobia imperialis & 19 & 0,0 & 100,0 & 26,3 & 68,4 & 5,3 & 0,0 & 0,0 & 0,0 & 5,3 & 5,3 & 15,8 & 73,7 \\
\hline Hylecoetus dermestoides & 196 & 0,0 & 100,0 & 98,0 & 2,0 & 0,0 & 0,0 & 15,8 & 23,5 & 21,9 & 11,2 & 27,6 & 0,0 \\
\hline
\end{tabular}




\begin{tabular}{|c|c|c|c|c|c|c|c|c|c|c|c|c|c|}
\hline Art & Individuen & \multicolumn{2}{|c|}{ Baumart } & \multicolumn{4}{|c|}{$\begin{array}{c}\text { Rindenklasse } \\
\text { Ri } 4 \text { Ri } 3 \text { Ri } 2 \text { Ri } 1\end{array}$} & \multirow{2}{*}{\begin{tabular}{|c|} 
\\
Bw 6 \\
4,0
\end{tabular}} & $\begin{array}{l}\text { Bohr } \\
\text { Bw } 5\end{array}$ & $\begin{array}{r}\text { widers } \\
\text { Bw } 4 \\
\end{array}$ & $\begin{array}{r}\text { tands } \\
\text { Bw } 3 \\
\end{array}$ & $\begin{array}{r}\text { klasse } \\
\text { Bw } 2 \\
\end{array}$ & Bw 1 \\
\hline Leptusa fumida & 201 & 13,4 & 86,6 & 84,1 & 7,0 & 3,0 & 6,0 & & 15,4 & 32,8 & 35,8 & 10,4 & 1,5 \\
\hline Malthinus frontalis & 38 & 18,4 & 81,6 & 40,5 & 2,7 & 18,9 & 37,8 & 0,0 & 0,0 & 7,9 & 13,2 & 28,9 & 50,0 \\
\hline Melanotus castanipes & 12 & 25,0 & 75,0 & 75,0 & 0,0 & 0,0 & 25,0 & 25,0 & 33,3 & 8,3 & 8,3 & 16,7 & 8,3 \\
\hline Micridium halidaii & 284 & 2,1 & 97,9 & 13,0 & 2,8 & 3,9 & 80,3 & 1,1 & 0,4 & 6,7 & 2,5 & 6,7 & 82,7 \\
\hline Microscydmus minimus & 23 & 4,3 & 95,7 & 54,5 & 4,5 & 9,1 & 31,8 & 13,0 & 8,7 & 4,3 & 13,0 & 21,7 & 39,1 \\
\hline Mycetophagus atomarius & 51 & 0,0 & 100,0 & 52,9 & 5,9 & 15,7 & 25,5 & 17,6 & 0,0 & 2,0 & 2,0 & 78,4 & 0,0 \\
\hline Orchesia undulata & 53 & 3,8 & 96,2 & 81,1 & 3,8 & 0,0 & 15,1 & 3,8 & 5,7 & 49,1 & 32,1 & 7,5 & 1,9 \\
\hline Orthoperus mundus & 39 & 2,6 & 97,4 & 79,5 & 5,1 & 10,3 & 5,1 & 7,7 & 10,3 & 25,6 & 0,0 & 46,2 & 10,3 \\
\hline Paromalus flavicornis & 484 & 3,7 & 96,3 & 81,8 & 6,6 & 7,7 & 3,9 & 2,3 & 14,7 & 44,4 & 13,2 & 23,1 & 2,3 \\
\hline Pediacus dermestoides & 36 & 2,8 & 97,2 & 54,3 & 2,9 & 0,0 & 42,9 & 8,3 & 22,2 & 2,8 & 5,6 & 8,3 & 52,8 \\
\hline Phloeocharis subtilissima & 18 & 33,3 & 66,7 & 44,4 & 16,7 & 5,6 & 33,3 & 11,1 & 16,7 & 0,0 & 33,3 & 16,7 & 22,2 \\
\hline Phloeonomus punctipennis & 64 & 26,6 & 73,4 & 96,9 & 1,6 & 0,0 & 1,6 & 1,6 & 53,1 & 23,4 & 4,7 & 17,2 & 0,0 \\
\hline Phloeopora corticalis & 10 & 90,0 & 10,0 & 90,0 & 0,0 & 0,0 & 10,0 & 0,0 & 20,0 & 50,0 & 20,0 & 10,0 & 0,0 \\
\hline Phloeopora testacea & 69 & 4,3 & 95,7 & 98,5 & 0,0 & 1,5 & 0,0 & 7,2 & 26,1 & 34,8 & 24,6 & 4,3 & 2,9 \\
\hline Phyllodrepa ioptera & 38 & 57,9 & 42,1 & 81,6 & 0,0 & 2,6 & 15,8 & 0,0 & 23,7 & 34,2 & 21,1 & 13,2 & 7,9 \\
\hline Plectophloeus nitidus & 12 & 25,0 & 75,0 & 75,0 & 8,3 & 8,3 & 8,3 & 16,7 & 25,0 & 25,0 & 8,3 & 8,3 & 16,7 \\
\hline Plegaderus dissectus & 95 & 1,1 & 98,9 & 71,6 & 6,3 & 7,4 & 14,7 & 2,1 & 10,5 & 30,5 & 12,6 & 30,5 & 13,7 \\
\hline Pteryx suturalis & 15 & 20,0 & 80,0 & 40,0 & 13,3 & 13,3 & 33,3 & 6,7 & 6,7 & 33,3 & 6,7 & 40,0 & 6,7 \\
\hline Ptilinus pectinicornis & 2705 & 0,9 & 99,1 & 19,4 & 28,1 & 11,2 & 41,2 & 0,0 & 1,7 & 4,5 & 0,4 & 21,5 & 71,7 \\
\hline Ptinella aptera & 536 & 0,2 & 99,8 & 99,3 & 0,4 & 0,2 & 0,2 & 3,9 & 56,3 & 25,2 & 3,0 & 11,2 & 0,4 \\
\hline Ptinella limbata & 10 & 20,0 & 80,0 & 90,0 & 0,0 & 10,0 & 0,0 & 20,0 & 0,0 & 40,0 & 20,0 & 10,0 & 10,0 \\
\hline Rhinosimus ruficollis & 14 & 28,6 & 71,4 & 92,9 & 7,1 & 0,0 & 0,0 & 0,0 & 28,6 & 35,7 & 28,6 & 7,1 & 0,0 \\
\hline Rhizophagus bipustulatus & 519 & 24,9 & 75,1 & 91,7 & 1,7 & 2,7 & 3,9 & 1,2 & 48,0 & 30,1 & 10,6 & 7,5 & 2,7 \\
\hline Rhizophagus dispar & 1507 & 7,4 & 92,6 & 88,2 & 2,7 & 2,2 & 7,0 & 1,5 & 34,9 & 20,8 & 12,9 & 28,5 & 1,4 \\
\hline Rhizophagus nitidulus & 119 & 0,0 & 100,0 & 100,0 & 0,0 & 0,0 & 0,0 & 10,1 & 52,9 & 20,2 & 3,4 & 13,4 & 0,0 \\
\hline Scydmoraphes minutus & 15 & 13,3 & 86,7 & 86,7 & 0,0 & 0,0 & 13,3 & 0,0 & 0,0 & 6,7 & 6,7 & 73,3 & 13,3 \\
\hline Sepedophilus bipunctatus & 13 & 0,0 & 100,0 & 15,4 & 0,0 & 0,0 & 84,6 & 0,0 & 0,0 & 7,7 & 0,0 & 84,6 & 7,7 \\
\hline Sepedophilus testaceus & 46 & 0,0 & 100,0 & 32,6 & 0,0 & 2,2 & 65,2 & 6,5 & 8,7 & 8,7 & 4,3 & 63,0 & 8,7 \\
\hline Sinodendron cylindricum & 35 & 0,0 & 100,0 & 15,2 & 0,0 & 6,1 & 78,8 & 2,9 & 0,0 & 5,7 & 0,0 & 54,3 & 37,1 \\
\hline Stenichnus godarti & 14 & 0,0 & 100,0 & 42,9 & 14,3 & 7,1 & 35,7 & 0,0 & 7,1 & 7,1 & 7,1 & 35,7 & 42,9 \\
\hline Taphrorychus bicolor & 135 & 0,0 & 100,0 & 99,3 & 0,0 & 0,7 & 0,0 & 2,2 & 60,7 & 29,6 & 2,2 & 5,2 & 0,0 \\
\hline Teredus cylindricus & 231 & 81,0 & 19,0 & 15,0 & 0,5 & 0,5 & 84,1 & 4,8 & 6,9 & 22,9 & 41,1 & 14,3 & 10,0 \\
\hline Vincenzellus ruficollis & 15 & 0,0 & 100,0 & 80,0 & 13,3 & 6,7 & 0,0 & 0,0 & 13,3 & 6,7 & 66,7 & 13,3 & 0,0 \\
\hline Xestobium rufovillosum & 39 & 20,5 & 79,5 & 2,6 & 0,0 & 10,3 & 87,2 & 5,1 & 0,0 & 2,6 & 10,3 & 7,7 & 74,4 \\
\hline Xyloterus domesticus & 28 & 64,3 & 35,7 & 100,0 & 0,0 & 0,0 & 0,0 & 0,0 & 32,1 & 39,3 & 28,6 & 0,0 & 0,0 \\
\hline Xyloterus signatus & 323 & 91,3 & 8,7 & 99,7 & 0,0 & 0,0 & 0,3 & 0,0 & 9,0 & 53,3 & 37,8 & 0,0 & 0,0 \\
\hline Mantelfläche & $23 \mathrm{~m}^{2}$ & 30,3 & 69,7 & 54,6 & 6,2 & 9,5 & 29,6 & 4,3 & 13,6 & 23,4 & 17,0 & 21,0 & 20,7 \\
\hline Rindenfläche & $14 \mathrm{~m}^{2}$ & 21,4 & 78,6 & 87,0 & 7,6 & 5,4 & $\mathbf{0 , 0}$ & 5,9 & 21,1 & 31,0 & 13,8 & 18,2 & 10,1 \\
\hline
\end{tabular}




\subsubsection{Sukzession der Artengemeinschaften an stehendem Buchentotholz}

Die Ähnlichkeit $\left(\mathrm{RE}_{\mathrm{ln}}\right)$ der in den 91 EFs-Proben vorgefundenen Artengemeinschaften ist in der Tabelle 4-66 dargestellt (s. a. Anhang 9a u. b). Die Anordnung der Proben folgt in der Tendenz einem Gradienten von frischem zu stärker zersetztem Material. Die größten Übereinstimmungen in der Artenvergesellschaftung wurden in vielen Fällen für Proben festgestellt, die von den gleichen Stammabschnitten stammten. Beispiele hierfür sind die Proben 08a-d/95, 02a,b/95 und 02a-f/96 sowie die Proben 03a-d/95 und 04a-f/96. Allem Anschein nach gestaltet sich das Erscheinen und das Verschwinden der Arten wie schon bei den STEProben als ein weitestgehend kontinuierlicher Prozeß bzw. läßt sich die Vielfalt besiedlungsrelevanter Faktorkombinationen nicht auf einen einzelnen Zersetzungsgradienten reduzieren.

Eine ähnliche Anordnung der Proben ergab sich auch als Ergebnis einer DCA. Die Abbildungen 4-46 und 4-47 zeigen die Ordinationsdiagramme für die Proben und Arten. Berechnungsgrundlage war die logarithmierte Schlüpfdichte von insgesamt 159 Arten, von denen aus Gründen der Übersichtlichkeit in erster Linie jene Arten dargestellt sind, die in größerer Anzahl festgestellt wurden. Tabelle 4-65 stellt die wichtigsten Ergebnisse verschiedener Korrespondenzanalysen vergleichend gegenüber.

Die Eigenwerte der abgebildeten Ordinationsachsen beliefen sich auf 0,388 für die erste und 0,250 für die zweite Achse, die der nicht dargestellten dritten und vierten Achse auf 0,215 und 0,179. Je nach gewähltem Ordinationsverfahren (CA, DCA) und zugrunde gelegtem Datensatz konnten die vier hypothetischen Variablen zwischen 14,1 und 33,5\% der beobachteten Streuung erklären.

Die vorgefundenen Unterschiede in den Artenvergesellschaftungen ließen sich zumindest teilweise durch die Dauer des Zersetzungsprozesses erklären. Allerdings ließen zahlreiche der stärker zersetzten Probestämme eine Einschätzung des Absterbezeitpunktes nicht zu. Die überwiegende Zahl dieser Proben sind gemeinsam mit älteren Totholzabschnitten im rechten Teil des Ordinationsdiagramms positioniert, während Proben von frischerem Material insbesondere im unteren linken Teil zu finden sind. Entsprechend ihrer Verteilung auf die Proben sind im linken Diagrammteil Arten wiedergegeben, die in erster Linie an berindeten und relativ frischen Stammabschnitten gefangen wurden, wie z. B. Ptinella aptera, Hylecoetus dermestoides, Rhinosimus ruficollis, Rhizophagus dispar und Rh. bipustulatus. Arten mit einer Präferenz für Totholzpilze und -mulm finden sich hingegen vermehrt im rechten Diagrammteil, wie z. B. Abraeus granulum, Corticaria longicollis, Cicones variegatus sowie verschiedene $C i s$-Arten. 
Tabelle 4-65: Ergebnisse verschiedener Korrespondenzanalysen mit den Fangdaten aus EFsProben an Buchentotholz

\begin{tabular}{|c|c|c|c|c|c|c|c|c|c|c|c|c|c|}
\hline & & $\begin{array}{l}\text { anungs- } \\
\text { dlage }\end{array}$ & $\begin{array}{l}\text { Eigenwerte }(\lambda) \text { der } \\
\text { Ordinationsachsen }\end{array}$ & $\begin{array}{c}\text { total } \\
\text { inertia }\end{array}$ & $\begin{array}{l}\text { cur } \\
\text { dat }\end{array}$ & $\begin{array}{r}\text { n. perc } \\
\text { spe } \\
\text { a (Ach }\end{array}$ & $\begin{array}{l}\text { c. var } \\
\text { ec. } \\
\text { asen } 1\end{array}$ & & $\begin{array}{r}\text { spec- env } \\
\text { (Achser }\end{array}$ & $\begin{array}{l}\text { v. corr } \\
\text { en } 1-4\end{array}$ & & $\begin{array}{r}\text { cum. p } \\
\text { of spe } \\
\text { rela } \\
\text { (Achs }\end{array}$ & $\begin{array}{l}\text { var. } \\
\text { env. } \\
\text { n } \\
1-2)\end{array}$ \\
\hline & Arten & Daten & $\begin{array}{llll}\lambda 1 & \lambda 2 & \lambda 3 & \lambda 4\end{array}$ & & 1 & 2 & 3 & 4 & 2 & 3 & 4 & 1 & 2 \\
\hline $\mathrm{C}$ & 159 & binär & $\begin{array}{llll}.372 .340 .269 & .257\end{array}$ & 7.904 & 4.7 & 9.0 & 12.4 & 15.7 & .248 .695 . & .506 & 391 & 2. & 17.6 \\
\hline A & 159 & In Dichte & $\begin{array}{llll}.388 & .366 & .273 & .253\end{array}$ & 6.765 & 5.7 & 11.1 & 15.2 & 18.9 & .504 .608 & .454 & .430 & 9.8 & 23.2 \\
\hline & 46 & binär & .242 .157 .139 .131 & 2.309 & 10.5 & 17.3 & 23.3 & 29.0 & .737 .622$. & .488 & .445 & 29.8 & 43.5 \\
\hline & 46 & ln Dichte & .313 .173 .147 .123 & 2.260 & 13.8 & 21.5 & 28.0 & 33.5 & .779 .537$. & .630 & .400 & 30.0 & 46.5 \\
\hline D & 159 & binär & 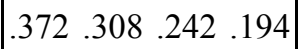 & 7.904 & 4.7 & 8.6 & 11.7 & 14.1 & .248 .696$. & יונד. & .500 & 1.6 & 16.7 \\
\hline $\mathrm{C}$ & 159 & In Dichte & .388 .250 .215 .179 & 6.765 & 5.7 & 9.4 & 12.6 & 15.2 & .504 .551 & .249 & .459 & 9.7 & 17.4 \\
\hline $\mathrm{A}$ & 46 & binär & .242 .131 .110 .100 & 2.309 & 10.5 & 16.2 & 21.0 & 25.3 & .737 .629$. & .209 & .495 & 29.8 & 42.3 \\
\hline & 46 & ln Dichte & .313 .142 .111 .087 & 2.260 & 13.8 & 20.1 & 25.0 & 28.9 & .779 .545 . & .561 & .458 & 36.8 & 45.3 \\
\hline
\end{tabular}


Tabelle 4-66: Ähnlichkeitsmatrix $\left(\mathrm{RE}_{\mathrm{ln}}\right)$ der EFs-Proben an stehendem Buchentotholz

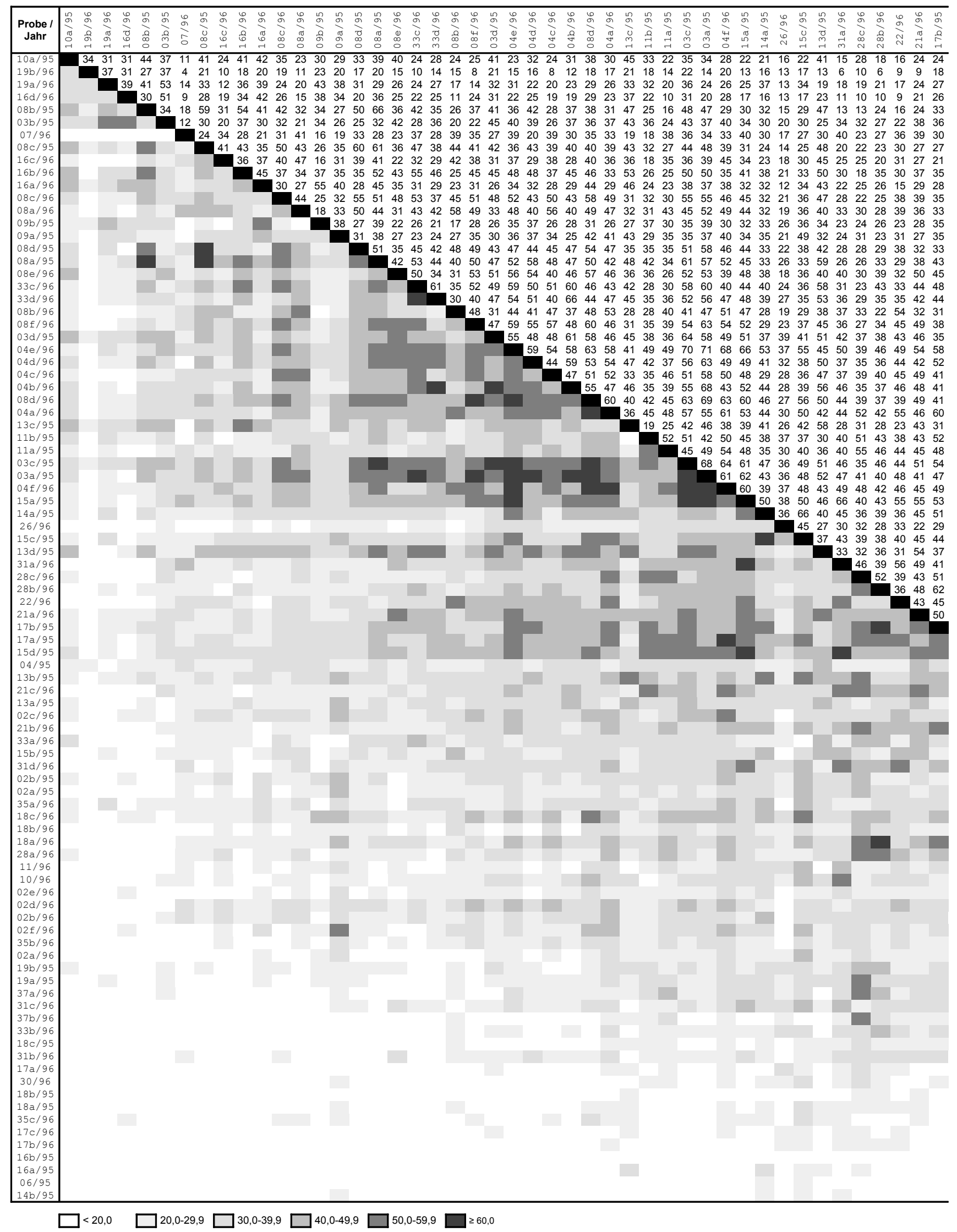


Tabelle 4-66 (Fortsetzung)

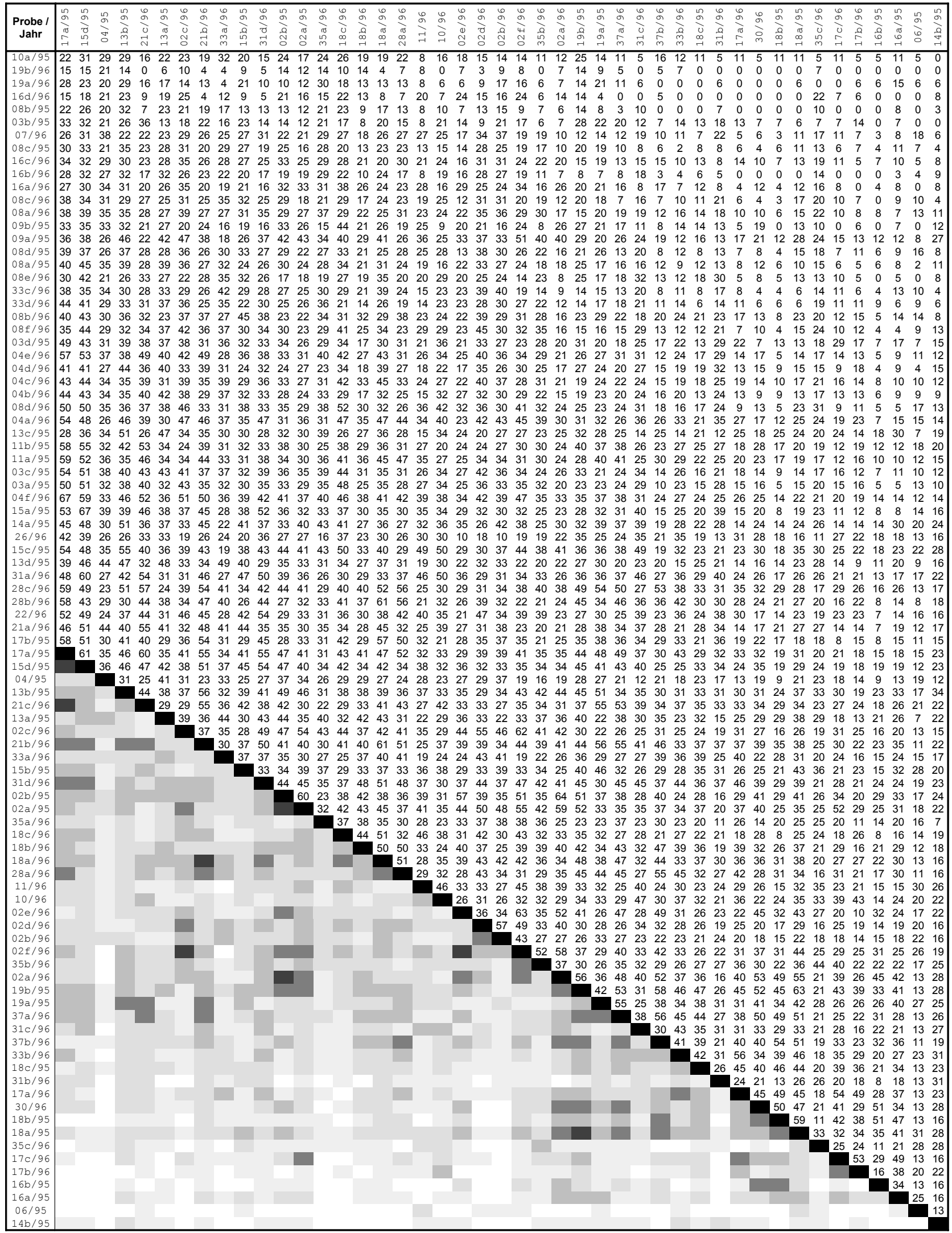




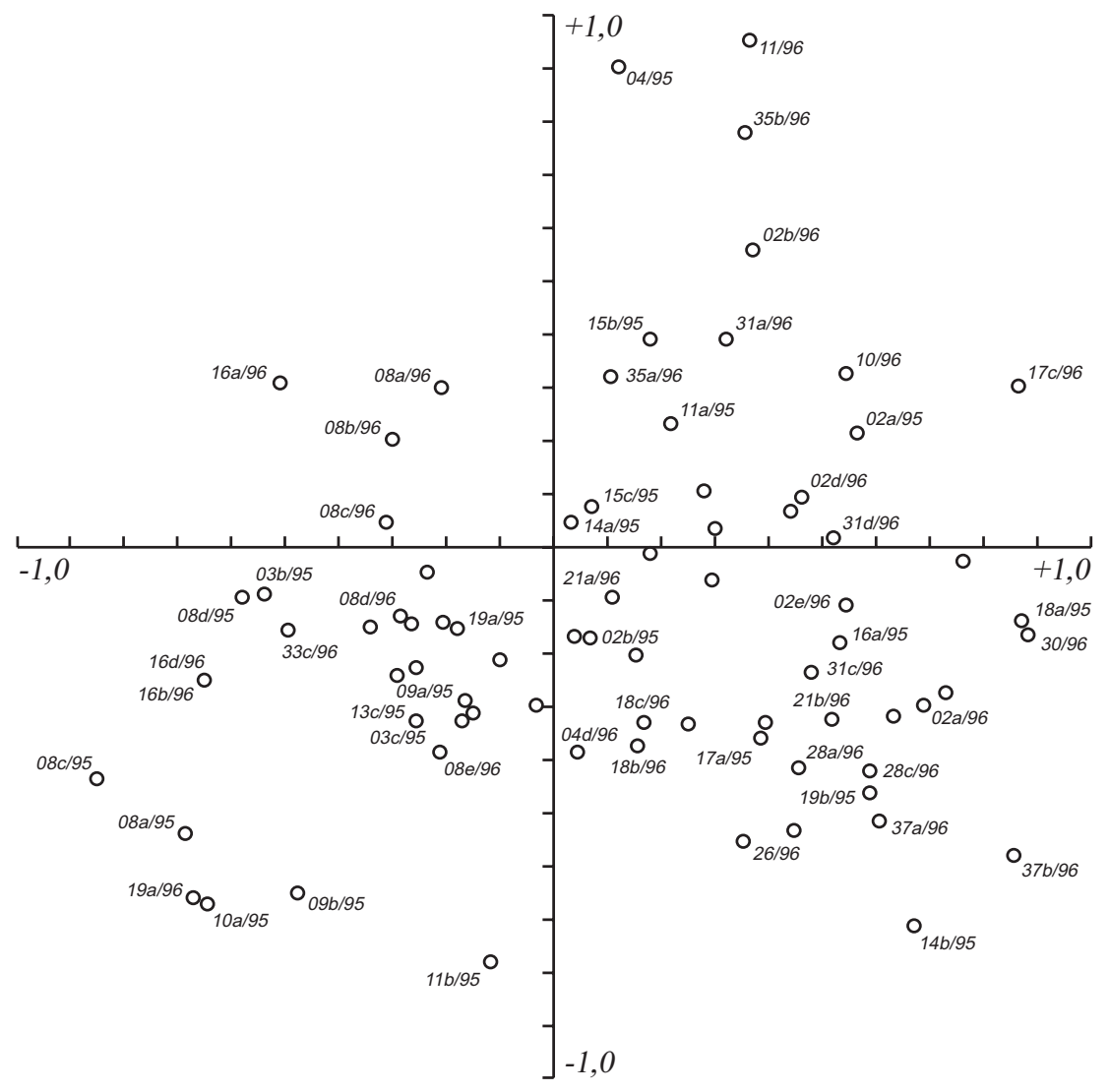

Abb. 4-46: Ordinationsdiagramm (DCA): EFs-Proben an stehendem Buchentotholz

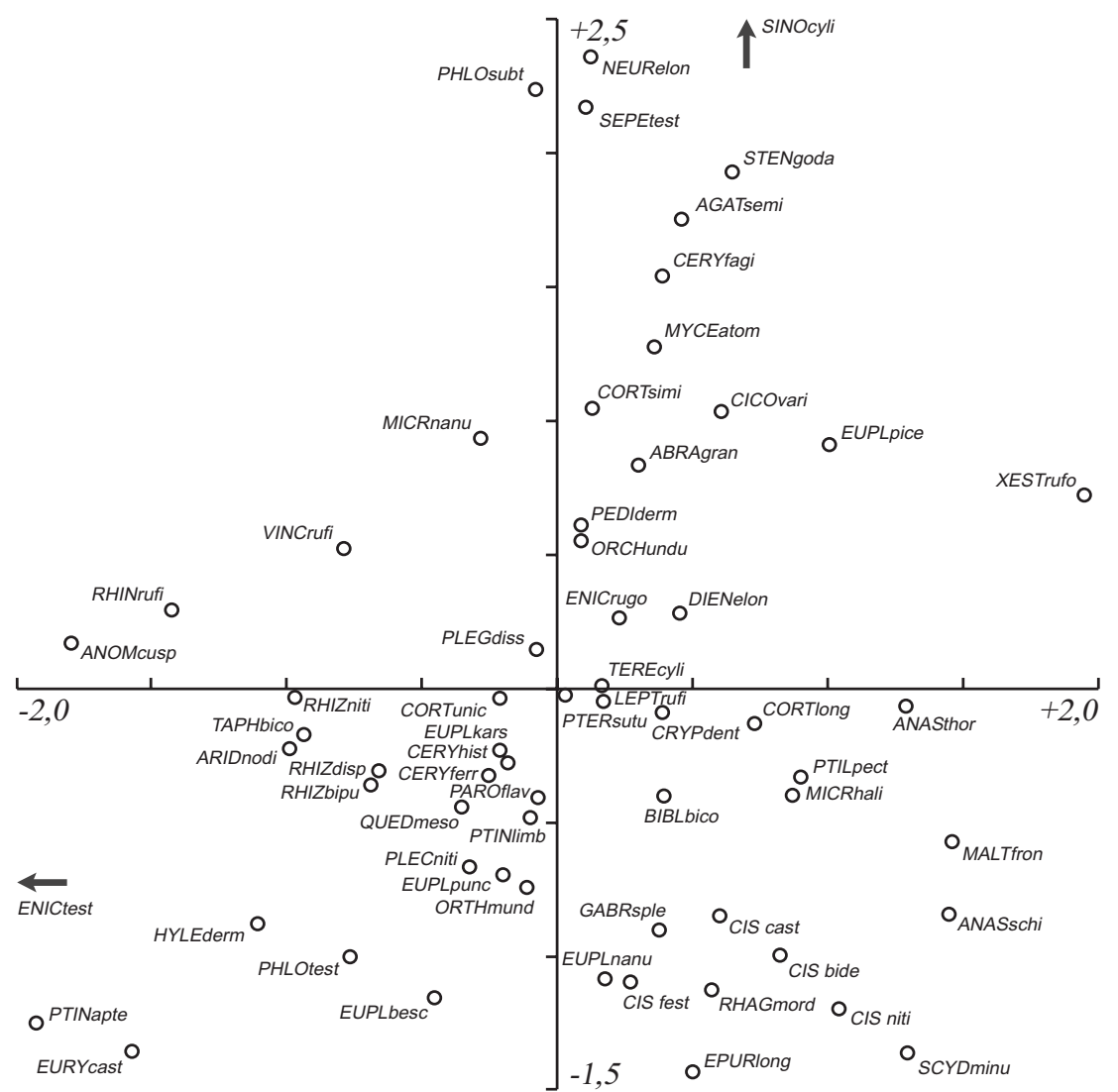

Abb. 4-47: Ordinationsdiagramm (DCA): Arten aus EFs-Proben an stehendem Buchentotholz 
Die Eigenwerte der Achsen und die Anordnung der Proben im Ordinationsdiagramm lassen aber auch erkennen, dass nicht ein einzelner stark dominierender Gradient das Vorkommen und die Häufigkeit der Arten bestimmte. Verschiedene kanonische Korrespondenzanalysen sollten darüber Aufschluß geben, welche der an den Fallenstandorten erhobenen Habitatvariablen bzw. Faktorkombinationen geeignet sind, einen nennenswerten Beitrag zur Erklärung der Streuung in der Vergesellschaftung der Arten zu leisten. Auch diesmal blieb der Faktor Absterbezeit aus den bereits genannten Gründen unberücksichtigt. Über die wichtigsten Ergebnisse informiert die Tabelle 4-67. Die Abbildungen 4-48 und 4-49 zeigen die Ergebnisse einer CCA, die auf den logarithmierten Schlüpfdichten von 46 Arten beruhen, die in wenigstens 10 der 91 EFs-Proben angetroffen worden waren. Für die 16 als passiv gekennzeichneten Proben lagen keine Angaben zu allen berücksichtigten Habitatvariablen vor, ihre Position im Diagramm wurde in einem zweiten Arbeitsschritt auf Grundlage ihrer Artenstruktur bestimmt.

Tabelle 4-67: Ergebnisse verschiedener kanonischer Korrespondenzanalysen mit den Fangdaten aus EFs- Proben an Buchentotholz

\begin{tabular}{|c|c|c|c|c|c|c|c|c|c|c|c|c|c|c|}
\hline & & $\begin{array}{l}\text { nungs- } \\
\text { dlage }\end{array}$ & $\begin{array}{l}\text { Eigenwerte }(\lambda) \text { der } \\
\text { Ordinationsachsen }\end{array}$ & $\begin{array}{c}\text { total } \\
\text { inertia }\end{array}$ & & $\begin{array}{r}\text { a. perc } \\
\text { spe } \\
\text { (Ach }\end{array}$ & $\begin{array}{l}\text { var. } \\
\text { c. } \\
\text { sen } 1\end{array}$ & & $\begin{array}{r}\text { spec } \\
(A\end{array}$ & $\begin{array}{l}\text { c- env } \\
\text { Achse }\end{array}$ & $\begin{array}{l}\text { v. corr } \\
\text { en } 1-4\end{array}$ & & $\begin{array}{r}\text { cum. } \\
\text { of sp } \\
\text { rel } \\
\text { (Ach }\end{array}$ & $\begin{array}{l}\text { var. } \\
\text { env. } \\
\text { n } \\
1-2)\end{array}$ \\
\hline & Arten & Daten & $\lambda 1 \quad \lambda 2 \quad \lambda 3 \quad \lambda 4$ & & 1 & 2 & 3 & 4 & 1 & 2 & 3 & 4 & 1 & 2 \\
\hline$C$ & 159 & binä1 & 252.154 .123 .110 & 7.173 & 3.5 & 5.7 & 7.4 & 8.9 & .878 & .860 & .859 & .827 & 23 & 48. \\
\hline $\mathrm{C}$ & 159 & ln Dichte & .294 .148 .118 .100 & 6.134 & 4.8 & 7.2 & 9.1 & 10.8 & .893 & .847 & .845 & .837 & 35 & 53.2 \\
\hline A & 70 & ln Dichte & $\mid$\begin{tabular}{|ccc}
.061 .061 .095 & .061
\end{tabular} & 3.149 & 8.1 & 11.2 & 13.7 & 15.7 & .871 & .787 & .791 & .793 & 4 & 62. \\
\hline & 46 & binär & |049. 060. 060. 178 & 2.295 & 7.8 & 10.4 & 13.0 & 15.1 & .835 & .743 & .742 & .683 & 4 & 59.5 \\
\hline & 46 & In Dic & \begin{tabular}{|ccc}
.079 .064 .043 \\
\end{tabular} & 2.202 & 10.9 & 14.5 & 17.4 & 19.4 & .863 & .800 & .754 & .733 & 5. & 67.7 \\
\hline$D$ & 159 & binär & 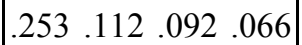 & 7.173 & 3.5 & 5.1 & 6.4 & 7.3 & .881 & .778 & .829 & .635 & 22 & 33.7 \\
\hline 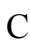 & 159 & ln Dichte & |057. 078. & 6.134 & 4.8 & 6.4 & 7.7 & 8.6 & .895 & .773 & .767 & .712 & 27 & 38.0 \\
\hline$C$ & 46 & binär & |033. 180.058 .045$. & 2.295 & 7.8 & 10.4 & 12.3 & 13.7 & .837 & .713 & .678 & .757 & & 49.6 \\
\hline & 46 & ln Dichte & 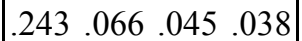 & 2.202 & 11.0 & 14.0 & 16.1 & 17.8 & .865 & .844 & .671 & .755 & 43.6 & 57.0 \\
\hline
\end{tabular}

Der Eigenwert der ersten Ordinationsachse belief sich auf 0,241, wohingegen den übrigen Achsen mit Werten zwischen 0,079 und 0,043 eine deutlich geringere Bedeutung zukam. Anhand der gemessenen Variablen ließ sich ein Faktorengradient fast ausschließlich entlang der ersten Achse ausmachen. 19,4\% der Gesamtvarianz der Arten konnten durch die vier Achsen der CCA erklärt werden. Eine Gegenüberstellung der Ergebnisse der CCA und der CA zeigt, dass die gemessenen Variablen geeignet waren, einen Beitrag zur Erklärung der Streuung in der Vergesellschaftung der Arten zu leisten. 


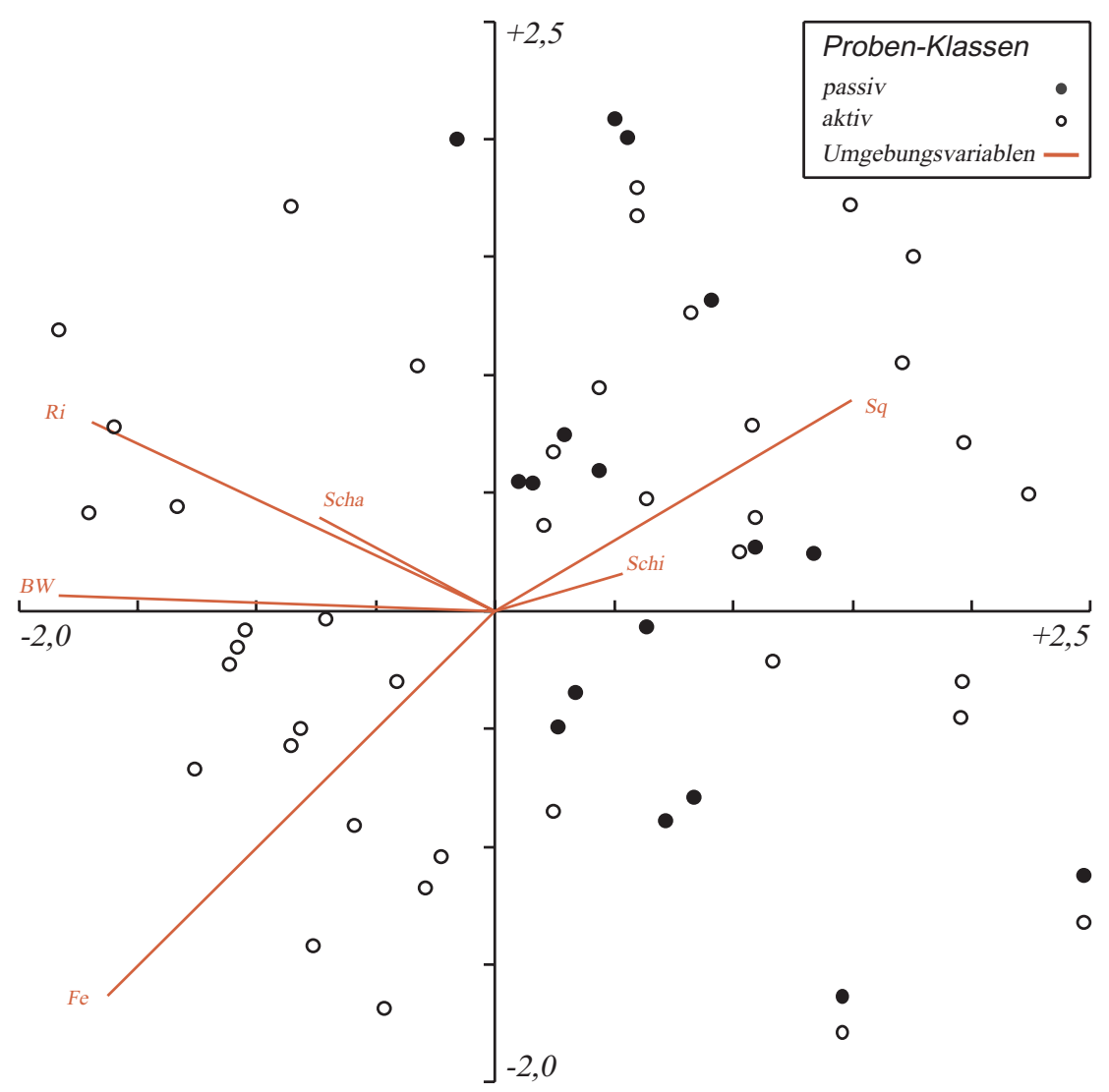

Abb. 4-48: Ordinationsdiagramm (CCA): EFs-Proben an stehendem Buchentotholz und Habitatvariablen

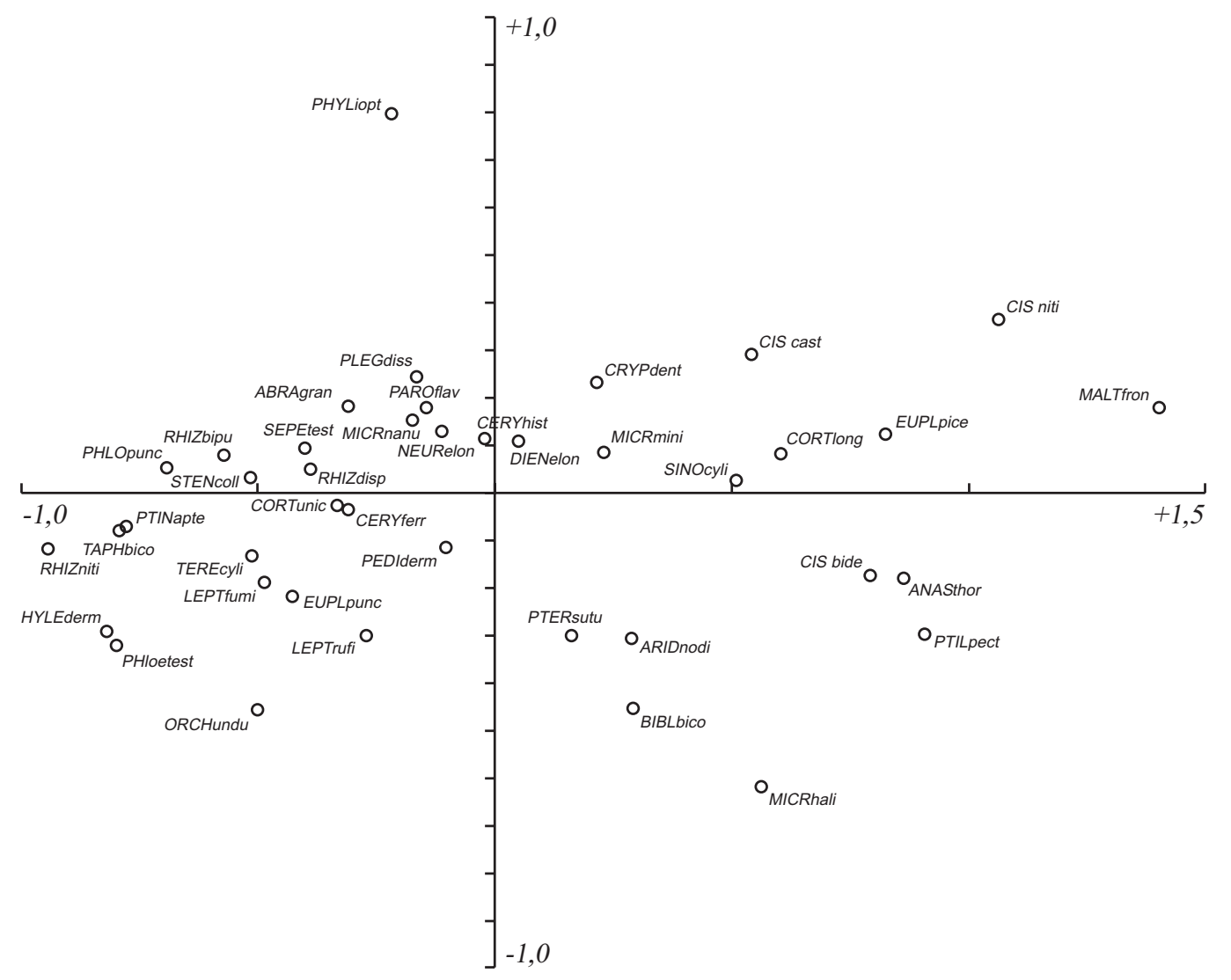

Abb. 4-49: Ordinationsdiagramm (CCA): Arten aus EFs-Proben an stehendem Buchentotholz 
Die erste Ordinationsachse war am engsten mit den Faktoren Bohrwiderstand, Rindenklasse, Feuchtegehalt und Saugfähigkeitsquotient korreliert (Tab. 4-68). Die höchsten Anteile zur Erklärung der Artenvergesellschaftungen lieferten die Variablen Bohrwiderstand und Feuchtegehalt, gefolgt von Berindung und Saugfähigkeitsquotient. Um die Bedeutung von Faktorkombinationen $\mathrm{zu}$ prüfen, wurden Korrelationen zwischen diesen untersucht (Tab. 4-69). Die Variablen Bohrwiderstand, Feuchte und Berindung waren miteinander positiv, der Faktor Saugfähigkeit mit den zuvorgenannten negativ korreliert. Als Ergebnis einer 'forward selection' ergab sich als günstigste Kombination eine Rangfolge von Bohrwiderstand, Rindenklasse, Feuchte, Beschattung, Saugfähigkeitsquotient, Beschirmung und Exposition.

Tabelle 4-68: Kanonische Koeffizienten und Korelationskoeffizienten

\begin{tabular}{|l|cc|cc|}
\hline & \multicolumn{2}{|c|}{ Kanonischer Koeffizient } & \multicolumn{2}{c|}{ Korrelationskoeffizient } \\
Variable & Achse 1 & Achse 2 & Achse 1 & Achse 2 \\
\hline Ri & $-0,61$ & $+0,51$ & $-0,74$ & $+0,32$ \\
Bw & $-0,41$ & $+0,52$ & $-0,80$ & $+0,03$ \\
Sq & $+0,09$ & $+0,33$ & $+0,65$ & $+0,35$ \\
Fe & $-0,32$ & $-1,03$ & $-0,71$ & $-0,64$ \\
Schi & $+0,04$ & $+0,09$ & $+0,23$ & $+0,06$ \\
Scha & $-0,25$ & $+0,53$ & $-0,32$ & $+0,16$ \\
\hline
\end{tabular}

Tabelle 4-69: Korrelationen zwischen den Variablen

\begin{tabular}{|c|c|c|c|c|c|}
\hline Variable & Bw & $\mathrm{Sq}$ & $\mathrm{Fe}$ & Schi & Scha \\
\hline $\mathbf{R i}$ & \multirow[t]{5}{*}{$+0,38$} & $-0,27$ & $+0,20$ & $-0,36$ & $-0,09$ \\
\hline Bw & & $-0,49$ & $+0,57$ & $-0,21$ & $+0,15$ \\
\hline Sq & & & $-0,61$ & $+0,02$ & $-0,37$ \\
\hline $\mathbf{F e}$ & & & & $-0,05$ & $+0,30$ \\
\hline Schi & & & & & $+0,39$ \\
\hline
\end{tabular}

In der linken Hälfte des Ordinationsdiagramms (Abb. 4-48) sind fast ausschließlich Proben zu finden, die an noch vollständig berindeten Stammabschnitten genommen wurden und den höheren Bw-Klassen zuzuordnen waren. Zudem wurden für sie in den meisten Fällen hohe Fe- und niedrige SQ-Werte festgestellt. Proben mit niedrigem Rindenprozent, geringem Bohrwiderstand und Feuchtegehalt sind dagegen in erster Linie in der rechten Diagrammhälfte positioniert. Während die Proben der rechten unteren Hälfte fast vollständig der BwKlasse 1 zuzuordnen waren, finden sich in der rechten oberen Hälfte vermehrt auch Proben der Bw-Klassen 2 und 3.

Die überwiegende Anzahl der Proben entfiel auf Stammabschnitte der Ri-Klasse 4. Hier wurde auch die höchste Arten- und Individuenzahl erfaßt. Mit 783 Ind. $/ \mathrm{m}^{2}$ war die Schlüpfdichte jedoch geringer als in den übrigen Ri-Klassen. Die höchste Dichte wurde für Proben der Ri-Klasse 3 registriert. Eine nicht geringe Anzahl der berindeten Stammabschnitte wies 
zugleich einen nur geringen Bohrwiderstand auf. So entfielen ca. $50 \%$ der Proben auf die Bw-Klassen 1 und 2, während die Klasse 6 nur einen geringen Anteil hatte. In letzterer wurde eine relativ geringe Artenzahl und Schlüpfdichte festgestellt, während die höchsten Werte auf Proben der Bw-Klasse 2 entfielen (Tab. 4-70).

Tabelle 4-70: Probenumfang, Arten- und Individuenzahl einzelner Bw-, Ri- und Fe-Klassen

\begin{tabular}{|c|c|c|c|c|c|c|c|c|c|c|c|c|c|c|c|}
\hline & \multicolumn{6}{|c|}{ Bohrwiderstandsklasse } & \multicolumn{4}{|c|}{ Rindenklasse } & \multicolumn{5}{|c|}{ Feuchteklasse } \\
\hline & & $6 \mathrm{Br}$ & Bw & Bw & 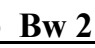 & 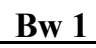 & Ri 4 & Ri 3 & Ri 2 & Ri 1 & Fe 4 & $4 \mathrm{Fe} 3$ & Fe 2 & Fe 1 & $\mathbf{0}$ \\
\hline & 4 & 2 & 8 & 10 & 1 & 27 & 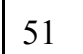 & 0 & & 10 & 7 & דת & 18 & -1 & 10 \\
\hline Mantelfl. $\left(\mathrm{dm}^{2}\right)$ & 71 & 243 & 324 & 174 & 348 & 431 & 943 & 129 & 215 & 285 & 120 & 616 & 218 & 375 & 262 \\
\hline Volumen $\left(\mathrm{dm}^{3}\right)$ & 136 & 441 & 626 & 315 & 665 & 761 & 1774 & 250 & 360 & 528 & 195 & 1159 & 379 & 685 & 525 \\
\hline Rindenfl. $\left(\mathrm{dm}^{2}\right)$ & 71 & 243 & 275 & 126 & 259 & 143 & 013 & 98 & 77 & 0 & 75 & 600 & 159 & 251 & 33 \\
\hline & 100 & 100 & 85 & 73 & 74 & 33 & 100 & 76 & 36 & 0 & 02 & 98 & 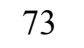 & 01 & 12 \\
\hline & 315 & 1917 & 2524 & 1003 & 4024 & 3686 & 7384 & 1369 & 2169 & 2468 & 836 & 4783 & 1571 & 4597 & 1682 \\
\hline Ind. $/ \mathbf{m}^{2}$ & 444 & 789 & 780 & 577 & 1156 & 855 & 783 & 1059 & 1008 & 867 & 698 & 777 & 721 & 1225 & 642 \\
\hline Arten & 57 & 89 & 86 & 64 & 98 & 81 & 137 & 60 & 74 & 82 & 46 & 128 & 79 & 72 & 77 \\
\hline
\end{tabular}

Den größten Anteil am Artenspektrum hatten die Zoophagen (Tab. 4-71). Auf sie entfielen in fast allen Bw-, Ri- und Fe-Klassen ca. $50 \%$ des Arteninventars. An den gefangenen Individuen waren die Räuber insgesamt mit 31,8 \% beteiligt. Ihr Individuenanteil in den einzelnen Faktorenklassen wies starke Schwankungen auf. An vollständig berindeten Stammabschnitten war dieser Konsumententyp mit 46,0 \% dominierend, während in den übrigen Ri-Klassen nur Anteile von 11,9 bis $16,7 \%$ registriert wurden. Mit 359,9 Ind. $/ \mathrm{m}^{2}$ lag die beobachtete Schlüpfdichte an den vollständig berindeten Stammabschnitten um etwa das dreifache höher als in Proben der Ri-Klasse 2. Dominierend waren die Räuber auch in den Stammabschnitten der Bw-Klassen 6, 5 und 3. Mit 496,1 Ind. $/ \mathrm{m}^{2}$ war die höchste Dichte für die Bw-Klasse $5 \mathrm{zu}$ verzeichnen, in den Klassen 4 bis 2 wurden in etwa übereinstimmende Werte registriert, während das Minimum mit nur 62,4 Ind. $/ \mathrm{m}^{2}$ und einem Individuenanteil von 7,3\% auf die Bw-Klasse 1 entfiel.

Mit 365,1 Ind. $/ \mathrm{m}^{2}$ und einem Individuenanteil von 43,1 \% waren die 50 mycetophagen Arten vorherrschend. Die höchste Artenzahl wurde in Proben der Ri-Klasse 4, die maximale Schlüpfdichte (719,8 Ind. $\left./ \mathrm{m}^{2}\right)$ und der höchste Individuenanteil (71,4 \%) in Stammabschnitten der Ri-Klasse 2 verzeichnet. Der Dominanzwert in den einzelnen Bw-Klassen variierte zwischen $27,5 \%$ in der Klasse 5 und $54,6 \%$ in der Klasse 2. In letzterer wurde mit 631,6 Ind. $/ \mathrm{m}^{2}$ die höchste, in der Bw-Klasse 6 mit 193,0 Ind. $/ \mathrm{m}^{2}$ die niedrigste Schlüpfdichte festgestellt.

Die xylophagen Arten erreichten mit 23,0 \% einen deutlich höheren Individuenanteil als an liegendem Buchentotholz. Die höchsten Dominanzwerte und Schlüpfdichten wurden in Proben der Ri-Klassen 3 und 1 gemessen. Mit sinkendem Bohrwiderstand erreichte dieser Konsumententyp einen steigenden Anteil am Fangergebnis. Nur die 10 Proben der Bw-Klasse 3 machten hiervon eine Ausnahme. Mit nur 10,4 Ind. $/ \mathrm{m}^{2}$ belief sich der Individuenanteil hier auf lediglich 1,8\%. Die höchsten Werte wurden mit einem Dominanzwert von 57,1\% und einer Schlüpfdichte von 488,1 Ind./ $\mathrm{m}^{2}$ in Proben der Bw-Klasse 1 verzeichnet. 
Tabelle 4-71: Anteile der Konsumententypen in den Bw-, Ri- und Fe-Klassen

\begin{tabular}{|c|c|c|c|c|c|c|c|c|c|c|c|c|c|c|c|c|}
\hline \multirow{2}{*}{\multicolumn{2}{|c|}{ Kon.typ }} & \multirow{2}{*}{\multicolumn{6}{|c|}{\begin{tabular}{|c|} 
Bohrwiderstandsklasse \\
Bw 6 Bw 5 Bw 4 Bw 3 Bw 2 Bw 1
\end{tabular}}} & \multicolumn{4}{|c|}{ Rindenklasse } & \multicolumn{4}{|c|}{ Feuchteklasse } & \multirow{3}{*}{\begin{tabular}{|l|} 
ges. \\
8
\end{tabular}} \\
\hline & & & & & & & & \multicolumn{4}{|c|}{ Ri 4 Ri 3 Ri 2 Ri 1} & \multicolumn{4}{|c|}{ Fe 4 Fe 3 Fe 2 Fe 1} & \\
\hline $\mathbf{A}$ & - & 2 & 6 & 2 & 5 & 4 & 3 & 7 & 2 & 3 & 4 & 1 & 6 & 3 & & \\
\hline $\mathbf{R}$ & $\mathbf{m}$ & 19 & 22 & 23 & 15 & 28 & 23 & 42 & 18 & 20 & 22 & 12 & 39 & 23 & 21 & 50 \\
\hline $\mathbf{T}$ & $\mathbf{p}$ & 1 & 4 & 3 & 1 & 2 & 4 & 5 & 1 & 2 & 3 & 0 & - & & 2 & 7 \\
\hline $\mathbf{E}$ & $\mathbf{s}$ & 1 & 4 & 2 & 1 & 2 & 0 & 6 & 0 & 2 & 1 & 1 & 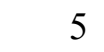 & 1 & 1 & 9 \\
\hline \multirow[t]{2}{*}{$\mathbf{N}$} & $\mathbf{x}$ & 4 & 6 & 11 & 8 & 11 & 15 & 14 & 8 & 12 & 13 & 8 & 16 & 8 & 10 & 23 \\
\hline & $\mathbf{z}$ & 30 & 47 & 45 & 34 & 51 & 36 & 63 & 31 & 35 & 39 & 24 & 57 & 43 & 35 & 76 \\
\hline D & - & & 1 & 2,1 & 7,6 & 1,0 & 0,3 & 2,4 & 1,6 & ,5 & 0,8 &, 1 & ,8 & 0,4 & 0,3 & 1,7 \\
\hline $\mathbf{O}$ & m & 43,5 & 27,5 & 49,8 & 39,2 & 54,6 & 35,1 & 41,4 & 27,0 & 71,4 & 32,8 & 58,6 & 31,5 & 51,4 & 56,5 & 43,1 \\
\hline $\mathbf{M}$ & p & ,6 & 0,3 & 0,2 & 0,1 & 0,1 & 0,2 & 0,2 & 0,2 & 0,2 & 0,2 & 0,0 & 0,3 & 0,1 & 0,1 & 0,2 \\
\hline I & $\mathbf{S}$ & 0,3 & 0,5 & 0,1 & 0,2 & 0,2 & 0,0 & 0,1 & 0,0 & 0,1 & 0,4 & 0,7 & 0,1 & 0,1 & 0,0 & 0,2 \\
\hline \multirow[t]{2}{*}{ N. } & $\mathbf{x}$ & 2,2 & 6,7 & 8,1 & 1,8 & 15,7 & 57,1 & 9,9 & 57,2 & 15,9 & 49,1 & 28,0 & 6,0 & 30,3 & 29,1 & 23,0 \\
\hline & $\mathbf{z}$ & 49,5 & 62,9 & 39,7 & 51,1 & 28,4 & 7,3 & 46,0 & 14,0 & 11,9 & 16,7 & 12,6 & 58,4 & 17,8 & 13,9 & 31,8 \\
\hline I & - & & 16,5 & 16,7 & 43,7 & 2 & 2,3 & 18,9 & 17,0 & 4,6 & 7,0 & 0,8 & 29,6 & 2,8 & 4,0 & 14, \\
\hline $\mathbf{N}$ & $\mathbf{m}$ & 193,0 & 216,8 & 388,9 & 226,1 & 631,6 & 300,3 & 324,2 & $285,4^{\prime}$ & 19,8 & 84,2 & 409,0 & $244,6=$ & 70,4 & 92,0 & 365,1 \\
\hline D. & p & 2,8 & 2,5 & 1,5 & 0,6 & 1,7 & 1,6 & 1 & 2,3 & 1,9 & 1,8 & 0,0 & 1,9 & 0,5 & 1,6 & 1,7 \\
\hline I & $\mathbf{s}$ & 1,4 & 3,7 & 0,6 & 1,2 & 2,6 & 0,0 & 1,2 & 0,0 & 0,9 & 3,5 & 5,0 & 0,8 & 0,5 & 0,3 & 1,4 \\
\hline $\mathbf{q}$ & $\mathbf{x}$ & 9,9 & 53,1 & 63,1 & 10,4 & 181,3 & 488,1 & 77,4 & 605,6 & 160,3 & 425,4 & 195,3 & 46,42 & 218,4 & 356,2 & 194,5 \\
\hline $\mathbf{m}$ & $\mathbf{z}$ & 219,7 & 496,1 & 309,4 & 295,2 & 327,9 & 62,4 & 359,9 & 148,5 & 120,4 & 145,1 & 87,6 & 453,4 & 128,5 & 170,8 & 269,4 \\
\hline
\end{tabular}

Ein Viertel der an stehendem Buchentotholz gefangenen Tiere wurde von 37 corticolen Arten gestellt (Tab. 4-72). Mit einem Individuenanteil von 39,1 \% und einer Schlüpfdichte von 305,9 Ind. $/ \mathrm{m}^{2}$ waren sie die dominierende Gruppe an Stammteilen der Ri-Klasse 4, während in den übrigen Ri-Klassen nur Dichten von 74,1 bis 97,4 Ind. $/ \mathrm{m}^{2}$ und Dominanzwerte von 7,6 bis 9,2 \% festgestellt wurden. In Proben der Bw-Klasse 5 hatten die Rindenbewohner einen Anteil von 59,9\% am Fangergebnis, die niedrigsten Werte waren für die Klassen 2 und 1 festzustellen. Im Ordinationsdiagramm der Abbildung 4-49 sind die corticolen Arten in ihrer Mehrheit in der linken Hälfte, vor allem im unteren Sektor, positioniert. Häufigster Vertreter der Rindenkäfer war Rhizophagus dispar. Die meisten Exemplare von diesem Räuber wurden in Proben der Bw-Klassen 5 bis 2 gefangen. 88 \% der Individuen stammten aus Proben der Ri-Klasse 4, aber auch in den Stammabschnitten der Ri-Klassen 3 und 1 zählte er zu den Hauptarten (Tab. 4-73). Der Borkenkäfer Taphrorychus bicolor wurde vor allem in Proben der Ri-Klasse 4, der Fe-Klasse 3 und den Bw-Klassen 5 und 4 mit der höchsten Schlüpfdichte in dem festeren Material, wo auch der höchste Dominanzwert registriert wurde, gefangen. Auch Rhizophagus nitidulus und Rh. bipustulatus waren in erster Linie an festem, berindeten und relativ feuchtem Material zu beobachten. Letztere war in Proben der Bw-Klasse 5 mit einem Individuenanteil von 12,3 \% die dritthäufigste Art. Mit 466 Exemplaren war Paromalus flavicornis der zweithäufigste Rindenkäfer am stehenden Buchentotholz. Die höchste Schlüpfdichte und der größte Dominanzwert war an berindeten Stammabschnitten der Bw-Klasse 4 bzw. der Fe-Klasse 3 festzustellen. Auch Corticeus unicolor hatte seinen Verteilungsschwerpunkt in Proben der Fe-Klasse 3. Die höchste Dichte wurde in 
Proben der Bw-Klasse 4 festgestellt. Mit Ausnahme der Klasse 1 trat er aber auch in den übrigen Bw-Klassen als Hauptart in Erscheinung. Von den 39 Proben, in denen die Art festgestellt wurde, gehörten $67 \%$ zur Ri-Klasse 4, aus denen $63 \%$ der insgesamt 375 Individuen schlüpften. $31 \%$ der Individuen wurden hingegen an Stammabschnitten der Ri-Klassen 1 und 2 gefangen, wo die spezifische Dichte sogar die höchsten Werte erreichte.

Tabelle 4-72: Anteile der Habitatpräferenztypen in den Bw-, Ri- und Fe-Klassen

\begin{tabular}{|c|c|c|c|c|c|c|c|c|c|c|c|c|c|c|c|c|}
\hline & & & Bohry & vid & tas & klasse & & & Rinden & klasse & & & euchte & eklasse & & \\
\hline & Habitat & Bw 6 & Bw 5 & B & BW 3 & B & Bw 1 & Ri 4 & Ri 3 & Ri 2 & Ri 1 & $\mathrm{Fe} 4$ & $\mathrm{Fe} 3$ & $\mathrm{Fe} 2$ & Fe 1 & ges. \\
\hline $\mathbf{A}$ & B & 4 & 7 & 7 & 5 & 8 & 4 & 9 & 4 & 6 & 8 & 1 & 0 & 9 & 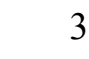 & 15 \\
\hline $\mathbf{R}$ & $\mathbf{E}$ & 9 & 8 & 6 & 4 & 5 & 7 & 11 & 3 & 6 & 6 & 5 & 8 & 9 & & 13 \\
\hline $\mathbf{T}$ & $\mathbf{F}$ & 0 & 4 & 0 & 0 & 1 & 0 & 5 & 0 & 0 & 0 & 0 & 4 & 0 & & 5 \\
\hline 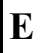 & $\mathbf{N}$ & 1 & 1 & 2 & 1 & 2 & 0 & 4 & 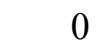 & 1 & 1 & 1 & 0 & 0 & & 6 \\
\hline $\mathbf{N}$ & $\mathbf{P}$ & 0 & 2 & 1 & 0 & 2 & 3 & 3 & - & 0 & -1 & 1 & 3 & 1 & & 4 \\
\hline$Z$ & $\mathbf{T}$ & 3 & 7 & 12 & 8 & 11 & 11 & 13 & 8 & 10 & 12 & 4 & 14 & 7 & 10 & 20 \\
\hline $\mathbf{A}$ & $\mathbf{T M}$ & 16 & 20 & 23 & 18 & 27 & 29 & 28 & 20 & 23 & 27 & 16 & 26 & 23 & 24 & 36 \\
\hline $\mathbf{H}$ & $\mathbf{T P}$ & 6 & 6 & 10 & 7 & 15 & 10 & 20 & 9 & 9 & 11 & 4 & 19 & 11 & 0 & 25 \\
\hline $\mathrm{L}$ & TR & 15 & 26 & 19 & 18 & 24 & 11 & 34 & 13 & 15 & 9 & 13 & 33 & 15 & 14 & 37 \\
\hline & $\mathbf{V}$ & 3 & 8 & 6 & 3 & 3 & 6 & 10 & 2 & 4 & 5 & 1 & 10 & 4 & 4 & 12 \\
\hline D & B & 8,3 & 1,9 & 0,8 & 1,5 & 0,6 & 0,8 & 1,4 & 0,5 & 0,5 & 1,2 & 0,1 & 1,6 & 1,7 & 0,3 & 1,1 \\
\hline $\mathbf{O}$ & $\mathbf{E}$ & 17,8 & 5,3 & 11,6 & 6,4 & 11,7 & 6,1 & 8,4 & 7,9 & 14,5 & 6,4 & 4,2 & 7,3 & 13,2 & 9,2 & 9,0 \\
\hline M & $\mathbf{F}$ & 0,0 & 0,2 & 0,0 & 0,0 & 0,0 & 0,0 & 0,1 & 0,0 & 0,0 & 0,0 & 0,0 & 0,1 & 0,0 & 0,0 & 0,0 \\
\hline I & $\mathbf{N}$ & 0,3 & 0,3 & 0,1 & 0,2 & 0,2 & 0,0 & 0,1 & 0,0 & 0,0 & 0,4 & 0,7 & 0,1 & 0,0 & 0,0 & 0,1 \\
\hline $\mathbf{N}$ & $\mathbf{P}$ & 0 & 0,3 & 0,0 & 0,0 & 0,1 & 0,2 & 0,1 & 0,1 & 0,0 & 0,3 & 0,5 & 0,1 & 0,1 & 0,0 & 0,1 \\
\hline $\mathbf{A}$ & $\mathbf{T}$ & 10,5 & 6,5 & 8,4 & 3,7 & 16,9 & 56,9 & 11,0 & 57,4 & 15,7 & 49,1 & 27,2 & 8,0 & 29,4 & 29,0 & 23,7 \\
\hline $\mathbf{N}$ & TN & 26,0 & 23,9 & 38,0 & 41,9 & 34,7 & 30,3 & 30,3 & 17,0 & 53,5 & 31,4 & 54,4 & 27,2 & 35,8 & 38,4 & 32,9 \\
\hline $\mathbf{Z}$ & TP & 4,8 & 0,7 & 6,5 & 2,2 & 16,1 & 3,1 & 9,0 & 7,1 & 7,8 & 1,9 & 2,4 & 1,9 & 9,5 & 14,4 & 7,2 \\
\hline & TR & 30,2 & 59,9 & 34,2 & 43,1 & 19,3 & 2,2 & 39,1 & 9,2 & 7,6 & 8,5 & 10,4 & 52,9 & 10,1 & 8,4 & 25,2 \\
\hline & $\mathbf{V}$ & 2,2 & 1,0 & 0,6 & 1,1 & 0,4 & 0,4 & 0,6 & 0,8 & 0,4 & 0,8 & 0,1 & 0,9 & 0,3 & 0,2 & 0,6 \\
\hline I & B & 36,6 & 14,8 & 5,9 & 8,6 & 6,6 & & 10,7 & 5,4 & 4,6 & 10,2 & 0,8 & 12,3 & 11,9 & 3,7 & 9,3 \\
\hline $\mathbf{N}$ & $\mathbf{E}$ & 78,9 & 42,0 & 90,6 & 36,81 & 134,8 & 52,4 & 65,5 & 83,51 & 145,9 & 55,5 & 29,2 & 56,8 & 95,0 & 113,2 & 76,1 \\
\hline D. & $\mathbf{F}$ & 0,0 & 1,6 & 0,0 & 0,0 & 0,3 & 0,0 & 0,5 & 0,0 & 0,0 & 0,0 & 0,0 & 0,6 & 0,0 & 0,3 & 0,3 \\
\hline$/$ & $\mathbf{N}$ & 1,4 & 2,5 & 0,6 & 1,2 & 2,6 & 0,0 & 1,0 & 0,0 & 0,5 & 3,5 & 5,0 & 0,5 & 0,0 & 0,3 & 1,3 \\
\hline $\mathbf{q}$ & $\mathbf{P}$ & 0,0 & 2,1 & 0,3 & 0,0 & 0,9 & 1,6 & 0,7 & 0,8 & 0,0 & 2,8 & 3,3 & 1,0 & 0,5 & 0,3 & 1,0 \\
\hline $\mathbf{m}$ & $\mathbf{T}$ & 46,5 & 51,4 & 65,2 & 21,31 & 196,0 & 486,7 & 86,1 & 607,91 & 158,54 & 425,7 & 189,5 & 62,42 & 212,03 & 54,9 & 200,4 \\
\hline & TM & 115,51 & 188,4 & 296,1 & 241,7 & 400,9 & 259,0 & 237,4 & 180,25 & 539,52 & 272,2 & 379,8 & 210,92 & $257,9<$ & 470,0 & 278,5 \\
\hline & TP & 21,1 & 5,3 & 50,4 & 12,7 & 186,2 & 26,7 & 70,4 & 75,0 & 78,5 & 16,2 & 16,7 & 14,5 & 68,8 & 176,7 & 61,4 \\
\hline & TR & 133,84 & 472,2 & 266,5 & 248,62 & 223,0 & 18,5 & 305,9 & 97,4 & 76,7 & 74,1 & 72,6 & 411,0 & 72,5 & 103,1 & 213,3 \\
\hline & $\mathbf{V}$ & 9,9 & 8,2 & 4,6 & 6,3 & 5,2 & 3,0 & 4,7 & 8,5 & 3,7 & 6,7 & 0,8 & 6,7 & 2,3 & 2,4 & 5,3 \\
\hline
\end{tabular}

In hoher Artenzahl traten auch die Mulmbewohner in Erscheinung, hinsichtlich der Individuenzahlen war dieser Präferenztyp sogar vorherrschend. Die höchste Schlüpfdichte wurde mit 539,5 Ind. $/ \mathrm{m}^{2}$ in den Proben der Ri-Klasse 2 gemessen, der Individuenanteil erreichte hier einen Wert von 53,5 \%. Die Dominanzwerte waren an Stammabschnitten der Bw-Klassen 4 
und 3, die Dichte an solchen der Klasse 2 am höchsten. Bestimmt wurde das Bild vor allem von Corticaria longicollis, der in 78 Proben mit insgesamt 2649 Individuen auftrat. Sämtliche Proben der Ri-Klassen 1 bis 3 und $75 \%$ derjenigen der Klasse 4 waren besiedelt. Die höchste Dichte wurde in Proben der Ri-Klasse 2 registriert, in denen sich der Dominanzwert auf über $50 \%$ belief. Aber auch in den übrigen Ri-Klassen erreichte C. longicollis den Status einer eudominanten Art. Nur eine sehr geringe Individuenzahl war in Stammabschnitten der BwKlassen 6 und 5 festzustellen, während sie in den Klassen 4 bis 2 die häufigste und in der Klasse 1 die zweithäufigste Art war. 67,6 \% der Individuen stammten aus Proben der BwKlassen 1 und 2, in letzteren war die höchste Schlüpfdichte zu verzeichnen. Ein Verteilungsschwerpunkt zeigte sich für Stammabschnitte der Fe-Klasse 1. Hier wurden $60 \%$ der Individuen und die max. Schlüpfdichte registriert. Ptinella aptera wurde fast ausschließlich in Proben der Ri-Klasse 4 festgestellt. Hier wurden 99,3\% der insgesamt 535 Individuen erfaßt und somit ein Dominanzwert von 7,2\% erreicht. Mehrheitlich wurde dieser Ptilidae in feuchteren Stammabschnitten der Bw-Klassen 5 und 4 nachgewiesen. In den Bw-Klassen 6 bis 4 und den Fe-Klassen 3 und 4 wurde der Status einer Hauptart erreicht. Ebenfalls in ihrer Mehrheit wurde Cerylon ferrugineum in Proben der Ri-Klasse 4 gefangen. 4,8 \% der auf diese Faktorenklasse entfallenden Fänge wurden von diesem Mulmbewohner gestellt. Im Vergleich zu Ptinella aptera wurde C. ferrugineum mit größerer Regelmäßigkeit auch an trockeneren Stammabschnitten festgestellt, die größte Individuenzahl und Schlüpfdichte war aber ebenfalls in Proben der Fe-Klasse 3 zu beobachten. Ein Verteilungsschwerpunkt ergab sich für die mittleren Bw-Klassen, die maximale Dichte entfiel auf die Klasse 3, für die auch der höchste Anteil am Gesamtfang registriert wurde. Aber auch in den Bw-Klassen 6 bis 4 zählte C. ferrugineum zu den Hauptarten. Als eine Art mit einem Verteilungsschwerpunkt in Stammteilen mit geringem Bohrwiderstand und weitestgehend fehlender Berindung zeigte sich Micridium halidaii. Von den 278 Exemplaren entfielen 84,5 bzw. 80,0\% auf diese beiden Klassen. $75,5 \%$ der Individuen waren in Proben der Fe-Klasse 4 zu finden, mit einem Dominanzwert von über $25 \%$ war M. halidaii die häufigste Art dieser Klasse.

Die lignicolen Arten waren mit einem Individuenanteil von 23,7 \% am Fangergebnis beteiligt. Hohe Schlüpfdichten wurden in den Proben der Ri-Klassen 3 und 1 mit 607,9 bzw. 425,7 Ind. $/ \mathrm{m}^{2}$ registriert. Die Dominanzwerte beliefen sich hier auf 57,4 bzw. 49,1\%. Deutlich niedriger lagen die Werte für die Ri-Klasse 4 mit 86,1 Ind. $/ \mathrm{m}^{2}$ und einem Individuenanteil von $11,0 \%$. In der Tendenz zeichnete sich eine steigende Schlüpfdichte der lignicolen Arten mit sinkendem Bohrwiderstand ab. Eine Ausnahme machten lediglich Proben der Bw-Klasse 3 mit der niedrigsten festgestellten Dichte von 21,3 Ind. $/ \mathrm{m}^{2}$ und einem Individuenanteil von nur 3,7 \%. Dagegen wurden an Stammabschnitten der Bw-Klasse 1 486,7 Ind./m² festgestellt, die mit 56,9 \% am Fangergebnis beteiligt waren. Mit einem Individuenanteil von 19,9 \% war Ptilinus pectinicornis als Vertreter dieses Präferenztyps die häufigste Art am stehenden Buchentotholz. Die höchsten Dichten waren in Proben der Ri-Klassen 3 und 1 zu beobachten, in denen mit Anteilen von 55,3 bzw. 44,0 auch die höchsten Dominanzwerte zu registrieren waren. Aber auch in den beiden übrigen Ri-Klassen hatte diese Art einen hohen Anteil am Gesamtfang. In der Tendenz trat dieser Anobiidae mit größerer Regelmäßigkeit bei sinkendem Bohrwiderstand auf. 72,4 \% der 2681 gefangenen Exemplare schlüpften aus Stammabschnitten der Bw-Klasse 1, weitere 21,7 \% der Klasse 2. Die Dominanzwerte betrugen hier 52,6 bzw. 14,5 \%. Nur ein geringer Anteil der Fänge entfiel auf die Fe-Klassen 3 und 4, die höchste Dichte auf Proben der Fe-Klasse 1. Die maximale in einer Einzelprobe registrierte Dichte wurde mit 3406 Ind. $/ \mathrm{m}^{2}$ an einem Stammabschnitt erfaßt, der nach der dendrochronologischen Analyse seit ca. 14 Jahren abgestorben war und der Ri-Klasse 3, der 
Bw-Klasse 1 und der Fe-Klasse 1 zuzurechnen war. Hylecoetus dermestoides wurde ausschließlich in Proben der Fe-Klasse 3 festgestellt, 98 \% der Individuen schlüpften aus Stammabschnitten der Ri-Klasse 4. In diesen Klassen wurde der Status einer Hauptart erreicht. Dagegen war eine enge Abhängigkeit der Schlüpfdichte vom Bohrwiderstand nicht erkennbar. Anaspis schilskyana erreichte die höchsten Dichten in Proben der Ri-Klassen 1 und 2. In Stammteilen der Bw-Klassen 5 und 6 wurde die Art nicht festgestellt, 56 \% der 75 Exemplare wurden in Proben der Bw-Klasse 1 registriert.

Tabelle 4-73: Dominante Arten in einzelnen Variablenklassen (Ind. \%)

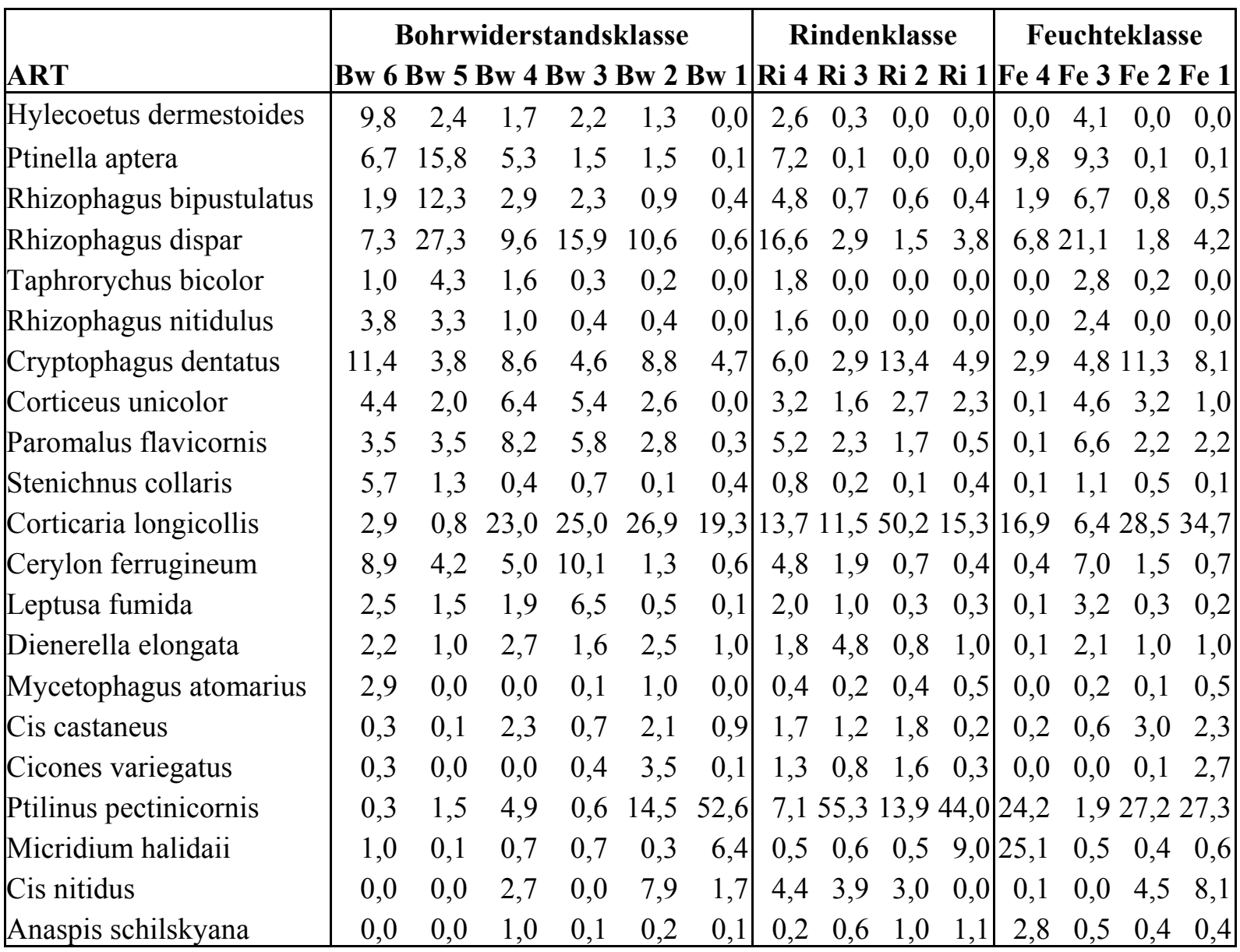

Die Besiedler von Totholzpilzen waren mit 25 Arten in den EFs-Proben vertreten. Sie traten in den Proben der Ri-Klassen 4 bis 2 in Dichten zwischen 70,4 und 78,5 Ind./ $/ \mathrm{m}^{2}$ auf und erreichten damit Individuenanteile zwischen 7,1 und 9,0 \%. Erheblich niedriger Lagen die entsprechenden Werte für die Ri-Klasse 1. Sehr groß waren die Unterschiede zwischen den einzelnen Bw-Klassen. Die geringste Dichte wurde für die Klasse 5 (5,3 Ind. $\left./ \mathrm{m}^{2}\right)$, die höchste für Klasse $2\left(186,2 \mathrm{Ind} . / \mathrm{m}^{2}\right)$ registriert. Entprechend groß waren die Abweichungen bei den Dominanzwerten, die zwischen 0,7 und 16,1\% variierten. Offensichtlich bestand zwischen Bohrwiderstand und Siedungsdichte der Pilzbewohner keine sehr enge Beziehung. Von größerer Bedeutung schien dagegen der Feuchtegehalt des Holzes zu sein. In Proben der Fe-Klassen 1 und 2 wurden mit Dichten von 176,7 bzw. 68,8 Ind. $/ \mathrm{m}^{2}$ und Individuenanteilen von 14,4 bzw 9,5\% deutlich höhere Werte festgestellt als in Proben der Klassen 3 und 4 mit 
Schlüpfdichten von 14,5 bzw. 16,7 Ind./m² und Dominanzwerten von nur 1,9 bzw. 2,4 \%. Als häufigster Vertreter dieser Gruppe wurde Cis nitidus mit 445 Exemplaren in 17 Proben festgestellt. In den Stammabschnitten der Ri-Klassen 2 bis 4 trat er als Hauptart auf, während in solchen der Ri-Klasse 1 fast keine Besiedlung zu beobachten war. Ein deutlicher Schwerpunkt der Verteilung lag in Proben der Fe-Klasse 1. 83,4 \% der Tiere entfielen auf diese Klasse, der Dominanzwert belief sich auf 8,1 \%. Für die Fe-Klasse 2 wurden bereits deutlich geringere Schlüpfdichten gemessen und in Proben der Fe-Klassen 3 und 4 wurde jeweils nur ein einzelnes Individuum gefangen. An festem Material der Bw-Klassen 6 und 5 fehlte $C$. nitidus vollständig, die höchste Schlüpfdichte und der höchste Dominanzwert wurden in der Klasse 2 registriert. Ähnlich stellte sich die Verteilung von Cis castaneus auf die Faktorenklassen dar. Insgesamt wurde er in einer höheren Anzahl von Proben, gleichzeitig aber auch in geringerer Dichte beobachtet. Sinkender Feuchtegehalt ging einher mit einer steigenden Schlüpfdichte. Im Vergleich zur vorherigen Art trat C. castaneus mit einer höheren Regelmäßigkeit auch im etwas feuchteren Material in Erscheinung. 25,3 \% der Individuen wurden in Proben der Fe-Klasse 2, 16,1 \% in solchen der Fe-Klasse 3 gefunden. Auch Cicones variegatus trat bevorzugt in Stämmen mit geringerem Feuchtegehalt auf. 84,9 \% der 146 gefangenen Exemplare schlüpften in Proben der Fe-Klasse 1, der erreichte Dominanzwert belief sich hier auf 2,7 \%. 95,2 \% der Individuen entstammten 9 Proben, die an Stammteilen der Bw-Klasse 2 genommen wurden. Nur an den Fängen dieser Bw-Klasse war C. variegatus als Hauptart beteiligt.

Auch die insgesamt 13 eurytopen Arten wurden in sinkenden Schlüpfdichten mit steigendem Feuchtegehalt der Stammabschnitte festgestellt. Dabei wurde der höchste Individuenanteil in der Fe-Klasse 3 mit 13,2 \% erreicht. Mit sinkendem Rindenprozent stiegen die Dichten zunächst an und erreichten ihr Maximum mit 145,9 Ind. $/ \mathrm{m}^{2}$ in Proben der Ri-Klasse 2, der Individuenanteil belief sich hier auf 14,5\%. Die niedrigste Dichte und der geringste Anteil war in Proben der Klasse 1 mit 55,5 Ind. $/ \mathrm{m}^{2}$ bzw. 6,4 \% zu verzeichnen. Für die einzelnen Bw-Klassen ergab sich ein sehr uneinheitliches Bild. Die Dichte variierte zwischen 36,8 (BwKlasse 3) und 134,8 Ind. $/ \mathrm{m}^{2}$ (Bw-Klasse 2), der Individuenanteil zwischen 5,3 (Bw-Klasse 5) und 17,8 \% (Bw-Klasse 6). Dominierende Art dieser Gruppe war Cryptophagus dentatus, der in allen Ri-, Bw- und Fe-Klassen zu den Hauptarten zählte. Von den 903 Individuen wurden 49,1 \% in Proben der Ri-Klasse 4 und 32,2 \% in Proben der Klasse 2 gefangen. In letzterer wurde mit 13,4 \% der höchste Dominanzwert und die höchste Schlüpfdichte gemessen. $C$. dentatus war in den meisten Proben der Fe-Klassen 1 bis 3 anwesend, nur ein sehr kleiner Teil der Tiere entfiel auf Stammabschnitte der Fe-Klasse 4. Besonders in den trockeneren Stammteilen wurden höhere Dichten registriert. Die Dominanzwerte in den einzelnen BwKlassen variierten zwischen 3,8 (Klasse 5) und 11,4 \% (Klasse 6). Der größte Teil der Fänge wurde dagegen in Proben der Bw-Klassen 4 und 2 erfaßt, in letzterer erreichte die Schlüpfdichte ihren höchsten Wert. Dienerella elongata wurde zu über $80 \%$ in Proben der RiKlassen 4 und 3 gewonnen. Die höchsten Schlüpfdichten und Dominanzwerte wurden für Stammabschnitte der Ri-Klasse 3 und der Fe-Klasse 3 gemessen. Die Verteilung der Individuen auf die Bw-Klassen war der von $C$. dentatus sehr ähnlich. Die höchsten Dichten wurden in den Klassen 4 und 2 registriert.

Die Bodenbewohner traten am stehenden Buchentotholz mit nur 15 Arten und einem Individuenanteil von 1,1 \% auf. Beide Werte lagen deutlich unter den an liegendem Buchentotholz ermittelten Anteilen. 


\subsubsection{Sukzession der Artengemeinschaften an stehendem Eichentotholz}

Die Berechnung der Ähnlichkeiten der Artengemeinschaften ließ in nur wenigen Fällen eine größere Übereinstimmung in der Struktur der Artenvergesellschaftungen sichtbar werden (Tab. 4-74, Anhang 10a und b). Fallengruppen, die sich untereinander durch hohe Ähnlichkeit bei gleichzeitig geringer Übereinstimmung mit den übrigen Proben auszeichneten, waren nicht zu identifizieren. Ein Teil der höheren Ähnlichkeitswerte war auch diesmal darauf zurückzuführen, dass mehrere Proben an den gleichen Stämmen genommen worden waren. Dies gilt z. B. für die Proben 20a-c, 7a-c und 23a-b. Die Proben 22a-c sind Beispiele dafür, dass die Artenvergesellschaftungen an unterschiedlichen Abschnitten des gleichen Stammes sehr unterschiedlich sein können. Die Übergänge in der Vergesellschaftung der Arten waren weitestgehend fließend. Die Reihenfolge der Proben spiegelt in der Tendenz fortschreitende Zersetzungszustände wieder. So sind im linken oberen Teil der Tabelle 4-74 in erster Linie Proben positioniert, die an relativ frischem Material genommen wurden, wie z. B. die Stämme 20, 22 und 23.

Die Daten aus den EFs-Proben wurden verschiedenen Verfahren der Korrespondenzanalyse unterzogen. Die wesentlichen Ergebnisse sind in der Tabelle 4-75 zusammengefaßt. Die Abbildungen 4-50 und 4-51 zeigen die Ordinationsdiagramme einer DCA auf der Grundlage der logarithmierten Schlüpfdichten von 104 Arten. Von diesen ist aus Gründen der Darstellbarkeit nur eine Auswahl wiedergegeben.

Der durch die vier hypothetischen Variablen erklärbare Anteil an der beobachteten Streuung im Datenmaterial betrug in Abhängigkeit von dem gewählten Verfahren und der Berechnungsgrundlage zwischen 16,8 und 35,5\%. Die Eigenwerte der in den Abbildungen 4-50 und 4-51 dargestellten Ordinationsachsen beliefen sich auf 0,464 und 0,334, die der dritten und vierten Achse betrugen 0,281 und 0,248. Entlang der ersten Achse war ein Gradient in Abhängigkeit von der Dauer des Zersetzungsprozesses erkennbar. Proben von relativ frischem Material sind vornehmlich am linken Rand des Diagramms positioniert. Entlang der Achse ist eine Tendenz $\mathrm{zu}$ fortgeschrittener Zersetzungsdauer $\mathrm{zu}$ beobachten. Entsprechend gestaltet sich die Positionierung der Arten. Im linken Bereich sind gehäuft Rindenbewohner und Frischholzbesiedler zu finden, wie z. B. Rhinosimus ruficollis, Dryocoetes villosus, Xyloterus signatus und Rhizophagus dispar. Dagegen treten am rechten Rand Mulmbewohner wie z. B. Aderus oculatus, Cerylon histeroides und Micridium halidaii stärker in Erscheinung. 
Tabelle 4-74: Ähnlichkeitsmatrix $\left(\mathrm{RE}_{\mathrm{ln}}\right)$ der EFs-Proben an stehendem Eichentotholz

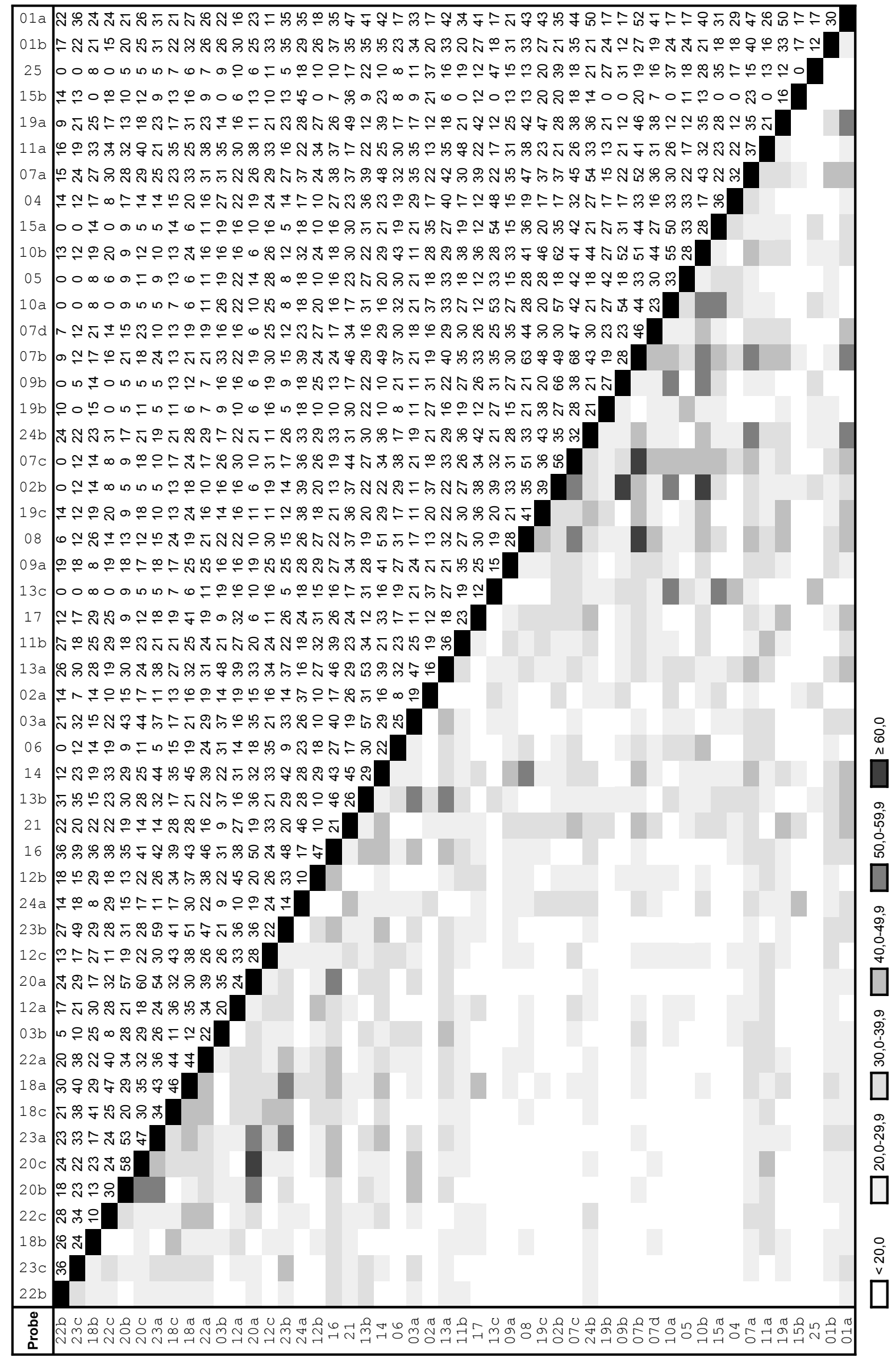


Tabelle 4-75: Ergebnisse verschiedener Korrespondenzanalysen mit den Fangdaten aus EFsProben an Eichentotholz

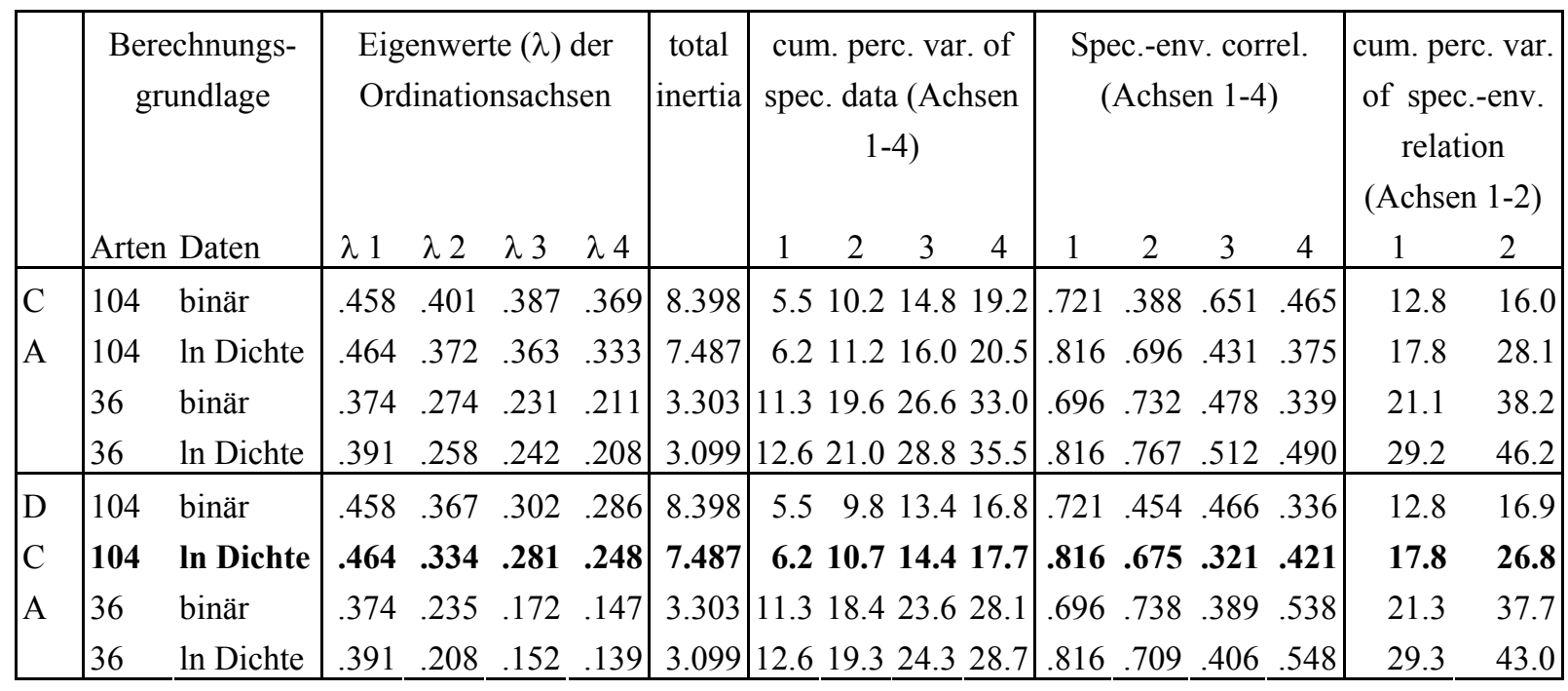

Um über die Bedeutung der an den Probenahmepunkten erhobenen Habitatvariablen für die Erklärung der vorgefundenen Artenvergesellschaftungen Auskunft zu erhalten, wurden die Fangergebnisse verschiedenen kanonischen Korrespondenzanalyseverfahren unterzogen. Die wichtigsten Ergebnisse sind in der Tabelle 4-76 zusammengefaßt. Die Abbildungen 4-52 und 4-53 zeigen die Ordinationsdiagramme einer CCA, basierend auf den Schlüpfdichten von 36 Arten, die in mindestens 5 der 50 Proben festgestellt worden waren. Proben, deren Position aufgrund fehlender Angaben zu einzelnen Habitatvariablen nachträglich auf der Basis der Artenstruktur festgelegt wurde, sind wiederum als passiv gekennzeichnet. 


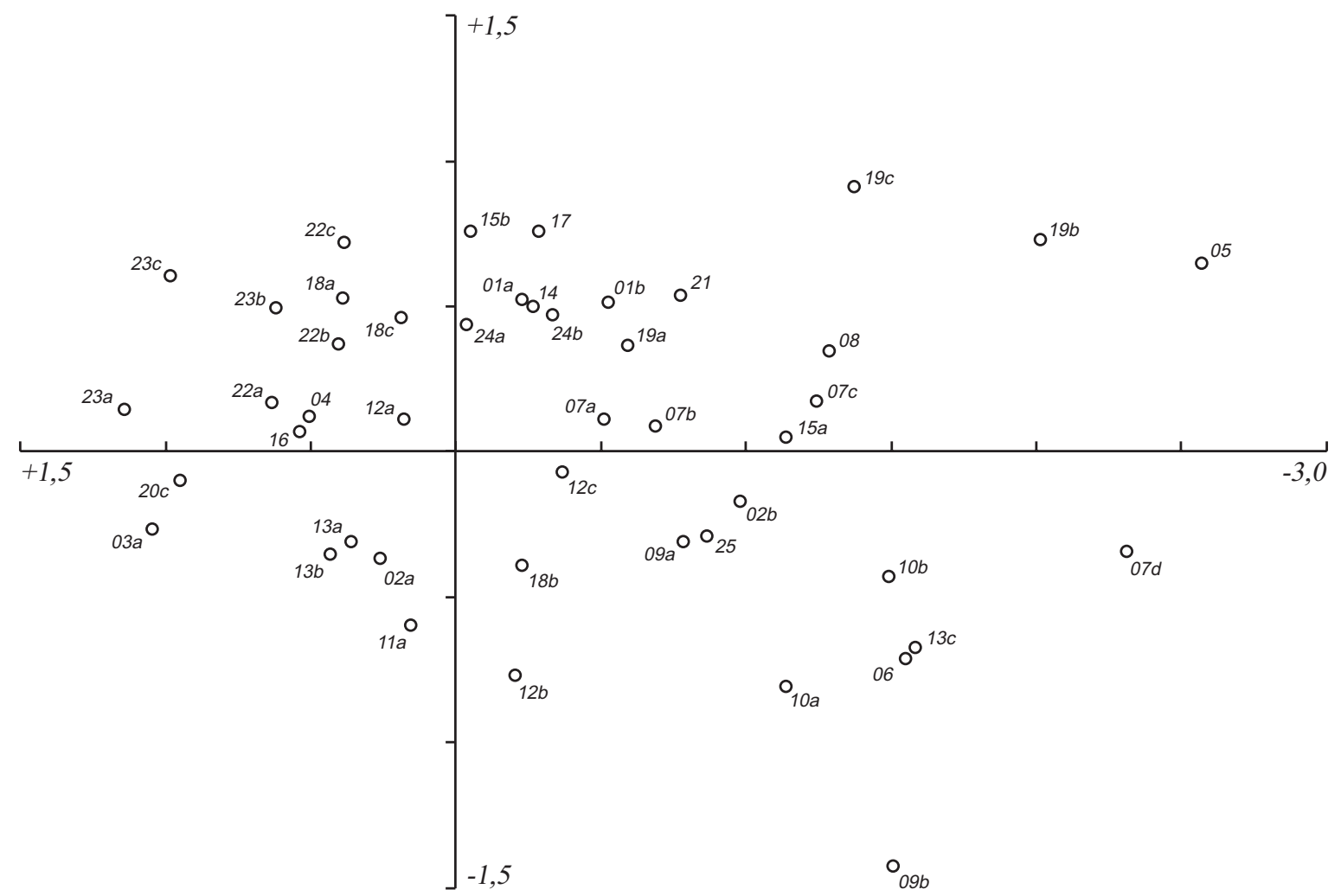

Abb. 4-50: Ordinationsdiagramm (DCA): EFs-Proben an stehendem Eichentotholz

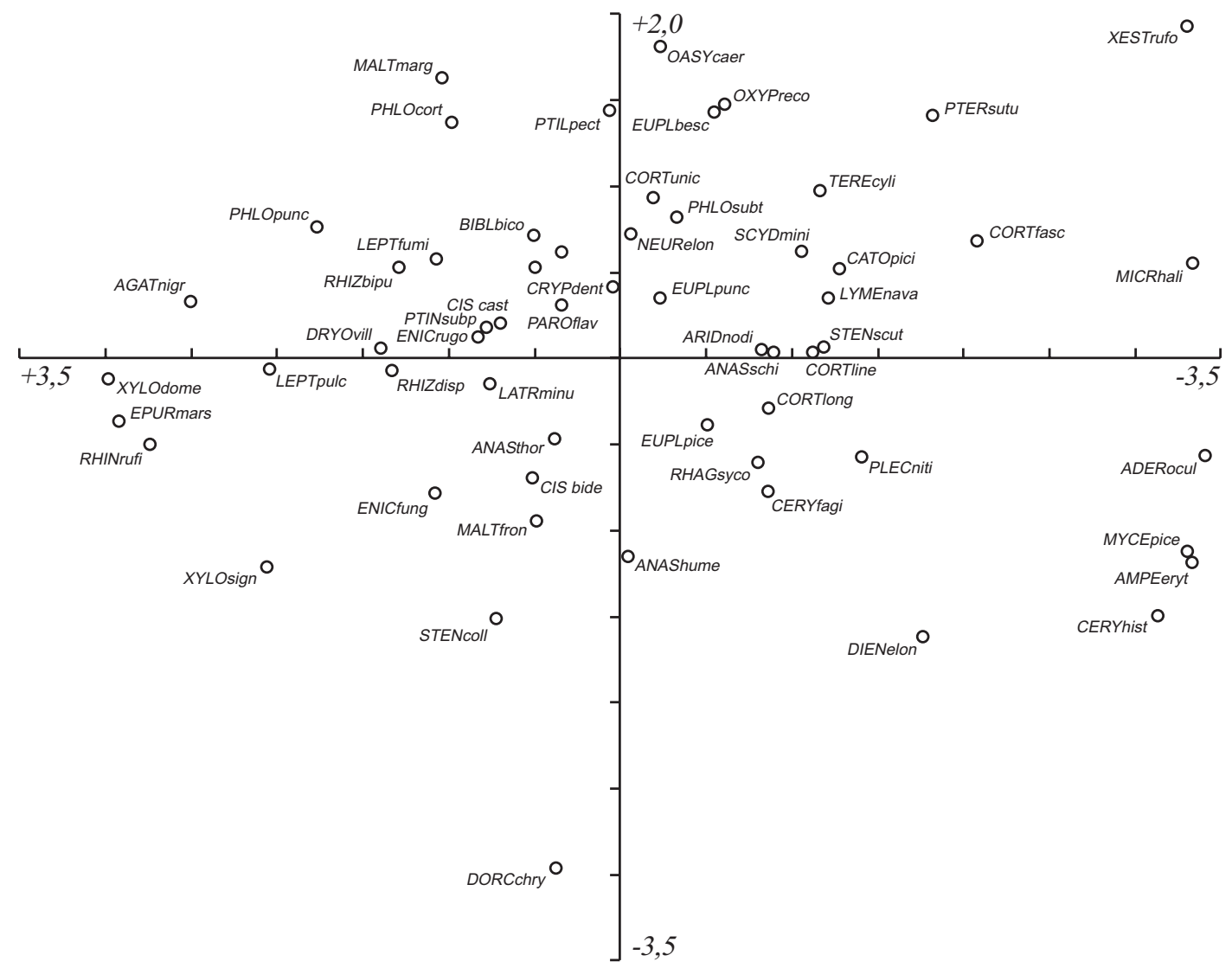

Abb. 4-51: Ordinationsdiagramm (DCA): Arten aus EFs-Proben an stehendem Eichentotholz 
Tabelle 4-76: Ergebnisse verschiedener kanonischer Korrespondenzanalysen mit den Fangdaten aus EFs- Proben an Eichentotholz

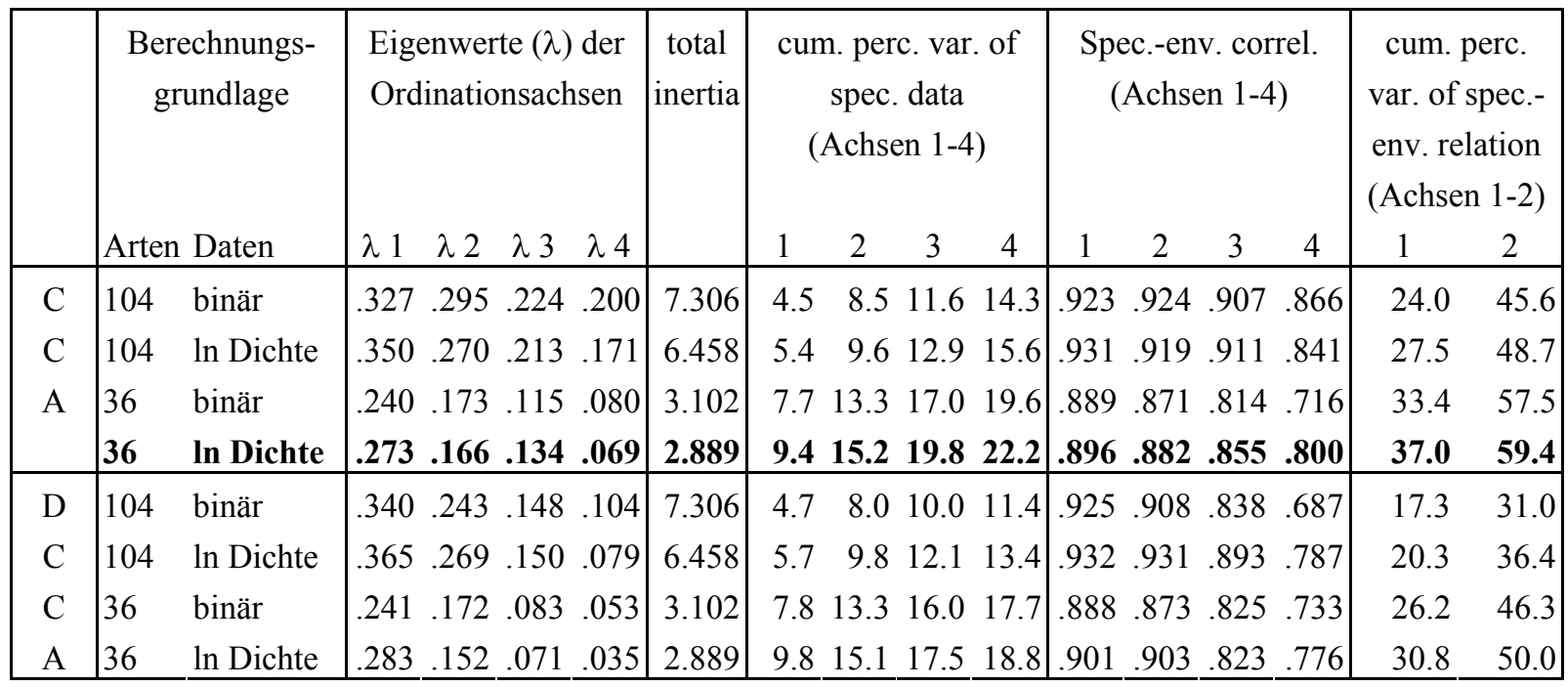

Die Eigenwerte der beiden dargestellten Ordinationsachsen beliefen sich auf 0,273 und 0,166. Gemeinsam erklärten die vier ausgeschiedenen Ordinationsachsen 22,2 \% der Gesamtvarianz. Die erste Achse war am engsten mit den Faktoren Berindung und Feuchtegehalt korreliert (Tab. 4-77). Diese beiden Faktoren trugen am stärksten zur Erklärung der Artenvergesellschaftungen bei, gefolgt von den Variablen Beschirmung, Bohrwiderstand, Saugfähigkeitsquotient und Beschattung. Unter Berücksichtigung der Korrelationen zwischen den Habitatvariablen (Tab. 4-78) ergab sich als Ergebnis einer 'forward selection' als günstigste Faktorenkombination eine Rangfolge von Rindenklasse, Feuchtegehalt, Saugfähigkeitsquotient und Beschirmung und schließlich dem Faktor Bohrwiderstand.

Tabelle 4-77: Kanonische Koeffizienten und Korrelationskoeffizienten

\begin{tabular}{|l|cc|cc|}
\hline & \multicolumn{2}{|c|}{ Kanonischer Koeffizient } & \multicolumn{2}{c|}{ Korrelationskoeffizient } \\
Variable & Achse 1 & Achse 2 & Achse 1 & Achse 2 \\
\hline Ri & $-0,52$ & $+1,17$ & $-0,84$ & $+0,41$ \\
Bw & $-0,12$ & $-0,48$ & $-0,45$ & $-0,16$ \\
Sq & $+0,30$ & $+0,51$ & $-0,07$ & $+0,39$ \\
Fe & $-0,72$ & $-0,50$ & $-0,83$ & $-0,15$ \\
Schi & $-0,28$ & $-0,55$ & $-0,45$ & $-0,03$ \\
Scha & $+0,07$ & $-0,49$ & $-0,30$ & $+0,51$ \\
\hline
\end{tabular}




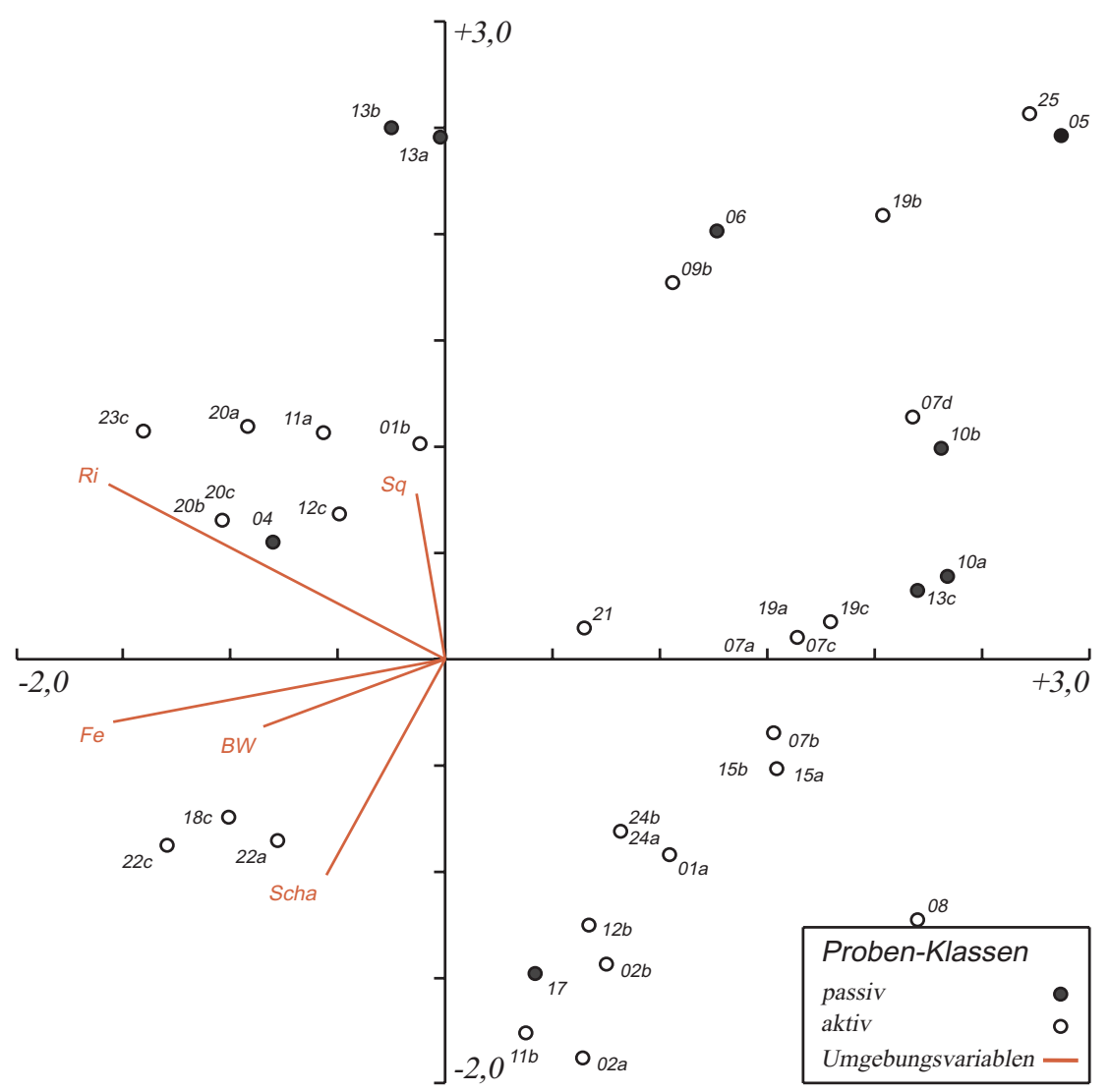

Abb. 4-52: Ordinationsdiagramm (CCA): EFs-Proben an stehendem Eichentotholz und Habitatvariablen

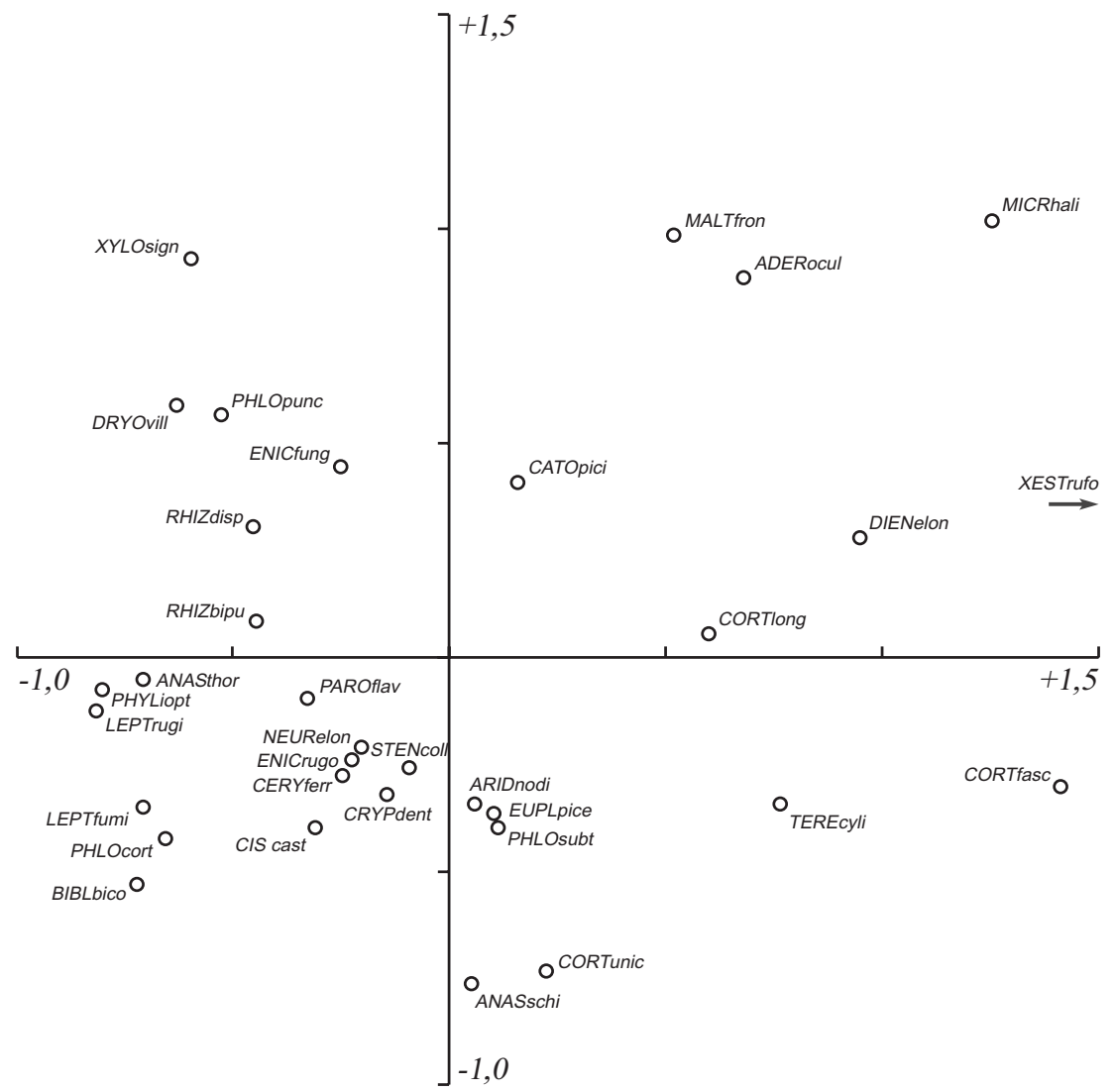

Abb. 4-53: Ordinationsdiagramm (CCA): Arten aus EFs-Proben an stehendem Eichentotholz 
Tabelle 4-78: Korrelationen zwischen den Variablen

\begin{tabular}{|c|c|c|c|c|c|}
\hline Variable & $\mathbf{B w}$ & $\mathrm{Sq}$ & $\mathbf{F e}$ & Schi & Scha \\
\hline $\mathbf{R i}$ & \multirow[t]{5}{*}{$+0,43$} & $+0,17$ & $+0,51$ & $+0,43$ & $+0,22$ \\
\hline Bw & & \multirow[t]{4}{*}{$-0,17$} & $+0,24$ & $-0,10$ & $+0,10$ \\
\hline $\mathbf{S q}$ & & & \multirow[t]{3}{*}{$+0,28$} & $+0,41$ & $+0,01$ \\
\hline $\mathrm{Fe}$ & & & & \multirow[t]{2}{*}{$+0,25$} & $+0,32$ \\
\hline Schi & & & & & $+0,24$ \\
\hline
\end{tabular}

Fast alle Proben gehörten entweder zur Ri-Klasse 4 oder 1 (Tab. 4-79). An unberindeten Stammabschnitten wurden 72, an den berindeten 88 Arten nachgewiesen. Deutlicher waren die Unterschiede in den Schlüpfdichten. Obwohl 55,9 \% der befangenen Oberfläche zur RiKlasse 1 gehörte, wurden hier nur 21,6 \% der Individuen gefangen. Die Schlüpfdichte war mit 1287,7 Ind. $/ \mathrm{m}^{2}$ in Proben der Ri-Klasse 4 um ein mehrfaches höher als an unberindeten Stammteilen (271,2 Ind. $/ \mathrm{m}^{2}$ ). Die in der Abbildung 4-52 zu beobachtende Separierung der Proben entlang der ersten Ordinationsachse ist vor allem auf diesen Faktor zurückzuführen. Proben der Ri-Klasse 4 sind fast ausschließlich in der linken, jene der Klasse 1 in der rechten Diagrammhälfte positioniert.

Gleichzeitig war die Mehrzahl der berindeten Stammabschnitte den Fe-Klassen 4 und 3 zuzurechnen, während die unberindeten Abschnitte mehrheitlich einen geringeren Feuchtegehalt aufwiesen. Mit abnehmendem Feuchtegehalt war die registrierte Schlüpfdichte rückläufig. Etwa ein Fünftel der beprobten Fläche entfiel auf die Fe-Klasse 4, in der aber 68,4 \% der Individuen erfaßt wurde $\left(2258,7 \mathrm{Ind} . / \mathrm{m}^{2}\right)$. Im Vergleich dazu wurde für die Fe-Klasse 2 lediglich eine Dichte von 175,0 Ind. $/ \mathrm{m}^{2}$ festgestellt.

Die Verteilung der Stichproben auf die Bw-Klassen erwies sich als sehr ungleichmäßig. In ihrer Mehrheit gehörten sie zu den Klassen 4 und 3. Nur ein geringer Anteil entfiel auf die Bw-Klassen 6, 5 und 1. Die höchsten Schlüpfdichten wurden in Proben der Klasse 4 registriert, die geringsten in Stammabschnitten mit nur geringem Bohrwiderstand. Im Ordinationsdiagramm der Abbildung 4-52 finden sich Proben mit höherem Bohrwiderstand vor allem im linken unteren Viertel, im rechten oberen Viertel dominieren dagegen die niedrigeren BwKlassen.

Tabelle 4-79: Probenumfang, Arten- und Individuenzahl einzelner Bw-, Ri- und Fe-Klassen

\begin{tabular}{|c|c|c|c|c|c|c|c|c|c|c|c|c|c|c|c|}
\hline & $\begin{array}{r}1 \\
\text { Bw } 6 \\
\end{array}$ & oh & $\begin{array}{l}\text { iders } \\
\text { Bw } 4\end{array}$ & $\begin{array}{l}\text { tand } \\
\text { Bw }\end{array}$ & w & & \begin{tabular}{|l} 
Rind \\
Ri 4 \\
\end{tabular} & $\begin{array}{l}\text { lenk } \\
\text { Ri } 3 \\
\end{array}$ & $\begin{array}{r}\text { lasse } \\
\text { Ri } 1 \\
\end{array}$ & $\mathrm{Fe} 4$ & $\begin{array}{l}\text { Feuc } \\
\text { Fe } 3 \\
\end{array}$ & $\begin{array}{l}\text { htekl } \\
\text { Fe } 2 \\
\end{array}$ & $\begin{array}{l}\text { lasse } \\
\text { Fe } 1 \\
\end{array}$ & . A. & ges. \\
\hline Prob & 2 & 5 & 14 & 16 & 10 & 3 & 20 & 1 & 29 & 10 & 11 & 12 & 5 & 11 & 50 \\
\hline Mantelfl. $\left(\mathbf{d m}^{2}\right)$ & 26 & 68 & 210 & 215 & 131 & 41 & 293 & 12 & 386 & 147 & 157 & 168 & 70 & 150 & 691 \\
\hline Volumen $\left(\mathbf{d m}^{3}\right)$ & 35 & 97 & 347 & 342 & 181 & 58 & 479 & 15 & 566 & 238 & 261 & 258 & 108 & 195 & 1059 \\
\hline 1. $\left(\mathrm{dm}^{2}\right)$ & 3 & 57 & 165 & 69 & 0 & 0 & 293 & 10 & & 129 & 75 & 25 & 31 & 45 & 303 \\
\hline Ri \% & 48 & 83 & 79 & 32 & 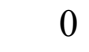 & 0 & 100 & 90 & 0 & 88 & 47 & 15 & 44 & 30 & 44 \\
\hline Indiv & 69 & 187 & 2783 & 1478 & 264 & 57 & 3773 & 18 & 1047 & 3309 & 618 & 294 & - 85 & 532 & 4838 \\
\hline Ind./m² & 265 & 275 & 1328 & 687 & 202 & 139 & 1288 & 157 & 271 & 2259 & 394 & 175 & 122 & 356 & 701 \\
\hline Arten & 24 & 42 & 71 & 66 & 31 & 15 & 88 & 5 & 72 & 62 & 63 & 48 & 25 & 43 & 112 \\
\hline
\end{tabular}


Die Zoophagen hatten in den einzelnen Ri-, Fe- und Bw-Klassen den höchsten Anteil an den festgestellten Artenspektren (Tab. 4-80). Der Artenanteil lag in den meisten Fällen zwischen 40 und $50 \%$. Hinsichtlich der Individuenzahlen belegte dieser Konsumententyp jedoch nur den dritten Rang. An berindeten Stammabschnitten war mit 135,2 Ind. $/ \mathrm{m}^{2}$ eine deutlich höhere Schlüpfdichte zu verzeichnen als an unberindeten (81,3 Ind. $\left./ \mathrm{m}^{2}\right)$. Umgekehrt verhielt es sich bei den auf diese Konsumentengruppe entfallenden Individuenanteilen. An unberindeten Stammteilen war ein Wert von 30,0 \%, an berindeten von nur 10,5 \% zu beobachten. Mit sinkendem Feuchtegehalt der Proben war die Schlüpfdichte rückläufig, gleichzeitig stieg der Dominanzwert von 10,5 \% (Fe-Kl.4) auf 36,7 \% an (Fe-Kl. 2). Die BwKlasse 4 wies zwar im Vergleich zu den übrigen Bw-Klassen die höchste Schlüpfdichte auf, gleichzeitig war der Individuenanteil der Räuber hier aber am geringsten (11,5\%). Die niedrigsten Schlüpfdichten wurden bei geringem Bohrwiderstand, die höchsten Dominanzwerte bei hohem Bohrwiderstand registriert.

Der zweithöchste Anteil am Artenspektrum entfiel auf die Mycetophagen. Ihr Artenanteil variierte in den einzelnen Ri-, Fe- und Bw-Klassen zwischen 20 und 30 \%. Die Schlüpfdichte lag an berindeten Stammteilen mit 257,0 Ind. $/ \mathrm{m}^{2}$ etwa doppelt so hoch wie an unberindeten. In letzteren waren die Pilzfresser mit einem Individuenanteil von 46,7 \% allerdings der vorherrschende Konsumententyp. Mit abnehmendem Feuchtegehalt war eine sinkende Schlüpfdichte zu verzeichnen. Der niedrigste Dominanzwert wurde mit 12,2 \% für die Fe-Klasse 4, der höchste mit 64,2 \% für die Fe-Klasse 3 gemessen. Die Individuenanteile in den BwKlassen lagen zwischen 19,4 (Klasse 4) und 69,7 \% (Klasse 2). Trotz des geringen Dominanzwertes wurde die höchste Schlüpfdichte in Proben der Bw-Klasse 4 registriert.

Tabelle 4-80: Anteile der Konsumententypen in den Bw-, Ri- und Fe-Klassen

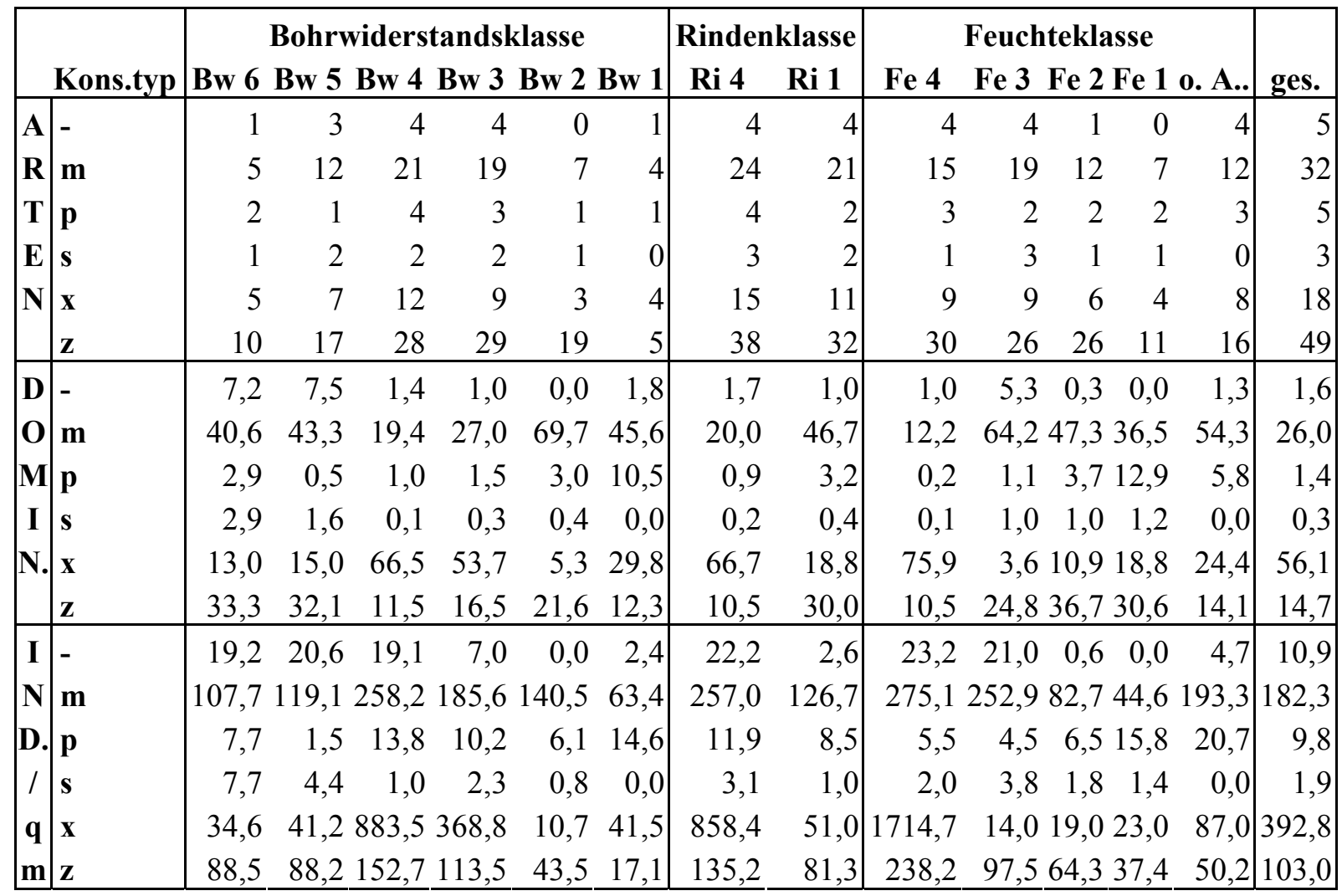


Den höchsten Individuenanteil (56,1 \%) in den Proben an stehendem Eichentotholz hatten die 18 xylophagen Arten. Die registrierten Schlüpfdichten und Dominanzwerte wurden vor allem von der Verteilung des Borkenkäfers Dryocoetes villosus geprägt, der $91 \%$ der Individuen dieses Konsumententyps stellte. Folglich waren Schlüpfdichte und Individuenanteil an berindeten Stammabschnitten besonders hoch (858,4 Ind. $/ \mathrm{m}^{2}$, 66,7 \%). In den Fe-Klassen 3 bis 1 lagen die Individuenanteile deutlich unter denen der zuvor genannten Konsumententypen. Dagegen erreichten Schlüpfdichte und Dominanzwert in Proben der Fe-Klasse 4 ein deutliches Maximum (1714,7 Ind. $/ \mathrm{m}^{2}$, 75,9 \%). In Proben der Bw-Klassen 4 und 3 waren jeweils über $50 \%$ der gefangenen Individuen diesem Konsumententyp zuzurechnen. In den übrigen Bw-Klassen lagen die Individuenanteile und Schlüpfdichten deutlich niedriger.

Die Rindenbewohner waren mit 25 Arten und einem Individuenanteil von 58,7 \% (411,6 Ind. $/ \mathrm{m}^{2}$ ) in den Proben vertreten (Tab. 4-81). Da 87 \% der Individuen dieser Gruppe von Dryocoetes villosus gestellt wurden, stimmten Anteile und Schlüpfdichten in den einzelnen Faktorenklassen in den wesentlichen Tendenzen mit den Ausführungen über die Konsumentengruppe der xylophagen Arten überein: hohe Schlüpfdichten und Dominanzwerte an berindeten Stammabschnitten der Fe-Klasse 4 bzw. den Bw-Klassen 4 und 3. Fast zwei Drittel der an berindeten Stammproben gefangenen Individuen entfielen auf D. villosus (Tab. 4-82), dessen spezifische Dichte hier 1166 Ind. $/ \mathrm{m}^{2}$ betrug. Von den 16 Proben, in denen dieser Borkenkäfer festgestellt wurde, gehörten 7 zur Fe-Klasse 4. In diesen wurden 96,4 \% der insgesamt 2471 Individuen gefangen, die Schlüpfdichte betrug 2246,2 Ind. $/ \mathrm{m}^{2}$. In Proben der Bw-Klassen 4 und 3 hatte D. villosus Individuenanteile von 61,3 und 51,3\%.

Mit 129 bzw. 112 Individuen zählten die beiden corticolen Arten Rhizophagus bipustulatus und $R h$. dispar zu den Hauptarten an stehendem Eichentotholz. Jeweils über $90 \%$ der Individuen wurden in Proben der Ri-Klasse 4 registriert und über $70 \%$ in Proben der Fe-Klasse 4. Rh. dispar zählte in Stammabschnitten der Bw-Klassen 4 und 3, Rh. bipustulatus zudem in denen der Klasse 5 zu den Hauptarten.

Die Mulmbewohner traten als artenreichste Gruppe in Erscheinung. Die 26 Arten hatten aber lediglich einen Individuenanteil von 14,5\%. Die Schlüpfdichte war an den berindeten Stammabschnitten mit 105,8 Ind. $/ \mathrm{m}^{2}$ geringfügig höher als an unberindeten $\left(97,9 \mathrm{Ind} . / \mathrm{m}^{2}\right)$. In letzteren wurde mit 36,1 \% jedoch ein deutlich höherer Dominanzwert erreicht. In Proben der Fe-Klassen 1 bis 3 waren die xylodetricolen Arten der klar vorherrschende Präferenztyp. Die höchste Schlüpfdichte und der höchste Dominanzwert wurde in Stammabschnitten der BwKlasse 2 registriert.

Fast drei Viertel der gefangenen Tiere wurden von Corticaria longicollis gestellt, der in 46 der 50 Proben festgestellt wurde. Die Schlüpfdichten waren in den Ri-Klassen sehr ähnlich. In Proben der Ri-Klasse 1 war C. longicollis mit einem Individuenanteil von 28,3 \% die häufigste Art, wohingegen an berindeten Stammteilen der Dominanzwert nur 5,7 \% betrug. In den Proben der Fe-Klasse 4 wurde eine nur geringe Schlüpfdichte registriert (25,3 Ind./ $\mathrm{m}^{2}$ ), deutlich höher lag der Wert für Proben der Fe-Klasse 3 (114,6 Ind. $\left./ \mathrm{m}^{2}\right)$. Mit sinkendem Feuchtegehalt waren die registrierten Dichten wieder rückläufig. Abgesehen von der Fe-Klasse 4 war C. longicollis in allen Fe-Klassen die häufigste Art. Die überwiegende Zahl der Individuen wurde an Stammabschnitten der Bw-Klassen 4 bis 2 gefangen, die höchste Schlüpfdichte und der höchste Dominanzwert wurde für Proben der Bw-Klasse 2 registriert. 
Tabelle 4-81: Anteile der Habitatpräferenztypen in den Bw-, Ri- und Fe-Klassen

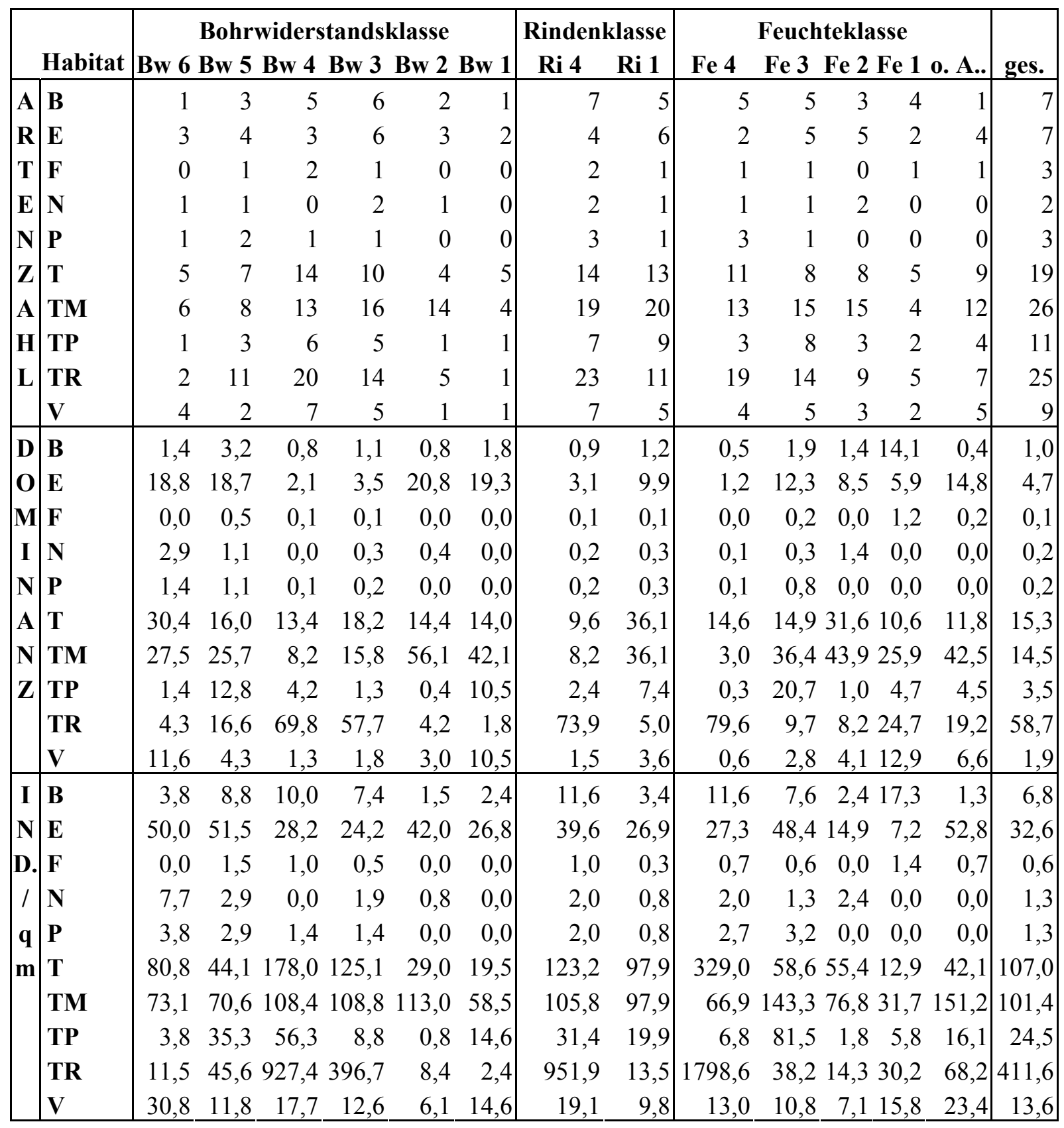

19 lignicole Arten waren mit einem Individuenanteil von 15,3 \% die zweithäufigste Präferentengruppe. An berindeten Stammteilen wurde eine Schlüpfdichte von 123,2, an unberindeten von 97,9 Ind. $/ \mathrm{m}^{2}$ registriert. Die Dominanzwerte beliefen sich auf 9,6 bzw. 36,1 \%. In Proben der Fe-Klasse 4 war mit 329,0 Ind. $/ \mathrm{m}^{2}$ eine deutlich höhere Schlüpfdichte zu verzeichnen als in den Stammteilen mit geringerem Feuchtegehalt. Der Individuenanteil erreichte mit 31,6 \% seinen höchsten Wert in Proben der Fe-Klasse 2. $87 \%$ der Individuen wurden an Stammteilen erfaßt, die zu den Bw-Klassen 4 und 3 zählten. Hier waren auch die höchsten Schlüpfdichten $\mathrm{zu}$ verzeichnen, während die niedrigsten Werte in Proben mit geringem Bohrwiderstand registriert wurden. Die Dominanzwerte in den Bw-Klassen 5 bis 1 variierten zwischen 13,4 und 18,2 \%. Nur in den wenigen auf die Klasse 6 entfallenden Proben waren die lignicolen Arten mit einem Individuenanteil von 30,4 \% vorherrschend. 
Der Borkenkäfer Xyloterus signatus war mit 295 Individuen der häufigste Vertreter dieser Gruppe. Abgesehen von wenigen Ausnahmen wurden die Tiere an berindeten Stammteilen der Fe-Klasse 4 und den Bw-Klasse 4 und 3 gefangen. Mit Individuenanteilen zwischen 6,2 \% (Bw-Klasse 4) und 8,7 \% (Fe-Klasse 4) war X. signatus in diesen Faktorenklassen jeweils die zweithäufigste Art.

In den Proben von rindenlosen Stammteilen war Lymexylon navale mit einem Individuenanteil von 12, $3 \%$ dritthäufigste Art. Insgesamt wurde diese Art jedoch in nur drei Proben festgestellt, 111 der 129 Individuen entstammten einer einzigen Probe, für die sich eine Schlüpfdichte von 634 Ind. $/ \mathrm{m}^{2}$ errechnete.

Tabelle 4-82: Dominante Arten in einzelnen Variablenklassen (Ind. \%)

\begin{tabular}{|l|rr|rrr|rrrr|}
\hline & \multicolumn{2}{|c|}{ Rindenklasse } & \multicolumn{3}{|c|}{ Feuchteklasse } & \multicolumn{4}{|c|}{ Bohrwiderstandsklasse } \\
ART & Ri 4 & Ri 1 & Fe 4 & Fe 3 & Fe 2+1 & Bw 6+5 & Bw 4 & Bw 3 & Bw 2+1 \\
\hline Dryocoetes villosus & 65,4 & 0,5 & 72,0 & 0,5 & 4,5 & 1,6 & 61,3 & 51,3 & 1,2 \\
Xyloterus signatus & 7,8 & 0,1 & 8,7 & 0,6 & 0,8 & 0,4 & 6,2 & 8,3 & 0,0 \\
Rhizophagus bipustulatus & 3,2 & 0,9 & 2,8 & 3,1 & 2,1 & 5,1 & 2,9 & 2,2 & 0,6 \\
Rhizophagus dispar & 2,7 & 1,0 & 2,5 & 1,3 & 2,4 & 1,2 & 2,5 & 2,4 & 1,2 \\
Lymexylon navale & 0,0 & 12,3 & 3,4 & 0,2 & 4,5 & 0,0 & 4,0 & 1,2 & 0,0 \\
Ptilinus pectinicornis & 0,0 & 2,2 & 0,0 & 1,1 & 0,0 & 7,0 & 0,0 & 0,4 & 0,0 \\
Aridius nodifer & 0,3 & 0,4 & 0,3 & 0,3 & 0,8 & 3,9 & 0,0 & 0,1 & 0,9 \\
Anaspis schilskyana & 0,1 & 0,4 & 0,1 & 0,3 & 0,0 & 2,3 & 0,1 & 0,0 & 0,0 \\
Cryptophagus dentatus & 2,4 & 2,5 & 0,9 & 9,2 & 1,6 & 12,1 & 1,9 & 1,8 & 1,6 \\
Corticaria longicollis & 5,7 & 28,3 & 1,1 & 29,1 & 33,0 & 12,5 & 6,0 & 12,9 & 40,8 \\
Teredus cylindricus & 0,3 & 16,7 & 1,5 & 10,5 & 14,2 & 6,3 & 1,7 & 6,3 & 9,3 \\
Cis castaneus & 1,9 & 2,7 & 0,2 & 12,9 & 0,3 & 8,6 & 2,3 & 0,9 & 0,0 \\
Dienerella elongata & 0,4 & 6,7 & 0,0 & 2,4 & 4,7 & 2,3 & 0,2 & 1,4 & 17,8 \\
Corticeus fasciatus & 0,0 & 2,8 & 0,1 & 1,3 & 4,0 & 1,6 & 0,1 & 0,9 & 2,8 \\
Cerylon ferrugineum & 1,0 & 2,0 & 1,0 & 1,5 & 0,8 & 3,9 & 1,1 & 1,1 & 0,0 \\
Phyllodrepa ioptera & 0,5 & 0,2 & 0,2 & 2,6 & 0,0 & 2,0 & 0,5 & 0,2 & 0,3 \\
Euplectus punctatus & 0,3 & 0,9 & 0,3 & 0,3 & 0,8 & 3,5 & 0,1 & 0,3 & 0,6 \\
Enicmus testaceus & 0,1 & 3,3 & 0,0 & 5,8 & 0,8 & 0,0 & 1,4 & 0,0 & 0,0 \\
Aderus oculatus & 0,1 & 2,0 & 0,0 & 0,3 & 0,3 & 0,8 & 0,0 & 0,0 & 6,5 \\
\hline
\end{tabular}

Als häufigste lignicole Art mit zoophager Ernährungsweise trat Teredus cylindricus in Erscheinung. Er war in fast jeder zweiten Probe festzustellen und mit einem Individuenanteil von 3,9\% die insgesamt vierthäufigste Art an stehendem Eichentotholz. 100 Exemplare wurden in nur 2 Proben registriert, die Schlüpfdichten betrugen hier 329 und $251 \mathrm{Ind} . / \mathrm{m}^{2} .175$ der 187 Individuen wurden an rindenlosen Stammabschnitten gefangen. Die spezifische Dichte belief sich hier auf 72,2 Ind. $/ \mathrm{m}^{2}$, der Dominanzwert betrug 16,7 \%. T. cylindricus war mit der größten Regelmäßigkeit in Proben der Fe-Klasse $2 \mathrm{zu}$ finden, in denen auch der höchste Dominanzwert verzeichnet wurde. $61 \%$ der Individuen wurden jedoch in Proben der Fe-Klassen 3 und 4 gefangen. Die spezifischen Dichten beliefen sich hier auf 92,2 bzw. 83,3 Ind. $/ \mathrm{m}^{2} .75 \%$ der Fänge entfielen auf Proben der Bw-Klassen 4 und 3, weitere $14 \%$ auf solche der Klasse 2. In letzterer wurde mit 10,2 \% der höchste Dominanzwert verzeichnet. 
Als zweithäufigster Räuber dieser Habitatpräferentengruppe war Corticeus fasciatus nachzuweisen. Die 29 Exemplare aus 9 Proben wurden fast ausnahmslos an seit mindestens 20 Jahren abgestorbenen Stämmen gefangen. Die Proben gehörten allesamt zur Ri-Klasse 1. Mit der größten Regelmäßigkeit war $C$. fasciatus in Proben der Fe-Klasse 2 zu beobachten, in denen sich der Individuenanteil auf 4,8 \% belief. $76 \%$ der Tiere wurden in Proben der BwKlassen 3 und 2 registriert.

Besiedler von Totholzpilzen waren mit 11 Arten und einem Individuenanteil von 3,5\% in den Proben vertreten. Die Schlüpfdichte war an berindeten, der Dominanzwert an unberindeten Stammabschnitten am höchsten. An Stammabschnitten der Fe-Klasse 3 wurden 76 \% der Individuen erfaßt. Mit einer Schlüpfdichte von 81,5 Ind. $/ \mathrm{m}^{2}$ und einem Individuenanteil von 20,7 \% waren die Totholzpilzkäfer der zweithäufigste Habitatpräferenztyp in dieser FeKlasse. In den übrigen Klassen lagen die Werte deutlich niedriger. In ihrer großen Mehrheit wurden die Tiere in Proben der Bw-Klasse 4 gefangen, in denen 56,3 Ind. $/ \mathrm{m}^{2}$ schlüpften. Mit 12,8 \% war der höchste Individuenanteil für die Bw-Klasse $5 \mathrm{zu}$ verzeichnen.

Vorherrschender Vertreter dieser Präferentengruppe war Cis castaneus, der in erster Linie an Proben der Ri-Klasse 4 gefangen wurde und hier auch in den höheren Schlüpfdichten auftrat, aber nur an unberindeten Stammabschnitten zu den Hauptarten zählte. Von den 100 Exemplaren wurden 80 in Proben der Fe-Klasse 3 gefangen. Mit einem Individuenanteil von 12,9 \% war C. castaneus die zweithäufigste Art dieser Klasse. Alle Nachweise entfielen auf die Bw-Klassen 5 bis 3, 65 \% der Individuen wurden in Proben der Bw-Klasse 4 festgestellt, in denen auch die höchste spezifische Dichte gemessen wurde, während die niedrigste Schlüpfdichte und der geringste Individuenanteil für die Klasse 3 registriert wurde. Mit 11,8 \% erreichte C. castaneus in Proben der Bw-Klasse 5 den höchsten Dominanzwert.

Als zweithäufigster Vertreter der xylofungicolen Arten wurde am stehenden Eichentotholz Enicmus testaceus gefangen, mit 39 Individuen allerdings in nur geringer Zahl, die zudem fast allesamt einer einzelnen Probe entstammten. Diese geringe Fangzahl genügte, um E. testaceus in Proben der Ri-Klasse 1 bzw. der Fe-Klasse 3 den Status einer Hauptart erreichen zu lassen.

Sieben als eurytop eingestufte Arten wurden in einer Dichte von 32,6 Ind.$/ \mathrm{m}^{2}$ festgestellt. Sie stellten 3,1\% der an berindeten und 9,9\% der an unberindeten Stammteilen gefangenen Individuen. Proben der Fe-Klasse 3 ergaben die höchste Schlüpfdichte $\left(48,4\right.$ Ind./ $\left.\mathrm{m}^{2}\right)$ und den höchsten Individuenanteil (12,3 \%). Für die einzelnen Bw-Klassen wurden Schlüpfdichten zwischen 24,2 (Klasse 3) und 51,5 Ind. $/ \mathrm{m}^{2}$ (Klasse 5) sowie Dominanzwerte zwischen 2,1 (Klasse 4) und 20,8\% (Klasse 2) registriert.

Individuenstärkster Vertreter dieser Gruppe war Cryptophagus dentatus mit 115 Exemplaren. Er war in jeweils 14 Proben von berindeten und von unberindeten Stammabschnitten vertreten und auch die Individuenanteile waren mit 2,4 bzw. 2,5 \% in den beiden Klassen sehr ähnlich. Die registrierte Schlüpfdichte lag an berindeten Stammteilen jedoch deutlich höher. Nur sehr wenige Individuen wurden in Proben der Fe-Klassen 1 und 2 festgestellt, während Schlüpfdichte und Individuenanteil ihre höchsten Werte in der Klasse 3 erreichten. $88 \%$ der Individuen wurden in Proben der Bw-Klassen 5 bis 3 erfaßt, aber nur bei hohem Bohrwiderstand trat $C$. dentatus als Hauptart in Erscheinung. Hier waren auch die registrierten Dichten am höchsten, während bei abnehmender Festigkeit des Materials auch die Schlüpfdichten rückläufig waren.

Anders sah die Verteilung von Dienerella elongata aus. Die meisten der insgesamt 89 Individuen wurden an unberindeten Stammabschnitten gefangen. Der Individuenanteil erreichte 
hier einen Wert von 6,7 \%, während dieser an berindeten Abschnitten nur 0,4\% betrug. $63 \%$ der Individuen entstammten Proben, für die der Feuchtegehalt nicht ermittelt werden konnte, die übrigen Exemplare wurden fast vollständig in Proben der Fe-Klassen 3 und 2 erfaßt, während kein Nachweis auf die Fe-Klasse 4 entfiel. Gering waren die Schlüpfdichten in Proben der Bw-Klassen 6 bis $4.55 \%$ der Tiere entstammten Proben der Bw-Klasse 2, weitere $22 \%$ solchen der Klasse 3. Schlüpfdichte und Dominanzwert waren bei geringem Bohrwiderstand am höchsten.

\subsection{Die Struktur der Artengemeinschaften in den Fensterfallenproben}

\subsubsection{Frequenz- und Aktivitätsdominanzstruktur}

Die große Mehrzahl der insgesamt 562 in Fensterfallen festgestellten Arten trat in nur wenigen Proben in Erscheinung (Tab. 4-83). 167 Arten (30\%) waren in nur einer Probe vertreten und 315 Arten (56\%) in weniger als 5 der 68 Proben. Sie stellten zusammen 3,8\% der gefangenen Individuen. 15,9 \% der Individuen verteilten sich dagegen auf nur 4 Arten, die in mindestens 60 Proben gefangen worden waren.

In vielen Fällen waren Arten mit hohem Frequenzprozent gleichzeitig diejenigen, die eine hohe Individuendominanz erlangten. Athous subfuscus (10,2 Ind./Probe), Cerylon ferrugineum (7,4 Ind./Probe), Meligethes denticulatus (18,4 Ind./Probe) und Rhizophagus bipustulatus (5,9 Ind./Probe), Arten, die in mindestens 60 Proben auftraten, nahmen auch in der Dominanzrangordnung eine führende Position ein.

Andere Arten wiederum waren zwar häufig, aber jeweils in nur geringer Zahl anzutreffen, so dass sie in der Dominanzstruktur von nachgeordneter Bedeutung waren. Cryptophagus dentatus sowie Aridius nodifer waren jeweils in 58 Proben präsent, in denen sie aber nur mit im Durchschnitt 3,8 bzw. 4,3 Individuen auftraten, so dass sie nur 1,3 bzw. 1,5\% des Gesamtfanges stellten. Weitere Beispiele sind Agriotes acuminatus (55 Proben, 3,3 Ind./Probe), Euplectus bescidicus (52 Proben, 2,9 Ind./Probe), Agathidium nigripenne (47 Proben, 2,4 Ind./Probe) und Pediacus dermestoides (44 Proben, 2,1 Ind./Probe).

Schließlich erreichte manche Art einen hohen Individuenanteil an den Gesamtfängen, indem sie in vergleichsweise wenigen Proben, in diesen aber in größerer Zahl auftrat. Beispiele hierfür sind Xyleborus dispar (37 Proben, 12,8 Ind./Probe) und Ptinella limbata (36 Proben, 8,8 Ind./Probe).

Die Dominanzstruktur erwies sich als relativ ausgeglichen. Durch die gewählten Klassengrenzen, denen eine logarithmische Relation zugrunde lag, sollte diesem Umstand Rechnung getragen werden, indem insbesondere bei den zahlreichen Arten mit niedrigen Individuenanteilen eine stärkere Differenzierung vorgenommen wurde (Tab. 4-84).

Bei der hier gewählten Klasseneinteilung war, bezogen auf den gesamten Untersuchungszeitraum, nur eine Art (Meligethes denticulatus) mit einem Individuenanteil von 6,9\% eudominant. 4 dominante Arten stellten gemeinsam 14,8 \% und 17 subdominante Arten weitere $27,5 \%$ der Individuen, so dass annähernd jedes 2. Käferindividuum zu den 22 Hauptarten zu 
zählen war. Die 20 rezedenten Arten trugen mit 17,3 \% zum Fangergebnis bei und die 29 subrezedenten Arten stellten weitere 13,3\% der Individuen. 491 Arten traten nur sporadisch in Erscheinung, davon 323 Arten mit weniger als 6 Exemplaren.

Tabelle 4-83: Frequenzstruktur

\begin{tabular}{|c|r|r|r|}
\hline Frequenz & \multicolumn{1}{|c|}{ Arten } & Ind. & Ind.\% \\
\hline$\geq 60$ & 4 & 2598 & 15,9 \\
$50-59$ & 13 & 3710 & 22,7 \\
$40-49$ & 16 & 2511 & 15,4 \\
$30-39$ & 24 & 3129 & 19,1 \\
$20-29$ & 26 & 1412 & 8,6 \\
$10-19$ & 70 & 1554 & 9,5 \\
$5-9$ & 94 & 816 & 5,0 \\
$<5$ & 315 & 628 & 3,8 \\
\hline
\end{tabular}

In den einzelnen Untersuchungsjahren stellte sich die Dominanzklassenstruktur als sehr ähnlich dar ${ }^{8}$. Jeweils 20 Hauptarten waren zusammen mit 48,2 bis 58,0 \% der Individuen am Fang beteiligt. Daneben traten 296 bis 391 Arten als Begleitarten in Erscheinung.

Tabelle 4-84: Dominanzklassenstruktur

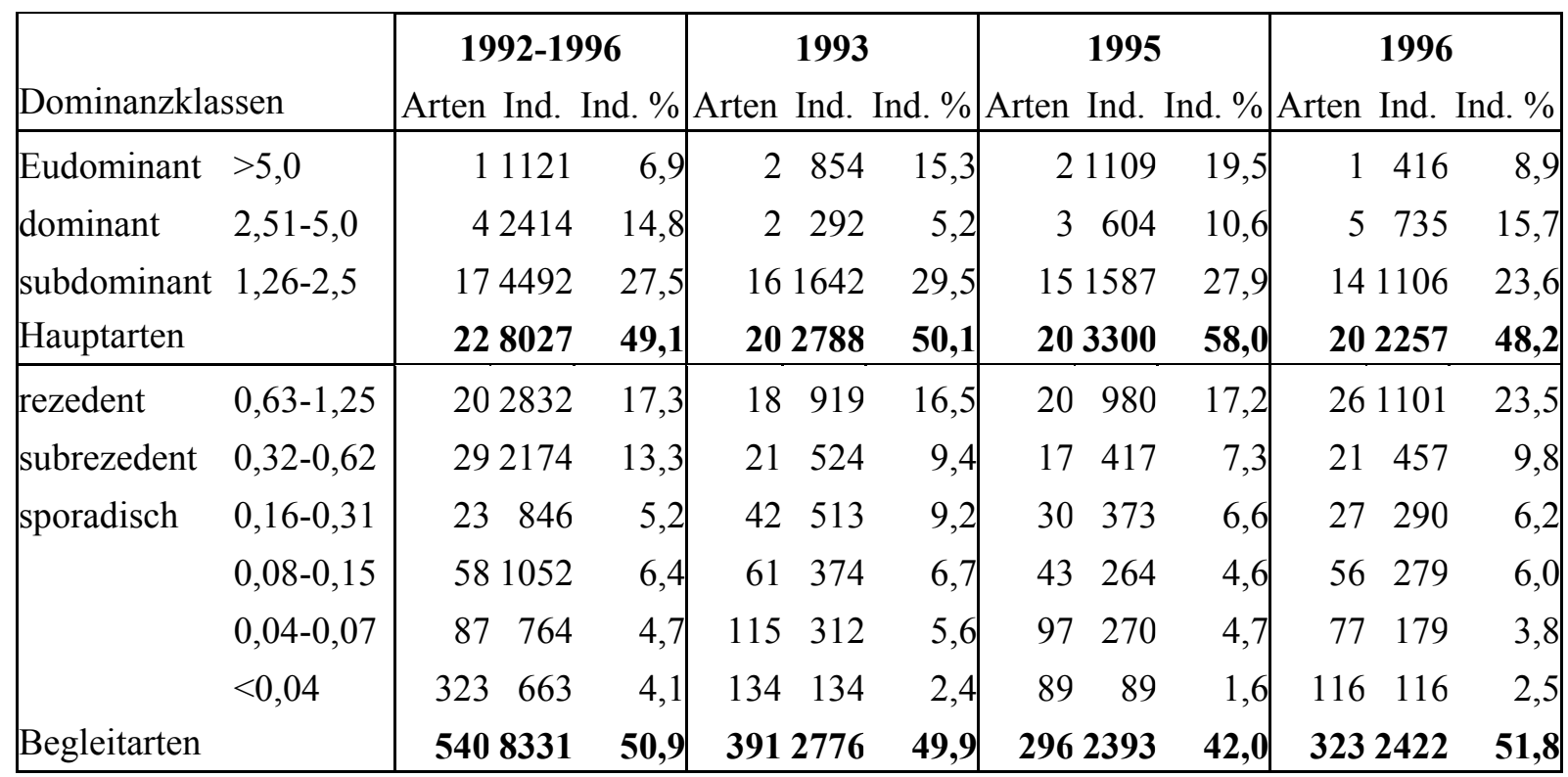

Insgesamt 35 Arten waren zumindest in einem Untersuchungsjahr mit einem Individuenanteil von mindestens 1,26 \% am Fangergebnis beteiligt, 22 von ihnen erreichten diesen Anteil auch hinsichtlich des Gesamtfangzeitraumes (Abb. 4-54). 17 Arten zählten in nur einem Jahr, 11 Arten in zwei Jahren und nur 7 Arten in drei Untersuchungsjahren zu den Hauptarten.

\footnotetext{
${ }^{8}$ Das Jahr 1992 bleibt hier wegen der geringen Fallenzahl und des späteren Aufbaus unberücksichtigt.
} 
Meligethes denticulatus

Hylecoetus dermestoides

Athous subfuscus

Xyleborus dispar

Cerylon ferrugineum

Rhizophagus bipustulatus

Ptinella limbata

Leptusa pulchella

Enicmus rugosus
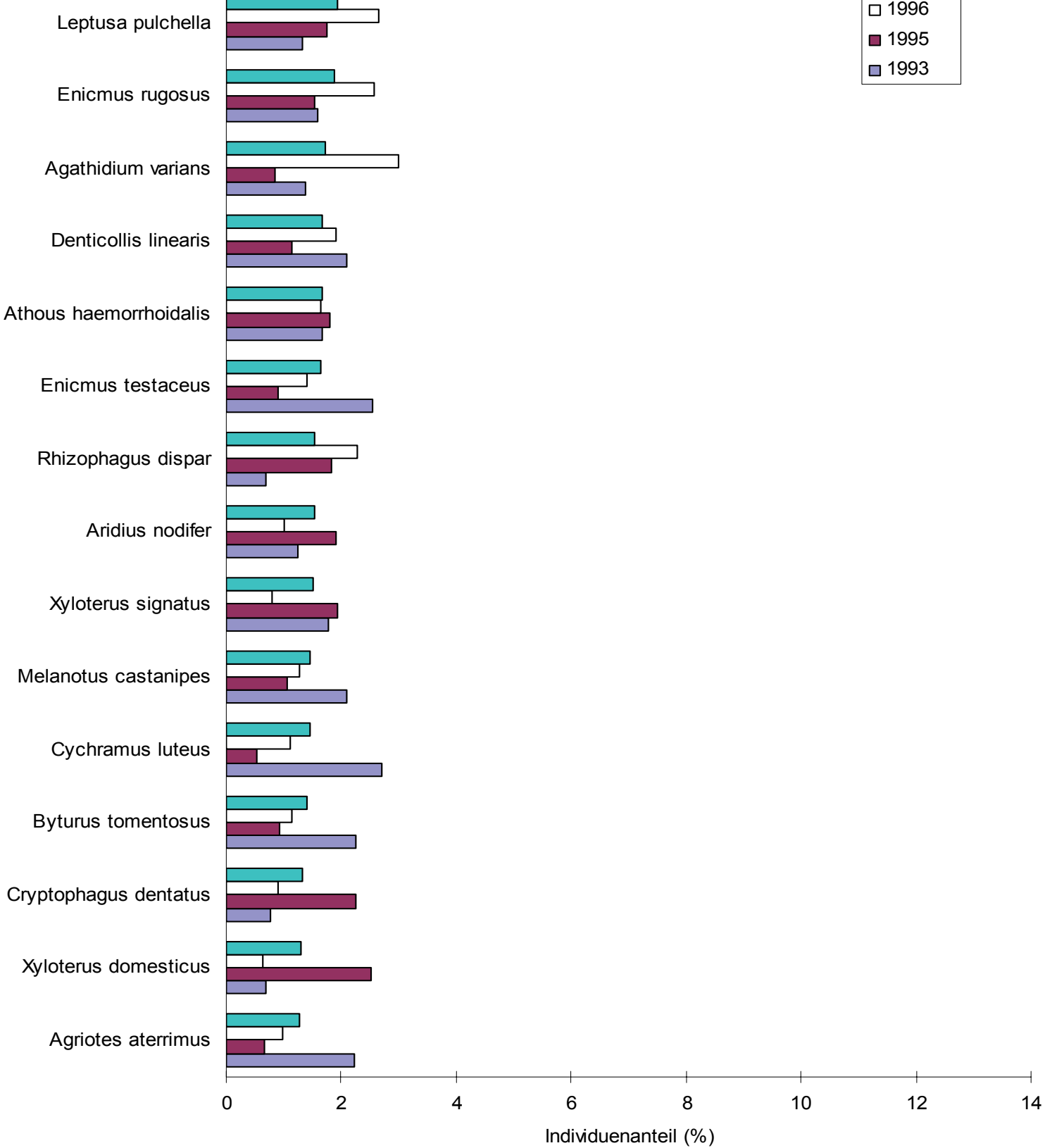

Abb. 4-54: Die 22 dominierenden Arten in den Fensterfallen 
Mit 6,9 \% Individuenanteil war Meligethes denticulatus die häufigste Art in den Fensterfallen. Die relative Häufigkeit variierte in den Einzeljahren zwischen 4,8 und 8,9 \%. Zweithäufigste Art und damit die vorherrschende Totholzkäferart der Fensterfallen war Hylecoetus dermestoides mit einem Individuenanteil von 5,0 \%. Keine andere Hauptart zeigte so deutliche Unterschiede im Individuenanteil der einzelnen Untersuchungsjahre. $86 \%$ der Individuen wurden im Jahr 1995 gefangen, in dem $H$. dermestoides mit 12,4 \% die mit Abstand häufigste Art repräsentierte, während 1993 nur 0,6 \% und 1996 1,7 \% des Gesamtfanges auf diese Art entfiel. Athous subfuscus war mit 4,0\% am Fangergebnis beteiligt. 1993 war er mit 7,6 \% die zweite eudominante Art dieses Jahres. In den beiden letzten Untersuchungsjahren reihte er sich unter die subdominanten Arten ein. Die holzbrütende Borkenkäferart Xyleborus dispar trat 1993 in nur einem einzigen Exemplar in den Fensterfallen auf, 1995 gehörte er mit einem Anteil von über 7\% zu den eudominanten Arten und wurde in der Häufigkeit nur von H. dermestoides übertroffen. An der Gesamtaktivität war er mit 2,9\% beteiligt. Cerylon ferrugineum erreichte in drei Untersuchungsjahren den Status einer Hauptart. Der Anteil an den Jahresfängen war mit Werten zwischen 2,3 und 3,3\% relativ konstant.

Die genannten Arten waren mit mindestens 2,51 \% Individuenanteil am Gesamtfang beteiligt. Darüber hinaus sind in Abbildung 4-54 auch die subdominanten Arten dargestellt. Weitere Informationen zur Frequenz und Aktivitätsdominanz der Arten in den FF-Proben können dem Anhang 11 entnommen werden.

\subsubsection{Flug- und Dominanzphänologie}

Die Anzahl der Hauptarten variierte in den einzelnen Fangperioden (ohne P1a) des Gesamtfangzeitraumes zwischen 15 und 20, der von diesen gestellte Individuenanteil zwischen 47,5 und 78,8 \%. Aufgrund der geringen Individuenzahlen zum Ende der Vegetationsperiode gerieten die auftretenden Arten rasch in die Position einer Hauptart. So waren im Fangzeitraum von Mitte September bis Anfang Oktober 28 \% der Arten zu dieser Fraktion zu zählen. Da während der 4 Untersuchungsjahre nur 283 Käferindividuen in diesem Zeitabschnitt gefangen wurden, sind selbst diejenigen Arten, die nur in einem Einzelexemplar auftraten, nicht mehr als sporadisch zu klassifizieren. Im Vergleich hierzu hatten in den Fangperioden bis Anfang August die Hauptarten einen Anteil von 6,1 bis 8,5\% an den in diesen Fangperioden festgestellten Artenzahlen. Der Individuenanteil der Hauptarten nahm vom Beginn der Vegetationsperiode zunächst ab, erreichte in der Fangperiode P4 mit 47,5 \% ein Minimum und stieg hiernach wieder kontinuierlich an.

Die Tabelle 4-85 gibt einen Überblick über die Hauptarten der einzelnen Fangperioden. Für die häufigsten Arten wird die saisonale Verteilung der Fänge (ohne 1992) in den Abbildungen 4-55a bis f dargestellt. Weitere Angaben zur Phänologie können dem Anhang 11 entnommen werden.

In der Fangperiode P1a, die ausschließlich Fänge des Jahres 1993 umfaßte, wurden 381 Käferindividuen aus 78 Arten gefangen. Mit Individuenanteilen von jeweils über $10 \%$ waren Aphodius prodromus, Rhizophagus bipustulatus und Meligethes aeneus vorherrschend. A. prodromus ist eine eurytope Art, deren Larven sich in Exkrementen entwickeln. Die Käfer schwärmen im zeitigen Frühjahr, nachdem die Imagines den Überwinterungsort verlassen haben. Die maximale Individuenzahl wurde auch 1993 in der Fangperiode P1b festgestellt. $M$. aeneus, der Rapsglanzkäfer, überwintert als Imago oft in der Streuauflage der Wälder. Zum 
Reifungsfraß suchen die Käfer im zeitigen Frühjahr verschiedene Blüten auf. Die Larven entwickeln sich in den Knospen von Kreuzblütlern. Anders als die zuvorgenannten Arten ist Rh. bipustulatus den eigentlichen Waldarten zuzurechnen. Er wird vor allem unter der Rinde abgestorbener Bäume gefunden, wo er als Räuber anderen Insekten nachstellt. 1993 fing sich fast ein Drittel der gefangenen Tiere in der Fangperiode P1a, das jährliche Maximum der Flugaktivität wurde jeweils in der Fangperiode P1b registriert. Desweiteren erreichten die Borkenkäferarten Xyloterus signatus und Xyl. domesticus und der Kurzdeckenflügler Philonthus cognatus höhere Anteile an den Fängen der Fangperiode P1a. Letztere gehört zu den häufigen Staphylinidenarten auf landwirtschaftlich genutzten Flächen, die im Frühjahr den Wald verläßt und im Herbst zur Überwinterung in diesen zurückkehrt.

Tabelle 4-85: Dominanzphänologie

\begin{tabular}{|c|c|c|c|c|c|c|c|c|c|}
\hline \multirow[b]{2}{*}{ ART } & \multicolumn{9}{|c|}{ Fangperiode } \\
\hline & P1a & P1b & $\mathbf{P 2}$ & $\mathbf{P 3}$ & P4 & P5 & P6 & P7 & P8 \\
\hline Aphodius prodromus & 11,02 & 2,60 & & & & & & & \\
\hline Rhizophagus bipustulatus & 11,02 & 4,49 & 1,38 & 1,46 & & & & & \\
\hline Meligethes aeneus & 10,24 & & & & & & & & \\
\hline Xyloterus signatus & 9,97 & 3,80 & & & & & & & \\
\hline Xyloterus domesticus & 5,51 & & 1,74 & 1,46 & & & & & \\
\hline Xantholinus longiventris & 4,20 & & & & & & & & \\
\hline Cortinicara gibbosa & 3,15 & 2,18 & & & & & & & \\
\hline Rhynchaenus fagi & 2,10 & & & & & & & & \\
\hline Corticarina fuscula & 1,31 & & & & & & & & \\
\hline Oulema melanopus & 1,31 & & & & & & & & \\
\hline Anotylus tetracarinatus & 1,31 & & & & & & & & \\
\hline Meligethes denticulatus & & 19,21 & 8,06 & & & & & & \\
\hline Xyleborus saxeseni & & 3,77 & & & & & & & \\
\hline Byturus tomentosus & & 3,21 & & & 1,66 & & & & \\
\hline Taphrorychus bicolor & & 2,60 & & 1,46 & & & & & \\
\hline Gabrius splendidulus & & 1,59 & & & & & & & \\
\hline Enicmus fungicola & & 2,58 & & & & & & & \\
\hline Hylecoetus dermestoides & & 1,46 & 12,95 & 5,74 & & & & & \\
\hline Athous subfuscus & & 2,74 & 7,53 & 6,69 & 1,72 & & & & \\
\hline Xyleborus dispar & & & 4,87 & 1,98 & 4,18 & 4,73 & & & \\
\hline Cerylon ferrugineum & & 1,49 & 3,62 & 4,35 & 4,24 & 2,53 & & & \\
\hline Athous haemorrhoidalis & & & 3,60 & 2,73 & & & & & \\
\hline Melanotus castanipes & & 1,62 & 2,98 & & & & & & \\
\hline Agriotes aterrimus & & 2,07 & 2,24 & & & & & & \\
\hline Corticarina similata & & & 1,93 & & & & & & \\
\hline Schizotus pectinicornis & & & 1,63 & & & & & & \\
\hline Rhizophagus perforatus & & & 1,38 & & & & & & \\
\hline Atomaria nigrirostris & & & 1,27 & & & & & & \\
\hline Rhizophagus dispar & & & & 4,95 & 2,63 & 2,72 & & & \\
\hline Euplectus bescidicus & & & & 2,53 & & & & & \\
\hline Denticollis linearis & & & 2,96 & 5,27 & & & & & \\
\hline Rhizophagus nitidulus & & & & 2,30 & & & & & \\
\hline
\end{tabular}




\begin{tabular}{|c|c|c|c|c|c|c|c|c|c|}
\hline \multirow[b]{2}{*}{ ART } & \multicolumn{9}{|c|}{ Fangperiode } \\
\hline & P1a & P1b & $\mathbf{P 2}$ & P3 & P4 & P5 & P6 & P7 & P8 \\
\hline Agriotes acuminatus & & & 1,93 & 2,18 & & & & & \\
\hline Agathidium confusum & & & 1,29 & 2,02 & & & & & \\
\hline Cis festivus & & & & 1,46 & & & & & \\
\hline Agathidium seminulum & & & & 1,43 & & & & & \\
\hline Leptusa pulchella & & & & & 8,37 & 5,90 & 2,88 & & \\
\hline Enicmus rugosus & & 2,21 & & & 4,13 & 5,19 & 3,44 & & \\
\hline Ptilinus pectinicornis & & & & & 3,59 & 1,30 & & & \\
\hline Enicmus testaceus & & 1,97 & 1,27 & 1,35 & 3,11 & 1,43 & 2,22 & & \\
\hline Aridius nodifer & & & & 1,74 & 2,68 & 3,57 & 3,66 & 2,97 & 4,95 \\
\hline Ptinella limbata & & & & & 1,82 & 11,09 & 12,42 & & \\
\hline Agathidium varians & & & & & 1,61 & 3,89 & 6,21 & 11,35 & 3,89 \\
\hline Ptinella aptera & & & & & & 3,37 & 1,55 & & \\
\hline Gyrophaena joyioides & & & & & & 1,69 & & & \\
\hline Antherophagus nigricornis & & & & & & 1,56 & & & \\
\hline Acrotrichis intermedia & & & & & & 1,56 & & & \\
\hline Bradycellus harpalinus & & & & & & & 5,10 & 4,59 & 2,83 \\
\hline Necrophorus vespilloides & & & & & & & 4,10 & 2,43 & \\
\hline Pteryx suturalis & & & & & & 2,14 & 2,66 & & \\
\hline Cryptophagus dentatus & & 1,78 & & & 1,98 & 1,43 & 2,11 & & \\
\hline Bibloporus bicolor & & & & & & & 2,00 & & \\
\hline Agaricochara latissima & & & & & & & & 9,73 & 8,83 \\
\hline Philonthus carbonarius & 1,84 & & & & & & 2,22 & 7,84 & 1,77 \\
\hline Phyllodrepa ioptera & & & & & & & & 4,59 & 1,41 \\
\hline Sphaeridium lunatum & & & & & & & 2,00 & 4,05 & \\
\hline Gyrophaena minima & & & & & & & & 3,78 & 2,83 \\
\hline Aphodius rufipes & & & & & & 1,75 & 1,33 & 2,97 & 1,41 \\
\hline Amischa analis & 1,31 & & & & & & 1,44 & 2,70 & 2,12 \\
\hline Phyllodrepoidea crenata & & & & & & & & 1,35 & 1,41 \\
\hline Anisotoma humeralis & & & & 1,27 & 1,56 & 1,36 & & 2,16 & \\
\hline Sphaeridium scarabaeoides & & & & & & & & 2,16 & \\
\hline Leptusa fumida & & & & & & & & 1,89 & \\
\hline Megasternum obscurum & & & & & & & & 1,35 & \\
\hline Cychramus luteus & & & & 1,98 & 4,24 & & & 1,89 & 28,98 \\
\hline Philonthus cognatus & 8,66 & & & & & & 2,11 & 4,86 & 5,65 \\
\hline Aphodius contaminatus & & & & & & & & & 5,65 \\
\hline Proteinus brachypterus & & & & & & & & & 3,18 \\
\hline Lordithon trinotatus & & & & & & & & & 2,12 \\
\hline Atheta amplicollis & & & & & & & & & 1,77 \\
\hline Ind.anteil Hauptarten & 72,97 & 61,37 & 62,61 & 54,35 & 47,53 & 57,20 & 57,43 & $\mathbf{7 2 , 7 0}$ & $\mathbf{7 8 , 8 0}$ \\
\hline
\end{tabular}




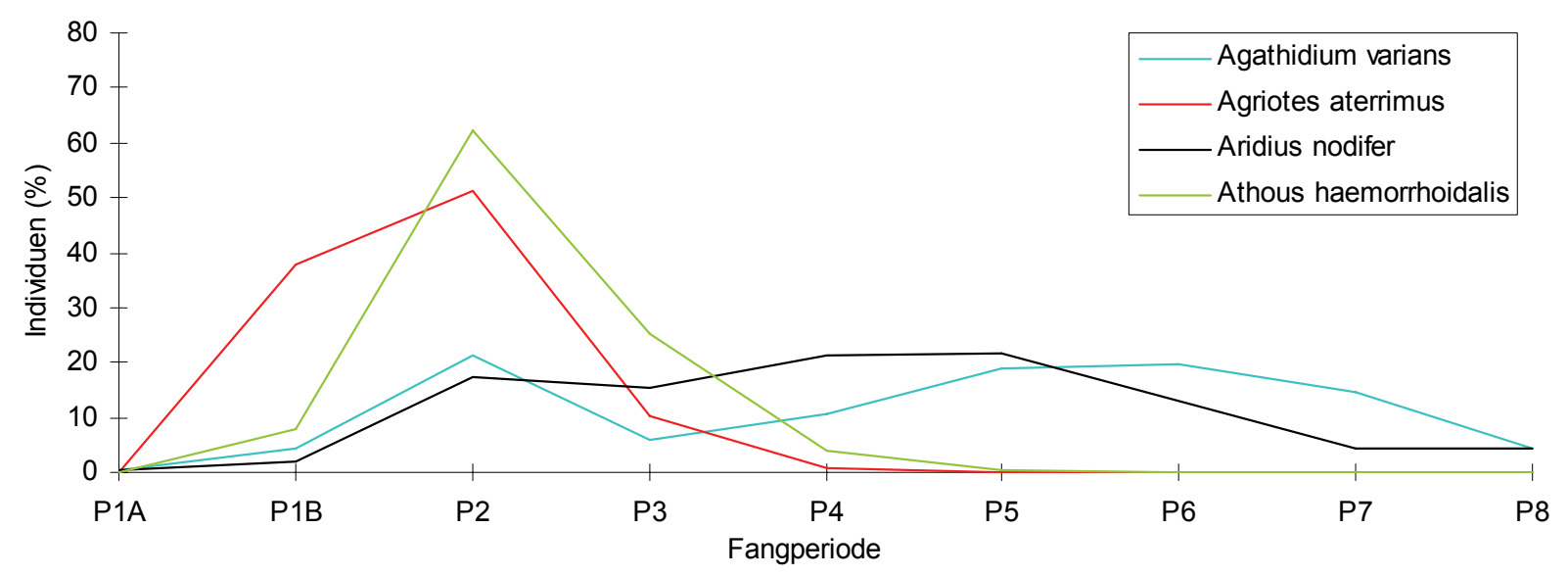

Abb. 4-55a: Flugphänologie dominierender Arten

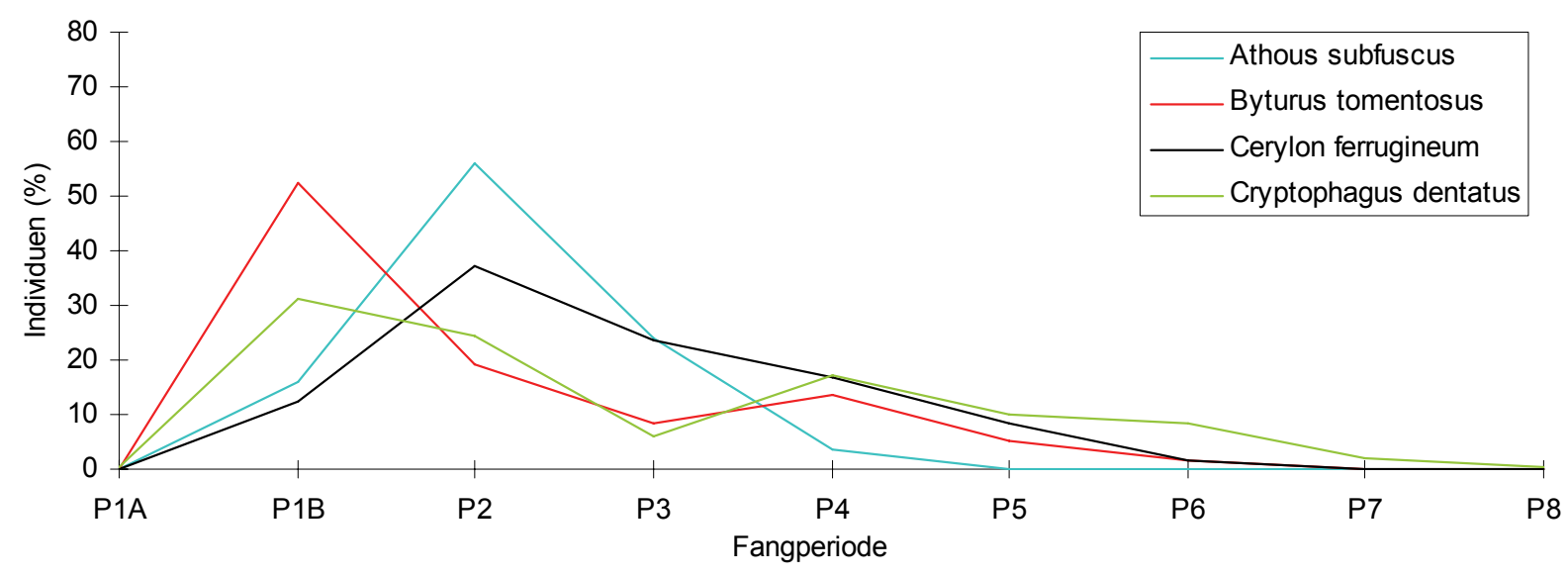

Abb. 4-55b: Flugphänologie dominierender Arten

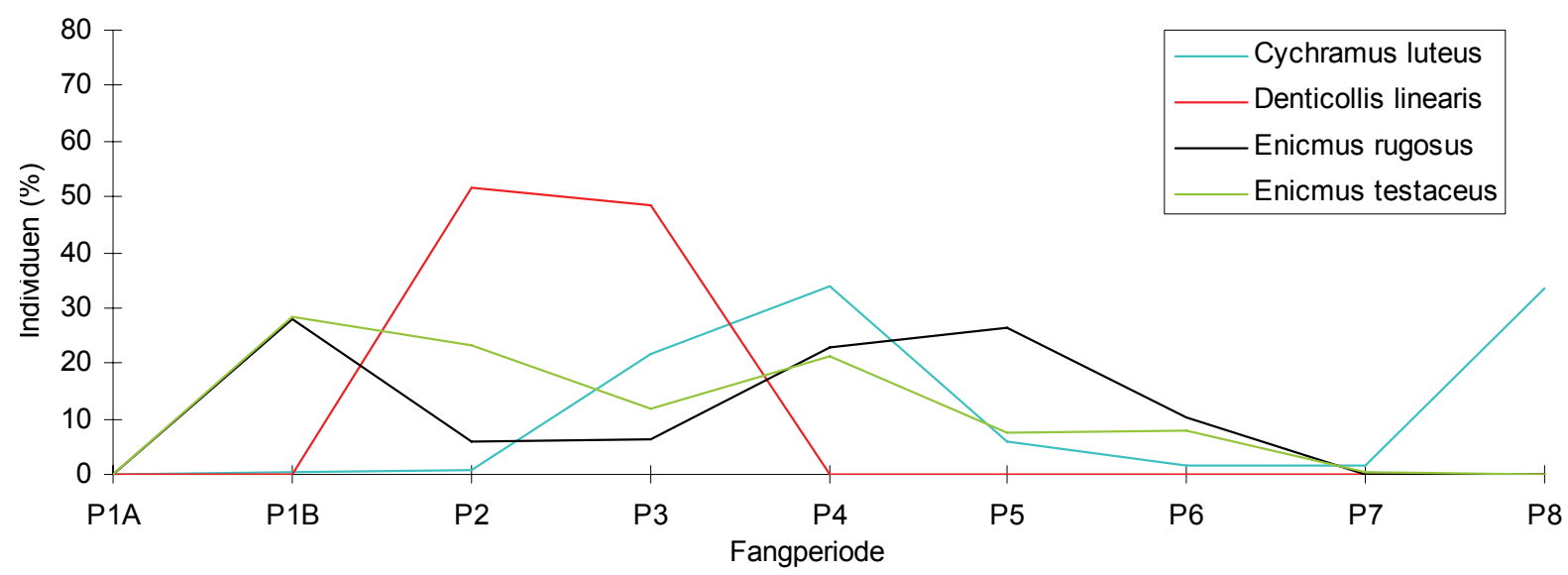

Abb. 4-55c: Flugphänologie dominierender Arten 


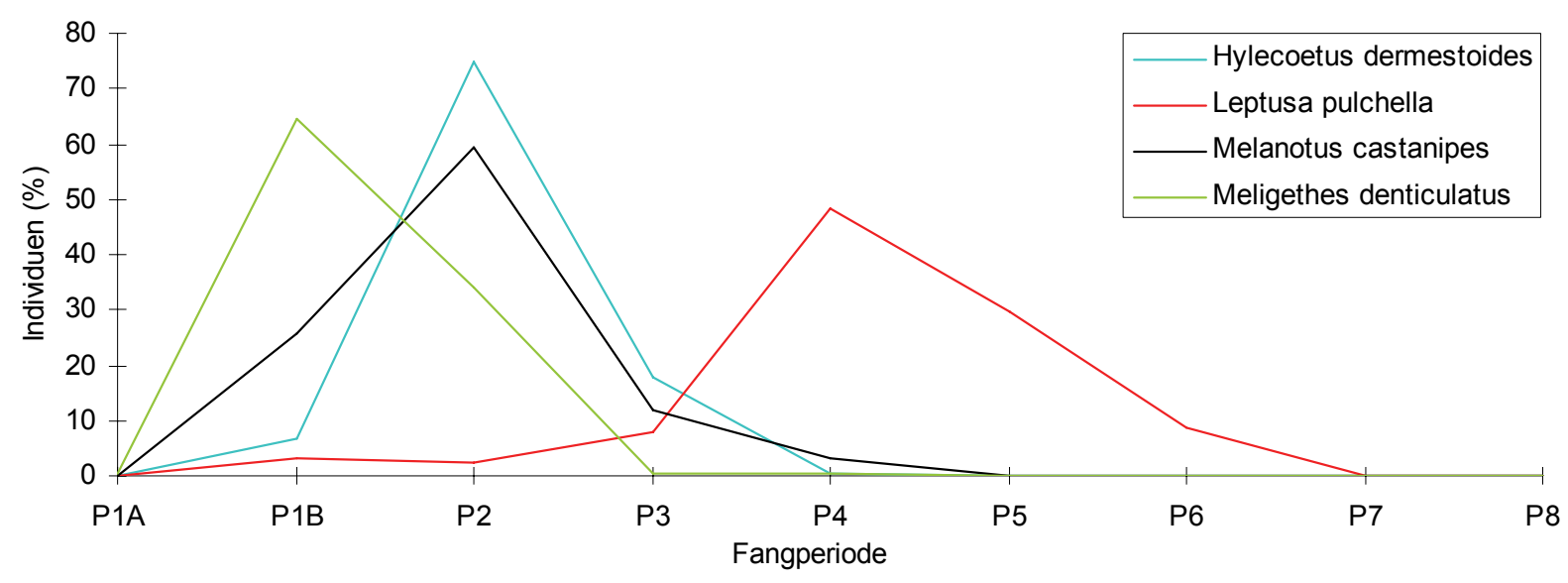

Abb. 4-55d: Flugphänologie dominierender Arten

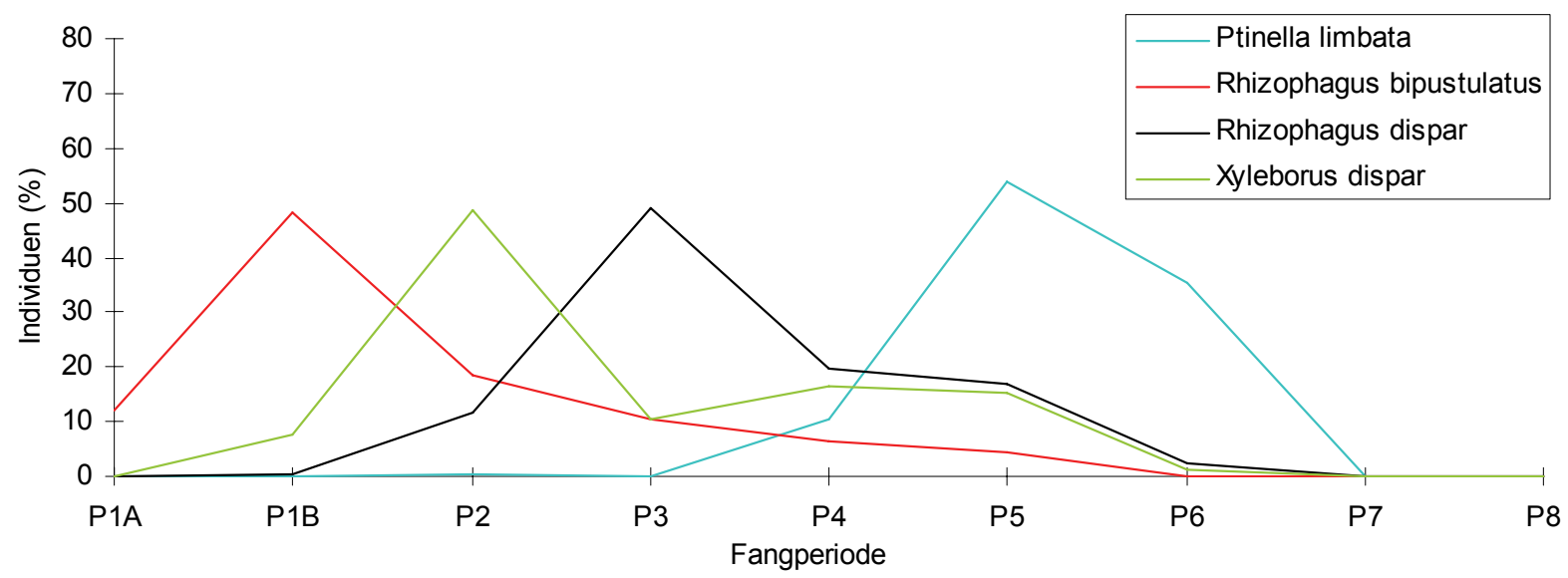

Abb. 4-55e: Flugphänologie dominierender Arten

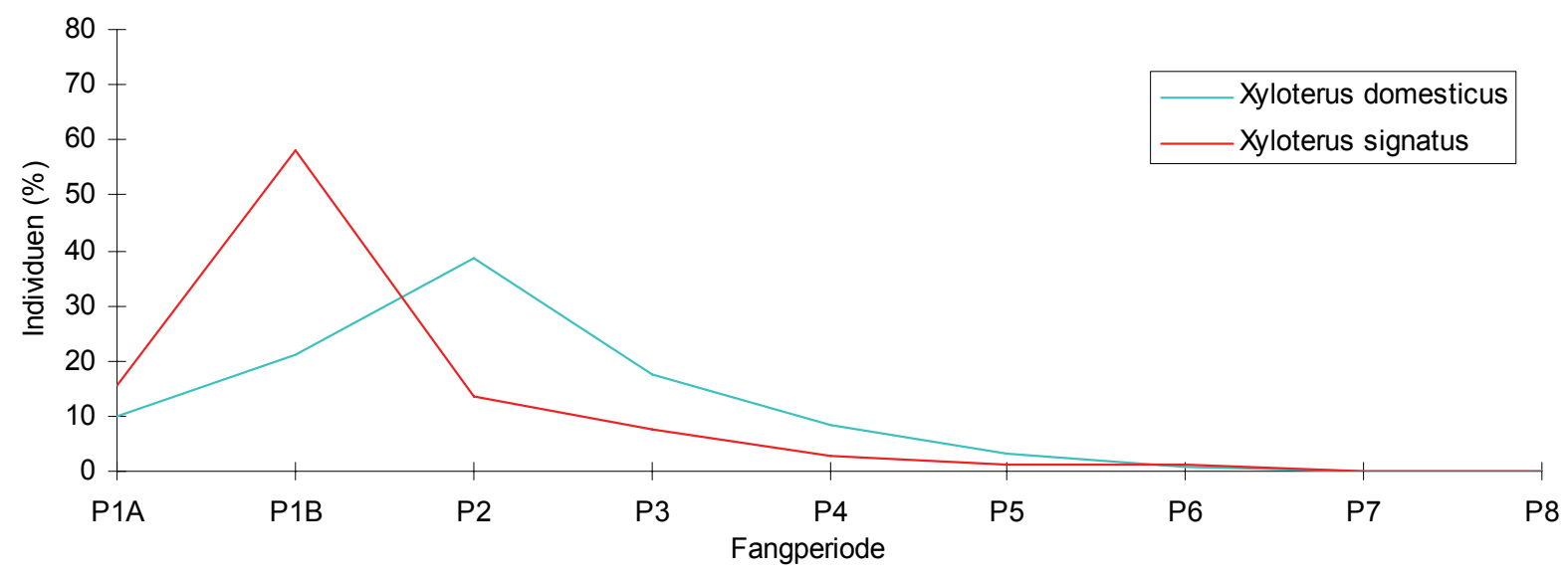

Abb. 4-55f: Flugphänologie dominierender Arten 
Meligethes denticulatus war in der Fangperiode P1b mit einem Anteil von 19,2\% die eindeutig vorherrschende Art. Der Käfer ist häufig in Wäldern, an Waldrändern und auf Lichtungen zu beobachten, wo sich die Larven in verschiedenen Rubus-Arten entwickeln. Sieht man vom letzten Untersuchungsjahr ab, konzentrierte sich die Flugaktivität zu 96 bzw. $95 \%$ auf den Zeitraum von Ende April bis Mitte Mai. Im letzten Jahr war das Aktivitätsmaximum verzögert, so dass $86 \%$ der Individuen in der darauffolgenden Fangperiode gefangen wurden. Mit einem Individuenanteil von 8,1 \% war M. denticulatus die zweithäufigste Art in der Fangperiode P2.

Die dominierende Art der Fangperiode P2 war Hylecoetus dermestoides mit einem Individuenanteil von 12,9 \%. Das Ergebnis wurde vor allem bestimmt durch das zahlreiche Auftreten dieser lignicolen Art im Jahr 1995, in dem sich der Anteil dieser Art in der Fangperiode P2 auf 25,0\% belief. Am Fang waren weibliche Tiere mit über $90 \%$ beteiligt. $75 \%$ der Flugaktivität entfielen auf die Fangperiode P2, wobei auch bei dieser Art für das Jahr 1996 ein etwas späteres Auftreten zu beobachten war. In der Fangperiode P3 trug H. dermestoides mit 5,7 \% zum Fangergebnis bei und war damit die zweithäufigste Art.

An den Fängen der Fangperiode P2 hatte Athous subfuscus einen Anteil von 7,5 \%, in der Fangperiode P3 war sie mit einem Individuenanteil von $6,7 \%$ die häufigste Art. Diese euryöke Waldart gehört zu den häufigsten Käferarten in Waldökosystemen. Die Imagines sind zahlreich auf der Vegetation zu beobachten. Die Larvenentwicklung findet vor allem im Boden statt, DERKSEN (1941) fand sie aber auch regelmäßig in Stümpfen. 54 \% der Flugaktivität wurden in der Fangperiode P2 registriert, $16 \%$ entfielen auf den vorangegangenen und $26 \%$ auf den sich anschließenden Fangzeitraum. Neben den beiden zuvor genannten Arten erreichten die Totholzkäferarten Denticollis linearis (5,3\%), Rhizophagus dispar $(4,9 \%)$ und Cerylon ferrugineum (4,4 \%) ebenfalls größere Anteile in der Fangperiode P3.

Als einzige eudominante Art in der Fangperiode P4 tratt Leptusa pulchella in Erscheinung. 1993 wurden $80 \%$ und $199570 \%$ des Jahresfanges in diesem Zeitraum festgestellt. 1996 wurde die Mehrzahl der Tiere in der folgenden Fangperiode gefangen, in der diese rindenbewohnende Staphylinidenart 5,9\% des Gesamtfanges stellte.

Ptinella limbata, ein mycetophager Totholzbewohner aus der Familie der Ptilidae, hatte in der Fangperiode P5 mit 11,1 \% den höchsten Individuenanteil. Mehr als die Hälfte der Individuen wurde in diesem Zeitraum gefangen. $35 \%$ der Tiere fingen sich in der Fangperiode P6, in der Pt. limbata mit einem Anteil von 12,4\% ebenfalls die dominierende Art war. In der Fangperiode P5 erreichte darüber hinaus Enicmus rugosus mit 5,2 \% einen nennenswerten Anteil am Fangergebnis.

Neben Pt. limbata erreichten in der Fangperiode P6 der Leiodidae Agathidium varians und der Carabidae Bradycellus harpalinus Anteile von über 6 bzw. 5 \%. Erstere Art konnte in allen Fangperioden in den Fensterfallen angetroffen werden. Nach einem ersten Aktivitätsmaximum in der Fangperiode P2 wurde in der zweiten Saisonhälfte ein erneuter Anstieg der Fangzahlen registriert. In der Fangperiode P7 entfielen 11,4 \% der gefangenen Käferindividuen auf diese Art. Die Hauptaktivität von B. harpalinus fiel in den August. Das Fangergebnis dieser Offenlandart beruhte vor allem auf den relativ hohen Fangzahlen eines Fallenstandortes aus den beiden ersten Untersuchungsjahren. Dieser befand sich unmittelbar an der Grenzlinie zwischen dem 'Neuenburger Urwald' und einer angrenzenden geräumten Windwurffläche. 
In den beiden letzten Fangperioden P7 und P8 wurden nur noch wenige Käferindividuen gefangen. Neben Adathidium varians erreichten die Kurzdeckenflügler Agaricochara latissima, Philonthus carbonarius und Ph. cognatus, Aphodius contaminatus aus der Familie der Scarabaeidae und der Nitidulidae Cychramus luteus höhere Anteile.

\subsubsection{Die trophische Struktur}

Die überwiegende Zahl der in Flugbarrierefallen nachgewiesenen Arten ernähren sich zoophag (Tab. 4-86, Abb. 4-56 u. 4-57). 223 räuberische Arten stellten gemeinsam 24,7 \% der Individuen. In den einzelnen Untersuchungsjahren lag die Artenzahl zwischen 121 und 159, während der Arten- und Individuenanteil nur geringfügig variierte. Die höchste Arten- und Individuenzahl wurde in den Fangperioden P1b und P2 registriert und ging in der Folgezeit kontinuierlich zurück (Tab. 4-87). Hinsichtlich der Artenzahl behielt diese Fraktion in allen Fangperioden ihre vorherrschende Stellung. Unter den zoophagen Arten hatten die Totholzbewohner, insbesondere corticole Arten, den größten Anteil. Sie stellten $37 \%$ der Arten und $72 \%$ der Individuen. Bodenbewohner trugen mit $29 \%$ der Arten und $16 \%$ der Individuen zum Fangergebnis dieses Konsumententyps bei. Unter den Arten mit räuberischer Ernährungsweise waren Cerylon ferrugineum, Rhizophagus bipustulatus und Rh. dispar die häufigsten.

Tabelle 4-86: Arten- und Individuenzahlen der Konsumententypen

\begin{tabular}{|l|rrrr|rrrr|}
\hline & \multicolumn{4}{|c|}{ A r t e n } & \multicolumn{4}{c|}{ I n d i v i d u e n } \\
Kons.-typ & $\mathbf{1 9 9 2 - 9 6}$ & $\mathbf{1 9 9 3}$ & $\mathbf{1 9 9 5}$ & $\mathbf{1 9 9 6}$ & $\mathbf{1 9 9 2 - 9 6}$ & $\mathbf{1 9 9 3}$ & $\mathbf{1 9 9 5}$ & $\mathbf{1 9 9 6}$ \\
\hline phytophag & 65 & 47 & 36 & 40 & 3375 & 1620 & 787 & 873 \\
mycetophag & 129 & 95 & 84 & 86 & 6410 & 1574 & 2853 & 1823 \\
saprophag & 52 & 43 & 31 & 28 & 601 & 328 & 94 & 146 \\
xylophag & 58 & 44 & 29 & 43 & 1408 & 528 & 411 & 457 \\
zoophag & 223 & 159 & 121 & 130 & 4044 & 1374 & 1391 & 1201 \\
o. Angabe & 35 & 23 & 15 & 16 & 520 & 140 & 157 & 179 \\
$\sum$ & $\mathbf{5 6 2}$ & $\mathbf{4 1 1}$ & $\mathbf{3 1 6}$ & $\mathbf{3 4 3}$ & $\mathbf{1 6 3 5 8}$ & $\mathbf{5 5 6 4}$ & $\mathbf{5 6 9 3}$ & $\mathbf{4 6 7 9}$ \\
\hline
\end{tabular}

Mit 39,2 \% erreichten die 129 mycetophagen Arten den höchsten Individuenanteil. Der Artenanteil lag in den einzelnen Untersuchungsjahren zwischen 23,1 und 26,6 \%, gleichzeitig variierte der Individuenanteil zwischen 28,3 \% im Jahr 1993 und 50,1 \% im vorletzten Untersuchungsjahr. Bis Mitte Juli blieb die Artenzahl in den einzelnen Fangperioden annähernd konstant und sank danach bis zum Ende der Vegetationszeit ab. Das Maximum gefangener Individuen dieses Konsumententyps fiel in die Fangperiode P2, der höchste Individuenanteil konnte in der Fangperiode P5 verzeichnet werden. $47 \%$ der in Fensterfallen gefangenen mycetophagen Arten und $64 \%$ der Individuen zeigen eine Präferenz für Totholz. Unter diesen waren die wenigen lignicolen pilzzüchtenden Arten, insbesondere Hylecoetus dermestoides und Xyleborus dispar, in größerer Individuenzahl vertreten als die artenreiche Gruppe der Totholzpilzkäfer. 
Sowohl hinsichtlich der Artenzahl als auch der Individuenzahl waren die Phytophagen die dritthäufigste Konsumentengruppe. Die 65 Arten stellten gemeinsam 20,6 \% der gefangenen Tiere. Während ihr Individuenanteil zwischen 14 und $29 \%$ in den Untersuchungsjahren variierte, blieb ihr Artenanteil annähernd konstant. Besonders zu Beginn der Vegetationszeit waren Phytophage zahlreich vertreten, in der Fangperiode P1b hatten sie einen Individuenanteil von $33 \%$. Unter den Arten mit vorwiegend phytophager Lebensweise dominierten Meligethes denticulatus und Athous subfuscus.

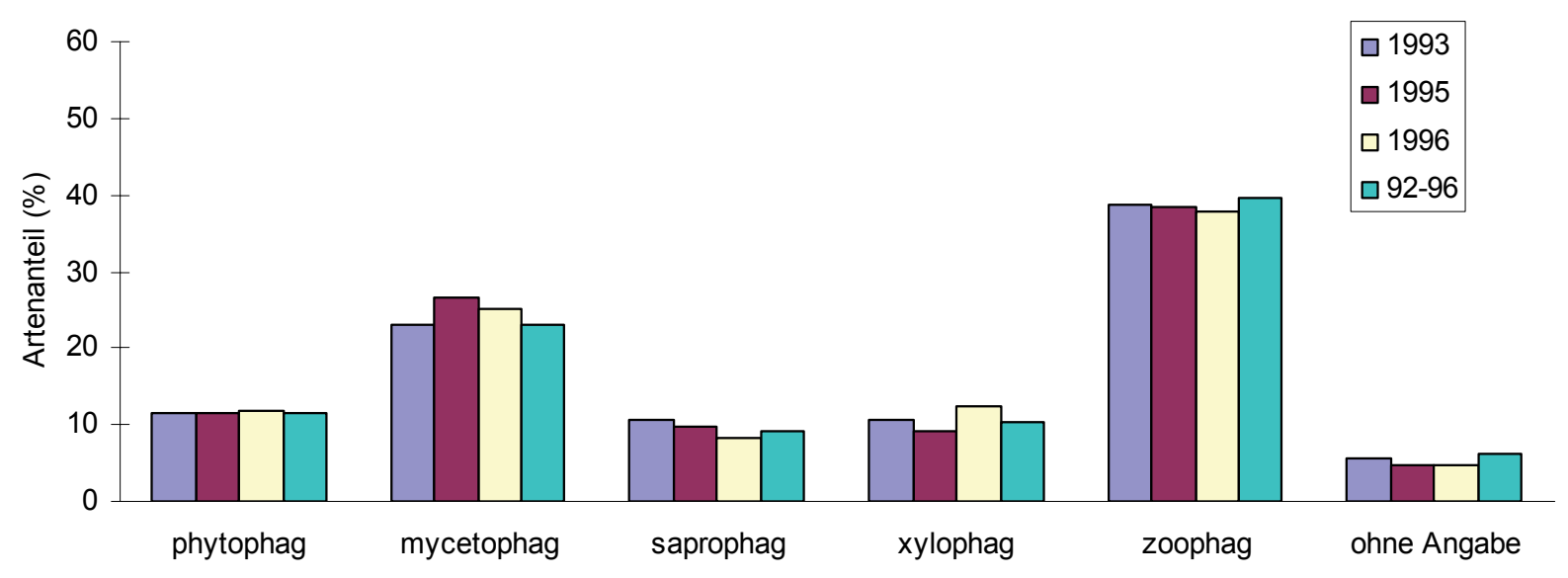

Abb. 4-56: Artenanteile der Konsumententypen

Jede zehnte Art und 8,6 \% der Individuen waren dem xylophagen Ernährungstyp zuzurechnen. Ihr jährlicher Individuenanteil variierte zwischen 7,2 und 9,8\%, die Artenzahl zwischen 29 und 44. Die höchste Artenzahl und der höchste Artenanteil wurden im Juni (P3) registriert. In dieser Fangperiode erreichte auch der Individuenanteil mit 15,9 \% sein Maximum, während die höchste Individuenzahl bereits in der vorangegangenen Fangperiode (P2) gefangen wurde. Die beiden Schnellkäferarten Denticollis linearis und Melanotus castanipes und der Borkenkäfer Taphrorychus bicolor hatten die größten Anteile an diesem Konsumententyp.

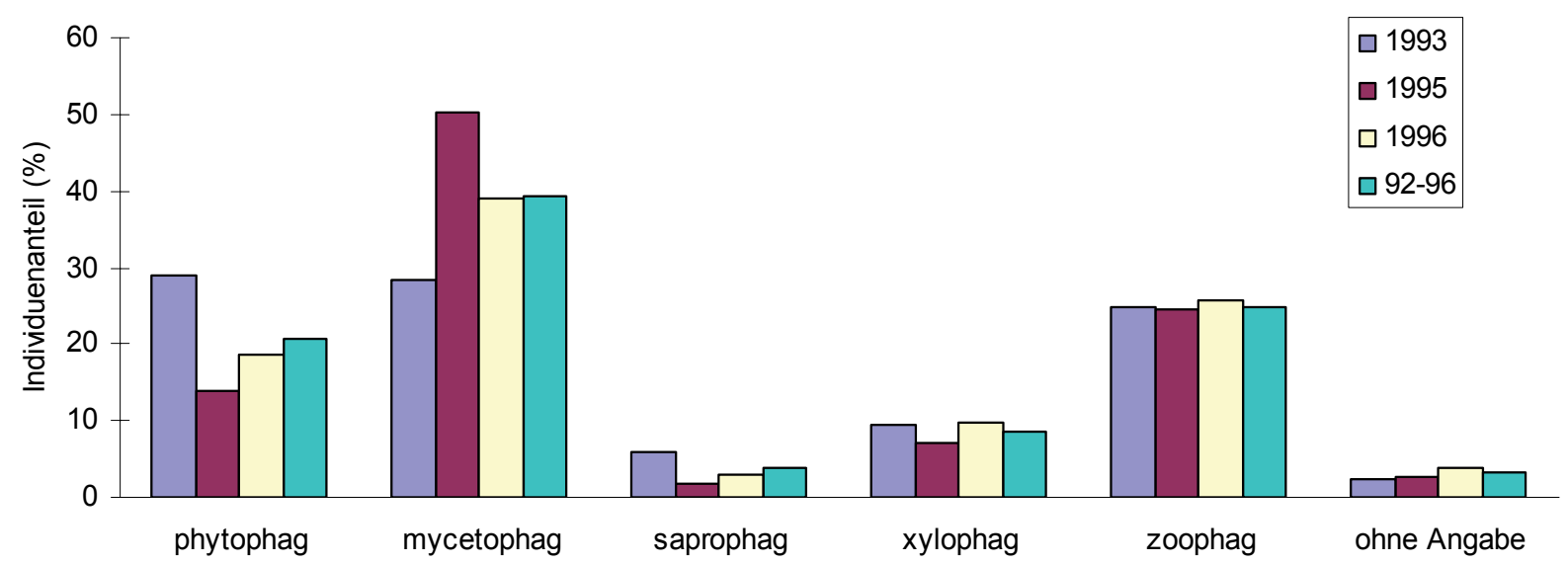

Abb. 4-57: Individuenanteile der Konsumententypen 
Mit 52 Arten stellten die Saprophagen nur 3,7\% der Individuen. Mit fast $6 \%$ war der Individuenanteil 1993 am höchsten und 1995 mit 1,7 \% am niedrigsten. 1993 war auch das einzige Untersuchungsjahr, in dem mit Aphodius prodromus eine saprophage Art den Status einer Hauptart $(1,9 \%)$ erreichte.

Für 35 Arten mit einem Individuenanteil von 3,2 \% konnte keine Zuordnung zu einem der aufgeführten Konsumentengilden getroffen werden.

Tabelle 4-87: Arten- und Individuenanteile der Konsumententypen in den Fangperioden

\begin{tabular}{|c|c|c|c|c|c|c|c|c|c|c|}
\hline & \multicolumn{9}{|c|}{ Fangperiode } \\
\hline & & \multirow{2}{*}{$\frac{\text { P1a }}{10}$} & \multirow{2}{*}{$\frac{\text { P1b }}{35}$} & \multirow{2}{*}{$\frac{\mathbf{P 2}}{32}$} & \multirow{2}{*}{$\frac{\mathbf{P 3}}{31}$} & \multirow{2}{*}{$\frac{\text { P4 }}{28}$} & \multirow{2}{*}{$\frac{\text { P5 }}{22}$} & \multirow{2}{*}{$\frac{\text { P6 }}{12}$} & \multirow{2}{*}{$\frac{\mathbf{P 7}}{11}$} & \multirow{2}{*}{ P8 } \\
\hline Ar- & phytophag & & & & & & & & & \\
\hline \multirow{6}{*}{$\begin{array}{l}\text { ten- } \\
\text { zahl }\end{array}$} & mycetophag & 24 & 69 & 69 & 69 & 73 & 62 & 52 & 27 & 15 \\
\hline & saprophag & 12 & 23 & 27 & 15 & 17 & 17 & 16 & 13 & 10 \\
\hline & xylophag & 1 & 18 & 28 & 36 & 29 & 22 & 9 & 1 & 0 \\
\hline & zoophag & 29 & 114 & 113 & 101 & 86 & 78 & 71 & 32 & 24 \\
\hline & o. Angabe & 2 & 16 & 13 & 10 & 12 & 10 & 7 & 6 & 4 \\
\hline & $\sum$ & 78 & 275 & 282 & 262 & 245 & 211 & 167 & 90 & 57 \\
\hline \multirow{6}{*}{$\begin{array}{l}\text { Ar- } \\
\text { ten- } \\
\text { an- } \\
\text { teil }\end{array}$} & phytophag & 12,8 & 12,7 & 11,3 & 11,8 & 11,4 & 10,4 & 7,2 & 12,2 & 7,0 \\
\hline & mycetophag & 30,8 & 25,1 & 24,5 & 26,3 & 29,8 & 29,4 & 31,1 & 30,0 & 26,3 \\
\hline & saprophag & 15,4 & 8,4 & 9,6 & 5,7 & 6,9 & 8,1 & 9,6 & 14,4 & 17,5 \\
\hline & xylophag & 1,3 & 6,5 & 9,9 & 13,7 & 11,8 & 10,4 & 5,4 & 1,1 & 0,0 \\
\hline & zoophag & 37,2 & 41,5 & 40,1 & 38,5 & 35,1 & 37,0 & 42,5 & 35,6 & 42,1 \\
\hline & o. Angabe & 2,6 & 5,8 & 4,6 & 3,8 & 4,9 & 4,7 & 4,2 & 6,7 & 7,0 \\
\hline \multirow{7}{*}{$\begin{array}{l}\text { In- } \\
\text { di- } \\
\text { vi- } \\
\text { du- } \\
\text { en- } \\
\text { zahl }\end{array}$} & phytophag & 68 & 1237 & 1283 & 448 & 160 & 69 & 69 & 30 & 11 \\
\hline & mycetophag & 104 & 1144 & 1846 & 834 & 834 & 875 & 467 & 151 & 155 \\
\hline & saprophag & 63 & 142 & 61 & 40 & 46 & 67 & 84 & 56 & 42 \\
\hline & xylophag & 3 & 260 & 456 & 401 & 193 & 74 & 18 & 3 & 0 \\
\hline & zoophag & 140 & 941 & 1053 & 766 & 432 & 341 & 212 & 95 & 64 \\
\hline & o. Angabe & 3 & 40 & 27 & 37 & 199 & 116 & 52 & 35 & 11 \\
\hline & $\Sigma$ & 381 & 3764 & 4726 & 2526 & 1864 & 1542 & 902 & 370 & 283 \\
\hline \multirow{6}{*}{$\begin{array}{l}\text { In- } \\
\text { di- } \\
\text { vi- } \\
\text { du- } \\
\text { en- } \\
\text { an- } \\
\text { teil }\end{array}$} & phytophag & 17,8 & 32,9 & 27,1 & 17,7 & 8,6 & 4,5 & 7,6 & 8,1 & 3,9 \\
\hline & mycetophag & 27,3 & 30,4 & 39,1 & 33,0 & 44,7 & 56,7 & 51,8 & 40,8 & 54,8 \\
\hline & saprophag & 16,5 & 3,8 & 1,3 & 1,6 & 2,5 & 4,3 & 9,3 & 15,1 & 14,8 \\
\hline & xylophag & 0,8 & 6,9 & 9,6 & 15,9 & 10,4 & 4,8 & 2,0 & 0,8 & 0,0 \\
\hline & zoophag & 36,7 & 25,0 & 22,3 & 30,3 & 23,2 & 22,1 & 23,5 & 25,7 & 22,6 \\
\hline & o. Angabe & 0,8 & 1,1 & 0,6 & 1,5 & 10,7 & 7,5 & 5,8 & 9,5 & 3,9 \\
\hline
\end{tabular}




\subsubsection{Biotopkomplexpräferenzen}

$53,7 \%$ der Arten und 68,3\% der Individuen wurden von Arten gestellt, die vor allem in Waldbiotopen angetroffen werden (Tab. 4-88, Abb. 4-58 u. 4-59). Die Artenanteile in den einzelnen Untersuchungsjahren variierten nur geringfügig (56,0 bis 59,5\%), etwas stärker waren die Schwankungen bei den Individuenanteilen (61,7 bis 73,9 \%). Die meisten Arten und Individuen wurden in der Fangperiode P2 von Mitte Mai bis Anfang Juni registriert. Die Arten- und Individuenanteile in den einzelnen Fangperioden zeigten im Jahresverlauf eine stetige Zunahme bis in den Juni, in dem die Waldarten 65,9\% der Arten und 82,4\% der Individuen stellten. Danach war ein kontinuierlicher Rückgang zu verzeichnen.

Tabelle 4-88: Arten- und Individuenzahlen der Biotopkomplexpräferenztypen

\begin{tabular}{|l|rrrr|rrrr|}
\hline & \multicolumn{5}{|c|}{ A r t e n } & \multicolumn{4}{c|}{ I n d i v i u e n } \\
Biotoptyp & $\mathbf{1 9 9 2 - 9 6}$ & $\mathbf{1 9 9 3}$ & $\mathbf{1 9 9 5}$ & $\mathbf{1 9 9 6}$ & $\mathbf{1 9 9 2 - 9 6}$ & $\mathbf{1 9 9 3}$ & $\mathbf{1 9 9 5}$ & $\mathbf{1 9 9 6}$ \\
\hline Eurytop & 175 & 125 & 89 & 98 & 4497 & 1835 & 1281 & 1235 \\
Feuchtbiotope & 55 & 35 & 31 & 25 & 328 & 158 & 120 & 48 \\
Offenlandbiotope & 29 & 21 & 11 & 15 & 365 & 140 & 83 & 90 \\
Waldbiotope & 302 & 230 & 185 & 204 & 11167 & 3431 & 4209 & 3305 \\
\hline
\end{tabular}

Die Bewohner verschiedener Offenlandbiotope wurden mit 29 Arten $(5,2 \%)$ und einem Individuenanteil von 2,2 \% festgestellt. In den Untersuchungsjahren wurden Artenanteile von 3,5 bis 5,1\% und Individuenanteile von 1,5 bis 2,5\% registriert, von denen allein auf Agriotes acuminatus 1,1\% entfielen. Dieser Elateridae war häufig an Waldinnen- und -außenrändern, sowie auf den zahlreichen Lichtungen des Bestandes zu beobachten. Im Spätsommer war der Laufkäfer Bradycellus harpalinus mit höheren Anteilen an den Fängen beteiligt.

Arten mit einem Verbreitungsschwerpunkt in Feuchtbiotopen verschiedenster Art stellten $2,0 \%$ der Individuen, aber 9,8 \% der Arten. Die Anteile der Individuen in den Einzeljahren beliefen sich auf 2,8, 2,1 und 1,0\%, die der Arten auf 8,5, 9,8 und 7,3\%. Sowohl die Artenals auch die Individuenzahl waren von Beginn der Fangsaison an bis zu dessen Ende rückläufig. In ihrer Mehrheit traten die Arten nur in Einzelexemplaren in Erscheinung. Begünstigt wird ihr Vorkommen im 'Neuenburger Urwald' durch mehrere feuchte Senken, die teilweise auch in den Sommermonaten nicht austrocknen. Sie sind Entwicklungsort u. a. von Agabus chalconotus, der häufigsten Feuchtbiotopart des Untersuchungsgebietes. 


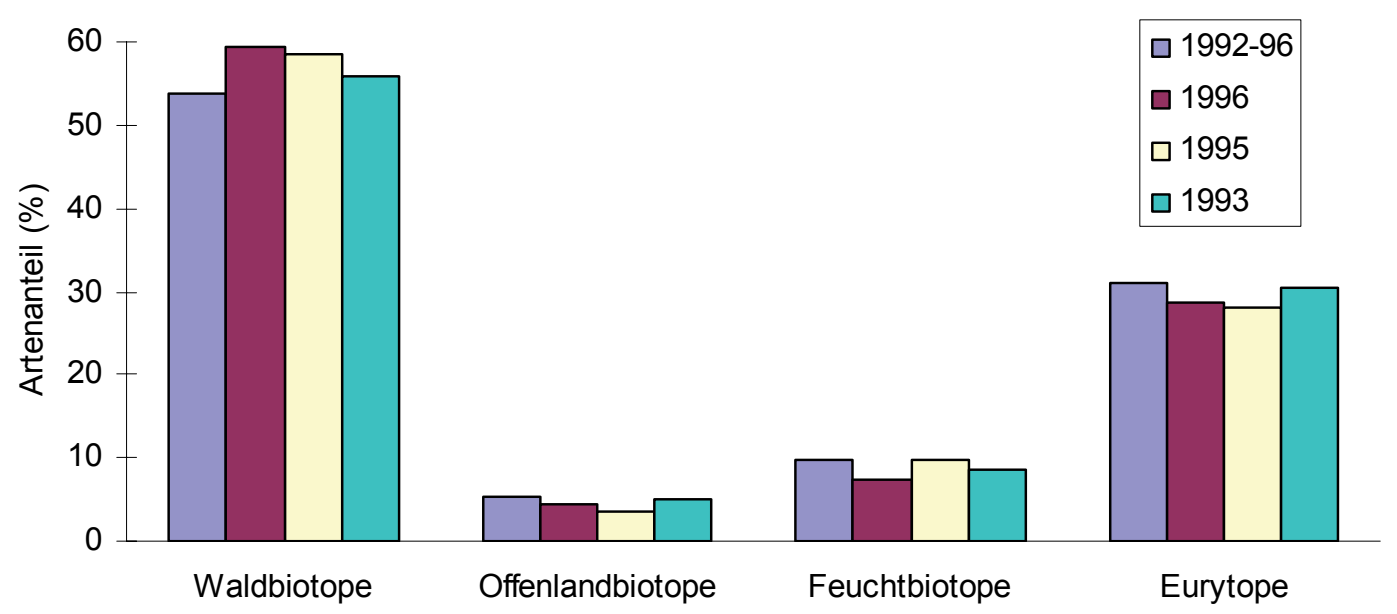

Abb. 4-58: Artenanteil der Biotoppräferenztypen

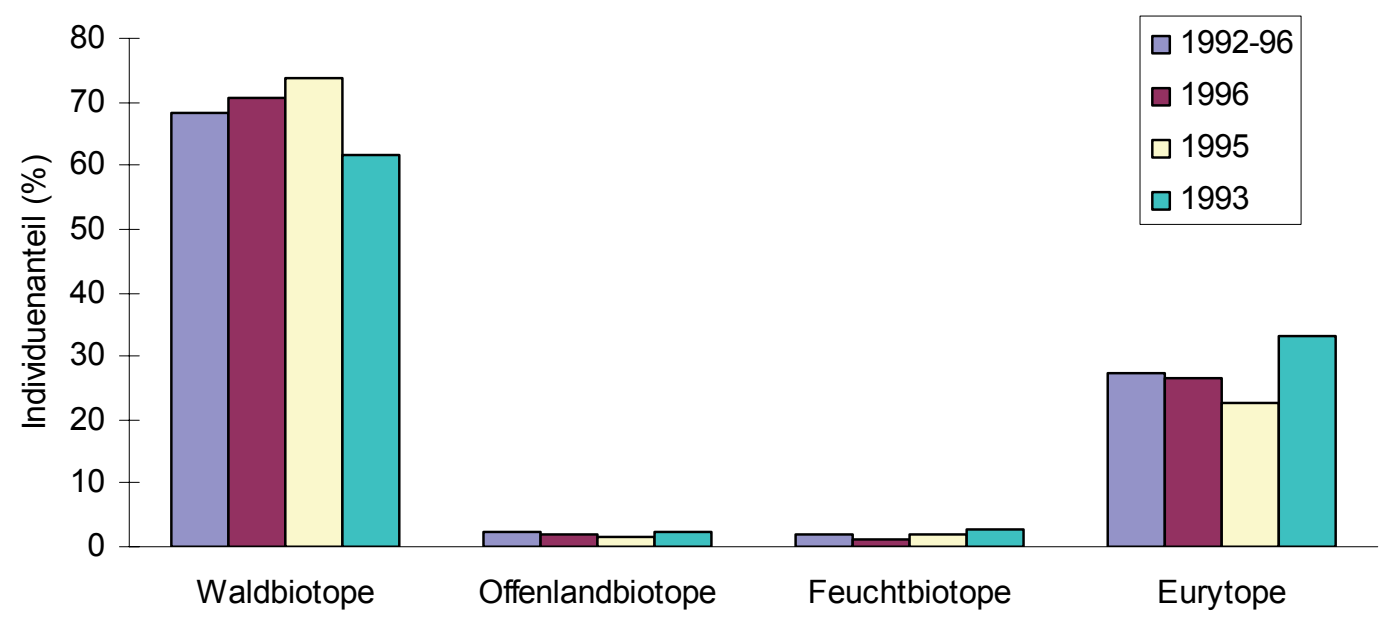

Abb. 4-59: Individuenanteil der Biotoppräferenztypen

Mit 4497 Individuen (27,5\%) aus 175 Arten (31,2\%) waren eurytope Arten zahlreich an den Fensterfallenfängen beteiligt. Der Artenanteil in den Untersuchungsjahren lag zwischen 28,2 und 30,4 \%, der Individuenanteil zwischen 22,5 und 33,0 \%. Die maximale Arten- und Individuenzahl wurde in der Fangperiode P1b registriert. Ihre relativen Anteile waren im Saisonverlauf zunächst rückläufig bis zur Fangperiode P3 und zeigten danach eine wieder ansteigende Tendenz bis zum Saisonende. Häufigster Vertreter dieser Gruppe war in allen Untersuchungsjahren Meligethes denticulatus. 


\subsubsection{Habitatpräferenzen}

Bewohner der Vegetation waren mit 3362 Individuen (20,6 \%) und 67 Arten (11,9\%) in den Fängen vertreten (Tab. 4-89, Abb. 4-60 u. 4-61). Ihr Artenanteil blieb in den Untersuchungsjahren sehr konstant und variierte um nur 0,5\%. Deutlichere Schwankungen zeigte dagegen ihr jährlicher Individuenanteil. Der höchste Wert wurde im Jahr 1993 mit 29,3 \% erreicht, der niedrigste 1995 mit 14,0 \%. Die höchsten Individuenzahlen und -anteile wurden im Zeitraum von April bis Anfang Juni (P1b u. P2) gezählt (Tab. 4-90). Der Individuenanteil dieser Fraktion in den Untersuchungsjahren und den einzelnen Fangperioden war in erster Linie durch zwei Arten, nämlich Meligethes denticulatus und Athous subfuscus, geprägt.

Bodenbewohnende Arten hatten in den Untersuchungsjahren Individuenanteile von 7,0 bis 7,9\%, während die Artenzahl zwischen 48 und 70 variierte. Insgesamt wurden 97 bodenbewohnende Arten - das entspricht einem Artenanteil von 17,3 \% - festgestellt. Sowohl Arten- als auch Individuenzahl zeigten während der Vegetationsperiode eine rückläufige Tendenz bis in den Juli (P4). Anschließend erfolgte eine geringfügige Zunahme in den Fangperioden P5 und P6, um danach bis zum Ende des jährlichen Untersuchungszeitraumes wieder deutlich zurückzugehen. Keine der bodenbewohnenden Arten erreichte an den Fängen der Fensterfallen einen hohen Anteil. Vertreten war diese Fraktion in den Fensterfallen in erster Linie durch Agathidium confusum.

Tabelle 4-89: Arten und Individuenzahlen der Habitatpräferenztypen

\begin{tabular}{|c|c|c|c|c|c|c|c|c|c|}
\hline \multirow{2}{*}{\multicolumn{2}{|c|}{ Habitat }} & \multicolumn{4}{|c|}{ Arten } & \multicolumn{4}{|c|}{ Individuen } \\
\hline & & $1992-96$ & 1993 & 1995 & 1996 & $1992-96$ & 1993 & 1995 & 1996 \\
\hline \multirow{4}{*}{\multicolumn{2}{|c|}{$\begin{array}{l}\text { Vegetation } \\
\text { Boden } \\
\text { Nester } \\
\text { Faulstoffe } \\
\end{array}$}} & 67 & 50 & 37 & 41 & 3362 & 1630 & 796 & 879 \\
\hline & & 97 & 70 & 48 & 49 & 1280 & 442 & 425 & 326 \\
\hline & & 19 & 14 & 7 & 9 & 132 & 64 & 14 & 53 \\
\hline & & 103 & 67 & 48 & 51 & 729 & 366 & 145 & 179 \\
\hline \multirow{6}{*}{$\begin{array}{l}\mathbf{T} \\
\mathbf{o} \\
\mathbf{t} \\
\mathbf{h} \\
\mathbf{o} \\
\mathbf{l} \\
\mathbf{z}\end{array}$} & $\mathbf{T}$ & 54 & 42 & 27 & 40 & 2717 & 470 & 1678 & 554 \\
\hline & TR & 63 & 49 & 50 & 54 & 2947 & 816 & 1143 & 947 \\
\hline & $\mathbf{T M}$ & 43 & 30 & 24 & 31 & 1643 & 442 & 495 & 678 \\
\hline & TP & 48 & 41 & 31 & 29 & 1547 & 577 & 339 & 567 \\
\hline & TS & 1 & 0 & 0 & 1 & 1 & 0 & 0 & 1 \\
\hline & $\sum$ Totholz & 209 & 162 & 132 & 155 & 8855 & 2305 & 3655 & 2747 \\
\hline \multirow{4}{*}{\multicolumn{2}{|c|}{$\begin{array}{l}\text { Pilze } \\
\text { Wasser } \\
\text { o. Präferenz } \\
\sum\end{array}$}} & 28 & 19 & 16 & 17 & 869 & 352 & 191 & 293 \\
\hline & & 16 & 12 & 12 & 6 & 160 & 103 & 47 & 10 \\
\hline & & 23 & 17 & 16 & 15 & 971 & 302 & 420 & 192 \\
\hline & & 562 & 411 & 316 & 343 & 16358 & 5564 & 5693 & 4679 \\
\hline
\end{tabular}


103 Arten (18,3 \%) gelten als Präferenten für Faulstoffe. Zusammen erreichten sie aber nur einen Anteil von 4,5\% an den gefangenen Individuen. Der vergleichsweise höhere Individuenanteil von 6,6 \% im Jahr 1993 war u. a. auf die in diesem Jahr verlängerte Fangzeit zurückzuführen. In der Fangperiode P1a waren 19,2 \% der Arten und 17,1 \% der Individuen den Faulstoffbewohnern zuzurechnen. Läßt man die Fänge dieser Fangperiode außen vor, betrug der Individuenanteil dieser Käfergruppe 5,8 \%. Der niedrigste Wert wurde 1995 mit 2,5 \% registriert. Sowohl Arten- als auch Individuenzahl waren im Frühjahr am höchsten. Im zeitigen Frühjahr war Aphodius prodromus die häufigste Art unter den Faulstoffbewohnern, während ab dem Spätsommer Aphodius rufipes vermehrt in Erscheinung trat.

Pilzbewohner waren mit 28 Arten in den Fensterfallen vertreten, wobei die an Totholzpilze gebundenen Arten hier nicht berücksichtigt sind. Diese wurden wegen ihrer Abhängigkeit von Totholz diesem Habitat zugeordnet. Die fungicolen Arten waren mit einem Anteil von $5 \%$ sowohl an der Arten- als auch der Individuenzahl beteiligt. Der Individuenanteil variierte zwischen 3,4 \% im Jahr 1995 und 6,3 \% in den Jahren 1993 und 1996. Die höchsten Individuenzahlen wurden in den Fangperioden P4 und P5 registriert, der Individuenanteil der Pilzbewohner kletterte in diesem Zeitraum auf über $10 \%$. Die drei häufigsten pilzbewohnenden Arten, und zwar auch in den einzelnen Untersuchungsjahren, waren Enicmus rugosus, Cychramus luteus und Agathidium seminulum.

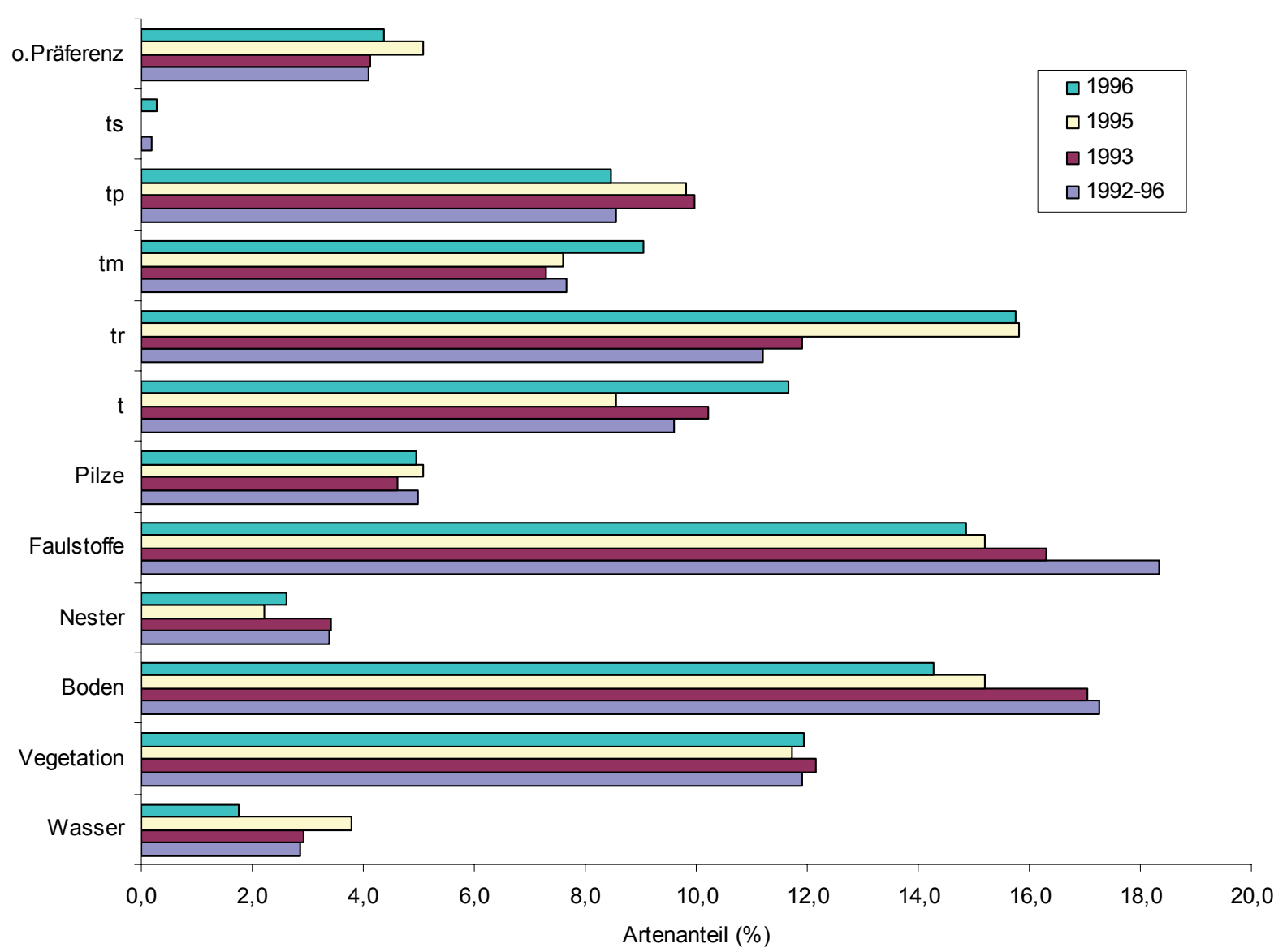

Abb. 4-60: Artenanteil der Habitatpräferenztypen 
Sowohl die Nest- als auch die Wasserbewohner hatten mit nur 19 bzw. 16 Arten einen geringen Anteil an den gefangenen Individuen. Für 23 Arten läßt sich keine Präferenz für bestimmte Lebensstätten erkennen. Sie sind regelmäßig in verschiedenen Habitaten zu finden und auch ihre Häufigkeit gibt keinen Hinweis auf Präferenzen. $4 \%$ der Arten und $6 \%$ der Individuen waren dieser Fraktion zuzurechnen. Am häufigsten fanden sich Individuen von Aridius nodifer und Cryptophagus dentatus in den Fallen, zwei mycetophage Arten, die auch regelmäßig an totem Holz zu finden sind.

Die Bewohner des Totholzes stellten sowohl hinsichtlich der Arten- als auch der Individuenzahl die größte Fraktion in den Fensterfallenfängen des 'Neuenburger Urwaldes'. 38 obligate Totholzarten wurden ausschließlich mit dieser Methode nachgewiesen. Insgesamt verteilten sich 8855 Individuen (54,1\%) auf 209 Arten (37,2 \%). Der Artenanteil an den Jahresfängen variierte zwischen 39,4 und 45,2 \%, der Individuenanteil zwischen 41,4 und 64,2 \%. Die höchste Individuenzahl wurde in der Fangperiode P2 registriert. In der Folgezeit gingen die Fänge kontinuierlich zurück. Artenzahl (131), Artenanteil (50 \%) und Individuenanteil (63\%) erreichten ihr Maximum im Juni (P3).

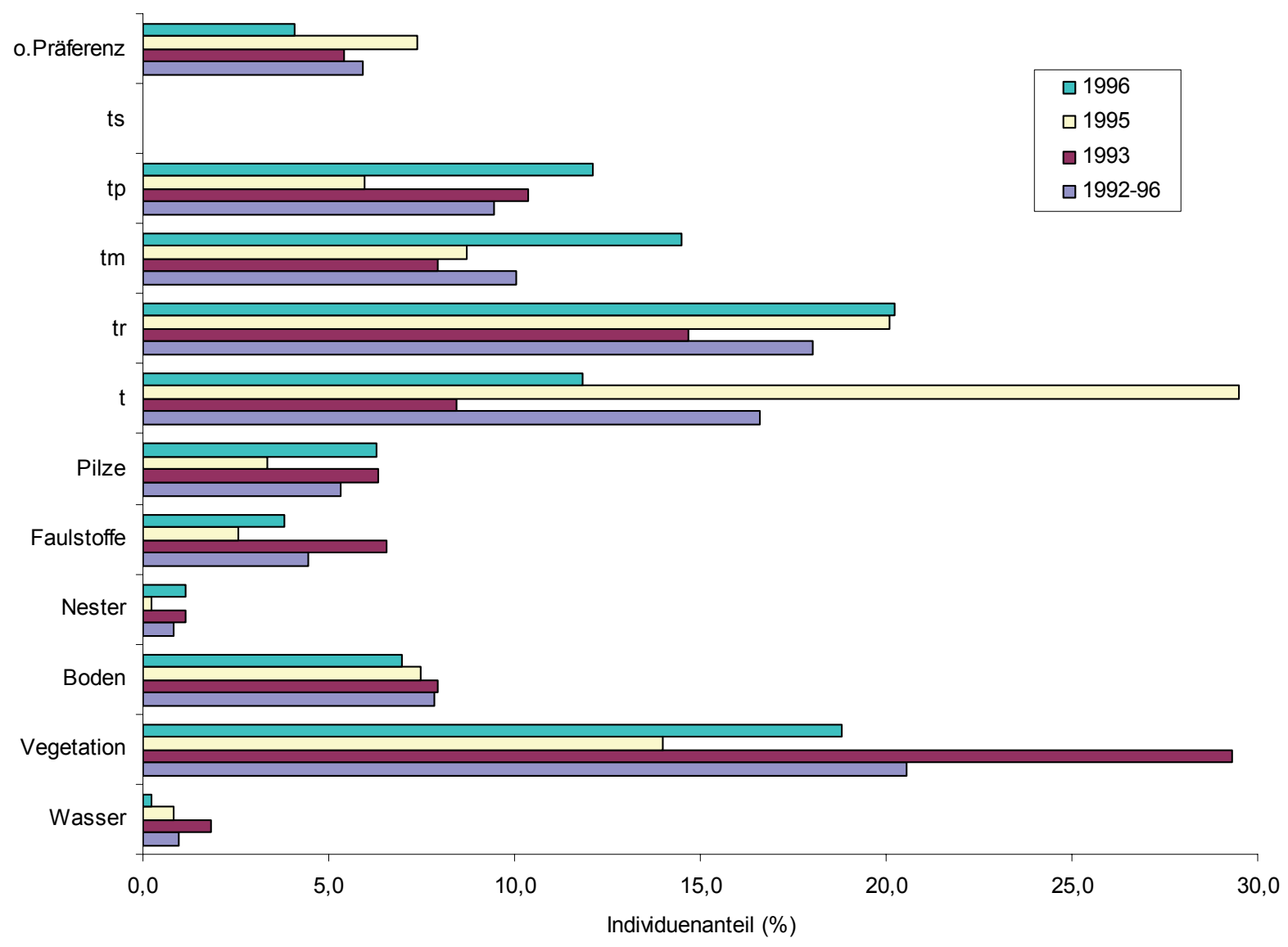

Abb. 4-61: Individuenanteil der Habitatpräferenztypen

Wie bei den den Fängen aus Eklektorproben, so wurde auch hier die Gruppe der Totholzbewohner nach den von ihnen bevorzugt besiedelten Strukturteilen weiter unterteilt. Die Abbildungen 4-62 bis 4-65 zeigen die Anteile der dominierenden Arten am Fangergebnis der 
jeweiligen Präferenztypen, in den Abbildungen 4-66 und 4-67 werden die Individuen- und Artenanteile der Konsumententypen innerhalb der einzelnen Präferenzgruppen dargestellt.

Die Rindenbewohner (TR) stellten in allen Untersuchungsjahren die artenreichste Fraktion unter den Totholzkäfern. Insgesamt wurden 63 corticole Arten registriert. Jeder dritte gefangene Totholzkäfer gehörte dieser Präferentengruppe an. Der Individuen- und Artenanteil am Gesamtfang belief sich auf 18,0 bzw. 11,2 \%. In den Einzeljahren waren die Rindenkäfer mit Individuenanteilen von 14,7 bis $20,2 \%$ beteiligt. Im Verlauf der Vegetationsperiode (ohne P1a) ging die Anzahl gefangener Rindenkäfer kontinuierlich zurück. Auch die Artenzahl war zum Ende der Vegetationsperiode deutlich reduziert. Dennoch blieben in allen Fangperioden die Rindenbesiedler die artenreichste Gruppe unter den Totholzkäfern. Die höchste Artenzahl wurde von Mitte Mai bis Anfang Juni (P2) festgestellt. Etwa zwei Drittel der corticolen Arten und Individuen werden zu den Zoophagen gezählt. Die Xylophagen stellten $21 \%$ der Arten und $14 \%$ der Individuen, $5 \%$ der Arten und Individuen wurden als mycetophag eingestuft. Rhizophagus bipustulatus (11,9\%), Leptusa pulchella (10,7\%), Rhizophagus dispar (8,6 \%) und Taphrorychus bicolor (6,5\%) waren die häufigsten corticolen Arten in den Fensterfallen (Abb. 4-62).

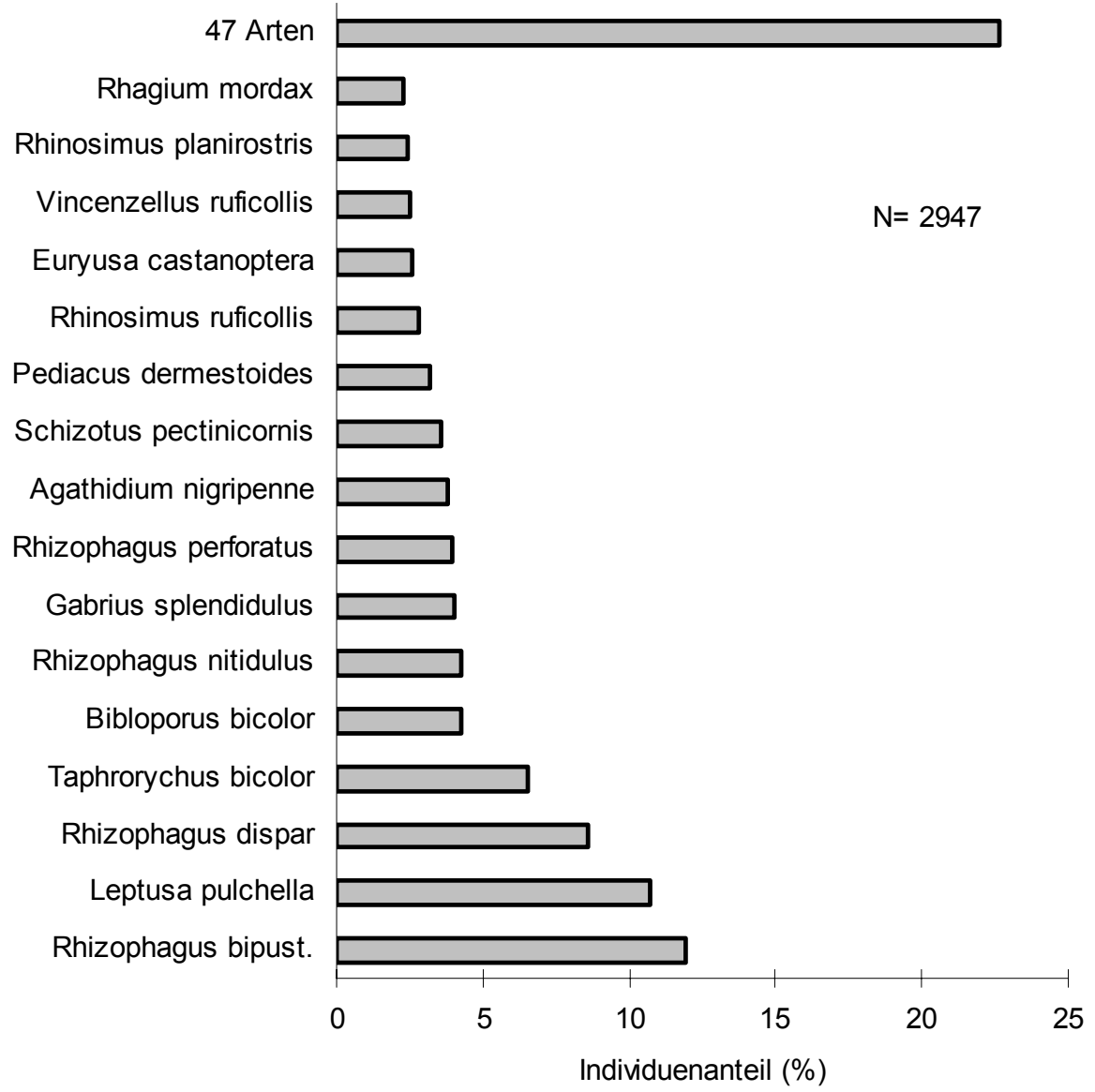

Abb. 4-62: Dominante corticole Arten (TR) 
Tabelle 4-90: Arten- und Individuenanteile der Habitatpräferenztypen in den Fangperioden

\begin{tabular}{|c|c|c|c|c|c|c|c|c|c|c|c|}
\hline & & & & & & F a n & peri & d e & & & \\
\hline & & Habitat & P1a & P1b & $\mathbf{P 2}$ & P3 & P4 & P5 & P6 & P7 & P8 \\
\hline Ar- & & setation & 8 & 30 & 38 & 37 & 32 & 26 & 14 & 8 & 2 \\
\hline ten- & & & 20 & 58 & 41 & 32 & 20 & 27 & 26 & 21 & 16 \\
\hline zahl & & ter & 1 & 7 & 6 & 5 & 7 & 6 & 5 & 2 & 0 \\
\hline & & Istoffe & 15 & 37 & 37 & 26 & 34 & 25 & 26 & 20 & 14 \\
\hline & $\mathbf{T}$ & $\mathbf{T}$ & 3 & 17 & 26 & 34 & 30 & 21 & 13 & 1 & 0 \\
\hline & о & TR & 11 & 47 & 49 & 44 & 38 & 41 & 25 & 11 & 5 \\
\hline & h & TM & 1 & 15 & 21 & 23 & 24 & 18 & 17 & 2 & 3 \\
\hline & $\mathbf{0}$ & TP & 7 & 27 & 32 & 29 & 26 & 19 & 17 & 8 & 4 \\
\hline & I & & 0 & 0 & 0 & 1 & 0 & 0 & 0 & 0 & 0 \\
\hline & $\mathbf{Z}$ & ETotholz & 22 & 106 & 128 & 131 & 118 & 99 & 72 & 22 & 12 \\
\hline & $\mathbf{P i}$ & & 2 & 11 & 10 & 9 & 11 & 13 & 9 & 8 & 7 \\
\hline & & sser & 3 & 11 & 8 & 8 & 8 & 2 & 3 & 1 & 1 \\
\hline & & räferenz & 7 & 15 & 14 & 14 & 15 & 13 & 12 & 8 & 5 \\
\hline & $\Sigma$ & & 78 & 275 & 282 & 262 & 245 & 211 & 167 & 90 & 57 \\
\hline Ar- & & setation & 10,3 & 10,9 & 13,5 & 14,1 & 13,1 & 12,3 & 8,4 & 8,9 & 3,5 \\
\hline ten- & & & 25,6 & 21,1 & 14,5 & 12,2 & 8,2 & 12,8 & 15,6 & 23,3 & 28,1 \\
\hline an- & & & 1,3 & 2,5 & 2,1 & 1,9 & 2,9 & 2,8 & 3,0 & 2,2 & 0,0 \\
\hline & & Istoffe & 19,2 & 13,5 & 13,1 & 9,9 & 13,9 & 11,8 & 15,6 & 22,2 & 24,6 \\
\hline & $\mathbf{T}$ & $\mathbf{T}$ & 3,8 & 6,2 & 9,2 & 13,0 & 12,2 & 10,0 & 7,8 & 1,1 & 0,0 \\
\hline & O & TR & 14,1 & 17,1 & 17,4 & 16,8 & 15,5 & 19,4 & 15,0 & 12,2 & 8,8 \\
\hline & h & TM & 1,3 & 5,5 & 7,4 & 8,8 & 9,8 & 8,5 & 10,2 & 2,2 & 5,3 \\
\hline & $\mathbf{0}$ & TP & 9,0 & 9,8 & 11,3 & 11,1 & 10,6 & 9,0 & 10,2 & 8,9 & 7,0 \\
\hline & l & TS & 0,0 & 0,0 & 0,0 & 0,4 & 0,0 & 0,0 & 0,0 & 0,0 & 0,0 \\
\hline & & ¿Totholz & 28,2 & 38,5 & 45,4 & 50,0 & 48,2 & 46,9 & 43,1 & 24,4 & 21,1 \\
\hline & $\mathbf{P i}$ & & 2,6 & 4,0 & 3,5 & 3,4 & 4,5 & 6,2 & 5,4 & 8,9 & 12,3 \\
\hline & & sser & 3,8 & 4,0 & 2,8 & 3,1 & 3,3 & 0,9 & 1,8 & 1,1 & 1,8 \\
\hline & & räferenz & 9,0 & 5,5 & 5,0 & 5,3 & 6,1 & 6,2 & 7,2 & 8,9 & 8,8 \\
\hline In- & & setation & 62 & 1213 & 1318 & 487 & 166 & 69 & 35 & 10 & 2 \\
\hline di- & & & 85 & 295 & 244 & 134 & 97 & 127 & 139 & 103 & 56 \\
\hline vi- & & & 2 & 19 & 13 & 11 & 28 & 38 & 16 & 5 & 0 \\
\hline du- & & Istoffe & 65 & 166 & 82 & 52 & 73 & 81 & 98 & 64 & 48 \\
\hline en- & $\mathbf{T}$ & $\mathbf{T}$ & 60 & 457 & 1200 & 544 & 278 & 152 & 25 & 1 & 0 \\
\hline $\mathbf{Z} \boldsymbol{\varepsilon}$ & O & TR & 61 & 780 & 695 & 542 & 414 & 292 & 130 & 24 & 9 \\
\hline & h & $\mathbf{T M}$ & 1 & 169 & 425 & 268 & 219 & 346 & 190 & 19 & 6 \\
\hline & $\mathbf{0}$ & TP & 12 & 308 & 363 & 239 & 215 & 159 & 121 & 92 & 38 \\
\hline & 1 & TS & 0 & 0 & 0 & 1 & 0 & 0 & 0 & 0 & 0 \\
\hline & $\mathbf{Z}$ & ETotholz & 134 & 1714 & 2683 & 1594 & 1126 & 949 & 466 & 136 & 53 \\
\hline & $\mathbf{P i}$ & & 2 & 109 & 89 & 123 & 199 & 160 & 56 & 29 & 102 \\
\hline & & sser & 8 & 25 & 64 & 30 & 24 & 3 & 4 & 1 & 1 \\
\hline & & räferenz & 23 & 223 & 233 & 95 & 151 & 115 & 88 & 22 & 21 \\
\hline & $\Sigma$ & & 381 & 3764 & 4726 & 2526 & 1864 & 1542 & 902 & 370 & 283 \\
\hline
\end{tabular}




\begin{tabular}{|c|c|c|c|c|c|c|c|c|c|c|c|}
\hline \multirow{2}{*}{\multicolumn{3}{|c|}{ Habitat }} & \multicolumn{9}{|c|}{ Fangperiode } \\
\hline & & & P1a & P1b & $\mathbf{P 2}$ & P3 & $\mathbf{P 4}$ & P5 & P6 & P7 & P8 \\
\hline \multirow{13}{*}{$\begin{array}{l}\text { In- } \\
\text { di- } \\
\text { vi- } \\
\text { du- } \\
\text { en- } \\
\text { an- } \\
\text { teil }\end{array}$} & \multirow{4}{*}{\multicolumn{2}{|c|}{$\begin{array}{l}\text { Vegetation } \\
\text { Boden } \\
\text { Nester } \\
\text { Faulstoffe }\end{array}$}} & 16,3 & 32,2 & 27,9 & 19,3 & 8,9 & 4,5 & 3,9 & 2,7 & 0,7 \\
\hline & & & 22,3 & 7,8 & 5,2 & 5,3 & 5,2 & 8,2 & 15,4 & 27,8 & 19,8 \\
\hline & & & 0,5 & 0,5 & 0,3 & 0,4 & 1,5 & 2,5 & 1,8 & 1,4 & 0,0 \\
\hline & & & 17,1 & 4,4 & 1,7 & 2,1 & 3,9 & 5,3 & 10,9 & 17,3 & 17,0 \\
\hline & \multirow{6}{*}{$\begin{array}{l}\mathbf{T} \\
\mathbf{0} \\
\mathbf{t} \\
\mathbf{h} \\
\mathbf{0} \\
\mathbf{l} \\
\mathbf{z}\end{array}$} & $\mathbf{T}$ & 15,7 & 12,1 & 25,4 & 21,5 & 14,9 & 9,9 & 2,8 & 0,3 & 0,0 \\
\hline & & TR & 16,0 & 20,7 & 14,7 & 21,5 & 22,2 & 18,9 & 14,4 & 6,5 & 3,2 \\
\hline & & TM & 0,3 & 4,5 & 9,0 & 10,6 & 11,7 & 22,4 & 21,1 & 5,1 & 2,1 \\
\hline & & TP & 3,1 & 8,2 & 7,7 & 9,5 & 11,5 & 10,3 & 13,4 & 24,9 & 13,4 \\
\hline & & TS & 0,0 & 0,0 & 0,0 & 0,0 & 0,0 & 0,0 & 0,0 & 0,0 & 0,0 \\
\hline & & ETotholz & 35,2 & 45,5 & 56,8 & 63,1 & 60,4 & 61,5 & 51,7 & 36,8 & 18,7 \\
\hline & \multicolumn{2}{|c|}{ Pilze } & 0,5 & 2,9 & 1,9 & 4,9 & 10,7 & 10,4 & 6,2 & 7,8 & 36,0 \\
\hline & \multicolumn{2}{|c|}{ Wasser } & 2,1 & 0,7 & 1,4 & 1,2 & 1,3 & 0,2 & 0,4 & 0,3 & 0,4 \\
\hline & \multicolumn{2}{|c|}{ o. Präferenz } & 6,0 & 5,9 & 4,9 & 3,8 & 8,1 & 7,5 & 9,8 & 5,9 & 7,4 \\
\hline
\end{tabular}

Lignicole Arten (T) waren mit 54 Arten in den Fensterfallen. Damit gehörten 9,6 \% der Arten bzw. jede vierte Totholzart dieser Fraktion an, die zusammen 16,6 \% der Individuen stellten. Bei der Gegenüberstellung der Ergebnisse einzelner Untersuchungsjahre waren deutliche Abweichungen zu verzeichnen. Während die Jahre 1993 und 1996 sowohl hinsichtlich der Artenzahl (42 bzw. 40), des Artenanteils (10,2 bzw. 11,7\%) als auch des Individuenanteils $(8,4$ bzw. 11,8 \%) größere Übereinstimmungen zeigten, wichen die Ergebnisse des Jahres 1995 hiervon deutlich ab. Es wurden nur 27 lignicole Arten erfaßt, die aber in deutlich höherer Individuenzahl auftraten. Mit einem Individuenanteil von 29,5 \% waren die lignicolen Arten 1995 die dominierende Präferentengruppe. Verursacht war dieser hohe Individuenanteil vor allem durch nur zwei Arten, die in diesem Jahr in besonders großer Anzahl in den Fensterfallen vertreten waren. Hylecoetus dermestoides war 1995 mit einem Individuenanteil von 19,3\% an den gefangenen Totholzkäfern beteiligt, während in den beiden anderen Jahren nur Anteile von 1,3 bzw. 3,0 \% registriert wurden. Auch Xyleborus dispar erreichte in diesem Jahr mit 11,1 \% der gefangenen Totholzkäfer seinen höchsten Anteil, während 1996 ein Anteil von 2,4\% und 1993 von nur 0,04\% von dieser Art gestellt wurde. Die hohe Individuenzahl lignicoler Arten in der Fangperiode P2 (Individuenanteil 25,4 \%) war ebenfalls zu einem nicht unerheblichen Anteil auf die Flugaktivität dieser beiden Arten zurückzuführen. Die Anzahl lignicoler Arten nahm bis in den Juni (P3) zu und ging dann stetig zurück. Ab Ende August wurde nur noch ein einziges Exemplar in den Fensterfallen festgestellt.

Die Mehrzahl der lignicolen Arten (76\%) gilt als xylophag, diese stellten jedoch nur $27 \%$ der Individuen. Zoophage waren mit $15 \%$ der Arten und nur $2 \%$ der Individuen beteiligt. Dagegen hatten die wenigen mycetophagen Arten (9\%) einen Individuenanteil von über $71 \%$ (Abb. 4-63). 


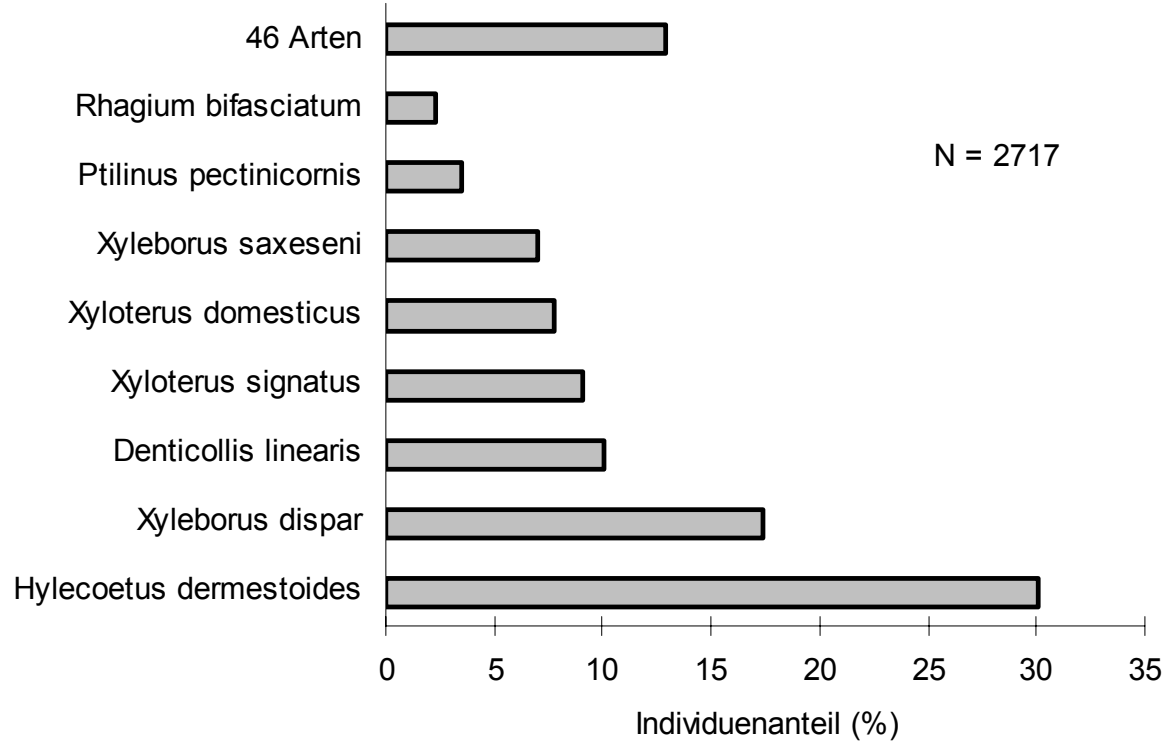

Abb. 4-63: Dominante lignicole Arten ( $\mathrm{T})$

Die Bewohner von Totholzmulm (TM) waren durch 43 Arten in den Fensterfallen repräsentiert. Ihr Artenanteil lag in den Untersuchungsjahren zwischen 7,3 und 9,0 \%, der Individuenanteil zwischen 7,9 und 14,5 \%. Die höchsten Artenzahlen wurden von Mitte Mai bis Mitte Juli (P2-P4) festgestellt. In den beiden folgenden Fangperioden waren die Mulmbewohner mit Anteilen von jeweils über $21 \%$ die individuenstärkste Präferentengruppe. Unter den Xylodetricolen sind räuberische Arten dominierend. Sie waren mit einem Artenanteil von $67 \%$ und einem Individuenanteil von $52 \%$ vertreten. $19 \%$ der Arten und $30 \%$ der Individuen gelten als mycetophag, 9 bzw. $16 \%$ als xylophag. Cerylon ferrugineum war mit 28,2 \% an den gefangenen Mulmbewohnern beteiligt. Ptinella limbata war mit einem Anteil von 19,4\%, Melanotus castanipes von 14,5\% und Euplectus bescidicus von 9,3\% vertreten (Abb. 4-64).

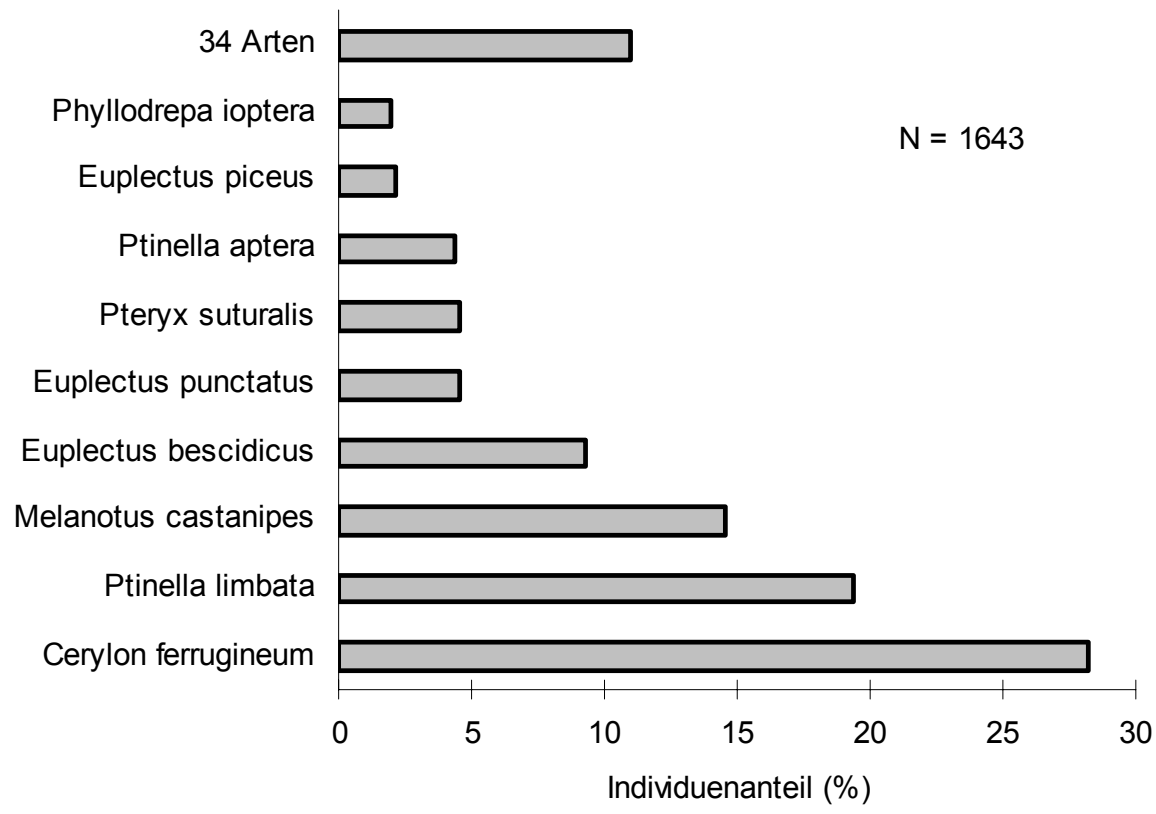

Abb. 4-64: Dominante xylodetricole Arten (TM) 
Viele xylobionte Arten besiedeln bevorzugt Pilze, die ihrerseits wiederum an Totholz gebunden sind (TP). Insgesamt fanden sich 48 Arten in den Flugbarrierefallen, die 9,5\% der Individuen stellten. Ihr Artenanteil lag in den einzelnen Untersuchungsjahren zwischen 8,5 und 10,0 \%, der Individuenanteil zwischen 6,0 und 12,1 \%. Arten- und Individuenzahl erreichten ihr Maximum in der Fangperiode P2, während der Individuenanteil die höheren Werte zum Ende der Vegetationsperiode hin erreichte. Die häufigsten Vertreter dieser Gruppe waren Agathidium varians (18,3\%), Enicmus testaceus $(17,4 \%)$, E. fungicola $(13,1 \%)$ und Anisotoma humeralis (10,4 \%) (Abb. 4-65).

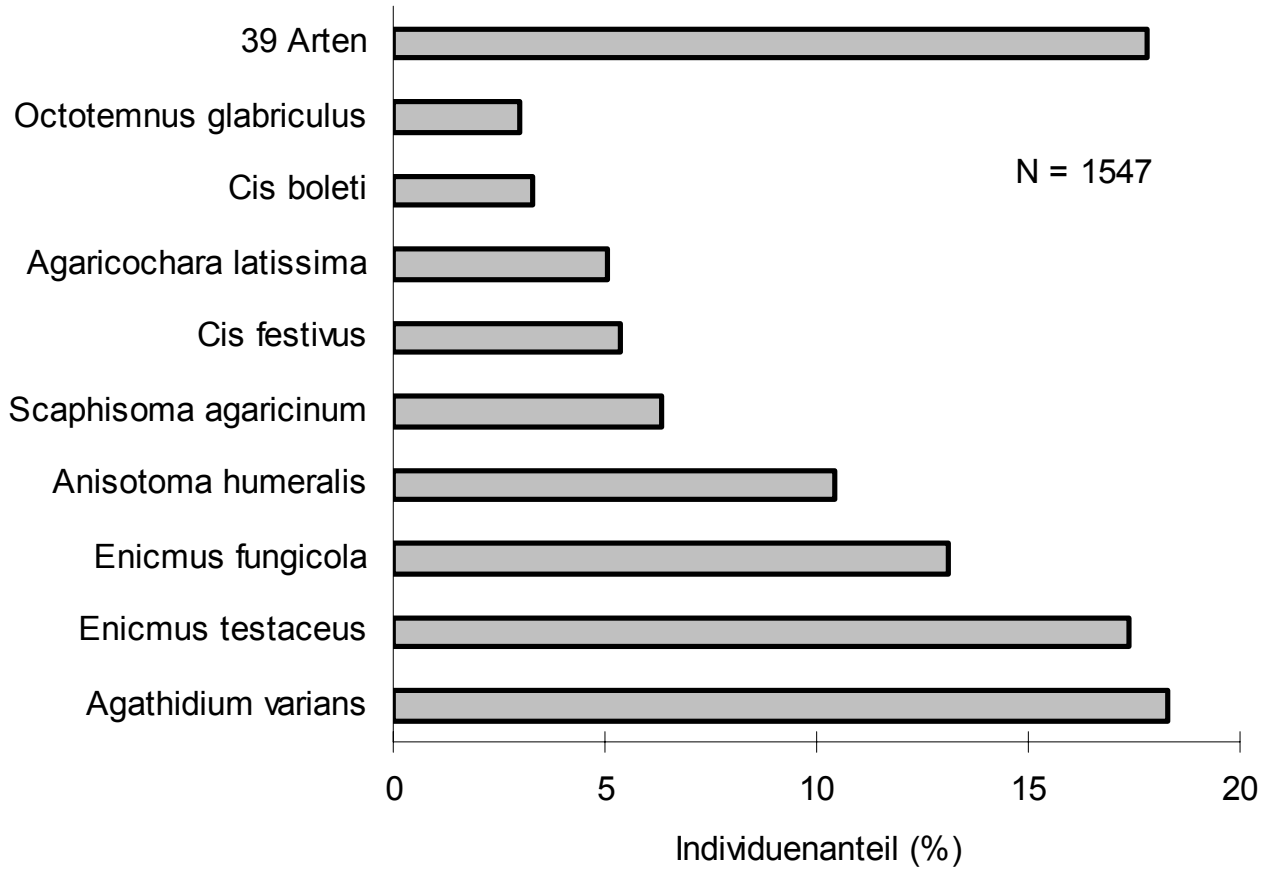

Abb. 4-65: Dominante xylofungicole Arten (TP) 


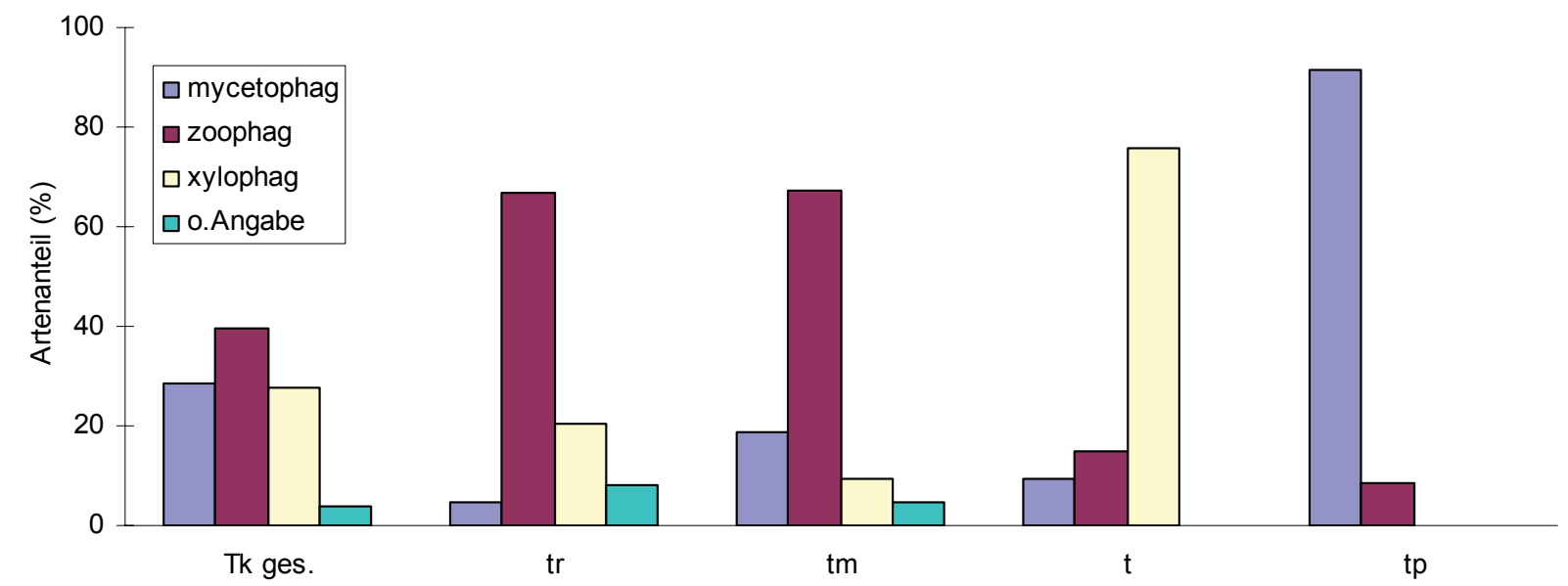

Abb. 4-66: Artenanteile der Konsumententypen an den Habitatpräferenzgilden

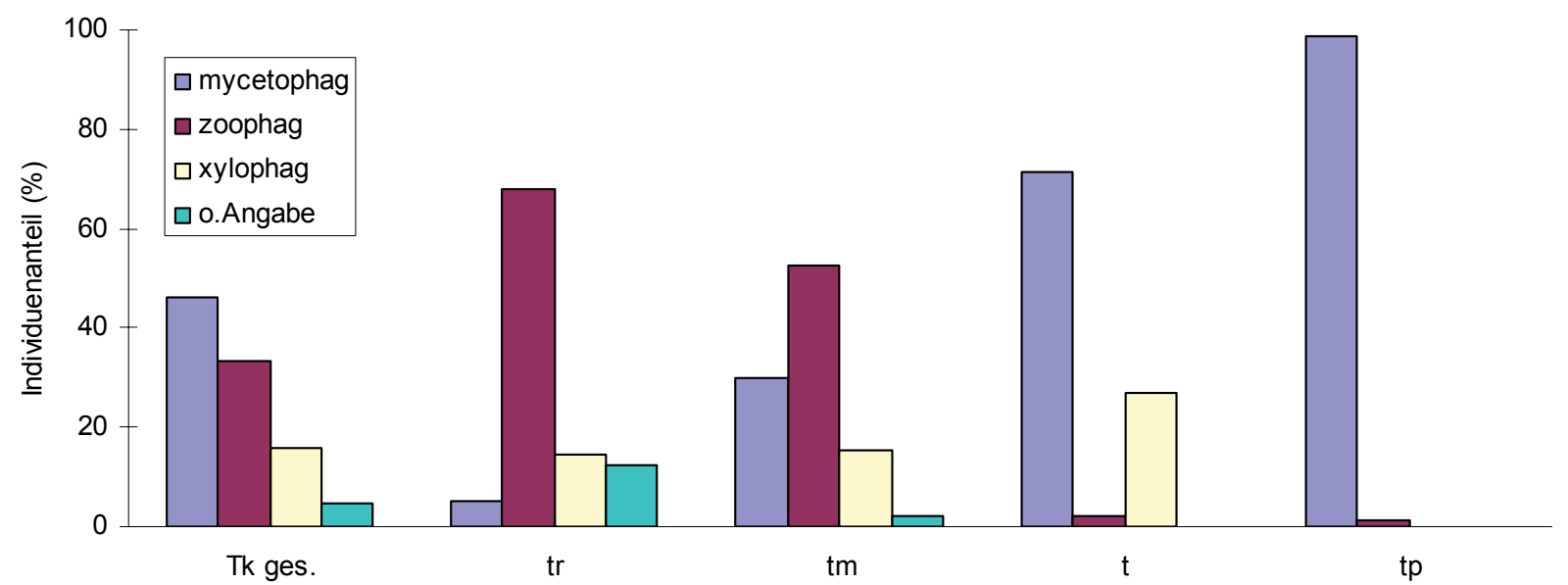

Abb. 4-67: Individuenanteile der Konsumententypen an den Habitatpräferenzgilden 


\section{Diskussion}

\subsection{Die Käferfauna des NSG 'Neuenburger Urwald'}

Im 'Neuenburger Urwald' wurden in vier Erfasssungsjahren 711 Käferarten aus 75 Familien festgestellt. Von diesen sind 260 Arten als xylobiont einzustufen.

Die große Mehrzahl der Käferarten trat in nur wenigen Proben und mit geringer Individuenzahl auf, während sehr wenige Arten in hoher Frequenz und mit hohem Individuenanteil vertreten waren. Elf Arten (1,5\%) mit einem Individuenanteil von mindestens $2 \%$ stellten gemeinsam fast die Hälfte der Individuen. Ein Viertel der Arten wurde dagegen nur mit einem Exemplar registriert.

Mit 6864 Individuen (10,6\%) war Rhizophagus dispar die dominierende Art im Gebiet, gefolgt von Corticaria longicollis (6,3 \%), Ptilinus pectinicornis (5,7 \%), Dryocoetes villosus (3,9\%), Xyloterus signatus (3,3\%), Hylecoetus dermestoides (3,1\%), Cryptophagus dentatus (2,8 \%), Orchesia undulata (2,7 \%), Cerylon ferrugineum $(2,7 \%)$, Rhizophagus bipustulatus (2,7\%) und Xyloterus domesticus (2,5\%)

Ein Blick auf diese Hauptarten läßt deutlich die Dominanz der Totholzkäfer in den Proben aus dem 'Neuenburger Urwald' erkennen. Sie stellten $37 \%$ der Arten und $79 \%$ der Individuen. Die Rindenbewohner (73 Arten) waren mit $34 \%$, die lignicolen Arten (64 Arten) mit $20 \%$, die Mulmbesiedler (55 Arten) mit $19 \%$ und die Besiedler von Totholzpilzen (62 Arten) mit $6 \%$ am Fangergebnis beteiligt, während die 4 succicolen Arten nur eine Nebenrolle spielten. Neben den xylobionten Käferarten waren noch Bodenbewohner, Faulstoffbewohner, Arten der Vegetation und eurytope Arten zahlreich vertreten, erreichten aber nur Individuenanteile von max. $6,4 \%$.

Die mit Abstand größte Zahl der Käferarten ernährt sich räuberisch. Die 299 Arten hatten einen Individuenanteil von 36 \%. Artenärmer, aber noch individuenreicher traten die mycetophagen Arten auf, die mit 149 Arten $39 \%$ der Individuen stellten. $15 \%$ der Individuen entfielen auf 76 xylophage Arten und $6 \%$ auf 82 phytophage Arten. Unter den Totholzkäfern hatten die Zoophagen (101Arten) und die Mycetophagen (71 Arten) ähnliche Individuenanteile (39 bzw. 38 \%). Die 76 xylophagen Arten stellten $19 \%$ der Individuen.

Untersuchungen zum Arteninventar xylobionter Käfer liegen aus zahlreichen Gebieten vor (z. B. ADELI 1961; RAUH 1993; KÖHLER 1996, 1999; BENSE \& GEIS 1998; DETSCH 1999a; SCHAFFRATH 1999; FLECHTNER, DOROW \& KOPELKE 2000; ELMER 2002). Ein Vergleich der im 'Neuenburger Urwald' erhobenen Ergebnisse mit diesen Untersuchungen ist jedoch nur unter Vorbehalt möglich, weil Unterschiede in der Zielsetzung, der Intensität und Dauer der Aufnahmen sowie in den eingesetzten Methoden und deren Anzahl von nicht unerheblicher Bedeutung für die Fangergebnisse sind. Zudem ist die Zuordnung der Arten zu einzelnen Habitatpräferenztypen und Konsumentengilden keineswegs immer eindeutig und stellt eine grobe Schematisierung der natürlichen Vielfältigkeit dar. Die Übergänge sind oft fließend. Dazu kommt, dass der Kenntnisstand über die Biologie und Ökologie der Mehrzahl der xylobionten Käferarten sehr lückenhaft ist (KÖHLER 2000). In der Konsequenz finden sich bei verschiedenen Autoren z. T. deutliche Unterschiede in der Einordnung der 
Arten, wodurch Vergleiche mit den Ergebnissen aus anderen Untersuchungen sehr erschwert werden.

KÖHLER (2000) hat die Daten aus über 130 Waldgebieten, vornehmlich Naturwaldreservaten und Naturschutzgebieten, zusammengestellt. Sowohl hinsichtlich der Gesamtartenzahl als auch der Artenzahl xylobionter Käfer nimmt der 'Neuenburger Urwald' eine vordere Position ein. Zudem zeigt der Vergleich, dass die xylofungicolen und xylodetricolen Arten etwas über-, die corticolen und lignicolen Arten etwas unterdurchschnittlich am Artenspektrum beteiligt sind.

Die Artenzahl xylobionter Käfer, insbesondere der Mulmbewohner, ist positiv mit der Naturnähe der Bestände korreliert (KÖHLER 1999). Xylodetricole Arten bevorzugen stabile Milieubedingungen und gelten als Charakterarten der Waldzerfallsphase (KÖHLER 1996). Sie werden daher als Indikatoren für naturnahe Waldbestände betrachtet (DOROW \& FLECHTNER 1999). Rindenkäfer sind dagegen in geringerem Maße an starkes Totholz und 'urwaldtypische' Strukturen gebunden (KÖHLER 1999). Pilzkäfer sind ihrerseits unmittelbar vom Artenreichtum der Pilze abhängig und deshalb besonders in geschlossenen Wäldern mit ihrem günstigen Mikroklima artenreich vertreten.

Aus Niedersachsen sind aktuell ca. 900, aus dem Weser-Ems-Gebiet ca. 600 xylobionte Käferarten gemeldet. Damit beherbergt der 'Neuenburger Urwald' etwa 29 \% der in Niedersachsen und ca. $43 \%$ der in der Weser-Ems-Region heimischen Totholzkäferfauna. Für einzelne Habitatpräferenzgilden liegen die Werte sogar noch deutlich höher. So wurden z. B. $53 \%$ der aus der Weser-Ems-Region gemeldeten Totholzpilzbesiedler im Untersuchungsgebiet registriert.

Die Artenzahlen aus dem 'Neuenburger Urwald' sind umso beeindruckender, bedenkt man, dass die Totholzkäferartenzahlen ein deutliches Süd-Nord-Gefälle erkennen lassen und das viele Arten, u. a. auf Grund der feucht-kühlen Klimabedingungen des nordwestdeutschen Tieflandes hier nicht mehr vorkommen (KÖHLER 2000). Auf der anderen Seite muß bei der Bewertung dieser Ergebnisse aber auch berücksichtigt werden, dass wohl nur wenige Waldgebiete so intensiv untersucht worden sind und der Erfassungsgrad insbesondere der saproxylen Arten daher als überdurchschnittlich einzustufen ist.

In Niedersachsen finden sich neben dem 'Neuenburger Urwald' weitere Waldgebiete, denen SPEIGHT (1989) eine potenzielle internationale Bedeutung für die Totholzfauna beimißt und bei denen es sich um Reste ehemaliger Hutewälder handelt. Aus einigen dieser Gebiete liegen Inventurergebnisse über die xylobionte Käferfauna vor. So wurde im bei Bremen gelegenen NSG 'Urwald Hasbruch', der hinsichtlich seiner Geschichte und Struktur eine große Ähnlichkeit mit dem 'Neuenburger Urwald' aufweist, eine fast identische Anzahl xylobionter Coleopteren (257 Arten) festgestellt (MÖLLER, BELLMANN \& ESSER 1995). Im 'Bentheimer Urwald' (Emsland) wurden im Rahmen einer weniger intensiven Untersuchung 153 xylobionte Arten registriert (MENKE 2001).

Der 'Neuenburger Urwald' ist Lebensraum zahlreicher seltener und gefährdeter Arten. In Ermangelung einer Roten Liste für die Käfer Niedersachsens wird den folgenden Ausführungen die Rote Liste gefährdeter Käfer in Deutschland (RLD) (GEISER 1998) zu Grunde gelegt.

$59 \%$ der in Deutschland beheimateten Totholzkäferarten sind in ihrem Bestand gefährdet (GEISER 1998). Besonders betroffen sind lignicole, xylofungicole und xylodetricole Arten. 
Viele Arten kommen heute nur noch reliktär in wenigen alten Wäldern mit hohem Totholzangebot und langer Biotoptradition vor. Als Ursachen für den hohen Gefährdungsgrad saproxyler Käfer wird v. a. auf das Fehlen von Altbäumen und großdimensioniertem und stark zersetztem Totholz, den Mangel an Totholzpilzen und Baumhöhlen sowie auf die Bedeutung der mikroklimatischen Bedingungen und die fehlende Biotoptradition verwiesen (GEISER 1994; KÖHLER 2000; BERG et al. 2002; SCHERZINGER 1996).

Insgesamt wurden im Untersuchungsgebiet 85 gefährdete Käferarten festgestellt. Das entspricht einem Anteil von $12 \%$ der nachgewiesenen Arten. 57 Arten wurden mit den Fensterfallen, 70 mit Eklektoren erfaßt. 53 Arten werden als 'gefährdet' (RLD 3), 25 als 'stark gefährdet' (RLD 2) und 7 Arten als 'vom Aussterben bedroht' (RLD 1) eingestuft. Legt man nur die Anzahl saproxyler Arten zu Grunde, beläuft sich der Anteil gefährdeter Arten auf insgesamt $25 \%$. Eine Liste der im 'Neuenburger Urwald' gefundenen gefährdeten Arten findet sich in Anhang 13.

Die große Mehrheit der festgestellten RLD-Arten ist streng an Totholz gebunden (65 Arten). Bezogen auf die einzelnen Gefährdungsklassen belief sich der Anteil saproxyler Arten auf $100 \%$ (RLD 1), $88 \%$ (RLD 2) und $70 \%$ (RLD 3). Unter den 65 gefährdeten xylobionten Käferarten sind Mulmbewohner und an Totholzpilze gebundene Arten besonders zahlreich. Sie stellten 22 (39\%) bzw. 21 (32\%) der gefangenen xylobionten RLD-Arten. 17 Arten $(26 \%)$ sind als lignicol, 4 Arten (6\%) als corticol und 1 Art (2\%) als succicol einzuordnen. Betrachtet man die Anteile gefährdeter Arten in den einzelnen Habitatpräferenzgilden, so zeigt sich, dass $40 \%$ der im 'Neuenburger Urwald' nachgewiesenen xylodetricolen und $34 \%$ der xylofungicolen Arten gefährdet sind. Für die übrigen Habitatpräferenztypen belaufen sich die Anteile auf $27 \%$ (lignicol), $17 \%$ (succicol) und $5 \%$ (corticol).

Neben den Artenzahlen ist auch die Häufigkeit, mit der einige der gefährdeten Arten im 'Neuenburger Urwald' festgestellt wurden, bemerkenswert. So war z. B. die vom Aussterben bedrohte Art Teredus cylindricus mit 334 Individuen die dritthäufiste RLD-Art im Untersuchungsgebiet. Der Rindenkäfer Pediacus dermestoides (RLD 1), aktuell aus nur vier Regionen in Deutschland gemeldet (KÖHLER \& KLAUSNITZER 1998), war mit 190 Individuen die achthäufigste RLD-Art.

Diese Zahlen belegen eindrucksvoll die Bedeutung eines großen und vielfältigen Totholzangebotes für das Vorkommen gefährdeter Käferarten. Auf Grund der Gesamtartenzahl und der vielen gefährdeten und faunistisch bemerkenswerten Arten ist das Gebiet auch überregional als sehr bedeutsamer Lebensraum für xylobionte Organismen, insbesondere für Besiedler von Totholzmulm und Totholzpilzen, einzuordnen.

\subsection{Gründe für den Artenreichtum xylobionter Coleopteren}

Der ungewöhnliche Artenreichtum der xylobionten Käferfauna sowie die hohe Zahl gefährdeter und faunistisch bemerkenswerter Arten findet seine Entsprechung in dem großen und vielfältigen Totholzangebot des 'Neuenburger Urwaldes'. Untersuchungen zur Artenausstattung unterschiedlich totholzreicher Bestände belegen die Abhängigkeit einer artenreichen Lebensgemeinschaft xylobionter Organismen (z. B. Moose, Flechten, Pilze und Insekten) von der Totholzausstattung eines Gebietes (SÖDERSTRÖM 1988a; ANDERSSON \& HYTTE- 
BORN 1991; BADER, JANSSON \& JONSSON 1995; CHRISTENSEN \& EMBORG 1996; ØKLAND 1996; HÅGVAR \& ØKLAND 1997; BENSE \& GEIS 1998; LINDBLAD 1998; KRUYS et al. 1999; MARTIKAINEN et al. 1999, 2000; MARTIKAINEN 2000; ØKLAND 2000, 2002; FERRIS, PEACE \& NEWTON 2000; SIPPOLA, LEHESVIRTA \& RENVALL 2001; SIPPOLA 2001; ÓDOR \& STANDOVÁR 2001; SIITONEN, PENTILLÄ \& KOTIRANTA 2001; SIMILÄ 2002; SIMILÄ et al. 2002; SVERDRUP-THYGESON 2002; HUMPHREY et al. 2002; SIMILÄ, KOUKI \& MARTIKAINEN 2003; SIPPOLA et al. 2004).

Im Vergleich zu Laubstreu ist Totholz eine räumlich, zeitlich und strukturell sehr variable Ressource. Wie zahlreiche waldkundliche Untersuchungen zeigen, variieren die Totholzvorräte in Waldökosystemen in einem Rahmen von mehreren hundert Festmetern.

Von zentraler Bedeutung sind Nutzungsgeschichte und -intensität eines Waldes, die sich sowohl in der Quantität als auch in der Struktur des Totholzangebotes niederschlagen. (ANDERSSON \& HYTTEBORN 1991; KIRBY, WEBSTER \& ANTCZAK 1991; BAADER, JANSSON \& JONSSON 1995; BRETZ GUBY \& DOBBERTIN 1996; FREEDMAN et al. 1996; GREEN \& PETERKEN 1997; BENEKE \& BUTLER MANNING 2003; CHRISTENSEN \& HAHN 2003). Der starke Einfluss der Bewirtschaftung auf das Totholzangebot wird durch die meist rasche Vorratsanreicherung in Naturwaldreservaten nach Nutzungsaufgabe belegt (BURGER 1999; DETSCH 1999b; KÖLBEL 1999; MEYER 1999).

Aber auch in ungenutzten Urwäldern ist eine große Variabilität der Totholzvorräte sowie in der Struktur des Angebotes zu beobachten (KORPEL 1995; LEIBUNDGUT 1993; BONCINA 2000; BOBIEC 2002; SANIGA \& SCHÜTZ 2002; CHRISTENSEN \& HAHN 2003; MEYER, TABAKU \& LÜPKE 2003). KORPEL (1997a) bezeichnet Totholz aber dennoch als „... beständige, der Menge und Struktur nach verhältnismäßig stabile Komponente.“ Totholz ist ein sehr dynamisches Strukturelement, das einem ständigen Wandel unterliegt. Neben Art und Geschwindigkeit der Zersetzung ist die Nachlieferung von entscheidender Bedeutung. In der oft festgestellten geklumpten Verteilung des Totholzangebotes und in den variierenden Anteilen der Zersetzungszustände spiegeln sich räumlich und zeitlich sehr variable Mortalitätsraten wieder. In der Konsequenz ist ein mehr oder weniger kleinräumiges Nebeneinander totholzreicher und -armer Flächen zu beobachten.

Neben der Nutzungsgeschichte und -intensität wirken sich zahlreiche weitere Faktoren auf das Totholzangebot aus. Menge und Struktur des Totholzangebotes sind $u$. a. auch von den klimatischen Bedingungen, der Standortproduktivität, der Waldgesellschaft, der Waldentwicklungsphase und dem vorherrschenden Störungsregime abhängig (FALINSKY 1986; KORPEL 1992, 1995, 1997a; PONTAILLER, FAILLE \& LEMÉE 1997; SIPPOLA, SIITONEN \& KALLIO 1998; BONCINA 2000; FRIDMAN \& WALHEIM 2000; SANIGA \& SCHÜTZ 2001; KRANKINA et al. 2002; MOUNTFORD 2002; BOBIEC 2002; ROUVINEN 2002; CHRISTENSEN \& HAHN 2003; LAIHO \& PRESCOTT 2004).

Voraussetzung für das Auftreten einer artenreichen Totholzkäfergemeinschaft ist das Angebot einer großen Habitatvielfalt. Xylobionte Käfer gelten als Struktur- und Milieuspezialisten (KÖHLER 1999), weshalb der Strukturvielfalt oft größere Bedeutung als der Totholzmenge beigemessen wird (KÖHLER 2000). Die Vielfalt der Mikrohabitate und Milieubedingungen und die Totholzmenge sind im Allgemeinen jedoch eng miteinander korreliert. So geht das hohe Totholzangebot der Zerfallsphase einher mit einer großen Vielfalt der Strukturen und Mikroklimabedingungen. Daher kann das Totholzvolumen als ein Indikator 
für die Bedeutung eines Gebietes für die Biodiversität der xylobionten Arten betrachtet werden (SCHUCK et al. 2004). Ausnahmen mögen großflächige Störungsereignisse darstellen, wie sie in unseren Regionen v. a. in Wirtschaftswäldern immer wieder auftreten. So zeichnen sich ungeräumte Windwurfflächen mit ihrem großen, aber relativ uniformen Totholzangebot nicht durch eine herausragende Artenvielfalt aus (OTTE 1989; KOPF \& FUNKE 1998; KOPF 2000; WERMELINGER, DUELI \& OBRIST 2002; DOROW 2002). In der Praxis ist eine Trennung der Bedeutung von Totholzmenge, Habiatvielfalt und -kontinuität kaum möglich (SIITONEN 2001).

Die Quantität des Habitatangebotes beeinflusst unmittelbar die räumliche Verteilung und damit dessen Konnektivität und Erreichbarkeit durch xylobionte Organismen (JONSELL 1999; SCHIEGG 2000a, 2000b; RUKKE 2000). Da Totholz ein vergänglicher Lebensraum ist, der von den Arten nur befristet nutzbar ist, sind sie auf die Besiedlung neu entstehender Totholzstrukturen angewiesen. Nur wenn die räumliche (Konnektivität) und zeitliche (Kontinuität) Vernetzung gewährleistet sind, können die Arten einen Lebensraum dauerhaft besiedeln.

In engem Zusammenhang hierzu steht die Beobachtung, dass die Verbreitung vieler an Totholz gebundenen Arten eine enge Abhängigkeit von Waldbeständen mit langer Biotoptradition erkennen läßt (GEISER 1989; SSYMANK 1994). Oft handelt es sich dabei um Reste ehemaliger Hudewälder (GEISER 1994). Auch der 'Neuenburger Urwald' beherbergt eine Anzahl von Arten, deren Verbreitung, zumindest im nordwestdeutschen Tiefland, auf 'historisch alte Wälder' beschränkt ist. Beispiele sind u. a. Teredus cylindricus und Pediacus dermestoides.

Die große Bedeutung der Biotoptradition für die Artenvielfalt und das Vorkommen gefährdeter Arten wird von vielen Autoren betont (GARLAND 1983; HARDING \& ALEXANDER 1993; BERG et al. 1994; GREEN \& PETERKEN 1997; ANGELSTAM 1997; ALEXANDER 1998; SIITONEN 2001; BUTLER, ALEXANDER \& GREEN 2002; ROUVINEN 2002). Beispiele für diese Abhängigkeit finden sich in den verschiedensten Organismengruppen, so z. B. bei Pilzen (BADER; JANSSON \& JONSSON 1995; LINDBLAD 1998; SIPPOLA, LEHESVIRTA \& RENVALL 2001; SVERDRUP-THYGESON \& LINDENMAYER 2003; STOCKLAND \& KAUSERUD 2004), Moosen und Flechten (OHLSON et al. 1997; EK, WASTEIN \& SVENSSON 2001) sowie Käfern (SIITONEN \& MARTIKAINEN 1994; NILSSON et al. 1995; NILSSON \& BARANOWSKI 1997; SIITONEN \& SAARISTO 2000; SVERDRUP-THYGESON 2001, 2002; JONSELL \& NORDLANDER 2002; RANIUS 2002). Die Abhängigkeit von der Biotoptradition ist oft so eng, dass verschiedene Arten als Indikatorarten für Habitatkontinuität vorgeschlagen worden sind (SSYMANK 1994; BREDESEN et al. 1997; THOR 1998; NORDÉN \& APPELQVIST 2001). Da Wälder mit langer Biotoptradition sich oft nicht nur durch einen großen Artenreichtum xylobionter Organismen auszeichnen, sondern auch besonders viele bestandsbedrohte Arten beherbergen, hat ALEXANDER $(1988,1998)$ aus dem Vorkommen gefährdeter xylobionter Käferarten einen 'Index of ecological continuity' als Indikator für alte Waldstandorte abgeleitet. Die Bindung an alte Waldstandorte und somit die Indikatorfunktion ist in vielen Fällen aber nur von regional beschränkter Gültigkeit.

Meistens handelt es sich bei den Arten mit enger Abhängigkeit von der Biotoptradition um solche, die an starkdimensioniertes Totholz fortgeschrittener Zersetzung oder sonstige seltene oder relativ stabile Totholzstrukturen wie Höhlen und Mulm gebunden sind, die mit beginnender Nutzung der Wälder rasch verschwinden (KÖHLER 1996; NILSSON \& 
BARANOWSKI 1997; JONSSON, JONSSEL \& NORDLANDER 2001). Die Ursachen der beobachteten Abhängigkeit sind in den meisten Fällen nicht geklärt (OHLSON et al. 1997; SVERDRUP-THYGESON 2002). Da das Fehlen dieser Arten in Waldbeständen oft nicht allein mit ihren Habitatansprüchen erklärt werden kann, wird die Ursache oft im geringen Dispersionsvermögen der Arten gesehen (GEISER 1989; NILSSON et al. 1995; NILSSON, HEDIN \& NIKLASSON 2001).

Das Dispersionsverhalten und die Effektivität der Lokalisation geeigneter Habitate sind als Anpassung der Arten an die Raum-Zeit-Dynamik des Angebotes in Urwäldern und somit des vorherrschenden natürlichen Störungsregimes zu sehen (JONSSEL 1999; BENGTSSON et al. 2000; NILLSON, HEDIN \& NIKLASSON 2001). Auf Grund des dort zur Verfügung stehenden großen Totholzangebotes bestand für viele Arten allem Anschein nach nur ein geringer Selektionsdruck zur Entwicklung effektiver Fernverbreitungsmechanismen (JONSSON 2000). So wird vielen xylobionten Käferarten ein nur geringes Ausbreitungsvermögen nachgesagt (GEISER 1989, 1994). Dies gilt besonders für die Besiedler relativ stabiler Strukturen (z. B. viele Mulm- und Höhlenbewohner), während für Bewohner kurzlebiger Habitate (z. B. zahlreiche Frischholzinsekten) auch größere Distanzen oft kein Hindernis darstellen (SANDERS 1987; KÖHLER 1996; NILSSON \& BARANOWSKI 1997; RANIUS \& NILSSON 1997; SCHIEGG PASINELLI 1999). Konkrete Untersuchungsergebnisse zum Dispersionsvermögen xylobionter Coleopteren liegen allerdings kaum vor. Eine der wenigen Ausnahmen sind die Arbeiten zum Migrationsverhalten von Osmoderma eremita (RANIUS \& HEDIN 2001; HEDIN \& RANIUS 2002).

Von einigen Arten ist bekannt, dass sie spezielle Strategien zur Ortung ihrer Entwicklungssubstrate entwickelt haben. Hierzu zählt die olfaktorische Wahrnehmung flüchtiger Substanzen ebenso wie die Reaktion auf Lockstoffe der eigenen oder anderer Arten. Beispiele sind das Pheromonsystem von Osmoderma eremita und einigen Dorcatoma-Arten, das Kairomonsystem von Elater ferrugineus sowie die Reaktion einiger Cis-Arten auf flüchtige Substanzen ihrer Wirtspilze (JONSELL \& NORDLANDER 1995; JONSSON, NORDLANDER \& JONSELL 1997; HÅGVAR 1999; FÄLDT et al. 1999; GUEVARA, RAYNER \& REYNOLDS 2000; JONSSON 2002; LARSSON et al. 2003; JONSSON et al. 2003; SVENSSON, LARSSON \& HEDIN 2004).

Die Quantität des Totholzangebotes ist nicht nur für dessen zeitliche Kontinuität und räumliche Vernetzung von Bedeutung, sondern wirkt sich auch unmittelbar auf die Populationsgrößen aus. Neben dem Dispersionsverhalten und zahlreichen weiteren Faktoren ist die Populationsgröße von Bedeutung für das Aussterberisiko einer Population, da kleine Populationen empfindlicher auf zufällige Schwankungen (demographische und genetische Stochastik, Umweltstochastik) reagieren (BAHL \& HENLE 1999; HENLE, POSCHOLD \& BAHL 1999; RUKKE 2000; SIITONEN 2001). Besonders gefährdet sind Arten mit hochspezialisierten Habitatansprüchen (KOMONEN 2003b). 


\subsection{Vergleich der Fangergebnisse von Fensterfallen und Totholzeklektoren}

Die zur Erfassung des Artenspektrums xylobionter Käfer eines Waldgebietes gewählten Erfassungsmethoden haben einen großen Einfluss auf die Ergebnisse. Ein Standardmethodenset, das Vergleiche aus verschiedenen Waldgebieten wesentlich erleichtern würde und zudem geeignet wäre, unterschiedliche Einflussfaktoren wie z. B. Totholzangebot, Waldgesellschaft etc. zu analysieren, existiert zur Zeit nicht. Im Folgenden sollen daher Ergebnisse sowie Eignung und Effizienz von Fensterfallen und Totholzeklektoren vergleichend gegenübergestellt werden.

Das größere Artenspektrum wurde mit Fensterfallen erfaßt, obwohl nur ein Viertel der gefangenen Individuen mit dieser Methode gefangen wurden. Von den 694 in Fallen nachgewiesenen Arten wurden 562 Arten in Fensterfallen und 457 Arten in Eklektoren festgestellt. Damit wurde nicht einmal die Hälfte der Arten in beiden Fallentypen registriert. Anders stellt sich die Situation bei den Totholzbewohnern dar, die in größerer Artenzahl in den Eklektoren gefangen wurden (219 Arten), während in den Fensterfallen 209 Arten vertreten waren. 168 Arten (65\%) wurden mit beiden Methoden nachgewiesen.

Obwohl mit den Fensterfallen insgesamt nur $17 \%$ der xylobionten Käferindividuen gefangen wurden, waren zahlreiche Arten in diesen in größerer Individuenzahl als in den Eklektoren vertreten. Beispiele unter den häufigen Arten sind Xyleborus dispar, Agathidium varians, Scaphisoma agaricinum, Melanotus castanipes, Enicmus fungicola und E. testaceus, von denen jeweils über $70 \%$ der Individuen in Fensterfallen gefangen wurden. Diese Beobachtung deutet einerseits auf eine sehr unterschiedliche Mobilität der Arten hin, kann aber auch z. T. darauf zurückgeführt werden, dass die von diesen Arten bevorzugten Habitate nicht in angemessem Umfang mit Eklektoren erfaßt wurden.

Die Dominanzklassenstruktur war in den Fensterfallen deutlich ausgeglichener als in den Eklektorproben. Traten in ersteren nur 6 Arten mit einem Individuenanteil von über $2 \%$ auf, waren es in letzteren 12 Arten. Der gemeinsame Individuenanteil dieser Arten belief sich auf $24 \%$ in den Fensterfallen und $59 \%$ in den Eklektoren. Betrachtet man nur die xylobionten Käferarten, fielen die Unterschiede weniger deutlich aus. Waren in den Eklektoren 12 Hauptarten mit einem Individuenanteil von zusammen $66 \%$ vertreten, waren es in den Fensterfallen 16 Arten, auf die $58 \%$ der Individuen entfielen. Sechs Totholzkäferarten, Hylecoetus dermestoides, Cerylon ferrugineum, Rhizophagus bipustulatus, Rh.dispar, Xyloterus signatus und X. domesticus, erreichten in beiden Fallentypen Anteile von über $2 \%$. Da die große Mehrheit der Arten in beiden Fallentypen in nur geringer Individuenzahl auftrat, sind für diese keine deutlichen Unterschiede in deren Individuenanteilen festzustellen. Größere Abweichungen zeigten sich bei einigen häufigen Arten. So erreichten Rhizophagus dispar, Corticaria longicollis, Ptilinus pectinicornis, Dryocoetes villosus und Orchesia undulata in den Eklektoren, Hylecoetus dermestoides und Agathidium varians in den Fensterfallen erheblich höhere Individuenanteile.

Deutliche Unterschiede sind in den Arten- und insbesondere den Individuenanteilen der Habitatpräferenztypen festzustellen. Mit Ausnahme der saproxylen Arten waren alle Habitatpräferenzgilden in den Fensterfallen in höherer oder annähernd identischer Artenzahl 
vertreten. Unter den Totholzkäfern waren die Artenanteile der Habitatpräferenztypen allerdings sehr ähnlich. Die Anteile der mit beiden Fallensystemen erfaßten Arten, läßt man die succicolen Arten wegen ihrer geringen Arten- und Individuenzahl unberücksichtigt, variierte zwischen $57 \%$ (xylofungicole Arten) und $74 \%$ (corticole Arten). Mit Ausnahme der Mulmbewohner, von denen 5 Arten exclusiv in Fensterfallen und 12 Arten exclusiv in Eklektoren auftraten, war die Zahl der ausschließlich in einem Fallentyp nachgewiesenen Arten sehr ähnlich. Größere Unterschiede zwischen den Fallensystemen waren bei den Individuenanteilen der Habiatpräferenztypen festzustellen. In den Fensterfallen waren die lignicolen und xylofungicolen Arten mit größeren, die corticolen und xylodetricolen Arten mit geringeren Individuenanteilen als in den Eklektoren vertreten.

Die Abweichungen in den Artenanteilen der einzelnen Konsumententypen an den xylobionten Arten fiel zwischen den Fallentypen gering aus (0,6-3,6 \%). Größere Abweichungen ergaben sich auch hier bei den Individuenanteilen (1,4-9,8\%). Die größten Abweichungen wurden bei den mycetophagen Arten registriert, die in den Fensterfallen die jeweils höheren Anteile hatten. Die xylophagen und zoophagen Arten hatten dagegen höhere Anteile in den Eklektoren.

Die vorangegangenen Gegenüberstellungen lassen viele Gemeinsamkeiten, aber auch deutliche Unterschiede in den Ergebnissen der beiden Fallensysteme erkennen. Über Unterschiede in der Struktur der Fangergebnisse berichten auch HAMMOND (1997) und HAMMOND, LANGOHR \& SPENCE (2004). Bei den Totholzkäferarten fielen die Abweichungen bei den Artenzahlen und -anteilen geringer aus als bei den Individuenzahlen und anteilen. Die deutlichsten Differenzen traten bei der Betrachtung einzelner Arten zu Tage. Diese Unterschiede sind zum einen darin begründet, dass die Emergenzfallen selektiv das Artenspektrum eines bestimmten Strukturelementes erfassen. Zum anderen fanden aufgrund der Zielsetzung bei weitem nicht alle Totholzstrukturen Berücksichtigung. Anders als die Ergebnisse der Eklektoren, die v. a. die Schlüpfdichten der saproxylen Arten widerspiegeln, messen Fensterfallen die Flugaktivitätsdichte der Arten. Die Fangergebnisse werden also bedeutend stärker vom Schwärmverhalten und von der Dispersionsneigung der Arten sowie von den Witterungsbedingungen beeinflusst.

Fensterfallen sind bislang nur selten in großer Anzahl eingesetzt worden. In der Regel werden sie nur ergänzend in Artenerfassungsprogramme aufgenommen (ALBRECHT 1990; DOROW, FLECHTNER \& KOPELKE 1992; KÖHLER 1996; WINTER et al. 1999). Untersuchungen unter Verwendung einer größeren Anzahl von Flugbarrierefallen stammen fast ausnahmslos aus dem skandinavischen Raum, wo unterschiedliche Typen von Fensterfallen zur vergleichenden Erfassung der Totholzkäferfauna von Waldökosystemen eingesetzt wurden, und um die Bedeutung verschiedener Einflussfaktoren (z. B. Totholzangebot, Biotoptradition) zu untersuchen (z. B. KAILA et al. 1994; ØKLAND 2000, 2002; SIPPOLA 2001; MARTIKAINEN et al. 2000; MARTIKAINEN 2001; MARTIKAINEN \& KOUKI 2003; SIMILÄ 2002). BAKKE (1999), ZÖRNER (2003) sowie HAMMOND, LANGOHR \& SPENCE (2004) haben in ihren Untersuchungen sowohl Fensterfallen als auch Totholzeklektoren eingesetzt. Die Ergebnisse zeigen die hohe Effektivität der Flugbarrierefallen zur Erfassung von Artenspektren. BUSSLER, MÜLLER \& SIMON (2004) sowie JUVONENLETTINGTON \& PULLIN (2001) heben ebenfalls die gute Eignung von Fensterfallen hervor und auch die Ergebnisse von IRMLER (1998) ,... deuten darauf hin, dass durch Fensterfallen repräsentative Ergebnisse über die Artenzusammensetzung in Wäldern oberhalb des Bodens erzielt werden können." 
In der vorliegenden Untersuchung wurden nach Kenntnis des Autors erstmals in Mitteleuropa sowohl Fensterfallen als auch Eklektoren in so großer Zahl eingesetzt, dass eine vergleichende Bewertung dieser beiden Fallensysteme möglich ist. Fensterfallen haben sich sowohl in den oben genannten Arbeiten als auch in dieser Untersuchung als geeignetes Instrument erwiesen, das Artenspektrum und wesentliche Strukturparameter der xylobionten Käferfauna eines Gebietes zu charakterisieren. Auch mit Totholzeklektoren kann ein hoher Erfassungsgrad erreicht werden. Anders als diese gewähren Fensterfallen zusätzlich einen Einblick in die Struktur der nicht-xylobionten Käferfauna. Außerdem werden Daten zur Phänologie der Flugaktivität und zum Dispersionsverhalten ermittelt. Auf Grund des deutlich geringeren personellen, materiellen und finanziellen Aufwandes sind Fensterfallen für die Inventur der Käferfauna eines Gebietes im Allgemeinen sowie der Totholzkäferfauna im Besonderen den Emergenzfallen vorzuziehen.

Anders als für Gebietsinventuren sind Totholzeklektoren für die in dieser Untersuchung im Vordergrund stehenden Fragestellungen jedoch unverzichtbar. Nur sie liefern die erforderlichen quantitativen Daten zur Struktur der Artengemeinschaften, Vergesellschaftung, Schlüpfdichte und -phänologie der xylobionten Arten und erlauben die enge Verbindung von Entwicklungsort und Habitateigenschaften. Dass das Eklektorprinzip den manuellen Methoden vorzuziehen ist, hat schon DERKSEN (1941) erkannt. Als Nachteil ist neben ihrer Störanfälligkeit die Veränderung des Mikroklimas und der Ausschluß einer Neubesiedlung zu sehen. Fraglich ist auch, wie exakt die Ergebnisse der Fangdosen die Arten- und Dichtestruktur der geschlüpften Tiere widerspiegeln (DOROW, FLECHTNER \& KOPELKE 1992; KOPF 2000). Von den in dieser Untersuchung eingesetzten Eklektortypen haben sich STE, EFs und mBE (Typ B) bewährt.

Die im 'Neuenburger Urwald' gewonnenen Ergebnisse erlauben auch eine Abschätzung des zur Erfassung eines repräsentativen Artenausschnittes in Waldökosystemen erforderlichen Methodenaufwandes. In den einzelnen Fensterfallen wurden von Mai bis Oktober zwischen 34 und 112 Arten, im Durchschnitt 77 Arten festgestellt. In den drei Untersuchungsjahren 1993, 1995 und 1996 wurden mit jeweils 20 Fensterkreuzfallen zwischen 56 und $73 \%$ sämtlicher bzw. zwischen 63 und 78 \% der xylobionten Käferarten festgestellt. $92 \%$ der Totholzkäferarten wurden in der ersten Vegetationshälfte bis Mitte Juli nachgewiesen. Aus der Artensummenkurve ergab sich, dass die Jahresfänge von ca. 30 Fallen erforderlich sind, um 80 bis $90 \%$ des in Flugbarrierefallen nachgewiesenen Artenspektrums zu erfassen.

Um einen hohen Erfassungsgrad zu gewährleisten, kann bei dem hier eingesetzten Fensterfallentyp der Einsatz von ca. 15 bis 20 Fensterfallen über einen Zeitraum von 2 Jahren, jeweils vom zeitigen Frühjahr bis etwa Mitte Juli, empfohlen werden. Eine solche allgemeine Empfehlung kann allerdings nicht den zahlreichen Faktoren (z. B. Gebietsgröße und Strukturvielfalt) gerecht werden, die von nicht unerheblicher Bedeutung für die erforderliche Fallenzahl sind.

Zur Erfassung der xylobionten Käferfauna werden vielfach manuelle Methoden wie z. B. Gesiebe und Klopfproben bzw. eine Kombination aus verschiedenen Fallentypen und manuellen Aufsammlungen angewandt und empfohlen. Ihre Eignung zur Erfassung des Artenspektrums der xylobionten Käferfauna eines Gebietes werden durch die so gewonnenen Ergebnisse belegt (KÖHLER 1996; DOROW \& FLECHTNER 1999; RAUH 1993). Eine Bewertung dieser Methoden ist schwierig, solange es an methodischen Vergleichsuntersuchnungen aus den gleichen Gebieten fehlt. 


\subsection{Die Bedeutung von Baumart, Lage und Zersetzungszustand}

Das Wissen über das Auftreten von saproxylen Käferarten in Abhängigkeit verschiedenster Einflussfaktoren beruht zum größten Teil auf der Summe zahlreicher Einzelbeobachtungen (z. B. SAALAS 1917, 1923; PALM 1951, 1959). Die Zahl der Untersuchungen, die quantitative Daten zur Struktur der saproxylen Käferartengemeinschaften an Totholz liefern, ist angesichts der Vielzahl der Einflussfaktoren sehr begrenzt (z. B. DERKSEN 1941; HOWDEN \& VOGT 1951; SCHIMITSCHEK 1953, 1954; DAJOZ 1966; FRANCH 1989; PFARR 1990; HILT 1992; LACZNY 1993; KLEINEVOSS 1995; ZÖRNER 2003; HAASE, TOPP \& ZACH 1998; SCHIEGG PASINELLE 1999; KOPF 2000; HÖLLING 2000; SCHIEGG 2001; FLECHTNER 2002; KAPPES \& TOPP 2004). Insbesondere vergleichende Studien zur Bedeutung von besiedlungsrelevanten Faktoren wie Baumart, Totholzform, Durchmesser, Mikroklima und Zersetzungszustand sind selten.

Die genannten Untersuchungen unterscheiden sich erheblich in den Zielsetzungen und den daraus resultierenden Erfassungs- und Analysemethoden sowie in der Intensität der Erhebungen von der hier vorgelegten Arbeit. In keiner dieser Untersuchungen wurde sowohl stehendes als auch liegendes Totholz unterschiedlicher Zersetzungszustände von verschiedenen Baumarten in vergleichbarer Intensität mit quantitativen Methoden untersucht. Insbesondere ist ein Mangel an Untersuchungen zur Sukzession der Käfergemeinschaften zu beklagen. Die Resultate älterer Arbeiten beruhen zudem in erster Linie auf manuellen Erfassungsmethoden, während Eklektoren erst in jüngerer Zeit verstärkt eingesetzt worden sind. Darüber hinaus sind die untersuchten Baumarten, Dimensionen und Totholzformen sowie die geographischen und klimatischen Bedingungen von großer Bedeutung für die Struktur und Sukzession der Artengemeinschaften. Ein Vergleich der nachfolgend diskutierten Ergebnisse mit den bisherigen Untersuchungen ist daher i. d. R. kaum möglich.

\subsubsection{Einfluss der Baumart auf die Vergesellschaftung saproxyler Coleopteren}

Verschiedentlich ist der Artenreichtum der xylobionten Arten mit dem Baumartenspektrum in Verbindung gebracht worden (STARZYK 1979; BENSE \& GEIS 1998; BARBALAT 1998; BAKKE 1999). Die Pflanzengesellschaften des 'Neuenburger Urwaldes' werden v. a. von Stieleiche sowie Rot- und Hainbuche beherrscht. In dieser Untersuchung wurden Eichen und Rotbuchen mit Eklektoren untersucht. An der Eiche wurden 149, an der Buche 199 saproxyle Käferarten festgestellt. 70 Arten wurden ausschließlich in den Eklektorproben mit Buchentotholz, aber nur 20 exclusiv an Eichentotholz festgestellt. Ein Vergleich der Artenzahlen und ihrer Gesamtschlüpfdichte ergab sowohl in den STE-Proben als auch in den EFs-Proben die jeweils höheren Werte für die Buche. Betrachtet man die jeweils dominierenden Hauptarten, waren insbesondere am stehenden Totholz deutliche Unterschiede auszumachen. Von den 16 Arten, die entweder in den EFs-Proben an Eiche oder an Buche als Hauptart registriert wurden, erreichten nur 4 diesen Status an beiden Baumarten.

Die hier beschriebenen Unterschiede können keineswegs allein auf den Faktor Baumart zurückgeführt werden. Die Stichprobenkollektive differierten noch in weiteren Merkmalen. 
Insgesamt waren Probenzahl, befangenes Volumen und befangene Fläche an der Buche deutlich höher als an der Eiche. Hinzu kommen Unterschiede in der Struktur der Stichprobenkollektive, z. B. bzgl. der berindeten Fläche und der Zersetzungszustände. Dabei waren die Unterschiede an stehendem Totholz größer als an liegendem.

Über Unterschiede in der Besiedlung von Eichen- und Buchentotholz berichten auch HAASE, TOPP \& ZACH (1998) sowie KAPPES \& TOPP (2004). Die von LACZNY (1993) an stehenden Eichen und Buchen ermittelten Ergebnisse weichen deutlich von den eigenen Untersuchungen ab. So liegen die von ihm ermittelten Käferdichten um ein vielfaches niedriger. Die Ursache hierfür dürfte v. a. in der Verwendung der Eklektoren als Lebendfallen zu sehen sein, so dass sich an dieser Stelle auch weitere Vergleiche erübrigen. Deutliche Differenzen zwischen verschiedenen Baumarten wurden auch von LINDHE \& LINDELÖW (2004) sowie JONSELL, NITTÉRUS \& STIGHÄL (2004) festgestellt.

In der Regel wird dem Faktor Baumart aber eine eher untergeordnete Rolle für die Verteilung der xylobionten Käferfauna beigemessen. Als besonders reich an xylobionten Käferarten gilt die Eiche (AMMER 1991; PALM 1959). Insgesamt ist der Anteil der Arten, die ausschließlich oder doch mit klarer Präferenz an einer einzelnen Gehölzart bzw. -gattung gefunden wird, aber nur gering. Für die Mehrzahl der Arten läßt sich lediglich eine Trennung in Laub- und Nadelholzbewohner erkennen (KLAUSNITZER 1996; BENSE \& GEIS 1998). KÖHLER (2000) beziffert die Zahl der streng an eine Laubholzgattung gebundenen Totholzkäferarten mit 147, von denen 31 auf Eiche und nur 3 auf Buche spezialisiert sind. Bei den wenigen Arten mit enger Baumartenbindung handelt es sich überwiegend um Besiedler der frühen Besiedlungsphase und ist bei den Xylophagen relativ häufiger zu finden als bei den mycetophagen und zoophagen Arten (PALM 1959; ELTON 1966; KÖHLER 2000). So finden sich z. B. in der Familie der Scolytidae vergleichsweise viele Vertreter mit einer deutlichen Bevorzugung bestimmter Baumarten. $\mathrm{Zu}$ diesem Zeitpunkt sind die holzchemischen und -physikalischen Unterschiede am deutlichsten ausgeprägt (COOKE \& RAYNER 1984; RAYNER \& BODDY 1988; DAJOZ 2000; BODDY 2001). Mit fortschreitender Zersetzung nimmt die Bedeutung der Baumart mehr und mehr ab (KÖHLER 1996; HAASE, TOPP \& ZACH 1998; DAJOZ 2000).

Es wäre allerdings voreilig, hieraus den Schluß zu ziehen, dass die Baumart für die Gesellschaftsstruktur der xylobionten Käfergemeinschaften ohne Belang ist. Auch im 'Neuenburger Urwald' wurden nur wenige Arten ausschließlich an einer Baumart festgestellt. Meist handelt es sich dabei um Begleitarten, die mit nur wenigen Individuen nachgewiesen wurden. Von den häufigeren Arten wurde Hypulus quercinus nur an Eiche und sieben Arten (Cicones variegatus, Cis boleti, Epuraea variegata, Euplectus nanus, Octotemnus glabriculus, Taphrorychus bicolor, Vinzencellus ruficollis) exclusiv an Buche festgestellt.

Dennoch zeigten die Ergebnisse der Korrespondenzanalysen mit den Fangergebnissen sowohl von liegendem als auch von stehendem Totholz klare Unterschiede in der Vergesellschaftung der Arten an den beiden untersuchten Baumarten auf, die sich nicht durch methodische Einflüsse erklären lassen. Das Ergebnis hing nicht davon ab, ob die Dichte der beteiligten Arten oder nur ihr Auftreten berücksichtigt wurde. Der Einfluss der Baumart war bei fortgeschrittenem Zersetzungszustand weniger ausgeprägt als bei relativ frischem Totholz. Auch bei der Analyse der Verteilung einzelner Arten auf die Baumarten zeigte sich ein nicht unerheblicher Einfluss. Von 95 saproxylen Arten, deren Verteilung genauer analysiert wurde, erfüllten 38 Arten (40\%) die 'Präferenz'kriterien für eine Baumart. 


\subsubsection{Unterschiede in der Besiedlung von stehendem und liegendem Totholz}

Deutlich größere Unterschiede als bei einem Vergleich der beiden Baumarten ergeben sich bei der Gegenüberstellung von liegendem und stehendem Totholz. Den folgenden Ausführungen liegen die mit Stammeklektoren (STE) und Emergenzfallen (EFs) erhobenen Daten zu Grunde. Möglicherweise ist ein Teil der Differenzen auf eine unterschiedliche Fangeffizienz der verwendeten Fallentypen zurückzuführen. Die Emergenzfallen am stehenden Totholz waren zudem weniger störanfällig und es wurden pro Falle kleinere Stammabschnitte befangen.

In den Stammeklektoren am liegenden Totholz wurden 331 Arten in einer Dichte von 171 Ind. $/ \mathrm{m}^{2}$ festgestellt. In den Emergenzfallen an stehendem Totholz wurde dagegen eine Dichte von 803 Ind. $/ \mathrm{m}^{2}$ ermittelt, die sich auf 201 Arten verteilten. Der Unterschied in der Artenzahl fiel geringer aus, wenn nur die obligaten Totholzbewohner berücksichtigt wurden (165 zu 132). Berücksichtigt man, dass nur ca. $15 \%$ der mit diesen beiden Fallentypen befangenen Totholzoberfläche auf das stehende Totholz entfiel, relativieren sich die unterschiedlichen Artenzahlen erheblich.

Von den insgesamt 377 Käferarten wurden 46 ausschließlich am stehenden und 176 ausschließlich am liegenden Material erfaßt. Die Artenüberlappung betrug demnach $41 \%$. 188 Arten wurden als obligate Totholzbewohner eingestuft. Für diese belief sich die Artenüberlappung auf $58 \%$, während 56 Arten exclusiv an liegendem und 23 exclusiv an stehendem Totholz erfaßt wurden.

Diese Zahlen zeigen, dass ein großer Teil der Differenz auf das zahlreichere Auftreten von Nicht-Totholzkäfern am liegenden Totholz zurückzuführen ist. Am liegenden Totholz gehörten 166 Arten (50\%), am stehenden 69 Arten (34\%) nicht zu den Xylobionten. Mit 85 bzw. $90 \%$ war der Individuenanteil der Totholzkäfer in den EFs-Proben aber nur geringfügig höher.

Unter den Totholzbewohnern stellten die lignicolen und die xylofungicolen Arten in beiden Totholzformen vergleichbar hohe Arten- und Individuenanteile. Die xylodetricolen Arten hatten am stehenden Totholz größere Anteile als am liegenden, bei den corticolen Arten verhielt es sich umgekehrt.

Am stehenden Totholz war der auf die Hauptarten entfallende Individuenanteil größer als an liegendem. Ihr gemeinsamer Anteil belief sich auf 64 bzw. $75 \%$. Vier Arten hatten an beiden Totholzformen einen Individuenanteil von über $2 \%$ (Rhizophagus dispar, Rh. bipustulatus, Corticaria longicollis und Cerylon ferrugineum). Dabei zeigten die Individuenanteile der Arten z. T. beträchtliche Differenzen. Rhizophagus dispar, Xyloterus domesticus und Xyl. signatus hatten an den liegenden, Cortiaria longicollis, Dryocoetes villosus und Ptilinus pectinicornis an den stehenden Stämmen deutlich größere Dominanzanteile.

Vergleicht man allerdings die Schlüpfdichten, so wurden auch viele derjenigen Arten, die am liegenden Totholz höhere Individuenanteile erreichten, an stehenden Stämmen in größerer Dichte festgestellt (z. B. Rhizophagus dispar). Von den 12 Hauptarten des liegenden Totholzes schlüpften nur Xyloterus domesticus, Ptinella limbata, Phloeonomus punctipennis, Pteryx suturalis und Aridius nodifer hier auch in größerer Dichte. 
Die Artenanteile der Konsumententypen an den gefangenen Totholzkäfern waren am stehenden und liegenden Totholz sehr ähnlich. Die Räuber waren klar die artenreichste Gruppe (49 und $43 \%$ ), gefolgt von den Mycetophagen (25 und $30 \%$ ) und den Xylophagen (21 und $24 \%$ ). Allerdings erreichten nur die Mycetophagen auch vergleichbare Individuenanteile (38\% und $34 \%$ ), während die Räuber am liegenden Totholz mit $56 \%$ einen deutlich höheren Individuenanteil als am stehenden (30\%) hatten. In den STE-Proben spielten die Xylophagen eine nur geringe Rolle (4\%), wohingegen sie mit einem Individuenanteil von $35 \%$ in den EFs-Proben dominierten. Betrachtet man die Dichten, erreichten alle Konsumentengruppen am stehenden Totholz höhere Werte.

Von Unterschieden in der Besiedlung von liegendem und stehendem Totholz ist wiederholt berichtet worden (z. B. SAALAS 1917; PALM 1959; HAMMOND, LANGOHR \& SPENCE 2001, 2004; GROVE 2002; SVERDRUP-THYGESON \& IMS 2002; JONSELL \& WESLIEN 2003). Auch DERKSEN (1941) fand deutliche Unterschiede in den Artengemeinschaften von stehenden und liegenden Buchenstämmen. Nur in der Nähe des Stammfußes war eine größere Übereinstimmung festzustellen. Nach dem Umstürzen dauerte es nur wenige Jahre, bis die Unterschiede weitgehend verschwunden waren, wie es auch für die Pilzbesiedlung beschrieben worden ist (RAYNER \& BODDY 1988). Eine strenge Bindung saproxyler Käfer an stehende oder liegende Stämme ist allerdings eher die Ausnahme (PALM 1959).

Die Unterschiede in der Besiedlung von stehendem und liegendem Totholz dürften ursächlich vor allem auf die unterschiedlichen kleinklimatischen Bedingungen sowie auf die abweichenden Substratqualitäten zurückzuführen sein. Dabei sind nur selten einfache und gerichtete Wirkketten zu beobachten, vielmehr stehen die Einzelfaktoren in wechselseitiger und variierender Abhängigkeit zueinander (z. B. Temperatur, Gas- und Wasserhaushalt, Pilzbesiedlung und Zersetzungszustand) (SVERDRUP-THYGESON \& IMS 2002; GROVE, MEGGS \& GOODWIN 2002; JONSELL \& WESLIEN 2003).

Der Einfluss klimatischer Faktoren für das Besiedlungsmuster saproxyler Coleopteren wird von zahlreichen Autoren hervorgehoben (SCHIMITSCHEK 1953, 1954; PALM 1959; GEISER 1989; KÖHLER 1991; BENSE \& GEIS 1998; DAJOZ 2000; SVERDRUPTHYGESON \& IMS 2002). Ihre Bedeutung spiegelt sich u. a. in der Verteilung der Arten in Abhängigkeit von der geographischen und Höhenlage wider (CAPECKI 1969; BENSE \& GEIS 1998; KÖHLER 2000; SIITONEN 2001; BERG et al. 2002; BRECHTEL \& KOSTENBADER 2002). Auch kleinräumige Unterschiede der Temperatur-, Luftfeuchte-, Strahlungsund Windverhältnisse wirken sich deutlich auf die Struktur der Artengemeinschaften aus (z. B. DERKSEN 1941; OTTE 1989; KAILA, MARTIKAINEN \& PUNTILLA 1997; KAHLEN 1997; RANIUS \& NILSSON 1997; HARZ \& TOPP 1999; NILSSON, HEDIN \& NIKLASSON 2001; MARTIKAINEN 2001; FLECHTNER 2002; KOMONEN 2003b; LINDHE \& LINDELÖW 2004).

Die Durchschnittswerte, die tages- und jahreszeitliche Amplitude und die Vielfalt der mikroklimatischen Bedingungen variieren $u$. a. in Abhängigkeit von der Waldgesellschaft (z. B. Kiefernwald, Buchenwald), der Waldentwicklungsphase (z. B. Verjüngungs-, Optimal-, Zerfallsphase) und der Hangneigung. Zusätzlich sind innerhalb der Bestände mikroklimatische Höhengradienten ausgebildet (GEIGER 1961; MITSCHERLICH 1981; EIMERN \& HÄCKEL 1984; DAJOZ 2000). Vergleichsweise viele saproxyle Käferarten mit einer Präferenz für Eiche oder Pionierbaumarten sind wärmeliebend, während an Buche gebundene Arten überwiegend an schattige Bedingugen angepaßt sind (GÄRDENFORS \& BARANOWSKI 1992; EHNSTRÖM 2001; RANIUS \& JANSSON 2002). 
In der Konsequenz sind die mikroklimatischen Bedingungen auch am und im Totholzobjekt sehr variabel und von verschiedenen Faktoren abhängig. So ist z. B. neben den Unterschieden zwischen sonnenexponierten und beschatteten Baumteilen auch ein durchmesserabhängiger Gradient im Holzquerschnitt zu beobachten. Mit zunehmendem Abstand zur Holzoberfläche sind geringere Temperaturmaxima sowie tages- und jahreszeitliche Schwankungen zu verzeichnen, so dass im Innern, inbesondere von starkdimensionierten Stämmen, deutlich stabilere mikroklimatische Bedingungen herrschen als in den oberflächennahen Schichten. Zusätzlich stellt sich am stehenden Totholz ein Vertikalgradient der mikroklimatischen Faktoren ein (SCHIMITSCHEK 1953a; DAJOZ 1966, 2000; HÖLLING 2000).

Die Mehrzahl der mitteleuropäischen Totholzkäferarten entwickelt sich v. a. an beschattetem Totholz. Nicht wenige Arten zeigen aber eine starke Vorliebe für klimatisch begünstigte Standorte bzw. Totholzstrukturen (KÖHLER 2000). GEISER (1994) schätzt den Anteil der Arten, die an Totholz mit mindestens $50 \%$ der örtlichen Sonneneinstrahlung gebunden sind, auf wenigstens $25 \%$. Viele Beispiele finden sich u. a. in den Familien der Bock- und Prachtkäfer (KLAUSNITZER \& SANDER 1981; BRECHTEL \& KOSTENBADER 2002). Besonders unter den gefährdeten Arten sind thermophile Arten sehr zahlreich vertreten (GEISER 1994).

Für die Erklärung der großen Unterschiede in den Artengesellschaften an stehendem und liegendem Totholz ist weniger die unmittelbare Wirkung des Mikroklimas auf die Organismen verantwortlich als vielmehr dessen Einfluss auf den Wasser- unnd Gashaushalt sowie auf die Pilzbesiedlung des Totholzes. Verschiedene Autoren haben auf die Unterschiede zwischen stehendem und liegendem Totholz in den mikroklimatischen Bedingungen und Feuchteverhältnissen hingewiesen und sehen hierin eine wesentliche Ursache für die unterschiedlichen Besiedlungsmuster der xylobionten Organismen (DERKSEN 1941; SCHIMITSCHEK 1953, 1954; BRAUNS 1951, 1954; VITÉ 1952; PALM 1959; CAPECKI 1969; KLAUSNITZER \& SANDER 1981; BENSE \& GEIS 1998; DAJOZ 2000; SVERDRUPTHYGESON \& IMS 2002; JONSELL \& WESLIEN 2003).

Bodenkontakt, Mikroklima und Holzfeuchte haben einen wesentlichen Einfluss auf die Verteilung und Vergesellschaftung der Pilzarten am Totholz (CARRUTHERS \& RAYNER 1979; COOKE \& RAYNER 1984; RAYNER \& BODDY 1988; JAHN 1990; RUNGE 1991; SCHMIDT 1994; BADER, JANSSON \& JONSSON 1995; RENVALL 1995; LINDBLAD 1998; BODDY 2001; SNÄLL \& JONSSON 2001; BERG et al. 2002; SIPPOLA et al. 2004). Zahlreiche xylobionte Käferarten entwickeln sich in den Fruchtkörpern bzw. ernähren sich vom Pilzmyzel im Holz (SCHEERPELTZ \& HÖFLER 1948; BENICK 1952; WILDING et al. 1989; HANSKI 1989; KOMONEN 2003a, 2003b). KÖHLER (2000) beziffert die Zahl der Totholzpilzarten mit 214, die der mycetophagen und xylomycetophagen Arten zusammen auf über 350. Aber auch für die übrigen Arten ist die Pilzbesiedlung wegen ihres Einflusses auf die Substratqualität ein wichtiger Faktor.

Die Beziehung zwischen Pilzbesiedlung und Käferartenvorkommen sowie die Rolle verschiedener Habitatvariablen ist in zahlreichen Arbeiten beschrieben worden (GILBERTSON 1984; ARAYA 1993; KAILA et al. 1994; THUNES 1994; ØKLAND 1995, 2002; THUNES \& WILLIASSEN 1997; FOSSLI \& ANDERSEN 1998; MIDTGAARD, RUKKE \& SVRDRUP-THYGESON 1998; EDMONDS \& MARRA 1999; HÅGVAR 1999; JONSELL 1999; REIBNITZ 1999; THUNES, MIDTGAARD \& GJERDE 2000; GUEVARA et al. 2000; RUKKE 2000, 2002; SVERDRUP-THYGESON 2001; KOMONEN 2001; MÜLLER et al. 2002; JONSELL \& NORDLANDER 2002; JONSSON et al. 2003). 
Liegendes Totholz ist für eine vielfältige Pilzflora von großer Bedeutung, während sich in stehendem Totholz, insbesondere sonnenexponierter Lage, oftmals nur eine vergleichsweise artenarme Pilzgesellschaft etabliert (RUNGE 1991; HØILAND \& BENDIKSEN 1996; RYDIN, DIECKMANN \& HALLINGBACK 1997; SCHLECHTE 2002; HEILMANNCLAUSEN \& CHRISTENSEN 2003). Daher werden viele der an Totholzpilze gebunden Käferarten mit größerer Wahrscheinlichkeit an liegenden und beschatteten Stämmen festgestellt (JONSELL 1999; JONSELL \& WESLIEN 2003). Eine positive Korrelation zwischen Pilzartenzahl und Käferartenvielfalt wurde von LINDHE \& LINDELÖW (2004) auch für stehendes Totholz beschrieben.

Im 'Neuenburger Urwald' waren allerdings keine großen Unterschiede in der Besiedlung von stehendem und liegendem Totholz mit xylofungicolen und mycetophagen Arten festzustellen. Die Gründe hierfür sind wahrscheinlich darin zu sehen, dass viele der stehenden Probestämme im Bestandesschatten standen, während verschiedene liegende Stämme keinen Bodenkontakt hatten und/oder in sonnenexponierten Bestandeslücken positioniert waren. Zudem waren die EFs-Fallen in relativ geringer Stammhöhe angebracht. In der Konsequenz ist zu vermuten, dass es große Überlappungen zwischen den Probekollektiven bzgl. den mikroklimatischen und Feuchtebedingungen gab.

Groß waren die Unterschiede hingegen bei den xylophagen Arten, die am stehenden Totholz deutlich höhere Individuenanteile stellten. Während an liegendem Totholz die in ihrer Mehrheit feuchtigkeitsliebenden Dipteren meistens die dominierende Insektenordnung darstellen (z. B. HILT 1992; KLEINEVOSS, TOPP \& BOHAC 1996; HAASE, TOPP \& ZACH 1998), treten diese an stehendem und sonnenexponiertem Totholz allem Anschein nach zugunsten der Coleopteren zurück (PFARR 1990; FLECHTNER 2002). Die reduzierte Pilzartenvielfalt und -aktivität findet ihren Niederschlag in einer geringeren Abbaugeschwindigkeit von stehendem Totholz (RAYNER \& BODDY 1988; BRANG 1989; KAHL 2003). In der Konsequenz ist für die Totholzkäfer, insbesondere für die xylophagen Arten, an stehendem Totholz eine i. d. R. größere Bedeutung für den Verlauf und die Geschwindigkeit des Abbauprozesses zu vermuten.

\subsubsection{Einfluss des Zersetzungszustandes auf die Besiedlung durch Käfer}

Mit voranschreitender Zersetzung des Totholzes geht eine kontinuierliche Veränderung der Substrateigenschaften einher (z. B. HARMON et al. 1986; MACKENSEN \& BAUHUS 1999; SCHÄFER 2002; MÜLLER-USING \& BARTSCH 2003; HÖVEMEYER \& SCHAUERMANN 2003). Daher kommt dem Zersetzungszustand eine große Bedeutung für das Auftreten der saproxylen Käferarten zu (PALM 1959; RAUH 1992; GEISER 1994; KÖHLER 2000). Unterschiede in der Struktur der Artengemeinschaften in Abhängigkeit vom Zersetzungsstadium sind wiederholt beschrieben worden (z. B. DERKSEN 1941; SCHIMITSCHEK 1953, 1954; DAJOZ 1966; HILT 1992; KLEINEVOSS, TOPP \& BOHAC 1996; ZÖRNER 2003; HAASE, TOPP \& ZACH 1998; KOPF 2000; FLECHTNER 2002; KAPPES \& TOPP 2004).

Probleme für die Vergleichbarkeit der eigenen Ergebnisse zur Sukzession der Käfergemeinschaften mit anderen Untersuchungen ergeben sich nicht nur auf Grund der bereits erwähnten Bedeutung von Einflussfaktoren wie Baumart, Totholzform, Durchmesser und Mi- 
kroklima, sondern auch aus grundsätzlichen Unterschieden in den gewählten Verfahren, die zur Beschreibung und Analyse des Sukzessionsprozesses gewählt worden sind. Anders als in der vorliegenden Untersuchung und bei DAJOZ (1966), wurden Käfersukzessionen bislang auf der Grundlage einer vorangegangenen Klassenbildung beschrieben. Als Klassifikationskriterium wurden dabei alternativ der Zersetzungsgrad (SCHIMITSCHEK 1953, 1954; HILT \& AMMER 1994), der Bohrwiderstand (KLEINEVOSS, TOPP \& BOHAC 1996; HAASE, TOPP \& ZACH 1998; KAPPES \& TOPP 2004) oder das Alter der abgestorbenen Bäume herangezogen (DERKSEN 1941, ZÖRNER 2003; KOPF 2000). Alle diese Ordnungskriterien können sich auf eine mehr oder weniger starke Korrelation mit dem Zersetzungszustand des Holzes, gemessen z. B. als Holzdichte, berufen (ARTHUR, TRITTON \& FAHEY 1993; ECKSTEIN \& SASS 1994; LEE 1998; HALE \& PASTOR 1998; NAESSET 1999; BODDY 2001; FRAVER, WAGNER \& DAY 2002; KAHL 2003; CREED, WEBSTER \& MORRISON 2004). Meist werden nur wenige Klassen unterschieden (z. B. ALBRECHT 1990; PFARR 1990; HILT 1992; KLEINEVOSS 1995; IRMLER, HELLLER \& WARNING 1996; MÜLLER-USING \& BARTSCH 2003; KAPPES \& TOPP 2004), so dass der sich über einen sehr langen Zeitraum erstreckende Abbauprozeß und die damit verbundenen Veränderungen in der Besiedlung nur in groben Zügen wiedergegeben werden können.

Bei der Einteilung in Zersetzungsgrade auf der Grundlage von wenigen, äußerlich leicht ansprechbaren Merkmalen, ist eine differenzierte Untergliederung des Abbauprozesses i. d. R. ausgeschlossen. Zudem ist nur die Einordnung der diskreten Werte auf einer Ordinalskala möglich. Ein Rückschluß auf den Zustand im Holzinneren ist kaum möglich (SCHIMITSCHEK 1953, 1954). Ein weiterer Nachteil ist darin zu sehen, dass die Klassenbreiten sehr unterschiedlich sind (HALE \& PASTOR 1998; HEILMANN-CLAUSEN 2001; KUULUVAINEN, SYRJANEN \& KALLIOLA 2001; ZIELONKA \& NIKLASSON 2001; HOLEKSA 2001; KRUYS, JONSSON \& STÅHL 2002; KRAIGHER et al. 2002; KAHL 2003). MÜLLER-USING (2005) beziffert z. B. die Verweildauer von liegenden Buchenstämmen in den Zersetzungsklassen mit 3,6 ( $\left.Z^{\circ} 1\right), 5,4\left(Z^{\circ} 2\right), 9,1\left(Z^{\circ} 3\right)$ und 15,7 ( $\left.Z^{\circ} 4\right)$ Jahren. Desweiteren ist kritisch anzumerken, dass diese Klassifikationssysteme baumarten- und totholzformspezifisch sind (MÜLLER-USING \& BARTSCH 2003). Ein Quervergleich zwischen Baumarten oder zwischen Totholzformen (z. B. stehender und liegender Stamm) ist damit weitgehend ausgeschlossen. Die Zuordnung der Objekte ist zudem nicht frei von subjektiven Einflüssen, die schon bei der Entwicklung bzw. bei der Auswahl des Klassifikationssystems beginnen. Eine Standardisierung in reproduzierbarer Form ist kaum möglich. Es sind viele Klasseneinteilungen beschrieben worden und meistens stehen sogar mehrere für die gleiche Baumart zur Verfügung, so dass auch aus diesem Grunde eine Vergleichbarkeit nicht gegeben ist (z. B. für Buchentotholz SCHIMITSCHEK 1953; SCHMITT 1992; HÖLLING 2000; KRAIGHER et al. 2002; MÜLLER-USING \& BARTSCH 2003; HEILMANNCLAUSEN \& CHRISTENSEN 2003).

Einige der Nachteile werden durch die Messung des Bohrwiderstandes vermieden bzw. gemindert, doch ist eine Beurteilung auf Grund der bestehenden Datenlage zur Zeit noch nicht möglich. Der Bohrwiderstand beschränkt sich nicht auf äußerliche Merkmale und kann damit auch Aussagen zum Zersetzungszustand des Holzinneren machen. Anders als bei der Zersetzungsgradeinteilung können zum einen Klassen gleicher Breite gebildet werden, zum anderen ist das Verfahren, abgesehen von der Wahl der Meßstelle, weitestgehend frei von subjektiven Einflüssen. Bei der Interpretation der Meßergebnisse ist allerdings zu beachten, dass diese nicht allein von der Dichte des Holzes, sondern auch von weiteren Faktoren wie z. 
B. der Holzfeuchte und der Art der Holzfäule beeinflusst werden (ECKSTEIN \& SASS 1994; KELKENBERG 1995; KAHL 2003).

Das Alter des Totholzes ist, soweit überhaupt Daten dazu vorliegen, als Klassifikationskriterium wenig geeignet, da die Abbaugeschwindigkeit zwischen den Untersuchungsobjekten in beträchtlichem Maße variieren kann, so dass bei gleichem Alter, selbst bei ähnlichen Zersetzungsbedingungen, sehr unterschiedliche Zersetzungszustände festgestellt wurden (BARBER \& LEAR 1984; LEE 1998; HALE \& PASTOR 1998; NAESSET 1999; JONSSON 2000; BODDY 2001; SCHÄFER 2002; KRAIGHER et al. 2002; GROVEN et al. 2002). Begründet ist dieses darin, dass die Totholzzersetzung ein hochgradig komplexer Prozeß ist, der von zahlreichen Faktoren beeinflusst wird. Da die Variation der Zersetzungszustände mit zunehmender Zeitdauer tendenziell zunimmt, kann höchstens in den frühen Zerzetzungsphasen eine noch relativ enge Korrelation zwischen Alter, Zersetzungszustand und Artenauftreten beobachtet werden, wie sie sich auch in den Ergebnissen von ZÖRNER (2003) widerspiegelt. Sowohl die in dieser Arbeit vorgestellten Ergebnisse als auch die von KOPF (2000) beschriebene Entwicklung der Ähnlichkeitswerte als auch die Beobachtungen von DERKSEN (1941), FRANCH (1989), DOROW (2002) und FLECHTNER (2002) machen deutlich, dass das Alter wenig geeignet ist, die Sukzession der Arten zu beschreiben und ihre Verteilung zu verstehen.

Die Beschreibung der Verteilung der Käferarten nach vorangegangener Zuordnung des Probenmaterials zu Zersetzungsklassen kann wichtige Hinweise zum Verständnis des Sukzessionsprozesses liefern. Diese Formen der direkten Gradientenanalyse sind aber mit einigen grundsätzlichen Schwierigkeiten behaftet, die über die gemachten Anmerkungen zu den Klassifikationssystemen hinausgehen. Zum einen stellt sich bei dieser Vorgehensweise immer die Frage, ob das richtige, d. h. das für die Vergesellschaftung der Arten primär verantwortliche Ordnungskriterium gewählt worden ist. Daher sollte in jedem Fall untersucht werden, wie eng das beobachtete Verteilungsmuster mit dem Merkmal korreliert ist, wie gut das Klassifikationskriterium also die Vergesellschaftung der Arten erklären kann. Dieser Analyseschritt wird leider meistens unterlassen.

Zum anderen sollte zudem stets die Möglichkeit in Erwägung gezogen werden, dass sich hinter einer beobachteten Veränderung der Häufigkeitsmuster weitere, mehr oder weniger voneinander unabhängige Gradienten verbergen, die erst gemeinsam einen nennenswerten Anteil zur Erklärung der erhobenen Daten beisteuern können. So konnte z. B. in Untersuchungen zur Sukzession der Pilze an Totholz gezeigt werden, dass neben dem Zersetzungszustand weitere Faktoren zur Erklärung der Artenvergesellschaftungen herangezogen werden müssen (RENVALL 1995; HOILAND \& BENDIKSEN 1996; HEILMANN-CLAUSEN 2001). Ein weiterer Nachteil einer vorgezogenen Klassenbildung ist, dass durch die Festlegung der Klassengrenzen und die daraus resultierende Abgrenzung von Sukzessionsstadien und -phasen deutlich unterscheidbare Einheiten mit klaren Grenzen suggeriert werden, auch wenn der Wechsel der Artengemeinschafen ein kontinuierlicher Prozeß ist.

Im Ergebnis bedeutet die Verwendung von Klassifikationssystemen der beschriebenen Art i. d. R. eine starke Vereinfachung des meist hochkomplexen Gesellschaftsmusters, was nicht immer als Nachteil zu betrachten ist, solange es der Fragestellung angemessen ist. Zur Beschreibung und zum Verständnis von Sukzessionsphänomenen ist die direkte Gradientenanalyse ein erster und keineswegs unbedeutender Schritt, solange man sich ihrer Restriktionen bewußt bleibt. 
Angesichts der Ergebnisse bisheriger Untersuchungen und des insgesamt geringen Kenntnisstandes zur Vergesellschaftung und Sukzession der xylobionten Käfer an Totholz sowie aus grundsätzlichen Erwägungen zur Gradientenanalyse erschien es ratsam, zunächst die Arten und ihre Vergesellschaftung für sich sprechen zu lassen. Daher war das Hauptaugenmerk der vorliegenden Untersuchung auf die möglichst unvoreingenommene Beschreibung der Artenvergesellschaftungen gerichtet. Hierzu wurden nicht hypothetisch bedeutsame Faktorengradienten, sondern die interne Datenstruktur der Artengemeinschaften selbst zum Ordnungsprinzip erhoben (Ordination). Die gemessenen Habitatvariablen wurden erst nachträglich herangezogen, um ihren Beitrag zur Erklärung der vorgefundenen Gemeinschaftsstruktur zu prüfen. Verfahren der Korrespondenzanalyse sind im Rahmen der Totholzforschung bislang erst vereinzelt verwendet worden (SETÄLÄ \& MARSHALL 1994; RENVALL 1995; HOILAND \& BENDIKSEN 1996; LINDBLAD 1998; HEILMANNCLAUSEN 2001; HÖVEMEYER \& SCHAUERMANN 2003; JONSELL, NITTÉRUS \& STIGHÄLL 2004). Zur Darstellung und Analyse der Sukzession xylobionter Coleopteren sind diese nach Kenntnis des Autors bislang noch nicht eingesetzt worden.

Die Resultate der durchgeführten Korrespondenzanalysen (CA, DCA) sind von der zugrunde liegenden Datenbasis abhängig. Die Unterschiede sind allerdings eher von geringer Bedeutung. Der Erklärungsanteil der hypothetischen Variablen steigt, wenn nur die häufiger auftretenden Arten berücksichtigt werden und wenn anstelle ihrer Anwesenheit auch Informationen zu ihrer Häufigkeit in die Berechnung einfließen.

Als Ergebnis der Berechnung von Ähnlichkeitsindices und der Korrespondenzanalysen läßt sich festhalten, dass sich die Sukzession an liegendem und stehendem Totholz deutlich unterscheiden und das die auftretenden Artenvergesellschaftungen sich jeweils als in hohem Grade variabel darstellen. Mit fortschreitender Zersetzung ist eine tendenzielle Zunahme der Variabilität der Gesellschaften zu beobachten, was u. a. in geringeren Ähnlichkeitswerten zum Ausdruck kommt. Die Struktur der Artengemeinschaften zeigt eine deutliche Abhängigkeit vom Zersetzungszutand des Totholzes und die Ordinationsdiagramme spiegeln das Aufteten und Verschwinden der einzelnen Arten im Sukzessionsprozess wieder. Der Wechsel der Artengemeinschaften vollzieht sich dabei offensichtlich in einem kontinuierlichen Prozeß. Folglich konnten auch keine separaten Sukzessionsstadien anhand der Artenvergesellschaftungen abgegrenzt werden. Vielmehr ist eine starke Überlappung im Auftreten der Arten zu verzeichnen, so dass sich anstatt einer regelmäßigen Abfolge der Artengemeinschaften eher Tendenzen in der Besiedlung zu verzeichnen sind.

Die Bedeutung der einzelnen am Totholzobjekt erhobenen Merkmale wurde mittels kanonischer Korrespondenzanalysen (CCA, DCCA) geprüft. Am liegenden Totholz trugen v. a. Berindung und Bohrwiderstand zur Erklärung der Streuung bei. Am stehenden Totholz spielte die Holzfeuchte eine größere Rolle. Faktoren, die keine oder eine nur geringe Beziehung zum Zersetzungszustand aufweisen wie z. B. die Überschirmung, waren von eher nachgeordneter Bedeutung. Dagegen zeigen Berindung, Bohrwiderstand und Holzfeuchte eine deutlich engere Beziehung zum Zersetzungszustand (ECKSTEIN \& SASS 1994; KRAIGHER et al. 2002; KAHL 2003). Insgesamt können die erhobenen Variablen aber nur einen kleinen Teil der Streuung in der Vergesellschaftung der Arten erklären, was u. a. darauf zurückzuführen ist, dass im Rahmen dieser Untersuchung nur einige wenige Einflussfaktoren erhoben werden konnten.

Die Ergebnisse der vorliegenden Untersuchung lassen in ihren Grundzügen gewisse Parallelen zu anderen Sukzessionsuntersuchungen erkennen. So berichtet z. B. auch DERK- 
SEN (1941) von einer großen Variabilität in der Vergesellschaftung der Arten und einer großen Überlappung in ihrem Auftreten im Sukzessionsprozeß, so dass für die Käfer , ... nur eine gewisse Ablösung der regelmäßig vorkommenden Arten innerhalb großer Zeiträume zu beobachten ..." war.

Ein hohes Maß an Variabilität in der Gesellschaftsstruktur wurde auch bei Untersuchungen zur Sukzession von Dipteren und Pilzen an Totholz festgestellt, die ebenfalls einen eher kontinuierlichen Artenwechsel erkennen ließen (z. B. RENVALL 1995; HOILAND \& BENDIKSEN 1996; HEILMANN \& CLAUSEN 2001; HÖVEMEYER \& SCHAUERMANN 2003).

Zur Interpretation der vorgestellten Ergebnisse, insbesondere der großen Variabilität der Artenvergesellschaftungen, müssen verschiedene mögliche Erklärungen in Betracht gezogen werden.

Eine sehr große Zahl von Käferarten ist an Totholz gebunden. Charakteristischerweise wird das Gros der Individuen aber nur von wenigen Arten gestellt, während die bei weitem überwiegende Zahl der Arten nur mit geringen Dichten in wenigen Proben in Erscheinung tritt. Vergleichbare Dominanzstrukturen sind auch für andere Organismengruppen beschrieben worden (z. B. PFARR 1990; HILT 1992; OWEN 1992; RENVALL 1995; KLEINEVOSS, TOPP \& BOHAC 1996; IRMLER, HELLER \& WARNING 1996, 1997; ZÖRNER 2003; HAASE, TOPP \& ZACH 1998; SCHIEGG PASINELLI 1999; SVERDRUP-THYGESON 2001; FLECHTNER 2002; HÖVEMEYER \& SCHAUERMANN 2003; HEILMANNCLAUSEN \& CHRISTENSEN 2003). Zudem lassen einige Käferarten ein ausgesprochen geklumptes Verteilungsmuster erkennen. Beispiele hierfür sind Dryocoetes villosus und Lymexylon navale, die in Einzelproben in großer Dichte auftraten, während sie in vielen anderen Stammabschnitten mit anscheinend vergleichbaren Eigenschaften fehlten. In diesem Zusammenhang ist auch die Beobachtung von DERKSEN (1941) zu sehen, dass selbst bei vergleichbaren Bedingungen eine Vorhersage über das Auftreten einzelner Arten nicht möglich war. Von einem aggregierten Auftreten einzelner Fliegenarten berichten HÖVEMEYER \& SCHAUERMANN (2003). Gerade auf das eher sporadische Auftreten der 'Begleitarten' und der geklumpten Verteilung einiger Arten ist ein großer Teil der Variabilität in der Gesellschaftsstruktur zurückzuführen. Ob in diesem Verteilungsmuster spezifische Habitatansprüche der Arten zum Ausdruck kommen, muß angesichts des allgemein geringen Kenntnisstandes zur Biologie und Ökologie der Mehrzahl xylobionter Arten (KLAUSNITZER 1995; KOMONEN 2003a) fraglich bleiben.

Das Auftreten vieler Arten steht vermutlich in einer eher lockeren und indirekten Abhängigkeit zum Zersetzungszustand der Totholzobjekte. Die Artenvergesellschaftung dürfte dagegen in hohem Maße von den Wechselwirkungen zwischen den Arten beeinflusst sein. So zählt ein großer Teil der festgestellten Arten zu den Zoophagen, deren Verteilung und Dichte entscheidend vom Beuteangebot (z. B. Dipterenlarven) gesteuert wird. Auf die Bedeutung der Pilzbesiedlung für zahlreiche saproxyle Arten wurde bereits oben hingewiesen.

Totholz ist zudem eine ausgesprochen heterogene Ressource. Aus der Vielzahl der Kombinationsmöglichkeiten von Substrateigenschaften und Milieubedingungen ergibt sich eine enorme Habiatvielfalt (GEISER 1994). Die Komplexität des Zersetzungsprozesses kommt auch darin zum Ausdruck, dass die Abbaugeschwindigkeit selbst vergleichbarer Totholzstrukturen und ähnlicher Zersetzungsbedingungen in einem hohen Maße variabel ist (z. B. 
JONSSON 2000; KRAIGHER et al. 2002; SCHÄFER 2002; MÜLLER-USING \& BARTSCH 2003; KAHL 2003).

Da die aktuelle Zusammensetzung einer Lebensgemeinschaft in einem Sukzessionsprozeß von der Aktivität der vorangegangenen Biozönose beeinflusst wird, ist wiederholt die Möglichkeit diskutiert worden, dass, z. B. in Abhängigkeit von Absterbeursache und Todeszeitpunkt, verschiedene Sukzessionspfade ('different pathways') beschritten werden können, die auch in einer zunehmenden Variabilität der Artenvergesellschaftungen mit fortschreitender Zersetzung zum Ausdruck kommen (RENVALL 1995; BODDY 2001; HEILMANNCLAUSEN \& CHRISTENSEN 2003).

Nicht nur zwischen, sondern auch innerhalb der einzelnen Totholzobjekte sind verschiedene Substratqualitäten und Ausprägungen der Milieubedingungen zu verzeichnen. Verschiedene Baumteile wie Borke, Kambialzone, Splint- und Kernholz unterscheiden sich erheblich in ihren holzphysikalischen, -anatomischen und -chemischen Eigenschaften, werden von verschiedenen Artengemeinschaften besiedelt und unterschiedlich schnell abgebaut, so dass hier mehrere, unterschiedlich lange Abbau- und Sukzessionsgradienten nebeneinander zu verzeichnen sind (SCHIMITSCHEK 1953, 1954; DAJOZ 1966, 2000; KÄÄRIK 1974; DEYRUP 1981; HAYES \& TICKEL 1984; HARMON et al. 1986; JAHN 1990; ARTHUR, TRITTON \& FAHEY 1993; SCHMITT 1994; SCHOWALTER, ZHANG \& SABIN 1998; MACKENSEN \& BAUHUS 1999; SIITONEN 2001).

Aber auch innerhalb dieser Baumteile können verschiedene Zersetzungszustände auf kleinstem Raum wechseln. So berichten z. B. DERKSEN (1941), SCHIMITSCHEK (1953, 1954) und BRAUNS (1954) von einem kleinräumigen Nebeneinander verschiedener Befallsfolgen. Insbesondere in stärker dimensioniertem Totholz ist daher eine zunächst zunehmende Heterogenität der Substrateigenschaften zu beobachten, die erst in späten Zersetzungsphasen wieder rückläufig ist und sich in einer entsprechenden Entwicklung der Biodiversität niederschlägt (HEILMANN-CLAUSEN \& CHRISTENSEN 2003; HÖVEMEYER \& SCHAUERMANN 2003; LINDHE \& LINDELÖW 2004). Diesem kleinräumigen Nebeneinander verschiedener Zersetzungszustände kann mit Eklektoren nur ungenügend Rechnung getragen werden.

Im Ergebnis stellt sich der Abbau von Totholz als hochgradig komplexer Prozeß dar, der von den Substratqualitäten, den Umweltbedingungen und den Aktivitäten der Lebensgemeinschaften gesteuert wird. Auch wenn die Zahl der Untersuchungen zur Totholzökologie in den letzten Jahren deutlich gestiegen ist, bleibt der Prozeß der Totholzzersetzung bislang noch weitgehend unverstanden. Sowohl im Hinblick auf die Bemühungen zum Schutz der Vielfalt xylobionter Coleopteren als auch bzgl. ihrer Vergesellschaftung, Sukzession und Funktion bleiben große Forschungslücken zu füllen. Hierbei muß in größerem Maße als bislang in den meisten Fällen geschehen durch die Wahl biologisch und ökologisch adäquater Meß- und Analysemethoden der Komplexität des Abbauprozesses Rechnung getragen werden. 


\section{Zusammenfassung}

Totholz ist ein wichtiges Strukturelement in Waldökosystemen, das zahlreiche Funktionen erfüllt und in verschiedene Prozesse involviert ist (HARMON et al. 1986; SAMUELSON, GUSTAFSSON \& INGELÖG 1994). Große Bedeutung kommt dem Totholz als Lebensraum für eine Vielzahl xylobionter Organismen zu. Allein mehr als 1300 Käferarten sind obligatorisch auf Totholz angewiesen (KÖHLER 2000).

Das Totholzangebot ist im Wirtschaftswald im Vergleich zu ungenutzten Wäldern deutlich reduziert. In der Konsequenz zählen die saproxylen Arten zu den am stärksten gefährdeten Organismengruppen.

Wegen seiner Biotoptradition und seines Totholzreichtums bietet das Naturschutzgebiet 'Neuenburger Urwald' diesen Arten besonders günstige Lebensraumbedingungen und somit ideale Voraussetzungen für Untersuchungen zur Struktur und Sukzession saproxyler Käfer.

Das Artenspektrum des Untersuchungsgebietes sowie die Vergesellschaftung der Arten an Eichen- und Buchentotholz in Abhängigkeit verschiedener Habitatvariablen wurde mittels Einsatz von Fensterfallen und Totholzeklektoren untersucht.

Im 'Neuenburger Urwald' wurden insgesamt 711 Käferarten festgestellt, von denen 85 Arten in der Roten Liste der Käfer Deutschlands (GEISER 1998) verzeichnet sind. Als xylobiont wurden 260 Arten eingestuft, von denen wiederum 65 Arten als gefährdet gelten.

Der größte Artenanteil entfiel auf die Konsumentengilde der Räuber, die aber bzgl. des Individuenanteils von den Mycetophagen leicht übertroffen wurden.

Der große Artenreichtum der xylobionten Käfer im 'Neuenburger Urwald' wird u. a. auf das große Totholzangebot und die Vielfalt der Habitatstrukturen sowie deren räumliche und zeitliche Vernetzung in Verbindung mit einer langen Biotoptradition zurückgeführt.

Mit Fensterfallen wurden 562 Käferarten erfaßt, von denen 209 Arten den obligaten Totholzbewohnern zuzurechnen sind. Die Mehrzahl der Arten war in nur geringer Individuenzahl in den Proben vertreten. Nur sechs Arten erreichten einen Individuenanteil von mindestens $2 \%$.

In Totholzeklektoren wurden 457 Käferarten festgestellt, von denen fast die Hälfte den Totholzbesiedlern zugerechnet wird. Während die meisten Arten auch hier nur geringe Individuenanteile erreichten, entfielen auf die zwölf Hauptarten gemeinsam 57 \% der Individuen.

Um das Artenspektrum und wesentliche Strukturparameter der xylobionten Käferfauna eines Gebietes zu erfassen, hat sich der Einsatz einer ausreichend großen Zahl an Fensterfallen als geeignetes Mittel erwiesen. Zur Beschreibung der Vergesellschaftung und Sukzession der Käfergemeinschaften an Totholz sowie zur Untersuchung der Ursachen ihrer Verteilung ist der Einsatz von Totholzeklektoren jedoch unabdingbar, da nur so der erforderliche Zusammenhang von Entwicklungs- bzw. Aufenthaltsort der Arten sowie der quantitativen Struktur der Vergesellschaftungen und den Habitateigenschaften hergestellt werden kann.

Sowohl die Baumart (Eiche, Buche) als auch die Lage des Totholzes (stehend, liegend) haben einen großen Einfluss auf die Struktur der Käferartengemeinschaften. Dieser zeigt sich weniger im exklusiven Auftreten von Arten als vielmehr in ihrer Häufigkeit und ihrer Vergesellschaftung. Mit fortschreitender Zersetzung war die Bedeutung des Faktors Baumart rückläufig. 
Mit fortschreitender Zersetzung des Totholzes ändert sich die Zusammensetzung der Käfergemeinschaften. Separate Sukzessionsstadien waren anhand der Käferzönosen nicht abzugrenzen. Der Wechsel der Arten vollzieht sich vielmehr in einem kontinuierlichen Prozeß. Die vorgefundenen Käfergemeinschaften sind in hohem Maße variabel. Eine große Regelmäßigkeit in der Abfolge der Arten war nicht zu beobachten.

Die unterschiedlichen methodischen Ansätze zur Beschreibung und Analyse des Sukzessionsprozesses werden diskutiert.

Von den an den Totholzobjekten erhobenen Variablen trugen v. a. Faktoren mit enger Beziehung zum Zersetzungszustand (Bohrwiderstand, Berindung, Holzfeuchte) zur Erklärung der Artenvergesellschaftungen bei. Insgesamt konnten diese aber nur einen Teil der Vielfalt der Gesellschaftsmuster erklären.

Zur Erklärung der großen Variabilität der Artenvergesellschaftungen wird der Einfluss der Dominanzstruktur, des aggregierten Auftretens vieler Arten, der Wechselwirkungen zwischen den beteiligten Organismengruppen und der Vielfalt der Kombinationsmöglichkeiten besiedlungsbestimmender Substrateigenschaften und Milieufaktoren diskutiert.

Der Abbau von Totholz und die Vergesellschaftung und Sukzession der Artengemeinschaften stellt sich als hochgradig komplexer Prozeß dar, der bislang noch weitgehend unverstanden ist. Weitere Struktur- und Kausalanalysen sind dringend erforderlich. Diese sind eine wesentliche Voraussetzung sowohl für die Untersuchung der Funktion der verschiedenen Organismengruppen im Abbauprozeß als auch für die Entwicklung geeigneter Strategien und Maßnahmen zum Schutz der xylobionten Arten. 


\section{Literaturverzeichnis}

ABBOTT, D. T. \& CROSSLEY, D. A. (1982): Woody litter decomposition following clearcutting. Ecology 63 (1): 35-42

ADELI, E. (1961): Zur Kenntnis der Insektenwelt des Naturschutzgebietes bei der Sababurg unter besonderer Berücksichtigung der Lagerholzbewohner und ihrer Lebensweise. Diss. Univ. Göttingen

ALBRECHT, L. (1990): Grundlagen, Ziele und Methodik der waldökologischen Forschung in Naturwaldreservaten. Schr.R. Naturwaldreservate in Bayern 1.

ALBRECHT, L. (1991): Die Bedeutung des toten Holzes im Wald. Forstw. Cbl. 110 (2): 106113

ALEXANDER, K. N. A. (1988): The development of an index of ecological continuity for deadwood associated beetles. Antenna 12: 69-71

ALEXANDER, K. N. A. (1998): The links between forest history and biodiversity: the invertebrate fauna of ancient pasture-woodlands in Britain and its conservation. In: Kirby, K. J.; Watkins, C. (Eds.): The ecological history of european forests, pp. 73-80.

ALEXANDER, K. N. A. (2003): The British saproxylic invertebrate fauna. Proc. of the second pan-European conference on saproxylic beetles. www.ptes.org/events/conferences/documents/Keith\%20Alexander.pdf

AMMER, U. (1991): Konsequenzen aus den Ergebnissen der Totholzforschung für die forstliche Praxis. Forstw. Cbl. 110: 149-157

ANDERSSON, L. J. \& HYTTEBORN, H. (1991): Bryophytes and decaying wood: a comparison between managed and natural forest. Holarctic Ecology 14 (2): 121-130

ANGELSTAM, P. (1997): Landscape analysis as a tool for the scientific management of biodiversity. Ecol. Bull. 46: 140-170

ANGELSTAM, P. \& MIKUSINSKI, G. (1994): Woodpecker assemblages in natural and managed boreal and hemiboreal forest - a review. Ann. Zool. Fennici 31: 157-172

ARAYA, K. (1993): Relationship between the decay types of dead wood and occurance of lucanid beetles (Coleoptera: Lucanidae). Applied Entomology and Zoology 28: 27-33

ARTHUR, M. A.; TRITTON, L. M. \& FAHEY, T. J. (1993): Dead boles and nutrients remaining 23 years after clear-felling of a northern hardwood forest. Can. J. For. Res. 23: $1298-1305$

AUSMUS, B. S. (1977): Regulation of wood decomposition rates by arthropod and annelid populations. Ecol. Bull. 25: 180-192

BADER, P.; JANSSON, S. \& JONSSON, B. G. (1995): Wood-inhabiting fungi and substratum decline in selectively logged boreal spruce forests. Biol. Cons. 72 (3): 355-362

BAHL, A. \& HENLE, K. (1999): Gefährdungsanalysen bei Tieren: Konzepte und Vorgehensweisen bei zoologischen Gefährdungsanalysen. In: Amler, K.; Bahl, A.; Henle, K.; Kaule, G.; Poschlod, P. \& Settele, J. (Hg.): Populationsbiologie in der Naturschutzpraxis: Isolation, Flächenbedarf und Biotopansprüche von Pflanzen und Tieren. S. 93-94 
BAKKE, A. (1999): High diversity of saproxylic beetles in a hemiboreal mixed forest in the south of Norway. Scand. J. For. Res. 14 (3): 199-208

BARBALAT, S. (1998): Importance of forest structures on four beetle families (Col.: Buprestidae, Cerambycidae, Lucanidae, and phytophagous Scarabaeidae) in the Areuse Gorges (Neuchatel, Switzerland). Revue Suisse de Zoologie 105 (3): 569-580

BARBER, B. L. \& LEAR, D. H. van (1984): Weight loss and nutrient dynamics in decomposing woody Loblolly Pine logging slash. Soil Sci. Soc. Am. J. 48: 906-910

BEHRE, G. F. (1989): Freilandökologische Methoden zur Erfassung der Entomofauna (Weiter- und Neuentwicklung von Geräten). J. ber. naturwiss. Ver. Wuppertal 42.

BENEKE, CH. \& BUTLER MANNING, D. (2003): Coarse woody debris in the Weberstedter Holz, a near natural beech forest in central Germany. www.flec.kvl.dk/natman $18 \mathrm{~S}$.

BENGTSSON, J.; NILSSON, S. G.; FRANC, A. \& MENOZZI, P. (2000): Biodiversity, disturbances, ecosystem function and mangement of European forests. For. Ecol. Managem. 132: 39-50

BENICK, L. (1952): Pilzkäfer und Käferpilze. Acta Zool. Fenn. 70: 250 pp

BENSE, U. \& GEIS, K.-U. (1998): Holzkäfer. Mitt. Forstl. Versuchs- u. Forschungsanstalt Baden-Württemberg, H. 203: 45-117

BERG, Å.; EHNSTRÖM, B.; GUSTAFSSON, L.; HALLINGBÄCK, T.; JONSELL, M. \& WESLIEN, J. (1994) : Threatened plant, animal, and fungus species in swedish forests: distribution and habitat associations. Cons. Biol. 8 (3): 718-731

BERG, Å.; GÄRDENFORS, U.; HALLINGBÄCK, T. \& NORÉN, M. (2002): Habitat preferences of red-listed fungi and bryophytes in woodland key habitats in southern Sweden - analyses of data from a national survey. Biodiv. Conserv. 11: 1479-1503

BLACKMAN, M. W. \& STAGE, H. H. (1924): On the succession of insects living in the bark and wood of dying, dead and decaying hickory. N.Y. State Coll. Forestry, Syracuse Techn. Publ. 17

BLUME, D. (1993): Die Bedeutung von Alt- und Totholz für unsere Spechte. Beih. Veröff. Naturschutz Landschaftspflge Bad.-Württ. 67: 157-162

BOBIEC, A. (2002): Living stands and dead wood in the Białowieza forest: suggestions for restoration management. For. Ecol. Managem. 165: 125-140

BODDY, L. (2001): Fungal community ecology and wood decomposition processes in angiosperms: from standing tree to complete decay of coarse woody debris. Ecol. Bull. 49: $43-56$

BODDY, L. B. \& SWIFT, M. J. (1984): Wood decomposition in an abandoned beech and oak coppiced woodland in SE England. III. Decomposition and turn over of twigs and branches. Holartic Ecology 7: 229-238

BONCINA, A. (2000): Comparison of structure and biodiversity in the Rajhenov virgin forest remnant and managed forest in the Dinaric region of Slovenia. Global Ecol. Biogeogr. 9 (3): 201-211

BRANG, P. (1989): Untersuchungen zur Zerfallsdynamik in unberührten Bergföhrenwäldern im schweizerischen Nationalpark. Schweiz. Z. Forstw. 140 (2): 155-163

BRAUNS, A. (1951): Die Feuchtigkeit bei der Humifizierung von Stöcken. Norddtsch. Holzwirtsch. 5 (30): 8 
BRAUNS, A. (1954): Die Sukzession der Dipterenlarven bei der Stockhumifizierung. Z. Morph. Ökol. Tiere 43: 313-320

BRECHTEL, F. (1991): Zur Lebensweise und Bestandessituation holzbewohnender Wespenarten in Mitteleuropa und Konsequenzen für ihren Schutz. NZ NRW Seminarberichte H. 10: 26-31

BRECHTEL, F. \& KOSTENBADER, H. (2002) (Eds.): Die Pracht- und Hirschkäfer BadenWürttembergs. Stuttgart

BREDESEN, B.; HAUGAN, R. AANDERAA, R.; LINDBLAD, I.; OKLAND, B. \& ROSOK, O. (1997): Wood-inhabiting fungi as indicators on ecological continuity within spruce forests of southestern Norway. Blyttia 55 (3): 131-140 (norwegisch, engl. sum.)

BRETZ GUBY, N. A. \& DOBBERTIN, M. (1996): Quantitative estimates of coarse woody debris and standing dead trees in selected Swiss forests. Global Ecology and Biogeography Letters (5): 327-341

BÜCHS, W. (1988): Stamm- und Rindenzoozönosen verschiedener Baumarten des Hartholzauenwaldes und ihr Indikatorwert für die Früherkennung von Baumschäden. Dissertation Bonn

BUNNELL, F. L.; HOUDE, I.; JOHNSTON, B. \& WIND, E. (2002): How dead trees sustain live organisms in western forests. USDA For. Serv. Gen. Tech. Rep. PSW-GTR-181, 291-318

BURGER, A. (1999): Lebensraum Totholz in Naturwaldreservaten der Rhön. AFZ/Der Wald 8: $390-391$

BURRICHTER, E. (1984): Baumformen als Relikte ehemaliger Extensivwirtschaft in Nordwestdeutschland. Drosera 1 (1-18) Oldenburg

BURSCHEL, P. (1992): Totholz und Wirtschaftswald. AFZ 21: 1143-1146

BURSELL, J. (2002): Winter abundance of hole-nesting birds in natural and managed woods of Zealand (Denmark). Acta Ornithologica Warsaw 37 (2): 67-74

BUSCHINGER, A. (1991): Lebensweise, Bestandessituation und Konsequenzen für den Schutz holzbewohnender Ameisen in Mitteleuropa. NZ NRW Seminarberichte H. 10: 36-38

BUSSLER, H.; MÜLLER, J. \& SIMON, U. (2004): Erfassung xylobionter Käfer in Waldökosystemen - Ein Methodenvergleich unter besonderer Berücksichtigung der Konenfauna. Naturschutz u. Landschaftsplanung 36 (7): 197-201

BUTLER, J.; ALEXANDER, K. N. A. \& GREEN, T. (2002): Decaying wood: an overview of its status and ecology in the United Kingdom and Continental Europe. USDA For. Serv. Gen. Tech. Rep. PSW-GTR-181: 11-19

CALDWELL, R. S. (1996): Macroinvertebrates and their relationship to coarse woody debris: with special reference to land snails. In: McMinn, J. W. \& Crossley, D. A. (Eds.); S. $49-54$

CAPECKI, Z. (1969): Insects damaging wood of the beech (Fagus sylvtica) on the area of its natural occurrence in Poland. Prace IBL No. 367 (poln., dtsch. Zus.)

CARRUTHERS, S. M. \& RAYNER, A. D. M. (1979): Fungal communities in decaying hardwood branches. Trans. Br. Mycol. Soc. 72 (2): 283-289 
CHRISTENSEN, M. \& EMBORG, J. (1996): Biodiversity in natural versus managed forest in Denmark. For. Ecol. Managem. 85: 47-51

CHRISTENSEN, M. \& HAHN, K. (2003): A study on dead wood in European beech forest reserves. Prepared by members of Work-package 2 in the Nat-Man project (Naturebased Management of beech in Europe) founded by the European Community $5^{\text {th }}$ Framework Programme. (www.flec.kvl.dk/natman (April 2004))

COOKE, R. C. \& RAYNER, A. D. M. (1984): Ecology of saprotrophic fungi. London

CREED, I. F.; WEBSTER, K. L. \& MORRISON, D. L. (2004): A comparison of techniques for measuring density and concentrations of carbon and nitrogen in coarse woody debris at different stages of decay. Can. J. For. Res. 34: 744-753

CRITES, S. \& DALE, M. R. T. (1998): Diversity and abundance of bryophytes, lichens, and fungi in relation to woody substrate and successional stage in aspen mixewood boreal forest. Can. J. Bot. 76 (4): 641-651

DAJOZ, R. (1966): Écologie et biologie des coléoptères xylophages de la hetraie. Vie et Milieu 17: Teil 1 und 2, S. 525-636 und 637-763.

DAJOZ, R. (2000): Insect and forests: the role and diversity of insects in the forest environment. London

DANIELS, J. A: (1991): Zur Bedeutung von Totholz für Moose und Flechten. NZ NRW Seminarberichte H. 10: 10-13

DE MEULDER, H. (1999): Experimenteel onderzoek naar de successie van paddestoelen op dood hout (6de vervolg). (Experimental research on the succession of fungi on dead wood (part 6)): AMK-Mededelingen 1999 (1): 2-10 (holländisch, engl. Sum.)

DERKSEN, W. (1941): Die Succession der pterygoten Insekten im abgestorbenen Buchenholz. Z. Morph. Ökol. Tiere 37: 683-734

DETSCH, R. (1999a): Der Beitrag von Wirtschaftswäldern zur Struktur- und Artenvielfalt: ein Vergleich ausgewählter waldökologischer Parameter aus Naturwaldreservaten und Wirtschaftswäldern des Hienheimer Forstes (Kehlheim, Niederbayern). Diss. Univ. München

DETSCH, R. (1999b): Vielfalt im Naturwald - Einfalt im Wirtschaftswald? Ergebnisse aus Strukturvergleichen von Naturwaldreservaten und Wirtschaftswäldern. NUA-Seminarbericht 4: 193-208

DEUTSCHER WETTERDIENST (1992, 1993, 1995, 1996): Monatlicher Witterungsbericht. Jahrg. 40, 41, 43, 44

DEYRUP, M. (1981): Deadwood decomposers. Natural History 90: 84-91

DIX, N. J. (1995): Fungal ecology. London

DOROW, W. (2002): Zoologische Untersuchungen auf der Sturmwurffläche - Tierordnungen, Heteroptera (Wanzen), Hymenoptera (Hautflügler). Mitt. d. Hess. Landesforstverwaltung 38: 79-115

DOROW, W. \& FLECHTNER, G. (1999): Ergebnisse umfassender Faunenuntersuchungen in montanen Buchenwäldern auf Basalt und Buntsandstein in Hessen. NUA Seminarberichte 4: 176-192

DOROW, W.; FLECHTNER, G. \& KOPELKE, J-P. (1992): Zoologische Untersuchungen Konzept. Mitt. d. Hess. Landesforstverwaltung 26 
ECKSTEIN, D. \& SASS, U. (1994): Bohrwiderstandsmessungen an Laubbäumen und ihre holzanatomische Interpretation. Holz als Roh- und Werkstoff 52: 279-286

EDMAN, M. \& JONSSON, B. G. (2001): Spatial pattern of downed logs and wood-decaying fungi in old-growth Picea abies forest. J. Veg. Sci. 12 (5): 609-620

EDMONDS, R. L. \& EGLITES, A. (1989): The role of the Douglas-fir beetle and wood borers in the decomposition of and the nutrient release from Douglas-fir logs. Can. J. For. Res. 19: 853-859

EDMONDS, R. L. \& MARRA, J. L. (1999): Decomposition of woody material: nutrient dynamics, invertebrate/fungi relationships and management in Northwest forests. USDA For. Serv., Gen. Techn. Rep. PNW-GTR-461, pp 68-79

EHNSTRÖM, B. (2001): Leaving dead wood for insects in boreal forests - suggestions for the future. Scand. J. For. Res. Suppl. 3: 91-98

EIMERN, J. VAN \& HÄCKEL, H. (1984): Wetter- und Klimakunde. Stuttgart

EK, T.; WADSTEIN, M. \& SVENSSON, L. (2001): Lang skoglig kontinuitet och nagra lavar I ostgotska sumpskogar (The importance of long forest continuity for some lichens in mixed swamp forests in Ostergotland, Sweden). Svensk Botanisk Tidskrift 95 (6): $357-$ 369 (swedish)

ELLENBERG, H. (1986): Vegetation Mitteleuropas mit den Alpen in ökologischer Sicht. Stuttgart (4. Aufl).

ELMER, M. (2002): Totholz im Wirtschaftswald als Lebensraum xylobionter Insekten. LÖBF-Mitteilungen 2002 (1): 36-42

ELTON, C. S. (1966): The pattern of animal community. London

ERDMANN, M. \& WILKE, H. (1997): Quantitative und qualitative Totholzerfassung in Buchenwirtschaftswäldern. Forstw. Cbl. 116: 16-28

ERICKSON, J. L. \& WEST, ST. D. (2003): Association of Bats with local structurs and landscape features of forested stands in western Oregon and Washington. Biodiv. Cons. 109 (1), 95-102

ESSEEN, P.-A. ; EHNSTRÖM, B. ; ERICSON, L. \& SJÖBERG, K. (1997): Boreal forests. Ecol. Bull. 46: 16-47

FÄLDT, J.; JONSELL, M.; NORDLANDER, G. \& BORG-KARLSON, A.-K. (1999): Volatiles of bracket fungi Fomitopsis pinicola and Fomes fomentarius and their functions as insect attractants. J- chem. Ecol. 25 (3): 567-590

FALINSKI, J. B. (1978): Uprooted trees, their distribution and influence in the primeval forest biotope. Vegetatio 38: 175-183

FALINSKI, J. B. (1986): Vegetation dynamics in temperate lowland primeval forests Ecological studies in Bialowieza forest. Geobotany 8, Junk Publisher, Dordrecht

FERRIS, R. \& HUMPHREY, J. W. (1999): A review of potential biodiversity indicators for application in British forests. Forestry 72 (4): 313-328

FERRIS, R.; PEACE, A. J. \& NEWTON, A. C. (2000): Macrofungal communities of lowland Scots pine (Pinus sylvestris L.) and Norway spruce (Picea abies (L.) Karsten) plantations in England: relationships with site factors and stand structure. For. Ecol. Managem. 131 (1/3): 255-267 
FLECHTNER, G. (2002): Die Rolle der Käfer beim Abbau von Buchen-Totholz in der Sturmwurffläche des Naturwaldreservates Weiherskopf/Vogelsberg. Mitt. D. Hess. Landesforstverwaltung 38: 123-145

FLECHTNER, G.; DOROW, W. H. O. \& KOPELKE, J.-P. (2000): Niddahänge östlich Rudingshaim - Zoologische Untersuchungen 1990-1992: Coleoptera (Käfer). Mitt. D. Hess. Landesforstverwaltung 32.

FOSSLI, T.-E. \& ANDERSEN, J. (1998): Host preference of Cisidae (Coleoptera) on treeinhabiting fungi in northern Norway. Entomologica Fennica 9: 65-78

FRANCH, J. (1989): Some regularities in invertebrate succession in different microhabitats on pine stumps. Orsis 4: 69-79

FRAVER, S.; WAGNER, R. \& DAY, M. (2002): Dynamics of coarse woody debris following gap harvesting in the Acadian forest of central Maine, U.S.A. Can. J. For. Res. 32 (12): 2094-2105

FREEDMAN, B.; ZELAZNY, V.; BEAUDETTE, D.; FLEMING, T.; FLEMING, S.; FORBES, G.; GERROW, J. S.; JOHNSON, G. \& WOODLEY, S. (1996): Biodiversity implications of changes in the quantity of dead organic matter in managed forests. Environmental Reviews 4 (3): 238-265

FREUDE, H.; HARDE, K. W. \& LOHSE, G. A. (1965-1983): Die Käfer Mitteleuropas. Bd. 1-11. Krefeld

FRIDMAN, J. \& WALHEIM, M. (2000): Amount, structure, and dynamics of dead wood on managed forestland in Sweden. For. Ecol. Managem. 131 (1-3): 23-36

FUNKE, W. (1971): Food and energy turnover of leaf-eating insects and their influence on primary production. Ecol. Studies 2: 81-93

GÄRDENFORS, U. \& BARANOWSKI, R. (1992): Skalbaggar anpassade till öppna respektive slutna ädellövskogar föredrar olika trädslag (Beetles living in open deciduous forest prefer different tree species than those living in dense forests). Entomologisk Tidskrift 113: 1-11 (Schwedisch, engl. Zusammenfassung)

GARLAND, S. P. (1983): Beetles as primary woodland indicators. The Sorby Record 21: 159-171

GAUCH, H. G. (1982): Multivariate analysis in community ecology. Cambridge

GEIGER, R. (1961): Das Klima der bodennahen Luftschicht. Ein Lehrbuch der Mikroklimatologie. Braunschweig

GEISER, R. (1989): Spezielle Käfer-Biotope, welche für die meisten übrigen Tiergruppen weniger relevant sind und daher in der Naturschutzpraxis zumeist übergangen werden. Schr.-R. f. Landschaftspflege u. Naturschutz 28: 268-276

GEISER, R. (1994): Artenschutz für holzbewohnende Käfer (Coleoptera xylobionta). Ber. ANL 18: 89-114

GEISER, R. (1998): Rote Liste der Käfer (Coleoptera). In: Binot, M.; Bless, R.; Boye, P.; Gruttke, H. \& Pretscher, P.: Rote Liste gefährdeter Tiere Deutschlands. Schr.R. Landschaftspflege u. Naturschutz 55: 168-230

GILBERTSON, R. L. (1984): Relationships between insects and wood-rotting fungi. In Wheeler, Q.; Blackwell, M. (Eds.): Fungus-Insects relationships: 130-165

GLEN, D. M. (1976): An emergence trap for bark-dwelling insects, its efficiency and effects on temperature. Ecol. Entom. 1: 91-94 
GREEN, P. \& PETERKEN, G. F. (1997): Variation in the amount of dead wood in the woodlamds of the Lower Wye Valley, UK in relation to the intensity of management. For. Ecol. Managem. 98 (3): 229-238

GROVE, S. J. (2002): Saproxylic insect ecology and the sustainable management of forests. Ann. Rev. Eco. System. 33: 1-23

GROVE, S. J.; MEGGS, J. \& GOODWIN, A. (2002): A review of biodiversity conservation issues relating to coarse woody debris management in the wet eucalyptus production forests of Tasmania. $72 \mathrm{~S}$.

GUEVARA, R.; HUTCHESON, K. A.; MEE, A. C.; RAYNER, A. D. M. \& REYNOLDS, S. E. (2000): Resource partitioning of the host fungus Coriolus versicolor by two ciid beetles: the role of odour components and host ageing. Oikos 91: 184-194

GUEVARA, R.; RAYNER, A. D. M. \& REYNOLDS, S. E. (2000): Orientation of specialist and generalist fungivorous ciid beetles of host and non-host odors. Physiol. Entomol. 25: $288-295$

HAASE, V.; TOPP, W. \& ZACH, P. (1998): Eichentotholz im Wirtschaftswald als Lebensraum für xylobionte Insekten. Z. Ökol. Nat.sch. 7: 137-153

HÅGVAR, S. (1999): Saproxylic beetles visiting sporocarps of Fomitopsis pinicola and Fomes fomentarius. Norwegian Journal of Entomology 46(1): 25-32

HÅGVAR, S. \& ØKLAND, B. (1997): Saproxylic beetle fauna associated with living sporocarps of Fomitopsis pinicola (Fr.) Karst. in four spruce forests with different management histories. Fauna Norv. (Ser. B) 44: 95-105

HAILA, Y. \& KOUKI, J. (1994) : The phenomen of biodiversity in concervation biology. Ann. Zool. Fennici 31: 5-18

HALE, C. M. \& PASTOR, J. (1998): Nitrogen content, decay rates, and decompositional dynamics of hollows versus solid hardwood logs in hardwood forests in Minnesota, USA. Can. J. For. Res. 28 (9): 1276-1285

HAMMOND, H. E. J. (1997): Arthropod biodiversity from Populus coarse woody material in north-central Alberta: a review of taxa and collection methods. The Canadian Entomologist 129: 1009 - 1033

HAMMOND, H. E. J.; LANGOR, D. W. \& SPENCE, J. R. (2001): Early colonization of Populus wood by saproxylic beetles (Coleoptera). Can. J. For. Res. 31: 1175-1183

HAMMOND, H. E. J.; LANGOR, D. W. \& SPENCE, J. R. (2004): Saproxylic beetles (Coleoptera) using aspen stands of western Canada: spatiotemporal variation and conservation of assemblages. Can. J. For. Res. 34: 1-19

HANSKI, I. (1989): Fungivory: Fungi, insects and ecology. In: Wilding et al. (Eds.): InsectFungus interactions. S. 25-68

HARDING, P. T. \& ALEXANDER, K. N. A. (1993): The saproxylic invertebrates of historic parklands: progress and problems. In: Kirby, K. J.; Drake, C. M. (1993, Eds.): Dead wood matters: the ecology and conservation of saproxylic invertebrates in Britain. Engl. Nat. Sci. 7: 58-73

HARMON, M. E. (2001): Moving towards a new paradigm for woody detrius management. Ecol. Bull. 49: 269-278

HARMON, M. E. ; FRANKLIN, J. F. ; SWANSON, F. J. ; SOLLINS, P. ; GREGORY, S. V. ; LATTIN, S. J. ; ANDERSON, N. H.; CLINE, S. P.; AUMEN, N. G.; SEDELL, J. 
R.; LIENKAEMPER, G. W.; CROMACK, K. JR. \& CUMMINS, K. W. (1986) : Ecology of coarse woody debris in temperate ecosystems. Adv. Ecol. Res. 15: 133-302

HARMON, M. E.; KRANKINA, O. N. \& SEXTON, J. (2000): Decomposition vectors: a new approach to estimating woody detritus decomposition dynamics. Can. J. For. Res. 30: 76-84

HARZ, B. \& TOPP, W. (1999): Totholz im Wirtschaftswald: eine Gefahrenquelle zur Massenvermehrung von Schadinsekten? Forstw. Cbl. 118: 302-313

HAYES, A. J. \& TICKELL, R. F. (1984): Colonization of coniferous stumps by cerambycid beetles (Coleoptera, Cerambycidae). Scot. For. 38: 17-32

HEDIN, J. \& RANIUS, T. (2002): Using radio telemetry to study dispersal of the beetle Osmoderma eremita, an inhabitant of tree hollows. Computers and Electronics in Agriculture 35 (2/3): 171-180

HEILMANN-CLAUSEN, J. (2001): A gradient analysis of communities of macrofungi and slime moulds on decaying beech logs. Mycd. Res 105 (5), 575-596

HEILMANN-CLAUSEN, J. \& CHRISTENSEN, M. (2003): Fungal diversity on decaying beech logs - implications for sustainable forestry. Biodiv. Cons. 12: 953-973

HENLE, K.; POSCHOLD, P. \& BAHL, A. (1999): Gefährdungsanalysen auf populationsbiologischer Grundlage: Einführung in Konzepte und Begriffe. In: Amler, K.; Bahl, A.; Henle, K.; Kaule, G.; Poschlod, P. \& Settele, J. (Hg.): Populationsbiologie in der Naturschutzpraxis: Isolation, Flächenbedarf und Biotopansprüche von Pflanzen und Tieren. S.46-52

HESMER, H. \& SCHROEDER, F. G. (1963): Waldzusammensetzung und Waldbehandlung im Niedersächsischen Tiefland westlich der Weser und der Münsterischen Bucht bis zum Ende des 18. Jahrhunderts. - Decheniana, Beiheft 11: 304 S., Bonn

HILL, M. O. (1973): Reciprocal averaging: an eigenvector method of ordination. J. Ecol. 61 (237-249)

HILL, M. O. (1979): DECORANA - A FORTRAN program for detrended correspondence analysis and reciprocal averaging. Ecology and systematics. Cornell University, Ithaca, New York 14850

HILL, M. O. \& GAUCH, H. G. (1980): Detrended correspondence analysis: an improved ordination technique. Vegetatio 42 (47-58)

HILT, M. (1992): Besiedlung von Fichten- und Eichentotholz durch Insekten - Vergleichende Untersuchungen an Käfern (Coleoptera) und Zweiflüglern (Diptera) im Allacher Forst (Forstamt München). Dipl.arb. Univ. München

HILT, M. \& AMMER, U. (1994): Totholzbesiedelnde Käfer im Wirtschaftswald - Fichte und Eiche im Vergleich. Forstw. Cbl. 113: 245-255

HØILAND, K. \& BENDIKSEN, E. (1996): Biodiversity of wood-inhabiting fungi in a boreal coniferous in Sør-Trøndelag county, Central Norway. Nord. J. Bot. 16: 643-659

HOLEKSA, J. (2001): Coarse woody debris in a Carpathian subalpine forest. Forstw. Cbl. 120: $256-270$

HÖLLING, D. (2000): Unterwuchs als wichtige Habitatqualität für xylobionte Käfer im Buchenwald. Diss. Univ. Bonn, Germany

HÖVEMEYER, K. (1998): Diptera associated with dead beech wood. Studia Dipterologica 5 (1): 113-122 
HÖVEMEYER, K. \& SCHAUERMANN, J. (2003): Succession of Diptera on dead beech wood: a 10-year study. Pedobiologia 47: 61-75

HOWDEN, H. F. \& VOGT, G. B. (1951): Insect communities of standing dead pine (Pinus virginiana). Annales Entomol. Soc. Am. 44: 581-595

HUMPHREY, J. W.; DAVEY, S.; PEACE, A. J.; FERRIS, R. \& HARDING, K. (2002): Lichens and bryophyte communities of planted and semi-natural forests in Britain : the influence of site type, stand structure and dead wood. Biol. Conserv. 107 (2): 165-180

HUTHA, V. (1979): Evaluation of different similarity indices as measures of succession in arthropod communities of the forest floor after clear-cutting. Oecologia (Berl.) 41: $11-23$

IRMLER, U. (1998): Die vertikale Verteilung flugaktiver Käfer (Coleoptera) in drei Wäldern Norddeutschlands. Faun.-ökol. Mitt. 7 (9-10): 387-404

IRMLER, U.; HELLER, K. \& WARNING, J. (1996): Age and tree species as factors influencing the populations of insects living in dead wood (Coleoptera, Diptera: Sciaridae, Mycetophilidae). Pedobiologia 40 (2): 134-148

IRMLER, U.; HELLER, K. \& WARNING, J. (1997): Kurzflügelkäfer (Co., Staphylinidae) an Totholz schleswig-holsteinischer Wälder. Faun.-ökol. Mitt. 7 (7-8): 307-318

JAHN, H. (1990): Pilze an Bäumen. Berlin. 2. Auflage

JANETSCHEK, H (1982): Ökologische Feldmethoden. Stuttgart

JANISCH, J. E. \& HARMON, M. E. (2002): Successional changes in live and dead wood carbon stores: Implications for net ecosystem productivity. Tree Physiology 22 (2-3): $77-89$

JONSELL, M. (1999): Insects on wood-decaying polypores: conservation aspects. Acta Universitatis Agriculturae Sueciae, Silvestria 93: $45 \mathrm{pp}$

JONSELL, M.; NITTÉRUS, K. \& STIGHÄLL, K. (2004): Saproxylic beetles in natural and man-made deciduous high stumps retained for conservation. Biol. Conserv. 118: 163173

JONSELL, M. \& NORDLANDER, G. (1995): Field attraction of Coleoptera to odours of the wood-decaying polyopores Fomitopsis pinicola and Fomes fomentarius. Ann. Zool. Fennici 32: 391-402

JONSELL, M. \& NORDLANDER, G. (2002): Insects in polypore fungi as indicator species: a comparison between forest sites differing in amounts and continuity of dead wood. For. Ecol. Manage. 157: 101-118

JONSELL, M. \& WESLIEN, J. (2003): Felled or standing retained wood: it makes a different for saproxylic beetles. For. Ecol. Manage. 175 (1-3): 425-435

JONSSON, B. G. (2000). Availability of coarse woody debris in a boreal old-growth Picea abies forest. J. Veg. Sci. 11: 51-56

JONSSON, M. (2002): Dispersal ecology of insects inhabiting wood-decaying fungi. Doctoral thesis Swedish University of Agricultural Sciences, Uppsala 2002

JONSSON, M.; JONSELL, M. \& NORDLANDER, G. (2001): Priorities in conservation biology: a comparison between two polypore-inhabiting beetles. Ecol. Bull. 49: 195-204 
JONSSON, M.; KINDVALL, O.; JONSELL, M. \& NORDLANDER, G. (2003): Modelling mating success of saproxylic beetles in relation to search behaviour, population density and substrate abundance. Animal Behaviour 65 (6): 1069-1076

JONSSON, M.; NORDLANDER, G. \& JONSELL, M. (1997): Pheromones affecting flying beetles colonizing the polypores Fomes fomentarius and Fomitopsis pinicola. Entom. Fennica 8: 161-165

JÜDES, U. (1991): Habitatgefüge und Habitatwahl der Waldfledermäuse - Folgerungen für die Forstwirtschaft. NZ NRW Seminarberichte H. 10: 54-56

JURGENSEN, M. F.; HARVEY, A. E.; GRAHAM, R. T.; PAGE-DUMROESE, D. S.; TONN, J. R.; LARSEN, M. J. \& JAIN, T. B. (1997): Impacts of timber harvesting on soil organic matter, nitrogen, productivity, and health of inland northwest forests. Forest Science 43 (2): 234-251

JUVONEN-LETTINGTON, A. \& PULLIN, A. S. (2001): The assessment of the saproxylic Coleoptera fauna of lowland parkland and wood pasture: an evaluation of sampling techniques. Antenna (London) 25 (2): 97-99

KÄÄRIK, A. A. (1974): Decomposition of wood. In: Dickinson, C. H.; Pugh, G. J. F. (eds): Biology of plant litter decomposition (Vol.1), S. 129-174, London, New York 1979, Academic Press

KAHL, T. (2003): Abbauraten von Fichtentotholz (Picea abies) (L.) Karst.) - Bohrwiderstandsmessungen als neuer Ansatz zur Bestimmung des Totholzabbaus, einer wichtigen Größe im Kohlenstoffhaushalt mitteleuropäischer Wälder. M.Sc. Univ. Jena

KAHLEN, M. (1997): Die Holz- und Rindenkäfer des Karwendels und angrenzender Gebiete. Natur in Tirol Sonderband 3

KAILA, L.; MARTIKAINEN, P. \& PUNTTILA, P. (1997): Dead trees left in clear-cuts benefit saproxylic Coleoptera adapted to natural disturbances in boreal forest. Biodiv. Conserv. $6(1): 1-18$

KAILA, L.; MARTIKAINEN, P.; PUNTTILA, P. \& YAKOVLEV, E. (1994): Saproxylic beetles (Coleoptera) on dead birch trunks decayed by different polypore species. Ann. Zool. Fennici 31: 97-197

KAPPES, H. \& TOPP, W. (2004): Emergence of coleoptera from deadwood in a managed broadleaved forest in central Europe. Biodiv. Conserv. 13: 1905-1924

KAYAHARA, G. J.; KLINKA, K. \& LAVKULICH, M. (1996): Effects of decaying wood on eluviation, podzolization, acidification, and nutrition in soils with different moisture regimes. Environmental Monitoring and Assessment 39: 485-492

KELKENBERG, J. (1995): Einfluß der Holzfeuchte auf die Dichtemessung mit dem RESISTOGRAPH 1410 - untersucht am Beispiel der Fichte (Picea abies (L.) Karst.). Dipl.arbeit Univ. Göttingen

KIRBY, K. J.; WEBSTER, S. D. \& ANTCZAK, A. (1991): Effects of forest management on stand structure and the quantity of fallen dead wood: some British and Polish examples. For. Ecol. Managem. 43 (1-2): 167-174

KLAUSNITZER, B. (1995): Die Hirschkäfer: Lucanidae. 2. überarb. Aufl. Magdeburg, Die neue Brehm-Bücherei 551

KLAUSNITZER, B. (1996): Gesunder Wald braucht totes Holz - Alt- und Totholz als Grundlage einer hohen Biodiversität. Insecta, Berlin (4): 5-22 
KLAUSNITZER, B. \& SANDER, F. (1981): Die Bockkäfer Mitteleuropas. Wittenberg

KLEINEVOSS, K. (1995): Buchen-Totholz im Wirtschaftswald als Lebensraum für saproxylische Insekten. Dipl.arb. Univ. Köln

KLEINEVOSS, K.; TOPP, W. \& BOHAC, J. (1996): Buchen-Totholz im Wirtschaftswald als Lebensraum für xylobionte Insekten. Z. Ökol. Nat.sch. 5: 85-95

KÖHLER, F. (1991): Anmerkungen zur ökologischen Bedeutung des Alt- und Totholzes in Naturwaldzellen. NZ NRW Seminarberichte 10: 14-18

KÖHLER, F. (1996): Käferfauna in Naturwaldzellen und Wirtschaftswald. Hg. Landesanstalt für Ökologie, Bodenordnung und Forsten / Landesamt für Agrarordnung NRW, LÖBFSchr.R. 18, 352 S.

KÖHLER, F. (1999): Totholzkäfer und Naturwaldreservate in Deutschland - Ein aktueller Überblick. NUA Seminarberichte 4: 165-175

KÖHLER, F. (2000): Totholzkäfer in Naturwaldzellen des nördlichen Rheinlandes. Hg. Landesanstalt für Ökologie, Bodenordnung und Forsten / Landesamt für Agrarordnung NRW, LÖBF-Schr.R. 6, 283 S.

KÖHLER, F. \& KLAUSNITZER, B. (1998)(Hg.): Verzeichnis der Käfer Deutschlands. Entom. Nachr. u. Ber., Beiheft 4

KÖLBEL, (1999): Totholz in Naturwaldreservaten und Urwäldern. LWF aktuell 18: 2-5

KOMONEN, A. (2001): Structure of insect communities inhabiting old-growth forest specialist bracket fungi. Ecol. Entom. 26: 63-75

KOMONEN, A. (2003 a): Hotspots of insect diversity in Boreal forests. Conserv. Biol. 17 (4): $976-981$

KOMONEN, A. (2003 b): Insects in wood-decaying fungi: ecology, diversity and response to forest management. Joensuu, $35 \mathrm{pp}$

KOOP, H. (1981): Vegetatiestructuur en dynamiek van twee natuurlijke bossen: het Neuenburger en Hasbrucher Urwald. Wageningen

KOOP, H. (1982): Waldverjüngung, Sukzessionsmosaik und kleinstandörtliche Differenzierung infolge spontaner Waldentwicklung. In: Dierschke, H. (Hg.): Struktur und Dynamik von Wäldern. Ber. d. int. Symp. d. int. Vereinigung f. Vegetationskde. in Rinteln 1981: $235-267$

KOOP, H. \& HILGEN, P. (1987): Forest dynamics and regeneration mosic shifts in unexploited beech (Fagus sylvatica) stands at Fontainebleau (France). For. Ecol. Manage. 20: $135-150$

KOPF, A. (2000): Untersuchungen zur Sukzession von Arthropodengesellschaften (insbes. Coleoptera) auf Fichten-Sturmwurfflächen in Baden-Württemberg. Diss. Universität Ulm

KOPF, A. \& FUNKE, W. (1998): Xylobionte Arthropoden. In: Fischer, A. (Hg.): Die Entwicklung von Wald-Biozönosen nach Sturmwurf. S. 282-291

KORPEL, S. (1992): Ergebnisse der Urwaldforschung für die Waldwirtschaft im Buchenökosystem. AFZ 21: 1148-1152

KORPEL, S. (1995): Die Urwälder der Westkarpaten. Stuttgart, Jena, New York

KORPEL, S. (1997 a): Totholz in Naturwäldern und Konsequenzen für Naturschutz und Forstwirtschaft. Forst \& Holz 52 (21): 619-624 
KORPEL, S. (1997 b): Erkenntnisse über Strukturdynamik und Entwicklungsprozesse der Urwälder in der Slowakei und ihre Anwendung in der naturnahen Waldwirtschaft. Beitr. Forstwirtsch. u. Landschaftsökologie 31: 151-155

KRAIGHER, H.; JURC, D.; KALAN, P.; KUTNAR, L.; LEVANIČ, T.; RUPEL, M. \& SMOLEJ, I. (2002): Beech coarse woody debris characteristics in two virgin forest reserves in southern Slovenia. Zbornik Gozdarstva in Lesarstva 69: 91-134

KRANKINA, O. N.; HARMON, M. E. \& GRIAZKIN, A. V. (1999): Nutrient stores and dynamics of woody detritus in a boreal forest: modelling potential implications at the stand level. Can. J. For. Res. 29: 20.32

KRANKINA, O. N.; HARMON, M. E.; KUKUEV, Y. A.; TREYFELD, R. F.; KASHPOR, N. N.; KRESNOV, V. G.; SKUDIN; V. M.; PROTASOV, N. A.; YATSKOV, M.; SPYCHER, G. \& POVAROV, E. D. (2002): Coarse woody debris in forest regions of Russia. Can. J. For. Res. 32: 768-778

KREBS, C. J. (1989): Ecological Methodology. New York

KRIEBITZSCH (1989): Der Neuenburger Urwald. Unveröff. Manuskript

KROGERUS, R. (1927): Beobachtungen über die Succession einiger Insektenbiocönosen in Fichtenstümpfen. Notulae Entomologicae 7: 121-126

KRUYS, N. \& JONSSON, B. G. (1999): Fine woody debris is important for species richness on logs in managed boreal spruce forests of northern Sweden, Can.J. For. Res. 29 (8): 1295-1299

KRUYS, N. ; FRIES, C ; JONSSON, B. G. ; LÄMÅS, T. \& STÅHL, G (1999): Wood-inhabiting cryptogams on dead Norway spruce (Picea abies) trees in managed Swedish boreal forests. Can. J. For. Res. 29 (2): 178-186

KRUYS, N. \& JONSSON, B. G. \& STÅHL, G (2002): A stage-based matrix model for decay-class dynamics of woody debris. Ecol. Appl. 12 (3): 773-781

KÜHNEL, S. (1999): Totholz im Bayerischen Staatswald - Ergebnisse der Totholzinventur. LWF-aktuell 18: 6-12

KÜSTER, H. (1995): Geschichte der Landschaft in Mitteleuropa - Von der Eiszeit bis zur Gegenwart. München, C. H. Beck

KÜSTER, H. (1998): Geschichte des Waldes - Von der Urzeit bis zur Gegenwart. München, C. H. Beck

KUULUVAINEN, T.; SYRJANEN, K. \& KALLIOLA, R. (2001): Logs in a pristine Picea abies forest: occurrence, decay stage distribution and spatial pattern. Ecol. Bull. 49: $105-113$

LACZNY, M. (1993): Zur Ökologie totholzbewohnender Coleopteren - Ein Beitrag zur Ökologie und Faunistik xylobionter Coleopteren in stehendem, starkvolumigen Laubbaumtotholz sowie Implikationen für den Naturschutz am Beispiel des Staatsforstes Göhrde. Dipl.arb. Univ. Hamburg

LAIHO, R. \& PRESCOTT, C. E. (2004): Decay and nutrient dynamics of coarse woody debris in northern coniferous forests: a synthesis. Can. J. For. Res. 34: 763-777

LAMBERT, R. L.; LANG, G. E.; REINERS, W. A. (1980): Loss of mass and chemical change in decaying boles of a subalpine balsam fir forest. Ecol. 61 (6): 1460-1473 
LARSSON, M. C.; HEDIN, J.; SVENSSON, G. P.; TOLASCH, T. \& FRANCKE, W. (2003): Characteristic odor of Osmoderma eremita identified as a male-released pheromone. J. Chem. Ecol. 29 (3): 575-587

LEE, P. (1998): Dynamics of snags in aspen-dominated midboreal forests. For. Ecol. Manage. 105 (1-3): 263-272

LEIBUNDGUT, H. (1982): Europäische Urwälder der Bergstufe. Bern

LEIBUNDGUT, H. (1993): Europäische Urwälder - Wegweiser zur naturnahen Waldwirtschaft. Bern

LINDBLAD, J. (1998): Wood-inhabiting fungi on fallen logs of Norway spruce: Relations to forest management and substrate quality. Nordic J. Bot. 18 (2): 243-255

LINDHE, A. (2004): Conservation through management - cut woods as substrate for saproxylic organisms. Doctoral thesis SLU Uppsala, Sweden

LINDHE, A. \& LINDELÖW, A. (2004): Cut high stumps of spruce, birch, aspen and oak as breeding substrates for saproxylic beetles. For. Ecol. Managem. 203 (1-3): 1-20

LOFROTH, E. (1998): The dead wood cycle. In: Voller, J. \& Harrison S. (Eds.): Conservation biology principles for forested landscapes. UBC Press, Vancouver, S. 185-214

LOHSE, G. A. \& LUCHT, W. (Hg.) (1989-1994): Die Käfer Mitteleuropas. Bd. 12-14. Krefeld

LUCHT, W. (1987): Die Käfer Mitteleuropas - Katalog. Krefeld

LUCHT, W. \& KLAUSNITZER, B. (1998): Die Käfer Mitteleuropas - Vierter Supplementband. Krefeld

MACKENSEN, J. \& BAUHUS, J. (1999): The decay of coarse woody debris. National Carbon Accounting System Technical Report No. 6; 46 pp.

MAMAEV, B. M. (1961) : The activity of large invertebrates as one of the principal factors in the breakdown of wood. Pedobiologia 1: 38-52 (in russisch)

MARTIKAINEN, P. (2000): Effects of forest management on beetle diversity, with implications for species conservation and forest protection. $\mathrm{PhD}$ thesis, Joensuu 2000

MARTIKAINEN, P. (2001): Conservation of threatened saproxylic beetles: significance of retained aspen Populus tremula on clearcut areas. Ecol. Bull. 49: 205-218

MARTIKAINEN, P. \& KOUKI, J. (2003): Sampling the rarest: threatened beetles in boreal forest biodiversity inventories. Biodiv. Cons. 12 (9): 1815-1831

MARTIKAINEN, P.; SIITONEN, J.; KAILA, L.; PUNTTILA, P. \& RAUH, J. (1999): Bark beetles (Coleoptera, Scolytidae) and associated beetle species in mature managed and old-growth forests in southern Finland. For. Ecol. Mnage. 116 (1-3): 233-245

MARTIKAINEN, P.; SIITONEN, J.; PUNTTILA, P.; KAILA, L. \& RAUH, J. (2000): Species richness of Coleoptera in mature managed and old-growth boreal forests in southern Finland. Biol. Cons. 94 (2), 199-209

MASER, CH. \& TRAPPE, J. M. (eds.) (1984): The seen and unseen world of the fallen tree. USDA For. Serv. Gen. Techn. Rep. PNW-164, Pac.North-west For. And Range Exp. Stn., Portland, OR 56p.

MCCAY, T. S.; HANULA, J. L.; LOEB, S. C.; LOHR, ST. M.; MCMINN; J. W. \& WRIGHT MILEY, B. D. (2002): The role of coarse woody debris in southeastern pine 
forests: preliminary results from a large-scale experiment. USDA For. Serv. Gen. Techn. Rep. PSW-GTR-181: 135-144

MENKE, N. (2001): Bestandsaufnahme der xylobionten Käfer im potenziellen FFH-Gebiet Nr. 059 'Bentheimer Urwald'. Unveröff. Gutachten

MEYER, P. (1999): Totholzuntersuchungen in nordwestdeutschen Naturwäldern: Methodik und erste Ergebnisse. Forstw. Cbl. 118: 176-180

MEYER, P.; TABAKU, V. \& LÜPKE, B. v. (2003): Die Struktur albanischer RotbuchenUrwälder - Ableitungen für eine naturnahe Buchenwirtschaft. Forstw. Cbl. 122: 47-58

MEYNEN, E. \& SCHMITTHÜSEN, J. (1959-62): Handbuch der naturräumlichen Gliederung Deutschlands II. Bad Godesberg

MIDTGAARD, F.; RUKKE, B. A. \& SVERDRUP-THYGESON, A. (1998): Habitat use of the fungivorous beetle Bolitophagus reticulatus (Coleoptera: Tenebrionidae): effects of basidiocarp size, humidity and competitors. Eur. J. Entom. 95 : 559-570

MITSCHERLICH, G. (1981): Wald, Wachstum und Umwelt. Eine Einführung in die ökologischen Grundlagen des Waldwachstums. Bd. 2: Waldklima und Wasserhaushalt. Frankfurt a. M.

MÖLLER, G. (1994): Alt- und Totholzlebensräume - Ökologie, Gefährdungssituation, Schutzmaßnahmen. Beitr. Forstwirtsch. Landschaftspfl. 28 (1): 7-15

MÖLLER, G.; BELLMAN, A. \& ESSER, J. (1995): Dendroentomologische Untersuchungen im Hasbruch bei Bremen unter besonderer Berücksichtigung der Pflege- und Entwicklungsplanung. Unveröff. Gutachten

MÖLLER, G. \& SCHNEIDER. M (1991): Kommentierte Liste ausgewählter Familien überwiegend holzbewohnender Käfer von Berlin-West mit Ausweisung der gefährdeten Arten (Rote Liste). In: Auhagen, A.; Platen, R. \& Sukopp, H. (Hg.) 1991: Rote Listen der gefährdeten Pflanzen und Tiere in Berlin. Landschaftsentwicklung und Unweltforschung 56: 373-420

MOUNTFORD, E. P. (2002): Fallen dead wood levels in the near-natural beech forest at La Tillaie reserve, Fontainbleau, France. Forestry (Oxford) 75 (2): 203-208

MÜHLENBERG, M. (1989): Freilandökologie. Heidelberg

MÜLLER, M. M.; VARAMA, M.; HEINONEN, J. \& HALLAKSELA, A.-M. (2002): Influence of insects on the diversify of fungi in decaying spruce wood in managed and natural forests. For. Ecol. Managem. 166 (1-3), 165-181

MÜLLER-USING, S. (2005): Totholzdynamik eines Buchenbestandes im Solling. Unveröff. Vortragsmanuskript.

MÜLLER-USING, S. \& BARTSCH, N. (2003): Totholzdynamik eines Buchenbestandes (Fagus sylvatica L.) im Solling. Nachlieferung, Ursache und Zersetzung von Totholz. Allg. Forst- u. Jagdztg. 174: 122- 130

NAESSET, E. (1999): Relationship between relative wood density of Picea abies logs and simple classification systems of decayed coarse woody debris. Scand. J. For. Res. 14 (5): 454-461

NFV (2005): Daten zur Totholzinventur auf einer Kernfläche im 'Neuenburger Urwald'. (unveröff.)

NICOLAI, V. (1995a): Ermittlung der Totholzfauna mittels Borkeneklektoren: Mitt. Dtsch. Ges. Allg. Angew. Ent. 9 (4-6): 755-761 
NILSSON, S. G.; ARUP, U. ; BARANOWSKI, R. \& EKMAN, St. (1995): Tree-dependent lichens and beetles as indicators in conservation forests. Cons. Biol. 9 (5): 1208-1215

NILSSON, S. G. \& BARANOWSKI, R. (1997): Habitat predictability and the occurrence of wood beetles in old-growth beech forests. Ecography 20: 491-498

NILSSON, S.G.; HEDIN, J. \& NIKLASSON, M. (2001): Biodiversity and its assessment in boreal and Nemoral forests. Scand. J. For. Res. Suppl.3, 10-26

NITZSCKE, H. (1932): Der Neuenburger Urwald bei Bockhorn in Oldenburg. Vegetationsbilder 23. Reihe (H.6/7), Jena

NORD, J. C. \& LEWIS, W. G. (1970): Two emergence traps for wood-boring insects. J. Georgia Ent. Soc. 5: 155-157

NORDÉN, B. \& APPELQVIST, T. (2001): Conceptual problems of ecological continuity and its bioindicators. Biod. Conserv. 10 (5): 779-791

ÓDOR, P. \& HEES, A. F. M. van (2004): Preference of wood-inhabiting bryophytes to decay phase, log size and habitat types in hungarian beech forests. Journ. Of Bryology 26 (2):79-95

ÓDOR, P. \& STANDOVÁR, T. (2001): Richness of bryophyte vegetation in near-natural and managed beech stands: the effects of management-induced differences in dead wood. Ecol. Bull. 49: 219-229

ÓDOR, P. \& STANDOVÁR, T. (2002): Substrate specificity and community structure of bryophyte vegetation in a near natural montane beech forest. Community Ecology 3 (1): $39-49$

OHLSON, M.; SODERSTROM, L.; HORNBERG, G.; ZACKRISSON, O. \& HERMANSSON, J. (1997): Habitat qualities versus long-term continuity as determinants of biodiversity in boreal old-growth swamp forests. Biol. Cons. 81 (3), 221-231

ØKLAND, B. (1995): Insect fauna compared between six polypore species in a southern Norwegian spruce forest. Fauna Norwegica Ser. B 42: 21-26

ØKLAND, B. (1996): Unlogged forests: important sites for preserving the diversity of Mycetophilids (Diptera: Sciaroidea). Biol. Conserv. 76: 297-310

ØKLAND, B. (2000): Management effects on the decomposer fauna of Diptera in spruce forests. Studia Dipterologica 7 (1): 213-223

ØKLAND, B. (2002): Canopy cover favours sporocarp-visiting beetles in spruce forest. Norw. J. Entom. 49 (1): 23-39

OLDENBURGISCHES STAATSMINISTERIUM (1938): Verordnung über das „Naturschutzgebiet Neuenburger Urwald" bei Neuenburg im Forstamt Varel, Amt Friesland. Amtliche Nachrichten 202 (115).

OTTE, J. (1989a): Ökologische Untersuchungen zur Bedeutung von Windwurfflächen für die Insektenfauna - Teil 1. Waldhygiene 17: 193-247

OTTE, J. (1989b): Ökologische Untersuchungen zur Bedeutung von Windwurfflächen für die Insektenfauna - Teil 2. Waldhygiene 18: 1-36

OTTO, H.-J. (1972): Das Ergebnis der Standortkartierung im pleistozänen Flachland Niedersachsens - Grundlage waldbaulicher Leitvorstellungen. Aus dem Walde 19, Hannover

OWEN, J. A. (1989): An emergence trap for insects breeding in dead wood. British Journal of Entomol. and Natural history 2: 65-67 
OWEN, J. A. (1992): Experience with an emergence trap for insects breeding in dead wood. British Journal of Entomol. and Natural history 5: 17-20

PALM, T. (1951): Die Holz- und Rinden-Käfer der nordschwedischen Laubbäume. Meddelanden fran Statens Skogsforskningsinstitut 40 (2), 1-241

PALM, T. (1959): Die Holz- und Rinden-Käfer der süd- und mittelschwedischen Laubbäume. Opuscula Entomologica Suppl. XVI, 1-374.

PALMER, M. (2002): Ordination methods for ecologists. http://www.okstate.edu/artsci/botany/ordinate oder http://ordination.okstate.edu/

PECHACEK, P. (1995): Spechte im Nationalpark Berchtesgaden. Forschungsbericht 31 der Nationalparkverwaltung Berchtesgaden. $182 \mathrm{~S}$.

PFARR, U. (1990): Fichten-Totholz im Spannungsfeld von Natur- und Forstschutz. Diss. Univ. München

PODANI, J. (1994): Multivarite data analysis in ecology and systematics: a methodological guide to the SYN-TAX 5.0 package. Ecological Computations Series (ECS): Vol. 6. The Hague

PONTAILLER, J.-Y.; FAILLE, A. \& LEMÉE, G. (1997) : Storms drive successional dynamics in natural forests: a case study in Fontainbleau forest (France) : For. Ecol. Manage. 98: $1-15$

POTT, R. \& HÜPPE, J. (1991): Die Hudelandschaften Nordwestdeutschlands. - Abh. Westf. Mus. Nat.kde 53 (1/2): 313 S., Münster

RAMBO, T. R. \& MUIR, P. S. (1998): Bryophyte associations with coarse woody debris and stand ages in Oregon. The Bryologist 101 (3), 366-376

RANIUS, T. (2002): Population ecology and conservation of beetles and pseudoscorpions living in hollow oaks in Sweden. Anim. Biodiv.Conserv. 25 (1): 53-68

RANIUS, T. \& Hedin, J. (2001): The dispersal rate of a beetle, Osmoderma eremita, living in tree hollows. Oecologia 126: 363-370

RANIUS, T. \& Jansson, N. (2002): A comparison of three methods to survey saproxylic beetles in hollow oaks. Biol. Cons. 11, 1759-1771

RANIUS, T. \& Nilsson, S. (1997) : Habitat of Osmoderma eremita Scop. (Coleoptera: Scarabaeidae), a beetle living in hollow trees. J. Ins. Conserv. 1: 193-204

RAUH, J. (1993): Faunistisch-ökologische Bewertung von Naturwaldreservaten anhand repräsentativer Tiergruppen. Schr.R. Naturwaldreservate in Bayern 2

RAYNER, A. D. M. \& BODDY, L. (1988): Fungal decomposition of wood - Its biology and ecology. New York

REIBNITZ, J. (1999): Verbreitung der Baumschwammfresser Südwestdeutschlands (Coleoptera: Cisidae). Mitt. Entom. Ver. Stuttgart 34: 3-74

REID, R. W. (1963): Biology of the mountain pine beetle, Dendroctonus monticolae Hopkins, in the East Kootenay Region of british Columbia. III. Interaction between the beetle and ist host, with emphasis on brood mortality and survival. Can. Ent. 95: 225238

RENKONEN, O. (1938): Statistisch-ökologische Untersuchungen über die terrestrische Käferwelt der finnischen Bruchmoore. Ann. Zool. Soc. Bot. Fenn. Vanamo 6: 1-231 
RENNER, K. (1991): Sukzession der Käferfauna an Alt- und Totholz von Laubbäumen in der halboffenen Landschaft. NZ NRW Seminarberichte H. 10: 19-21

RENVALL, P. (1995): Community structure and dynamics of woodrotting Basidiomycetes on decomposing conifer trunks in northern Finland. Karstenia 35: 1-51

RINN, F.; SCHWEINEGRUBER, F.-H. \& SCHÄR, E. (1994): RESISTOGRAPH drill resistance profiles and high-resolution X-ray density charts of different species of wood - a comparative evaluation. Nachdruck

RINN, F.; SCHWEINEGRUBER, F.-H. \& SCHÄR, E. (1996): RESISTOGRAPH and X-ray density charts of wood comparative evaluation of drill resistance profiles and X-ray density charts of different wood species. Holzforschung 50 (4): 303-311

RÖHRIG, E. (1991): Totholz im Wald. Forstl. Umschau 34 (4): 259-270

ROUVINEN, S. (2002): Amount, diversity and spatio-temporal availability of dead wood in old forests in boreal Fennoscandia. University of Joensuu, Faculty of Forestry, Research Notes 140, $53 \mathrm{pp}$

RUKKE, B. A. (2000): Effects of habitat fragmentation: increased isolation and reduced habitat size reduces the incidence of dead wood fungi beetles in a fragmented forest landscape. Ecography 23: 492-502

RUKKE, B.A. (2002): Fungivorous beetles in basidiocarps of Fomes fomentarius respond differently to microhbitat variables. Eur. J. Entom. 99 (1) : 43-52

RUNGE, A. (1975): Pilzsukzession auf Laubholzstümpfen. Zeitschr. F. Pilzkunde 41: 31-38

RUNGE, A. (1991): Zur Sukzession der Pilzbesiedlung auf Totholz. NZ NRW Seminarberichte H. 10: 6-9

RYDIN, H. ; DIECKMANN, M. \& HALLINGBACK, T. (1997): Biological characteristics, habitat associations and distribution of macrofungi in Sweden. Cons. Biol. 11 (3): 628640

SAALAS, U. (1917): Die Fichtenkäfer Finnlands I. Ann. Acad. Sci. Fenn. A8: 547 pp.

SAALAS, U. (1923): Die Fichtenkäfer Finnlands II. Ann. Acad. Sci. Fenn. A22: 746 pp.

SAMUELSON, J.; GUSTAFSSON, L. \& INGELÖG, T. (1994): Dying and dead trees. A review of their importance for biodiversity. Schr.R. Uppsala Rapport 4306, 109 pp.

SANDERS, W. (1987): Untersuchungen über die Aktivitätsdichte des Buchdruckers Ips typographus in Laubwäldern und in offener Landschaft. J. appl. Entomol. 103 (3): 240-249

SANDSTRÖM, U. (1992): Cavities in trees: their ocurence, formation and importance for hole-nesting birds in relation to silvicultural practise. Schr.R. Uppsala Rapport 23

SANIGA, M. \& SCHÜTZ, J. P. (2001): Dynamik des Totholzes in zwei gemischten Urwäldern der Westkarpaten im pflanzengeographischen Bereich der Tannen-Buchen- und der Buchenwälder in verschiedenen Entwicklungsstadien (Deadwood dynamics in two mixed virgin forests in the West carpathians in the phytogeografic domain of pure beech and mixed fir-beech forests). Schweiz. Z. Forstw. 152 (10): 407-416

SANIGA, M. \& SCHÜTZ, J. P. (2002): Relation of dead wood coarse within the developement cycle of selected virgin forests in Slowakia. J. For. Sci. Prague 48 (12): 513-528

SCHÄFER, M. (2002): Zersetzung der sturmgeworfenen Buchenstämme im Naturwaldreservat Weiherskopf seit 1990. Mitt. D. Hess. Landesforstverwaltung 38: 49-60 
SCHAFFRATH, U. (1999): Zur Käferfauna am Edersee (Insecla, Coleoplera) Phlippia 9 (1); $1-94$

SCHEERPELTZ, O. \& HÖFLER, K. (1948): Käfer und Pilze. Wien. Verlag f. Jugend und Volk

SCHERZINGER, W. (1982): Die Spechte im Nationalpark Bayerischer Wald. Sch.R. Bayer. StMELF, H.9

SCHERZINGER, W. (1996): Naturschutz im Wald. Stuttgart

SCHIEGG, K. (2000a): Are there saproxylic beetle species characteristic of high dead wood connectivity? Ecography 23 (5): 579-587

SCHIEGG, K. (2000b): Effects of dead wood volume and connectivity on saproxylic insect species diversity. Ecoscience 7 (3): 290-298

SCHIEGG, K. (2001): Saproxylic insect diversity of beech: limbs are richer than trunks. For. Ecol. Managem. 149: 295-304

SCHIEGG PASINELLI, K. (1999): Limiting factors of saproxylic insects: habitat relationships of an endangered ecological group. Diss. ETH Zürich

SCHIMTSCHEK, E. (1953 a): Forstentomologische Studien im Urwald Rotwald. Teil I. Z. angew. Ent. 34: 178-215

SCHIMTSCHEK, E. (1953 b): Forstentomologische Studien im Urwald Rotwald. Teil II. Z. angew. Ent. 34: 513-542

SCHIMTSCHEK, E. (1954): Forstentomologische Studien im Urwald Rotwald. Teil III. Z. angew. Ent. 35: 1-54

SCHLECHTE, G. B. (2002): Sukzession holzzerstörender Pilze auf der Windwurffläche. Mitteilungen der Hess. Landesforstverwaltung 38: 61-78

SCHMIDT, O. (1994): Holz- und Baumpilze: Biologie, Schäden, Schutz, Nutzen. Berlin u.a.

SCHMITT, M. (1992): Buchen-Totholz als Lebensraum für xylobionte Käfer - Untersuchungen im Naturwaldreservat „Waldhaus“ und zwei Vergleichsflächen im Wirtschaftswald (Forstamt Ebrach, Steigerwald). Waldhygiene 19: 97-191

SCHÖNBORN, W. (1961): Zoozönotische Struktur- und Konnexitätsanalyse in Kiefernstümpfen. Biol. Zbl. 80: 645-663

SCHOWALTER, T. D.; ZHANG, Y. L. \& SABIN, T. E. (1998): Decomposition and nutrient dynamics of oak Quercus ssp. logs after five year of decomposition. Ecography 21: 3-10

SCHUCK, A.; MEYER, P.; MENKE, N.; LIER, M. \& LINDNER, M. (2004): Forest biodiversity indicators: Dead wood - a proposed approach towards operationalising the MCPFE indicator. In: Marchetti, M (Ed.): Monitoring and indicators of forest biodiversity in Europe - From ideas to operationality. EFI-Proceedings No. 51: 49-77

SEASTEDT, T. R.; REDDY, M. V. \& CLINE, S. P. (1989): Microarthropods in decaying wood from temperate coniferous and deciduous forests. Pedobiologia 33 (2): 69-77

SETÄLÄ, H. \& MARSHALL, U. G. (1994): Stumps as a habitat for Collenmola during succession from clear-cuts in old-growth Douglas-fir forests. Pedobiologia 38: 307-326

SHOROHOVA, E. V. \& SHOROHOV, A. A. (2001): Coarse woody debris dynamics and stores in a boreal virgin spruce forest. Ecol. Bull. 49: 129-135

SIITONEN, J. (2001): Forest management, coarse woody debris and saproxylic organisms: Fennoscandian boreal forests as an example. Ecol. Bull. 49: 11-41 
SIITONEN, J. \& MARTIKAINEN, P. (1994): Occurence of rare and threatened insects living on decaying Populus tremulae: A comparison between Finnish and Russian Karelia. Scand. J. For. Res. 9: 185-191

SIITONEN, J.; PENTILLÄ, R. \& KOTIRANTA, H. (2001): Coarse woody debris, polyporous fungi and saproxylic insects in an old-growth spruce forest in Vodlozero National Park, Russian Karelia. Ecol. Bull. 49: 231-242

SIITONEN, J. \& SAARISTO, L. (2000): Habitat requirements and conservation of Phyto kolwensis, a beetle species of old-growth boreal forest. Biol. Cons. 94 (2), 211-220

SIMILÄ, M. (2002): Patterns of beetle species diversity in Fennoscandian boreal forests. Diss. Univ. of Joensuu

SIMILÄ, M.; KOUKI, J. \& MARTIKAINEN, P. (2003): Saproxylic beetles in managed and seminatural scots pine forests: Quality of dead wood matters. For. Ecol. Managem. 174 (1-3), 364-381

SIMILÄ, M.; KOUKI, J.; MARTIKAINEN, P. \& UOTILA, A. (2002): Conservation of beetles in boreal pine forests: the effects of forest age and natuaralness on species assemblages. Biol. Conserv. 106 (1): 19-27

SIPPOLA, A.-L. (2001): Forest structure and biodiversity in northern boreal forests: effects of regeneration cutting on flying beetles and wood-decomposing fungi. Arctic Centre Reports 35

SIPPOLA, A.-L.; LEHESVIRTA, T. \& RENVALL, P. (2001): Effects of selective logging on coarse woody debris and diversity of wood-decaying polypores in eastern Finland. Ecol. Bull. 49: 243-254

SIPPOLA, A.-L.; SIITONEN, J. \& KALLIO, R. (1998): Amount and quality of coarse woody debris in natural and managed coniferous forests near the timberline in Finnish Lapland. Scand. J. For. Res. 13 (2): 204-214

SIPPOLA, A.-L.; SIMILÄ, M.; MONKKONEN, M. \& JOKIMAKI, J. (2004): Diversity of polyporous fungi (polyporaceae) in northern boreal forests: Effects of forest site type and logging intensity. Scand. J. For. Res. 19 (2): 152-163

SMILAUER, P. (1992): CanoDraw user's guide vs. 3.0. New York

SNÄLL, T. \& JONSSON, B. G. (2001): Edge effects on six polyporous fungi used as oldgrowth indicators in Swedish boreal forest. Ecol. Bull. 49: 255-262

SÖDERSTRÖM, L. (1988a): The occurrence of epixylic bryophyte and lichen species in an old natural and manged forest stand in northeast Sweden. Biol. Cons. 45: 169-178

SÖDERSTRÖM, L. (1988b): Sequence of bryophytes and lichens in relation to substrate variables of decaying coniferous wood in northern Sweden. Nord. J. Bot. 8: 89-97

SØRENSEN, T. (1948): A method of establishing groups of equal amplitude in plant sociology based on similarity of species content. Kong. Dansk. Vidensk. Selsk. Biol. Skr. (Kopenhagen) 5 (4): 1-34

SOUTHWOOD, T. R. E. (1978): Ecological Methods. London

SPEIGHT, M. C. D. (1989): Saproxylic invertebrates and their conservation. Council of Europe, Nature and Environment Series No.42

SSYMANK, A. (1994): Indikatorarten der Fauna für historisch alte Wälder. NNA Ber. 7 (3): 134-141 
STARZYK, J. R. (1979): Cerambycidae communities (Col., Cerambycidae) occring in various phytosociological forest types of Niepolomice Forest near Krakow. Z. angew. Entom. 88: 44-55

STOKLAND, J. N. (2001): The coarse woody debris profile: an archive of recent forest history and an important biodiversity indicator. Ecoll. Bull. 49: 71-83

STOKLAND, J. N. \& KAUSERUD, H. (2004): Phellinus nigrolimitatus: A wood-decomposing fungus highly influenced by forestry. For. Ecol. Managem. 187 (2-3): 333-343

SVENSSON, G. P.; LARSSON, M. C. \& HEDIN, J. (2004): Attraction of the larval predator Elater ferrugineus to the sex pheromene of its prey, Osmoderma eremita, and its implication for conservation biology. J. Chem. Ecol. 30 (2): 353-363

SVERDRUP-THYGESON, A. (2001): Can 'continuity indicator species' predict species richness or red-listed species of saproxylic beetles. Biodiv. Cons. 10, 815-832

SVERDRUP-THYGESON, A. (2002): Key habitats in the norwegian production forest: A case study. Scand. J. For. Res. 17, 166-171

SVERDRUP-THYGESON, A. \& IMS, R. A. (2002): The effect of forest clear-cutting in Norway on the community of saproxylic beetles in aspen. Biol. Cons. 106 (3): 347-357

SVERDRUP-THYGESON, A. \& LINDENMAYER, D. B. (2003): Ecological continuity and assumed indicator fungi in boreal forest: the importance of the landscape matrix. For. Ecol. Manage. 174: 353-363

SVERDRUP-THYGESON, A. \& MIDTGAARD, F. (1998): Fungus-infected trees as islands in boreal forest: spatial distribution of the fungivorous beetle Bolitophagus reticulatus (Coleoptera: Tenebrionidae). Ecoscience 5 (4): 486-493

SWIFT, M. J. (1977 a): The ecology of wood decomposition. Sci. Progr. (Oxford) 64: 175199

SWIFT, M. J. (1977 b): The role of fungi and animals in the immobilization and release of nutrient elements from decomposing branh-wood. Ecol. Bull. 25: 193-202

SWIFT, M. J.; BODDY, L. B. \& HEALEY, I. N. (1984): Wood decomposition in a abandoned beech and oak coppiced woodland in SE England. II. The standding crop of wood on the forest floor with particular reference to its invasion by Tipula flavolineata and other animals. Holartic Ecology 7: 218-228

TAAKE, K.-H. (1991): Zur Besiedlung von Althölzern und Fledermauskästen durch Waldfledermäuse. NZ NRW Seminarberichte 10: 57-58

TABAKU,V. \& MEYER, P. (1999): Lückenmuster albanischer und mitteleuropäischer Buchenwälder unterschiedlicher Nutzungsintensität. Forstarchiv 70: 87-97

TARASOV, M. E. \& BIRDSEY, R. A. (2001): Decay rate and potential storage of coarse woody debris in the Leningrad region. Ecol. Bull. 49: 137-147

TAUX, K. (1986): Die Oldenburgischen Naturschutzgebiete. Oldenburg

TER BRAAK, C. J. F. (1986): Canonical correspondence analysis: a new eigenvector method for multivariate direct gradient analysis. Ecology 67 (5): 1167-1179

TER BRAAK, C. J. F. (1987): The analysis of vegetation-environment relationships by canonical correspondence analysis. Vegetatio 69: 69-77

TER BRAAK, C. J. F. (1988): CANOCO - a FORTRAN program for canonical community ordination by [partial] [detrended] [canonical] correspondence analysis, principal 
components analysis and redundancy analysis (version 2.1). Agricultural Mathematics Group, Wageningen

TER BRAAK, C. J. F. (1990): Update notes: CANOCO Version 3.1. Agricultural Mathematics Group, Wageningen

TER BRAAK, C. J. F. (1994): Canonical community ordination. Part I: Basic theory and linear methods. Ecoscience 1 (2): 127-140

TER BRAAK, C. J. F. (1995): Ordination. In Jongman, R. H. G.; Ter Braak, C. J. F. \& Tongeren, O. F. R.van (Hg.) (2.Aufl): Data analysis in community and landscape ecology. Cambridge

TER BRAAK, C. J. F. \& PRENTICE, I. C. (1988): A theory of gradient analysis. Advances in ecological research 18: 127-140

TER BRAAK, C. J. F. \& VERDONSCHOT, P. F. M. (1995): Canonical correspondence analysis and related multivariate methods in aquatic ecology. Aquatic Sciences 57 (3): 255-289

THOR, G. (1998): Red-listed lichens in Sweden: habitats, threats, protection, and indicator value in boreal coniferous forests. Biodiv. Conserv. 7: 59-72

THUNES, K. H. (1994): The coleopteran fauna of Piptoporus betulinus and Fomes fomentarius (Aphyllophorales: Polyporaceae) in western Norway. Entom. Fennica 5: 157-168

THUNES, K. H.; MIDTGAARD, F. \& GJERDE, I. (2000): Diversity of coleoptera of the bracket fungi Fomitopsis pinicola in a Norwegian spruce forest. Biodiv. Cons. 9: 833852

THUNES, K. H. \& WILLASSEN, E. (1997): Species composition of beetles (Coleoptera) in bracket fungi Piptoporus betulinus and Fomes fomentarius (Aphyllophorales: Polyporaceae): an explorative approach with cannonical correspondence analysis. J. Nat. Hist. 31: 471-486

TONGEREN, O. F. R. van (1995): Cluster analysis. In Jongman, R. H. G.; Ter Braak, C. J. F. \& Tongeren, O. F. R.van (Hg.): Data analysis in community and landscape ecology. Cambridge

UTSCHIK, H. 1991: Beziehungen zwischen Totholzreichtum und Vogelvielfalt in Wirtschaftswäldern. Forstw. Cbl. 110 (1991), 135-148

VITÉ, J. P. (1952): Die holzzerstörenden Insekten Mitteleuropas - Textband

WALANKIEWICZ, W. (2002): The number and composition of snags in the pine-spruce stands of the Bialowieza National Park, Poland. USDA for. Serv. Gen. Tech. Rep. PSW-GTR-181: 489-500

WALTER, H. (1979): Allgemeine Geobotanik. Stuttgart

WEHAGE, J. (1930): Deutsche Urwälder. Mitt. d. Deutsch. Dendrol. Ges. 42

WERMELINGER, B.; DUELLI, P. \& OBRIST, M. K. (2002): Dymanics of saproxylic beetles (Coleoplera) in windthrow areas in alpine spruce forests. For. Snow Landsc. Res. 77 (1/2), 133-148

WESTRICH, P. (1991): Wildbienen als Bewohner von Totholz. NZ NRW Seminarberichte H. 10: $32-35$

WHITTAKER, R. H. (1967): Gradient analysis of vegetation. Biol. Rev. 42 (2): 207-264 
WILDING, N.; COLLINS, N. M.; HAMMOND, P. M \& WEBBER, J. F. (1989): Insectfungus interactions. London, Academic Press, $344 \mathrm{pp}$

WINTER, K. (1991): Untersuchungen über die xylobionte Käferfauna in Niedersachsen. Ber. a. d. norddeutschen Naturschutzakademie (NNA-Berichte) 4 (2): 157-162

WINTER, K.; BOGENSCHÜTZ, H.; DORDA, D.; DOROW, W. H. O.; FLECHTNER, G.; GRAEFE, U.; KÖHLER, F.; MENKE, N.; SCHAUERMANN, J.; SCHUBERT, H.; SCHULZ, U. \& TAUCHERT, J. (1999): Programm zur Untersuchung der Fauna in Naturwäldern. IHW-Verlag, Eching

WINTERHOFF, W. (2001): Die Großpilz-Fruchtkörpersukzession auf toten Kiefern im Bannwald „Franzosenbusch“. In: Bücking, W. (ed.): Die Bannwälder „Franzosenbusch” und „Kartoffelacker” in der Schwetzinger Hardt. Ber. Freiburger Forstl. Forsch. 29: 126-147

WOLDA (1981): Similarity indices, sample size and diversity. Oecologia 50: 296-302

YATSKOV, M.; HARMON, M. E. \& KRANKINA, O. N. (2003): A chronosequence of wood decomposition in the boreal forests of Russia. Can. J. For. Res. 33 (7): 1211-1226

ZHONG, H. \& SCHOWALTER, T. D. (1989): Conifer bole utilization by wood-boring beetles in western Oregon. Can. J. For. Res. 19: 943-947

ZIELONKA, T. \& NIKLASSON, M. (2001): Dynamics of dead wood and regeneration pattern in natural spruce forest in the Tatra Mountains, Poland. Ecol. Bull. 49: 159-163

ZÖRNER, M. (2003): Untersuchungen zur xylobionten Käferfauna an der Stieleiche (Quercus robur Linnaeus, 1753) unter besonderer Berücksichtigung des Gesundheitszustandes der Bäume und der Höhenexposition. Diss. Univ. Hamburg. 


\section{Anhang}

\section{Anhang 1: Fangbeginn und Leerungstermine}

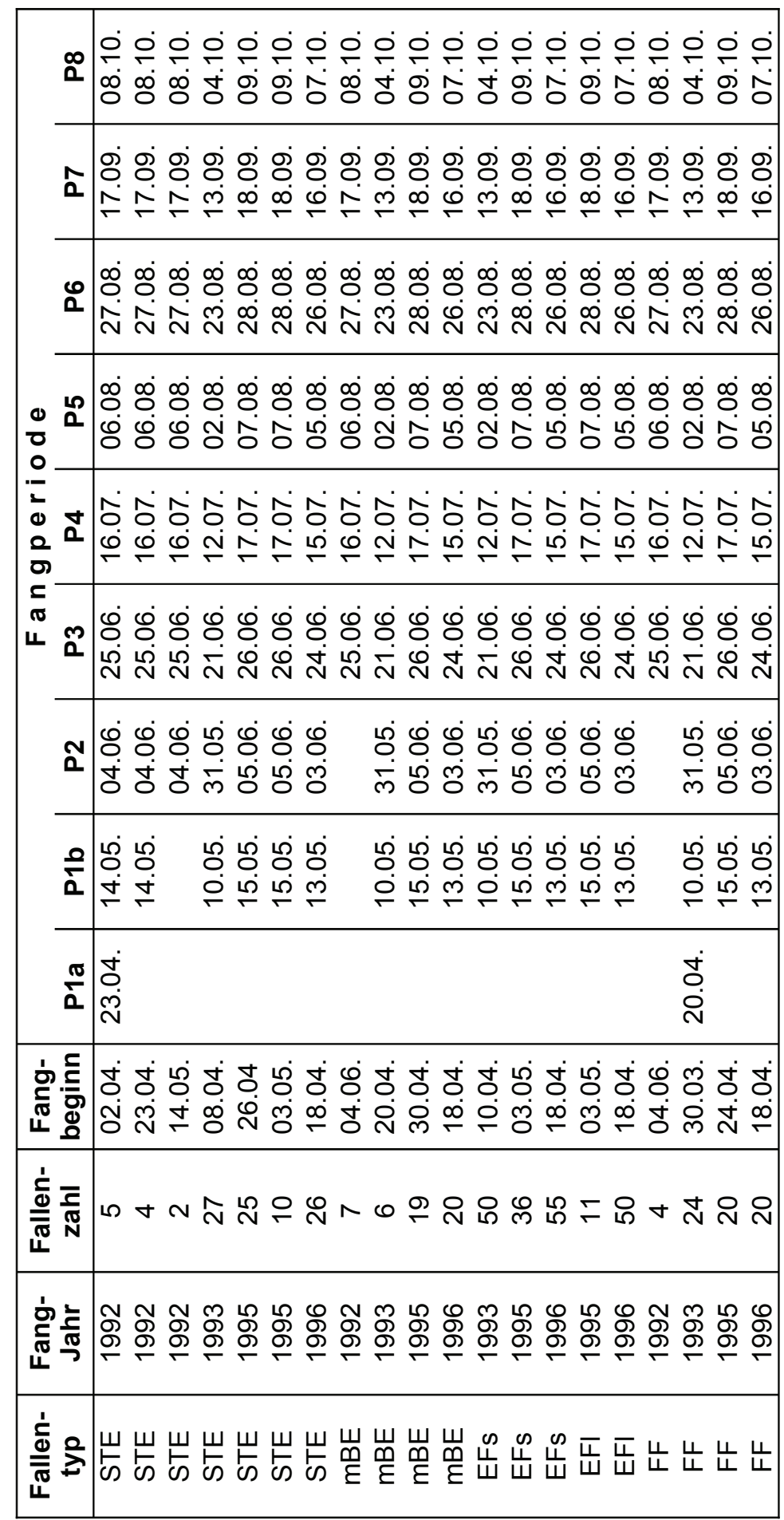


Anhang 2: Stichprobenumfang der Eklektoren

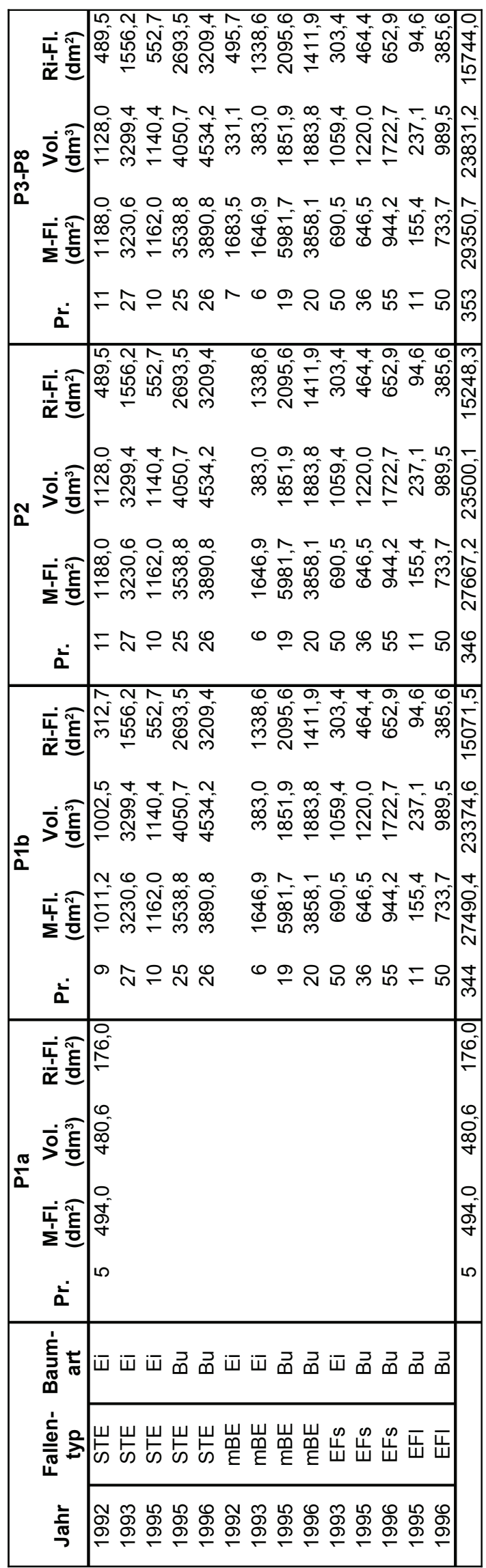


Anhang 3: Käferfamilien in verschiedenen Eklektortypen an Eiche und Buche

\begin{tabular}{|c|c|c|c|c|c|c|c|c|c|c|}
\hline \multirow[b]{3}{*}{ FAMILIE } & \multicolumn{10}{|c|}{$\bar{E} \mathrm{iche}$} \\
\hline & \multicolumn{3}{|c|}{ STE } & \multicolumn{3}{|c|}{$\mathrm{mBE}$} & \multicolumn{3}{|c|}{ EFs } & \multirow{2}{*}{$\begin{array}{l}\text { Sum } \\
\text { Ind. }\end{array}$} \\
\hline & Ind. & Ind. \% & |rten & Ind. & nd. \% & irten & Ind. II & nd. \% & Arten & \\
\hline Carabidae & 191 & 2,2 & 9 & 68 & 3,0 & 7 & 20 & 0,4 & 3 & 279 \\
\hline Hydraenidae & 0 & 0,0 & 0 & 0 & 0,0 & 0 & 0 & 0,0 & 0 & 0 \\
\hline Hydrophilidae & 2 & 0,0 & 2 & 0 & 0,0 & 0 & 0 & 0,0 & 0 & 2 \\
\hline Histeridae & 14 & 0,2 & 3 & 0 & 0,0 & 0 & 21 & 0,4 & 4 & 35 \\
\hline Silphidae & 0 & 0,0 & 0 & 0 & 0,0 & 0 & 0 & 0,0 & 0 & 0 \\
\hline Leptinidae & 3 & 0,0 & 1 & 0 & 0,0 & 0 & 0 & 0,0 & 0 & 3 \\
\hline Cholevidae & 28 & 0,3 & 5 & 2 & 0,1 & 1 & 8 & 0,2 & 1 & 38 \\
\hline Leiodidae & 69 & 0,8 & 7 & 3 & 0,1 & 1 & 4 & 0,1 & 2 & 76 \\
\hline Scydmaenidae & 429 & 5,0 & 9 & 2 & 0,1 & 2 & 27 & 0,6 & 5 & 458 \\
\hline Ptilidae & 560 & 6,5 & 6 & 0 & 0,0 & 0 & 13 & 0,3 & 5 & 573 \\
\hline Scaphidiidae & 21 & 0,2 & 2 & 15 & 0,7 & 2 & 0 & 0,0 & 0 & 36 \\
\hline Staphylinidae & 993 & 11,6 & 74 & 322 & 14,2 & 31 & 116 & 2,4 & 15 & 1431 \\
\hline Pselaphidae & 138 & 1,6 & 10 & 5 & 0,2 & 3 & 39 & 0,8 & 7 & 182 \\
\hline Cantharidae & 47 & 0,5 & 4 & 9 & 0,4 & 6 & 11 & 0,2 & 2 & 67 \\
\hline Malachiidae & 0 & 0,0 & 0 & 0 & 0,0 & 0 & 0 & 0,0 & 0 & 0 \\
\hline Melyridae & 4 & 0,0 & 2 & 10 & 0,4 & 2 & 2 & 0,0 & 1 & 16 \\
\hline Trogositidae & 1 & 0,0 & 1 & 0 & 0,0 & 0 & 1 & 0,0 & 1 & 2 \\
\hline Lymexylonidae & 120 & 1,4 & 1 & 2 & 0,1 & 1 & 129 & 2,7 & 1 & 251 \\
\hline Elateridae & 83 & 1,0 & 11 & 27 & 1,2 & 11 & 9 & 0,2 & 5 & 119 \\
\hline Eucnemidae & 1 & 0,0 & 1 & 0 & 0,0 & 0 & 0 & 0,0 & 0 & 1 \\
\hline Throscidae & 4 & 0,0 & 1 & 7 & 0,3 & 1 & 1 & 0,0 & 1 & 12 \\
\hline Buprestidae & 1 & 0,0 & 1 & 1 & 0,0 & 1 & 7 & 0,1 & 1 & 9 \\
\hline Clambidae & 1 & 0,0 & 1 & 0 & 0,0 & 0 & 0 & 0,0 & 0 & 1 \\
\hline Scirtidae & 0 & 0,0 & 0 & 0 & 0,0 & 0 & 0 & 0,0 & 0 & 0 \\
\hline Dermestidae & 0 & 0,0 & 0 & 0 & 0,0 & 0 & 1 & 0,0 & 1 & 1 \\
\hline Cerylonidae & 230 & 2,7 & 3 & 48 & 2,1 & 2 & 64 & 1,3 & 3 & 342 \\
\hline Sphaerosomatidae & 0 & 0,0 & 0 & 0 & 0,0 & 0 & 0 & 0,0 & 0 & 0 \\
\hline Nitidulidae & 59 & 0,7 & 11 & 15 & 0,7 & 5 & 2 & 0,0 & 1 & 76 \\
\hline Kateridae & 0 & 0,0 & 0 & 0 & 0,0 & 0 & 0 & 0,0 & 0 & 0 \\
\hline Rhizophagidae & 1704 & 19,9 & 6 & 10 & 0,4 & 4 & 244 & 5,0 & 4 & 1958 \\
\hline Cucujidae & 7 & 0,1 & 2 & 1 & 0,0 & 1 & 2 & 0,0 & 2 & 10 \\
\hline Silvanidae & 1 & 0,0 & 1 & 0 & 0,0 & 0 & 0 & 0,0 & 0 & 1 \\
\hline Cryptophagidae & 314 & 3,7 & 9 & 4 & 0,2 & 1 & 116 & 2,4 & 2 & 434 \\
\hline Phalacridae & 0 & 0,0 & 0 & 0 & 0,0 & 0 & 0 & 0,0 & 0 & 0 \\
\hline Laemophloeidae & 0 & 0,0 & 0 & 0 & 0,0 & 0 & 0 & 0,0 & 0 & 0 \\
\hline Lathridiidae & 974 & 11,4 & 13 & 23 & 1,0 & 5 & 685 & 14,2 & 10 & 1682 \\
\hline Merophysiidae & 0 & 0,0 & 0 & 0 & 0,0 & 0 & 0 & 0,0 & 0 & 0 \\
\hline Mycetophagidae & 2 & 0,0 & 2 & 2 & 0,1 & 1 & 10 & 0,2 & 2 & 14 \\
\hline Colydiidae & 84 & 1,0 & 1 & 0 & 0,0 & 0 & 187 & 3,9 & 1 & 271 \\
\hline Corylophidae & 45 & 0,5 & 1 & 1 & 0,0 & 1 & 1 & 0,0 & 1 & 47 \\
\hline Endomychidae & 0 & 0,0 & 0 & 0 & 0,0 & 0 & 0 & 0,0 & 0 & 0 \\
\hline Coccinellidae & 2 & 0,0 & 2 & 4 & 0,2 & 2 & 1 & 0,0 & 1 & 7 \\
\hline Sphindidae & 2 & 0,0 & 1 & 53 & 2,3 & 1 & 0 & 0,0 & 0 & 55 \\
\hline Cisidae & 14 & 0,2 & 4 & 20 & 0,9 & 1 & 112 & 2,3 & 4 & 146 \\
\hline Anobiidae & 52 & 0,6 & 4 & 22 & 1,0 & 4 & 38 & 0,8 & 3 & 112 \\
\hline Ptinidae & 1 & 0,0 & 1 & 2 & 0,1 & 1 & 4 & 0,1 & 1 & 7 \\
\hline Oedemeridae & 0 & 0,0 & 0 & 0 & 0,0 & 0 & 0 & 0,0 & 0 & 0 \\
\hline Salpingidae & 69 & 0,8 & 2 & 7 & 0,3 & 3 & 4 & 0,1 & 1 & 80 \\
\hline Pyrochroidae & 16 & 0,2 & 2 & 18 & 0,8 & 2 & 0 & 0,0 & 0 & 34 \\
\hline Scraptiidae & 23 & 0,3 & 4 & 46 & 2,0 & 7 & 33 & 0,7 & 5 & 102 \\
\hline Aderidae & 0 & 0,0 & 0 & 530 & 23,3 & 1 & 23 & 0,5 & 1 & 553 \\
\hline Mordellidae & 0 & 0,0 & 0 & 0 & 0,0 & 0 & 0 & 0,0 & 0 & 0 \\
\hline Melandryidae & 39 & 0,5 & 3 & 884 & 38,9 & 1 & 2 & 0,0 & 1 & 925 \\
\hline Tetratomidae & 2 & 0,0 & 1 & 0 & 0,0 & 0 & 0 & 0,0 & 0 & 2 \\
\hline Alleculidae & 1 & 0,0 & 1 & 0 & 0,0 & 0 & 0 & 0,0 & 0 & 1 \\
\hline Tenebrionidae & 31 & 0,4 & 2 & 24 & 1,1 & 1 & 44 & 0,9 & 2 & 99 \\
\hline Trogidae & 0 & 0,0 & 0 & 0 & 0,0 & 0 & 0 & 0,0 & 0 & 0 \\
\hline Scarabaeidae & 1 & 0,0 & 1 & 0 & 0,0 & 0 & 0 & 0,0 & 0 & 1 \\
\hline Lucanidae & 4 & 0,0 & 1 & 9 & 0,4 & 1 & 0 & 0,0 & 0 & 13 \\
\hline Cerambycidae & 11 & 0,1 & 3 & 46 & 2,0 & 3 & 4 & 0,1 & 3 & 61 \\
\hline Chrysomelidae & 1 & 0,0 & 1 & 15 & 0,7 & 1 & 1 & 0,0 & 1 & 17 \\
\hline Anthribidae & 0 & 0,0 & 0 & 0 & 0,0 & 0 & 0 & 0,0 & 0 & 0 \\
\hline Scolytidae & 2123 & 24,8 & 6 & 0 & 0,0 & 0 & 2785 & 57,6 & 4 & 4908 \\
\hline Rhynchitidae & 1 & 0,0 & 1 & 0 & 0,0 & 0 & 0 & 0,0 & 0 & 1 \\
\hline Apionidae & 1 & 0,0 & 1 & 0 & 0,0 & 0 & 0 & 0,0 & 0 & 1 \\
\hline Curculionidae & 52 & 0,6 & 7 & 16 & 0,7 & 6 & 67 & 1,4 & 4 & 135 \\
\hline Gesamtergebnis & 8576 & 100,0 & 248 & 2273 & 100,0 & 124 & 4838 & 100,0 & 112 & 15687 \\
\hline
\end{tabular}


Anhang 3: (Fortsetzung)

\begin{tabular}{|c|c|c|c|c|c|c|c|c|c|c|c|c|c|}
\hline \multicolumn{13}{|c|}{ Buche } & \multirow{3}{*}{$\begin{array}{c}\text { Eklektoren } \\
\text { gesamt } \\
\text { Ind. }\end{array}$} \\
\hline \multicolumn{3}{|c|}{ STE } & \multicolumn{3}{|c|}{$\mathrm{mBE}$} & \multicolumn{3}{|c|}{$\mathrm{EFs}$} & \multicolumn{3}{|c|}{$\mathrm{EFI}$} & \multirow{2}{*}{$\begin{array}{l}\text { Sum } \\
\text { Ind. }\end{array}$} & \\
\hline Ind. & Ind. \% & Arten & Ind. & Ind. \% & Arten & Ind. & Ind. \% & arten & Ind. & nd. \% & Arten & & \\
\hline 184 & 1,3 & 12 & 34 & 0,7 & 11 & 12 & 0,1 & 3 & 16 & 2,5 & 7 & 246 & 525 \\
\hline 1 & 0,0 & 1 & 0 & 0,0 & 0 & 0 & 0,0 & 0 & 0 & 0,0 & 0 & 1 & 1 \\
\hline 2 & 0,0 & 2 & 6 & 0,1 & 2 & 2 & 0,0 & 2 & 2 & 0,3 & 2 & 12 & 14 \\
\hline 212 & 1,5 & 3 & 50 & 1,0 & 3 & 617 & 4,6 & 5 & 4 & 0,6 & 2 & 883 & 918 \\
\hline 2 & 0,0 & 1 & 7 & 0,1 & 2 & 0 & 0,0 & 0 & 1 & 0,2 & 1 & 10 & 10 \\
\hline 0 & 0,0 & 0 & 0 & 0,0 & 0 & 6 & 0,0 & 1 & 0 & 0,0 & 0 & , & \\
\hline 16 & 0,1 & 2 & 0 & 0,0 & 0 & 10 & 0,1 & 1 & 1 & 0,2 & & 27 & 65 \\
\hline 141 & 1,0 & 10 & 77 & 1,5 & 5 & 13 & 0,1 & 3 & 9 & 1,4 & 3 & 240 & 316 \\
\hline 432 & 3,2 & 9 & 17 & 0,3 & 3 & 177 & 1,3 & 8 & 20 & 3,1 & 5 & 646 & 1104 \\
\hline 1036 & 7,6 & 6 & 99 & 2,0 & 6 & 834 & 6,2 & 5 & 80 & 12,5 & 6 & 2049 & 2622 \\
\hline 15 & 0,1 & 3 & 38 & 0,8 & 2 & 0 & 0,0 & 0 & 0 & 0,0 & 0 & 53 & 89 \\
\hline 1812 & 13,2 & 76 & 1456 & 28,8 & 64 & 476 & 3,5 & 30 & 108 & 16,9 & 28 & 3852 & 5283 \\
\hline 183 & 1,3 & 11 & 93 & 1,8 & 7 & 126 & 0,9 & 8 & 8 & 1,3 & 1 & 410 & 592 \\
\hline 8 & 0,1 & 3 & 43 & 0,9 & 9 & 42 & 0,3 & 3 & 13 & 2,0 & 2 & 106 & 173 \\
\hline 0 & 0,0 & 0 & 3 & 0,1 & 1 & 0 & 0,0 & 0 & 0 & 0,0 & 0 & 3 & 3 \\
\hline 0 & 0,0 & 0 & 6 & 0,1 & 1 & 1 & 0,0 & 1 & 0 & 0,0 & 0 & 7 & 23 \\
\hline 0 & 0,0 & 0 & 0 & 0,0 & 0 & 1 & 0,0 & 1 & 0 & 0,0 & 0 & 1 & \\
\hline 767 & 5,6 & 2 & 39 & 0,8 & 2 & 196 & 1,5 & 1 & 72 & 11,3 & 1 & 1074 & 1325 \\
\hline 77 & 0,6 & 8 & 234 & 4,6 & 13 & 25 & 0,2 & 6 & 5 & 0,8 & 3 & 341 & 460 \\
\hline 22 & 0,2 & 3 & 96 & 1,9 & . & 26 & 0,2 & 2 & 1 & 0,2 & 1 & 145 & 146 \\
\hline 8 & 0,1 & 1 & 71 & 1,4 & 2 & 1 & 0,0 & 1 & 0 & 0,0 & 0 & 80 & 92 \\
\hline 0 & 0,0 & 0 & 0 & 0,0 & 0 & 0 & 0,0 & 0 & 0 & 0,0 & 0 & 0 & 9 \\
\hline 0 & 0,0 & 0 & 1 & 0,0 & 1 & 0 & 0,0 & 0 & 0 & 0,0 & & 1 & \\
\hline 1 & 0,0 & 1 & 6 & 0,1 & 4 & 0 & 0,0 & 0 & 0 & 0,0 & 0 & 7 & \\
\hline 1 & 0,0 & 1 & 0 & 0,0 & 0 & 1 & 0,0 & 1 & 0 & 0,0 & 0 & 2 & 3 \\
\hline 705 & 5,1 & 3 & 70 & 1,4 & 3 & 485 & 3,6 & 3 & 6 & 0,9 & 3 & 1266 & 1608 \\
\hline 9 & 0,1 & 1 & 1 & 0,0 & 1 & 1 & 0,0 & 1 & 0 & 0,0 & 0 & 11 & 11 \\
\hline 146 & 1,1 & 8 & 27 & 0,5 & 6 & 36 & 0,3 & 5 & 16 & 2,5 & 3 & 225 & 301 \\
\hline 0 & 0,0 & 0 & 2 & 0,0 & 1 & 0 & 0,0 & 0 & 0 & 0,0 & & 2 & 2 \\
\hline 4608 & 33,6 & 6 & 130 & 2,6 & 6 & 1906 & 14,2 & 5 & 99 & 15,5 & 3 & 6743 & 8701 \\
\hline 44 & 0,3 & 3 & 13 & 0,3 & 2 & 39 & 0,3 & 3 & 8 & 1,3 & 2 & 104 & 114 \\
\hline 6 & 0,0 & 1 & 4 & 0,1 & 1 & 1 & 0,0 & 1 & 3 & 0,5 & 1 & 14 & 15 \\
\hline 268 & 2,0 & 9 & 104 & 2,1 & 8 & 904 & 6,7 & 2 & 15 & 2,4 & 2 & 1291 & 1725 \\
\hline 0 & 0,0 & 0 & 1 & 0,0 & 1 & 0 & 0,0 & 0 & 0 & 0,0 & 0 & T & \\
\hline 6 & 0,0 & 2 & 2 & 0,0 & 1 & 3 & 0,0 & 1 & 0 & 0,0 & & 11 & 11 \\
\hline 697 & 5,1 & 12 & 281 & 5,6 & 12 & 2961 & 22,0 & 11 & 47 & 7,4 & 6 & 3986 & 5668 \\
\hline 0 & 0,0 & 0 & 0 & 0,0 & 0 & 1 & 0,0 & & 0 & 0,0 & & 1 & \\
\hline 18 & 0,1 & 3 & 5 & 0,1 & 2 & 60 & 0,4 & 5 & 2 & 0,3 & & 85 & 99 \\
\hline 15 & 0,1 & 3 & 2 & 0,0 & 1 & 190 & 1,4 & 2 & 2 & 0,3 & 2 & 209 & 480 \\
\hline 65 & 0,5 & 1 & 12 & 0,2 & 1 & 38 & 0,3 & 1 & 0 & 0,0 & 0 & 115 & 162 \\
\hline 4 & 0,0 & 1 & 0 & 0,0 & 0 & 0 & 0,0 & 0 & 0 & 0,0 & & 4 & 4 \\
\hline 0 & 0,0 & 0 & 8 & 0,2 & 6 & 2 & 0,0 & 2 & 0 & 0,0 & ( & 10 & 17 \\
\hline 17 & 0,1 & 1 & 3 & 0,1 & 1 & 1 & 0,0 & 1 & 0 & 0,0 & 0 & 21 & 76 \\
\hline 207 & 1,5 & 8 & 114 & 2,3 & 8 & 705 & 5,2 & 11 & 5 & 0,8 & 4 & 1031 & 1177 \\
\hline 271 & 2,0 & 3 & 733 & 14,5 & 6 & 2732 & 20,3 & 4 & 8 & 1,3 & 1 & 3744 & 3856 \\
\hline 1 & 0,0 & 1 & 1 & 0,0 & . & 1 & 0,0 & 1 & 0 & 0,0 & 0 & 3 & 10 \\
\hline 0 & 0,0 & 0 & 0 & 0,0 & 0 & 2 & 0 & 1 & 0 & 0,0 & 0 & 2 & 2 \\
\hline 177 & 1,3 & 4 & 24 & 0,5 & 4 & 25 & 0,2 & 2 & 1 & 0,2 & 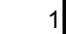 & 227 & 307 \\
\hline 32 & 0,2 & 2 & 34 & 0,7 & 1 & 3 & 0,0 & 1 & 2 & 0,3 & 2 & 71 & 105 \\
\hline 20 & 0,1 & 3 & 127 & 2,5 & 7 & 97 & 0,7 & 2 & 0 & 0,0 & 0 & 244 & 346 \\
\hline 0 & 0,0 & 0 & 0 & 0 , & 0 & 10 & 0 & & 0 & 0 & & 10 & 563 \\
\hline 2 & 0,0 & 2 & 11 & 2 & 2 & 0 & 0,0 & 0 & 1 & 0,2 & 1 & 14 & 14 \\
\hline 169 & 1,2 & 1 & 647 & 12,8 & 4 & 51 & 0,4 & 1[ & 4 & 0,6 & 1 & 871 & 1796 \\
\hline 0 & 0,0 & 0 & 2 & 0 , & 1 & 0 & 0 & & 0 & 0,0 & & , & 4 \\
\hline 1 & 0,0 & 1 & o & 2 & 2 & 2 & 0,0 & 2 & 0 & 0,0 & 0 & 11 & 12 \\
\hline 296 & 2,2 & 2 & 61 & 1,2 & 1 & 379 & 2,8 & 4 & 0 & 0,0 & 0 & 736 & 835 \\
\hline 0 & 0,0 & 0 & 0 & 0,0 & 0 & 1 & 0 & 1 & 0 & 0,0 & & 1 & 1 \\
\hline 1 & 0,0 & 1 & 4 & 0,1 & 1 & 12 & 0,1 & 1 & 0 & 0,0 & 0 & 17 & 18 \\
\hline 22 & 0,2 & 1 & 54 & 1,1 & 1 & 35 & 0,3 & 1 & 1 & 0,2 & 1 & 112 & 125 \\
\hline 28 & 0,2 & 3 & 25 & 0,5 & 7 & 8 & 0,1 & 2 & 2 & 0,3 & 2 & 63 & 124 \\
\hline 1 & 0,0 & t & 6 & 0,1 & 4 & 1 & 0 & 1 & 2 & 0,3 & 1 & 10 & 27 \\
\hline 0 & 0,0 & 0 & 4 & 0,1 & 2 & 0 & 0,0 & 0 & 0 & 0,0 & 0 & 4 & 4 \\
\hline 919 & 6,7 & 8 & 71 & 1,4 & 5 & 187 & 1,4 & 6 & 71 & 11,1 & 4 & 1248 & 6156 \\
\hline 0 & 0,0 & 0 & 1 & 0,0 & 1 & 0 & 0,0 & & 0 & 0,0 & & 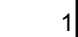 & 2 \\
\hline 1 & 0,0 & 1 & 1 & 0,0 & 1 & 0 & 0,0 & 0 & 0 & 0,0 & 0 & 2 & \\
\hline 19 & 0,1 & 6 & 79 & 1,6 & 8 & 24 & 0,2 & 5 & 3 & 0,5 & 2 & 125 & 260 \\
\hline 3695 & 100,0 & 257 & 5048 & 100,0 & 262 & $\begin{array}{l}13469 \\
\end{array}$ & 100,0 & 173 & 638 & 100,0 & 107 & 32850 & 48537 \\
\hline
\end{tabular}


Anhang 4: Schlüpfphänologie der Arten (Eklektoren 1992-96)

\begin{tabular}{|c|c|c|c|c|c|c|c|c|c|c|c|c|c|c|}
\hline & & & & & $\operatorname{lng~}$ & erio & & & & & & & & \\
\hline$\stackrel{\frac{\mathfrak{\alpha}}{\alpha}}{\frac{\mathfrak{\alpha}}{\alpha}}$ & 宫 & $\overleftarrow{a}$ & $\frac{m}{\Sigma}$ & $\mathfrak{\Sigma}$ & $\dddot{q}$ & $\dot{a}$ & $\stackrel{2}{0}$ & $\ddot{\Omega}$ & $\hat{a}$ & $\stackrel{\infty}{0}$ & 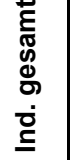 & 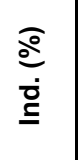 & 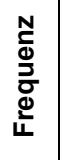 & 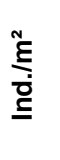 \\
\hline Carabus coriaceus & 01-.004-.001-. & & & & & & & 4 & 1 & 1 & 6 & 0,01 & 6 & 0,8 \\
\hline Carabus problematicus & 01-.004-.010-. & & 3 & 8 & 3 & 4 & 7 & 32 & 20 & 24 & 101 & 0,21 & 27 & 3,2 \\
\hline Carabus granulatus & 01-.004-.012-. & & 2 & & & & & & & 1 & 3 & 0,01 & 3 & 0,7 \\
\hline Leistus rufomarginatus & 01-.006-.002-. & & & & 2 & 4 & 1 & & 1 & 10 & 18 & 0,04 & 12 & 1,0 \\
\hline Nebria brevicollis & 01-.007-.006-. & & & 1 & 1 & 1 & & 3 & 5 & & 11 & 0,02 & 8 & 0,8 \\
\hline Notiophilus biguttatus & 01-.009-.008-. & & 3 & & & & & & & & 3 & 0,01 & 3 & 0,6 \\
\hline Tachyta nana & 01-.028-.001-. & & & & 1 & & & & & & 1 & 0,00 & 1 & 0,8 \\
\hline Bembidion lunulatum & 01-.029-.103-. & & 1 & & & & & & & & 1 & 0,00 & 1 & 8,0 \\
\hline Stomis pumicatus & 01-.049-.001-. & & & & & & 1 & & & & 1 & 0,00 & 1 & 6,3 \\
\hline Pterostichus minor & 01-.051-.022-. & & 1 & & & & & & & & 1 & 0,00 & 1 & 0,6 \\
\hline Pterostichus oblongopunctatus & 01-.051-.024-. & 1 & 32 & 50 & 50 & 12 & 3 & & 2 & 8 & 158 & 0,33 & 68 & 1,5 \\
\hline Pterostichus niger & 01-.051-.026-. & & 11 & 12 & 11 & 32 & 33 & 68 & 13 & 5 & 185 & 0,38 & 59 & 2,5 \\
\hline Abax parallelepipedus & 01-.053-.002-. & & & 2 & 7 & 4 & 1 & 3 & & & 17 & 0,04 & 9 & 1,1 \\
\hline Agonum muelleri & 01-.062-.009-. & & & & & & & & 1 & & 1 & 0,00 & 1 & 0,7 \\
\hline Amara similata & 01-.065-.008-. & & 2 & 1 & & & & & 2 & & 5 & 0,01 & 5 & 0,7 \\
\hline Amara communis & 01-.065-.014-. & & 1 & & 1 & & & & & & 2 & 0,00 & 2 & 1,4 \\
\hline Amara aenea & 01-.065-.021-. & & 2 & 1 & 1 & & & & & 2 & 6 & 0,01 & 4 & 2,0 \\
\hline Dromius quadrimaculatus & 01-.079-.012-. & & & 2 & & & 1 & 1 & & & 4 & 0,01 & 4 & 0,5 \\
\hline Dromius spilotus & 01-.079-.013-. & & & & & 1 & & & & & 1 & 0,00 & 1 & 0,2 \\
\hline Hydraena spec. & 07-.001-.000-. & & & & & & & & & 1 & 1 & 0,00 & 1 & 0,7 \\
\hline Sphaeridium lunatum & 09-.002-.004-. & & 1 & 2 & & 1 & & & 1 & 1 & 6 & 0,01 & 6 & 0,4 \\
\hline Cercyon melanocephalus & 09-.003-.008-. & & 1 & & & & & & & & 1 & 0,00 & 1 & 0,8 \\
\hline Cercyon unipunctatus & 09-.003-.013-. & & & 1 & & & & & & & 1 & 0,00 & 1 & 0,6 \\
\hline Cercyon pygmaeus & 09-.003-.017-. & & & & 1 & & & & & & 1 & 0,00 & 1 & 7,7 \\
\hline Megasternum obscurum & 09-.004-.001-. & & 1 & & & & & 1 & 2 & 1 & 5 & 0,01 & 5 & 1,1 \\
\hline Plegaderus dissectus & 10-.002-.004-. & & 26 & 21 & 36 & 84 & 62 & 27 & 3 & 4 & 263 & 0,54 & 88 & 2,9 \\
\hline Abraeus granulum & $10-.005-.001-$. & & 3 & 1 & 18 & 19 & 25 & 19 & 3 & 3 & 91 & 0,19 & 47 & 2,4 \\
\hline Gnathoncus rotundatus & 10-.009-.001-. & & & & & & & & & 1 & 1 & 0,00 & 1 & 6,9 \\
\hline Gnathoncus nannetensis & 10-.009-.002-. & & & & & 1 & & & & & 1 & 0,00 & 1 & 5,6 \\
\hline Gnathoncus buyssoni & 10-.009-.004-. & & & & 1 & & & & & & 1 & 0,00 & 1 & 5,0 \\
\hline Paromalus flavicornis & 10-.020-.001-. & & 66 & 113 & 127 & 100 & 75 & 33 & 25 & 22 & 561 & 1,16 & 96 & 9,3 \\
\hline Oeceoptoma thoracica & 12-.004-.001-. & & & & 2 & & & & & & 2 & 0,00 & 1 & 1,9 \\
\hline Phosphuga atrata & 12-.009-.001-. & & 7 & 1 & & & & & & & 8 & 0,02 & 7 & 1,0 \\
\hline Leptinus testaceus & 13-.001-.001-. & & 2 & 1 & & & & 6 & & & 9 & 0,02 & 2 & 5,8 \\
\hline Nemadus colonoides & 14-.002-.001-. & & 1 & & & & & & & & 1 & 0,00 & 1 & 1,1 \\
\hline Nargus velox & 14-.005-.001-. & & & & & & & & & 1 & 1 & 0,00 & 1 & 0,6 \\
\hline Nargus wilkini & 14-.005-.003-. & & & & & & & & 1 & 2 & 3 & 0,01 & 2 & 1,8 \\
\hline Catops nigrita & 14-.011-.012-. & & 1 & & & & & & & & 1 & 0,00 & 1 & 0,7 \\
\hline Catops fuliginosus & 14-.011-.017-. & & & & & & & & & 6 & 6 & 0,01 & 3 & 0,7 \\
\hline Catops picipes & 14-.011-.020-. & & & & 2 & & & & 1 & 50 & 53 & 0,11 & 27 & 2,4 \\
\hline Anisotoma humeralis & 16-.007-.001-. & & 23 & 10 & 11 & 13 & 12 & 15 & & & 84 & 0,17 & 12 & 4,7 \\
\hline Anisotoma orbicularis & 16-.007-.005-. & & & 1 & 3 & 6 & 4 & & & 1 & 15 & 0,03 & 8 & 1,5 \\
\hline Amphicyllis globus & 16-.009-.001-. & & & 1 & & & & & & & 1 & 0,00 & 1 & 0,7 \\
\hline Agathidium varians & 16-.011-.003-. & & 2 & 2 & & 1 & & 1 & 1 & & 7 & 0,01 & 4 & 1,0 \\
\hline Agathidium rotundatum & 16-.011-.007-. & & 3 & 1 & & 6 & 1 & & & 1 & 12 & 0,02 & 9 & 1,0 \\
\hline Agathidium confusum & 16-.011-.008-. & & & 1 & 3 & 1 & & & & & 5 & 0,01 & 3 & 1,1 \\
\hline Agathidium nigrinum & 16-.011-.010-. & & & 1 & & & & & & 1 & 2 & 0,00 & 2 & 1,2 \\
\hline Agathidium nigripenne & 16-.011-.013-. & & 24 & 30 & 16 & 12 & 1 & 2 & & & 85 & 0,18 & 20 & 3,8 \\
\hline Agathidium seminulum & 16-.011-.015-. & & 5 & 14 & 15 & 13 & 6 & 4 & 1 & 4 & 62 & 0,13 & 40 & 1,4 \\
\hline Agathidium badium & 16-.011-.018-. & & 4 & 13 & 8 & 9 & 4 & 4 & 1 & & 43 & 0,09 & 25 & 1,3 \\
\hline Euthiconus conicicollis & 18-.001-.001-. & & 1 & & 2 & 2 & 2 & & & & 7 & 0,01 & 7 & 0,8 \\
\hline Euthia linearis & 18-.002-.004-. & & & 1 & & & & & & & 1 & 0,00 & 1 & 0,9 \\
\hline
\end{tabular}




\begin{tabular}{|c|c|c|c|c|c|c|c|c|c|c|c|c|c|c|}
\hline & & & & & $\mathrm{ngp}$ & eric & & & & & & & & \\
\hline$\stackrel{\frac{\mathfrak{\alpha}}{\alpha}}{\frac{\alpha}{\alpha}}$ & 辛 & $\frac{\nwarrow}{\Omega}$ & $\frac{m}{\alpha}$ & $\mathfrak{\Sigma}$ & $\dddot{2}$ & $\dot{A}$ & $\stackrel{2}{0}$ & $\dddot{2}$ & $\hat{a}$ & $\stackrel{\infty}{0}$ & 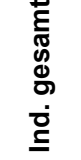 & $\begin{array}{l}\overparen{\mathfrak{o}} \\
\stackrel{0}{\mathrm{o}}\end{array}$ & 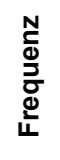 & 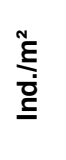 \\
\hline Cephennium thoracicum & 18-.004-.003-. & & 5 & 5 & 7 & 1 & & & 1 & & 19 & 0,04 & 7 & 2,1 \\
\hline Neuraphes elongatulus & 18-.005-.001-. & & 72 & 59 & 61 & 89 & 36 & 33 & 16 & 46 & 412 & 0,85 & 85 & 4,8 \\
\hline Neuraphes carinatus & 18-.005-.005-. & & & & 1 & & & & & & 1 & 0,00 & 1 & 7,7 \\
\hline Scydmoraphes helvolus & 18-.006-.003-. & & & & 1 & 2 & 1 & & & & 4 & 0,01 & 4 & 0,8 \\
\hline Scydmoraphes minutus & 18-.006-.004-. & & & 1 & 5 & 3 & 2 & 1 & 2 & 1 & 15 & 0,03 & 9 & 9,4 \\
\hline Stenichnus scutellaris & 18-.007-.003-. & & 1 & 4 & 9 & 1 & 3 & & 1 & & 19 & 0,04 & 14 & 1,8 \\
\hline Stenichnus godarti & 18-.007-.005-. & & 1 & 2 & 8 & 7 & 2 & & 2 & & 22 & 0,05 & 15 & 2,3 \\
\hline Stenichnus collaris & 18-.007-.008-. & & 42 & 73 & 168 & 122 & 74 & 28 & 5 & 7 & 519 & 1,07 & 117 & 5,2 \\
\hline Microscydmus nanus & 18-.008-.001-. & & 1 & 1 & 11 & 18 & 12 & 8 & 3 & 3 & 57 & 0,12 & 43 & 1,5 \\
\hline Microscydmus minimus & 18-.008-.002-. & & 4 & & 5 & 1 & 12 & 5 & & 1 & 28 & 0,06 & 23 & 2,7 \\
\hline Ptenidium gressneri & 21-.002-.001-. & & & & 1 & & & & & & 1 & 0,00 & 1 & 5,4 \\
\hline Ptenidium intermedium & 21-.002-.004-. & & & & 1 & 1 & 1 & & & & 3 & 0,01 & 3 & 1,1 \\
\hline Micridium halidaii & 21-.005-.003-. & & 1 & 1 & 233 & 65 & 33 & 7 & & 2 & 342 & 0,70 & 61 & 9,3 \\
\hline Ptinella limbata & 21-.012-.002-. & & 7 & 1 & 10 & 197 & 347 & 142 & 22 & 24 & 750 & 1,55 & 73 & 8,1 \\
\hline Ptinella aptera & 21-.012-.004-. & & 3 & 2 & 22 & 99 & 471 & 143 & 25 & 24 & 789 & 1,63 & 64 & 12,0 \\
\hline Pteryx suturalis & 21-.013-.001-. & & 8 & 12 & 21 & 173 & 222 & 197 & 33 & 30 & 696 & 1,43 & 94 & 6,1 \\
\hline Acrotrichis insularis & 21-.019-.012-. & & 1 & & 1 & & 2 & & 2 & & 6 & 0,01 & 4 & 2,3 \\
\hline Acrotrichis intermedia & 21-.019-.015-. & & 7 & 3 & 7 & 5 & 5 & 4 & 2 & 1 & 34 & 0,07 & 27 & 0,8 \\
\hline Acrotrichis fascicularis & 21-.019-.021-. & & 1 & & & & & & & & 1 & 0,00 & 1 & 0,3 \\
\hline Scaphidium quadrimaculatum & 22-.002-.001-. & & 24 & 5 & 2 & 2 & 13 & 2 & 1 & 2 & 51 & 0,11 & 14 & 1,6 \\
\hline Scaphisoma agaricinum & 22-.003-.001-. & & 3 & 1 & 6 & 4 & 1 & 1 & 1 & & 17 & 0,04 & 11 & 0,7 \\
\hline Scaphisoma balcanicum & 22-.003-.007-. & & 1 & 8 & 3 & 1 & 2 & 3 & 3 & & 21 & 0,04 & 6 & 3,4 \\
\hline Phloeocharis subtilissima & 23-.005-.001-. & 3 & 92 & 42 & 27 & 15 & 7 & 16 & 8 & 6 & 216 & 0,45 & 89 & 1,9 \\
\hline Megarthrus sinuatocollis & 23-.008-.004-. & & 1 & & & & & & & & 1 & 0,00 & 1 & 1,1 \\
\hline Proteinus brachypterus & 23-.009-.004-. & & 1 & & & & & & & 15 & 16 & 0,03 & 8 & 1,8 \\
\hline Proteinus atomarius & 23-.009-.005-. & & 2 & & & & & & & & 2 & 0,00 & 1 & 0,8 \\
\hline Phyllodrepa melanocephala & 23-.014-.001-. & & 3 & & & & & & & & 3 & 0,01 & 1 & 3,4 \\
\hline Phyllodrepa nigra & 23-.014-.004-. & & & & 1 & 1 & & & & & 2 & 0,00 & 2 & 0,6 \\
\hline Phyllodrepa ioptera & 23-.014-.012-. & & 61 & 70 & 11 & 1 & 1 & 9 & 25 & 25 & 203 & 0,42 & 71 & 1,8 \\
\hline Phyllodrepa gracilicornis & 23-.014-.015-. & & 5 & 9 & 3 & & & 2 & 1 & 1 & 21 & 0,04 & 7 & 1,2 \\
\hline Omalium rivulare & 23-.015-.005-. & & & 1 & & & & & & & 1 & 0,00 & 1 & 0,8 \\
\hline Phloeonomus monilicornis & 23-.016-.001-. & & 2 & 3 & 1 & & & & & & 6 & 0,01 & 4 & 1,1 \\
\hline Phloeonomus planus & 23-.016-.003-. & & 4 & 1 & & & & & & & 5 & 0,01 & 5 & 0,7 \\
\hline Phloeonomus punctipennis & 23-.016-.006-. & 1 & 388 & 236 & 224 & 171 & 82 & 76 & 39 & 24 & 1241 & 2,56 & 153 & 5,5 \\
\hline Xylodromus testaceus & 23-.017-.004-. & & & 4 & & & & & & & 4 & 0,01 & 3 & 0,5 \\
\hline Phyllodrepoidea crenata & 23-.023-.001-. & & 5 & & & & & & 5 & 3 & 13 & 0,03 & 8 & 1,0 \\
\hline Lathrimaeum atrocephalum & 23-.025-.002-. & & 3 & & & & & & & & 3 & 0,01 & 2 & 1,0 \\
\hline Lathrimaeum unicolor & 23-.025-.003-. & & 7 & & & & & & & & 7 & 0,01 & 7 & 0,8 \\
\hline Olophrum piceum & 23-.026-.001-. & & & & & & & & & 1 & 1 & 0,00 & 1 & 5,6 \\
\hline Lesteva longelytrata & 23-.032-.003-. & & 1 & & & & & & & & 1 & 0,00 & 1 & 0,5 \\
\hline Syntomium aeneum & 23-.040-.001-. & 1 & 2 & 3 & 1 & & & & & & 7 & 0,01 & 5 & 1,7 \\
\hline Coprophilus striatulus & 23-.042-.001-. & & & 3 & 1 & & & & & & 4 & 0,01 & 4 & 0,5 \\
\hline Carpelimus corticinus & 23-.046-.017-. & & 2 & & & & & & & & 2 & 0,00 & 2 & 0,6 \\
\hline Carpelimus gracilis & 23-.046-.030-. & & 1 & & & & & & & & 1 & 0,00 & 1 & 0,7 \\
\hline Carpelimus elongatulus & 23-.046-.032-. & & & & 1 & & & & & & 1 & 0,00 & 1 & 6,7 \\
\hline Anotylus tetracarinatus & 23-.0481.022-. & & & & & 1 & 1 & & & & 2 & 0,00 & 1 & 1,4 \\
\hline Stenus fossulatus & 23-.055-.006-. & & 3 & & & & & & & & 3 & 0,01 & 2 & 1,1 \\
\hline Rugilus rufipes & 23-.061-.003-. & & & & 1 & & & & 1 & & 2 & 0,00 & 2 & 0,4 \\
\hline Lathrobium fulvipenne & 23-.068-.021-. & & 1 & & 2 & 1 & & 1 & & & 5 & 0,01 & 5 & 0,8 \\
\hline Lathrobium brunnipes & 23-.068-.023-. & & & 2 & & & 1 & & 3 & 1 & 7 & 0,01 & 6 & 1,0 \\
\hline Nudobius lentus & 23-.078-.001-. & & 1 & & 5 & 1 & & & & 1 & 8 & 0,02 & 4 & 1,2 \\
\hline Xantholinus linearis & 23-.080-.010-. & & & & & & & & & 1 & 1 & 0,00 & 1 & 0,8 \\
\hline Xantholinus longiventris & 23-.080-.015-. & & & & & & & & & 1 & 1 & 0,00 & 1 & 0,9 \\
\hline Atrecus affinis & 23-.081-.001-. & & & 1 & 4 & 4 & 1 & 2 & 2 & & 14 & 0,03 & 14 & 0,7 \\
\hline Othius punctulatus & 23-.082-.001-. & & 2 & 2 & 1 & & & & & & 5 & 0,01 & 4 & 0,6 \\
\hline Othius myrmecophilus & 23-.082-.005-. & & 3 & 2 & & & & 2 & 8 & 3 & 18 & 0,04 & 11 & 1,3 \\
\hline
\end{tabular}




\begin{tabular}{|c|c|c|c|c|c|c|c|c|c|c|c|c|c|c|}
\hline & & & & & $\mathbf{n g}$ & erio & & & & & & & & \\
\hline 占 & 各 & $\frac{\nwarrow}{\Omega}$ & $\stackrel{m}{a}$ & $\Sigma$ & $\dddot{q}$ & a & $\stackrel{2}{2}$ & 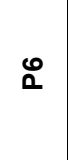 & $\hat{a}$ & $\stackrel{\infty}{\infty}$ & 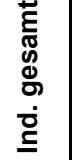 & 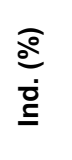 & 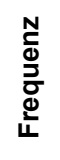 & 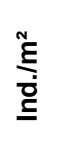 \\
\hline Philonthus cognatus & 23-.088-.023-. & & 1 & 6 & 3 & & & & & 4 & 14 & 0,03 & 5 & 1,9 \\
\hline Philonthus succicola & 23-.088-.026-. & & & & 1 & & & & & & 1 & 0,00 & 1 & 0,6 \\
\hline Philonthus decorus & 23-.088-.029-. & & 1 & & & & & & & & 1 & 0,00 & 1 & 0,4 \\
\hline Philonthus carbonarius & 23-.088-.039-. & & 2 & 1 & 1 & & & & 5 & 3 & 12 & 0,02 & 11 & 0,6 \\
\hline Philonthus varians & 23-.088-.044-. & & & & & & & & & 2 & 2 & 0,00 & 1 & 1,5 \\
\hline Philonthus fimetarius & 23-.088-.047-. & & 1 & & & & & & & & 1 & 0,00 & 1 & 0,6 \\
\hline Philonthus marginatus & 23-.088-.073-. & & 1 & & & & & & & 1 & 2 & 0,00 & 2 & 0,7 \\
\hline Gabrius splendidulus & 23-.090-.009-. & & 109 & 61 & 36 & 25 & 11 & 8 & 7 & 2 & 259 & 0,53 & 82 & 2,2 \\
\hline Ocypus olens & 23-.099-.001-. & & & & & & & 1 & & 1 & 2 & 0,00 & 2 & 0,8 \\
\hline Ocypus compressus & 23-.099-.020-. & & & & & & 3 & 2 & 1 & 1 & 7 & 0,01 & 5 & 0,8 \\
\hline Quedius lateralis & 23-.104-.005-. & & 1 & & & & & & 7 & 7 & 15 & 0,03 & 10 & 0,8 \\
\hline Quedius cruentus & 23-.104-.013-. & & & & & 1 & & & & & 1 & 0,00 & 1 & 1,1 \\
\hline Quedius brevicornis & 23-.104-.014-. & & & 1 & & & & & & 1 & 2 & 0,00 & 2 & 6,7 \\
\hline Quedius mesomelinus & 23-.104-.016-. & & & 2 & 4 & 1 & 1 & & 2 & 3 & 13 & 0,03 & 11 & 1,1 \\
\hline Quedius scitus & 23-.104-.020-. & & 7 & 3 & 1 & & & & & & 11 & 0,02 & 8 & 1,6 \\
\hline Habrocerus capillaricornis & 23-.107-.001-. & & 3 & 1 & 2 & 1 & 4 & 1 & 2 & 1 & 15 & 0,03 & 10 & 1,1 \\
\hline Mycetoporus clavicornis & 23-.109-.017-. & & & 1 & & & & & & & 1 & 0,00 & 1 & 0,7 \\
\hline Mycetoporus niger & 23-.109-.021-. & & 1 & & & & & & & & 1 & 0,00 & 1 & 0,4 \\
\hline Lordithon trinotatus & 23-.111-.006-. & & 21 & 5 & & 1 & & & 4 & 10 & 41 & 0,08 & 22 & 1,2 \\
\hline Lordithon lunulatus & 23-.111-.007-. & & & & & & & & 1 & & 1 & 0,00 & 1 & 7,1 \\
\hline Bolitobius cingulata & 23-.112-.001-. & & & 2 & & 1 & 1 & & & & 4 & 0,01 & 3 & 1,1 \\
\hline Bolitobius inclinans & 23-.112-.003-. & & 1 & & & & 1 & 1 & & & 3 & 0,01 & 3 & 0,9 \\
\hline Sepedophilus littoreus & 23-.113-.001-. & & 3 & 3 & 3 & 1 & 2 & & & 2 & 14 & 0,03 & 11 & 0,9 \\
\hline Sepedophilus testaceus & 23-.113-.002-. & & 41 & 67 & 101 & 82 & 14 & 19 & 19 & 33 & 376 & 0,77 & 80 & 4,5 \\
\hline Sepedophilus bipunctatus & 23-.113-.005-. & & 4 & 1 & 1 & 2 & 5 & & & & 13 & 0,03 & 5 & 15,7 \\
\hline Tachyporus hypnorum & 23-.114-.007-. & & & & & & & & & 1 & 1 & 0,00 & 1 & 0,8 \\
\hline Tachyporus chrysomelinus & 23-.114-.008-. & & 1 & & & & & & & 1 & 2 & 0,00 & 2 & 0,7 \\
\hline Tachyporus dispar & 23-.114-.0081. & & 2 & 1 & & & & & & 1 & 4 & 0,01 & 4 & 0,8 \\
\hline Tachyporus ruficollis & 23-.114-.012-. & & & 3 & & 1 & 1 & & & & 5 & 0,01 & 5 & 1,6 \\
\hline Holobus apicatus & 23-.1261.002-. & & 2 & 7 & & & & 1 & & & 10 & 0,02 & 4 & 0,8 \\
\hline Cypha hanseni & 23-.1262.013-. & & & & 1 & & 1 & & & 2 & 4 & 0,01 & 4 & 0,4 \\
\hline Gyrophaena gentilis & 23-.130-.009-. & & & 1 & 1 & & & & & & 2 & 0,00 & 2 & 0,8 \\
\hline Gyrophaena minima & 23-.130-.011-. & & & 2 & 2 & & & & 1 & 1 & 6 & 0,01 & 5 & 0,7 \\
\hline Gyrophaena joyioides & 23-.130-.021-. & & 2 & 9 & & & & & & & 11 & 0,02 & 5 & 1,7 \\
\hline Agaricochara latissima & 23-.1301.001-. & & 6 & & & & & & & 3 & 9 & 0,02 & 4 & 1,5 \\
\hline Placusa tachyporoides & 23-.132-.003-. & & & 1 & & & & & & & 1 & 0,00 & 1 & 0,7 \\
\hline Placusa pumilio & 23-.132-.006-. & & 1 & & 4 & 2 & & 1 & & & 8 & 0,02 & 5 & 1,0 \\
\hline Homalota plana & 23-.133-.001-. & & 11 & 1 & 2 & 2 & 1 & & & & 17 & 0,04 & 6 & 3,0 \\
\hline Anomognathus cuspidatus & 23-.134-.001-. & & 84 & 25 & 39 & 14 & 14 & 4 & 2 & 6 & 188 & 0,39 & 43 & 2,8 \\
\hline Leptusa pulchella & 23-.141-.001-. & & 29 & 24 & 14 & 41 & 40 & 17 & 1 & 1 & 167 & 0,34 & 67 & 1,7 \\
\hline Leptusa fumida & 23-.141-.004-. & & 177 & 106 & 161 & 115 & 45 & 293 & 31 & 9 & 937 & 1,93 & 162 & 4,7 \\
\hline Leptusa ruficollis & 23-.141-.006-. & & 31 & 11 & 6 & 5 & 2 & 5 & 6 & 1 & 67 & 0,14 & 37 & 4,9 \\
\hline Euryusa castanoptera & 23-.142-.001-. & & 70 & 31 & 31 & 22 & 7 & 4 & & & 165 & 0,34 & 36 & 3,4 \\
\hline Bolitochara obliqua & 23-.147-.001-. & & 113 & 86 & 58 & 46 & 71 & 47 & 6 & 5 & 432 & 0,89 & 53 & 4,1 \\
\hline Autalia impressa & 23-.148-.001-. & & & & & 2 & & & & & 2 & 0,00 & 2 & 0,4 \\
\hline Amischa analis & 23-.168-.001-. & 1 & 18 & 6 & 4 & 1 & 2 & 1 & & 1 & 34 & 0,07 & 29 & 0,9 \\
\hline Amischa soror & 23-.168-.004-. & & 2 & 3 & 2 & & 1 & & & 1 & 9 & 0,02 & 8 & 0,6 \\
\hline Amischa decipiens & 23-.168-.007-. & & 2 & 3 & 3 & 1 & & & & & 9 & 0,02 & 8 & 0,5 \\
\hline Geostiba circellaris & 23-.180-.003-. & & & & 1 & 3 & 1 & & 1 & & 6 & 0,01 & 5 & 1,4 \\
\hline Dinaraea aequata & 23-.182-.002-. & & 28 & 16 & 15 & 3 & 3 & 1 & & & 66 & 0,14 & 28 & 1,2 \\
\hline Dinaraea linearis & 23-.182-.003-. & & 16 & 10 & 6 & 10 & 4 & 1 & & & 47 & 0,10 & 22 & 1,5 \\
\hline Dadobia immersa & 23-.184-.001-. & & 9 & 2 & 5 & 1 & 1 & 1 & & & 19 & 0,04 & 12 & 0,6 \\
\hline Liogluta oblongiuscula & 23-.187-.007-. & & 2 & & & & & & & & 2 & 0,00 & 2 & 0,4 \\
\hline Atheta elongatula & 23-.188-.004-. & & & 1 & & & & 1 & & & 2 & 0,00 & 2 & 1,0 \\
\hline Atheta terminalis & 23-.188-.008-. & & & & & 1 & & & & & 1 & 0,00 & 1 & 6,9 \\
\hline Atheta parca & 23-.188-.024-. & & & & & & 2 & & & & 2 & 0,00 & 2 & 0,4 \\
\hline
\end{tabular}




\begin{tabular}{|c|c|c|c|c|c|c|c|c|c|c|c|c|c|c|}
\hline & & & & & $\mathbf{n g}$ & eric & & & & & & & & \\
\hline$\frac{\mathfrak{r}}{\frac{\alpha}{\alpha}}$ & 总 & $\frac{\varangle}{a}$ & $\frac{m}{\Sigma}$ & $\mathfrak{\Sigma}$ & $\dddot{a}$ & $a^{2}$ & $\stackrel{n}{\alpha}$ & $\stackrel{0}{0}$ & $\hat{a}$ & $\stackrel{\infty}{0}$ & 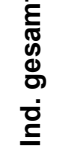 & $\begin{array}{l}\text { ఏ } \\
\text { ఏ் }\end{array}$ & $\begin{array}{l}\mathbf{N} \\
\frac{\mathbf{d}}{\mathbf{U}} \\
\frac{\vec{Z}}{\mathrm{~d}} \\
\stackrel{\underline{L}}{\mathbf{L}}\end{array}$ & $\begin{array}{l}\stackrel{N}{E} \\
\stackrel{0}{\delta}\end{array}$ \\
\hline Atheta nigricornis & 23-.188-.045-. & & & 3 & 1 & & 1 & & & & 5 & 0,01 & 3 & 0,8 \\
\hline Atheta harwoodi & 23-.188-.046-. & & 6 & 10 & 1 & 1 & 2 & 1 & & & 21 & 0,04 & 13 & 1,3 \\
\hline Atheta sodalis & 23-.188-.109-. & & 6 & 7 & 6 & & 6 & 6 & 2 & & 33 & 0,07 & 23 & 0,7 \\
\hline Atheta pallidicornis & 23-.188-.111-. & & 4 & 5 & 2 & 3 & & 1 & & 1 & 16 & 0,03 & 12 & 0,7 \\
\hline Atheta picipes & 23-.188-.126-. & & 3 & & & & & & & & 3 & 0,01 & 3 & 0,5 \\
\hline Atheta fungi & 23-.188-.136-. & & 8 & 2 & & 2 & & & & & 12 & 0,02 & 10 & 0,6 \\
\hline Atheta amplicollis & 23-.188-.1362. & & 2 & & & & & 1 & & & 3 & 0,01 & 3 & 0,4 \\
\hline Atheta dadopora & 23-.188-.155-. & & 1 & & & & & & & & 1 & 0,00 & 1 & 0,4 \\
\hline Atheta sordidula & 23-.188-.158-. & & 2 & & 1 & & & & & & 3 & 0,01 & 3 & 0,5 \\
\hline Atheta celata & 23-.188-.159-. & 1 & & & & & & & & & 1 & 0,00 & 1 & 1,1 \\
\hline Atheta ravilla & 23-.188-.183-. & & 1 & 1 & & 1 & & 1 & & & 4 & 0,01 & 4 & 0,4 \\
\hline Atheta pilicornis & 23-.188-.196-. & & 1 & 4 & 1 & 1 & 2 & 18 & 4 & & 31 & 0,06 & 9 & 1,4 \\
\hline Atheta britanniae & 23-.188-.198-. & & 1 & 2 & & & & & & & 3 & 0,01 & 2 & 2,3 \\
\hline Atheta crassicornis & 23-.188-.199-. & & 10 & 1 & & 1 & 2 & & & & 14 & 0,03 & 6 & 0,9 \\
\hline Atheta macrocera & 23-.188-.202-. & & & & & 1 & & & & 1 & 2 & 0,00 & 2 & 0,7 \\
\hline Atheta nigripes & 23-.188-.208-. & & 3 & & & & & & & & 3 & 0,01 & 3 & 0,5 \\
\hline Atheta atramentaria & 23-.188-.210-. & & & & & & & & & 1 & 1 & 0,00 & 1 & 0,4 \\
\hline Acrotona obfuscata & 23-.1881.005-. & & & & 1 & & & & & & 1 & 0,00 & 1 & 0,6 \\
\hline Acrotona consanguinea & 23-.1881.006-. & & 1 & & & & & & & & 1 & 0,00 & 1 & 0,6 \\
\hline Thamiaraea cinnamomea & 23-.194-.001-. & & & 4 & 13 & 2 & 2 & & & & 21 & 0,04 & 6 & 2,2 \\
\hline Phloeopora testacea & 23-.201-.004-. & & 39 & 13 & 20 & 12 & 4 & 8 & 1 & 1 & 98 & 0,20 & 42 & 2,6 \\
\hline Phloeopora corticalis & 23-.201-.006-. & & 20 & 5 & 6 & 5 & 1 & & & & 37 & 0,08 & 17 & 1,4 \\
\hline Ilyobates nigricollis & 23-.203-.003-. & & & & & 3 & 1 & 1 & & & 5 & 0,01 & 4 & 0,8 \\
\hline Calodera aethiops & 23-.204-.005-. & & 2 & 1 & & 2 & 2 & & & & 7 & 0,01 & 7 & 0,4 \\
\hline Mniusa incrassata & 23-.219-.001-. & 1 & 20 & 14 & 3 & 5 & 3 & 1 & & 2 & 49 & 0,10 & 31 & 1,4 \\
\hline Oxypoda vittata & 23-.223-.007-. & & 1 & & & & & & & & 1 & 0,00 & 1 & 0,7 \\
\hline Oxypoda arborea & 23-.223-.0331. & & & & 3 & 1 & & & & & 4 & 0,01 & 3 & 0,8 \\
\hline Oxypoda alternans & 23-.223-.034-. & & & & 1 & & & & & 2 & 3 & 0,01 & 3 & 0,5 \\
\hline Oxypoda brachyptera & 23-.223-.046-. & & & & 1 & & & & & & 1 & 0,00 & 1 & 0,9 \\
\hline Oxypoda annularis & 23-.223-.049-. & & & 2 & & & & & & & 2 & 0,00 & 2 & 0,7 \\
\hline Oxypoda recondita & 23-.223-.058-. & & 9 & 3 & & & & 1 & & & 13 & 0,03 & 13 & 0,9 \\
\hline Ischnoglossa prolixa & 23-.228-.001-. & & 1 & 1 & & & & 1 & & & 3 & 0,01 & 3 & 0,8 \\
\hline Dexiogya corticina & 23-.229-.001-. & & & & & & 2 & & & & 2 & 0,00 & 2 & 0,7 \\
\hline Haploglossa villosula & 23-.234-.002-. & & & & 2 & & & & & & 2 & 0,00 & 2 & 0,7 \\
\hline Tinotus morion & 23-.235-.001-. & & & 1 & & & & & & & 1 & 0,00 & 1 & 1,1 \\
\hline Aleochara lanuginosa & 23-.237-.021-. & & & 1 & & & & & & & 1 & 0,00 & 1 & 1,1 \\
\hline Aleochara ruficornis & 23-.237-.038-. & & & & & & 1 & & & & 1 & 0,00 & 1 & 5,3 \\
\hline Bibloporus bicolor & 24-.002-.002-. & & 22 & 27 & 27 & 27 & 14 & 38 & 10 & 10 & 175 & 0,36 & 87 & 1,7 \\
\hline Bibloporus minutus & 24-.002-.003-. & & 3 & 3 & 5 & 3 & 1 & 4 & 1 & 1 & 21 & 0,04 & 17 & 0,6 \\
\hline Bibloplectus minutissimus & 24-.005-.006-. & & & & 1 & & & & & & 1 & 0,00 & 1 & 0,6 \\
\hline Euplectus nanus & 24-.006-.001-. & & & 5 & 8 & 4 & 10 & 3 & & & 30 & 0,06 & 16 & 4,2 \\
\hline Euplectus piceus & 24-.006-.003-. & & 8 & 12 & 20 & 9 & 7 & 8 & 6 & 8 & 78 & 0,16 & 46 & 1,7 \\
\hline Euplectus bescidicus & 24-.006-.007-. & & 5 & 12 & 23 & 14 & 8 & 2 & & & 64 & 0,13 & 38 & 1,1 \\
\hline Euplectus punctatus & 24-.006-.013-. & & 17 & 27 & 39 & 24 & 14 & 10 & 2 & 1 & 134 & 0,28 & 67 & 2,1 \\
\hline Euplectus karsteni & 24-.006-.015-. & & 2 & 2 & 9 & 15 & 8 & 2 & & & 38 & 0,08 & 25 & 1,1 \\
\hline Plectophloeus nitidus & 24-.008-.006-. & & 5 & 2 & 1 & 13 & 7 & 5 & & 3 & 36 & 0,07 & 26 & 2,0 \\
\hline Batrisodes venustus & 24-.015-.002-. & & & 2 & & & & & & & 2 & 0,00 & 2 & 0,9 \\
\hline Brachygluta fossulata & 24-.021-.001-. & & 1 & & 1 & 4 & 1 & 2 & & & 9 & 0,02 & 3 & 2,5 \\
\hline Brachygluta haematica & 24-.021-.007-. & & & & 1 & 1 & 1 & & 1 & & 4 & 0,01 & 2 & 1,6 \\
\hline Cantharis pellucida & 27-.002-.008-. & & & 2 & 1 & & & & & & 3 & 0,01 & 3 & 1,6 \\
\hline Cantharis decipiens & 27-.002-.025-. & & 24 & 47 & 6 & & & & & & 77 & 0,16 & 37 & 1,8 \\
\hline Rhagonycha lignosa & 27-.005-.008-. & & 2 & 1 & & 1 & & & & & 4 & 0,01 & 4 & 0,6 \\
\hline Rhagonycha gallica & 27-.005-.014-. & & & & 1 & 1 & & & & & 2 & 0,00 & 2 & 0,4 \\
\hline Malthinus punctatus & 27-.008-.001-. & & & & 2 & & & & & & 2 & 0,00 & 1 & 0,5 \\
\hline Malthinus frontalis & 27-.008-.010-. & & 4 & 25 & 14 & 1 & & & & & 44 & 0,09 & 23 & 6,0 \\
\hline Malthodes minimus & 27-.009-.012-. & & & & & & 2 & & & & 2 & 0,00 & 2 & 0,3 \\
\hline
\end{tabular}




\begin{tabular}{|c|c|c|c|c|c|c|c|c|c|c|c|c|c|c|}
\hline \multirow{2}{*}{$\stackrel{\leftarrow}{\frac{\alpha}{4}}$} & \multirow[b]{2}{*}{ 嵒 } & \multicolumn{9}{|c|}{ Fangperiode } & \multirow{2}{*}{ 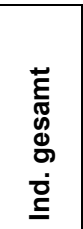 } & \multirow[b]{2}{*}{$\begin{array}{l}\overparen{\varrho} \\
\stackrel{0}{\grave{g}}\end{array}$} & \multirow[b]{2}{*}{ 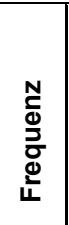 } & \multirow[b]{2}{*}{$\begin{array}{l}\stackrel{N}{\xi} \\
\stackrel{0}{0} \\
\underline{\Xi}\end{array}$} \\
\hline & & $\overleftarrow{\Omega}$ & $\frac{m}{i}$ & $\mathfrak{N}$ & $\ddot{a}$ & a & $\stackrel{\llcorner}{\alpha}$ & $\ddot{Q}$ & $\hat{a}$ & $\infty$ & & & & \\
\hline Malthodes guttifer & 27-.009-.015-. & & 1 & & 1 & 22 & 6 & & & & 30 & 0,06 & 18 & 1,1 \\
\hline Malthodes marginatus & 27-.009-.016-. & & 7 & 1 & & & & & & & 8 & 0,02 & 5 & 1,4 \\
\hline Malthodes brevicollis & 27-.009-.032-. & & & & 1 & & & & & & 1 & 0,00 & 1 & 0,3 \\
\hline Malachius bipustulatus & 29-.006-.0032. & & & 2 & 1 & & & & & & 3 & 0,01 & 2 & 0,4 \\
\hline Dasytes caeruleus & 30-.005-.005-. & & 19 & 2 & & & & & & & 21 & 0,04 & 14 & 0,8 \\
\hline Dasytes aerosus & 30-.005-.009-. & & 1 & 1 & & & & & & & 2 & 0,00 & 2 & 0,5 \\
\hline Thymalus limbatus & 321.009-.001-. & & & & & 1 & 1 & 1 & & & 3 & 0,01 & 3 & 1,6 \\
\hline Hylecoetus dermestoides & 33-.001-.001-. & & 216 & 821 & 151 & 5 & & & & & 1193 & 2,46 & 46 & 25,2 \\
\hline Lymeylon navale & 33-.002-.001-. & & & & & 118 & 12 & 2 & & & 132 & 0,27 & 6 & 16,6 \\
\hline Ampedus erythrogonus & 34-.001-.004-. & & 4 & 8 & 7 & 6 & 5 & & & & 30 & 0,06 & 16 & 1,8 \\
\hline Ampedus brunnicornis & 34-.001-.013-. & & & 1 & & & & & & & 1 & 0,00 & 1 & 6,7 \\
\hline Ampedus nigerrimus & 34-.001-.014-. & & & 2 & 7 & 6 & & & & & 15 & 0,03 & 11 & 1,0 \\
\hline Ampedus sanguineus & 34-.001-.015-. & & & 1 & & & & & & & 1 & 0,00 & 1 & 0,8 \\
\hline Ampedus pomorum & 34-.001-.019-. & & 7 & 7 & 18 & 12 & 2 & 1 & & & 47 & 0,10 & 28 & 0,8 \\
\hline Ampedus nigroflavus & 34-.001-.021-. & & 2 & & & & & & & & 2 & 0,00 & 2 & 0,6 \\
\hline Procraerus tibialis & 34-.004-.001-. & & 1 & 8 & 4 & & & & & & 13 & 0,03 & 8 & 1,9 \\
\hline Agriotes aterrimus & 34-.010-.001-. & & 2 & & 1 & & & & & & 3 & 0,01 & 3 & 0,4 \\
\hline Agriotes acuminatus & 34-.010-.003-. & & 2 & 4 & 6 & 1 & & & & & 13 & 0,03 & 13 & 0,4 \\
\hline Agriotes lineatus & 34-.010-.009-. & & 1 & & & & & & & & 1 & 0,00 & 1 & 8,0 \\
\hline Melanotus castanipes & 34-.016-.003-. & & 7 & 24 & 57 & 11 & 2 & 1 & & & 102 & 0,21 & 63 & 1,0 \\
\hline Calambus bipustulatus & 34-.030-.001-. & & 1 & & 1 & & & & & & 2 & 0,00 & 2 & 0,4 \\
\hline Hypoganus inunctus & 34-.031-.001-. & & & 9 & 6 & 2 & 2 & & & & 19 & 0,04 & 9 & 2,1 \\
\hline Denticollis linearis & 34-.033-.004-. & & & 64 & 78 & 1 & & & & & 143 & 0,29 & 64 & 1,3 \\
\hline Stenagostus rhombeus & 34-.038-.002-. & & & & & 3 & 3 & & & & 6 & 0,01 & 4 & 0,7 \\
\hline Athous haemorrhoidalis & 34-.041-.001-. & & 1 & 8 & 5 & 2 & & & & & 16 & 0,03 & 12 & 0,8 \\
\hline Athous subfuscus & 34-.041-.003-. & & 10 & 16 & 17 & 3 & & & & & 46 & 0,09 & 32 & 0,8 \\
\hline Melasis buprestoides & 36-.001-.001-. & & & & 2 & 1 & & & & & 3 & 0,01 & 2 & 0,5 \\
\hline Eucnemis capucina & 36-.003-.001-. & & & & & 3 & & & & & 3 & 0,01 & 1 & 1,3 \\
\hline Dirhagus lepidus & 36-.008-.004-. & & & & 60 & 22 & & & & & 82 & 0,17 & 3 & 10,2 \\
\hline Hylis olexai & 36-.011-.001-. & & & & & & 20 & 21 & & & 41 & 0,08 & 8 & 6,3 \\
\hline Xylophilus corticalis & 36-.012-.001-. & & & & 1 & 7 & 5 & 4 & & & 17 & 0,04 & 13 & 0,7 \\
\hline Trixagus dermestoides & 37-.001-.002-. & & 1 & 21 & 17 & 24 & 16 & 8 & 3 & 1 & 91 & 0,19 & 29 & 1,5 \\
\hline Trixagus carinifrons & 37-.001-.003-. & & & & & & & & & 1 & 1 & 0,00 & 1 & 0,3 \\
\hline Agrilus biguttatus & 38-.020-.003-. & & & & & 5 & 2 & & & & 7 & 0,01 & 4 & 11,2 \\
\hline Agrilus sulcicollis & 38-.020-.007-. & & & & 2 & & & & & & 2 & 0,00 & 2 & 0,6 \\
\hline Clambus punctulum & 381.002-.002-. & & & 2 & & & & & & & 2 & 0,00 & 2 & 0,5 \\
\hline Cyphon coarctatus & 40-.003-.001-. & & & & & 2 & & & & & 2 & 0,00 & 2 & 0,4 \\
\hline Cyphon ochraceus & 40-.003-.006-. & & & & 1 & & & & & & 1 & 0,00 & 1 & 0,3 \\
\hline Cyphon padi & 40-.003-.011-. & & & & & & & & & 1 & 1 & 0,00 & 1 & 0,6 \\
\hline Prionocyphon serricornis & 40-.004-.001-. & & & & & 1 & & 2 & & & 3 & 0,01 & 3 & 0,4 \\
\hline Trogoderma angustum & 45-.003-.001-. & & 1 & & 1 & & & & & & 2 & 0,00 & 2 & 1,3 \\
\hline Anthrenus museorum & 45-.008-.010-. & & & & & 1 & & & & & 1 & 0,00 & 1 & 6,3 \\
\hline Cerylon fagi & 492.002-.001-. & & 28 & 31 & 28 & 24 & 14 & 6 & 5 & 5 & 141 & 0,29 & 65 & 1,6 \\
\hline Cerylon histeroides & 492.002-.002-. & & 26 & 39 & 46 & 48 & 7 & 2 & 8 & 19 & 195 & 0,40 & 76 & 2,6 \\
\hline Cerylon ferrugineum & 492.002-.003-. & & 221 & 340 & 294 & 190 & 102 & 37 & 45 & 43 & 1272 & 2,62 & 157 & 7,6 \\
\hline Sphaerosoma pilosum & 493.001-.007-. & & 1 & & 1 & 4 & 5 & & & & 11 & 0,02 & 9 & 0,8 \\
\hline Meligethes denticulatus & 50-.008-.003-. & & & 1 & & & & & & & 1 & 0,00 & 1 & 0,4 \\
\hline Meligethes aeneus & 50-.008-.014-. & & 5 & 1 & & & & 1 & & & 7 & 0,01 & 7 & 0,6 \\
\hline Epuraea neglecta & 50-.009-.005-. & & & 1 & 2 & 2 & & 1 & & & 6 & 0,01 & 5 & 0,7 \\
\hline Epuraea marseuli & 50-.009-.015-. & & 9 & 14 & 5 & 6 & & 1 & 1 & & 36 & 0,07 & 17 & 1,9 \\
\hline Epuraea pygmaea & 50-.009-.016-. & & 3 & 1 & & & & & & & 4 & 0,01 & 4 & 0,7 \\
\hline Epuraea longula & 50-.009-.017-. & & 11 & 13 & 3 & & & & & & 27 & 0,06 & 10 & 1,9 \\
\hline Epuraea unicolor & 50-.009-.027-. & & 20 & 4 & & & 1 & & 1 & & 26 & 0,05 & 10 & 1,7 \\
\hline Epuraea variegata & 50-.009-.028-. & & 21 & 19 & 31 & 7 & 6 & 8 & 2 & 1 & 95 & 0,20 & 25 & 3,1 \\
\hline Cychramus luteus & 50-.019-.002-. & & & & 8 & 1 & 1 & & 1 & 3 & 14 & 0,03 & 4 & 1,7 \\
\hline Cryptarcha strigata & 50-.020-.001-. & & & 2 & 10 & & 1 & 1 & & & 14 & 0,03 & 5 & 1,4 \\
\hline
\end{tabular}




\begin{tabular}{|c|c|c|c|c|c|c|c|c|c|c|c|c|c|c|}
\hline & & & & & ang & oric & $\mathrm{de}$ & & & & & & & \\
\hline 占 & 竞 & $\frac{\pi}{\alpha}$ & $\stackrel{m}{\Sigma}$ & $\mathfrak{\Sigma}$ & $\dddot{a}$ & Z & $\stackrel{2}{\alpha}$ & ஜ & $\hat{a}$ & $\stackrel{\infty}{0}$ & 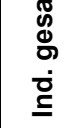 & $\begin{array}{l}\stackrel{0}{0} \\
\stackrel{0}{\circ}\end{array}$ & $\begin{array}{l}\overline{\mathbf{d}} \\
\bar{\Xi} \\
\bar{d}\end{array}$ & 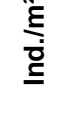 \\
\hline Cryptarcha undata & 50-.020-.002-. & & & & 1 & 1 & & & & & 2 & 0,00 & 1 & 0,9 \\
\hline Glischrochilus quadriguttatus & 50-.021-.001-. & & 45 & 20 & 1 & & & & & 1 & 67 & 0,14 & 17 & 3,2 \\
\hline Glischrochilus hortensis & 50-.021-.002-. & & & 1 & & & & & & & 1 & 0,00 & 1 & 0,8 \\
\hline Pityophagus ferrugineus & 50-.022-.001-. & & & & 1 & & & & & & 1 & 0,00 & 1 & 0,8 \\
\hline Brachypterus urticae & 501.003-.001-. & & & & & & & & 1 & 1 & 2 & 0,00 & 2 & 0,5 \\
\hline Rhizophagus ferrugineus & 52-.001-.004-. & & 2 & 7 & 27 & 29 & 5 & 3 & 1 & & 74 & 0,15 & 32 & 1,3 \\
\hline Rhizophagus perforatus & 52-.001-.006-. & & 4 & 25 & 8 & 12 & 1 & 2 & 1 & 3 & 56 & 0,12 & 26 & 1,3 \\
\hline Rhizophagus dispar & 52-.001-.008-. & 9 & 1111 & 1032 & 1296 & 1227 & 738 & 540 & 196 & 463 & 6612 & 13,62 & 203 & 40,7 \\
\hline Rhizophagus bipustulatus & 52-.001-.009-. & 3 & 483 & 286 & 247 & 195 & 84 & 30 & 10 & 34 & 1372 & 2,83 & 173 & 8,6 \\
\hline Rhizophagus nitidulus & 52-.001-.010-. & & 95 & 144 & 153 & 50 & 45 & 51 & 8 & 27 & 573 & 1,18 & 79 & 6,3 \\
\hline Rhizophagus cribratus & 52-.001-.013-. & & & & 9 & 1 & 1 & 1 & & 2 & 14 & 0,03 & 9 & 0,8 \\
\hline Monotoma longicollis & 53-.001-.009-. & & & & & 1 & & & & & 1 & 0,00 & 1 & 8,7 \\
\hline Uleiota planata & 53-.012-.001-. & & 2 & 1 & 3 & & & 1 & 2 & 1 & 10 & 0,02 & 6 & 2,2 \\
\hline Pediacus depressus & 53-.015-.001-. & & & & 2 & 3 & & 1 & & & 6 & 0,01 & 6 & 0,6 \\
\hline Pediacus dermestoides & 53-.015-.002-. & 1 & 43 & 38 & 8 & 3 & & & 1 & 3 & 97 & 0,20 & 56 & 1,6 \\
\hline Silvanus bidentatus & 531.006-.001-. & & 3 & 4 & 4 & 4 & & & & & 15 & 0,03 & 10 & 1,0 \\
\hline Cryptophagus pubescens & 55-.008-.019-. & & & & & & & & 1 & & 1 & 0,00 & 1 & 0,3 \\
\hline Cryptophagus saginatus & 55-.008-.021-. & & & & 1 & & & & & & 1 & 0,00 & 1 & 0,8 \\
\hline Cryptophagus dentatus & 55-.008-.027-. & 2 & 190 & 237 & 242 & 214 & 192 & 206 & 171 & 162 & 1616 & 3,33 & 204 & 8,4 \\
\hline Cryptophagus distinguendus & 55-.008-.030-. & & & & & & 1 & & & & 1 & 0,00 & 1 & 5,9 \\
\hline Cryptophagus scanicus & 55-.008-.034-. & & 1 & & & 5 & & 1 & 1 & 3 & 11 & 0,02 & 5 & 2,3 \\
\hline Cryptophagus lycoperdi & 55-.008-.040-. & & & & & 5 & 2 & 1 & 1 & 1 & 10 & 0,02 & 5 & 1,0 \\
\hline Micrambe abietis & 55-.0081.005-. & & & & & & & & 1 & & 1 & 0,00 & 1 & 0,3 \\
\hline Atomaria pusilla & 55-.014-.011-. & & & & & & 1 & & & & 1 & 0,00 & 1 & 0,7 \\
\hline Atomaria fuscata & 55-.014-.014-. & & 2 & & 1 & & & & & & 3 & 0,01 & 3 & 1,3 \\
\hline Atomaria turgida & 55-.014-.033-. & & 1 & & & & & & & & 1 & 0,00 & 1 & 0,7 \\
\hline Atomaria testacea & 55-.014-.036-. & & & 1 & & & & & & & 1 & 0,00 & 1 & 0,5 \\
\hline Atomaria umbrina & 55-.014-.038-. & & 1 & 2 & & 1 & & & & & 4 & 0,01 & 2 & 1,1 \\
\hline Atomaria nigrirostris & 55-.014-.045-. & & 6 & 3 & 19 & 2 & & & & & 30 & 0,06 & 15 & 1,2 \\
\hline Atomaria linearis & 55-.014-.046-. & & & & & 1 & & & & & 1 & 0,00 & 1 & 0,6 \\
\hline Atomaria pulchra & 55-.014-.051-. & & 8 & 7 & & & & & & & 15 & 0,03 & 13 & 0,8 \\
\hline Atomaria bella & 55-.014-.054-. & & & 14 & 11 & 1 & 1 & & & 1 & 28 & 0,06 & 18 & 1,2 \\
\hline Olibrus aeneus & 56-.002-.001-. & & 1 & & & & & & & & 1 & 0,00 & 1 & 0,4 \\
\hline Cryptolestes duplicatus & 561.004-.001-. & & 1 & & & 1 & 1 & & & & 3 & 0,01 & 3 & 0,4 \\
\hline Cryptolestes ferrugineus & 561.004-.005-. & & & & & 1 & 4 & & 1 & 2 & 8 & 0,02 & 5 & 2,5 \\
\hline Latridius minutus & 58-.003-.0021. & & & & & & 3 & & & 1 & 4 & 0,01 & 4 & 1,9 \\
\hline Enicmus fungicola & 58-.004-.010-. & & 30 & 4 & & 2 & & 1 & & & 37 & 0,08 & 12 & 3,9 \\
\hline Enicmus rugosus & 58-.004-.012-. & & 22 & 8 & 4 & 8 & 6 & 10 & 16 & 16 & 90 & 0,19 & 33 & 2,1 \\
\hline Enicmus testaceus & 58-.004-.013-. & & 4 & 4 & 6 & 14 & 17 & 9 & 1 & & 55 & 0,11 & 13 & 5,2 \\
\hline Enicmus transversus & 58-.004-.014-. & & 12 & 2 & 1 & & 1 & & & & 16 & 0,03 & 12 & 1,0 \\
\hline Dienerella elongata & 58-.0041.001-. & & 55 & 43 & 75 & 69 & 68 & 81 & 55 & 55 & 501 & 1,03 & 94 & 9,1 \\
\hline Dienerella clathrata & 58-.0041.0021. & & & 3 & 4 & 1 & 3 & 2 & 1 & 1 & 15 & 0,03 & 5 & 3,1 \\
\hline Stephostethus lardarius & 58-.0061.001-. & & & 1 & & & & & & & 1 & 0,00 & 1 & 1,1 \\
\hline Stephostethus angusticollis & 58-.0061.002-. & & 1 & 1 & & 1 & 2 & 1 & & & 6 & 0,01 & 6 & 0,7 \\
\hline Aridius nodifer & 58-.0063.003-. & & 14 & 32 & 41 & 150 & 190 & 159 & 91 & 47 & 724 & 1,49 & 104 & 4,9 \\
\hline Corticaria linearis & 58-.007-.016-. & & 7 & 2 & 3 & 1 & & 2 & 1 & & 16 & 0,03 & 9 & 1,2 \\
\hline Corticaria longicollis & 58-.007-.018-. & 1 & 604 & 399 & 412 & 579 & 652 & 842 & 409 & 187 & 4085 & 8,42 & 234 & 24,7 \\
\hline Corticaria elongata & 58-.007-.021-. & & & 1 & 2 & 4 & 2 & & & & 9 & 0,02 & 9 & 0,7 \\
\hline Corticarina similata & 58-.008-.002-. & & 5 & 14 & 25 & 7 & 7 & 7 & 9 & 6 & 80 & 0,16 & 35 & 1,1 \\
\hline Corticarina fuscula & 58-.008-.005-. & & 2 & 2 & & 1 & 1 & & 6 & 5 & 17 & 0,04 & 13 & 0,8 \\
\hline Cortinicara gibbosa & 58-.0081.001-. & 1 & 4 & 2 & & 1 & 1 & 1 & & 2 & 12 & 0,02 & 10 & 0,7 \\
\hline Holoparamecus caularum & 581.001-.001-. & & & & & & & & & 1 & 1 & 0,00 & 1 & 5,9 \\
\hline Triphyllus bicolor & 59-.002-.001-. & & & & & & & 2 & 1 & & 3 & 0,01 & 2 & 2,1 \\
\hline Litargus connexus & 59-.003-.001-. & & 12 & 3 & & & & 1 & 1 & & 17 & 0,04 & 10 & 1,1 \\
\hline Mycetophagus quadripustulatus & 59-.004-.001-. & & & 1 & & & & & & & 1 & 0,00 & 1 & 5,1 \\
\hline Mycetophagus piceus & 59-.004-.003-. & & 5 & & & & 1 & & & & 6 & 0,01 & 1 & 40,0 \\
\hline
\end{tabular}




\begin{tabular}{|c|c|c|c|c|c|c|c|c|c|c|c|c|c|c|}
\hline & & & & & $\mathrm{ngp}$ & pric & $\mathrm{de}$ & & & & & & & \\
\hline 占 & 구 & $\overleftarrow{\Delta}$ & $\frac{m}{i}$ & 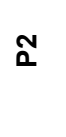 & $\dddot{2}$ & I & $\stackrel{2}{\alpha}$ & $\dddot{\Omega}$ & $\hat{a}$ & $\stackrel{\infty}{\alpha}$ & 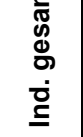 & $\begin{array}{l}\widehat{\varrho} \\
\stackrel{0}{\grave{g}}\end{array}$ & 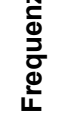 & 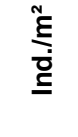 \\
\hline Mycetophagus atomarius & 59-.004-.006-. & & 16 & 14 & 16 & 6 & 9 & 8 & 2 & & 71 & 0,15 & 20 & $\overline{4,8}$ \\
\hline Mycetophagus populi & 59-.004-.010-. & & & & & & & 1 & & & 1 & 0,00 & 1 & 5,9 \\
\hline Cicones variegatus & 60-.014-.001-. & & 21 & 63 & 23 & 22 & 6 & 11 & 6 & 3 & 155 & 0,32 & 18 & 19,3 \\
\hline Bitoma crenata & 60-.016-.001-. & & & 1 & & 1 & & & & & 2 & 0,00 & 2 & 0,6 \\
\hline Teredus cylindricus & 60-.020-.001-. & & 24 & 44 & 82 & 58 & 48 & 33 & 12 & 22 & 323 & 0,67 & 70 & 8,1 \\
\hline Orthoperus mundus & 601.008-.004-. & & 7 & 8 & 19 & 20 & 24 & 35 & 41 & 8 & 162 & 0,33 & 51 & 2,6 \\
\hline Endomychus coccineus & 61-.013-.001-. & & & & & & & 3 & & 1 & 4 & 0,01 & 1 & 1,8 \\
\hline Nephus bipunctatus & 62-.0081.004-. & & & & & & & & & 1 & 1 & 0,00 & 1 & 0,4 \\
\hline Chilocorus renipustulatus & 62-.012-.002-. & & & & & & & & & 1 & 1 & 0,00 & 1 & 6,5 \\
\hline Aphidecta obliterata & 62-.017-.001-. & & 1 & & & & & & & & 1 & 0,00 & 1 & 7,4 \\
\hline Adalia decempunctata & 62-.023-.002-. & & 1 & & & & & & & & 1 & 0,00 & 1 & 0,8 \\
\hline Adalia bipunctata & 62-.023-.003-. & & 2 & & & & & & & & 2 & 0,00 & 2 & 0,3 \\
\hline Coccinella septempunctata & 62-.025-.003-. & & 1 & 1 & & & & & & & 2 & 0,00 & 2 & 0,5 \\
\hline Coccinella quinquepunctata & 62-.025-.005-. & & & & & & & & & 1 & 1 & 0,00 & 1 & 0,4 \\
\hline Propylea quatuordecimpunctata & 62-.032-.001-. & & 3 & 2 & 1 & & & & & 2 & 8 & 0,02 & 7 & 0,5 \\
\hline Arpidiphorus orbiculatus & 63-.002-.001-. & & 1 & 8 & 21 & 39 & 4 & 3 & & & 76 & 0,16 & 18 & 2,4 \\
\hline Octotemnus glabriculus & 65-.001-.001-. & & 15 & 18 & 17 & 8 & 39 & 15 & 27 & 47 & 186 & 0,38 & 20 & 6,2 \\
\hline Sulcacis bidentulus & 65-.005-.002-. & & & & & & 2 & & & 1 & 3 & 0,01 & 3 & 0,6 \\
\hline Cis nitidus & 65-.006-.002-. & & 69 & 61 & 73 & 64 & 71 & 47 & 70 & 45 & 500 & 1,03 & 33 & 21,2 \\
\hline Cis glabratus & 65-.006-.004-. & & & & & 1 & & & & & 1 & 0,00 & 1 & 4,9 \\
\hline Cis hispidus & 65-.006-.007-. & & 2 & 2 & 3 & 2 & & & & & 9 & 0,02 & 6 & 0,9 \\
\hline Cis boleti & 65-.006-.011-. & & 5 & 2 & 4 & 1 & 3 & 12 & 5 & 7 & 39 & 0,08 & 12 & 2,4 \\
\hline Cis castaneus & 65-.006-.015-. & & 20 & 35 & 64 & 48 & 45 & 54 & 24 & 13 & 303 & 0,62 & 62 & 9,3 \\
\hline Cis bidentatus & 65-.006-.017-. & & 9 & 8 & 9 & 8 & 12 & 11 & 10 & 2 & 69 & 0,14 & 23 & 9,0 \\
\hline Cis pygmaeus & 65-.006-.024-. & & & & & & & 1 & & & 1 & 0,00 & 1 & 6,7 \\
\hline Cis festivus & 65-.006-.028-. & 1 & 13 & 11 & 14 & 9 & 2 & 2 & 1 & 1 & 54 & 0,11 & 21 & 2,5 \\
\hline Ennearthron cornutum & 65-.007-.002-. & & 1 & 5 & 2 & 1 & & 2 & 1 & & 12 & 0,02 & 11 & 0,8 \\
\hline Hedobia imperialis & 68-.001-.002-. & & & 12 & 10 & 1 & & & & & 23 & 0,05 & 7 & 2,6 \\
\hline Dryophilus pusillus & 68-.003-.003-. & & & & 1 & & & & & & 1 & 0,00 & 1 & 0,3 \\
\hline Xestobium plumbeum & 68-.005-.001-. & & 82 & 2 & & & & & & & 84 & 0,17 & 3 & 9,1 \\
\hline Xestobium rufovillosum & 68-.005-.002-. & & 31 & 48 & 14 & 2 & 4 & & & 1 & 100 & 0,21 & 23 & 6,9 \\
\hline Anobium costatum & 68-.012-.005-. & & 28 & 2 & & & & & & & 30 & 0,06 & 5 & 1,8 \\
\hline Ptilinus pectinicornis & 68-.014-.001-. & & & & 66 & 2252 & 1188 & 80 & 9 & 5 & 3600 & 7,42 & 133 & 22,7 \\
\hline Dorcatoma flavicornis & 68-.022-.001-. & & & & & & 1 & 1 & & & 2 & 0,00 & 2 & 0,4 \\
\hline Dorcatoma chrysomelina & 68-.022-.003-. & & & & 1 & 4 & 5 & 4 & 1 & & 15 & 0,03 & 9 & 1,8 \\
\hline Dorcatoma robusta & 68-.022-.007-. & & & & 1 & & & & & & 1 & 0,00 & 1 & 4,5 \\
\hline Ptinus fur & 69-.008-.005-. & & 2 & & & & & & & 1 & 3 & 0,01 & 3 & 0,5 \\
\hline Ptinus subpilosus & 69-.008-.013-. & & 2 & 1 & 2 & & 1 & & & 1 & 7 & 0,01 & 7 & 1,3 \\
\hline Ischnomera cyanea & 70-.007-.0021. & & 2 & & & & & & & & 2 & 0,00 & 1 & 12,1 \\
\hline Lissodema quadripustulatum & 711.001-.002-. & & & & 3 & 8 & & 1 & 2 & & 14 & 0,03 & 4 & 1,2 \\
\hline Vincenzellus ruficollis & 711.005-.001-. & & 17 & 18 & 32 & 29 & 32 & 18 & 6 & 2 & 154 & 0,32 & 22 & 5,6 \\
\hline Rhinosimus planirostris & 711.006-.002-. & & 6 & & 2 & 3 & & 1 & 1 & & 13 & 0,03 & 11 & 0,5 \\
\hline Rhinosimus ruficollis & 711.006-.003-. & & 18 & 14 & 10 & 7 & 5 & 12 & 17 & 43 & 126 & 0,26 & 28 & 4,2 \\
\hline Pyrochroa coccinea & 72-.001-.001-. & & & 29 & 9 & & & & & & 38 & 0,08 & 19 & 1,6 \\
\hline Schizotus pectinicornis & 72-.002-.001-. & & 6 & 46 & 15 & & & & & & 67 & 0,14 & 21 & 1,2 \\
\hline Anaspis humeralis & 73-.004-.001-. & & 5 & 1 & 1 & 1 & & & & & 8 & 0,02 & 3 & 1,4 \\
\hline Anaspis frontalis & 73-.004-.009-. & & 1 & 1 & & & & & & & 2 & 0,00 & 2 & 0,4 \\
\hline Anaspis maculata & 73-.004-.010-. & & 2 & 5 & & 1 & & & & & 8 & 0,02 & 6 & 0,6 \\
\hline Anaspis schilskyana & 73-.004-.011-. & & 3 & 89 & 44 & 49 & 8 & 2 & & & 195 & 0,40 & 58 & 2,8 \\
\hline Anaspis thoracica & 73-.004-.012-. & & 1 & 50 & 31 & 7 & 1 & 3 & & & 93 & 0,19 & 48 & 1,7 \\
\hline Anaspis rufilabris & 73-.004-.019-. & & 24 & 4 & & & & & & & 28 & 0,06 & 18 & 1,1 \\
\hline Anaspis flava & 73-.004-.022-. & & 1 & 10 & & 1 & & & & & 12 & 0,02 & 7 & 0,8 \\
\hline Aderus oculatus & 74-.002-.007-. & & & & 49 & 432 & 62 & 13 & 7 & & 563 & 1,16 & 10 & 155,1 \\
\hline Tomoxia biguttata & 79-.001-.001-. & & & & & 1 & & 1 & & & 2 & 0,00 & 2 & 1,2 \\
\hline Mordellistena variegata & 79-.011-.053-. & & & & & & & 1 & & & 1 & 0,00 & 1 & 0,4 \\
\hline Mordellochroa abdominalis & 79-.012-.001-. & & 1 & 8 & 2 & & & & & & 11 & 0,02 & 4 & 1,0 \\
\hline
\end{tabular}




\begin{tabular}{|c|c|c|c|c|c|c|c|c|c|c|c|c|c|c|}
\hline & & & & & $\mathrm{ng}$ & eric & & & & & & & & \\
\hline$\stackrel{\frac{\mathfrak{\alpha}}{\alpha}}{\frac{\alpha}{\alpha}}$ & 辛 & $\frac{\nwarrow}{a}$ & $\frac{m}{\alpha}$ & $\mathfrak{\Sigma}$ & $\dddot{m}$ & $\dot{Z}$ & $\stackrel{\infty}{\alpha}$ & $\dddot{2}$ & $\hat{a}$ & $\stackrel{\infty}{0}$ & 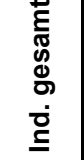 & $\begin{array}{l}\overparen{\mathfrak{o}} \\
\stackrel{0}{\mathrm{o}}\end{array}$ & 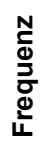 & 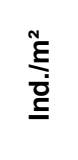 \\
\hline Hallomenus binotatus & 80-.004-.001-. & & & & & & 1 & & & & 1 & 0,00 & 1 & 0,7 \\
\hline Orchesia minor & 80-.005-.004-. & & & & & & & & 2 & 1 & 3 & 0,01 & 1 & 1,3 \\
\hline Orchesia undulata & 80-.005-.006-. & & 31 & 22 & 14 & 151 & 866 & 414 & 191 & 65 & 1754 & 3,61 & 74 & 14,2 \\
\hline Hypulus quercinus & 80-.013-.001-. & & 16 & 8 & & & & & & & 24 & 0,05 & 5 & 3,4 \\
\hline Melandrya caraboides & 80-.016-.001-. & & & 1 & 3 & & & & & & 4 & 0,01 & 4 & 0,4 \\
\hline Conopalpus testaceus & 80-.018-.001-. & & & & 2 & 8 & & & & & 10 & 0,02 & 3 & 1,2 \\
\hline Tetratoma desmaresti & 801.001-.002-. & & & 1 & & & & & 1 & & 2 & 0,00 & 1 & 2,3 \\
\hline Tetratoma ancora & 801.001-.003-. & & 1 & 1 & & & & & & & 2 & 0,00 & 2 & 0,3 \\
\hline Allecula morio & 82-.001-.002-. & & & & & 1 & & & & & 1 & 0,00 & 1 & 6,7 \\
\hline Pseudocistela ceramboides & 82-.005-.001-. & & & & & 1 & & 1 & & & 2 & 0,00 & 2 & 0,8 \\
\hline Mycetochara linearis & 82-.008-.011-. & & & & & 1 & 7 & 1 & & & 9 & 0,02 & 5 & 1,4 \\
\hline Scaphidema metallicum & 83-.019-.001-. & & & & 1 & & & & & & 1 & 0,00 & 1 & 5,9 \\
\hline Corticeus unicolor & 83-.023-.001-. & & 87 & 190 & 283 & 110 & 69 & 28 & 13 & 9 & 789 & 1,63 & 99 & 7,2 \\
\hline Corticeus fasciatus & 83-.023-.008-. & & 6 & 8 & 12 & 6 & 7 & 1 & 3 & 1 & 44 & 0,09 & 17 & 4,6 \\
\hline Tribolium castaneum & 83-.025-.002-. & & 1 & & & & & & & & 1 & 0,00 & 1 & 5,9 \\
\hline Trox scaber & 841.001-.004-. & & & 1 & & & & & & & 1 & 0,00 & 1 & 5,1 \\
\hline Gnorimus variabilis & 85-.050-.002-. & & & & & 8 & 10 & & & & 18 & 0,04 & 5 & 3,4 \\
\hline Sinodendron cylindricum & 86-.005-.001-. & & & & 38 & 62 & 24 & 1 & & & 125 & 0,26 & 33 & 2,6 \\
\hline Rhagium bifasciatum & 87-.011-.001-. & & 10 & 2 & 14 & 1 & & & & & 27 & 0,06 & 10 & 1,4 \\
\hline Rhagium sycophanta & 87-.011-.002-. & & 1 & & & & & & & & 1 & 0,00 & 1 & 6,7 \\
\hline Rhagium mordax & 87-.011-.003-. & & 14 & 3 & 3 & & & & & & 20 & 0,04 & 14 & 1,4 \\
\hline Alosterna tabacicolor & 87-.024-.001-. & & & 1 & 2 & & & & & & 3 & 0,01 & 1 & 4,7 \\
\hline Strangalia maculata & 87-.029-.007-. & & & & & 2 & & & & & 2 & 0,00 & 2 & 0,4 \\
\hline Strangalia melanura & 87-.029-.010-. & & & & & 1 & & & & & 1 & 0,00 & 1 & 0,2 \\
\hline Clytus arietis & 87-.058-.003-. & & & & 1 & & & & & & 1 & 0,00 & 1 & 0,3 \\
\hline Mesosa nebulosa & 87-.071-.002-. & & 4 & & & & & & & & 4 & 0,01 & 1 & 0,9 \\
\hline Pogonocherus hispidus & 87-.075-.002-. & & 1 & 1 & & & 1 & & & & 3 & 0,01 & 3 & 0,5 \\
\hline Leiopus nebulosus & 87-.078-.001-. & & & 22 & 26 & 1 & 3 & & & & 52 & 0,11 & 8 & 3,1 \\
\hline Saperda scalaris & 87-.082-.004-. & & & 5 & 3 & 2 & & & & & 10 & 0,02 & 6 & 1,1 \\
\hline Oulema melanopus & 88-.0061.005-. & & & & & & & & 1 & 1 & 2 & 0,00 & 2 & 1,2 \\
\hline Cryptocephalus pusillus & 88-.017-.071-. & & & & 1 & 8 & 6 & 1 & & & 16 & 0,03 & 6 & 0,9 \\
\hline Gonioctena quinquepunctata & 88-.035-.011-. & & 1 & & & 1 & & & & & 2 & 0,00 & 2 & 0,4 \\
\hline Phyllotreta undulata & 88-.049-.005-. & & & 1 & & & & & & & 1 & 0,00 & 1 & 6,9 \\
\hline Phyllotreta striolata & 88-.049-.010-. & & 1 & & & & & & & & 1 & 0,00 & 1 & 0,8 \\
\hline Chaetocnema laevicollis & 88-.066-.004-. & & 4 & & & & & & & & 4 & 0,01 & 3 & 1,4 \\
\hline Chaetocnema hortensis & 88-.066-.017-. & & & & & & & & & 1 & 1 & 0,00 & 1 & 0,2 \\
\hline Enedreutes sepicola & 90-.006-.001-. & & & & & & & 1 & & & 1 & 0,00 & 1 & 0,2 \\
\hline Choragus sheppardi & 90-.015-.002-. & & & & & & 1 & 2 & & & 3 & 0,01 & 2 & 0,4 \\
\hline Scolytus intricatus & 91-.001-.003-. & & & & 1 & & & & & & 1 & 0,00 & 1 & 1,1 \\
\hline Hylastes opacus & 91-.004-.002-. & & 1 & & & & & & & & 1 & 0,00 & 1 & 0,7 \\
\hline Hylurgops palliatus & 91-.005-.002-. & & 1 & & & & & & & & 1 & 0,00 & 1 & 1,2 \\
\hline Crypturgus hispidulus & 91-.020-.002-. & & & & 1 & & & & & & 1 & 0,00 & 1 & 0,4 \\
\hline Dryocoetes villosus & 91-.024-.002-. & & 27 & 1640 & 763 & 40 & 14 & 18 & 6 & 1 & 2509 & 5,17 & 22 & 328,7 \\
\hline Cryphalus abietis & 91-.026-.004-. & & 1 & & & & & & & & 1 & 0,00 & 1 & 0,8 \\
\hline Taphrorychus bicolor & 91-.031-.003-. & & 48 & 42 & 68 & 18 & 13 & 15 & 4 & 2 & 210 & 0,43 & 34 & 6,0 \\
\hline Pityogenes chalcographus & 91-.032-.001-. & & 1 & & 2 & & & & & & 3 & 0,01 & 3 & 1,1 \\
\hline Ips typographus & 91-.035-.004-. & & & 1 & & & & & & & 1 & 0,00 & 1 & 6,5 \\
\hline Xyleborus saxeseni & 91-.036-.004-. & & 112 & 10 & 5 & 8 & 3 & & & & 138 & 0,28 & 7 & 12,3 \\
\hline Xyloterus domesticus & 91-.038-.001-. & & 614 & 272 & 218 & 84 & 52 & 51 & 42 & 63 & 1396 & 2,88 & 34 & 39,4 \\
\hline Xyloterus signatus & 91-.038-.002-. & & 152 & 77 & 154 & 932 & 274 & 287 & 14 & 4 & 1894 & 3,90 & 36 & 43,5 \\
\hline Deporaus betulae & 923.007-.004-. & & 1 & 1 & & & & & & & 2 & 0,00 & 2 & 0,5 \\
\hline Protapion fulvipes & 925.021-.002-. & & & & & 1 & & & 1 & & 2 & 0,00 & 2 & 1,6 \\
\hline Ischnopterapion loti & 925.034-.001-. & & & & 1 & & & & & & 1 & 0,00 & 1 & 0,7 \\
\hline Otiorhynchus singularis & 93-.015-.104-. & & & & & & 1 & & & & 1 & 0,00 & 1 & 0,6 \\
\hline Caenopsis waltoni & 93-.019-.002-. & & & & & & 2 & & & & 2 & 0,00 & 1 & 1,4 \\
\hline Phyllobius calcaratus & 93-.021-.015-. & & & 1 & 1 & & & & & & 2 & 0,00 & 2 & 0,9 \\
\hline
\end{tabular}




\begin{tabular}{|c|c|c|c|c|c|c|c|c|c|c|c|c|c|c|}
\hline & & & & & $\mathbf{n g}$ & erio & & & & & & & & \\
\hline 占 & 竞 & 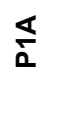 & $\frac{m}{\Sigma}$ & $\mathfrak{\Sigma}$ & $\dddot{q}$ & a & $\stackrel{n}{\alpha}$ & 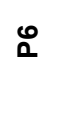 & $\hat{a}$ & $\stackrel{\infty}{a}$ & $\begin{array}{l}\bar{\Phi} \\
\Phi \\
\Phi \\
\dot{\Xi} \\
\text { த் }\end{array}$ & 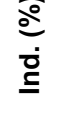 & $\begin{array}{l}\frac{D}{d} \\
\frac{0}{\vec{\sigma}} \\
\frac{d}{L}\end{array}$ & 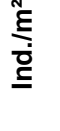 \\
\hline Phyllobius argentatus & 93-.021-.019-. & & 3 & 8 & 3 & 3 & & & & & 17 & 0,04 & 14 & $\overline{0,7}$ \\
\hline Polydrusus undatus & 93-.027-.016-. & & 1 & 2 & 2 & & & & & & 5 & 0,01 & 5 & 0,3 \\
\hline Barypeithes pellucidus & 93-.037-.011-. & & & & 1 & & & & & & 1 & 0,00 & 1 & 0,7 \\
\hline Strophosoma melanogrammum & 93-.040-.002-. & & 5 & 8 & 1 & 4 & 6 & 1 & 8 & 30 & 63 & 0,13 & 29 & 1,6 \\
\hline Strophosoma capitatum & 93-.040-.003-. & & & & & & & & 1 & 1 & 2 & 0,00 & 2 & 0,3 \\
\hline Sitona lepidus & 93-.044-.016-. & & & & & & & & & 1 & 1 & 0,00 & 1 & 5,9 \\
\hline Tychius picirostris & 93-.104-.019-. & & 1 & & & & & & & & 1 & 0,00 & 1 & 4,8 \\
\hline Curculio pyrrhoceras & 93-.110-.011-. & & 2 & 2 & 1 & 2 & & & & & 7 & 0,01 & 6 & 0,5 \\
\hline Coeliodes dryados & 93-.157-.003-. & & 1 & & & & & & & & 1 & 0,00 & 1 & 7,4 \\
\hline Coeliodes erythroleucos & 93-.157-.008-. & & 2 & & & & & & & & 2 & 0,00 & 2 & 0,4 \\
\hline Ceutorhynchus floralis & 93-.163-.0601. & & 1 & 2 & & 1 & & & & & 4 & 0,01 & 3 & 1,3 \\
\hline Rhynchaenus quercus & 93-.180-.005-. & & 9 & & & & & & & & 9 & 0,02 & 7 & 2,9 \\
\hline Rhynchaenus fagi & 93-.180-.013-. & 2 & 106 & 11 & 1 & 6 & 3 & 2 & 5 & 6 & 142 & 0,29 & 73 & 1,7 \\
\hline
\end{tabular}




\section{Anhang 5: Auftreten der Arten in STE- und EFs-Proben an Eiche und Buche}

\begin{tabular}{|c|c|c|c|c|c|c|c|c|c|c|c|c|c|c|c|c|c|c|c|c|c|}
\hline \multirow{2}{*}{ EDV } & \multirow{2}{*}{ Art } & \multirow{2}{*}{\multicolumn{4}{|c|}{$\begin{array}{c}\text { Frequenz } \\
\text { STE STE EFs EFs }\end{array}$}} & \multirow{2}{*}{\multicolumn{4}{|c|}{$\begin{array}{c}\text { Frequenz (\%) } \\
\text { STE STE EFs EFs }\end{array}$}} & \multirow{2}{*}{$\begin{array}{c}\text { STE } \\
\text { Ei }\end{array}$} & \multicolumn{3}{|c|}{ Individuen } & \multirow{2}{*}{\multicolumn{4}{|c|}{$\begin{array}{c}\text { Individuen (\%) } \\
\text { STE STE EFs EFs }\end{array}$}} & \multicolumn{4}{|c|}{ spez. Dichte (Ind./m2) } \\
\hline & & & & & & & & & & & STE & EFs & EFs & & & & & \multicolumn{4}{|c|}{ STE STE EFs EFs } \\
\hline 01-.004-.001-. & Carabus coriaceus & 1 & 3 & 1 & & 2,1 & 5,9 & 2,0 & & 1 & 3 & 1 & & 0,0 & 0,0 & 0,0 & & 0,8 & 0,8 & 6,3 & \\
\hline 01-.004-.012-. & Carabus granulatus & 1 & 2 & & & 2,1 & 3,9 & & & 1 & 2 & & & 0,0 & 0,0 & & & 1,1 & 0,6 & & \\
\hline 01-.006-.002-. & Leistus rufomarginatus & 9 & & & & 18,8 & & & & 15 & & & & 0,2 & & & & 1,4 & & & \\
\hline 01-.028-.001-. & Tachyta nana & & 1 & & & & 2,0 & & & & 1 & & & & 0,0 & & & & 0,8 & & \\
\hline 01-.051-.024-. & Pterostichus oblongopunctatus & 27 & 27 & & 1 & 56,3 & 52,9 & & 1,1 & 83 & 54 & & 1 & 1,0 & 0,4 & & 0,0 & 2,7 & 1,4 & & 7,7 \\
\hline 01-.051-.026-. & Pterostichus niger & 26 & 24 & & 2 & 54,2 & 47,1 & & 2,2 & 62 & 104 & & 4 & 0,7 & 0,8 & & 0,0 & 2,0 & 3,0 & & 12,9 \\
\hline 01-.053-.002-. & Abax parallelepipedus & & 3 & & & & 5,9 & & & & 4 & & & & 0,0 & & & & 1,0 & & \\
\hline 01-.062-.009-. & Agonum muelleri & & 1 & & & & 2,0 & & & & 1 & & & & 0,0 & & & & 0,7 & & \\
\hline 01-.079-.012-. & Dromius quadrimaculatus & & & 2 & & & & 4,0 & & & & 2 & & & & 0,0 & & & & 7,3 & \\
\hline 07-.001-.000-. & Hydraena spec. & & 1 & & & & 2,0 & & & & 1 & & & & 0,0 & & & & 0,7 & & \\
\hline 09-.002-.004-. & Sphaeridium lunatum & & & & 1 & & & & 1,1 & & & & 1 & & & & 0,0 & & & & 6,1 \\
\hline 09-.003-.008-. & Cercyon melanocephalus & 1 & & & & 2,1 & & & & 1 & & & & 0,0 & & & & 0,8 & & & \\
\hline 09-.003-.013-. & Cercyon unipunctatus & & 1 & & & & 2,0 & & & & 1 & & & & 0,0 & & & & 0,6 & & \\
\hline 09-.004-.001-. & Megasternum obscurum & 1 & 1 & & 1 & 2,1 & 2,0 & & 1,1 & 1 & 1 & & 1 & 0,0 & 0,0 & & 0,0 & 1,4 & 0,7 & & 4,5 \\
\hline 10-.002-.004-. & Plegaderus dissectus & 2 & 24 & 1 & 42 & 4,2 & 47,1 & 2,0 & 46,2 & 2 & 127 & 1 & 94 & 0,0 & 0,9 & 0,0 & 0,7 & 0,8 & 3,5 & 8,0 & 12,5 \\
\hline 10-.005-.001-. & Abraeus granulum & 2 & 13 & 1 & 26 & 4,2 & 25,5 & 2,02 & 28,6 & 3 & 26 & 1 & 55 & 0,0 & 0,2 & 0,0 & 0,4 & 1,1 & 1,3 & 6,9 & 12,4 \\
\hline 10-.009-.001-. & Gnathoncus rotundatus & & & 1 & & & & 2,0 & & & & 1 & & & & 0,0 & & & & 6,9 & \\
\hline 10-.009-.002-. & Gnathoncus nannetensis & & & & 1 & & & & 1,1 & & & & 1 & & & & 0,0 & & & & 5,6 \\
\hline 14-.005-.003-. & Nargus wilkini & 2 & & & & 4,2 & & & & 3 & & & & 0,0 & & & & 1,8 & & & \\
\hline 14-.011-.012-. & Catops nigrita & 1 & & & & 2,1 & & & & 1 & & & & 0,0 & & & & 0,7 & & & \\
\hline 14-.011-.017-. & Catops fuliginosus & & 1 & & & & 2,0 & & & & 4 & & & & 0,0 & & & & 3,1 & & \\
\hline 14-.011-.020-. & Catops picipes & 12 & 5 & 7 & 2 & 25,0 & 9,8 & 14,0 & 2,2 & 22 & 12 & 8 & 10 & 0,3 & 0,1 & 0,2 & 0,1 & 1,6 & 1,7 & 8,4 & 32,3 \\
\hline 16-.007-.001-. & Anisotoma humeralis & 5 & 1 & 2 & & 10,4 & 2,0 & 4,0 & & 14 & 1 & 2 & & 0,2 & 0,0 & 0,0 & & 2,3 & 0,6 & 6,8 & \\
\hline 16-.007-.005-. & Anisotoma orbicularis & 2 & 5 & & & 4,2 & 9,8 & & & 2 & 12 & & & 0,0 & 0,1 & & & 1,0 & 1,9 & & \\
\hline 16-.009-.001-. & Amphicyllis globus & & 1 & & & & 2,0 & & & & 1 & & & & 0,0 & & & & 0,7 & & \\
\hline 16-.011-.003-. & Agathidium varians & 2 & 1 & & & 4,2 & 2,0 & & & 5 & 1 & & & 0,1 & 0,0 & & & 2,1 & 0,8 & & \\
\hline 16-.011-.007-. & Agathidium rotundatum & 6 & 3 & & & 12,5 & 5,9 & & & 6 & 6 & & & 0,1 & 0,0 & & & 1,0 & 1,2 & & \\
\hline 16-.011-.008-. & Agathidium confusum & & 3 & & & & 5,9 & & & & 5 & & & & 0,0 & & & & 1,1 & & \\
\hline 16-.011-.010-. & Agathidium nigrinum & & 1 & & & & 2,0 & & & & 1 & & & & 0,0 & & & & 0,7 & & \\
\hline 16-.011-.013-. & Agathidium nigripenne & 2 & 10 & 1 & 3 & 4,2 & 19,6 & 2,0 & 3,3 & 2 & 66 & 2 & 6 & 0,0 & 0,5 & 0,0 & 0,0 & 1,0 & 4,1 & 12,9 & 10,7 \\
\hline 16-.011-.015-. & Agathidium seminulum & 21 & 9 & & 5 & 43,8 & 17,6 & & 5,5 & 32 & 15 & & 5 & 0,4 & 0,1 & & 0,0 & 1,3 & 1,2 & & 6,1 \\
\hline 16-.011-.018-. & Agathidium badium & 6 & 17 & & 2 & 12,5 & 33,3 & & 2,2 & 8 & 33 & & 2 & 0,1 & 0,2 & & 0,0 & 1,2 & 1,3 & & 8,1 \\
\hline 18-.001-.001-. & Euthiconus conicicollis & 2 & 3 & & 1 & 4,2 & 5,9 & & 1,1 & 2 & 3 & & 1 & 0,0 & 0,0 & & 0,0 & 0,9 & 0,7 & & 6,3 \\
\hline 18-.002-.004-. & Euthia linearis & 1 & & & & 2,1 & & & & 1 & & & & 0,0 & & & & 0,9 & & & \\
\hline
\end{tabular}




\begin{tabular}{|c|c|c|c|c|c|c|c|c|c|c|c|c|c|c|c|c|c|c|c|c|}
\hline \multirow{2}{*}{ EDV } & \multirow{2}{*}{ Art } & \multirow{2}{*}{\multicolumn{3}{|c|}{$\begin{array}{c}\text { Frequenz } \\
\text { STE STE EFs EFs }\end{array}$}} & \multirow{2}{*}{\multicolumn{4}{|c|}{$\begin{array}{c}\text { Frequenz (\%) } \\
\text { STE STE EF }\end{array}$}} & \multicolumn{4}{|c|}{ Individuen } & \multicolumn{4}{|c|}{ Individuen (\%) } & \multicolumn{4}{|c|}{ spez. Dichte (Ind./m2) } \\
\hline & & & & & & & & & STE & STE & EFs & EFs & STE & STE & EFs I & EFs & STE & STE & EFs & EFs \\
\hline 18-.004-.003-. & Cephennium thoracicum & 5 & 2 & & 10,4 & 3,9 & & & 16 & 3 & & & 0,2 & 0,0 & & & 2,7 & 1,0 & & \\
\hline 18-.006-.003-. & Scydmoraphes helvolus & 1 & 2 & & 2,1 & 3,9 & & & 1 & 2 & & & 0,0 & 0,0 & & & 0,8 & 0,7 & & \\
\hline 18-.006-.004-. & Scydmoraphes minutus & & & 27 & & & 4,0 & 7,7 & & & 2 & 13 & & & 0,0 & 0,1 & & & 7,1 & 9,9 \\
\hline 18-.007-.008-. & Stenichnus collaris & 32 & 37 & $\begin{array}{ll}6 & 33 \\
\end{array}$ & 66,7 & 72,5 & 12,03 & 36,3 & 140 & 278 & 9 & 79 & 1,6 & 2,0 & 0,2 & 0,6 & 3,8 & 5,2 & 10,5 & 14,3 \\
\hline 18-.008-.001-. & Microscydmus nanus & 1 & 9 & 23 & 2,1 & 17,6 & & 25,3 & 1 & 9 & & 30 & 0,0 & 0,1 & & 0,2 & 0,7 & 0,7 & & 7,8 \\
\hline 18-.008-.002-. & Microscydmus minimus & & 2 & $1 \quad 17$ & & 3,9 & 2,0 & 18,7 & & 2 & 1 & 22 & & 0,0 & 0,0 & 0,2 & & 0,9 & 6,9 & 7,7 \\
\hline 21-.002-.001-. & Ptenidium gressneri & & & 1 & & & & 1,1 & & & & 1 & & & & 0,0 & & & & 5,4 \\
\hline 21-.002-.004-. & Ptenidium intermedium & & 2 & 1 & & 3,9 & 2,0 & & & 2 & 1 & & & 0,0 & 0,0 & & & 0,8 & 6,7 & \\
\hline 21-.013-.001-. & Pteryx suturalis & 26 & 42 & $3 \quad 11$ & 54,2 & 82,4 & 6,0 & 12,1 & 240 & 421 & 3 & 12 & 2,8 & 3,1 & 0,1 & 0,1 & 7,9 & 6,9 & 6,6 & 6,6 \\
\hline 21-.019-.012-. & Acrotrichis insularis & 2 & & & 4,2 & & & & 2 & & & & 0,0 & & & & 0,9 & & & \\
\hline 21-.019-.015-. & Acrotrichis intermedia & 8 & 13 & & 16,7 & 25,5 & & & 9 & 18 & & & 0,1 & 0,1 & & & 0,9 & 0,9 & & \\
\hline 22-.002-.001-. & Scaphidium quadrimaculatum & 1 & 4 & & 2,1 & 7,8 & & & 1 & 5 & & & 0,0 & 0,0 & & & 0,8 & 0,9 & & \\
\hline 22-.003-.001-. & Scaphisoma agaricinum & & 7 & & & 13,7 & & & & 9 & & & & 0,1 & & & & 0,9 & & \\
\hline 22-.003-.007-. & Scaphisoma balcanicum & 5 & 1 & & 10,4 & 2,0 & & & 20 & 1 & & & 0,2 & 0,0 & & & 4,0 & 0,8 & & \\
\hline 23-.005-.001-. & Phloeocharis subtilissima & 25 & 40 & 9 & 52,1 & 78,4 & 10,0 & 9,9 & 68 & 114 & 6 & 12 & 0,8 & 0,8 & 0,1 & 0,1 & 2,3 & 1,9 & 9,0 & 8,0 \\
\hline 23-.009-.004-. & Proteinus brachypterus & 2 & 4 & 1 & 4,2 & 7,8 & & 1,1 & 4 & 5 & & 1 & 0,0 & 0,0 & & 0,0 & 1,5 & 0,9 & & 5,0 \\
\hline 23-.014-.001-. & Phyllodrepa melanocephala & 1 & & & 2,1 & & & & 3 & & & & 0,0 & & & & 3,4 & & & \\
\hline 23-.014-.012-. & Phyllodrepa ioptera & 15 & 13 & $10 \quad 10$ & 31,3 & 25,5 & 20,0 & 11,0 & 51 & 16 & 22 & 16 & 0,6 & 0,1 & 0,5 & 0,1 & 2,6 & 0,8 & 16,1 & 9,3 \\
\hline 23-.025-.002-. & Lathrimaeum atrocephalum & 1 & 1 & & 2,1 & 2,0 & & & 2 & 1 & & & 0,0 & 0,0 & & & 1,4 & 0,7 & & \\
\hline 23-.025-.003-. & Lathrimaeum unicolor & 4 & 3 & & 8,3 & 5,9 & & & 4 & 3 & & & 0,0 & 0,0 & & & 0,8 & 0,7 & & \\
\hline 23-.026-.001-. & Olophrum piceum & & & 1 & & & & 1,1 & & & & 1 & & & & 0,0 & & & & 5,6 \\
\hline 23-.040-.001-. & Syntomium aeneum & 3 & & & 6,3 & & & & 5 & & & & 0,1 & & & & 1,4 & & & \\
\hline 23-.042-.001-. & Coprophilus striatulus & & 1 & & & 2,0 & & & & 1 & & & & 0,0 & & & & 0,6 & & \\
\hline 23-.046-.017-. & Carpelimus corticinus & 1 & 1 & & 2,1 & 2,0 & & & 1 & 1 & & & 0,0 & 0,0 & & & 0,7 & 0,6 & & \\
\hline 23-.046-.030-. & Carpelimus gracilis & & 1 & & & 2,0 & & & & 1 & & & & 0,0 & & & & 0,7 & & \\
\hline 23-.0481.022-. & Anotylus tetracarinatus & 1 & & & 2,1 & & & & 2 & & & & 0,0 & & & & 1,4 & & & \\
\hline 23-.055-.006-. & Stenus fossulatus & & 2 & & & 3,9 & & & & 3 & & & & 0,0 & & & & 1,1 & & \\
\hline 23-.061-.003-. & Rugilus rufipes & & 1 & & & 2,0 & & & & 1 & & & & 0,0 & & & & 0,9 & & \\
\hline 23-.068-.021-. & Lathrobium fulvipenne & 1 & 4 & & 2,1 & 7,8 & & & 1 & 4 & & & 0,0 & 0,0 & & & 1,1 & 0,7 & & \\
\hline 23-.068-.023-. & Lathrobium brunnipes & 2 & 3 & & 4,2 & 5,9 & & & 2 & 4 & & & 0,0 & 0,0 & & & 0,7 & 1,1 & & \\
\hline 23-.078-.001-. & Nudobius lentus & & 2 & 1 & & 3,9 & & 1,1 & & 6 & & 1 & & 0,0 & & 0,0 & & 1,9 & & 5,0 \\
\hline 23-.080-.010-. & Xantholinus linearis & & 1 & & & 2,0 & & & & 1 & & & & 0,0 & & & & 0,8 & & \\
\hline 23-.080-.015-. & Xantholinus longiventris & 1 & & & 2,1 & & & & 1 & & & & 0,0 & & & & 0,9 & & & \\
\hline 23-.081-.001-. & Atrecus affinis & 5 & 5 & 1 & 10,4 & 9,8 & & 1,1 & 5 & 5 & & 1 & 0,1 & 0,0 & & 0,0 & 0,9 & 0,7 & & 6,7 \\
\hline 23-.082-.001-. & Othius punctulatus & 1 & 2 & & 2,1 & 3,9 & & & 2 & 2 & & & 0,0 & 0,0 & & & 1,5 & 0,6 & & \\
\hline 23-.082-.005-. & Othius myrmecophilus & 8 & 3 & & 16,7 & 5,9 & & & 13 & 5 & & & 0,2 & 0,0 & & & 1,5 & 1,1 & & \\
\hline 23-.088-.023-. & Philonthus cognatus & & 1 & 1 & & 2,0 & & 1,1 & & 1 & & 1 & & 0,0 & & 0,0 & & 0,9 & & 5,0 \\
\hline 23-.088-.026-. & Philonthus succicola & & 1 & & & 2,0 & & & & 1 & & & & 0,0 & & & & 0,6 & & \\
\hline
\end{tabular}




\begin{tabular}{|c|c|c|c|c|c|c|c|c|c|c|c|c|c|c|c|c|c|c|c|c|}
\hline \multirow{2}{*}{ EDV } & \multirow{2}{*}{ Art } & \multirow{2}{*}{\multicolumn{3}{|c|}{ 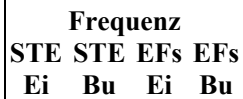 }} & \multirow{2}{*}{\multicolumn{4}{|c|}{$\begin{array}{c}\text { Frequenz }(\%) \\
\text { STE STE EFs EFs }\end{array}$}} & \multirow{2}{*}{$\begin{array}{c}\text { STE } \\
\text { Ei } \\
\end{array}$} & \multicolumn{3}{|c|}{ Individuen } & \multicolumn{4}{|c|}{ Individuen (\%) } & \multicolumn{4}{|c|}{ spez. Dichte (Ind./m2) } \\
\hline & & & & & & & & & & STE & EFs & EFs & STE & STE & EFs & EFs & STE & STE & EFs & EFs \\
\hline 23-.088-.044-. & Philonthus varians & & 1 & & & 2,0 & & & & 2 & & & & 0,0 & & & & 1,5 & & \\
\hline 23-.088-.073-. & Philonthus marginatus & 1 & & & 2,1 & & & & 1 & & & & 0,0 & & & & 0,7 & & & \\
\hline 23-.090-.009-. & Gabrius splendidulus & 14 & 34 & 1 & 29,2 & 66,7 & 2,0 & 5,5 & 22 & 170 & 1 & 5 & 0,3 & 1,2 & 0,0 & 0,0 & 1,4 & 3,3 & 6,3 & 5,8 \\
\hline 23-.104-.005-. & Quedius lateralis & 3 & & 1 & 6,3 & & 2,0 & & 3 & & 1 & & 0,0 & & 0,0 & & 0,9 & & 8,0 & \\
\hline 23-.104-.013-. & Quedius cruentus & 1 & & & 2,1 & & & & 1 & & & & 0,0 & & & & 1,1 & & & \\
\hline 23-.104-.014-. & Quedius brevicornis & & & 2 & & & & 2,2 & & & & 2 & & & & 0,0 & & & & 6,7 \\
\hline 23-.104-.016-. & Quedius mesomelinus & 1 & & 5 & 2,1 & & & 5,5 & 1 & & & 7 & 0,0 & & & 0,1 & 0,7 & & & 7,8 \\
\hline 23-.104-.020-. & Quedius scitus & 2 & 2 & 4 & 4,2 & 3,9 & & 4,4 & 2 & 2 & & 7 & 0,0 & 0,0 & & 0,1 & 0,7 & 0,6 & & 10,0 \\
\hline 23-.112-.001-. & Bolitobius cingulata & 1 & 2 & & 2,1 & 3,9 & & & 1 & 3 & & & 0,0 & 0,0 & & & 0,8 & 1,3 & & \\
\hline 23-.112-.003-. & Bolitobius inclinans & 2 & 1 & & 4,2 & 2,0 & & & 2 & 1 & & & 0,0 & 0,0 & & & 0,8 & 1,1 & & \\
\hline 23-.113-.001-. & Sepedophilus littoreus & 4 & 7 & & 8,3 & 13,7 & & & 4 & 10 & & & 0,0 & 0,1 & & & 0,8 & 1,0 & & \\
\hline 23-.113-.002-. & Sepedophilus testaceus & 17 & 40 & 16 & 35,4 & 78,4 & & 17,6 & 47 & 273 & & 46 & 0,5 & 2,0 & & 0,3 & 2,6 & 4,7 & & 17,3 \\
\hline 23-.113-.005-. & Sepedophilus bipunctatus & & & 5 & & & & 5,5 & & & & 13 & & & & 0,1 & & & & 15,7 \\
\hline 23-.114-.008-. & Tachyporus chrysomelinus & 1 & 1 & & 2,1 & 2,0 & & & 1 & 1 & & & 0,0 & 0,0 & & & 0,8 & 0,6 & & \\
\hline $23-.114-.012-$. & Tachyporus ruficollis & 1 & 1 & 1 & 2,1 & 2,0 & & 1,1 & 1 & 1 & & 1 & 0,0 & 0,0 & & 0,0 & 0,7 & 0,8 & & 6,1 \\
\hline $23-.1261 .002-$. & Holobus apicatus & & 1 & & & 2,0 & & & & 7 & & & & 0,1 & & & & 4,6 & & \\
\hline $23-.1262 .013-$. & Cypha hanseni & 1 & 1 & & 2,1 & 2,0 & & & 1 & 1 & & & 0,0 & 0,0 & & & 0,8 & 0,6 & & \\
\hline 23-.130-.009-. & Gyrophaena gentilis & & & 1 & & & & 1,1 & & & & 1 & & & & 0,0 & & & & 5,6 \\
\hline 23-.134-.001-. & Anomognathus cuspidatus & 7 & 15 & 1 & 14,6 & 29,4 & 2,0 & 6,6 & 8 & 55 & 1 & 13 & 0,1 & 0,4 & 0,0 & 0,1 & 1,0 & 2,4 & 8,7 & 12,7 \\
\hline 23-.141-.001-. & Leptusa pulchella & 26 & 25 & 3 & 54,2 & 49,0 & 6,0 & 3,3 & 70 & 69 & 3 & 4 & 0,8 & 0,5 & 0,1 & 0,0 & 2,4 & 1,8 & 6,4 & 7,9 \\
\hline 23-.141-.004-. & Leptusa fumida & 27 & 41 & $12 \quad 41$ & 56,3 & 80,4 & 24,0 & 45,1 & 74 & 207 & 27 & 174 & 0,9 & 1,5 & 0,6 & 1,3 & 2,4 & 3,4 & 15,2 & 23,9 \\
\hline $23-.141-.006-$. & Leptusa ruficollis & 4 & 3 & $\begin{array}{ll}12 & 17 \\
\end{array}$ & 8,3 & 5,9 & 24,0 & 18,7 & 8 & 5 & 22 & 31 & 0,1 & 0,0 & 0,5 & 0,2 & 1,9 & 1,1 & 13,1 & 10,6 \\
\hline 23-.142-.001-. & Euryusa castanoptera & 6 & 20 & 1 & 12,5 & 39,2 & 2,0 & 6,6 & 24 & 121 & 1 & 12 & 0,3 & 0,9 & 0,0 & 0,1 & 3,1 & 3,9 & 6,3 & 10,5 \\
\hline 23-.147-.001-. & Bolitochara obliqua & 7 & 26 & 1 & 14,6 & 51,0 & & 1,1 & 55 & 101 & & 1 & 0,6 & 0,7 & & 0,0 & 8,1 & 2,6 & & 6,3 \\
\hline 23-.168-.001-. & Amischa analis & 11 & 10 & 1 & 22,9 & 19,6 & 2,0 & & 14 & 11 & 1 & & 0,2 & 0,1 & 0,0 & & 1,1 & 0,8 & 6,3 & \\
\hline 23-.168-.004-. & Amischa soror & 1 & 2 & 2 & 2,1 & 3,9 & & 2,2 & 1 & 2 & & 2 & 0,0 & 0,0 & & 0,0 & 0,9 & 0,5 & & 5,6 \\
\hline 23-.168-.007-. & Amischa decipiens & & 3 & & & 5,9 & & & & 4 & & & & 0,0 & & & & 1,1 & & \\
\hline $23-.180-.003-$. & Geostiba circellaris & 5 & & & 10,4 & & & & 6 & & & & 0,1 & & & & 1,4 & & & \\
\hline $23-.182-.002-$. & Dinaraea aequata & 5 & 12 & & 10,4 & 23,5 & & & 18 & 16 & & & 0,2 & 0,1 & & & 3,6 & 0,9 & & \\
\hline 23-.182-.003-. & Dinaraea linearis & 4 & 13 & & 8,3 & 25,5 & & & 9 & 28 & & & 0,1 & 0,2 & & & 2,4 & 1,5 & & \\
\hline 23-.184-.001-. & Dadobia immersa & & 4 & & & 7,8 & & & & 5 & & & & 0,0 & & & & 0,7 & & \\
\hline 23-.188-.004-. & Atheta elongatula & 2 & & & 4,2 & & & & 2 & & & & 0,0 & & & & 1,0 & & & \\
\hline 23-.188-.024-. & Atheta parca & & 1 & & & 2,0 & & & & 1 & & & & 0,0 & & & & 0,9 & & \\
\hline 23-.188-.045-. & Atheta nigricornis & 2 & & & 4,2 & & & & 4 & & & & 0,0 & & & & 1,6 & & & \\
\hline 23-.188-.046-. & Atheta harwoodi & 2 & 6 & & 4,2 & 11,8 & & & 3 & 12 & & & 0,0 & 0,1 & & & 1,4 & 1,3 & & \\
\hline 23-.188-.109-. & Atheta sodalis & 4 & 7 & & 8,3 & 13,7 & & & 5 & 14 & & & 0,1 & 0,1 & & & 1,0 & 1,5 & & \\
\hline 23-.188-.111-. & Atheta pallidicornis & 1 & 7 & & 2,1 & 13,7 & & & 1 & 11 & & & 0,0 & 0,1 & & & 1,2 & 1,0 & & \\
\hline 23-.188-.126-. & Atheta picipes & & 1 & & & 2,0 & & & & 1 & & & & 0,0 & & & & 1,1 & & \\
\hline
\end{tabular}




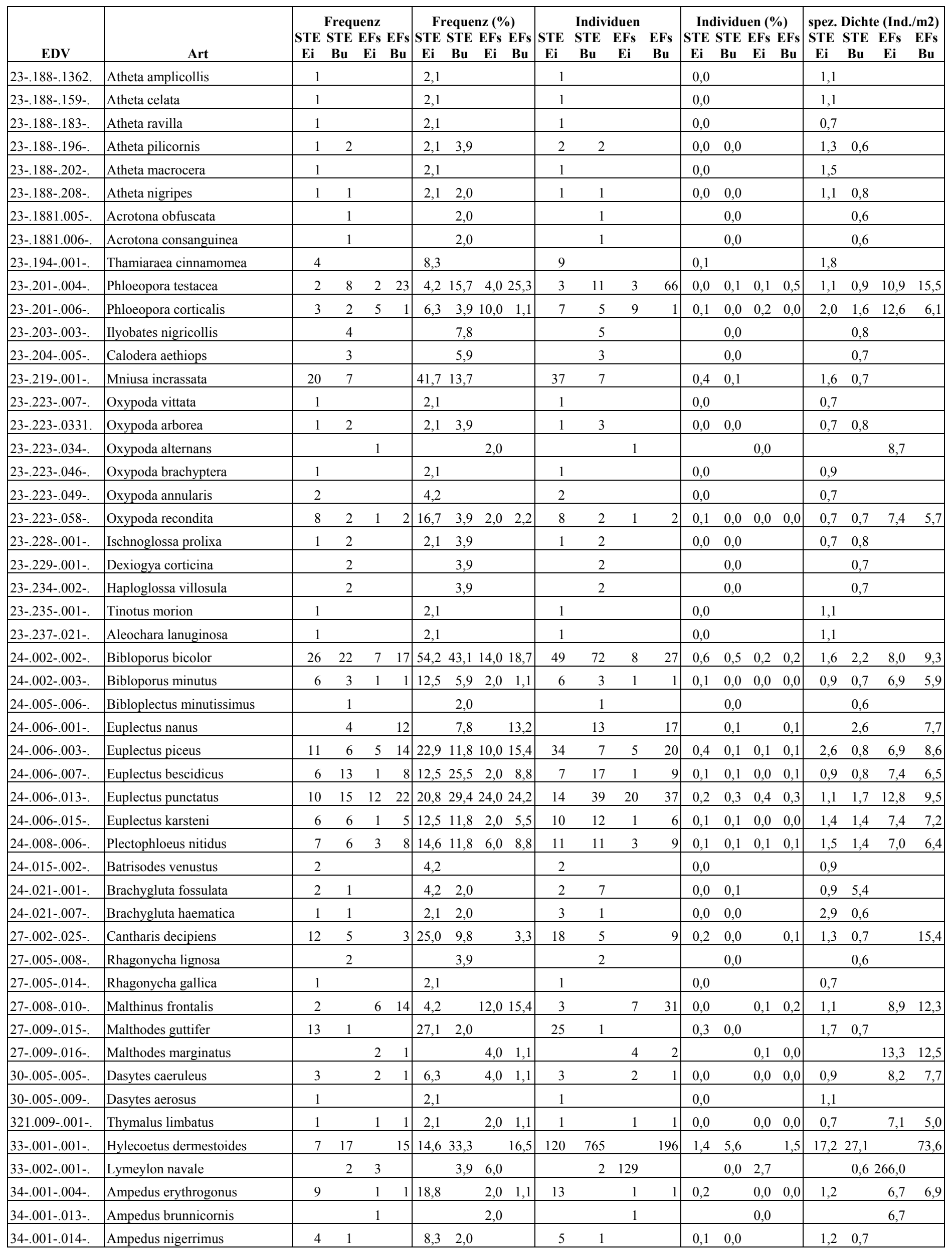




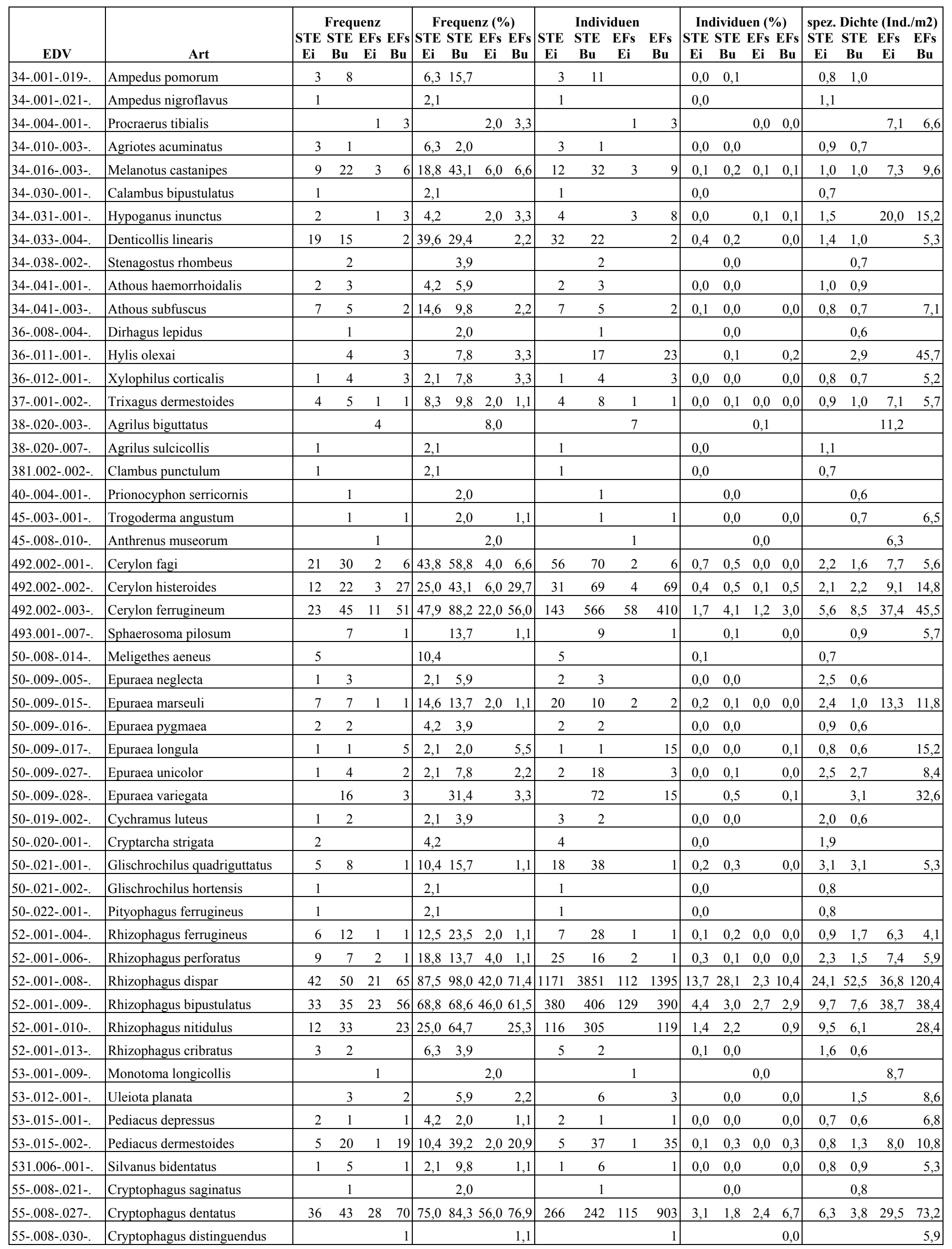




\begin{tabular}{|c|c|c|c|c|c|c|c|c|c|c|c|c|c|c|c|c|c|c|c|c|c|}
\hline \multirow{2}{*}{ EDV } & \multirow{2}{*}{ Art } & \multirow{2}{*}{\multicolumn{4}{|c|}{$\begin{array}{c}\text { Frequenz } \\
\text { STE STE EFs EFs }\end{array}$}} & \multirow{2}{*}{\multicolumn{4}{|c|}{$\begin{array}{c}\text { Frequenz (\%) } \\
\text { STE STE EFs EFs }\end{array}$}} & \multirow{2}{*}{$\begin{array}{c}\text { STE } \\
\text { Ei }\end{array}$} & \multicolumn{3}{|c|}{ Individuen } & \multirow{2}{*}{\multicolumn{4}{|c|}{$\begin{array}{c}\text { Individuen (\%) } \\
\text { STE STE EFs EFs }\end{array}$}} & \multicolumn{4}{|c|}{ spez. Dichte (Ind./m2) } \\
\hline & & & & & & & & & & & STE & EFs & EFs & & & & & \multicolumn{4}{|c|}{ STE STE EFs EFs } \\
\hline 55-.008-.034-. & Cryptophagus scanicus & 4 & & 1 & & 8,3 & & 2,0 & & 10 & & 1 & & 0,1 & & 0,0 & & 2,2 & & 8,7 & \\
\hline 55-.014-.011-. & Atomaria pusilla & 1 & & & & 2,1 & & & & 1 & & & & 0,0 & & & & 0,7 & & & \\
\hline 55-.014-.014-. & Atomaria fuscata & 1 & 1 & & & 2,1 & 2,0 & & & 1 & 1 & & & 0,0 & 0,0 & & & 1,1 & 0,8 & & \\
\hline \begin{tabular}{|l} 
55-.014-.038-. \\
\end{tabular} & Atomaria umbrina & & 1 & & & & 2,0 & & & & 3 & & & & 0,0 & & & & 2,4 & & \\
\hline 55-.014-.045-. & Atomaria nigrirostris & 1 & 9 & & & 2,1 & 17,6 & & & 1 & 11 & & & 0,0 & 0,1 & & & 0,8 & 0,8 & & \\
\hline 55-.014-.051-. & Atomaria pulchra & 4 & 7 & & & 8,3 & 13,7 & & & 6 & 7 & & & 0,1 & 0,1 & & & 1,2 & 0,7 & & \\
\hline 55-.014-.054-. & Atomaria bella & 17 & 1 & & & 35,4 & 2,0 & & & 27 & 1 & & & 0,3 & 0,0 & & & 1,2 & 0,7 & & \\
\hline 561.004-.001-. & Cryptolestes duplicatus & & 1 & & & & 2,0 & & & & 1 & & & & 0,0 & & & & 0,7 & & \\
\hline 58-.004-.012-. & Enicmus rugosus & 1 & 14 & 6 & 4 & 2,1 & 27,5 & 12,0 & 4,4 & 1 & 51 & 7 & 8 & 0,0 & 0,4 & 0,1 & 0,1 & 1,1 & 2,4 & 8,0 & 12,3 \\
\hline \begin{tabular}{|l} 
58-.004-.013-. \\
\end{tabular} & Enicmus testaceus & 1 & 2 & 3 & 5 & 2,1 & 3,9 & 6,0 & 5,5 & 1 & 3 & 39 & 8 & 0,0 & 0,0 & 0,8 & 0,1 & 0,8 & 1,0 & 85,7 & 9,4 \\
\hline 58-.004-.014-. & Enicmus transversus & 3 & 1 & 2 & 1 & 6,3 & 2,0 & 4,0 & 1,1 & 4 & 1 & 2 & 1 & 0,0 & 0,0 & 0,0 & 0,0 & 1,0 & 0,7 & 7,5 & 5,4 \\
\hline 58-.0041.001-. & Dienerella elongata & 15 & 16 & 17 & 44 & 31,3 & 31,4 & 34,0 & 48,4 & 109 & 54 & 89 & 247 & 1,3 & 0,4 & 1,8 & 1,8 & 5,6 & 2,3 & 37,8 & 31,9 \\
\hline 58-.0041.0021. & Dienerella clathrata & 1 & 2 & & 2 & 2,1 & 3,9 & & 2,2 & 5 & 6 & & 4 & 0,1 & 0,0 & & 0,0 & 3,7 & 1,9 & & 11,0 \\
\hline 58-.0061.001-. & Stephostethus lardarius & 1 & & & & 2,1 & & & & 1 & & & & 0,0 & & & & 1,1 & & & \\
\hline 58-.0061.002-. & Stephostethus angusticollis & & & & 3 & & & & 3,3 & & & & 3 & & & & 0,0 & & & & 5,5 \\
\hline \begin{tabular}{|l}
$58-.0063 .003-$. \\
\end{tabular} & Aridius nodifer & 29 & 35 & 5 & 13 & 60,4 & 68,6 & 10,0 & 14,3 & 247 & 272 & 15 & 31 & 2,9 & 2,0 & 0,3 & 0,2 & 7,1 & 5,3 & 23,4 & 14,0 \\
\hline 58-.007-.016-. & Corticaria linearis & 5 & 2 & 1 & & 10,4 & 3,9 & 2,0 & & 10 & 4 & 1 & & 0,1 & 0,0 & 0,0 & & 1,8 & 1,4 & 7,4 & \\
\hline 58-.007-.018-. & Corticaria longicollis & 41 & 45 & 46 & 78 & 85,4 & 88,2 & 92,08 & 85,7 & 582 & 295 & 521 & 2649 & 6,8 & 2,2 & 10,81 & 19,7 & 12,4 & 4,5 & 82,4 & 197,2 \\
\hline 59-.003-.001-. & Litargus connexus & 1 & 1 & 1 & 2 & 2,1 & 2,0 & 2,0 & 2,2 & 1 & 1 & 4 & 5 & 0,0 & 0,0 & 0,1 & 0,0 & 1,1 & 0,6 & 34,8 & 14,7 \\
\hline 59-.004-.001-. & Mycetophagus quadripustulatus & & & & 1 & & & & 1,1 & & & & 1 & & & & 0,0 & & & & 5,1 \\
\hline 59-.004-.003-. & Mycetophagus piceus & & & 1 & & & & 2,0 & & & & 6 & & & & 0,1 & & & & 40,0 & \\
\hline 59-.004-.006-. & Mycetophagus atomarius & 1 & 6 & & 10 & 2,1 & 11,8 & & 11,0 & 1 & 16 & & 51 & 0,0 & 0,1 & & 0,4 & 1,1 & 2,0 & & 29,6 \\
\hline 59-.004-.010-. & Mycetophagus populi & & & & 1 & & & & 1,1 & & & & 1 & & & & 0,0 & & & & 5,9 \\
\hline 60-.014-.001-. & Cicones variegatus & & 4 & & 13 & & 7,8 & & 14,3 & & 8 & & 146 & & 0,1 & & 1,1 & & 1,4 & & 64,9 \\
\hline 60-.016-.001-. & Bitoma crenata & & 2 & & & & 3,9 & & & & 2 & & & & 0,0 & & & & 0,6 & & \\
\hline 60-.020-.001-. & Teredus cylindricus & 17 & 4 & 24 & 22 & 35,4 & 7,8 & 48,02 & 24,2 & 84 & 5 & 187 & 44 & 1,0 & 0,0 & 3,9 & 0,3 & 3,9 & 0,9 & 56,8 & 11,2 \\
\hline 601.008-.004-. & Orthoperus mundus & 12 & 14 & 1 & 17 & 25,0 & 27,5 & 2,0 & 18,7 & 45 & 65 & 1 & 38 & 0,5 & 0,5 & 0,0 & 0,3 & 3,4 & 3,0 & 8,0 & 12,7 \\
\hline 61-.013-.001-. & Endomychus coccineus & & 1 & & & & 2,0 & & & & 4 & & & & 0,0 & & & & 1,8 & & \\
\hline 62-.012-.002-. & Chilocorus renipustulatus & & & & 1 & & & & 1,1 & & & & 1 & & & & 0,0 & & & & 6,5 \\
\hline 62-.017-.001-. & Aphidecta obliterata & & & 1 & & & & 2,0 & & & & 1 & & & & 0,0 & & & & 7,4 & \\
\hline 62-.025-.003-. & Coccinella septempunctata & 1 & & & & 2,1 & & & & 1 & & & & 0,0 & & & & 0,7 & & & \\
\hline 62-.032-.001-. & Propylea quatuordecimpunctata & 1 & & & 1 & 2,1 & & & 1,1 & 1 & & & 1 & 0,0 & & & 0,0 & 0,7 & & & 5,9 \\
\hline 63-.002-.001-. & Arpidiphorus orbiculatus & 1 & 10 & & 1 & 2,1 & 19,6 & & 1,1 & 2 & 17 & & 1 & 0,0 & 0,1 & & 0,0 & 1,8 & 1,1 & & 7,7 \\
\hline 65-.001-.001-. & Octotemnus glabriculus & & 16 & & 1 & & 31,4 & & 1,1 & & 122 & & 1 & & 0,9 & & 0,0 & & 5,2 & & 6,5 \\
\hline 65-.005-.002-. & Sulcacis bidentulus & & & & 1 & & & & 1,1 & & & & 1 & & & & 0,0 & & & & 5,9 \\
\hline 65-.006-.002-. & Cis nitidus & 1 & 12 & & 17 & 2,1 & 23,5 & & 18,7 & 1 & 50 & & 445 & 0,0 & 0,4 & & 3,3 & 1,1 & 2,9 & & 143,9 \\
\hline 65-.006-.004-. & Cis glabratus & & & & 1 & & & & 1,1 & & & & 1 & & & & 0,0 & & & & 4,9 \\
\hline 65-.006-.007-. & Cis hispidus & & 3 & & 1 & & 5,9 & & 1,1 & & 4 & & 1 & & 0,0 & & 0,0 & & 0,9 & & 5,3 \\
\hline
\end{tabular}




\begin{tabular}{|c|c|c|c|c|c|c|c|c|c|c|c|c|c|c|c|c|c|c|c|c|c|}
\hline \multirow[b]{2}{*}{ EDV } & \multirow[b]{2}{*}{ Art } & \multirow{2}{*}{\multicolumn{4}{|c|}{$\begin{array}{c}\text { Frequenz } \\
\text { STE STE EFs EFs }\end{array}$}} & \multirow{2}{*}{\multicolumn{4}{|c|}{$\begin{array}{c}\text { Frequenz (\%) } \\
\text { STE STE EFs EFs }\end{array}$}} & \multirow[b]{2}{*}{$\begin{array}{c}\text { STE } \\
\text { Ei }\end{array}$} & \multicolumn{3}{|c|}{ Individuen } & \multicolumn{4}{|c|}{ Individuen (\%) } & \multicolumn{4}{|c|}{ spez. Dichte (Ind./m2) } \\
\hline & & & & & & & & & $\begin{array}{c}\text { EFs } \\
\text { Bu }\end{array}$ & & $\begin{array}{c}\text { STE } \\
\text { Bu }\end{array}$ & $\begin{array}{c}\text { EFs } \\
\text { Ei }\end{array}$ & $\begin{array}{c}\text { EFs } \\
\text { Bu }\end{array}$ & $\begin{array}{c}\text { STE } \\
\text { Ei }\end{array}$ & $\begin{array}{l}\text { STE } \\
\text { Bu }\end{array}$ & $\begin{array}{c}\text { EFs } \\
\text { Ei }\end{array}$ & $\begin{array}{c}\text { EFs } \\
\text { Bu }\end{array}$ & $\begin{array}{c}\text { STE } \\
\text { Ei }\end{array}$ & $\begin{array}{c}\text { STE } \\
\text { Bu }\end{array}$ & $\begin{array}{c}\text { EFs } \\
\text { Ei }\end{array}$ & $\begin{array}{c}\text { EFs } \\
\text { Bu }\end{array}$ \\
\hline 65-.006-.015-. & Cis castaneus & 2 & 3 & 14 & 38 & 4,2 & 5,92 & 28,0 & 41,8 & 3 & 5 & 100 & 186 & 0,0 & 0,0 & 2,1 & 1,4 & 1,3 & 1,1 & 50,5 & 27,3 \\
\hline 65-.006-.024-. & Cis pygmaeus & & & & 1 & & & & 1,1 & & & & 1 & & & & 0,0 & & & & 6,7 \\
\hline 65-.006-.028-. & Cis festivus & 5 & 1 & 3 & 6 & 10,4 & 2,0 & 6,0 & 6,6 & 6 & 1 & 3 & 6 & 0,1 & 0,0 & 0,1 & 0,0 & 1,1 & 0,8 & 6,3 & 5,5 \\
\hline 68-.005-.002-. & Xestobium rufovillosum & 2 & 2 & 5 & 9 & 4,2 & 3,9 & 10,0 & 9,9 & 46 & 2 & 8 & 31 & 0,5 & 0,0 & 0,2 & 0,2 & 17,3 & 0,7 & 13,0 & 21,6 \\
\hline 68-.014-.001-. & Ptilinus pectinicornis & 4 & 39 & 3 & 49 & 8,3 & 76,5 & 6,05 & 53,8 & 4 & 268 & 24 & 2681 & 0,0 & 2,0 & 0,5 & 19,9 & 0,8 & 4,7 & 59,33 & 316,7 \\
\hline 68-.022-.001-. & Dorcatoma flavicornis & 1 & & & & 2,1 & & & & 1 & & & & 0,0 & & & & 0,8 & & & \\
\hline 68-.022-.003-. & Dorcatoma chrysomelina & 1 & 1 & 5 & & 2,1 & 2,0 & 10,0 & & 1 & 1 & 6 & & 0,0 & 0,0 & 0,1 & & 0,7 & 0,6 & 7,6 & \\
\hline 68-.022-.007-. & Dorcatoma robusta & & & & 1 & & & & 1,1 & & & & 1 & & & & 0,0 & & & & 4,5 \\
\hline 711.001-.002-. & Lissodema quadripustulatum & & 1 & & & & 2,0 & & & & 1 & & & & 0,0 & & & & 0,7 & & \\
\hline 711.005-.001-. & Vincenzellus ruficollis & & 13 & & 7 & & 25,5 & & 7,7 & & 136 & & 15 & & 1,0 & & 0,1 & & 6,8 & & 13,5 \\
\hline 711.006-.002-. & Rhinosimus planirostris & 2 & 2 & & & 4,2 & 3,9 & & & 2 & 2 & & & 0,0 & 0,0 & & & 0,8 & 0,6 & & \\
\hline 711.006-.003-. & Rhinosimus ruficollis & 5 & 9 & 3 & 8 & 10,4 & 17,6 & 6,0 & 8,8 & 67 & 38 & 4 & 10 & 0,8 & 0,3 & 0,1 & 0,1 & 11,4 & 2,7 & 8,4 & 6,9 \\
\hline 72-.001-.001-. & Pyrochroa coccinea & 3 & 10 & & 3 & 6,3 & 19,6 & & 3,3 & 15 & 14 & & 3 & 0,2 & 0,1 & & 0,0 & 5,0 & 0,9 & & 5,3 \\
\hline 72-.002-.001-. & Schizotus pectinicornis & 1 & 4 & & & 2,1 & 7,8 & & & 1 & 18 & & & 0,0 & 0,1 & & & 1,1 & 3,4 & & \\
\hline 73-.004-.010-. & Anaspis maculata & & & 1 & & & & 2,0 & & & & 1 & & & & 0,0 & & & & 6,3 & \\
\hline 73-.004-.011-. & Anaspis schilskyana & 7 & 6 & 6 & 19 & 14,6 & 11,8 & 12,02 & 20,9 & 11 & 9 & 8 & 75 & 0,1 & 0,1 & 0,2 & 0,6 & 1,3 & 1,0 & 9,5 & 23,4 \\
\hline 73-.004-.012-. & Anaspis thoracica & 3 & 8 & 9 & 14 & 6,3 & 15,7 & 18,0 & 15,4 & 6 & 10 & 15 & 22 & 0,1 & 0,1 & 0,3 & 0,2 & 1,9 & 0,8 & 12,0 & 8,7 \\
\hline 73-.004-.019-. & Anaspis rufilabris & 5 & 1 & 5 & & 10,4 & 2,0 & 10,0 & & 5 & 1 & 8 & & 0,1 & 0,0 & 0,2 & & 0,8 & 0,8 & 11,3 & \\
\hline 80-.013-.001-. & Hypulus quercinus & 5 & & & & 10,4 & & & & 24 & & & & 0,3 & & & & 3,4 & & & \\
\hline 801.001-.002-. & Tetratoma desmaresti & 1 & & & & 2,1 & & & & 2 & & & & 0,0 & & & & 2,3 & & & \\
\hline 82-.001-.002-. & Allecula morio & & & & 1 & & & & 1,1 & & & & 1 & & & & 0,0 & & & & 6,7 \\
\hline 82-.008-.011-. & Mycetochara linearis & 1 & 1 & & 1 & 2,1 & 2,0 & & 1,1 & 1 & 1 & & 1 & 0,0 & 0,0 & & 0,0 & 0,7 & 0,6 & & 6,1 \\
\hline 83-.019-.001-. & Scaphidema metallicum & & & & 1 & & & & 1,1 & & & & 1 & & & & 0,0 & & & & 5,9 \\
\hline 83-.023-.001-. & Corticeus unicolor & 9 & 29 & 5 & 39 & $18,8=$ & 56,9 & 10,0 & 42,9 & 20 & 294 & 15 & 375 & 0,2 & 2,1 & 0,3 & 2,8 & 2,2 & 6,8 & 19,1 & 54,1 \\
\hline 83-.023-.008-. & Corticeus fasciatus & 4 & 2 & 9 & 2 & 8,3 & 3,9 & 18,0 & 2,2 & 11 & 2 & 29 & 2 & 0,1 & 0,0 & 0,6 & 0,0 & 2,2 & 0,7 & 22,2 & 6,7 \\
\hline 83-.025-.002-. & Tribolium castaneum & & & & 1 & & & & 1,1 & & & & 1 & & & & 0,0 & & & & 5,9 \\
\hline 841.001-.004-. & Trox scaber & & & & 1 & & & & 1,1 & & & & 1 & & & & 0,0 & & & & 5,1 \\
\hline 85-.050-.002-. & Gnorimus variabilis & 1 & 1 & & 1 & 2,1 & 2,0 & & 1,1 & 1 & 1 & & 12 & 0,0 & 0,0 & & 0,1 & 0,8 & 0,8 & & 85,7 \\
\hline 86-.005-.001-. & Sinodendron cylindricum & 2 & 7 & & 10 & 4,2 & 13,7 & & 11,0 & 4 & 22 & & 35 & 0,0 & 0,2 & & 0,3 & 1,5 & 2,3 & & 22,1 \\
\hline 87-.011-.001-. & Rhagium bifasciatum & 1 & 2 & & 1 & 2,1 & 3,9 & & 1,1 & 1 & 13 & & 1 & 0,0 & 0,1 & & 0,0 & 1,0 & 4,2 & & 6,9 \\
\hline 87-.011-.002-. & Rhagium sycophanta & & & 1 & & & & 2,0 & & & & 1 & & & & 0,0 & & & & 6,7 & \\
\hline 87-.011-.003-. & Rhagium mordax & & 7 & & 6 & & 13,7 & & 6,6 & & 12 & & 7 & & 0,1 & & 0,1 & & 1,1 & & 6,5 \\
\hline 87-.078-.001-. & Leiopus nebulosus & 2 & & 1 & & 4,2 & & 2,0 & & 9 & & 1 & & 0,1 & & 0,0 & & 4,2 & & 6,7 & \\
\hline 87-.082-.004-. & Saperda scalaris & 1 & 1 & 1 & & 2,1 & 2,0 & 2,0 & & 1 & 3 & 2 & & 0,0 & 0,0 & 0,0 & & 1,1 & 1,9 & 13,3 & \\
\hline \begin{tabular}{|l} 
88-.0061.005-. \\
\end{tabular} & Oulema melanopus & & 1 & 1 & & & 2,0 & 2,0 & & & 1 & 1 & & & 0,0 & 0,0 & & & 0,7 & 6,7 & \\
\hline 88-.049-.005-. & Phyllotreta undulata & & & & 1 & & & & 1,1 & & & & 1 & & & & 0,0 & & & & 6,9 \\
\hline 88-.049-.010-. & Phyllotreta striolata & 1 & & & & 2,1 & & & & 1 & & & & 0,0 & & & & 0,8 & & & \\
\hline 91-.001-.003-. & Scolytus intricatus & 1 & & & & 2,1 & & & & 1 & & & & 0,0 & & & & 1,1 & & & \\
\hline
\end{tabular}


Anhang 5: Auftreten der Arten in STE- und EFs-Proben an Eiche und Buche

\begin{tabular}{|c|c|c|c|c|c|c|c|c|c|c|c|c|c|c|c|c|c|c|c|c|c|}
\hline \multirow{2}{*}{ EDV } & \multirow{2}{*}{ Art } & \multirow{2}{*}{\multicolumn{4}{|c|}{$\begin{array}{c}\text { Frequenz } \\
\text { STE STE EFs EFs }\end{array}$}} & \multirow{2}{*}{\multicolumn{4}{|c|}{$\begin{array}{c}\text { Frequenz (\%) } \\
\text { STE STE EFs EFs }\end{array}$}} & \multirow{2}{*}{$\begin{array}{c}\text { STE } \\
\text { Ei }\end{array}$} & \multicolumn{3}{|c|}{ Individuen } & \multirow{2}{*}{\multicolumn{4}{|c|}{$\begin{array}{c}\text { Individuen (\%) } \\
\text { STE STE FFs FFs }\end{array}$}} & \multicolumn{4}{|c|}{ spez. Dichte (Ind./m2) } \\
\hline & & & & & & & & & & & STE & EFs & EFs & & & & & & & EFs & EFs \\
\hline 91-.005-.002-. & Hylurgops palliatus & 1 & & & & 2,1 & & & & 1 & & & & 0,0 & & & & 1,2 & & & \\
\hline 91-.026-.004-. & Cryphalus abietis & & 1 & & & & 2,0 & & & & 1 & & & & 0,0 & & & & 0,8 & & \\
\hline 91-.031-.003-. & Taphrorychus bicolor & & 10 & & 19 & & 19,6 & & 20,9 & & 46 & & 135 & & 0,3 & & 1,0 & & 3,0 & & 38,7 \\
\hline 91-.036-.004-. & Xyleborus saxeseni & 1 & 4 & 1 & & 2,1 & 7,8 & 2,0 & & 5 & 124 & 1 & & 0,1 & 0,9 & 0,0 & & 3,3 & 20,2 & 6,3 & \\
\hline 91-.038-.001-. & Xyloterus domesticus & 4 & 14 & 4 & 3 & 8,3 & 27,5 & 8,0 & 3,3 & 558 & 718 & 18 & 10 & 6,5 & 5,2 & 0,4 & 0,1 & 117,1 & 31,8 & 28,8 & 17,9 \\
\hline 91-.038-.002-. & Xyloterus signatus & 13 & 9 & 8 & 1 & 27,1 & 17,6 & 16,0 & 1,1 & 1533 & 26 & 295 & 28 & 17,9 & 0,2 & 6,1 & 0,2 & 93,8 & 1,8 & 241,8 & 164,7 \\
\hline 923.007-.004-. & Deporaus betulae & 1 & & & & 2,1 & & & & 1 & & & & 0,0 & & & & 0,7 & & & \\
\hline $925.021-.002-$. & Protapion fulvipes & 1 & & & & 2,1 & & & & 1 & & & & 0,0 & & & & 1,6 & & & \\
\hline 93-.021-.015-. & Phyllobius calcaratus & & 1 & & & & 2,0 & & & & 1 & & & & 0,0 & & & & 0,8 & & \\
\hline 93-.021-.019-. & Phyllobius argentatus & 4 & 1 & & & 8,3 & 2,0 & & & 4 & 1 & & & 0,0 & 0,0 & & & 0,8 & 0,6 & & \\
\hline 93-.037-.011-. & Barypeithes pellucidus & 1 & & & & 2,1 & & & & 1 & & & & 0,0 & & & & 0,7 & & & \\
\hline 93-.040-.002-. & Strophosoma melanogrammum & 3 & 10 & 3 & 4 & 6,3 & 19,6 & 6,0 & 4,4 & 4 & 12 & 3 & 6 & 0,0 & 0,1 & 0,1 & 0,0 & 1,1 & 0,8 & 6,7 & 8,5 \\
\hline 93-.044-.016-. & Sitona lepidus & & & & 1 & & & & 1,1 & & & & 1 & & & & 0,0 & & & & 5,9 \\
\hline 93-.104-.019-. & Tychius picirostris & & & & 1 & & & & 1,1 & & & & 1 & & & & 0,0 & & & & 4,8 \\
\hline 93-.110-.011-. & Curculio pyrrhoceras & 3 & & & & 6,3 & & & & 4 & & & & 0,0 & & & & 0,9 & & & \\
\hline 93-.157-.003-. & Coeliodes dryados & & & 1 & & & & 2,0 & & & & 1 & & & & 0,0 & & & & 7,4 & \\
\hline $93-.163-.0601$. & Ceutorhynchus floralis & 2 & & & & 4,2 & & & & 2 & & & & 0,0 & & & & 0,7 & & & \\
\hline 93-.180-.005-. & Rhynchaenus quercus & 1 & & 3 & 2 & 2,1 & & 6,0 & 2,2 & 2 & & 4 & 2 & 0,0 & & 0,1 & 0,0 & 2,3 & & 9,0 & 5,4 \\
\hline
\end{tabular}


Anhang 6: Artenaffinität: Sørensen-Quotient (SQ) für Artenpaarungen

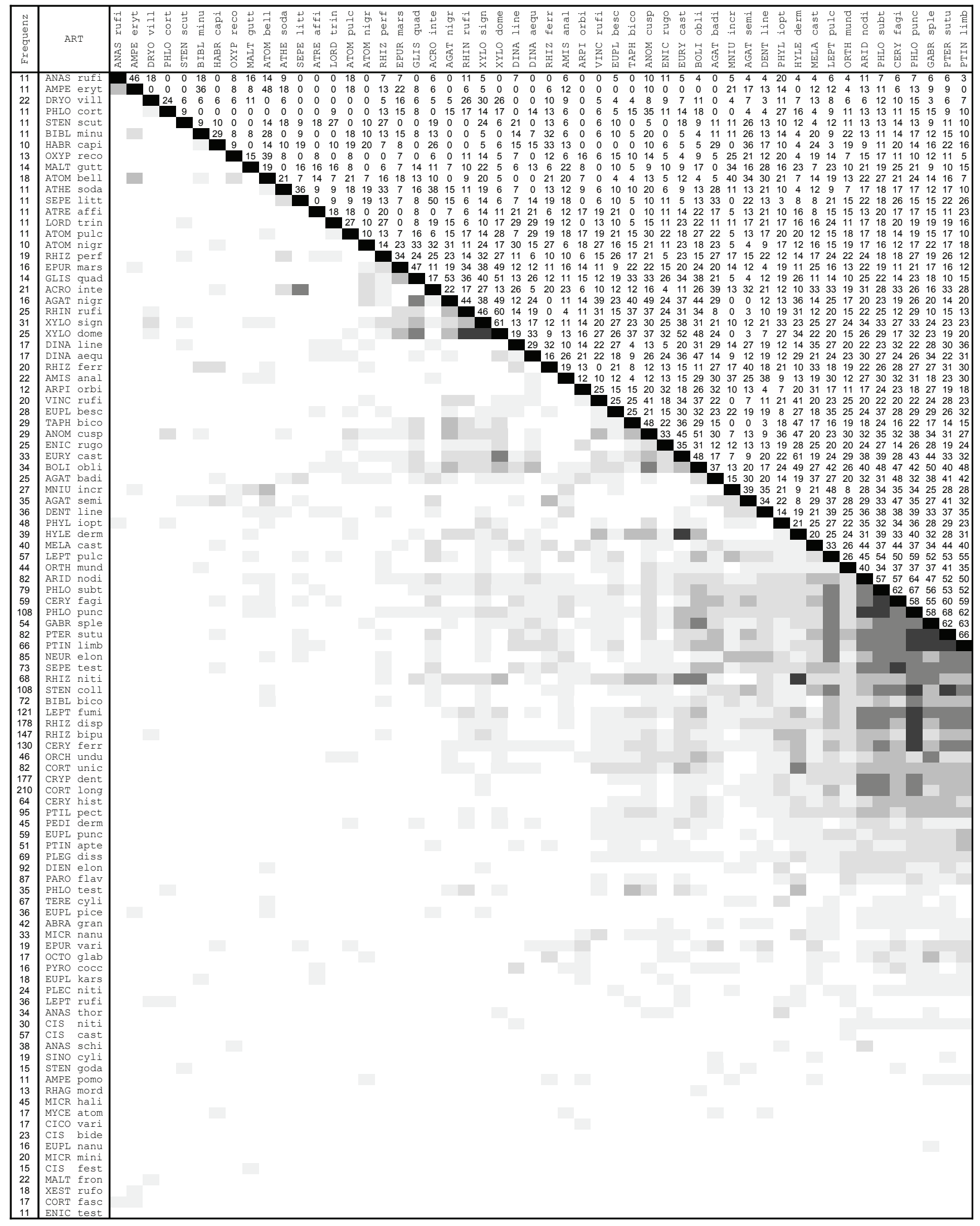

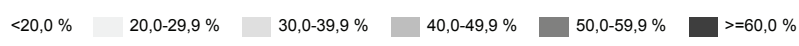




\section{Anhang 6 (Fortsetzung)}

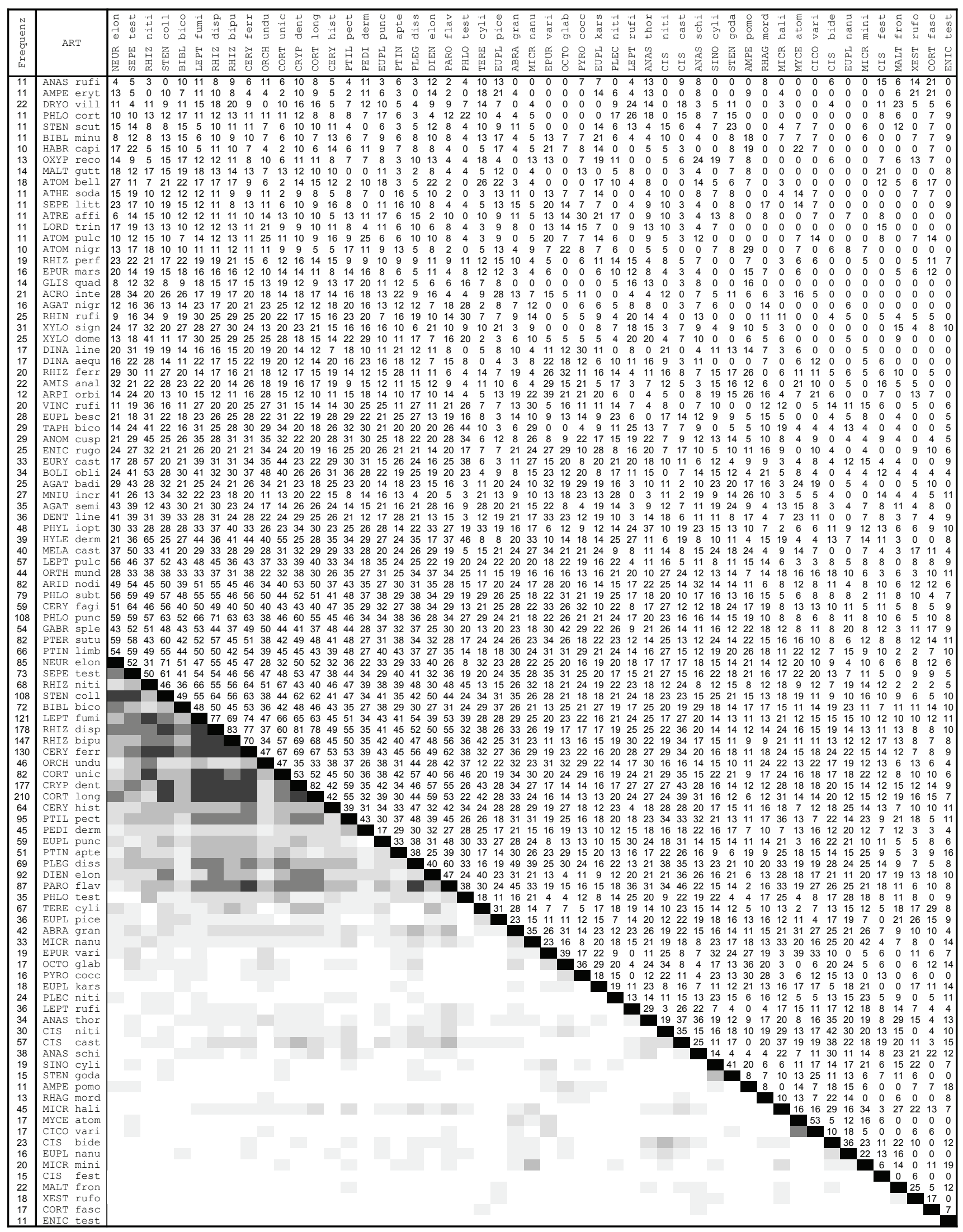




\section{Anhang 7a: Probenähnlichkeit: Sørensen-Quotient (SQ) für STE-Proben an Buche}

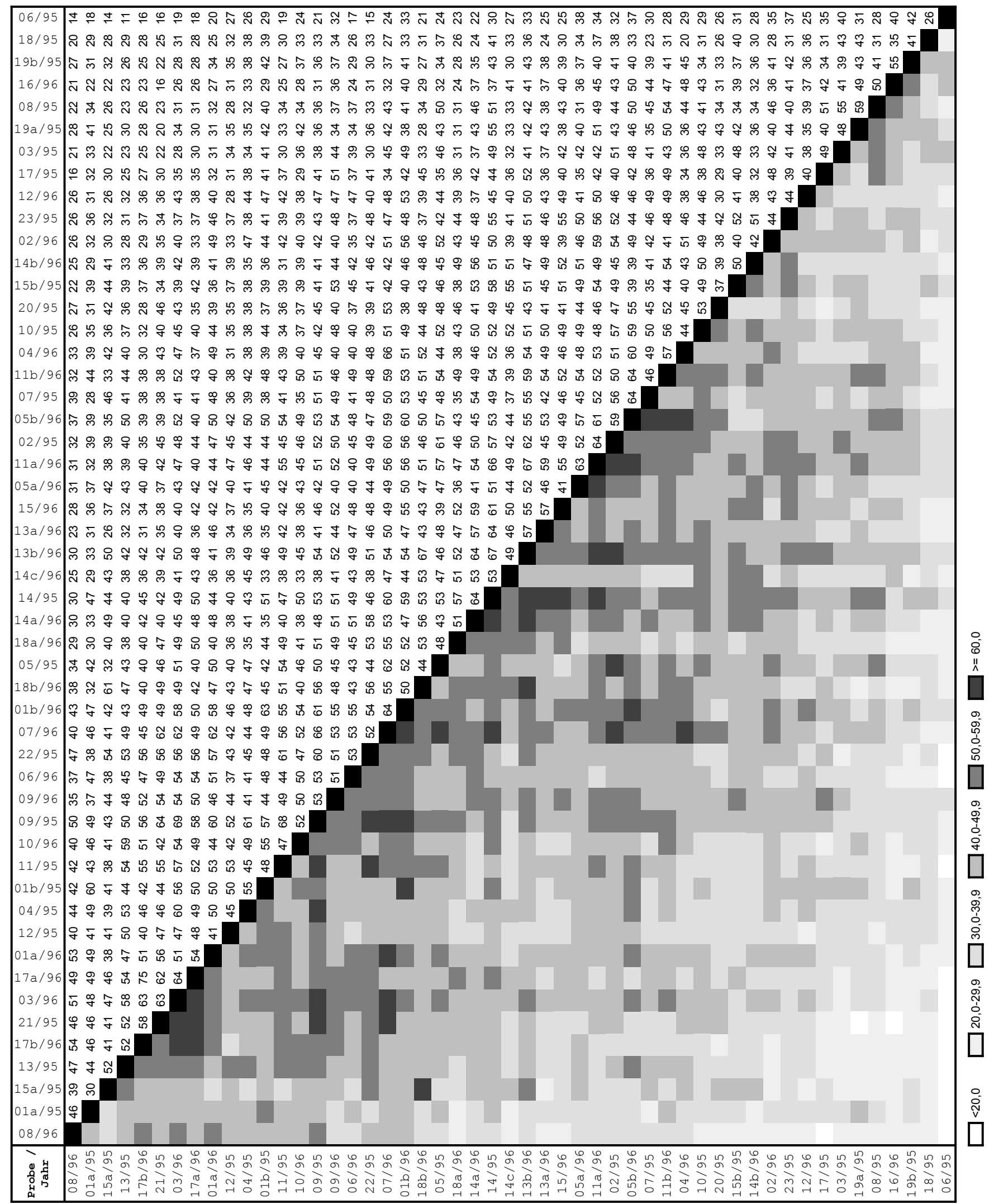




\section{Anhang 7b: Probenähnlichkeit: Renkonen-Zahl (RE) für STE-Proben an Buche}

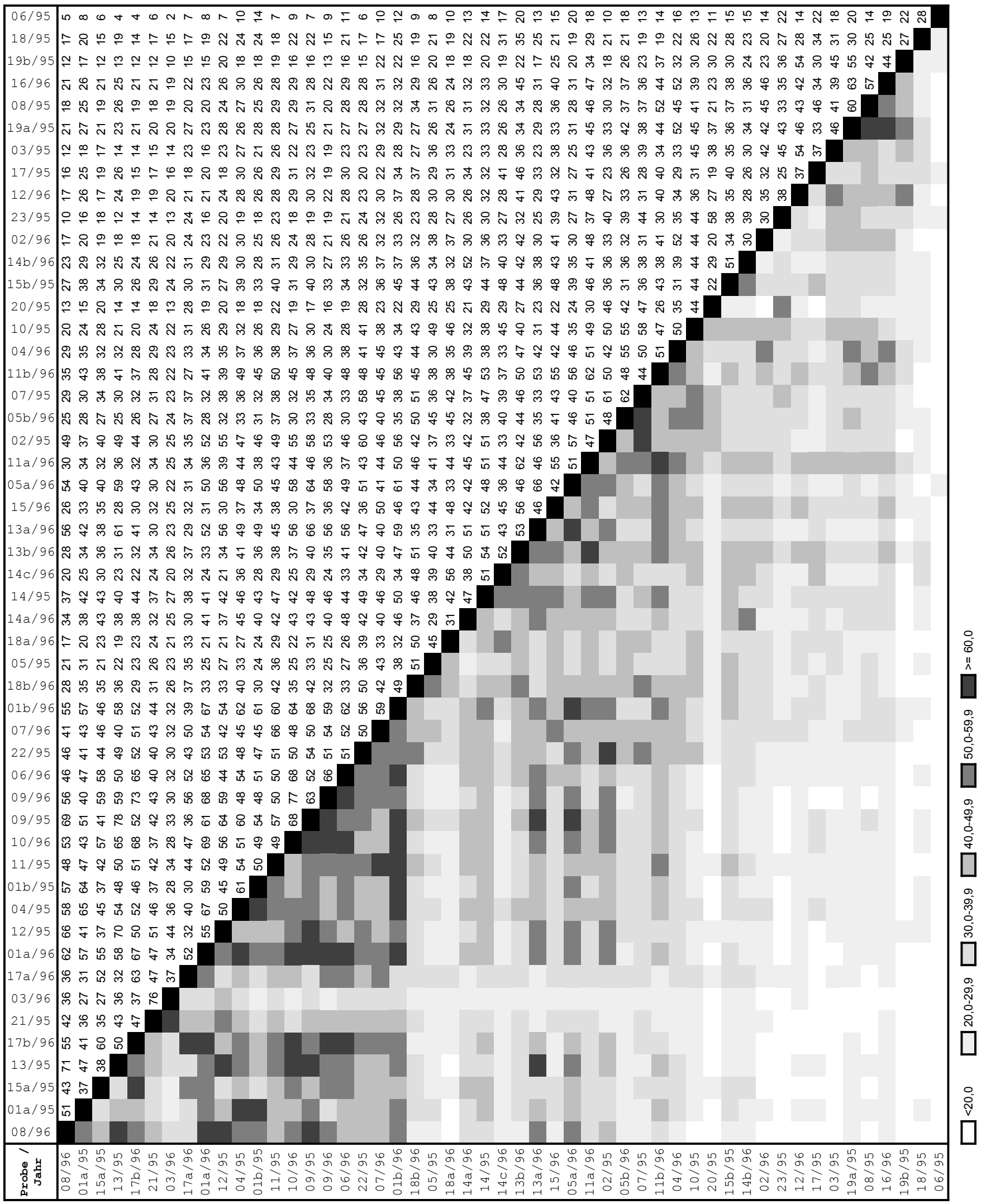




\section{Anhang 8a: Probenähnlichkeit: Sørensen-Quotient (SQ) für STE-Proben an Eiche}

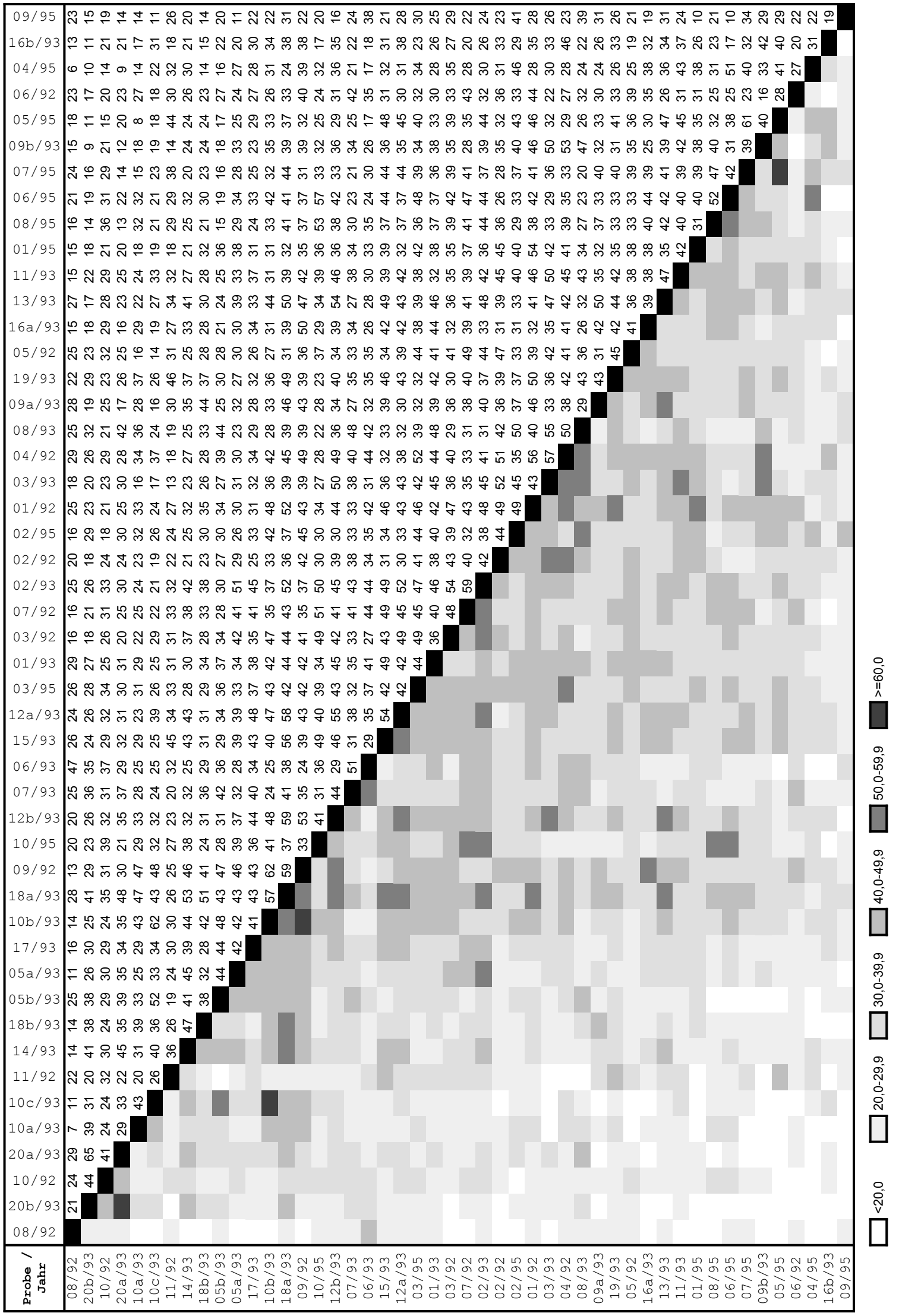


Anhang 8b: Probenähnlichkeit: Renkonen-Zahl (RE) für STE-Proben an Eiche

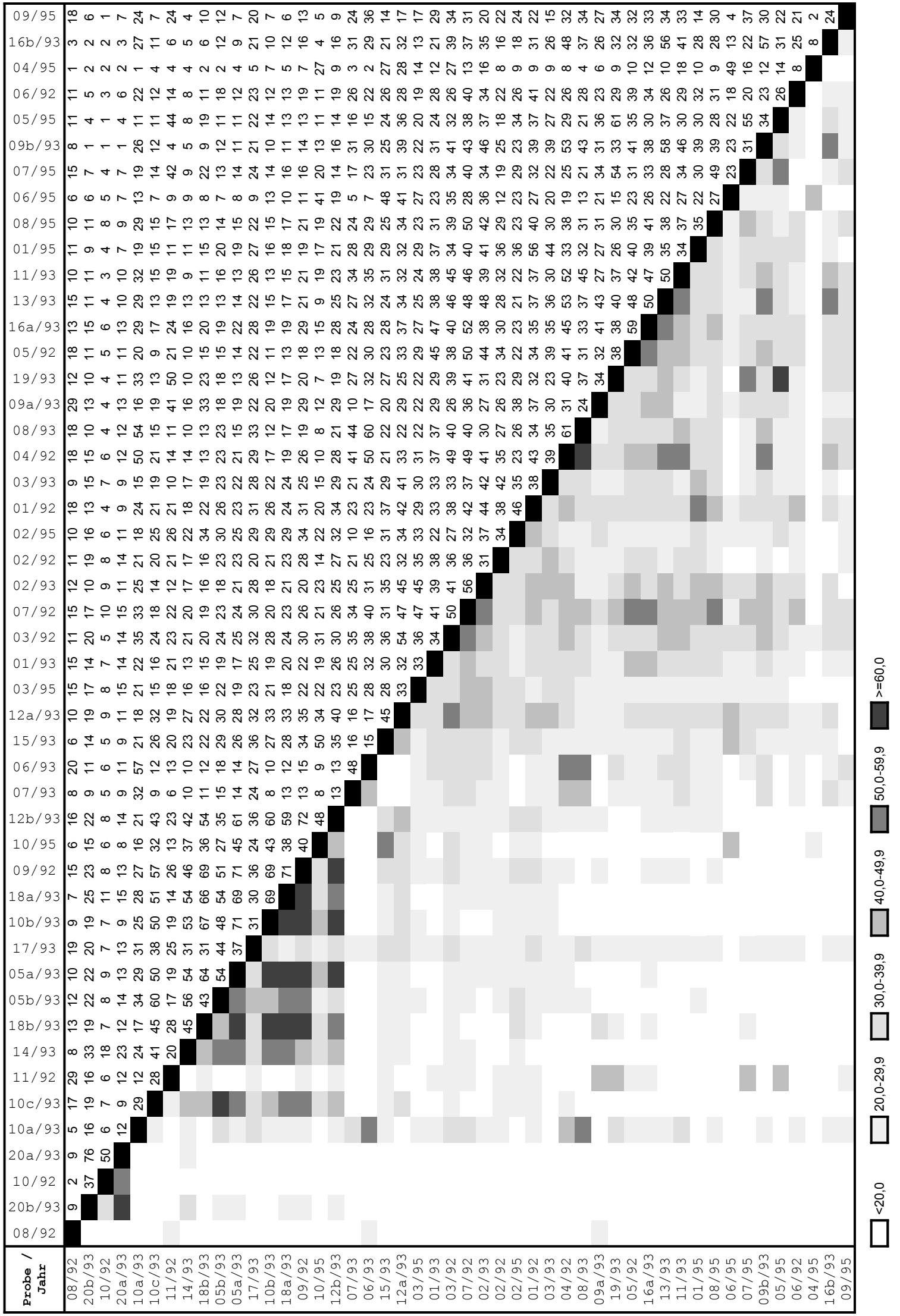


Anhang 9a: Probenähnlichkeit: Sørensen-Quotient (SQ) für EFs-Proben an Buche

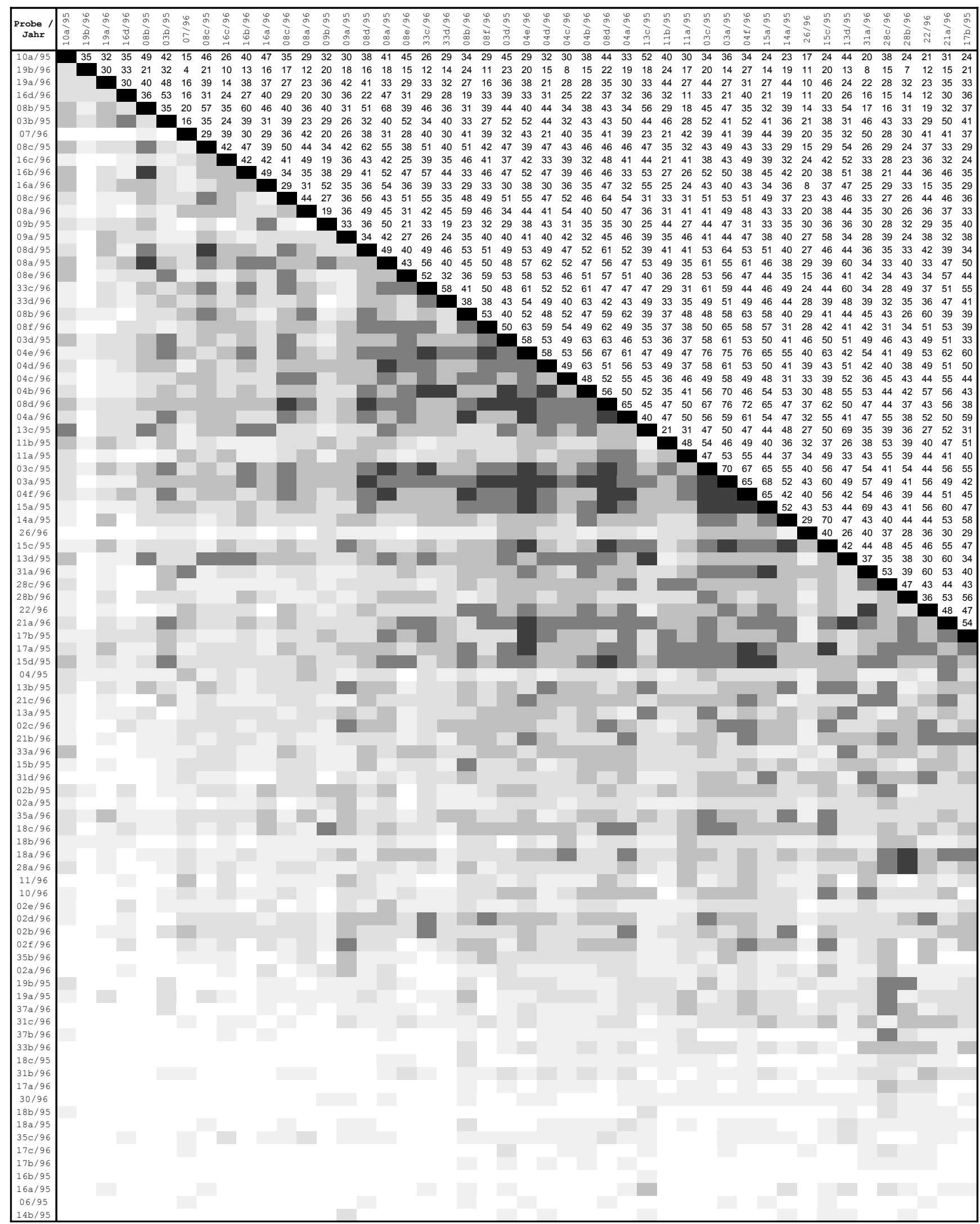

$\square^{<20,0} \quad \square^{20,0-29,9} \quad \square_{30,0-39,9}^{30,0-49,9} \quad \square_{50,0-59,9}^{40,0-69,9}$ 


\section{Anhang 9a (Fortsetzung)}

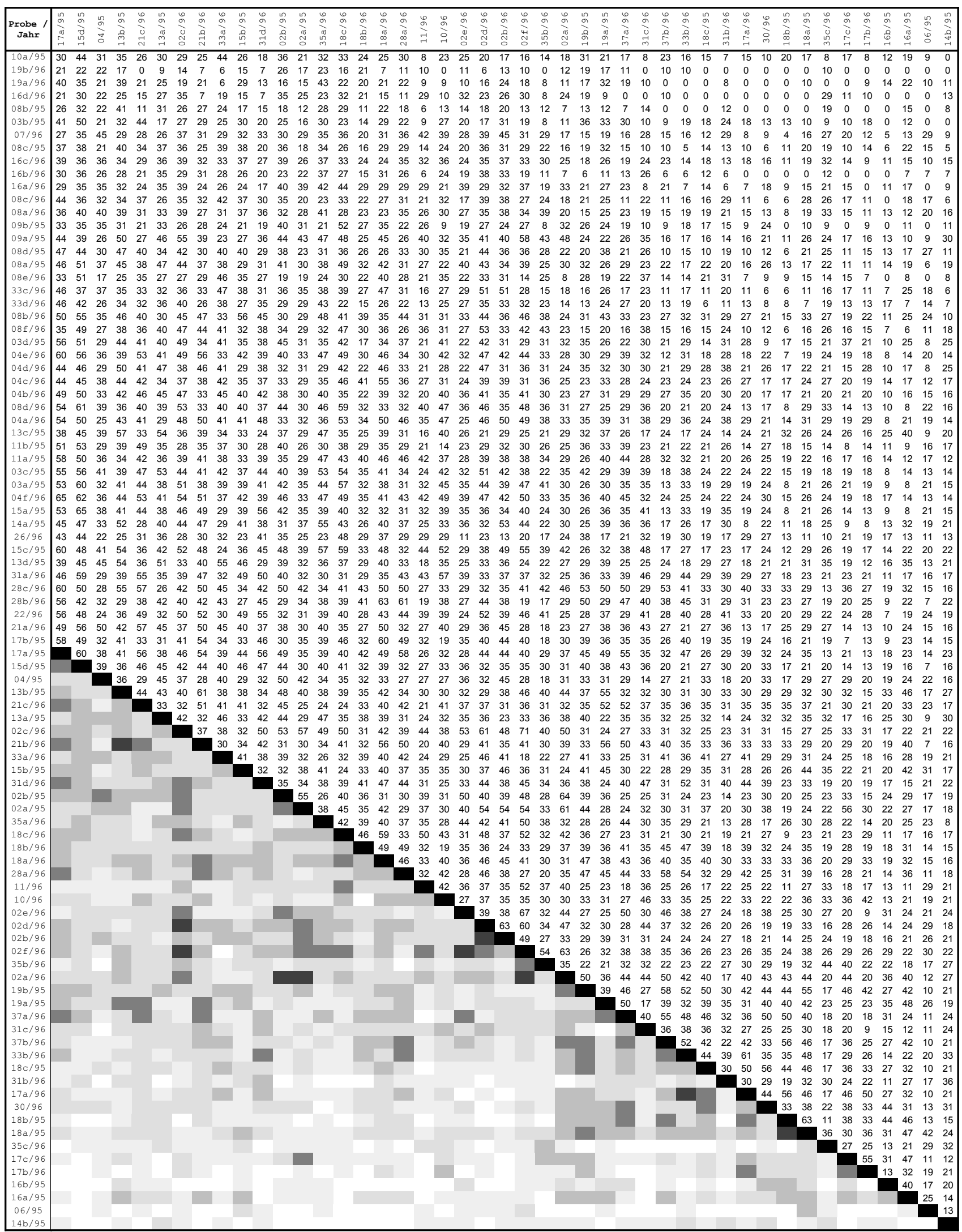


Anhang 9b: Probenähnlichkeit: Renkonen-Zahl (RE) für EFs-Proben an Buche

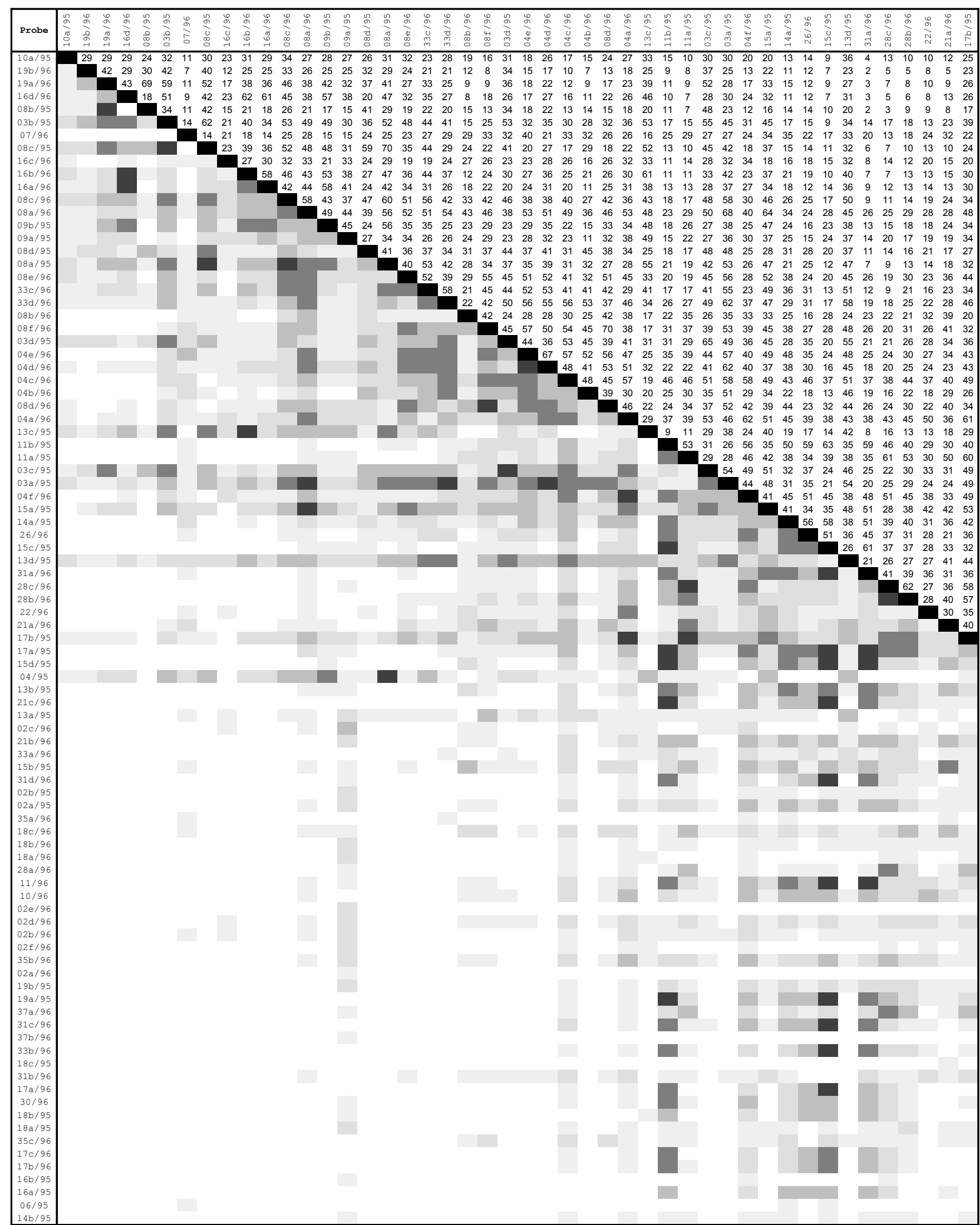

$\square_{<20,0 \quad}^{20,0-29,9} \quad \square_{30,0-39,9}^{40,0-49,9} \quad \square_{50,0-59,9}^{460,0-69,9}$ 
Anhang 9b (Fortsetzung)

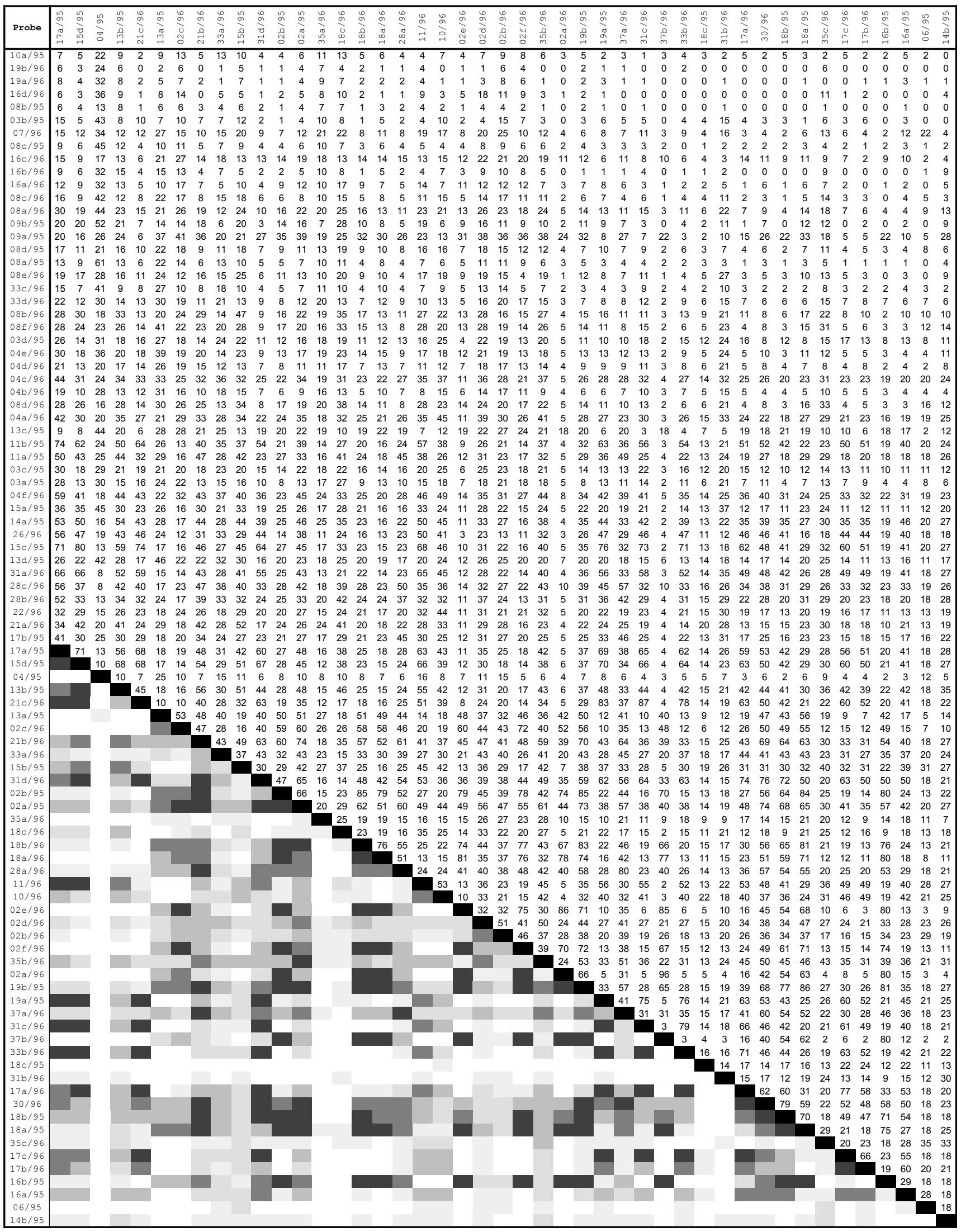




\section{Anhang 10a: Probenähnlichkeit: Sørensen-Quotient (SQ) für EFs- Proben an Eiche}

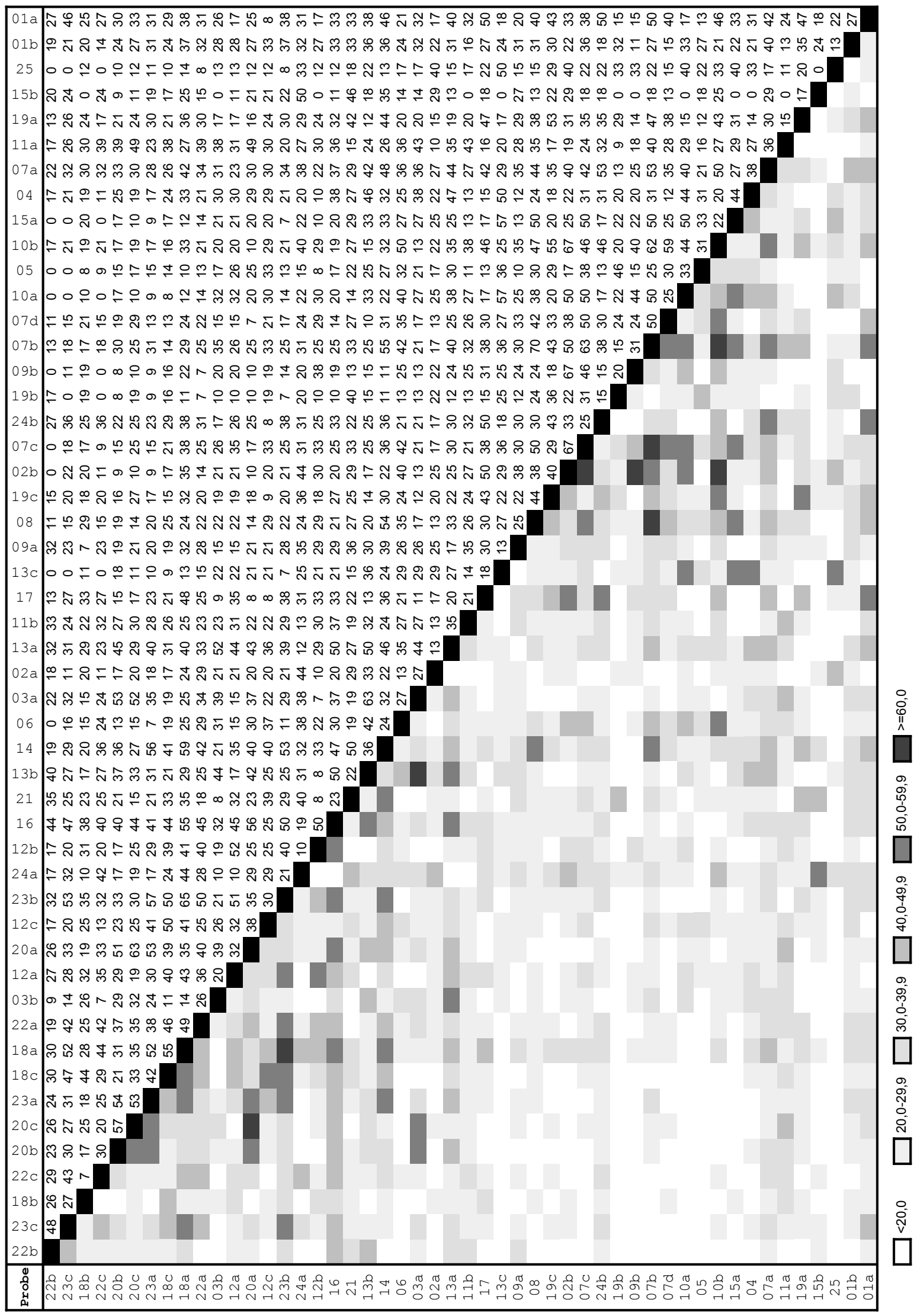




\section{Anhang 10b: Probenähnlichkeit: Renkonen-Zahl (RE) für EFs-Proben an Eiche}

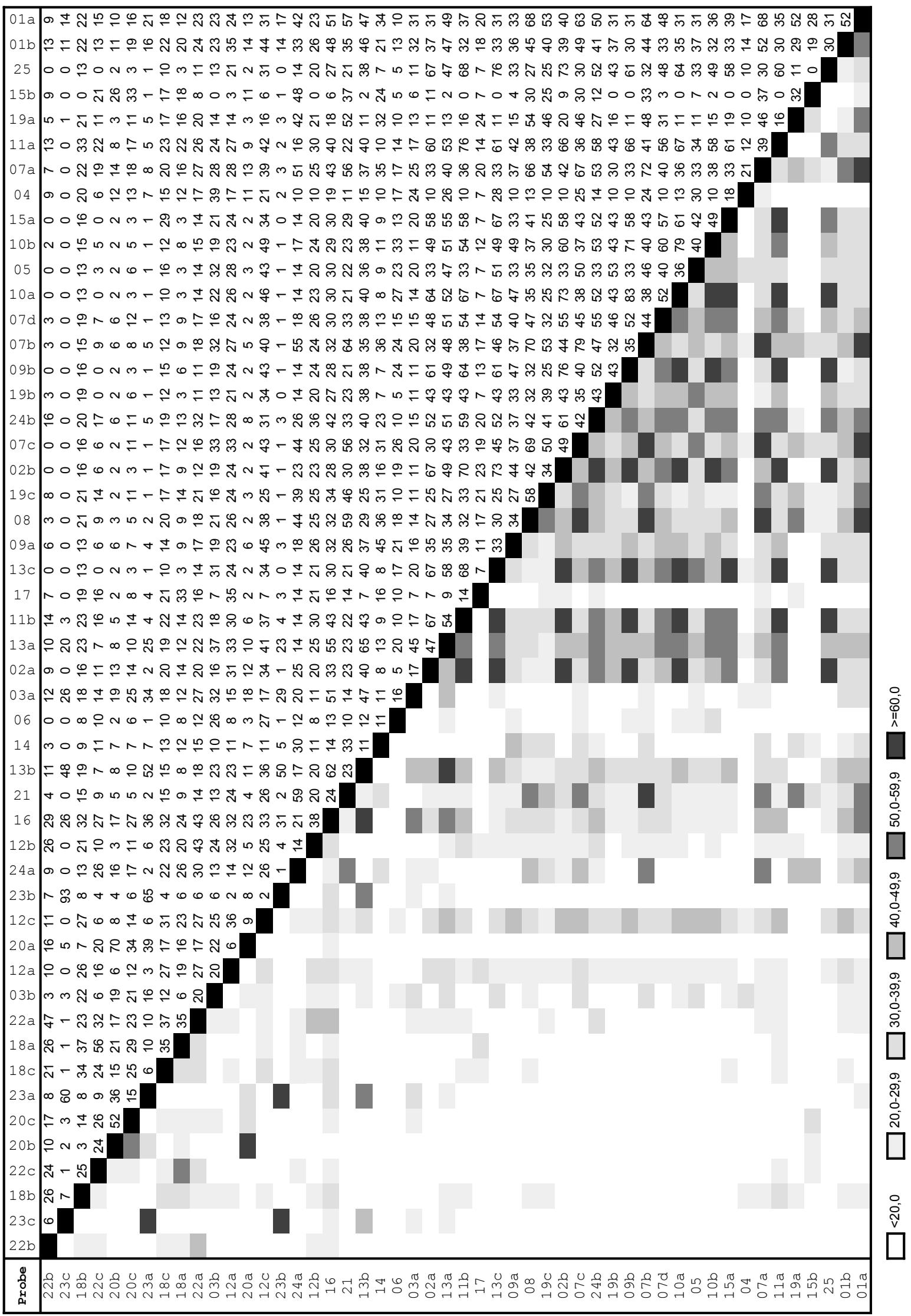


Anhang 11: Flugphänologie der Arten (Fensterfallen)

\begin{tabular}{|c|c|c|c|c|c|c|c|c|c|c|c|c|c|}
\hline & & & & & $\mathbf{a n}$ & $\mathrm{pe}$ & od & & & & & & \\
\hline ART & EDV & $\frac{\varangle}{\Delta}$ & $\frac{m}{\alpha}$ & $\mathfrak{\Sigma}$ & $\dddot{q}$ & $\bar{a}$ & $\stackrel{2}{0}$ & $\stackrel{\circ}{2}$ & $\hat{a}$ & $\stackrel{\infty}{\infty}$ & 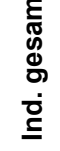 & 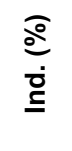 & 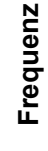 \\
\hline Notiophilus biguttatus & 01-.009-.008-. & & & & & & & & & 1 & 1 & 0,01 & 1 \\
\hline Elaphrus cupreus & 01-.012-.002-. & & 1 & & & & & & & & 1 & 0,01 & 1 \\
\hline Loricera pilicornis & 01-.013-.001-. & & & & & 1 & & & & & 1 & 0,01 & 1 \\
\hline Clivina fossor & 01-.015-.001-. & & 1 & & & & & & & & 1 & 0,01 & 1 \\
\hline Trechus obtusus & 01-.021-.007-. & & & & & & 1 & 1 & & & 2 & 0,01 & 2 \\
\hline Bembidion deletum & 01-.029-.042-. & & 12 & 1 & 1 & & & & & & 14 & 0,09 & 10 \\
\hline Bembidion lunulatum & 01-.029-.103-. & 1 & 1 & 1 & & & 1 & & & & 4 & 0,02 & 4 \\
\hline Asaphidion flavipes & 01-.030-.004-. & 1 & 3 & & 1 & & & & & & 5 & 0,03 & 5 \\
\hline Anisodactylus binotatus & 01-.037-.001-. & & 1 & 1 & & & & 1 & & & 3 & 0,02 & 3 \\
\hline Harpalus quadripunctatus & 01-.041-.047-. & & & & 1 & & & & & & 1 & 0,01 & 1 \\
\hline Stenolophus teutonus & 01-.042-.001-. & & 1 & & & & & & & & 1 & 0,01 & 1 \\
\hline Bradycellus harpalinus & 01-.045-.005-. & & & & & & 12 & 46 & 17 & 8 & 83 & 0,51 & 18 \\
\hline Acupalpus flavicollis & 01-.046-.002-. & & 1 & & & & & & & & 1 & 0,01 & 1 \\
\hline Acupalpus exiguus & 01-.046-.010-. & & 1 & 2 & & & & & & & 3 & 0,02 & 3 \\
\hline Pterostichus strenuus & 01-.051-.011-. & & 4 & 1 & & & & & & & 5 & 0,03 & 5 \\
\hline Pterostichus minor & 01-.051-.022-. & & 1 & & & & & & & & 1 & 0,01 & 1 \\
\hline Agonum muelleri & 01-.062-.009-. & 3 & 2 & & & & 2 & 4 & 2 & 3 & 16 & 0,10 & 12 \\
\hline Agonum fuliginosum & 01-.062-.028-. & & 1 & & & & & & & & 1 & 0,01 & 1 \\
\hline Amara plebeja & 01-.065-.001-. & & 5 & 1 & & & 2 & & 1 & & 9 & 0,06 & 8 \\
\hline Amara similata & 01-.065-.008-. & & 1 & 2 & & 1 & & & 1 & & 5 & 0,03 & 5 \\
\hline Amara communis & 01-.065-.014-. & & 4 & & & & & & & & 4 & 0,02 & 4 \\
\hline Amara lunicollis & 01-.065-.018-. & & 2 & 1 & & & & & & & 3 & 0,02 & 3 \\
\hline Amara aenea & 01-.065-.021-. & 4 & 10 & & & & & 1 & & & 15 & 0,09 & 10 \\
\hline Amara familiaris & 01-.065-.026-. & 1 & 9 & & 2 & & & 2 & 2 & 1 & 17 & 0,10 & 13 \\
\hline Amara tibialis & 01-.065-.029-. & & & & & & & & 1 & & 1 & 0,01 & 1 \\
\hline Badister bullatus & 01-.070-.002-. & & & 1 & & & & & & & 1 & 0,01 & 1 \\
\hline Badister lacertosus & 01-.070-.003-. & & & & 1 & & & & & & 1 & 0,01 & 1 \\
\hline Dromius quadrimaculatus & 01-.079-.012-. & & & & & & 1 & & & & 1 & 0,01 & 1 \\
\hline Hydroporus umbrosus & 04-.008-.004-. & & & & & & & 2 & & & 2 & 0,01 & 1 \\
\hline Hydroporus palustris & 04-.008-.009-. & & 1 & & 1 & 1 & & & & & 3 & 0,02 & 3 \\
\hline Hydroporus planus & 04-.008-.019-. & & & 2 & 2 & & & & & & 4 & 0,02 & 3 \\
\hline Hydroporus memnonius & 04-.008-.027-. & & 1 & 4 & 1 & & & & & & 6 & 0,04 & 5 \\
\hline Agabus chalconotus & 04-.023-.003-. & 3 & 3 & 49 & 19 & 3 & & & & & 77 & 0,47 & 25 \\
\hline Agabus bipustulatus & 04-.023-.009-. & & 1 & & & 5 & & & & & 6 & 0,04 & 3 \\
\hline Hydraena spec. & 07-.001-.000-. & & 1 & & & & & 1 & & & 2 & 0,01 & 2 \\
\hline Helophorus grandis & 09-.0011.008-. & 3 & 4 & 1 & & & & & & & 8 & 0,05 & 7 \\
\hline Helophorus aequalis & 09-.0011.0091. & & 3 & 4 & 2 & 5 & & & 1 & & 15 & 0,09 & 11 \\
\hline Helophorus brevipalpis & 09-.0011.0152. & & 1 & 2 & 2 & 3 & 1 & & & & 9 & 0,06 & 6 \\
\hline Helophorus flavipes & 09-.0011.022-. & 2 & 6 & & 2 & 5 & & & & 1 & 16 & 0,10 & 11 \\
\hline Helophorus obscurus & 09-.0011.0221. & & 2 & & & 1 & & & & & 3 & 0,02 & 3 \\
\hline Helophorus minutus & 09-.0011.028-. & & & & & 1 & & & & & 1 & 0,01 & 1 \\
\hline Sphaeridium bipustulatum & 09-.002-.001-. & & 1 & & & & & & & & 1 & 0,01 & 1 \\
\hline Sphaeridium scarabaeoides & 09-.002-.003-. & 2 & 1 & & & & 1 & 1 & 8 & & 13 & 0,08 & 11 \\
\hline Sphaeridium lunatum & 09-.002-.004-. & & 8 & 2 & & 3 & 11 & 18 & 15 & 2 & 59 & 0,36 & 30 \\
\hline Cercyon impressus & 09-.003-.005-. & & 1 & 4 & & 1 & 2 & 1 & & & 9 & 0,06 & 8 \\
\hline Cercyon melanocephalus & 09-.003-.008-. & & 1 & & & & 2 & & & 1 & 4 & 0,02 & 4 \\
\hline Cercyon lateralis & 09-.003-.011-. & & & 10 & & & & 1 & 1 & & 12 & 0,07 & 7 \\
\hline Cercyon pygmaeus & 09-.003-.017-. & & 1 & 2 & & & 1 & & 1 & 1 & 6 & 0,04 & 6 \\
\hline Cercyon convexiusculus & 09-.003-.021-. & & & 1 & & & & & & & 1 & 0,01 & 1 \\
\hline Megasternum obscurum & 09-.004-.001-. & & 1 & & 1 & 1 & 2 & 1 & 5 & 3 & 14 & 0,09 & 11 \\
\hline Hydrobius fuscipes & 09-.008-.001-. & & 2 & 1 & & & 2 & 1 & & & 6 & 0,04 & 6 \\
\hline Anacaena limbata & 09-.010-.002-. & & & 1 & & & & & & & 1 & 0,01 & 1 \\
\hline
\end{tabular}




\begin{tabular}{|c|c|c|c|c|c|c|c|c|c|c|c|c|c|}
\hline & & & & & an & pe & od & & & & & & \\
\hline ART & EDV & $\frac{\varangle}{a}$ & $\stackrel{m}{a}$ & $\mathbf{\Sigma}$ & $\dddot{\Omega}$ & a & $\stackrel{n}{\alpha}$ & $\dddot{0}$ & $\hat{a}$ & $\stackrel{\infty}{0}$ & 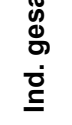 & 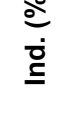 & 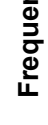 \\
\hline Plegaderus dissectus & 10-.002-.004-. & & & 2 & 4 & 4 & 4 & 1 & & & 15 & 0,09 & 13 \\
\hline Abraeus granulum & 10-.005-.001-. & & & & & 1 & & 1 & & & 2 & 0,01 & 2 \\
\hline Gnathoncus buyssoni & 10-.009-.004-. & & 1 & & & & & & & & 1 & 0,01 & 1 \\
\hline Paromalus flavicornis & 10-.020-.001-. & & & & 1 & 1 & 7 & 1 & & & 10 & 0,06 & 6 \\
\hline Margarinotus ventralis & 10-.029-.005-. & & & & & 1 & & & & & 1 & 0,01 & 1 \\
\hline Margarinotus striola & 10-.029-.008-. & & & & 1 & & & & & & 1 & 0,01 & 1 \\
\hline Margarinotus marginatus & 10-.029-.013-. & & 1 & 1 & & & & & & & 2 & 0,01 & 2 \\
\hline Necrophorus humator & 12-.001-.002-. & 1 & 6 & 1 & & & & & & & 8 & 0,05 & 8 \\
\hline Necrophorus vespilloides & 12-.001-.006-. & & & 3 & 12 & 3 & 5 & 37 & 9 & & 69 & 0,42 & 22 \\
\hline Thanatophilus sinuatus & 12-.003-.002-. & & 1 & & & & & & & & 1 & 0,01 & 1 \\
\hline Oeceoptoma thoracica & 12-.004-.001-. & & & & 1 & & & 2 & & & 3 & 0,02 & 3 \\
\hline Xylodrepa quadrimaculata & 12-.006-.001-. & & 1 & & & & & & & & 1 & 0,01 & 1 \\
\hline Nargus velox & 14-.005-.001-. & & & & & & & & 1 & & 1 & 0,01 & 1 \\
\hline Nargus wilkini & 14-.005-.003-. & & 2 & & & & & & 1 & & 3 & 0,02 & 3 \\
\hline Sciodrepoides watsoni & 14-.010-.001-. & & 1 & 4 & 4 & 5 & 2 & 2 & & & 18 & 0,11 & 15 \\
\hline Sciodrepoides fumatus & 14-.010-.002-. & & & & & & & 1 & & & 1 & 0,01 & 1 \\
\hline Catops tristis & 14-.011-.007-. & 2 & 2 & & & 1 & & 1 & & & 6 & 0,04 & 5 \\
\hline Catops nigrita & 14-.011-.012-. & & & & & & 1 & & & & 1 & 0,01 & 1 \\
\hline Catops fuscus & 14-.011-.016-. & & & & 1 & & & & & & 1 & 0,01 & 1 \\
\hline Catops fuliginosus & 14-.011-.017-. & & 1 & & & & & & & & 1 & 0,01 & 1 \\
\hline Colon spec. & 15-.001-.000-. & & & & & 1 & & & & & 1 & 0,01 & 1 \\
\hline Leiodes oblonga & 16-.003-.0131. & & & & & & & & 2 & 2 & 4 & 0,02 & 3 \\
\hline Leiodes polita & 16-.003-.020-. & & & & & & 1 & & & & 1 & 0,01 & 1 \\
\hline Colenis immunda & 16-.004-.001-. & & & & & 1 & 1 & & & & 2 & 0,01 & 2 \\
\hline Anisotoma humeralis & 16-.007-.001-. & & 26 & 37 & 32 & 29 & 21 & 8 & 8 & & 161 & 0,98 & 52 \\
\hline Anisotoma castanea & 16-.007-.003-. & & & 1 & 4 & 3 & 2 & 1 & & & 11 & 0,07 & 8 \\
\hline Anisotoma orbicularis & 16-.007-.005-. & & & 4 & 1 & 3 & 1 & & & & 9 & 0,06 & 8 \\
\hline Agathidium varians & 16-.011-.003-. & 1 & 11 & 56 & 16 & 30 & 60 & 56 & 42 & 11 & 283 & 1,73 & 55 \\
\hline Agathidium rotundatum & 16-.011-.007-. & & & 9 & 6 & 8 & 9 & 1 & & & 33 & 0,20 & 16 \\
\hline Agathidium confusum & 16-.011-.008-. & & & 61 & 51 & 22 & 15 & 6 & 4 & 1 & 160 & 0,98 & 38 \\
\hline Agathidium nigripenne & 16-.011-.013-. & 3 & 8 & 34 & 21 & 23 & 16 & 5 & 1 & 2 & 113 & 0,69 & 47 \\
\hline Agathidium atrum & 16-.011-.014-. & 1 & 1 & & & & & & & & 2 & 0,01 & 2 \\
\hline Agathidium seminulum & 16-.011-.015-. & & 9 & 46 & 36 & 17 & 11 & 1 & & & 120 & 0,73 & 46 \\
\hline Agathidium badium & 16-.011-.018-. & & 1 & 3 & & & & & & & 4 & 0,02 & 4 \\
\hline Euthiconus conicicollis & 18-.001-.001-. & & & & 2 & & & & & & 2 & 0,01 & 2 \\
\hline Neuraphes elongatulus & 18-.005-.001-. & & 5 & 2 & & & 2 & & & & 9 & 0,06 & 7 \\
\hline Stenichnus scutellaris & 18-.007-.003-. & & 3 & 2 & 2 & 2 & 2 & 1 & & & 12 & 0,07 & 10 \\
\hline Stenichnus godarti & 18-.007-.005-. & & & & & & & 1 & & & 1 & 0,01 & 1 \\
\hline Stenichnus collaris & 18-.007-.008-. & & & 4 & & 1 & & & & & 5 & 0,03 & 4 \\
\hline Microscydmus nanus & 18-.008-.001-. & & & & & 7 & 13 & 2 & & & 22 & 0,13 & 15 \\
\hline Microscydmus minimus & 18-.008-.002-. & & & & & 3 & 2 & & & & 5 & 0,03 & 4 \\
\hline Ptenidium gressneri & 21-.002-.001-. & & & & 4 & 1 & & & & & 5 & 0,03 & 4 \\
\hline Ptenidium laevigatum & 21-.002-.002-. & & & 1 & 1 & 1 & & & & & 3 & 0,02 & 2 \\
\hline Ptenidium intermedium & 21-.002-.004-. & & & 3 & 1 & 4 & 4 & & & & 12 & 0,07 & 8 \\
\hline Ptenidium nitidum & 21-.002-.014-. & & 1 & 2 & 2 & 3 & 1 & & 2 & & 11 & 0,07 & 10 \\
\hline Micridium halidaii & 21-.005-.003-. & & & & & 6 & 4 & & & & 10 & 0,06 & 8 \\
\hline Euryptilium saxonicum & 21-.007-.002-. & & & & & 1 & & & & & 1 & 0,01 & 1 \\
\hline Ptinella limbata & 21-.012-.002-. & & & 1 & & 34 & 171 & 112 & & & 318 & 1,94 & 36 \\
\hline Ptinella aptera & 21-.012-.004-. & & & & & 6 & 52 & 14 & & & 72 & 0,44 & 18 \\
\hline Pteryx suturalis & 21-.013-.001-. & & & & & 18 & 33 & 24 & & & 75 & 0,46 & 26 \\
\hline Acrotrichis grandicollis & 21-.019-.001-. & & & & & & & & 1 & & 1 & 0,01 & 1 \\
\hline Acrotrichis sericans & 21-.019-.005-. & & & & & & 1 & & & & 1 & 0,01 & 1 \\
\hline Acrotrichis pumila & 21-.019-.008-. & & 1 & & & & & & & & 1 & 0,01 & 1 \\
\hline Acrotrichis insularis & 21-.019-.012-. & & 1 & & & & & & & & 1 & 0,01 & 1 \\
\hline Acrotrichis intermedia & 21-.019-.015-. & 1 & 13 & 16 & 15 & 18 & 24 & 10 & 1 & & 98 & 0,60 & 33 \\
\hline Scaphidium quadrimaculatum & 22-.002-.001-. & & 5 & 1 & 1 & 2 & 1 & & & & 10 & 0,06 & 8 \\
\hline
\end{tabular}


Anhang 11: Flugphänologie der Arten (Fensterfallen)

\begin{tabular}{|c|c|c|c|c|c|c|c|c|c|c|c|c|c|}
\hline & & & & & a n & pe & od & & & & & & \\
\hline ART & EDV & $\frac{\varangle}{\Delta}$ & $\frac{m}{i}$ & $\mathbb{N}$ & $\dddot{m}$ & $\dot{Z}$ & $\stackrel{n}{0}$ & ஜ & $\hat{a}$ & $\stackrel{\infty}{a}$ & 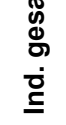 & $\begin{array}{l}\stackrel{0}{\circ} \\
\text { ْ̊ }\end{array}$ & $\begin{array}{l}\bar{\Xi} \\
\overline{\mathrm{d}} \\
\overline{\mathrm{d}}\end{array}$ \\
\hline Scaphisoma agaricinum & 22-.003-.001-. & & 14 & 15 & 28 & 16 & 18 & 7 & & & 98 & 0,60 & 43 \\
\hline Scaphisoma balcanicum & 22-.003-.007-. & & 2 & 2 & 4 & 4 & & & & & 12 & 0,07 & 9 \\
\hline Phloeocharis subtilissima & 23-.005-.001-. & & & 3 & 1 & & & & & 1 & 5 & 0,03 & 2 \\
\hline Megarthrus depressus & 23-.008-.001-. & & & 1 & & & 2 & & & & 3 & 0,02 & 3 \\
\hline Megarthrus sinuatocollis & 23-.008-.004-. & 1 & & & & 1 & & & & & 2 & 0,01 & 2 \\
\hline Proteinus brachypterus & 23-.009-.004-. & 1 & & & & & & & 1 & 9 & 11 & 0,07 & 10 \\
\hline Proteinus macropterus & 23-.009-.006-. & & & 1 & & & & & & & 1 & 0,01 & 1 \\
\hline Phyllodrepa ioptera & 23-.014-.012-. & & & & 2 & & & 10 & 17 & 4 & 33 & 0,20 & 11 \\
\hline Phyllodrepa gracilicornis & 23-.014-.015-. & & 1 & 1 & & & & & 2 & & 4 & 0,02 & 3 \\
\hline Omalium rivulare & 23-.015-.005-. & 1 & & & & & & & & & 1 & 0,01 & 1 \\
\hline Phloeonomus planus & 23-.016-.003-. & & 1 & 1 & & & 1 & & & & 3 & 0,02 & 3 \\
\hline Phloeonomus punctipennis & 23-.016-.006-. & & 11 & 1 & 1 & 4 & 1 & 2 & 1 & & 21 & 0,13 & 16 \\
\hline Xylodromus concinnus & 23-.017-.003-. & & 1 & & & & & & & & 1 & 0,01 & 1 \\
\hline Xylodromus testaceus & 23-.017-.004-. & & & 1 & & & & & & & 1 & 0,01 & 1 \\
\hline Phyllodrepoidea crenata & 23-.023-.001-. & & & & & & & 1 & 5 & 4 & 10 & 0,06 & 9 \\
\hline Lathrimaeum unicolor & 23-.025-.003-. & & & 1 & & & & & & & 1 & 0,01 & 1 \\
\hline Lesteva punctata & 23-.032-.001-. & & & & 1 & & & & & & 1 & 0,01 & 1 \\
\hline Lesteva longelytrata & 23-.032-.003-. & & 3 & 3 & & & & & & & 6 & 0,04 & 5 \\
\hline Syntomium aeneum & 23-.040-.001-. & & & & & 11 & 6 & & & & 17 & 0,10 & 11 \\
\hline Coprophilus striatulus & 23-.042-.001-. & 2 & 1 & 1 & & 1 & & & & & 5 & 0,03 & 5 \\
\hline Carpelimus corticinus & 23-.046-.017-. & & 1 & & & & & & 1 & & 2 & 0,01 & 2 \\
\hline Carpelimus elongatulus & 23-.046-.032-. & & 3 & & 1 & & 1 & & & & 5 & 0,03 & 5 \\
\hline Oxytelus laqueatus & 23-.048-.008-. & 1 & 1 & 1 & 1 & 1 & 1 & & 1 & & 7 & 0,04 & 6 \\
\hline Anotylus rugosus & 23-.0481.003-. & & 2 & 1 & & & & & & & 3 & 0,02 & 3 \\
\hline Anotylus mutator & 23-.0481.008-. & & & 1 & 1 & 3 & & 1 & & & 6 & 0,04 & 5 \\
\hline Anotylus tetracarinatus & 23-.0481.022-. & 5 & 2 & 2 & & 3 & & & & & 12 & 0,07 & 10 \\
\hline Platystethus arenarius & 23-.049-.001-. & & & & 2 & 1 & 1 & & & 2 & 6 & 0,04 & 5 \\
\hline Stenus fossulatus & 23-.055-.006-. & & 8 & 4 & 4 & & & & & & 16 & 0,10 & 13 \\
\hline Stenus canaliculatus & 23-.055-.041-. & & 1 & & & & & & & & 1 & 0,01 & 1 \\
\hline Stenus pusillus & 23-.055-.050-. & & 1 & & & & & & & & 1 & 0,01 & 1 \\
\hline Stenus fulvicornis & 23-.055-.070-. & & & & 1 & & & & & & 1 & 0,01 & 1 \\
\hline Stenus impressus & 23-.055-.094-. & & & & & & & & & 1 & 1 & 0,01 & 1 \\
\hline Rugilus rufipes & 23-.061-.003-. & & & 1 & & & & & & & 1 & 0,01 & 1 \\
\hline Lathrobium fulvipenne & 23-.068-.021-. & & & 1 & & & & & & & 1 & 0,01 & 1 \\
\hline Nudobius lentus & 23-.078-.001-. & & 2 & 1 & & & & & & & 3 & 0,02 & 3 \\
\hline Xantholinus linearis & 23-.080-.010-. & & & & & & & 1 & & & 1 & 0,01 & 1 \\
\hline Xantholinus longiventris & 23-.080-.015-. & 16 & 15 & & & & & 1 & 3 & 1 & 36 & 0,22 & 21 \\
\hline Atrecus affinis & 23-.081-.001-. & & & & & 1 & & & & & 1 & 0,01 & 1 \\
\hline Philonthus subuliformis & 23-.088-.006-. & & & & & & 1 & & & & 1 & 0,01 & 1 \\
\hline Philonthus laminatus & 23-.088-.020-. & 1 & 1 & & & & & & & & 2 & 0,01 & 2 \\
\hline Philonthus cognatus & 23-.088-.023-. & 33 & 21 & 9 & 2 & 1 & 3 & 19 & 18 & 16 & 122 & 0,75 & 29 \\
\hline Philonthus addendus & 23-.088-.027-. & & & & & 1 & & & & & 1 & 0,01 & 1 \\
\hline Philonthus decorus & 23-.088-.029-. & 1 & 1 & & & & & & & & 2 & 0,01 & 2 \\
\hline Philonthus rotundicollis & 23-.088-.033-. & & 1 & & & & & & & & 1 & 0,01 & 1 \\
\hline Philonthus carbonarius & 23-.088-.039-. & 7 & 9 & 7 & 2 & & 17 & 20 & 29 & 5 & 96 & 0,59 & 35 \\
\hline Philonthus cruentatus & 23-.088-.041-. & & & & & & & 1 & 1 & & 2 & 0,01 & 2 \\
\hline Philonthus varians & 23-.088-.044-. & & & & & & 1 & 2 & 1 & 1 & 5 & 0,03 & 5 \\
\hline Philonthus splendens & 23-.088-.046-. & & & & 1 & & & 1 & & & 2 & 0,01 & 2 \\
\hline Philonthus fimetarius & 23-.088-.047-. & & & & & 2 & & & & & 2 & 0,01 & 2 \\
\hline Philonthus quisquiliarius & 23-.088-.053-. & & & 1 & & & & & & & 1 & 0,01 & 1 \\
\hline Philonthus sanguinolentus & 23-.088-.058-. & & & 1 & & & & 2 & & & 3 & 0,02 & 3 \\
\hline Philonthus marginatus & 23-.088-.073-. & & & & & 1 & & & & 3 & 4 & 0,02 & 4 \\
\hline Gabrius splendidulus & 23-.090-.009-. & 1 & 60 & 31 & 13 & 8 & 5 & 1 & & & 119 & 0,73 & 46 \\
\hline Gabrius subnigritulus & 23-.090-.024-. & & & & & 2 & & & & & 2 & 0,01 & 2 \\
\hline Ontholestes murinus & 23-.092-.002-. & & 2 & & & 1 & & 1 & & & 4 & 0,02 & 4 \\
\hline Quedius infuscatus & 23-.104-.003-. & & & 1 & & & & & & & 1 & 0,01 & 1 \\
\hline
\end{tabular}




\begin{tabular}{|c|c|c|c|c|c|c|c|c|c|c|c|c|c|}
\hline & & & & & a n & per & od & & & & & & \\
\hline ART & EDV & $\frac{\varangle}{a}$ & $\frac{m}{a}$ & $\mathbf{\Sigma}$ & $\dddot{q}$ & $\stackrel{+}{a}$ & $\tilde{n}$ & ஜ & $\hat{a}$ & $\stackrel{\infty}{0}$ & 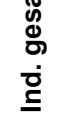 & 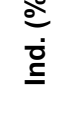 & 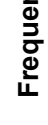 \\
\hline Quedius cruentus & 23-.104-.013-. & & & & & 2 & 3 & & & & 5 & 0,03 & 2 \\
\hline Quedius mesomelinus & 23-.104-.016-. & & 1 & 1 & & & & 2 & 2 & & 6 & 0,04 & 5 \\
\hline Quedius maurus & 23-.104-.018-. & & & 1 & & & & & & & 1 & 0,01 & 1 \\
\hline Quedius scitus & 23-.104-.020-. & & 5 & 1 & 1 & & & & & & 7 & 0,04 & 5 \\
\hline Quedius fuliginosus & 23-.104-.025-. & & 2 & & & & & & & & 2 & 0,01 & 2 \\
\hline Quedius tristis & 23-.104-.027-. & & & & & & & & & 1 & 1 & 0,01 & 1 \\
\hline Quedius fumatus & 23-.104-.048-. & 1 & 3 & 1 & & & & 1 & & & 6 & 0,04 & 5 \\
\hline Quedius lucidulus & 23-.104-.055-. & & & 1 & & & & & & & 1 & 0,01 & 1 \\
\hline Habrocerus capillaricornis & 23-.107-.001-. & & 2 & & & & & & & & 2 & 0,01 & 2 \\
\hline Trichophya pilicornis & 23-.108-.001-. & & 1 & 1 & & & & & & & 2 & 0,01 & 2 \\
\hline Mycetoporus lepidus & 23-.109-.008-. & 1 & 1 & & 3 & 9 & 3 & 1 & 1 & & 19 & 0,12 & 11 \\
\hline Mycetoporus niger & 23-.109-.021-. & 1 & & & & & & & & & 1 & 0,01 & 1 \\
\hline Lordithon thoracicus & 23-.111-.003-. & & & & & & 1 & & 1 & 1 & 3 & 0,02 & 3 \\
\hline Lordithon trinotatus & 23-.111-.006-. & & 1 & 1 & & & & 1 & 1 & 6 & 10 & 0,06 & 8 \\
\hline Lordithon lunulatus & 23-.111-.007-. & & & 4 & 3 & 1 & 1 & & & & 9 & 0,06 & 5 \\
\hline Bolitobius cingulata & 23-.112-.001-. & & 1 & & & & & & & & 1 & 0,01 & 1 \\
\hline Sepedophilus littoreus & 23-.113-.001-. & & 7 & 8 & 7 & 1 & & & & & 23 & 0,14 & 13 \\
\hline Sepedophilus testaceus & 23-.113-.002-. & & 4 & 2 & 2 & 1 & 1 & & & & 10 & 0,06 & 9 \\
\hline Tachyporus nitidulus & 23-.114-.001-. & & 1 & & & & & & & & 1 & 0,01 & 1 \\
\hline Tachyporus obtusus & 23-.114-.002-. & & & & & 1 & & & & & 1 & 0,01 & 1 \\
\hline Tachyporus hypnorum & 23-.114-.007-. & 1 & 1 & & & & 1 & & & 1 & 4 & 0,02 & 4 \\
\hline Tachyporus chrysomelinus & 23-.114-.008-. & & 1 & & & & & & & & 1 & 0,01 & 1 \\
\hline Tachyporus dispar & 23-.114-.0081. & 2 & 10 & 1 & & & 1 & & 3 & 2 & 19 & 0,12 & 13 \\
\hline Tachyporus transversalis & 23-.114-.013-. & & & & 1 & & & & & & 1 & 0,01 & 1 \\
\hline Tachyporus pusillus & 23-.114-.015-. & & 1 & & & & & & & & 1 & 0,01 & 1 \\
\hline Tachinus humeralis & 23-.117-.004-. & & & & & 1 & & & & & 1 & 0,01 & 1 \\
\hline Tachinus subterraneus & 23-.117-.006-. & & 1 & & & & & & & & 1 & 0,01 & 1 \\
\hline Tachinus pallipes & 23-.117-.010-. & & & 1 & & & & & & & 1 & 0,01 & 1 \\
\hline Tachinus signatus & 23-.117-.013-. & & 1 & & & & & 1 & & & 2 & 0,01 & 2 \\
\hline Tachinus laticollis & 23-.117-.014-. & & & & 1 & 3 & & & & & 4 & 0,02 & 4 \\
\hline Tachinus marginellus & 23-.117-.015-. & & & & & 1 & & & & & 1 & 0,01 & 1 \\
\hline Tachinus elongatus & 23-.117-.020-. & 1 & 3 & & & & & & & & 4 & 0,02 & 3 \\
\hline Oligota parva & 23-.126-.004-. & & & & & & 1 & & & & 1 & 0,01 & 1 \\
\hline Cypha longicornis & 23-.1262.001-. & & 1 & & & & & & & & 1 & 0,01 & 1 \\
\hline Cypha laeviuscula & 23-.1262.005-. & & & 1 & & & 1 & & & & 2 & 0,01 & 2 \\
\hline Gyrophaena affinis & 23-.130-.004-. & & & & & & 1 & & & & 1 & 0,01 & 1 \\
\hline Gyrophaena gentilis & 23-.130-.009-. & & & & 1 & & & & & & 1 & 0,01 & 1 \\
\hline Gyrophaena minima & 23-.130-.011-. & & & 1 & 2 & 6 & 13 & 7 & 14 & 8 & 51 & 0,31 & 25 \\
\hline Gyrophaena fasciata & 23-.130-.016-. & & & & & & & & 1 & & 1 & 0,01 & 1 \\
\hline Gyrophaena joyioides & 23-.130-.021-. & & & 4 & 3 & 7 & 26 & 6 & 1 & 1 & 48 & 0,29 & 20 \\
\hline Agaricochara latissima & 23-.1301.001-. & & 2 & 11 & & 1 & & 3 & 36 & 25 & 78 & 0,48 & 17 \\
\hline Cyphaea curtula & 23-.131-.001-. & & 1 & 1 & 1 & & & & & & 3 & 0,02 & 3 \\
\hline Placusa depressa & 23-.132-.002-. & & & 1 & & & & & & & 1 & 0,01 & 1 \\
\hline Placusa tachyporoides & 23-.132-.003-. & & & & & & 2 & & & & 2 & 0,01 & 1 \\
\hline Placusa pumilio & 23-.132-.006-. & & 2 & 1 & & 1 & 2 & & & & 6 & 0,04 & 4 \\
\hline Homalota plana & 23-.133-.001-. & & 1 & 4 & & 1 & 5 & 2 & & & 13 & 0,08 & 12 \\
\hline Anomognathus cuspidatus & 23-.134-.001-. & & 29 & 4 & 7 & 9 & 5 & 2 & 1 & & 57 & 0,35 & 25 \\
\hline Leptusa pulchella & 23-.141-.001-. & & 9 & 7 & 26 & 156 & 91 & 26 & & & 315 & 1,93 & 59 \\
\hline Leptusa fumida & 23-.141-.004-. & & 3 & 3 & 1 & 1 & 2 & 11 & 7 & & 28 & 0,17 & 15 \\
\hline Leptusa ruficollis & 23-.141-.006-. & & 4 & 1 & & 1 & & & & & 6 & 0,04 & 3 \\
\hline Euryusa castanoptera & 23-.142-.001-. & & 22 & 21 & 19 & 4 & 4 & 5 & & 1 & 76 & 0,46 & 33 \\
\hline Bolitochara obliqua & 23-.147-.001-. & 2 & 5 & 2 & & 1 & & & & & 10 & 0,06 & 9 \\
\hline Autalia longicornis & 23-.148-.002-. & & & & 1 & & & & & & 1 & 0,01 & 1 \\
\hline Autalia rivularis & 23-.148-.003-. & & 1 & & & & & 1 & & & 2 & 0,01 & 2 \\
\hline Ischnopoda atra & 23-.154-.006-. & & & & & & 1 & & & & 1 & 0,01 & 1 \\
\hline Aloconota gregaria & 23-.166-.014-. & & 3 & & & 1 & & & & & 4 & 0,02 & 4 \\
\hline
\end{tabular}


Anhang 11: Flugphänologie der Arten (Fensterfallen)

\begin{tabular}{|c|c|c|c|c|c|c|c|c|c|c|c|c|c|}
\hline & & & & & a n & pe & od & & & & & & \\
\hline ART & EDV & $\frac{\varangle}{\alpha}$ & $\frac{m}{\grave{\alpha}}$ & $\mathfrak{\Sigma}$ & $\dddot{q}$ & $\dot{Z}$ & $\stackrel{n}{\alpha}$ & ஜ & $\hat{a}$ & $\stackrel{\infty}{a}$ & 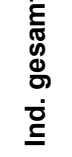 & $\begin{array}{l}\overparen{\varrho} \\
\stackrel{0}{\Xi}\end{array}$ & 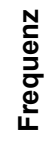 \\
\hline Aloconota languida & 23-.166-.015-. & & 1 & & & & & & & & 1 & 0,01 & 1 \\
\hline Amischa analis & 23-.168-.001-. & 5 & 38 & 20 & 4 & 9 & 5 & 13 & 10 & 6 & 110 & 0,67 & 45 \\
\hline Amischa cavifrons & 23-.168-.002-. & & & 1 & 1 & & & & & & 2 & 0,01 & 2 \\
\hline Amischa soror & 23-.168-.004-. & & 1 & 3 & 1 & & 2 & 1 & 2 & 1 & 11 & 0,07 & 8 \\
\hline Amischa decipiens & 23-.168-.007-. & & 3 & 4 & & 1 & 1 & 1 & 1 & & 11 & 0,07 & 10 \\
\hline Neohilara subterranea & 23-.173-.001-. & & & & & & 2 & & & & 2 & 0,01 & 2 \\
\hline Dinaraea aequata & 23-.182-.002-. & & 2 & & 1 & 1 & & & & & 4 & 0,02 & 4 \\
\hline Dinaraea linearis & 23-.182-.003-. & & & & 1 & & & 1 & & & 2 & 0,01 & 2 \\
\hline Dadobia immersa & 23-.184-.001-. & & 4 & 3 & 2 & & 3 & & & & 12 & 0,07 & 7 \\
\hline Plataraea brunnea & 23-.186-.005-. & & & 3 & & & & & & & 3 & 0,02 & 3 \\
\hline Atheta elongatula & 23-.188-.004-. & & & & & 1 & 1 & & & & 2 & 0,01 & 2 \\
\hline Atheta melanocera & 23-.188-.015-. & & & 1 & & & & & & & 1 & 0,01 & 1 \\
\hline Atheta palustris & 23-.188-.020-. & 1 & & & & & & 1 & & & 2 & 0,01 & 2 \\
\hline Atheta parca & 23-.188-.024-. & & & & 1 & & & & & & 1 & 0,01 & 1 \\
\hline Atheta deformis & 23-.188-.025-. & & & 1 & & & & & & & 1 & 0,01 & 1 \\
\hline Atheta monticola & 23-.188-.038-. & & & & 1 & & & & & & 1 & 0,01 & 1 \\
\hline Atheta nigricornis & 23-.188-.045-. & & 2 & 2 & & 1 & 4 & & & & 9 & 0,06 & 6 \\
\hline Atheta harwoodi & 23-.188-.046-. & & & & & & 9 & 1 & & & 10 & 0,06 & 8 \\
\hline Atheta palleola & 23-.188-.063-. & & 1 & & & & & & & & 1 & 0,01 & 1 \\
\hline Atheta benickiella & 23-.188-.064-. & & 7 & & 1 & 1 & & & & & 9 & 0,06 & 9 \\
\hline Atheta boreella & 23-.188-.092-. & & & 1 & & & & 1 & & & 2 & 0,01 & 2 \\
\hline Atheta sodalis & 23-.188-.109-. & & & 1 & 2 & 1 & & & & & 4 & 0,02 & 3 \\
\hline Atheta gagatina & 23-.188-.110-. & & 1 & & & & & & & & 1 & 0,01 & 1 \\
\hline Atheta orphana & 23-.188-.134-. & & & & & & 1 & & & & 1 & 0,01 & 1 \\
\hline Atheta fungi & 23-.188-.136-. & & 8 & 2 & 2 & 4 & 4 & 1 & & 3 & 24 & 0,15 & 18 \\
\hline Atheta negligens & 23-.188-.1361. & & 3 & 1 & 1 & & & & & & 5 & 0,03 & 5 \\
\hline Atheta amplicollis & 23-.188-.1362. & 3 & 8 & 4 & & 1 & & 1 & 3 & 5 & 25 & 0,15 & 18 \\
\hline Atheta dadopora & 23-.188-.155-. & & & & & & & 1 & & & 1 & 0,01 & 1 \\
\hline Atheta sordidula & 23-.188-.158-. & & & & & & & & & 1 & 1 & 0,01 & 1 \\
\hline Atheta castanoptera & 23-.188-.165-. & & & 1 & & & & & & & 1 & 0,01 & 1 \\
\hline Atheta laticollis & 23-.188-.179-. & & & & & & & 1 & & & 1 & 0,01 & 1 \\
\hline Atheta ravilla & 23-.188-.183-. & & & & 1 & & & & & & 1 & 0,01 & 1 \\
\hline Atheta pilicornis & 23-.188-.196-. & & 1 & & & & & & & & 1 & 0,01 & 1 \\
\hline Atheta britanniae & 23-.188-.198-. & & & & & 1 & & & 2 & & 3 & 0,02 & 3 \\
\hline Atheta crassicornis & 23-.188-.199-. & & & & & & 1 & & & & 1 & 0,01 & 1 \\
\hline Atheta macrocera & 23-.188-.202-. & & & & & & & & 1 & 1 & 2 & 0,01 & 2 \\
\hline Atheta nigripes & 23-.188-.208-. & & & & & & & 2 & 1 & 1 & 4 & 0,02 & 2 \\
\hline Atheta atramentaria & 23-.188-.210-. & & 1 & & 1 & & & & & & 2 & 0,01 & 2 \\
\hline Atheta longicornis & 23-.188-.223-. & & & & 1 & & & & & & 1 & 0,01 & 1 \\
\hline Acrotona aterrima & 23-.1881.011-. & & & & 1 & & & & & & 1 & 0,01 & 1 \\
\hline Phloeopora testacea & 23-.201-.004-. & & 24 & 3 & 1 & 2 & 3 & & & & 33 & 0,20 & 16 \\
\hline Phloeopora corticalis & 23-.201-.006-. & 2 & 24 & 4 & 1 & 5 & 4 & & & & 40 & 0,24 & 25 \\
\hline Calodera aethiops & 23-.204-.005-. & & 4 & 1 & 2 & & & & & & 7 & 0,04 & 6 \\
\hline Meotica marchica & 23-.213-.006-. & & & & 1 & & & & & & 1 & 0,01 & 1 \\
\hline Oxypoda procerula & 23-.223-.003-. & & 2 & & & & & & & & 2 & 0,01 & 2 \\
\hline Oxypoda umbrata & 23-.223-.018-. & 1 & & & & & & & 1 & & 2 & 0,01 & 2 \\
\hline Oxypoda arborea & 23-.223-.0331. & & & 1 & 3 & & & 2 & & & 6 & 0,04 & 5 \\
\hline Oxypoda alternans & 23-.223-.034-. & & & & & & & & & 2 & 2 & 0,01 & 2 \\
\hline Oxypoda brachyptera & 23-.223-.046-. & & & 1 & 1 & & & 1 & & & 3 & 0,02 & 3 \\
\hline Oxypoda recondita & 23-.223-.058-. & & 1 & & & & & & & & 1 & 0,01 & 1 \\
\hline Ischnoglossa prolixa & 23-.228-.001-. & & 1 & & & 1 & 1 & & & & 3 & 0,02 & 3 \\
\hline Haploglossa villosula & 23-.234-.002-. & & 7 & 2 & 2 & 2 & 1 & & & & 14 & 0,09 & 11 \\
\hline Tinotus morion & 23-.235-.001-. & & & 1 & & & & 2 & & & 3 & 0,02 & 3 \\
\hline Aleochara lanuginosa & 23-.237-.021-. & 1 & & & 2 & 3 & 2 & 1 & 1 & & 10 & 0,06 & 9 \\
\hline Bibloporus bicolor & 24-.002-.002-. & & 32 & 22 & 28 & 13 & 10 & 18 & 2 & 1 & 126 & 0,77 & 44 \\
\hline Bibloporus minutus & 24-.002-.003-. & & 10 & 3 & 5 & 4 & 1 & 7 & 1 & & 31 & 0,19 & 24 \\
\hline
\end{tabular}




\begin{tabular}{|c|c|c|c|c|c|c|c|c|c|c|c|c|c|}
\hline & & & & & an & $\overline{p e r}$ & $\overline{o d}$ & & & & & & \\
\hline ART & EDV & $\frac{\pi}{n}$ & $\frac{m}{i}$ & $\Sigma$ & $\dddot{q}$ & a & $\stackrel{\curvearrowleft}{\alpha}$ & $\dddot{0}$ & $\hat{a}$ & $\stackrel{\infty}{a}$ & 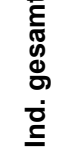 & $\begin{array}{l}\overparen{\varrho} \\
\stackrel{0}{\mathrm{~g}}\end{array}$ & 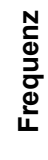 \\
\hline Euplectus nanus & 24-.006-.001-. & & & & & 1 & & 1 & & & 2 & 0,01 & 1 \\
\hline Euplectus piceus & 24-.006-.003-. & & 1 & 14 & 7 & 4 & 4 & 5 & & & 35 & 0,21 & 23 \\
\hline Euplectus bescidicus & 24-.006-.007-. & & 14 & 35 & 64 & 23 & 12 & 4 & & & 152 & 0,93 & 52 \\
\hline Euplectus punctatus & 24-.006-.013-. & & 16 & 22 & 14 & 10 & 9 & 4 & & & 75 & 0,46 & 35 \\
\hline Euplectus karsteni & 24-.006-.015-. & & & 1 & 1 & 4 & 1 & & & & 7 & 0,04 & 7 \\
\hline Plectophloeus nitidus & 24-.008-.006-. & & & & & & 3 & 1 & & & 4 & 0,02 & 4 \\
\hline Batrisodes venustus & 24-.015-.002-. & & & 1 & & & & & & & 1 & 0,01 & 1 \\
\hline Bythinus burrelli & 24-.017-.002-. & & & & 2 & & & & & & 2 & 0,01 & 1 \\
\hline Bryaxis puncticollis & 24-.018-.008-. & & & & 1 & & & & & & 1 & 0,01 & 1 \\
\hline Tychus niger & 24-.019-.001-. & 1 & 1 & 2 & 1 & 1 & 1 & 1 & 1 & & 9 & 0,06 & 8 \\
\hline Brachygluta haematica & 24-.021-.007-. & & & 2 & 1 & & & 1 & & & 4 & 0,02 & 3 \\
\hline Podabrus alpinus & 27-.001-.001-. & & & 3 & 5 & & & & & & 8 & 0,05 & 7 \\
\hline Cantharis pellucida & 27-.002-.008-. & & & 2 & 2 & 1 & & & & & 5 & 0,03 & 5 \\
\hline Cantharis nigricans & 27-.002-.018-. & & & & 7 & & & & & & 7 & 0,04 & 7 \\
\hline Cantharis decipiens & 27-.002-.025-. & & 2 & 15 & 5 & & & & & & 22 & 0,13 & 15 \\
\hline Rhagonycha fulva & 27-.005-.002-. & & & & & 3 & 4 & 8 & 1 & & 16 & 0,10 & 11 \\
\hline Rhagonycha lignosa & 27-.005-.008-. & & 1 & 11 & 13 & 2 & 1 & & & & 28 & 0,17 & 20 \\
\hline Rhagonycha gallica & 27-.005-.014-. & & & & 1 & 5 & & 1 & & & 7 & 0,04 & 7 \\
\hline Malthinus punctatus & 27-.008-.001-. & & & & & & & 1 & & & 1 & 0,01 & 1 \\
\hline Malthinus seriepunctatus & 27-.008-.002-. & & & & & 1 & 1 & 1 & & & 3 & 0,02 & 3 \\
\hline Malthodes guttifer & 27-.009-.015-. & & & & & & 2 & & & & 2 & 0,01 & 2 \\
\hline Malachius bipustulatus & 29-.006-.0032. & & & 1 & 1 & 2 & & & & & 4 & 0,02 & 4 \\
\hline Dasytes caeruleus & 30-.005-.005-. & & 5 & 1 & & & & & 1 & & 7 & 0,04 & 6 \\
\hline Dasytes plumbeus & 30-.005-.008-. & & & 1 & 1 & 7 & 4 & 3 & & & 16 & 0,10 & 11 \\
\hline Dasytes aerosus & 30-.005-.009-. & & 1 & 1 & 1 & & 1 & & & & 4 & 0,02 & 4 \\
\hline Phloiophilus edwardsi & 301.001-.001-. & 1 & & & & & & & & & 1 & 0,01 & 1 \\
\hline Thymalus limbatus & 321.009-.001-. & & & 1 & 1 & & & & & & 2 & 0,01 & 2 \\
\hline Hylecoetus dermestoides & 33-.001-.001-. & & 55 & 612 & 145 & 4 & & & & & 816 & 4,99 & 52 \\
\hline Lymeylon navale & 33-.002-.001-. & & & & & & 1 & & & & 1 & 0,01 & 1 \\
\hline Ampedus erythrogonus & 34-.001-.004-. & & 2 & 6 & 5 & 1 & & & & & 14 & 0,09 & 13 \\
\hline Ampedus brunnicornis & 34-.001-.013-. & & 1 & & & & & & & & 1 & 0,01 & 1 \\
\hline Ampedus nigerrimus & 34-.001-.014-. & & 2 & 11 & 3 & 2 & & & & & 18 & 0,11 & 17 \\
\hline Ampedus sanguineus & 34-.001-.015-. & & & & 1 & & & & & & 1 & 0,01 & 1 \\
\hline Ampedus pomorum & 34-.001-.019-. & & 2 & 2 & 4 & 5 & & & & & 13 & 0,08 & 10 \\
\hline Ampedus nigroflavus & 34-.001-.021-. & & & & 1 & & & & & & 1 & 0,01 & 1 \\
\hline Agriotes aterrimus & 34-.010-.001-. & & 78 & 106 & 24 & 2 & & & & & 210 & 1,28 & 42 \\
\hline Agriotes acuminatus & 34-.010-.003-. & & 31 & 91 & 55 & 4 & 2 & & & & 183 & 1,12 & 55 \\
\hline Agriotes lineatus & 34-.010-.009-. & & 1 & 1 & 2 & & & & & & 4 & 0,02 & 4 \\
\hline Adrastus pallens & 34-.015-.004-. & & & & & 2 & & & & & 2 & 0,01 & 2 \\
\hline Melanotus castanipes & 34-.016-.003-. & & 61 & 141 & 29 & 8 & & & & & 239 & 1,46 & 55 \\
\hline Ctenicera pectinicornis & 34-.022-.003-. & & & 1 & & & & & & & 1 & 0,01 & 1 \\
\hline Anostirus castaneus & 34-.026-.003-. & & 1 & & & 1 & & & & & 2 & 0,01 & 2 \\
\hline Calambus bipustulatus & 34-.030-.001-. & & & & 2 & & & & & & 2 & 0,01 & 2 \\
\hline Hypoganus inunctus & 34-.031-.001-. & & & 3 & 2 & & & & & & 5 & 0,03 & 4 \\
\hline Denticollis linearis & 34-.033-.004-. & & & 140 & 133 & & & & & & 273 & 1,67 & 53 \\
\hline Athous haemorrhoidalis & 34-.041-.001-. & & 22 & 170 & 69 & 11 & 1 & & & & 273 & 1,67 & 43 \\
\hline Athous vittatus & 34-.041-.002-. & & & 16 & 6 & 2 & & & & & 24 & 0,15 & 12 \\
\hline Athous subfuscus & 34-.041-.003-. & & 103 & 356 & 169 & 32 & 1 & & & & 661 & 4,04 & 65 \\
\hline Melasis buprestoides & 36-.001-.001-. & & 2 & 7 & 5 & & & & & & 14 & 0,09 & 13 \\
\hline Dirhagus lepidus & 36-.008-.004-. & & & & 1 & & 2 & & & & 3 & 0,02 & 3 \\
\hline Hylis olexai & 36-.011-.001-. & & & & & & 6 & 3 & & & 9 & 0,06 & 7 \\
\hline Xylophilus corticalis & 36-.012-.001-. & & & & & 2 & 3 & 1 & & & 6 & 0,04 & 6 \\
\hline Trixagus dermestoides & 37-.001-.002-. & & & 2 & 2 & 20 & 7 & & & & 31 & 0,19 & 20 \\
\hline Trixagus carinifrons & 37-.001-.003-. & & & & 1 & 1 & 1 & 1 & & & 4 & 0,02 & 4 \\
\hline Agrilus biguttatus & 38-.020-.003-. & & & & 1 & & & & & & 1 & 0,01 & 1 \\
\hline Clambus punctulum & 381.002-.002-. & & 1 & 2 & 1 & 1 & & & & & 5 & 0,03 & 5 \\
\hline
\end{tabular}


Anhang 11: Flugphänologie der Arten (Fensterfallen)

\begin{tabular}{|c|c|c|c|c|c|c|c|c|c|c|c|c|c|}
\hline & & & & & ang & pe & od & & & & & & \\
\hline ART & EDV & $\overleftarrow{a}$ & $\frac{m}{\alpha}$ & $\mathfrak{\Sigma}$ & $\dddot{a}$ & $\dot{Z}$ & $\stackrel{n}{0}$ & ஜ & $\hat{a}$ & $\stackrel{\infty}{0}$ & 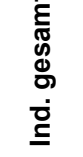 & $\begin{array}{l}\overparen{\varrho} \\
\stackrel{0}{\Xi}\end{array}$ & 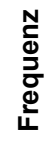 \\
\hline Clambus nigrellus & 381.002-.008-. & & & 2 & 1 & 2 & & & & & 5 & 0,03 & 3 \\
\hline Microcara testacea & 40-.002-.001-. & & & 1 & 1 & 1 & 1 & & & & 4 & 0,02 & 3 \\
\hline Cyphon coarctatus & 40-.003-.001-. & & & 2 & 7 & 2 & & 2 & & & 13 & 0,08 & 5 \\
\hline Cyphon palustris & 40-.003-.002-. & & & & 1 & & & & & & 1 & 0,01 & 1 \\
\hline Cyphon ochraceus & 40-.003-.006-. & & 1 & 2 & 3 & 3 & 3 & 1 & & & 13 & 0,08 & 10 \\
\hline Cyphon padi & 40-.003-.011-. & & 3 & 1 & & & & & & 1 & 5 & 0,03 & 4 \\
\hline Dryops ernesti & 42-.002-.002-. & & & & 1 & & & & & & 1 & 0,01 & 1 \\
\hline Cytilus sericeus & 47-.010-.001-. & & 1 & 2 & & & & & & & 3 & 0,02 & 3 \\
\hline Byturus tomentosus & 49-.001-.001-. & & 121 & 44 & 19 & 31 & 12 & 4 & & & 231 & 1,41 & 39 \\
\hline Byturus ochraceus & 49-.001-.002-. & & 1 & 16 & 12 & 1 & & & & & 30 & 0,18 & 21 \\
\hline Cerylon fagi & 492.002-.001-. & & & 5 & 5 & 2 & & & & & 12 & 0,07 & 10 \\
\hline Cerylon histeroides & 492.002-.002-. & & & 2 & 7 & 5 & 1 & 1 & & & 16 & 0,10 & 12 \\
\hline Cerylon ferrugineum & 492.002-.003-. & & 56 & 171 & 110 & 79 & 39 & 8 & & 1 & 464 & 2,84 & 63 \\
\hline Meligethes denticulatus & 50-.008-.003-. & 3 & 723 & 381 & 5 & 5 & 1 & 1 & 2 & & 1121 & 6,85 & 61 \\
\hline Meligethes aeneus & 50-.008-.014-. & 39 & 34 & 14 & 13 & 13 & 6 & 5 & & & 124 & 0,76 & 42 \\
\hline Meligethes sulcatus & 50-.008-.024-. & & 1 & & & & & & & & 1 & 0,01 & 1 \\
\hline Meligethes difficilis & 50-.008-.026-. & & & 1 & & & & & & & 1 & 0,01 & 1 \\
\hline Meligethes ochropus & 50-.008-.028-. & & & & & 1 & & & & & 1 & 0,01 & 1 \\
\hline Meligethes brunnicornis & 50-.008-.030-. & & & 1 & & 1 & 1 & & 2 & & 5 & 0,03 & 5 \\
\hline Meligethes viduatus & 50-.008-.034-. & & & 1 & & & & 1 & & & 2 & 0,01 & 2 \\
\hline Meligethes ovatus & 50-.008-.039-. & 1 & & & 1 & & & & & & 2 & 0,01 & 2 \\
\hline Meligethes nigrescens & 50-.008-.058-. & & & & 1 & & & & & & 1 & 0,01 & 1 \\
\hline Epuraea neglecta & 50-.009-.005-. & & 1 & 1 & 5 & & 1 & & & & 8 & 0,05 & 7 \\
\hline Epuraea marseuli & 50-.009-.015-. & & 5 & 6 & 1 & 1 & 4 & 1 & & & 18 & 0,11 & 16 \\
\hline Epuraea pygmaea & 50-.009-.016-. & & & 2 & & & & & & & 2 & 0,01 & 2 \\
\hline Epuraea longula & 50-.009-.017-. & & 1 & 4 & 2 & 2 & 2 & & 1 & & 12 & 0,07 & 9 \\
\hline Epuraea terminalis & 50-.009-.020-. & & 1 & & 1 & 1 & 1 & & & & 4 & 0,02 & 3 \\
\hline Epuraea unicolor & 50-.009-.027-. & & & 2 & & 1 & 2 & & & & 5 & 0,03 & 4 \\
\hline Epuraea variegata & 50-.009-.028-. & & 4 & 3 & & 1 & & & & & 8 & 0,05 & 7 \\
\hline Epuraea depressa & 50-.009-.033-. & & 6 & 5 & 3 & 4 & & & & & 18 & 0,11 & 14 \\
\hline Soronia grisea & 50-.013-.002-. & & & & 1 & & & & & & 1 & 0,01 & 1 \\
\hline Pocadius ferrugineus & 50-.015-.001-. & & 1 & & & & 1 & 1 & & & 3 & 0,02 & 3 \\
\hline Cychramus luteus & 50-.019-.002-. & & 1 & 2 & 50 & 79 & 14 & 4 & 7 & 82 & 239 & 1,46 & 52 \\
\hline Glischrochilus quadriguttatus & 50-.021-.001-. & 3 & 4 & 5 & 2 & & & 4 & 1 & & 19 & 0,12 & 13 \\
\hline Brachypterus urticae & 501.003-.001-. & & & & 1 & & & & 1 & & 2 & 0,01 & 2 \\
\hline Rhizophagus depressus & 52-.001-.003-. & & 3 & 7 & & & & & & & 10 & 0,06 & 7 \\
\hline Rhizophagus ferrugineus & 52-.001-.004-. & & 12 & 11 & 1 & 3 & 4 & & & & 31 & 0,19 & 21 \\
\hline Rhizophagus perforatus & 52-.001-.006-. & & 27 & 65 & 21 & 4 & & & & & 117 & 0,72 & 36 \\
\hline Rhizophagus dispar & 52-.001-.008-. & & 1 & 29 & 125 & 49 & 42 & 6 & & & 252 & 1,54 & 47 \\
\hline Rhizophagus bipustulatus & 52-.001-.009-. & 42 & 169 & 65 & 37 & 23 & 15 & & & 1 & 352 & 2,15 & 60 \\
\hline Rhizophagus nitidulus & 52-.001-.010-. & & 6 & 43 & 58 & 12 & 1 & 5 & & & 125 & 0,76 & 37 \\
\hline Rhizophagus cribratus & 52-.001-.013-. & & 1 & 9 & 6 & 4 & 2 & 1 & & & 23 & 0,14 & 15 \\
\hline Monotoma picipes & 53-.001-.005-. & & & & & & 1 & & & & 1 & 0,01 & 1 \\
\hline Monotoma longicollis & 53-.001-.009-. & & & & & 1 & & & & & 1 & 0,01 & 1 \\
\hline Uleiota planata & 53-.012-.001-. & & 2 & & & & & & & & 2 & 0,01 & 2 \\
\hline Pediacus dermestoides & 53-.015-.002-. & 2 & 29 & 31 & 15 & 12 & 3 & & 1 & & 93 & 0,57 & 44 \\
\hline Silvanus bidentatus & 531.006-.001-. & & & 4 & 2 & 2 & 2 & & & & 10 & 0,06 & 8 \\
\hline Tritoma bipustulata & 54-.001-.001-. & & & 3 & 3 & & & 1 & & & 7 & 0,04 & 7 \\
\hline Triplax russica & 54-.002-.003-. & & 1 & 2 & & & 1 & & & & 4 & 0,02 & 3 \\
\hline Cryptophagus acutangulus & 55-.008-.007-. & 1 & & & & & & & & & 1 & 0,01 & 1 \\
\hline Cryptophagus lapponicus & 55-.008-.016-. & & & & 3 & 1 & & 3 & 2 & & 9 & 0,06 & 8 \\
\hline Cryptophagus pubescens & 55-.008-.019-. & & & & & 1 & & 2 & & & 3 & 0,02 & 2 \\
\hline Cryptophagus dentatus & 55-.008-.027-. & 1 & 67 & 52 & 16 & 37 & 22 & 19 & 4 & 1 & 219 & 1,34 & 58 \\
\hline Cryptophagus distinguendus & 55-.008-.030-. & & 1 & & & & & & & & 1 & 0,01 & 1 \\
\hline Cryptophagus scanicus & 55-.008-.034-. & & & & & & & & & 2 & 2 & 0,01 & 1 \\
\hline Cryptophagus scutellatus & 55-.008-.039-. & & 1 & & & & & & & & 1 & 0,01 & 1 \\
\hline
\end{tabular}




\begin{tabular}{|c|c|c|c|c|c|c|c|c|c|c|c|c|c|}
\hline & & & & & $\overline{a n}$ & $\overline{p e r}$ & $\overline{o d}$ & & & & & & \\
\hline ART & EDV & $\frac{\mathbb{a}}{a}$ & $\frac{m}{i}$ & $\mathfrak{\Sigma}$ & $\dddot{q}$ & I & $\stackrel{\curvearrowleft}{\alpha}$ & $\mathscr{Q}$ & $\hat{a}$ & $\stackrel{\infty}{a}$ & 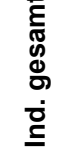 & $\begin{array}{l}\widehat{\varrho} \\
\stackrel{0}{\Xi}\end{array}$ & 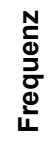 \\
\hline Cryptophagus lycoperdi & 55-.008-.040-. & & 3 & & & & & 4 & & & 7 & 0,04 & 7 \\
\hline Cryptophagus pilosus & 55-.008-.042-. & 2 & 8 & 4 & 3 & 1 & 4 & 2 & & 1 & 25 & 0,15 & 13 \\
\hline Antherophagus nigricornis & 55-.011-.001-. & & & & 2 & 17 & 24 & 9 & 3 & & 55 & 0,34 & 30 \\
\hline Antherophagus pallens & 55-.011-.003-. & & & & & 2 & & & & & 2 & 0,01 & 2 \\
\hline Caenoscelis subdeplanata & 55-.012-.001-. & & 1 & 2 & 1 & & & & & & 4 & 0,02 & 4 \\
\hline Atomaria impressa & 55-.014-.001-. & & 7 & 1 & & & & & & & 8 & 0,05 & 5 \\
\hline Atomaria ornata & 55-.014-.006-. & 1 & 5 & 6 & 2 & & & & & & 14 & 0,09 & 10 \\
\hline Atomaria fuscata & 55-.014-.014-. & & 2 & & 1 & 1 & 3 & 4 & & & 11 & 0,07 & 8 \\
\hline Atomaria lewisi & 55-.014-.016-. & & 5 & & & & & 2 & & & 7 & 0,04 & 5 \\
\hline Atomaria basalis & 55-.014-.021-. & & 1 & & 1 & & & 1 & & & 3 & 0,02 & 3 \\
\hline Atomaria rubella & 55-.014-.024-. & & & & & & 1 & & & & 1 & 0,01 & 1 \\
\hline Atomaria atricapilla & 55-.014-.025-. & & 1 & & 1 & & & & & & 2 & 0,01 & 2 \\
\hline Atomaria analis & 55-.014-.028-. & & 1 & 1 & & & & & & & 2 & 0,01 & 2 \\
\hline Atomaria nitidula & 55-.014-.0281. & & & 1 & & & & & & & 1 & 0,01 & 1 \\
\hline Atomaria turgida & 55-.014-.033-. & & 24 & 14 & 14 & & 3 & & & & 55 & 0,34 & 23 \\
\hline Atomaria testacea & 55-.014-.036-. & & 6 & 7 & 3 & 2 & & 2 & 1 & & 21 & 0,13 & 18 \\
\hline Atomaria puncticollis & 55-.014-.044-. & & 1 & & & & & & & & 1 & 0,01 & 1 \\
\hline Atomaria nigrirostris & 55-.014-.045-. & 1 & 21 & 60 & 11 & 3 & 2 & & & & 98 & 0,60 & 31 \\
\hline Atomaria linearis & 55-.014-.046-. & & & & & & 1 & & & & 1 & 0,01 & 1 \\
\hline Atomaria pulchra & 55-.014-.051-. & & 2 & & & 1 & & & & & 3 & 0,02 & 3 \\
\hline Atomaria atrata & 55-.014-.052-. & & & & & & 2 & 1 & & & 3 & 0,02 & 3 \\
\hline Atomaria procerula & 55-.014-.053-. & 3 & & & & & & & & & 3 & 0,02 & 3 \\
\hline Atomaria bella & 55-.014-.054-. & & 3 & 13 & 8 & & & & & & 24 & 0,15 & 13 \\
\hline Cryptolestes duplicatus & 561.004-.001-. & & & 1 & & & & & & & 1 & 0,01 & 1 \\
\hline Latridius minutus & 58-.003-.0021. & & 2 & 1 & & & & & & & 3 & 0,02 & 3 \\
\hline Enicmus fungicola & 58-.004-.010-. & & 97 & 55 & 28 & 20 & 2 & 1 & & & 203 & 1,24 & 49 \\
\hline Enicmus rugosus & 58-.004-.012-. & & 83 & 18 & 21 & 77 & 80 & 31 & & & 310 & 1,90 & 59 \\
\hline Enicmus testaceus & 58-.004-.013-. & & 74 & 60 & 34 & 58 & 22 & 20 & 1 & & 269 & 1,64 & 53 \\
\hline Enicmus transversus & 58-.004-.014-. & 1 & 25 & 6 & 5 & 2 & 7 & 5 & 1 & & 52 & 0,32 & 35 \\
\hline Enicmus histrio & 58-.004-.015-. & & 1 & 1 & 1 & 1 & & & 1 & & 5 & 0,03 & 5 \\
\hline Dienerella elongata & 58-.0041.001-. & & & & 1 & & & & & & 1 & 0,01 & 1 \\
\hline Cartodere constricta & 58-.005-.0011. & & & & 1 & & & & & & 1 & 0,01 & 1 \\
\hline Stephostethus lardarius & 58-.0061.001-. & & & & & 1 & & & & & 1 & 0,01 & 1 \\
\hline Stephostethus angusticollis & 58-.0061.002-. & & & 14 & & 7 & 3 & 3 & & & 27 & 0,17 & 20 \\
\hline Stephostethus rugicollis & 58-.0061.007-. & 1 & & & & & & & & & 1 & 0,01 & 1 \\
\hline Aridius norvegicus & 58-.0063.0011. & & & & & & 1 & 1 & & & 2 & 0,01 & 2 \\
\hline Aridius nodifer & 58-.0063.003-. & 1 & 4 & 39 & 44 & 50 & 55 & 33 & 11 & 14 & 251 & 1,53 & 58 \\
\hline Corticaria punctulata & 58-.007-.002-. & & 1 & & & & & & & & 1 & 0,01 & 1 \\
\hline Corticaria linearis & 58-.007-.016-. & & 2 & & & & & & & & 2 & 0,01 & 2 \\
\hline Corticaria longicollis & 58-.007-.018-. & 1 & 1 & & & & & & & & 2 & 0,01 & 2 \\
\hline Corticaria elongata & 58-.007-.021-. & & & & & & 1 & & & & 1 & 0,01 & 1 \\
\hline Corticarina similata & 58-.008-.002-. & 1 & 7 & 91 & 11 & 17 & 1 & 2 & 1 & & 131 & 0,80 & 34 \\
\hline Corticarina fuscula & 58-.008-.005-. & 5 & 16 & 4 & 1 & 6 & 5 & 6 & & & 43 & 0,26 & 29 \\
\hline Cortinicara gibbosa & 58-.0081.001-. & 12 & 82 & 9 & 5 & 4 & 4 & 9 & 1 & 3 & 129 & 0,79 & 43 \\
\hline Triphyllus bicolor & 59-.002-.001-. & & & & & & & 2 & & & 2 & 0,01 & 2 \\
\hline Litargus connexus & 59-.003-.001-. & 1 & 3 & & & 1 & 2 & & & & 7 & 0,04 & 7 \\
\hline Mycetophagus piceus & 59-.004-.003-. & & 9 & 2 & 2 & 3 & & 1 & & & 17 & 0,10 & 14 \\
\hline Mycetophagus atomarius & 59-.004-.006-. & 1 & 12 & 5 & 4 & 2 & & & & & 24 & 0,15 & 19 \\
\hline Typhaea stercorea & 59-.005-.001-. & & & & & 2 & & & & & 2 & 0,01 & 2 \\
\hline Cicones variegatus & 60-.014-.001-. & & 4 & & & & & & & & 4 & 0,02 & 4 \\
\hline Bitoma crenata & 60-.016-.001-. & & 8 & 3 & 2 & & 1 & 1 & & & 15 & 0,09 & 7 \\
\hline Teredus cylindricus & 60-.020-.001-. & & & & 3 & 4 & 3 & 1 & & & 11 & 0,07 & 7 \\
\hline Orthoperus atomus & 601.008-.003-. & & & & & & 1 & & & & 1 & 0,01 & 1 \\
\hline Orthoperus mundus & 601.008-.004-. & & & 2 & & 3 & 1 & & & & 6 & 0,04 & 6 \\
\hline Adalia decempunctata & 62-.023-.002-. & 1 & & & & & & & & & 1 & 0,01 & 1 \\
\hline Coccinella septempunctata & 62-.025-.003-. & 3 & 10 & 2 & 2 & & 3 & 1 & 1 & & 22 & 0,13 & 14 \\
\hline
\end{tabular}


Anhang 11: Flugphänologie der Arten (Fensterfallen)

\begin{tabular}{|c|c|c|c|c|c|c|c|c|c|c|c|c|c|}
\hline & & & & & a n & pe & od & & & & & & \\
\hline ART & EDV & $\frac{\varangle}{\Delta}$ & $\frac{m}{i}$ & $\mathfrak{\Sigma}$ & $\dddot{q}$ & $\dot{a}$ & $\stackrel{n}{0}$ & ஜ & $\hat{a}$ & $\stackrel{\infty}{a}$ & 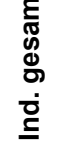 & 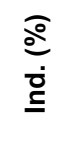 & 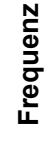 \\
\hline Propylea quatuordecimpunctata & 62-.032-.001-. & & 5 & 2 & & & 1 & 3 & 1 & & 12 & 0,07 & 11 \\
\hline Sphindus dubius & 63-.001-.001-. & & & & 1 & 2 & & & & & 3 & 0,02 & 2 \\
\hline Arpidiphorus orbiculatus & 63-.002-.001-. & & & 2 & 4 & 5 & 7 & 2 & & & 20 & 0,12 & 16 \\
\hline Octotemnus glabriculus & 65-.001-.001-. & & 2 & 16 & 4 & 8 & 6 & 8 & 1 & 1 & 46 & 0,28 & 12 \\
\hline Cis nitidus & 65-.006-.002-. & & & 4 & 5 & 9 & 3 & & & & 21 & 0,13 & 15 \\
\hline Cis glabratus & 65-.006-.004-. & & 1 & 2 & & 1 & & & & & 4 & 0,02 & 3 \\
\hline Cis hispidus & 65-.006-.007-. & & 3 & & & & & & & & 3 & 0,02 & 2 \\
\hline Cis boleti & 65-.006-.011-. & & 1 & 24 & 9 & 5 & 6 & 4 & 2 & & 51 & 0,31 & 26 \\
\hline Cis rugolosus & 65-.006-.0111. & & & & & 1 & & & & 1 & 2 & 0,01 & 2 \\
\hline Cis castaneus & 65-.006-.015-. & & & & 2 & & & & & & 2 & 0,01 & 1 \\
\hline Cis bidentatus & 65-.006-.017-. & & 3 & 4 & & & & & & & 7 & 0,04 & 5 \\
\hline Cis alni & 65-.006-.022-. & & 2 & 1 & 1 & & & & & & 4 & 0,02 & 4 \\
\hline Cis festivus & 65-.006-.028-. & & 15 & 22 & 37 & 5 & 3 & & 1 & & 83 & 0,51 & 29 \\
\hline Ennearthron cornutum & 65-.007-.002-. & & & & 1 & 1 & 1 & 1 & & & 4 & 0,02 & 4 \\
\hline Xestobium plumbeum & 68-.005-.001-. & & & 2 & & & & & & & 2 & 0,01 & 2 \\
\hline Xestobium rufovillosum & 68-.005-.002-. & & 1 & & & & & & & & 1 & 0,01 & 1 \\
\hline Anobium costatum & 68-.012-.005-. & & 12 & 7 & 1 & 2 & & & & & 22 & 0,13 & 13 \\
\hline Ptilinus pectinicornis & 68-.014-.001-. & & & & 8 & 67 & 20 & & & & 95 & 0,58 & 38 \\
\hline Dorcatoma flavicornis & 68-.022-.001-. & & & & & & & 1 & & & 1 & 0,01 & 1 \\
\hline Dorcatoma chrysomelina & 68-.022-.003-. & & & & & 4 & 4 & 1 & & & 9 & 0,06 & 7 \\
\hline Dorcatoma robusta & 68-.022-.007-. & & & 1 & 1 & & & & & & 2 & 0,01 & 1 \\
\hline Ptinus subpilosus & 69-.008-.013-. & & & 1 & & & & & & & 1 & 0,01 & 1 \\
\hline Lissodema quadripustulatum & 711.001-.002-. & & & & & & 1 & 1 & & & 2 & 0,01 & 2 \\
\hline Vincenzellus ruficollis & 711.005-.001-. & & 35 & 31 & 6 & 1 & & & & & 73 & 0,45 & 23 \\
\hline Rhinosimus planirostris & 711.006-.002-. & 1 & 14 & 25 & & 11 & 15 & 5 & & & 71 & 0,43 & 35 \\
\hline Rhinosimus ruficollis & 711.006-.003-. & 2 & 15 & 28 & 10 & 5 & 13 & 9 & 1 & & 83 & 0,51 & 40 \\
\hline Pyrochroa coccinea & 72-.001-.001-. & & & 8 & 2 & 2 & & & & & 12 & 0,07 & 5 \\
\hline Schizotus pectinicornis & 72-.002-.001-. & & 18 & 77 & 10 & & & & & & 105 & 0,64 & 22 \\
\hline Anaspis humeralis & 73-.004-.001-. & & 1 & 1 & & & 1 & & & & 3 & 0,02 & 3 \\
\hline Anaspis frontalis & 73-.004-.009-. & & & 7 & 15 & 2 & & & & & 24 & 0,15 & 9 \\
\hline Anaspis maculata & 73-.004-.010-. & & 1 & 7 & 10 & & & & & & 18 & 0,11 & 13 \\
\hline Anaspis schilskyana & 73-.004-.011-. & & & 1 & 3 & 2 & & & & & 6 & 0,04 & 6 \\
\hline Anaspis thoracica & 73-.004-.012-. & & & 7 & 5 & & & 1 & & & 13 & 0,08 & 9 \\
\hline Anaspis rufilabris & 73-.004-.019-. & & 2 & 3 & 5 & 2 & & & & & 12 & 0,07 & 7 \\
\hline Anaspis flava & 73-.004-.022-. & & & & & 1 & & & & & 1 & 0,01 & 1 \\
\hline Aderus oculatus & 74-.002-.007-. & & & & 2 & 2 & 6 & & & & 10 & 0,06 & 8 \\
\hline Omonadus floralis & 75-.0043.002-. & & & & & & & & 1 & & 1 & 0,01 & 1 \\
\hline Tomoxia biguttata & 79-.001-.001-. & & & & 6 & 7 & 2 & & & & 15 & 0,09 & 8 \\
\hline Mordellochroa abdominalis & 79-.012-.001-. & & & 1 & & & & & & & 1 & 0,01 & 1 \\
\hline Hallomenus binotatus & 80-.004-.001-. & & & & 1 & 1 & 1 & 3 & 1 & & 7 & 0,04 & 4 \\
\hline Orchesia undulata & 80-.005-.006-. & 1 & 9 & 4 & 3 & 3 & 3 & 7 & & & 30 & 0,18 & 19 \\
\hline Abdera affinis & 80-.007-.001-. & & & 1 & & & & & & & 1 & 0,01 & 1 \\
\hline Hypulus quercinus & 80-.013-.001-. & & 3 & 2 & 1 & & & & & & 6 & 0,04 & 6 \\
\hline Melandrya caraboides & 80-.016-.001-. & & & 1 & 14 & 2 & & & & & 17 & 0,10 & 11 \\
\hline Conopalpus testaceus & 80-.018-.001-. & & & & & 1 & & & & & 1 & 0,01 & 1 \\
\hline Tetratoma ancora & 801.001-.003-. & 3 & 2 & 4 & 1 & & & & & & 10 & 0,06 & 7 \\
\hline Pseudocistela ceramboides & 82-.005-.001-. & & & & 1 & & & & & & 1 & 0,01 & 1 \\
\hline Mycetochara linearis & 82-.008-.011-. & & & 3 & 7 & 1 & & & & & 11 & 0,07 & 9 \\
\hline Corticeus unicolor & 83-.023-.001-. & & 2 & 6 & 17 & & & & & & 25 & 0,15 & 14 \\
\hline Corticeus fasciatus & 83-.023-.008-. & & & & 1 & 1 & & & & & 2 & 0,01 & 2 \\
\hline Anoplotrupes stercorosus & 842.005-.001-. & & & & & 2 & & & & & 2 & 0,01 & 2 \\
\hline Onthophagus similis & 85-.014-.018-. & 1 & & & & & & & & & 1 & 0,01 & 1 \\
\hline Aphodius fossor & 85-.019-.004-. & & & 1 & 1 & & & & & & 2 & 0,01 & 2 \\
\hline Aphodius haemorrhoidalis & 85-.019-.005-. & & & & & & & & 1 & & 1 & 0,01 & 1 \\
\hline Aphodius rufipes & 85-.019-.012-. & & & & & 14 & 27 & 12 & 11 & 4 & 68 & 0,42 & 22 \\
\hline Aphodius maculatus & 85-.019-.022-. & & & & & & 3 & 2 & & & 5 & 0,03 & 3 \\
\hline
\end{tabular}




\begin{tabular}{|c|c|c|c|c|c|c|c|c|c|c|c|c|c|}
\hline & & & & & $\overline{a n ?}$ & $\overline{p e r}$ & $\overline{o d}$ & & & & & & \\
\hline ART & EDV & $\frac{\mathbb{a}}{a}$ & $\frac{m}{i}$ & $\mathfrak{\Sigma}$ & $\dddot{m}$ & I & $\stackrel{\curvearrowleft}{\alpha}$ & $\ddot{q}$ & $\hat{a}$ & $\stackrel{\infty}{a}$ & 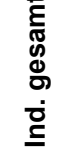 & $\begin{array}{l}\overparen{\varrho} \\
\stackrel{0}{\mathrm{~g}}\end{array}$ & 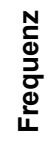 \\
\hline Aphodius pusillus & 85-.019-.024-. & & & 1 & & & & & & & 1 & 0,01 & 1 \\
\hline Aphodius contaminatus & 85-.019-.039-. & & & & & & & & & 16 & 16 & 0,10 & 12 \\
\hline Aphodius prodromus & 85-.019-.044-. & 42 & 98 & 7 & & & & & 1 & 3 & 151 & 0,92 & 36 \\
\hline Aphodius fimetarius & 85-.019-.060-. & 4 & 3 & 4 & 3 & & & & & & 14 & 0,09 & 11 \\
\hline Aphodius ater & 85-.019-.066-. & & 2 & 4 & & & & & & & 6 & 0,04 & 6 \\
\hline Serica brunnea & 85-.025-.001-. & & & & & 3 & 14 & 4 & & & 21 & 0,13 & 18 \\
\hline Melolontha melolontha & 85-.033-.002-. & & & 1 & & & & & & & 1 & 0,01 & 1 \\
\hline Phyllopertha horticola & 85-.037-.001-. & & & & 3 & & & & & & 3 & 0,02 & 2 \\
\hline Sinodendron cylindricum & 86-.005-.001-. & & & & 22 & 13 & 1 & & & & 36 & 0,22 & 23 \\
\hline Rhagium bifasciatum & 87-.011-.001-. & & 6 & 31 & 23 & 3 & & & & & 63 & 0,39 & 30 \\
\hline Rhagium mordax & 87-.011-.003-. & & 32 & 18 & 10 & 7 & 1 & & & & 68 & 0,42 & 29 \\
\hline Grammoptera ruficornis & 87-.023-.002-. & & & 1 & 1 & 1 & & & & & 3 & 0,02 & 3 \\
\hline Alosterna tabacicolor & 87-.024-.001-. & & & 2 & 6 & 3 & 1 & & & & 12 & 0,07 & 7 \\
\hline Judolia cerambyciformis & 87-.028-.002-. & & & & 4 & 4 & & & & & 8 & 0,05 & 6 \\
\hline Strangalia quadrifasciata & 87-.029-.006-. & & & & & & 2 & 2 & & & 4 & 0,02 & 2 \\
\hline Strangalia maculata & 87-.029-.007-. & & & & & & 1 & & & & 1 & 0,01 & 1 \\
\hline Strangalia aethiops & 87-.029-.009-. & & & & & 1 & & & & & 1 & 0,01 & 1 \\
\hline Strangalia melanura & 87-.029-.010-. & & & & 1 & 17 & 4 & 1 & & & 23 & 0,14 & 10 \\
\hline Pyrrhidium sanguineum & 87-.054-.001-. & & 1 & & & & & & & & 1 & 0,01 & 1 \\
\hline Clytus arietis & 87-.058-.003-. & & & & 3 & & & & & & 3 & 0,02 & 3 \\
\hline Pogonocherus hispidus & 87-.075-.002-. & & & & & 1 & & & & & 1 & 0,01 & 1 \\
\hline Leiopus nebulosus & 87-.078-.001-. & & & & 2 & & & & & & 2 & 0,01 & 2 \\
\hline Oulema gallaeciana & 88-.0061.003-. & & 2 & & & & & & & & 2 & 0,01 & 2 \\
\hline Oulema melanopus & 88-.0061.005-. & 5 & 2 & & & & 1 & & & & 8 & 0,05 & 6 \\
\hline Cryptocephalus pusillus & 88-.017-.071-. & & & & & 1 & & & & & 1 & 0,01 & 1 \\
\hline Gastrophysa polygoni & 88-.028-.001-. & & & & 2 & & & & & & 2 & 0,01 & 2 \\
\hline Gonioctena quinquepunctata & 88-.035-.011-. & & & 1 & & & & & & & 1 & 0,01 & 1 \\
\hline Phratora laticollis & 88-.036-.004-. & & & & & & 1 & & & & 1 & 0,01 & 1 \\
\hline Lochmaea capreae & 88-.042-.001-. & & 10 & & 1 & & 1 & & & & 12 & 0,07 & 7 \\
\hline Anthribus albinus & 90-.010-.001-. & & & & & & 1 & & & & 1 & 0,01 & 1 \\
\hline Scolytus intricatus & 91-.001-.003-. & & & 1 & 13 & 2 & 2 & & & & 18 & 0,11 & 8 \\
\hline Hylastes cunicularius & 91-.004-.003-. & & 5 & & & & & & & & 5 & 0,03 & 5 \\
\hline Dendroctonus micans & 91-.008-.001-. & & & & 1 & & & & & & 1 & 0,01 & 1 \\
\hline Crypturgus hispidulus & 91-.020-.002-. & & 2 & 1 & & & 1 & & & & 4 & 0,02 & 4 \\
\hline Dryocoetes autographus & 91-.024-.001-. & & 13 & 17 & 6 & 3 & 3 & & & & 42 & 0,26 & 23 \\
\hline Dryocoetes villosus & 91-.024-.002-. & & & 7 & 14 & 22 & 1 & 4 & & & 48 & 0,29 & 18 \\
\hline Dryocoetes alni & 91-.024-.003-. & & & & 1 & & & & & & 1 & 0,01 & 1 \\
\hline Cryphalus abietis & 91-.026-.004-. & 3 & 6 & 1 & & & & & & & 10 & 0,06 & 9 \\
\hline Ernoporicus fagi & 91-.027-.001-. & & 13 & 13 & 6 & 2 & 1 & & & & 35 & 0,21 & 16 \\
\hline Taphrorychus bicolor & 91-.031-.003-. & & 98 & 30 & 37 & 10 & 10 & 4 & 3 & & 192 & 1,17 & 39 \\
\hline Pityogenes chalcographus & 91-.032-.001-. & & & 1 & & & & & & & 1 & 0,01 & 1 \\
\hline Xyleborus dispar & 91-.036-.001-. & & 37 & 230 & 50 & 78 & 73 & 5 & & & 473 & 2,89 & 37 \\
\hline Xyleborus saxeseni & 91-.036-.004-. & & 142 & 14 & 3 & 17 & 12 & 1 & & & 189 & 1,16 & 34 \\
\hline Xyloterus domesticus & 91-.038-.001-. & 21 & 45 & 82 & 37 & 18 & 7 & 2 & & & 212 & 1,30 & 40 \\
\hline Xyloterus signatus & 91-.038-.002-. & 38 & 143 & 33 & 19 & 7 & 3 & 3 & & & 246 & 1,50 & 48 \\
\hline Lasiorhynchites sericeus & 923.003-.001-. & & 1 & & & & & & & & 1 & 0,01 & 1 \\
\hline Caenorhinus aequatus & 923.004-.005-. & & & 1 & & & 1 & & & & 2 & 0,01 & 1 \\
\hline Deporaus betulae & 923.007-.004-. & & 1 & 6 & 1 & 1 & & & & & 9 & 0,06 & 8 \\
\hline Phyllobius calcaratus & 93-.021-.015-. & & & 1 & 1 & & & & & & 2 & 0,01 & 2 \\
\hline Phyllobius argentatus & 93-.021-.019-. & & 6 & 34 & 27 & 16 & 1 & & & & 84 & 0,51 & 32 \\
\hline Polydrusus cervinus & 93-.027-.011-. & & & & 1 & 1 & & & & & 2 & 0,01 & 2 \\
\hline Polydrusus undatus & 93-.027-.016-. & & 12 & 11 & 18 & 10 & 2 & & & & 53 & 0,32 & 31 \\
\hline Strophosoma melanogrammum & 93-.040-.002-. & & & 1 & 1 & & 1 & 1 & & & 4 & 0,02 & 3 \\
\hline Strophosoma capitatum & 93-.040-.003-. & & & 3 & 1 & & & & & & 4 & 0,02 & 4 \\
\hline Sitona lepidus & 93-.044-.016-. & & & & & & 1 & & & & 1 & 0,01 & 1 \\
\hline Notaris acridulus & 93-.092-.004-. & & & & & 1 & & & & & 1 & 0,01 & 1 \\
\hline
\end{tabular}




\begin{tabular}{|c|c|c|c|c|c|c|c|c|c|c|c|c|c|}
\hline \multirow[b]{2}{*}{ ART } & \multirow[b]{2}{*}{ EDV } & \multicolumn{9}{|c|}{ Fangperiode } & \multirow[b]{2}{*}{ 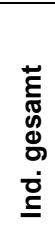 } & \multirow[b]{2}{*}{ 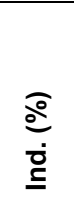 } & \multirow[b]{2}{*}{ 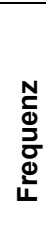 } \\
\hline & & $\frac{\nwarrow}{\alpha}$ & $\frac{m}{\alpha}$ & $\tilde{\Sigma}$ & $\dddot{q}$ & $a^{+}$ & $\stackrel{n}{n}$ & $\ddot{\square}$ & $\hat{a}$ & $\stackrel{\infty}{0}$ & & & \\
\hline Anthonomus rubi & 93-.106-.015-. & & & & & 1 & & & & & 1 & 0,01 & 1 \\
\hline Curculio pyrrhoceras & 93-.110-.011-. & & & 1 & 1 & 2 & 1 & & & & 5 & 0,03 & 4 \\
\hline Coeliodes erythroleucos & 93-.157-.008-. & 2 & 2 & & & & & & & & 4 & 0,02 & 4 \\
\hline Ceutorhynchus floralis & 93-.163-.0601. & & 2 & & & & & 1 & & & 3 & 0,02 & 3 \\
\hline Rhynchaenus fagi & 93-.180-.013-. & 8 & 31 & 11 & 4 & 4 & 5 & 2 & 1 & 1 & 67 & 0,41 & 30 \\
\hline Rhynchaenus salicis & 93-.180-.025-. & & & & & 1 & & & & & 1 & 0,01 & 1 \\
\hline
\end{tabular}

\section{Anhang 12: Liste aktualisierter Artnamen und EDV-Codes}

\begin{tabular}{|c|c|c|c|}
\hline EDV & ART & EDV neu & ART neu \\
\hline 01-.000-.000-. & Familie Carabidae & & \\
\hline 01-.029-.101-. & Bembidion mannerheimi Sahlb.,1827 & 01-.029-.101-. & Bembidion mannerheimii Sahlb.,1827 \\
\hline 01-.041-.047-. & Harpalus quadripunctatus Dej.,1829 & 01-.041-.047-. & Harpalus laevipes Zett.,1828 \\
\hline 01-.063-.002-. & Platynus assimilis (Payk.,1790) & 01-.0631.003-. & Limodromus assimilis (Payk.,1790) \\
\hline 01-.065-.021-. & Amara aenea (Geer, 1774) & 01-.065-.021-. & Amara aenea (DeGeer, 1774) \\
\hline 01-.079-.013-. & Dromius spilotus (III.,1798) & 01-.0791.001-. & Calodromius spilotus (III.,1798) \\
\hline 04-.000-.000-. & Familie Dytiscidae & & \\
\hline 04-.023-.003-. & Agabus chalconotus (Panz.,1796) & 04-.023-.003-. & Agabus chalconatus (Panz.,1796) \\
\hline 12-.000-.000-. & Familie Silphidae & & \\
\hline 12-.004-.001-. & Oeceoptoma thoracica (L.,1758) & 12-.004-.001-. & Oiceoptoma thoracica (L.,1758) \\
\hline 14-.000-.000-. & Familie Cholevidae & & \\
\hline 14-.011-.012-. & Catops nigrita Er.,1837 & 14-.0111.001-. & Apocatops nigritus Er.,1837 \\
\hline 22-.000-.000-. & Familie Scaphidiidae & & \\
\hline 22-.002-.001-. & Scaphidium quadrimaculatum OI.,1790 & 23-.0022.001-. & Scaphidium quadrimaculatum OI.,1790 \\
\hline 22-.003-.001-. & Scaphisoma agaricinum (L.,1758) & 23-.0023.001-. & Scaphisoma agaricinum (L.,1758) \\
\hline 22-.003-.007-. & Scaphisoma balcanicum Taman.,1954 & 23-.0023.007-. & Scaphisoma balcanicum Taman.,1954 \\
\hline 23-.000-.000-. & Familie Staphylinidae & & \\
\hline 23-.016-.001-. & Phloeonomus monilicornis (Gyll.,1810) & 23-.0161.001-. & Xylostiba monilicornis (Gyll.,1810) \\
\hline 23-.016-.003-. & Phloeonomus planus (Payk., 1792) & 23-.0162.001-. & Phloeostiba planus (Payk.,1792) \\
\hline 23-.017-.003-. & Xylodromus concinnus (Marsh.,1802) & 23-.017-.003-. & Xylodromus brunnipennis (Steph.,1834) \\
\hline 23-.025-.002-. & Lathrimaeum atrocephalum (Gyll.,1827) & 23-.025-.002-. & Anthobium atrocephalum (Gyll.,1827) \\
\hline 23-.025-.003-. & Lathrimaeum unicolor (Marsh.,1802) & 23-.025-.003-. & Anthobium unicolor (Marsh.,1802) \\
\hline 23-.032-.003-. & Lesteva longelytrata (Goeze,1777) & 23-.032-.003-. & Lesteva longoelytrata (Goeze,1777) \\
\hline 23-.046-.032-. & Carpelimus elongatulus Er.,1839 & 23-.046-.032-. & Carpelimus elongatulus (Er.,1839) \\
\hline 23-.061-.003-. & Rugilus rufipes Germ.,1836 & 23-.061-.003-. & Rugilus rufipes (Germ.,1836) \\
\hline 23-.1261.002-. & Holobus apicatus Er.,1837 & 23-.1261.002-. & Holobus apicatus (Er.,1837) \\
\hline 23-.131-.001-. & Cyphaea curtula (Er.,1837) & 23-.131-.001-. & Cyphea curtula (Er.,1837) \\
\hline 23-.154-.006-. & Ischnopoda atra (Grav.,1806) & 23-.1541.001-. & Thinonoma atra (Grav.,1806) \\
\hline 23-.166-.015-. & Aloconota languida (Er.,1837) & 23-.1651.001-. & Disopora languida (Er.,1837) \\
\hline 23-.168-.002-. & Amischa cavifrons (Shp.,1869) & 23-.168-.002-. & Amischa bifoveolata (Mannh.,1830) \\
\hline 23-.168-.004-. & Amischa soror $(\mathrm{Kr} ., 1856)$ & 23-.168-.004-. & Amischa nigrofusca (steph.,1832) \\
\hline 23-.223-.018-. & Oxypoda umbrata (Gyll.,1810) & 23-.223-.018-. & Oxypoda brevicornis (Steph.,1832) \\
\hline 30-.000-.000-. & Familie Melyridae & & \\
\hline 30-.005-.005-. & Dasytes caeruleus (Geer,1774) & 30-.005-.005-. & Dasytes cyaneus (F.,1775) \\
\hline 30-.005-.009-. & Dasytes aerosus Kiesw.,1867 & 30-.005-.009-. & Dasytes aeratus Steph.,1830 \\
\hline 301.000-.000-. & Familie Phloiophilidae & & \\
\hline 301.001-.001-. & Phloiophilus edwardsi Steph.,1830 & 301.001-.001-. & Phloiophilus edwardsii Steph.,1830 \\
\hline 321.000-.000-. & Familie Trogositidae & & \\
\hline 321.009-.001-. & Thymalus limbatus (F.,1787) & 322.004-.001-. & Thymalus limbatus (F.,1787) \\
\hline 34-.000-.000-. & Familie Elateridae & & \\
\hline 34-.010-.001-. & Agriotes aterrimus (L.,1761) & 34-.0101.001-. & Ectinus aterrimus (L.,1761) \\
\hline
\end{tabular}




\begin{tabular}{|c|c|c|c|}
\hline EDV & ART & EDV neu & ART neu \\
\hline $36-.000-.000-$. & Familie Eucnemidae & & \\
\hline 36-.011-.001-. & Hylis olexai $($ Palm, 1955) & 36-.011-.001-. & Hylis olexai Palm,1955 \\
\hline 49-.000-.000-. & Familie Byturidae & & \\
\hline 49-.001-.001-. & Byturus tomentosus (Geer, 1774) & 49-.001-.001-. & Byturus tomentosus (DeGeer,1774) \\
\hline $50-.000-.000-$ & Familie Nitidulidae & & \\
\hline 50-.008-.034-. & Meligethes viduatus (Heer,1841) & 50-.008-.034-. & Meligethes pedicularius (Gyll.,1808) \\
\hline 50-.009-.033-. & Epuraea depressa (III.,1798) & 50-.009-.033-. & Epuraea aestiva (L.,1758) \\
\hline 53-.000-.000-. & Familie Cucujidae & & \\
\hline 53-.001-.005-. & Monotoma picipes Hbst.,1793 & 52-.0001.005-. & Monotoma picipes Hbst.,1793 \\
\hline 53-.001-.009-. & Monotoma longicollis (Gyll.,1827) & 52-.0011.009-. & Monotoma longicollis (Gyll.,1827) \\
\hline 53-.012-.001-. & Uleiota planata (L.,1761) & 531.011-.001-. & Uleiota planata (L.,1761) \\
\hline 58-.000-.000-. & Familie Lathridiidae & & \\
\hline 58-.004-.015-. & Enicmus histrio Tomlin, 1910 & 58-.004-.015-. & Enicmus histrio Joy Tomlin, 1910 \\
\hline 58-.0061.001-. & Stephostethus lardarius (Deg.,1775) & 58-.0061.001-. & Stephostethus lardarius (DeGeer,1775) \\
\hline 58-.0063.0011. & Aridius norvegicus (Belon, 1887) & 58-.005-.0041-. & Cartodere norvegica (Strand, 1940) \\
\hline 58-.0063.003-. & Aridius nodifer (Westw.,1839) & 58-.005-.0031-. & Cartodere nodifer (Westw.,1839) \\
\hline $581.000-.000-$. & Familie Merophysiidae & & \\
\hline $581.001-.001-$. & Holoparamecus caularum Aube, 1843 & 61.0011-.001-. & Holoparamecus caularum (Aube,1843) \\
\hline 60-.000-.000-. & Familie Colydiidae & & \\
\hline 60-.020-.001-. & Teredus cylindricus (OI.,1790) & 491.002-.001-. & Teredus cylindricus (OI.,1790) \\
\hline 65-.000-.000-. & Familie Cisidae & & \\
\hline 65-.006-.022-. & Cis alni Gyll., 1813 & 65-.0061.001-. & Orthocis alni (Gyll.,1813) \\
\hline 65-.006-.024-. & Cis pygmaeus (Marsh.,1802) & 65-.0061.004-. & Orthocis pygmaeus (Marsh.,1802) \\
\hline 65-.006-.028-. & Cis festivus (Panz.,1793) & 65-.0061.008-. & Orthocis festivus (Panz.,1793) \\
\hline 68-.000-.000-. & Familie Anobiidae & & \\
\hline $\begin{array}{l}\text { 68-.005-.002-. } \\
711.000-.000-\end{array}$ & $\begin{array}{l}\text { Xestobium rufovillosum }(\text { Geer, } 1774) \\
\text { Familie Salpingidae }\end{array}$ & 68-.005-.002-. & Xestobium rufovillosum (DeGeer,1774) \\
\hline $711.001-.002-$. & Lissodema quadripustulatum (Marsh.,1802) & 711.001-.002-. & Lissodema denticolle (Gyll.,1813) \\
\hline 711.006-.002-. & Rhinosimus planirostris (F.,1787) & 711.006-.002-. & Salpingus planirostris (F.,1787) \\
\hline 711.006-.003-. & Rhinosimus ruficollis (L.,1761) & 711.006-.003-. & Salpingus ruficollis $(\mathrm{L} ., 1761)$ \\
\hline 73-.000-.000-. & Familie Scraptiidae & & \\
\hline 73-.004-.010-. & Anaspis maculata Fourcr.,1785 & 73-.004-.010-. & Anaspis maculata (Fourcr.,1785) \\
\hline 73-.004-.011-. & Anaspis schilskyana Csiki,1915 & 73-.004-.011-. & Anaspis marginicollis Lindberg, 1925 \\
\hline 74-.000-.000-. & Familie Aderidae & & \\
\hline 74-.002-.007-. & Aderus oculatus (Panz.,1796) & 74-.003-.002-. & Euglenes oculatus (Payk.) \\
\hline 79-.000-.000-. & Familie Mordellidae & & \\
\hline 79-.001-.001-. & Tomoxia biguttata (Gyll.,1827) & 79-.001-.001-. & Tomoxia bucephala Costa, 1854) \\
\hline 85-.000-.000-. & Familie Scarabaeidae & & \\
\hline 85-.019-.066-. & Aphodius ater (Geer,1774) & 85-.019-.066-. & Aphodius ater (DeGeer,1774) \\
\hline 85-.025-.001-. & Serica brunnea (L.,1758) & 85-.025-.001-. & Serica brunna (L.,1758) \\
\hline 87-.000-.000-. & Familie Cerambycidae & & \\
\hline 87-.011-.003-. & Rhagium mordax (Geer,1775) & 87-.011-.003-. & Rhagium mordax (DeGeer,1775) \\
\hline 87-.024-.001-. & Alosterna tabacicolor (Geer, 1775) & 87-.024-.001-. & Alosterna tabacicolor (DeGeer, 1775) \\
\hline 87-.028-.002-. & Judolia cerambyciformis (Schrk.,1781) & 87-.0281.001-. & Pachytodes cerambyciformis (Schrk.,1781) \\
\hline 87-.029-.006-. & Strangalia quadrifasciata $(\mathrm{L} ., 1758)$ & $87-.027-.0031$. & Leptura quadrifasciata $($ L., 1758$)$ \\
\hline 87-.029-.007-. & Strangalia maculata (Poda,1761) & $87-.027-.0041$ & Leptura maculata (Poda,1761) \\
\hline 87-.029-.009-. & Strangalia aethiops (Poda,1761) & $87-.027-.0061$ & Leptura aethiops (Poda,1761) \\
\hline 87-.029-.010-. & Strangalia melanura (L.,1758) & 87-.0293.001-. & Stenurella melanura (L.,1758) \\
\hline 88-.000-.000-. & Familie Chrysomelidae & & \\
\hline 88-.036-.004-. & Phratora laticollis Suffr., 1851 & 88-.036-.004-. & Phratora laticollis (Suffr.,1851) \\
\hline 88-.049-.005-. & Phyllotreta undulata Kutsch.,1860 & 88-.049-.005-. & Phyllotreta undulata (Kutsch.,1860) \\
\hline 93-.000-.000-. & Familie Curculionidae & & \\
\hline 93-.040-.003-. & Strophosoma capitatum $($ Geer, 1775$)$ & 93-.040-.003-. & Strophosoma capitatum (DeGeer,1775) \\
\hline 93-.180-.025-. & Rhynchaenus salicis (L.,1758) & 93-.1802.004-. & Tachyerges salicis $(\mathrm{L} ., 1758)$ \\
\hline
\end{tabular}




\section{Anhang 13: Rote-Liste-Deutschland (RLD)-Arten}

\begin{tabular}{|c|c|c|c|c|c|c|c|c|}
\hline \multirow[b]{2}{*}{ EDV } & \multirow[b]{2}{*}{ ART } & \multicolumn{3}{|c|}{ Individuen } & \multirow[b]{2}{*}{ KON } & \multirow[b]{2}{*}{ BIO } & \multirow[b]{2}{*}{ HAB } & \multirow[b]{2}{*}{ RLD } \\
\hline & & FF & Ekl. & Sum & & & & \\
\hline 01-.032-.001-. & Patrobus australis Sahlb.,1875 & & & 1 & $\mathrm{z}$ & $\mathrm{F}$ & $\mathrm{B}$ & 2 \\
\hline 01-.046-.010-. & Acupalpus exiguus (Dej.,1829) & 3 & & 3 & z & $\mathrm{F}$ & B & 3 \\
\hline 10-.002-.004-. & Plegaderus dissectus Er., 1839 & 15 & 263 & 278 & $\mathrm{z}$ & W & TM & 3 \\
\hline 10-.005-.001-. & Abraeus granulum Er., 1839 & 2 & 91 & 93 & $\mathrm{z}$ & W & TM & 3 \\
\hline 14-.002-.001-. & Nemadus colonoides (Kr.,1851) & & 1 & 1 & $\mathrm{~s}$ & W & $\mathrm{N}$ & 3 \\
\hline 16-.003-.0131. & Leiodes oblonga (Er.,1845) & 4 & & 4 & $\mathrm{~m}$ & W & $\mathrm{P}$ & 3 \\
\hline 16-.011-.010-. & Agathidium nigrinum Sturm,1807 & & 2 & 2 & $\mathrm{~m}$ & W & $\mathrm{P}$ & 3 \\
\hline 18-.001-.001-. & Euthiconus conicicollis (Fairm.Lab.,1855) & 2 & 7 & 9 & $z$ & W & TM & 1 \\
\hline 18-.002-.004-. & Euthia linearis Muls.,1861 & & 1 & 1 & $\mathrm{z}$ & $\mathrm{E}$ & $\mathrm{E}$ & 2 \\
\hline 18-.006-.004-. & Scydmoraphes minutus (Chaud.,1845) & & 15 & 15 & $\mathrm{z}$ & W & TM & 2 \\
\hline 18-.008-.002-. & Microscydmus minimus (Chaud.,1845) & 5 & 28 & 33 & $\mathrm{z}$ & W & TM & 3 \\
\hline 21-.002-.001-. & Ptenidium gressneri Er.,1845 & 5 & 1 & 6 & $\mathrm{~m}$ & W & TM & 3 \\
\hline 21-.005-.003-. & Micridium halidaii (Matth.,1868) & 10 & 342 & 352 & $\mathrm{~m}$ & W & TM & 3 \\
\hline 22-.003-.007-. & Scaphisoma balcanicum Taman.,1954 & 12 & 21 & 33 & $\mathrm{~m}$ & W & TP & 3 \\
\hline 23-.014-.001-. & Phyllodrepa melanocephala $(\mathrm{F} ., 1787)$ & & 3 & 3 & - & W & $\mathrm{N}$ & 3 \\
\hline 23-.014-.004-. & Phyllodrepa nigra (Grav.,1806) & & 2 & 2 & - & W & $\mathrm{N}$ & 3 \\
\hline 23-.014-.015-. & Phyllodrepa gracilicornis (Fairm.Lab.,1856) & 4 & 21 & 25 & - & W & TM & 2 \\
\hline 23-.017-.004-. & Xylodromus testaceus (Er.,1840) & 1 & 4 & 5 & - & W & $\mathrm{N}$ & 3 \\
\hline 23-.023-.001-. & Phyllodrepoidea crenata (Grav.,1802) & 10 & 13 & 23 & - & W & TR & 3 \\
\hline 23-.104-.003-. & Quedius infuscatus Er.,1840 & 1 & & 1 & $z$ & W & TM & 2 \\
\hline 23-.104-.014-. & Quedius brevicornis Thoms., 1860 & & 2 & 2 & $\mathrm{z}$ & W & TM & 3 \\
\hline 23-.117-.020-. & Tachinus elongatus Gyll.,1810 & 4 & & 4 & $z$ & $E$ & $\mathrm{~F}$ & 3 \\
\hline 23-.1261.002-. & Holobus apicatus Er.,1837 & & 10 & 10 & $z$ & W & TP & 3 \\
\hline 23-.1262.013-. & Cypha hanseni (Palm,1949) & & 4 & 4 & - & W & TM & 2 \\
\hline 23-.1301.001- & Agaricochara latissima (Steph.,1832) & 78 & 9 & 87 & $\mathrm{~m}$ & W & TP & 3 \\
\hline 23-.131-.001-. & Cyphaea curtula (Er.,1837) & 3 & & 3 & - & W & TR & 2 \\
\hline 23-.187-.007-. & Liogluta oblongiuscula (Shp.,1869) & & 2 & 2 & z & W & B & 3 \\
\hline 23-.188-.092-. & Atheta boreella Brundin, 1948 & 2 & & 2 & z & W & $\mathrm{F}$ & 3 \\
\hline 23-.194-.001-. & Thamiaraea cinnamomea (Grav.,1802) & & 21 & 21 & z & W & TS & 3 \\
\hline 23-.213-.006-. & Meotica marchica Benick,1954 & 1 & & 1 & $\mathrm{z}$ & - & $\mathrm{B}$ & 2 \\
\hline 23-.223-.0331. & Oxypoda arborea Zerche,1994 & 6 & 4 & 10 & $\mathrm{z}$ & W & TP & 1 \\
\hline 23-.223-.058-. & Oxypoda recondita Kr.,1856 & 1 & 13 & 14 & $\mathrm{z}$ & W & TM & 3 \\
\hline 24-.005-.006-. & Bibloplectus minutissimus (Aubé,1833) & & 1 & 1 & $\mathrm{z}$ & $\mathrm{F}$ & B & 3 \\
\hline 24-.006-.007-. & Euplectus bescidicus Rtt.,1881 & 152 & 64 & 216 & $z$ & W & TM & 2 \\
\hline 301.001-.001-. & Phloiophilus edwardsi Steph.,1830 & 1 & & 1 & z & W & $T$ & 2 \\
\hline 321.009-.001-. & Thymalus limbatus (F.,1787) & 2 & 3 & 5 & $\mathrm{~m}$ & W & TP & 3 \\
\hline 33-.002-.001-. & Lymexylon navale (L.,1758) & 1 & 132 & 133 & $\mathrm{x}$ & W & $T$ & 3 \\
\hline 34-.001-.004-. & Ampedus erythrogonus (Müll.,1821) & 14 & 30 & 44 & z & W & TM & 3 \\
\hline 34-.001-.013-. & Ampedus brunnicornis Germ.,1844 & 1 & 1 & 2 & z & W & TM & 1 \\
\hline 34-.001-.014-. & Ampedus nigerrimus (Lacord.,1835) & 18 & 15 & 33 & z & W & TM & 3 \\
\hline 34-.001-.021-. & Ampedus nigroflavus (Goeze,1777) & 1 & 2 & 3 & z & W & $T$ & 3 \\
\hline 34-.004-.001-. & Procraerus tibialis (Lacord.,1835) & & 13 & 13 & $\mathrm{x}$ & W & $\mathrm{T}$ & 2 \\
\hline 34-.031-.001-. & Hypoganus inunctus (Lacord.,1835) & 5 & 19 & 24 & $\mathrm{x}$ & W & $\mathrm{T}$ & 3 \\
\hline 34-.038-.002-. & Stenagostus rhombeus (Ol.,1790) & & 6 & 6 & $\mathrm{x}$ & W & TM & 3 \\
\hline 36-.003-.001-. & Eucnemis capucina Ahr.,1812 & & 3 & 3 & $\mathrm{x}$ & W & $T$ & 3 \\
\hline 36-.008-.004-. & Dirhagus lepidus (Rosh.,1847) & 3 & 82 & 85 & $\mathrm{x}$ & W & $T$ & 3 \\
\hline 36-.011-.001-. & Hylis olexai $(P a l m, 1955)$ & 9 & 41 & 50 & $\mathrm{x}$ & W & $T$ & 3 \\
\hline 36-.012-.001-. & Xylophilus corticalis (Payk.,1800) & 6 & 17 & 23 & $\mathrm{x}$ & W & $T$ & 2 \\
\hline 40-.004-.001-. & Prionocyphon serricornis (Müll.,1821) & & 3 & 3 & $\mathrm{~s}$ & W & $\mathrm{V}$ & 3 \\
\hline 53-.015-.002-. & Pediacus dermestoides (F.,1792) & 93 & 97 & 190 & z & W & TR & 1 \\
\hline
\end{tabular}




\begin{tabular}{|c|c|c|c|c|c|c|c|c|}
\hline \multirow[b]{2}{*}{ EDV } & \multirow[b]{2}{*}{ ART } & \multicolumn{3}{|c|}{ Individuen } & \multirow[b]{2}{*}{ KON } & \multirow[b]{2}{*}{ BIO } & \multirow[b]{2}{*}{ HAB } & \multirow[b]{2}{*}{ RLD } \\
\hline & & FF & Ekl. & Sum & & & & \\
\hline 55-.008-.016-. & Cryptophagus lapponicus Gyll.,1827 & 9 & & 9 & $\mathrm{~m}$ & W & $\mathrm{N}$ & 3 \\
\hline 55-.014-.001-. & Atomaria impressa Er.,1846 & 8 & & 8 & $\mathrm{~m}$ & $\mathrm{~F}$ & B & 3 \\
\hline 55-.014-.052-. & Atomaria atrata Rtt.,1875 & 3 & & 3 & $\mathrm{~m}$ & W & TP & 2 \\
\hline 55-.014-.053-. & Atomaria procerula Er.,1846 & 3 & & 3 & $\mathrm{~m}$ & W & TP & 3 \\
\hline 55-.014-.054-. & Atomaria bella Rtt.,1875 & 24 & 28 & 52 & $\mathrm{~m}$ & W & TP & 2 \\
\hline 58-.004-.013-. & Enicmus testaceus (Steph.,1830) & 269 & 55 & 324 & $\mathrm{~m}$ & W & TP & 2 \\
\hline 59-.002-.001-. & Triphyllus bicolor (F.,1792) & 2 & 3 & 5 & $\mathrm{~m}$ & W & TP & 3 \\
\hline 59-.004-.003-. & Mycetophagus piceus (F.,1792) & 17 & 6 & 23 & $\mathrm{~m}$ & W & TP & 3 \\
\hline 59-.004-.010-. & Mycetophagus populi F.,1798 & & 1 & 1 & $\mathrm{~m}$ & W & TP & 2 \\
\hline 60-.014-.001-. & Cicones variegatus (Hellw.,1792) & 4 & 155 & 159 & $\mathrm{z}$ & W & TP & 3 \\
\hline 60-.020-.001-. & Teredus cylindricus (OI.,1790) & 11 & 323 & 334 & $\mathrm{z}$ & W & $T$ & 1 \\
\hline 62-.0081.004-. & Nephus bipunctatus (Kug.,1794) & & 1 & 1 & $z$ & $\mathrm{O}$ & $\mathrm{V}$ & 3 \\
\hline 65-.005-.002-. & Sulcacis bidentulus (Rosh.,1847) & & 3 & 3 & $\mathrm{~m}$ & W & TP & 2 \\
\hline 65-.006-.004-. & Cis glabratus Mell.,1848 & 4 & 1 & 5 & $\mathrm{~m}$ & W & TP & 3 \\
\hline 65-.006-.024-. & Cis pygmaeus (Marsh.,1802) & & 1 & 1 & $\mathrm{~m}$ & W & TP & 3 \\
\hline 68-.022-.001-. & Dorcatoma flavicornis (F.,1792) & 1 & 2 & 3 & $\mathrm{x}$ & W & $T$ & 3 \\
\hline 68-.022-.003-. & Dorcatoma chrysomelina Sturm,1837 & 9 & 15 & 24 & $\mathrm{x}$ & W & $T$ & 3 \\
\hline 68-.022-.007-. & Dorcatoma robusta Strand,1938 & 2 & 1 & 3 & $\mathrm{~m}$ & W & TP & 2 \\
\hline 73-.004-.011-. & Anaspis schilskyana Csiki,1915 & 6 & 195 & 201 & $\mathrm{x}$ & W & $T$ & 2 \\
\hline 74-.002-.007-. & Aderus oculatus (Panz.,1796) & 10 & 563 & 573 & $\mathrm{x}$ & W & TM & 2 \\
\hline 80-.007-.001-. & Abdera affinis (Payk.,1799) & 1 & & 1 & $\mathrm{~m}$ & W & TP & 2 \\
\hline 80-.013-.001-. & Hypulus quercinus (Quensel,1790) & 6 & 24 & 30 & $\mathrm{x}$ & W & TM & 2 \\
\hline 80-.016-.001-. & Melandrya caraboides (L.,1761) & 17 & 4 & 21 & $\mathrm{x}$ & W & $\mathrm{T}$ & 3 \\
\hline 801.001-.002-. & Tetratoma desmaresti Latr.,1807 & & 2 & 2 & $\mathrm{~m}$ & W & TP & 1 \\
\hline 801.001-.003-. & Tetratoma ancora F.,1790 & 10 & 2 & 12 & $\mathrm{~m}$ & W & TP & 3 \\
\hline 82-.001-.002-. & Allecula morio (F.,1787) & & 1 & 1 & $\mathrm{x}$ & W & TM & 3 \\
\hline 82-.005-.001-. & Pseudocistela ceramboides (L.,1761) & 1 & 2 & 3 & $\mathrm{x}$ & W & TM & 2 \\
\hline 83-.023-.008-. & Corticeus fasciatus F., 1790 & 2 & 44 & 46 & $\mathrm{z}$ & W & $T$ & 2 \\
\hline 85-.019-.022-. & Aphodius maculatus Sturm, 1800 & 5 & & 5 & $\mathrm{~s}$ & W & $\mathrm{F}$ & 3 \\
\hline 85-.050-.002-. & Gnorimus variabilis (L., 1758) & & 18 & 18 & $\mathrm{x}$ & W & TM & 1 \\
\hline 86-.005-.001-. & Sinodendron cylindricum (L.,1758) & 36 & 125 & 161 & $\mathrm{x}$ & W & $T$ & 3 \\
\hline 87-.011-.002-. & Rhagium sycophanta (Schrk.,1781) & & 1 & 1 & $\mathrm{x}$ & W & TR & 3 \\
\hline 87-.071-.002-. & Mesosa nebulosa (F.,1781) & & 4 & 4 & $\mathrm{x}$ & W & $T$ & 3 \\
\hline 90-.015-.002-. & Choragus sheppardi Kirby,1818 & & 3 & 3 & $\mathrm{x}$ & W & TP & 3 \\
\hline 93-.019-.002-. & Caenopsis waltoni (Boh.,1843) & & 2 & 2 & $\mathrm{p}$ & 0 & $\mathrm{~B}$ & 2 \\
\hline
\end{tabular}


Anhang 13: Rote-Liste-Deutschland (RLD)-Arten 


\section{Abkürzungsverzeichnis}

\begin{tabular}{|c|c|}
\hline Abkürzung & Bedeutung \\
\hline $\mathrm{B}$ & Habitat: Bodenstreubewohner \\
\hline Bio & Biotop \\
\hline $\mathrm{Bu}$ & Buche \\
\hline $\mathrm{Bw}$ & Bohrwiderstand \\
\hline CA & Correspondence Analysis \\
\hline $\mathrm{CCA}$ & Canonical Correspondence Analysis \\
\hline DCA & Detrendet Correspondence Analysis \\
\hline DCCA & Detrendet Canonical Correspondence Analysis \\
\hline $\mathrm{E}$ & Habitat: eurytop \\
\hline EFl & Emergenzfalle an liegendem Totholz \\
\hline EFs & Emergenzfalle an stehendem Totholz \\
\hline $\mathrm{Ei}$ & Eiche \\
\hline $\mathrm{F}$ & Biotop: Feuchtbiotope; Habitat: Faulstoffbewohner \\
\hline Fam & Familie \\
\hline $\mathrm{Fe}$ & Feuchte \\
\hline FF & Fensterfalle \\
\hline $\mathrm{h}$ & hochliegendes Totholz \\
\hline $\mathrm{Hab}$ & Habitat \\
\hline Ind & Individuen \\
\hline Kon & Konsumenten \\
\hline 1 & liegendes Totholz mit Bodenkontakt \\
\hline $\mathrm{m}$ & Konsumententyp: mycetophag \\
\hline $\mathrm{mBE}$ & modifizierter Bodeneklektor \\
\hline $\mathrm{M}-\mathrm{Fl}$ & Mantelfläche \\
\hline $\mathrm{N}$ & Habitat: Nestbewohner \\
\hline $\mathrm{O}$ & Biotop: Offenlandbiotope \\
\hline $\mathrm{P}$ & Fangperiode (P1a-P8); Habitat: Pilzbewohner (o. TP) \\
\hline $\mathrm{p}$ & Konsumententyp: phytophag \\
\hline $\operatorname{Pr}$ & Probe \\
\hline $\mathrm{RE}$ & Renkonenzahl \\
\hline $\mathrm{RE}_{\mathrm{ln}}$ & Renkonenzahl nach Logarithmierung der Daten \\
\hline $\mathrm{Ri}$ & Rinde \\
\hline Ri-Fl & Rindenfläche \\
\hline $\mathrm{S}$ & Konsumententyp: saprophag (einschl. necro- und coprophag) \\
\hline Scha & Beschattung \\
\hline Schi & Überschirmung \\
\hline $\mathrm{Sq}$ & Saugfähigkeitsquotient \\
\hline SQ & Sørensenquotient \\
\hline STE & Stammeklektor \\
\hline $\mathrm{T}$ & Habitat: festes Holz (lignicol) \\
\hline TK & Totholzkäfer \\
\hline $\mathrm{TM}$ & Habitat: Holzmulm (xylodetricol) \\
\hline $\mathrm{TP}$ & Habitat: Totholzpilze (xylofungicol) \\
\hline TR & Habitat: Totholzrinde (corticol) \\
\hline TS & Habitat: Baumsaft (succicol) \\
\hline $\mathrm{V}$ & Habitat: Vegetationsbesiedler \\
\hline Vol & Volumen \\
\hline $\mathrm{W}$ & Biotop: Waldbiotope; Habitat: Wasserbewohner \\
\hline $\mathrm{x}$ & Konsumententyp: xylophag \\
\hline $\mathrm{z}$ & Konsumententyp: zoophag \\
\hline$\lambda$ & Eigenwert der Ordinationsachse \\
\hline
\end{tabular}




\section{Lebenslauf}

\section{Persönliche Daten:}

$\begin{array}{ll}\text { Name: } & \text { Menke } \\ \text { Vorname: } & \text { Norbert } \\ \text { Geburtsdatum: } & 10.10 .1961 \\ \text { Geburtsort: } & \text { Emstek } \\ \text { Familienstand: } & \text { Ledig }\end{array}$

\section{Schulausbildung:}

1968 - 72:

Grundschule Halen

$1972-82$ :

Clemens-August-Gymnasium Cloppenburg.

1982:

Abitur

\section{Wehr- und Zivildienst:}

1982 - 83: $\quad$ Wehrdienst; Zivildienst beim Deutschen Roten Kreuz und der Johanniter Unfallhilfe.

\section{Praktikum:}

1985 - 86: $\quad$ Fachhochschulpraktikum in der Niedersächsischen

Landesforstverwaltung, Forstamt Neuenburg.

\section{Hochschulausbildung:}

1986 - 91: $\quad$ Studium der Forstwissenschaft an der Georg-August-Universität Göttingen

Schwerpunkt: Biologie und Ökologie. Abschluß: Dipl.-Forstw.

\section{Weiterbildung:}

2002 - 03: $\quad$ Computergestützte Führungs- und Planungstechniken für

Naturwissenschaftler. Praktikum in einem Landschaftsplanungsbüro.

\section{Berufliche Tätigkeiten:}

$1983-84$ :

$1992-94:$

1995 - 97:

$1998-00:$

2000 - 05:

Seit 2005:
Transportbegleiter bei der Johanniter-Unfallhilfe in Ahlhorn.

Wissenschaftlicher Angestellter am Institut für Forstzoologie der Universität Göttingen.

Wissenschaftlicher Angestellter am Institut für Forstzoologie der Universität Göttingen.

Wissenschaftlicher Mitarbeiter bei der Stiftung Naturschutz Freistatt. Freiberufliche Gutachtertätigkeit und Mitarbeit in einem Planungsbüro. Wissenschaftlicher Angestellter an der Nordwestdeutschen Forstlichen Versuchsanstalt. 